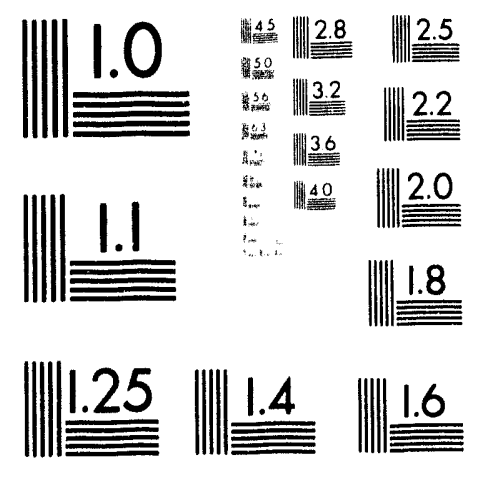



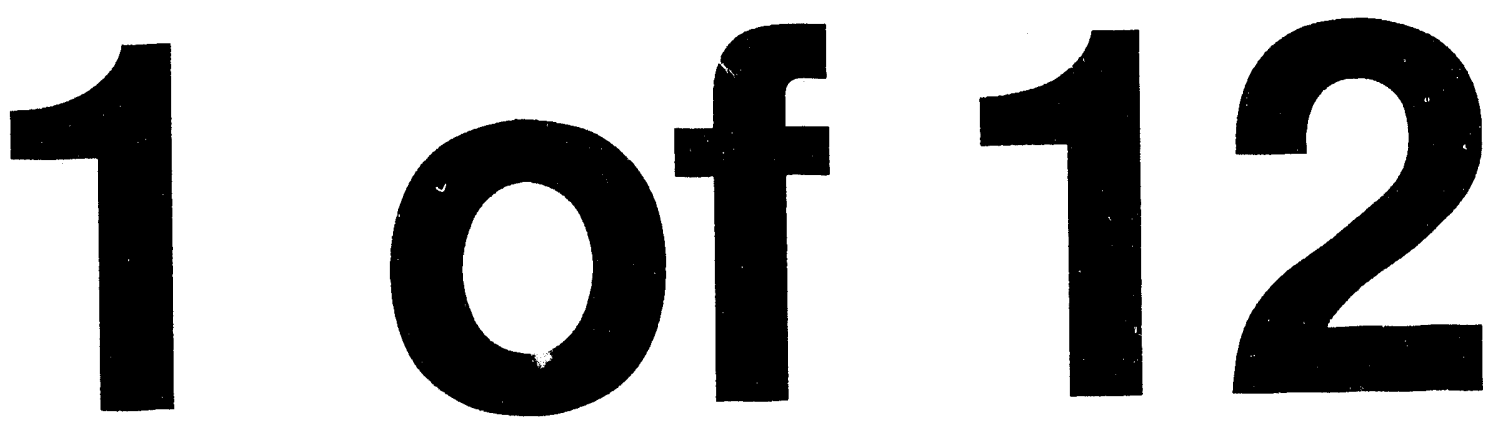


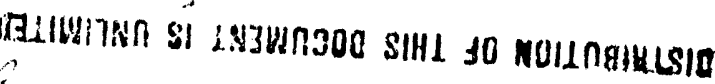

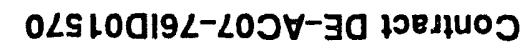

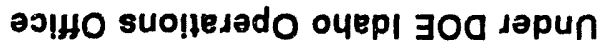

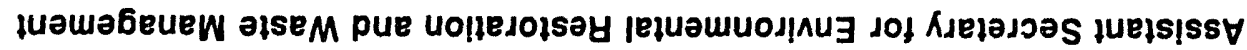

র́bsau jo juawuedəa 's'n

әul sol pasedadd

191949

SLtE8 OyepI 'sןleJ OuepI

'oul 'oyep $589 \exists$

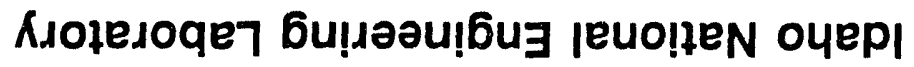

E66L Alnr pays!|qnd

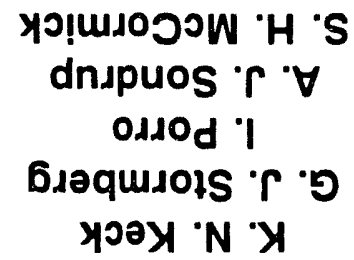

0 ×คว

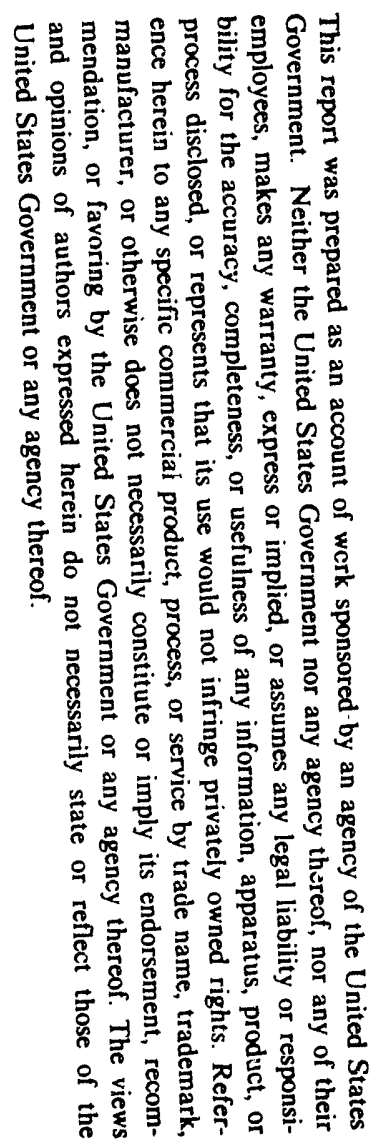

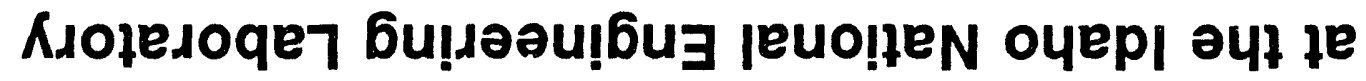

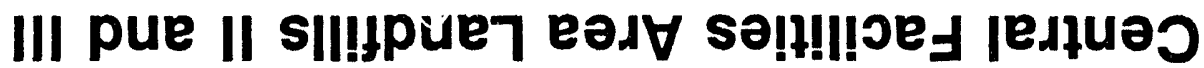

:ZL-t l!u

epuәpp $\forall$ pue ueld Y10M

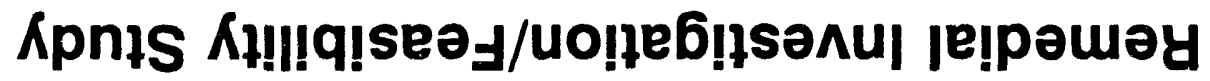

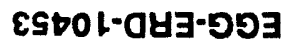




\section{Remedial Investigation/Feasibility Study Work Plan and Addenda for Operable Unit 4-12: \\ Central Facilities Area Landfills II and III at the Idaho National Engineering Laboratory}

July 1993

.3eviewed by:
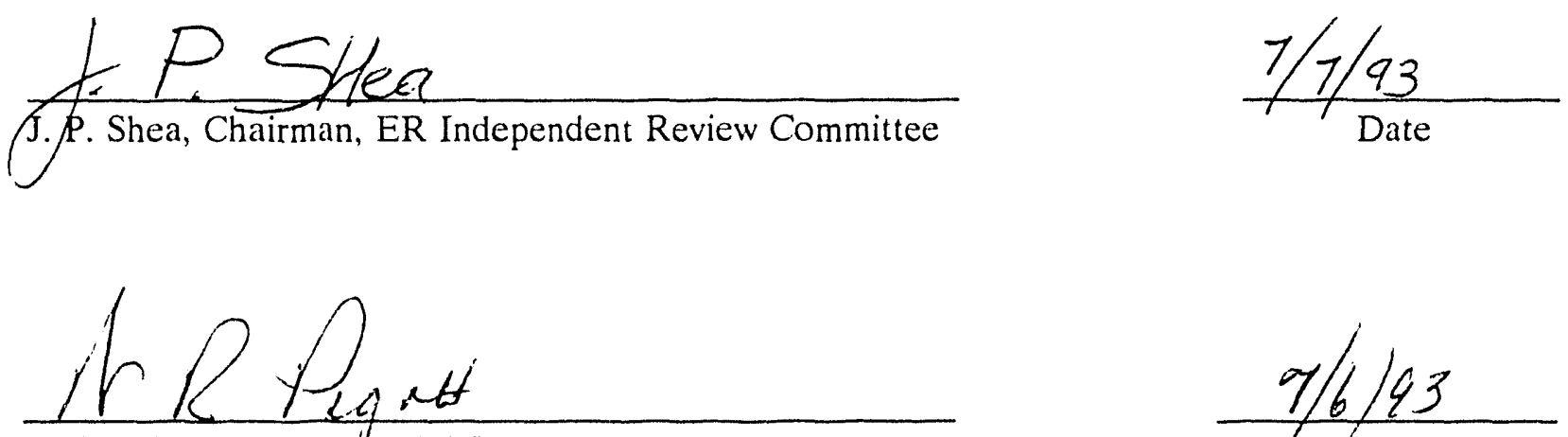

W. R. Pigott. Managdr. WAG 4

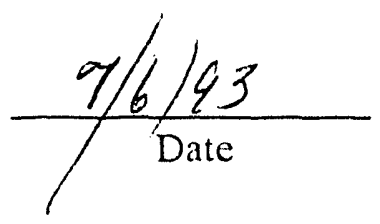

Approved by:

Ref Rom RPW

R. P. Wadkins, Unit Manage, WAGs 1, 4, and 5

$7 / 6 / 4.3$

Date 


\section{ABSTRACT}

This document is divided into two main sections-the Work Plan and the addenda. The Work Plan describes the regulatory history and physical setting of Operable Unit 4-12, previous sampling activities, and data. It also identifies a preliminary conceptual model, preliminary remedial action alternatives, and preliminary applicable or relevant and appropriate requirements. In addition, the Work Plan discusses data gaps and data quality objectives for proposed remedial investigation activities. Also included are tasks identified for the remedial investigation/feasibility study (RI/FS) and a schedule of RI/FS activities. The addenda include details of the proposed field activities (Field Sampling Plan), anticipated quality assurance activities (Quality Assurance Project Plan), policies and procedures to protect RI/FS workers and the environment during field investigations (Health and Safety Plan), and policies, procedures, and activities that the Department of Energy will use to involve the public in the decision-making process concerning CFA Landfills II and III RI/FS activities (Community Relations Plan). 


\section{PREFACE}

Central Facilities Area Landfills II and III at the Idaho National Engineering Laboratory (INEL) have been identified as Operable Unit 4-12 and are being investigated pursuant to the Comprehensive Environmental Response, Compensation, and Liability Act of 1980 (CERCLA); the Superfund Amendments and Reauthorization Act of 1986; the National Oil and Hazardous Substances Pollution Contingency Plan (40 CFR 300) (EPA 1990a); and applicable Department of Energy orders, directives, and policies. To assist in remedial investigation/feasibility study (RI/FS) planning, the following documents have been prepared.

Document Title

RI/FS Work Plan (seven sections)

\section{Purpose}

Describes operable unit based on existing knowledge. Identifies data necessary for risk assessment and remedial planning and identifies data quality objectives. Conceptualizes proposed RI/FS data collection activities.

RI Sampling and Analysis Plan (two parts):

Part I-Ficld Sampling Plan

Describes in detail proposed ficld activities to fill data gaps identified in the Work Plan.

Describes quality assurance protocols to be implemented in the field and laboratory during data collection, analysis, and reporting.

Describes activities to be implemented and protocols to be followed during RI/FS activities to protect the health and safety of $\mathrm{RI} / \mathrm{FS}$ workers.

Community Relations Plan

Describes INEL activities to be implemented to ensure compliance with public involvement requirements of CERCLA. 


\section{EXECUTIVE SUMMARY}

The remedial investigation/feasibility study (RI/FS) Work Plan for Operable Unit (OU) 4-12: Central Facilities Area (CFA) Landfills II and III at the Idaho National Engineering Laboratory (INEL) was prepared for the U.S. Department of Energy Idaho Field Office (DOE-ID). This document presents the initial evaluation of existing data and background information for the CFA landfills and defines the scope and objectives of the RI/FS activities proposed for implementation at the site. The Work Plan and addenda document the RI/FS scoping process, identify site investigations and feasibility studies, assign responsibilities for RI/FS activities, and present the RI/FS schedule.

Subsequent to listing the INEL on the National Priority List (NPL) and with the development of a Federal Facility Agreement/Consent Order, DOE, EPA, and the State of Idaho decided that CFA Landfills II and III should be remediated through the Comprehensive Environmental Response, Compensation, and Liability Act (CERCLA)-driven RI/FS process. CFA Landfills II and III are considered CERCLA landfills that are primarily composed of cafetcria, office, and construction waste, and to a lesser extent hazardous waste.

The INEL is located approximately $42 \mathrm{mi}(68 \mathrm{~km})$ west of Idaho Falls, Idaho, and occupies $890 \mathrm{mi}^{2}\left(2,305 \mathrm{~km}^{2}\right)$ of the northwestern portion of the Eastern Snake River Plain. The CFA landiflls are located in the south-central portion of the INEL, approximately $0.5 \mathrm{mi}(0.8 \mathrm{~km})$ north of CFA proper. CFA Landfill II, in use from 1970 until 1982, was a fill operation in the southwestern portion of an abandoned gravel pit. The perimeter of the landfill encompasses 14 acres (6 hectares) with a maximum depth of approximately $15 \mathrm{ft}(5 \mathrm{~m})$ in the southern end; the north end of the landfill is not quite as deep. At present, the landfill is covered with generally coarse soil materials and revegetated with crested wheatgrass, and is level with the surrounding ground surface.

Landfill III was opened in October of 1982 when operations at CFA Landfill II were terminated. In 1984. the cut and fill operation on the east was terminated and designated as the inactive portion of Landfill III. The operation was moved to the west where it is designated the active portion of the landfill and is currently operational but is not considered part of the OU being investigated under this RI/FS. The active portion of Landfill III will be investigated under appropriate regulatory programs for active landfills or under the final WAG 4 comprehensive RI/FS, OU 4-13. The dimensions of the inactive portion of Landilll III are $200 \mathrm{ft}(61 \mathrm{~m})$ wide by 2.40() $\mathrm{ft}(7.32 \mathrm{~m}) \mathrm{long}$ and $12 \mathrm{ft}(4 \mathrm{~m})$ deep. The individual trenches were cut $24 \mathrm{ft}(7 \mathrm{~m})$ wide, $12 \mathrm{lt}(4 \mathrm{~m})$ deep, and about $2,400 \mathrm{lt}(7.32 \mathrm{~m}) \mathrm{long}$. The waste was spread in the trenches and compacted into layers no more than $2 \mathrm{fl}(10.6 \mathrm{~m})$ thick, with cach layer covered with at least 6 in. $(15 \mathrm{~cm})$ of soil. A linal layer of cover material. compacted to a uniform depth of $2 \mathrm{ft}(0.6 \mathrm{~m})$, was placed over the entire surlace of each filled trench. The cover consists of coarse soil materials that have been reseeded with crested whealgrass.

Contaminants detected to date at the CFA landfills have included relatively low eoncentrations of organics in the shallow soil gas, organics including methane in soil gas collected at depth from boreholes, semivolatile and volatile organics in the soil, and chromium and tritium in the groundwater from both upgradient and downgradient wells. The souree of this groundwater contamination is most likely due to past waste dispossal practices at two upgradient 
facilities-the Idaho Chemical Processing Plant and the Test Reactor Area, not the CFA landfills. Additionally, the dedicated sampling pumps in the landfill monitoring wells, which are manufactured, in part, with high chromium stainless steel, are also considered a possible but perhaps minor contributing source of the particulate chromium. This hypothesis will be evaluated by removing the dedicated sampling pumps from both upgradient and downgradient wells and by collecting groundwater for metals analysis.

Containment has been identified as the most likely response action at these landfills because (a) : hese CERCLA landfills are primarily composed of cafeteria, office, and construction waste, anc to a lesser exteni hazardous wastes; and (b) the volume and heterogeneity of the waste within these landfills will make treatment impractical. Therefore, site characterization strategies have been limited to focus on the data required to evaluate containment and to determine if unacceptable risks exist to human health and the environment.

The specific objectives of the CFA Landfills II and III RI include:

- Perform a topographic survey of Landfill III to (a) identify topographic anomalies, (b) document erosional features, and (c) determine the adequacy of the existing landfill cover for controlling erosion from surface runon/runoff.

- Perform geophysical surveys to (a) determine the perimeter boundary of CFA Landfill III and the boundaries of the trenches within the landfill, (b) determine the depth and topography of underlying basalt (both landfills), (c) define areas containing high concentrations of metal (Landfill III), and (d) determine overburden cover thickness (Landfill III).

- Perform surface soil sampling of the Landfill II and III cover soils for volatile organic, semivolatile organic, and metai analyses to (a) determine the distribution and concentration of potential contamination in surface soils, (b) determine if the surficial soils should be included in source control actions for the landfills, and (c) characterize direct exposure risks.

- Perform subsurface soil sampling at Landfill II and, if present, free liquid/leachate sampling within the landfill for volatile orginic, semivolatile organic, and metals analyses to (a) determine the nature and concentration of leachable contaminants, (b) determine the nature and concentration of contaminants in any leachate or free liquid that may be present, (c) provide input for fate and transport modeling, and (d) reduce the uncertainty associated with available source term in mation. The need for subsurface soil sampling at Landfill III will be evaluated based .... the surface soil and soil gas sampling results from Landfill III.

- Perform a cover investigation at Landfill III to (a) determine the permeability of the existing cover, (b) determine the thickness of the cover, (c) evaluate potential for infiltration, and (d) evaluate the physical properties of the geologic unit governing the transport of contaminants. To accomplish these cover investigation objectives, soil will be collected from the landfill cover to measure porosity, capillary pressure/moisture content relationships, hydraulic conductivity, moisture content, and particle size. 
- Perform a soil gas survey at both landfills to (a) identify areas within the landfill containing high concentrations of explosive or toxic landfill gas to perform an assessment of human health risks due to air toxics and explosive hazards, (b) evaluate the feasibility of gas collection and treatment, (c) evaluate remedial actions, and (d) estimate concentrations of selected volatile organics being emitted to the atmosphere to characterize direct exposure risks. These objectives will be accomplished by collecting shallow soil gas samples, collecting soil gas samples from the existing instrumented boreholes, and collecting gas emissions from the landfill surface with a surface flux chamber and analyzing the samples for volatile organics.

- Perform a groundwater investigation to (a) determine the distribution and concentration of potential contaminants in the aquifer to characterize ingestion exposure risks, (b) determine groundwater flow direction and rate, (c) measure depth to water, and (d) correct measured depth to water for potential borehole deviation effects and confirm up and downgradient locations of existing monitor wells. These objectives will be accomplished by removing the existing pumps from the wells and collecting groundwater samples for volatile organics, metals, and common anions analyses, by taking water level measurements, and perforıning borehole deviation logging.

Identified preliminary remedial activities for CFA Landfills II and III include no action, access restriction, containment, surface controls (grading the landfill surface), passive collection of landfill gas, leachate collection/monitoring and treatment, groundwater monitoring, and potential "hot-spot" removal. Selection of remedial alternatives will consider all ARARs and practicable technologies for landfills.

The tasks to be carried out as part of the RI/FS for the CFA landfills are the following:

- Project planning

- Community relations

- Ficld investigations

- Sample analysis and data validation

- Risk assessment

- Treatability studies (if necessary)

- Preparation of a remedial investigation report

- Development, screening, and detailed analysis of alternatives

- Preparation of a feasibility study report

- Preparation of the Proposed Plan and Record of Decision. 


\section{CONTENTS}

\section{REMEDIAL INVESTIGATION/FEASIBILITY STUDY WORK PLAN}

1. Introduction

2. Site Background and Physical Setting

3. Initial Evaluation

4. Work Plan Rationale

5. RI/FS Tasks

6. Schedule

7. References

Appendix A-Shallow Soil Gas Survey Results for Landfill II. Model Description and Approach for Estimating Air Concentrations, and Soil Gas Survey of Instrumented Boreholes

Appendix B-Analytical Results for Subsurface Soil Samples at Landfills II and III

Appendix C-Validated Groundwater Quality Data for Landfills II and III

Appendix D-End-of-Well Reports for Landfills II and III

Appendix E-Baseline and Background Concentrations of Trace Elements in Surface Soils

Appendix F-Resolutions of Idaho Department of Health and Welfare and Environmental

Protection Agency Review Comments of the Draft RI/FS Work Plan and Addenda

\section{REMEDIAL INVESTIGATION/FEASIBILITY STUDY WORK PLAN ADDENDA}

Remedial Investigation Sampling and Analysis Plan

Part I-Ficld Sampling Plan

Part II-Quality Assurance Project Plan

Health and Safety Plan

Community Relations Plan 


\title{
Remedial Investigation/Feasibility Study Work Plan for Operable Unit 4-12: Central Facilities Area Landfills II and III at the Idaho National Engineering Laboratory
}

\author{
K. N. Keck \\ G. J. Stormberg \\ I. Porro \\ A. J. Sondrup \\ S. H. McCormick
}

Published July 1993

Idaho National Engineering Laboratory

EG\&G Idaho, Inc.

Idaho Falls, Idaho 83415 


\section{CONTENTS}

ACRONYMS $\ldots \ldots \ldots \ldots \ldots \ldots \ldots \ldots \ldots \ldots \ldots \ldots \ldots \ldots \ldots \ldots \ldots \ldots \ldots \ldots$

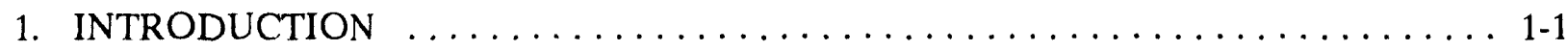

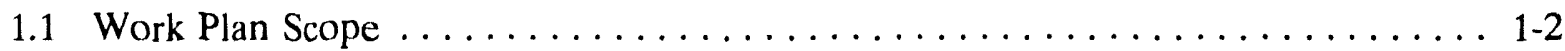

1.2 Operable Unit $4-12$ Regulatory History $\ldots \ldots \ldots \ldots \ldots \ldots \ldots \ldots \ldots \ldots \ldots \ldots \ldots \ldots$

2. SITE BACKGROUND AND PHYSICAL SETTING $\ldots \ldots \ldots \ldots \ldots \ldots \ldots \ldots \ldots$

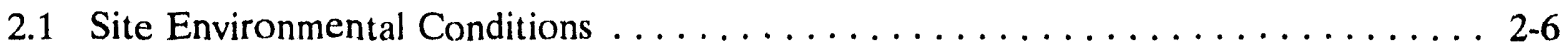

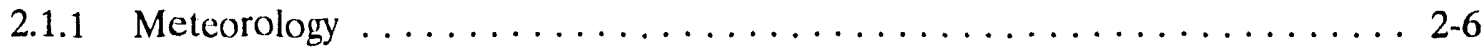

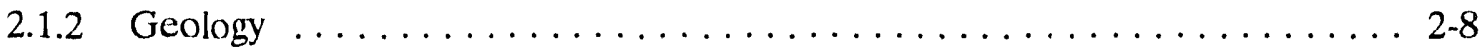

2.1.3 Hydrology ........................... 2-14

2.1 .4 Ecology ............................... 2-23

2.1.5 Archaeology ........................... 2-23

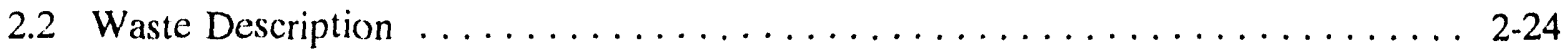

2.2.1 Waste Disposal Practices . . . . . . . . . . . . . . . . . . 2-24

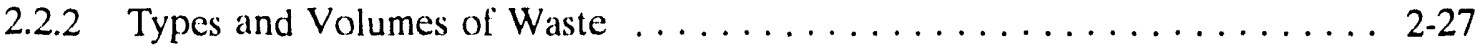

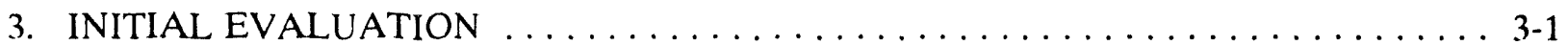

3.1 Summary and Evaluation of Previous Sampling Activities $\ldots \ldots \ldots \ldots \ldots \ldots \ldots$.

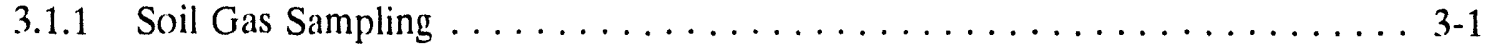

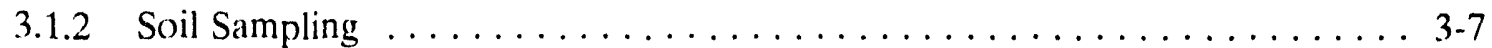

3.1.3 Vadose Zone Moisture Monitoring Data ................ 3-10

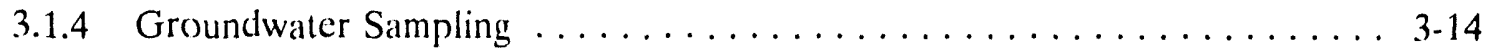

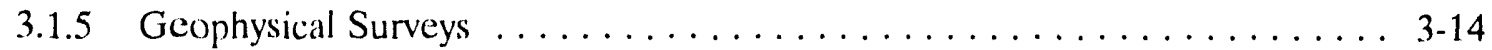

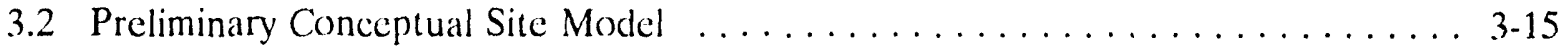

3.3 Preliminary Public Health and Environmental Impacts $\ldots \ldots \ldots \ldots \ldots \ldots \ldots$

3.4 Preliminary Remedial Action Alternatives $\ldots \ldots \ldots \ldots \ldots \ldots \ldots \ldots \ldots \ldots$

3.5 Preliminary Applicable or Relevant and Appropriate Requirements . . . . . . . 3-18

4. WORK PLAN RATIONALE $\ldots \ldots \ldots \ldots \ldots \ldots \ldots \ldots \ldots \ldots \ldots \ldots \ldots \ldots \ldots \ldots$

4.1 Remedial Investigation and Feasibility Study Objectives $\ldots \ldots \ldots \ldots \ldots \ldots \ldots$ 4-2 
4.2 Data Quality Objectives

5. RI/FS TASKS

5.1 Project Planning $\ldots \ldots \ldots \ldots \ldots \ldots \ldots \ldots \ldots \ldots \ldots \ldots \ldots \ldots \ldots \ldots \ldots \ldots \ldots$

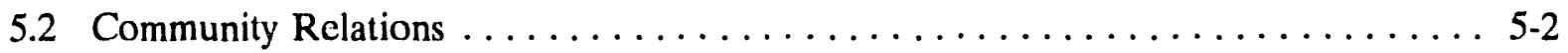

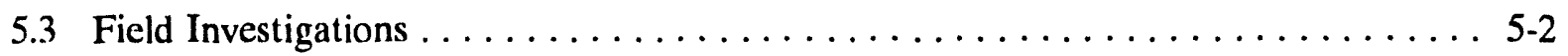

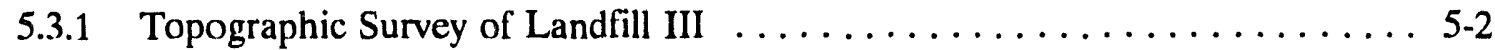

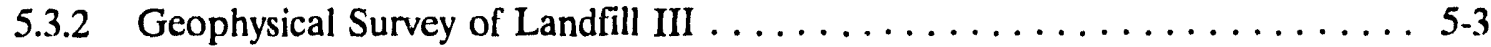

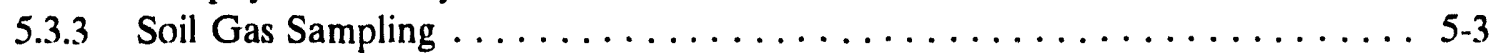

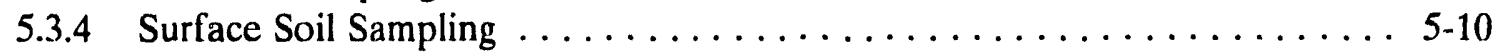

5.3.5 Cover Characterization of Landfill III .................. 5-10

5.3.6 Water Level Measurements and Borehole Deviation Logging . . . . . . . 5-12

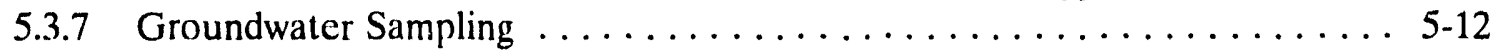

5.3.8 Subsurface Soil Sampling of Landfill II $\ldots \ldots \ldots \ldots \ldots \ldots \ldots \ldots \ldots \ldots \ldots$

5.4 Sample Analysis and Data Validation $\ldots \ldots \ldots \ldots \ldots \ldots \ldots \ldots \ldots \ldots \ldots$

5.5 Data Evaluation $\ldots \ldots \ldots \ldots \ldots \ldots \ldots \ldots \ldots \ldots \ldots \ldots \ldots \ldots \ldots \ldots \ldots$

5.6 Contaminant Fate and Transport Modeling $\ldots \ldots \ldots \ldots \ldots \ldots \ldots \ldots \ldots \ldots$

5.6.1 Modeling of Landfill Cover Performance ............... 5-17

5.6 .2 Contaminant Transport Modeling $\ldots \ldots \ldots \ldots \ldots \ldots \ldots \ldots \ldots \ldots \ldots \ldots \ldots$

5.7 Risk Assessment . . . . . . . . . . . . . . . . . . . . . . . 5-19

5.7 .1 Human Health Evaluation . . . . . . . . . . . . . . . . 5-19

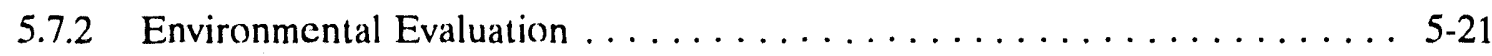

5.7.3 Uncertainty Analysis in the Risk Assessment Process . . . . . . . . . . 5-21

5.8 Treatability Studies $\ldots \ldots \ldots \ldots \ldots \ldots \ldots \ldots \ldots \ldots \ldots \ldots \ldots \ldots \ldots \ldots \ldots \ldots \ldots \ldots \ldots .22$

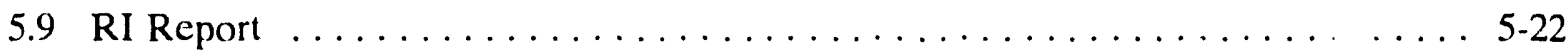

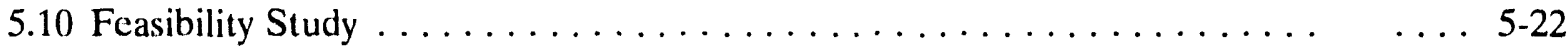

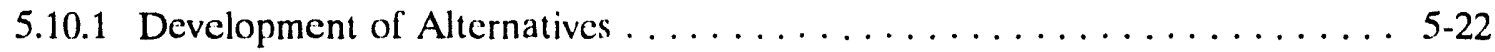

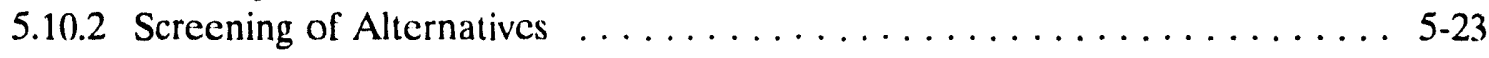

5.10 .3 Detailed Analysis of Alternatives . . . . . . . . . . . . . . $5-24$

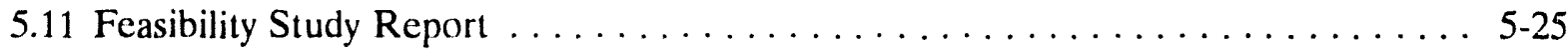

5.12 Proposed Plan and Record of Decision . . . . . . . . . . . . $5-26$ 


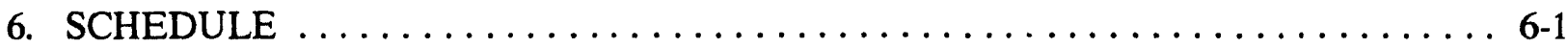

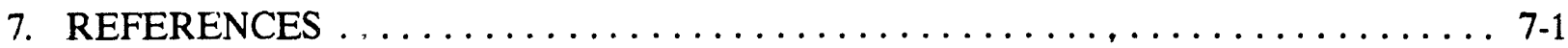

Appendix A-Shallow Soil Gas Survey Results for Landfill II, and Model Description and Approach for Estimating Air Concentrations $\ldots \ldots \ldots \ldots \ldots \ldots \ldots \ldots \ldots \ldots \ldots \ldots$

Appendix B-Analytical Results for Subsurface Soil Samples at Landfills II and III ...... B-1 Appendix C-Validated Groundwater Quality Data for Landfills II and III . . . . . . . C-1 Appendix D-End-of-Well Reports for Landfills II and III $\ldots \ldots \ldots \ldots \ldots \ldots \ldots \ldots \ldots$ Appendix E-Baseline and Background Concentrations of Trace Elements in Surface Soils .. E-1 Appendix F-Resolutions of Idaho Department of Health and Welfare and Environmental Protection Agency Review Comments of the Draft RI/FS Work Plan and Addenda ..... F-1

\section{FIGURES}

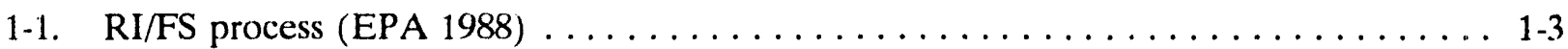

2-1. Location map of the INEL $\ldots \ldots \ldots \ldots \ldots \ldots \ldots \ldots \ldots \ldots \ldots \ldots \ldots \ldots \ldots \ldots \ldots \ldots \ldots$

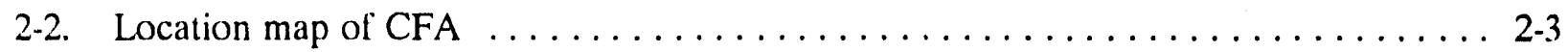

2-3. Location map of CFA Landfills II and III $\ldots \ldots \ldots \ldots \ldots \ldots \ldots \ldots \ldots \ldots \ldots$

2-4. CFA Landfill II topography estimated by ordinary point kriging $\ldots \ldots \ldots \ldots \ldots$

2-5. Location of the INEL within the context of southern Idaho geologic features $\ldots . . .2-9$

2-6. Generalized geologic map of the INEL area, showing the surficial distribution of major

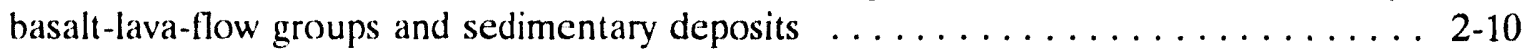

2-7. Strategic cross sections $A-A^{\prime}$ and $B-B^{\prime}$ through the CFA landfills $\ldots \ldots \ldots \ldots 2-12$

2-8. Map showing surface water features near or on the INEL $\ldots \ldots \ldots \ldots \ldots \ldots$

2-9. Water table map for the Snake River Plain Aquifer below the INEL . . . . . . 2-18

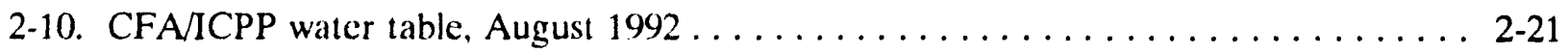

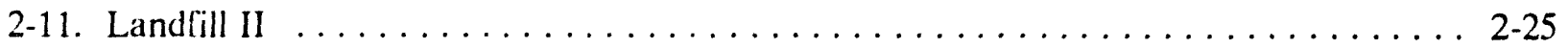

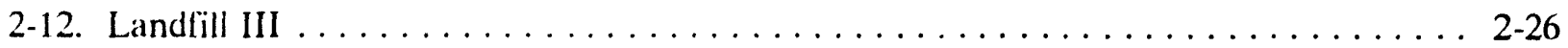


3-1. Map of CFA Landfill II showing the location of neutron access boreholes and boreholes used for gas sampling and moisture monitoring.

3-2. Map of CFA Landfill III showing the location of neutron access boreholes and boreholes for gas sampling and moisture monitoring $\ldots \ldots \ldots \ldots \ldots \ldots \ldots \ldots$

3-3. Preliminary conceptual model for CFA Landfills II and III $\ldots \ldots \ldots \ldots \ldots \ldots \ldots$

5-1. Geophysical survey grids for (a) seismic refraction, (b) induced electromagnetic measurements, and (c) magnetic field measurements $\ldots \ldots \ldots \ldots \ldots \ldots \ldots \ldots$

$5-2$. Seismic refraction grid for Landfill II $\ldots \ldots \ldots \ldots \ldots \ldots \ldots \ldots \ldots \ldots \ldots$

5-3. Shallow soil gas sample locations for Landfill III $\ldots \ldots \ldots \ldots \ldots \ldots \ldots \ldots$

5-4. Shallow soil gas sample locations for Landfill II $\ldots \ldots \ldots \ldots \ldots \ldots \ldots \ldots \ldots$

5-5. Location of boreholes to be used in borehole gas sampling $\ldots \ldots \ldots \ldots \ldots \ldots$

5-6. Location of wells to be used for water level measurement and groundwater sampling

5-7. Subsurface soil boring sites - Landfill II

\section{TABLES}

2-1. Average, highest, and lowest total monthly and annual precipitation at CFA from January 1950 to December $1988 \ldots \ldots \ldots \ldots \ldots \ldots \ldots \ldots \ldots \ldots \ldots \ldots \ldots \ldots$

2-2. Depths of clay in surficial sediments observed in shallow boreholes at Landfills II

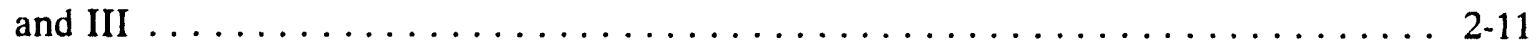

2-3. Depths of clay in sedimentary interbeds observed in monitoring wells at Landfills II

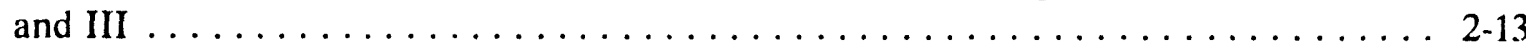

2-4. Transmissivity and hydraulic conductivity values for wells in the CFA area, based on

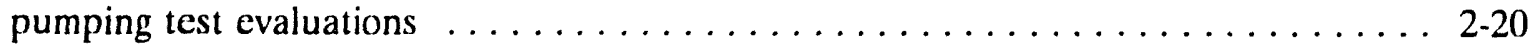

2-5. Categories of waste from the IWMIS and INWMIS data bases $\ldots \ldots \ldots \ldots \ldots 2-29$

2-6. Estimated waste description for CFA Landfill II $\ldots \ldots \ldots \ldots \ldots \ldots \ldots \ldots$

2-7. Estimatıd waste description for CFA Landfill III $\ldots \ldots \ldots \ldots \ldots \ldots \ldots \ldots$

3-1. Summary of characterization and sampling activities conducted at CFA Landfills II

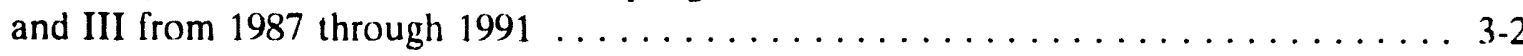


3-2. Unvalidated soil gas sampling results for the May 1988 sampling of borehole gas ports

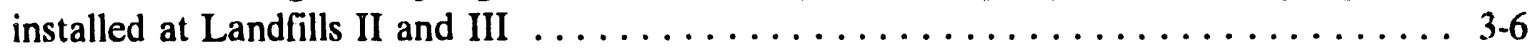

3-3. Unvalidated shallow borehole soil sampling analytical results, with corresponding sample

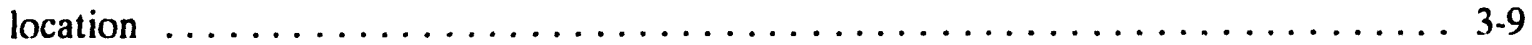

3-4. Risk-based screening of carcinogenic and noncarcinogenic soil contaminants $\ldots \ldots$ 3-10

3-5. Boreholes used for neutron probe access tubes and access tube depths $\ldots \ldots \ldots \ldots 3-11$

3-6. Locations and depths of heat dissipation blocks installed at Landfills II and III . . . . 3-12

3-7. Locations and depths of salinity probes installed at Landfills II and III . . . . . . 3-13

3-8. Preliminary chemical-specific ARARs for CFA Landfills II and III . . . . . . . . 3-20

3-9. Preliminary location-specific ARARs for CFA Landfills II and III $\ldots \ldots \ldots \ldots \ldots$

3-10. Preliminary action-specific ARARs for CFA Landfills II and III . . . . . . . 3-22

3-11. DOE and exccutive orders to be considered $\ldots \ldots \ldots \ldots \ldots \ldots \ldots \ldots \ldots \ldots \ldots \ldots$

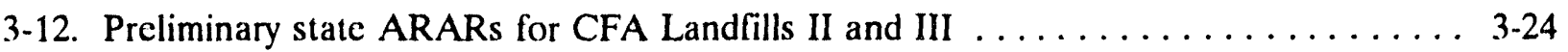

4-1. Remedial investigation objectives for CFA Landfills II and III $\ldots \ldots \ldots \ldots \ldots \ldots$

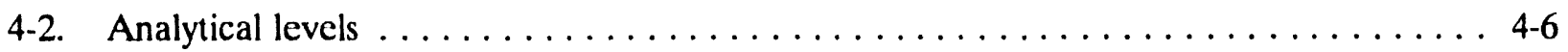

4-3. Data quality objectives summary for CFA Landfills II and III RI/FS . . . . . . . 4 4-7

5-1. Wells to be measured on a monthly basis for water level data $\ldots \ldots \ldots \ldots \ldots \ldots$

5-2. Existing aquifer wells to be sampled $\ldots \ldots \ldots \ldots \ldots \ldots \ldots \ldots \ldots \ldots \ldots \ldots \ldots \ldots$

6-1. Summary working schedule for the Operable Unit $4-12$ RIFS $\ldots \ldots \ldots \ldots \ldots \ldots$ 


\section{ACRONYMS}

ACGIH American Conference of Governmental Industrial Hygienists

ARA

Auxiliary Reactor Area

ARARs applicable or relevant and appropriate requirements

ARDC Administrative Records and Document Control

ASTM American Society for Testing and Materials

bls below land surface

BRA baseline risk assessment

CERCLA Comprehensive Environmental Response, Compensation, and Liability Act

CFA Central Facilities Area

CFR Code of Federal Regulations

C.LP Contract Laboratory Program

COCA Consent Order and Compliance Agreement

cpm counts per minute

CRP Community Relations Plan

CRQL contract-required quantitation limits

DCA dichloroethane

DMP Data Management Plan

DOE U.S. Department of Energy

DOE-ID U.S. Department of Energy Idaho Field Office

DOT U.S. Department of Transportation

DQOs data quality objectives

EA evaluation of alternatives

EBR ! erimental Breeder Reactor

ECAO Environmental Criteria and Assessment Office

ECD electron capture detector

EDA Engineering Design of Alternatives

EEM Environmental Evaluation Manual

EM terrain conductivity

EPA U.S. Environmental Protection Agency 
ERA ecological risk assessment

ERAA evaluation of remedial action alternatives

ER Environmental Restoration

ESRP Eastern Snake River Plain

FFA/CO Federal Facility Agreement/Consent Order

FR Federal Register

FSP Field Sampling Plan

GPR ground penetrating radar

HELP hydrologic evaluation of landfill performance

HHEM Human Health Evaluation Manual

HP health physics technician

HRS Hazard Ranking System

ICPP Idaho Chemical Processing Plant

IDHW Idaho Department of Health and Welfare

INEL Idaho National Engineering Laboratory

INWMIS Industrial Nonradiological Waste Management Information System

IPMP Implementing Program Management Plan

IRC Independent Review Committee

IWMIS Industrial Waste Management Information System

LEL lower explosive limit

MCL maximum contaminant level

msl mean sea level

NAPLs nonaqueous phase liquids

NCP National Contingency Plan

NOAA National Oceanic and Atmospheric Administration

NPL National Priorities List

NRF Naval Reactor Facility

OSHA Occupational Safety and Health Administration

OU operable unit

PARCC precision, accuracy, representativeness, completeness, comparability

PCBs polychlorinated biphenyls 


\begin{tabular}{|c|c|}
\hline PCE & tetrachloroethylene \\
\hline PD & program directive \\
\hline PMP & Project Management Plan \\
\hline $\mathrm{ppb}$ & parts per billion \\
\hline QA & quality assurance \\
\hline QAPjP & Quality Assurance Project Plan \\
\hline $\mathrm{QA} / \mathrm{QC}$ & quality assurance/quality control \\
\hline $\mathrm{QC}$ & quality control \\
\hline RA & risk assessment \\
\hline RAGS & Risk Assessment Guidance for Superfund \\
\hline RCRA & Resource Conservation and Recovery Act \\
\hline $\mathrm{RI} / \mathrm{FS}$ & remedial investigation/icasibility study \\
\hline ROD & Record of Decision \\
\hline RWMC & Radioactive Waste Management Complex \\
\hline SAP & Sampling and Analysis Plan \\
\hline SARA & Superfund Amendments and Reauthorization Act \\
\hline SC & site characterization \\
\hline SOP & standard operating procedure \\
\hline SOW & statement of work \\
\hline SPERT & Special Power Excursion Reactor Test \\
\hline SVOCs & semivolatile organic compounds \\
\hline TAN & Test Area North \\
\hline TBC & To Be Considered \\
\hline TCA & trichlorocthane \\
\hline TCE & trichloroethylene \\
\hline TCL & target compound list \\
\hline TCLP & toxicity characteristic leaching procedure \\
\hline TEM & time-domain electromagnetic induction \\
\hline TLV & threshold limit value \\
\hline TOC & total organic carbon \\
\hline TOX & total organic halogen \\
\hline
\end{tabular}


TRA

TWA

USGS

VOA

VOC

WAG

WHS
Test Reactor Area

time weighted average

United States Gcological Survey

volatile organic analysis

volatile organic compound

waste area group

worker health and safety 


\section{Remedial Investigation/Feasibility Study Work Plan and Addenda for Operable Unit 4-12: \\ Central Facilities Area Landfills II and III at the Idaho National Engineering Laboratory}

\section{INTRODUCTION}

This scrtion discusses the organization and scope of the Central Facilities Area (CFA) Landfills II and III Remedial Investigation/Feasibility Study (RI/FS) Work Plan and presents the regulatory history of Operable Unit (OU) 4-12.

The Work Plan is organized into seven sections:

1. Introduction-provides the scope of the Work. Plan and addenda, and reviews the regulatory history of OU 4-12.

2. Site Background and Physical Setting-describes OU 4-12 based on existing site data.

3. Initial Evaluation-summarizes previous sampling activities and data, presents a preliminary conceptual model, identifies preliminary remedial action alternatives, and identifies preliminary applicable or relevant and appropriate requirements (ARARs).

4. Work Plan Rationale-discusses data gaps and describes site-specific objectives and data quality objectives for proposed remedial investigation activities.

5. RI/FS Tasks-describes tasks identified for the RI/FS.

6. Schedule-provides a schedule of RI/FS activities.

7. References-lists references cited in the above sections.

Documents (addenda) prepared and included with the Work Plan are as follows:

- Remedial Investigation Sampling and Analysis Plan-is comprised of a Field Sampling Plan, which describes proposed lield activities, and a Quality Assurance Project Plan (QAPjP), which describes anticipated quality assurance (QA) activities.

- Health and Safety Plan-describes policies and procedures to protect RI/FS workers and the environment during lield investigations.

- Community Relations Plan Supplement-describes policies, procedures, and activities that the Department of Energy (DOE) will use to involve the public in the decisionmaking process concerning CFA Landfills II and III RI/FS activities. 


\subsection{Work Plan Scope}

The overall goals of the RI/FS for OU 4-12: CFA Landfills II and III are to complete a field program at the site for collecting data required to determine if unacceptable risks exist to current/future human health and the environment, and to develop and evaluate remedial action alternatives that are most practicable for landfill sites, if unacceptable risks exist. In connection with the RI/FS, this Work Plan was prepared to do the following:

- $\quad$ Document the RI/FS process

- Describe OU 4-12 on the basis of existing information

- Identify additional data needed to make decisions required by the Comprehensive Environmental Response, Compensation, and Liability Act of 1980 (CERCLA)

- Identify site investigations needed to obtain additional data

- Identify fate and transport modeling and the baseline risk assessment methodologies

- Identify responsibilitics for RI/FS activities

- Present the RI/FS schedule.

The DOE (which manages the Idaho National Engineering Laboratory (INEL) and is the lead agency], the Environmental Protection Agency (EPA), and the Idaho Department of Health and Welfare (IDHW) initiated the RI/FS pursuant to CERCLA. Section 120 and the Federal Facility Agreement/Consent Order (FFA/CO). The RI/FS process is shown in Figure 1-1. The final product of this study will be a single, integrated set of documents, which includes a remedial investigation report and a feasibility study.

\subsection{Operable Unit 4-12 Regulatory History}

CFA Landfills II and III were initially governed by hazardous waste regulations promulgated under the Resource Conservation and Recovery Act (RCRA). This act requires all land disposal units operational after 1980 to have a groundwater monitoring system to detect potential releases of hazardous constituents into the environment, aceording to requirements of Code of Federal Regulations (CFR) 40 CFR 265.90, Subpart F. CFA Landfills II and III were identified in the Consent Order and Compliance Agreement (COCA) (EPA 1987a) for remedial action under RCRA. While under COCA provisions, groundwater monitoring and closure plans were developed and a facility investigation was initiated.

On July 14, 1989, EPA proposed placing the INEL on the National Priorities List (NPL) of the National Contingency Plan (NCP) (54 FR 29820) (EPA 1990a). This was done using Hazard Ranking System (HRS) procedures found in the NCP. The INEL's score was 51.91. Data that support listing the INEL as an NPL site are found in the Federal Facilities Docket, EPA Headquarters, Washington, D.C. As a federal facility, the INEL is eligible for the NPL pursuant 


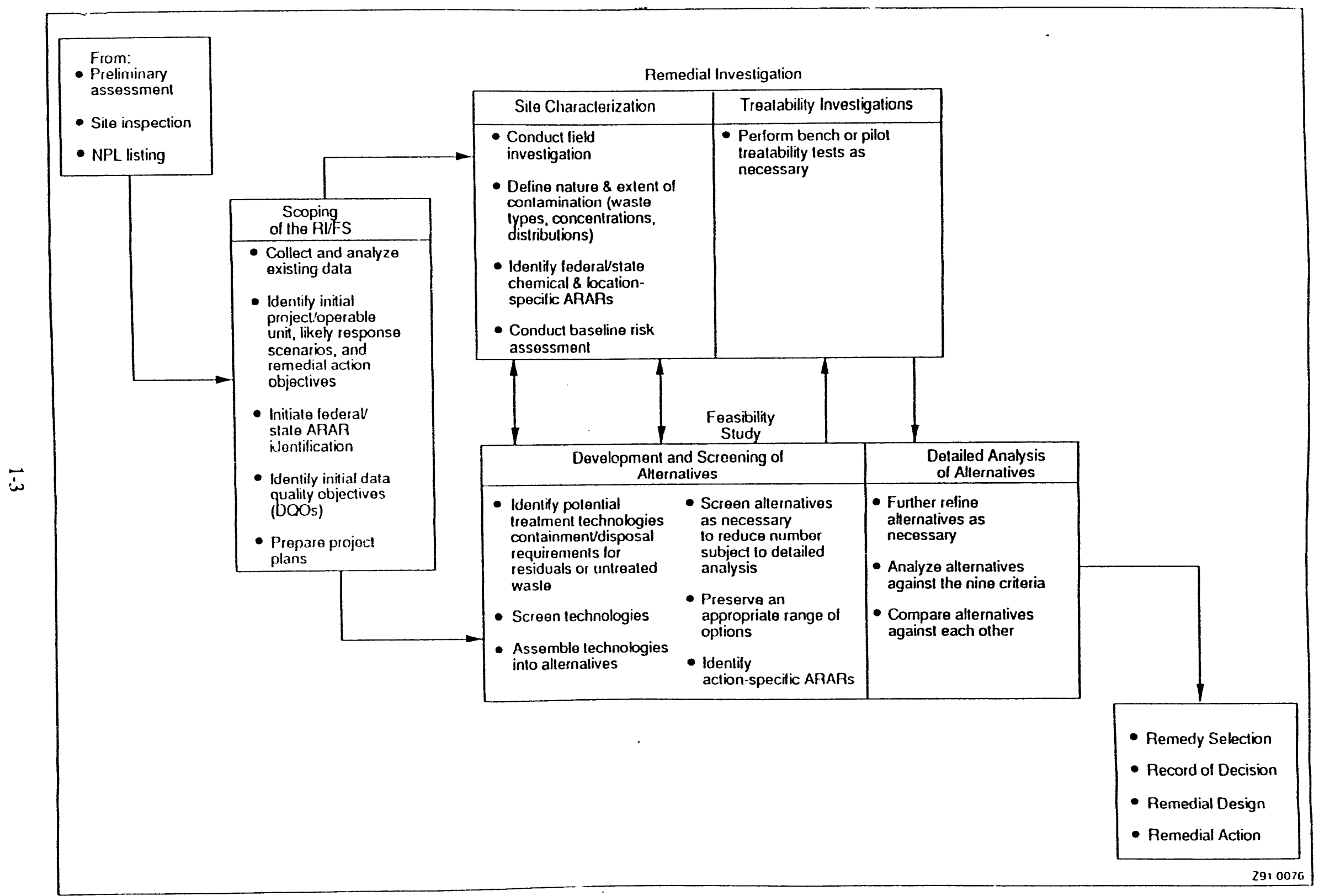

Figure 1-1. RI/FS process (EPA, 1988). 
to the NCP by 40 CFR 300.66(c)(2). After considering public input during a 60 -day comment period following the proposed INEL listing, EPA issued a final rule listing the INEL Site. The rule was published in the Federal Register, November 21, 1989.

Subsequent to listing the INEL on the NPL and with the development of the FFA/CO (effective date December 9, 1991), DOE, EPA, and IDHW decided that CFA Landfills II and III should be remediated through the CERCLA-driven RI/FS process. CFA Landfills II and III are considered CERCLA landfills that are primarily composed of calcteria, office, and construction wastes, and to a lesser extent hazardous wastes. The FFA/CO establishes the procedural framework and schedule for developing, prioritizing, implementing, and monitoring response actions at the INEL in accordance with CERCLA, RCRA, and the Idaho Hazardous Waste Management Act. OU 4-12 as defined here, includes all of Landfill II, the inactive portion of Landfill III, and the various media associated with each landfill. The active portion of Landfill III and inactive Landfill I are not considered part of OU 4-12; therefore, they will not be assessed in this RI/FS. Landfill I has been addressed as a Track 2 investigation under the FFA/CO, and the active portion of Landlill III will be addressed under appropriate regulatory programs for active landfills or under the final WAG 4 comprehensive RI/FS, OU 4-13. 


\section{SITE BACKGROUND AND PHYSICAL SETTING}

The INEL is located approximately $42 \mathrm{mi}(68 \mathrm{~km})$ west of Idaho Falls, Idaho, and occupies $890 \mathrm{mi}^{2}\left(2,305 \mathrm{~km}^{2}\right)$ of the northwestern portion of the Eastern Snake River Plain (ESRP) (Figure 2-1). Although the INEL is operated by DOE primarily for nuclear reactor research, it also supports other government-sponsored projects such as energy, defense, and environmental and ecological research.

The CFA is located in the south-central portion of the INEL (Figure 2-2). The original facilities at CFA were built in the 1940s and 1950s to house Naval Gunnery Range personnel. The facilities have been modified over the years to fit the changing needs of the INEL and now provide four major types of functional space: craft, office, service, and laboratory.

CFA Landfills II and III are located approximately $0.5 \mathrm{mi}(0.8 \mathrm{~km})$ north of CFA proper (Figure 2-3). CFA Landfill II was opened in 1970 to dispose of INEL construction, cafeteria, and office wastes. To a lesser extent, hazardous wastes such as waste oil, solvents, and paint were also disposed of at the landfill. After the closure of Landfill II in September 1982, Landfill III was opened to handle the same types of waste.

Landfill II was operational from 1970 to September 1982 and was used as the primary landfill for the disposal of cafeteria, office, and construction waste for the INEL during this period. The perimeter of Landfill II encompasses approximately 14 acres (6 hectares), and the maximum depth of the landfill is approximately $15 \mathrm{ft}(5 \mathrm{~m})$ (ICF Technology, Inc. 1989). The existing cover material on Landfill II, ranging in thickness from 6 in. $(15 \mathrm{~cm})$ to over $3 \mathrm{ft}(0.9 \mathrm{~m})$, is gently undulating due to differential settling of the waste unit and maintains a sparse stand of crested wheatgrass. Much of the cover material is desert pavement consisting of gravel with interstitial sand and silt fines. The western, southern, and southeastern boundaries of the cover are relatively flush with the adjacent ground surface. On the north and northeastern boundaries, the cover material slopes north and east down into the floor of the existing gravel pit. The elevation across the landfill surface ranges from $4,927(1,501 \mathrm{~m})$ to $4,934 \mathrm{ft}(1,503 \mathrm{~m})$, with a gentle downward slope to the northeast (Figure 2-4).

Landfill III opened in October of 1982 (when the operations at CFA Landfill II were terminated) and was operational until December 4, 1984. Landfill III consists of a series of northsouth trending trenches and is located approximately $0.5 \mathrm{mi}(0.8 \mathrm{~km})$ west of the Lincoln Boulevard and Portland Avenue intersection and just north of Portland Avenue. The landfill perimeter boundary is known from a surveyed grid depicted on blueprint maps of the area, but is not marked in the field. Based on this information, the area of Landfill III is considered to be approximately 11 acres (5 hectares). At the time of closure, the cover surface was compacted to a uniform depth of $2 \mathrm{ft}(0.6 \mathrm{~m})$ and is generally llush with the adjacent ground surface. No further cover maintenance has been performed since closure of the landfill. The existing cover on Landfill III, at an average elevation of $4,940 \mathrm{ft}(1,505 \mathrm{~m})$, is also gently undulating due to differential settling of the waste unit and maintains a sparse stand of crested wheatgrass. A spatial survey of the landfill cover thickness and its topography has not been performed. 


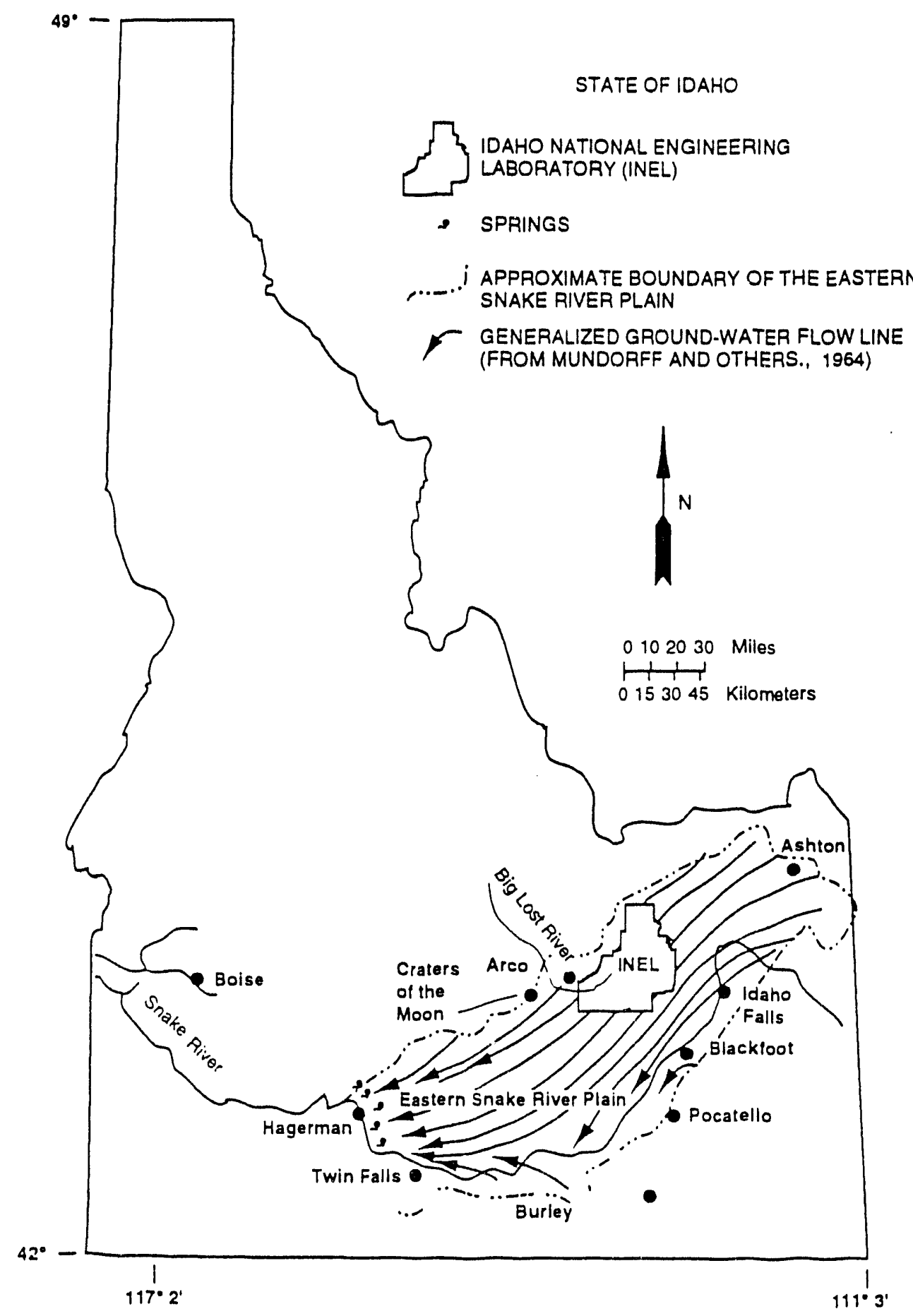

Figure 2-1. Location map of the INEL. 


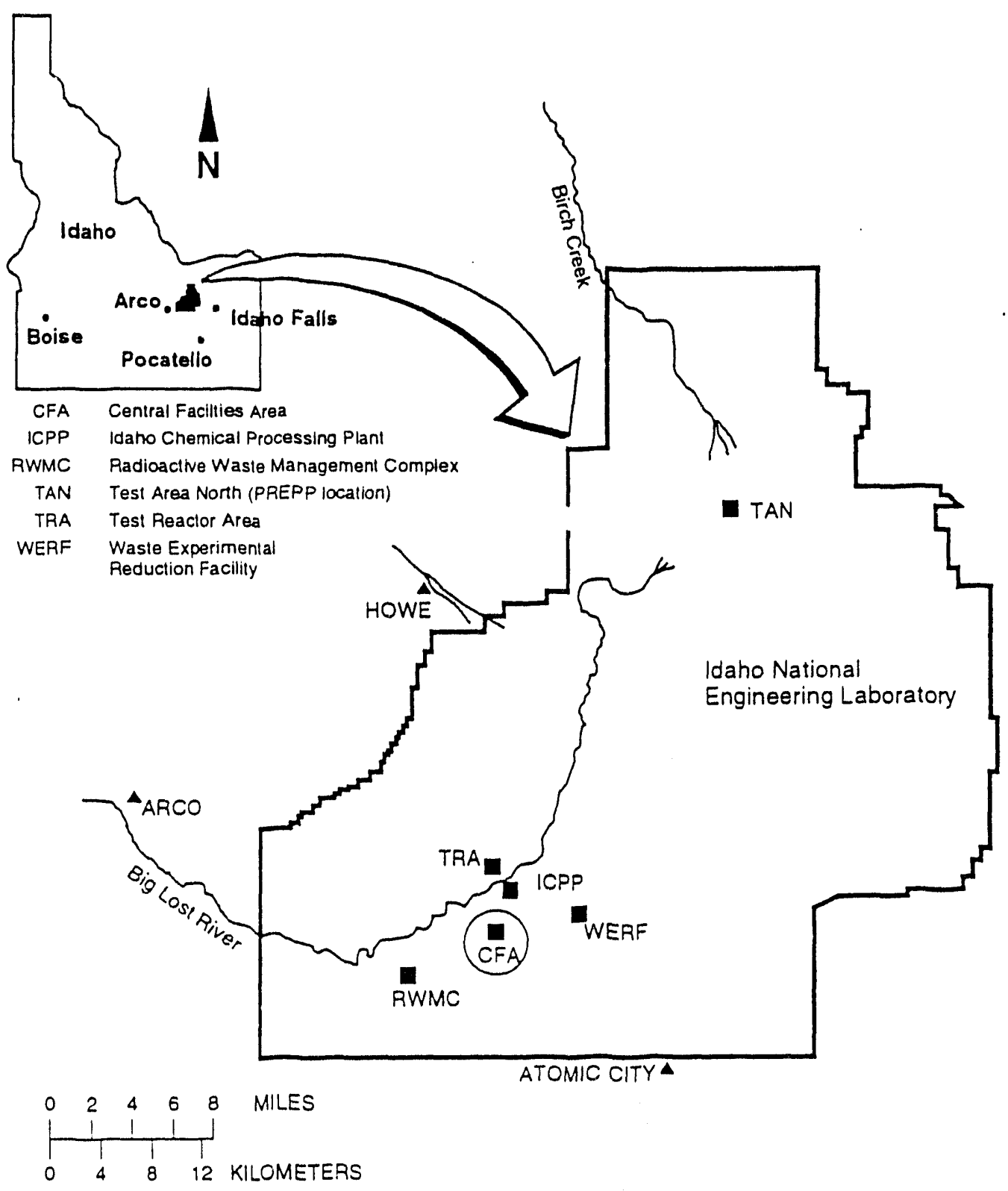

R92 1390

Figure 2-2. Location map of CFA. 


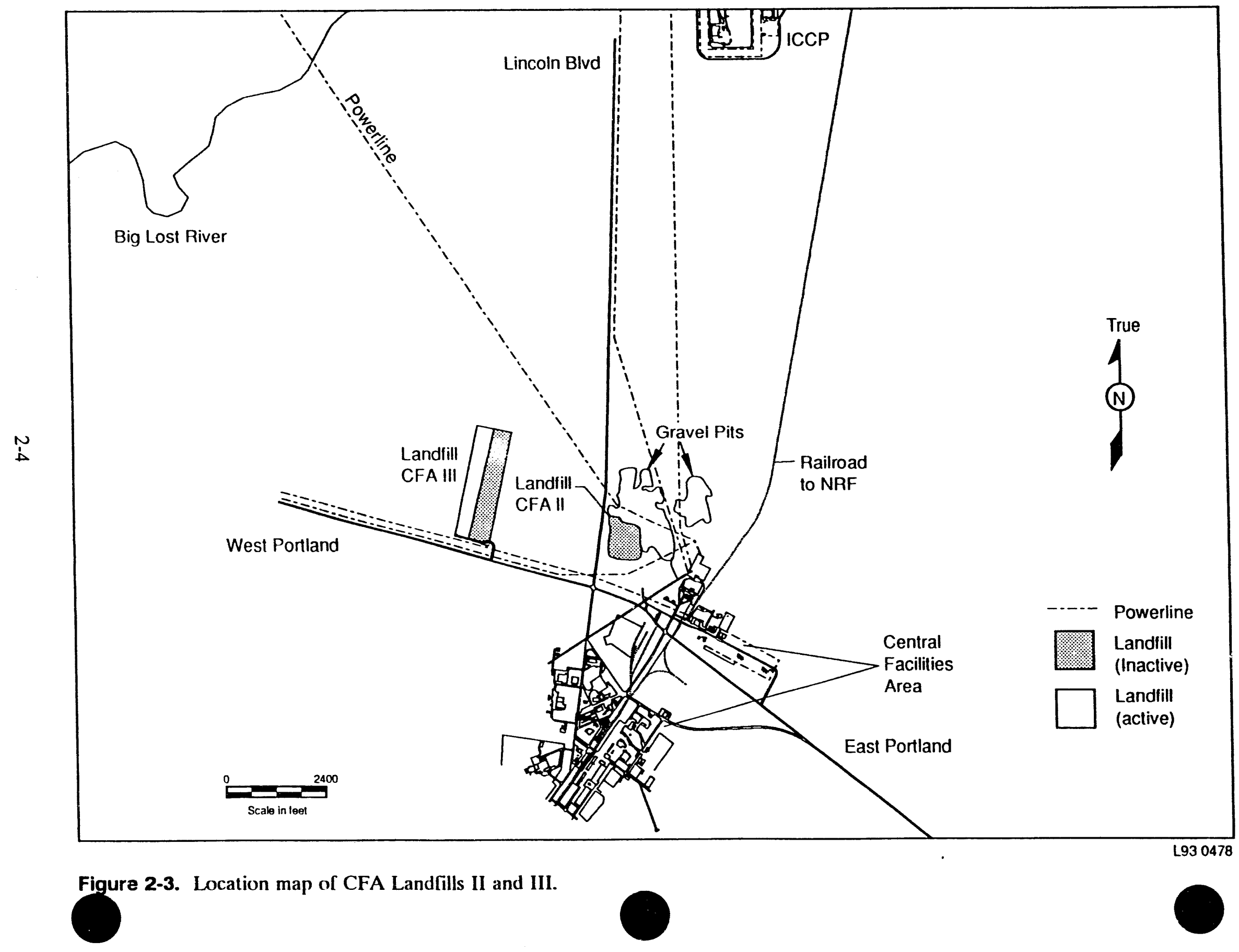




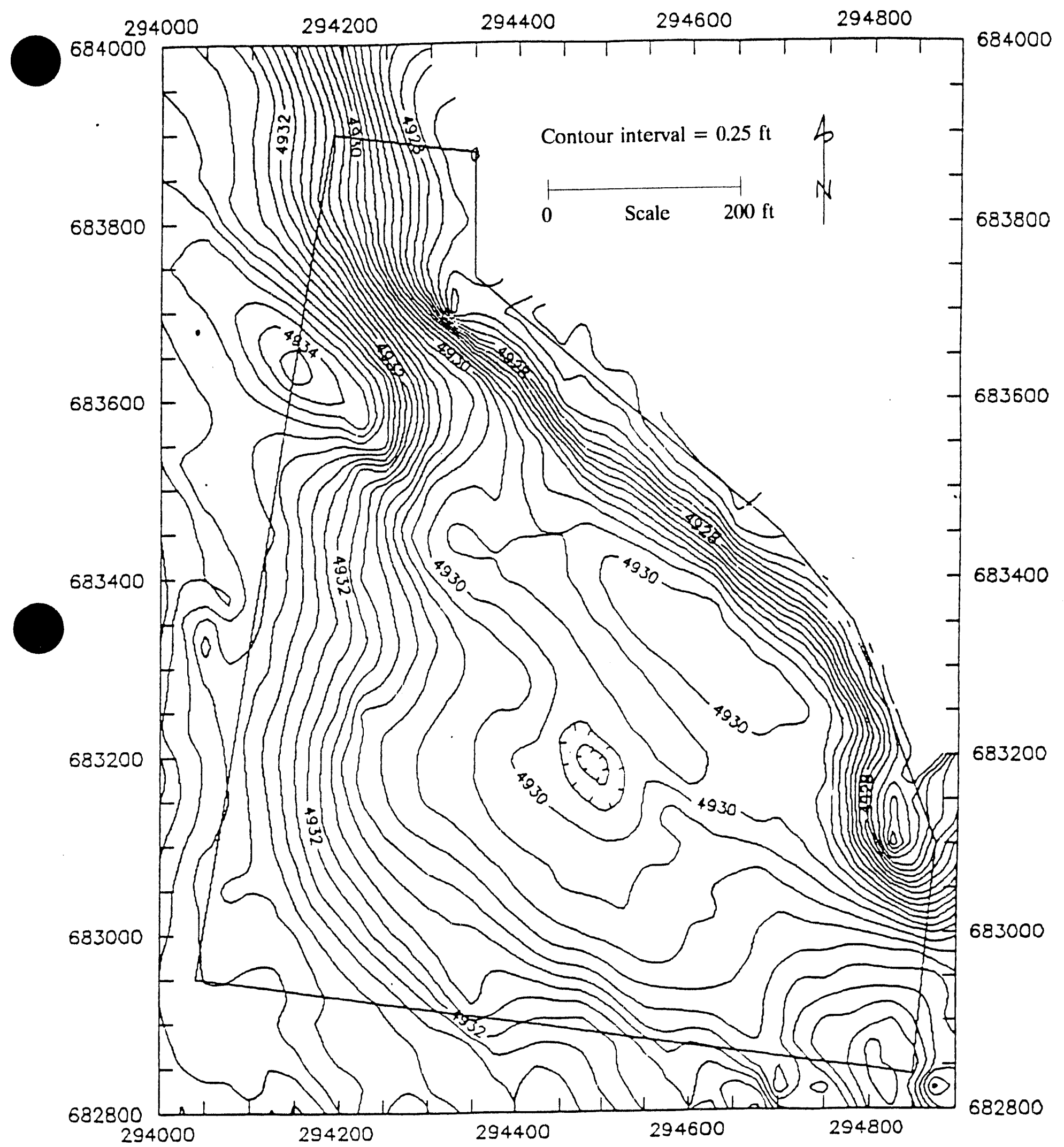

Figure 2-4. CFA Landfill II topography estimated by ordinary point kriging (Miller et al. 1990). 


\subsection{Site Environmental Conditions}

The following sections describe the regional and site-specific environmental conditions, including meteorology, geology, surface water, vadose zone, groundwater, ecology, and archaeology.

\subsubsection{Meteorology}

Meteorological data has been collected from a U.S. Weather Bureau station located at CFA since 1949. The National Oceanic and Atmospheric Administration (NOAA) maintains a full range of hourly and daily climatological observations at this station. NOAA publishes these data annually and in summary reports specific for the climatography of the INEL. Data recorded at CFA is summarized below and includes air temperature, atmospheric humidity, precipitation, wind speed, and atmospheric pressure (Clawson et al. 1989).

The monthly average air temperature at CFA for the period January 1950 through December 1988 ranged from $16.1^{\circ} \mathrm{F}\left(-9^{\circ} \mathrm{C}\right)$ in January to $68.0^{\circ} \mathrm{F}\left(20^{\circ} \mathrm{C}\right)$ in July. The highest maximum air temperature recorded at $\mathrm{CFA}$ during this period was $101^{\circ} \mathrm{F}\left(38^{\circ} \mathrm{C}\right)$, while the coldest minimum was $-47^{\circ} \mathrm{F}\left(-44^{\circ} \mathrm{C}\right)$. On average, $42 \%$ of the days in a year contain a freeze/thaw cycle, where the maximum air temperature exceeds $32^{\circ} \mathrm{F}\left(0^{\circ} \mathrm{C}\right)$ and the minimum air temperature is at or below $32^{\circ} \mathrm{F}\left(0^{\circ} \mathrm{C}\right)$. Inversion conditions (warmer air temperature with increasing altitude) and lapse conditions (cooler air temperature with increasing altitude) occur approximately $46 \%$ and $54 \%$ of the time, respectively.

The average hourly relative humidity from the six-year period of record from 1956 to 1961 was calculated using wet bulb temperatures recorded at CFA. The highest relative humidity is observed in the winter, with the average midday relative humidity at about $55 \%$. The lowest is observed in the summer, when the midday average is approximately $18 \%$. An absolute maximum relative humidity value of $100 \%$ was observed in every month of the year except July and the lowest observed was $4 \%$ in July and August. This is indicative of the very dry summers experienced at the INEL.

Another measure of atmospheric humidity is pan evaporation. While these types of measurements have not been made at the INEL, an estimate of pan evaporation rates was calculated using regression analysis from Class-A pan evaporation data obtained from stations in southeastern Idaho (Clawson et al. 1989). Results of the analysis suggest that total pan evaporation at CFA is approximately 43 in. $(109 \mathrm{~cm})$ per year, with a range of approximately 40 to $46 \mathrm{in}$. (102 to $117 \mathrm{~cm}$ ). Consumptive use (evapotranspiration) by sparse native vegetation found at the CFA landfills ranges from 6 to 9 in. (15 to $23 \mathrm{~cm}$ ) per year (Wood et al. 1989b).

Precipitation has been measured at CFA sine 1950. The average total monthly and annual precipitation for the period of record, January 1950) to December 1988, is shown in Table 2-1. During the summer, most precipitation is in the form of rain showers or thunderstorms. In the spring and fall. there are periods of rain or snow: during the winter, most precipitation is in the form of snow. The majority of precipitation occurs in May and June, with an average precipitation for each of these months of $1.2 \mathrm{in.}(3 \mathrm{~cm})$. The annual average total precipitation is 8.71 in. $(22 \mathrm{~cm})$. Precipitation amounts in excess of $1 \mathrm{in.}(2.54 \mathrm{~cm})$ per day have been recorded 
Table 2-1. Average, highest, and lowest total monthly and annual precipitation at CFA from January 1950 to December $1988 .^{a}$

\begin{tabular}{lccc}
\hline \multicolumn{1}{c}{ Month } & $\begin{array}{c}\text { Average } \\
\text { (in.) }\end{array}$ & $\begin{array}{c}\text { Highest } \\
\text { (in.) }\end{array}$ & $\begin{array}{c}\text { Lowest } \\
\text { (in.) }\end{array}$ \\
\hline January & 0.69 & 2.56 & 0.00 \\
February & 0.64 & 2.40 & 0.00 \\
March & 0.60 & 1.44 & 0.07 \\
April & 0.73 & 2.50 & 0.00 \\
May & 1.20 & 4.42 & 0.07 \\
June & 1.18 & 3.89 & 0.02 \\
July & 0.53 & 2.29 & 0.00 \\
August & 0.57 & 3.27 & 0.00 \\
September & 0.63 & 3.52 & 0.00 \\
October & 0.52 & 1.67 & 0.00 \\
November & 0.68 & 1.74 & 0.00 \\
December & 0.75 & 3.43 & 0.02 \\
Annual & & & 4.50 \\
\hline Clawson et al. 1989. & & 14.40 & \\
\hline
\end{tabular}

cight times at CFA, with the maximum being $1.64 \mathrm{in.}(4 \mathrm{~cm})$. The maximum observed hourly precipitation observed at CFA is $0.54 \mathrm{in} .(1.37 \mathrm{~cm})$.

The predominant llow direction of wind across the ESRP is from the southwest to northeast. Prefrontal winds are almost always from the southwest, although nocturnal drainage winds are generally from the northeast. The wind at CFA is distinctly channeled and occurs most frequently from the west-southwest. Little wind originates in the southeast or northwest, and the frequency of calm periods is greatest in the winter and lowest in the spring. Average wind speed at the 20-ft (6-m) level at CFA ranged from $5.1 \mathrm{mph}(8.2 \mathrm{kph})$ in December to $9.3 \mathrm{mph}$ (15 kph) in March and April for the period 1950 through 1964. The highest hourly average wind speed recorded for CFA at the 20-ft (6-m) level was $67 \mathrm{mph}(108 \mathrm{kph})$, and the maximum instantaneous gust at the same level was $78 \mathrm{mph}(125.5 \mathrm{kph})$. 
Atmospheric pressure data have been recorded nearly continuously at CFA since February of 1950. The data indicate that the average pressure at the station is $25.06 \mathrm{in} .(63.7 \mathrm{~cm})$ of mercury. The monthly average atmospheric pressure ranges from 24.98 in. $(63.45 \mathrm{~cm}$ ) (April) to 25.13 in. $(63.83 \mathrm{~cm})$ (December). The extreme lowest and highest atmospheric pressures ever recorded were 24.26 and 25.69 in. (61.62 and $65.25 \mathrm{~cm})$, respectively.

\subsubsection{Geology}

CFA Landfills II and III are located on the ESRP, a low-lying volcanic region of basalt lava beds within the rugged, basin and range mountains of southern Idaho (Eaton 1982; Malde 1991; and Hackett and Smith 1992) (see Figure 2-5). Extension of the earth's crust produced normal faults and uplift of the basin and range mountains that surround the ESRP. Although it is surrounded by a parabolic region of mountain uplift and historical seismicity, as shown by many small earthquakes, and by large earthquakes in 1959 (Hebgen Lake, Montana) and 1983 (Borah Peak, Idaho) (Anders et al. 1989), the ESRP and its margins are relatively aseismic.

The CFA landfills are located on Big Lost River alluvial deposits (Figure 2-6), consisting of sands, gravels, silts, and clays. The characteristics of surficial sediments near the CFA landfills, including their mineralogical compositions, cation-exchange and sorption capacities, and grain-size characteristics are summarized in Ansley et al. (1988). In some places, the surficial sediments are composed of two layers: an upper, relatively coarse layer of sands and gravels 10 to $40 \mathrm{ft}(3$ to $12 \mathrm{~m})$ thick, and a lower, clay-rich layer [0 to $8 \mathrm{ft}(0$ to $2.4 \mathrm{~m})$ thick] above bedrock. Table $2-2$ indicates where clay was observed during drilling of shallow boreholes. Complete borehole logs are included in Appendix D.

Geologic cross sections through the CFA landfills are: (Figure 2-7) show a number of basalt lava flows and thin sedimentary interbeds in the vadose zone. Basalt lava-flow groups, separated by sedimentary interbeds, are composed of numerous basalt lava flows that erupted from one or more vents. From bottom to top, each lava flow is typically composed of a basal zone of highly permeable rubble; a lower vesicular zone; a dense, massive, and jointed central zone; an upper vesicular zone; and a cap of slabby lava crust. Interbedded sediments are largely composed of fine-grained silts of eolian origin and clays, silts, sands, and relatively uncommon gravels deposited by streams such as the Big Lost River. Subsurface sedimentary interheds are lithologically similar to surficial sediments, and past depositional processes and systems are therefore inferred to have been similar to those of recent times. Table 2-3 indicates where interbed clay was observed during drilling of monitoring wells around Landfills II and IIl. Complete well logs are included in Appendix D. Interbeds may be important in protecting the aquifer from contamination, especially when clays are the dominant fraction. Such interbeds will impede the downward migration of water and contaminants to the water table by virtue of their very low permeability and high adsorptive capacity. However, many of the interbeds shown in Figure 2-7 and observed in the wells at the landfills are thin and discontinuous, confounding subsurface correlations between drillholes. 


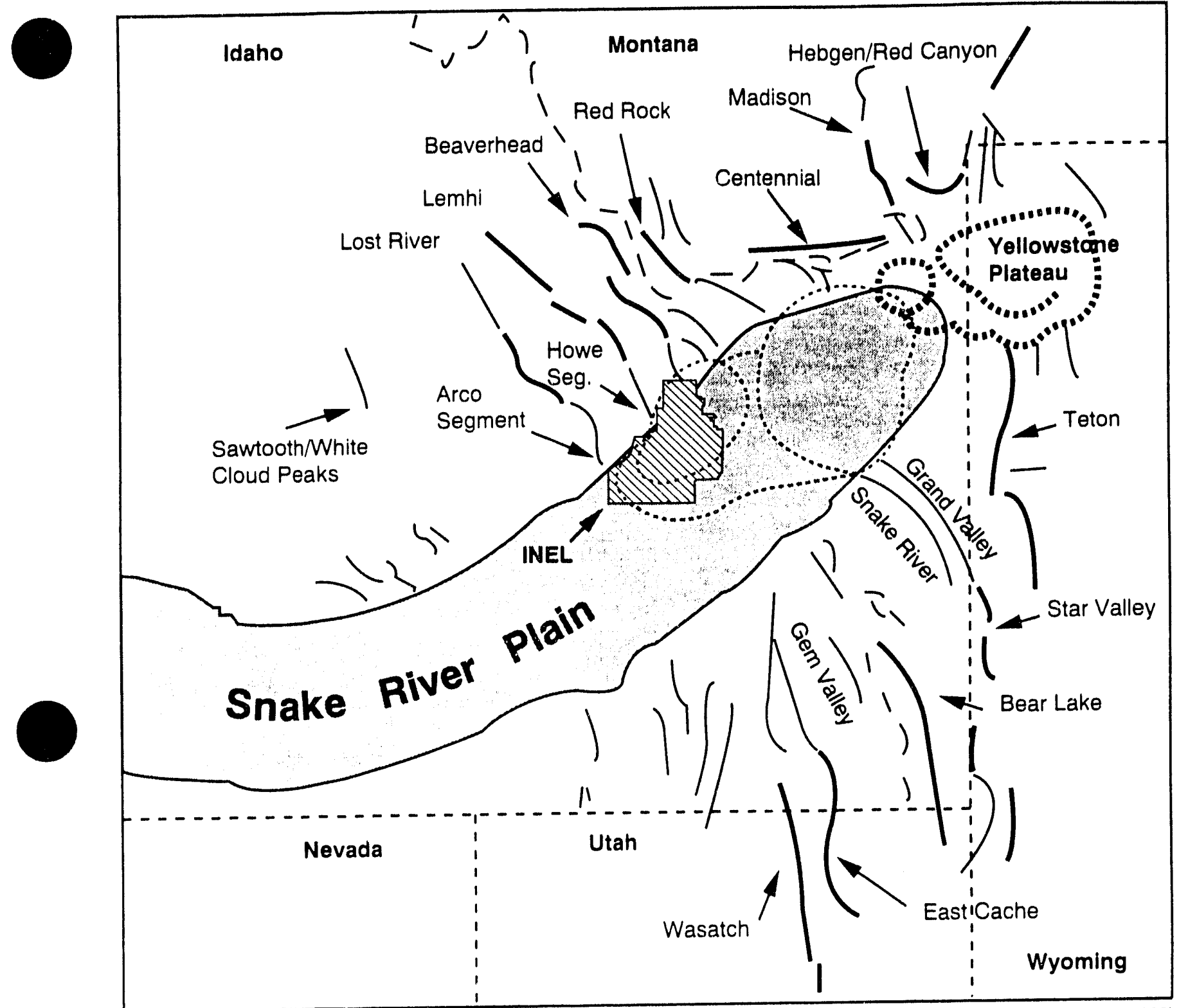

(Map modified from Anders et al., 1989 and Hackett and Morgan, 1988)

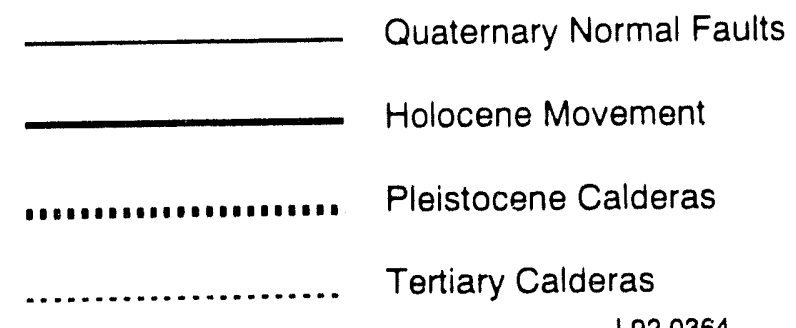

L92 0364

Figure 2-5. Location of the INEL within the context of southern Idaho geologic leatures. 


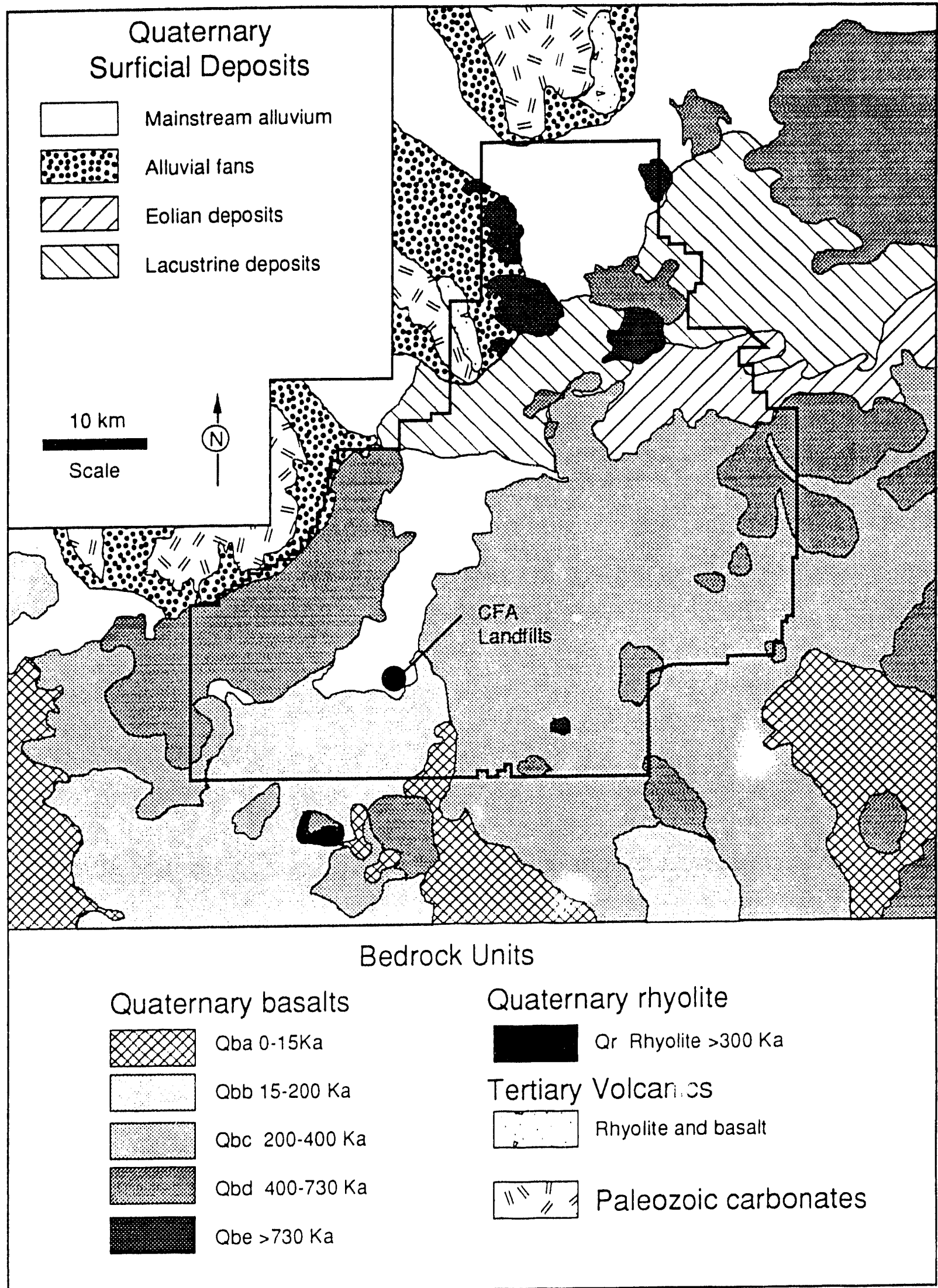

L930421

Figure 2-6. Gencralized geologic map of the IN: . area, showing the surficial distribution of major basalt-lava-flow groups and sedimentary deposits (Kuntz et al. 1990; Scott 1982). 
Table 2-2. Depths of clay in surficial sediments observed in shallow boreholes at Landfills II and III.

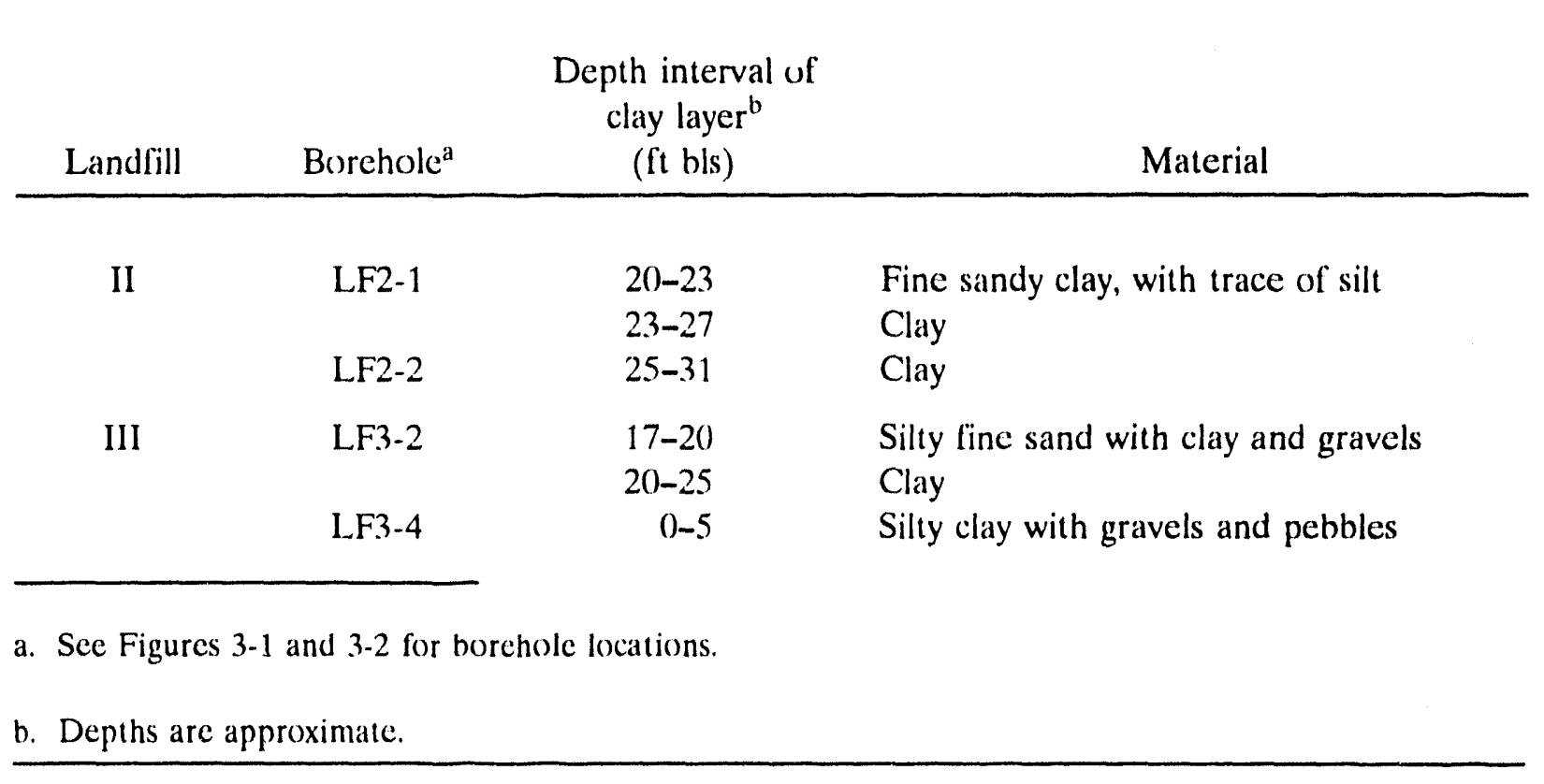




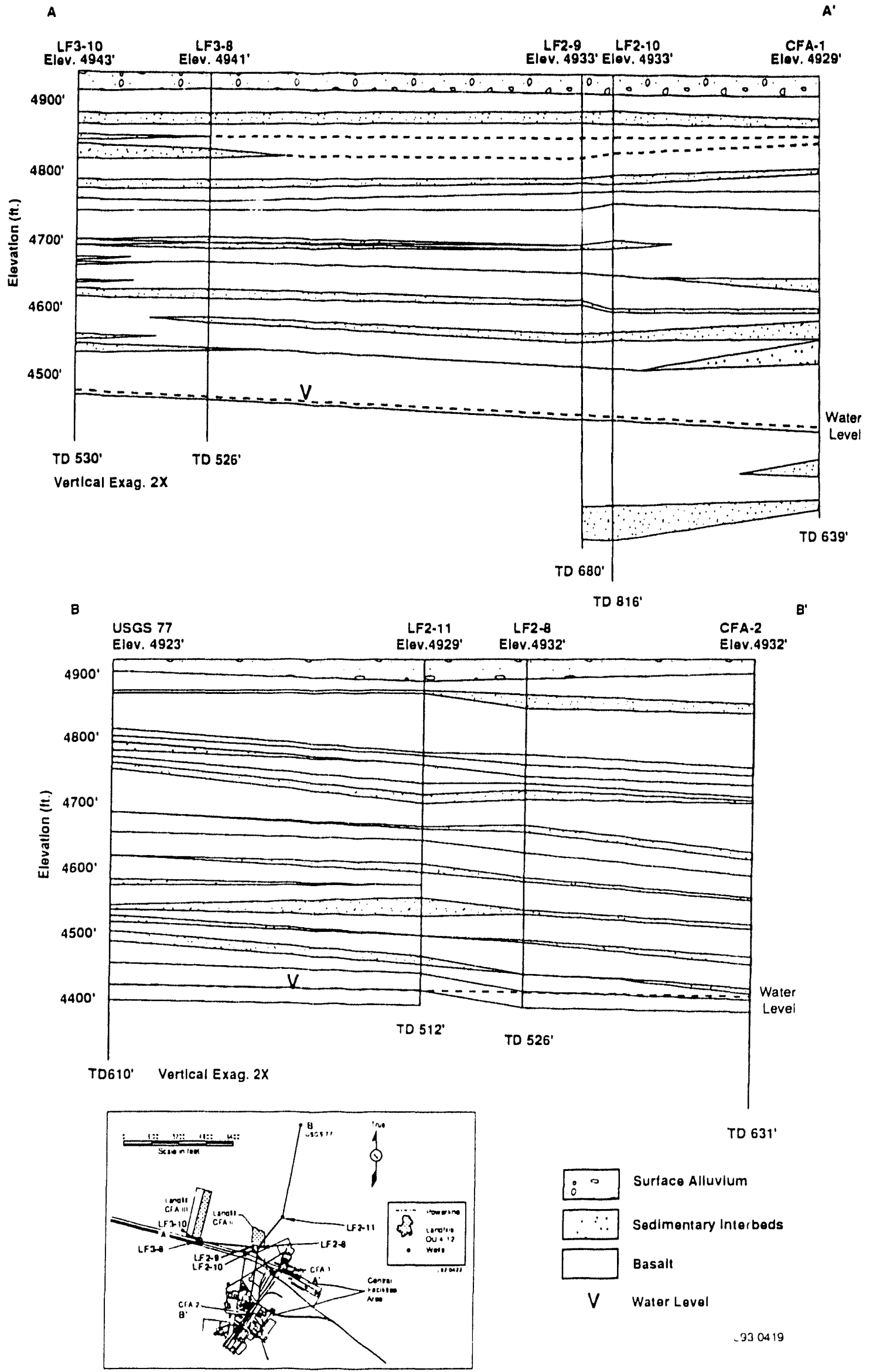

Figure 2-7 Strategic cross sections $A-A^{\prime}$ and $B-B^{\prime}$ through the $C F A$ landfills. 
Table 2-3. Depths of clay in sedimentary interbeds observed in monitoring wells at Landfills II and III.

\begin{tabular}{|c|c|c|c|}
\hline Landfill & $\begin{array}{c}\text { Monitoring } \\
\text { well }^{\mathrm{a}}\end{array}$ & $\begin{array}{l}\text { Depth interval of } \\
\text { clay layer } \\
\text { (ft bls) }\end{array}$ & Material \\
\hline \multirow[t]{4}{*}{ II } & LF2-08 & $\begin{array}{l}185-200 \\
372-385\end{array}$ & $\begin{array}{l}\text { Clay } \\
\text { Sandy, clayey silt }\end{array}$ \\
\hline & LF2-09 & $\begin{array}{r}45-65 \\
370-385 \\
625-645\end{array}$ & $\begin{array}{l}\text { Sand, clay } \\
\text { Silt and clay } \\
\text { Silt and clay }\end{array}$ \\
\hline & LF2-10 & $\begin{array}{r}50-65 \\
148-149\end{array}$ & $\begin{array}{l}\text { Clay with trace of silt and sand } \\
\text { Clay }\end{array}$ \\
\hline & LF2-12A & $195-197$ & Clay, sandy \\
\hline \multirow[t]{5}{*}{ III } & LF3-08 & $\begin{array}{l}150-167 \\
185-200\end{array}$ & $\begin{array}{l}\text { Silt/clay } \\
\text { Silt/clay }\end{array}$ \\
\hline & LF3-10 & $\begin{array}{r}55-70 \\
90-97 \\
150-190 \\
240-250\end{array}$ & $\begin{array}{l}\text { Sand, cinders changing to sand with } 25 \% \text { clay } \\
\text { Sand with } 20 \% \text { clay } \\
\text { Sand with } 0-3 \% \text { clay } \\
\text { Sand with } 20-30 \% \text { clay }\end{array}$ \\
\hline & LF3-11 & $405-415$ & Sand with silt and clay \\
\hline & LF3-11A & $\begin{array}{l}128-135 \\
190-192 \\
352-362 \\
410-420\end{array}$ & $\begin{array}{l}\text { Clay, silty with basalt } \\
\text { Clay/silt } \\
\text { Sand, clay } \\
\text { Sand with clay and silt }\end{array}$ \\
\hline & USGS-85 & $\begin{array}{r}55-65 \\
95-100 \\
145-165 \\
170-200 \\
298-302 \\
345-355 \\
515-520 \\
612-622\end{array}$ & $\begin{array}{l}\text { Clay } \\
\text { Clay and basalt } \\
\text { Basalt and clay } \\
\text { Basalt and clay } \\
\text { Clay } \\
\text { Clay } \\
\text { Broken basalt and clay } \\
\text { Clay }\end{array}$ \\
\hline $\begin{array}{l}\text { See Figur } \\
\text { Depths a }\end{array}$ & $\begin{array}{l}-6 \text { for well locat } \\
\text { pproximate. }\end{array}$ & & \\
\hline
\end{tabular}




\subsubsection{Hydrology}

This section describes the regional and site-specific surface water, vadose zone, and groundwater hydrology.

2.1.3.1 Surface Water. Surface water on the INEL consists mainly of three streams draining intermountain valleys to the north and northwest of the Site: the Big Lost River, the Little Lost River, and Birch Creek (Figure 2-8). The INEL lies within the Pioneer Basin, a closed topographic depression into which these three streams drain. Streamflows are often depleted before reaching the INEL by irrigation diversions, hydropower diversions, and infiltration losses along the channel bed. When water does flow onto the INEL, it either evaporates or infiltrates into the ground because the Pioneer Basin is a closed topographic depression (i.e., it has no outlet).

Streamflows from the Little Lost River a... Birch Creek very seldom reach the INEL and would have no effeet on the CFA landfills. The Little Lost River drains the slopes of the Lemhi and Lost River ranges. Water in the Little Lost River is diverted for irrigation north of Howe, Idaho, and does not llow onto the INEL. Birch Creck originales from springs below Gilmore Summit in the Beaverhead Mountains and llows in a southeasterly direction onto the Snake River Plain. The water in the creek is diverted north of the INEL for irrigation and hydropower purposes. In the winter months when the water is not used for irrigation. llows are returned to a man-made channel on the INEL, $4 \mathrm{mi}(6.4 \mathrm{~km})$ north of Test Area North, where it recharges the Snake River Plain Aquifer by infiltration.

The Big Lost River is the major surface water feature on the INEL. Its waters are impounded and regulated by Mackay Dam, located approximitely $4 \mathrm{mi}(6.4 \mathrm{~km})$ northwest of Mackay, Idaho. The Big Lost River flows from the dam southeastward through the Big Lost River valley, past Arco and onto the ESRP. Streamflows are often depleted before reaching the INEL by irrigation diversions and infiltration losses along the river. When flow in the Big Lost River actually reaches the INEL, it is either diverted at the INEL Diversion Dam (see Figure 2-8) or flows northward across the INEL in a shallow, gravel-filled channel to its terminus at the Lost River sinks where its llow is lost to evaporation and infiltration. Gates placed on two large, corrugated sted culverts, which are $6 \mathrm{lt}(1.8 \mathrm{~m})$ in diameter, control $17 \mathrm{nw}$; less than $90(0) \mathrm{ft}^{3} / \mathrm{sec}$ $\left(25.5 \mathrm{~m}^{3} / \mathrm{sec}\right.$ ) of low is permilled through the diversion dam. downst . Im onto the INEL (Lamke 1969). All flow of the Big Lost River that enters onto the INEL is recharged to the subsurface except for evaporation losses. Recharge effects from the Big Lost River can be very pronounced in the Snake River Plain Aquiler and in perched water beneath the river. Due to the prolonged drought conditions in southeastern Idaho over the last five years and increased irrigation demands for the water upstream, the Big Lost River has not flowed onto the INEL since 1986.

The Big Lost River is approximately $1.5 \mathrm{mi}(2.4 \mathrm{~km})$ northwest of the CFA landfills at its nearest point. The distance of the CFA landfills to the Big Lost River channel, local topography between the CFA landfills and the channel, infiltration rates of the surface alluvium and basalt, and intermittent to nonexistent llows in the Big Lost River channel all suggest that the Big Lost kiver would not have any effect on the landlills. There is no potential impact of the landfills on the Big Lost River either, as runoff from the cover of the landfills infiltrates the desert floor with 


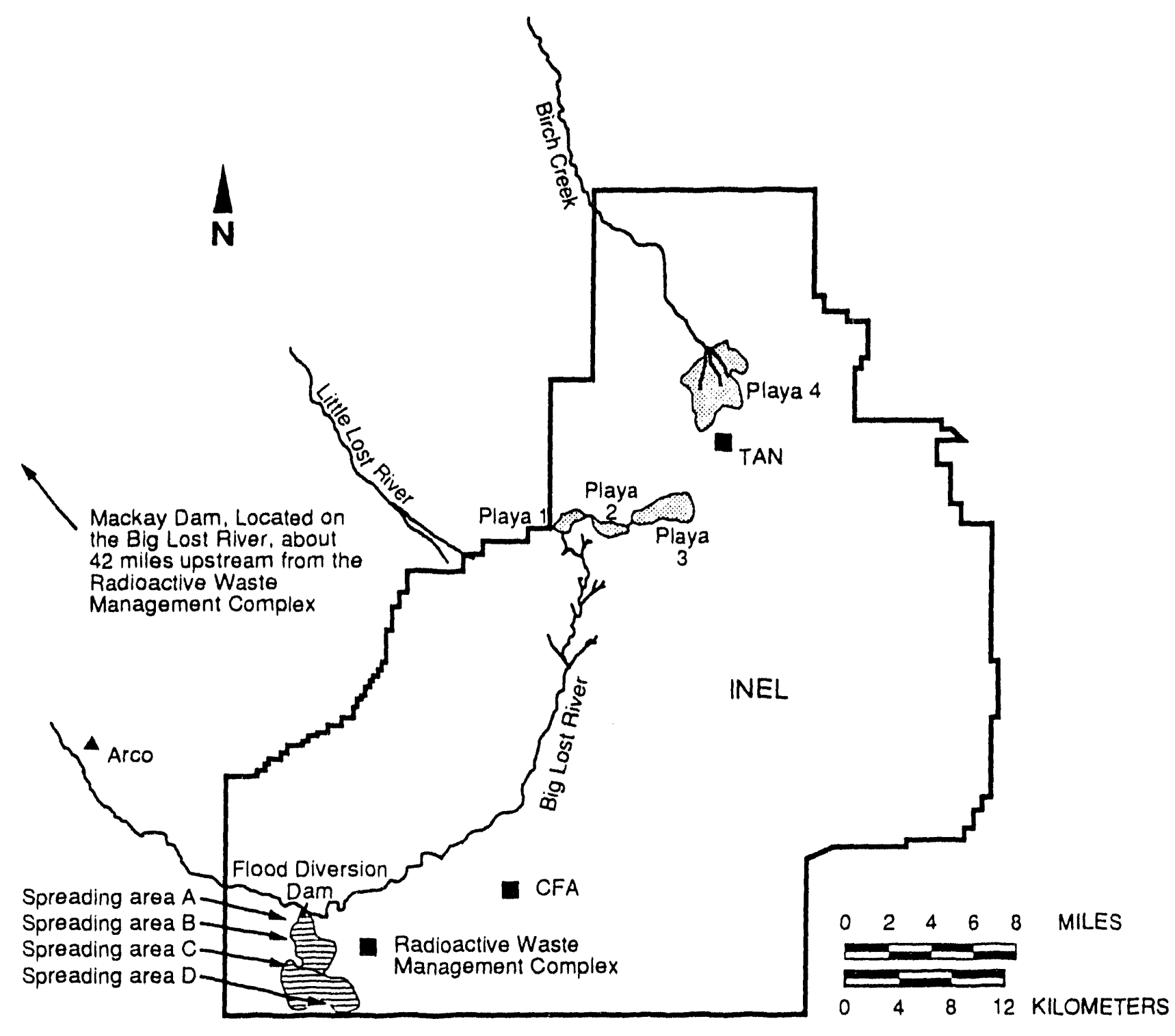

L93 0033

Figure 2-8. Map showing surface water features near or on the INEL. 
no discharge to the Big Lost River. Groundwater at the landfills is at a depth of approximately $480 \mathrm{ft}(146 \mathrm{~m})$ below the landfill surface and does not discharge to the Big Lost River.

Other sources of surface water on the INEL consist of precipitation in the form of rain or snow and the subsequent melting of the snow. Precipitation on the INEL is light, and there is little runoff, even locally, except during heavy rainstorms or rapid snow melting (Nace et al. 1956). The average annual precipitation at CFA is $8.71 \mathrm{in.}(22.12 \mathrm{~cm})$ (Clawson et al. 1989). The evapotranspiration rates are relatively high (greater than $80 \%$ of the available water); therefore, very little water is available to infil ate the surface soil cover of the landfills or to provide significant runon/runoff' (Anderson $\therefore$ al. 1987).

Water that can result in ponding conditions at the INEL may be experienced with the simultancous occurrence of melting snow and spring rains. Snowmelt runoff on the INEL occurs in January, February, and March, while runoff from the surrounding mountains generally occurs in May or June. The onset of a prolonged freeze usually takes place in late November, lasting hree months or morc, and ending in late February or early March. If the ground is frozen when snowmelt occurs, ponding of water may result because the infiltration capacity of the soil is greatly reduced.

2.1.3.2 Vadose Zone. The vadose zone is that portion of the subsurface that extends from the land surface down through the subsurface to the water table. The vadose zone at Landfills II and III is approximately $480 \mathrm{ft}(146 \mathrm{~m})$ thick and is composed of surface sediments, horizontal basalt flows, and interbedded sediments (Figure 2-7). The surface sediments are composed primarily of sands and gravels that are poorly sorted and contain very few fine-grained materials.

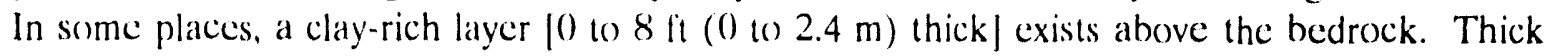
sequences of interfingering basalt flows comprise the bulk of the vadose zone. Considerable variation oceurs in the characteristics of the hasalts. The basalts may be fine or coarse-grained, vesicular or nonvesicular, fractured or jointed. Some fractures and vesicles may be filled with sedimentary material or secondary calcite. Considerable variation also occurs among the sedimentary interbeds found among the basall llows. Some incerbeds are continuous beneath the area of the landfills, while others are thin and discontinuous. These interbeds consist of sands, silts, and clays. They may he compacted due $t 0$ original deposition and subsequent overburden pressures. Interbeds with relatively high clay content may provide some measure of defense against possible migrating leachate from the landfills. Additionally, there are local pockets or lenses of sand, silt, and clay within the lava tlows that were deposited in topographic lows during periods of minimal volcanic activity. Since the average annual precipitation is relatively low $[8.71 \mathrm{in} .(22.12 \mathrm{~cm})]$ and the evapotranspiration rates may be as much as $8(0) .10(1 \%)$ of the precipitation, the potential infiltration rates are expected to be low, less than $1.5 \mathrm{in}$. $(3.8 \mathrm{~cm})$ per year (Anderson et al. 1991).

2.1.3.3 Groundwater. The Snake River Plain Aquifer is one of the largest and most productive groundwater resourees in the United States. The aquiler is listed as a Class I aquifer, and EPA has recently designated it as a sole source aquiler. The Snake River Plain Aquifer is defined as the series of saturated basalt llows and interlayered pyroclastic and sedimentary materials that underlie the ESRP. The Snitie River Plain Ayuifer is approximately $200 \mathrm{mi}$ $\left(322 \mathrm{~km}\right.$ ) long, 40 to $60 \mathrm{mi}(64 \mathrm{t0} 97 \mathrm{~km})$ wide, and covers an area of $9,600 \mathrm{mi}^{2}\left(24,853 \mathrm{~km}^{2}\right)$. It extends from Bliss, Idaho, on the southwest (o) near Ashton. Idaho, northeast of the INEL. 
Aquifer boundaries are formed by contacts with less permeable rocks at the margins of the plain (Mundorff et al. 1964).

Permeability of the aquifer is controlled by the distribution of highly fractured basalt flow tops and interflow zones with some additional permeability contributed by vesicles and intergranular pore spaces. The variety and degree of interconnected water-bearing zones complicates the direction of groundwater movement locally throughout the aquifer (Barraclough et al. 1981). Although a single lava flow may not be a good aquifer, a series of flows may include several excellent water-bearing zones. If the sequence of basalt llows beneath the Snake River Plain is considered to constitute a single aquifer, it is one of the world's most productive (Mundorff et al. 1964).

Robertson et al. (1974) estimated that as much as 2 billion acre-ft of water may be in storage in the aquifer, of which about 500 million acre- $\mathrm{ft}$ are recoverable. The aquifer discharges about 7.6 million acre-ft of water annually to springs and rivers. Pumpage from the aquifer for irrigation totals about 1.9 million acre-ft annually (Hackett et al. 1986). Groundwater, withdrawn from wells and springs, supplies $100 \%$ of the drinking water consumed within the ESRP.

Recharge to the aquifer occurs mostly through infiltration of irrigation water $(5.1$ million acre-ft) and from valley underflow (1.5 million acre-ft) from the $35,000 \mathrm{mi}^{2}\left(90,610 \mathrm{~km}^{2}\right)$ of recharge area in the surrounding mountains to the north and northeast of the plain (Hackett et al. 1986). Recharge from river seepage amounts to about 1.3 million acre-ft, and direct recharge from precipitation falling on the plain is estimated at 0.8 million acre-ft/year (Hackett et al. 1986).

The United States Geological Survey (USGS) has maintained a groundwater monitoring network at the INEL to characterize the occurrence, movement, and quality of water and to delineate the movement of lacility-related wastes in the Snake River Plain Aquifer since 1949. This network consists of a series of wells from which periodic water-level and water-quality data are obtained. Data from the monitoring network are on file at the USGS's INEL Project Office.

While under COCA provisions, nine groundwater monitoring wells were installed upgradient and downgradient from the CFA landfills. These nine wells, the two CFA production wells, and an additional 17 USGS wells will be monitored for water quality and/or water levels during the CFA Landlills II and III RI/FS (see Table 5-1 and Figure 5-6).

The regional tlow bencath the INEL is south-southwest, although the local direction of groundwater flow may be affected by recharge from streams. surface water spreading areas, and inhomogeneities in the aquifer. Across the southern INEL, the average gradient of the water table is approximately $2 \mathrm{ft} / \mathrm{mi}$ or $0.00038 \mathrm{ft} / \mathrm{ft}$ (Lewis and Goldstein 1982). Depth to water varies from about $200 \mathrm{ft}(61 \mathrm{~m})$ in the northeast corner of the INEL to $1,000 \mathrm{ft}(305 \mathrm{~m})$ in the southeast corner. The depth to water at the CFA landfills varies from about $476 \mathrm{ft}(145 \mathrm{~m})$ at LF2-8 to just over 486 ft $(148 \mathrm{~m}$ ) at LF3-8 (Wood et al. 1989a and 1989b). Water table contours for the Snake River Plain Aquiler below the INEL are depicted in Figure 2-9.

Aquifer tests have been conducted on wells completed in the Snake River Plain Aquifer to determine the wells' suitability for water supply and to support regional studies conducted by the 


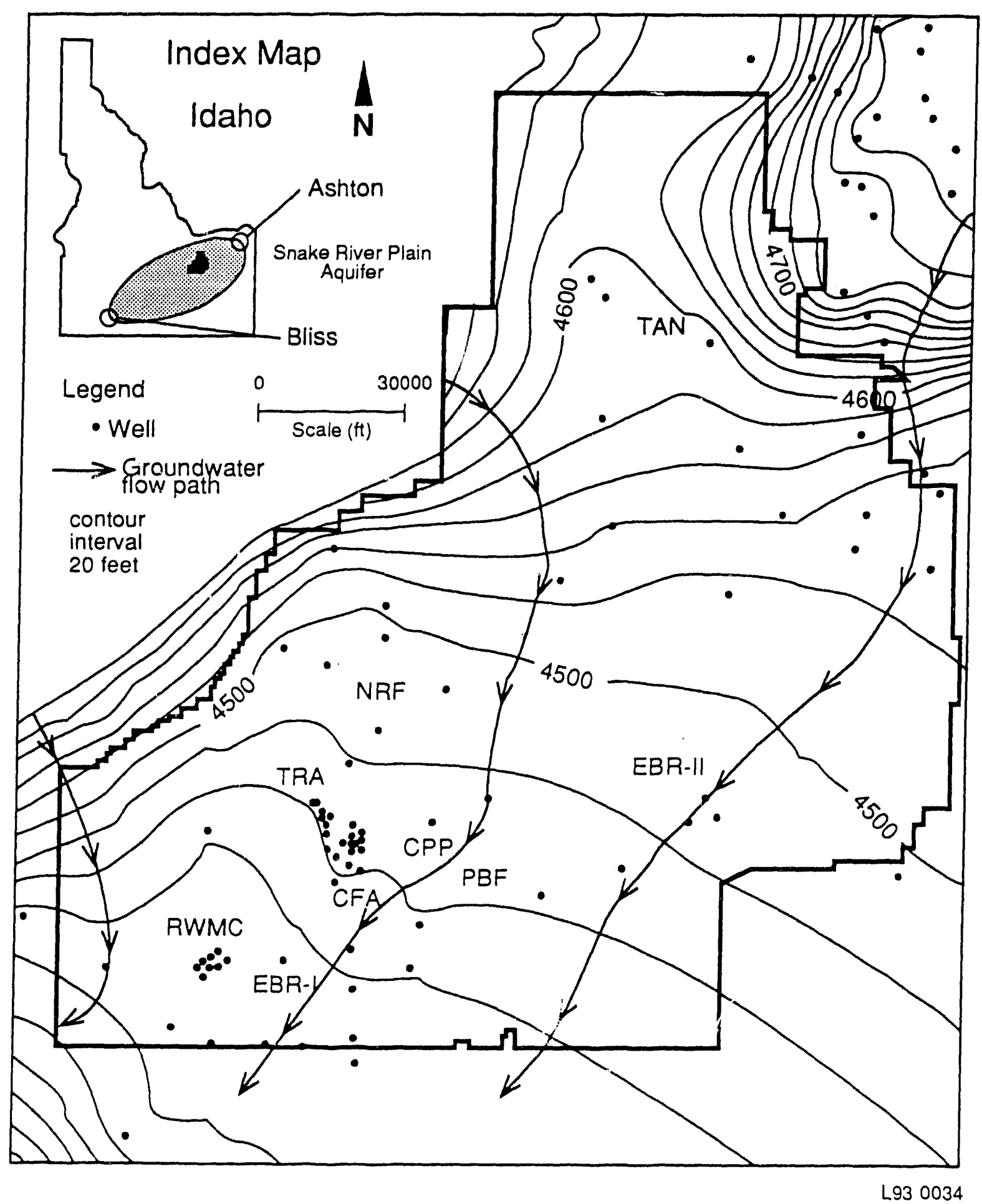

Figure 2-9. Water table map for the Snake River Plain Aquifer below the INEL (Source: Environmental Restoration Information System, May 1989). 
USGS (Mundorff et al. 1964; Wood 1989; and Ackerman 1991). Data from those tests have been compiled and were used to estimate the transmissivity of the aquifer at the CFA landfills. Ackerman's transmissivity calculations range from a low of $10 \mathrm{ft}^{2}$ /day in USGS-114 to a high of $760,000 \mathrm{ft}^{2} /$ day in CPP-4, with a median value of $56,000 \mathrm{ft}^{2} /$ day at USGS-82. This is much lower than the 270,000 to $400,000 \mathrm{ft}^{2} /$ day transmissivity Robertson et al. (1974) estimated for the regional aquifer at the INEL. Perhaps this is due to the short open interval in the wells rather than a local decrease in transmissivity. None of the wells tested fully penetrate the aquifer; therefore, the transmissivity of the local aquifer in the vicinity of CFA may be somewhat higher. The results of the aquifer tests demonstrate that the aquifer is not homogeneous and isotropic, and that there is considerable variation in the transmissivity and hydraulic conductivity at CFA (Table 2-4).

Aquifer storativity has not been calculated for wells in the vicinity of the CFA landfills. Robertson et al. (1974) estimated that the regional aquifer underlying the INEL has a storativity between 0.01 and 0.06 .

Figure 2-10 is a local water table map of the CFA-ICPP area for August 1992. It is apparent from Figure 2-10 that at a small scale and small contour interval the water table surface of the Snake River Plain aquifer is much more complicated than it appears on the regional water table map (Figure 2-9). Low hydraulic heads have been observed consistently in the past in several wells south of the ICPP, suggesting they are constant, not temporal, phenomena. Determining the cause of the hydraulic head variations and estimating the direction and rate of groundwater flow in the Snake River Plain aquifer at the local scale is made difficult by the complexity of the subsurface geology and its interactions with the groundwater.

A relatively simple explanation for the observed variations in hydraulic head may be related to deviations in well plumbness and trueness. Well plumbness is a measure of how close to vertical a well is, and well trueness is a measure of how closely the well approximates a straight line. Deviations in either plumbness or trueness increase the depth to water as measured in the well, because the well does not follow an exact vertical line from land surface to the water table. A deviation of only 5 degrees in well plumbness will add nearly $2 \mathrm{ft}(0.6 \mathrm{~m})$ to the length of a well drilled to a geologic horizon $480 \mathrm{ft}(146 \mathrm{~m})$ bls.

The problem of interpreting observed water level measurements is further complicated by the lack of sufficient well control at local scales and by the various interpretations that can be made of the data. For example, the elevation contours shown in Figure 2-10 are just one interpretation of the measured water level data. Other conligurations of elevation contours may be just as valid and may lead to different conclusions regarding the direction and rate of groundwater flow.

Local complexities in the hydrology and geology, however, appear to be overshadowed by larger-scale, regional effects. Aquifer contaminant plumes originating at ICPP have been monitored over time and mapped by the USGS (Pittman et al. 1988). These observations represent, essentially, the monitoring of large-scale tracer tests. The contaminants have been observed to move from the ICPP to CFA and beyond. The shape and distribution of the plumes suggest that the groundwater moves down the maximum gradient of the regional water table. The direction of groundwater llow in the CFA-ICPP area is in a south south-westerly direction. 
Table 2-4. Transmissivity and hydraulic conductivity values for wells in the CFA area, based on pumping test evaluations. ${ }^{\text {a }}$

\begin{tabular}{|c|c|c|c|c|}
\hline $\begin{array}{l}\text { Well } \\
\text { name }\end{array}$ & $\begin{array}{l}\text { Completion } \\
\text { zone } \\
\text { (ft bls) }\end{array}$ & Date of test & $\begin{array}{l}\text { Transmissivity } \\
\left(\mathrm{ft}^{2} / \text { day }\right)\end{array}$ & $\begin{array}{l}\text { Hydraulic } \\
\text { conductivity } \\
(\mathrm{ft} / \text { day })\end{array}$ \\
\hline CFA-2 & $\begin{array}{l}521-651 \\
661-681\end{array}$ & $2 / 27 / 51$ & 170 & 1.1 \\
\hline CPP-1 & $\begin{array}{l}459.9-485.9 \\
527.4-576.8\end{array}$ & $8 / 12 / 81$ & 73,000 & 970 \\
\hline CPP-2 & $\begin{array}{l}458.3-483.3 \\
551.1-600.25\end{array}$ & $8 / 14 / 81$ & 160,000 & 2200 \\
\hline CPP-3 & $\begin{array}{l}412-452 \\
490-593\end{array}$ & $9 / 27 / 51$ & 760,000 & 7400 \\
\hline USGS-37 & $507-571.5$ & $7 / 7 / 87$ & 16,000 & 250 \\
\hline USGS-40 & $456-678.8$ & $7 / 28 / 87$ & 87,000 & 390 \\
\hline USGS-43 & $450.5-675.8$ & $7 / 29 / 87$ & 80,000 & 360 \\
\hline USGS-51 & $475-659$ & $6 / 26 / 87$ & 2,900 & 16 \\
\hline USGS-57 & $477-732$ & $6 / 24 / 87$ & 28,000 & 110 \\
\hline USGS-76 & $457-718$ & $6 / 10 / 87$ & 190,000 & 750 \\
\hline USGS-82 & $469-561$ & $6 / 26 / 87$ & 56,000 & 610 \\
\hline USGS-111 & $440-600$ & $5 / 20 / 87$ & 22 & 0.16 \\
\hline USGS-112 & $432-56.3$ & $5 / 26 / 87$ & 64,000 & 670 \\
\hline USGS-113 & $445-564$ & $6 / 1 / 87$ & 190,000 & 2000 \\
\hline USGS-114 & $440-564$ & $5 / 21 / 87$ & 10 & 0.10 \\
\hline USGS-115 & 44()$-581$ & $5 / 22 / 87$ & 32 & 0.26 \\
\hline USGS-116 & $400-580$ & $5 / 29 / 87$ & 150 & 1.18 \\
\hline
\end{tabular}




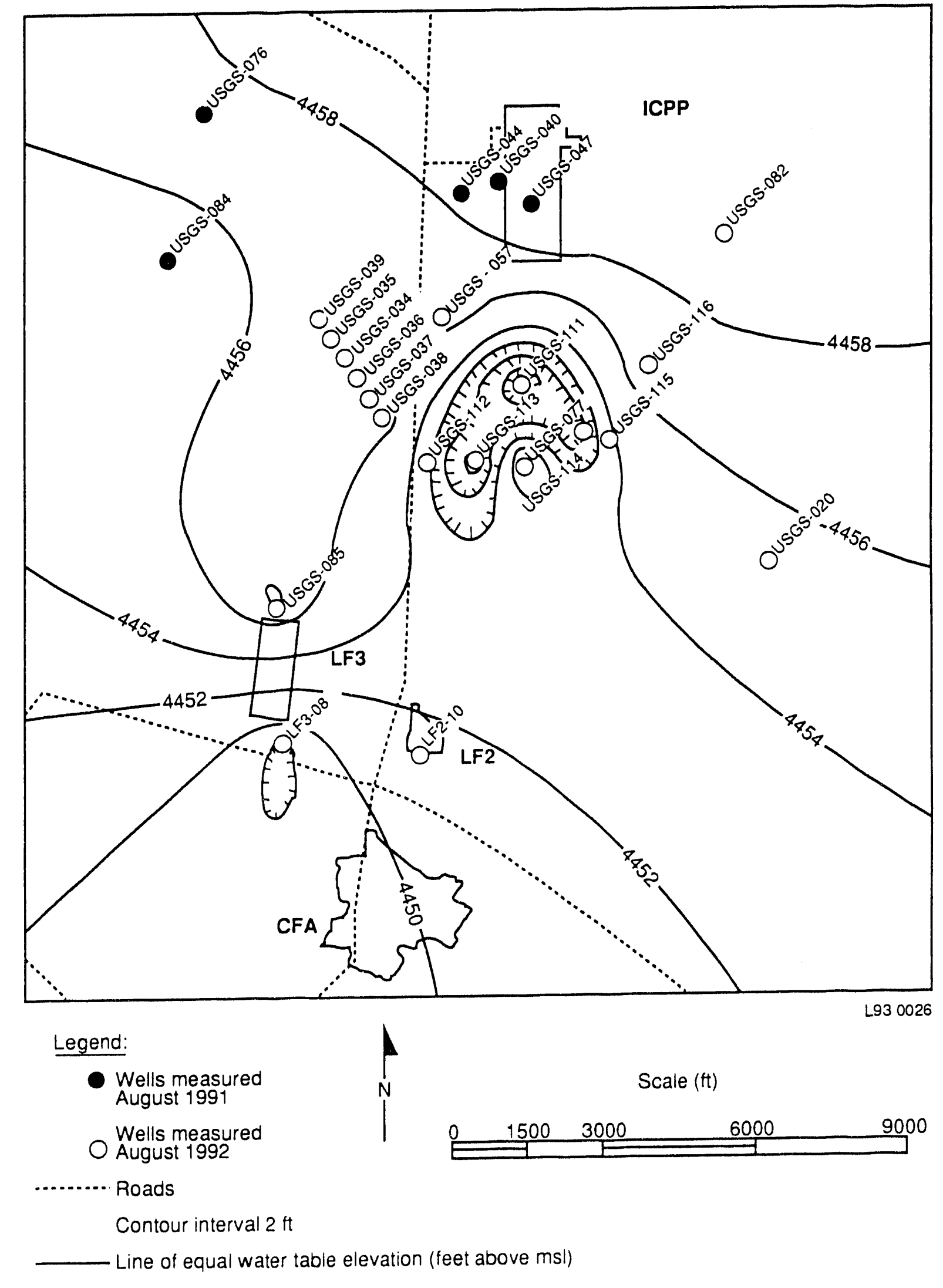

Figure 2-10. CFA/ICPP water table, August 1992. 
Groundwater flow velocity in the vicinity of the CFA landfills has been calculated using contaminants as tracers. Barraclough et al. (1967) used a peak tritium discharge to the ICPP disposal well in December 1961 to calculate groundwater flow rates south of ICPP. Based on the arrival of this peak at USGS-85, just north of Landfill III, Barraclough et al. (1967) calculated an average groundwater flow rate of about $11 \mathrm{ft} /$ day $(3.4 \mathrm{~m} /$ day $)$ between the ICPP disposal well and USGS-85. Because of apparent problems associated with well plumbness and trueness, the direction of groundwater flow and gradient cannot be accurately determined; consequently, the rate of flow cannot be calculated using Darcy's law.

There is an apparent decrease in head across the sedimentary interbed from 630 to $675 \mathrm{ft}$ (192 to $206 \mathrm{~m}$ ). The average difference in water levels across the interbed is $2.34 \mathrm{ft}(0.7 \mathrm{~m})$, as measured in wells LF2-8 and LF2-9, when compared with LF2-10. A 2.34-ft (0.7-m) difference is about what would be expected if LF2-8 and LF2-9 are nearly plumb and true, and if LF2-10 deviates about 5 degrees from vertical.

Water in the Snake River Plain Aquifer shows a chemical composition reflecting the source area of the recharge (Robertson et al. 1974). Recharge from the north and northwest is derived from clastic and carbonate sedimentary rocks and is, therefore, a calcium bicarbonate-type water. Recharge from the east is derived from silicious volcanic rocks and is somewhat higher in sodium, fluoride, and silica. Groundwater at the CFA landfills is of the calcium bicarbonate-type indicative of recharge from the north and northwest.

Documented instances of groundwater degradation at the INEL have occurred from past waste disposal practices. For example, radionuclide and chemical constituents detected in the Snake River Plain Aquifer include tritium, strontium-90, cobalt-60, cesium-137, plutonium-238, plutonium-239, plutonium-240 (undivided), americium-241, total chromium, sodium, chloride, and nitrate (Orr and Cecil 1991). Locally, a tritium plume and elevated total chromium have been detected in the groundwater collected from monitoring wells upgradient and downgradient from the CFA landfills. The source of this groundwater contamination is probably due to past waste disposal practices at ICPP and TRA, two facilities upgradient of the CFA landfills. From 1952 to 1988, about 30.900 $\mathrm{Ci}$ of tritium contained in waste water from ICPP and TRA operations, was disposed to wells and infiltration ponds at these facilities (Mann and Cecil 1990). From 1952 to 1964, an estimated 11,000 kilograms of chromium were contained in wastewater disposed to an unlined infiltration pond at TRA and from 1965 to 1972, an estimated 14,100 kilograms of chromium were contained in wastewater injected directly into the Snake River Plain aquifer through a disposal well (Mann and Knobel 1988). Continued monitoring and remediation of this groundwater contamination will be addressed in the INEL Site-wide RI/FS and is outside the scope of this operable unit.

The sources of drinking water for site employees at CFA consists of two production wells (CFA-1 and CFA-2), both downgradient of the CFA landfills. Water from these wells meets Federal drinking water standards based on groundwater quality results for March 1993. Tritium was detected in CFA-1 at 16,700$) \mathrm{pCi} / \mathrm{ml}$ and in CFA-2 at 15,700$) \mathrm{pCi} / \mathrm{ml}$. Trichloroethylene was detected at 0.6 and $1.0 \mu \mathrm{g} / \mathrm{L}$ in CF.4-1 and CFA-2, respectively. The drinking water standards for tritium and trichloroethylene are $21.100 \mathrm{pCi} / \mathrm{ml}$ and $5 \mu \mathrm{g} / \mathrm{L}$, respectively. The groundwater at the CFA landfills is not used as a public drinking water source. 


\subsubsection{Ecology}

The INEL is located in a sagebrush ecosystem of the kind commonly found in the cold desert region of the Great Basin. The sagebrush ecosystem is characterized by shrubs with an understory of perennial grasses and forbs (Anderson et al. 1978). The INEL includes over 20 vegetation communities and almost 400 plant species. All plant species at the INEL have been compiled in a computer data system (Floyd and Anderson 1983), and additional plant summaries are available for the Site (Cholewa and Henderson 1983; Floyd and Anderson 1983; Marlette and Anderson 1983; Arthur et al. 1983; McBride et al. 1978; and Shumar 1983).

The INEL also supports a diverse population of birds, mammals, amphibians, and reptiles. These fauna have been extensively studied, and the reader is referred to available documents for detailed results of this research (Connelly 1982; Connelly and Ball 1983; Craig et al. 1979, 1983; Gates 1983; Gleason 1978; Groves and Keller 1983; Halford and Markham 1983; Johnson and Anderson 1983; Markham 1987; McBride et al. 1978; Peterson and Best 1983; Reynolds and Rose 1978; Stafford and Barr 1983; Stafford 1984; Stoddart 1983; Wilde and Keller 1978; Arthur et al. 1983). In a 1985-1986 breeding bird survey, it was found that a greater species diversity and density of birds was observed near INEL facilities than is typically found in and around the INEL Site due to the presence of lawns, deciduous trees, and ponds that attract waterfowl, shorebird species, and other birds (Markham 1987).

The bald eagle (Haliacetus leucocephalus) and the peregrine falcon (Falco peregrinus) are the only animals observed on the INEL that are classified by the U.S. Fish and Wildlife Service as endangered (FWS 1992). Bald eagles winter on or near the INEL. The peregrine falcon has been observed infrequently in the northern portion of the INEL. The ferruginous hawk (Buteo regalis), long-billed curlew (Numenius americanus), and Townsend's big-eared bat (Plecotus townsendi) are candidate species for the list of threatened and endangered species that appear on the INEL (FWS 1992). The merlin (Faleo columbarius), which is considered a rare breeding and year-round resident species (Reynolds et al. 1986), is listed as a species of special concern in Idaho (Moseley and Groves 199()). In addition, the plant species Oxytheca (Oxytheca dendroidea Nutt.), found at CFA and to the northeast (Cholewa and Henderson 1984), is currently listed by the State of Idaho as imperiled (Moseley and Groves 1990). There are no known species listed by the U.S. Fish and Wildlife Service (5) CFR 17.11, 17.12) as endangered or threatened that reside year-round on the INEL, and no known critical habitats exist (Reynolds et al. 1986; FWS 1992).

\subsubsection{Archaeology}

The borrow pit area northeast of CFA was surveyed for cultural resources in July 1985 (Reed el al. 1987). The survey covered 340 acres (1.38 hectares) in the western portion of Section 31, T.N R30E, the southeast quarter of Section 36, T3N, R29, and the northeast quarter of Section 1, T2N R29E, Butte County. The area is bounded on the west by Lincoln Blvd., on the north and east by a dirt road, and on the south by the CFA substation. Government railroad tracks dissect the eastern hall of the areat. The unexcavated portions were surveyed in 5()-ft $(15-\mathrm{m})$ transects. and the interiors of the pits and the exposed wall profiles were spot-checked for archaeological materials. No cultural resourees were encountered in the course of the survey, and archacological clearance of the area was recommended. 


\subsection{Waste Description}

This section summarizes the waste disposal practices at OU 4-12: CFA Landfills II and III and provides information on the types and volume of waste disposed to these landfills. Waste at OU 4-12 consists of wastes disposed to Landfill II from 1970 to 1982 and to Landfill III from 1982 to 1984 . The waste description has been determined from the Industrial Nonradioactive Waste Management Information System (INWMIS), formerly referred to as the Industrial Waste Management Information System (IWMIS), interviews with knowledgeable individuals, reports, and other information related to waste disposal at Landfills II and III.

\subsubsection{Waste Disposal Practices}

Landfill II was operational from September 1970 to September 1982. It occupies the southwest corner of an existing gravel pit that was opened up in the early 1950s (Figure 2-11). Waste disposal began in September of 1970 in the far southwes: vorner of the pit. It was standard practice for a single operator to be assigned to the landfill during the day to receive and log in waste. Waste from INEL facilitics or subcontractors was placed in the landfill randomly or in "low spots," and was then compacted by a D-8 Caterpillar tractor into layers or cells that were 12 to $24 \mathrm{in.} \mathrm{(30} \mathrm{to} 61 \mathrm{~cm}$ ) thick. The compacted waste was covered with at least 6 to $8 \mathrm{in}$. (15 to $20 \mathrm{~cm}$ ) of coarse soil material (sandy gravel) at the end of the day. Material for the intermediate cover was scraped from the bottom of the pit and from a previously unexcavated area north of the landfill. After the landfill operation eeased, overburden material, previously stockpiled during the opening of the pit, was used for cover material. Cover material thickness ranges from 6 in. $(15 \mathrm{~cm})$ to over $3 \mathrm{ft}(0.9 \mathrm{~m})$ and consists predominantly of sand and gravel, with measured saturated hydraulic conductivities ranging from 0.002 to $0.0025 \mathrm{~cm} / \mathrm{sec}$. (Miller et al. 1990). Total thickness of the waste is 12 to $15 \mathrm{ft}(3.7$ to $4.6 \mathrm{~m})$ in the south and slightly thinner toward the north. The landfill was lilled from the bottom of the pit up to the present elevation and covers approximately 14 acres (6 hectares). Since waste was deposited randomly in low spots, it is impossible to estimate where specific wastes were deposited based on the known dates of disposal recorded in the INWMIS.

Landfill III opened in October 1982 after Landfill II was closed, and operated as a cut-andfill trench until December 1984. The trenches, trending north-south, were cut $24 \mathrm{ft}(7 \mathrm{~m})$ wide, $12 \mathrm{lt}(4 \mathrm{~m})$ deep, and $2.4(0) \mathrm{lt}(7.32 \mathrm{~m}) \mathrm{long}$ (Figure 2-12). A series of six trenches are defined as the closed part of Landfill III, an area of approximately 11 acres (5 hectares), with all areas west of these trenches defined as the still active portion of the landlill. Waste disposal in the closed part of Landfill III ecased in December 1984. Waste was placed in trenches as they were excavated. The eastern-most trench was the first to be excavated and was started from the south end. All other trenches in the landlill were excavated in the same manner.

Solid nonradioactive waste disposed to the CFA landfills was generated by the following facilities: Auxiliary Reactor Area (ARA), CFA, ICPP, Experimental Breeder Reactor (EBR) II, Naval Reactor Facility (NRF). Special Power Excursion Reactor Test (SPERT). Test Area North (TAN), and TRA. Solid waste was collected from the facilities in 4-yd ${ }^{3}, 6-\mathrm{yd}^{3}$, or 8 -yd $\mathrm{d}^{3}$ dumpster containers. Approximately 100 contitiners were distributed throughout these facilities usually in locations designated for nonradioactive waste. The dumpsters were usually collected weekly and 


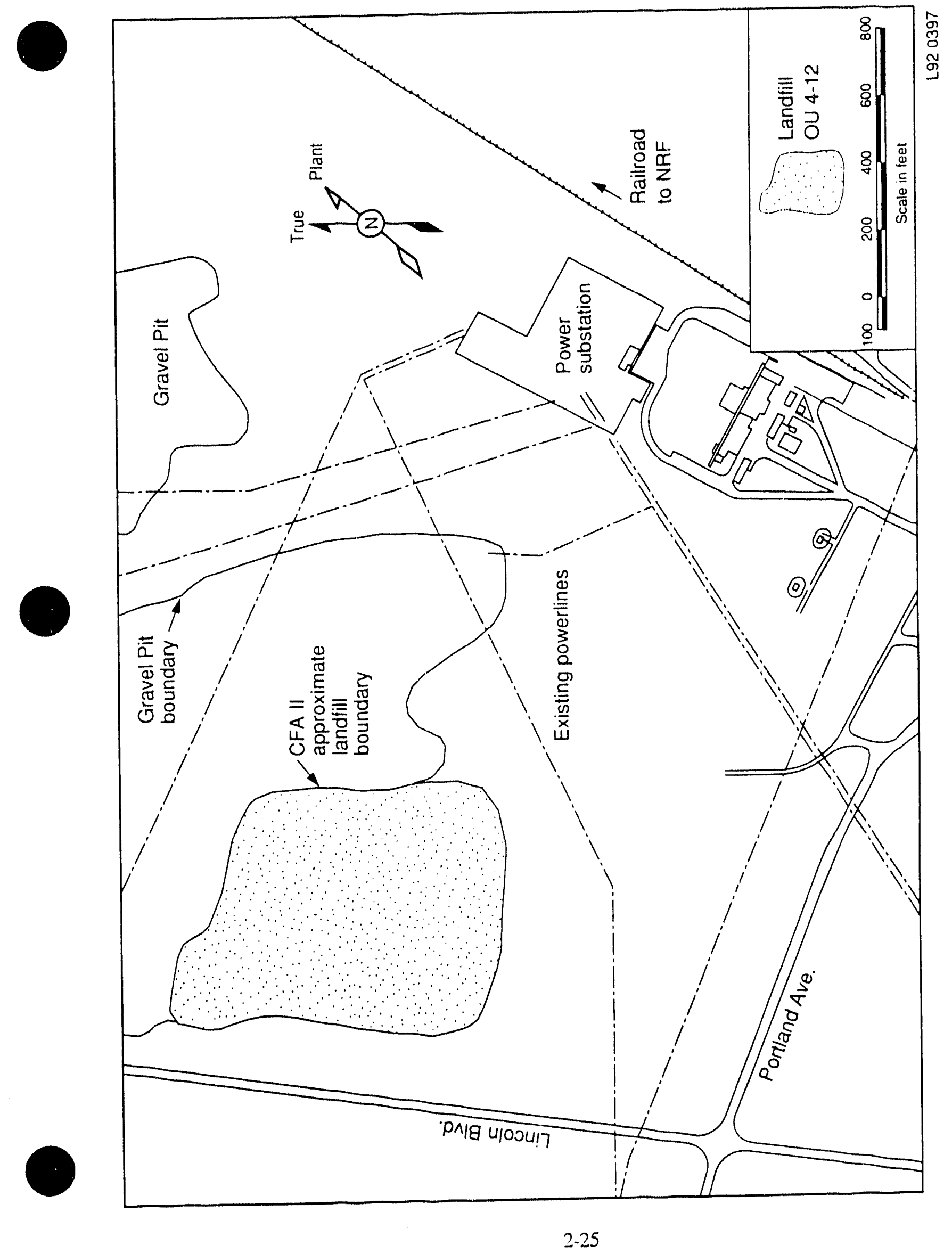

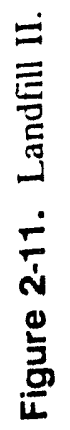




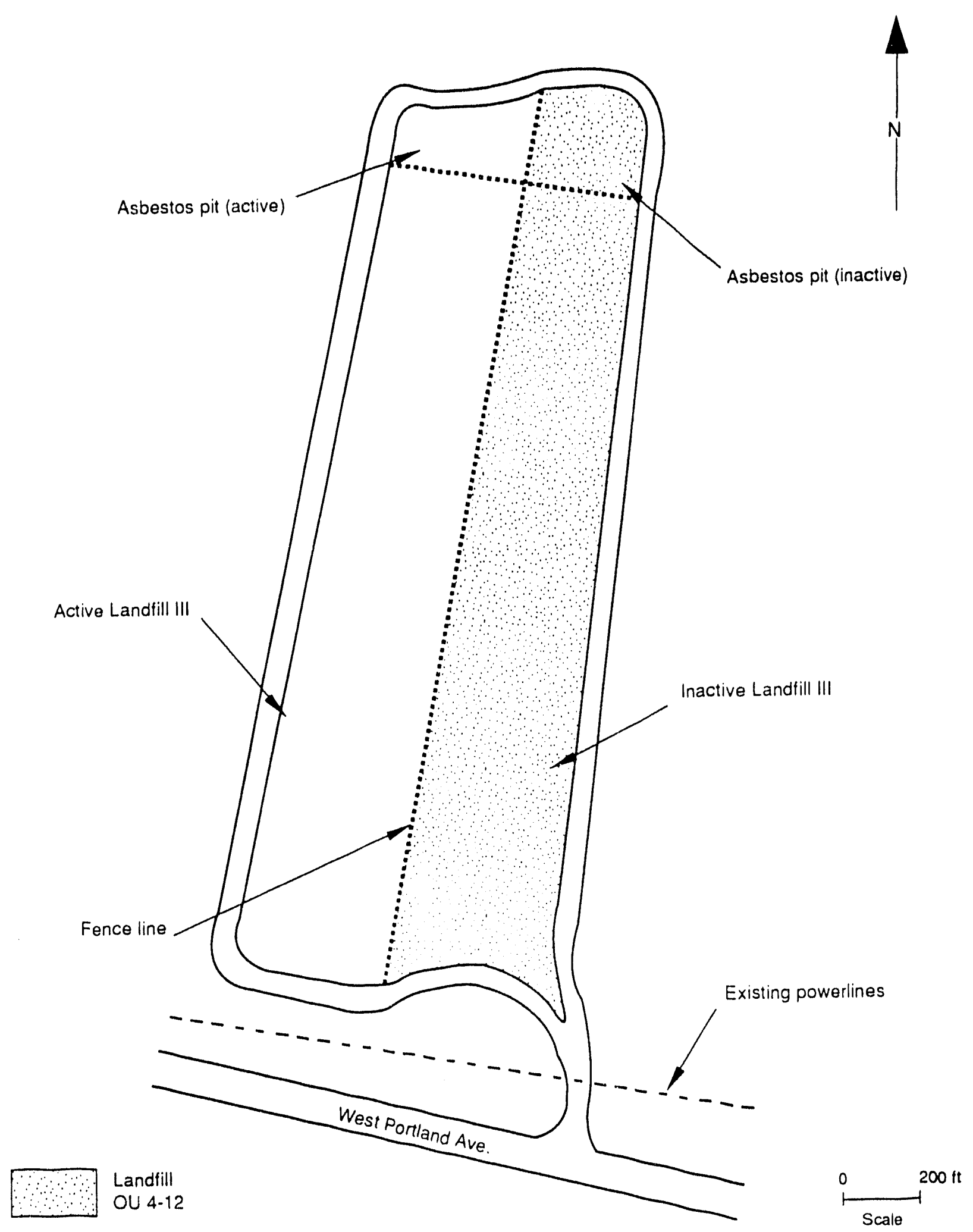

Figure 2-12. Landfill III. 
transported to the CFA landfill for waste disposal. Waste from the cafeterias at ICPP, NRF, and TRA was collected two to three times per week. Cafeteria waste would have been collected in 4 -yd ${ }^{3}$ dumpster containers. Demolition and construction wastes were disposed to the landfill directly by subcontractors responsible for the project. Records show no indication of wastes being segregated within the landfills.

Prior to disposal of any waste material at the CFA landfills, the waste must be screened by a Radiological Control Technician for beta- and gamma-emitting radionuclides and for alphaemitting radionuclides to determine if the waste material is above radioactive background levels. Radioactive background is usually 100 counts per minute (cpm) beta/gamma at the INEL, but will vary among individual site facilities. If the waste material is less than $100 \mathrm{cpm}$ beta/gamma over established background, the waste material is considered nonradioactive and may be disposed of at the CFA landfill. If screening of the waste material results in a reading over $100 \mathrm{cpm}$ over background, the waste may not be disposed of at the CFA landlill. DOE-ID form 130, "Nonradioactive Solid Waste Log," must be lilled out by the individual responsible for disposal of the waste and a record maintained attesting to the fulfillment of the CFA landfill disposal requirement of less than $100 \mathrm{cpm}$ above background, prior to waste disposal. Interviews with former landfill personnel employed during 1970 1o 1984 indicate waste generation, collection and disposal was performed as stated above during this period. There was little change in the process described above until Landfill III was abandoned in 1984.

\subsubsection{Types and Volumes of Waste}

Information regarding waste disposal al Landfills II and III was originally stored in the Industrial Waste Management Information System (IWMIS), which is a computerized data base that was started in 1971. The IWMIS data hase was based on waste summary reports for INEL lacilities that disposed waste to the landlills. The summary reports were based on information from DOE-ID form 130, "Nonradioactive Solid Waste Log," completed by generators of the waste or landfill operators. The original forms no longer exist in the INEL records storage because the retention time on this type of document was likely 3 years, and the forms may not have been submitted for permanent storage.

The amounts or volumes of waste were estimaled at the lime of disposal, and the information was entered on the forms, which were collected by landfill personnel until the end of the month. The boltom copy was then sent to the lacility representative where the waste was generated. The facility summaries were collected and entered in the IWMIS data base. The bottom lorms were difficult $t o$ read which resulted in some lost information. The original data in IWMIS represents monthly totals from each INEL facility disposing waste to the landfills.

The dala base was upgraded and its name was changed 10 INWMIS in 1991 to incorporate greater detail for future disposal records and $t o$ serve as the official record for all types of nonradiological waste stored or disposed at the INEL. INWMIS incorporated data from IWMIS but changed the organization of data in certain waste categories. The INWMIS data base provides a description of the waste, the souree area that the waste came from, the date the waste wass aceepted, the type of waste, and the volume of the waste aleepted. The predominant waste types entering the landlills were construction, oflice, and calceria waste. Review of the INWMIS data base indicates that the major types of waste aceepled at the landfills include trash, cafeteria 
garbage, wood and scrap lumber, masonry concrete, scrap metal, weeds and grass, dirt and gravel, asphalt, and asbestos. "Other" landfill wastes include waste oil, solvents, chemicals, and paint. Table 2-5 lists the nine original waste categories tabulated under the IWMIS and the revised waste categories currently in use under the INWMIS. Where appropriate, original IWMIS data were shifted to the proper category now listed in the INWMIS. IWMIS data, which were originally collected in English units, were converted to metric units in INWMIS. The INWMIS data base (column 2 of Table 2-5) will be used as the main source of information regarding waste disposal at Landfills II and III. Tables 2-6 and 2-7 show, by waste category, the amounts and types of wastes thought to be disnosed to the landfills. The information in Tables 2-6 and 2-7 is from the INWMIS data base an from information gathered from facility records and interviews.

A detailed description of waste disposed to Landfill II by category was made in 1970 (Commander, 1971). As part of this study, a waste collection survey was conducted from September 20 to November 7, 1970, which described characteristics of wastes in INWMIS Category 1-trash and sweepings, and Category 2-cafeteria garbage. The description given to trash and sweepings was, "A mixture of highly combustible waste such as paper, cardboard cartons, wood boxes, and floor sweepings from commercial and industrial activities. The mixture contains up to $10 \%$ by weight of plastic bags, coated paper, laminated paper, treated corrugated cardboard. oily rags, and plastic or rubber scraps. This type of waste contains $10 \%$ moisture and $5 \%$ noncombustible solids..." The description given to cafeteria garbage was, "Garbage such as animal and vegetable wastes from restaurants, hotels, hospitals, markets and similar installations. This type of waste contains up to $70 \%$ moisture and up to $5 \%$ noncombustible solids..." Categories 1 and 2 in INWMIS represent $73 \%$ of the total waste disposed to Landfill II and $85 \%$ of the total solid waste disposed to Landfill III.

Additional information on chemical wastes disposed to the landfills was gathered from reports or interviews and is presented below in greater detail.

Boron Solutions. There are three entries in the data base that indicate boron solutions were disposed in the landfill. These entries are summarized helow.

$\begin{array}{lcc}\text { Date } & \text { Volume (L) } & \text { Source } \\ 01 / 31 / 72 & 1,760.16 & \text { NRF } \\ 02 / 29 / 72 & 567.80 & \\ 05 / 31 / 72 & 5,621.17 & \\ & & \\ \text { Trital } & 7,949.13(2,100 \mathrm{gal}) & \end{array}$

According to NRF personnel and available information. all waste shipments out of the facility to the landfill during this period were carefully monitored to ensure the waste was not radiologically contaminated. Information related to the actual use of boron solutions at NRF is classified. It is well known that boron has been used in the form of boric acid for criticality control reactors. Assuming that a $40 \%$ boric acid solution was used at NRF, the amount of boric acid potentially disposed to the landfill is estimated to be $7,949 \mathrm{~L} \times 0.40=3,179 \mathrm{~L}$ ( $840 \mathrm{gal}$ ). The boron solutions could also have bee: disposed in the landfill before use in a reactor as in the case of a shipment of boric acid (219 kg) from ANL in April 1980. 
Table 2-5. Categories of waste from the IWMIS and INWMIS data bases.

IWMIS

1. Trash $\left(\mathrm{ft}^{3}\right)$

2. Cateteria garbage $\left(\mathrm{ft}^{3}\right)$

3. Wood and scrap lumber $\left(\mathrm{ft}^{3}\right)$

4. Masonry and concretc

5. Scrap metal

6. Oil

7. Solvents

8. Chemicals

Liquids (gal)

Solids (lb)

9. Others

Liquids (gal)

Solids $\left(\mathrm{ft}^{3}\right)$
INWMIS

1. Trash and sweepings $\left(\mathrm{m}^{3}\right)$

2. Cafeteria garbage $\left(\mathrm{m}^{3}\right)$

3. Wood and scrap lumber $\left(\mathrm{m}^{3}\right)$

4. Masonry and concrete $\left(\mathrm{m}^{3}\right)$

5. Scrap metal $\left(\mathrm{m}^{3}\right)$

6. Weeds, grass, and trees

7. Dirt and gravel

8. Asphalt

9. Asbestos

10. Other - must specify

96. Oil (L)

97. Solvents (L)

98. Chemicals

Liquids (L)

Solids $(\mathrm{kg})$ 
Table 2-6. Estimated waste description for CFA Landfill II.

\begin{tabular}{|c|c|c|c|c|c|}
\hline $\begin{array}{l}\text { INWMIS } \\
\text { waste } \\
\text { calegory }\end{array}$ & Type of waste & Source & $\begin{array}{l}\text { Solid } \\
\text { volume } \\
\mathrm{m}^{3} \\
\left(\mathrm{ft}^{3}\right)\end{array}$ & $\begin{array}{l}\text { Solid } \\
\text { amount } \\
\mathrm{kg} \\
\text { (lb) }\end{array}$ & $\begin{array}{l}\text { Liquid } \\
\text { volume } \\
\text { L } \\
\text { (gal) }\end{array}$ \\
\hline 1. Trash & $\begin{array}{l}\text { - office trash, paper, } \\
\text { cardboard, plastic, } \\
\text { glass, etc. }\end{array}$ & $\begin{array}{l}\text { - INEL facilities, dumpster } \\
\text { containers }\end{array}$ & $\begin{array}{c}219,166 \\
(7,738,912)\end{array}$ & & \\
\hline $\begin{array}{l}\text { 2. Cafeleria } \\
\text { garbage }\end{array}$ & $\begin{array}{l}\text { - used grease } \\
\text { - soybean oil } \\
\text { - vegetable oil } \\
\text { - food waste } \\
\text { - up to } 70 \% \text { moisture }\end{array}$ & $\begin{array}{l}\text { - CFA cafeteria, CF-662 } \\
\text { - TRA, TAN, and ICPP satellite } \\
\text { cafeterias }\end{array}$ & $\begin{array}{c}30,986 \\
(1,094,138)\end{array}$ & & \\
\hline $\begin{array}{l}\text { 3. Wood, } \\
\text { scrap lumber }\end{array}$ & $\begin{array}{l}\text { - wood and scrap } \\
\text { lunber } \\
\text { - scrap lumber }\end{array}$ & $\begin{array}{l}\text { - INEL facilities, construction and } \\
\text { demolition subcontractors } \\
\text { - CFA receiving warchouse, CF- } 674 \\
\text { - CFA carpenter shop, CF- } 654\end{array}$ & $\begin{array}{c}14,585 \\
(515,007)\end{array}$ & & \\
\hline $\begin{array}{l}\text { 4. Masonry, } \\
\text { concrete }\end{array}$ & $\begin{array}{l}\text { - used masonry } \\
\text { - used concrete }\end{array}$ & $\begin{array}{l}\text { - INEL facilities, construction and } \\
\text { demolition subcontractors }\end{array}$ & $\begin{array}{c}13,484 \\
(476,130)\end{array}$ & & \\
\hline \multirow[t]{3}{*}{$\begin{array}{l}\text { 5. Scrap } \\
\text { metal }\end{array}$} & - scrap metal & $\begin{array}{l}\text { INI:L facilities, construction and } \\
\text { demolition subcontractors }\end{array}$ & $\begin{array}{c}5,470 \\
(193,149)\end{array}$ & & \\
\hline & $\begin{array}{l}\text { - scrap metal from } \\
\text { welding, pipe fitting } \\
\text { - shect metal } \\
\text { operations }\end{array}$ & $\begin{array}{l}\text { - CFA multicraft shops, CF-621, CI: } \\
622 \\
\text { - CFA machine shop CF- } 640\end{array}$ & & & \\
\hline & $\begin{array}{l}\text { - metal vehicle parts } \\
\text { - wheels, mufflers, } \\
\text { bearings, vehicle } \\
\text { batteries, etc. }\end{array}$ & $\begin{array}{l}\text { CFA vehicle maintenance shop, } \\
C F-(665\end{array}$ & & & \\
\hline $\begin{array}{l}\text { 6. Weeds, } \\
\text { grass, trees }\end{array}$ & $\begin{array}{l}\text { - weeds, grass, and } \\
\text { trees }\end{array}$ & $\begin{array}{l}\text { - } \triangle N L, N R F, \text { TRA, } \triangle R A . C F A, \\
\text { ICPP, WMC } \\
\text { - grounds maintenance waste }\end{array}$ & $\begin{array}{c}711 \\
(25,106)\end{array}$ & & \\
\hline $\begin{array}{l}\text { 7. Dirt, } \\
\text { gravel }\end{array}$ & - dirl, gravel & $\begin{array}{l}\text { - NRI: TRA, ARA, CFA, IC.PP, } \\
\text { PBS: } \\
\text { - construction and demolotion } \\
\text { subcontractors }\end{array}$ & $\begin{array}{c}4,906 \\
(173,234)\end{array}$ & & \\
\hline 8. Asphall & - used asphalt & $\begin{array}{l}\text { - NRE: TRA, ARA, CFA, ICPP, } \\
\text { PBF: } \\
\text { - construction and demolition } \\
\text { subcontractors }\end{array}$ & $\begin{array}{c}1,231 \\
(43,468)\end{array}$ & & \\
\hline 9. Asbestos & $\begin{array}{l}\text { - alsbestos } \\
\text { - ashestos coated } \\
\text { materals such as } \\
\text { pipes. elc }\end{array}$ & $\begin{array}{l}-A N I, N R F, T R A, C F A, P B Y \\
\text { TAN }\end{array}$ & $\begin{array}{c}617 \\
(21,681)\end{array}$ & & \\
\hline
\end{tabular}


Table 2-6. (continued).

\begin{tabular}{|c|c|c|c|c|c|}
\hline $\begin{array}{l}\text { INWMIS } \\
\text { waste } \\
\text { category }\end{array}$ & Type of waste & Source & $\begin{array}{l}\text { Solid } \\
\text { volume } \\
\mathrm{m}^{3} \\
\left(\mathrm{ft}^{3}\right)\end{array}$ & $\begin{array}{l}\text { Solid } \\
\text { amount } \\
\text { kg } \\
\text { (lb) }\end{array}$ & $\begin{array}{c}\text { Liquid } \\
\text { volume } \\
\text { L } \\
\text { (gal) }\end{array}$ \\
\hline \multirow[t]{7}{*}{$\begin{array}{l}\text { 10. Other as } \\
\text { specified }\end{array}$} & $\begin{array}{l}\text { - asphall, concrete, } \\
\text { grass, dirl, sod, rock }\end{array}$ & $\begin{array}{l}\text { - ANL, NRF, TRA, CFA, ICPP, } \\
\text { PBF } \\
\text { - construction and demolition } \\
\text { subcontractors }\end{array}$ & $\begin{array}{c}31 \\
(1,095)\end{array}$ & & \\
\hline & - cans, bottles & - CFA & $\begin{array}{c}1 \\
(35)\end{array}$ & & \\
\hline & $\begin{array}{l}\text { - tar, buckets, plastic, } \\
\text { metal }\end{array}$ & $\cdot A N L$ & $\begin{array}{c}2 \\
(70)\end{array}$ & & \\
\hline & $\begin{array}{l}\text { - barrels, crates, } \\
\text { buckels }\end{array}$ & $-I C P P$ & $\begin{array}{c}14 \\
(494)\end{array}$ & & \\
\hline & $\begin{array}{l}\text { - consıruction } \\
\text { materials, waste, }\end{array}$ & - ANL, NRF, TRA, PBF & $\begin{array}{c}2,443 \\
(86,264)\end{array}$ & & \\
\hline & - misc & $\cdot \operatorname{TRA}$ & $\begin{array}{c}9 \\
(318)\end{array}$ & & \\
\hline & - unknown & - ARA, CFA, ICPP & $\begin{array}{c}203 \\
(7,168)\end{array}$ & & \\
\hline \multirow[t]{3}{*}{ Oil } & waste oil sludge & $\begin{array}{l}\text { - CFA vehicle maintenance shop } \\
\text { sump, CF-66.5 }\end{array}$ & & & $\begin{array}{l}1,529 \\
(404)\end{array}$ \\
\hline & & - NRF equipment & & & $\begin{array}{l}29,215 \\
(7,718)\end{array}$ \\
\hline & - waste oil & $\begin{array}{l}\text { - CFA vehicle maintenance shop, } \\
\text { CF- } 60.5\end{array}$ & & & \\
\hline \multirow[t]{3}{*}{ Solvents } & - paint in cans & - NRF operations & & & $\begin{array}{c}95 \\
(25)\end{array}$ \\
\hline & - paint thinner & & & & $\begin{array}{c}401 \\
(106)\end{array}$ \\
\hline & - solvents & & & & $\begin{array}{l}208 \\
(55)\end{array}$ \\
\hline \multirow[t]{4}{*}{ Chemicals } & - chromate solutions & $\begin{array}{l}\text { - NRF water treatment systems, } \\
\text { secondary reactor coosling system }\end{array}$ & & $\begin{array}{c}0.35 \\
(0.77)\end{array}$ & $\begin{array}{l}24,681 \\
(6,520)\end{array}$ \\
\hline & - boron Solutions & - NRF reactor cosoling systems & & & $\begin{array}{c}7,949 \\
(2,100)\end{array}$ \\
\hline & - morpholine & - NRF solvents & & & $\begin{array}{l}359 \\
(95)\end{array}$ \\
\hline & - boric Acid & - ANI & & $\begin{array}{r}219 \\
(99)\end{array}$ & \\
\hline
\end{tabular}


Table 2-6. (continued).

\begin{tabular}{|c|c|c|c|c|c|}
\hline $\begin{array}{l}\text { INWMIS } \\
\text { waste } \\
\text { category }\end{array}$ & Type of waste & Source & $\begin{array}{c}\text { Solid } \\
\text { volume } \\
\mathrm{m}^{3} \\
\left(\mathrm{ft}^{3}\right)\end{array}$ & $\begin{array}{c}\text { Solid } \\
\text { amount } \\
\text { kg } \\
\text { (lb) }\end{array}$ & $\begin{array}{c}\text { Liquid } \\
\text { volume } \\
\text { L } \\
\text { (gal) }\end{array}$ \\
\hline & - chromium 3 & - ANL & & $\begin{array}{c}268 \\
(591)\end{array}$ & \\
\hline & - zircalloy turnings & $-A N I$ & & $\begin{array}{c}7 \\
(16)\end{array}$ & \\
\hline & $\begin{array}{l}\text { - calcium } \\
\text { hyperchlorate }\end{array}$ & - CFA & & $\begin{array}{c}73 \\
(161)\end{array}$ & \\
\hline & - misc chemicals & - NRF & & $\begin{array}{c}2,190 \\
(4,828)\end{array}$ & \\
\hline & $\begin{array}{l}\text { - methylene } \\
\text { dithiocynate }\end{array}$ & $-N R F$ & & $\begin{array}{c}23 \\
(51)\end{array}$ & \\
\hline & - soda ash & $-N R F$ & & $\begin{array}{c}4,128 \\
(9,100)\end{array}$ & \\
\hline & - mercury & - TAN & & $\begin{array}{c}2 \\
(4)\end{array}$ & \\
\hline & - resin & - TRA, ion exchange resin & & $\begin{array}{c}17,585 \\
(38,733)\end{array}$ & \\
\hline
\end{tabular}


Table 2-7. Estimated waste description for CFA Landfill III.

\begin{tabular}{|c|c|c|c|c|c|}
\hline $\begin{array}{l}\text { INWMIS } \\
\text { waste } \\
\text { category }\end{array}$ & Type of waste & Source & $\begin{array}{l}\text { Solid } \\
\text { volume } \\
\mathrm{m}^{3} \\
\left(\mathrm{ft}^{3}\right)\end{array}$ & $\begin{array}{l}\text { Solid } \\
\text { amount } \\
\text { kg } \\
\text { (lb) }\end{array}$ & $\begin{array}{l}\text { Liquid } \\
\text { volume } \\
\text { L } \\
\text { (gal) }\end{array}$ \\
\hline $\begin{array}{l}\text { 1. Trash, } \\
\text { sweepings }\end{array}$ & $\begin{array}{l}\text { office trash, paper, } \\
\text { cardboard, plastic, glass, } \\
\text { etc }\end{array}$ & $\begin{array}{l}\text { - INEL facilities, dumpster } \\
\text { containers }\end{array}$ & $\begin{array}{c}39,613 \\
(1,398,764)\end{array}$ & & \\
\hline \multirow[t]{2}{*}{$\begin{array}{l}\text { 2. Cafeteria } \\
\text { garbage }\end{array}$} & $\begin{array}{l}\text { used grease - soybean } \\
\text { oil, vegetable oil }\end{array}$ & $\begin{array}{l}\text { - CFA cafeteria, CF- } 662 \\
\text { - TRA, TAN, and ICPP satellite } \\
\text { cafeterias }\end{array}$ & $\begin{array}{c}8,238 \\
(290,890)\end{array}$ & & \\
\hline & $\begin{array}{l}\text { food waste - organic } \\
\text { material with up to } 70 \% \\
\text { moisture }\end{array}$ & $\begin{array}{l}\text { - CFA cafeteria, CF- } 662 \\
\text { - TRA, TAN, and ICPP satellite } \\
\text { cafeterias }\end{array}$ & & & \\
\hline \multirow[t]{2}{*}{$\begin{array}{l}\text { 3. Wood, } \\
\text { scrap lumber }\end{array}$} & wood and scrap lumber & $\begin{array}{l}\text { - INEL facilities, construction and } \\
\text { demolition subcontractors }\end{array}$ & $\begin{array}{c}7,500 \\
(264,830)\end{array}$ & & \\
\hline & scrap lumber & $\begin{array}{l}\text { - CFA receiving warehouse, CF. } \\
674 \\
\text { - CFA carpenter shop, CF- } 6.54\end{array}$ & & & \\
\hline \multirow[t]{2}{*}{$\begin{array}{l}\text { 4. Masonry, } \\
\text { concrete }\end{array}$} & used masonry & $\begin{array}{l}\text { - INEL facilities, construction and } \\
\text { denmolition subcontractors }\end{array}$ & $\begin{array}{c}4,802 \\
(169,562)\end{array}$ & & \\
\hline & used concrete & $\begin{array}{l}\text { - INEL facilities, construction and } \\
\text { demolition subcontractors }\end{array}$ & & & \\
\hline \multirow[t]{3}{*}{$\begin{array}{l}\text { 5. Scrap } \\
\text { Metal }\end{array}$} & scrap metal & $\begin{array}{l}\text { - INEL facilities, construc ion and } \\
\text { demolition subcontractors }\end{array}$ & $\begin{array}{c}1,055 \\
(37,252)\end{array}$ & & \\
\hline & $\begin{array}{l}\text { scrap metal from } \\
\text { welding, pipe fitting, and } \\
\text { sheet metal operations }\end{array}$ & $\begin{array}{l}\text { - CFA multicraft shop, CF- } 621 \\
\text { and } 622 \\
\text { - CFA machine shop, CF- } 640\end{array}$ & & & \\
\hline & $\begin{array}{l}\text { metal vehicle parts such } \\
\text { as wheels, mufflers, } \\
\text { bearings, vehicle } \\
\text { batteries, etc. }\end{array}$ & $\begin{array}{l}\text { - CFA vehicle maintenance shop, } \\
\text { CF-665 }\end{array}$ & & & \\
\hline $\begin{array}{l}\text { 6. Weeds, } \\
\text { grass, trees }\end{array}$ & $\begin{array}{l}\text { - weeds, grass, and trees } \\
\text { from landscape } \\
\text { maintenance operations }\end{array}$ & - INEIL facilities & $\begin{array}{c}3.52 \\
(12,429)\end{array}$ & & \\
\hline $\begin{array}{l}\text { 7. Dirt, } \\
\text { gravel }\end{array}$ & $\begin{array}{l}\text { - dirt and gravel from } \\
\text { maintenance, } \\
\text { construction and } \\
\text { demolition projects }\end{array}$ & - INEL facilities & $\begin{array}{c}1,204 \\
(42,514)\end{array}$ & & \\
\hline 8. Asphalt & $\begin{array}{l}\text { - waste asphalt from } \\
\text { maintenance, } \\
\text { construction and } \\
\text { denolition projects }\end{array}$ & - INLEL facilities & $\begin{array}{c}938 \\
(33,121)\end{array}$ & & \\
\hline
\end{tabular}


Table 2-7. (continued).

\begin{tabular}{|c|c|c|c|c|c|}
\hline $\begin{array}{l}\text { INWMIS } \\
\text { waste } \\
\text { category }\end{array}$ & Type of waste & Source & $\begin{array}{l}\text { Solid } \\
\text { volume } \\
\mathrm{m}^{3} \\
\left(\mathrm{ft}^{3}\right)\end{array}$ & $\begin{array}{l}\text { Solid } \\
\text { amount } \\
\mathrm{kg} \\
\text { (lb) }\end{array}$ & $\begin{array}{l}\text { Liquid } \\
\text { volume } \\
\mathrm{L} \\
\text { (gal) }\end{array}$ \\
\hline 9. Asbestos & $\begin{array}{l}\text { - asbestos } \\
\text { - asbestos coated } \\
\text { materials such as pipes, } \\
\text { etc. }\end{array}$ & - INEL facilities & $\begin{array}{c}103 \\
(3,637)\end{array}$ & & \\
\hline \multirow[t]{9}{*}{$\begin{array}{l}\text { 10. Other - } \\
\text { as specified }\end{array}$} & $\begin{array}{l}\text { - waste asphalt and } \\
\text { gravel }\end{array}$ & $\cdot \mathrm{CFA}$ & $\begin{array}{c}1,401 \\
(49,470)\end{array}$ & & \\
\hline & $\begin{array}{l}\text { - sod, dirl, grass, weeds, } \\
\text { gravel }\end{array}$ & - CFA, ICPP & $\begin{array}{c}111 \\
(3,919)\end{array}$ & & \\
\hline & - dirt, rock & - INEL facilities & $\begin{array}{c}115 \\
(4,060)\end{array}$ & & \\
\hline & - outdated drugs & - CIA dispensary, CF-603 & $\begin{array}{c}1 \\
(35)\end{array}$ & & \\
\hline & - rooting material & - CFA & $\begin{array}{c}4 \\
(141)\end{array}$ & & \\
\hline & - tires & $\begin{array}{l}\text { - CFA vehicle maintenance shop, } \\
\text { CF- } 66.5\end{array}$ & $\begin{array}{c}3 \\
(106)\end{array}$ & & \\
\hline & - barrels & $-I C P P, P B F$ & $\begin{array}{c}157 \\
(5,543)\end{array}$ & & \\
\hline & - buckeı boxes & - ICPP & $\begin{array}{c}1 \\
(35)\end{array}$ & & \\
\hline & - misc & $-I C P P$ & $\begin{array}{c}1.3 \\
(4.59)\end{array}$ & & \\
\hline \multirow[t]{3}{*}{ Sump sludge } & - waste oil sludge & $\begin{array}{l}\text { - CFA vehicle maintenance shop } \\
\text { sump, CF-665 }\end{array}$ & & & $\begin{array}{l}1,530 \\
(404)\end{array}$ \\
\hline & - used oil & $\begin{array}{l}\text { CFA vehicle maintenance shop, } \\
\text { CF- } 60.5\end{array}$ & & & $\begin{array}{l}15 \\
(4)\end{array}$ \\
\hline & - asphalı & $-W M C^{a}$ & & & $\begin{array}{c}379 \\
(100)\end{array}$ \\
\hline \multirow[t]{2}{*}{$\begin{array}{l}\text { Outdated } \\
\text { medications }\end{array}$} & - ouidaled medications & - CFA dispensary, CF-603 & & $\begin{array}{c}3 \\
(7)\end{array}$ & \\
\hline & - sodium nitrate & - TRA & & $\begin{array}{c}187 \\
(412)\end{array}$ & \\
\hline Paint & $\begin{array}{l}\text { - outdaled pain in } 1-10 \\
5 \text {-gal cans }\end{array}$ & $-\mathrm{NRF}$ & & & $\begin{array}{c}65 \\
(17)\end{array}$ \\
\hline
\end{tabular}


Chromate Solutions. There are three entries in the data base indicating chromates or chromate solutions were disposed in Landfill II. These entries are summarized below.

\begin{tabular}{lcl} 
Date & Volume (L) & Source \\
\cline { 2 - 3 } $05 / 31 / 72$ & 624.6 & NRF \\
$11 / 30 / 72$ & $15,898.3$ & \\
$05 / 31 / 73$ & $11,249.1$ & \\
Total & $17,771.7(4,695 \mathrm{gal})$ &
\end{tabular}

Chromate solutions and chromates were typically used in water treatment systems as a corrosion inhibitor. The chromate solutions from NRF were likely used in secondary cooling systems and cooling towers and were not radioactively contaminated. The chromate solution would have been delivered to Landfill II as a liquid in 55-gal drums or as part of solid materials used for spill cleanup. Spills at NRF were typically absorbed with kitty litter (diatomaceaous earth) or rags and sealed in a 55-gal drum. The chromate solutions listed in INWMIS were likely in a solid absorbed form. However, if we assume that all chromates disposed to the landfill were liquid $(17,771.7 \mathrm{~L}$ or $4,695 \mathrm{gal}$ ), with an assumed concentration of $14 \mathrm{ppm}$ (the typical concentration of chromate solutions used in water treatment system for the reactors in use at that time), the total amount of chromium disposed to Landfill II is estimated to be 17,771 $\mathrm{L} \times 14 \mathrm{mg} / \mathrm{L}=248,794 \mathrm{mg}(0.25 \mathrm{~kg})$. Another entry in INWMIS indicates $268 \mathrm{~kg}(591 \mathrm{lb})$ was disposed to Landfill II from Argonne National Laboratory (ANL). It is assumed that similar cleanup, containerization and disposal methods were used for this disposal.

Morpholine. One entry in the data base indicates that $359.6 \mathrm{~L}(95 \mathrm{gal})$ of morpholine was disposed in Landfill II on 07/31/71. Information related to the actual use of morpholine at NRF is classified. Morpholine $\left(\mathrm{C}_{4} \mathrm{H}_{9} \mathrm{NO}\right)$ compounds are used as corrosion inhibitors, antioxidants, plasticizers, viscosity improvers, insecticides, fungicides, local anesthetics, and antiseptics (Merck 1989). Morpholine is also a solvent for resins, waxes, and dyes. According to NRF personnel, morpholine was likely disposed because it had exceeded the recommended shelf life.

Sump Sludge. One entry in the data base indicates disposal of $1,529 \mathrm{~L}$ ( $404 \mathrm{gal}$ ) of sump sludge to Landfill II in October 1982 from Central Facilities Area. The source of the sump sludge was likely the sumps at the vehicle maintenance shop. The sump was periodically cleaned and the sludge was disposed at the landfill. During 1970, $350 \mathrm{gal}$ of Trichlorocthylene (TCE) was used at the CF-655 shop (Hogg, 1971). Trichloroethylene was used in a parts washer in the shop. The overflow from this parts washer went to the sewage system via the sump. Other than the $350 \mathrm{gal}$ of TCE used in 1970, no records exist indicating the quantity of TCE used over time or how often the parts washer overflowed to the sewage system and the sump.

Waste Oil Sludge. Three entries in the data base shown below indicate disposal of waste oil to Landfill II.

$\begin{array}{lll}\text { Date } & \text { Volume (L) } & \text { Source } \\ 066 / 30 / 74 & 13,589.23 & \text { NRF } \\ 07 / 31 / 74 & 4,542.36 & \\ 10 / 31 / 74 & 11,083.36 & \\ \text { Total } & 29,214.72(7,718 \text { gal }) & \end{array}$


The NRF facility summary of waste for 1974 indicates disposal of 7,788 gal of waste oils and solvents. The source of the waste oils and solvents was likely equipment associaled with operations rather than vehicle waste oil because no vehicle servicing occurred at NRF during this period.

Trichloroethylene (TCE). Although TCE was not listed as a known chemical disposed at Landfill II, it is possible that it may have been. This is based on references cited in reports and on interviews. A reference noted that 120 drums $(6,600 \mathrm{gal})$ of TCE may have been disposed in the landfill (ICF Technology 1989). Equipment operaiors also believe that TCE may have reen disposed to the landfill. It is known that $1,325 \mathrm{~L}(350 \mathrm{gal})$ of TCE was used in the CFA shop during 1970 in small cleaning processes and in a circulating-parts washer (Hogg 1971). The parts washer overflowed to the CFA shop sump, which overflowed to the CFA sewer system. Consequently, TCE may have been present in the sump sludge that was disposed to the landfill. TCE was also disposed as spent solvent in small containers to the dumpsters during this period. While the reference to 120 drums cannot be confirmed, it can be assumed that TCE was disposed in the landfills because it was used at the CFA shop and landlill personnel suggest possible disposal.

The overall reliability of the above waste descriptions based on the INWMIS data base, interviews, and reports is considered to be low to medium. Some of the uncertainty in the waste descriptions is discussed below.

- The amounts and volumes of waste reported in INWMIS under all categories may be as much as $30 \%$ low hecause many of the DOE ID-130 forms were impossible to read and the information was not included in the facility summaries that were entered into INWMIS. Because of lost or unreadable records, it may be that other waste types were also disposed at the landfills.

- The total volume of waste reported in INWMIS is greater than the available space in the landfills because the volumes of waste that were reported were estimated, not weighed or measured to ensure accuracy. Also, waste was compacted and covered daily with soil further reducing the total volume, which leads to further uncertainty. The total volume of waste in INWMIS for both landfills indicate that these estimates were $14 \%$ higher than the available space in Landfill II and $80 \%$ higher than the available space in Landiill III.

- The characteristics of wastes in the calcgories labeled "trash and sweepings" and "cafeteria garbage" was taken from a study of wastes disposed to the landfill in 1970. It is uncertain whether the characteristics of these wastes remained constant from 1970 to 1984. Also, liquids disposed to dumpsters in small containers, in some cases, may fall under this category and would have heen included in the total.

Although the reliability of the waste descriptions may $n \cdot l$ be very high, the waste descriptions do indicate the general cattegories of waste typically disposed to these landfills. 


\section{INITIAL EVALUATION}

This section summarizes and evaluates the characterization activities conducted to date at CFA Landfills II and III, presents a preliminary conceptual model for the site, discusses preliminary public health and environmental impacts, presents preliminary remedial action alternatives, and presents preliminary ARARs.

\subsection{Summary and Evaluation of Previous Sampling Activities}

Table $3-1$ is a comprehensive list of sampling and characterization activities conducted at CFA Landfills II and III from 1987 through 1991. The following sections discuss the results of some of these sampling events with supporting data provided in Appendices A through E.

\subsubsection{Soil Gas Sampling}

Soil gas sampling of gas ports installed in boreholes (see Figures 3-1 and 3-2) drilled in and around Landfills II and III was conducted in May of 1988 (Tracer Research Corp. 1988). The results of this sampling effort are presented in Table 3-2. The data indicate the presence of trichlorocthane (TCA) in Landfill III at a concentration of $16 \mu \mathrm{g} / \mathrm{L}$ at a depth of $11.5 \mathrm{ft}(3.5 \mathrm{~m})$ and at concentrations of $1 \mu \mathrm{g} / \mathrm{L}$ or less at Landfill II. Trichloroethylene (TCE) was found at Landilll II at concentrations of 6 and $12 \mu \mathrm{g} / \mathrm{L}$ at depths of 19 and $22 \mathrm{ft}(5.8$ and $6.7 \mathrm{~m})$, respectively. At Landfill III, TCE concentrations were $2 \mu \mathrm{g} / \mathrm{L}$. Tetrachloroethylene (PCE) was found in Landfill II at concentrations of 2 and $4 \mu \mathrm{g} / \mathrm{L}$ at depths of 19 and $22 \mathrm{ft}(5.8$ and $6.7 \mathrm{~m})$, respectively. Landfill III had a PCE concentration of $2 \mu \mathrm{g} / \mathrm{L}$ at a depth of $11.5 \mathrm{ft}(3.5 \mathrm{~m})$ bls. Benzene was found in Landfill II at concentrations of 130 and $66 \mu \mathrm{g} / \mathrm{L}$ at depths of 19 and $22 \mathrm{ft}$ $(5.8$ and $6.7 \mathrm{~m})$, respectively, and was not detected at Landfill III. The contaminants carbon tetrachloride and chloroform were found in some boreholes at concentrations well below $1 \mu \mathrm{g} / \mathrm{L}$. Methane, a common landfill gas, was found in both landfills with a maximum concentration of $40,000 \mu \mathrm{g} / \mathrm{L}$ in Landfill II. This concentration of methane ( $6.1 \%$ by volume) is above the lower explosive limit (LEL) of $5 \%$ hy volume. This suggests a buildup of methane at concentrations that could potentially pose an explosion hazard.

Once in the soil gas, volatile organic compounds tend to diffuse vertically and horizontally through the soil to the ground surface where they dissipate into the atmosphere. The soil gas contamination acts as a source and the above ground atmosphere acts as a sink, with a concentration gradient between the two. The soil gas coneentration gradient between the source and ground surface is often affected by hydrologic and geologic anomalies in the vadose zone. Since the soil gas was collected at a depth below the land surface it is important to determine what concentration may be found in the ambient air above the landfill, if the soil gas was to migrate to the surface. Preliminary modeling of the potential migration of the maximum concentration for each contaminant found in the soil gas at the landfills to the landfill surface, using conservative assumptions and a box model, indicate that the ambient air concentration would be below $1 \mu \mathrm{g} / \mathrm{L}$ for all contaminants modeled (see Appendix A.2). The modeled ambient air concentrations for each contaminant were compared to the threshold limit value-time-weighted average (TLV-TWA), which is the allowable airborne concentration of a material to which most workers can be exposed during a normal 8 -hr workday or 40-hr workweck without adverse effects 
Table 3-1. Summary of characterization and sampling activities conducted at CFA Landfills II and III from 1987 through 1991.

I. Drilling:

A. Drilling of 4 deep wells and completion of 2 as monitoring wells. Wells

$1987-1988$ LF2-8 and LF3-8 were completed (see End-of-Well reports, Appendix D). Wells LF2-10 and LF2-9 were not completed. Augering and installation of shallow instrumented boreholes and neutron access tubes in and around Landfills II and III (LF2-1 through LF2-7 and LF3-1 through LF3-7).

B. Completion of monitoring well LF 2-9 drilled in 1988. Drilling and installation of one additional monitoring well LF 2-11 upgradient of Landfill II (see End-of-Well reports, Appendix D).

C. Drilling and completion of 4 deep monitoring wells around Landfills II and III (LF2-12, LF3-9, LF3-10, and LF3-11) (see End-of-Well reports, Appendix D). Two boreholes were drilled and abandoned (LF2-12A and LF3-11A).

II. Soil Gas Sampling:

A. Tracer Research Corporation (report):

Sampling of soil gas during drilling of deep wells (LF 2-8 and LF 2-9) and from shallow boreholes (see I.A above) for dichloromethane, trichloroethane, carbon tetrachloride, trichloroethene, tetrachloroethene, methane, benzene, total hydrocarbon, and chloroform. Samples from Landfills II and III (unvalidated - see Table 3-2).

B. EG\&G Idaho sampling of soil gas from shallow gas ports for methane and 34 volatile organic constituents. Samples from Landfills II and III.

C. ICF Technology Incorporated (report):

Shallow soil/gas sampling ( $<5 \mathrm{ft}$ ) on a regularly-spaced grid over Landfill II for 1,1-dichloroethene, trans-1,2-dichloroethene, chloroform, carbon tetrachloride, 1,1,2-trichloroethane, 1,1,2,2-tetrachloroethane, methylene chloride, 1,1-dichloroethane, 1,1,1-trichloroethane, 1,1,2trichloroethene, and 1,1,2,2-tetrachloroethene (unvalidated - see Appendix A).

III. Vadose Z moisture Monitoring:

Data from soil salinity sensors, heat dissipation blocks, and neutron probe access tubes in Landfills II and III. Instruments read monthly. 
Table 3-1. (continued).

IV. Groundwater Sampling:

A. USGS sampling and data, RESL analyses (USGS 85, CFA-1, and

1962 to CFA-2).

present

B. USGS sampling and data, Denver analyses (unvalidated). 1962 to

present

C. Quarterly groundwater sampling from 8 deep wells for volatile organics,

Feb 1990 semivolatile organics, ICP metals, pesticides, PCB's, nitrate, fluoride, coliform bacteria, TOC, TOX, sulfate, chloride, herbicides, and

May 1990 radiological constituents (validated - see Appendices C.1-C.5).

Oct 1990

Apr 1991

Jul 1991

V. Soil and Interbed Sampling:

A. Science Applications International Corp. (letter report):

1988 Soil sampling from land surface to the top of uppermost basalt in and around Landfills II and III for volatile organics, semivolatile organics, trace metals, pesticides, and PCBs (unvalidated - see Appendix B).

B. University of Idaho (report):

Surficial soil sampling over Landfill II for hydraulic and physical soil properties.

C. Uppermost interbed sampling during drilling of 4 deep wells for hydraulic and mechanical properties (samples not analyzed).

VI. Drilling and Sampling Wastes:

A. Samples collected from drill cuttings and development water

$1987-1990$ accumulated during drilling of 4 deep wells around Landfills II and III. Analyzed for TCLP metals, corrosivity, reactivity, ignitability, TCLP volatile organics, TCLP semivolatile organics, TCLP herbicides, TCLP pesticides, and radiological contaminants.

VII. Geophysics:

A. ICF Technology Incorporated (report):

Nov 1989

Geophysical surveys including time-domain electromagnetic induction (TEM), ground penetrating radar (GPR), and terrain conductivity (EM) were conducted across the surface of Landfill II.

B. US Geological Survey:

Downhole logging conducted on all deep monitoring wells including gamma, gamma-gamma, caliper, borehole TV, and some neutron logs (see Appendix D). 


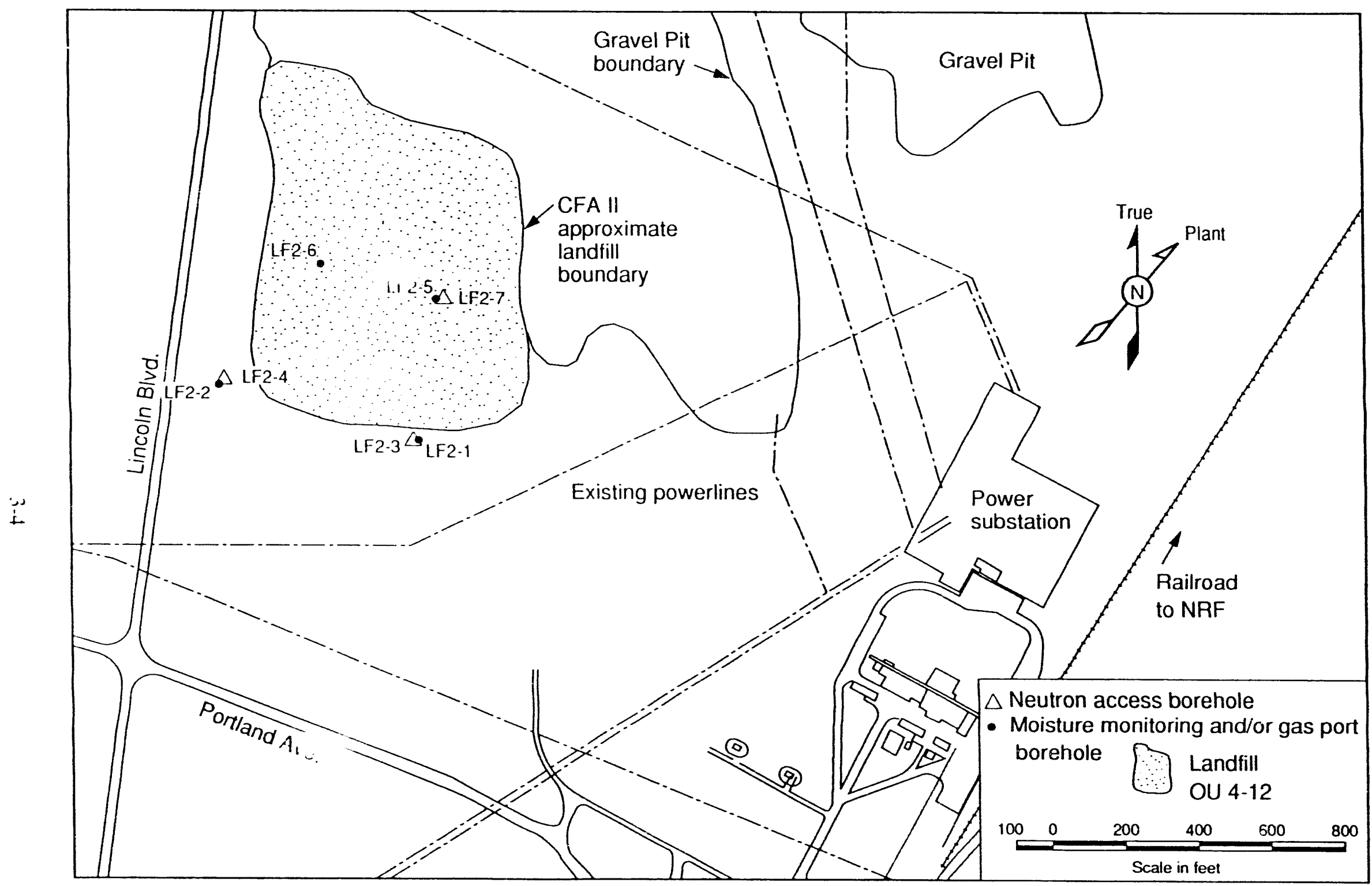

1930418

Figure 3-1. Map of CFA Landfill II showing the location of neutron access boreholes and boreholes used for gas sampling and moisture monitoring. 


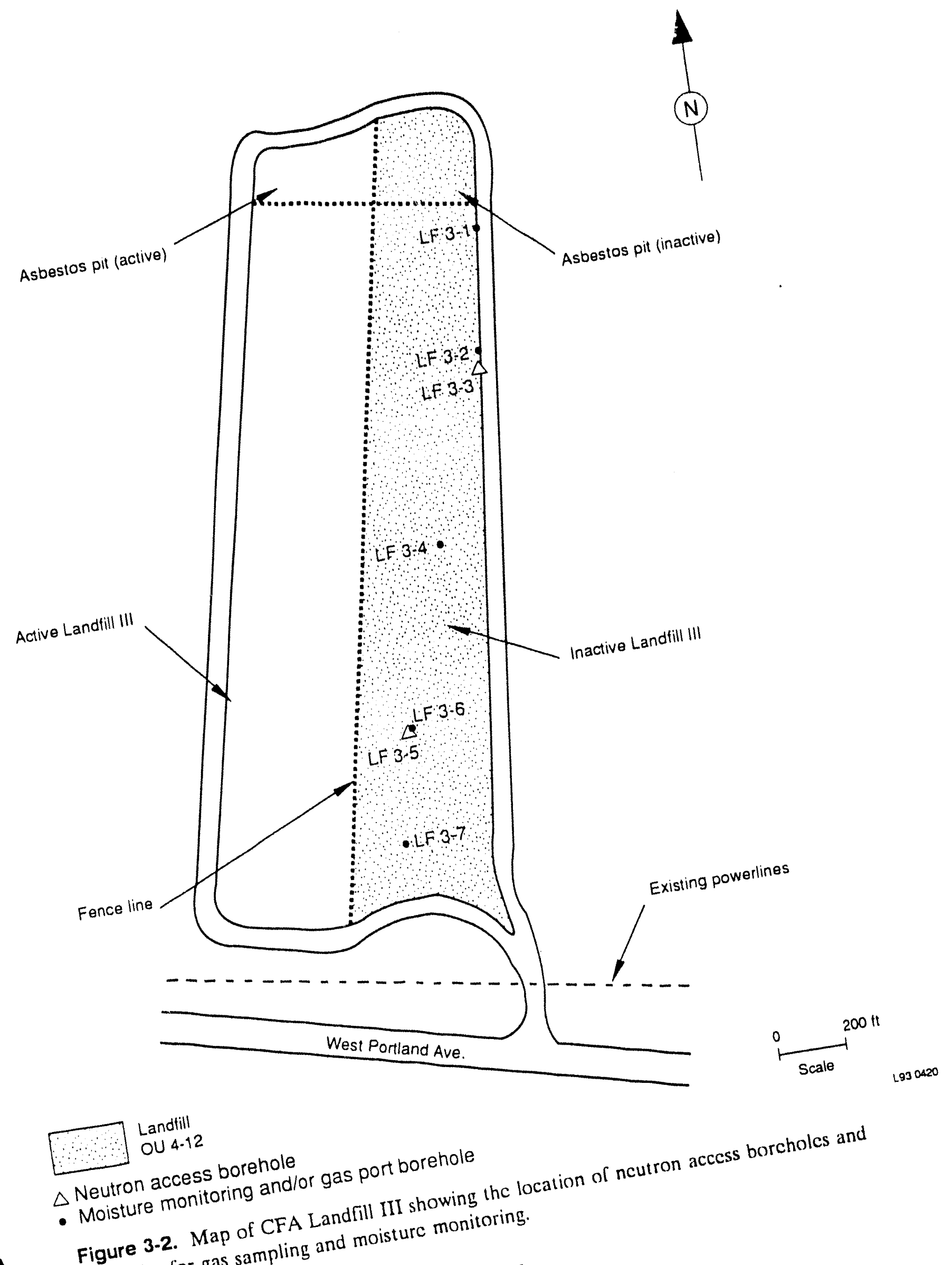

Figure 3-2. Map or gampling and mosture mas sor
borcholes 
Table 3-2. Unvalidated soil gas sampling results for the May 1988 sampling of borehole gas ports installed at Landfills II and III.

\begin{tabular}{|c|c|c|c|c|c|c|c|c|c|c|c|}
\hline Sample & $\begin{array}{l}\text { Sample } \\
\text { depth }\end{array}$ & Date & $\begin{array}{c}\mathrm{CI}_{2} \mathrm{Cl}_{2} \\
(\mu \mathrm{g} /)\end{array}$ & $\begin{array}{l}\text { TCA } \\
(\mu \mathrm{g} /)\end{array}$ & $\begin{array}{l}\mathrm{CCl}_{4} \\
(\mu \mathrm{g} /)\end{array}$ & $\begin{array}{l}\text { ТСE } \\
(\mu \mathrm{g} /)\end{array}$ & $\begin{array}{l}\text { PCE } \\
(\mu \mathrm{g} /)\end{array}$ & $\begin{array}{l}\mathrm{CH}_{4} \\
(\mu \mathrm{g} /)\end{array}$ & $\begin{array}{l}\text { Benzene } \\
(\mu g /)\end{array}$ & $\begin{array}{l}\mathrm{CHCl}_{3} \\
(\mu \mathrm{g} /)\end{array}$ & $\begin{array}{c}\text { Total } \\
\text { hydroc. } \\
(\mu \mathrm{g} /)\end{array}$ \\
\hline $\mid F-02-01$ & $27 \mathrm{ft}$ & $5-13$ & $<0.07^{\mathrm{a}}$ & 0.4 & 0.07 & 0.2 & 0.5 & 81 & $<0.03$ & 0.05 & 0.8 \\
\hline $1,1=-02-(02$ & $31 \mathrm{ft}$ & $5-13$ & $<0.07$ & 1 & $<0.0002$ & 0.6 & 2 & 10 & $<0.03$ & 0.02 & 1 \\
\hline $1 . F-02-0.5$ & $22 \mathrm{ft}$ & $5-13$ & $<0.07$ & 0.5 & $<0.000) 2$ & 12 & 4 & 40,000 & 66 & $<0.002$ & 180 \\
\hline $1.1=-02-(x)$ & $19 \mathrm{ft}$ & $5-13$ & $<0.07$ & 0.9 & $<0.0002$ & 6 & 2 & 14.000 & 130 & 0.2 & 200 \\
\hline IF-(1)3-04 & $11.5 \mathrm{ft}$ & $5-13$ & $<7$ & 16 & $<0.002$ & 2 & 2 & 10 & $<0.07$ & $N A^{b}$ & 6 \\
\hline
\end{tabular}

a. The sample value is less than the detection limit.

b. NA - Not analyzed. 
as defined by the American Conference of Governmental Industrial Hygienists (ACGIH 1990). All ambient air concentrations were well below the TLV-TWA. The ambient air concentrations were also below the general public screening value of 0.01 times the compound-specific TLV-TWA, suggesting no adverse health effects to workers and the general public. The downward migration of these VOCs to the groundwater will be evaluated during the RI.

Soil gas sampling of these boreholes for methane and VOCs was again conducted in November of 1988, and March, May, and November of 1989 (see Appendix A). A preliminary evaluation of the data indicates that methane gas was detected above the lower explosive limit in the central and southern portion of Landfill II. Methane gas was also detected at Landfill III, but all samples were well below the lower explosive limit. The data has not been validated and the $\mathrm{QA} / \mathrm{QC}$ procedures of sampling and analysis are not available. Therefore, conclusions with regard to health risks for VOCs cannot be made because the reliability of the data is suspect. For purposes of this work plan, the above data will be used only for project planning.

In 1989, ICF Technology Incorporated conducted a shallow soil gas survey $\mid<5 \mathrm{ft}(<1.5 \mathrm{~m})]$ across the surface of Landfill II and the surrounding area. Soil gas was sampled on $50-\mathrm{ft}(15-\mathrm{m})$ centers within the landfill boundary and on $100-\mathrm{ft}(30-\mathrm{m})$ centers outside the landfill boundary for a total of 179 regularly spaced sample locations. Samples were analyzed according to EPA Method 601 on a gas chromatograph equipped with an electron capture detector (ECD), using direct injection instead of purge and trap. Analytical results for the 11 chlorinated organics analyzed in the survey are presented in Appendix A.1.

The data indicate the presence of some chlorinated organics, particularly chloroform, 1,1dichloroethane (1,1-DCA), 1,1,1-trichloroethane (1,1,1-TCA), tetrachloroethylene (PCE) in low concentrations in the central and southwestern portion of the landfill. Chloroform was found in the central portion of the landfill in the highest concentration $(357 \mu \mathrm{g} / \mathrm{L})$. A chloroform concentration of $225 \mu \mathrm{g} / \mathrm{L}$ was also found in the southwest quadrant of the landfill with lower concentrations found to the northwest. 1,1-DCA was found in the west central portion of the landfill at a concentration of $82 \mu \mathrm{g} / \mathrm{L}$ and at lower concentrations southwestward. 1,1,1-TCA was found at a maximum concentration of $67 \mu \mathrm{g} / \mathrm{L}$ with lower concentrations extending southeastnorthwest across the site. PCE was found at a maximum concentration of $111 \mu \mathrm{g} / \mathrm{L}$ in the southwest with lower concentrations in the southeast corner of the landfill. It should be noted that the field crew found that Level D protection was appropriate during the entire sampling phase and at no time encountered total organic vapor readings from the landfill surface in excess of $5 \mathrm{ppm}$.

Preliminary fate and transport modeling of the migration of the maximum concentration of contaminants found in the landfill to the land surface indicated ambient air concentrations well below the compound specific TLV-TWA and the general public safe screening value of 0.01 times the compound specific TLV-TWA (see Appendix A.2).

\subsubsection{Soil Sampling}

Eight soil samples were collected during the drilling of boreholes in 1987 at Landfills II and III at depths varying from 1 lo $2.3 \mathrm{lt}(0.3107 \mathrm{~m}$ ) (see Figures $3-1$ and $3-2$ for borehole locations). The statigraphic logs for these borcholes can be found in Appendix D. It is important 
to note here that no leachate was encountered during this sampling investigation. The soil samples were analyzed for semivolatiles, volatile organic compounds and metals. The summary data tables are presented in Appendix B. The data has not been validated and the QA/QC procedures of the sampling and analysis are not available. Three of the four batches of samples analyzed for semivolatiles exceeded the extraction holding times. The reliability of the data is suspect and will be presented here as a limited investigation. Benzoic acid and 4-methylphenol were the only semivolatile compounds found in the soil. A total of four volatile organic compounds (acetone, 2-butanone, xylene, and methylene chloride) were positively identified in the soil collected from the landfills. Table 3-3 summarizes the semivolatile and volatile organic compounds found in the borchole soils. It is interesting to note that five of the above mentioned compounds were found in one borehole (LF2-5) at a depth of $1 \mathrm{ft}(0.3 \mathrm{~m})$ below land surface. Methylene chloride is present at low concentrations in both landfills, but was also found in corresponding blank samples indicating sample contamination. However, some of the soil concentrations do exceed ten times the maximum concentration detected in the blank sample. Therefore, they should be considered positive results for methylene chloride in soils. The inorganic data were all within the common range expected for soils of this area (DOE 1992) (sec Appendix E).

A risk-based screening of the soils' maximum volatile organic concentrations for each contaminant found in the landfills was performed (see Table 3-4). The screening process compared the maximum concentrations to risk-based concentrations based on standard default exposure factors and the soil ingestion pathway (EPA 1991b). For soil, the default screening level at which carcinogenic contaminants can be eliminated is less than or cqual to $10^{-7}$ risk (based on the soil ingestion pathway) and for noncarcinogens the screening concentration is less than or equal to 0.1 hazard quotient (EPA 1991b). The lower target risk of $1 \times 10^{-7}$ is used in this screening for soil because additional pathways (i.e., dermal and inhalation) which are not accounted for in the calculations, could result in significantly higher exposure for some chemicals. All contaminants were below the screcning risk-based concentrations.

A preliminary evaluation of the fate and transport of these soil contaminants to the groundwater was made using the code GW SCREEN (Rood 1992). Soil concentrations found in Landfills II and III were modeled to the groundwater. The resulting groundwater concentrations were then compared to the risk-based groundwater concentrations for the water ingestion and inhalation pathways. For groundwater, the default screening level at which carcinogenic contaminants can be eliminated is less than or equal to $10^{-6}$ risk and for noncarcinogens the screening concentration is less than or equal to 0.1 hazard quotient (EPA 1991b). Results indicate that for all contaminants, with the excevion of chromium (Landfills II and III) and methylene chloride (Landfill III), modeled groundwater concentrations were below the risk-based concentration for the particular contaminant. In the case of chromium, soil eoncentrations were for total chromium with no differentiation between chromium III and chro um VI; therefore, a conservative assumption of $100 \% \mathrm{chr}$. nium VI was made for this preliminary data evaluation. This evaluation suggests that methylenc chloride and chromium may be contaminants of potential concern in the soil.

Drill cuttings brought to the surface during the drilling operations were sampled and analyzed for Toxicity Characteristic Leaching Procedure (TCLP) RCRA hazardous constituents for land disposal. All drilling wastes were found to be nonhazardous and in compliance with RCRA requirements for land disposal. 
Table 3-3. Unvalidated shallow borehole soil sampling analytical results, with corresponding sample location. All units are in $\mu \mathrm{g} / \mathrm{kg}^{\mathrm{a}}$

\begin{tabular}{lcccccccc}
\hline Sample location & LF2-1 & LF2-3 & LF2-5 & LF2-6 & LF3-2 & LF3-4 & LF3-6 & LF3-7 \\
\hline & & & & & & & & \\
Sample depth (bls) ${ }^{\mathrm{b}}$ & $17 \mathrm{ft}$ & $23 \mathrm{ft}$ & $1 \mathrm{ft}$ & $1 \mathrm{ft}$ & $12 \mathrm{ft}$ & $10 \mathrm{ft}$ & $17 \mathrm{ft}$ & $15 \mathrm{ft}$ \\
\hline
\end{tabular}

\begin{tabular}{ll} 
& \\
Contaminant & Concentration \\
\hline
\end{tabular}

4-methyl phenol 110

(cresol)

Benzoic acid $\quad 90$

Acetone $\quad 800$

2-butanone 73

i. (methyl ethyl ketone)

Xylene

Methylene chloride

$\begin{array}{lll}8 & 12 & 60\end{array}$

60

17

4

(dichloroethane)

a. Summary data tables are provided in Appendix B.

b. bls = below land surface. 
Table 3-4. Risk-based screening of carcinogenic and noncarcinogenic soil contaminants.

\begin{tabular}{lccc}
\multicolumn{1}{c}{$\begin{array}{c}\text { Maximum } \\
\text { Contaminant } \\
(\mathrm{mg} / \mathrm{kg})\end{array}$} & $\begin{array}{c}\text { Noncarcinogen risk- } \\
\text { based concentration } \\
(0.1 \text { hazard quotient }) \\
(\mathrm{mg} / \mathrm{kg})\end{array}$ & $\begin{array}{c}\text { Carcinogen risk-based } \\
\text { concentration } \\
\left(10^{-7} \mathrm{screening} \mathrm{value}\right) \\
(\mathrm{mg} / \mathrm{kg})\end{array}$ \\
$\begin{array}{l}\text { 4-methylphenol } \\
\text { (cresol) }\end{array}$ & 0.110 & 1,000 & $\mathrm{NA}$ \\
$\begin{array}{l}\text { Benzoic acid } \\
\text { Acetone }\end{array}$ & 0.090 & 100,000 & $\mathrm{NA}$ \\
2-butanone & 0.800 & 3,000 & $\mathrm{NA}$ \\
Xylene & 0.073 & 1,000 & $\mathrm{NA}$ \\
Methylene chloride & 0.004 & 50,000 & $\mathrm{NA}$ \\
\hline & 0.073 & 2,000 & 9 \\
NA - Not applicable, the contaminant is not a carcinogen. & \\
\hline
\end{tabular}

\subsubsection{Vadose Zone Moisture Monitoring Data}

During December 1987, a shallow drilling program was implemented at CFA Landfills II and III. The objectives of the program included monitoring hydraulic behavior of the surficial sediments to quantify the amounts and rates of water movement into and through the sediments. Neutron moisture probe access tubes, heat dissipation sensors, and salinity sensors were installed in the shallow boreholes at Landfills II and III. Data from these instruments were collected on roughly a monthly basis from January 1988 to January 1991. Data from January 1988 to September 1988 have been analyzed and the results are reported in Ansley et al. (1988).

Neutron moisture probes indirectly measure moisture content of soils by emitting fast neutrons into the soil and counting the neutrons thermalized by collisions with hydrogen. The counts are correlated to water content because the majority of hydrogen in most soils exists as part of the water molecule. Table 3-5 identifies the boreholes at each landfill that were equipped with neutron probe access tubes and their depth of installation. The borehole locations are shown in Figures 3-1 and 3-2 for Landfills II and III, respectively. Analyses of the first nine months of neutron probe data (Ansley et al. 1988) led to the conclusion that large changes in moisture content occurred near the surface but little or no change occurred at depth [depths ranged from approximately 18 to $23 \mathrm{ft}(5.5$ to $7 \mathrm{~m})]$. There was no clear evidence of a massive, deeply penetrating downward moving pulse of water. However, the report concluded that evidence of downward migration of water was found in data from a few of the neutron moisture 
Table 3-5. Boreholes used for neutron probe access tubes and access tube depths.

\begin{tabular}{|c|c|c|}
\hline Landfill & Borehole & $\begin{array}{c}\text { Depth } \\
\text { (ft) }\end{array}$ \\
\hline \multirow[t]{3}{*}{ II } & LF2-03 & 23.0 \\
\hline & LF2-04 & 22.7 \\
\hline & LF2-07 & 18.2 \\
\hline \multirow[t]{2}{*}{ III } & LF3-03 & 18.7 \\
\hline & LF3-05 & 22.5 \\
\hline
\end{tabular}

probe holes. Net recharge entering the waste was estimated to be 0.22 in. $(0.56 \mathrm{~cm})$ or approximately $6 \%$ of the $3.77 \mathrm{in} .(9.58 \mathrm{~cm})$ of precipitation falling between November 1987 and September 1988.

Heat dissipation blocks are used to measure matric potential. Heat dissipation blocks are operated by running a fixed electric current through a wire. The more moisture present, the greater the heat capacity of the block and the lower the temperature change. The heat dissipation blocks were calibrated in terms of matric potential because of the different water holding characteristics of different sediments at a given matric potential. Table 3-6 identifies the boreholes and depths where heat dissipation blocks were installed at each landfill. The borehole locations are shown in Figures 3-1 and 3-2 for Landfills II and III, respectively. Large variability in measured matric potentials made interpretations of the first nine months of data difficult (Ansley et al., 1988) and no conclusions were reached regarding water movement based on this data.

Salinity probes are used to measure the electrical conductance of soil water. Conductance is proportional to the dissolved solids or salts in the water. Leachate from landfills is expected to be much higher in dissolved solids than natural soils. Therefore, the salinity probes were used to monitor for migration of leachate from the landfill. Table 3-7 identifies the boreholes and depths where salinity probes were installed at each landfill. The borehole locations are shown in Figure 3-1 and 3-2 for Landfills II and III, respectively. An evaluation of the salinity probe data indicated that the probes underwent a period of equilibration with the soil lasting until late summer of 1988 (Ansley et al. 1988). Subsequent readings were within the range of values typical for saline desert soils. It was concluded that none of the probes indicated significant migration of leachates with high dissolved solids.

Neutron probe, heat dissipation sensor, and salinity probe data from slightly more than two additional years is stored in a database and remains unanalyzed. These data will be evaluated during the RI to verify the conclusions of the 1988 report. Additional neutron moisture probe data will also be collected during the RI. 
Table 3-6. Locations and depths of heat dissipation blocks installed at Landfills II and III.

\begin{tabular}{|c|c|c|c|}
\hline Landfill & Borehole & Instrument & $\begin{array}{c}\text { Depth } \\
(\mathrm{ft})\end{array}$ \\
\hline \multirow[t]{10}{*}{ II } & LF2-01 & H0670 & 6.17 \\
\hline & & $\mathrm{H} 1250$ & 6.17 \\
\hline & & $\mathrm{H} 1215$ & 11.17 \\
\hline & & H1227 & 11.17 \\
\hline & & $\mathrm{H} 0642$ & 16.17 \\
\hline & & H1223 & 16.17 \\
\hline & & H0628 & 21.17 \\
\hline & & H1249 & 21.17 \\
\hline & & H0604 & 26.17 \\
\hline & & H0637 & 26.17 \\
\hline \multirow[t]{10}{*}{ II } & LF2-02 & $\mathrm{H} 06.3 .3$ & 6.00 \\
\hline & & H0643 & 6.00 \\
\hline & & $\mathrm{H} 0770$ & 12.00 \\
\hline & & H1219 & 12.00 \\
\hline & & H0775 & 18.00 \\
\hline & & H1234 & 18.00 \\
\hline & & $\mathrm{H} 1221$ & 24.00 \\
\hline & & H1236 & 24.00 \\
\hline & & H0690 & 30.00 \\
\hline & & H0766 & 30.00 \\
\hline \multirow[t]{2}{*}{ II } & LF2-05 & H0687 & 20.58 \\
\hline & & $\mathrm{H} 1222$ & 20.58 \\
\hline \multirow[t]{2}{*}{ II } & LF2-06 & Hô603 & 18.25 \\
\hline & & $\mathrm{H} 1217$ & 18.25 \\
\hline \multirow[t]{10}{*}{ III } & LF3-02 & H0646 & 4.58 \\
\hline & & $\mathrm{H} 0777$ & 4.58 \\
\hline & & H1235 & 8.58 \\
\hline & & H 1252 & 8.58 \\
\hline & & $\mathrm{H}(0645$ & 14.67 \\
\hline & & $\mathrm{H}(0773$ & 14.67 \\
\hline & & $\mathrm{H}(0610$ & 18.67 \\
\hline & & $\mathrm{H} 1226$ & 18.67 \\
\hline & & H0605 & 23.67 \\
\hline & & H1229 & 23.67 \\
\hline
\end{tabular}


Table 3-6. (continued).

\begin{tabular}{|c|c|c|c|}
\hline Landfill & Borehole & Instrument & $\begin{array}{c}\text { Depth } \\
(\mathrm{ft})\end{array}$ \\
\hline \multirow[t]{8}{*}{ III } & LF3-06 & H0666 & 3.92 \\
\hline & & H0774 & 3.92 \\
\hline & & $\mathrm{H} 1216$ & 7.92 \\
\hline & & $\mathrm{H} 1224$ & 7.92 \\
\hline & & H0640 & 11.92 \\
\hline & & H1218 & 11.92 \\
\hline & & H0641 & 15.92 \\
\hline & & $\mathrm{H} 1220$ & 15.92 \\
\hline
\end{tabular}

Table 3-7. Locations and depths of salinity probes installed at Landfills II and III.

\begin{tabular}{cccc} 
Landfill & Borehole & Instrument & $\begin{array}{c}\text { Depth } \\
(\mathrm{ft})\end{array}$ \\
\hline II & LF2-01 & S1374 & 21.17 \\
& & $\mathrm{~S} 1401$ & 26.17 \\
II & LF2-02 & $\mathrm{S} 1364$ & 30.00 \\
& & $\mathrm{~S} 1406$ & 20.58 \\
II & LF2-05 & $\mathrm{S} 1367$ & 18.25 \\
II & LF2-06 & $\mathrm{S} 1361$ & 11.83 \\
III & LF3-01 & $\mathrm{S} 1399$ & 16.83 \\
& & $\mathrm{~S} 1398$ & 18.67 \\
III & LF3-02 & $\mathrm{S} 1992$ & 23.67 \\
& & $\mathrm{~S} 1397$ & 6.00 \\
III & LF3-04 & $\mathrm{S} 1396$ & 10.92 \\
& & $\mathrm{~S} 1402$ & 11.92 \\
III & LF3-06 & $\mathrm{S} 1400$ & 15.92 \\
& & $\mathrm{~S} 1393$ & 6.42 \\
III & LF3-07 & $\mathrm{S} 1989$ & 11.50 \\
\hline
\end{tabular}




\subsubsection{Groundwater Sampling}

Groundwater samples were collected by EG\&G Idaho Geosciences personnel from monitoring wells located around CFA Landfills II and III quarterly from February 1990 through July 1991. Appendices C.1-C.5 contain the results tables for the validated groundwater data collected from these wells. The volatile organic compound 1,1,1-trichloroethane was detected in both upgradient and downgradient wells at concentrations of $s 1 \mu \mathrm{g} / \mathrm{L}$. Monitoring of these wells by others have also detected 1,1,1-trichloroethane in these same wells at concentrations below $s 1 \mu \mathrm{g} / \mathrm{L}$ (Barrash and Marts 1991). The screeni: concentration for 1,1,1-trichloroethane, a noncarcinogen, is $200 \mu \mathrm{g} / \mathrm{L}$. Other volatile organics were detected in the groundwater but were also detected in the trip and field blanks. No semivolatile compounds were detected in the groundwater samples. Tritium and chromium were both found in the groundwater at concentrations above their respective maximum contaminant levels (MCLs).

Tritium, which has an MCL of $20 \mathrm{pCi} / \mathrm{mL}$, was found in both upgradient and d wwngradient wells at concentrations ranging from 39 to $48 \mathrm{pCi} / \mathrm{mL}$. However, the CFA landfills art not considered the source of the tritium. A tritium plume has developed in the Snake River Plain Aquifer since the 1950s from the disposal of liquid wastes at the INEL. The principal sources of tritium in the aquifer have been from the injection of liquid wastes in the disposal well at ICPP and the discharge of wastewater to the infiltration ponds at ICPP and TRA; both facilities are upgradient of the landfills (Orr and Cecil 1991). Groundwater contamination from ICPP and TRA will be addressed in the INi Site-wide (WAG 10) RI/FS and/or other operable unit RIs being conducted at the INEL and will not be discussed further in this RI/FS.

Total chromium in unfiltured samples (dissolved and particulate fractions) was found in both the upgradient and downgradient wells above the MCL of $50 \mu \mathrm{g} / \mathrm{L}$. Chromium concentrations for filtered samples (dissolved fraction) were generally below the MCL. ICPP and TRA, which are both upgradient from the CFA landfills, have discharged chromium to injection wells and infiltration ponds. The past disposal practices at these facilities have resulted in elevated chromium concentrations in the aquifer (Orr and Cecil 1991). In addition, the dedicated sampling pumps (Hydrostar model) in the landfill monitoring wells are considered to also be a minor contributing source of the particulate chromium. This hypothesis is supported by the fact that these pumps are constructed partly with high chromium stainless steel and that the filtered sample concentrations are much lower than the unfillered sample concentrations.

\subsubsection{Geophysical Surveys}

Several geophysical surveys ere conducted on Landill II by ICF Technology Inc., in 1989 as an irmovative research demons,ration to confirm the subsurface lithology and depth of consolicided rock, characterize the vertical and horizontal extent of the landfill body and the location of buried metal objects. The survey methods used included: an electromagnetic induction (terrain conductivity) survey to locate buried metal, ground penetrating radar (GPR) to further characterize th netal areas found, and time-domain electromagnetic induction (TEM) to determine subsuilace stratigraphy.

The terrain conductivity (EM) survey identilied the boundaries of the landfill and the locations of buried metal objects. Metal was found widely distributed throughout the landfill, with 
no significant areas free of metal. No additional information concerning metal was obtained from the GPR survey. The TEM survey was a research effort that resulted in inconclusive results. The depth to basalt was not conclusively defined by this survey method. In summary the geophysical surveys performed at CFA Landfill II identified the landfill boundaries and detected buried metal throughout the entire landfill.

\subsection{Preliminary Conceptual Site Model}

The preliminary conceptual model for CFA Landfills II and III is presented in Figure 3-3. The identification of the specific sources, pathways, and receptors for the model are based on an evaluation of past disposal practices, a review of the waste inventory at the landfills, and the results of soil gas sampling, soil moisture monitoring, geophysical data, chemical analytical data for soil from the cover and waste, and groundwater sampling. This model will be refined as additional information is obtained about the site during the remedial investigation phase of the project.

\subsection{Preliminary Public Health and Environmental Impacts}

There have been no known releases from the landfill site that would pose a threat to INEL workers or the public. The landfills are located within the INEL on federally owned land that has been withdrawn from public use by DOE. Public access to the INEL is limited to two federal highways and three state highways. Other roads within the INEL boundary are restricted to use by INEL personnel and visitors on official business. No resident populations are located within the INEL Site boundary and the nearest towns are Atomic City and Arco, $7 \mathrm{mi}(11 \mathrm{~km})$ southeast and $17.5 \mathrm{mi}(28 \mathrm{~km})$ west of CFA, respectively. There are no immediate public health concerns from the landfills.

Groundwater sampling has shown no known contamination of the groundwater that can be directly attributable to the landfills. Production wells at CFA are used for drinking water by INEL workers and visitors. There is no use of the water by the general public as the INEL is a controlled site with limited access. The water from the CFA production wells is tested regularly and meets Federal drinking water standards based on March 1993 data.

The existing soil and vegetative cover for the landfills protect the INEL workers and visitors from direct contact with the underlying waste and contaminated soil. Subsurface soil sampling for chemical analytical data at the landfills has not indicated any contamination that would pose a threat to workers or the public.

Soil gas sampling has indicated the presence of methane, a common by-product of landfills, and some volatile organics at low concentrations. Preliminary fate and transport modeling of these soil gas vapors to the landfill surface indicate that concentrations are well below TLV-TWA. Methane gas was found at Landfill II above the lower explosive limit and this poses a potential explosion hazard. 


\begin{tabular}{cccc}
\hline $\begin{array}{c}\text { Contaminant } \\
\text { Source }\end{array}$ & $\begin{array}{c}\text { Contaminant } \\
\text { Release/Transport }\end{array}$ & $\begin{array}{c}\text { Affected } \\
\text { Media }\end{array}$ & $\begin{array}{c}\text { Exposure } \\
\text { Route }\end{array}$ \\
\hline
\end{tabular}

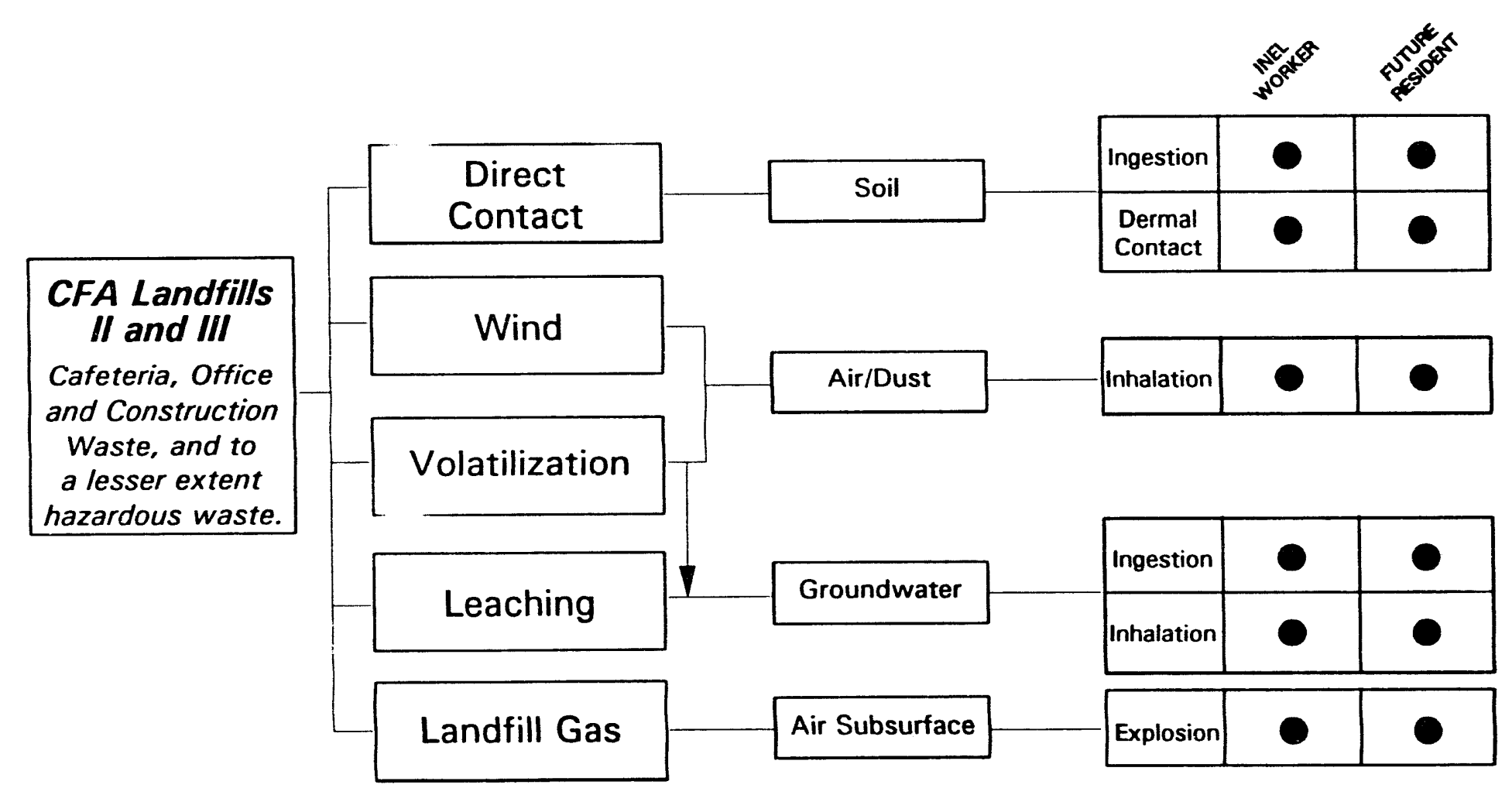

Figure 3-3. Preliminary conceptual model for CFA Landfills II and III. 


\subsection{Preliminary Remedial Action Alternatives}

The number of practical remedial actions available for municipal landfills is limited. They are based on previous experience, engineering judgement, and the NCP expectations.

Containment technologies are generally appropriate for waste that poses a relatively low, longterm threat or where treatment is impractical. Remedial action alternatives for landfill sites are generally limited to source control by capping and possibly removal or treatment of "hot spots," groundwater extraction and treatment, and landfill-gas control. As required by the NCP, the noaction alternative is included and involves no additional activities, thereby providing a baseline for evaluating other alternatives. Most of the remedial action alternatives applicable to the CFA landfills, including the no action alternative, can and would include groundwater monitoring.

Preliminary remedial action alternatives considered for the CFA landfills include:

- No action.

- Access restriction-intended to prevent or reduce exposure to on-site contamination. This may be accomplished through fencing to physically limit access to the landfill site, by signs posted to warn trespassers of potential hazards, and through deed restrictions to notify any potential purchaser of the landfill property that the land was used for waste disposal and the land use must be restricted in order to ensure the integrity of the waste containment system.

- Containment-containment refers to technologies that isolate the landfill contents and mitigate offsite migration through the use of engineering controls. A landfill cover or cap which may consist of a native soil cover, single barrier (i.e., clay), or composite barrier (i.e., clay plus flexible membrane liner) may be considered.

- Hot-spot removal-removal of contaminated soils that represent discrete accessible locations within the landfill where a waste type or mixture of wastes present a principal threat to human health or the environment.

- Surface controls-surface control technologies are designed to control and direct site runoff and to prevent offsite surface water from running onto the site. Examples of surface controls include: grading the landiill surface cover, which modifies topography in order to promote positive drainage and control the flow of surface water; and the establishment of vegetation to stabilize the soil surface of the landfill site and promote evapotranspiration.

- Passive collection of landfill gas-to direct subsurface llow to points of controlled release through the use of high-permeability systems.

- Leachate collection/monitoring and treatment-to minimize or eliminate the migration of leachate to the groundwater system.

- Groundwater monitoring - to detect potential future releases to the groundwater. 


\subsection{Preliminary Applicable or Rel vant and Appropriate Requirements}

This section identifies the preliminary list of applicable or relevant and appropriate requirements (ARARs) based on Federal and State-of-Idaho requirements. These ARARs will be refined throughout the RI/FS process at CFA.

The "applicable" requirements are defined as

"Standards, standards of control, and other substantive environmental protection requirements, criteria, or limitations promulgated under federal or state law that specifically address a hazardous substance, pollutant, contaminant, remedial action, location, or other circumstance at a CERCLA site."

"Relevant and appropriate" requirements are defined as

"Standards, standards of control, and other substantive environmental protection requirements, criteria, or limitations promulgated under federal or state law that, while not "applicable" to a hazardous substance, pollutant, contaminant, remedial action, location, or other circumstance at a CERCLA site, address problems or situations sufficiently similar to those encountered at the CERCLA site such that their use is well suited to the particular site."

The determination that a requirement is relevant and appropriate is a two-step process: (a) determine if a requirement is relevant and (b) determine if a requirement is appropriate. In general, this involves a comparison of a number of site-specific lactors, including the characteristics of the proposed remedial action, the hazardous substances present at the site, or the physical eircumstances of the site, with the requirements in the statutory or regulatory requirement (EPA 1988h and EPA 1989d). In some cases, a requirement may be relevant, but not appropriate, given site-spe. lic circumstances; such a requirement would not be an ARAR for the site. In some cases it is possible for only part of a requirement to be considered relevant and appropriate.

In addition to ARARs, agencies are to identify advisories or guidance that may be considered during the evaluation of proposed remedial actions. The guidance or advisories are identified as "To Be Considered (TBC)." The TBC, although not an ARAR, may be used in establishing a cleanup standard when one does not exist [40CFR.30(0.40)(g)(3)].

There are three types of ARARs to be identified: chemical-specilic, location-specific, and action-specific. Chemical-specific ARARs are established chemical standards under State or Federal law, such as drinking water standards. Location-specific ARARs are requirements specific to the geographical or physical setting of the site. Action-specific ARARs are technology- or activity-based requirements under State or Federal law and depend on the proposed remedial alternatives. 
The potential ARARs specific to CFA Landfills II and III that have been identified thus far in the RI/FS process are included in this section. As additional information becomes known concerning the nature and extent of contamination, ARARs will be further refined. There are generally four phases of the RI/FS process during which ARARs are identified and evaluated: scoping, site characterization, development of remedial alternatives, and design of alternatives.

Chemical-specific requirements are usually technology- or risk-based numerical limitations or methodologies that, when applied to site-specific conditions, result in the establishment of acceptable chemical concentrations. If a chemical has more than one requirement (whether State or Federal), the most stringent requirement generally is the identified ARAR. Table 3-8 is a preliminary list of the chemical-specific ARARs identified for CFA Landfills II and III.

Location-specific ARARs are restrictions that determine the conduct or type of activities that may occur in special locations. The special location category includes the 100-year floodplain, archaeologically or historically significant areas, wetlands, and seismically active areas. Table 3-9 identifies the preliminary location-specific ARARs identified for the CFA Landfills II and III.

Action-specific ARARs are usually technology- or activity-based requirements or limitations on actions taken with respect to hazardous substances. These requirements typically define acceptable treatment, storage, and disposal procedures for hazardous substances during the implementation of the response action. The requirements generally set performance or design standards for specific activities such as managing hazardous wastes at municipal landfill sites. Potential action-specific ARARs anticipated for landfill sites are shown in Table 3-10.

A category of to-be-considered guidance has also been initially identified for CFA Landfills II and III. This category identifies criteria, advisories, guidance, or policies that do not meet the definition of ARARs but may assist in determining what is protective. TBCs for the CFA include:

- $\quad$ EPA guidance documents /e.g., A Guide to Delisting RCRA Waste for Superfund Remedial Responses (OSWER, 9347.3-09FS, September 199())]

- DOE orders

- Executive orders

- Proposed state air standards and/or guidances

- Safe Drinking Water Act; Final Determination (EPA) of Sole Source Designation for the Eastern Snake River Aquifer, Southern Idaho (56 FR 506.34)

- Proposed standards under the Safe Drinking Water Act

- Conducting Remedial Investigations/Feasibility Studies for CERCLA Municipal Landfill Sites (EPA/540/P-91/(0)1) 
Table 3-8. Preliminary chemical-specific ARARs for CFA-Landfills II and III.

Statute

Regulation

Safe Drinking Water Act

Resource Conservation and Recovery Act

Emergency Planning and Community

Right-to-Know Act of 1986

Clean Air Act
40 CFR 141, "National Primary Drinking Water Standards"

40 CFR 141, "Maximum Contaminant Level Goals"

40 CFR 143, "National Secondary Drinking Water Standards"

40 CFR 264, "Maximum Concentration Limits" (IDAPA § 16.01.5008)

40 CFR 355, "Emergency Planning and Notification under CERCLA"

40 CFR Part 50, "National Primary and Secondary Ambient Air Quality Standards"

- State-of-Idaho Water Quality Plan (Idaho Ground Water Quality Plan (IDHW et al. 1991).

Specific DOE and executive orders that will be considered are listed in Table 3-11.

Potential State ARARs must also be considered for the CFA landfills. In general, in order for a State requirement to be considered an ARAR, it must:

- Be promulgated (be legally enforceable and of general applicability)

- Be identified to EPA in a timely manner

- Be more stringent than Federal requirements.

Even if the State standard meets these conditions, it may be waived if it is found not to have been applied uniformly and consistently throughout the State. See Table 3-12 for a list of preliminary State ARARs. 
Table 3-9. Preliminary location-specific ARARs for CFA-Landfills II and III.

Clean Air Act

Archaeological Resources Protection Act

Preservation of American Antiquities Act

National Historic Preservation Act

National Archeological and Historic

Preservation Act

Archacological Resource

Protection Act

Migratory Bird Treaty Act

Bald and Golden Eagle Protection Act

Endangered Species Act
40 CFR Part 53, "Prevention of Significant Deterioration

36 CFR Part 7, "Protection of Archeological Resources"

36 CFR Part 296, "Protection of Archeological Resources; Uniform Regulations

4.3 CFR Part 3, "Preservation of American Antiquities"

36 CFR Part 800

36 CFR Part 296, "Protection of Archeological Resources"

36 CFR Part 7, "Protection of Archaeological Resources"

50 CFR Part 20, "Migratory Bird Protection"

50 CFR Part 22, "Bald and Golden Eagle Protection Act"

50 CFR Part 17, "Endangered and Threatened Wildlife and Plants"

50 CFR Part 225, "Federal/State Cooperation in the Conservation of Endangered and Threatened Species"

50 CFR Part 226, "Designated Critical Habitat" 50 CFR Part 402, "Interagency Cooperation" 
Table 3-10. Preliminary action-specitic ARARs for CFA-Landfills II and III.

Statute

Regulation

Resource Conservation and Recovery Act

40 CFR Part 257, "Criteria for Classification of Solid Waste Disposal Facilities and Practices"

40 CFR Part 258, "Criteria for Municipal Solid Waste Landfills"

Clean Air Act

40 CFR Part 50, "National Primary and Secondary Ambient Air Quality Standards"

40 CFR Part 61, "National Emission Standards for Hazardous Air Pollutants"

40 CFR Part 200, "Standards for Performance for New Stationary Sources"

Clean Water Art

40 CFR Part 230, "Guidelines for Specification of Disposal Sites for Dredged or Fill Material"

Atomic Energy Act and Energy

10 CFR Part 61, Subpart D, "Technical Requirements for Reorganization Act Land Disposal Facilities 
Table 3-11. DOE and executive orders to be considered.

DOE Orders

5480.1B Environment, Safety, and Health Program for DOE Operations

5480.4 Environmental Protection, Safety, and Health Protection Standards

$5400.5 \quad$ Radiation Protection of the Public and Environment

5482.1B Environment, Safety, and Health Appraisal Program

5480.10 Contractor Industrial Hygiene Program

Executive Orders

11989

Off-Road Vehicles on Public Lands

11991 and 11514

Protection and Enhancement of Environmental Quality

11543

Protection and Enhancement of the Cultural Environment

12088

Federal Compliance with Pollution Control Standards

12316

Response to Environmental Damage

12.342

Environmental Safeguards on Activities for Animal Damage and Control on Federal Lands

12580 Superfund Implementation 
Table 3-12. Preliminary state ARARs for CFA-Landfills II and III.

Potential State ARARs

Environmental Protection and Health Act (EPHA)

Hazardous Waste Management Act of 1983

Protection of Natural Resources

Toxic Substances, Air Quality

Air Pollution Permits to Construct and Operating Permits

Visible Emissions

Portable Equipment

Fugitive Dust

New Source Performance Standards

Antidegradation Policy

Short Term Activity Exemption

Subsurface Waste Disposal Facility

Hazardous Waste Management System: General

Identification and Listing of Hazardous Wastes

Land Disposal Restrictions

Idaho Solid Waste Management Regulations

Standards for Owners and Operators of Hazardous Waste Treatment, Storage, and Disposal Facilities

\section{Citation}

Idaho Code \$39-101 through 119

Idaho Code $\$ 39-4401$ to 4432

Idaho Code \$67-5801 through 5804

IDAPA $\$ 16 .($ ) i.1011, 01

IDAPA $\$ 16.01 .1012$

IDAPA $\$ 16.01 .1201$

IDAPA $\$ 1601.1013$

IDAPA $\$ 16.01,1251$ to 1253

IDAPA $\$ 16.01 .1951$

IDAPA $\$ 16.01 .2051$

IDAPA $\$ 16.01 .2301$

IDAPA $\$ 16.01 .2460$

IDAPA $\$ 16.01 .5004$

IDAPA $\$ 16.01 .5005$

IDAPA $\$ 16.01 .5011$

IDAPA $\$ 16.01 .60144,01$

IDAPA § 16.01 .50018 


\section{WORK PLAN RATIONALE}

Containment has been identified as the most likely response action at these landfills because (a) these CERCLA landfills are primarily composed of cafeteria, office, and construction wastes and to a lesser extent hazardous wastes; and (b) the volume and heterogeneity of the waste within these landfills will make treatment impractical. Therefore, the site characterization strategies have been limited to focus on the data required to evaluate alternatives that are most practicable for CERCLA landfill sites (i.e., containment) and to determine if unacceptable risks exist to human health and the environment.

Existing data for Landfill II, as discussed in Section 3.1, consists of topographic data, soil cover thickness data, cover soils physical and hydraulic properties data, geophysical data (terrain conductivity, ground penetrating radar, time-domain electromagnetic induction), shallow soil gas [<5 ft $(1.5 \mathrm{~m})$ at 179 locations], and borehole soil gas data, limited subsurface soil chemical data, vadose zone moisture monitoring data, and groundwatei quality data from both upgradient and downgradient wells. Data gaps are (a) the nature and concentration of organic and metals contamination in surface soils, (b) the nature and concentration of methane and volatile organics in shallow soil gas, instrumented borehole soil gas, and landfill emissions, (c) the nature and concentration of contaminants in subsurface soil and, if present, leachate contamination, (d) an evaluation of existing moisture monitoring data, (e) the source of particulate chromium in the groundwater, which will be evaluated by removing the dedicated sampling pumps from both upgradient and downgradient wells and by collecting groundwater for total metals and $\mathrm{Cr}^{6+}$ analysis and other parameters, $(f)$ the groundwater flow regime at CFA, and $(\mathrm{g})$ depth and topography of underlying basalt.

Existing data for Landfill III, also discussed in Section 3.1, includes instrumented borehole soil gas data, subsurface soil chemical data, vadose zone moisture monitoring data, and groundwater quality data from both upgradient and downgradient wells. Data gaps are (a) topographic data to identify topographic anomalies, document erosional features, and determine the adequacy of the existing cover for controlling erosion from surface runon/runoff, (b) geophysical data to determine the perimeter boundary of the landfill, the boundaries of the trenches within the landfill, the depth and topography of the underlying basalt, and the depth of the overburden cover material, and to define areas containing high concentrations of metal, (c) the nature and concentration of organic and metals contamination in surface soils, (d) the nature and concentration of methane and volatile organics in shallow soil gas, instrumented borchole soil gas, and landfill emissions, (e) an evaluation of existing moisture monitoring data, (f) the source of particulate chromium in the groundwater, which will be evaluated by removing the dedicated sampling pumps from both upgradient and downgradient wells and by collecting groundwater for total metals and $\mathrm{Cr}^{6+}$ analysis and other parameters, and $(\mathrm{g})$ the groundwater flow regime at $\mathrm{CFA}$.

The following sections discuss the overall and site-specific objectives for the RI/FS of CFA Landfills II and III and the data quality objectives to ensure data of known and appropriate quality are obtained. 


\subsection{Remedial Investigation and Feasibility Study Objectives}

The overall goals of this RI/FS are to:

- Complete a field program for collecting data to quantify the presence and magnitude of contamination in surface and subsurface soils, landfill gas, and the groundwater.

- Determine if unacceptable risks exist to human health and the environment.

- Develop and evaluate remedial action alternatives if unacceptable risks are identified.

The specific objectives of the CFA Landfills II and III RI and the necessary actions to accomplish these objectives are shown in Table 4-1.

\subsection{Data Quality Objectives}

The data to be collected during the RI will be used for site characterization, risk assessment, evaluation of alternatives, engineering design of alternatives, and worker health and safety. The following briefly summarizes each general purpose category:

- Site characterization (SC) - Data will need to be acquired from surface and subsurface soil, landfill gas, and groundwater to supplement existing information so that the nature and magnitude of contamination at the CFA landfills can be further defined.

- Risk Assessment (RA) - Data will need to be acquired in order to evaluate the threat posed by contaminants at Landfills II and III to the local human population and environment. Samples collected from surface and subsurface soil, soil gas surveys and groundwater sampling tasks will be used to characterize direct exposure risks to potential receptors. These data will also be required for the fate and transport modeling to support the risk assessment.

- Evaluation of remedial action alternatives (تRAA) - Data will be required to evaluate various remedial technologies that may be implemented at the site. Data may include physical, chemical, and/or hydrogeologic data, which will be obtained during various laboratory and field studies.

- Engineering design of alternatives (EDA) - Data may need to be collected to support design of selected remedial alternatives. Data may include physical, chemical and/or hydrogeological data obtained during various laboratory and field studies.

- Worker health and safety (WHS) - Data are required to establish the level of protection needed for investigators or workers at the CFA landfill sites during RI/FS field investigations and to determine if there is a concern for the population in the vicinity of the site. Data collection will focus on exposure levels for contaminants of concern. 
Table 4-1. Remedial investigation objectives for CFA Landfills II and III.

\begin{tabular}{|c|c|c|}
\hline Activity & Objective & Action \\
\hline \multirow[t]{3}{*}{$\begin{array}{l}\text { Topographic survey } \\
\text { (Landfill III only) }\end{array}$} & $\begin{array}{l}\text { Identify topographic } \\
\text { anomalies }\end{array}$ & Topographic survey \\
\hline & Document erosional features & \\
\hline & $\begin{array}{l}\text { Determine the adequacy of } \\
\text { the existing landfill cover for } \\
\text { controlling erosion from } \\
\text { surface runon/runoff }\end{array}$ & \\
\hline \multirow[t]{4}{*}{$\begin{array}{l}\text { Geophysical survey } \\
\text { (Landfills II and III) }\end{array}$} & $\begin{array}{l}\text { Determine the perimeter } \\
\text { boundary of the landfill and } \\
\text { the boundaries of the } \\
\text { trenches within the landfill } \\
\text { (Landfill III) }\end{array}$ & $\begin{array}{l}\text { Terrain conductivity and } \\
\text { magnetics }\end{array}$ \\
\hline & $\begin{array}{l}\text { Determine the depth and } \\
\text { topography of the underlying } \\
\text { basalt (both landfills) }\end{array}$ & Seismic refraction \\
\hline & $\begin{array}{l}\text { Define areas containing high } \\
\text { concentrations of metal } \\
\text { (Landfill III) }\end{array}$ & $\begin{array}{l}\text { Terrain conductivity and } \\
\text { magnetics } \\
\text { Ground penetrating radar }\end{array}$ \\
\hline & $\begin{array}{l}\text { Map depth of overburden } \\
\text { cover material (Landfill III) }\end{array}$ & \\
\hline \multirow[t]{3}{*}{$\begin{array}{l}\text { Surficial soil sampling } \\
\text { (Landfills II and III) }\end{array}$} & $\begin{array}{l}\text { Determine the presence and } \\
\text { concentration of } \\
\text { contamination in surface soils }\end{array}$ & $\begin{array}{l}\text { Sample surface soils and } \\
\text { analyze for VOCs, SVOCs, } \\
\text { and metals, including cyanide } \\
\text { at Landfill II }\end{array}$ \\
\hline & $\begin{array}{l}\text { Determine if the surficial soils } \\
\text { should be included in the } \\
\text { source control actions for the } \\
\text { landfill }\end{array}$ & \\
\hline & $\begin{array}{l}\text { Characterize direct exposure } \\
\text { risks }\end{array}$ & \\
\hline
\end{tabular}


Table 4-1. (continued).

\begin{tabular}{lll}
\hline \multicolumn{1}{c}{ Activity } & \multicolumn{1}{c}{ Objective } & \multicolumn{1}{c}{ Action } \\
\hline $\begin{array}{l}\text { Cover investigation } \\
\text { (Landfill III only) }\end{array}$ & $\begin{array}{l}\text { Evaluate the physical } \\
\text { properties of the geologic } \\
\text { unit governing transport of } \\
\text { contaminants and evaluate } \\
\text { potential for infiltration }\end{array}$ & $\begin{array}{l}\text { Collect data on hydraulic } \\
\text { conductivity, porosity, soil } \\
\text { moisture content, particle } \\
\text { size, density, cover thickness, } \\
\text { capillary pressure-moisture } \\
\text { content relationship }\end{array}$ \\
& $\begin{array}{l}\text { Collect data on soil } \\
\text { characteristics to determine if } \\
\text { onsite soil can be used as fill } \\
\text { material and to determine } \\
\text { placement of a potential } \\
\text { cover }\end{array}$ & \\
& &
\end{tabular}

Soil gas survey

(Landfills II and III)
Evaluate existing cover to determine physical soil properties and thickness

Determine extent of vegetation cover, any vegetative stress, and erosion

Identify areas within the landfill containing high concentrations of explosive or toxic landfill gas to perform an assessment of human health risks due to air toxics and explosive hazards

Evaluate the feasibility of gas collection and treatment

\section{Vegetation survey}

Collect soil gas samples from the cover, instrumented boreholes, and landfill surface emissions
Obtain flow-related data using a surface flux chamber, estimate emission rates, and perform air modeling

Evaluate remedial actions

Estimate concentrations of selected VOCs being emitted to the atmosphere to characterize direct exposure risks
Collect and analyze ambient air samples with a surface flux chamber 
Table 4-1. (continued).

\begin{tabular}{lll}
\hline \multicolumn{1}{c}{ Activity } & \multicolumn{1}{c}{ Objective } & \multicolumn{1}{c}{ Action } \\
\hline $\begin{array}{l}\text { Potential for leachate } \\
\text { (Landfills II and III) }\end{array}$ & $\begin{array}{l}\text { Estimate potential movement } \\
\text { of water through landfills }\end{array}$ & $\begin{array}{l}\text { Perform water balance } \\
\text { calculations on landfills }\end{array}$ \\
& $\begin{array}{l}\text { Evaluate existing moisture } \\
\text { monitoring data and collect } \\
\text { neutron probe moisture data }\end{array}$ \\
$\begin{array}{l}\text { Subsurface soil sampling } \\
\text { (Landfill II only) }^{\mathrm{a}}\end{array}$ & $\begin{array}{l}\text { Determine nature and } \\
\text { concentration of contaminants } \\
\text { in and below the landfill }\end{array}$ & $\begin{array}{l}\text { Sample subsurface soils and } \\
\text { landfill material for VOCs, } \\
\text { SVOCs, and metals including } \\
\text { cyanide }\end{array}$ \\
& &
\end{tabular}

Provide data for modeling migration of contaminants from the landfill

Determine presence and nature of leachate

Sample leachate for VOCs, SVOCs, and metals, if present. Inspect toe of slope for seepage and sample for VOCs, SVOCs, and metals including cyanide, if leachate is present.

Groundwater Investigation (Landfills II and III)

Determine source of chromium contamination and Remove existing pumps from Landfill wells and collect the potential for other siterelated contamination of the aquifer groundwater samples and analyze for VOCs, metals including cyanide, and common anions

Characterize ingestion exposure risks

Determine depth to groundwater, groundwater flow direction and rate

Take water level mersurements

Correct measured depth to water for potential borehole Borehole deviation logging deviation effects

a. The need for subsurface soil sampling at Landfill III will be evaluated by DOE, EPA, and IDHW based on the results of surface soil sampling and the soil gas survey at Landfill III. 
In order to achieve the established DQOs, the various tasks of the RI/FS will require different levels of data quality. The assignment of analytical levels to the various data types are based on the intended use of the data and the QA/QC protocols available for the test methods being considered. Analytical levels applicable to the data needs at the CFA landfills are defined in Table 4-2. The types of soil, groundwater, and landfill gas samples to be collected for a sufficient representation of the conditions at the site; the chemicals of concern for which the samples are to be analyzed; and the methods and analytical levels are summarized in Table 4-3. Additional data input requirements and the RI task or source of the input data for modeling tasks are discussed in Section 5. Statements pertaining to the precision, accuracy, representativeness, completeness, and comparability parameters for these methods can be found in the QAPJP.

Table 4-2. Analytical levels ${ }^{\mathrm{a}}$

Level I Analyses done by on-site instrumentation primarily used for monitoring air for health and safety purposes (e.g., organic vapor monitoring instruments). Limited quantitative information can be gathered along with limited qualitative information (e.g., presence of volatile organics, not which compound is present).

Level II Analyses done by field instrumentation or in a mobile laboratory that provides qualitative as well as quantitative results (e.g., portable x-ray fluorescence or gas chromatograph). Data from these analyses can be used for site characterization and monitoring during remedial activities.

Level III Analyses done by any approved laboratory procedure [i.e., approved by American Society for Testing and Materials (ASTM), EPA, the ER Independent Review Committee (ER IRC), the USGS, etc.]. Data from these analyses can be used to confirm analyses performed by Level II techniques, evaluate engineering design, etc.

Level IV Chemical analyses done by any EPA-approved method or any radiological analyses by methods as specified by the Radiological Statement of Work. The laboratory deliverables consist of an EPA Contract Laboratory Program (CLP) type data package or the data package for radiological analyses specified by the Statement of Work for radiological analyses.

Level V Analyses done by modified approved methods. The EPA Special Analytical Services and ER IRC-approved methods for experimental analyses in unusual matrices are examples of Level $V$ support. These data are also used for decisions requiring the highest level of confidence in the data.

a. EG\&G Idaho, Environmental Restoration. 
Table 4-3. Data quality objectives summary for CFA Landfills II and III RI/FS.

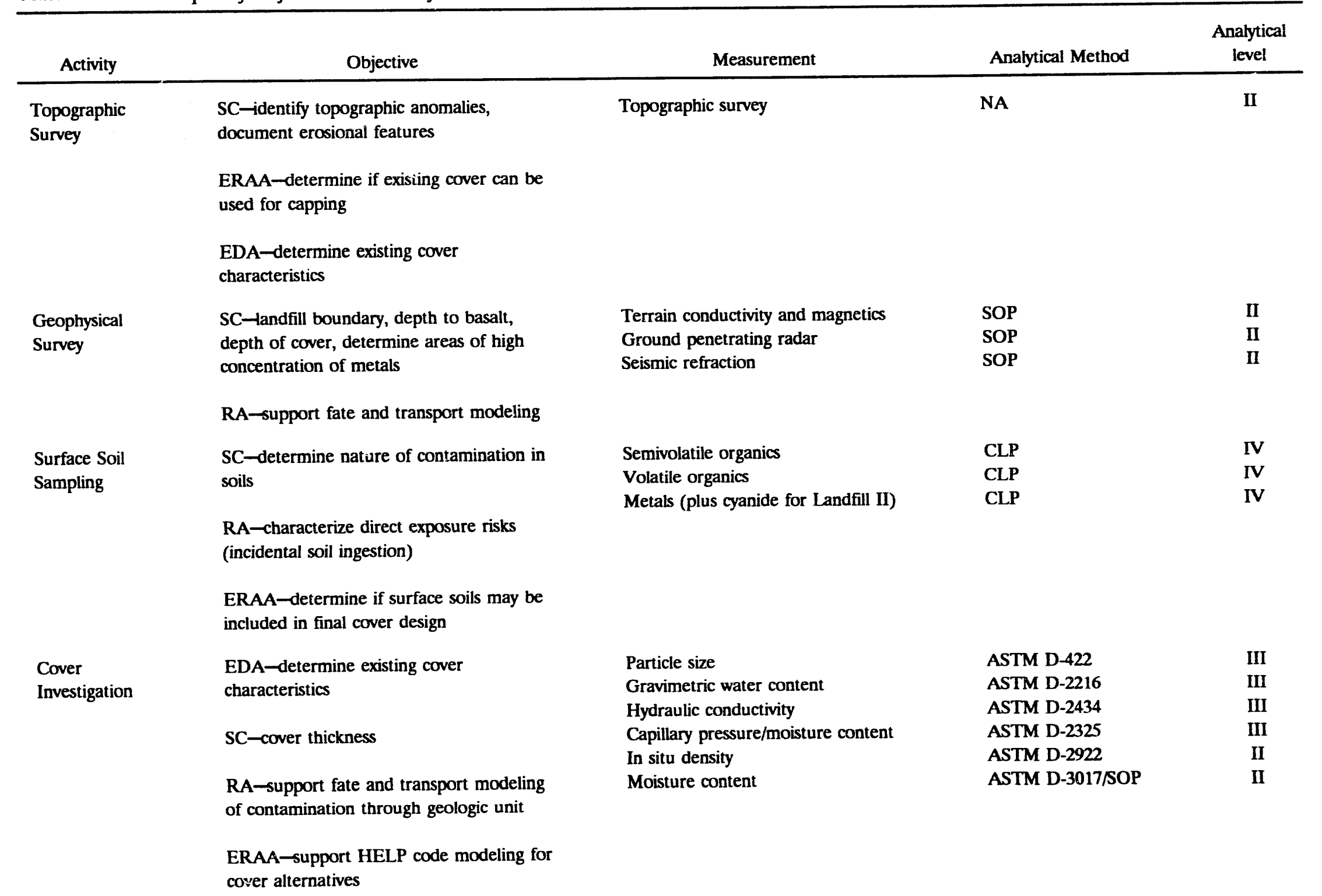


Table 4-3. (continued).

\begin{tabular}{|c|c|c|c|c|}
\hline Activity & Objective & Measurement & Analytical Method & $\begin{array}{c}\text { Analytical } \\
\text { level }\end{array}$ \\
\hline \multirow[t]{2}{*}{ Soil gas survey } & $\mathrm{SC} \rightarrow$ nature and extent of contamination & $\begin{array}{l}\text { Borehole gas-VOCs, methane } \\
\text { Surface soil gas-VOCs, methane }\end{array}$ & $\begin{array}{l}\text { EPA } 601 \\
\text { EPA } 601\end{array}$ & $\begin{array}{l}\text { III } \\
\text { III }\end{array}$ \\
\hline & $\begin{array}{l}\text { RA-evaluate human health risks due to } \\
\text { air toxics and explosive hazards and } \\
\text { characterize direct exposure }\end{array}$ & Surface flux-chamber-VOCs, methane & EPA TO-14 & III \\
\hline ' & ERAA $\rightarrow$ treatment of landfill gas & & & \\
\hline \multirow{4}{*}{$\begin{array}{l}\text { Potential for } \\
\text { Leachate }\end{array}$} & SC-estimate potential movement of water & Water balanice calculations & NA & II $^{\mathbf{a}}$ \\
\hline & through landfills & Existing moisture data & NA & $\mathrm{II}^{\mathbf{a}}$ \\
\hline & & Neutron probe moisture monitoring & SOP & II \\
\hline & $\begin{array}{l}\text { ERAA-determine if existing cover is } \\
\text { adequate for maximizing } \\
\text { evapotranspiration }\end{array}$ & & & \\
\hline \multirow{4}{*}{$\begin{array}{l}\text { Subsurface Soil } \\
\text { and Leachate } \\
\text { Sampling }\end{array}$} & $\mathrm{SC}-$ nature and concentration of & VOCs & CLP & IV \\
\hline & contaminants & SVOCs & CLP & IV \\
\hline & & Metals and cyanide & CLP & IV \\
\hline & RA-support fate and transport modeling & & & \\
\hline \multirow{9}{*}{$\begin{array}{l}\text { Groundwater } \\
\text { Investigation }\end{array}$} & SC-identify extent and type of & VOCs & EPA 524.2 & IV \\
\hline & contamination, determine groundwater & Metals & CLP & IV \\
\hline & flow direction and rate & Common anions & EPA 300 & IV \\
\hline & & Alkalinity & EPA 310.1 & IV \\
\hline & RA-assess risks due to ingestion & $\mathrm{Cr}^{6+}$ & SW 846-7195 & IV \\
\hline & & $\mathrm{pH}$, conductivity, temperature & SOP & I \\
\hline & & Water level & SOP & I \\
\hline & & Borehole deviation logging & SOP & I \\
\hline & & Cyanide & CLP & IV \\
\hline
\end{tabular}

a. Based on previously collected data from neutron probe, salinity, and heat dissipation blocks.

SOP - Standard operating procedure (see Appendix B of the Field Sampling Plan)

NA - Not applicable 


\section{RI/FS TASKS}

This section presents the tasks that will be carried out as part of this RI/FS. The RI/FS tasks identified by the EPA in "Guidance for Conducting Remedial Investigations and Feasibility Studies under CERCLA" provide consistent reporting and allow more effective monitoring of RI/FS projects (EPA 1988a). The tasks to be carried out as part of the RI/FS for the CFA Landfills II and III site are the following:

1. Project Planning

2. Community relations

3. Field investigations

4. Sample analysis and data validation

5. Data evaluation

6. Contaminant fate and transport modeling

7. Risk assessment

8. Treatability studies (if necessary)

9. RI report

10. Feasibility study (including alternative identification, screening, and detailed analysis)

11. Feasibility study report

12. Proposed Plan and Record of Decision.

A more detailed description of each of the Rl/FS tasks is provided in the following sections.

\subsection{Project Planning}

The project planning process, of which this Work Plan is a part, involves efforts to initiate the RI/FS. Included in this task are limited field investigation activities, existing data evaluation, development of the Work Plan, obtaining approvals for the Work Plan, budget and schedule preparation, preparation of the sampling and analysis plan, preparation of the health and safety plan, project management and agency coordination, and meetings between the EPA, the DOE, and the IDHW.

Development of the RI/FS Work Plan includes formulation of DQOs, identification of the necessary RI/FS tasks, and preparation of budgets and schedules for implementing the RI/FS tasks. Results of the existing data evaluation, with the exception of the vadose zone moisture monitoring data that will be evaluated during the RI, are presented in Section 3.

A sampling and analysis plan has been prepared in conjunction with this Work Plan and includes a Field Sampling Plan (FSP) and a Quality Assurance Project Plan (QAPjP), which are included as addenda to this document. The FSP defines sampling locations, collection procedures, and the equipment necessary for sampling and testing. The QAPjP specifies the analytical procedures and the methods for analytical choices and data reduction, validation, and reporting. The guiding Data Management Plan (DMP) for this RI/FS will be the Data Management Plan for the EG\&G Idaho Environmental Restoration Department (EG\&G Idaho, 1992). The health and safety plan was prepared from historical information, OSHA regulations, and INEL health and safety policies, and is also provided as an addendum to this Work Plan. 
The guiding project management plan (PMP) for the Operable Unit 4-12 RI/FS will be the Implementing Program Management Plan for the EG\&G Idaho Environmental Restoration Department (EG\&G Idaho, 1991).

\subsection{Community Relations}

Community relations ensure that the community understands the actions taken during the remedial response activities and has the opportunity to provide input on the RI/FS program. A programmatic community relations plan (CRP) has been prepared and is attached as an addendum to this Work Plan. The CRP will guide the actions taken to ensure appropriate public involvement in agency decision making.

\subsection{Field Investigations}

This section addresses the planned field investigations and data development activities that were identified through the scoping process to fill data needs identified in Section 4. These activities can be grouped into the following subtasks:

- Topographic survey (Landfill III)

- Geophysical survey (Landfills II and III)

- Soil gas surveys (Landfills II and III)

- Surface soil sampling (Landfills II and III)

- Cover characterization (Landfill III)

- Water level measurements and borehole deviation surveys (Landfills II and III)

- Groundwater sampling (Landfills II and III)

- Subsurface soil sampling (Landfill II).

All sample locations will be staked and surveyed. The following text outlines these activities with specific details provided in the FSP.

\subsubsection{Topographic Survey of Landfill III}

A topographic map of Landfill III will be prepared to (a) identify topographic anomalies, (b) document erosional features, and (c) determine the adequacy of existing landfill cover for controlling erosion from surface runon/runoff. The field survey will use the sample locations from the shallow soil gas investigation (Section 5.3.3.1) as the initial survey grid. Horizontal control for the survey will be class 3 [distance accurate to $0.007 \mathrm{ft}(0.002 \mathrm{~m})$ ], and the vertical control will be $3 \mathrm{rd}$ order $[0.05 \mathrm{ft}(0.015 \mathrm{~m})]$. If this survey grid does not provide adequate horizontal and vertical control to compile a topographic map with $1 \mathrm{ft}$ contour intervals at a scale of 1 in. $(2.54 \mathrm{~cm})$ equals $100 \mathrm{ft}(30.6 \mathrm{~m})$, then additional survey points will be necessary to further define the topography of the site. Locations for additional survey points will be determined in the field based on the surveyor's judgement. A topographic map covering the 11 acres (4 hectares) of the site and immediate surrounding area will be prepared consisting of contour intervals not to exceed $1 \mathrm{ft}(0.3 \mathrm{~m})$ and with a scale of $1 \mathrm{in} .(2.54 \mathrm{~cm})$ equal $100 \mathrm{ft}(30.6 \mathrm{~m})$. A map of the surfacewater drainage system in this area will also be prer red. 


\subsubsection{Geophysical Survey of Landfills II and III}

A combination of geophysical surveys will be performed at Landfill III. The objectives of the surveys are to (a) determine the perimeter boundary of Landfill III and the boundaries of the trenches in the landfill, (b) determine the depth and topography of the underlying basalt, (c) define areas containing high concentrations of metal, and (d) determine overburden cover thickness. The depth and topography of the underlying basalt at Landfill II will also be determined.

Data from the geophysical surveys will be used to generate maps showing the landfill boundaries, topography of the basalt surface, the distribution of electrically conductive bodies, the distribution of buried magnetic material, and overburden cover thickness. This data will be used to confirm the historical landfill boundaries, as input data to the fate and transport modeling studies, and for comparison to soil gas and soil contaminant data.

Seismic refraction methods will be used to determine the thickness of surficial sediments and the general topography of the underlying basalt at Landfills II and III. At Landfill III, data will be collected on profiles across the short dimension of the landfill and will provide depth estimates every $16.4 \mathrm{ft}(5 \mathrm{~m})$ along each profile with $98 \mathrm{ft}(30 \mathrm{~m})$ between profiles (Figure 5-1a). For Landfill II, data will be collected to provide depth estimates every $16.4 \mathrm{ft}(5 \mathrm{~m})$ along each profile with $100 \mathrm{ft}(30.6 \mathrm{~m})$ between profiles (Figure 5-2). Induced electromagnetic measurements will be used to map concentrations of conductive materials such as steel drums, scrap metal, and areas of elevated soil moisture at Landfill III. These measurements will be made on a regular grid of $\mathbf{3}$ $\mathrm{ft}(1 \mathrm{~m})$ by $13 \mathrm{ft}(4 \mathrm{~m})$ over the entire landfill area (Figure 5-1b). Magnetic field measurements will be used to map the distribution of buried ferrous objects at Landfill III. These measurements will be made on a regular grid of $1.6 \mathrm{ft}(0.5 \mathrm{~m})$ by $3 \mathrm{ft}(1 \mathrm{~m})$ over the entire survey area (Figure 5-1c). These sampling grids should allow for the clear definition of the landfill area and the individual trenches making up Landfill III. Ground penetrating radar will be used to deterınine the overburden cover thickness at Landfill III. Additionally, several random boreholes will be augered through the cover material to confirm the results of the ground penetrating radar.

\subsubsection{Soil Gas Sampling}

A combination of soil gas sampling techniques will be used at the landfills to (a) identify areas within the landfill containing high concentrations of explosive or toxic landfill gas to perform an assessment of human health risks due to air toxics and explosive hazards, (b) evaluate the feasibility of gas collection and treatment, (c) evaluate remedial actions, and (d) estimate concentrations of selected VOCs being emitted to the atmosphere to characterize direct exposure risks by inhalation. This will be accomplished through a shallow soil gas survey, soil gas collection from the instrumented boreholes, and by sampling with a surface-flux chamber.

5.3.3.1 Shallow Soil Gas Survey - Landfill II and III. The objectives of the shallow soil gas survey at Landfills II and III are to (a) define potential source areas for volatile organics, (b) determine the concentration and distribution of methane, and (c) delineate vapor phase contaminant migration. The results of this survey and existing data obtained from the 1989 shallow soil gas survey at Landfill II will be used to provide information for contaminant fate and transport modeling in support of a qualitative baseline risk assessment for the inhalation pathway. 


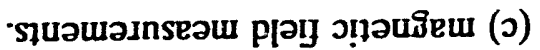

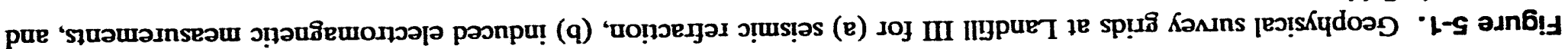
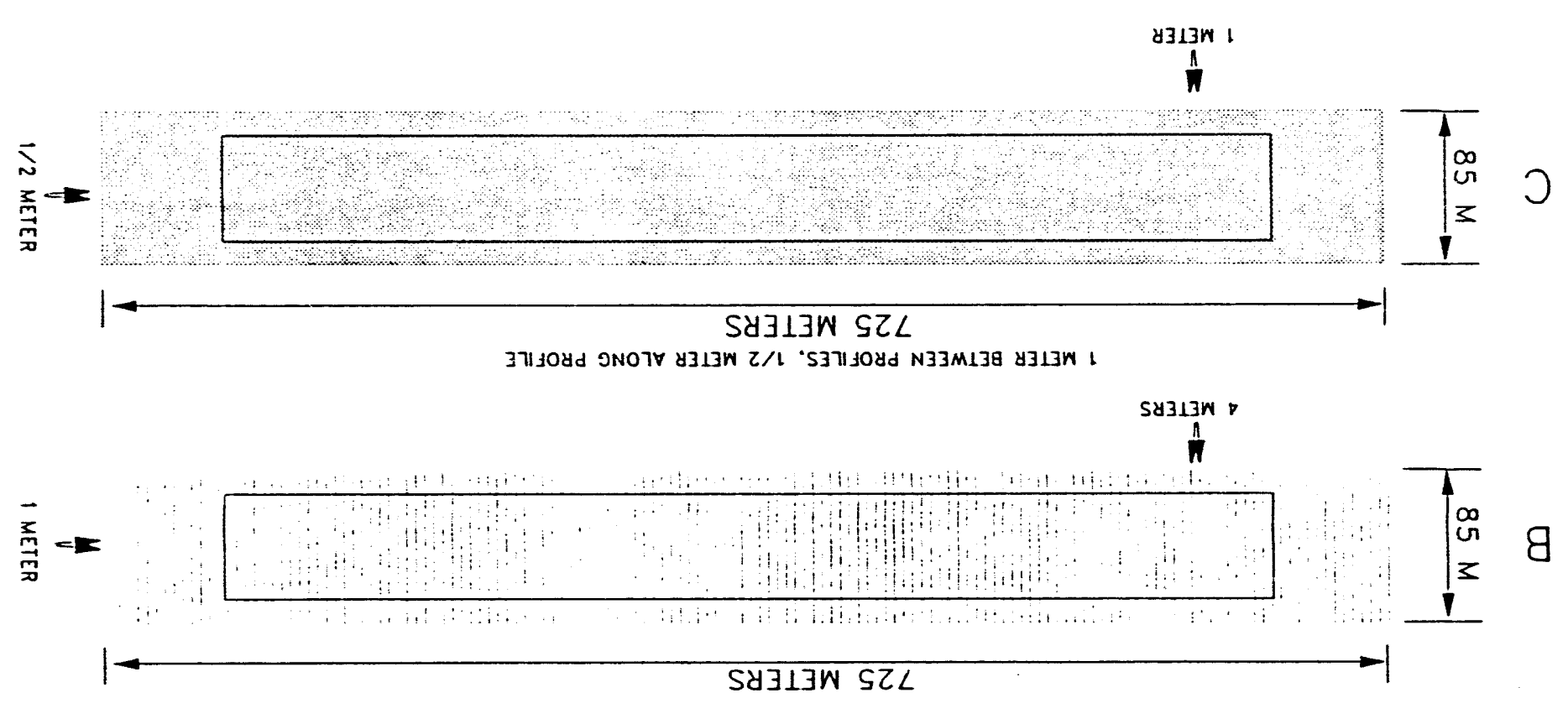

3า1 108d 9NO

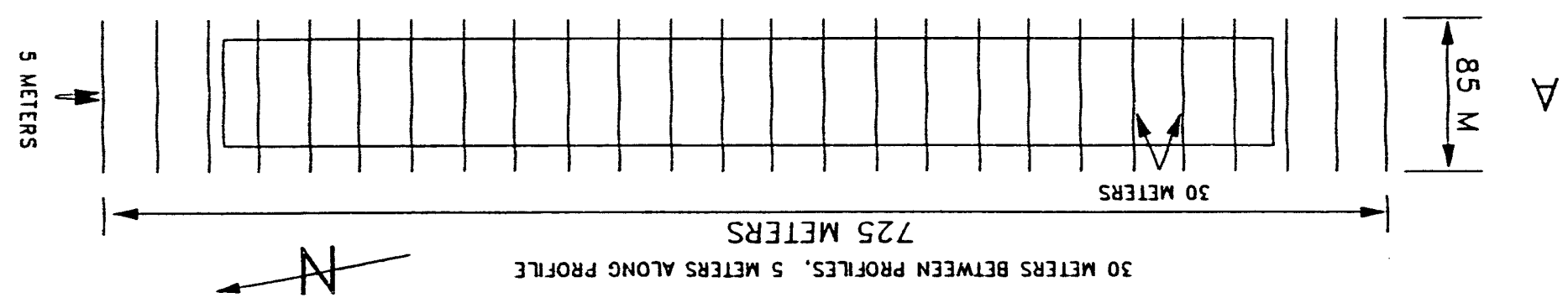



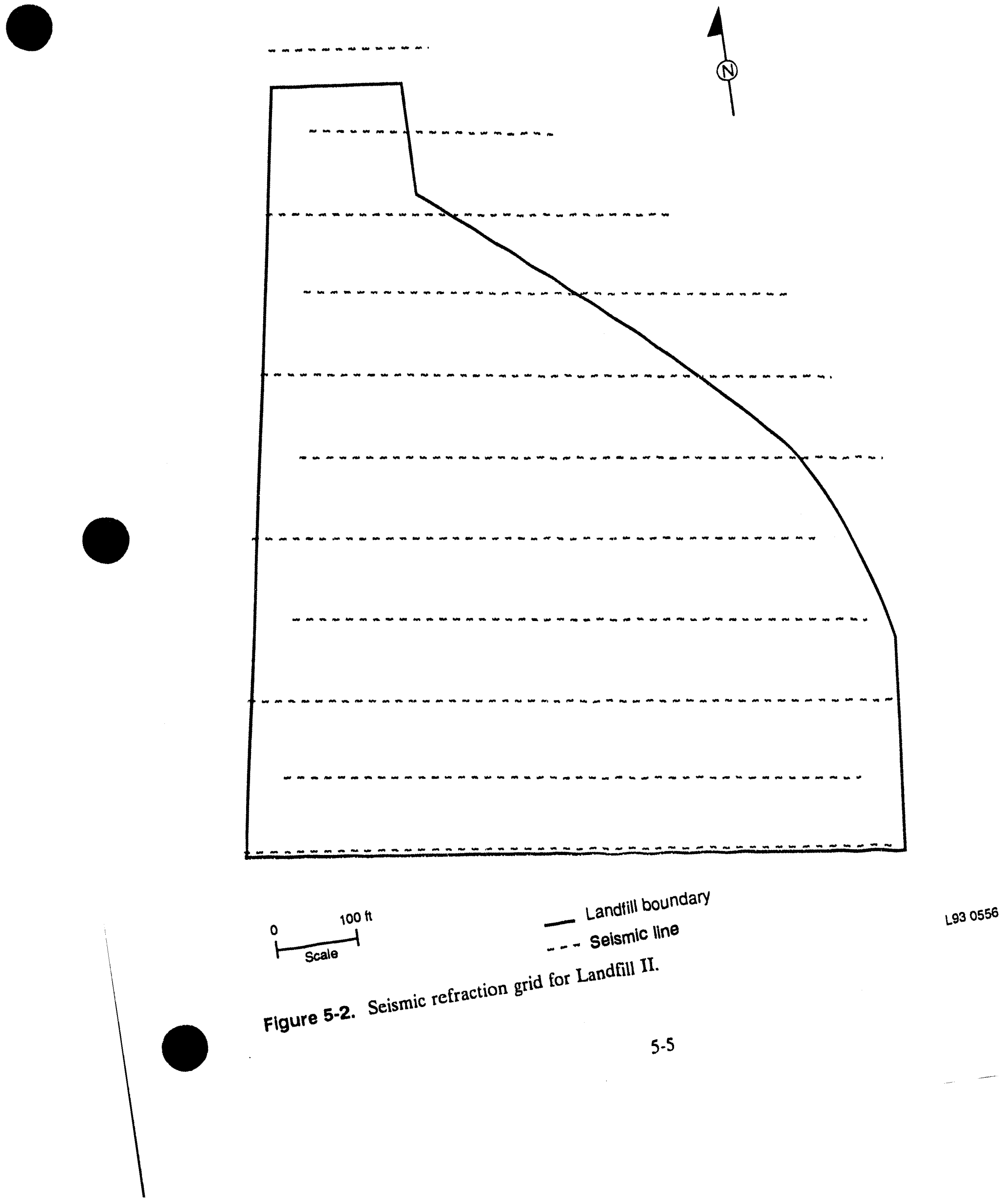


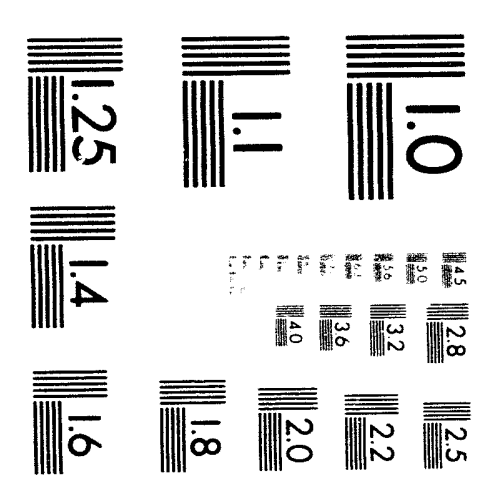



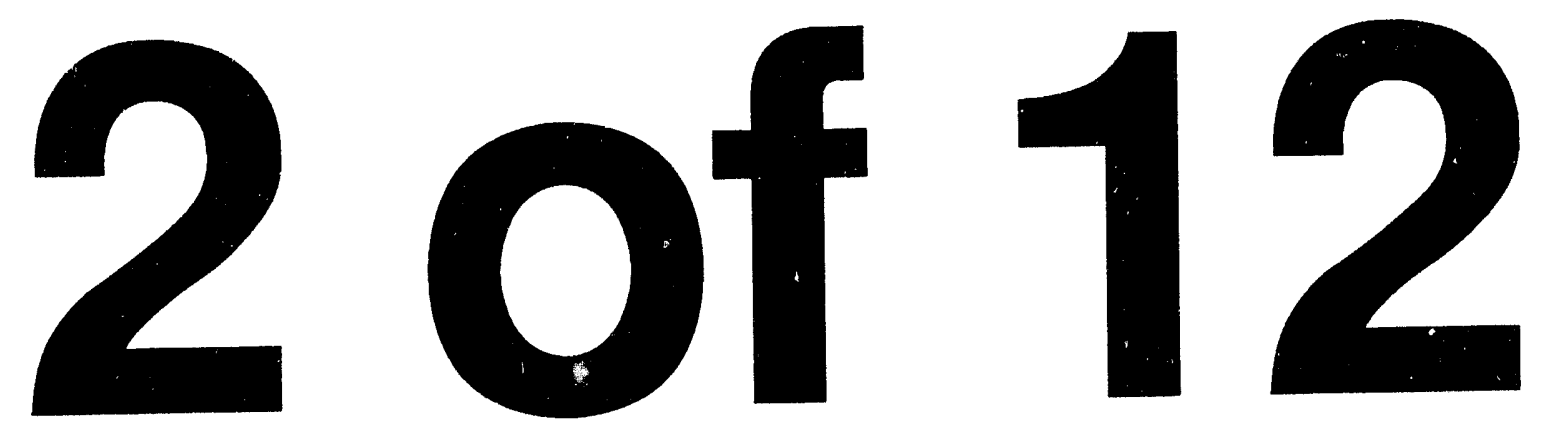
A regularly spaced sampling grid on $100-\mathrm{ft}(30.6-\mathrm{m})$ centers will be used over an area completely enclosing landfill III and along the perimeter of the landfill (Figure 5-3). The regularly spaced grid and the grid size used for the soil gas sampling is based on the nature of the landfill with regularly spaced trenches and no known hot spots (EPA 1991a). A regularly spaced sampling grid on $100-\mathrm{ft}(30.6-\mathrm{m})$ centers will also be used over an area completely enclosing Landfill II (Figure 5-4) (EPA 1991a).

To collect the soil gas samples, a 0.5 to 1 -in. $(1.27$ to $2.54-\mathrm{cm})$ hole will be driven to a depth of approximately $4 \mathrm{ft}(1.29 \mathrm{~m})$ using a drive rod or electric hammer drill. A stainless steel probe will be inserted to the full depth of the hole and sealed off from the atmosphere. A sample of insitu soil gas will be withdrawn through the probe to purge ambient air from the sampling system. A second sample of soil gas will then be withdrawn through the probe and trapped in a preevacuated glass vial at two atmospheres of pressure. The self-sealing vial will then be detached from the sampling system, packaged, and sent for laboratory analysis. All samples collected will be analyzed according to EPA Method 601 using either an electron capture detector or a flame ionization detector. Details of the sampling program and QA/QC samples and procedures are provided in the FSP.

5.3.3.2 Borehole Soil Gas Survey. A total of nine shallow boreholes instrumented with gas ports have been installed in Landfills II and III (Figure 5-5). In May 1988, soil gas samples were collected from these boreholes and several volatile organics were detected at low to moderate concentrations. Methane was detected in several boreholes above the lower explosive limit in Landfill II. During the RI, borehole soil gas samples will be collected from these existing instrumented boreholes to (a) determine the concentration of vapor phase volatile organics and methane in the subsurface soils, (b) evaluate the feasibility of gas collection and treatment, and (c) evaluate potential remedial actions. All samples will be collected and analyzed as described for the shallow soil gas survey. Data collected will be used for fate and transport modeling and the baseline risk assessment.

5.3.3.3 Surface Flux Chamber Sampling. VOC emission rates from the surface of Landfills II and III will be determined using an emission flux chamber (EPA 1986). The chamber is placed on the soil surface and air of known purity is swept through the chamber at a known, constant rate. Air samples are collected at the exit of the chamber and VOC concentrations are determined by gas chromatography. The VOC emission rate is calculated as:

$E_{i}=\frac{Q C_{i}}{A}$

where

$E_{i}=$ emission rate of compound $i, \mu \mathrm{g} / \mathrm{m}^{2}-\mathrm{s}$

$\mathrm{C}_{\mathrm{i}}=$ concentration of compound $\mathrm{i}$ in exhaust gas, $\mu \mathrm{g} / \mathrm{m}^{3}$

$\mathrm{Q}=$ volumetric air flow rate, $\mathrm{m}^{3} / \mathrm{s}$

$\mathrm{A}=$ chamber surface area, $\mathrm{m}^{2}$ 


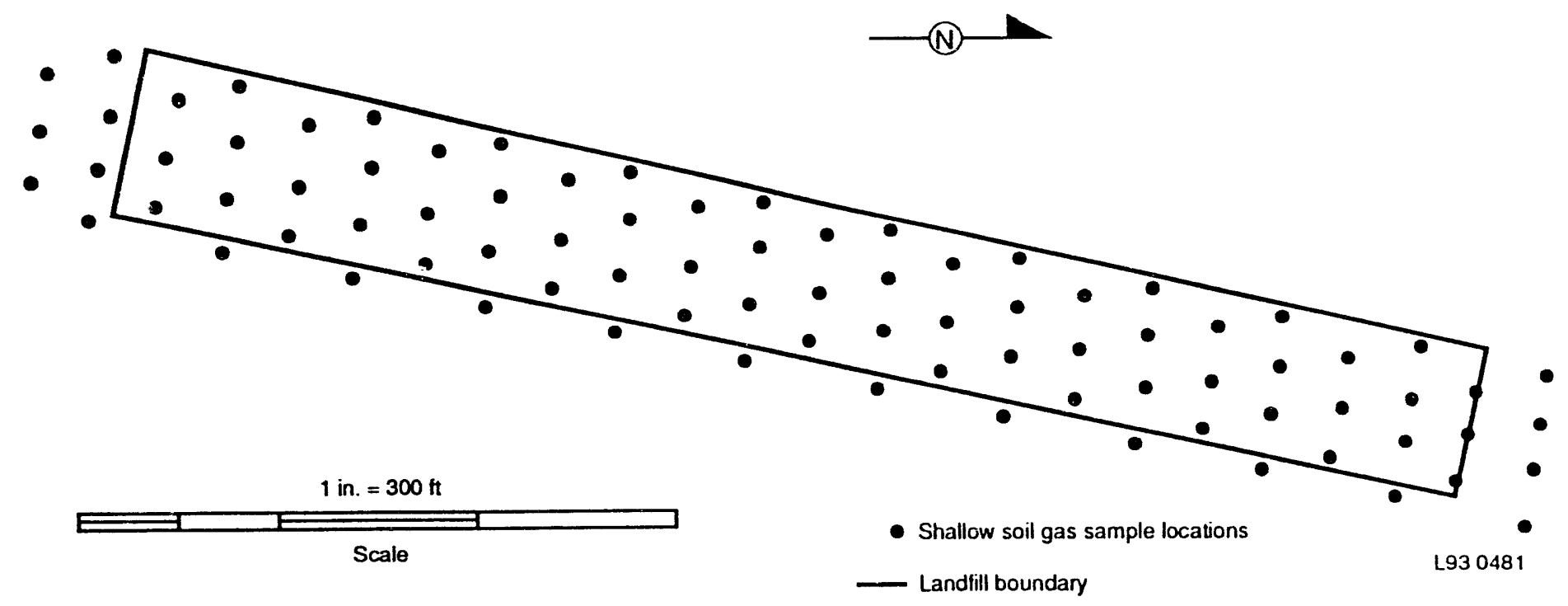

Figure 5-3. Shallow soil gas sample locations for Landfill III. 


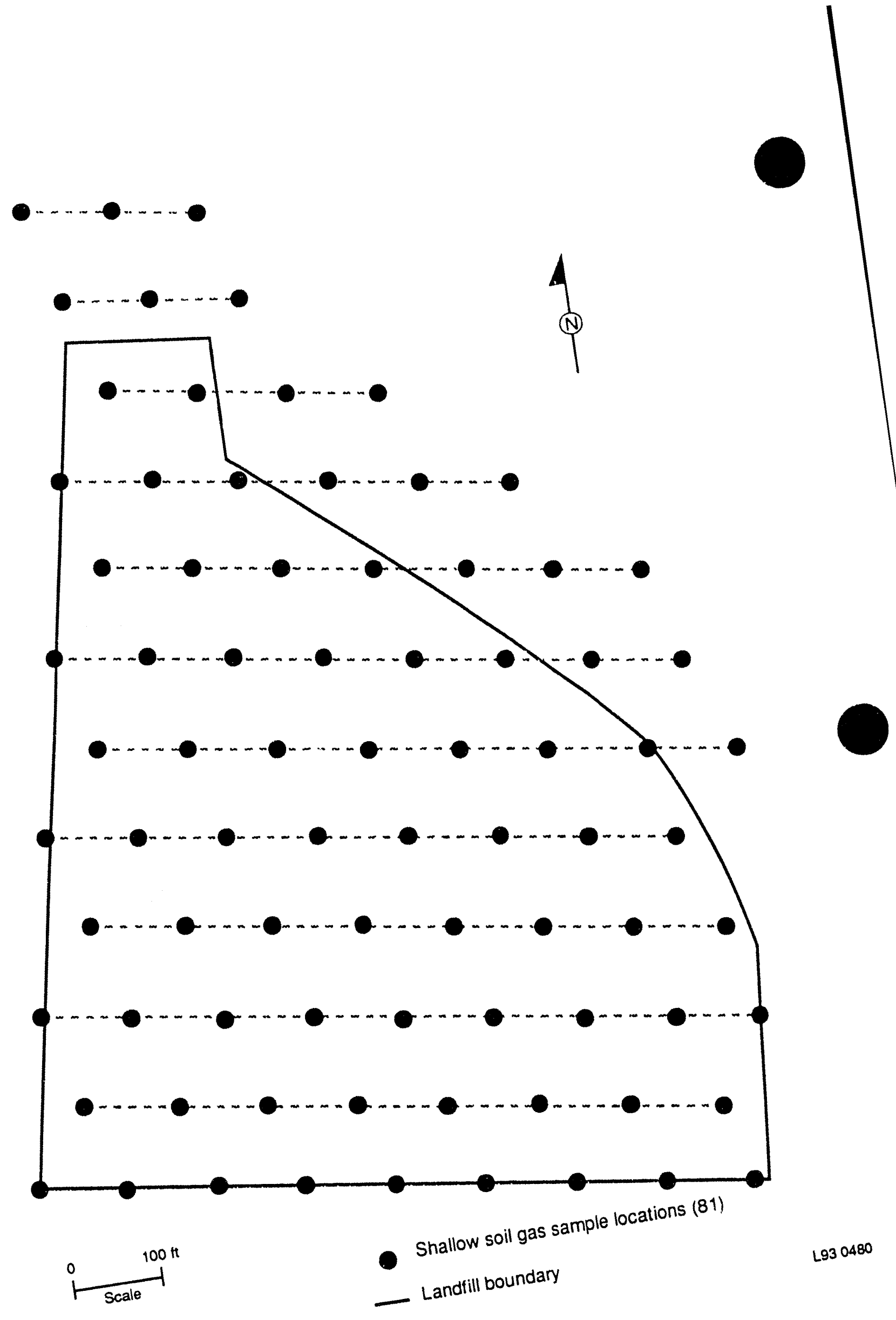



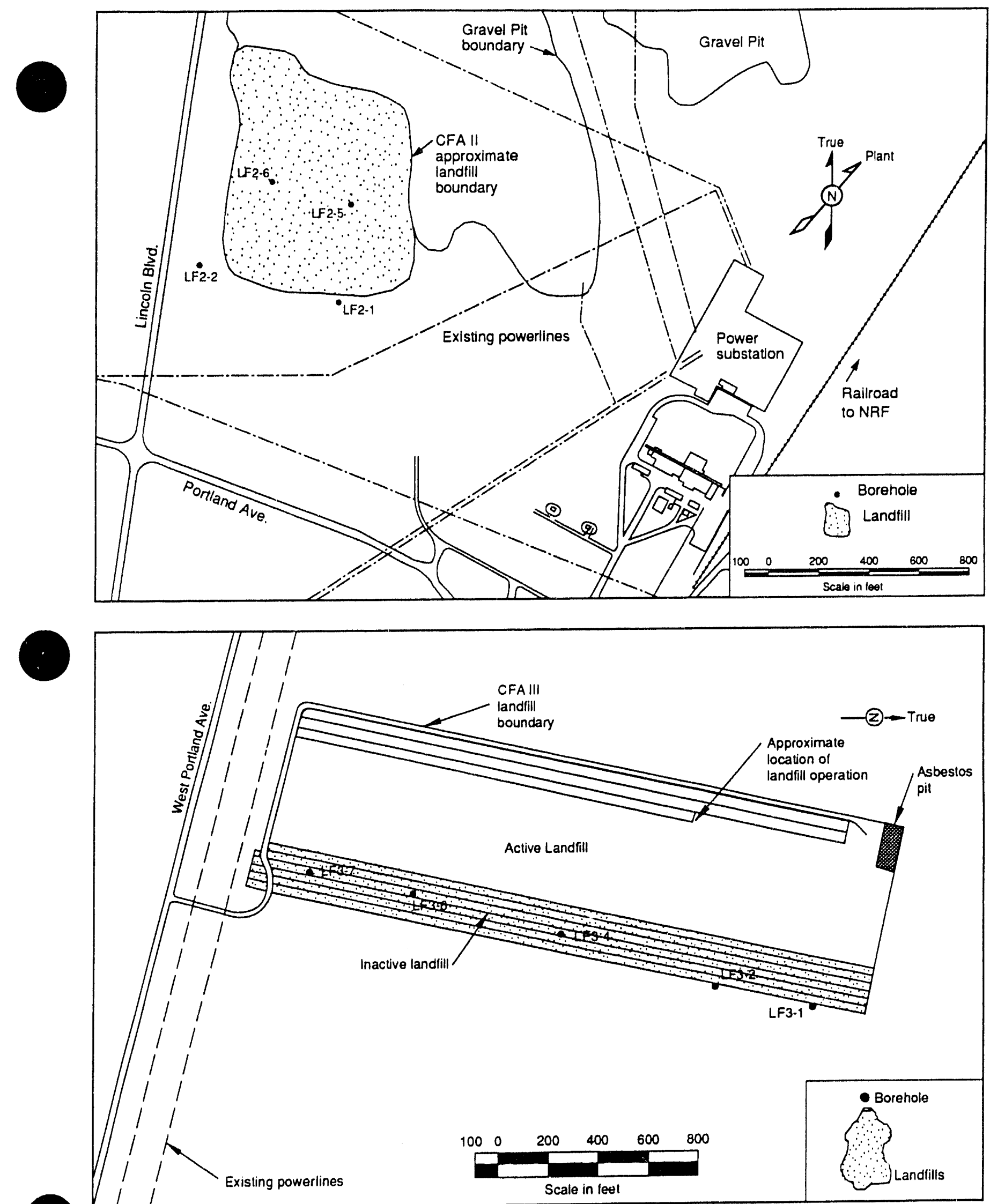

Figure 5-5. Location of boreholes to be used in borehole gas sampling.

L93 0027 
A minimum of eight sampling locations per landfill will be selected to determine the VOC emission rate measurements for each landfill. Sampling locations will be selected based on any hot spots identified during the shallow soil gas survey. If the shallow soil gas data are not available at the time of sampling or hot spots are not identified, sample locations will be randomly selected. All samples collected will be analyzed according to EPA method TO-14 (EPA 1988c). Details of the sampling program and QA/QC samples and procedures are provided in the FSP.

\subsubsection{Surface Soil Sampling}

Surface soil will be collected from each landfill for analysis of volatile organics, semivolatile organics, and metals. This data will be collected to (a) determine the distribution and concentration of potential contamination in surface soils, (b) determine if the surficial soils should be included in the source control actions for the landfills, and (c) characterize direct exposure risks for the landfills. At least one sample per acre will be collected for analysis. The actual sampling locations will correspond to randomly sclected grid points from among the points that were established for the shallow soil gas survey. Before sampling is initiated, the soils exposed at the surface will be examined visually by a soil scientist for evidence of staining, signs of vegetation stress, and differences in soil type to aid in sample site selection. The geophysical surveys may also provide data on anomalies or zones of surficial soil that warrant investigation. The results from soil gas collected in FY-88 from the instrumented boreholes and from the shallow soil gas survey performed on CFA Landfill II in FY-89 will also be used to select sample sites.

Samples for CLP metals (including cyanide for Landfill II samples) will be collected from the 0 to 6 -in. $(0$ to $15-\mathrm{cm})$ depth to characterize direct exposure risks. Samples for volatile organics and semivolatile organics will be collected from the 18 to 24 -in. (46 to $61-\mathrm{cm}$ ) depth because these compounds tend to volatilize from the soil at shallower depths. Surface soil samples will be collected using stainless steel trowels or shovels, hand augers, or soil sampling Shelby tubes.

\subsubsection{Cover Characterization of Landfill ill}

Because the existing soil cover may be used as a component of the final cover system, further investigation of the cover material is warranted. The objectives of the landfill cover characterization are to (a) determine the permeability of the existing cover, (b) determine the thickness of the cover, (c) evaluate the physical properties of the geologic unit governing the transport of contaminants, (d) evaluate potential for infiltration, and (e) evaluate remedial alternatives. The cover material on Landfill II was investigated in 1989 (Miller et al. 1990).

The tasks associated with the Landfill III cover characterization are summarized below, with detailed descriptions of the activities presented in the Field Sampling Plan.

5.3.5.1 Field Measurements of the Soil Cover. At least one sample per acre for a total of 12 sampling sites will be selected across CFA Landfill III (EPA 1991a). Actual sampling sites will be the same as those identified for the surface soil sampling (Section 5.3.4). The physical and hydraulic properties of the soil cover across the landfill wili be determined. Field measurements of the soil cover from each of the selected sites will include the following: 
- In-situ density and moisture content using a nuclear density gauge (neutron densometer) following ASTM D-2922 and ASTM D-3017. Measurements will be taken at 4- and 8-in. (10- and $20-\mathrm{cm})$ depths. Additionally, the moisture content of the soil cover and the landfill soils will be monitored over time using a neutron probe moisture gauge following SOP. Probe measurements will be made using the existing neutron probe access tubes at Landfill III (LF3-03 and LF3-05). Similar measurements will be made at Landfill II in neutron probe access tubes LF2-03, LF2-04, and LF2-07. The toe slope of Landfill II will also be examined weekly (beginning in May) for the presence of leachates. If leachate is present, samples will be collected for volatile organic, semivolatile organic, and metals (including cyanide) analysis.

- If the geophysical survey using ground penetrating radar was unsuccessful in determining the cover thickness, cover thickness will be determined using gasolinepowered and/or hand augers. Augering will continue until either waste is encountered or the auger cannot be advanced due to hole conditions. The borehole and soil cuttings will be surveyed periodically by an Industrial Hygienist using field screening instruments. Wastes encountered will not be removed from the borehole and each hole will be back filled with a mixture of native soil and bentonite. Cover material thickness at each site will be recorded with the results presented graphically in a plan view map of Landfill III.

5.3.5.2 Collection of Soil Samples for Laboratory Analysis. Soil samples will be collected for subsequent laboratory testing for (a) moisture content, (b) particle size analysis, and (c) hydraulic testing. Eight sample sites will be chosen randomly from among those selected for surface soil sampling (see Section 5.3.4) to represent the various types of cover material encountered at the landfill. Undisturbed soil samples will be collected for the hydraulic properties tests.

Samples for gravimetric moisture content and particle size analysis will be collected at approximately $1-\mathrm{ft}(0.3-\mathrm{m})$ depth increments using a 3.5 -in. $(9-\mathrm{cm})$ bucket auger. At each $1-\mathrm{ft}$ $(0.3-\mathrm{m})$ increment, the sample volume will be mixed and a subsample collected for gravimetric water content analysis in accordance with ASTM D-2216. The remaining sample will be collected for particle size analysis according to ASTM D-422.

Undisturbed soil samples will also be collected using thin-walled tubes in accordance with ASTM D-1587. Samples will be collected after removing the upper 3 to 4 in. (8 to $10 \mathrm{~cm}$ ) of dry cohesionless soil from the sample site by driving the sampler a minimum of 8 in. $(20 \mathrm{~cm})$ into the ground. Upon retrieval, the samples will be sealed and shipped to the laboratory for analysis of saturated hydraulic conductivity using constant-head permeability techniques (ASTM D-2434), capillary pressure-moisture content relationships according to ASTM D-2325, and porosity.

5.3.5.3 In-situ Drainage Tests. To estimate the total in-situ water holding capacity of the cover materials at Landfill III, in-situ drainage tests will be conducted. The goal is to provide an upper limit on the amount of water stored in the soil after saturation and a subsequent drainage period. These tests will be performed at the same sites chosen for collection of samples for laboratory analysis of soil physical and hydraulic properties (see Section 5.3.5.2). Each test will be performed by adding a volume of water sufficient to wet the cover material to approximately 1 to 
$2 \mathrm{ft}(0.3$ to $0.6 \mathrm{~m})$. Two to three days will be allowed for gravitational drainage. Samples will be obtained using a bucket auger, with samples collected at $1-\mathrm{ft}(0.3-\mathrm{m})$ increments through the wetted depth. Samples will be packaged and shipped to the laboratory for gravimetric water content analysis according to ASTM D-2216.

\subsubsection{Water Level Measurements and Borehole Deviation Logging}

The regional groundwater flow at CFA is in a south-to-southwest direction. However, regional flow may be affected by infiltration from percolation ponds and aquifer heterogeneities. These effects could have a direct bearing on the direction and rate of contaminant migration. Thus, it is important to understand the groundwater flow system at CFA. Monthly water level measurements will be collected from available wells (Table 5-1 and Figure 5-6), within approximately a $1-\mathrm{mi}(1.6-\mathrm{km})$ radius of the CFA landfills. Monthly water level measurements will begin in May 1993 and continue through December 1993.

A second-order survey of all wells on the INEL is being conducted to establish a common datum for INEL wells. The wells identified in Table 5-1 will be included in this survey to ensure that all wells used in this investigation reference the same datum.

Borehole deviation logs will be run by the USGS on the landfill wells (LF designator), and if possible, the USGS wells identified in Table 5-1. Prior to conducting deviation surveys, the dedicated sampling pumps installed in the wells will be removed. Information obtained from the logging effort will be used to correct the measured depth to water for borehole deviation effects.

\subsubsection{Groundwater Sampling}

The objectives of groundwater sampling at CFA Landfills II and III are to (a) determine whether or not the particulate chromium in the up and down gradient wells near the landfills is from the sampling pumps (Hydrostar model), (b) provide verification of existing water quality data, which indicates that the landfills are not a source of groundwater contamination, and (c) characterize ingestion exposure risks.

Three rounds of groundwater samples will be collected and analyzed from a network of 10 monitoring wells located both upgradient and downgradient from CFA Landfills II and III and from two production wells used for drinking water at CFA (see Table 5-2 and Figure 5-6). The first sampling event will take place in the May-June time frame, with the second and third events targeted for July and October, respectively. The results of each sampling event will be evaluated by DOE, EPA, and IDHW in order to determine the scope and/or need of each subsequent event. Analytical results from the first two rounds of sampling will be used in the baseline risk assessment, and the results from the third sampling event will be used for confirmation.

Prior to sampling, the dedicated Hydrostar sampling pumps will be removed from the wells and each well purged using a portable submersible pump until the $\mathrm{pH}$, temperature, and conductivity of the purge water has stabilized as defined in the FSP. The production wells will be

purged and sampled using the existing production well pumps. Samples from the production wells will be collected from an existing sample port. 
Table 5-1. Wells to be measured on a monthly basis for water level data.

$\begin{array}{lll}\text { LF } 2-08 & \text { USGS-020 } & \text { USGS-082 } \\ \text { LF 2-09 } & \text { USGS-034 } & \text { USGS-085 } \\ \text { LF 2-10 } & \text { USGS-035 } & \text { USGS-111 } \\ \text { LF } 2-11 & \text { USGS-036 } & \text { USGS-112 } \\ \text { LF 2-12 } & \text { USGS-037 } & \text { USGS-113 } \\ \text { LF 3-08 } & \text { USGS-038 } & \text { USGS-114 } \\ \text { LF 3-09 } & \text { USGS-039 } & \text { USGS-115 } \\ \text { LF 3-10 } & \text { USGS-057 } & \text { USGS-116 } \\ \text { LF 3-11 } & \text { USGS-077 } & \end{array}$

Table 5-2. Existing aquifer wells to be sampled.

Landfill II

Downgradient - LF 2-8, LF 2-9, LF 2-10, LF 2-12

Upgradient - LF 2-11

Landfill III

Downgradient - LF 3-8, LF 3-9, LF 3-10

Upgradient - USGS-85, LF 3-11

Production Wells:

CFA-1, CFA-2

Groundwater samples will be collected for analysis of volatile organics (EPA 524.2), CLP metals, $\mathrm{Cr}^{6+}$ (SW 846-7195), cyanide, nitrates, sulfates, chloride, fluoride, and alkalinity. Samples collected for metals and $\mathrm{Cr}^{6+}$ analysis will include both filtered and unfiltered samples. A sample from each well will also be collected for analysis of gamma radiological activity to determine offsite shipping requirements. The analyses to be performed and the desired analytical support level are presented in the FSP and in Table 5-1 of the QAPjP.

\subsubsection{Subsurface Soil Sampling of Landfill II}

Subsurface soil samples will be collected for analysis of volatile organics, semivolatile organics, and metals (including cyanide). The data will be collected to (a) determine the nature and concentration of leachable contaminants within and below the waste unit, and (b) determine if leachate is present in or below the landfill. The analytical results will be used as input to fate and transport modeling and to reduce the uncertainty associated with the source term information for Landfill II. The need for subsurface soil sampling at Landfill III will be evaluated by DOE, EPA, and IDHW based on surface soil and soil gas results from Landfill III.

Seven auger holes will be drilled and continuously sampled (with a split-spoon sampler) from land surface to the top of basalt (Figure 5-7). Borehole sites were selected primarily from the results of the 1989 shallow soil gas survey and also to provide spatial coverage across the landfill. 


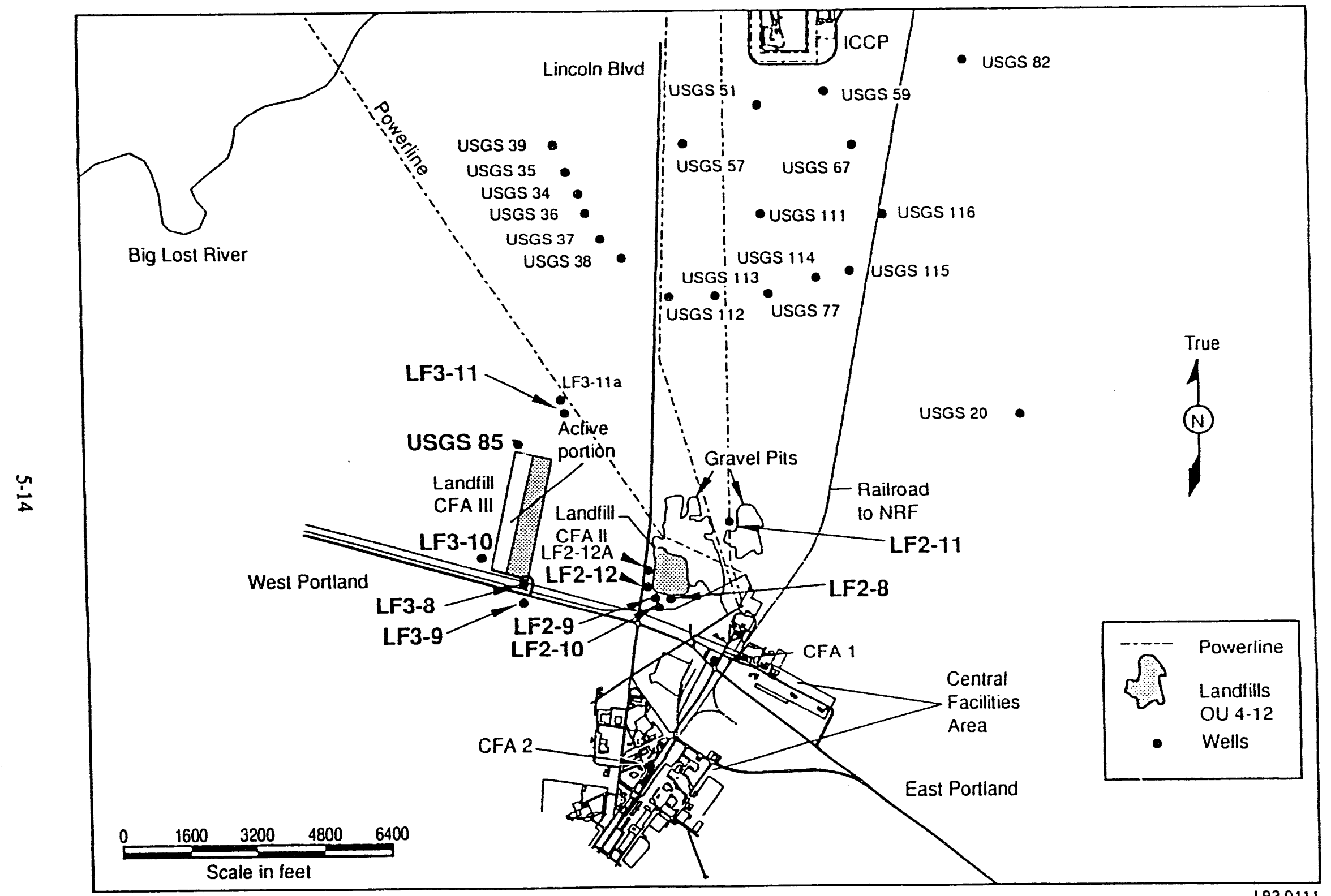

Figure 5-6. Location of wells to be used for water level measurement and groundwater sampling (bold).

L92 0414 


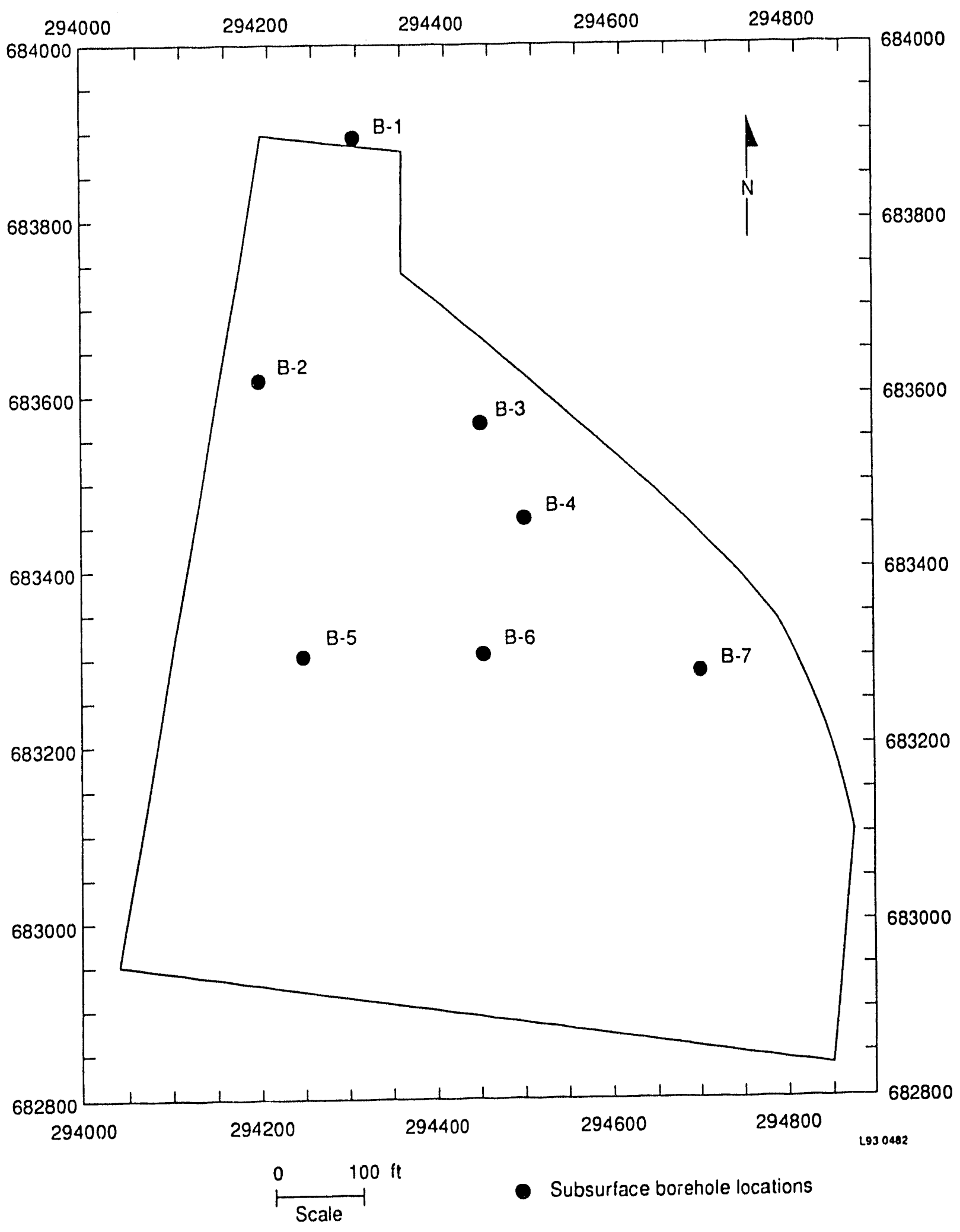

Figure 5-7. Subsurface soil boring sitcs-Landfill II. 
A minimum of two samples per borehole will be submitted for analysis: one from within the waste unit and one from below the waste unit.

If leachate is encountered during subsurface soil sampling at Landfill II, the leachate will be sampled for the same analytes as the soil samples and these boreholes will also be completed as leachate monitoring wells.

A detailed discussion of field screening, soil and leachate sampling, and leachate well installation is provided in the FSP.

Four site comparison samples will be submitted along with the subsurface soil samples for semivolatile organic and metals analysis. The site comparison samples are soils that were spiked with semivolatile and metal analytes in April 1992 by the EPA. The data from these analyses will be used to evaluate analytical precision and accuracy.

\subsection{Sample Analysis and Data Validation}

This task involves efforts relating to the analysis and validation of samples, including the development of sample management and QC procedures. The methods and protocols that will be used in the analysis of samples collected from the CFA landfills are described in the SAP, an addendum to this Work Plan.

Data from samples collected from the CFA landfills will be validated using the EG\&G Idaho ER Sample Management Office's Standard Operating Procedures (SOPs) (12.1.1 - 12.1.5). The process of validating data is also discussed in the ER Program Directive (PD) 2.4. Once data are validated through this process, it will be transferred to the ER Data Management System described in the DMP. Quality-assured data or results shall be submitted to EPA and IDHW as they become available but no later than 120 days after collection.

\subsection{Data Evaluation}

Data evaluation will consist of reduction, tabulation, and evaluation of the data from the RI. Data evaluation will begin after data validation has been completed. The objectives of data evaluation are to (a) assess the need for additional sampling, and (b) perform data interpretation.

Data evaluation to assess the need for additional sampling will be performed for the following field investigation tasks: surface and subsurface soil sampling, soil gas surveys, and groundwater sampling. Once data are provided by the interim reports for these field investigations, another scoping session may be held to evaluate adequacy of the data for meeting RI/FS data needs (i.e., remedial selection, risk assessment, and remedial design data needs). Validated data will be evaluated to determine if quantity and quality are sufficient to meet the objectives discussed in Section 4 and the precision, accuracy, representativeness, completeness, and comparability (PARCC) goals identified in the QAPjP.

The data interpretation process will involve the reduction of data into maps, tables, graphs, and figures, and the use of subsurface fate and transport models to acquire a better understanding of the nature and extent of contamination at the site. 


\subsection{Contaminant Fate and Transport Modeling}

Hydrologic modeling will be used to evaluate landfill cover performance and for the development of remedial alternatives, and contaminant fate and transport modeling will be used to support the baseline risk assessment, both of which are discussed in greater detail in the following subsections.

\subsubsection{Modeling of Landfill Cover Performance}

The efficiency of the landfill covers will be evaluated using EPA's computer model HELP (Hydrologic Evaluation of Landfill Performance). HELP is a quasi-two dimensional hydrologic model of vertical water movement through the landfill cover. The model accounts for the effects of surface water runoff, evapotranspiration, soil moisture storage, and lateral flow through drainage layers to predict the rate of water infiltration through the cover. The HELP code will be used to determine the adequacy of the existing landfill covers under the no-action alternative and to evaluate potential new landfill cover designs.

HELP code data input requirements include (a) 10 years of daily precipitation data and the mean monthly temperature for this period (these data will be obtained from the NOAA CFA weather station), (b) the evaporative zone depth, which will be obtained from the literature, and (c) the soil characteristics (i.e., thickness, porosity, soil/water content, etc.), which will be obtained from field investigations conducted under this RI. HELP code default parameters will also be used.

\subsubsection{Contaminant Transport Modeling}

This section describes the approach for modeling potential contaminant migration at CFA Landfills II and III. In general, modeling contaminant migration for use in the baseline risk assessment will be based on existing waste inventory information, empirical data, and soil gas data collected from the landfill cover, shallow boreholes, and surface flux chamber, and surface and subsurface soil contaminant concentration data. The basic strategy will be to employ simple modeling methods based on the above data to obtain a quantitative understanding of the risk posed by migration of contaminants at the CFA landfills. The modeling will be done using different approaches for the various modes of transport occurring at the landfills. For this study, effort will be focused on (a) contaminants that leach from the landfill and migrate with the natural water flow in the vadose zone and groundwater systems, (b) gas phase contaminants that migrate to land surface and are released into the atmosphere, (c) gas phase contaminants that migrate downward through the vadose zone and enter the aquifer, and (d) liquid phase contaminant migration to the aquifer (if necessary). When liquid, gas or dissolved phase contamination reaches the aquifer it is assumed to be moved and dispersed by the flow of groundwater. Gas phase contaminants released to the atmosphere are subject to wind and atmospheric dispersion. Specific details regarding models and approaches will be addressed in a technical memorandum which will be available in August 1993.

5.6.2.1 Fate and Transport of Leachable Contaminants. The level of modeling necessary to predict the movement of the leachable compounds is limited by the amount and 
quality of available data. Subsurface flow and transport is difficult to simulate even with the most sophisticated models and appropriate amounts of data. Information concerning the initial inventory of contaminants and their spatial variability appears to be incomplete and may not be available or reliable. Therefore, leachate release and transport will be simulated with the computer code GWSCREEN (Rood 1992). This approach will effectively and conservatively represent contaminant migration from the landfills for the baseline risk assessment. The analytical results of surface and subsurface soil sampling (and leachates if present) collected during this RI and from existing waste inventory records will be used to support this modeling effort. Standard default parameters for GWSCREEN will be used to describe the aquifer and vadose zone.

5.6.2.2 Fate and Transport of Gas Phase Contaminants. Gas phase contaminants can migrate upward from the landfill and be released into the atmosphere or can migrate downward through the vadose zone and enter the groundwater. Vadose zone transport of gas phase contaminants can occur by molecular diffusion, mechanical dispersion, and advection. Sorption onto the porous solid and vapor-liquid partitioning can also affect the transport. The vapor or gas phase will move predominantly by diffusion in the absence of significant pressure gradients. The migration rate depends on chemical specific properties and the geologic environment.

As a first approach to modeling gas phase contaminant migration at the CFA landfills, each pathway can be evaluated using a steady-state analytic model. Contaminant fluxes or emissions from the vadose zone to the air can be predicted using soil gas data and diffusion analysis, or may be derived from flux chambers, which measure the emission rate. An analysis for receptors in the proximity of the landfill can be done using a simple box model. A box model assumes gas emissions from the soil are in equilibrium with mechanisms transporting contaminants away from the landfill area and are completely mixed in a control volume above the emission area. If necessary, a more complete analysis for atmospheric transport of gas phase contaminants can be done with an EPA-approved atmospheric dispersion model such as CAP-88 (EPA 1989c) or GENII (Napier et al. 1988). These models are generally used for receptors located downwind of the emission area. After the preliminary modeling is completed, DOE, EPA, and IDHW will evaluate the need for a more complete analysis of atmosphere transport using models such as CAP-88 or GEN II.

Transport of gas phase contaminants in the vadose zone to the aquifer can be modeled using a diffusion analysis similar to that proposed for the air pathway. This steady-state analysis assumes a source capable of maintaining current soil gas concentration levels in the landfill. These concentrations will be determined from soil gas samples collected from shallow soils and boreholes during the RI. Compounds in the gas phase will move predominantly by gas diffusion, especially deeper in the vadose zone where pressure changes due to gas generation and atmospheric pumping are not important. The rate of migration will depend on the vertical concentration gradient, chemical specific properties, and the geologic environment. If the gradient as determined from the shallow soil gas and borehole samples is indicative of significant downward movement, then vadose zone transport of gas phase contaminants will be further examined using conservative parameter estimates and the method described. Once the gas phase contaminants reach the aquifer, the same type of box model proposed for atmospheric transport in close proximity to the landfill would be used. Groundwater transport to downgradient receptors would be done using the same model used for saturated groundwater transport of the leachate. If groundwater concentrations using this method are deemed significant, then a more 
complete examination of paramcters would be appropriate before the results are included in the risk assessment.

5.6.2.3 Fate and Transport of Liquid Phase Contaminants. Some contaminants at the lancifills may have been disposed in solution or as separate nonaqueous phase liquids (NAPLs). Movement of contaminants disposed in solution will be governed by the same processes that control movement of leachate. However, the rate of movement in the vadose zone could be different depending on the amount of solution, disposal location, and the container failure rate. The passage of contaminated solution through the surrounding sediments may result in the loss of contaminants from the soil water to the sediments by sorption. Release of the sorbed contaminants is then controlled by leaching. Modeling NAPL transport is not planned for this RI. However, if during subsurface soil sampling, NAPL is encountered, the need for fate and transport modeling of separate phase contaminants will be evaluated by DOE, EPA, and IDHW.

\subsection{Risk Assessment}

A baseline risk assessment (BRA) will be conducted for the CFA Landfills II and III RI/FS and will, in addition to the ARARs, aid in the decision-making process for developing remediation goals for the landfills. The BRA is initiated to help determine whether the contaminants of concern at the site pose a current or potential future risk to human health and the environment and are significant enough to warrant remedial action. Because options for remedial action at municipal landfill sites are limited, it may be possible to streamline or limit the scope of the BRA (EPA 1991a). This may be achieved by (a) using the conceptual site model and RI-generated data to perform a qualitative risk assessment that identifies the contaminants of concern in the affected media, their concentrations, and their hazardous properties that may pose a risk through the various routes of exposure, and (b) identifying pathways that are an obvious threat to human health or the environment by comparing RI-derived contaminant concentration levels to standards that are potential chemical-specific ARARs for the action. Ultimately, it will be necessary to demonstrate that the final remedy, once implemented, will in fact address all pathways and contaminants of concern.

The BRA is comprised of a human health evaluation and an environmental evaluation. The CFA Landfills II and III BRA will follow guidance provided in EPA's Risk Assessment Guidance For Superfund, Volume I: Human Health Evaluation Manual (RAGS HHEM) (EPA 1989a) and Volume II: Environmental Evaluation Manual (EEM) (EPA 1989b). Supplemental guidance prepared specifically for EPA Region 10 will also be followed (EPA 1991b) as will guidance on risk assessments for municipal landfills (EPA 1991a).

\subsubsection{Human Health Evaluation}

The goal of the human health evaluation is to provide a framework for developing the risk information necessary to assist in making decisions regarding remedial actions at the site. The evaluation will involve a BRA to determine the potential adverse health effects (both current and future) caused by the contaminants of concern at the site under the no-action alternative. A preliminary conceptual model for CFA Landfills II and III is presented in Figure 3-3. The identification of sources, pathways, and receptors for the model are based on an evaluation of 
past disposal practices, a review of the waste inventory at the landfills, and an evaluation of past sampling activities. This model will be refined based on additional data collected and evaluated during the remedial investigation phase.

The BRA involves a four-step process consisting of data collection and evaluation, exposure assessment, toxicity assessment, and risk characterization.

5.7.1.1 Data Collection and Evaluation. This step involves gathering and analyzing site data relevant to the human health evaluation and identifying the contaminants at the site that are the focus of the risk assessment process. Existing data for the CFA landfill are summarized in Section 3 and the appendices of this Work Plan. Additional data proposed for collection and analysis during the RI include groundwater, surface soil, subsurface soil and soil gas and will also be evaluated for use in the BRA for evaluating the exposure concentrations from the ingestion of groundwater, dermal contact, incidental ingestion of soil, and inhalation pathways, as well as explosion hazards. The first two rounds of groundwater sampling data will be used to support the BRA and fate and transport modeling. Due to the tight schedule of receiving validated data for use in the BRA, the third round of groundwater sampling data will be used as historical confirmation of previous data collected and discussed in the uncertainty section of the BRA. QA/QC measures for data collected during this RI in support of the BRA are presented in the QAPjP, an addendum to this Work Plan.

The BRA will be evaluated based on the 1988 through 1991 data that qualifies for a quantitative risk assessment and the proposed data to be collected in 1993. A preliminary list of contaminants of concern and their chemical-specific ARARs and risk-based concentrations will be determined based on a review of the existing data and the 1993 RI data. A screening of this preliminary contaminant list will be made to focus the BRA on important chemicals. The data will be evaluated against analytical methods used, quantitation limits, qualified and coded data, sample blank contamination, natural background elements, essential nutrients, and risk-based concentrations in a systematic manner in accordance with EPA guidance (EPA ;989a and EPA 1991b).

5.7.1.2 Exposure Assessment. An exposure assessment will be conducted to estimate the magnitude of actual and/or potential human exposures, the frequency and duration of these exposures, and the pathways by which humans at or in the vicinity of the CFA landfill site nay be exposed. Exposure estimates will be made for both current and future land-use assumptions. The exposure assessment will involve analyzing contaminants of concern, identifying exposed populations, identifying all potential pathways of exposure, estimating exposure point concentrations for specific pathways based on environmental monitoring data and fate and transport modeling, and estimating contaminant intakes for specific pathways. The result of the exposure assessment will be the determination of pathway-specific intakes for current and future exposures to the contaminants of concern.

The exposure assessment will refine the conceptual model presented in Section 3.2.2 based on an extensive review of the existing site data and the 1993 RI tasks. Figure 3-3 outlines the potential contaminated media, exposure pathways, and human receptors. The current industrial scenario will evaluate the ingestion of groundwater pumped from CFA Production Wells \#1 and 2 , inhalation of volatiles from the use of this water, incidental soil ingestion, dermal contact, 
inhalation of dust, and the inhalation of vapors emanating from the landfill surface to INEL workers. The future residential scenario will evaluate the ingestion of groundwater, inhalation of volatiles from the use of the water, incidental soil ingestion, dermal contact, and inhalation of volatiles and dust. The calculation of risks will be for both the average and the reasonable maximum exposure as defined in the RAGS HHEM. Fate and transport modeling will be used to determine future concentrations at the source and the operable unit boundary. The potential for explosion hazard will also be evaluated by comparing methane soil gas data to the lower explosive limit for methane.

5.7.1.3 Toxicity Assessment. Toxicity assessment summarizes the critical toxicity information for a chemical and will be conducted prior to the risk charccterization process. Toxicity information together with the exposure assessment results are used to characterize risks. The toxicity information consists of values that describe the degree of toxicity of a chemical.

The primary source of data for reference doses and cancer slope factors will be the Integrated Risk Information System maintained by EPA. Secondary sources include the Health Effects Assessment Summary Tables (EPA 1990b), Agency for Toxic Substances and Disease Control Toxicological Profiles, EPA water quality criteria documents, and EPA health advisories. For chemicals not listed in EPA guidance, toxicity information is also available through the Environmental Criteria and Assessment Office (ECAO). The ECAO will be contacted if insufficient information exists in EPA's guidance documents or data bases.

5.7.1.4 Risk Characterization. The final step in the overall risk assessment process is to integrate the results of the exposure assessment and the toxicity assessment in an estimate of risk to humans from the site. The toxicity and exposure assessments are summarized and integrated into quantitative expressions of risk. Noncarcinogenic effects are characterized by comparing projected intakes of substances to toxicity values. The carcinogenic effects or probability that an individual will develop cancer over a lifetime of exposure are estimated from projected intakes and chemical-specific dose-response relationships.

\subsubsection{Environmental Evaluation}

As part of the risk assessment process, the impacts of the no-action alternative on the natural environment needs to be evaluated. This evaluation, referred to as an ecological risk assessment (ERA), will be a qualitative assessment of the actual or potential effects of the landfill on plants and animals other than people and domesticated species (EPA 1989b). The ERA will follow guidance provided in the RAGS EEM (EPA 1989b). A detailed evaluation of possible environmental impacts associated with operable units in WAG 4 will be addressed in the WAG 4 comprehensive RI/FS or the WAG-10 Site-wide RI/FS.

\subsubsection{Uncertainty Analysis in the Risk Assessment Process}

The risk assessment process is an inexact science, complete with uncertainties and limitations. Many uncertainties exist in the determination of factors related to risk such as toxicity values, cancer incidence rates, and exposure scenarios. These inherent uncertainties associated with the risk assessment process will be discussed qualitatively in the RI report in accordance with the HHEM. This discussion will provide the decision maker with the necessary information to 
make an informed decision concerning the need for action and remedy selection to reduce risks at the site.

\subsection{Treatability Studies}

Treatability studies are not currently planned for the CFA Landfills II and III RI/FS. The preliminary evaluation of existing data presented in Section 3 has not indicated any contaminants or areas within the landfill that would require special treatment processes. The preliminary remedial action alternatives identificd in Section 4 of this Work Plan do not indicate the need for any laboratory, bench, or pilot scale treatability studies. However, should further site characterization indicate the need for laboratory studies, a test plan for the studies will be prepared and presented to the EPA and the IDHW for review and approval. The test plan will identify the types and goals of the studies, the level of effort needed, a schedule for completion, and the data management guidelines. A report summarizing the testing program and its results would be prepared and presented in the final FS report.

\subsection{RI Report}

A report summarizing $\mathrm{RI}$ activities and findings will be prepared and submitted to DOE, EPA, and the IDHW for review and comment. Additionally, interim reports may be produced as a result of activities planned for the RI. These reports are intended to facilitate preliminary interpretations of data and timely dissemination of data, and would be informational in nature. These include preliminary interpretations of each of the following investigation tasks:

- Deviation logging and water level measurements

- Geophysical surveys

- Soil gas surveys

- Surface and subsurface sampling

- Groundwater sampling

- Identification of contaminants of concern

- Exposure assessment.

The RI report will be prepared in accordance with the current RI/FS Guidance (EPA 1988a).

\subsection{Feasibility Study}

The feasibility study ( $r$ S) consists of the development of alternatives, a screening of alternatives, and a detailed analysis of alternatives. Based on a review of practical remedial technologies for municipal landfill sites and the actual characteristics of the CFA Landfills II and III, a range of alternatives will be developed.

\subsubsection{Development of Alternatives}

Alternatives for remediation are developed by assembling combinations of technologies, and the media to which they would be applied, into alternatives that address contamination on a site- 
wide basis for the Operable Unit. Because many landfill sites share similar characteristics, they lend themselves to remediation by similar technologies. The EPA has established a number of expectations as to the types of remedial alternatives that should be developed during the detailed analysis stage, and these are listed in the NCP. For landfill sites, it is expected that:

- The principal threats posed by a site will be treated wherever practical, such as in the case of remediation of areas of highly toxic or mobile material that pose potential principal threats.

- Engineering controls (such as containment) will be used for waste that poses a relatively low long-term threat or where treatment is impractical.

- A combination of methods will be used as appropriate to achieve protection of human health and the environment. An example of combined methods for municipal landfill sites would be treatment of hot spots in conjunction with containment (capping) of the landfill contents.

- Institutional controls, such as deed restrictions, may be used to supplement engineering controls, as appropriate, to prevent exposure to hazardous wastes.

- Innovative technologies will be considered when such technologies offer the potential for superior treatment performance or lower costs for performance similar to that of demonstrated technologies.

- Groundwater will be returned to beneficial uses whenever practical, within a reasonable time, given the particular circumstances of the site.

In developing the remedial action alternatives, remedial action objectives need to be identified. The primary remedial action objectives for remediating landfill sites include:

- Preventing direct contact with the landfill contents

- Reducing contaminant leaching to groundwater

- Controlling surface water runoff and erosion

- Remediating hot spots

- Collecting and treating contaminated groundwater and leachate

- Controlling and treating landfill gas

- Remediating contaminated surface water and sediments

- Remediating contaminated wetland areas.

\subsubsection{Screening of Alternatives}

Based on the above remedial action objectives for CERCLA landfill sites and EPA expectations outlined in the NCP, the following points should be considered in order to streamline the development of remedial action alternatives: 
- Generally, the most practical remedial alternative for landfills is containment (capping). Depending on site characteristics, containment could range from a soil cover to a multicomponent impermeable cap.

- Treatment of soils and wastes may be practical for hot spots. Consolidation of hot spot materials under landfill cap is a potential alternative in cases when treatment is not practical or necessary.

- Extraction and treatment of contaminated groundwater and leachate may be required to control offsite migration of wastes. Additionally, extraction and treatment of leachate from landfill contents may be required. Collection and treatment may be necessary indefinitely because of continued contaminant loadings from the landfill.

- Constructing an active landfill gas collection and treatment system should be considered where (a) existing or planned homes or buildings may be adversely affected through either explosion or inhalation hazards, (b) final use of the site includes allowing public access, (c) the landfill produces excessive odors, or (d) it is necessary to comply with ARARs. Most landfills will require at least a passive gas collection system (venting) to prevent buildup of pressure below the cap and to prevent damage to vegetative cover.

The EPA has made available a list of technologies practical for use at CERCLA landfills based on a review of selected remedies in the records of decision (RODs) the EPA has signed through FY 1989. The feasibility study will consider these and other potentially appropriate technologies that will satisfy remedial objectives and ARARs for the landfill sites.

\subsubsection{Detailed Analysis of Alternatives}

During detailed analysis, the potential remedial action alternatives identified for the landfill sites that are carried through the initial screening process are further refined, as appropriate, and analyzed in detail with respect to the nine evaluation criteria. The evaluation criteria have been divided into three groups based on the function of the criteria during the remedy selection. The three groups include the threshold criteria, the balancing criteria, and the modifying criteria.

The threshold criteria relate to statutory requirements that each alternative must satisfy in order to be eligible for selection. These include:

- Overall protection of human health and the environment

- Compliance with ARARs, including chemical-specific, location-specific, and actionspecific ARARs.

The balancing criteria are the technical criteria that are considered during the detailed analysis. These include:

- Long-term effectiveness and permanence

- Reduction of toxicity, mobility, or volume through treatment

- $\quad$ Short-term effectiveness 
- Implementability

- Cost.

The modifying criteria are formally assessed after the public comment period. However, state or community views are considered during the feasibility study to the extent they are known. The modifying criteria are as follows:

- State/support agency acceptance

- Community acceptance.

Developing remedial alternatives based on NCP expectations and focusing on alternatives successfully implemented at other landfill sites will help to direct the alternatives selection effort. This Work Plan outlines the necessary information required to develop remedial alternatives that will mitigate current and potential threats to human health and the environment at the CFA Landfills II and III.

\subsection{Feasibility Study Report}

Following completion of the detailed analysis of alternatives, a draft FS report will be prepared. The report will summarize FS activities and RI site characterization results and will be prepared in accordance with RI/FS guidance (EPA 1988a). The FS report will contain the following elements:

- Objectives of remedial response

- Actions of general response

- Analysis of ARARs

- Description of potential remedial technologies and development of alternatives

- Description of screening methodology

- Results of screening evaluations

- Description of the detailed alternative evaluation process

- Results of the detailed alternative analysis

- Summary and comparison of alternatives.

The analysis of individual alternatives will include information on

- Assumptions made during the analysis

- Implementation requirements

- Compliance with ARARs

- Risk-based exposure scenarios for alternatives

- Quantities of material to be treated

- Concentrations of constituents to be treated per unit volume

- Time requirements

- $\quad$ Sizing requirements

- Cost.

The analysis of individual alternatives will be presented in a tabular format combined with a narrative discussion. 
Information on environmental impacts will be prepared as necessary to supplement the information contained in the RI and FS reports. If at any time during the RI/FS it is determined that the remedial alternatives being considered will have significant impacts, additional information will be added to the RI/FS as necessary to comply with requirements for environmental analysis that is consistent with DOE policy.

\subsection{Proposed Plan and Record of Decision}

The RI/FS process culminates with the Proposed Plan and the ROD. The Proposed Plan will summarize the information presented in the RI/FS Report for the remedial alternatives that were evaluated. The Proposed Plan will describe the preferred alternative selected by DOE, EPA, and IDHW for the CFA Landfills II and III remedial action, so that the plan can be sent out for public review. Public comments will be considered by the DOE, the EPA, and the IDHW before selection of the linal remedy, and the official response to the public comments and a detailed description of the selected alternative will be presented in the ROD. Other public involvement activities are detailed in the site-specific RI/FS Community Relations Plan, attached as an addendum to this Work Plan.

At the INEL, draft RODs will be prepared for signature by the DOE, the EPA, and the IDHW. Following finalization of the ROD, the DOE will initiate remedial design, remedial action, monitoring during remedial actions as required, and post-remedial action operations and maintenance (il the selected remedy requires operations and maintenance) in accordance with the remedial design/remedial action process. 


\section{SCHEDULE}

Table 6-1 below is the working schedule for the Operable Unit 4-12 RI/FS. The schedule shows the planned dates for all FFA/CO deliverables, review periods, and field activities.

Table 6-1. Summary working schedule for the Operable Unit 4-12 RI/FS.

1. DOE submits draft RI/FS Scope of Work ${ }^{a}$ to EPA/IDHW:

2. DOE submits draft RI/FS Work Plan ${ }^{a}$ to EPA/IDHW:

3. EPA/IDHW completes review of draft RI/FS Work Plan:

4. DOE submits draft final RI/FS Work Plan to EPA/IDHW:

5. RI/FS Work Plan becomes final:

6. RI/FS tasks planning and mobilization for field work:

7. 14-day notice to EPA/IDHW of field activities:

8. Groundwater sampling

Sample collection - first round:

Analysis and validation - first round:

Transmit groundwater data to EPA/IDHW - first round:

Sample coliection - second round:

Analysis and validation - second round:

Transmit groundwater data to EPA/IDHW - second round:

Sample collection - third round:

Analysis and validation - third round:

Transmit groundwater data to EPA/IDHW - third round:

9. Topographic survey and map:

10. Geophysical survey and data evaluation:

11. Soil cover investigation

Field investigation:

Analysis and validation of physical samples:

Transmit soil cover data to EPA/IDHW by:

12. Soil gas survey

Sample collection:

Analysis and validation:

Transmit soil gas survey data to EPA/IDHW by:
August 31, 1992

January 29, 1993

March 15, 1993

May 1993

July 1993

January to March 1993

March 1993

June 1993

June to July 1993

July 1993

July 1993

July to September 1993

September 1993

October 1993

October to December 1993

December 1993

May to June 1993

May to July 1993

July 1993

July to September 1993

October 1993

June to August 1993

July to September 1993

October 1993 
Table 6-1. (continued).

13. Subsurface investigation

Sample collection:

August 1993

Analysis and validation:

August to September 1993

Transmit data to EPA/IDHW:

October 1993

14. Water level measurements (monthly) beginning:

May 1993

15. Neutron probe moisture measurements (monthly) beginning:

June 1993

16. Inspection/sampling of Landfill II toe slope (weekly) beginning:

May 1993

17. Perform fate and transport modeling-risk assessment:

September to December 1993

18. Prepare/review RI report with risk assessment:

December 1993 to May 1994

19. DOE submits draft RI report ${ }^{b}$ to EPA/IDHW:

May 19, 1994

20. EPA/IDHW completes review of draft RI report:

July 5, 1994

21. DOE submits draft RI/FS report ${ }^{a}$ to EPA/IDHW:

August 24, 1994

22. EPA/IDHW completes review of draft RI/FS:

October 7, 1994

23. DOE submits draft final RI/FS to EPA/IDHW:

November 25, 1994

24. RI/FS report becomes final:

December 26, 1994

25. DOE submits draft Proposed Plan ${ }^{b}$ to EPA/IDHW:

January 4, 1995

26. EPA/IDHW completes review of draft Proposed Plan:

February 2, 1995

27. Public comment period:

April to May 1995

28. DOE submits draft ROD ${ }^{a}$ to EPA/IDHW:

July 11, 1995

a. Primary documents per FFA/CO.

b. Secondary documents per FFA/CO. 


\section{REFERENCES}

Ackerman, D. J., 1991, "Transmissivity of the Snake River Plain Aquifer at the Idaho National Engineering Laboratory, Idaho," U.S. Geological Survey Water-Resources Investigations Report 91-4058, DOE/ID-22097.

ACGIH (American Conference of Governmental Industrial Hygienists), 1989, Threshold Limit Values and Biological Exposure Indices for 1990-1991, Cincinnati, OH.

Anders, M. H., J. W. Geissman, L. A. Piety, and J. T. Sullivan, 1989, "Parabolic Distribution of Circum-Eastern Snake River Plain Seismicity and Latest Quaternary Faulting: Migratory Pattern and Association with the Yellowstone Hotspot," Journal of Geophysical Research, 94, pp. 1589-1621.

Anderson, J. E., R. J. Jepson, and K. E. Holte, 1978, "Trends in Vegetation Development on the Idaho National Engineering Laboratory Site," Ecological Studies on the Idaho National Engineering Site, 1978 Progress Report, IDO-12087, pp. 144-166.

Anderson, J. E., R. S. Nowak, T. D. Ratzlaft, and O. D. Markham, 1991, Managing Soil Moisture on Waste Burial Sites, Radiological and Environmental Science Laboratory, Idaho National Engineering Laboratory, Idaho Falls, ID.

Anderson, J. E., M. L. Shumar, N. L. Toft, and R. S. Nowak, 1987, "Control of the Soil Water Balance by Sagebrush and Three Perennial Grasses in a Cold-Desert Environment," Arid Soil Research and Rehabilitation, 1, pp. 229-244.

Ansley, S. L., L. C. Hull, and S. M. Burns, 1988, Shallow Drilling Report for CFA Landfills II and III-FY-1988, Characterization of Surficial Sediments, EGG-ER-8291, Rev. 1.

Arthur, W. J., D. K. Halford, and J. W. Connelly, 1983, Idaho National Engineering Laboratory Radioecology and Ecology Programs, 1983 Progress Report, DOE/ID-12098, Springfield, VA.

Balfour, W. D., C. E. Schmidt, and B. M. Eklund, 1987, "Sampling Approaches for the Measurement of Volatile Compounds at Hazardous Waste Sites," Journal of Hazardous Materials, 14, pp. 135-148.

Barraclough, J. T., B. D. Lewis, and R. G. Jensen, 1981, Hydraulic Conditions at the Idaho National Engineering Laboratory, Idaho Emphasis: 1974-1978, U.S. Geologic Survey WaterResources Investigation Open File Report 81-526.

Barraclough, J. T., W. E. Teasdale, J. B. Robertson, and R. G. Jensen, 1967, "Hydrology of the National Reactor Testing Station, Idaho, 1966," U.S. Geological Survey Open-File Report TID-4500. 
Barrash, W. and K. Marts, 1991, Duplicate Sampling of Groundwater Collected in the Area Between the Idaho Chemical Processing Plant and Central Facilities Area, INEL, State of Idaho INEL Oversight Program Technical Report (OPTR) 91-02, April.

Cholewa, A. F. and D. M Henderson, 1983, "A Survey and Assessment of the Rare Vascular Plants of the Idaho National Engineering Laboratory Site," Great Basin Naturalist, 44, pp. $140-144$.

Cholewa, A. F. and D. M Henderson, 1984, A Survey and Assessment of the Rare Vascular Plants of the Idaho National Engineering Laboratory Site, DOE/ID-12100.

Clawson, K. L., G. E. Start, and N. R. Ricks, 1989, Climatography of the Idaho National Engineering Laboratory 2nd Edition, DOE/ID-12118, U.S. Department of Commerce, National Oceanic and Atmospheric Administration, Environmental Research Laboratories, Air Resources Laboratory, Field Research Division, Idaho Falls, ID, p. 155.

Commander J. C., 1971, Solid Waste Disposal Methodology Study, ANCR-001, January.

Connelly, J. W., 1982, An Ecological Study of Sage Grouse in Southeastern Idaho, Ph.D. dissertation, Washington State University, Pullman, WA.

Connelly, J. W. and I. J. Ball, 1983, "Sage Grouse on the Idaho National Environmental Research Park," Idaho National Engineering Laboratory Radioecology and Ecology Programs, 1983 Progress Report, DOE/ID-12098, NTIS, Springfield, VA, pp. 347-356.

Craig, T. H., E. H. Craig, and L. R. Powers, 1983, "Raptor Studies on the Idaho National Engineering Laboratory," Idaho National Engineering Laboratory Radioecology and Ecology Programs, 1983 Progress Report, DOE/ID-12098, NTIS, Springfield, VA, pp. 309-315.

Craig, T. H., D. K. Halford, and O. D. Markham, 1979, "Radionuclide Concentrations in Nesting Raptors near Nuclear Facilities," Wilson Bull., 91, pp. 72-77.

DOE, 1992, Track 1 Sites: Guidance for Assessing Low Probability Hazard Sites at the INEL, DOE/ID-10340 (92), Rev. 1.

Eaton, G. P., 1982, "The Basin and Range Province: Origin and Tectonic Significance," Annual Reviews of Earth and Planetary Science, 10, pp. 409-440.

EG\&G Idaho, 1991, Implementing Program Management Plan for the EG\&G Idaho Envrionmental Restoration Program, Rev. 1, EGG-WM-8676, Idaho National Engineering Laboratory, Idaho Falls, ID.

EG\&G Idaho, 1992, Data Management Plan for the EG\&G Idaho Environmental Restoration Department, Rev. 0, EGG-ERD-10227, Idaho National Engineering Laboratory, Idaho Falls, ID. 
EPA, 1986, Measurement of Gaseous Emission Rates from Land Surfaces Using an Emission Isolation Flux Chamber, EPA/600/8-86/M8, Environmental Monitoring Systems Laboratory, Office of Research and Development, U.S. Environmental Protection Agency, Las Vegas, NV 89114.

EPA, 1987a, "Consent Order and Compliance Agreement 1085-10-07-3008," in the matter of: United States Environmental Protection Agency and the United States Department of Energy, Idaho National Engineering Laboratory, ID4890008952, proceeding under Section 300 8(h) of the Resource Conservation and Recovery Act, 42 USC, Section 6928(h).

EPA, 1987b, Data Quality Objectives for Remedial Response Activities: Development Process, EPA/540/G-87/003, Office of Emergency and Remedial Response and Office of Waste Programs Enforcement, Washington, DC.

EPA, 1988a, "Guidance for Conducting Remedial Investigations and Feasibility Studies Under CERCLA," OSWER Directive 9335.3-01, Office of Emergency and Remedial Response and Office of Solid Waste and Emergency Response.

EPA, 1988b, CERCLA Compliance with Other Laws Manual, EPA/540/G-89/006.

EPA, 1988c, Compendium of Methods for the Determination of Toxic Organic Compounds in Ambient Air, U.S. Environmental Protection Agency, Atmospheric Research and Exposure Assessment Laboratory, Office of Research and Development, U.S. Environmental Protection Agency, Research Triangle Park, NC 27711.

EPA, 1989a, Risk Assessment Guidance for Superfund, Volume I: Human Health Evaluation Manual, Interim Final, EPA/540/1-89/001, Office of Solid Waste and Emergency Response, Washington, DC.

EPA, 1989b, Risk Assessment Guidance for Superfund, Volume II: Environmental Evaluation Manual, Interim Final, EPA/540/1-89/001, Office of Emergency and Remedial Response, Washington, DC.

EPA, 1989c, The Clean Air Assessment Package-1988, CAP-88, Office of Radiation Programs, Washington, DC.

EPA, 1989d, CERCLA Compliance with Other Laws Manual, EPA/540/G-89/009.

EPA, 1990a, "National Oil and Hazardous Substances Pollution Contingency Plan," Federal Register, Volume 55, No. 46, pp. 8813-8865.

EPA, 1990b, Health Effects Assessment Summary Tables, Third Quarter-FY 1990, PB 90-921103, OSWER (OS-230).

EPA, 1991a, Conducting Remedial Investigations/Feasibility Studies for CERCLA Municipal Landfill Sites, EPA/540/P-91/001, Office of Emergency Remedial Response, Washington, DC. 
EPA, 1991b, Region 10 Supplemental Risk Assessment Guidance for Superfund.

Floyd, D. A. and J. E. Anderson, 1983, "Baseline Vegetation Data for a Controlled Burn Site," Idaho National Engineering Laboratory Radioecology and Ecology Programs, 1983 Progress Report, DOE/ID12098, NTIS, Springfield, VA.

FWS (U.S. Fish and Wildlife Service), 1992, letter to DOE/ID, "Listed and Proposed Endangered and Threatened Species That May Occur Within the Area of the Idaho National Engineering Laboratory," No. SP1-4-92-SP-419 (File \#506.0000), dated April 17, 1992.

Gates, R. J., 1983, Sage Grouse, Lagomorph, and Pronghorn Use of Sagebrush Grass Burn Site on the Idaho National Engineering Laboratory, M.S. thesis, Montana State University, Bozeman, MT.

Gleason, R. S., 1978, "Ecology of Burrowing Owls on the Idaho National Engineering Laboratory Site," Ecological Studies on the Idaho National Engineering Site, 1978 Progress Report, IDO-12087.

Groves, C. R. and B. L. Keller, 1983, "Population Ecology of Small Mammals on the Radioactive Waste Management Complex, Idaho National Engineering Laboratory," Idaho National Engineering Laboratory Radioecology and Ecology Programs, 1983 Progress Report, DOE/ID12098, NTIS, Springfield, VA, pp. 21-46.

Hackett, W., J. Pelton, and C. Brockway, 1986, Geohydrologic Story of the Eastern Snake River Plain and the INEL, U.S. Department of Energy, Idaho National Engineering Laboratory, Idaho Falls, ID.

Hackett, W. R. and R. P. Smith, 1992, "Quaternary Volcanism, Tectonics, and Sedimentation in the INEL Area," Utah Geological Survey Miscellaneous Publication, 92-3, pp. 1-18.

Hackett, W. R. and L. A. Morgan, 1988, Explosive Basaltic and Rhyolitic Volcanism of the Eastern Snake River Plain, Idaho, in Link, P. K., and Hackett, W. R., editors, Guidebook to the Geology of Central and Southern Idaho: Idaho Geological Survey Bulletin 27.

Halford, D. K. and O. D. Markham, 1983, "Iodine-129 in Muscle from Waterfowl Using the Test Reactor Leaching Ponds in Southeastern Idaho," Idaho National Engineering Laboratory Radioecology and Ecology Programs, 1983 Progress Report, DOE/ID-12098, NTIS, Springfield, VA, pp. 83-88.

Hogg G. W., 1971, A Survey of NRTS Waste Management Practices, Volume 1 \& 2, ICP-1042, September.

ICF Technology, Inc., 1989, "CFA Landfill II, Idaho National Engineering Laboratory, Idaho Falls, Idaho," Innovative Technology Demonstration, Fairfax, VA, November 16. 
Johnson, R. D. and J. E. Anderson, 1983, "Relationships of Black-Tailed Jack Rabbits Diets to Vegetal Composition of Habitats," Idaho National Engineering Laboratory Radioecology and Ecology Programs, 1983 Progress Report, DOE/ID-12098, NTIS, Springfield, VA.

Kuntz, M. A., B. Skipp, M. A. Lanphere, W. E. Scott, K. L. Pierce, G. B. Dalrymple, L. A. Morgan, D. E. Champion, G. F. Embree, R. P. Smith, W. R. Hackett, and D. W. Rodgers, 1990, "Revised Geologic Map of the INEL and Adjoining Areas, Eastern Idaho," U.S. Geological Survey Open-File Report 90-333.

Lamke, A. D., 1969, "Stage-Discharge Relations on Big Lost River within National Reactor Testing Station, Idaho," U.S. Geological Survey Open-File Report, IDO-22050, p. 28.

Lewis, B. D. and F. J. Goldstein, 1982, "Evaluation of a Predictive Groundwater Solute-Transport Model at the Idaho National Engineering Laboratory," U.S. Geological Sunvey-Water Resources Investigations Report.

Malde, H. E., 1991, "Quaternary Geology and Structural History of the Snake River Plain, Idaho and Oregon," in "Quaternary Nonglacial Geology; Conterminous United States," edited by R. B. Morrison, The Geology of North America, K-2, Geological Society of America, Boulder, Colorado, pp. 251-281.

Mann, L. J., and L. L. Knobel, 1988, "Concentrations of Nine Trace Metals in Ground Water at the Idaho National Engineering Laboratory, Idaho," U.S. Geological Survey Open-File Report 88-332, DOE/ID-22075, Idaho Falls, ID.

Mann, L. J., and L. D. Cecil, 1990, "Tritium in Ground Water at the Idaho National Engineering Laboratory, Idaho," U.S. Geological Survey, Water Resources Investigations Report 90-4090, Idaho Falls, ID.

Markham, O. D., 1987, Summaries of the Idaho National Engineering Laboratory Radioecology and Ecology Program Research Projects, DOE-ID-12111, Radiological and Environmerital Sciences Laboratory, U.S. Department of Energy, Idaho Falls, ID.

Marlette, G. M. and J. E. Anderson, 1983, "Stability and Succession in Crested Wheatgrass Seeding on the Idaho National Engineering Laboratory," Idaho National Engineering Laboratory Radioecology and Ecology Programs, 1983 Progress Report, O. D. Markham (ed.), DOE/ID-12098, NTIS, Springfield, VA, pp. 127-146.

McBride, R., N. R. French, A. H. Dahl, and J. E. Detmer, 1978, "Vegetation Types and Surface of the Idaho National Engineering Laboratory Site," Ecological Studies on the Idaho National Engineering Laboratory Site, 1978 Progress Report, IDO-12087, NTIS, Springfield, VA.

Merck, 1989, The Merck Index: An Encylopedia of Chemicals, Drugs, and Biologicals, 11th edition, Merck \& Co., Inc. 
Miller, S. M., J. E. Hammel, and L. F. Hall, 1990, Characterization of Soil Cover and Estimation of Water Infiltration of Central Facilities Area Landfill II, Idaho National Engineering Laboratory (INEL), Idaho Water Resources Research Institute, University of Idaho, Moscow, ID.

Moseley, R. and C. Groves, 1990, Rare, Threatened, and Endangered Plants and Animals of Idaho, Natural Heritage Section Nongame and Endangered Wildlife Program, Idaho Department of Fish and Game.

Mundorff, M. J., E. G. Crosthwaite, and Chabot Kilburn, 1964, Groundwater for Imigation in the Snake River Basin in Idaho, Water Supply Paper 1654, U.S. Geological Survey.

Nace, R. L., M. Deutsch, and P. T. Voegeli, 1956, Geography, Geology, and Water Resources of the National Reactor Testing Station, Idaho: Part 2, Geography and Geology, IDO-22033, U.S. Geological Survey, Boise, ID.

Napier, B. A., D. L. Strenge, R. A. Peloquin, and J. V. Ramsdell, 1988, GENII-The Hanford Environmental Radiation Software System, Report PNL-6584, Battelle-Pacific Northwest Laboratory.

Orr, B. R. and L. D. Cecil, 1991, "Hydrologic Conditions and Distribution of Selected Chemical Constituents in Water, Snake River Plain Aquifer, Idaho National Engineering Laboratory, Idaho 1986 to 1988," U.S. Geological Survey-Water Resources Investigations Report 91-4047, DOE/ID-22096.

Peterson, K. L. and L. B. Best, 1983, "Effects of Prescribed Burning on Nongame Birds Breeding in Sagebrush Communities-Pre-Burn Phase," Idaho National Engineering Laboratory Radioecology and Ecology Programs, 1983 Progress Report, O. D. Markham (ed.), DOE/ID-12098, NTIS, Springfield, VA, pp. 202-210.

Pittman, J. R., R. G. Jensen, and P. R. Fischer, 1988, "Hydrologic Conditions at the Idaho National Engineering Laboratory, 1982 to 1985," U.S. Geological Survey Water Resources Investigations Report 89-4008, DOE/ID-22078.

Reed, W. G., J. W. Ross, B. L. Ringe, and R. N. Holmes, 1987, "Archaeological Investigations on the INEL: 1984-1985," Swanson/Crabtree Anthropological Research Laboratory Reports of Investigations, 87-1, Pocatello, ID.

Reynolds, T. D., J. W. Connelly, D. K. Halford, and W. J. Arthur, 1986, "Vertebrate Fauna of the Idaho National Environmental Research Park," Great Basin Naturalist, 46-3, pp. 513-527.

Reynolds, T. D. and F. L. Rose, 1978, "Pronghorn Antelope Use of the INEL National Environmental Research Park," Ecological Studies on the Idaho National Engineering Laboratory Site, 1978 Progress Report, IDO-12087.

Robertson, J. B., R. Schoen, and J. T. Barraclough, 1974, "The Influence of Liquid Waste Disposal on the Geochemistry of Water at the National Reactor Testing Station, Idaho: 1952-1970," U.S. Geological Survey Open-File Report 22053. 
Rood, A. S., 1992, "GWSCREEN: A Semi-Analytical Model for Assessment of the Groundwater Pathway from Surface of Buried Contamination: Theory and User's Manual," EGG-GEO-10158, EG\&G Idaho, Inc., Idaho Falls, ID.

Scott, W. E., 1982, "Surficial Geologic Map of the Eastern Snake River Plain and Adjacent Areas, Idaho and Wyoming," U.S. Geological Survey Miscellaneous Investigations Map I-1372.

Shumar, M. L., 1983, "Sagebrush Distributions on the Idaho National Engineering Laboratory," Idaho National Engineering Laboratory Radioecology and Ecology Programs, 1983 Progress Report, DOE/ID-12098, NTIS Springfield, VA, pp. 157-161.

Stafford, M. P., 1984, Surface-Dwelling Coleoptera Inhabiting S'Sagebrush Communities in Southeastern Idaho, M.S. thesis, University of Idaho, Mrasow, ID.

Stafford, M. P. and W. F. Barr, 1983, "Insects Associated with Sagebrush Communities," Idaho National Engineering Laboratory Radioecology and Ecology Programs, 1983 Progress Report, DOE/ID-12098, NTIS, Springfield, VA.

Stoddart, L. C., 1983, "Relative Abundance of Coyotes, Lagamorphs, and Rodents on the Idaho National Engineering Laboratory," Idaho National Engineering Laboratory Radioecology and Ecology Programs, 1983 Progress Report, DOE/ID-12098, NTIS Springficld, VA, pp. 268-277.

Tracer Research Corp., 1988, "Shallow Soil Gas Investigation at the Idaho National Engineering Laboratory (INEL)," Idaho Falls, Idaho.

Wilde, D. B. and B. L. Keller, 1978, "An Analysis of Pygmy Rabbit Populations on the Idaho National Engineering Laboratory Site," Ecological Studies on the Idaho National Engineering Laboratory Site, 1978 Progress Report, IDO-12087, pp. 305-316.

Wood, T. R., 1989, Test Area North Pumping Tests, EGG-ER-8438, EG\&G Idaho, Inc., Idaho Falls, ID.

Wood, T. R., L. C. Hull, and M. H. Doornbos, 1989a, Groundwater Monitoring Plan and Interim Status Report for Central Facilities Landfill II, EGG-ER-8496, EG\&G Idaho, Inc., Idaho Falls, ID.

Wood, T. R. , L. C. Hull, and M. H. Doornbos, 1989b, Groundwater Monitoring Plan and Interim Status Report for Central Facilities Landfill III, EGG-ER-8521, EG\&G Idaho, Inc., Idaho Falls, ID. 


\section{Appendix A.1}

\section{Shallow Soil Gas Survey Results for CFA Landfill II}




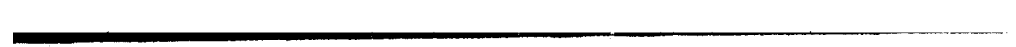

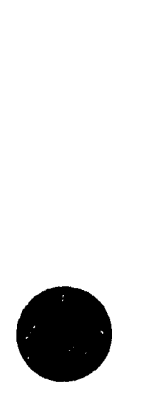

-

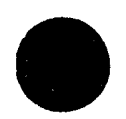

A.1-2 


\section{Appendix A.1}

\section{Shallow Soil Gas Survey Results for CFA Landfill II}

This appendix contains a complete table of the analytical results for the soil gas analyses conducted at CFA Landfill II and a map of soil gas sample locations. 


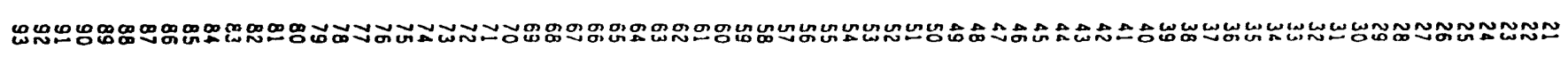

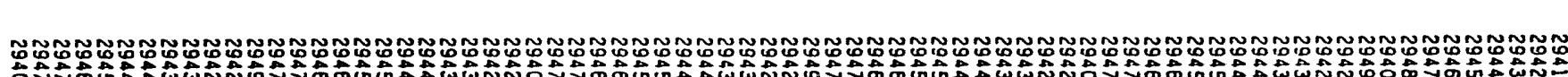

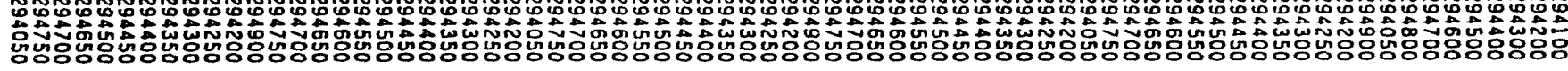

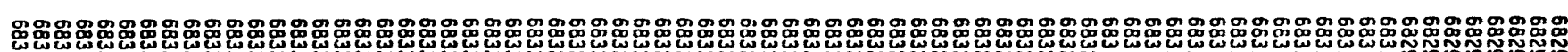

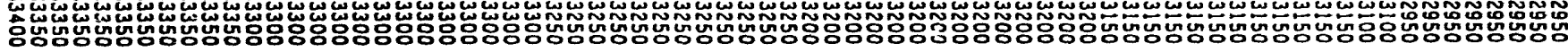

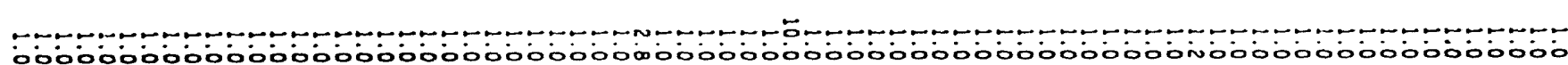

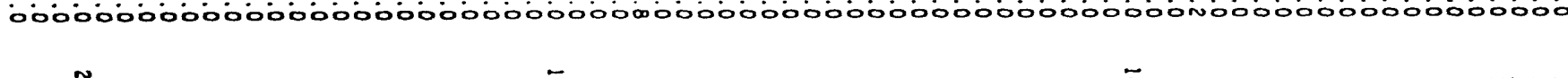
50un 200

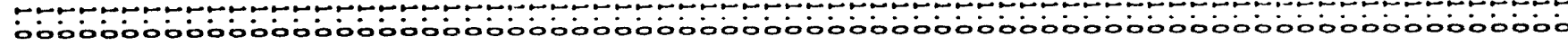

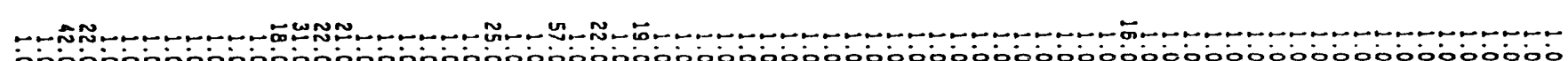

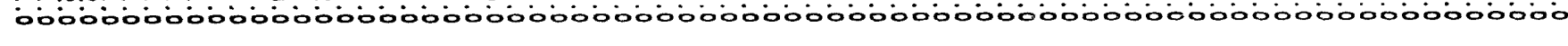

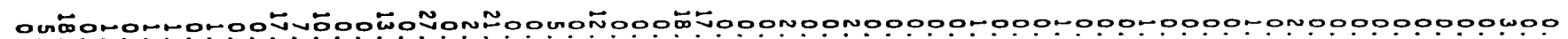
б8

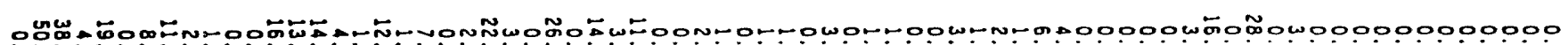

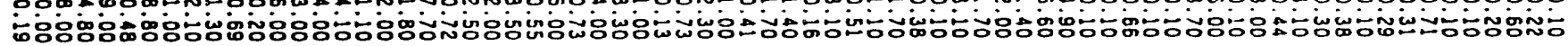

0000000000000000000000000000000000000000000000000000000000000000000000000

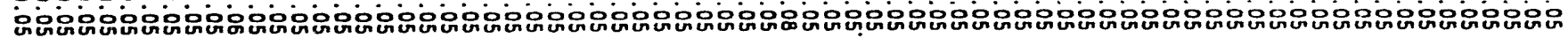

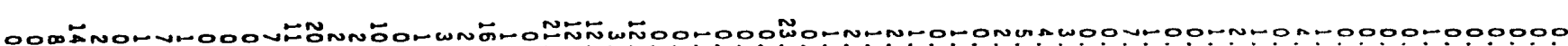

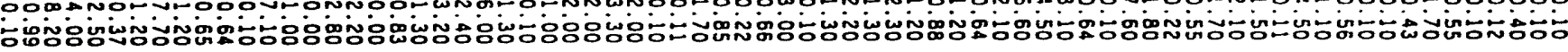
0000000000000000000000000000000000000000000000000000000000000000000000 бо боб

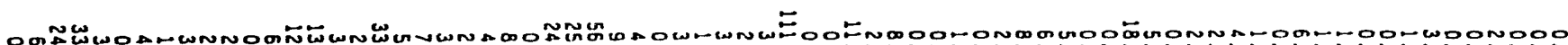

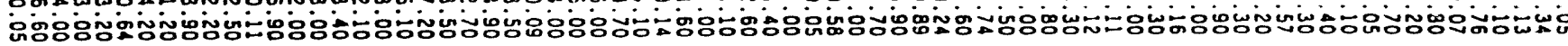

000000000000000000000000000000000000000000000000000000000000000000000000

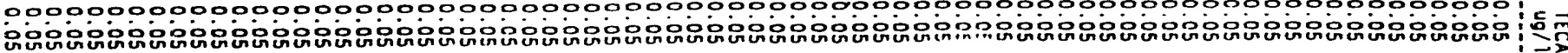




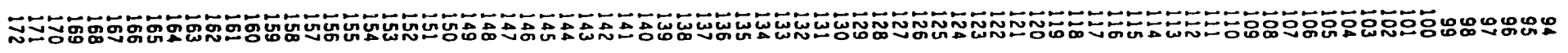

WWW

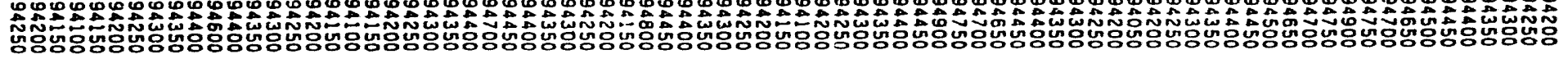

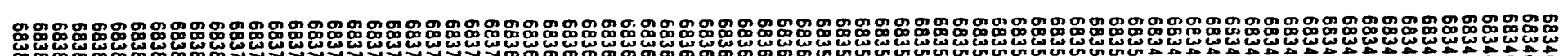
-

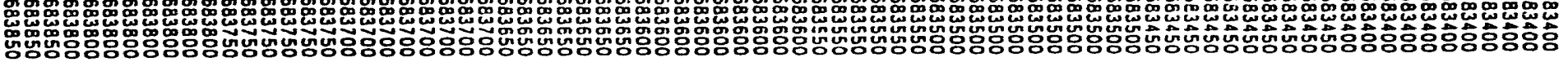

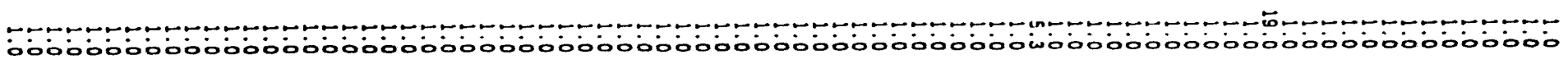
1:-:-:-: 2._. 00000000000000000000000000000000000000000000000000000000000000000000000000

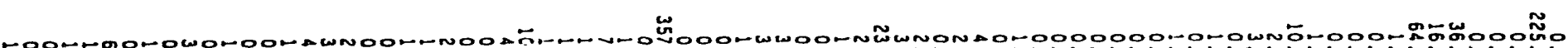

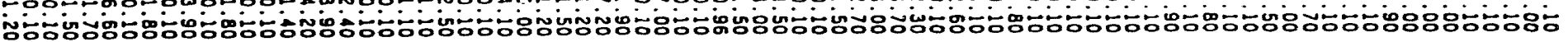

WWAN 0 Oด

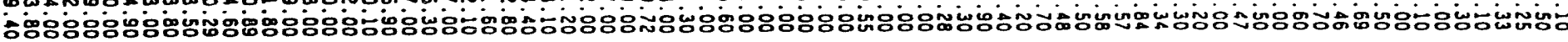

0000000000000000000000000000000000000000000000000 000000000000000000000000000000 bo

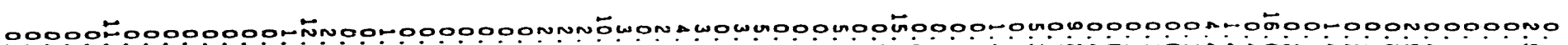

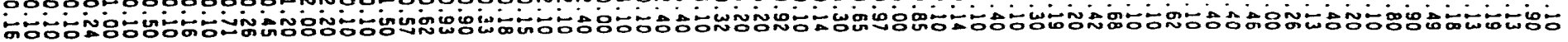
0000000000000000000000000000000000000000000000000000000000000000000000000000000

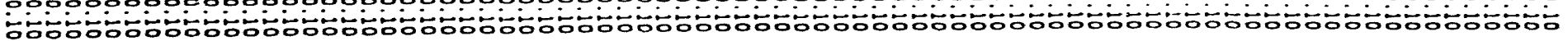

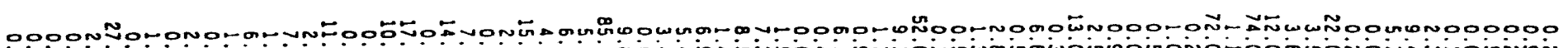

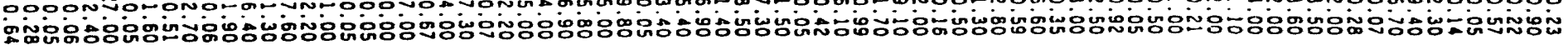
0000000000000000000000000000000000000000000000000000000000000000000000000000000

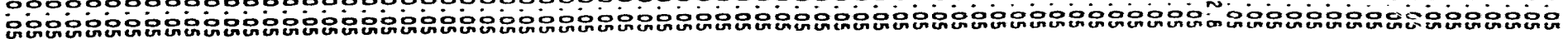




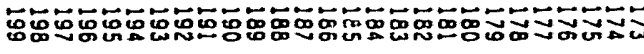

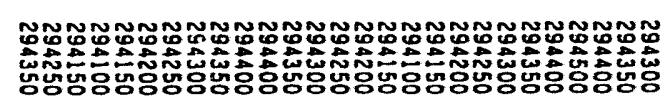

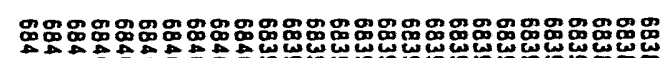

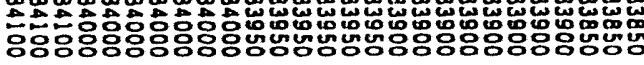

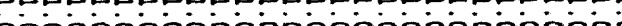

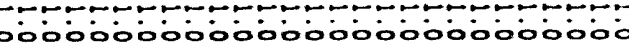

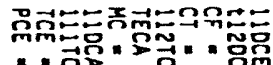

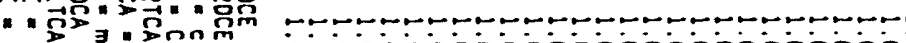

-

nition

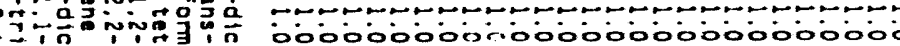

0000m

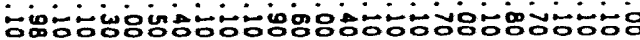

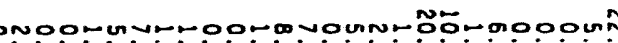

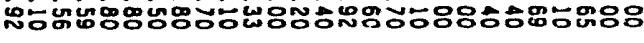

000000000000000000000000000 -

0000000000000000000 ton00000n

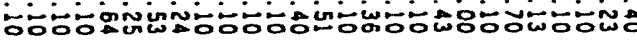

000000000000000000000000000 \%ั5050ั0

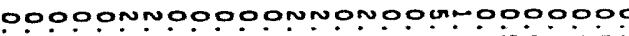

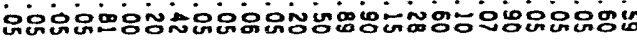

000000000000000000000000000

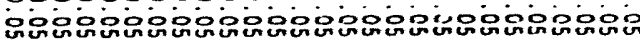

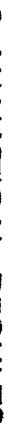




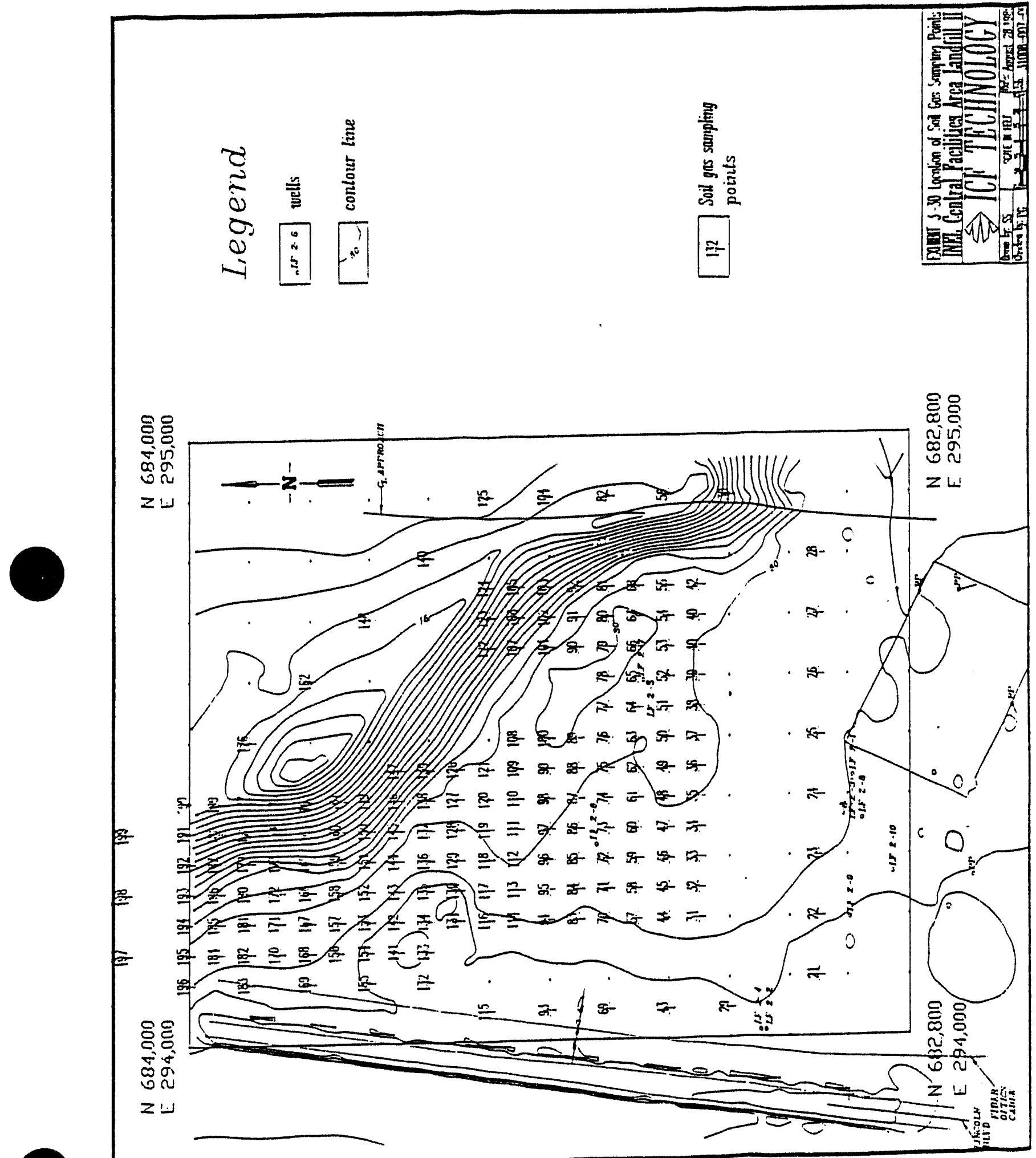

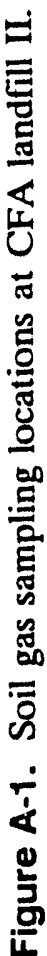


Appendix A.2

Model Description and Approach for Estimating Air Concentrations 
A.2-2 


\section{Appendix A.2}

\section{Model Description and Approach for Estimating Air Concentrations}

A vapor emission model was combined with a simple box model to yield an estimate of atmospheric concentrations of VOC vapors at a receptor location near the source. This was done by performing a mass balance on a control volume (box) of air immediately above a contaminated area. Mass (contaminant) enters the volume uniformly from below at the soil-atmosphere interface and is completely mixed before exiting the volume by means of an ambient wind. This general process is shown in Figure A.2-1. Mass influx to the volume occurs by vapor emission from the soil surface. This emission is the product of upward transport of vapor phase contaminants through the surface soils. The wind direction is assumed to perpendicular to the width (W) of the box and parallel to the ground.

A number of models have been developed lor estimating emission rates from covered landfills (EPA, 1990a). The Farmer model (Farmer et al., 1978; Farmer et al., 1980) was used because it represents the most conservative of the diffusion dominated models. Diffusion is believed to be the primary subsurface transport process; however, $i$ is possible that advection due to changes in barometric pressure could be important. The Farmer model is based on Fick's First Law of steady diffusion and assumes that vapor emission rates are limited by diffusion of vapors upward through the surface soil cover to a plane surface of constant concentration. The Farmer equation also makes use of the Millington (1959) formulation of the diffusion coefficient to account for the air-filled soil porosity being less than the total porosity due to the presence of moisture. The air-filled porosity can be determined from knowledge of the total porosity and either the moisture content or degree of water saturation.

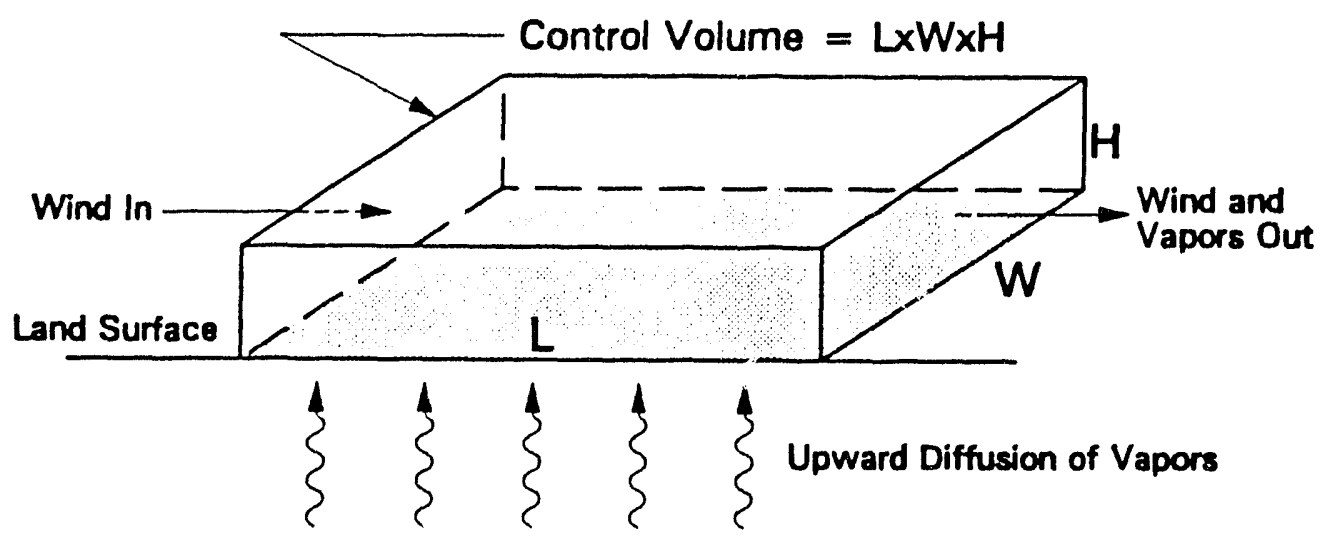

Figure A.2-1. Diagram of control volume and the general mass flows. 
The Farmer equation is:

$E_{i}=A_{e} D_{i}\left(\frac{\phi_{a}^{10 / 3}}{\phi_{t}^{2}}\right) \frac{\left(C_{i}-C_{s}\right)}{z}$

where

$$
\begin{aligned}
& E_{i}=\text { emission rate of component } \mathrm{i}(\mathrm{mg} / \mathrm{s}) \\
& A_{e}=\text { exposed area }=\operatorname{LxW}\left(\mathrm{m}^{2}\right) \\
& D_{i}=\text { vapor diffusion coefficient of component } \mathrm{i} \text { in air }\left(\mathrm{m}^{2} / \mathrm{s}\right) \\
& \phi_{a}=\text { air filled porosity }\left(\mathrm{m}^{3} / \mathrm{m}^{3}\right) \\
& \phi_{t}=\text { total porosity }\left(\mathrm{m}^{3} / \mathrm{m}^{3}\right) \\
& C_{i}=\text { concentration of } \mathrm{i} \text { at the bottom of the soil layer }\left(\mathrm{mg} / \mathrm{m}^{3}\right) \\
& C_{s}=\text { concentration of } \mathrm{i} \text { at soil surface }\left(\mathrm{mg} / \mathrm{m}^{3}\right) \\
& z=\text { depth of the soil layer }(\mathrm{m}) .
\end{aligned}
$$

$C_{i}$ is not necessarily the concentration at the bottom of the soil layer but the concentration at some known depth. For Landfill II, concentrations from soil-gas surveys (ICF Technology Inc. 1989 and Tracer Research Corp., 1988) were used where gas samples were taken at various depths. The rate at which mass exits the control volume shown in Figure A.2-1 due to wind can be expressed as follows:

$u_{m} A_{w} C_{i_{a}}$

where

$$
\begin{aligned}
& u_{m}=\text { the mean wind speed through the region }(\mathrm{m} / \mathrm{s}) \\
& A_{w}=\text { the area perpendicular to the wind direction }=W x H\left(\mathrm{~m}^{2}\right) \\
& C_{i_{a}}=\text { the mean air concentration of component } \mathrm{i}\left(\mathrm{mg} / \mathrm{m}^{3}\right) .
\end{aligned}
$$

In a steady condition, the mass flow rate into the volume, $E_{i}$, is equal to the mass flow rate out (Equation A.2-1 = Equation A.2-2). 
$A_{e} D_{i}\left(\frac{\phi_{a}^{10 \beta}}{\phi_{b}^{2}}\right) \frac{\left(C_{i}-C_{s}\right)}{z}=u_{m} A_{w} C_{i_{a}}$

From this the mean air concentration of component $i$ can be estimated. This is expressed in Equation A.2-4 where the dimensions of the different areas, $A_{e}$ and $A_{w}$, have been entered.

$$
C_{i_{a}}=\frac{D_{i}}{u_{m}}\left(\frac{\phi_{a}^{10 / 3}}{\phi_{t}^{2}}\right) \frac{\left(C_{i}-C_{s}\right)}{z} \frac{L}{H}
$$

The equation can be simplified by assuming a conservative scenario where the soil is assumed completely dry $\left(\phi_{a}=\phi_{t}\right)$ and the concentration at the soil surface $\left(C_{s}\right)$ equals zero. Completely dry soil means there is no moisture to block available pore space to vapor transport. A surface concentration of zero implies a concentration boundary layer of zero thickness and thus the concentration gradient or driving force for diffusion is a maximum. These assumptions result in the following expression.

$$
C_{i_{a}}=\frac{D_{i} \phi_{t}^{4 / 3}}{u_{m}} \frac{C_{i}}{z} \frac{L}{H}
$$

Notice the air concentration does not depend on the width of the source area. This is because the wind direction is perpendicular to the width and there is no dispersion. Therefore, any increase in width would only add to the total amount of VOCs entering the atmosphere while not increasing the concentration.

Several parameters are necessary to solve for the air concentration as expressed in Equations A.2-4 and A.2-5. Except for the diffusion coefficient in air, all are site specific and need to measured or estimated. $C_{i}, \phi_{i}$, and $z$ were determined from measurements while $L, H$, and $u_{m}$ were conservatively estimated. Table A.2-1 contains a summary of the parameters used in the calculations and the references. The calculations were performed for chloroform, methylene chloride, and benzene.

The effective length of the source, $L$, was determined from inspection of recent soil-gas survey results (ICF Technology Inc., 1989). Results from the soil-gas survey indicate there are high concentration areas or "hot spots" located above certain areas. Only the highest concentrations were used in the calculations.

The emission rate for an entire landfill is a complicated function of the emission area. This area and rate would be difficult to determine and was considered beyond the scope of a preliminary screening analysis. This analysis chose rather to focus on the most highly concentrated area and build conservatism into the analysis by assuming the highest concentration measured was spread over an area, or length in this case, larger than indicated in the soil-gas survey results (see Appendix A). The length, $L$, of the contaminated area was estimated to be greater than the distance across an average "hot spot" as determined by visual examination of the 
Table A.2-1. Parameters for calculating mean air concentrations.

\begin{tabular}{|c|c|c|}
\hline Parameter & Value & Reference \\
\hline$D_{i}$ & $\begin{array}{l}8.3 \times 10^{-6} \mathrm{~m}^{2} / \mathrm{s} \text { chloroform } \\
1.0 \times 10^{-5} \mathrm{~m}^{2} / \mathrm{s} \text { methylene } \mathrm{Cl} \\
9.3 \times 10^{-6} \mathrm{~m}^{2} / \mathrm{s} \text { benzene }\end{array}$ & Lugg, 1968 \\
\hline$\phi_{t}$ & 0.42 & Schroeder et al., 1988 \\
\hline$C_{i}$ & $\begin{array}{l}357 \mathrm{mg} / \mathrm{m}^{3} \text { chloroform (II) } \\
130 \mathrm{mg} / \mathrm{m}^{3} \text { benzene (II) } \\
6 \mathrm{mg} / \mathrm{m}^{3} \text { trichloroethylene } \\
111 \mathrm{mg} / \mathrm{m}^{3} \text { tetrachloroethylene } \\
82 \mathrm{mg} / \mathrm{m}^{3} 1,1 \text { dichloroethane } \\
60 \mathrm{mg} / \mathrm{m}^{3} 1,1,1 \text { trichloroethane }\end{array}$ & $\begin{array}{l}\text { ICF Technology, Inc., } 1989 \\
\text { Tracer Research Corp., } 1988 \\
\text { Tracer Research Corp., } 1988 \\
\text { ICF Technology, Inc., } 1989 \\
\text { ICF Technology, Inc., } 1989 \\
\text { ICF Technology, Inc., } 1989\end{array}$ \\
\hline$z$ & $\begin{array}{l}1.2 \mathrm{~m} \text { chloroform (II) } \\
5.8 \mathrm{~m} \text { benzene (II) } \\
6.7 \mathrm{~m} \text { trichloroethylene } \\
1.2 \mathrm{~m} \text { tetrachloroethyl } \mathrm{ne} \\
1.2 \mathrm{~m} 1,1 \text { dichloroethane } \\
1.2 \mathrm{~m} 1,1,1 \text { trichloroethane }\end{array}$ & $\begin{array}{l}\text { ICF Technology, Inc., } 1989 \\
\text { Tracer Research Corp., } 1988 \\
\text { Tracer Research Corp., } 1988 \\
\text { ICF Technology, Inc., } 1989 \\
\text { ICF Technology, Inc., } 1989 \\
\text { ICF Technology, Inc., } 1989\end{array}$ \\
\hline$L$ & $100 \mathrm{~m}$ & ICF Technology, Inc., 1989 \\
\hline$H$ & $1.5 \mathrm{~m}$ & $\begin{array}{l}\text { This study, which estimated approximate } \\
\text { average breathing height }\end{array}$ \\
\hline$u_{m}$ & $2 \mathrm{~m} / \mathrm{s}$ & Memorandum from G.E. Start, 1989 \\
\hline $\begin{array}{l}\text { (II) } \\
\text { (III) }\end{array}$ & $\begin{array}{l}\text { fill II. } \\
\text { fill III. }\end{array}$ & \\
\hline
\end{tabular}

soil-gas survey contour plots (ICF Technology Inc., 1989). By using this approach, it was felt that the maximum air concentration at the landfill could be conservatively estimated without having to consider all emission sources and does not depend on the wind direction or orientation of the landfill.

\section{Concentration Results}

Air concentrations for the contaminants calculated using Equation A.2-5 are presented in Table A.2-2. These concentrations were compared with the Time Weighted Average-Threshold Limit Values (TWA-TLVs) (American Conference of Governmental Industrial Hygienists, 1990) and found to be less than $1 / 100$ of the TWA/TLV which is the comparison generally used for public exposures. 
Table A.2-2. Comparison of estimated air concentrations to TWA-TLVs.

\begin{tabular}{lccc}
\hline \multicolumn{1}{c}{ Contaminant } & $\begin{array}{c}\text { Calculated air } \\
\text { concentration } \\
\left(\mathrm{mg} / \mathrm{m}^{3}\right)\end{array}$ & $\begin{array}{c}\text { TWA-TLV } \\
\left(\mathrm{mg} / \mathrm{m}^{3}\right)\end{array}$ & $\begin{array}{r}\text { 0.01 Times } \\
\text { TWA-TLV } \\
\left(\mathrm{mg}^{3} \mathrm{~m}^{3}\right)\end{array}$ \\
\hline Chloroform & 0.104 & 49 & 0.49 \\
Benzene & 0.009 & 32 & 0.32 \\
Trichloroethylene & 0.0007 & 269 & 2.69 \\
Tetrachloroethylene & 0.0309 & 339 & 3.39 \\
1, 1-Dichloroethane & 0.0263 & 810 & 8.10 \\
1, 2, 1-Trichloroethane & 0.0166 & 1910 & 19.10 \\
\hline
\end{tabular}

\section{Discussion of Uncertainties}

The effect of varying parameters other than porosity can easily be determined because of the linear relationship between concentration and all other parameters. For example, doubling the source length $(L)$, or soil-gas concentration $\left(C_{i}\right)$, would double the air concentration. Although not calculated, this could then double the risk estimate and/or hazard quotient. On the other hand, doubling the wind speed $\left(u_{m}\right)$, or the height of the mixing region $(H)$ would cut the air concentration estimate in half because it is inversely proportional to those parameters.

The calculated contaminant air concentrations for the contaminants detected should be interpreted with caution due to the depth at which some of the samples were taken. This analysis assumes a linear gradient between the measurement at depth and the surface. It is possible that for deeper measurements, the gradient could reverse as it approaches the surface. This means the concentration would be a maximum at a shallower depth than where the measurement was taken. If this were the case, a steeper concentration gradient would exist and increase the flux into the control volume. This situation however is not likely to significantly increase air concentrations above current estimates.

The length of the source is expected to remain comparable if not decrease. The wind speed can and will change as atmospheric conditions change. That is why the parameter was conservatively estimated to simulate relatively stagnant conditions. The height of the mixing region was also conservatively estimated. Therefore, this analysis should yield a fairly accurate estimate of air concentrations that a receptor around the landfill could be exposed to in a worstcase scenario.

The primary limitations of this analysis include: (1) a diffusion dominated emission rate, and (2) not directly accounting for emissions from other areas. It is possible that barometric pumping could enhance the emission rate on days of low barometric pressure, but it could also inhibit it on high pressure days and over time the effects would likely average out. As for emissions from other areas it is believed that contributions from other sources to the air loading at a receptor location near the landfill or anywhere else would be small (because of dilution/dispersion effects) 
yet not negligible. If the other areas were accounted for and a more realistic estimate of the total source area is included, the conservative parameter estimates that went into this analysis should be relaxed to more realistic values.

In summary, this fate and transport modeling of soil gas concentrations found at depth at the landfills suggest the resultant ambient air concentrations for all contaminants were well below the compound-specific TWA-TLVs for workers and well below 0.01 times the compound-specific TWA-TLV for public receptors. 


\section{REFERENCES}

American Conference of Governmental Industrial Hygienists (ACGIH), Threshold Limit Values and Biological Exposure Indices for 1989-1990, Cincinnati, Ohio, 1989.

EPA, Air/Superfund National Technical Guidance Study Series, Volume II-Estimation of Baseline Air Emissions at Superfund Sites, EPA-450/1-89-002a, August 1990.

Farmer, W. J., M. S. Yang, J. Letey, W. F. Spencer, and M. H. Roulier, Land Disposal of Hexachlorobenzene Wastes: Controlling Vapor Movement in Soils, In: Land Disposal of Hazardous Wastes, Proceedings of the Fourth Annual Research Symposium, U.S. EPA Municipal Environmental Research Laboratory, San Antonio, TS, March 6-8, 1978.

Farmer, W. J., M. S. Yang, J. Letey, and W. F. Spencer, Land Disposal of Hexachlorobenzene Wastes: Controlling Vapor Movement in Soils, EPA-600/2-80-119, U.S. EPA Municipal Environmental Research Laboratory, Cincinnati, OH, 1980.

ICF Technology Incorporated, 1989, Innovative Technology Demonstration, Idaho National Engineering Laboratory (INEL), CFA Landfill II, Idaho Falls, Idaho.

Lugg, G. A., Diffusion Coefficients of Some Organic and Other Vapors in Air, Anal. Chem., Vol. 40, No. 7, 1968.

Memorandum from G. E. Start, U.S. Department of Commerce, National Oceanic and Atmospheric Administration, to Les Finch, WINCO, "Interim Guidance for Dispersion Calculations at ICPP," March 24, 1989.

Millington, R. J., "Gas Diffusion in Porous Media," Science, Vol. 130, 1959.

Schroeder, P. R., R. L. Peyton, B. M. McEnroe, and J. W. Sjostrom, 1988, The Hydrologic Evaluation of Landfill Performance (HELP) Model, Volume III, User's Guide for Version 2, Hazardous Waste Engineering Research Laboratory, Office of Research and Development, U.S. Environmental Protection Agency, Cincinnati, Ohio, 1988.

Tracer Research Corp., 1988, Shallow Soil Gas Investigation at the Idaho National Engineering Laboratory (INEL), Idaho Falls, Idaho. 
Appendix A.3

CFA Landfills II and III Borehole Soil Gas Data 
A.3-2
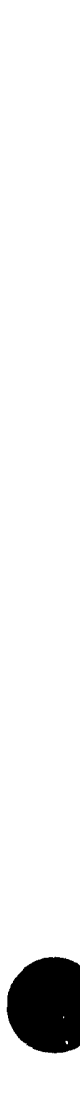


\section{INTEROFFICE CORRESPONDENCE}

Date: $\quad$ Apri1 1, 1989

To: $\quad$ S. L. Ansley

From: W. F. Bauer LUPB

Subject: REPORT OF RESULTS FOR METHANE ANALYSIS OF SOIL GASES wfb-09-89

Following are the results of the analyses for methane in the soil gas samples that were submitted to us on March 6, 1989 and March 8, 1989. All analyses were performed on a Perkin-Elmer Sigma $2 B$ gas chromatograph. The chromatographic column was a six foot stainless steel column packed with 5 Angstrom molecular sieve : Detection was via a flame lonization detector. Calibration was performed by repeated injections of different volumes of a methane standards certified to within 2\%. All samples and standards were reduced to atmospheric pressure prior to injection. Concentration calculations were performed via a standard curve of volume methane injected or concentration in $\mathrm{ppm} v / \mathrm{v}$ versus detector response. Detection 1 imits were determined as 3 times the standard deviation of the $y$-intercept. Sample dilutions or concentrations were done where necessary by simply injecting smaller or larger volumes of the sample as necessary.

Because of the uncertainties due to sampling, transport, variations in laboratory temperature and pressure, and the difficulties of accurately injecting differing volumes of a gas, the the results should be considered to have an uncertainty of $\pm 20 \%$. Actual standard deviations estimated from the regression curve were at or less than $\pm 5 \%$ and standard deviations estimated from injecting varying volumes of the same sample were on the order of $\pm 15 \%$.

The concentrations in the $\mathrm{mg} / \mathrm{m}^{3}$ units were calculated using corrections to the molar volume with the laboratory temperature and pressure. The corrected molar volume was approximately $28.81 /$ mole. 
S. L. Ansley

Apri1 11, 1989

WFB-05-89

Page 2

\begin{tabular}{|c|c|c|c|}
\hline Sample & Analysis Date & $\begin{array}{c}\text { Methane } \\
\mathrm{ppm}(v / v)\end{array}$ & $\begin{array}{c}\text { Methane } \\
\mathrm{mg} / \mathrm{m}^{3}\end{array}$ \\
\hline Laboratory Air & $3-7-89$ & 5 & 3 \\
\hline $99.18 \mathrm{ppm}$ standard & $3-7-89$ & 95 & 46 \\
\hline MLF202MAR89 & $3-7-89$ & 8 & 4 \\
\hline MLF201MAR89 & $3-7-89$ & 169 & 82 \\
\hline MLF206MAR89 & $3-7-89$ & 13000 & 6300 \\
\hline MLF203MAR89 & $3-7-89$ & 57000 & 28000 \\
\hline MLF208MAR89 & $3-7-89$ & 6 & 3 \\
\hline Laboratory Air & $3-10-89$ & $<10$ & $<5$ \\
\hline $99.18 \mathrm{ppm}$ Standard & $3-10-89$ & 90 & 44 \\
\hline MLF3X7MAR89 & $3-10-89$ & 31 & 15 \\
\hline MLF304MAR89 & $3-10-89$ & $<10$ & $<5$ \\
\hline MLF307MAR89 & $3-10-89$ & $<10$ & $<5$ \\
\hline MLF306MAR89 & $3-10-89$ & 97 & 47 \\
\hline MLF301MAR89 & $3-10-89$ & $<10$ & $<5$ \\
\hline MLF302MAR89 & $3-10-89$ & 19 & 9 \\
\hline \multicolumn{4}{|c|}{$\begin{array}{l}\text { If you have any questions or require any additional data for these } \\
\text { analyses, call me at } 6-1180 \text { or } 6-0484 \text { and I will be happy to supply them } \\
\text { for you. }\end{array}$} \\
\hline
\end{tabular}




\section{INTEROFFICE CORRESPONDENCE}

Date: June 1, 1989

To: $\quad$ S. L. Ansley

From:

W. F. Bauer LUPB

Subject: REPORT OF RESULTS FOR METHANE ANALYSIS OF SOIL GASES wfb-09-89

Attached are the results of the analyses for methane in the soil gas samples that were submitted to us on 5-23-89. All analyses were performed on a Perkin-Elmer Sigma 1 gas chromatograph. The chromatographic column was a 6 foot stainless steel column packed with 5 Angstrom molecular sieve held at approximately $50^{\circ} \mathrm{C}$. The carrier gas was helium flowing at 40 $\mathrm{mL} / \mathrm{min}$ and detection was via a flame ionization detector. Calibration was performed by repeated injections of different volumes of methane gas standards certified by Scott Specialty Gases to within 2\%. A11 samples and standards were reduced to atmospheric pressure prior to injection. concentration calculations were performed via a standard curve of yolume methane injected versus detector response. Detection limits were determined as 3 times the standard deviation of the $y$-intercept of this curve. Sample dilutions or concentrations were done where necessary by simply injecting smaller or larger volumes of the sample as necessary.

The concentrations in the $\mathrm{mg} / \mathrm{m}^{3}$ units were calculated using corrections to the molar volume with the laboratry temperature and pressure. The corrected molar volume was approximately $28.81 /$ mole.

If you have any questions or require any additional data for these analyses, call me at 6-1180 or 6-0484 and I will be happy to supply them for you.

ph

Attachment:

As Stated

cc: W. A. Propp

J. W. Owens $丁 60$

C. A. Allen

Central Files

W. F. Bauer Ltr File (2) 


\begin{tabular}{|c|c|c|c|c|c|c|}
\hline SAMPLE & $\begin{array}{l}\text { Analysis } \\
\text { Date }\end{array}$ & $n^{a}$ & $\begin{array}{l}\text { Methane } \\
\mathrm{ppm}(\mathrm{v} / \mathrm{v})\end{array}$ & $\begin{array}{l}\text { Methąne } \\
\mathrm{mg} / \mathrm{m}^{3}\end{array}$ & $\%$ RSD & $\%$ Error \\
\hline 105 ppm standard & $5-24-89$ & 3 & 106 & 59 & $1.8^{b}$ & 0.6 \\
\hline Laboratory Air & $5-24-89$ & 2 & 2.4 & 1.3 & $12.8^{C}$ & \\
\hline MLF20TMAY89 & $5-24-89$ & 1 & 0.8 & 0.4 & & \\
\hline MLF307MAY89 & $5-24-89$ & 1 & 20.7 & 11.5 & $2.6^{d}$ & \\
\hline MLF306MAY89 & $5-24-89$ & 1 & 8.5 & 4.7 & $6.5^{d}$ & \\
\hline MLF308MAY89 & $5-24-89$ & 1 & 107.3 & 59.5 & $0.5^{d}$ & \\
\hline MLF201MAY89 & $5-24-89$ & 1 & 8.8 & 4.9 & $6.3^{d}$ & \\
\hline MLF202MAY89 & $5-24-89$ & 1 & 6.2 & 3.4 & $9.0^{d}$ & \\
\hline MLF304MAY89 & $5-24-89$ & 1 & 1.2 & 0.7 & $45.7^{d}$ & \\
\hline MLF311MAY89 & $5-24-89$ & 1 & 1.1 & 0.6 & $52.5^{d}$ & \\
\hline MLF301MAY89 & $5-24-89$ & 1 & 2.2 & 1.2 & $25.4^{d}$ & \\
\hline $1.066 \% \mathrm{CH}_{4}$ & $5-25-89$ & 3 & 10620 & 5920 & $1.0^{b}$ & -0.4 \\
\hline MLF206MAY892 & $5-25-89$ & 2 & 5530 & 3080 & $2.4^{C}$ & \\
\hline MLF207MAY89 & $5-25-89$ & 3 & 97000 & 54300 & $1.2^{b}$ & \\
\hline MLF206MAY893 & $5-25-89$ & 1 & 22360 & 12480 & $0.2^{d}$ & \\
\hline MLF206MAY891 & $5-25-89$ & 2 & 18480 & 10310 & $0.1^{c}$ & \\
\hline $0.9888 \% \mathrm{CH}_{4}$ & $5-25-89$ & 1 & 9830 & 5480 & $0.2^{d}$ & -0.6 \\
\hline
\end{tabular}

aThe number of replicate injections.

b The reported mean concentration and the percent RSD were calculated from replicate injections of the same sample.

CThe percent RSD is actually an RPD from calculated from duplicate measurements as is the reported mean concentration.

dThe percent RSD values are estimated from the errors present in the analytical curve and are determined by the linear regression. 
Date: June 27,1989

To: S. L. Ansley

From: W. F. Bauer UJ

Subject: REVIEW OF VOLATILE ORGANIC DATA FROM SOIL GAS SAMPLES WFB-11-89

Listed below are several comments based upon our review of the data supplied by EG\&G Environmental Chemistry for the analys is of volatile hydrocarbons in soil gas. In general, the analyses and data appear to be reasonable. The data were from samples collected at the end of November 1988 and in March of 1989. Data were reviewed using the "guidelines" within the Contract Compliance Screening (CCS) procedures for Volatile Organic Analysis (VOA) pubtished by the EPA. The CCS procedures and the requirements therein may not necessarily best fit all aspects of these analyses either because of the modifications to the procedure or because of the scope of the work (and level of reporting) actually contracted for.

1) Based upon our experience, we can say that the precision AND accuracy of gas (gas-tight) syringes tends to vary, sometimes dramatically, from syringe to syringe. This places some doubt upon the use of quantitative data derived from samples that were taken and stored in a number of different syringes. This doubt increases with the length of time that the sample is stored in the syringe. Some samples from Landfill 3 that were taken in March 1989 were held for up to 6 days. These analyses were performed using a modified version of EPA Method 624 which states a holding time of 7 days for aqueous VOA samples. Alternate sampling procedures should be pursued.

2) No initial calibration data were available. This data was apparently not asked for or required by the scope of work. For future reference, this data should be requested for each new calibration with a summary form (Form VI) in each data package covered by that calibration. This data is useful in data review for assessing the quality of the calibration and thus the data for the samples and continuing calibrations (standards) associated with it. Other useful forms which, since they were not required, were missing were the method blank summary (Form IV), the surrogate recovery form (Form II) and the case narrative (ROA will suffice).

3) No raw or summarized data other than the report of analysis (ROA) was present for samples LF301NOV88 thru LF307NOV88. A11 of these samples were collected on $11 / 28 / 88$ and run on $11 / 29 / 89$. 
S. L. Ansley

June 27,1989

WFB-11-89

Page 2 of 2

4) The column of numbers for the sample LF206NOV88 on the December ROA should be labeled with the sample id of LF205NOV88 and visa versa.

5) The ROA does not reflect an estimated concentration ( $f$ lagged $B, J$ ) of methylene chloride at $3.7 \mathrm{mg} / \mathrm{m}^{3}$ for sample LF201NOV88FB.

6) The ROA does not reflect an eştimated concentration (flagged $\mathrm{J}$ ) of tetrachloroethene at $1.0 \mathrm{mg} / \mathrm{m}^{3}$ for sample LF201NOV88FB.

7) A method blank was run for the samples analyzed on $12 / 01 / 88$ but not with the samples run on $11 / 29 / 88$ putting their results even further into doubt. All positive results for this day should be flagged as estimated since they may be argued to be noncompliant by CCS procedures. If it is assumed that the 12/01/88 method blank is valid for the day in question, then all positive hits for acetone, 2-butanone, and methylene chloride should also be flagged with a B.

8) Sample LF2-08MAR89 is missing the volatile organics analysis sheet (Form I).

9) The percent difference (\%D) calculated for the relative response factor (RRF) for ethylbenzene during the continuing calibration of $3 / 6 / 89$ at $13: 28$ is $42.21 \%$ which is far outside of the allowable range of $25 \% \mathrm{D}$. No samples with this continuing calibration contain hits of ethylbenzene, but all samples could be possibly be regarded as noncompliant by strict adherence to the CCS procedures.

10) The ROA for the March samples contains two typing errors:

* The toluene concentration for LF3-04MAR89 should read $0.2 \mathrm{mg} / \mathrm{m}^{3}$.

* The method blank on page 4 reports the acetone concentration of $0.41 \mathrm{ppm}$ instead of $0.83 \mathrm{ppm}$.

11) p-Dioxane was also noted in the method blank and in several of the samples for the November series of samples. This compound was not listed on the report forms for any of the analyses. This raises a question of the source and importance of this compound.

$\mathrm{ph}$

cc: D. L. Miller J Jom

J. W. Owens

L. J. Peterson-Wright

Central Files

W. F. Bauer File 
EG\&G Idaho, Enviromental Chemistry, ROA

EG\&G Idaho, Ine.

Enviromental Chemistry

P.O. Box 1625, MS 4123

Idaho Falls, 1083415

This report of analysis (ROA) presents the analytical results for the analyses you requested. Sample identification, sampling information, and laboratory analysis information are listed in a colum for each sample. Anaiytical results are listed by analytical parameter below the sample identification information. Acronyms and abbreviations used are defined at the end of the report. A letter and/or letter number flag imediately to the right of a parameter, method, POL and units, result, or any other entry indicates an amplifying corment also provided at the end of the report.

The analytical data (analysis data sheets, chromatograms, mass spectra, quantitation reports) are enclosed.

REPORT OF ANALYSIS

Prepared for:

Report Identification:
ROA Number:
65
Date:
$03 / 20 / 89$
Charge No.: $\quad 3 R I F 04030$

Shannon L. Ansley

EG\&G Idaho, Inc.

.0. 80x 1625, MS 2212

Page:

1 


Sampling Site:
Sample Type:
Customer Sample ID:
Sampling Date:
Sampl ing Time:
Oate Received:
Lab Sample ID:
Date Extracted:
Date Analyzed:
Comments:

\section{Parameter}

...........

Volatile Organic Compounds

Method

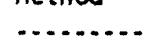

\begin{abstract}
POL and Units
\end{abstract} (...................

\section{EPA-624 M2}

2-8utanone

Chloroethane

1, 1-Dichloroethane

1,2-Dichloroethene (Total) EPA-624

M2

EPA-624 M2

Tetrachloroethene

Tolvene

1,1,1-Trichloroethane

Trichloroethene

1,1,2-Trichloro-1,2,2trifluoroethane

Others - Tentatively Identified Compounds

Others - Comment $A 3$

EPA-624 MZ
EPA-624 M2

EPA-624 M2

$$
\begin{array}{r}
\text { CFA-LF2 } \\
25 \cdot \mathrm{ml} \\
\text { soil gas }
\end{array}
$$

Method Blk

03/06/89

03/06/89

Lab Blank

$$
\text { thil gas }
$$

Comment A3

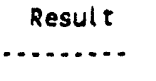

$$
\text { CFA-LF2 }
$$$$
25 \cdot \mathrm{ml}
$$

soil gas

LF2-07MARB9

03/06/89

$11: 38$

$03 / 06 / 89$

9m1A0341

03/06/89

03/06/89

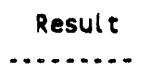

CFA-LF2

$25-\mathrm{ml}$

soil gas

LF2-OBMAR89

03/06/89

$11: 50$

03/06/89

9M1A0342

$03 / 06 / 89$

$03 / 06 / 89$

Result
CFA-LFZ

$25-\mathrm{ml}$

soil gas

LF2-01MAR89

03/06/89

$12: 03$

03/06/89

9MIA0343

03/06/89

03/06/89

\section{Result}

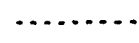

Comment $N_{1}$

$2.0 \mathrm{mg} / \mathrm{m}^{3}$

$1.0 \mathrm{mg} / \mathrm{m}^{3}$

$40 \mathrm{mg} / \mathrm{m}^{3} B, E 28.0 \mathrm{mg} / \mathrm{m}^{3} \mathrm{~B}$

$0.83 \mathrm{ppm}$

$0.41 \mathrm{ppm} \mathrm{J}$

$17 \mathrm{ppm} \quad B, E 2 \quad 3.3 \mathrm{ppm} B$

NA

NA

$2.0 \mathrm{mg} / \mathrm{ms}^{3}$

$2.0 \mathrm{mg} / \mathrm{m3}$

$0.67 \mathrm{ppm}$

BPOL

BPOL

$0.67 \mathrm{ppm}$

BPOL

BPQL

NA

NA

NA

NA

$0.74 \mathrm{ppm}$

BPOL

BPQL

BPQL

BPQL

$0.24 \mathrm{pom}$

$1.0 \mathrm{mg} / \mathrm{m}^{3}$

0.25 ppm

EPA-624 M2

$2.0 \cdot 4.0 \mathrm{mg} / \mathrm{m}^{3} \quad \mathrm{T3}$ $0.3-0.5$ ppm 13

$2.0-4.0 \mathrm{mg} / \mathrm{m}^{3} \mathrm{~T} 3 \mathrm{see}$ results
BPQL

BPOL

BPOL

BPOL

BPQL

BPOL

BPQL

BPOL

BPQL

BPOL

BPOL

BPOL

BPQL

BPOL

BPQL

BPOL

BPQL

BPQL

BPOL

BPOL

BPOL

BPOL

BPOL

BPOL

$3.0 \mathrm{mg} / \mathrm{ms} \mathrm{B2}^{3}, \mathrm{~d} 2$

0.4 ppm B2, $J 2$

NA

on attached

form IE

See results

on atrached

Form IE

See resulis

on atrached

form IE

(VOA-TIC)

NA

NA

NA

NA

NA

NA

NA

NA

NA

NA

NA

NA

NA

NA

(VOA-TIC)

BPOL

BPQL 


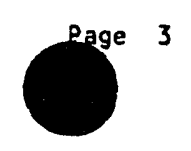

Parameter

..........

Volatile Organic Compounds

Acetone

2-Butanone

Chloroethane

1,1-Dichloroethane

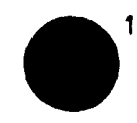

1,2-0ichloroethene (Total) EPA-624 M2

Methylene chloride

Tolvene

1,1,1-Trichlor oethane

Trichloroethene

1, 1,2-irichloro-1,2,2-

erifluoroethane

Others - Tentatively

identified Compounds

Others - Comment $A 3$
EPA-624 M2
Sampling site:

Sample Type:

Customer Sample 10:

Sampling Date:

Date Received:

Lab Sample 10:

Date Analyzed:

Remarks :

\section{Method}

.........

PQL and Units

.................
Sampl ing Time:

Date Extracted:

$$
\begin{array}{r}
\text { CFA-LF2 } \\
25 \cdot \mathrm{ml} \\
\text { soil gas }
\end{array}
$$

Comment $A 3$

$$
\text { EPA-624 M2 }
$$

EPA-624 M2

EPA-624 M

$2.0 \mathrm{mg} / \mathrm{m3}$

$0.83 \mathrm{ppm}$

$2.0 \mathrm{mg} / \mathrm{m}^{3}$

$0.67 \mathrm{ppm}$

$2.0 \mathrm{mg} / \mathrm{ms}^{3}$

$0.67 \mathrm{ppm}$

$2.0 \mathrm{mg} / \mathrm{m}^{3}$

$0.74 \mathrm{ppm}$

$1.0 \mathrm{mg} / \mathrm{ms}^{3}$

0.24 ppm

$1.0 \mathrm{mg} / \mathrm{m}^{3}$

$0.25 \mathrm{ppm}$

$1.0 \mathrm{mg} / \mathrm{m}^{3}$

$0.28 \mathrm{ppm}$

$1.0 \mathrm{mg} / \mathrm{m}^{3}$

$0.14 \mathrm{ppm}$

$1.0 \mathrm{mg} / \mathrm{m}^{3}$

$0.26 \mathrm{ppm}$

EPA-624 M2

$1.0 \mathrm{mg} / \mathrm{m}^{3}$

$0.18 \mathrm{ppm}$

EPA-624 M2

$1.0 \mathrm{mg} / \mathrm{m}^{3}$

$0.18 \mathrm{ppm}$

EPA-624 M2

$2.0 \cdot 4.0 \mathrm{mg} / \mathrm{m}^{3} \mathrm{T3}$ $0.3 \cdot 0.5 \mathrm{ppm} T 3$

BPQL

BPQL

BPOL

BPQL

BPOL

BPOL

BPOL

BPQL

BPQL

BPOL

BPOL

BPOL

BPOL

BPQL

BPOL

BPOL

BPOL

BPOL

BPOL

EPA-624 M2

$2.0 \cdot 4.0 \mathrm{mg} / \mathrm{m}^{3} \mathrm{~T} 3$

See result on attached

form IE

(VOA-TIC)

BPOL

$$
\begin{array}{r}
\text { CFA-LF2 } \\
25 \cdot m l \\
\text { soil gas }
\end{array}
$$

$03 / 06 / 89$

$12: 25$

03/06/89

9N1A0344

03/06/89

03/06/89

Result

..........
CFA-LF2

$25-\mathrm{ml}$

soil gas

LF2. OGMAR89

03/06/89

$12: 45$

$03 / 06 / 89$

911 10345

$03 / 06 / 89$

$03 / 06 / 89$

Result

........
CFA-LF2

25-ml

soil gas

LF2.05MAR89

$03 / 06 / 89$

$12: 58$

03/06/89

9MIA0346

03/06/89

$03 / 06 / 89$

Result

.........

Comment N1

NA

NA

1.7 ppm

BPOL

BPOL

$1.0 \mathrm{mg} / \mathrm{m}^{3} \quad B, J \quad N A$

BPOL $0.33 \mathrm{ppm}$ B, J NA

BPOL $\quad 4.0 \mathrm{mg} / \mathrm{m}^{3} \quad \mathrm{NA}$

BPQL $\quad 1.5 \mathrm{pPm} \quad$ NA

BPOL $\quad 4.0 \mathrm{mg} / \mathrm{ms}^{3} \quad$ NA

BPQL $0.97 \mathrm{pPm}$ NA

BPQL $\quad 38 \mathrm{mg} / \mathrm{m}^{3} \quad$ NA

BPOL $\quad 9.4 \mathrm{pPm}$ NA

BPQL $\quad 2.0 \mathrm{mg} / \mathrm{m3} \quad \mathrm{NA}$

BPQL $\quad 0.57 \mathrm{ppm}$ NA

BPOL $\quad 2.0 \mathrm{mg} / \mathrm{m3} \quad$ NA

BPOL $\quad 0.29 \mathrm{ppm}$ NA

BPQL $\quad 0.50 \mathrm{mg} / \mathrm{m}^{3} \mathrm{~J}$ NA

BPOL $0.13 \mathrm{ppm} J$ NA

BPQL BPOL NA

BPOL BPOL NA

BPOL $\quad 3.0 \mathrm{mg} / \mathrm{mb}^{3} \quad \mathrm{NA}$

BPOL 0.54 pPm NA

BPOL $34 \mathrm{mg} / \mathrm{m}^{3} \quad B 2, \mathrm{~J} 2$ NA

BPQL $\quad 4.3 \mathrm{pPm} \quad \mathrm{B2}, \mathrm{J} 2$ NA

See results NA

on atrached NA

Form IE

(VOA-TIC)

BPOL

BPOL

BPQL 


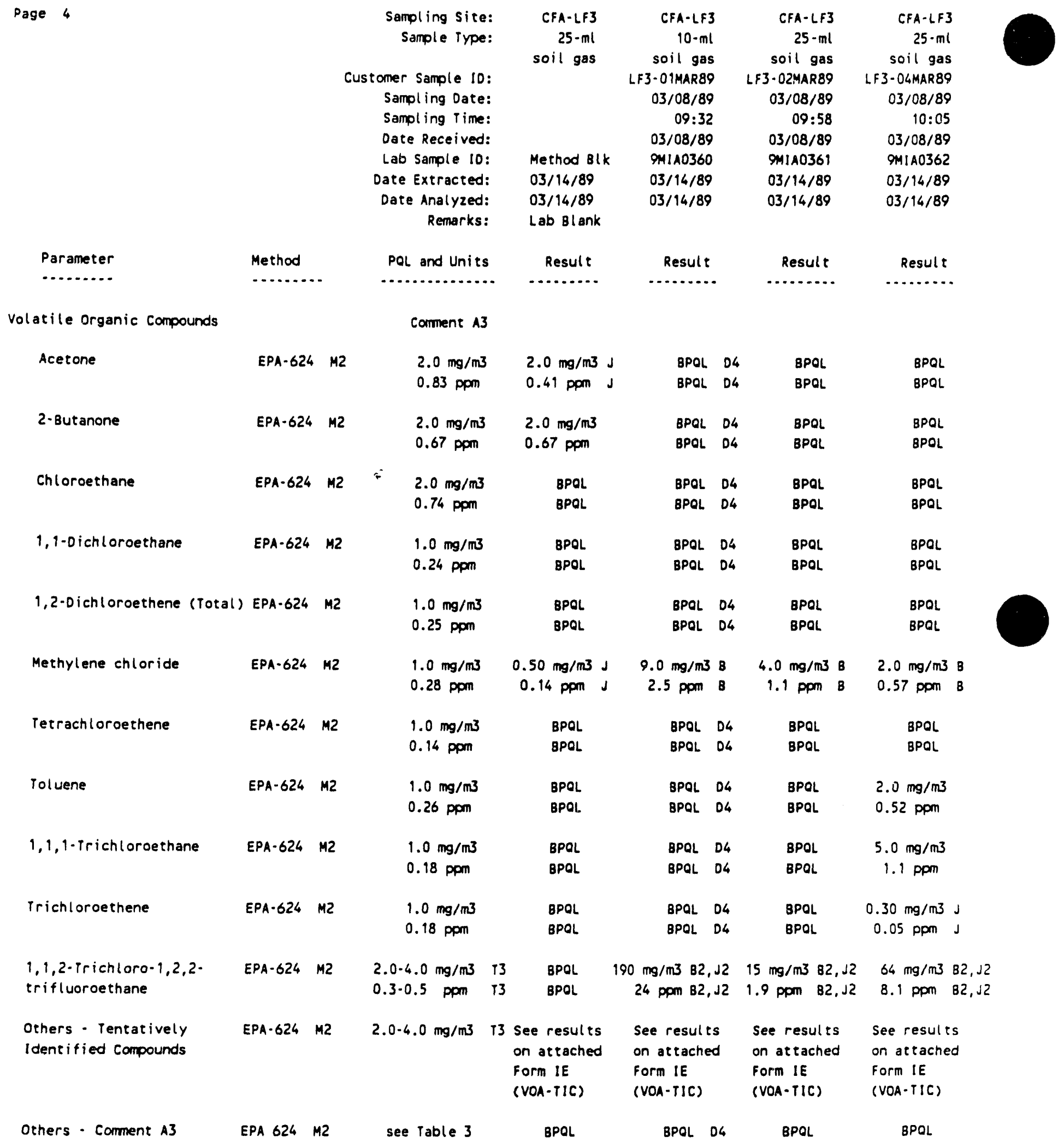

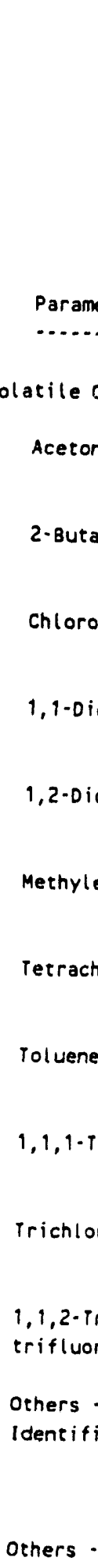




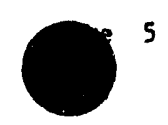

Sampling site:
Sample Type:
Customer Sample 10:
Sampl ing Date:
Sampl ing Time:
Date Received:
Lab Sample 10:
Date Extracted:
Date Analyzed:
Remarks:

Parameter

...........

Volatile Organic Compounds

Acetone

2-Butanone

Chloroethane

1,1-Dichloroethane

1,2-Dichloroethene (Total) EPA-624 M2

Methylene chloride

Tetrachloroethene

Tolvene

1,1,1-Trichloroethane

Trichloroethene

1,1,2-Irichloro-1,2,2-

erifluoroethane

Others - Tentatively

Identified compounds

others - Comment $A 3$
EPA $624 \quad M 2$ see Table 3
EPA-624 M2

EPA-624 M2

Method

EPA-624 M2

EPA-624 M2

EPA-624 M2

EPA-624 M2

EPA-624 M2

EPA-624 M2

EPA-624 M2

EPA-624 M2

EPA-624 M2

$2.0 \mathrm{mg} / \mathrm{ms}^{3}$

$0.83 \mathrm{ppm}$

$2.0 \mathrm{mg} / \mathrm{m}^{3}$

$0.67 \mathrm{ppm}$

$2.0 \mathrm{mg} / \mathrm{m}^{3}$

$0.74 \mathrm{ppm}$

$1.0 \mathrm{mg} / \mathrm{m}^{3}$

$0.24 \mathrm{ppm}$

$1.0 \mathrm{mg} / \mathrm{m}^{3}$

$0.25 \mathrm{ppm}$

$1.0 \mathrm{mg} / \mathrm{ms}^{3}$

0.28 ppm

$1.0 \mathrm{mg} / \mathrm{m}^{3}$

$0.14 \mathrm{ppm}$

$1.0 \mathrm{mg} / \mathrm{m}^{3}$

$0.26 \mathrm{ppm}$

$1.0 \mathrm{mg} / \mathrm{m}^{3}$

$0.18 \mathrm{ppm}$

$1.0 \mathrm{mg} / \mathrm{m}^{3}$

$0.18 \mathrm{ppm}$

$2.0-4.0 \mathrm{mg} / \mathrm{m}^{3} \mathrm{T3}$

$0.3 \cdot 0.5$ ppm T3

$2.0 \mathrm{mg} / \mathrm{m}^{3}$

$0.83 \mathrm{ppm}$

$2.0 \mathrm{mg} / \mathrm{m}^{3}$

$0.67 \mathrm{ppm}$

BPQL

BPOL

BPQL

BPQL

BPQL

BPQL

$0.50 \mathrm{mg} / \mathrm{m} \mathrm{J}$
$0.14 \mathrm{ppm} \mathrm{J}$

BPOL

BPOL

BPOL

BPQL

BPQL

BPQL

BPOL

8POL.

BPOL

BPOL
BPOL D4

BPQL 04

BPQL DA

BPOL D4

BPQL D4

BPQL D4

BPQL $04 \quad 1.0 \mathrm{mg} / \mathrm{ms}^{3}$

BPQL $04 \quad 0.24$ ppm

BPOL D4 BPQL

BPOL O4 BPQL

\section{$2.0 \mathrm{mg} / \mathrm{m}^{3}$}

$0.57 \mathrm{ppm}$

BPOL OC

BPOL 04

$4.0 \mathrm{mg} / \mathrm{m3}$

$1.9 \mathrm{ppm}$

BPOL

BPOL

$0.40 \mathrm{mg} / \mathrm{m}^{3} \mathrm{~J}$

$0.60 \mathrm{mg} / \mathrm{ms} \mathrm{J}$

$0.10 \mathrm{ppm} \mathrm{J}$

$0.16 \mathrm{ppom} J$

$2.0 \mathrm{mg} / \mathrm{m}^{3}$

$5.0 \mathrm{mg} / \mathrm{m}^{3}$

$0.43 \mathrm{ppm}$

$1.1 \mathrm{ppm}$

BPQL $04 \quad 0.40 \mathrm{mg} / \mathrm{m}^{3} \mathrm{~J}$

BPOL D4 $0.07 \mathrm{ppm} \mathrm{J}$

$72 \mathrm{mg} / \mathrm{m}^{3} \quad 82, \mathrm{~J} 2 \quad 34 \mathrm{mg} / \mathrm{m}^{3} \quad \mathrm{B2}, \mathrm{J} 2 \quad 190 \mathrm{mg} / \mathrm{m}^{3} \quad B 2, \mathrm{~J} 2$

$9.1 \mathrm{ppm} \quad 82, \mathrm{~J} 2 \quad 4.3 \mathrm{ppm} \quad 82, \mathrm{~J} 2 \quad 24 \mathrm{ppm} \quad 82, \mathrm{~J} 2$

3 See results

on attached

Form IE

(VOA-TIC)
BPOL DG BPQL

BPQL
BPOL 04

BPOL

BPQL DG 


$\begin{array}{rrr}\text { Sampling site: } & \text { CFA-LF3 } & \text { CFA-LF3 } \\ \text { Sample Type: } & 25 \cdot \mathrm{ml} & 25-\mathrm{ml} \\ & \text { soil gas } & \text { soil gas } \\ \text { Customer Sample 10: } & & \text { LF3-08MAR89 } \\ \text { Sampl ing Date: } & & 03 / 08 / 89 \\ \text { Sampling Time: } & & 11: 08 \\ \text { Date Received: } & & 03 / 08 / 89 \\ \text { Lab Sample 10: } & \text { Method BlK } & 9 M 110366 \\ \text { Date Extracted: } & 03 / 14 / 89 & 03 / 14 / 89 \\ \text { Date Analyzed: } & 03 / 14 / 89 & 03 / 14 / 89 \\ \text { Remarks: } & \text { Lab Blank } & \end{array}$

\begin{tabular}{|c|c|c|c|c|}
\hline Parameter & Method & PQL and Units & Result & Result \\
\hline$\ldots \ldots$ & ......... & $\ldots \ldots \ldots \ldots$ & $\ldots \ldots \ldots$ & $\ldots \ldots$ \\
\hline
\end{tabular}

Volatile Organic Compounds

Comment 13

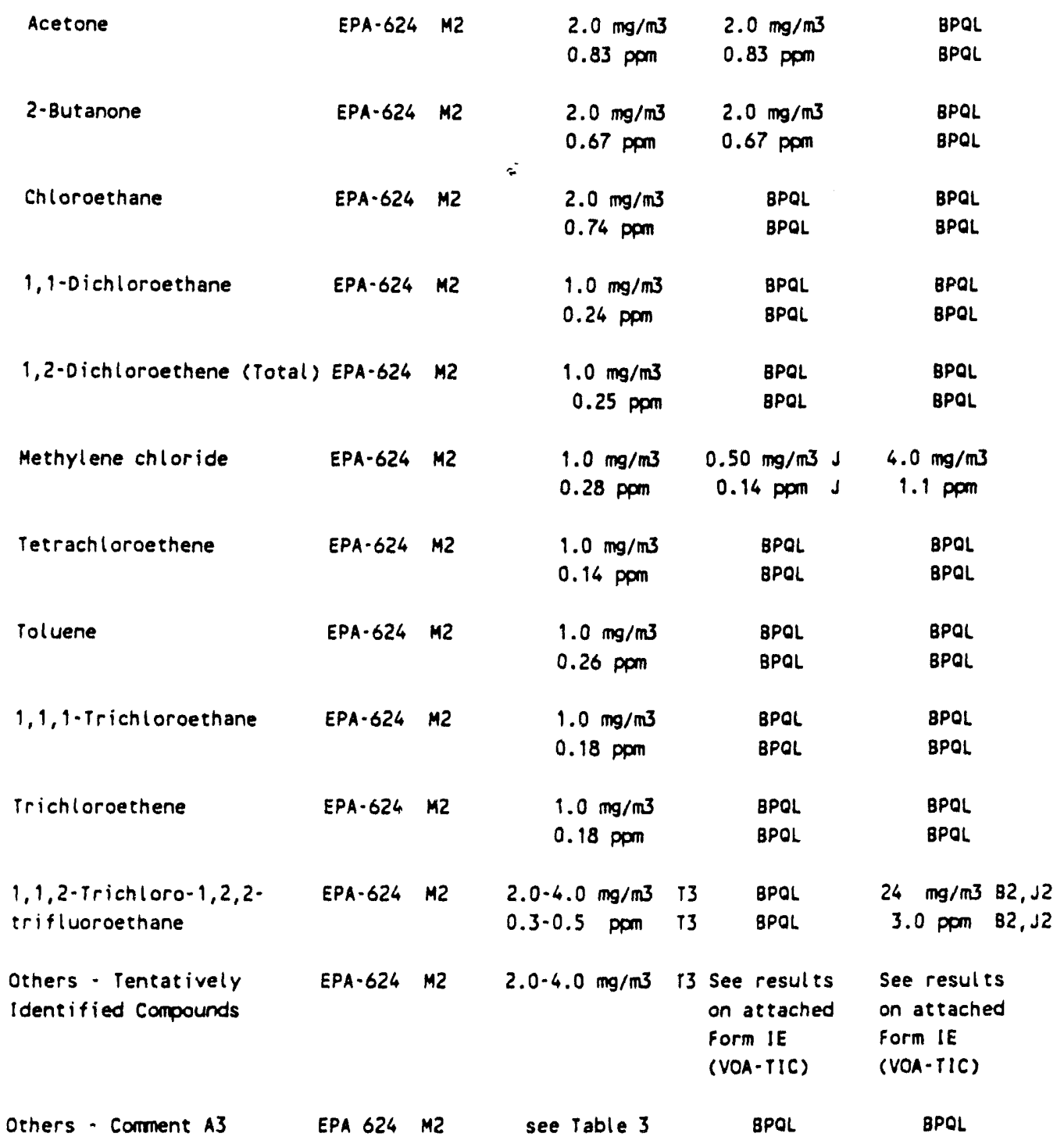


EG\&G Idaho, Enviromental Chemistry, ROA

Legend:

BPQL = Below practical quantitation level. See POL definition for further explanation of practical quantitation level.

NA $=\quad$ Not analyzed.

POL = Practical quantitation level. This is the lowest concentration reliably measurable (i.e., $33 \%$ maximum uncertainty in precision and accuracy at the one standard deviation confidence level) for normal samples during routine laboratory operations.

Comments:

Target volatile organic compounds and their practical quantitation levels (pols) are provided in table 3 . The instrument was calibrated to quantitatively measure these compounds, therefore compounds reported as below PQL (BPQL) are unlikely to have been present above their PaLs.

The presence of this compound in the associated laboratory method blank indicates the analyte found in the sample may be partially or completely a result of laboratory contamination.

This compound is a suspected laboratory contaminant. The presence of this compound in the sample may be partially or completely a result of laboratory contamination.

The sample analyzed was not full $25 \mathrm{ml}$; therefore, the normal laboratory pols could not be achieved. The normal POLs are those listed in the POL and units colum and in Table 3 . The elevated PoLs can be determined by multiplying the POL for a $25 \mathrm{ml}$ sample by 25 then dividing by the actual sample volume; The specific multiplication factors (MF) are as follows: for sample LF3-01MAR89 MF $=25 / 10=2.5$, for sample LF3-06MAR89 MF $=25 / 21.5=1.2$, and for sample LF3-X7MAR89 MF $=25 / 10=2.5$.

E2 The concentration reported for this sample exceeded the calibration range of the instrument and shoud be

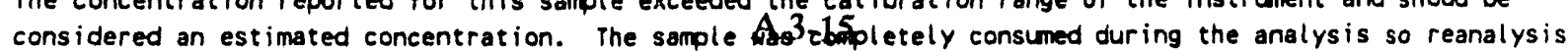
was not possible. The most likely result of this analytical difficulty is that the reported concentration underestimates the actual concentration.

Indicates estimated value. The concentration is below the PQL, but the compound was detected in the sample.

$\$ 2$ Indicates estimated value. These volatile organic compounds are not target compounds si.e., tentatively identified compounds), and consequently the instrument is not calibrated to quantitatively measure their abundances. An estimated calibration factor is assumed to quant ify these compounds, therefore the reported concentrations are estimated.

M2. A modification of EPA Method 624 was used since the method is designed for water samples. The Pols listed apply to a volume of $25 \mathrm{~mL}$. The results are reported in units of $\mathrm{mg} / \mathrm{m} 3$ and ppm. The ppm unit is on a volume compound per volume air basis and is determined by multiplying the weight of compound per volume of air concentration by $24.0 \mathrm{~L}$ (volume of 1.0 mole of gas at normal local temperature and pressure) and dividing by the compound's molecular weight ( $g / m o l e)$. Variations in temperature or pressure could introduce up to $25 \%$ uncertainty in the values reported in pom units. 
Page 8

N1 This sample was lost during analysis before it was possible to obtain any reportable analytical results. The initial analysis attempt consumed all of the sample so reanalysis was not possible.

i3 Tentatively identified compounds. These volatile organic compounds are not target compounds, and consequently the instrument is not calibrated to quantitatively measure their abundances. The estimated practical quantitation level for these compounds is 2.0 to $4.0 \mathrm{mg} / \mathrm{m} 3$ for gas samples. 
Estó idaho, ine.

Environsental Chesistry Unit

iaola 3. Hethod sensativities for volatile organic coepounds by purge and trap scins analysis

\begin{tabular}{|c|c|c|}
\hline Cospound & $\begin{array}{r}25.0 \\
\text { Practical } \\
\text { (ag/as) }\end{array}$ & $\begin{array}{c}\text { AL Alr Saoples } \\
\text { Guantitation Levelt } \\
\text { (DDs) }\end{array}$ \\
\hline Acetone & 2.0 & 0.83 \\
\hline Benizne & 1.0 & 0.31 \\
\hline Bronodichloronethane & 1.0 & 0.15 \\
\hline Brosotore & 1.0 & 0.094 \\
\hline Broconethane & 2.0 & 0.51 \\
\hline 2-Butanone & 2.0 & 0.67 \\
\hline Carbon disultide & 1.0 & 0.32 \\
\hline Carbon tetrachlorade & 1.0 & 0.16 \\
\hline Chlorobenzene & 1.0 & 0.21 \\
\hline Chloroethane & 2.0 & 0.74 \\
\hline Chlorotor & 1.0 & 0.20 \\
\hline Chlorocethane & 2.0 & 1.0 \\
\hline Oibrosochloronethane & 1.0 & 0.11 \\
\hline $1, d$-0ichloroethane & 1.0 & 0.24 \\
\hline 1,2-0ichloroethane & 1.0 & 0.24 \\
\hline 1, 1 -Dichloroethene & 1.0 & 0.24 \\
\hline 1,2-0ichloroethene (total) & 1.0 & 0.24 \\
\hline 1,2-Dichloropropane & 1.0 & 0.19 \\
\hline cis-1,3-oichloropropene & 1.0 & 0.22 \\
\hline trans-1,3-Dichloropropene & 1.0 & 0.22 \\
\hline Ethylbenzene & 1.0 & 0.22 \\
\hline 2-Hexanone & 2.0 & 0.48 \\
\hline Methylene chloride & 1.0 & 0.28 \\
\hline 1-riethyl-2-pentanone & 2.0 & 0.18 \\
\hline Styrene & 1.0 & 0.23 \\
\hline $1,1,2,2$-Tetrachloroethane & 1.0 & 0.14 \\
\hline Tetrachloroethene & 1.0 & 0.14 \\
\hline Toluene & 1.0 & 0.26 \\
\hline 1,1,1-Irichlor oethane & 1.0 & 0.18 \\
\hline $1,1,2$-irichloroethane & 1.0 & 0.18 \\
\hline Trichloroethene & 1.0 & 0.18 \\
\hline Viayl acatate & 2.0 & 0.26 \\
\hline Vingl chloride & 2.0 & $0 . \pi$ \\
\hline Iylenes (total) & 1.0 & 0.077 \\
\hline
\end{tabular}

1 Practical quantitation level is the lowest concentration reliably aeasurable li.e., 52 oaxiaus uncertainty in precision and accuracy at the one standard deviation confidence levell for noraal saptes during routine laboratory operations. 
Lad Nama:

\section{isb cocia:}

Case No.:

Matsix: (soil/water)

sample we/vol: $(g / D)$

-Level: (10w/med)

- Moistura: not dac. colum: (pack/cap)
Coneract:

Sas Yo.:
Method Blank

SDC NO.:
Lab Sample ID:

Lab Fle Is: $>M B C E E A$

Date Received:

Date Aralyzed:

Dliueion Factor:

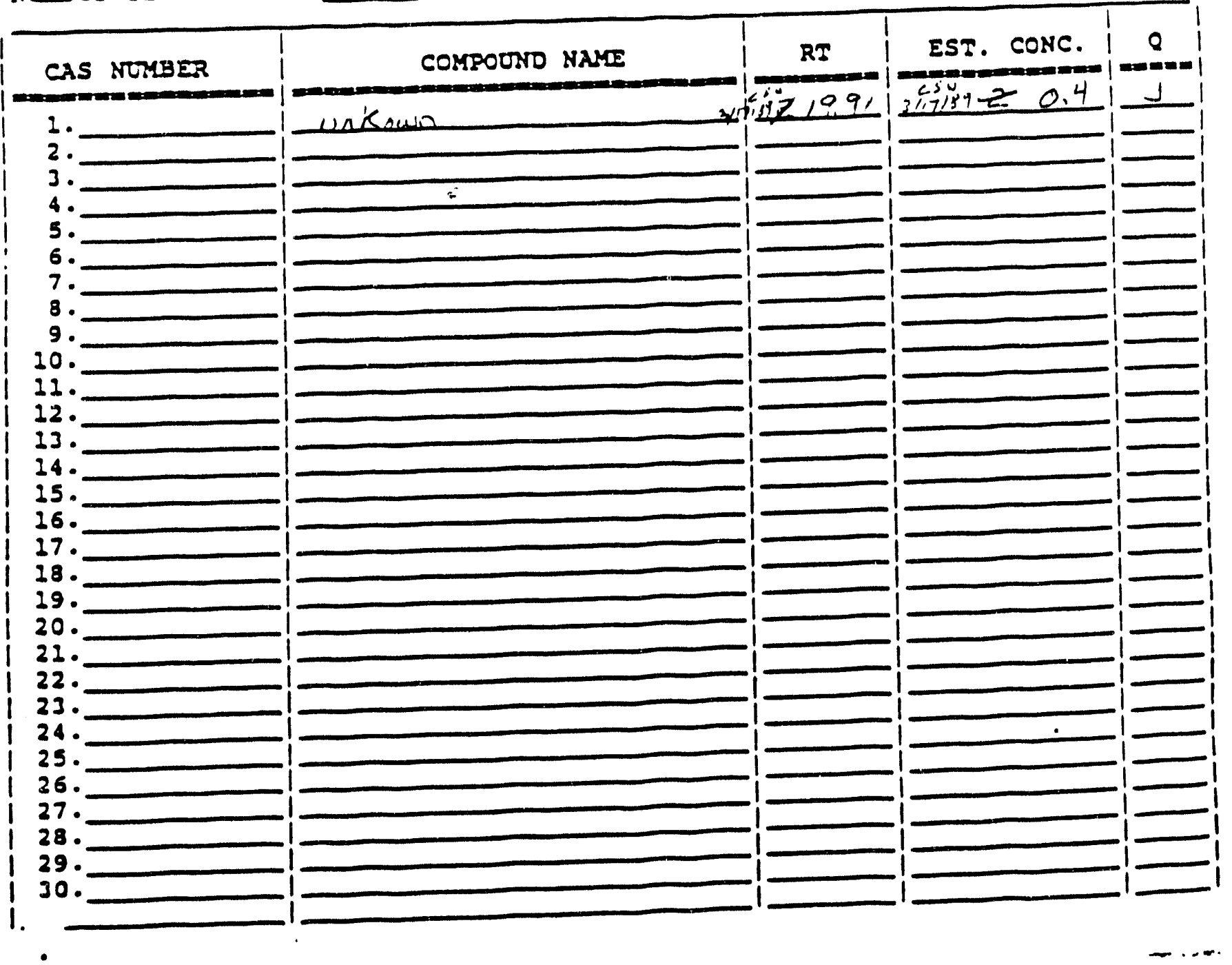


Lab Nase:

cocia:

Aatzix: (soil/watas)

Saspla we/vol:

$(g / \geq C)$

-Lavel: (low/med)

r Yoiseuza: not dac.

Colum: (pack/cap)

\section{Case No.:}

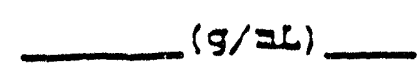

Conezace:

SAS No.:
I LFZ-ORMARS9:

soc No.:
Lab Sample ID:

Lab F1. I0: $\quad>M \phi_{C} E B$

Cate Recalved:

Date Aralyzed:

Dlluelon Faceor:
Number racs sound: 2
CONCENTRATION UNITS:
(ug/L of $4 \mathrm{~g} / \mathrm{kg}) \mathrm{mg} / \mathrm{m}^{3}$
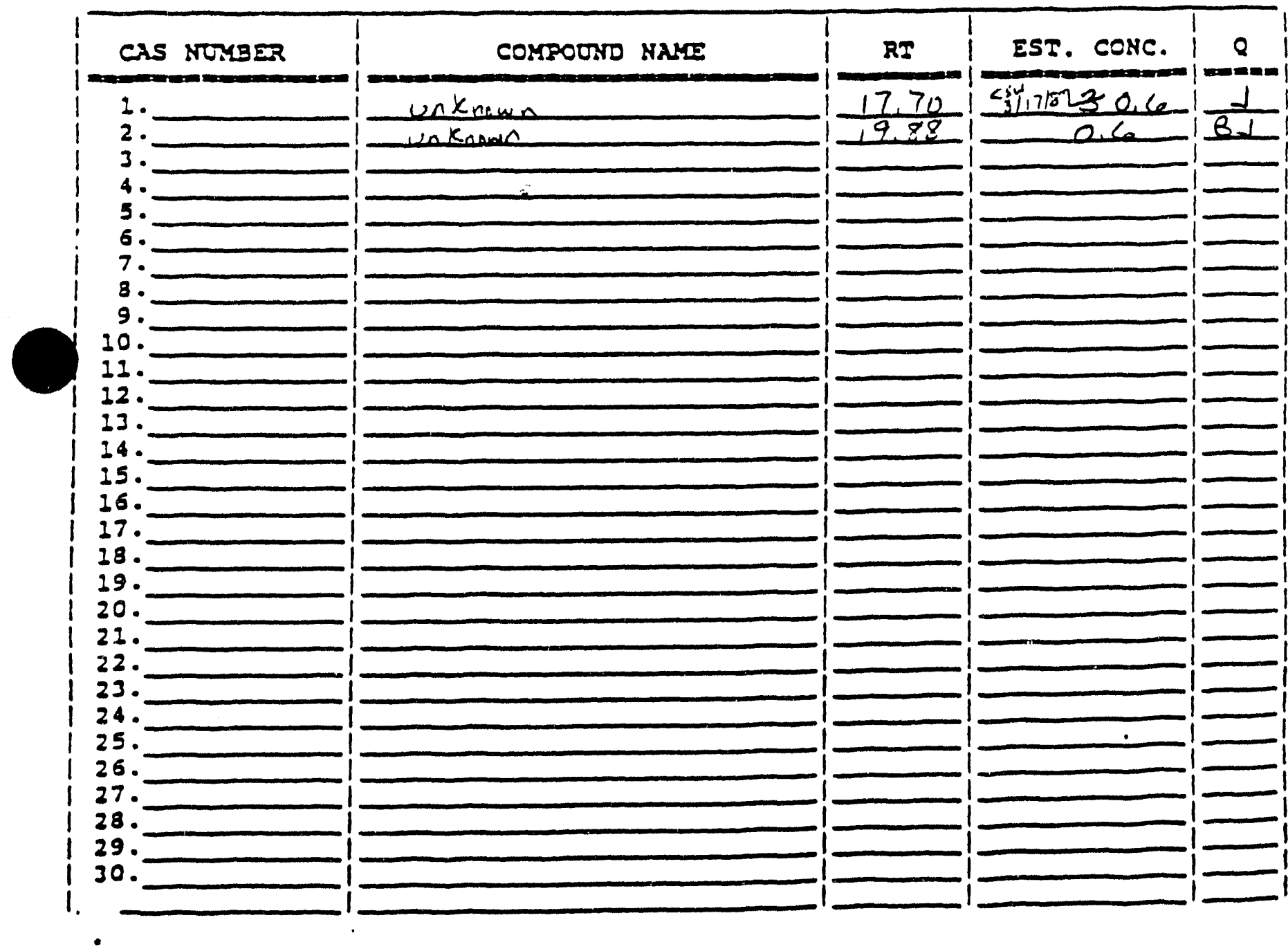

TORY I VOA-TIC

2/87 ReV. 
Lab Name:

in cocia:

Case No.:

Matzix: (soil/water)

sasple we/vol: $(g /=1)$

-Level: (low/zed)

t Yoistura: not dec.

colum: (pack/cap)
Conesacs:

SAS YO.:

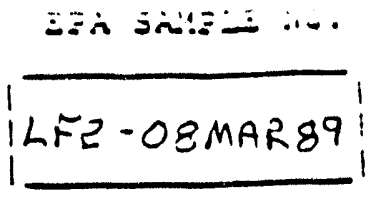

SDC No.:
La Saple ID:

Lab F11. 50: $>M \phi C E C$

Date Recaived:

Date drabyzed:

Dilution Factor:

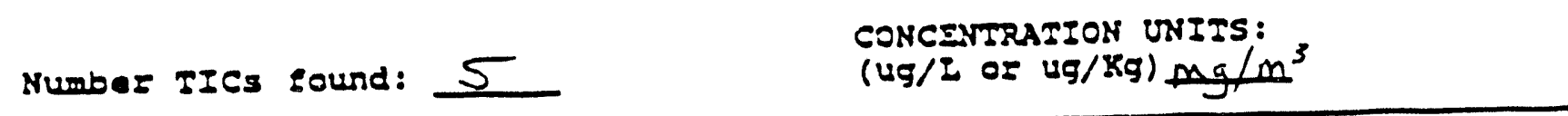

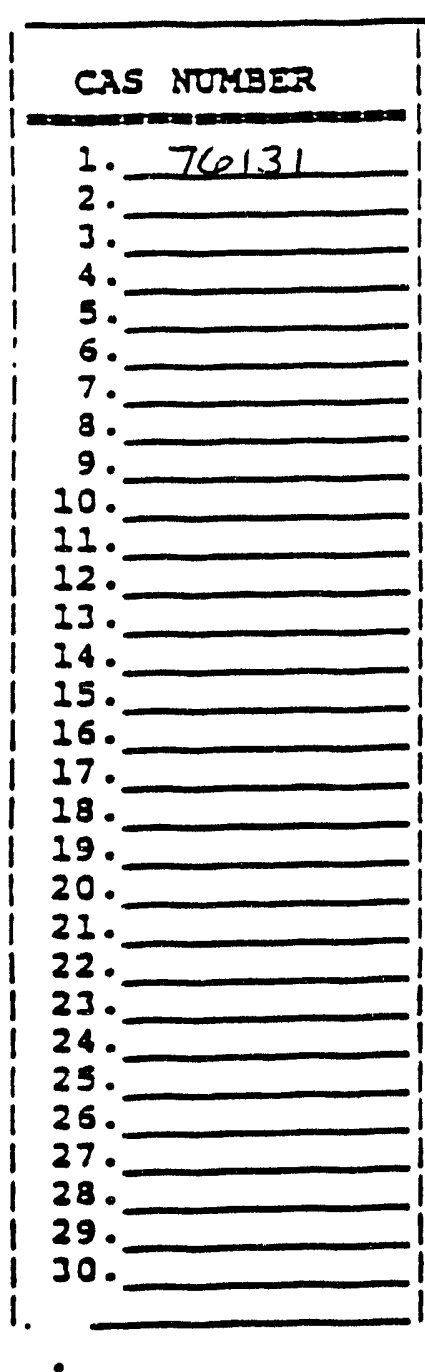
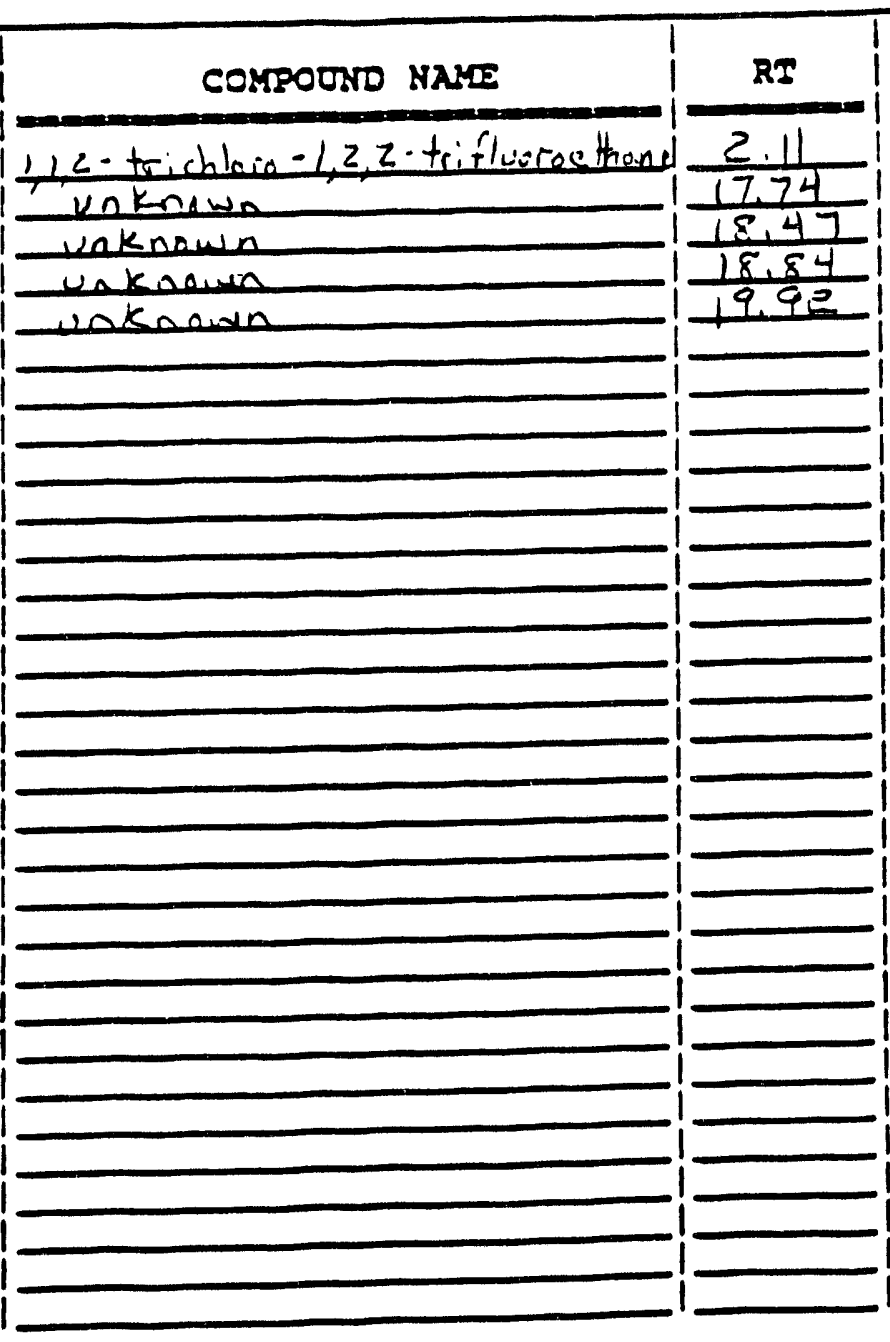

EST. CONC. 0

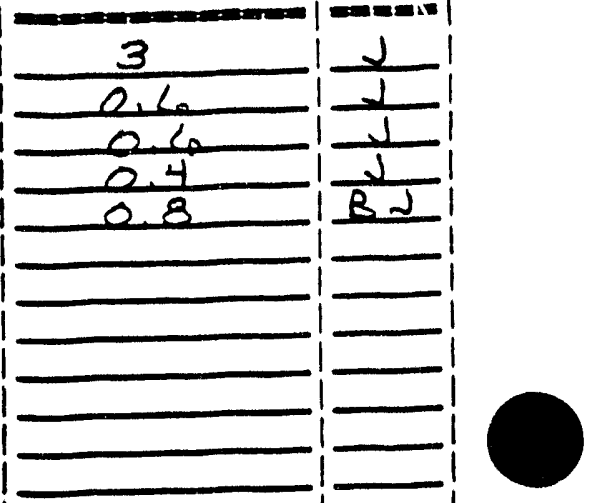


Lao Naze:

cocia:

Matzix: (soil/vater)

Sasple we/vol:

$(g / 2)$

contract:

sas tro.:

Lab sampl ID:

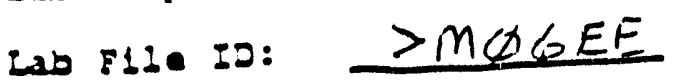

Date Recaived:

Date Aralyzad:

Dilution Factor:

poistuza: not dec.

Coluan: (pack/cap)

CONCENIRATION UNITS:

Numer IICs found: 1

(ug/L of ug/ Kg) $\mathrm{mg} / \mathrm{m}^{\mathrm{J}}$

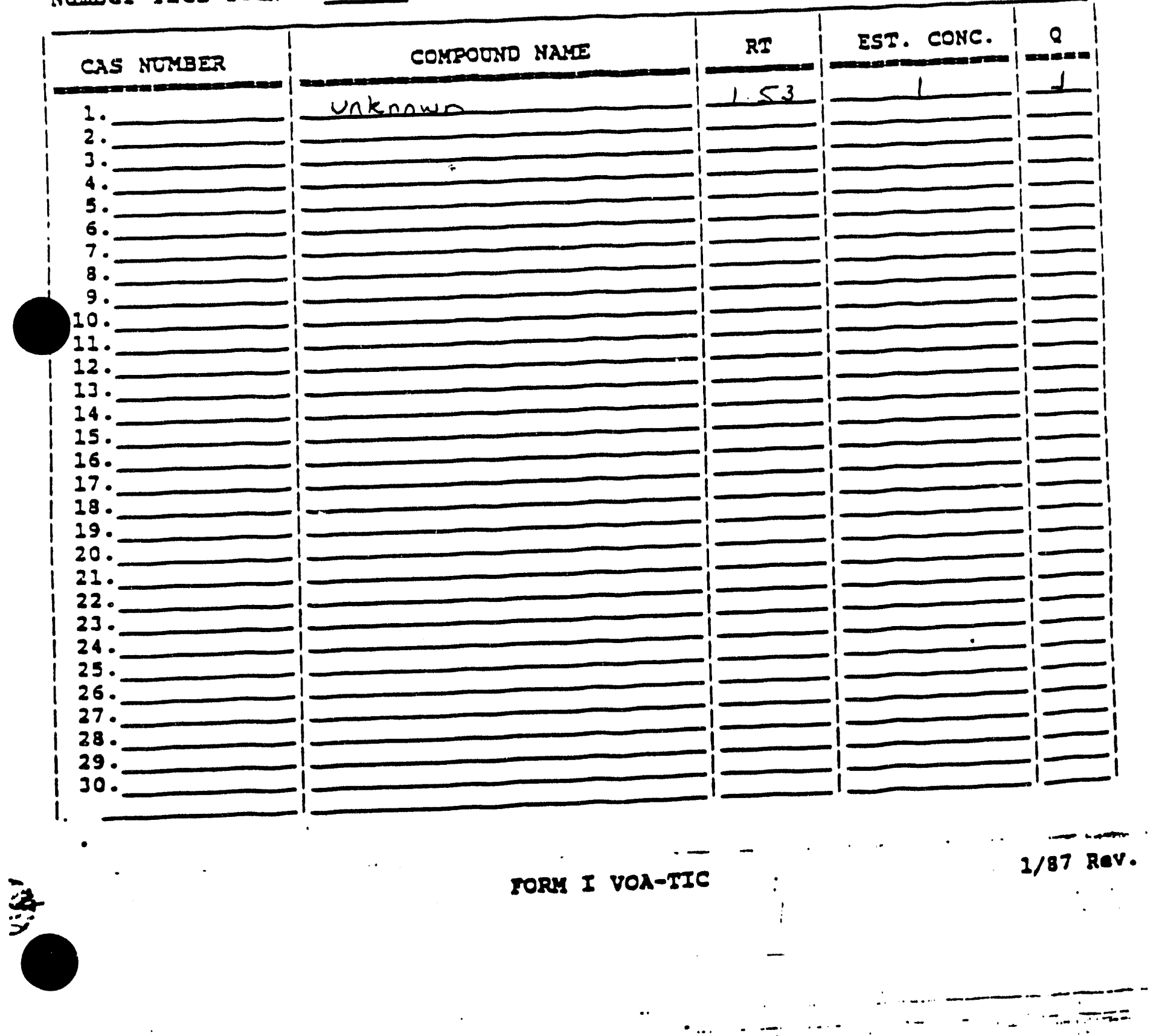


Lao Yame:

lab cocie:

Casa No.:

Matzix: (soil/yates)

sampla we/vol: $(g / 2)$

-Lavel: (low/zed)

Y Yoisedza: not dec.

colum: (pack/cap)

Number racs sound: 18 conezace:

sas No.:
SDC No.:

Lab Sasple ID:

ia 5:1. 20: $>M D 6 E F$

Dase Recaived:

Date Aralyzed:

ollueton Factor:

CONCENIRATION UNITS:

( $4 \mathrm{~g} / \mathrm{L}$ of $\mathrm{ug} / \mathrm{Kg}) \mathrm{mg} \mathrm{gm}^{3}$

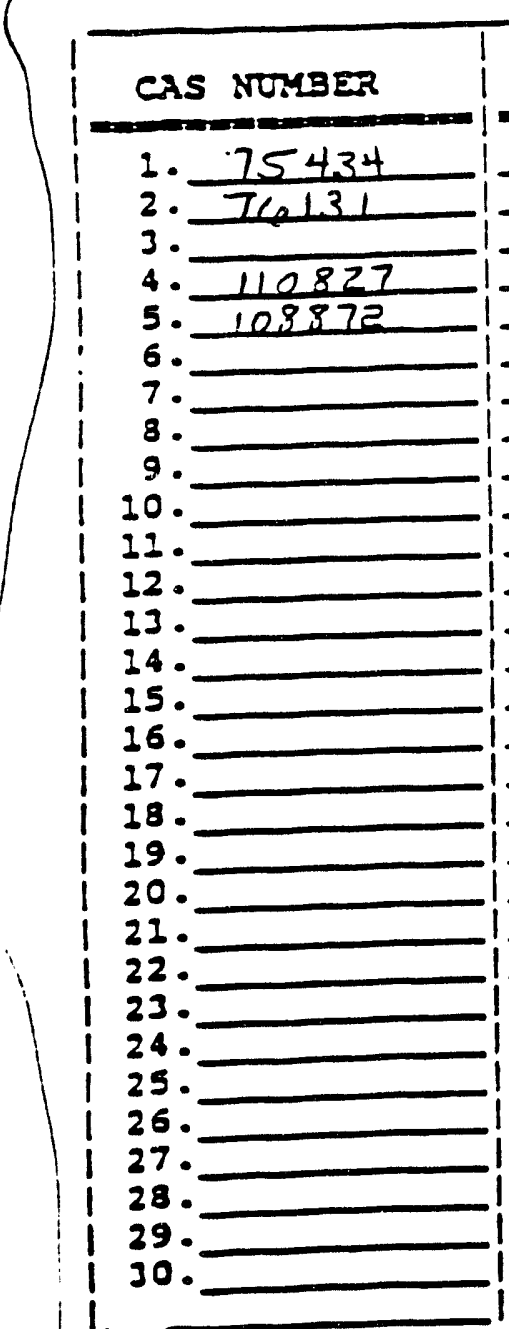

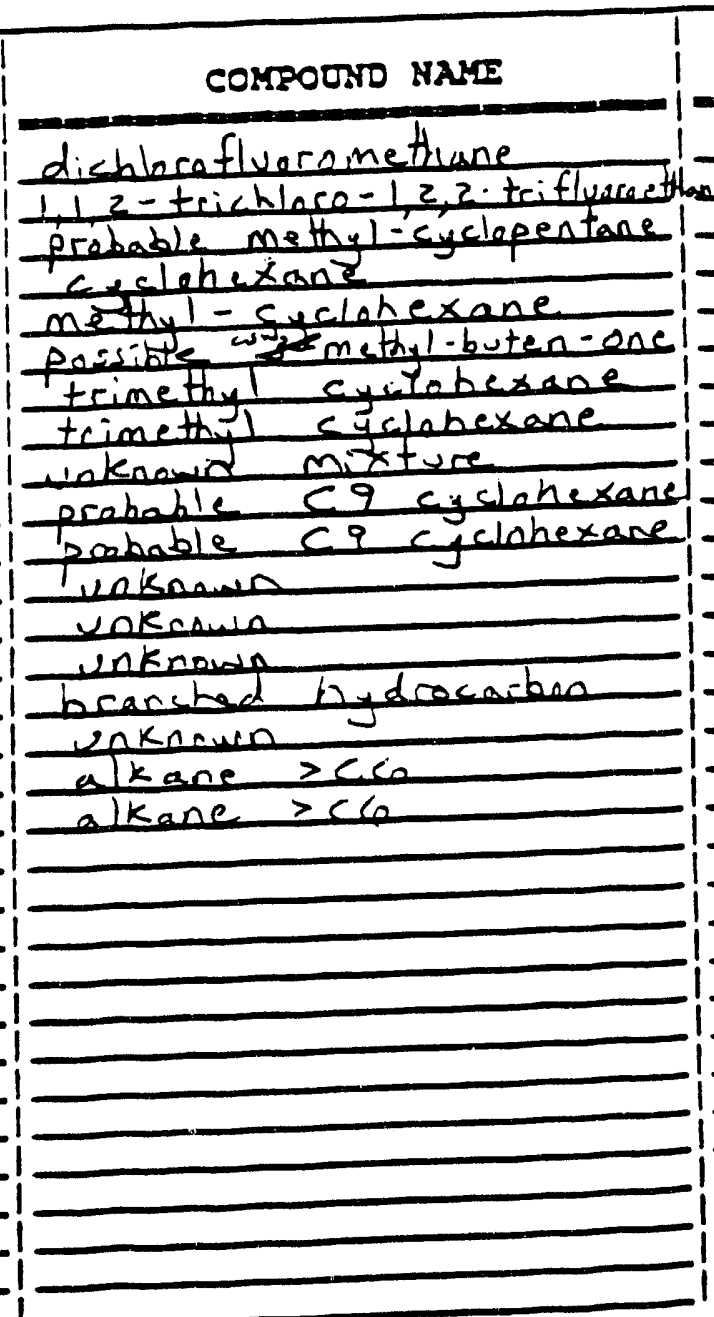

Sece addition to quent report roRs I vOA-IIC kianen Smith. this * note is not for your info.

$\eta T K$
EST. CONC. 0$\}$ EST. CONC. $\mid$\begin{tabular}{c|}
0 \\
-1
\end{tabular} 
Lab Name:

Cocie:

Matzix: (soiz/water)

Sample we/vol:

$(g / 25)$

-Lavel: (low/zed)

t yoiseuza: not dec.

Colus: (pack/cap)

Contzacs:

SAS No.:

SDG NO.:

Lab Sample ID:

Lab E:LE I0: >MIHEA

Dare Recoived:

Data dralyzed:

Dilueton Facsor:

CONCENTRATION UNITS:

(Ug/I of Ug/Kg)

Number IICs found: 1

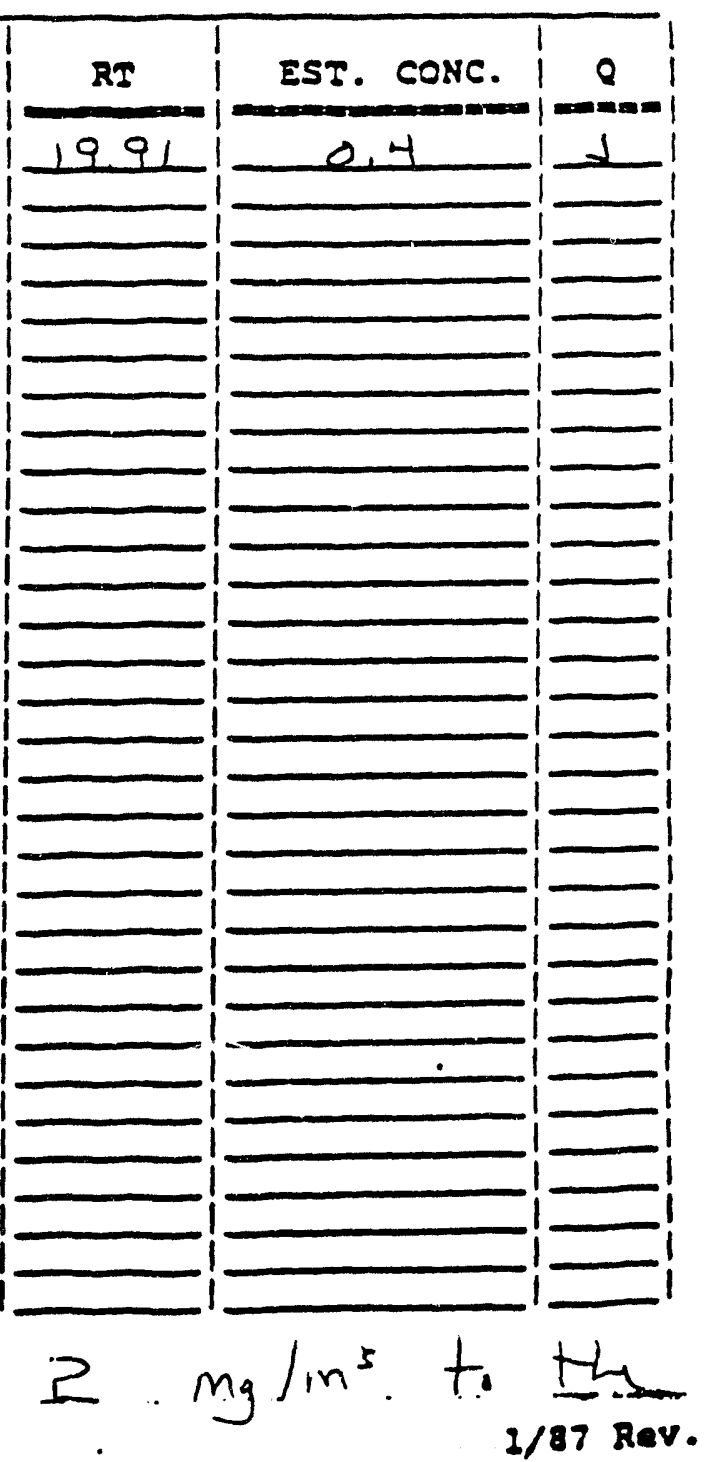
form $\perp A$

TORA I VOA-TIC

1/87 Rev. 
Lao Yame:

is cocia:

Casa No.:

Yatsix: (soil/yater)

sampla we/vol: $\quad 10 \quad(g / 2) M L$

- Lavel: (Low/ged)

roistyza: not dec.

colum: (pack/cap)

Number rICs found: 4 conesace:

SAS NO.:

Lab Sample ID:

Lab 5110 Is: $>M 14 E B$

Daen Recaired:

Daze Aralyzed:

Dilueton Factor:

CONCENTRATION UNITS

(ug/L of ug/ $\mathrm{kg}$ ) $\mathrm{mg}_{\mathrm{g}} \mathrm{m}^{3}$

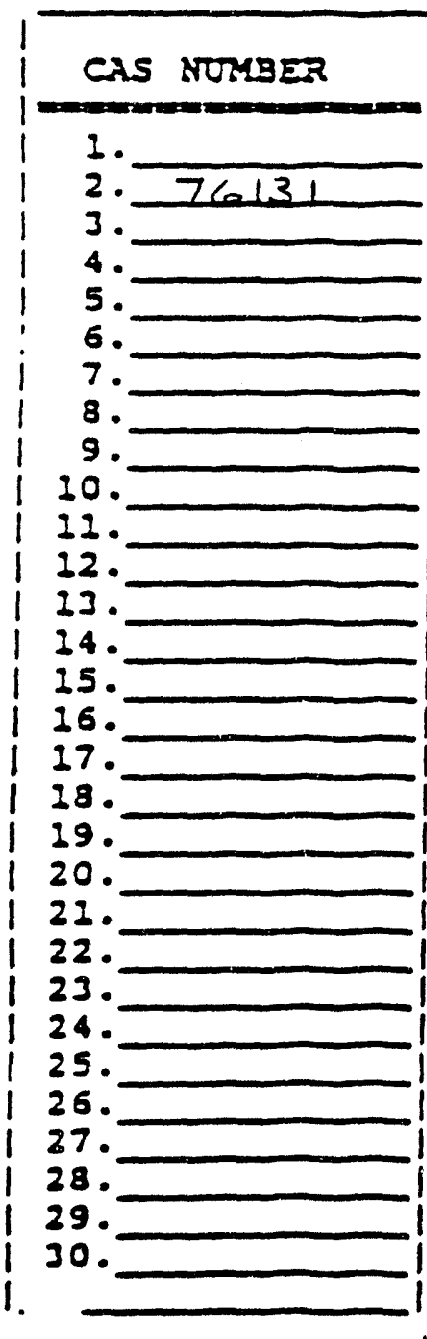

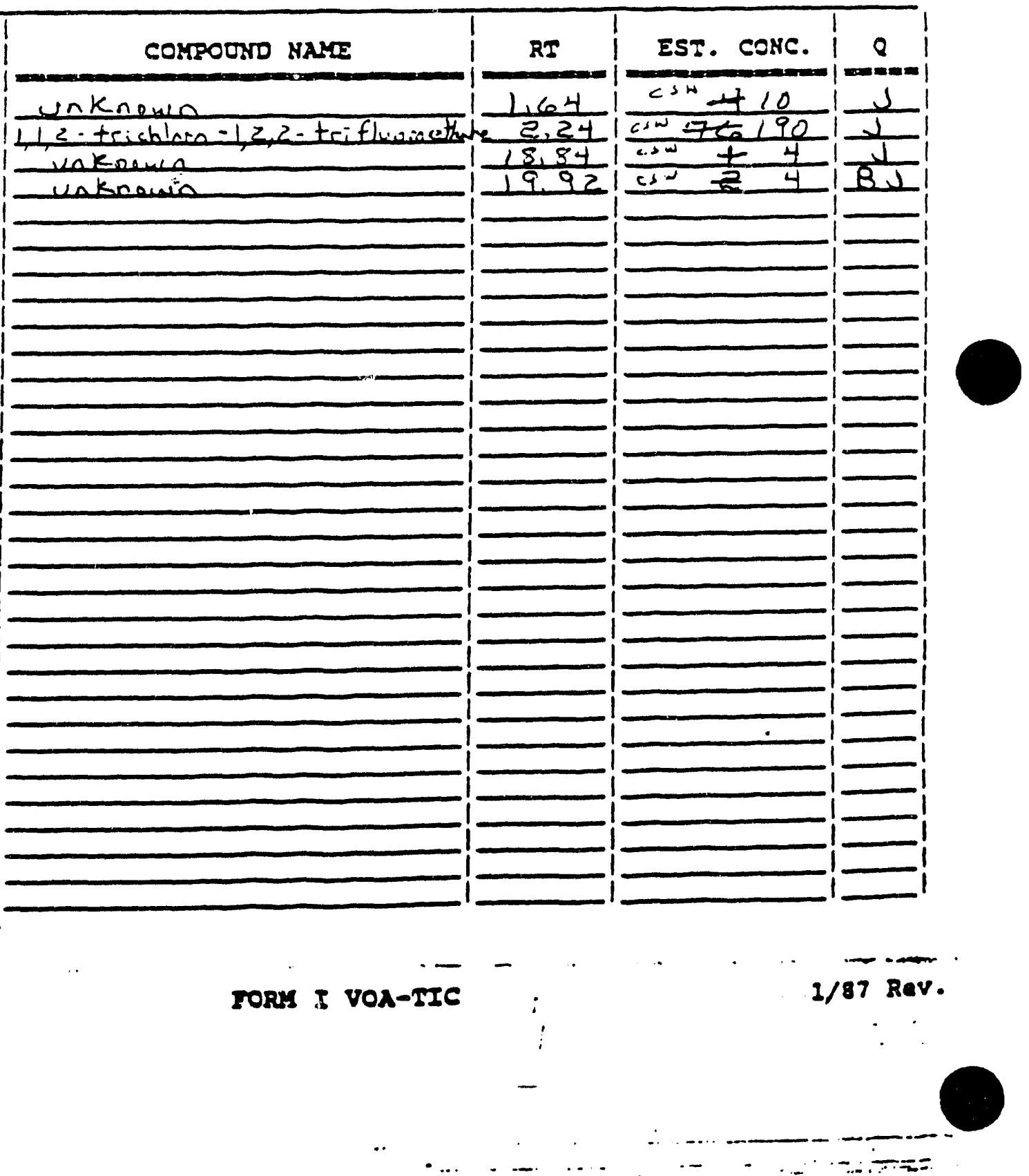


L30 Naze:

Cocie:

Yatzix: (soil/watar)

Case No.:

Saspla we/vol: $(g / \geq \mathcal{L})$

-Lavel: (30w/zed)

\& roistuga: not dec.

colus: (pack/cap) conesace:

SAS No.:

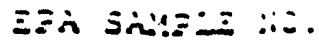

LF3-02 MAR 89:

SDC No.:

Lab Sample ID:

Lab F11. ID: $>M / 4 E C$

Dare Recelved:

Date Nralyzed:

Diluelon Factor:

CONCENTRATION UNITS:

Number IICs found: 2

(ug/I of ug/ $\mathrm{kg}$ ) $\mathrm{mg} / \mathrm{m}^{3}$
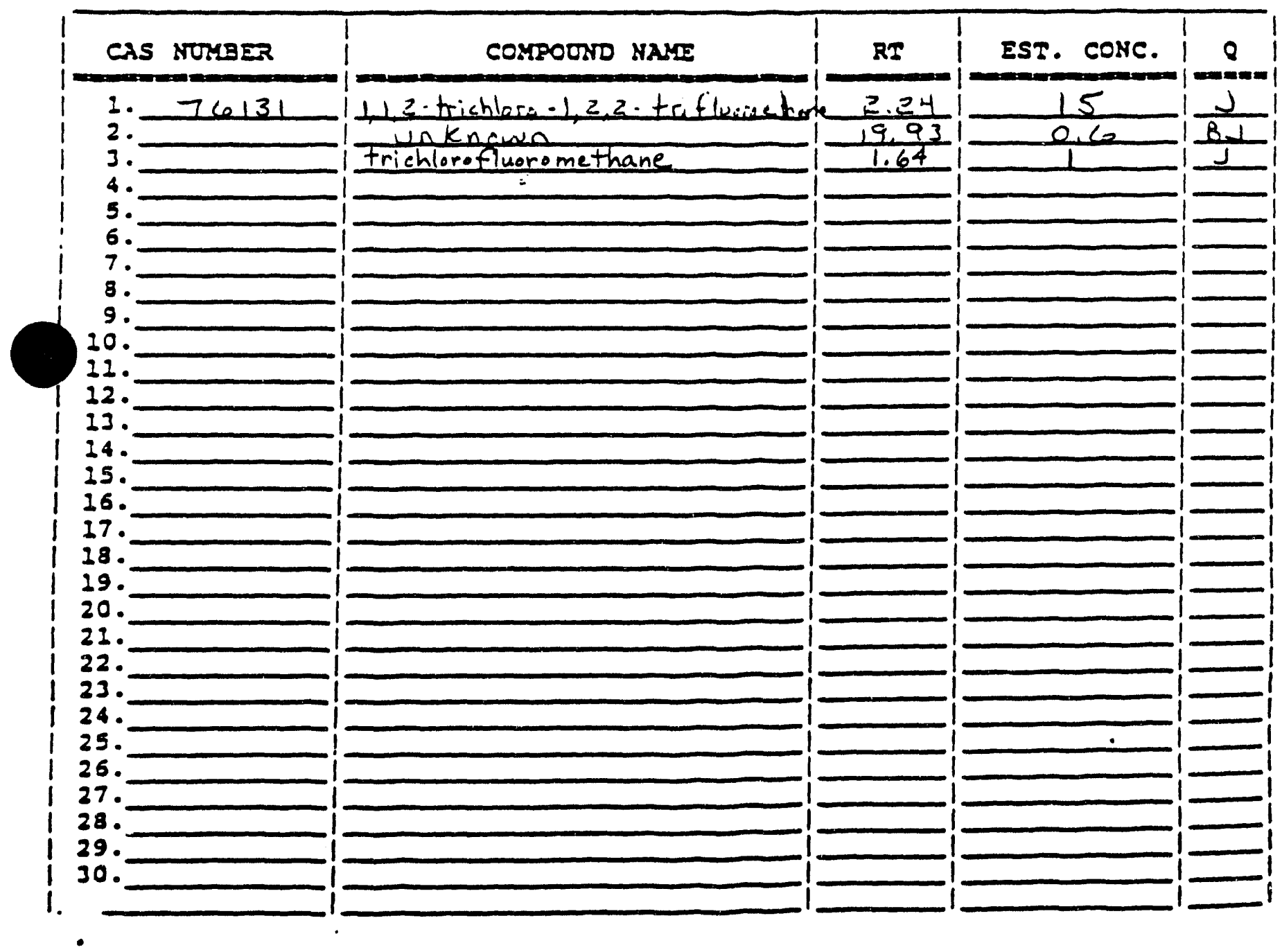
LaD Naze:

wb Cocia:

Case No.:

Matsix: (soil/water)

Sample we/vol: $(g / 25)$

-Laval: (low/zed)

I noiseura: not dec.

Coluan: (pack/cap)

Numer IICs found:
Contract:

sas No.: SDC NO.:

Lab SAmple ID:

La: E. I0: 2MIHEP

Date Recelved:

Date Analyzad:

DiIuefon Factor:

CONCENTRATION UNITS:

(ug/L or $u g / \mathrm{kg}) \mathrm{mg} \mathrm{m}^{3}$

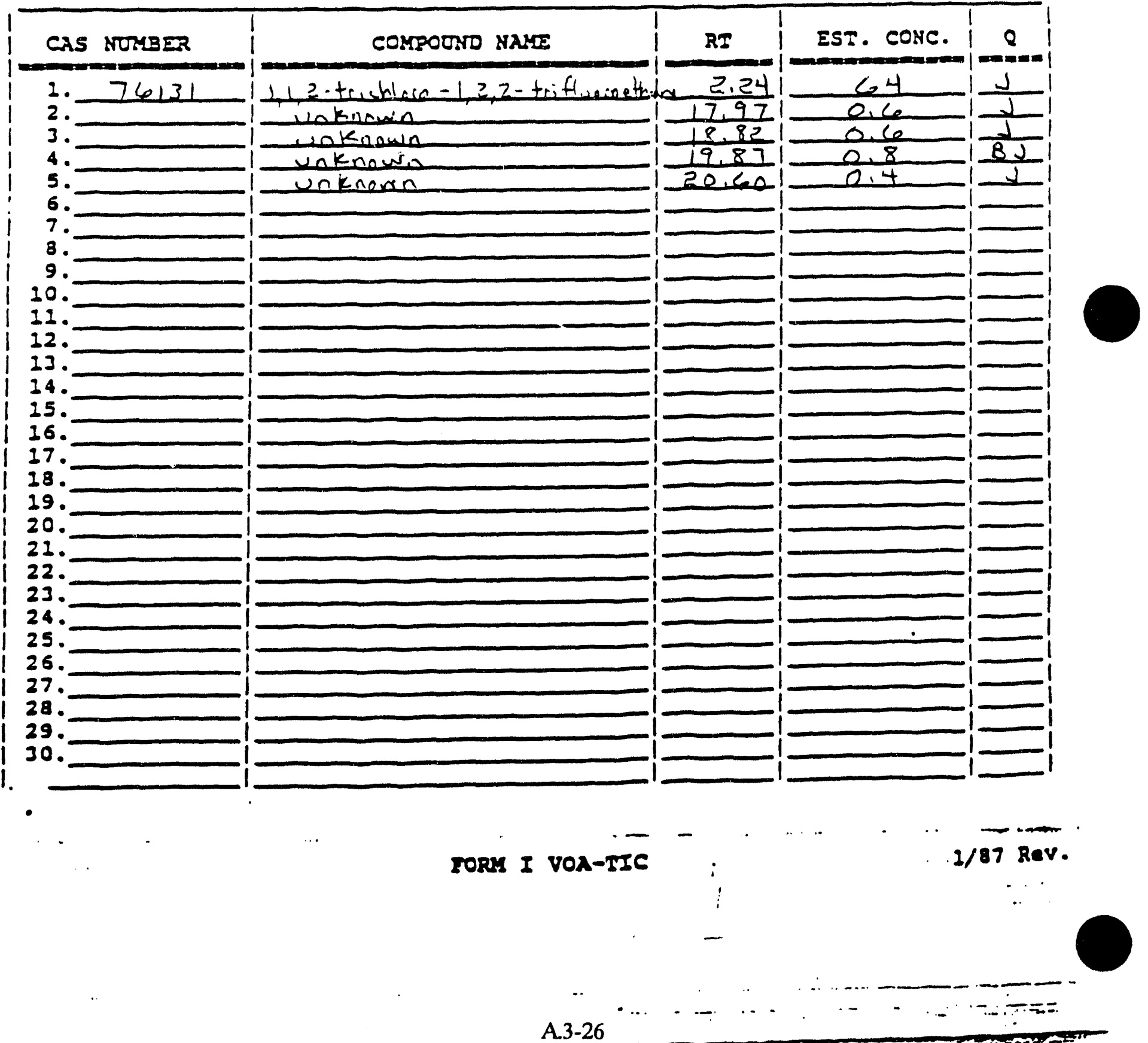


Lab Na=e:

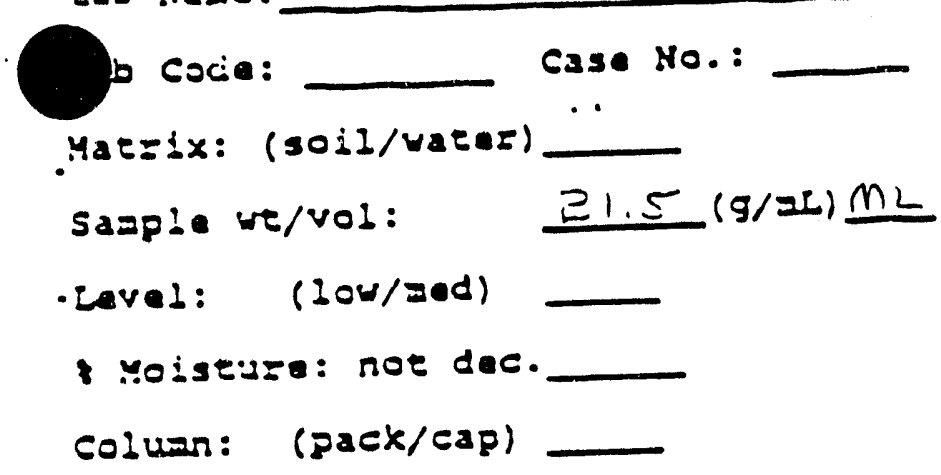

\section{contracs:}

SAS No.:
SDC NO.:

Lab Sample ID:

La El10 I0: >MHEE

cate Recaived:

Date dnalyzad:

Dilueion Factor:
CONCENTRATION UNITS:

(ug/L of ug/ $\mathrm{Kg}$ ) $\mathrm{mg} / \mathrm{m}^{3}$

Number rICs found: 1
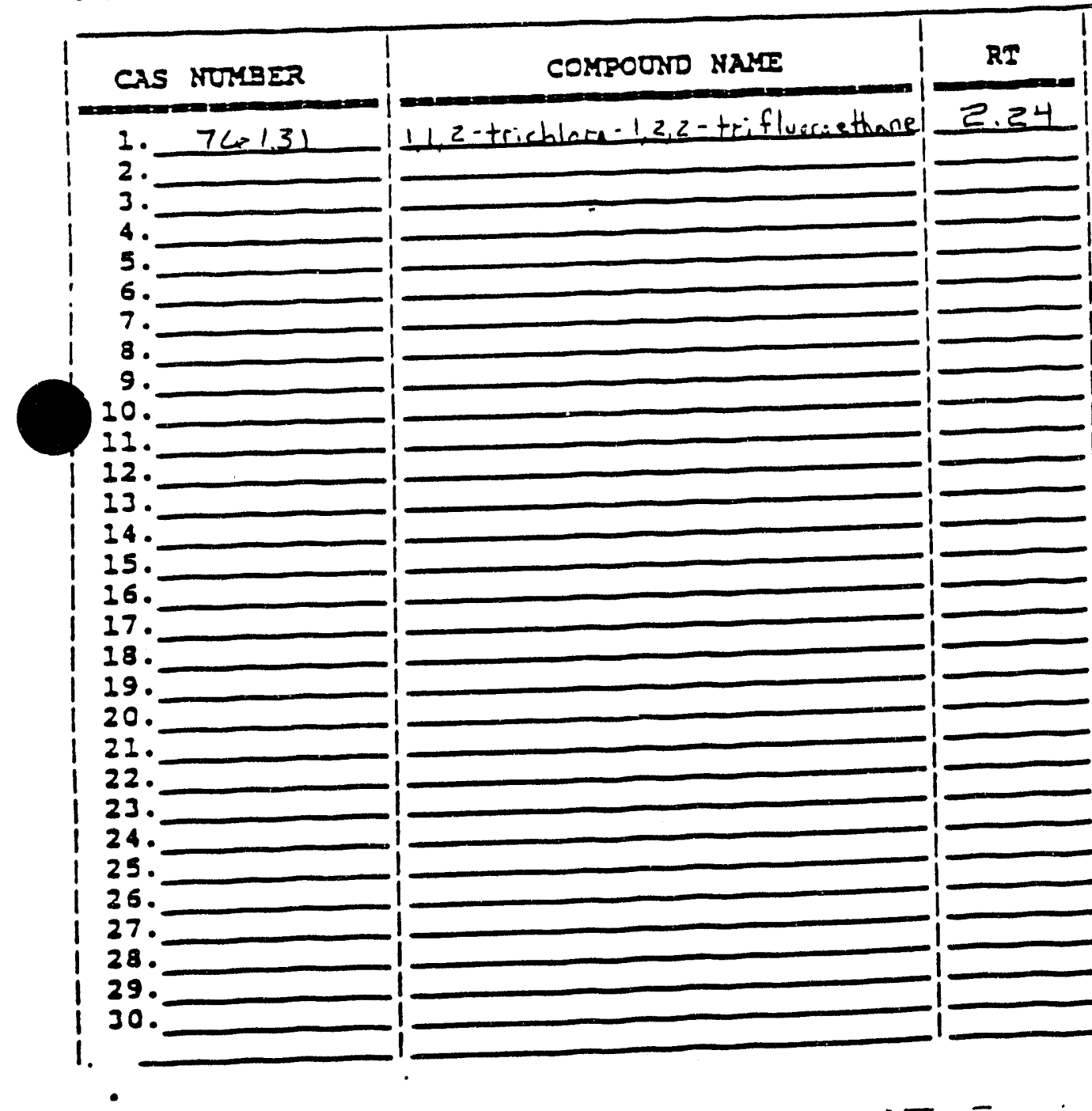

EST. CONC. $Q$ ESI. CONC. $1=0=0$ 72

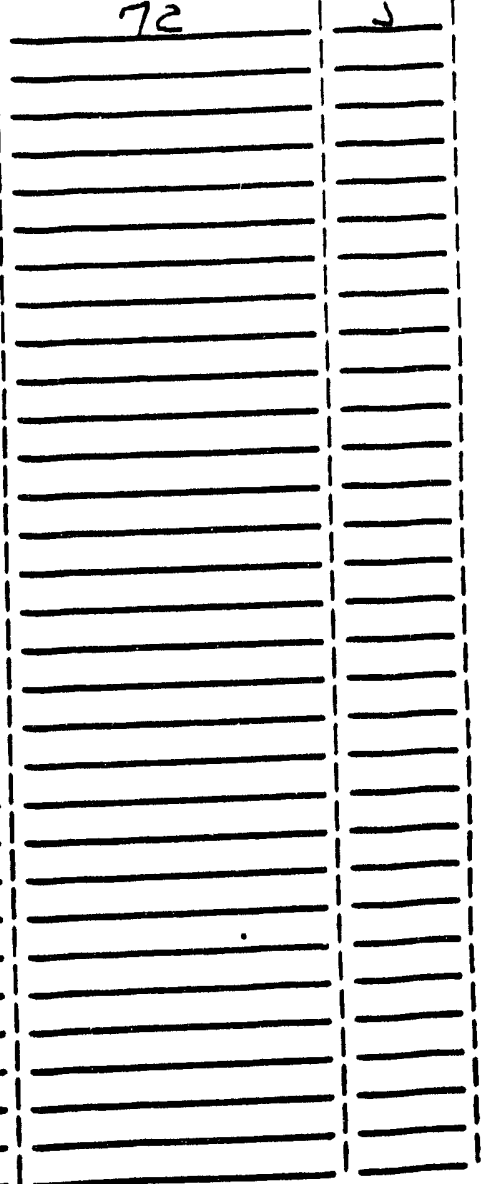

TORA I VOA-TIC

1/87 Rev. 
LaD Mama:

ib cocia:

Case No.:

Matsix: (soil/yatar) sample we/vol: $\quad 25(g / 2 L) M L$

- cove1: (10w/med)

soisture: not dec.

column: (pack/cap)

contrac:
SAS Na.: SDe No.:

Lab sampla ID:

Iab F:1. IJ: $>M 14 E F$

Date Recalvad:

Date Analyzed:

Dilueton Faceor:

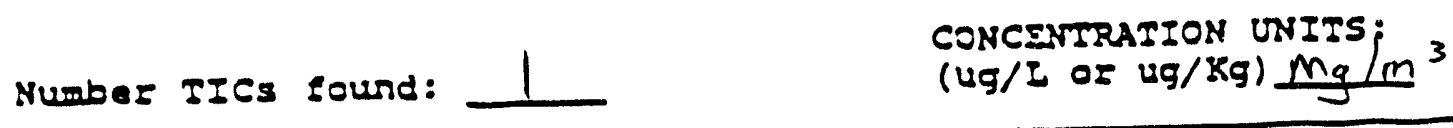

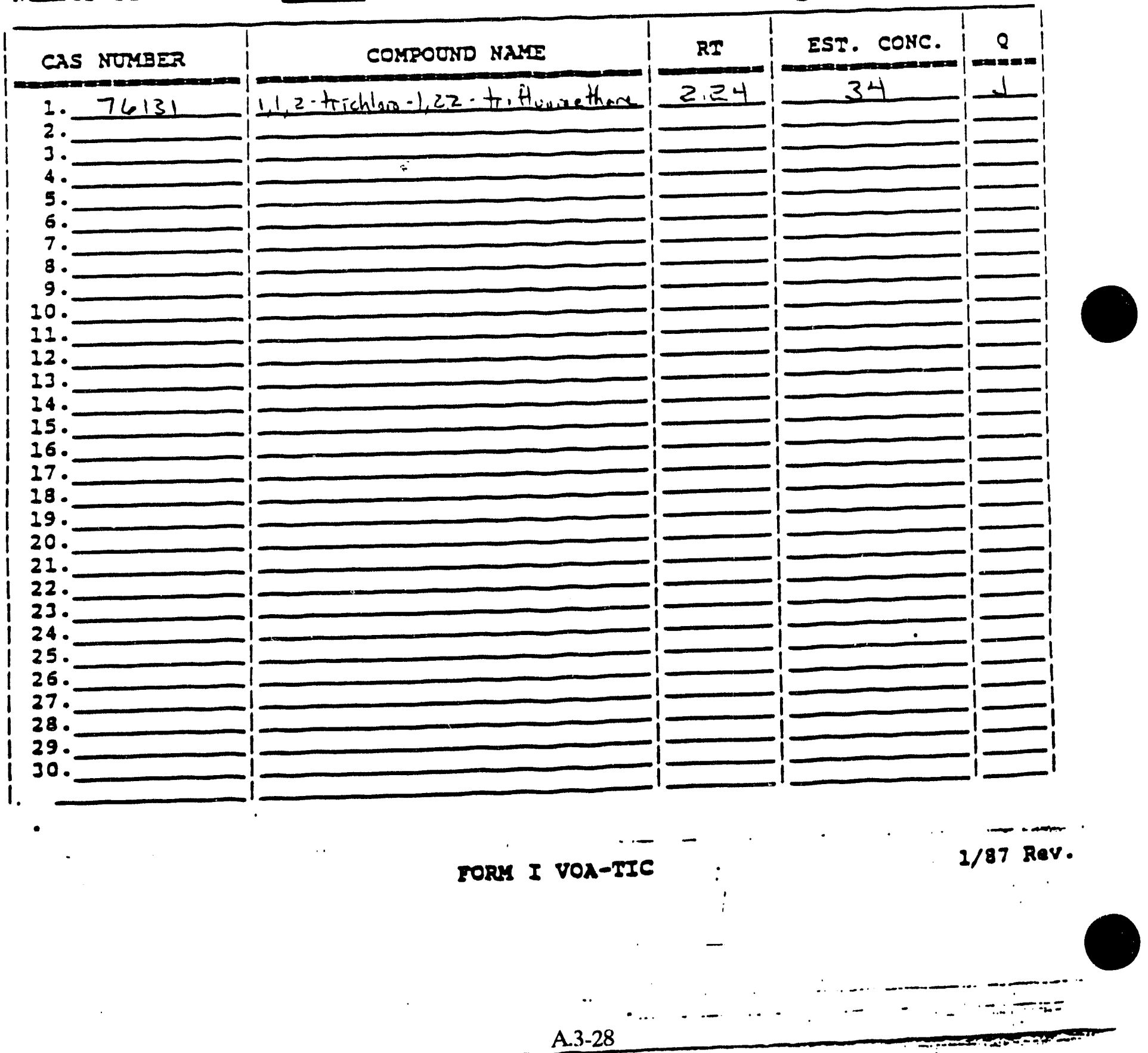


Ldo Name:

bocia:

Maesix: (soil/water)

Sazple we/vol:

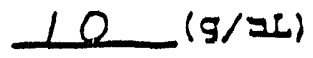

-Lavel: (Iow/aed)

r Moisejza: not dac.

colum: (pack/cap)

conesace:

SAS No.: SDC NO.:

Lab sample ID:

Fi: 50: $2 M 14 E C_{x}$

Date Recal'sed:

Dace Aralyzed:

DIIuelon Eactor:

CONCENTRATION UNITS:

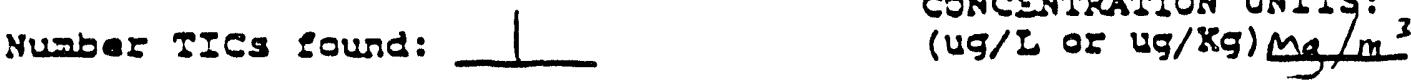

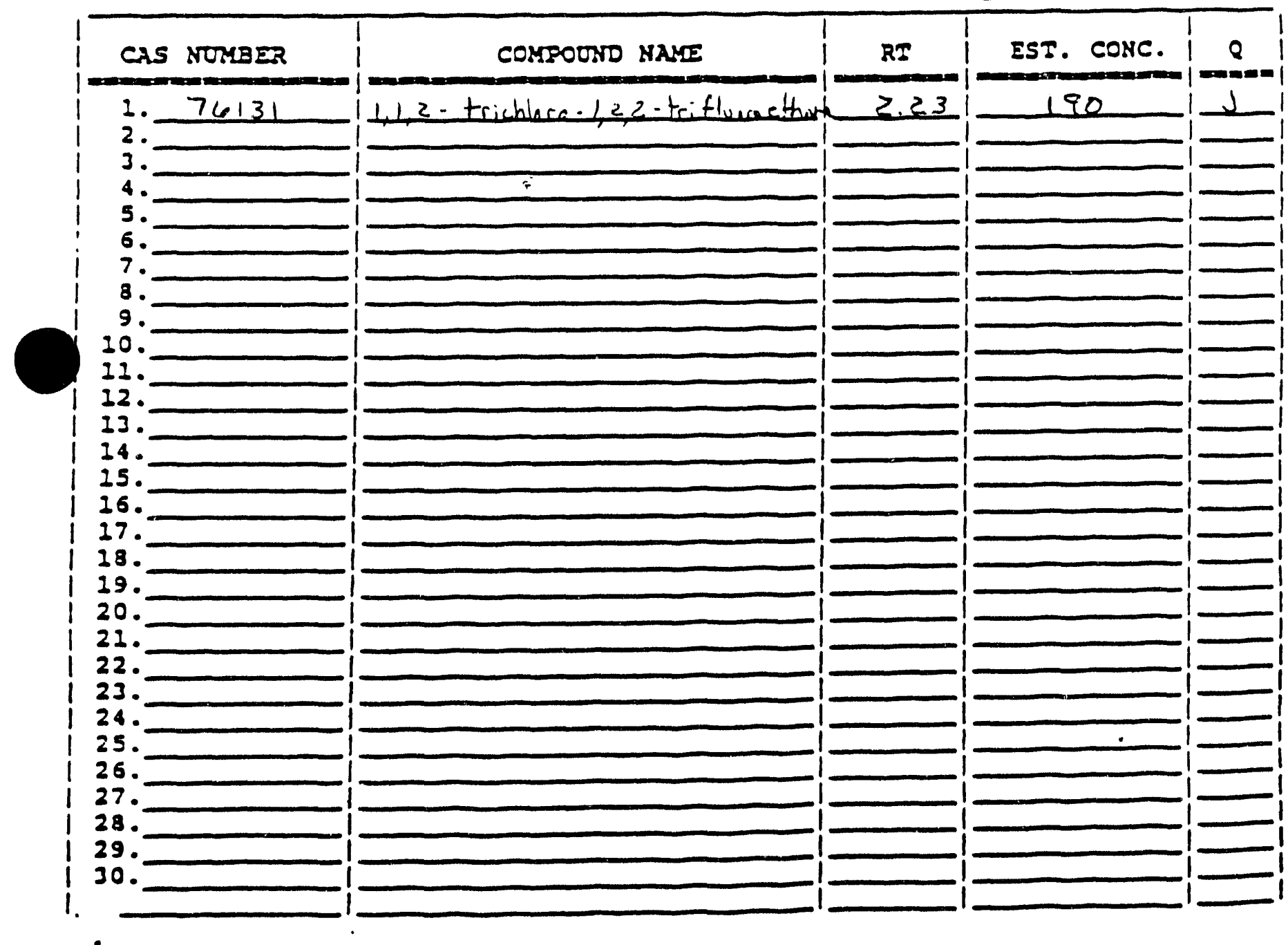


Lao Name:

Lacia:

Case No.:

Matzix: (soil/water)

Sazple we/vol: $25(9 /=2) M L$

-Lavel: (low/zed)

1 Yoistuza: not dec.

colun: (pack/cap)
Contsacs:

sas so.:

SDC No.:

Lap Sazple ID:

Fi2: I0: PMIHEH

Date Received:

Data rralyzed:

Dilueton Factor:

CONCENTRATION UNITS:

Number IICs found:

(ug/L of ug/ Kg) $\mathrm{mg} / \mathrm{m}^{3}$

$\left\{\begin{array}{l}\text { CAS NOMBER } \\ 1.76131 \\ 3 . \overline{ } \\ 6.1 \\ 7 . \\ 9 . \\ 10 . \\ 11 . \\ 12 . \\ 13 . \\ 14 . \\ 15 . \\ 16 . \\ 17 . \\ 18 . \\ 19 . \\ 20 . \\ 21 . \\ 22 . \\ 23 . \\ 24 . \\ 25 .\end{array}\right.$
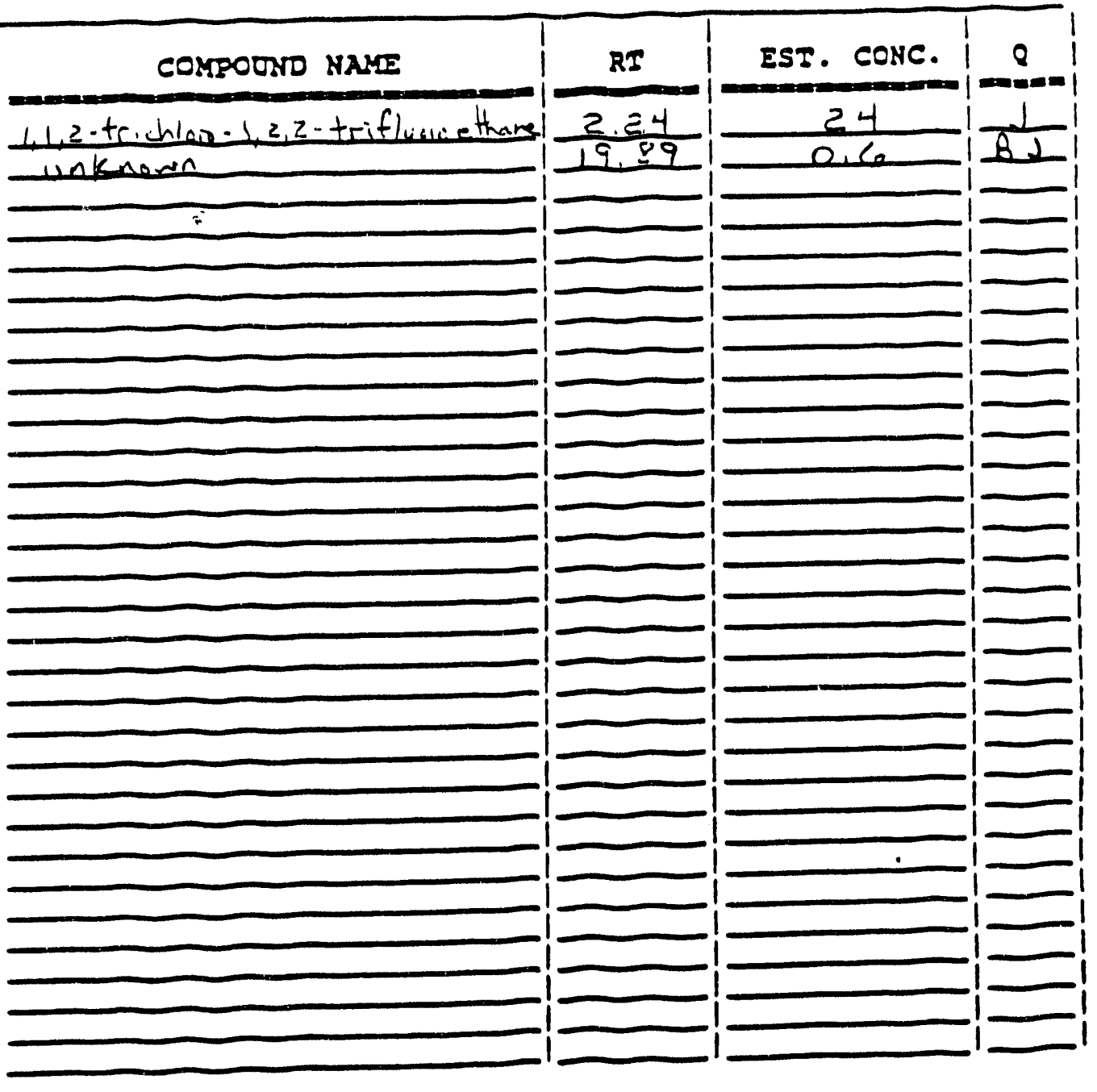
EG\&G Idaho, Environmental Chemistry, ROA

EG\&G Idaho, Inc.

Environmental Chemistry

P.O. Box 1625, MS 4123

Idaho Falls, ID 83415

This report of analysis (ROA) presents the analytical results for the analyses you requested. Sample identification, sampling information, and laboratory analysis information are listed in a colum for each sample. Analytical results are listed by analytical parameter below the sample identification information. Acronyms and abbreviations used are defined at the end of the report. A letter and/or letter number flag immediately to the right of a parameter, method, PQL and units, result, or any other entry indicates an amplifying corment also provided at the end of the report.

REPORT OF ANALYSIS

Prepared for:

Shannon Ansley

EG\&G Idaho, ine.

P.O. Box 1625, MS 2107

Idaho Falls, 1083415
Report Identification:

ROA Number:

Date:

110

Charge No.:

Page:

1 
Page 2

Parameter

..........

Volatile Organic Compounds

\begin{tabular}{|c|c|c|c|c|}
\hline Acetone & $E P A-624$ & M2 & & $\begin{array}{l}2.0 \mathrm{mg} / \mathrm{m} \\
0.83 \mathrm{ppm}\end{array}$ \\
\hline 2-Butanone & EPA-624 & M2 & $\therefore$ & $\begin{array}{l}2.0 \mathrm{mg} / \mathrm{m} \\
0.67 \mathrm{ppm}\end{array}$ \\
\hline Chloroethane & EPA-624 & M2 & & $\begin{array}{l}2.0 \mathrm{mg} / \mathrm{m} \\
0.74 \mathrm{ppm}\end{array}$ \\
\hline 1,1-Dichloroethane & EPA- 624 & M2 & & $\begin{array}{l}1.0 \mathrm{mg} / \mathrm{m} \\
0.24 \mathrm{ppm}\end{array}$ \\
\hline 1,1 -Dichloroethene & $E P A-624$ & M2 & & $\begin{array}{l}1.0 \mathrm{mg} / \mathrm{m} \\
0.24 \mathrm{ppm}\end{array}$ \\
\hline 1,2 Dichloroethene (total) & $E P A-624$ & $M 2$ & & $\begin{array}{l}1.0 \mathrm{mg} / \mathrm{m} \\
0.25 \mathrm{ppm}\end{array}$ \\
\hline 1,2-0ichloropropane & EPA- 624 & M2 & & $\begin{array}{l}1.0 \mathrm{mg} / \mathrm{m} \\
0.21 \mathrm{ppm}\end{array}$ \\
\hline Ethylbenzene & EPA-624 & M2 & & $\begin{array}{l}1.0 \mathrm{mg} / \mathrm{m} \\
0.23 \mathrm{ppm}\end{array}$ \\
\hline Methylene chloride & $E P A-624$ & M2 & & $\begin{array}{l}1.0 \mathrm{mg} / \mathrm{m} \\
0.28 \mathrm{ppm}\end{array}$ \\
\hline 4-Methyl-2-pentanone & EPA-624 & $M 2$ & & $\begin{array}{l}2.0 \mathrm{mg} / \mathrm{m} \\
0.48 \mathrm{ppm}\end{array}$ \\
\hline Tetrach loroethene & EPA- 624 & $M 2$ & & $\begin{array}{l}1.0 \mathrm{mg} / \mathrm{m} \\
0.14 \mathrm{ppm}\end{array}$ \\
\hline Toluene & EPA-624 & $M 2$ & & $\begin{array}{l}1.0 \mathrm{mg} / \mathrm{m} \\
0.26 \mathrm{ppm}\end{array}$ \\
\hline $1,1,1$-Trichloroethane & EPA- 624 & $M 2$ & & $\begin{array}{l}1.0 \mathrm{mg} / \mathrm{m} \\
0.18 \mathrm{ppm}\end{array}$ \\
\hline
\end{tabular}

Method ...........
Sampling Site: Sample Type:

Customer Sample ID: Sampling Date: Sampling Time: Date Received: Lab Sample ID: Date Extracted: Date Analyzed: Comments:

PQL and Units ..................

Comment A3

$.0 \mathrm{mg} / \mathrm{m} 3$

$0.6 \mathrm{mg} / \mathrm{m} 3 \mathrm{~J}$

0.25 ppm J

BPQL

$3.0 \mathrm{mg} / \mathrm{m}^{3} \mathrm{~B}$

$21.0 \mathrm{mg} / \mathrm{m}^{3} \mathrm{~B}, 05, \mathrm{~J}$ $1.24 \mathrm{ppm}$ B $8.67 \mathrm{ppm} \quad B, 05, \mathrm{~J}$

$1.3 \mathrm{mg} / \mathrm{m}^{3} \mathrm{~J}$ $0.43 \mathrm{ppm} J$

$1.3 \mathrm{mg} / \mathrm{m}^{3} \mathrm{~B}, \mathrm{~J} \quad 1.2 \mathrm{mg} / \mathrm{m}^{3} \mathrm{~B}, \mathrm{~J} 34.0 \mathrm{mg} / \mathrm{m3} \quad B, 05, \mathrm{~J}$ 0.43 ppm B,J 0.40 ppm B,J 11.3 ppm B,05,J

BPQL

BPQL

BPOL

$<50.0 \mathrm{mg} / \mathrm{m}^{3}$ D., $\mathrm{d}$

< $18.6 \mathrm{ppm} 05, \mathrm{~J}$

BPQL

BPQL

$0.3 \mathrm{mg} / \mathrm{m3} J$ 0.07 ppm J

$1.0 \mathrm{mg} / \mathrm{m}^{3} \mathrm{~J}<25.0 \mathrm{mg} / \mathrm{m}^{3} 05, \mathrm{~J}$

BPOL

BPQL

BPQL

BPQL 0.24 ppm $J<6.07$ ppm $05, J$

BPQL $<25.0 \mathrm{mg} / \mathrm{m}^{3} 05, \mathrm{~J}$ < 6.07 ppm 05,J

BPQL $<25.0 \mathrm{mg} / \mathrm{m}^{3} \mathrm{DS}, \mathrm{J}$

< 6.07 ppm D5,J

BPQL $\quad<25.0 \mathrm{mg} / \mathrm{m}^{3} 05, \mathrm{~J}$

< $5.31 \mathrm{ppm} 05, \mathrm{~J}$

BPQL

BPOL

BPQL $\quad<25.0 \mathrm{mg} / \mathrm{m} 305, \mathrm{~J}$ $<6.07$ ppm $05, \mathrm{~J}$

$0.8 \mathrm{mg} / \mathrm{m}^{3} \mathrm{~J} \quad 1.0 \mathrm{mg} / \mathrm{m}^{\circ} \mathrm{B}, \mathrm{J} \quad 1.2 \mathrm{mg} / \mathrm{m}^{3} \mathrm{~B} \quad 29.0 \mathrm{mg} / \mathrm{m}^{3} \mathrm{~B}, 05$ 0.2 ppm $\rfloor$ $0.28 \mathrm{ppm} \quad \mathrm{B}, \mathrm{J} \quad 0.34 \mathrm{ppm} B$ 8.19 ppm B,05

BPQL

BPQL

BPOL $<50.0 \mathrm{mg} / \mathrm{m}^{3} 05, \mathrm{~J}$ $<18.6$ ppm 05,J

BPQL

$0.3 \mathrm{mg} / \mathrm{m}^{3} \mathrm{~J}$

$1.1 \mathrm{mg} / \mathrm{m}^{3} \mathrm{~J}<25.0 \mathrm{mg} / \mathrm{m}^{3} \mathrm{DS}, \mathrm{J}$ $0.04 \mathrm{ppm} J$

0.16 ppm $J<6.07$ ppm DS, J

BPQL

BPQL

BPOL

< $25.0 \mathrm{mg} / \mathrm{m}^{3} 05, \mathrm{~J}$

$<6.07$ ppm 05.J

BPQL
$0.5 \mathrm{mg} / \mathrm{mB}^{\mathrm{J}}$

$1.0 \mathrm{mg} / \mathrm{ms}$ $0.09 \mathrm{ppm} J$
$0.18 \mathrm{ppm}$
$<25.0 \mathrm{mg} / \mathrm{m}^{3} \mathrm{DS}, \mathrm{J}$ < 6.07 ppm 05, J 
EG\&G Idaho, Environmental Chemistry, ROA 3

$$
\begin{array}{r}
\text { Sampling Site: } \\
\text { Sample Type: } \\
\text { Customer Sample ID: } \\
\text { Sampling Date: } \\
\text { Sampl ing Time: } \\
\text { Date Received: } \\
\text { Lab Sample ID: } \\
\text { Date Extracted: } \\
\text { Date Analyzed: } \\
\text { Comments: }
\end{array}
$$

Parameter

-..........

Method

PQL and Units (..................

Corment A3

Volatile Organic Compounds

Trichloroethene

EPA-624 M2

$1.0 \mathrm{mg} / \mathrm{mm}^{3}$

$0.18 \mathrm{ppm}$

Vinyl Chloride

EPA.624 M2 $\approx 2.0 \mathrm{mg} / \mathrm{m}^{3}$

Xylenes (total)

EPA-624 M2

$1.0 \mathrm{mg} / \mathrm{m}^{3}$

$0.22 \mathrm{ppm}$

Tentatively Identified Compounds

Comment T3
C6 cyclic hydrocarbon

EPA-624

Hexane

Trichlorofluorome thane

$E P A-624$

EPA-624

$4 \quad \mathrm{M2}$

$2 \begin{aligned} & 2.0-4.0 \mathrm{mg} / \mathrm{m3} \mathrm{T3} \\ & 0.6-1.1 \mathrm{ppm}\end{aligned}$

Trichlorotrifluoroethane

EPA-624

M2

$0-4.0 \mathrm{mg} / \mathrm{m} 3 \mathrm{T3}$

0.3-0.5 ppm

EPA-624 M2
Others - Comment A3

see Table 3
BPQL

BPQL

BPQL

$05 / 22 / 89$

$05 / 22 / 89$

Lab Blank

Result

-.........
Method Blk

$$
\text { Landfill }
$$
$25 \mathrm{~mL}$

Soil gas

LF201MAY89

05/22/89

$10: 49$

05/22/89

$9 M 1 A 0896$

$05 / 22 / 89$

Result
-
$05 / 22 / 89$
Landfill

$1 \mathrm{~mL}$

Soil gas

LF206MAY89

05/22/89

$11: 17$

$05 / 22 / 89$

$9 M 1 A 0898$

$\begin{array}{ll}05 / 22 / 89 & 05 / 22 / 89 \\ 05 / 22 / 89 & 05 / 22 / 89\end{array}$

$\begin{array}{ll}05 / 22 / 89 & 05 / 22 / 89 \\ 05 / 22 / 89 & 05 / 22 / 89\end{array}$

Result Result 
Page 4

Parameter

-.........

Volatile Organic Compounds

\begin{tabular}{|c|c|c|c|c|}
\hline Acetone & EPA. 624 & M2 & & $\begin{array}{l}2.0 \mathrm{mg} / \mathrm{m}^{3} \\
0.83 \mathrm{ppm}\end{array}$ \\
\hline 2-Butanone & EPA- 624 & M2 & $\therefore$ & $\begin{array}{l}2.0 \mathrm{mg} / \mathrm{m}^{3} \\
0.67 \mathrm{ppm}\end{array}$ \\
\hline Chloroethane & $E P A-624$ & M2 & & $\begin{array}{l}2.0 \mathrm{mg} / \mathrm{m} 3 \\
0.74 \mathrm{ppm}\end{array}$ \\
\hline 1,1-Dichloroethane & EPA- 624 & M2 & & $\begin{array}{l}1.0 \mathrm{mg} / \mathrm{m}^{3} \\
0.24 \mathrm{ppm}\end{array}$ \\
\hline 1,1-Dichloroethene & $E P A-624$ & M2 & & $\begin{array}{l}1.0 \mathrm{mg} / \mathrm{m}^{3} \\
0.25 \mathrm{ppm}\end{array}$ \\
\hline 1,2 Dichloroethene (total) & EPA-624 & M2 & & $\begin{array}{l}1.0 \mathrm{mg} / \mathrm{m} 3 \\
0.25 \mathrm{ppm}\end{array}$ \\
\hline 1,2-Dichloropropane & EPA- 624 & M2 & & $\begin{array}{l}1.0 \mathrm{mg} / \mathrm{m} 3 \\
0.21 \mathrm{ppm}\end{array}$ \\
\hline Ethylbenzene & $E P A-624$ & $M 2$ & & $\begin{array}{l}1.0 \mathrm{mg} / \mathrm{m} 3 \\
0.23 \mathrm{ppm}\end{array}$ \\
\hline Methylene chloride & $E P A-624$ & M2 & & $\begin{array}{l}1.0 \mathrm{mg} / \mathrm{m}^{3} \\
0.28 \mathrm{ppm}\end{array}$ \\
\hline 4-Methyl-2-pentanone & EPA-624 & M2 & & $\begin{array}{l}2.0 \mathrm{mg} / \mathrm{m} 3 \\
0.48 \mathrm{ppm}\end{array}$ \\
\hline Tetrachloroethene & EPA-624 & M2 & & $\begin{array}{l}1.0 \mathrm{mg} / \mathrm{m} 3 \\
0.14 \mathrm{ppm}\end{array}$ \\
\hline Toluene & EPA- 624 & M2 & & $\begin{array}{l}1.0 \mathrm{mg} / \mathrm{m} 3 \\
0.26 \mathrm{ppm}\end{array}$ \\
\hline $1,1,1$-Trichloroethane & EPA-624 & M2 & & $\begin{array}{l}1.0 \mathrm{mg} / \mathrm{m} 3 \\
0.18 \mathrm{ppm}\end{array}$ \\
\hline
\end{tabular}

Sampling Site: Sample Type:

Customer Sample 10: Sampling Date: Sampl ing Time: Date Received: Lab Sample 10: Date Extracted: Date Analyzed: Remarks:

Method M......... POL and Units
Comment A3

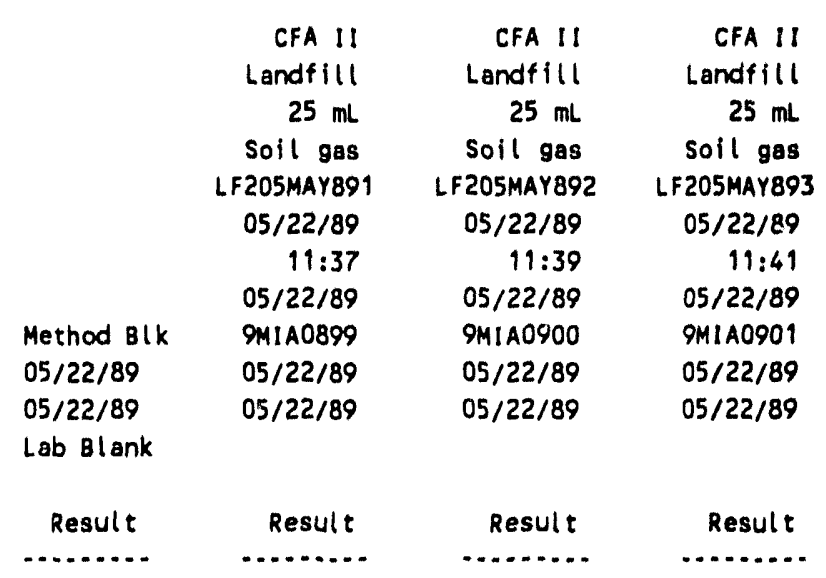

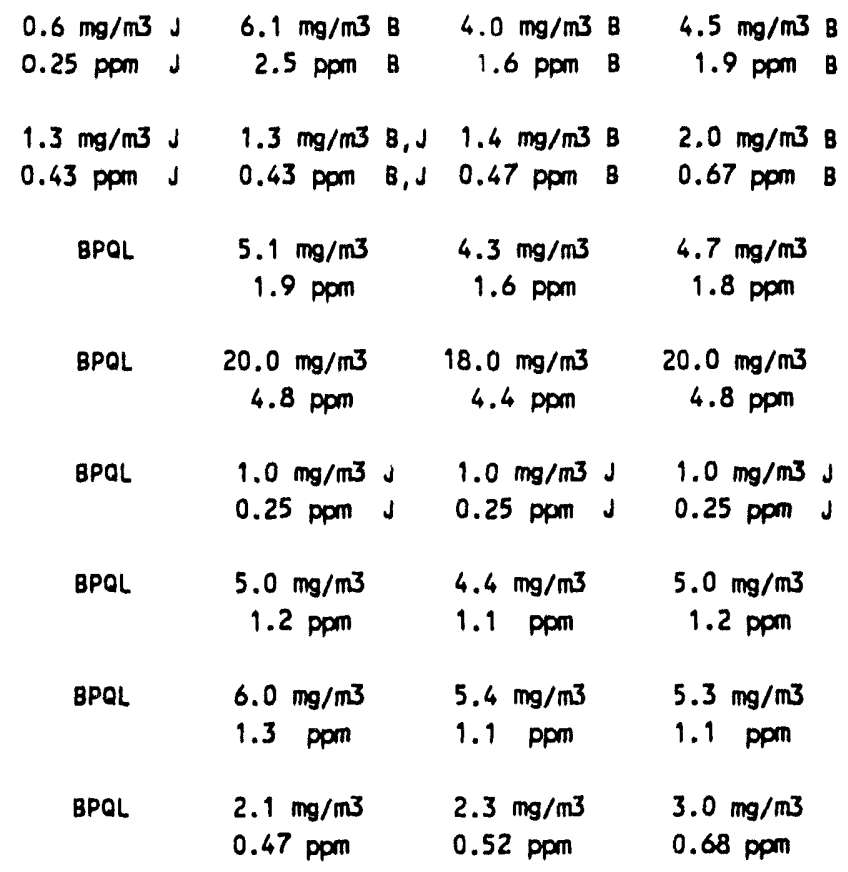

$0.8 \mathrm{mg} / \mathrm{m}^{3} \mathrm{~J} 55.0 \mathrm{mg} / \mathrm{m}^{3}$ B,E1 $50.0 \mathrm{mg} / \mathrm{m}^{3}$ B,E1 $53.0 \mathrm{mg} / \mathrm{m}^{3}$ B,E1 $0.23 \mathrm{ppm} J 15.5 \mathrm{ppm}$ B,E1 $14.1 \mathrm{ppm}$ B,E1 $15.0 \mathrm{ppm}$ B,E1

BPQL $B P Q L$

BPQL

$11.0 \mathrm{mg} / \mathrm{m3}$

$2.64 \mathrm{ppm}$

BPQL

$4.3 \mathrm{mg} / \mathrm{m}^{3}$

$4.3 \mathrm{mg} / \mathrm{m}^{3}$

$5.0 \mathrm{mg} / \mathrm{m3}$

$0.62 \mathrm{ppm}$

$0.62 \mathrm{ppm}$

$0.72 \mathrm{ppm}$

BPQL

$12.0 \mathrm{mg} / \mathrm{m} 3$

$13.0 \mathrm{mg} / \mathrm{m} 3$

$16.0 \mathrm{mg} / \mathrm{m3}$

$3.1 \mathrm{ppm}$

$3.4 \mathrm{ppm}$

$4.2 \mathrm{ppm}$

BPQL

BPQL

BPQL

BPQL 


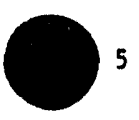

\begin{tabular}{|c|c|c|c|c|}
\hline Sampling site: & & $\begin{array}{r}\text { CFA II } \\
\text { Landfill }\end{array}$ & CFA II & $\begin{array}{r}\text { CFA II } \\
\text { Landfill }\end{array}$ \\
\hline Sample Type: & & $25 \mathrm{~mL}$ & $25 \mathrm{~mL}$ & $25 \mathrm{~mL}$ \\
\hline Istomer Sample 10 : & & L205MAY891 & LF205MAY892 & LF205MAY893 \\
\hline Sampl ing Date: & & $05 / 22 / 89$ & $05 / 22 / 89$ & $05 / 22 / 89$ \\
\hline Sampling Time: & & $11: 37$ & $11: 39$ & $11: 41$ \\
\hline Date Received: & & $05 / 22 / 89$ & $05 / 22 / 89$ & $05 / 22 / 89$ \\
\hline Lab Sample 10: & Method Blk & 9M1A0899 & 9MIA0900 & SMIA0901 \\
\hline Date Extracted: & $05 / 22 / 89$ & $05 / 22 / 89$ & $05 / 22 / 89$ & $05 / 22 / 89$ \\
\hline \multirow{2}{*}{$\begin{array}{l}\text { Date Analyzed: } \\
\text { Comments: }\end{array}$} & $05 / 22 / 89$ & $05 / 22 / 89$ & $05 / 22 / 89$ & $05 / 22 / 89$ \\
\hline & Lab Blank & & & \\
\hline PQL and Units & Result & Result & Result & Result \\
\hline n & $\ldots \ldots$ & .......... & $\cdots \ldots \ldots$ & \\
\hline
\end{tabular}

Parameter

P..........

Volatile Organic Compounds

Trichloroethene

Vinyl Chloride

xylenes (total)

EPA-624 M2

Tentatively Identified Compounds

Co Cyclic hydrocarbon

Hexane

EPA-624 M2

Trichlor of luoromethane

EPA-624 M2

Trichlorotrifluoroethane

EPA.624

M2

Others - Comment A3

EPA-624 M2
Comment A3

$$
\begin{aligned}
& 1.0 \mathrm{mg} / \mathrm{m} 3 \\
& 0.18 \mathrm{ppm}
\end{aligned}
$$

BPOL

$15.0 \mathrm{mg} / \mathrm{m3}$

$14.0 \mathrm{mg} / \mathrm{m} 3$

$15.0 \mathrm{mg} / \mathrm{m3}$ $2.7 \mathrm{ppm}$

$2.6 \mathrm{ppm}$

$2.7 \mathrm{ppm}$

BPQL

$13.0 \mathrm{mg} / \mathrm{m3}$

$12.0 \mathrm{mg} / \mathrm{m} 3$

$8.0 \mathrm{mg} / \mathrm{m3}$

$4.99 \mathrm{ppm}$

$4.6 \mathrm{ppm}$

$3.1 \mathrm{ppm}$

8PQL

$17.0 \mathrm{mg} / \mathrm{m}^{3}$

$16.0 \mathrm{mg} / \mathrm{m3}$

$21.0 \mathrm{mg} / \mathrm{m3}$

$0.22 \mathrm{ppm}$

$3.84 \mathrm{ppm}$

$3.61 \mathrm{ppm}$

$4.74 \mathrm{ppm}$
Comment i3
$2.0-4.0 \mathrm{mg} / \mathrm{m}^{3} \mathrm{T3}$ 0.6-1.1 ppm

$2.0 \cdot 4.0 \mathrm{mg} / \mathrm{m} 3 \mathrm{T3}$ 0.6-1.1 ppm
2.0
0.6

$\mathrm{ppm}$

BPQL J2

$2.0-4.0 \mathrm{mg} / \mathrm{m}^{3}$
$0.3-0.7 \mathrm{pgm}$

$2.0-4.0 \mathrm{mg} / \mathrm{m3} \mathrm{T3}$ 0.3-0.5 ppm

BPQL J2

BPQL J2

$33.0 \mathrm{mg} / \mathrm{m}^{3} \mathrm{~J} 2$

$\begin{array}{cc}38.0 \mathrm{mg} / \mathrm{m} 3 & \mathrm{~J} 2 \\ 6.6 \mathrm{ppm} & \mathrm{J} 2\end{array}$

BPQL J2

BPQL J2

BPOL
See results on at tached Form VOA-TIC for sample 9MIA0900

See results on at tached Form VOA-TIC for sample 9MIA0901 
Sampling site: Sample Type:

Customer Sample ID: Sampling Date: Sampl ing Time: Date Received: Lab Sample ID: Date Extracted: Date Analyzed: Remarks :

\section{Parameter}

...........

\section{Method}

Volatile Organic Compounds

\begin{tabular}{|c|c|c|c|c|}
\hline Acetone & $E P A-624$ & M2 & & $\begin{array}{l}2.0 \mathrm{mg} / \mathrm{m} 3 \\
0.83 \mathrm{ppm}\end{array}$ \\
\hline 2-8utanone & $E P A-624$ & M2 & $=$ & $\begin{array}{l}2.0 \mathrm{mg} / \mathrm{m}^{3} \\
0.67 \mathrm{ppm}\end{array}$ \\
\hline Chloroe thane & $E P A-624$ & $M 2$ & & $\begin{array}{l}2.0 \mathrm{mg} / \mathrm{m3} \\
0.74 \mathrm{ppm}\end{array}$ \\
\hline 1,1-Dichloroethane & $E P A-624$ & $M 2$ & & $\begin{array}{l}1.0 \mathrm{mg} / \mathrm{m} 3 \\
0.24 \mathrm{ppm}\end{array}$ \\
\hline 1,1-Dichloroethene & EPA- 624 & $M 2$ & & $\begin{array}{l}1.0 \mathrm{mg} / \mathrm{m} 3 \\
0.24 \mathrm{ppm}\end{array}$ \\
\hline 1,2 Dichloroethene (total) & $E P A=624$ & $M 2$ & & $\begin{array}{l}1.0 \mathrm{mg} / \mathrm{m3} \\
0.25 \mathrm{ppm}\end{array}$ \\
\hline 1,2-o ichloropropane & $E P A-624$ & M2 & & $\begin{array}{l}1.0 \mathrm{mg} / \mathrm{m} 3 \\
0.19 \mathrm{ppm}\end{array}$ \\
\hline Ethylbenzene & $E P A-624$ & $M 2$ & & $\begin{array}{l}1.0 \mathrm{mg} / \mathrm{m3} \\
0.23 \mathrm{ppm}\end{array}$ \\
\hline Methylene chloride & EPA- 624 & M2 & & $\begin{array}{l}1.0 \mathrm{mg} / \mathrm{m}^{3} \\
0.28 \mathrm{ppm}\end{array}$ \\
\hline 4-Methyl-2-pentanone & $E P A-624$ & M2 & & $\begin{array}{l}2.0 \mathrm{mg} / \mathrm{m}^{3} \\
0.48 \mathrm{ppm}\end{array}$ \\
\hline Tetrachloroethene & EPA- 624 & $M 2$ & & $\begin{array}{l}1.0 \mathrm{mg} / \mathrm{m}^{3} \\
0.14 \mathrm{ppm}\end{array}$ \\
\hline Toluene & $E P A-024$ & M2 & & $\begin{array}{l}1.0 \mathrm{mg} / \mathrm{m}^{3} \\
0.26 \mathrm{ppm}\end{array}$ \\
\hline $1,1,1-$ Trichloroethane & $E P A-624$ & M2 & & $\begin{array}{l}1.0 \mathrm{mg} / \mathrm{m}^{3} \\
0.18 \mathrm{ppm}\end{array}$ \\
\hline
\end{tabular}

Landfill

$$
22 \mathrm{~mL}
$$

Soil gas

LF208MAY89

05/22/89

$11: 49$

05/22/89

Method Blk 9MJA0902

05/22/89 05/22/89

05/22/89 05/22/89

Lab Blank
PQL and Units $\quad$ Result $\quad$ Result

$0.6 \mathrm{mg} / \mathrm{m} 3 \mathrm{~J} \quad 0.8 \mathrm{mg} / \mathrm{m}^{3} \mathrm{~B}, 05, \mathrm{~J} 2$

$0.25 \mathrm{ppm} J \quad 0.33 \mathrm{ppm} B, 05, \mathrm{~J} 2$

$1.3 \mathrm{mg} / \mathrm{m}^{3} \mathrm{~J} \quad 2.0 \mathrm{mg} / \mathrm{m} 3 \mathrm{~B}, 05, \mathrm{~J} 2$

$0.430 .43 \mathrm{~J} \quad 0.67$ pom B,05, J2

BPQL $\quad<2.3 \mathrm{mg} / \mathrm{m}^{3} 05, \mathrm{~J}$

$<0.86$ ppm D5,J

BPOL $<1.1 \mathrm{mg} / \mathrm{m} 305, \mathrm{~J}$

$<0.42$ ppm 05, J

BPQL $\quad<1.1 \mathrm{mg} / \mathrm{m}^{3} 05, \mathrm{~J}$

$<0.41$ ppm $05, J$

BPOL $<1.1 \mathrm{mg} / \mathrm{m3} 05, \mathrm{~J}$

$<0.41$ ppm 05,J

BPQL $<1.1 \mathrm{mg} / \mathrm{m}^{3} 05, \mathrm{~J}$

$<0.23$ ppm 05, J

BPOL $<1.1 \mathrm{mg} / \mathrm{m}^{3} \mathrm{DS}, \mathrm{J}$

$<0.25$ ppm 05,J

$0.8 \mathrm{mg} / \mathrm{m}^{3} \mathrm{~J} \quad 1.0 \mathrm{mg} / \mathrm{m}^{3} \mathrm{~B}, 05$

$0.23 \mathrm{ppm} J \quad 0.28 \mathrm{ppm} B, 05$

BPQL $\quad<2.3 \mathrm{mg} / \mathrm{m}^{3} 05, \mathrm{~J}$

$<0.55$ ppm D5, J

BPQL $<1.1 \mathrm{mg} / \mathrm{m} 3 \mathrm{DS}, \mathrm{J}$

$<0.16 \mathrm{ppm}$ DS, J

BPQL $<1.1 \mathrm{mg} / \mathrm{m}^{3} 05, \mathrm{~J}$

$<0.29$ ppm $05, \mathrm{~J}$

BPQL $<1.1 \mathrm{mg} / \mathrm{m}^{3} 05, \mathrm{~J}$

$<0.20$ ppm D, $\mathrm{J}$ 
EG\&G Idaho, Environmental Chenistry, ROA

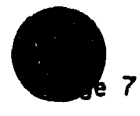

Sampling site: Sample Type:

Customer Sample 10: Sampling Date: Sampl ing Time: Date Received: Lab Sample 1D: Date Extracted: Date Analyzed: Comments:

Parameter ..........

Method ..........

Volatile Organic Compounds

Trichloroethene

EPA-624 M2

vinyl Chloride

EPA-624 M2

Xylenes (total)

EPA-624 M2

Tentat ively Ident ified Compounds

C6 Cyclic hydrocarbon

EPA-624 M2

Hexane

EPA-624 M2

Trichlorofluorome thane

EPA-624 M2

Trichlorotrifluoroethane

EPA-624 M2

Others - Comment $A 3$

EPA-624 M2
POL and Units

-..................

Comment A3

$$
\begin{aligned}
& 1.0 \mathrm{mg} / \mathrm{m}^{3} \\
& 0.18 \mathrm{ppm}
\end{aligned}
$$

$2.0 \mathrm{mg} / \mathrm{m}^{3}$

$0.77 \mathrm{ppm}$

$1.0 \mathrm{mg} / \mathrm{m}^{3}$

$0.077 \mathrm{ppm}$

Comment 13

$2.0-4.0 \mathrm{mg} / \mathrm{m}^{3} \mathrm{T3}$ 0.6-1.1 ppm

BPQL $J 2<2.3 .4 .6 \mathrm{mg} / \mathrm{m}^{3} 05, \mathrm{~J} 2$

$<0.7-1.3$ ppm DS, J2

$2.0-4.0 \mathrm{mg} / \mathrm{m}^{3} \mathrm{i} 3 \quad 2.0 \mathrm{mg} / \mathrm{m}^{3} \mathrm{~J} 2$

$34.0 \mathrm{mg} / \mathrm{m3}^{3} \quad B, 05, \mathrm{~J} 2$

0.6-1.1 ppm

$0.6 \mathrm{ppm} \sqrt{2}$

9.5 ppm B,05,J2

$2.0 \cdot 4.0 \mathrm{mg} / \mathrm{m}^{3} \mathrm{r3}$

$0.3-0.7 \mathrm{ppm}$

BPQL $\mathrm{J} 2<2.3-4.6 \mathrm{mg} / \mathrm{m}^{3} 05, \mathrm{~J} 2$

$<0.4-0.8 \mathrm{ppm} \quad 05, \mathrm{~J} 2$

$2.0 \cdot 4.0 \mathrm{mg} / \mathrm{m}^{3} \mathrm{T3}$ $0.3-0.5 \mathrm{ppm}$

BPQL $\mathrm{J} 2<2.3-4.6 \mathrm{mg} / \mathrm{m3} \quad 05, \mathrm{~J} 2$

$<0.3-0.6 \mathrm{ppm} 05 . \mathrm{J} 2$

see Table 3

$B P Q L ~ J 2<2.0-4.0 \mathrm{mg} / \mathrm{m}^{3} 05 . \mathrm{J} 2$ 
EG\&G Idaho, Environmental Chemistry, ROA

Page 8

Legend:

BPQL = Below practical quantitation level. See PQL definition for further explanation of practical quantitation level.

PQL = Practical quantitation level. This is the lowest concentration reliably measurable (i.e., 33\% maximum uncertainty in precision and accuracy at the one standard deviation confidence level) for normal samples during routine laboratory operations.

Comments:

A3 Target volatile organic compounds and their practical quantitation levels (POLs) are provided in Table 3. The instrument was calibrated to quantitatively measure these compounds, therefore compounds reported as below PQL (BPQL) are unlikely to have been present above their POLs.

B

The presence of this element/compound in the associated laboratory method blank indicates the analyte found in the sample may be partially or completely a result of laboratory contamination.

D5 The sample analyzed was not a full $25 \mathrm{~mL}$; therefore, the normal laboratory PQLs could not be achieved. The normal PQLs are those listed in the PQL and units colum and in Table 3 . The elevated POLs can be determined by multiplying the POL for a $25 \mathrm{~mL}$ sample by 25 then dividing by the actual sample volume; The specific multiplication factors (MF) are as follows: for sample LF206MAY89 MF $=25 / 1=25.0$ and for sample LF208MAY89 MF $=25 / 22 \div 1.1$.

The concentration reported for this sample exceeded the calibration range of the instrument and should be considered an estimated concentration. The most likely result of this analytical dificulty is that the reported concentration underestimates the actual concentration.

Indicates estimated value. The concentration is below the PQL, but the compound was detected in the sample.

Indicates estimated value. These volatile organic compounds are not target compounds (i.e., tentatively identified compounds), and consequently the instrument is not calibrated to quantitatively measure their abundances. An estimated calibration factor is assumed to quantify these compounds, therefore the reported concentrations are estimated.

A modification of EPA Method 624 was used since the method is designed for water samples. The PQLs listed apply to a volume of $25 \mathrm{~mL}$. The results are reported in units of $\mathrm{mg} / \mathrm{m3}$ and ppm. The ppm unit is on a volume compound per volume air basis and is determined by multiplying the weight of compound per volume of air concentration by $24.0 \mathrm{~L}$ (volume of $1.0 \mathrm{~mole}$ of gas at normal local temperature and pressure) and dividing by the compound's molecular weight $(\mathrm{g} / \mathrm{mole})$. Variations in temperature or pressure could introduce up to $25 \%$ uncertainty in the values repc ted in ppm units.

Tentatively identified compounds. These volatile organic compounds are not target compounds, and consequently the instrument is not calibrated to quantitatively measure their abundances. The estimated practical quantitation level for these compounds is 2.0 to $4.0 \mathrm{mg} / \mathrm{m} 3$ for gas samples.

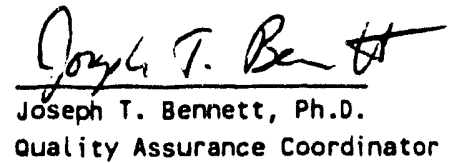

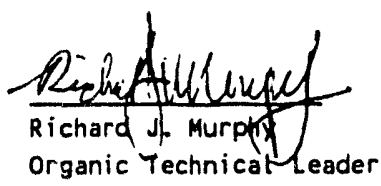


Est6 Idaho, ine.

Environaental Cheastry Unit

Table 3. Hethod sensitivities for volatile organic ceapounss oy jurge and trap SC/ns analysis

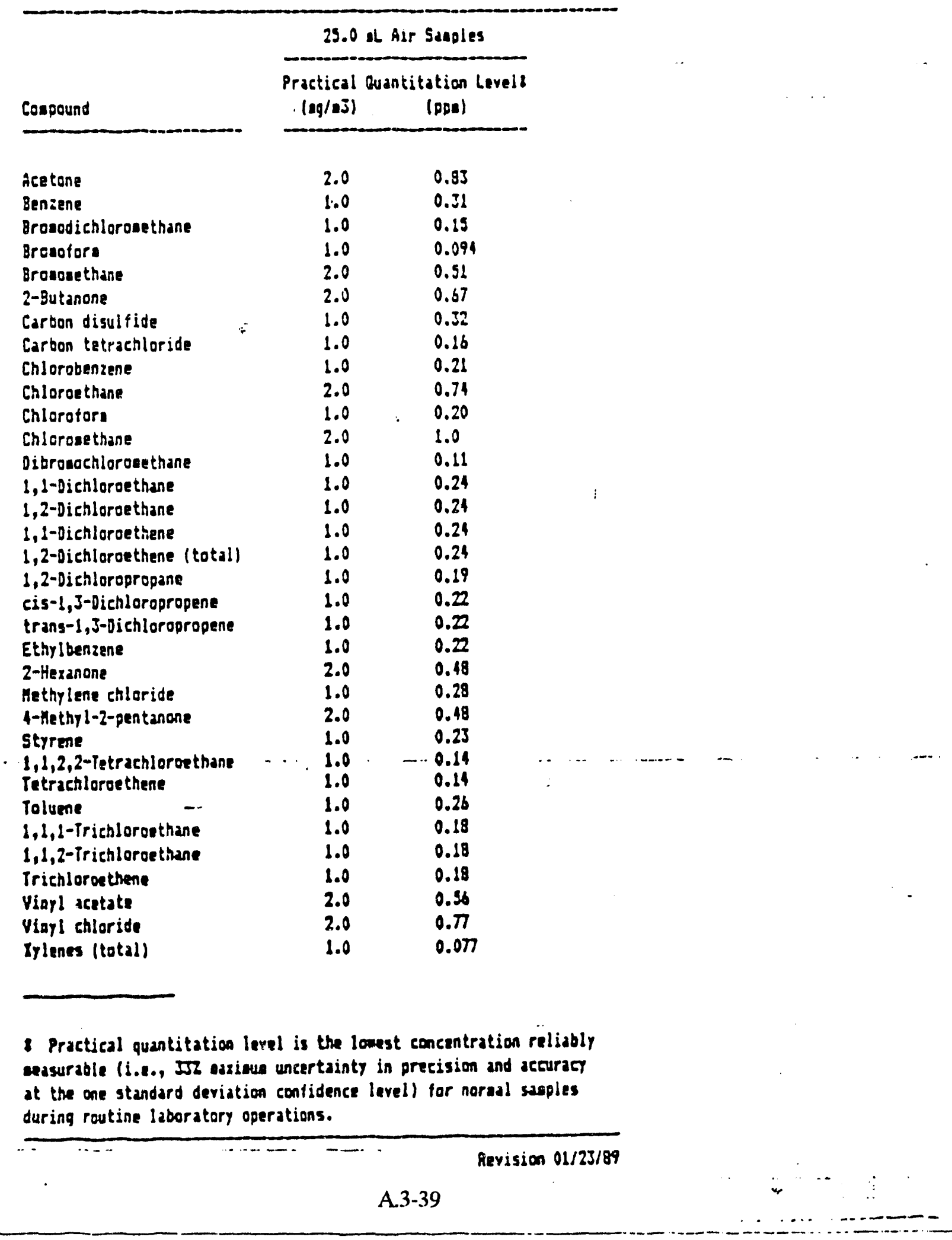




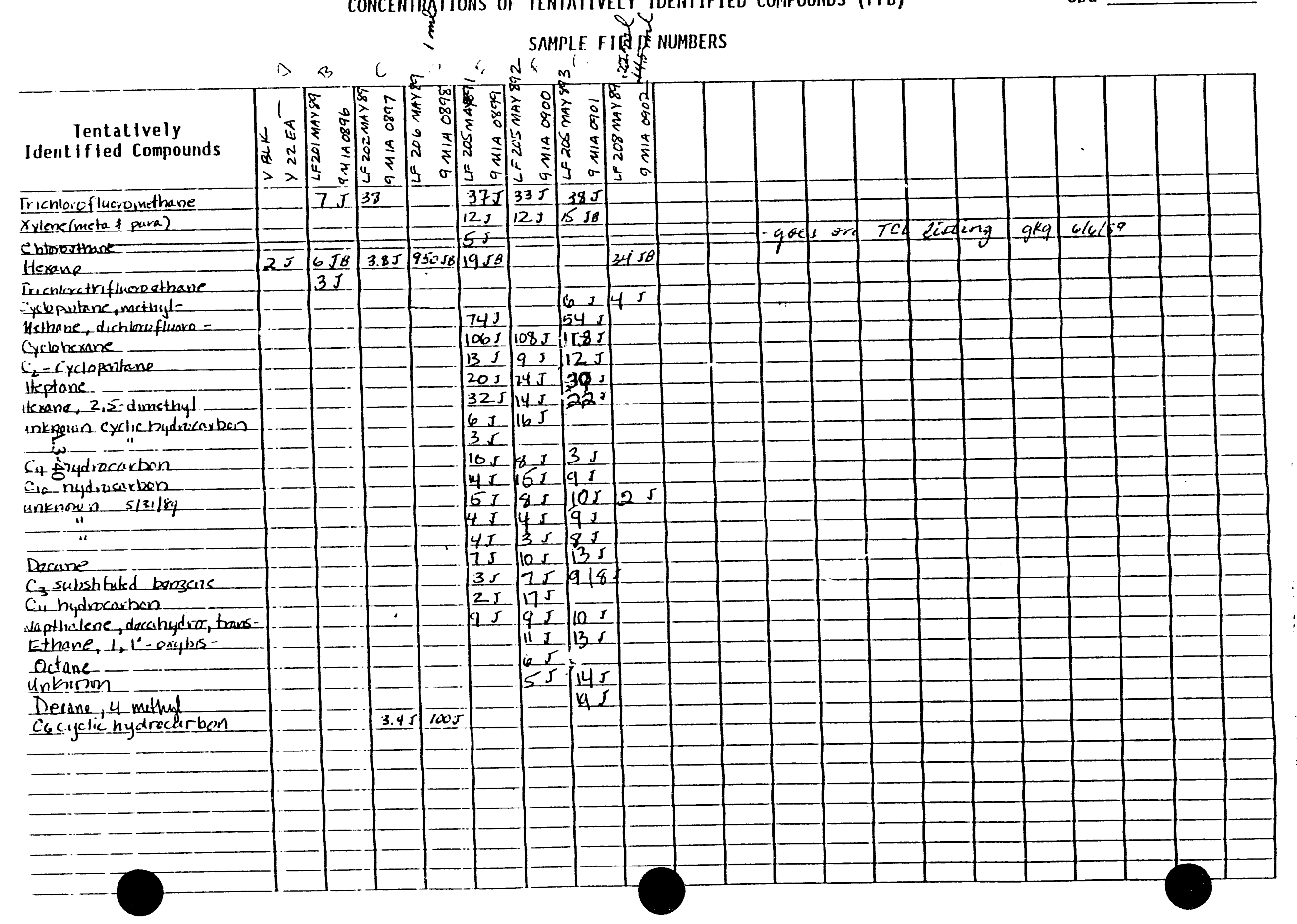


EG\&G Idaho, Environmental Chemistry, ROA

EG\&G Idaho, Inc.

Environmental Chemistry

P.O. Box 1625, MS 4123

Idaho Falls, 1083415

This report of analysis (ROA) presents the analytical results for the analyses you requested. Sample identification, sampling information, and laboratory analys is information are listed in a colum for each sample. Analytical results are listed by analytical parameter below the sample identification information. Acronyms and abbreviations used are defined at the end of the report. A letter and/or letter number flag immediately to the right of a parameter, method, $\mathrm{PQL}$ and units, result, or any other entry indicates an amplifying comment also provided at the end of the report.

REPORT OF ANALYSIS

Prepared for:

Shannon Ansley

EG\&G Idaho, Inc.

P.O. Box 1625, MS 2107

IJaho Falls, ID 83415
Report Identification:

ROA Number:

Date:

109

Charge No.:

$6 / 28 / 89$

3RIF04031

Page:

1 
EG\&G Idaho, Environmental Chemistry, ROA

Page 2

Parameter

..........

Volatile Organic Compounds

Acetone
Benzene
2-Butanone

Chlorobenzene

1,1-Dichloroethane

2-Hexanone

Methylene chloride

Tetrachloroethene

Tolvene

1,1,1-Trichloroethane

Trichloroethene

xylene (total)

Others - Corment A3

Sampling Site:
Sample Type:
Customer Sample ID:
Sampl ing Date:
Date Received:
Lab Sample 1D:
Date Extracted:
Date Analyzed:
Remarks:
POL and Units
.........................

Corment A3

EPA-624 M2

$2.0 \mathrm{mg} / \mathrm{m}^{3}$

$0.83 \mathrm{ppm}$

EPA-624 M2

$1.0 \mathrm{mg} / \mathrm{m} 3$

$0.31 \mathrm{ppm}$

EPA-624 M2

$2.0 \mathrm{mg} / \mathrm{m}^{3}$ $0.67 \mathrm{ppm}$

EPA-624 M2

$1.0 \mathrm{mg} / \mathrm{m} 3$ $0.21 \mathrm{ppm}$

EPA-624 M2

$1.0 \mathrm{mg} / \mathrm{m} 3$ $0.24 \mathrm{ppm}$

EPA-624 M2

$2.0 \mathrm{mg} / \mathrm{m}^{3}$ $0.48 \mathrm{ppm}$

EPA-624 M2

$1.0 \mathrm{mg} / \mathrm{m} 3$ $0.28 \mathrm{ppm}$

EPA-624 M2

$1.0 \mathrm{mg} / \mathrm{m} 3$

$0.14 \mathrm{ppm}$

EPA-624 M2

$1.0 \mathrm{mg} / \mathrm{m} 3$ $0.26 \mathrm{ppm}$

EPA-624 M2

$1.0 \mathrm{mg} / \mathrm{m}^{3}$ $0.18 \mathrm{ppm}$

EPA-624 M2

$1.0 \mathrm{mg} / \mathrm{m}^{3}$ $0.18 \mathrm{ppm}$

EPA-624 M2

$1.0 \mathrm{mg} / \mathrm{m}^{3}$ $0.077 \mathrm{ppm}$

EPA $624 \quad M 2$

$\begin{array}{lr} & \begin{array}{r}\text { Landfill } \\ 2 \mathrm{~mL}\end{array} \\ & \text { soil gas } \\ \text { LF302MAY89 } \\ 05 / 23 / 89 \\ & 05 / 23 / 89 \\ & 9 M 1 A 0954 \\ \text { Method Blk } & 05 / 24 / 89 \\ 05 / 24 / 89 & 05 / 24 / 89 \\ 05 / 24 / 89 & \\ \text { Lab Blank } & \\ & \\ \text { Result } & \text { Result } \\ \ldots . . . . . . . & \ldots . . . . . . .\end{array}$

Landfill

$25 \mathrm{~mL}$

soil gas

LF304MAY89

05/23/89

05/23/89

9M!A0955

$05 / 24 / 89$

05/24/89

Analytical

Problem

Result

\section{$0.5 \mathrm{mg} / \mathrm{m3} \mathrm{J} \quad 5.0 \mathrm{mg} / \mathrm{m} 3 \quad B, D 5, \mathrm{~J}$}

$0.21 \mathrm{ppm} J \quad 2.1 \mathrm{ppm} B, 05, J$

BPQL $\quad 12.5 \mathrm{mg} / \mathrm{m}^{3} \quad 05, \mathrm{~J}$

< 3.9 ppm D5, J

$2.0 \mathrm{mg} / \mathrm{m3} \mathrm{J}$

$17.0 \mathrm{mg} / \mathrm{m3} \quad B, 05, \mathrm{~J}$

$0.67 \mathrm{ppm} \mathrm{J}$

$5.7 \mathrm{ppm} \quad \mathrm{B}, 05, \mathrm{~J}$

BPQL

$<12.5 \mathrm{mg} / \mathrm{m}^{3} \quad 05, \mathrm{~J}$

< $2.7 \mathrm{ppm} 05, \mathrm{~J}$

BPQL

$<12.5 \mathrm{mg} / \mathrm{m}^{3} \quad 05, \mathrm{~J}$

$<3.0 \mathrm{ppm} \mathrm{D5,J}$

$0.6 \mathrm{mg} / \mathrm{m} 3 \mathrm{~J}<25.0 \mathrm{mg} / \mathrm{m}^{3} 05, \mathrm{~J}$

$0.14 \mathrm{ppm} J<6.0 \mathrm{ppm} \quad 05, J$

$0.1 \mathrm{mg} / \mathrm{m} 3 \mathrm{~J}$

$2.0 \mathrm{mg} / \mathrm{m3} \quad 8,05, \mathrm{~J}$

$0.03 \mathrm{ppm} J$

$0.02 \mathrm{ppm} \quad \mathrm{B}, 05, \mathrm{~J}$

BPQL

$<12.5 \mathrm{mg} / \mathrm{m}^{3} \quad 05, \mathrm{~J}$

$<1.8 \mathrm{ppm}$ D5.J

BPQ

$<12.5 \mathrm{mg} / \mathrm{m} 305, \mathrm{~J}$

< $3.3 \mathrm{ppm} 05, \mathrm{~J}$

BPOL < $12.5 \mathrm{mg} / \mathrm{m} 305, \mathrm{~J}$

< $2.2 \mathrm{ppm}$ DS,J

BPQL $<12.5 \mathrm{mg} / \mathrm{m3}$ D5, J

$<2.3 \mathrm{ppm} 05 . \mathrm{J}$

BPOL $<12.5 \mathrm{mg} / \mathrm{m3}$ DS,J

$<0.94$ ppm DS,J

BPQL

BPQL 05
NR N1

NR N1

NR N1

NR $\quad$ 1

NR N1

NR N1

NR N1

NR N1

NR N1

NR N1

NR N1

NR N1

NR $\quad N 1$ 
Sampling site: Sample Type:

Customer Sample 1D: Sampling Date: Date Received: Lab Sample 10: Date Extracted: Date Analyzed: Remarks:

Parameter Pa........

Volatile Organic Compounds

$$
\text { Acetone }
$$

Benzene

2-Butanone

Chlorobenzene

1,1-Dichloroethane

2-Hexanone

Methylene chloride

Tetrachloroethene

Toluene

1,1,1-Trichloroethane

Trichloroethene

Xylene (total)

Others - Comment A3

\section{Method}

Method
Comment A3

$2.0 \mathrm{mg} / \mathrm{m} 3$ $0.83 \mathrm{ppm}$

$1.0 \mathrm{mg} / \mathrm{m} 3$ $0.31 \mathrm{ppm}$ $\begin{array}{ll}\text { EPA-624 M2 } & 2.0 \mathrm{mg} / \mathrm{m3} \\ & 0.67 \mathrm{ppm}\end{array}$ $\begin{array}{ll}\text { EPA-624 M2 } & 2.0 \mathrm{mg} / \mathrm{mm}^{3} \\ & 0.67 \mathrm{ppm}\end{array}$

$\begin{array}{ll}\text { EPA.624 M2 } & 1.0 \mathrm{mg} / \mathrm{m3} \\ & 0.21 \mathrm{pPm}\end{array}$

$\begin{array}{ll}\text { EPA.624 M2 } & 1.0 \mathrm{mg} / \mathrm{m}^{3} \\ & 0.21 \mathrm{ppm}\end{array}$

EPA-624 M2

$1.0 \mathrm{mg} / \mathrm{m} 3$

$0.24 \mathrm{ppm}$

$2.0 \mathrm{mg} / \mathrm{m3}$ $0.48 \mathrm{ppm}$

$1.0 \mathrm{mg} / \mathrm{m}^{3}$

$0.28 \mathrm{ppm}$

$1.0 \mathrm{mg} / \mathrm{m}^{3}$ $0.14 \mathrm{ppm}$ $0.26 \mathrm{ppm}$

EPA-624 M2

EPA-624 M2

$1.0 \mathrm{mg} / \mathrm{m}^{3}$ $0.18 \mathrm{ppm}$

EPA-624 M2

$1.0 \mathrm{mg} / \mathrm{m}^{3}$

$0.18 \mathrm{ppm}$

EPA-624 M2

$1.0 \mathrm{mg} / \mathrm{m} 3$ $0.077 \mathrm{ppm}$

EPA $624 \quad M 2$

\begin{tabular}{|c|c|c|c|}
\hline & CFA III & CFA 111 & CFA III \\
\hline & Landfill & Landfill & Landf $i l l$ \\
\hline & $\begin{array}{r}25 \mathrm{~mL} \\
\text { soil gas }\end{array}$ & $\begin{array}{r}25 \mathrm{~mL} \\
\text { soil gas }\end{array}$ & $\begin{array}{r}25 \mathrm{~mL} \\
\text { LFsoil gas }\end{array}$ \\
\hline & LF306MAY89 & LF307MAY89 & LF309MAY89 \\
\hline & $05 / 23 / 89$ & $05 / 23 / 89$ & $05 / 23 / 89$ \\
\hline & $05 / 23 / 89$ & $05 / 23 / 89$ & $05 / 23 / 89$ \\
\hline Method Blk & 9MIA0956 & 9MIA0957 & 9M1A0958 \\
\hline $05 / 24 / 89$ & $05 / 24 / 89$ & $05 / 24 / 89$ & $05 / 24 / 89$ \\
\hline $05 / 24 / 89$ & $05 / 24 / 89$ & $05 / 24 / 89$ & $05 / 24 / 89$ \\
\hline Lab Blank & & & \\
\hline Result & Result & Result & Result \\
\hline & - & , & \\
\hline
\end{tabular}

see Table 3

$0.5 \mathrm{mg} / \mathrm{m}^{3} \mathrm{~J}$

$0.7 \mathrm{mg} / \mathrm{m}^{3} \mathrm{~B}, \mathrm{~J} \quad 2.0 \mathrm{mg} / \mathrm{m3} \quad B, J \quad 1.0 \mathrm{mg} / \mathrm{m}^{3} \mathrm{~B}, \mathrm{~J}$ $0.21 \mathrm{ppm} \mathrm{J}$ 0.29 ppm B,J 0.83 ppm B,J 0.41 ppm B,J

BPQL

BPQL

BPQL

$11.0 \mathrm{mg} / \mathrm{m3}$

$3.4 \mathrm{ppm}$

$2.0 \mathrm{mg} / \mathrm{m}^{3} \mathrm{~J}$ $0.67 \mathrm{ppm} \mathrm{J}$

$2.0 \mathrm{mg} / \mathrm{m} 3 \mathrm{~B}, J \quad 2.0 \mathrm{mg} / \mathrm{m3} B, J \quad 1.0 \mathrm{mg} / \mathrm{m}^{3} \mathrm{~B}, J$

BPOL

$B P Q L$

$2.0 \mathrm{mg} / \mathrm{m}^{3}$

BPQL

BPOL

$4.0 \mathrm{mg} / \mathrm{ms}$

$0.42 \mathrm{ppm}$

$0.6 \mathrm{mg} / \mathrm{m3} \mathrm{J}$

$0.14 \mathrm{ppm} \mathrm{J}$

$0.1 \mathrm{mg} / \mathrm{m3} \mathrm{J}$

$0.03 \mathrm{ppm} J$

BPQL

BPQL

BPQL
BPOL

BPQL

BPQL
$0.96 \mathrm{ppm}$

BPQL

BPQL

BPQL

$0.3 \mathrm{mg} / \mathrm{m}^{3} B, J \quad 0.4 \mathrm{mg} / \mathrm{m}^{3} B, J \quad 0.6 \mathrm{mg} / \mathrm{m}^{3} \mathrm{~B}, \mathrm{~J}$ 0.08 ppm B,J 0.11 ppm B,J 0.17 ppm B,J

BPQL

BPQL

BPQL

BPQL

BPQL

$5.0 \mathrm{mg} / \mathrm{m}^{3}$

$1.3 \mathrm{ppm}$

BPQL

$18.0 \mathrm{mg} / \mathrm{m3}$

BPQL

\section{$3.2 \mathrm{ppm}$}

BPQL

$1.0 \mathrm{mg} / \mathrm{m}^{3}$

$0.2 \mathrm{mg} / \mathrm{m3} \mathrm{J}$

$0.18 \mathrm{ppm}$

$0.04 \mathrm{ppm} J$

BPQL

$1.0 \mathrm{mg} / \mathrm{m}^{3}$

$0.08 \mathrm{ppm}$

BPQL

BPAL BPQL

BPOL 


\begin{tabular}{|c|c|c|}
\hline Sampling site: & & Landfill \\
\hline Sample Type: & & $14.5 \mathrm{~mL}$ \\
\hline & & soil gas \\
\hline Customer Sample ID: & & LF310MAY89 \\
\hline Sampling Date: & & $05 / 23 / 89$ \\
\hline Date Received: & & $05 / 23 / 89$ \\
\hline Lab Sample ID: & Method BIk & 9MIA0959 \\
\hline Date Extracted: & $0.5 / 24 / \varepsilon$ & $05 / 24 / 89$ \\
\hline Date Analyzed: & $05 / 24 / 89$ & $05 / 24 / 89$ \\
\hline Remarks: & Lab Blank & \\
\hline PQL and Units & Result & Result \\
\hline 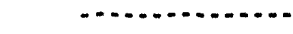 & $\cdots-\infty \ldots$ & \\
\hline
\end{tabular}

Volatile Organic Compounds

\begin{tabular}{|c|c|c|c|}
\hline Acetone & EPA- 624 & M2 & $\begin{array}{l}2.0 \mathrm{mg} / \mathrm{m3} \\
0.83 \mathrm{ppm}\end{array}$ \\
\hline Benzene & EPA-624 & M2 & $\begin{array}{l}1.0 \mathrm{mg} / \mathrm{m} 3 \\
0.31 \mathrm{ppm}\end{array}$ \\
\hline 2-Butanone & EPA- 624 & M2 & $\begin{array}{l}2.0 \mathrm{mg} / \mathrm{m3} \\
0.67 \mathrm{ppm}\end{array}$ \\
\hline Chlorobenzene & EPA-624 & M2 & $\begin{array}{l}1.0 \mathrm{mg} / \mathrm{ml} \\
0.21 \mathrm{ppm}\end{array}$ \\
\hline 1,1-Dichloroethane & EPA- 624 & M2 & $\begin{array}{l}1.0 \mathrm{mg} / \mathrm{m} 3 \\
0.24 \mathrm{ppm}\end{array}$ \\
\hline 2-Hexanone & EPA-624 & $M 2$ & $\begin{array}{l}2.0 \mathrm{mg} / \mathrm{m}^{3} \\
0.48 \mathrm{ppm}\end{array}$ \\
\hline Methylene chloride & EPA- 624 & M2 & $\begin{array}{l}1.0 \mathrm{mg} / \mathrm{m} 3 \\
0.28 \mathrm{ppm}\end{array}$ \\
\hline Tetrachloroethene & EFA- 624 & M2 & $\begin{array}{l}1.0 \mathrm{mg} / \mathrm{m} 3 \\
0.14 \mathrm{ppm}\end{array}$ \\
\hline Toluene & EPA-624 & M2 & $\begin{array}{l}1.0 \mathrm{mg} / \mathrm{m} 3 \\
0.26 \mathrm{ppm}\end{array}$ \\
\hline $1,1,1-$ Trichloroethane & EPA-624 & M2 & $\begin{array}{l}1.0 \mathrm{mg} / \mathrm{m} 3 \\
0.18 \mathrm{ppm}\end{array}$ \\
\hline Trichloroethene & EPA- 624 & M2 & $\begin{array}{l}1.0 \mathrm{mg} / \mathrm{m} 3 \\
0.18 \mathrm{ppm}\end{array}$ \\
\hline Xylene (total) & EPA- 624 & M2 & $\begin{array}{l}1.0 \mathrm{mg} / \mathrm{m} 3 \\
0.077 \mathrm{ppm}\end{array}$ \\
\hline Others - Comment $A 3$ & EPA 624 & M2 & see Table 3 \\
\hline
\end{tabular}

$$
\begin{aligned}
& 0.5 \mathrm{mg} / \mathrm{m} 3 \mathrm{~J} \quad 0.9 \mathrm{mg} / \mathrm{m}^{3} \quad B, 05, \mathrm{~J} \\
& 0.21 \mathrm{ppm} J \quad 0.4 \mathrm{ppm} B, 05, J \\
& \text { BPQL }<1.72 \mathrm{mg} / \mathrm{m3} \quad 05, \mathrm{~J} \\
& <0.53 \text { ppm 05,J } \\
& 2.0 \mathrm{mg} / \mathrm{m}^{3} \mathrm{~J} \quad 2.4 \mathrm{mg} / \mathrm{m}^{3} \quad B, 05, \mathrm{~J} \\
& 0.67 \mathrm{ppm} J \quad 0.80 \mathrm{ppm} B, 05, J \\
& \text { BPQL }<1.72 \mathrm{mg} / \mathrm{m3} 05, \mathrm{~J} \\
& <0.37 \text { ppm D5,J } \\
& \text { BPQL }<1.72 \mathrm{mg} / \mathrm{m3} 05, \mathrm{~J} \\
& <0.42 \text { ppm D5, J }
\end{aligned}
$$

$0.6 \mathrm{mg} / \mathrm{m3} \mathrm{J}<3.4 \mathrm{mg} / \mathrm{m} 305, \mathrm{~J}$ 0.14 ppm $J<0.81$ ppm D5, J $\begin{array}{lllll}0.1 \mathrm{mg} / \mathrm{m} 3 & \mathrm{~J} & 0.4 & \mathrm{mg} / \mathrm{m} 3 & B, 05, \mathrm{~J} \\ 0.03 \mathrm{ppm} & \mathrm{J} & 0.11 \mathrm{ppm} & B, 05, \mathrm{~J}\end{array}$

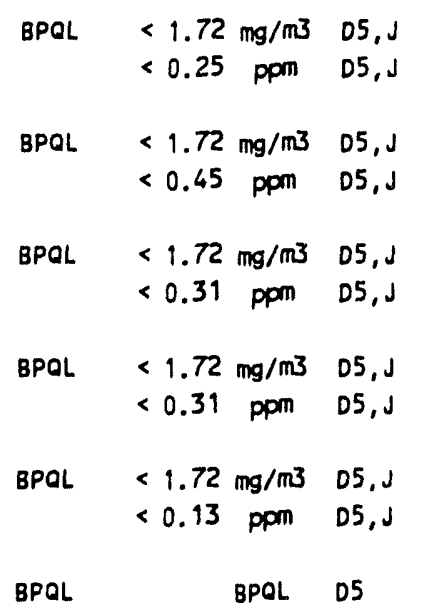


EG\&G Idaho, Environmental Chemistri, ROA

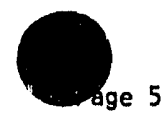

5

Legend:

BPQL = Below practical quantitation level. See PQL definition for further explanation of practical quantitation level.

PQL = Practical quantitation level. This is the lowest concentration reliably measurable (i.e., $33 \%$ maximum uncertainty in precision and accuracy at the one standard deviation confidence (evel) for normal samples during routine laboratory operations.

Corments :

Target volatile organic compounds and their practical quantitation levels (PQLs) are provided in Table 3. The instrument was calibrated to quantitatively measure these compounds, therefore compounds reported as below PQL (BPQL) are unlikely to have been present above their PQLs.

B

The presence of this element/compound in the associated laboratory method blank indicates the analyte found in the sample may be partially or completely a result of laboratory contamination.

The sample analyzed was not a full $25.0 \mathrm{ml}$; therefore, the normal laboratory pols could not be achieved. The normal PQLs are those listed in the PQL and units colum and in Table 3 . The elevated PQLs can be determined by multiplying the PQL for a $25.0 \mathrm{ml}$ sample by 25.0 then dividing by the actual sample volume; The specific multiplication factars (MF) are as follows: for sample 9MIA0954 MF $=25.0 / 2.0=12.5$ and for sample 9 MIA0959 MF $=25.0 / 14.5=1.72$. A result below the PQL is identified by $<X X$ where the $X X$ value is the PQL.

Indicates estimated value. The concentration is below the PQL, but the compound was detected in the sample.

A modification of EPA Method 624 was used since the method is designed for water samples. The PQLs listed apply to a volume of $25 \mathrm{~mL}$. The results are reported in units of $\mathrm{mg} / \mathrm{m3}$ and ppm. The ppm unit is on a volume compound per volume air basis and is determined by multiplying the weight of compound per volume of air concentration by $24.0 \mathrm{~L}$ (volume of 1.0 mole of gas at normal local temperature and pressure) and dividing by the compound's molecular weight $(\mathrm{g} / \mathrm{mole})$. Variations in temperature or pressure could introduce up to $25 \%$ uncertainty in the values reported in ppm units.

This sample was lost during analysis before it was possible to obtain any reportable analytical results. The initial analysis attempt consumed all of the sample so reanalys is was not possible.

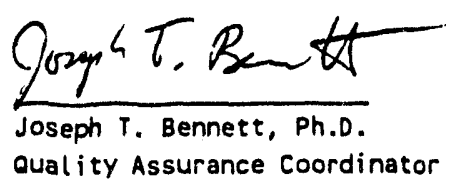

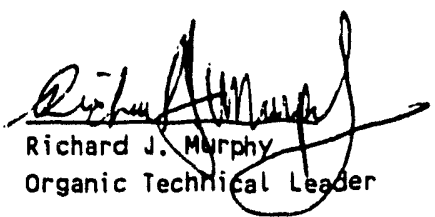


Egts idaho, Inc.

Environaental Chessistr Unst

Table 3. Method sensitivities for volatile organic conoounss or jurge and trap scins analysis

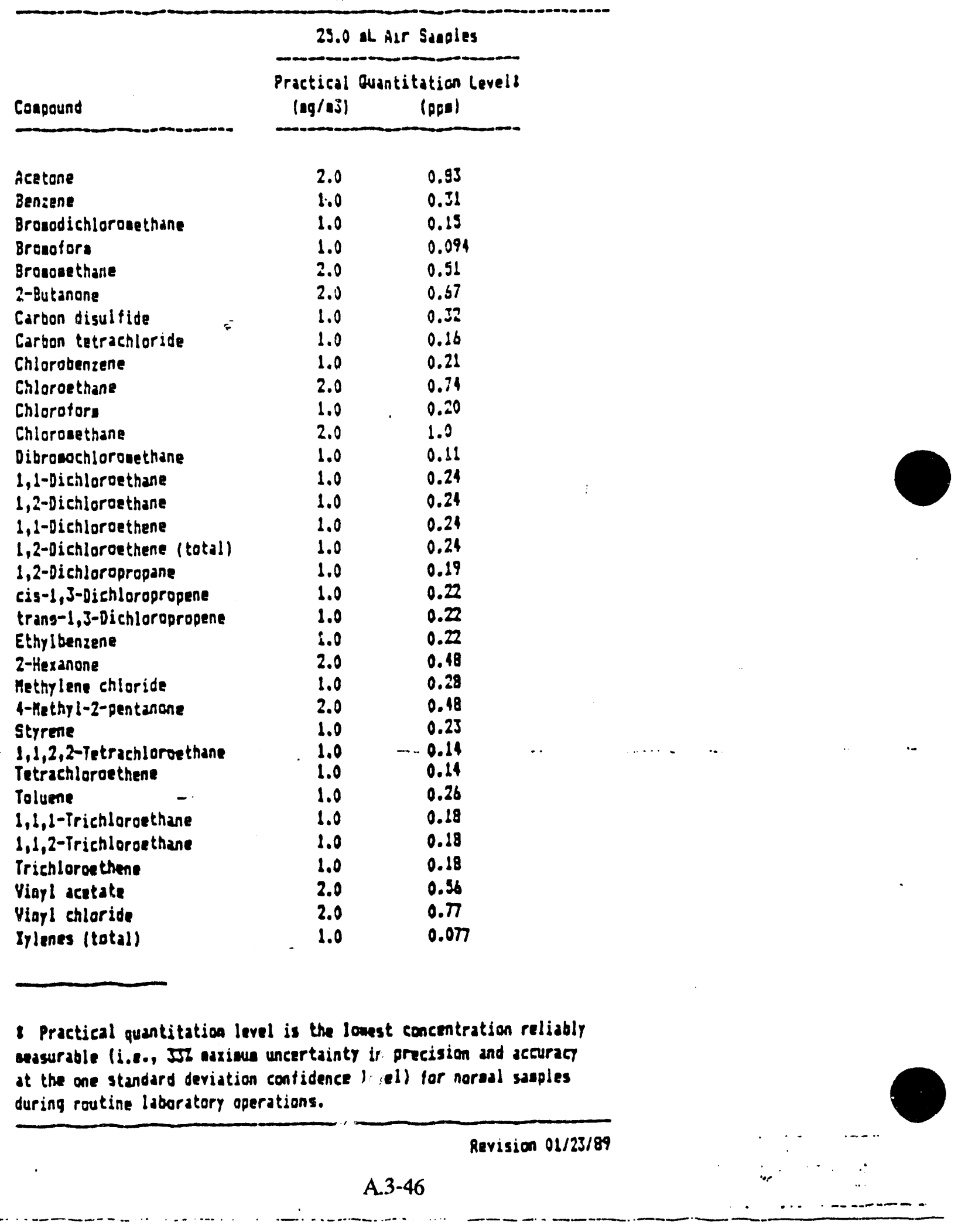


Appendix B

Analytical Results for Subsurface Soil Samples at Landfills II and III

B-1 
-

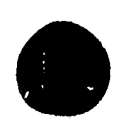

B-2

$$
\text { B-2 }
$$




\section{ANALYTICAL REPORT}

EG\&G IDAHO

SAIC PROJECT NO. 2-8XX-07-667

B-3 


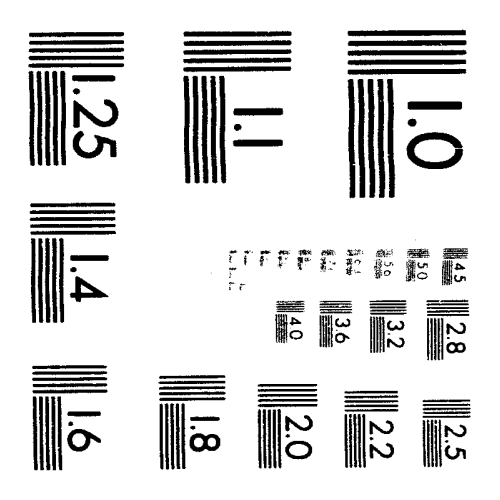



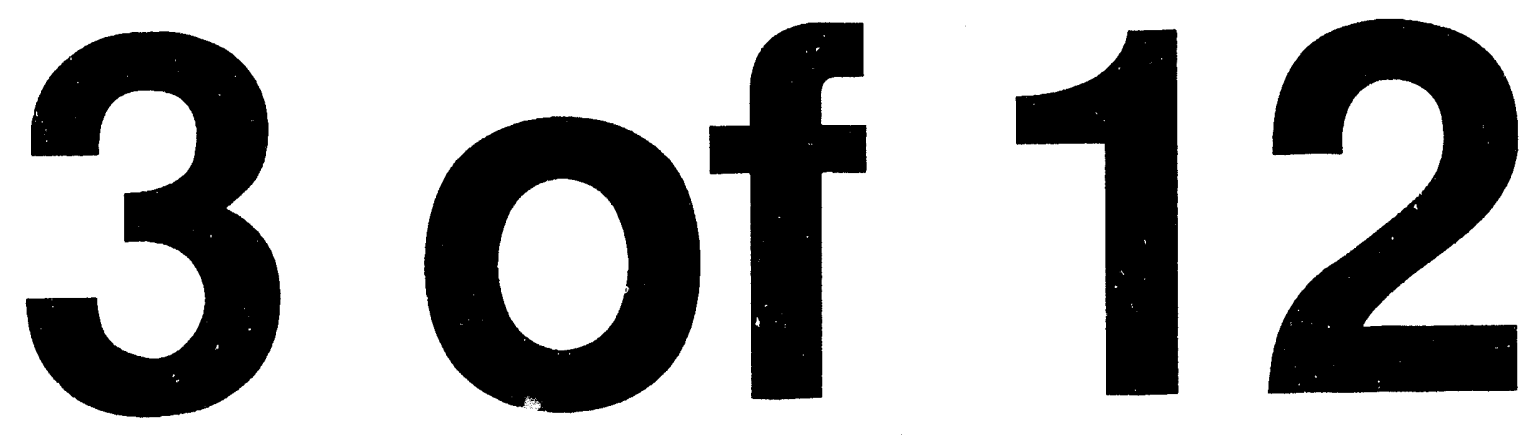
Release of the data contained in this basdcopy bas been autborized by the Luboratory Manager or bis designee, as verified by the following igature.

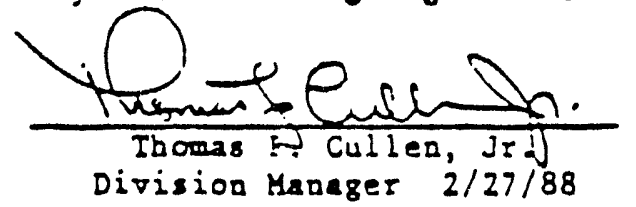


INEL LANDFILL CFA II and III FACILITIES

LABORATORY/FIELD SAMPLING

\begin{tabular}{llllc}
\hline $\begin{array}{c}\text { Lab } \\
\text { Sample Nos. }\end{array}$ & \multicolumn{1}{c}{$\begin{array}{c}\text { Field } \\
\text { Samples }\end{array}$} & $\begin{array}{c}\text { Sample } \\
\text { Depth }\end{array}$ & $\begin{array}{c}\text { Sampling } \\
\text { Date }\end{array}$ & $\begin{array}{c}\text { Sample } \\
\text { Media }\end{array}$ \\
\hline 87346010 & FBI & & $12 / 08 / 87$ & Water \\
87346011 & LF 3-6-FB & & $12 / 11 / 87$ & Water \\
87346012 & Equip. Blank & & $12 / 10 / 87$ & Water \\
87346013 & LF 3-7 & $15 \mathrm{ft}$ & $12 / 10 / 87$ & Soil \\
87346014 & LF 3-6 & $17 \mathrm{ft}$ & $12 / 11 / 87$ & Soil \\
87350026 & LF 3-4-FB & & $12 / 12 / 87$ & Water \\
87350027 & LF 3-4 & $10 \mathrm{ft}$ & $12 / 12 / 87$ & Soil \\
87350028 & LF 3-2-FB & & $12 / 14 / 87$ & Water \\
87350029 & LF 3-2S & $12 \mathrm{ft}$ & $12 / 14 / 87$ & Soil \\
87352018 & LF 2-1 & $17 \mathrm{ft}$ & $12 / 16 / 87$ & Soil \\
87352019 & LF 2-1-FB & & $12 / 15 / 87$ & Water \\
87352020 & LF 2-3S & $23 \mathrm{ft}$ & $12 / 17 / 87$ & Soil \\
87352021 & LF 2-3-FB & & $12 / 17 / 87$ & Water \\
87352022 & Equip. Blank 2 & & $12 / 15 / 87$ & Water \\
87362001 & LF 2-5-FB & & $12 / 21 / 87$ & Water \\
87362002 & LF 2-5 & $1 \mathrm{ft}$ & $12 / 21 / 87$ & Soil \\
87362003 & LF 2-6 & & $12 / 21 / 87$ & Soil \\
87362004 & Equip. Blank 3 & & $12 / 21 / 87$ & Water \\
\hline
\end{tabular}




\section{NOTES FOR ORGANICS RESULTS}

U -- $\quad$ indicates compound was analyzed for but not detected.

$\mathrm{J}$-- $\quad$ indicates an estimated value. This flag is used when estimating the concentration of tentatively identified compounds or when a compound is identified but the concentration is less than the sample quantitation limit.

B -- $\quad$ analyte found in the associated blank as well as in the sample.

D -- identifies all compounds identified in an analysis at a secondary dilution factor.

\section{NOTES FOR INORGANICS RESULTS}

U .- $\quad$ indicates compound was analyzed for but not detected.

B -- $\quad$ indicates the reported value is less than the Contract Required Detection Limit (CRDL) but greater than the Instrument Detection Limit (IDL).

E -- $\quad$ indicates a value estimated or not reported due to the presence of interference.

S -- $\quad$ indicates value determined by Method of Standard Addition.

M -- $\quad$ indicates duplicate injection precision not met.

$\mathrm{N}$-- $\quad$ indicates matrix spike sample recovery is not within control limits.

W -- $\quad$ indicates post-digestion spike for furnace AA analysis is out of control limits (85-115\%) while sample absorbance is less than $50 \%$ of spike absorbance.

* .. $\quad$ indicates duplicate analysis is not within control limits.

+ indicates that the correlation coefficient for Method of Standard Addition is less than 0.995 . 
PRELIMINARY UNVALIDATED RESULTS

VOLATILE ORGANICS ABOVE DETECTION LIMITS

LANDFILL \#2

\begin{tabular}{lccc}
\hline \multicolumn{1}{c}{ Analyte } & Sample ID & $\begin{array}{c}\text { Concentration } \\
\text { (ppb) }\end{array}$ & Qualifier \\
\hline 2-Butanone & LF2-5 & 73 & \\
Acetone & LF2-5 & 800 & B \\
Methylene chloride & LF2-1 & 8 & B \\
Methylene chloride & LF2-3s & 12 & B \\
Methylene chloride & LF2-5 & 60 & B \\
Methylene chloride & LF2-6 & 17 & B \\
\hline
\end{tabular}

\section{EQUIPMENT AND FIELD BLANK EVALUATION VOLATILE ORGANICS \\ LANDFILL \#2}

\begin{tabular}{llcc}
\hline \multicolumn{1}{c}{ Analyte } & Sample ID & $\begin{array}{c}\text { Concentration } \\
\text { (ppb) }\end{array}$ & Qualifier \\
\hline Methylene chloride & Equipblk2 & 6 & B \\
Chloroform & Equipblk3 & 3 & J \\
Methylene chloride & LF2-1-FB & 3 & B \\
Methylene chloride & LF2-3-FB & 4 & B \\
Chloroform & LF2-3-FB & 3 & J \\
Methylene chloride & LF2-5-FB & 1 & B \\
Chloroform & LF2-5-FB & 3 & J \\
\hline
\end{tabular}


PRELIMINARY UNVALIDATED RESULTS

VOLATILE ORGANICS ABOVE DETECTION LIMITS

LANDFILL \#3

\begin{tabular}{lccc}
\hline Analyte & Sample ID & $\begin{array}{c}\text { Concentration } \\
(\mathrm{ppb})\end{array}$ & Qualifier \\
\hline Methylene chloride & LF3-2s & 4 & B \\
Methylene chloride & LF3-4 & 10 & B \\
Methylene chloride & LF3-6 & 73 & B \\
Methylene chloride & LF3-7 & 6 & B \\
Xylene & LF3-4 & 4 & J \\
\hline
\end{tabular}

EQUIPMENT AND FIELD BLANK EVALUATION

VOLATILE ORGANICS

LANDFILL \#3

\begin{tabular}{lccc}
\hline \multicolumn{1}{c}{ Analyte } & Sample ID & $\begin{array}{c}\text { Concentration } \\
(\mathrm{ppb})\end{array}$ & Qualifier \\
\hline & Equipblk1 & 10 & B \\
Acetone & LF3-2-FB & 2 & B \\
Methylene chloride & & & \\
\hline
\end{tabular}


PRELIMINARY UNVALIDATED RESULTS

SEMIVOLATILE ORGANICS ABOVE DETECTION LIMITS

LANDFILL \#2 \& 3

\begin{tabular}{lccc}
\hline Analyte & Sample ID & $\begin{array}{c}\text { Concentration } \\
(\mathrm{ppb})\end{array}$ & Qualifier \\
\hline & & & \\
4-Methylphenol & LF2-5 & 110 & $\mathrm{~J}$ \\
Benzoic acid & LF2-5 & 90 & $\mathrm{~J}$ \\
\hline
\end{tabular}

\section{EQUIPMENT AND FIELD BLANK EVALUATION SEMIVOLATILE ORGANICS \\ LANDFILL \#2 \& 3}

\begin{tabular}{cccc}
\hline \multicolumn{1}{c}{ Analyte } & Sample ID & $\begin{array}{c}\text { Concentration } \\
(\mathrm{ppb})\end{array}$ & Qualifier \\
\hline Bis (2-ethylhexyl)phthalate & Equipblk3 & 40 & B \\
Bis (2-ethylhexyl)phthalate & LF3-2-FB & 15 & \\
Bis (2-ethylhexyl)phthalate & LF3-4-FB & 25 & \\
Bis (2-ethylhexyl)phthalate & LF3-6-FB & 20 & \\
Di-n-octylphthalate & LF3-2-FB & 120 & \\
\hline
\end{tabular}


PRELIMINARY UNVALIDATED RESULTS

INORGANICS ABOVE DETECTION LIMITS

LANDFILL \#2

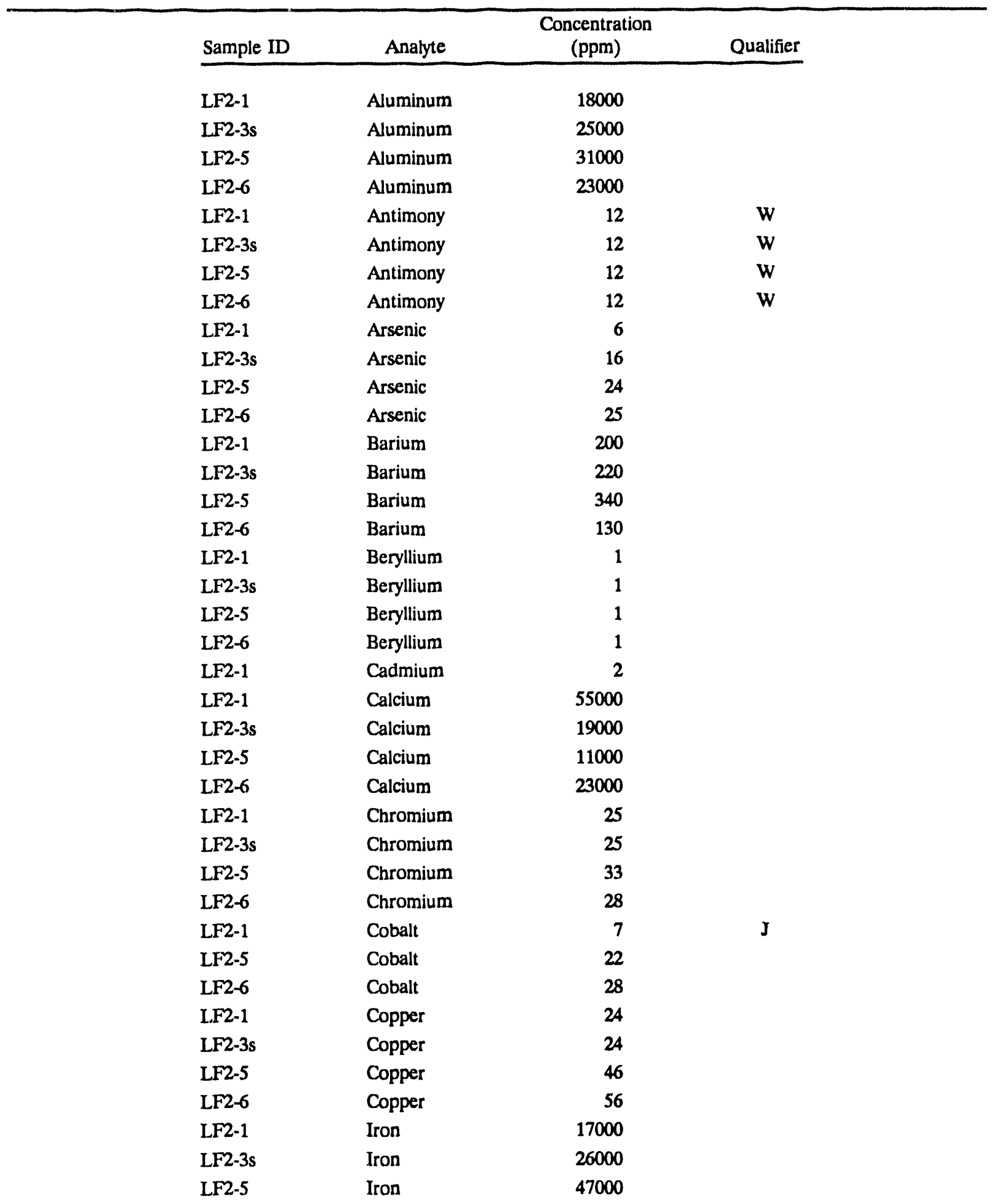


PRELIMINARY UNVALIDATED RESULTS

INORGANICS ABOVE DETECTION LIMITS

LANDFILL \#2 (CONT.)

\begin{tabular}{|c|c|c|c|c|}
\hline \multirow{35}{*}{ - } & Sample ID & Analyte & $\begin{array}{c}\text { Concentration } \\
\text { (ppm) }\end{array}$ & Qualifier \\
\hline & LF2-6 & Iron & 46000 & \\
\hline & LF2-1 & Lead & 14 & \\
\hline & LF2-3s & Lead & 19 & \\
\hline & LF2-5 & Lead & 16 & \\
\hline & LF2-6 & Lead & 12 & \\
\hline & LF2-1 & Magnesium & 8500 & \\
\hline & LF2-3s & Magnesium & 8900.00 & \\
\hline & LF2-5 & Magnesium & 12000.00 & \\
\hline & LF2-6 & Magnesium & 15000.00 & \\
\hline & LF2-1 & Manganese & 190.00 & \\
\hline & LF2-3s & Manganese & 750.00 & \\
\hline & LF2-5 & Manganese & 840.00 & \\
\hline & LF2-6 & Manganese & 830.00 & \\
\hline & LF2-1 & Mercury & 0.02 & J \\
\hline & LF2-3s & Nickel & 26.00 & \\
\hline & LF2-5 & Nickel & 28.00 & \\
\hline & LF2-6 & Nickel & 26.00 & \\
\hline & LF2-1 & Potassium & 7700.00 & \\
\hline & LF2-3s & Potassium & 5600.00 & \\
\hline & LF2-5 & Potassium & 5100.00 & \\
\hline & LF2-6 & Potassium & 3900.00 & \\
\hline & LF2-1 & Selenium & 3.00 & $\mathbf{S}$ \\
\hline & LF2-3s & Selenium & 3.00 & $\mathbf{S}$ \\
\hline & LF2-5 & Selenium & 1.00 & W \\
\hline & LF2-6 & Selenium & 1.00 & w \\
\hline & LF2-1 & Silver & 0.10 & $\mathbf{J}$ \\
\hline & LF2-1 & Vanadium & 45.00 & \\
\hline & LF2-3s & Vanadium & 30.00 & \\
\hline & LF2-5 & Vanadium & 67.00 & \\
\hline & LF2-6 & Vanadium & 81.00 & \\
\hline & LF2-1 & Zinc & 100.00 & \\
\hline & LF2-3s & Zinc & 40.00 & \\
\hline & LF2-5 & Zinc & 32.00 & \\
\hline & LF2-6 & Zinc & 31.00 & \\
\hline
\end{tabular}


EQUIPMENT AND FIELD BLANK EVALUATION

INORGANICS

LANDFILL \#2

\begin{tabular}{llcc}
\hline Sample ID & Analyte & $\begin{array}{r}\text { Concentration } \\
(\mathrm{ppm})\end{array}$ & Qualifier \\
\hline Equipblk1 & Selenium & 5.00 & $\mathrm{~W}$ \\
Equipblk2 & Selenium & 5.00 & $\mathrm{~W}$ \\
Equipblk3 & Selenium & 5.00 & $\mathrm{~W}$ \\
FB-1 & Selenium & 5.00 & \\
LF2-1-FB & Selenium & 5.00 & W \\
LF2-3-FB & Selenium & 5.00 & W \\
LF2-5-FB & Selenium & 5.00 & W \\
LF2-3-FB & Calcium & 9500.00 & \\
\hline
\end{tabular}




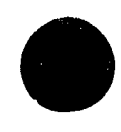

PRELIMINARY UNVALIDATED RESULTS

INORGANICS ABOVE DETECTION LIMITS

LANDFILL \#3

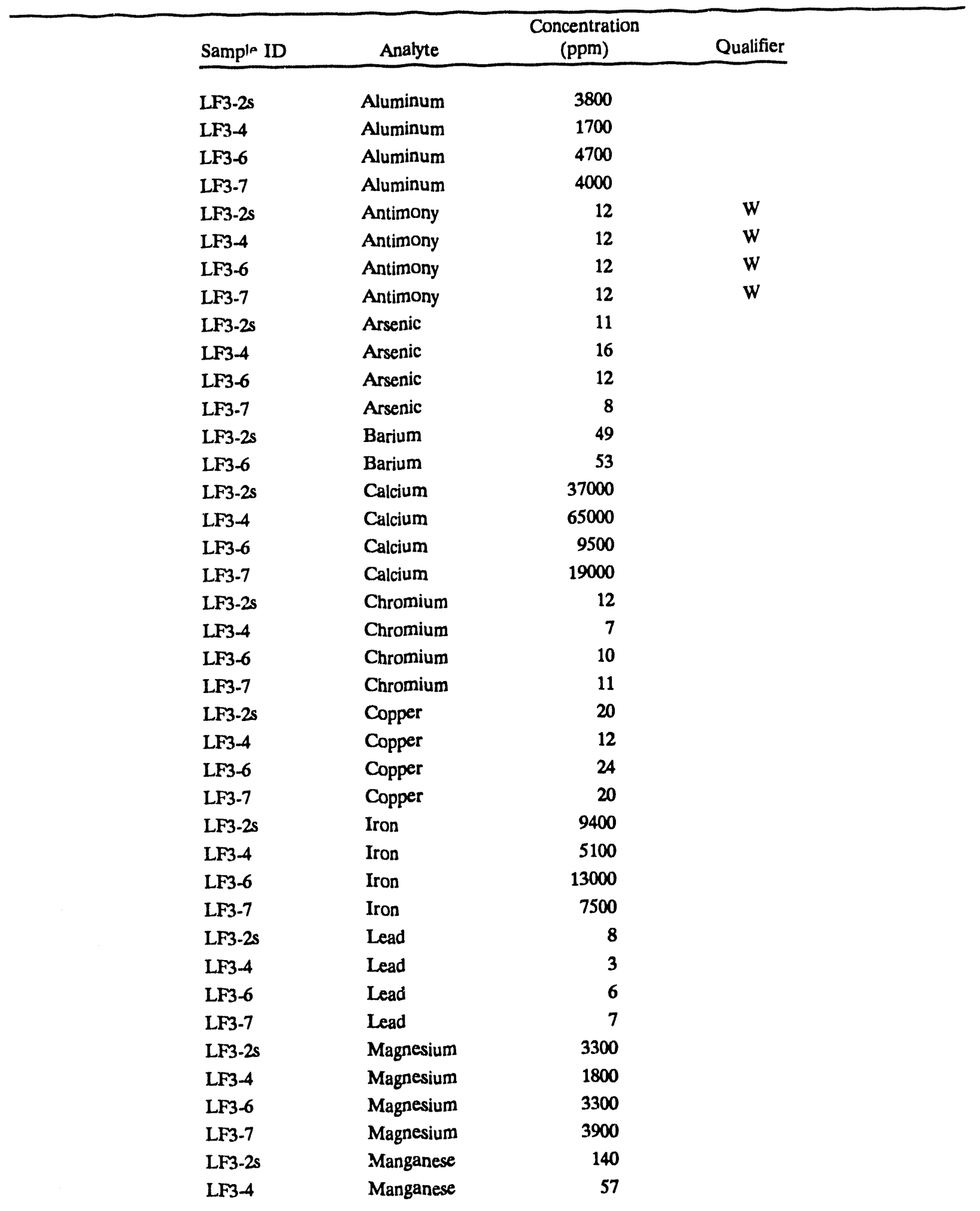


PRELIMINARY UNVALIDATED RESULTS

INORGANICS ABOVE DETECTION LIMITS

LANDFILL \#3 (CONT.)

\begin{tabular}{llcc}
\hline Sample ID & \multicolumn{1}{c}{ Analyte } & $\begin{array}{c}\text { Concentration } \\
(\mathrm{ppm})\end{array}$ & Qualifier \\
\hline LF3-6 & Manganese & 140 & \\
LF3-7 & Manganese & 150 & \\
LF3-2s & Nickel & 11 & \\
LF3-6 & Nickel & 12 & \\
LF3-7 & Nickel & 13 & \\
LF3-2-FB & Selenium & 5.00 & W \\
LF3-4-FB & Selenium & 5.00 & W \\
LF3-6-FB & Selenium & 5.00 & W \\
\hline
\end{tabular}




\section{EQUIPMENT AND FIELD BLANK EVACUATION INORGANICS \\ LANDFILL \#3}

\begin{tabular}{llcc}
\hline Sample ID & Analyte & $\begin{array}{c}\text { Concentration } \\
(\mathrm{ppm})\end{array}$ & Qualifier \\
\hline LF3-2-FB & Selenium & 5.00 & $\mathrm{~W}$ \\
LF3-4-FB & Selenium & 5.00 & W \\
LF3-6-FB & Selenium & 5.00 & $\mathrm{~W}$ \\
\hline
\end{tabular}


PRELIMINARY UNVALIDATED RESULTS

PESTICIDES ABOVE DETECTION LIMITS

LANDFILL \#2 AND 3

\begin{tabular}{llcc}
\hline Sample ID & Analyte & $\begin{array}{c}\text { Concentration } \\
(\mathrm{ppb})\end{array}$ & Qualifier \\
\hline No detects & & \\
\hline
\end{tabular}


Appendix C.1

\section{Validated Groundwater Quality Data for CFA Landfills II and III}

\section{February 1990}


-
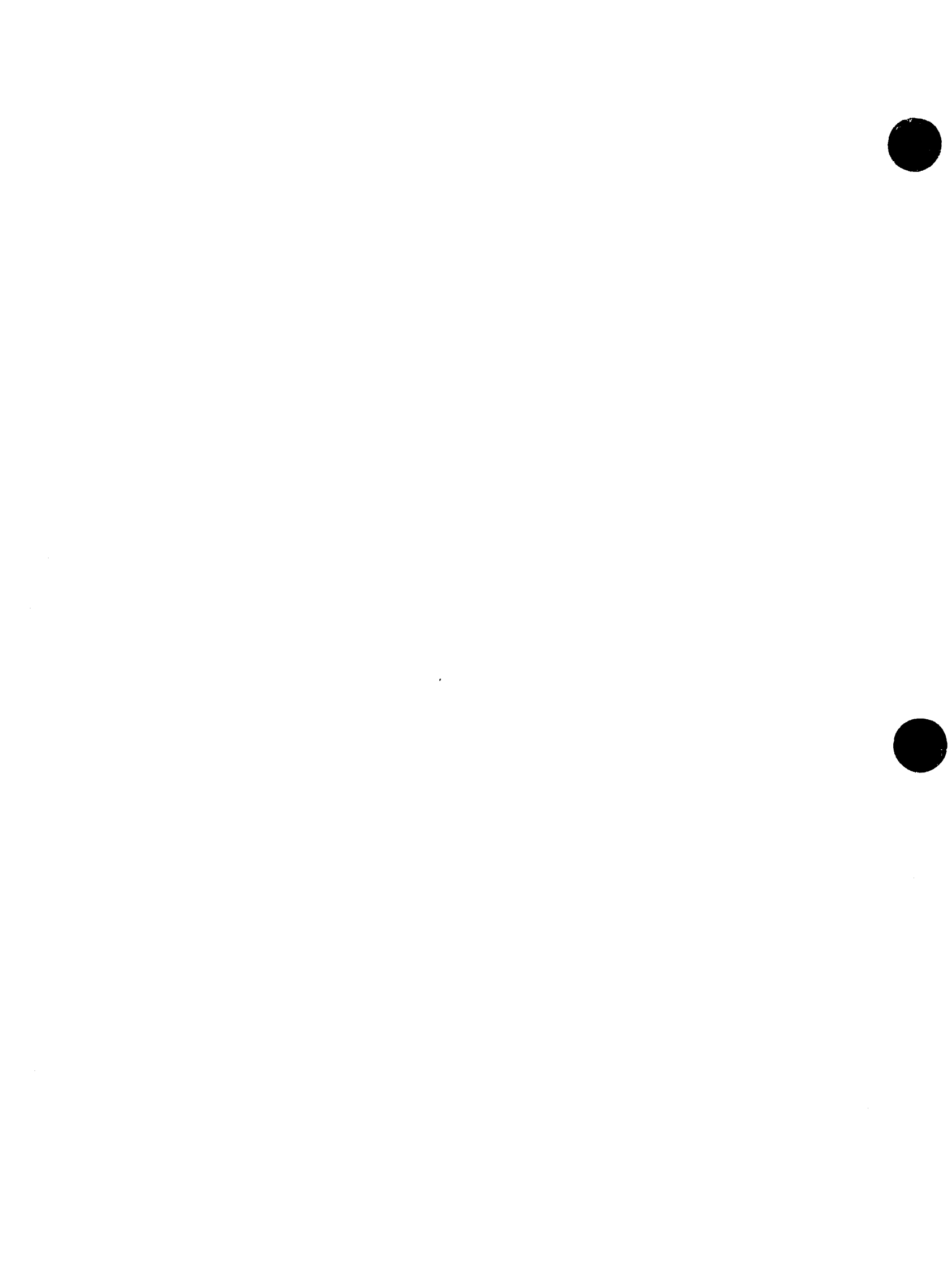

C.1-2 


\section{DATA QUALIFIER DEFINITIONS}

Organic Analysis Data Flags

A - Indicates that a tentatively identified compound is a suspected aldol-condensation product.

B - Used when the analyte is found in the associated blank as well as in the sample.

C - Applies to pesticide results where the identification has been confirmed by GC/MS.

D - Identifies all compounds identified in an analysis at a secondary dilution factor.

E - Identifies compounds which have concentrations that exceed the calibration range of the GC/MS instrument for that specific analysis.

J - Indicates as estimated value.

U - Indicates compound was analyzed for but not detected.

\section{Organic Validation Data Qualifiers}

J - The analyte was positively identified in the sample, but the associated numerical value may not be an accurate representation of the amount actually present in the environmental sample. The data should be seriously considered for decision making and are usable for many purposes.

N - Presumptive evidence of the presence of the material.

NJ - Presumptive evidence of the presence of the material at an estimated quantity.

R - The data are unusable (may or may not be present). Resampling and reanalysis is necessary for verification.

U - The material was analyzed for but was not detected. The associated numerical value is the sample quantitation limit.

UJ - The material was analyzed for, but was not detected. The sample quantitation limit is an estimated quantity.

Reference: Environmental Restoration Department, Sample Management Office Standard Operating Procedures 12.1.4 and 12.1.5 (EG\&G Idaho). 


\section{DATA QUALIFIER DEFINITIONS}

\section{Inorganic Analysis Data Flags}

B - Value is less than the CRDL, but greater than the IDL.

E - Value is estimated because of the presence of interference.

N - Spiked sample recovery not within control limits.

NR - Analyte was not required to be analyzed.

S - Value was determined by the method of standard additions (MSA).

U - Analyte was analyzed for but not detected.

W - Post digestion spike for Furnace AA analysis is out of control limits (85\% to $115 \%$ ), while sample abscrbance is less than $50 \%$ of spike absorbance.

* - Duplicate analysis not within control limits.

\section{Methods}

A - Flame AA.

CV - Manual Cold Vapor AA.

F - Furnace AA.

NR - Not run, analyte not required to be analyzed.

P - Inductively Coupled Plasma - Atomic Emission Spectrometry.

\section{Inorganic Validation Data Qualifiers}

J - The analyte was analyzed for and was positively identified, but the associated numerical value may not be consistent with the amount actually present in the environmental sample.

R - The data are unusable.

U - The material was analyzed for, but was not detected above the level of the associated value. The associated value is either the sample quantitation limit or the sample detection limit.

UJ - The material was analyzed for, but was not detected. The associated value is an estimate and may not accurately reflect the instrument detection limit in the sample matrix. 
CFA Landfills II and III fY89 Wells - 1st Quarter February 1990 S\&A Data Document

TABLE _-_-- CFA LANDFILLS II AND III FY89 HELLS - 524.2 VOLATILE ORGANIC DATA

Page 1 of 2

\begin{tabular}{|c|c|c|c|c|c|}
\hline $\begin{array}{l}\text { AREA } \\
\text { LOCATION } \\
\text { TYPE OF LOCATION } \\
\text { SAMPLE NUMBER } \\
\text { MEDIA } \\
\text { UNITS } \\
\text { SDG NUMBER } \\
\end{array}$ & $\begin{array}{r}\text { CFA } \\
\text { 03-LF3-8 } \\
\text { DOWN GRAD HELL } \\
\text { CFAO101A } \\
\text { GROUND HATER } \\
\text { Ug/L } \\
\text { CFAO101A } \\
\end{array}$ & $\begin{array}{r}\text { CFA } \\
\text { O2-LF2-8 } \\
\text { DOUN GRAD HELL } \\
\text { CFAO201A } \\
\text { LROUND WATER } \\
\text { Ug/L } \\
\text { CFA0101A } \\
\end{array}$ & $\begin{array}{r}\text { CFA } \\
\text { O2-LF2-9 } \\
\text { DOWN GRAD HELL } \\
\text { CFAO301A } \\
\text { GROUND HATER } \\
\text { Ug/L } \\
\text { CFAO101A } \\
\end{array}$ & $\begin{array}{r}\text { CFA } \\
\text { O2-LF2-9 } \\
\text { DOWN GRAD HELL } \\
\text { CFAO302A } \\
\text { GROUND WATER } \\
\text { Ug/L } \\
\text { CFAO101A } \\
\end{array}$ & $\begin{array}{r}\text { CFA } \\
\text { 02-LF2-11 } \\
\text { UPGRADIENT WELL } \\
\text { CFAO401A } \\
\text { GROUND WATER } \\
\text { Ug/L } \\
\text { CFA0101A }\end{array}$ \\
\hline $\begin{array}{l}\text { FIELD MEASUREMENTS } \\
\text { pH } \\
\text { Conductivity (uS) } \\
\text { Temperature (C) }\end{array}$ & $\begin{array}{r}8.0 \\
0.6 \\
10.4\end{array}$ & $\begin{array}{r}9.1 \\
0.6 \\
10.3\end{array}$ & $\begin{array}{r}9.1 \\
0.6 \\
10.4\end{array}$ & $\begin{array}{r}9.1 \\
0.6 \\
10.4\end{array}$ & $\begin{array}{r}7.6 \\
0.7 \\
10.1\end{array}$ \\
\hline $\begin{array}{l}\text { TARGET COMPOUNDS } \\
1,1,1-\text { Trichloroethane } \\
1,2-\text { ibromo-3-chloropropane }\end{array}$ & 0.5 UR & 0.5 UR & $\begin{array}{l}1 \\
0.5 \text { UR }\end{array}$ & 0.5 UR & 0.5 UR \\
\hline $\begin{array}{l}\text { Dilution Factor } \\
\text { Total (Allowed) Hold Time }\end{array}$ & $\begin{array}{r}1.000 \\
14(14) d\end{array}$ & $\begin{array}{c}1.000 \\
15(14) d^{*}\end{array}$ & $15(14) d^{*}$ & $15(14) d^{\star}$ & $\begin{array}{r}1.000 \\
14(14) d\end{array}$ \\
\hline
\end{tabular}


CFA Landfills II and III FY89 Hells - 1st Quarter February 1990 S\&A Data Document

TABLE _-_-_ CFA LANDFILLS II AND III FY89 WELLS - 524.2 Volatille ORgANic dATA (Continued)

\begin{tabular}{l} 
AREA \\
LOCATION \\
TYPE OF LOCATION \\
SAMPLE NUMBER \\
MEDIA \\
UN!TS \\
SDG MUMBER \\
\hline
\end{tabular}

CFA
O2-LF2-11
UPGRADIENT WELL
CFAO402A
GROUND MATER
Ug/L
CFAO101A

FIELD MEASUREMENTS

pH

Conductivity (uS)

Temperature (C)

TARGET COMPOUNDS

1,2-Dibromo-3-chloropropane

$0-2$ UR

Dilution Factor

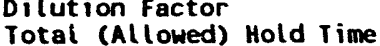

0.5 UR

1.000

$9-11-92$ 
TABLE -. CFA LANDFILLS II AND III FY89 WELLS - 524.2 VOLATILE ORGANIC FIELD BLANK DATA

Page 1 of 1

AREA

LOCATION

TYPE OF LOCAT

SAMPLE NUMBER

UHITS

SOG MUMBER

$$
\begin{array}{r}
\text { CFA } \\
\text { ASTM TYPEII H20 } \\
\text { OO-FIELD BLANK } \\
\text { CFA0901A } \\
\text { HATER } \\
\text { Ug/L } \\
\text { CFA0101A }
\end{array}
$$

TARGET COMPOUNDS

chloroform

1,2-D ibromo-3-chloropropane

Dilution Factor

Total (Allowed) Hold Time

$$
\begin{gathered}
3 \\
0.5 \text { UR } \\
15(14) d^{\star}
\end{gathered}
$$

$9-11-92$ 
CFA Landfills II and III FY89 Wells - 1st Quarter February 1990 S\&A Data Document

TABLE _--- CFA LANDFILLS II AND III FYB9 WELLS - 524.2 VOLATILE ORGANIC IRIP BLANK DATA

Page 1 of 2

\begin{tabular}{|c|c|c|c|c|c|}
\hline $\begin{array}{l}\text { AREA } \\
\text { LOCATION } \\
\text { TYPE OF LOCATION } \\
\text { SAMPLE NUMBER } \\
\text { MEDIA } \\
\text { UNITS } \\
\text { SDG MUMBER } \\
\end{array}$ & $\begin{array}{r}\text { CFA } \\
\text { QC } \\
\text { DO-TRIP BLANK } \\
\text { CFA0501A } \\
\text { WATER } \\
\text { Ug/L } \\
\text { CFA0101A } \\
\end{array}$ & $\begin{array}{r}\text { CFA } \\
\text { OC } \\
\text { O0-TRIP BLANK } \\
\text { CFA0601A } \\
\text { WATER } \\
\text { Ug/L } \\
\text { CFA0101A } \\
\end{array}$ & $\begin{array}{r}\text { CFA } \\
\text { OC } \\
\text { O0-TRIP BLANK } \\
\text { CFAOT01A } \\
\text { UATER } \\
\text { Ug/L } \\
\text { CFA0101A } \\
\end{array}$ & $\begin{array}{r}\text { CFA } \\
\text { QC } \\
\text { O0-TRIP BLANK } \\
\text { CFA0B01A } \\
\text { WATER } \\
\text { Ug/L } \\
\text { CFA0101A } \\
\end{array}$ & $\begin{array}{r}\text { CFA } \\
\text { QC } \\
\text { O0-TRIP BLANK } \\
\text { CFA0802A } \\
\text { HATER } \\
\text { Ug/L } \\
\text { CFA0101A } \\
\end{array}$ \\
\hline $\begin{array}{l}\text { TARGET COAPOUHDS } \\
\text { Methylene chloride } \\
\text { Chloroform } \\
1,2 \text {-D ibromo-3-chloropropane }\end{array}$ & $\begin{array}{l}\ldots . . \\
0.5 \text { UR }\end{array}$ & $\begin{array}{c}9 \\
0.5 \text { UR }\end{array}$ & $\begin{array}{c}4 \\
0.5 \text { UR }\end{array}$ & 0.5 UR & 0.5 UR \\
\hline $\begin{array}{l}\text { Dilution Factor } \\
\text { Total (Allowed) Hold time }\end{array}$ & $\begin{array}{c}1.000 \\
15^{(14) d^{\star}}\end{array}$ & $\begin{array}{c}1.000 \\
15(14) d^{\star}\end{array}$ & $\begin{array}{c}1.000 \\
15(14) d^{\star}\end{array}$ & $\begin{array}{c}1.000 \\
15(14) d^{\star}\end{array}$ & $\begin{array}{r}1.000 \\
14(14) d\end{array}$ \\
\hline
\end{tabular}

9-11-92 

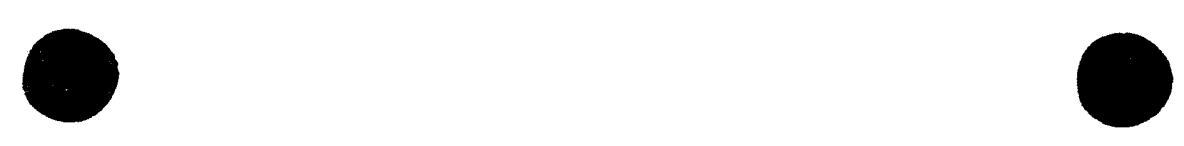

CFA Landfills II and III FY89 Wells - Ist Quarter February 1990 S\&A Data Document

TABLE --:- CFA LAHDFILLS II AND III FY89 WELLS - 524.2 VOLATILE ORGANIC tRIP BLANK DATA (Continued)

LOCATION

TYPE OF LOCATIO

SAMPLE MUMBER

MEDIA

UNITS

CFA
OC
OC

OO-TRIP BLANK
CFA0803A

CFA0803A
HATER TFAO804A
HATER

Ug/L
CFAO101A
CFA01014

TARGET COMPOUNDS

Methylene Chloríde

Chloroform

1,2-Dibromo-3-chloropropane

3

0.5 UR

3

Dilution Factor

Total (Allowed) Hold Time

1.000

$14(14) d$

0.5 UR

1.000

9-11-92 
CFA Landfills II and III FY89 Wells - 1st Quarter February 1990 S\&A Data Document

TABLE _--- CFA LANDFILLS II AND III FY89 hells - SEMIVOLATILE APPENDIX IX ORGANIC DATA

Page 1 of 2

\begin{tabular}{|c|c|c|c|c|c|}
\hline $\begin{array}{l}\text { AREA } \\
\text { LOCATION } \\
\text { TYPE OF LOCATION } \\
\text { SAMPLE NUMBER } \\
\text { MEDIA } \\
\text { UNITS } \\
\text { SOG NUMBER } \\
\end{array}$ & $\begin{array}{r}\text { CFA } \\
\text { 03-LF3-8 } \\
\text { DOWN GRAD HELL } \\
\text { CFAO101D } \\
\text { GROUND WATER } \\
\text { Ug/L } \\
\text { CFAOTO1D } \\
\end{array}$ & $\begin{array}{r}\text { CFA } \\
03-L F 3-8 \\
\text { DOWN GRAD WELL } \\
\text { CFA0101DRE } \\
\text { GROUND WATER } \\
\text { Ug/L } \\
\text { CFAO101D } \\
\end{array}$ & $\begin{array}{r}\text { CFA } \\
\text { O2-LF2-8 } \\
\text { DOWN GRAD HELL } \\
\text { CFAO201D } \\
\text { GROUND WATER } \\
\text { Ug/L } \\
\text { CFAO101D } \\
\end{array}$ & $\begin{array}{r}\text { CFA } \\
\text { O2-LF2-9 } \\
\text { DOWN GRAD WELL } \\
\text { CFA0301D } \\
\text { GROUND WATER } \\
\text { Ug/L } \\
\text { CFAO101D } \\
\end{array}$ & $\begin{array}{r}\text { CFA } \\
\text { O2-LF2-9 } \\
\text { DOWN GRAD WELL } \\
\text { CFAO302D } \\
\text { GROUND WATER } \\
\text { Ug/L } \\
\text { CFAO1010 } \\
\end{array}$ \\
\hline $\begin{array}{l}\text { FIELD MEASUREMENTS } \\
\text { pH } \\
\text { Conductivity (uS) } \\
\text { Temperature (C) }\end{array}$ & $\begin{array}{r}8.0 \\
0.6 \\
10.4\end{array}$ & $\begin{array}{r}8.0 \\
0.6 \\
10.4\end{array}$ & $\begin{array}{r}9.1 \\
0.6 \\
10.3\end{array}$ & $\begin{array}{r}9.1 \\
0.6 \\
10.4\end{array}$ & $\begin{array}{r}9.1 \\
0.6 \\
10.4\end{array}$ \\
\hline IARGET COMPOUNDS & & & & & \\
\hline None Detected & & & & & \\
\hline $\begin{array}{l}\text { Dilution Factor } \\
\text { Extraction (Allowed) Hold rime } \\
\text { Analytical (Allowed) Hold time }\end{array}$ & $\begin{array}{l}1.000 \\
17(7) d^{\star} \\
5(40) d\end{array}$ & $\begin{array}{l}1.000 \\
17(7) d^{\star} \\
7(40) d\end{array}$ & $\begin{array}{l}1.000 \\
18(7) d^{\star} \\
4(40) d\end{array}$ & $\begin{array}{l}1.000 \\
18(7) d^{\star} \\
4(40) d\end{array}$ & $\begin{array}{l}1.000 \\
18(7) d^{\star} \\
4(40) d\end{array}$ \\
\hline
\end{tabular}

$9-11-92$ 

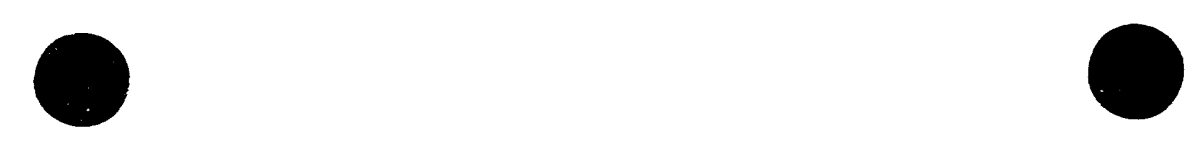

CFA Landfills II and III FY89 Wells - 1st Quarter February 1990 S\&A Data Document

TABLE _._- CFA LANDFILLS II AND 111 FYBg Wells - SEMIVOLATILE APPENDIX IX ORGANIC DATA (Continued)

\begin{tabular}{|c|c|c|}
\hline $\begin{array}{l}\text { AREA } \\
\text { LOCATION } \\
\text { TYPE OF LOCAIION } \\
\text { SAMPLE NUMBER } \\
\text { MEDIA } \\
\text { UNITS } \\
\text { SDG NUMBER } \\
\end{array}$ & $\begin{array}{r}\text { CFA } \\
\text { O2-LF2-11 } \\
\text { UPGRADIENT WELL } \\
\text { CFAO4010 } \\
\text { GROUND WATER } \\
\text { Ug/L } \\
\text { CFA0101D } \\
\end{array}$ & $\begin{array}{r}\text { CFA } \\
\text { O2-LF2-11 } \\
\text { UPGRADIENT HELL } \\
\text { CFA0402D } \\
\text { GROUND HATER } \\
\text { Ug/L } \\
\text { CFA0101D } \\
\end{array}$ \\
\hline $\begin{array}{l}\text { FIELD MEASUREMENTS } \\
\mathrm{pH} \\
\text { Conductivity (uS) } \\
\text { Temperature (C) }\end{array}$ & $\begin{array}{r}7.6 \\
0.7 \\
10.1\end{array}$ & $\begin{array}{r}7.6 \\
0.7 \\
10.1\end{array}$ \\
\hline IARGET COMPOUNDS & & \\
\hline None Detected & & \\
\hline $\begin{array}{l}\text { Dilution factor } \\
\text { Extraction (A!lowed) Hold Time } \\
\text { Analytical (Allowed) Hold Time }\end{array}$ & $\begin{array}{l}1.000 \\
18(7) d^{\star} \\
5(40) d\end{array}$ & $\begin{array}{l}1.000 \\
17(7) d^{*} \\
7(40) d\end{array}$ \\
\hline
\end{tabular}


CFA Landfills II and III FY89 Wells - 1st Quarter February 1990 S\&A Data Document

TABLE _-_- CFA LANDFILLS II AND $111 \mathrm{FY} 89$ Wells - SEMIVOLATILE APPENDIX IX ORGANIC FIELD BLANK DATA

Page 1 of 1

\begin{tabular}{lr} 
AREA & QC \\
LOCATION & OO-FIELD BLANK \\
TYPE DF LOCATION & ASTM TYPEII H20 \\
SAMPLE NUMBER & CFAO901D \\
MEDIA & WATER \\
UNIIS & U9/L \\
SDG NUMBER & CFAO1010 \\
\hline
\end{tabular}

FIELD MEASUREMENTS

$\mathrm{pH}$

Conductivity (uS)

Temperature (C)

IARGET COMPOUNDS

None Detected

Dilution Factor

Extraction (Allowed) Hold Time

1.000

Analytical (Allowed) Hold Time

$18(7) \mathrm{d}^{*}$

$9-11-92$ 
CFA Landfills II and III fY89 Mells - 1st Quarter February 1990 seA Data Document

TABLE _-_-_ CFA LANDFILLS II AND III FY89 WELLS - INORGANIC DATA

Page 1 of 1

\begin{tabular}{|c|c|c|c|c|c|c|}
\hline $\begin{array}{l}\text { AREA } \\
\text { LOCATION } \\
\text { TYPE OF LOCAIION } \\
\text { SAMPLE NUMBER } \\
\text { MEDIA } \\
\text { UNITS } \\
\text { SOG NUMBER } \\
\end{array}$ & $\begin{array}{r}\text { CFA } \\
\text { 03-LF3-8 } \\
\text { DOWN GRAD WELL } \\
\text { CFAO101H } \\
\text { GROUND WATER } \\
\text { Ug/L } \\
\text { CFAO101H } \\
\end{array}$ & $\begin{array}{r}\text { CFA } \\
\text { O2-LF2-8 } \\
\text { DOWN GRAD MELL } \\
\text { CFAO2O1H } \\
\text { GROUND WATER } \\
\text { Ug/L } \\
\text { CFAO101H } \\
\end{array}$ & $\begin{array}{r}\text { CFA } \\
\text { O2-LF2-9 } \\
\text { DONN GRAD WELL } \\
\text { CFAO301H } \\
\text { GROUND HATER } \\
\text { Ug/L } \\
\text { CFA0101H } \\
\end{array}$ & $\begin{array}{r}\text { CFA } \\
\text { O2-LF2-9 } \\
\text { DOWN GRAD WELL } \\
\text { CFAO302H } \\
\text { GROUND WATER } \\
\text { Ug/L } \\
\text { CFAO101H } \\
\end{array}$ & $\begin{array}{r}\text { CFA } \\
\text { O2-LF2-11 } \\
\text { UPGRADIENT WELL } \\
\text { CFAO401H } \\
\text { GROUND WATER } \\
\text { Ug/L } \\
\text { CFAO101H } \\
\end{array}$ & $\begin{array}{r}\text { CFA } \\
\text { O2-LF2-11 } \\
\text { UPGRADIENT WELL } \\
\text { CFAO402H } \\
\text { GROUND WATER } \\
\text { Ug/L } \\
\text { CFAO101H } \\
\end{array}$ \\
\hline $\begin{array}{l}\text { F1ELD MEASUREMENTS } \\
\text { pH } \\
\text { Conduct ivity (uS) } \\
\text { Temperature (C) }\end{array}$ & $\begin{array}{r}8.0 \\
0.6 \\
10.4\end{array}$ & $\begin{array}{r}9.1 \\
0.6 \\
10.9\end{array}$ & $\begin{array}{r}9.1 \\
0.6 \\
10.3\end{array}$ & $\begin{array}{r}9.1 \\
0.6 \\
10.3\end{array}$ & $\begin{array}{r}7.6 \\
0.7 \\
10.1\end{array}$ & $\begin{array}{r}7.6 \\
0.7 \\
10.1\end{array}$ \\
\hline $\begin{array}{l}\text { ANALYTES } \\
\text { Arsenic } \\
\text { Barium } \\
\text { Cadmium } \\
\text { Chromium } \\
\text { Iron }\end{array}$ & $\begin{array}{c}80.0 \text { B } \\
\cdots \cdots \\
312 \\
5080\end{array}$ & $\begin{array}{l}147 \text { B } \\
206 \\
1100\end{array}$ & $\begin{array}{l}\cdots-. \\
1298 \\
\cdots- \\
293 \\
4190\end{array}$ & $\begin{array}{l}\cdots- \\
130 \text { B } \\
\cdots-- \\
307 \\
4370\end{array}$ & $\begin{array}{l}\cdots \\
121 \text { B } \\
\cdots-. \\
379 \\
5410\end{array}$ & $\begin{array}{r}\cdots \\
121 \\
\cdots \\
358 \\
4500\end{array}$ \\
\hline $\begin{array}{l}\text { Lead } \\
\text { Manganese } \\
\text { Mercury } \\
\text { Seleniun } \\
\text { Silver }\end{array}$ & $\begin{array}{c}2.38 \\
44.0 \\
\cdots \\
\cdots \\
\cdots\end{array}$ & $\begin{array}{c}\cdots \\
16.0 \\
\cdots \\
\cdots \\
\cdots\end{array}$ & $\begin{array}{c}\cdots \\
33.0 \\
\cdots \\
\cdots \\
\cdots\end{array}$ & $\begin{array}{c}\cdots .- \\
36.0 \\
\cdots \\
\cdots \\
\cdots\end{array}$ & $\begin{array}{c}\ldots 6 \\
\ldots \\
\ldots \\
\ldots\end{array}$ & $\begin{array}{c}\cdots \\
66.0 \\
\cdots \\
\cdots\end{array}$ \\
\hline Sodium & 38100 & 35700 & 36200 & 37400 & 42000 & 42300 \\
\hline $\begin{array}{l}\text { Total (Allowed) Hold Time } \\
\text { Total (Allowed) Hold Time } \\
\text { Total (Allowed) Hold Time }\end{array}$ & $\begin{array}{l}17(180) d \\
14(26) d \\
17(180) d\end{array}$ & $\begin{array}{r}17(180) d \\
14(26) d \\
17(180) d\end{array}$ & $\begin{array}{l}17(180) d \\
15(26) d \\
17(180) d\end{array}$ & $\begin{array}{l}17(180) d \\
14(26) d \\
17(180) d\end{array}$ & $\begin{array}{l}17(180) d \\
14(26) d \\
17(180) d\end{array}$ & $\begin{array}{l}17(180) d \\
14(26) d \\
17(180) d\end{array}$ \\
\hline
\end{tabular}
a. ICP
b. CVAAS
c. GFAAS
$9-11-92$ 
CFA Landfills II and III FY89 Wells - 1st Quarter February 1990 S\&A Dato Document

TABLE _-_- CFA LANDFILLS II AND 111 FY89 MELLS - INORGANIC FIELD BLANK DATA

Page 1 of 1

\section{AREA}

LOCATION

TYPE OF LOCATION

SAMPLE NUMBER

MEDIA

UDITS NUMBER

\section{ANALYIES}

Arsenic

Barium

Cadmium

Chromium

I ron

Lead

Manganese

Mercury

Selenium

Silver

Sodiun

Total (Allowed) Hold Timea Total (Allowed) Hold Time ${ }^{b}$ Total (Allowed) Hold Time ${ }^{c}$
a. ICP
b. CVAAS
c. GFAAS
9-11-92

ASTM TYPEII HZO

OO-FIELD BLANK

CFA0901H

WATER

CFAO101H

-..

-..

$-$.

$79.0 \mathrm{~B}$

-..

-..

$\cdots$

340

$17(180) d$

$15(26) d$

$17(180) d$ 
CFA Landfills 11 and III FY89 Wells - 1st Quarter February 1990 S\&A Data Document - Method Validation Level A

TABLE _-_-_ CFA LANDFILLS 11 AND 111 FY89 WELLS - NON-METALS DATA

Page 1 of 1

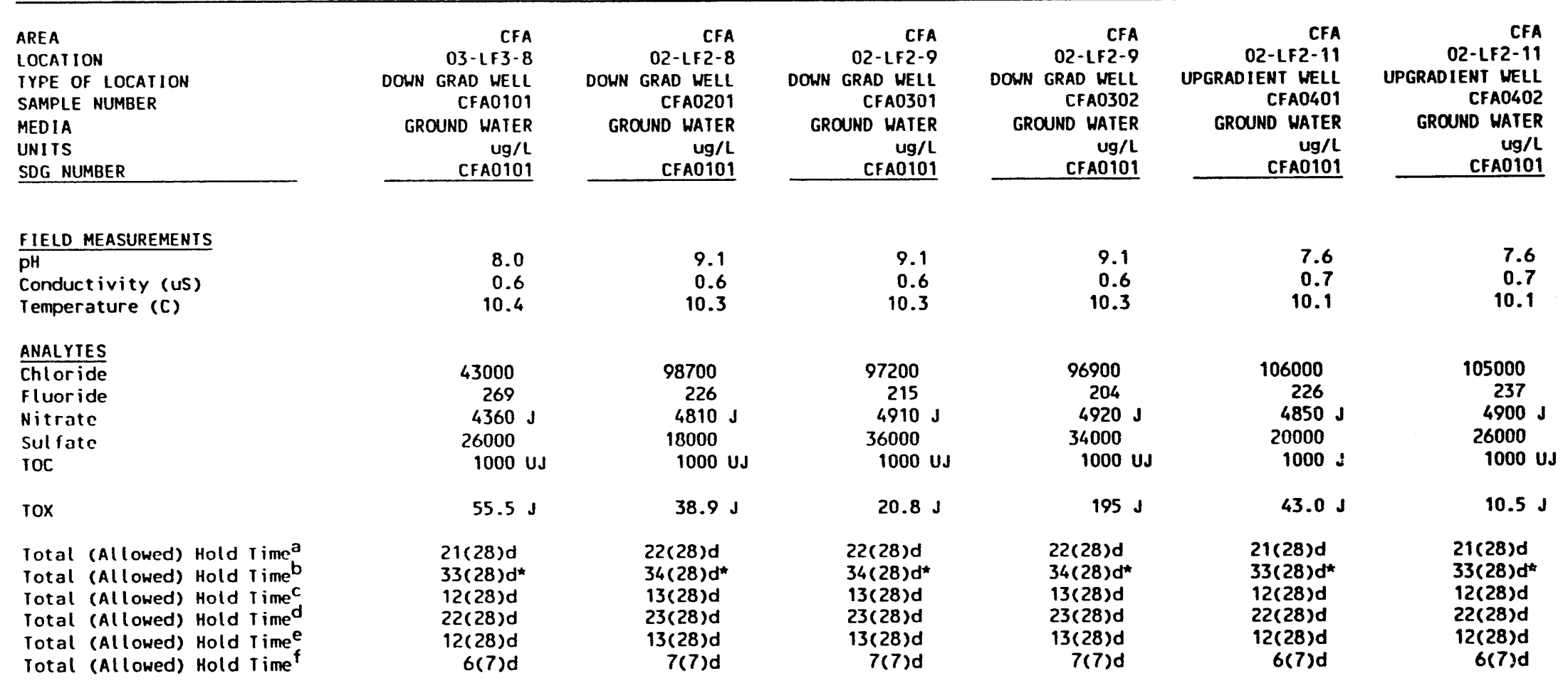

\footnotetext{
a. Chloride - Method 9251

b. Fluoride - Method 340.3

c. Nitrate - Method 353.1

d. Sulfate - Nethod 9036

e. TOC - Method 9060

f. TOX - Methad 9020

9-22-92
} 
CFA Landfills 11 and III FY89 Hells - 1st Quarter February 1990 S\&A Data Document - Method Validation Level A

TABLE _._- CFA LANDFILLS II AND III FY89 WELLS - NON-METALS FIELD BLANK DATA

Page 1 of 1

\begin{tabular}{|c|c|c|}
\hline \multicolumn{2}{|l|}{ AREA } & CFA \\
\hline \multirow{2}{*}{\multicolumn{2}{|c|}{$\begin{array}{l}\text { LOCATION } \\
\text { TYPE OF LOCATION }\end{array}$}} & ASTM TYPEII HZO \\
\hline & & OO-FIELD BLANK \\
\hline \multicolumn{2}{|l|}{ SAMPLE NUMBER } & CFA0901 \\
\hline \multicolumn{2}{|l|}{ MEDIA } & HATER \\
\hline \multicolumn{2}{|l|}{ UNITS } & $\mathrm{ug} / \mathrm{L}$ \\
\hline \multicolumn{2}{|l|}{ SDG NUMBER } & CFA0101 \\
\hline \multicolumn{3}{|l|}{ ANALYIES } \\
\hline \multicolumn{2}{|l|}{ Chloride } & 1400 \\
\hline \multirow{2}{*}{\multicolumn{2}{|c|}{$\begin{array}{l}\text { Fluoride } \\
\text { Nitrate }\end{array}$}} & -.- \\
\hline & & $23.8 \mathrm{~J}$ \\
\hline Sulfate & & $\cdots$ \\
\hline \multicolumn{2}{|l|}{ TOC } & $1000 \mathrm{U}$ \\
\hline \multicolumn{2}{|l|}{ TOX } & 30.8 \\
\hline \multirow{6}{*}{\multicolumn{2}{|c|}{$\begin{array}{l}\text { Total (Allowed) Hold Timea } \\
\text { Total (Allowed) Hold Time } \\
\text { Total (Allowed) Hold Time } \\
\text { Total (Allowed) Hold Time } \\
\text { Total (All owed) Hold Time } \\
\text { Total (All owed) Hold Timef }\end{array}$}} & $22(28) d$ \\
\hline & & $34(28) d^{\star}$ \\
\hline & & $13(28) d$ \\
\hline & & $23(28) d$ \\
\hline & & $13(28) d$ \\
\hline & & $7(7) d$ \\
\hline
\end{tabular}
a. Chloride - Method 9251
b. Fluoride - Method 340.3
c. Nitrate - Method 353.1
d. Sulfate - Method 9036
e. TOC - Method 9060
f. TOX - Method 9020

$9-22-92$

$$
\begin{aligned}
& 1400 \\
& \cdots-. \\
& 23.8 \mathrm{~J} \\
& \cdots-. \\
& 1000 \mathrm{UJ} \\
& 30.8 \\
& \\
& 22(28) \mathrm{d} \\
& 34(28) \mathrm{d}^{\star} \\
& 13(28) \mathrm{d} \\
& 23(28) \mathrm{d} \\
& 13(28) \mathrm{d} \\
& 7(7) \mathrm{d}
\end{aligned}
$$


CFA Landfills il and III FY89 Wells - 1st Quarter February 1990 S\&A Data Document

TABLE _-_- CFA LANDFILLS II AND III FY89 WELLS - ORGANOCHLORINE PESTICIDE APPENDIX IX DATA

Page 1 of 2

\begin{tabular}{|c|c|c|c|c|c|c|}
\hline $\begin{array}{l}\text { AREA } \\
\text { LOCATION } \\
\text { TYPE OF LOCATION } \\
\text { SAMPLE NUMBER } \\
\text { MEDIA } \\
\text { UNITS } \\
\text { SOG NUMBER } \\
\end{array}$ & $\begin{array}{r}\text { CFA } \\
\text { 03-LF3-8 } \\
\text { DOWN GRAD WELL } \\
\text { CFAO101G } \\
\text { GROUND WATER } \\
\text { Ug/L } \\
\text { CFA0101G } \\
\end{array}$ & $\begin{array}{r}\text { CFA } \\
\text { 03-LF3-8 } \\
\text { DOWN GRAD WELL } \\
\text { CFA0101GDL } \\
\text { GROUND WATER } \\
\text { Ug/L } \\
\text { CFA0101G } \\
\end{array}$ & $\begin{array}{r}\text { CFA } \\
\text { O2-LF2-8 } \\
\text { DOWN GRAD HELL } \\
\text { CFAO201G } \\
\text { GROUND WATER } \\
\text { Ug/L } \\
\text { CFAO101G } \\
\end{array}$ & $\begin{array}{r}\text { CFA } \\
02-L F 2-8 \\
\text { DOWN GRAD WELL } \\
\text { CFA0201GDL } \\
\text { GROUND WATER } \\
\text { Ug/L } \\
\text { CFA0101G } \\
\end{array}$ & $\begin{array}{r}\text { CFA } \\
\text { 02-LF2-9 } \\
\text { DOWN GRAD WELL } \\
\text { CFAO301G } \\
\text { GROUND WATER } \\
\text { Ug } / L \\
\text { CFAO101G } \\
\end{array}$ & $\begin{array}{r}\text { CFA } \\
02-L F 2-9 \\
\text { DOWN GRAD WELL } \\
\text { CFA0301GDL } \\
\text { GROUND WATER } \\
\text { Ug/L } \\
\text { CFA0101G } \\
\end{array}$ \\
\hline $\begin{array}{l}\text { FIELD MEASUREMENTS } \\
\text { pH } \\
\text { Conductivity (uS) } \\
\text { Temperature (C) }\end{array}$ & $\begin{array}{r}8.0 \\
0.6 \\
10.4\end{array}$ & $\begin{array}{r}8.0 \\
0.6 \\
10.4\end{array}$ & $\begin{array}{r}9.1 \\
0.6 \\
10.3\end{array}$ & $\begin{array}{r}9.1 \\
0.6 \\
10.3\end{array}$ & $\begin{array}{r}9.1 \\
0.6 \\
10.3\end{array}$ & $\begin{array}{r}9.1 \\
0.6 \\
10.3\end{array}$ \\
\hline $\begin{array}{l}\text { TARGET COMPOUNDS } \\
\text { gamma-BHC (Lindane) } \\
\text { Endrin } \\
\text { Methoxychlor } \\
\text { Toxaphene } \\
\text { Aroclor-1016 }\end{array}$ & $\begin{array}{c}0.04 \mathrm{Ux} \\
\ldots \\
\cdots \\
0.5 \\
0.50 \mathrm{ux}\end{array}$ & $\begin{array}{r}0.40 \text { UD } \\
0.60 \text { UD } \\
18 \text { UD } \\
24 \text { UD } \\
5 \text { UD }\end{array}$ & $\begin{array}{l}0.04 \text { UX } \\
0.06 \text { UX } \\
1.80 \text { Ux } \\
2.40 \text { UX } \\
0.50 \text { ux }\end{array}$ & $\begin{array}{r}1 \text { UD } \\
1.50 \text { UD } \\
45 \text { UD } \\
60 \text { UD } \\
13 \text { UD }\end{array}$ & $\begin{array}{c}0.04 \mathrm{Ux} \\
\ldots \\
\ldots \\
\ldots . \\
0.50 \mathrm{ux}\end{array}$ & $\begin{array}{r}0.40 \text { UD } \\
0.60 \text { UD } \\
18 \text { UD } \\
24 \text { UD } \\
5 \text { UD }\end{array}$ \\
\hline $\begin{array}{l}\text { Aroclor }-1221 \\
\text { Aroclor }-1232 \\
\text { Aroclor }-1242 \\
\text { Arocl or }-1248 \\
\text { Aroclor-1254 }\end{array}$ & $\begin{array}{c}0.50 \mathrm{Ux} \\
0.50 \mathrm{Ux} \\
\ldots \\
\ldots \\
\ldots\end{array}$ & $\begin{array}{r}5 \text { UD } \\
5 \text { UD } \\
6.50 \text { UD } \\
5 \text { UD } \\
10 \text { UD }\end{array}$ & $\begin{array}{l}0.50 \text { UX } \\
0.50 \text { UX } \\
0.65 \text { UX } \\
0.50 \text { UX } \\
1 \text { UX }\end{array}$ & $\begin{array}{l}13 \text { UD } \\
13 \text { UD } \\
16 \text { UD } \\
13 \text { UD } \\
25 \text { UD }\end{array}$ & $\begin{array}{c}0.50 \text { UX } \\
0.50 \text { UX } \\
\ldots \\
\ldots \\
\ldots-\end{array}$ & $\begin{array}{r}5 \text { UD } \\
5 \text { UD } \\
6.50 \text { UD } \\
5 \text { UD } \\
10 \text { UD }\end{array}$ \\
\hline $\begin{array}{l}\text { Dilution Factor } \\
\text { Extraction (Allowed) Hold Time } \\
\text { Analytical (Allowed) Hold Tine }\end{array}$ & $\begin{array}{r}1.0 \\
4(7) d \\
22(40) d\end{array}$ & $\begin{array}{r}10.0 \\
4(7) d \\
22(40) d\end{array}$ & $\begin{array}{r}1.0 \\
5(7) d \\
23(40) d\end{array}$ & $\begin{array}{r}25.0 \\
5(7) d \\
23(40) d\end{array}$ & $\begin{array}{r}1.0 \\
5(7) d \\
23(40) d\end{array}$ & $\begin{array}{r}10.0 \\
5(7) d \\
22(40) d\end{array}$ \\
\hline
\end{tabular}


CFA Landfills II and III FY89 Wells - 1st Quarter February 1990 S\&A Data Document

TABLE _-_- CFA LANDFILLS II AND III FYBg WELLS - ORGANOCHLORINE PESTICIDE APPENDIX IX DATA (Cont inUed)

Page 2 of 2

\begin{tabular}{|c|c|c|c|c|c|c|}
\hline $\begin{array}{l}\text { AREA } \\
\text { LOCAIION } \\
\text { TYPE OF LOCATION } \\
\text { SAMPLE NUMBER } \\
\text { MEDIA } \\
\text { UNITS } \\
\text { SDG NUMBER } \\
\end{array}$ & $\begin{array}{r}\text { CFA } \\
\text { O2-LF2-9 } \\
\text { DOWN GRAD WELL } \\
\text { CFA0302G } \\
\text { GROUND WATER } \\
\text { Ug/L } \\
\text { CFAO101G } \\
\end{array}$ & $\begin{array}{r}\text { CFA } \\
\text { 02-LF2-9 } \\
\text { DOWN GRAD WELL } \\
\text { CFA0302GDL } \\
\text { GROUND WATER } \\
\text { Ug/L } \\
\text { CFA0101G } \\
\end{array}$ & $\begin{array}{r}\text { CFA } \\
\text { 02-LF2-11 } \\
\text { UPGRADIENT WELL } \\
\text { CFAO401G } \\
\text { GROUND WATER } \\
\text { Ug } / 1 \\
\text { CFA0101G } \\
\end{array}$ & $\begin{array}{r}\text { CFA } \\
\text { O2-LF2-11 } \\
\text { UPGRADIENT WELL } \\
\text { CFA0401GDL } \\
\text { GROUND WATER } \\
\text { Ug/L } \\
\text { CFAO101G } \\
\end{array}$ & $\begin{array}{r}\text { CFA } \\
\text { 02-LF2-11 } \\
\text { UPGRADIENT WELL } \\
\text { CFAO402G } \\
\text { GROUND WATER } \\
\text { Ug } / L \\
\text { CFAO101G } \\
\end{array}$ & $\begin{array}{r}\text { CFA } \\
\text { O2-LL2-11 } \\
\text { UPGRADIENT WELL } \\
\text { CFA0402GDL } \\
\text { GROUND WATER } \\
\text { Ug/L } \\
\text { CFAO101G } \\
\end{array}$ \\
\hline $\begin{array}{l}\text { FIELD MEASUREMENIS } \\
\text { pH } \\
\text { Conduct ivity (uS) } \\
\text { Temperature (C) }\end{array}$ & $\begin{array}{r}9.1 \\
0.6 \\
10.3\end{array}$ & $\begin{array}{r}9.1 \\
0.6 \\
10.3\end{array}$ & $\begin{array}{r}7.6 \\
0.7 \\
10.1\end{array}$ & $\begin{array}{r}7.6 \\
0.7 \\
10.1\end{array}$ & $\begin{array}{r}7.6 \\
0.7 \\
10.1\end{array}$ & $\begin{array}{r}7.6 \\
0.7 \\
10.1\end{array}$ \\
\hline $\begin{array}{l}\text { TARGET COMPOUNDS } \\
\text { gamma-BHC (Lindane) } \\
\text { Endrin } \\
\text { Methoxychlor } \\
\text { Toxaphene } \\
\text { Aroclor-1016 }\end{array}$ & $\begin{array}{c}0.04 \mathrm{ux} \\
\ldots \\
\ldots \\
\ldots . \\
0.50 \mathrm{ux}\end{array}$ & $\begin{array}{r}0.40 \text { UD } \\
0.60 \text { UD } \\
18 \text { UD } \\
24 \text { UD } \\
5 \text { UD }\end{array}$ & $\begin{array}{c}0.04 \text { Ux } \\
\ldots \\
\cdots \\
0.50 \\
0.5 x\end{array}$ & $\begin{array}{r}0.40 \text { UD } \\
0.60 \text { UD } \\
18 \text { UD } \\
24 \text { UD } \\
5 \text { UD }\end{array}$ & $\begin{array}{c}0.04 \mathrm{Ux} \\
\ldots \\
\cdots \\
0.50 \\
0.5 x\end{array}$ & $\begin{array}{r}1 \text { UD } \\
1.50 \text { UD } \\
45 \text { UD } \\
60 \text { UD } \\
13 \text { UD }\end{array}$ \\
\hline $\begin{array}{l}\text { Aroclor }-1221 \\
\text { Aroclor }-1232 \\
\text { Aroclor }-1242 \\
\text { Aroclor }-1248 \\
\text { Aroclor }-1254\end{array}$ & $\begin{array}{c}0.50 \text { UX } \\
0.50 \text { UX } \\
0.65 \text { ux } \\
\ldots \\
\ldots\end{array}$ & $\begin{aligned} 5 & \text { UD } \\
5 & \text { UD } \\
6.50 & \text { UD } \\
5 & \text { UD } \\
10 & \text { UD }\end{aligned}$ & $\begin{array}{c}0.50 \text { ux } \\
0.50 \text { ux } \\
\ldots . . \\
\ldots \\
\cdots\end{array}$ & $\begin{array}{r}5 \text { UD } \\
5 \text { UD } \\
6.50 \text { UD } \\
5 \text { UD } \\
10 \text { UD }\end{array}$ & $\begin{array}{c}0.50 \text { UX } \\
0.50 \text { UX } \\
0.65 \text { UX } \\
\ldots . . \\
\ldots\end{array}$ & $\begin{array}{l}13 \text { UD } \\
13 \text { UD } \\
16 \text { UD } \\
13 \text { UD } \\
25 \text { UD }\end{array}$ \\
\hline $\begin{array}{l}\text { Dilution Factor } \\
\text { Extraction (Allowed) Hold Time } \\
\text { Analytical (Allowed) Hold Time }\end{array}$ & $\begin{array}{r}1.0 \\
5(7) d \\
23(40) d\end{array}$ & $\begin{array}{r}10.0 \\
5(7) d \\
23(40) d\end{array}$ & $\begin{array}{r}1.0 \\
4(7) d \\
22(40) d\end{array}$ & $\begin{array}{r}10.0 \\
4(7) d \\
22(40) d\end{array}$ & $\begin{array}{r}1.0 \\
4(7) d \\
22(40) d\end{array}$ & $\begin{array}{r}25.0 \\
4(7) d \\
22(40) d\end{array}$ \\
\hline
\end{tabular}

9-11-92 
CFA Landfills 11 and III FY89 Wells - 1st Quarter February 1990 S\&A Data Document

TABLE _-_-_ CFA LANDFILLS II AND III FY89 WELLS - ORGANOCHLORINE PESTICIDE APPENDIX IX FIELD BLANK DATA

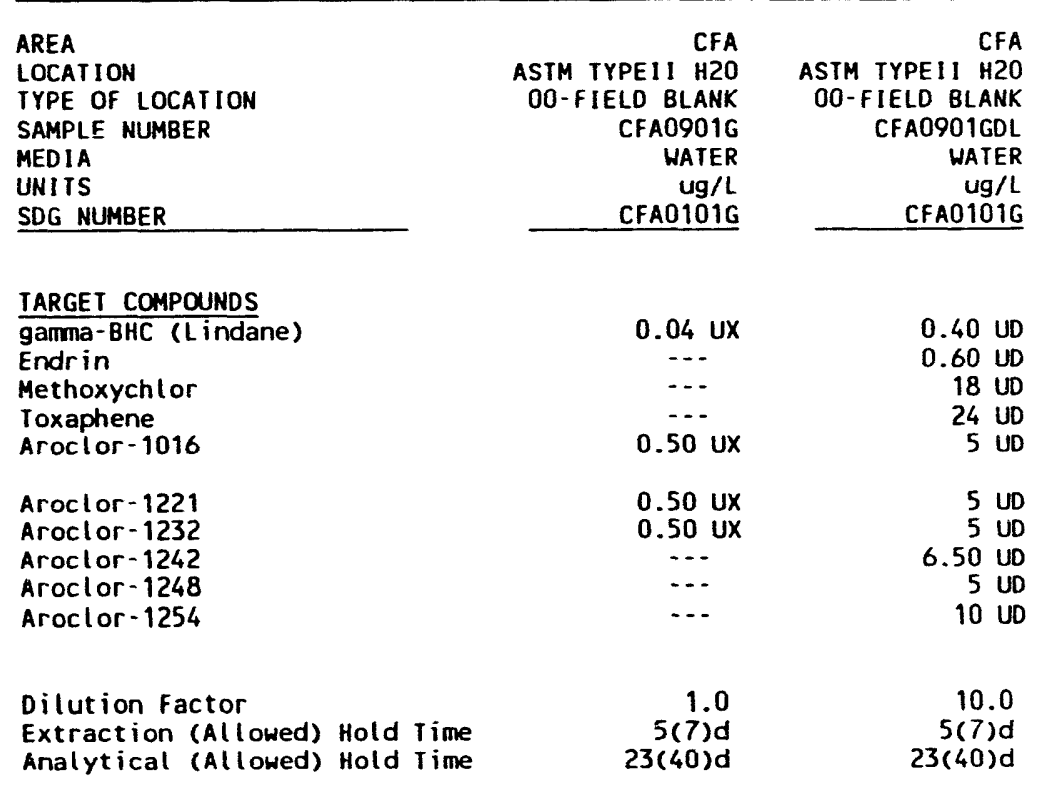


CFA Landfills 11 and 111 FY89 Wells - 1st Quarter February 1990 S\&A Data Document

TABLE _.- CFA LANDFILLS II AND 111 FY89 HELLS - ORGANOCHLORINE HERBICIDE APPENDIX IX DATA

Page 1 of 1

AREA

LOCATION

IYPE OF LOCATION

SAMPLE NUMBER

MEDIA

MEDIA

SDG NUMBER

\section{FIELD MEASUREMENTS}

PH

Conductivity (US)

IARGET COMPOUNDS

None Detected

Dilution Factor

Extraction (Allowed) Hold Time

Analytical (Allowed) Hold time

$\stackrel{\overbrace{}}{\text { ì }}$

$9-14-92$

CFA
03-LF3-8
DOWN GRAD HEL
CFAO101
GROUND HATER
Ug/
CFAO101


8.0
0.6
10.4

8.0
0.6
10.4

0.4

1.0
$4(7) d$

$4(7) d$
$15(40) d$
CFA
02-LF2-8
DOWN GRAD WELL

GROUND WATER

Ug/L
CFA01012

CFA
$02-L F 2-9$
DOWN GRAD HELL

GRAD WELL

CFAO3012

Ug/L

CFA
$02-L F 2-9$

DOWN GRAD HELL

CFA03022

GROUND WATER

Ug/L
CFA01012

CFA
02-LF2-11
UPGRADIENT HELL

GRADIENT WELL

GROUND WATER

Ug/L
CFA01012

9.1
0.6

0.6
10.3

0.6
10.3

10.3
1.0
$5(7) d$

$15(40) d$
1.0
$5(7) d$
$15(40) d$
9.1

7.6
0.7

10.1

10.3

1.0

$5(7) d$
$15(40) d$

CFA
$02-L F 2-11$

UPGRADIENT WELL

GROUND WATER

Ug/L
CFAO1012

7.6
0.7

10.1

(7)d 
CFA Landfills 11 and III FY89 Wells - 1st Quarter February 1990 S\&A Data Document

TABLE CFA LANDFILLS II AND III FY89 WELLS - ORGANOCHLORINE HERBICIDE APPENDIX IX FIELD BLANK DATA

Page 1 of 1

\begin{tabular}{lr} 
AREA & CFA \\
LOCATION & ASTM TYPEII H20 \\
TYPE OF LOCATION & HO-FIELD BLANK \\
SAMPLE NUMBER & CFA09012 \\
MEDIA & HATER \\
UNITS & Ug/L \\
SDG NUMBER & CFAO1012 \\
\hline
\end{tabular}

TARGET COMPOUNDS

None Detected

Dilution Factor

Extraction (Allowed) Hold Time

Analytical (Allowed) Hold Time

$15(40) d$

$9-11-92$ 
CFA Landfills II and III FY89 Wells - 1st Quarter February 1990 S\&A Data Document - Method Validation Level C

TABLE _.. CFA LANDFILLS II AND 111 FY89 WELLS - ANALYSIS RESULTS FOR GAMMA-EMITTING RADIONUCLIDES

Page 1 of 1

Date: $\underline{03 / 05 / 90}$

Lab Name: EGGRML

Report No.: STCS00990
Case No.:

SDG NO.: CFA01012

\begin{tabular}{|c|c|c|c|c|c|c|c|c|c|c|c|c|c|c|}
\hline $\begin{array}{l}\text { Field } \\
\text { Sample No. }\end{array}$ & Location & $\begin{array}{c}\text { Lab } \\
\text { Sample ID }\end{array}$ & $\begin{array}{l}\text { Sample } \\
\text { Matrix }\end{array}$ & $\begin{array}{l}\text { Anal } \\
\text { Type }\end{array}$ & $\begin{array}{l}\text { Sample } \\
\text { Value }\end{array}$ & $\begin{array}{l}\text { Sample } \\
\text { Error }\end{array}$ & Units & $\begin{array}{l}\text { Anal } \\
\text { Date }\end{array}$ & $\begin{array}{l}\text { Sample } \\
\text { Date }\end{array}$ & $\begin{array}{l}\text { Sample } \\
\text { size }\end{array}$ & Yield & $\begin{array}{l}\text { Detector } \\
\text { ID }\end{array}$ & DOF & ASL \\
\hline CFA01012 & $03-$ LF3-8 & $A 6020790041$ & WATER & GAMMA & ND & & $\mathrm{pCi} / \mathrm{mL}$ & $02 / 07 / 90$ & $02 / 07 / 90$ & 500.0000 & 0.0 & RML A6 PG-13 & & \\
\hline CFA02012 & $02-L F 2-8$ & A5020690051 & WATER & GAMMA & ND & & $\mathrm{pCi} / \mathrm{mL}$ & $02 / 06 / 90$ & $02 / 06 / 90$ & 540.0500 & 0.0 & RML A5 PG-1 & & \\
\hline CFA03012 & $02-L F 2-9$ & A6020690052 & WATER & GAMMA & ND & & $\mathrm{pCi} / \mathrm{mL}$ & $02 / 06 / 90$ & $\underline{02} / 06 / 90$ & 540.0000 & 0.0 & RML A6 PG-13 & & \\
\hline CFA03022 & $02-L F 2-9$ & A60208990038 & WATER & GAMMA & ND & & $\mathrm{pCi} / \mathrm{mL}$ & $02 / 08 / 90$ & $02 / 06 / 90$ & 530.0000 & 0.0 & RML AG PG-13 & & \\
\hline CFA04012 & $02-L F 2-11$ & A5020790040 & WATER & GAMMA & NDD & & $\mathrm{pCi} / \mathrm{mL}$ & $02 / 07 / 90$ & $02 / 07 / 90$ & 520.0000 & 0.0 & RML A5 PG-1 & & \\
\hline CFA04022 & $02-L F 2-11$ & A5020890037 & WATER & GAMMA & NND & & $p C i / m L$ & $02 / 08 / 90$ & $02 / 07 / 90$ & 520.0000 & 0.0 & RML A5 PG-1 & & \\
\hline CFA09012 & O0-FIELD BLANK & A5020990035 & WATER & GAMMA & ND & & $p c i / m L$ & $02 / 09 / 90$ & $02 / 06 / 90$ & 540.0000 & 0.0 & RML A5 PG-1 & & \\
\hline & & & & & & & & & & & & & & \\
\hline
\end{tabular}

$9-20-92$ 
CFA Landfills II and III FY89 Wells - 1st Quarter February 1990 S\&A Data Document - Method Validation Level C

TABLE _-_-_ CFA LANDFILLS II AND III FY89 WELLS - ANALYSIS RESULTS FOR GROSS ALPHA

Page 1 of 1

Date: $\underline{03 / 21 / 90}$

Lab Name: EGGRML

Report No.: STCS00990
Case No.:

SDG HO.: CFA01012

\begin{tabular}{|c|c|c|c|c|c|c|c|c|c|c|c|c|c|c|}
\hline $\begin{array}{c}\text { Field } \\
\text { Sample No. }\end{array}$ & Location & $\begin{array}{l}\text { Lab } \\
\text { Sample ID }\end{array}$ & $\begin{array}{l}\text { Sample } \\
\text { Matrix }\end{array}$ & $\begin{array}{l}\text { Anal } \\
\text { Type }\end{array}$ & $\begin{array}{l}\text { Sample } \\
\text { Value }\end{array}$ & $\begin{array}{l}\text { Sample } \\
\text { Error }\end{array}$ & Units & $\begin{array}{l}\text { Anal } \\
\text { Date }\end{array}$ & $\begin{array}{l}\text { Sample } \\
\text { Date }\end{array}$ & $\begin{array}{l}\text { Sample } \\
\text { Size }\end{array}$ & Yield & $\begin{array}{c}\text { Detector } \\
\text { ID }\end{array}$ & DoF & ASL \\
\hline CFA01011 & $03-L F 3-8$ & $\underline{G}$ & WATER & ALPHA & $5.00 E-03$ & $2.00 \mathrm{E}-03$ & $p C i / m L$ & $\underline{02 / 28 / 90}$ & $02 / 07 / 90$ & NP & 0.0 & & & \\
\hline CFA01011 & $03-L F 3-8$ & $\underline{G}$ & WATER & ALPHA & $0.00 E-03$ & $3.00 E-03$ & $\mathrm{pci} / \mathrm{mL}$ & $03 / 19 / 90$ & $02 / 07 / 90$ & NP & 0.0 & & & \\
\hline CFA02011 & $02-L F 2-8$ & $\underline{B}$ & WATER & ALPHA & $4.00 E-03$ & $3.00 E-03$ & $p c i / m t$ & $02 / 26 / 90$ & $02 / 06 / 90$ & NP & 0.0 & & & \\
\hline CFA03011 & 02-LF2-9 & $F$ & WATER & ALPHA & $2.00 E-03$ & $2.00 E-03$ & $\mathrm{pCi} / \mathrm{mL}$ & $02 / 26 / 90$ & $\underline{02 / 06 / 90}$ & NP & 0.0 & & & \\
\hline CFA03011 & $02-L F 2-9$ & $\mathbf{F}$ & WATER & ALPHA & $4.00 E-03$ & $2.00 E-03$ & $\mathrm{pCi} / \mathrm{mL}$ & $02 / 28 / 90$ & $02 / 06 / 90$ & NP & 0.0 & & & \\
\hline CFA03021 & 02-LF2-9 & $E$ & WATER & ALPKA & $2.00 \mathrm{E}-03$ & $3.00 E-03$ & $\mathrm{cci} / \mathrm{mL}$ & $02 / 26 / 90$ & $\underline{02 / 06 / 90}$ & NP & 0.0 & & & \\
\hline CFA04011 & 02-LF2-11 & c & HATER & ALPHA & $9.00 E-03$ & $4.00 E-03$ & $\mathrm{pCi} / \mathrm{mL}$ & $02 / 26 / 90$ & $02 / 07 / 90$ & NP & 0.0 & & & \\
\hline CFAO4021 & 02-LF2-11 & $\underline{D}$ & WATER & ALPHA & $0.00 E-04$ & $2.00 E-04$ & $\mathrm{pCi} / \mathrm{mL}$ & $02 / 26 / 90$ & $02 / 07 / 90$ & NP & 0.0 & & 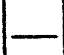 & \\
\hline CFA04021 & $02-$ LF2-11 & $\underline{D}$ & WATER & ALPHA & $1.40 E-03$ & $2.00 E-03$ & $\mathrm{pCi} / \mathrm{mL}$ & $02 / 28 / 90$ & $02 / 07 / 90$ & NP & 0.0 & & & \\
\hline CFA09011 & & A & WATER & ALPHA & $9.00 E-04$ & $6.00 E-04$ & $\mathrm{pCi} / \mathrm{mL}$ & $02 / 26 / 90$ & $02 / 06 / 90$ & NP & 0.0 & & & - \\
\hline
\end{tabular}

NP - Information Not Provided by Laboratory

9-20-92 
CFA Landfills II and III FY89 Wells - 1st Ouarter February 1990 S\&A Data Document - Method Validation Level C

TABLE _-_-_ CFA LANDFILLS II AND III FY89 WELLS - ANALYSIS RESULTS FOR GROSS BETA

Page 1 of 1

Date: $\underline{03 / 21 / 90}$

Lab Name: EGGRML

Report No.: ST-CS-009-90
Case No.:

SDG No.: CFA01012

\begin{tabular}{|c|c|c|c|c|c|c|c|c|c|c|c|c|c|c|}
\hline $\begin{array}{c}\text { Field } \\
\text { Sample No. }\end{array}$ & Location & $\begin{array}{l}\text { Lab } \\
\text { Sample ID }\end{array}$ & $\left|\begin{array}{l}\text { Sample } \\
\text { Matrix }\end{array}\right|$ & $\begin{array}{l}\text { Anal } \\
\text { Type }\end{array}$ & $\begin{array}{l}\text { Sample } \\
\text { value }\end{array}$ & $\begin{array}{l}\text { Sample } \\
\text { Error }\end{array}$ & Units & $\begin{array}{l}\text { Anal } \\
\text { Date }\end{array}$ & $\begin{array}{l}\text { Sample } \\
\text { Date }\end{array}$ & $\begin{array}{l}\text { Sample } \\
\text { size }\end{array}$ & rield & $\begin{array}{c}\text { Detector } \\
\text { ID }\end{array}$ & DOF & ASL \\
\hline CFA01011 & $03-L F 3-8$ & $\underline{G}$ & WATER & BETA & $1.10 E-02$ & $0.20 E-02$ & $\mathrm{pCi} / \mathrm{mL}$ & $02 / 28 / 90$ & $02 / 07 / 90$ & NP & 0.0 & & & \\
\hline CFA01011 & 03-LF3-8 & $\underline{G}$ & WATER & BETA & $1.20 E-02$ & $0.20 E-02$ & $\mathrm{pCi} / \mathrm{mL}$ & $03 / 19 / 90$ & $02 / 07 / 90$ & NP & 0.0 & & & \\
\hline CFA02011 & $02-L F 2-8$ & B & WATER & BETA & $9.00 E-03$ & $2.00 E-03$ & $\mathrm{pCi} / \mathrm{mL}$ & $02 / 26 / 90$ & $02 / 06 / 90$ & NP & 0.0 & & & \\
\hline CFA03011 & 02-LF2-9 & F & WATER & BETA & $2.20 E-02$ & $0.30 E-02$ & pci/mL & $02 / 26 / 90$ & $02 / 06 / 90$ & NP & 0.0 & & - & \\
\hline CFA03011 & 02-LF2-9 & $F$ & WATER & BETA & $2.00 E-02$ & $0.30 E-02$ & $\mathrm{pCi} / \mathrm{ml}$ & $02 / 28 / 90$ & $02 / 06 / 90$ & NP & 0.0 & & & \\
\hline CFA03021 & 02-LF:2-9 & $\underline{E}$ & WATER & BETA & $7.00 \mathrm{E}-03$ & $2.00 E-03$ & $\mathrm{pCi} / \mathrm{mL}$ & $02 / 26 / 90$ & $02 / 06 / 90$ & NP & 0.0 & & $\longrightarrow$ & \\
\hline CFA04011 & 02-LF2-11 & c & WATER & BETA & $1.50 \mathrm{E}-02$ & $0.30 E-02$ & $\mathrm{pCi} / \mathrm{mL}$ & $02 / 26 / 90$ & $02 / 07 / 90$ & NP & 0.0 & & - & \\
\hline CFA04021 & $02-L F 2-11$ & D & WATER & BETA & $2.30 E-02$ & $0.30 E-02$ & $\mathrm{pci} / \mathrm{mL}$ & $02 / 26 / 90$ & $02 / 07 / 90$ & NP & 0.0 & & & \\
\hline CFA04021 & $02-L F 2-11$ & $\underline{0}$ & WATER & BETA & $1.40 E-02$ & $0.20 E-02$ & $\mathrm{pCi} / \mathrm{mL}$ & $02 / 28 / 90$ & $02 / 07 / 90$ & NP & 0.0 & & - & \\
\hline CFA09011 & 00-FIELD BLANK & A & WATER & BETA & $1.40 E-03$ & $1.00 E-03$ & $\mathrm{pci} / \mathrm{mL}$ & $02 / 26 / 90$ & $\underline{02 / 06 / 90}$ & NP & 0.0 & & - & \\
\hline
\end{tabular}

NP - Information Not Provided by Laboratory

$9-20-92$ 
CFA Landfills II and III FY89 Wells - 1st Quarter February 1990 S\&A Data Document - Method Validation Level C

TABLE _-:- CFA LANDFILLS II AND III FY89 WELLS - ANALYSIS RESULTS FOR TRITIUM

Page 1 of 1

Date: $03 / 16 / 90$

Lab Name: EGGRML

Case No.:

Report No.: STCS00990

SDG NO.: CFA01012

\begin{tabular}{|c|c|c|c|c|c|c|c|c|c|c|c|c|c|c|}
\hline $\begin{array}{c}\text { Field } \\
\text { Sample No. }\end{array}$ & Location & $\begin{array}{l}\text { Lab } \\
\text { Sample } 10\end{array}$ & $\begin{array}{l}\text { Sample } \\
\text { Matrix }\end{array}$ & $\begin{array}{l}\text { Anal } \\
\text { Type }\end{array}$ & $\begin{array}{l}\text { Sample } \\
\text { Value }\end{array}$ & $\begin{array}{l}\text { Sample } \\
\text { Error }\end{array}$ & Units & $\begin{array}{l}\text { Anal } \\
\text { Date }\end{array}$ & $\begin{array}{l}\text { Sample } \\
\text { Date }\end{array}$ & $\begin{array}{l}\text { Sample } \\
\text { Size }\end{array}$ & Yield & $\begin{array}{c}\text { Detector } \\
\text { ID }\end{array}$ & DOF & ASL \\
\hline CFA01015 & 03-LF3-8 & 7 & WATER & IRITIUM & $4.20 E+01$ & $0.14 E+01$ & $\mathrm{pCi} / \mathrm{mL}$ & $02 / 23 / 90$ & $02 / 06 / 90$ & 10.0000 & 0.0 & & - & \\
\hline CFA02015 & $02-L F 2-8$ & 2 & WATER & IRITIUM & $4.20 E+01$ & $0.14 E+01$ & $\mathrm{pci} / \mathrm{mL}$ & $02 / 23 / 90$ & $02 / 06 / 90$ & 10.0000 & 0.0 & & & \\
\hline CFA03015 & 02-LF2-9 & 6 & WATER & TRITIUM & $4.40 \mathrm{E}+01$ & $0.15 E+01$ & $\mathrm{pCi} / \mathrm{mL}$ & $02 / 23 / 90$ & $02 / 06 / 90$ & 10.0000 & 0.0 & & & \\
\hline CFA03025 & 02-LF2-9 & 5 & WATER & TRITIUM & $4.40 E+01$ & $0.15 E+01$ & $\mathrm{pCi} / \mathrm{mL}$ & $02 / 23 / 90$ & $02 / 06 / 90$ & 10.0000 & 0.0 & & & \\
\hline CFA04015 & $02-L F 2-11$ & 3 & WATER & IRITIUM & $4.80 E+01$ & $0.20 E+01$ & $\mathrm{pCi} / \mathrm{mL}$ & $02 / 23 / 90$ & $02 / 07 / 90$ & 10.0000 & 0.0 & & & \\
\hline CFA04015 & $02-L F 2-11$ & 3 & WATER & TRITIUM & $4.80 E+01$ & $0.20 E+01$ & $\mathrm{pCi} / \mathrm{mL}$ & $02 / 26 / 90$ & $\underline{02 / 07 / 90}$ & 10.0000 & 0.0 & & & \\
\hline CFA04025 & $02-L F 2-11$ & 4 & WATER & TRITIUM & $4.70 E+01$ & $0.20 E+01$ & $\mathrm{pci} / \mathrm{mL}$ & $02 / 23 / 90$ & $02 / 07 / 90$ & 10.0000 & 0.0 & & - & - \\
\hline CFA09015 & 00-FIELD BLANK & 1 & WATER & IRITIUM & $1.20 E-01$ & $0.80 E-01$ & $\mathrm{pCi} / \mathrm{mL}$ & $02 / 23 / 90$ & $02 / 06 / 90$ & 10.0000 & 0.0 & & & \\
\hline
\end{tabular}

9-20-92 


\title{
Appendix C.2
}

\section{Validated Groundwater Quality Data} for CFA Landfills II and III

\author{
May 1990
}



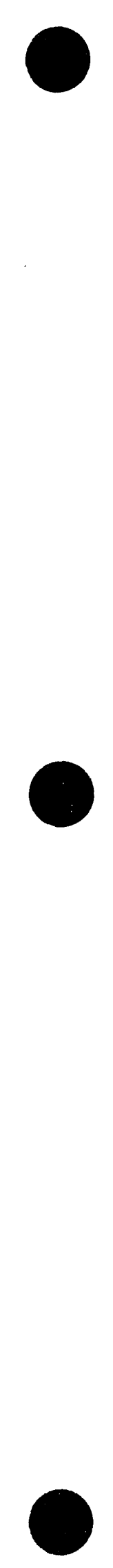

\section{C. $2-2$}




\section{DATA QUALIFIER DEFINITIONS}

\section{Organic Analysis Data Flags}
A - Indicates that a tentatively identified compound is a suspected aldol-condensation product.
B - Used when the analyte is found in the associated blank as well as in the sample.
C - Applies to pesticide results where the identification has been confirmed by GC/MS.
D - Identifies all compounds identified in an analysis at a secondary dilution factor.
E - Identifies compounds which have concentrations that exceed the calibration range of the GC/MS instrument for that specific analysis.
J - Indicates as estimated value.
U - Indicates compound was analyzed for but not detected.

\section{Organic Validation Data Qualifiers}

J - The analyte was positively identified in the sample, but the associated numerical value may not be an accurate representation of the amount actually present in the environmental sample. The data should be seriously considered for decision making and are usable for many purposes.

$\mathbf{N}$ - Presumptive evidence of the presence of the material.

NJ - Presumptive evidence of the presence of the material at an estimated quantity.

R - The data are unusable (may or may not be present). Resampling and reanalysis is necessary for verification.

$\mathbf{U}$ - The material was analyzed for but was not detected. The associated numerical value is the sample quantitation limit.

UJ - The material was analyzed for, but was not detected. The sample quantitation limit is an estimated quantity.

Reference: Environmental Restoration Department, Sample Management Office Standard Operating Procedures 12.1.4 and 12.1.5 (EG\&G Idaho). 


\section{DATA QUALIFIER DEFINITIONS}

Inorganic Analysis Data Flags

B - Value is less than the CRDL, but greater than the IDL.

E - Value is estimated because of the presence of interference.

N - Spiked sample recovery not within control limits.

NR - Analyte was not required to be analyzed.

S - Value was determined by the method of standard additions (MSA).

U - Analyte was analyzed for but not detected.

W - Post digestion spike for Furnace AA analysis is out of control limits (85\% to 115\%), while sample absorbance is less than $50 \%$ of spike absorbance.

* - Duplicate analysis not within control limits.

$\underline{\text { Methods }}$

A - Flame AA.

CV - Manual Cold Vapor AA.

F - Furnace AA.

NR - Not run, analyte not required to be analyzed.

P - Inductively Coupled Plasma - Atomic Emission Spectrometry.

\section{Inorganic Validation Data Qualifiers}

J - The analyte was analyzed for and was positively identified, but the associated numerical value may not be consistent with the amount actually present in the environmental sample.

R - The data are unusable.

U - The material was analyzed for, but was not detected above the level of the associated value. The associated value is either the sample quantitation limit or the sample detection limit.

UJ - The material was analyzed for, but was not detected. The associated value is an estimate and may not accurately reflect the instrument detection limit in the sample matrix. 
CFA Landfills II and III FY89 Wells - 2nd Quarter May 1990 S8A Data Document

TABLE _. CFA LANDFILLS II AND 111 FY89 WELLS - 524.2 VOLATILE ORGANIC DATA

Page 1 of 2

\begin{tabular}{l} 
AREA \\
LOCATION \\
TYPE OF LOCATION \\
SAMPLE NUMBER \\
MEDIA \\
UNITS \\
SDG MUMBER \\
\hline
\end{tabular}

\section{FIELD MEASUREMENTS}

pH

Conductivity (uS)

Temperature (C)

TARGET COMPQUNDS
Methylene chloride
Chloroform

1,1,1-Trichloroethane

1,1,1-Trice

Dilution Factor

Total (Allowed) Hold Time

\begin{tabular}{rr} 
CFA & CFA \\
O3-LF3-8 & O2-LF2-8 \\
DOWN GRAD HELL & DOWN GRAD WELL \\
CFA1001A & CFA1101A \\
GROUND HATER & GROUND WATER \\
Ug/L & Ug/L \\
CFA1001A & CFA1001A \\
\hline
\end{tabular}

$\begin{array}{r}\text { CFA } \\ \text { O2-LF2-9 } \\ \text { DOWN GRAD HELL } \\ \text { CFA12011 } \\ \text { GROUND HATER } \\ \text { Ug/L } \\ \text { CFA1001A } \\ \hline\end{array}$

$\begin{array}{r}\text { CFA } \\ \text { O2-LF2-9 } \\ \text { DOWN GRAD WELL } \\ \text { CFA1202A } \\ \text { GROUND WATER } \\ \text { Ug/L } \\ \text { CFA1001A } \\ \hline\end{array}$

$\begin{array}{r}\text { CFA } \\ \text { O2-LF2-11 } \\ \text { UPGRADIENT HELL } \\ \text { CFA1301A } \\ \text { GROUND HATER } \\ \text { Ug/L } \\ \text { CFA1001A } \\ \hline\end{array}$

9-11-92

$\begin{array}{cc}8.2 & 8.8 \\ 0.5 & 0.6 \\ 12.5 & 12.0 \\ & \\ \cdots-. & \cdots . . \\ 0.5 \mathrm{~J} & 0.5 \\ \cdots & 0.6 \\ 1.000 & 0.5 \\ 8(14) d & 1.000 \\ & 8(14) d\end{array}$

9.1
0.5

0.5
12.1

9.1
0.5

12.1

7.6
0.6

0.6
11.0

$0.2 \mathrm{~J}$

$0.6 \mathrm{~J}$

$0.2 \mathrm{~J}$

$0.2 J$

1.000

$7(14) \mathrm{d}$

1.000

$8(14) d$

-..

5

1.000 $8(14) d$ 
CFA Landfills II and III FYB9 Wells - 2nd Quarter May 1990 S\&A Data Document

TABLE _-_- CFA LANDFILLS II AND III FY89 helLS - 524.2 vOLATILE ORgANIC DATA (Continued)

AREA

LOCATION

TYPE OF LOCATION

SAMPLE NUMBER

MEDIA

SDG MUMBER

$\begin{array}{r}\text { CFA } \\ \text { O2-LF2-11 } \\ \text { UPGRADIENT HELL } \\ \text { CFA1302A } \\ \text { GROUNO WATER } \\ \text { U9/L } \\ \text { CFA1001A } \\ \hline\end{array}$

FIELD MEASUREMENTS

$\mathrm{PH}$

Conductivity (uS)

Temperature (C)

IARGET COMPOUNDS

Methylene chloride

Chloroform

1,1,1-Trichloroethane

Toluen

ấ

Total (Allowed) Hold Time

7.6
0.6

0.6
11.0

$-.-$

0.5

1.000

$8(14) d$

9-11-92 
CFA Landfills II and III FY89 Mells - 2nd Quarter May 1990 S\&A Data Document

TABLE _-_-_ CFA LAMDFILLS 11 AND 111 FY89 WELLS - 524.2 VOLATILE ORGANIC FIELD BLANK DATA

AREA

LOCATION

TYPE OF LOCATION

SAMPLE MUMBER

MEDIA

UNITS

\section{CFA}

ASTM TYPEII HZO

O0-FIELD BLANK

CFA1801A
WATER

UAR

CFA1001A

\section{TARGET COMPOUHDS}

None detected.

Dilution Factor

Total (Allowed) Hold Time

1.000

$7(14) d$

9-11-92 
CFA Landfills II and III FY89 Wells - 2nd Quarter May 1990 S\&A Data Document

TABLE -. CFA LANDFILLS 11 AND III FY89 heLLS - 524.2 VOLATILE ORGANIC TRIP BLANK DATA

Page 1 of 1

\begin{tabular}{l} 
AREA \\
LOCATION \\
TYPE OF LOCATION \\
SAMPLE MUMBER \\
MEDIA \\
UNITS \\
SDG MUMBER \\
\hline
\end{tabular}

\section{TARGET COMPOUNDS}

Chloromethane

Methylene Chloride

Chloroform

Bromodichloromethane

Toluene

xylene (total)

$1,3,5$-Irimethylbenzene

1,2,4-Trimethylbenzene

Dilution Factor

Iotal (Allowed) Hold Time

\begin{tabular}{|c|c|}
\hline $\begin{array}{r}\text { CFA } \\
\text { OC } \\
\text { 00-TRIP BLANK } \\
\text { CFA1401A } \\
\text { WATER } \\
\text { Ug/L } \\
\text { CFA1001A } \\
\end{array}$ & $\begin{array}{r}\text { CF } \\
\text { 00-TRIP BLAN } \\
\text { CFA1501 } \\
\text { WATE } \\
\text { Ug } \\
\text { CFA1001 } \\
\end{array}$ \\
\hline$\ldots$ & - - \\
\hline $0.4 \mathrm{~J}$ & $0.4 \mathrm{~J}$ \\
\hline$\cdots$ & $\cdots$ \\
\hline .. & $\ldots$ \\
\hline$\cdots$ & $\ldots$ \\
\hline $\begin{array}{r}1.000 \\
9(14) d\end{array}$ & $9(14) d$ \\
\hline
\end{tabular}

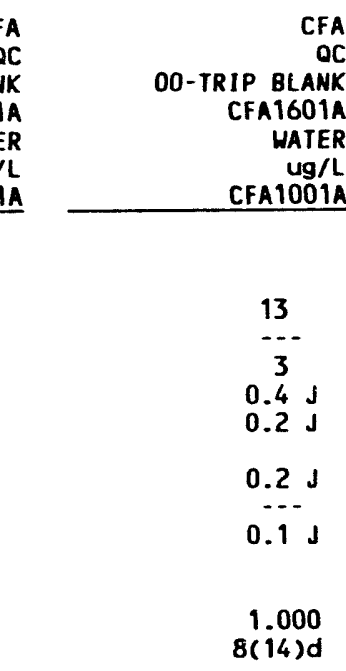

CFA
OC

CFA
OC
ANK

00-TRIP BLANK

CFA1701A

WATER

CFA1001A

00-TRIP BLANK

OO-TRIP BLANK

WATER

WATER

UG/L

$9-11-92$ 
CFA Landfills 11 and 111 fY89 Wells - 2nd Quarter May 1990 S\&A Data Document

TABLE _-_- CFA LANDFILLS II AND III fY89 hELLS - SEMIVOLATILE APPENOIX IX ORGANIC DATA

Page 1 of 2

\begin{tabular}{l} 
AREA \\
LOCATION \\
TYPE OF LOCATION \\
SAMPLE NUMBER \\
MEDIA \\
UNIIS \\
SDG NUMBER \\
\hline
\end{tabular}

\section{FIELD MEASUREMENTS \\ $\mathrm{pH}$ \\ Conductivity (uS) \\ Temperature (C)}

TARGET COMPOUNDS

\section{None Detected}

Dilution Factor

Extraction (Allowed) Hold Time

Analytical (Allowed) Hold Time

$9-14-92$

\begin{tabular}{rrr} 
CFA & CFA & CFA \\
O3-LF3-8 & O2-LF2-8 & O2-LF2-9 \\
DOWN GRAD HELL & DOWN GRAD HELL & DOWN GRAD HELL \\
CFA1001D & CFA11010 & CFA1201D \\
GROUND HATER & GROUND HATER & GROUND WATER \\
UG/L & Ug/L & Ug/L \\
CFA1001D & CFA10010 & CFA10010 \\
\hline
\end{tabular}

\section{2}

12.5

8.8
0.6

0.6
12.0

9.1
0.5

0.5
12.1

1.000

$4(7) d$

$14(40) d$

1.000

$4(7) d$
$14(40) d$

$\begin{array}{r}\text { CFA } \\ \text { O2-LF2-9 } \\ \text { DOWN GRAD WELL } \\ \text { CFA1202D } \\ \text { GROUND WATER } \\ \text { Ug/L } \\ \text { CFA1O01D } \\ \hline\end{array}$

CFA

CFA13010

CFA 1301D
GROUND WATER

Ug/L
CFA1001D 
CFA Landfills II and 111 FY89 Hells - 2nd Quarter May 1990 S\&A Data Document

TABLE -.-- CFA LANDFILLS II AND III FY89 WELLS - SEMIVOLATILE APPENDIX IX ORGANIC DATA (Cont inued)

Page 2 of 2

AREA

LOCATION

TYPE OF LOCATION

SAMPLE NUMBER

MEDIA

SDG NUMBER

FIELD MEASUREMENTS

PH

Conductivity (uS)

Temperature (C)

TARGET COMPOUNDS

None Detected

Dilution Factor

Extraction (Allowed) Hold Time

Analytical (Allowed) Hold Time

$9-14-92$
CFA
$02-L F 2-11$

UPGRADIENT WELL

CFA1302D

GROUND WAIER

CFA1001D

\section{6}

0.6
11.0

1.000
$3(7) d$

$3(7) d$
$14(40) d$ 
CFA Landfills II and III FY89 Wells - 2nd Quarter May 1990 S\&A Data Document

TABLE

CFA LANDFILLS II AND III FY89 WELLS - SEMIVOLATILE APPENDIX IX ORGANIC FIELD BLANK DATA

Page 1 of 1

\section{AREA}

LOCATION

TYPE OF LOCATION

SAMPLE NUMBER

MEDIA

SDG NUMBER

\section{IARGET COMPOUNDS}

\section{None Detected}

Dilution Factor

Extraction (Allowed) Hold Time

Analytical (Allowed) Hold time

1.000

$4(7) d$
$14(40) d$
ASTM TYPEII H2O

OO-FIELD BLANK

CFA18010

UG/L

CFA10010

$9-14-92$ 
CFA Landfills 11 and III FY89 Wells - 2nd Ouarter May 1990 S\&A Data Document

TABLE _-_- CFA LANDFILLS II AND III FY89 WELLS - INORGANIC DATA

Page 1 of 2

\begin{tabular}{|c|c|c|c|c|c|c|}
\hline $\begin{array}{l}\text { AREA } \\
\text { LOCATION }\end{array}$ & $\begin{array}{r}\text { CFA } \\
03-L F 3-8\end{array}$ & $\begin{array}{r}\text { CFA } \\
03-\text { LF3-8 }\end{array}$ & $\begin{array}{r}\text { CFA } \\
02-\text { LF2-8 }\end{array}$ & $\begin{array}{r}\text { CFA } \\
02-L F 2-8\end{array}$ & $\begin{array}{r}\text { CFA } \\
02-L F 2-9\end{array}$ & $\begin{array}{r}\text { CFA } \\
\text { 02-LF2-9 }\end{array}$ \\
\hline $\begin{array}{l}\text { TYPE OF LOCATION } \\
\text { SAMPLE NUMBER } \\
\text { MEDIA }\end{array}$ & $\begin{array}{r}\text { DOWN GRAD WELL } \\
\text { CFA1OOTH } \\
\text { GROUND HATER }\end{array}$ & $\begin{array}{r}\text { DOWN GRAD WELL } \\
\text { CFA1OO1M } \\
\text { GROUND WATER }\end{array}$ & $\begin{array}{r}\text { DOWN GRAD WELL } \\
\text { CFA1101H } \\
\text { GROUND HATER }\end{array}$ & $\begin{array}{r}\text { DOWN GRAD VELL } \\
\text { CFA1101M } \\
\text { GROUND WATER }\end{array}$ & $\begin{array}{r}\text { DOWN GRAD WELL } \\
\text { CFA1201H } \\
\text { GROUND MATER }\end{array}$ & $\begin{array}{r}\text { DON GRAD WELL } \\
\text { CFA1ZOIM } \\
\text { GROND MATER }\end{array}$ \\
\hline $\begin{array}{l}\text { UNITS } \\
\text { SDG NUMBER }\end{array}$ & $\begin{array}{r}u g / L \\
\text { CFA1001H } \\
\end{array}$ & $\begin{array}{r}u g / L \\
\text { CFA1001M } \\
\end{array}$ & $\begin{array}{r}u g / L \\
\text { CFA1001H } \\
\end{array}$ & $\begin{array}{r}u g / L \\
\text { CFA1001M } \\
\end{array}$ & $\begin{array}{r}\mathrm{Ug} / \mathrm{L} \\
\text { CFA1001H } \\
\end{array}$ & $\begin{array}{r}u g / L \\
\text { CFA1001M } \\
\end{array}$ \\
\hline \multicolumn{7}{|l|}{ FIELD MEASUREMENTS } \\
\hline $\begin{array}{l}\text { pH } \\
\text { Conductivity (US) } \\
\text { Temperature (C) }\end{array}$ & $\begin{array}{r}8.2 \\
0.5 \\
12.5\end{array}$ & $\begin{array}{r}8.2 \\
0.5 \\
12.5\end{array}$ & $\begin{array}{r}8.8 \\
0.6 \\
12.0\end{array}$ & $\begin{array}{r}8.8 \\
0.6 \\
12.0\end{array}$ & $\begin{array}{r}9.1 \\
0.5 \\
12.1\end{array}$ & $\begin{array}{r}9.1 \\
0.5 \\
12.1\end{array}$ \\
\hline \multicolumn{7}{|l|}{ ANALYTES } \\
\hline $\begin{array}{l}\text { Arsenic } \\
\text { Barium } \\
\text { Cadmium } \\
\text { Chromium } \\
\text { Iron }\end{array}$ & $\begin{array}{c}1.1 \text { B } \\
86.0 \text { B } \\
-\ldots- \\
589 \text { NJ } \\
11900\end{array}$ & $\begin{array}{c}1.4 \mathrm{~B} \\
82.0 \mathrm{~B} \\
1.0 \mathrm{UWJ} \\
10.0 \mathrm{WJ} \\
200 \mathrm{U}\end{array}$ & $\begin{array}{l}1.3 \mathrm{~B} \\
142 \mathrm{~B} \\
1.0 \mathrm{UWJJ} \\
285 \mathrm{NJ} \\
1540\end{array}$ & $\begin{array}{l}101 \mathrm{~B} \\
1.0 \mathrm{UWU} \\
14.0 \mathrm{~J} \\
160 \mathrm{U}\end{array}$ & $\begin{array}{l}1.3 \mathrm{~B} \\
152 \mathrm{~B} \\
1.0 \mathrm{uWJ} \\
263 \mathrm{NJ} \\
4700\end{array}$ & $\begin{array}{l}83.0 \text { B } \\
1.0 \text { UnU } \\
567 \mathrm{~J} \\
230 \mathrm{U}\end{array}$ \\
\hline $\begin{array}{l}\text { Lead } \\
\text { Manganese }\end{array}$ & $\begin{array}{l}1.4 B \\
217^{B}\end{array}$ & $\begin{array}{l}1.0 \mathrm{~B} \\
200^{-1}\end{array}$ & $\begin{array}{r}11.2 \\
153\end{array}$ & $\overline{214}$ & 154 & 213 \\
\hline Mercury & $0.10 \mathrm{~B}$ & $\cdots$ & $\cdots$ & $\cdots$ & $\cdots$ & $\cdots$ \\
\hline $\begin{array}{l}\text { Selenium } \\
\text { silver }\end{array}$ & $\begin{array}{l}2.0 \text { UUUJ } \\
7.0 \text { UNUJ }\end{array}$ & $\begin{array}{l}2.0 \text { und } \\
7.0 \text { uUJ }\end{array}$ & $\begin{array}{l}2.0 \text { UWUJ } \\
7.0 \text { UNUJ }\end{array}$ & $\begin{array}{l}2.8 \text { BWJ } \\
7.0 \mathrm{WJJ}\end{array}$ & $\begin{array}{l}2.0 \text { und } \\
7.0 \text { unus }\end{array}$ & $7.0 \mathrm{wJ}$ \\
\hline Sodium & $36300 \mathrm{EJ}$ & $36400 \mathrm{EJ}$ & $35800 \mathrm{EJ}$ & $35700 \mathrm{EJ}$ & $37600 \mathrm{EJ}$ & $35900 \mathrm{EJ}$ \\
\hline $\begin{array}{l}\text { Total (Allowed) Hold Time } \\
\text { Total (Allowed) Hold Time } \\
\text { Total (Allowed) Hold Time }\end{array}$ & $\begin{array}{r}7(180) d \\
6(26) d \\
7(180) d\end{array}$ & $\begin{array}{r}7(180) d \\
6(26) d \\
7(180) d\end{array}$ & $\begin{array}{l}8(180) d \\
7(26) d \\
8(180) d\end{array}$ & $\begin{array}{l}8(180) d \\
7(26) d \\
8(180) d\end{array}$ & $\begin{array}{r}7(180) d \\
6(26) d \\
7(180) d\end{array}$ & $\begin{array}{l}7(180) d \\
6(26) d \\
7(180) d\end{array}$ \\
\hline
\end{tabular}
a. ICP
b. CVAAS
c. GFAAS
$9-11-92$ 
CFA Landfills II and III FY89 Wells - 2nd Ouarter May 1990 S\&A Data Document

TABLE _--- CFA LANDFILLS 11 AND 111 FY89 WELLS - INORGANIC DATA (Continued)

Page 2 of 2

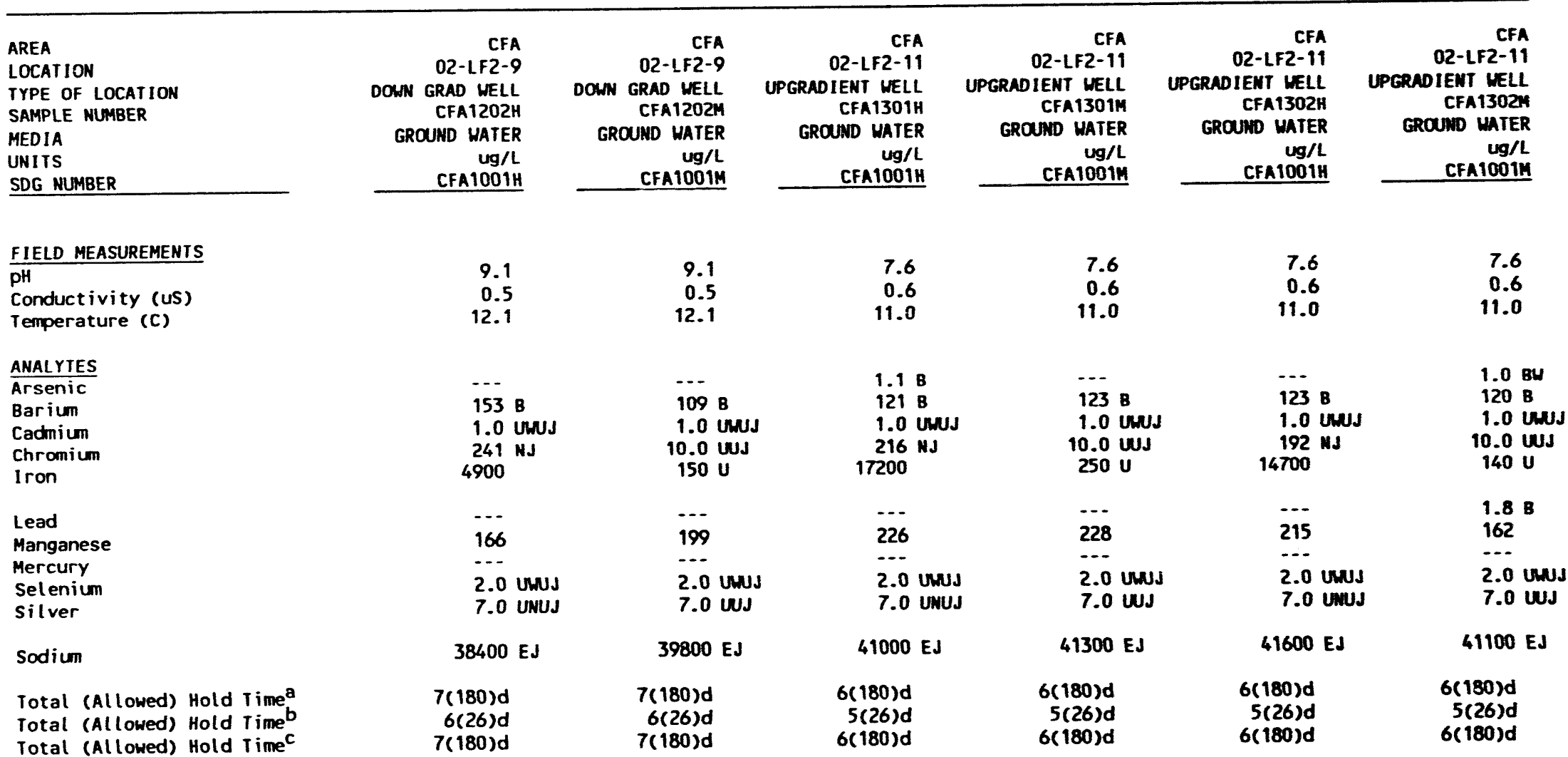
a. ICP
b. CVAAS
c. GFAAS

$9-11-92$ 
CFA Landfills 11 and 111 fY89 Hells - 2nd Quarter May 1990 S\&A Date Document

TABLE -. CFA LANDFILLS II AND III FYB9 WELLS - INORGANIC FIELD BLANK DATA

\begin{tabular}{|c|c|c|}
\hline AREA & ac & ac \\
\hline LOCATION & ASTM TYPEII HZO & ASTM TYPEII HZO \\
\hline TYPE OF LOCATION & OO-FIELD BLANK & O0-FIELD BLANK \\
\hline SAMPLE NUMBER & CFA1801H & CFA1801M \\
\hline MEDIA & WATER & WATER \\
\hline UNITS & $u g / L$ & $u g / L$ \\
\hline SDG NUMBER & CFA1001H & CFA1001M \\
\hline \multicolumn{3}{|l|}{ ANALYIES } \\
\hline Arsenic &.- & $\cdots$ \\
\hline Barium & $33.0 \mathrm{~B}$ & $5.0 \mathrm{~B}$ \\
\hline Cadmium & $\ldots$ & -.. \\
\hline Chromium & 10.0 UNUJ & $10.0 \mathrm{us}$ \\
\hline Iron & $40.0 \mathrm{BU}$ & $180 \mathrm{U}$ \\
\hline Lead & 3.2 & $1.6 \mathrm{~B}$ \\
\hline Manganese & 122 & 233 \\
\hline Mercury & $-\cdot$ & -.. \\
\hline Selenium & $\ldots$ & 2.0 UMJ \\
\hline silver & 7.0 UNUS & $7.0 \mathrm{uss}$ \\
\hline Sodium & 256 UJ & 256 UEJ \\
\hline Total (Allowed) Hold Time & $8(180) d$ & $8(180) d$ \\
\hline Total (Allowed) Hold Timeb & $7(26) d$ & $7(26) d$ \\
\hline Total (Allowed) Hold Time & $8(180) d$ & $8(180) d$ \\
\hline
\end{tabular}
a. ICP
b. CVAAS
c. GFAAS

$9-11-92$ 
CFA Landfills 11 and III FY89 Hells - 2nd Quarter May 1990 S\&A Data Document - Method Validation Level A

tABlE _-_-_ CFA LANDFILLS $I 1$ AND $I 11$ FY89 WELLS - NON-METALS DATA

Page 1 of 1

\begin{tabular}{|c|c|c|c|c|c|c|}
\hline $\begin{array}{l}\text { AREA } \\
\text { LOCATION }\end{array}$ & $\begin{array}{r}\text { CFA } \\
03-L F 3-8\end{array}$ & $\begin{array}{r}\text { CFA } \\
02-L F 2-8\end{array}$ & $\begin{array}{r}\text { CFA } \\
02-L F 2-9\end{array}$ & $\begin{array}{r}\text { CFA } \\
02-L F 2-9\end{array}$ & $\begin{array}{r}\text { CFA } \\
02-L F 2-11\end{array}$ & $\begin{array}{r}\text { CFA } \\
02-L F 2-11\end{array}$ \\
\hline $\begin{array}{l}\text { LOCATION } \\
\text { IYPE OF LOCATIION } \\
\text { SAMPLE NUMBER }\end{array}$ & $\begin{array}{r}03-L F 3-8 \\
\text { DOWN GRAD WELL } \\
\text { CFA1001 }\end{array}$ & $\begin{array}{r}\text { 02-LF2-8 } \\
\text { DOWN GRAD HELL } \\
\text { CFA1101 }\end{array}$ & $\begin{array}{r}\text { DOWN GRAD WELL } \\
\text { CFA1201 }\end{array}$ & $\begin{array}{r}\text { DOWN GRAD WELL } \\
\text { CFA1202 }\end{array}$ & $\begin{array}{r}\text { UPGRADIENT WELL } \\
\text { CFA1301 }\end{array}$ & $\begin{array}{l}\text { UPGRADIENT WELL } \\
\text { CFA1302 }\end{array}$ \\
\hline MEDIA & GROUND WATER & GROUND WATER & GROUND HATER & GROUND WATER & GROUND WATER & GROUND WATER \\
\hline $\begin{array}{l}\text { UNI IS } \\
\text { SDG NUMBER }\end{array}$ & $\begin{array}{r}u g / L \\
\text { CFA1001 } \\
\end{array}$ & $\begin{array}{r}\text { ug/L } \\
\text { CFA1001 } \\
\end{array}$ & $\begin{array}{r}u g / L \\
\text { CFA1001 } \\
\end{array}$ & $\begin{array}{r}u g / L \\
\text { CFA1001 } \\
\end{array}$ & $\begin{array}{r}u g / L \\
\text { CFA1001 } \\
\end{array}$ & $\begin{array}{r}u g / L \\
\text { CFA1001 } \\
\end{array}$ \\
\hline \multicolumn{7}{|l|}{ FIELD MEASUREMENTS } \\
\hline $\begin{array}{l}\text { pH } \\
\text { Conductivity (uS) } \\
\text { Temperature (C) }\end{array}$ & $\begin{array}{r}8.2 \\
0.5 \\
12.5\end{array}$ & $\begin{array}{r}8.8 \\
0.6 \\
12.0\end{array}$ & $\begin{array}{r}9.1 \\
0.5 \\
12.1\end{array}$ & $\begin{array}{r}9.1 \\
0.5 \\
12.1\end{array}$ & $\begin{array}{r}7.6 \\
0.6 \\
11.0\end{array}$ & $\begin{array}{r}7.6 \\
0.6 \\
11.0\end{array}$ \\
\hline \multicolumn{7}{|l|}{ ANALYTES } \\
\hline Fluoride & $\begin{array}{r}66200 \\
246\end{array}$ & $\begin{array}{r}80500 \\
210\end{array}$ & $\begin{array}{r}80700 \\
234\end{array}$ & $\begin{array}{r}78900 \\
210\end{array}$ & $\begin{array}{r}86200 \\
222\end{array}$ & $\begin{array}{r}86400 \\
234\end{array}$ \\
\hline Nitrate & 4440 & $\begin{array}{r}210 \\
5330\end{array}$ & 5580 & 5960 & 5540 & 5820 \\
\hline Sulfate & 34000 & 25000 & 28000 & 32000 & 32000 & 32000 \\
\hline IOC & 1000 UJ & $1000 \mathrm{UJ}$ & $1000 \mathrm{UJ}$ & $1000 \mathrm{UJ}$ & $1000 \mathrm{UJ}$ & 1000 Us \\
\hline rox & $12.8 \mathrm{~J}$ & $129 \mathrm{~J}$ & $25.7 \mathrm{~J}$ & $95.7 \mathrm{~J}$ & $40.9 \mathrm{~J}$ & $41.8 \mathrm{~J}$ \\
\hline Total (Allowed) Hold Time a & $16(28) d$ & $17(28) d$ & $16(28) d$ & $16(28) d$ & $15(28) d$ & $15(28) d$ \\
\hline Total (Allowed) Hold Timeb & $23(28) d$ & $24(28) d$ & $23(28) d$ & $23(28) d$ & $22(28) d$ & $22(28) d$ \\
\hline Total (Allowed) Hold Time c & $6(28) d$ & $7(28) d$ & $6(28) \mathrm{d}$ & $6(28) d$ & $5(28) d$ & $5(28) d$ \\
\hline Total (Allowed) Hold Timed & $12(28) d$ & $13(28) d$ & $12(28) \mathrm{d}$ & $12(28) d$ & $11(28) d$ & $11(28) d$ \\
\hline Total (Allowed) Hold Time & $20(28) d$ & $21(28) d$ & $20(28) d$ & $20(28) d$ & $19(28) d$ & $19(28) d$ \\
\hline Total (Allowed) Hold Time ${ }^{t}$ & $6(7) d$ & $7(7) d$ & $6(7) d$ & $6(7) d$ & $5(7) d$ & $5(7) d$ \\
\hline
\end{tabular}

\footnotetext{
a. Chloride - Method 9251

b. Fluoride - Method 340.3

c. Nitrate - Method 353.1

d. Sulfate - Method 9036

e. TOC - Method 9060

f. Tox - Method 9020

9-22-92
} 
AREA

LOCATION

TYPE OF LOCATION

SAMP OF LOCAT

MEDIA

UNIIS

SOG NUMBER

\section{ANALYIES}

Chloride

Fluoride

Nitrate

Sul fate

IOC

Tox

Total (Allowed) Hold Iime

Total (Allowed) Hold Timeb

Total (Allowed) Hold Timec

rotal (Allowed) Hold Timed

rotal (Allowed) Hold Time

Total (Allowed) Hold Timef

Chloride - Method 9251

b. Fluoride - Method 340.3

c. Nitrate - Method 353.1

d. Sulfate - Method 9036

e. TOC - Method 9060
f. TOX - Method 9020

9-22-92
OO-FIELD BLANK

CFA1801

WATER

CFA1001

91.0

-.-

1000 Us

$5.0 \mathrm{UJ}$

$17(28) d$

$24(28) d$

$7(28) d$

$13(28) d$

(128)

$21(28) d$ 
CFA Landfills II and III FY89 Wells - 2nd Quarter May 1990 S\&A Data Document

TABLE --_- CFA LANDFILLS II AND III FY89 WELLS - ORGANOCHLORINE PESTICIDE APPENDIX IX DATA

Page 1 of 1

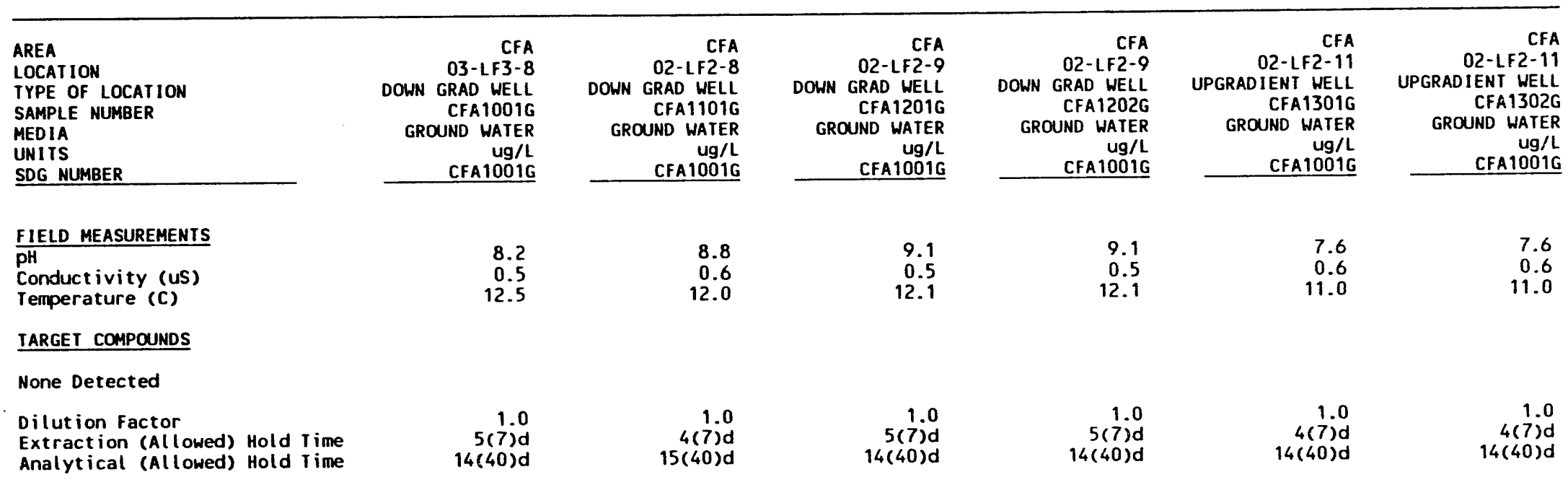

9-11-92 
CFA Landfills II and III FY89 Wells - 2nd Quarter May 1990 S\&A Data Document

TABLE _-_- CFA LANDFILLS II AND III FY89 WELLS - ORGANOCHLORINE PESTICIDE APPENDIX IX FIELD BLANK DATA

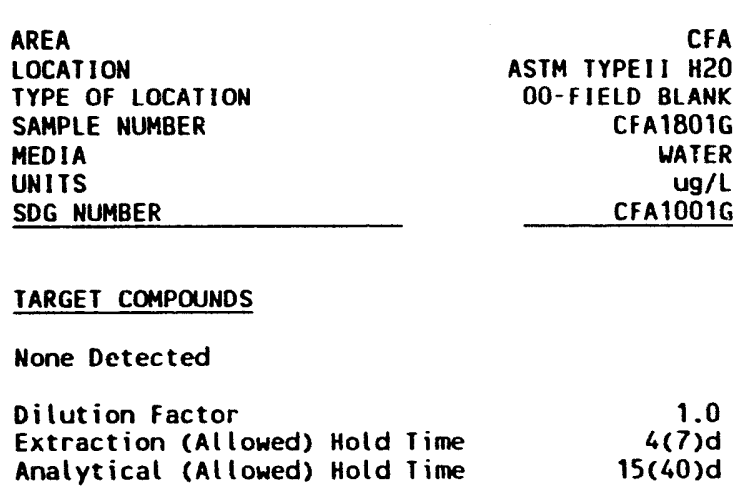

$\stackrel{\overbrace{}}{\stackrel{\oplus}{\infty}}$

$9-11-92$ 
CFA Landfills 11 and III FY89 Hells - 2nd Quarter May 1990 S\&A Data Document

TABLE _-_- CFA LANDFILLS II AND 111 FY89 WELLS - ORGANOCHLORINE HERBICIDE APPENDIX IX DATA

Page 1 of 1

\begin{tabular}{|c|c|c|c|c|c|c|}
\hline $\begin{array}{l}\text { AREA } \\
\text { LOCATION } \\
\text { IYPE OF LOCATION } \\
\text { SAMPLE NUMBER } \\
\text { MEDIA } \\
\text { UNITS } \\
\text { SDG NUMBER } \\
\end{array}$ & $\begin{array}{r}\text { CFA } \\
\text { O3-LF3-8 } \\
\text { DOWN GRAD WELL } \\
\text { CFA 10011 } \\
\text { GROUND WATER } \\
\text { Ug/L } \\
\text { CFA10012 } \\
\end{array}$ & $\begin{array}{r}\text { CFA } \\
\text { O2-LF2-8 } \\
\text { DOWN GRAD WELL } \\
\text { CFA11012 } \\
\text { GROUND HATER } \\
\text { Ug/L } \\
\text { CFA10012 } \\
\end{array}$ & $\begin{array}{r}\text { CFA } \\
\text { O2-LF2-9 } \\
\text { DOWN GRAD HELL } \\
\text { CFA12012 } \\
\text { GROUND WATER } \\
\text { Ug/L } \\
\text { CFA10012 } \\
\end{array}$ & $\begin{array}{r}\text { CFA } \\
\text { 02-LF2-9 } \\
\text { DOWN GRAD WELL } \\
\text { CFA1202Z } \\
\text { GROUND HATER } \\
\text { Ug/L } \\
\text { CFA1001Z } \\
\end{array}$ & $\begin{array}{r}\text { CFA } \\
\text { 02-LF2-11 } \\
\text { UPGRADIENT WELL } \\
\text { CFA13012 } \\
\text { GROUND WATER } \\
\text { Ug/L } \\
\text { CFA10012 } \\
\end{array}$ & $\begin{array}{r}\text { CFA } \\
\text { O2-LF2-11 } \\
\text { UPGRADIENT HELL } \\
\text { CFA13022 } \\
\text { GROUND WATER } \\
\text { Ug/L } \\
\text { CFA10012 } \\
\end{array}$ \\
\hline $\begin{array}{l}\text { FIELD MEASUREMENTS } \\
\text { pH } \\
\text { Conductivity (uS) } \\
\text { Temperature (C) }\end{array}$ & $\begin{array}{r}8.2 \\
0.5 \\
12.5\end{array}$ & $\begin{array}{r}8.8 \\
0.6 \\
12.0\end{array}$ & $\begin{array}{r}9.1 \\
0.5 \\
12.1\end{array}$ & $\begin{array}{r}9.1 \\
0.5 \\
12.1\end{array}$ & $\begin{array}{r}7.6 \\
0.6 \\
11.0\end{array}$ & $\begin{array}{r}7.6 \\
0.6 \\
11.0\end{array}$ \\
\hline \multicolumn{7}{|l|}{ IARGET COMPOUNDS } \\
\hline \multicolumn{7}{|l|}{ None Detected } \\
\hline $\begin{array}{l}\text { Dilution Factor } \\
\text { Extraction (Allowed) Hold Time } \\
\text { Analytical (Allowed) Hold Time }\end{array}$ & $\begin{array}{r}1.0 \\
4(7) d \\
9(40) d\end{array}$ & $\begin{array}{r}1.0 \\
4(7) d \\
9(40) d\end{array}$ & $\begin{array}{r}1.0 \\
4(7) d \\
9(40) d\end{array}$ & $\begin{array}{r}1.0 \\
4(7) d \\
9(40) d\end{array}$ & $\begin{array}{r}1.0 \\
3(7) d \\
9(40) d\end{array}$ & $\begin{array}{r}1.0 \\
3(7) d \\
9(40) d\end{array}$ \\
\hline
\end{tabular}

9-14-92 
CFA Landfills 11 and 111 FY89 Wells - 2nd Quarter May 1990 S\&A Data Document

TABLE _-_- CFA LANDFILLS $\|$ AND $\| 1 /$ FY89 HELLS - ORGANOCHLORINE HERBICIDE APPENDIX IX FIELD BLANK DATA

AREA

LOCATION

TYPE OF LOCATION

SAMPLE NUMBER

MEDIA

UNI TS
SDG NUMBER

TARGET COMPOUNDS

\section{None Detected}

Dilution Factor

Extraction (Allowed) Hold Time Analytical (Allowed) Hold Time

$\begin{array}{r}\text { CFA } \\ \text { ASTM TYPEII H20 } \\ \text { O0-FIELD BLANK } \\ \text { CFA18012 } \\ \text { WATER } \\ \text { Ug/L } \\ \text { CFA10012 } \\ \hline\end{array}$

\section{CFA}

BLANK

UATER
$\mathrm{ug}$

FA10012

9-14-92
1.0
$4(7) d$

$9(40) d$ 



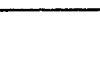

\section{C}

Lab Name: EGGRML

Report No.: CFA10011
Date: $05 / 29 / 90$

Case No.:

SDG No.: CFA10011

\begin{tabular}{|c|c|c|c|c|c|c|c|c|c|c|c|c|c|c|}
\hline $\begin{array}{c}\text { Field } \\
\text { Sample No. }\end{array}$ & Location & $\begin{array}{c}\text { Lab } \\
\text { Sample ID }\end{array}$ & $\begin{array}{l}\text { Sample } \\
\text { Matrix }\end{array}$ & $\begin{array}{l}\text { Anal } \\
\text { Type }\end{array}$ & $\begin{array}{l}\text { Sample } \\
\text { Value }\end{array}$ & $\begin{array}{l}\text { Sample } \\
\text { Error }\end{array}$ & Units & $\begin{array}{l}\text { Anal } \\
\text { Date }\end{array}$ & $\begin{array}{l}\text { Sample } \\
\text { Date }\end{array}$ & $\begin{array}{l}\text { Sample } \\
\text { Size }\end{array}$ & Yield & $\begin{array}{c}\text { Detector } \\
\text { ID }\end{array}$ & DQF & ASL \\
\hline CFA10011 & 03-LF3-8 & $B$ & WATER & ALPHA & $-2.00 E-03$ & $3.00 E-03$ & $\mathrm{pCi} / \mathrm{mL}$ & $07 / 03 / 90$ & $05 / 30 / 90$ & NP & 0.0 & & & \\
\hline CFA11011 & $02-L F 2-8$ & D & WATER & ALPHA & $0.00 E-03$ & $4.00 E-03$ & $\mathrm{pCi} / \mathrm{mL}$ & $07 / 03 / 90$ & $05 / 29 / 90$ & NP & 0.0 & & & \\
\hline CFA12011 & 02-LF2-9 & A & WATER & ALPHA & $-2.00 E-03$ & $3.00 E-03$ & $\mathrm{pCi} / \mathrm{mL}$ & $07 / 03 / 90$ & $05 / 30 / 90$ & NP & 0.0 & & & \\
\hline CFA12021 & 02-LF2-9 & c & WATER & ALPHA & $-2.00 E-03$ & $3.00 \mathrm{E}-03$ & $\mathrm{pCi} / \mathrm{mL}$ & $07 / 03 / 90$ & $05 / 30 / 90$ & NP & 0.0 & & & \\
\hline CFA 13011 & $02-$ LF2-11 & $\underline{G}$ & WATER & ALPHA & $-2.00 E-03$ & $2.00 \mathrm{E}-03$ & $\mathrm{pCi} / \mathrm{mL}$ & $07 / 03 / 90$ & $05 / 31 / 90$ & NP & 0.0 & & & \\
\hline CFA 13021 & $02-L F 2-11$ & $\underline{F}$ & WATER & ALPHA & $0.00 E-03$ & $4.00 E-03$ & $\mathrm{pCi} / \mathrm{mL}$ & $07 / 03 / 90$ & $05 / 31 / 90$ & NP & 0.0 & & & \\
\hline CFA18011 & OO-FIELD BLANK & $\underline{E}$ & WATER & ALPHA & $0.00 E-03$ & $2.00 E-03$ & $\mathrm{pCi} / \mathrm{mL}$ & $07 / 03 / 90$ & $05 / 29 / 90$ & NP & 0.0 & & & \\
\hline
\end{tabular}

NP - Information Not Provided by Laboratory

9-20-92 
CFA Landfills II and III FY89 Wells - 2nd Quarter May 1990 S\&A Data Document - Method Validation Level C

TABLE _-_-_ CFA LANDFILLS II AND III FY89 WELLS - ANALYSIS RESULTS FOR GROSS BETA

Page 1 of 1

Date: $\underline{05 / 29 / 90}$

Lab Name: EGGRML

Case No.:

Report No.: CFA10011

SDG No.: CFA10011

\begin{tabular}{|c|c|c|c|c|c|c|c|c|c|c|c|c|c|c|}
\hline $\begin{array}{c}\text { Field } \\
\text { Sample No. }\end{array}$ & Location & $\begin{array}{l}\text { Lab } \\
\text { Sample ID }\end{array}$ & $\begin{array}{l}\text { Sample } \\
\text { Matrix }\end{array}$ & $\begin{array}{l}\text { Anal } \\
\text { Type }\end{array}$ & $\begin{array}{l}\text { Sample } \\
\text { Value }\end{array}$ & $\begin{array}{l}\text { Sample } \\
\text { Error }\end{array}$ & Units & $\begin{array}{l}\text { Anal } \\
\text { Date }\end{array}$ & $\begin{array}{c}\text { Sample } \\
\text { Date }\end{array}$ & $\begin{array}{l}\text { Sample } \\
\text { size }\end{array}$ & Yield & $\begin{array}{c}\text { Detector } \\
\text { ID }\end{array}$ & DOF & ASL \\
\hline CFA10011 & $03-L F 3-8$ & $\underline{B}$ & WATER & BETA & $1.90 E-02$ & $0.50 E-02$ & $\mathrm{pCi} / \mathrm{mL}$ & $07 / 03 / 90$ & $05 / 30 / 90$ & NP & 0.0 & & & \\
\hline CFA11011 & $02-L F 2-8$ & $\underline{D}$ & WATER & BETA & $1.30 E-02$ & $0.50 E-02$ & $\mathrm{pci} / \mathrm{mL}$ & $07 / 03 / 90$ & $05 / 29 / 90$ & NP & 0.0 & & & \\
\hline CFA12011 & 02-LF2-9 & A & WATER & BETA & $2.80 E-02$ & $0.60 \mathrm{E}-02$ & $\mathrm{pCi} / \mathrm{mL}$ & $07 / 03 / 90$ & $05 / 30 / 90$ & NP & 0.0 & & & \\
\hline CFA12021 & 02-LF2-9 & $\underline{c}$ & WATER & BETA & $2.10 E-02$ & $0.60 E-02$ & $\mathrm{pCi} / \mathrm{mL}$ & $07 / 03 / 90$ & $05 / 30 / 90$ & NP & 0.0 & & & \\
\hline CFA13011 & $02-1 F 2-11$ & $\underline{G}$ & WATER & BETA & $1.40 E-02$ & $0.40 E-02$ & $\mathrm{pCi} / \mathrm{mL}$ & $07 / 03 / 90$ & $05 / 31 / 90$ & NP & 0.0 & & & \\
\hline CFA13021 & $02-L F 2-11$ & $\underline{F}$ & WATER & BETA & $4.10 E-02$ & $0.90 E-02$ & $\mathrm{pCi} / \mathrm{mL}$ & $07 / 03 / 90$ & $\underline{05 / 31 / 90}$ & NP & 0.0 & & & \\
\hline CFA18011 & OO-FIELD BLANK & E & WATER & BETA & $8.00 E-03$ & $4.00 E-03$ & $\mathrm{pCi} / \mathrm{mL}$ & $07 / 03 / 90$ & $05 / 29 / 90$ & NP & 0.0 & & 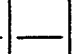 & \\
\hline
\end{tabular}

NP - Information Not Provided by Laboratory

9-20-92 
CFA Landfills II and III FY89 Wells - 2nd Quarter May 1990 S\&A Data Document - Method Validation Level C

TABLE _-_- CFA LANCFILLS II AND III FY89 WELLS - ANALYSIS RESULTS FOR TRITIUM

Page 1 of 1

Date: $\underline{05 / 29 / 90}$

Lab Name: EGGRML

Report No.: CFA10015
Case No.:

SDG NO.: $\quad$ CFA10015

\begin{tabular}{|c|c|c|c|c|c|c|c|c|c|c|c|c|c|c|}
\hline $\begin{array}{l}\text { Field } \\
\text { Sample No. }\end{array}$ & Location & $\begin{array}{c}\text { Lab } \\
\text { Sample ID }\end{array}$ & $\left|\begin{array}{l}\text { Sample } \\
\text { Matrix }\end{array}\right|$ & $\begin{array}{l}\text { Anal } \\
\text { Type }\end{array}$ & $\begin{array}{l}\text { Sample } \\
\text { Value }\end{array}$ & $\begin{array}{l}\text { Sample } \\
\text { Error }\end{array}$ & Units & $\begin{array}{l}\text { Anal } \\
\text { Date }\end{array}$ & $\begin{array}{l}\text { Sample } \\
\text { Date }\end{array}$ & $\begin{array}{l}\text { Sample } \\
\text { Size }\end{array}$ & rield & $\begin{array}{c}\text { Detector } \\
\text { ID }\end{array}$ & DQF & ASL \\
\hline CFA10015 & $03-L F 3-8$ & 2 & WATER & IRITIUM & $4.10 E+01$ & $0.16 E+01$ & $\mathrm{pCi} / \mathrm{mL}$ & $06 / 01 / 90$ & $05 / 29 / 90$ & 10.0000 & 0.0 & & & \\
\hline CFA11015 & $02-$ LF2-8 & 4 & WATER & IRITIUM & $3.90 E+01$ & $0.16 E+01$ & $p \mathrm{Ci} / \mathrm{mL}$ & $06 / 01 / 90$ & $05 / 30 / 90$ & 10.0000 & 0.0 & & & \\
\hline CFA12015 & $02-$ LF2-9 & 3 & WATER & IRITIUM & $4.40 E+01$ & $0.17 E+01$ & $\mathrm{pCi} / \mathrm{mL}$ & $06 / 01 / 90$ & $05 / 30 / 90$ & 10.0000 & 0.0 & & & - \\
\hline CFA12025 & $02-L F 2-9$ & 1 & WATER & IRITIUM & $4.20 E+01$ & $0.17 E+01$ & pCi $/ \mathrm{mL}$ & $06 / 01 / 90$ & $05 / 30 / 90$ & 10.0000 & 0.0 & & & - \\
\hline CFA13015 & $02-$ L.F2-11 & 7 & WATER & IRITIUM & $4.60 E+01$ & $0.20 E+01$ & pci $i / m L$ & $06 / 01 / 90$ & $05 / 29 / 90$ & 10.0000 & 0.0 & & & _ \\
\hline CFA 13025 & $02-L F 2-11$ & 6 & WATER & IRITIUM & $4.80 E+01$ & $0.20 E+01$ & $\mathrm{pCi} / \mathrm{mL}$ & $06 / 01 / 90$ & $05 / 31 / 90$ & 10.0000 & 0.0 & & & - \\
\hline CFA18015 & OO-FIELD BLANK & 5 & WATER & IRITIUM & $-9.00 E-03$ & $7.00 E+01$ & $p c i / m L$ & $06 / 01 / 90$ & $05 / 31 / 90$ & 10.0000 & 0.0 & & & - \\
\hline
\end{tabular}

9-20-92 


\section{Appendix C.3}

\section{Validated Groundwater Quality Data for CFA Landfills II and III}

October 1990 

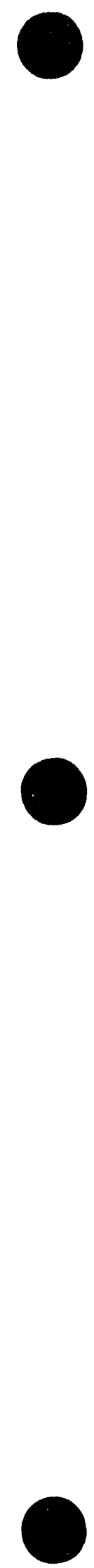

C.3-2 


\section{DATA QUALIFIER DEFINITIONS}

\section{Organic Analysis Data Flags}

A - Indicates that a tentatively identified compound is a suspected aldol-condensation product.

B - Used when the analyte is found in the associated blank as well as in the sample.

C - Applies to pesticide results where the identification has been confirmed by GC/MS.

D - Identifies all compounds identified in an analysis at a secondary dilution factor.

E - Identifies compounds which have concentrations that exceed the calibration range of the GC/MS instrument for that specific analysis.

J - Indicates as estimated value.

$\mathbf{U}$ - Indicates compound was analyzed for but not detected.

\section{Organic Validation Data Qualifiers}

J - The analyte was positively identified in the sample, but the associated numerical value may not be an accurate representation of the amount actually present in the environmental sample. The data should be seriously considered for decision making and are usable for many purposes.

N - Presumptive evidence of the presence of the material.

NJ - Presumptive evidence of the presence of the material at an estimated quantity.

R - The data are unusable (may or may not be present). Resampling and reanalysis is necessary for verification.

U - The material was analyzed for but was not detected. The associated numerical value is the sample quantitation limit.

UJ - The material was analyzed for, but was not detected. The sample quantitation limit is an estimated quantity.

Reference: Environmental Restoration Department, Sample Management Office Standard Operating Procedures 12.1.4 and 12.1.5 (EG\&G Idaho). 


\section{DATA QUALIFIER DEFINITIONS}

Inorganic Analysis Data Flags

B - Value is less than the CRDL, but greater than the IDL.

E - Value is estimated because of the presence of interference.

N - Spiked sample recovery not within control limits.

NR - Analyte was not required to be analyzed.

S - Value was determined by the method of standard additions (MSA).

U - Analyte was analyzed for but not detected.

W - Post digestion spike for Furnace AA analysis is out of control limits (85\% to $115 \%$ ), while sample absorbance is less than $50 \%$ of spike absorbance.

* Duplicate analysis not within control limits.

Methods

A - Flame AA.

CV - Manual Cold Vapor AA.

F - Furnace AA.

NR - Not run, analyte not required to be analyzed.

P - Inductively Coupled Plasma - Atomic Emission Spectrometry.

\section{Inorganic Validation Data Qualifiers}

J - The analyte was analyzed for and was positively identified, but the associated numerical value may not be consistent with the amount actually present in the environmental sample.

R - The data are unusable.

U - The material was analyzed for, but was not detected above the level of the associated value. The associated value is either the sample quantitation limit or the sample detection limit.

UJ - The material was analyzed for, but was not detected. The associated value is an estimate and may not accurately reflect the instrument detection limit in the sample matrix. 
CFA Landfills II and III FY89 Wells - 3rd Quarter October 1990 S\&A Data Document

TABLE -. CFA LANDFILLS II AND III FY89 WELLS - 524.2 VOLATILE ORGANIC DATA

Page 1 of 2

\begin{tabular}{l} 
AREA \\
LOCATION \\
TYPE OF LOCATION \\
SAMPLE NUMBER \\
MEDIA \\
UMITS \\
SDG MUMBER \\
\hline
\end{tabular}

\section{FIELD MEASUREMENTS}

Conduct ivity (uS;

Temperature (C)

TARGET COMPOUNDS

1,1,1-Trichloroethane

Benzene

Trichloroethene

Toluene

1,2-D ibromo-3-chloropropane Naphthalene

Dilution Factor

Total (Allowed) Hold Time

$\begin{array}{rr}\text { CFA } & \text { CFA } \\ \text { O3-LF3-8 } & \text { O2-LF2-8 } \\ \text { DOUN GRAD WELL } & \text { DOWN GRAD WELL } \\ \text { CFA1901A } & \text { CFA2001A } \\ \text { GROUND WATER } & \text { GROUND WATER } \\ \text { Ug/L } & \text { Ug/L } \\ \text { CFA2001A } & \text { CFA2001A }\end{array}$

CFA
O2-LF2-9
DONM GRAD WELL
CFA2101A
GROUND WATER
UG/L
CFA2001A

CFA
O2-LF2-9
DOWN GRAD UELL
CFA2102A
GROUND WATER
Ug/L
CFA2001A

$\begin{array}{r}\text { CFA } \\ \text { O2-LF2-11 } \\ \text { UPGRADIENT WELL } \\ \text { CFA2201A } \\ \text { GROUND HATER } \\ \text { Ug/L } \\ \text { CFAZOOIA } \\ \hline\end{array}$

$\begin{array}{rr}8.4 & 9.1 \\ 0.5 & 0.6 \\ 11.7 & 11.7\end{array}$

\section{9}

$\begin{array}{rr}9.6 & 0.6 \\ 11.7 & 11.5\end{array}$

8.9
0.6

0.6
11.5

8.0
0.7

0.6
0.5

$0.1 \mathrm{~J}$

$0.3 \mathrm{~J}$

$0.1 \mathrm{~J}$

$0.2 \mathrm{~J}$
0.5

$0.5 \mathrm{~s}$

0.5 UR

0.5 UR

1.000
$11(14) d$

$0.4 \mathrm{~J}$

-..

-..

$0.3 \mathrm{~J}$

-..-

$0.1 \mathrm{~J}$

0.5 UR

0.5 UR

1.000

1.000

1.000
$12(14) d$

$13(14) d$

1.000

$11(14) \mathrm{d}$

8.7
0.7
11.0

$0.3 \mathrm{~J}$

$0.3 \mathrm{~J}$

-..

0.5 UR

1000

$11(14) d$

9-11-92 
CFA Landfills II and III FY89 Wells - 3rd Quarter October 1990 S\&A Data Document

TABLE CFA LANDFILLS $\|$ AND 11 fY89 WELLS - 524.2 volatile ORganic DATA

(Cont inued)

OCATIO

TYPE OF LOCATION

SAMPLE NUMBER

MEDIA

SDG MUMBER

\section{CFA}

UPGRADIENT UELL

CFA2202A

GROUND WATER

Ug/L

FIELD MEASUREMENTS

PH

Temperature (C)

\section{TARGET COMPOUNDS}

1,1,1-Trichloroethane

Trichloroethene

Toluene

\begin{tabular}{c}
8.0 \\
0.7 \\
11.0 \\
$0.3 \mathrm{~J}$ \\
\hdashline.- \\
$\cdots-$. \\
0.5 UR \\
$\cdots$ \\
$10(14) \mathrm{d}$
\end{tabular}

$\begin{array}{lr}\begin{array}{l}\text { 1,2-Dibromo-3-chloropropane } \\ \text { Naphthal ene }\end{array} & 0.5 \text { UR } \\ \text { Dilution Factor } & 1.000 \\ \text { Total (Allowed) Hold Time } & 10(14) \mathrm{d}\end{array}$

9-11-92 
CFA Landfills II and III FY89 Wells - 3rd Quarter October 1990 S\&A Data Document

TABLE

CFA LANDFILLS II AND III FY89 WELLS - 524.2 VOLATILE ORGANIC FIELD BLANK DATA

\section{ARÉA}

LOCATION

TYPE OF LOCATION

SAMPLE MUMBER

MEDIA

SDG NUMBER

$\begin{array}{r}\text { CFA } \\ \text { ASTM TYPEII H2O } \\ \text { O0-FIELD BLANK } \\ \text { CFA2701A } \\ \text { MATER } \\ \text { Ug/L } \\ \text { CFA2001A } \\ \hline\end{array}$

TARGET COMPOUNDS

Chloroform

Bromodichloromethane

1,2-D ibromo-3-chloropropane

$0.3 \mathrm{~J}$

0.5 UR

Dilution Factor

Total (Allowed) Hold Time

1.000

$12(14) d$

9-11-92

\section{!}

BLANK

UATER 
CFA Lendfills II and III fY89 Wells - 3rd Quarter October 1990 S\&A Data Document

TABLE -. CFA LANDFILLS II AND III FY89 velLS - 524.2 VOLATILE ORGANIC IRIP BLANK DATA

\begin{tabular}{l} 
AREA \\
LOCATION \\
TYPE OF LOCATION \\
SAMPLE MUMBER \\
MEDIA \\
UNITS \\
SDG MUMBER \\
\hline
\end{tabular}

TARGET COMPOUNDS

Chloroform

Benzene

1,4-Dichlorobenzene

1,2-Dichlorobenzene

1,2-Dibromo-3-chloropropane

Naph thalene

\section{Dilution Factor}

Total (Allowed) Hold Time

$9-11-92$

\begin{tabular}{|c|c|c|}
\hline $\begin{array}{r}\text { CFA } \\
\text { QC } \\
\text { 00-TRIP BLANK } \\
\text { CFA2301A } \\
\text { UATER } \\
\text { Ug/L } \\
\text { CFA2001A } \\
\end{array}$ & $\begin{array}{r}\text { CFA } \\
\text { QC } \\
\text { O0-TRIP BLANK } \\
\text { CFA2401A } \\
\text { UATER } \\
\text { Ug/L } \\
\text { CFA2001A } \\
\end{array}$ & $\begin{array}{r}\text { CFA } \\
\text { QC } \\
\text { O0-TRIP BLANK } \\
\text { CFA2501A } \\
\text { WATER } \\
\text { Ug } / L \\
\text { CFA2001A } \\
\end{array}$ \\
\hline $\begin{array}{l}12 \\
\cdots \\
\cdots \\
\cdots \\
\cdots\end{array}$ & $\begin{array}{l}12 \\
\cdots- \\
\cdots- \\
\cdots- \\
-.\end{array}$ & $\begin{array}{l}12 \\
0.3 \mathrm{~J} \\
0.1 \\
0.1 \mathrm{~J} \\
0.1 \mathrm{~J}\end{array}$ \\
\hline 0.5 UR & 0.5 UR & $\begin{array}{l}0.5 \text { UR } \\
0.1 \mathrm{~J}\end{array}$ \\
\hline $\begin{array}{r}1.000 \\
10(14) d\end{array}$ & $\begin{array}{r}1.000 \\
12(14) d\end{array}$ & $\begin{array}{r}1.000 \\
11(14) d\end{array}$ \\
\hline
\end{tabular}

A

12

$0.1 \mathrm{~J}$

$0.1 \mathrm{j}$

5 UR

$1(14) d$ 
CFA Landfills II and III FY89 Wells - 3rd Quarter October 1990 S\&A Data Document

TABLE _-_- CFA LANDFILLS II AND 111 FY89 WELLS - SEMIVOLATILE APPENDIX IX ORGANIC dATA

Page 1 of 4

\begin{tabular}{l} 
AREA \\
LOCATION \\
TYPE OF LOCATION \\
SAMPLE NUMBER \\
MEDIA \\
UNITS \\
SOG MUMBER \\
\hline
\end{tabular}

\section{FIELD MEASUREMENTS}

Conductivity (uS)

Temperature (C)

TARGET COMPOUNDS

2 -chlorophenol

2- - Methylophenol

4-Methylphenol

2-Nitrophenol

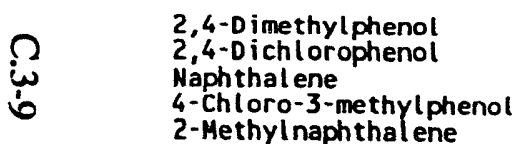

\section{$2,4,6$-Trichlorophenol \\ 2. $c h$ loronaph \\ 2-Chloronaphthalen}

Acenaph thene

2,4-Dini trophenol
4-Nitrophenol

4-Nitrophent
Fluorene

4,6-Dinitro-2-methylphenol

Pentachlorophenol

Phenanthrene

Anthracene

Pyrene

Pyrene
Benzo(a) anthracene

\section{Chrysene}

Benzo(b)f luoranthene

Benzo(k)fluoranthene

Indeno( $1,2,3-c d)$ pyrene

$9-11-92$

$\begin{array}{rr}\text { CFA } & \text { CFA } \\ \text { O3-LF3-8 } & \text { O2-LF2-8 } \\ \text { DOWN GRAD WELL } & \text { DFA19010 } \\ \text { GROUND WATER } & \text { CFA WELL } \\ \text { UG/L } & \text { GROUND WATER } \\ \text { CFA20001A } & \text { Ug/L } \\ \text { CFA2001A }\end{array}$

8.4
0.5
11.7

10 UJ

10 UJ

10 UJ

10 us

$10 \mathrm{UJ}$

10 UJ

10 UJ

$10 \mathrm{us}$

50 UJ

10 UJ

10 UJ

50 UJ

50 UJ

10 UJ

50 UJ

$10 \mathrm{UJ}$

10 US

$10 \mathrm{UJ}$

$10 \mathrm{UJ}$

10 UJ

10 UJ

$10 \mathrm{UJ}$
9.1
0.6
11.7

10 UJ

10 UJ

10 UJ

$10 \mathrm{UJ}$

10 UJ

10 us

$10 \mathrm{UJ}$

$10 \mathrm{Us}$

50 UJ

$10 \mathrm{UJ}$

$10 \mathrm{UJ}$

$50 \mathrm{UJ}$

10 UJ

$50 \mathrm{UJ}$

$10 \mathrm{UJ}$

10 U.

$10 \mathrm{UJ}$

$10 \mathrm{UJ}$

10 UJ

10 UJ

10 UJ

$10 \mathrm{UJ}$

$\begin{array}{r}\text { CFA } \\ \text { O2-LF2-8 } \\ \text { DOWN GRAD WELL } \\ \text { CFA2OO1DRE } \\ \text { GROUND WATER } \\ \text { Ug/L } \\ \text { CFA2001A } \\ \hline\end{array}$

9.1
0.6
11.7

$10 \mathrm{US}$

$10 \mathrm{UJ}$

10 UJ

10 UJ

10 UJ

10 UJ

$10 \mathrm{us}$

$10 \mathrm{UJ}$
$50 \mathrm{UJ}$

$50 \mathrm{UJ}$

10 UJ

$10 \mathrm{UJ}$

50 UJ

20
$\mathrm{UJ}$

50 UJ

10 UJ

$10 \mathrm{UJ}$

$10 \mathrm{UJ}$

10 us

10 US

10 UJ

$10 \mathrm{UJ}$
CFA
F2-9

CFA
DOWN GRAD WELL DOWN GRAD LF2-9 CFA2102D
GROUND HATER CFA2001A CFAZOOIA

.

8.9
0.6

11.5

10 UJ

10 UJ

10 UJ

$10 \mathrm{UJ}$

10 UJ

10 UJ

$10 \mathrm{UJ}$

$10 \mathrm{UJ}$

10 us

10 UJ

$10 \mathrm{UJ}$

50 UJ

$50 \mathrm{UJ}$

10 UJ

50 UJ

10 us

10 UJ

10 UJ

$10 \mathrm{UJ}$

$10 \mathrm{UJ}$

$10 \mathrm{UJ}$

10 UJ

$10 \mathrm{UJ}$

10 US

$10 \mathrm{UJ}$

10 US

10 UJ

$10 \mathrm{UJ}$

$10 \mathrm{UJ}$ 
CFA Landfills II and III FY89 Wells - 3rd Quarter October 1990 S\&A Data Document

TABLE _-_-_ CFA LANDFILLS II AND III FY89 WELLS - SEMIVOLATILE APPENDIX IX ORGANIC DATA

Page 2 of 4

\begin{tabular}{|c|c|c|c|c|c|}
\hline $\begin{array}{l}\text { AREA } \\
\text { LOCATION } \\
\text { TYPE OF LOCATION } \\
\text { SAMPLE NUMBER } \\
\text { MEDIA } \\
\text { UNITS } \\
\text { SDG NUMBER } \\
\end{array}$ & $\begin{array}{r}\text { CFA } \\
\text { 03-LF3-8 } \\
\text { DOWN GRAD HELL } \\
\text { CFA1901D } \\
\text { GROUND WATER } \\
\text { Ug/L } \\
\text { CFA2001A } \\
\end{array}$ & $\begin{array}{r}\text { CFA } \\
\text { 02-LF2-8 } \\
\text { DOWN GRAD WELL } \\
\text { CFA20010 } \\
\text { GROUND WATER } \\
\text { Ug/L } \\
\text { CFAZOO1A } \\
\end{array}$ & $\begin{array}{r}\text { CFA } \\
\text { 02-LF2-8 } \\
\text { DOWN GRAD WELL } \\
\text { CFA2001DRE } \\
\text { GROUND WATER } \\
\text { Ug/L } \\
\text { CFA2001A } \\
\end{array}$ & $\begin{array}{r}\text { CFA } \\
\text { O2-LF2-9 } \\
\text { DOWN GRAD WELL } \\
\text { CFA21010 } \\
\text { GROUND WATER } \\
\text { Ug/L } \\
\text { CFA2001A }\end{array}$ & $\begin{array}{r}\text { CFA } \\
\text { O2-LF2-9 } \\
\text { DOWN GRAD WELL } \\
\text { CFA2102D } \\
\text { GROUND WATER } \\
\text { Ug/L } \\
\text { CFA2001A } \\
\end{array}$ \\
\hline $\begin{array}{l}\text { Dibenz }(a, h) \text { anthracene } \\
\text { Benzo(g,h,i perylene } \\
\text { 3-Methylphenol } \\
\text { 2,6-Dichlorophenol } \\
\text { 1,3-Dinitrobenzene }\end{array}$ & $\begin{array}{l}10 \text { UJ } \\
10 \text { UJ } \\
10 \text { UJ } \\
10 \text { UJ } \\
20 \text { UXJ }\end{array}$ & $\begin{array}{l}10 \mathrm{UJ} \\
10 \mathrm{UJ} \\
10 \mathrm{UJ} \\
10 \mathrm{UJ} \\
20 \mathrm{UXJ}\end{array}$ & $\begin{array}{l}10 \mathrm{UJ} \\
10 \mathrm{UJ} \\
10 \mathrm{UJ} \\
10 \mathrm{UJ} \\
20 \mathrm{UXJ}\end{array}$ & $\begin{array}{l}10 \mathrm{UJ} \\
10 \mathrm{UJ} \\
10 \mathrm{UJ} \\
10 \mathrm{UJ} \\
20 \mathrm{UXJ}\end{array}$ & $\begin{array}{l}10 \mathrm{UJ} \\
10 \mathrm{UJ} \\
10 \mathrm{UJ} \\
10 \mathrm{UJ} \\
20 \mathrm{UXJ}\end{array}$ \\
\hline $\begin{array}{l}\text { 2,3,4,6-Tetrachlorophenol } \\
\text { 3-Methylchol anthrene }\end{array}$ & $\begin{array}{l}10 \mathrm{UXJ} \\
10 \mathrm{UXJ}\end{array}$ & $\begin{array}{l}10 \text { UXJ } \\
10 \text { UXJ }\end{array}$ & $\begin{array}{l}10 \mathrm{UXJ} \\
10 \mathrm{UXJ}\end{array}$ & $\begin{array}{l}10 \mathrm{UXJ} \\
10 \mathrm{UXJ}\end{array}$ & $\begin{array}{l}10 \text { UXJ } \\
10 \mathrm{UXJ}\end{array}$ \\
\hline $\begin{array}{l}\text { Dilution Factor } \\
\text { Extraction (Allowed) Hold Time } \\
\text { Analytical (Allowed) Hold TIme }\end{array}$ & $\begin{array}{c}1.000 \\
10(7) d^{\star} \\
39(40) d^{-}\end{array}$ & $\begin{array}{c}1.000 \\
12(7) d^{\star} \\
13(40) d\end{array}$ & $\begin{array}{c}1.000 \\
12(7) d^{\star} \\
39(40) d\end{array}$ & $\begin{array}{c}1.000 \\
10(7) d^{\star} \\
44(40) d^{\star}\end{array}$ & $\begin{array}{l}1.000 \\
10(7) d^{\star} \\
39(40) d\end{array}$ \\
\hline
\end{tabular}

9-11-92 
CFA Landfills 11 and 111 FY89 Wells - 3rd Quarter October 1990 S\&A Data Document

TABLE _--:- CFA LANDFILLS II AND III FY89 WELLS - SEMIVOLATILE APPENDIX IX ORgANIC DATA (Cont inued)

\begin{tabular}{l} 
AREA \\
LOCATION \\
TYPE OF LOCATION \\
SAMPLE NUMBER \\
MEDIA \\
UNITS \\
SDG NUMBER \\
\hline
\end{tabular}

\begin{tabular}{rr} 
CFA & CFA \\
O2-LF2-11 & O2-LF2-11 \\
UPGRADIENT WELL & UPGRADIENT WELL \\
CFA22010 & CFA2202D \\
GROUND HATER & GROUND WATER \\
UgLL & Ug/L \\
CFA2001A & CFA2001A \\
\hline
\end{tabular}

\section{FIELD MEASUREMENTS}

Conductivity (uS)

Temperature (C)

TARGET COMPOUNDS

Phenol

2-Chlorophenol

2-Methylpheno

2-Methylpheno

2,4-Dimethylphenol

2,4-Dichloropheno

Naphthalene
4-Chloro-3-methylphenol $\quad \begin{aligned} & \text { 2-Methylnaphthalene } \\ & \text { J }\end{aligned}$

\section{$2,4,6$ - Trichlorophenol
$2,4,5$ - Trichlorophenol \\ $2,4,5$-Trichloropheno
2-Chiloronaph thal ene}

Acenaphthylene

Acenaph thene

2,4-Dinitrophenol
4-Nitrophenol

Fluorene

4,6-D initro-2-methylphenol

Pentachlorophenol

\subsection{7}

\section{Phenanthrene}

Anthracene

Fluoranthen

Pyrene
Benzo(a) anthracene

Chrysene

Benzo(b)fluoranthene

Benzo(k)fluoranthene

Benzo(a) pyrene
Indeno( $1,2,3-c d)$ pyrene

11.0

8.0
0.7

10 UR $\quad 10$ UR

U UR

UR

10 UR

10 UR
10 UR
10 UR

10 UR

10 UR

10 UR

50 UR

10 UR

10 UR

50 UR

50 UR

10 UR

50 UR

CFA

10 UR $\quad 10$ UR

10 UR 10 UR

10 UR

1O UR

10 UR

10 UR

UR UR

10 UR

10 UR

10 UR

10 UR

10 UR

$9-11-92$ 
CFA Landfills II and III FY89 Hells - 3rd Quarter October 1990 S\&A Data Document

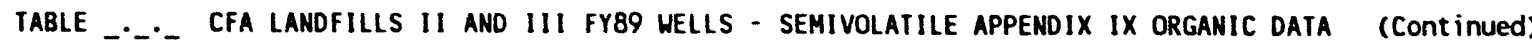

AREA

TYPE OF LOCATION

SAMPLE NUMBER

MEDIA

SDG NUMBER

Dibenz $(a, h)$ anthracene

Benzo( $(9, h, i)$ perylen

-Methyl phenol

1, -Dichlorophenol

$2,3,4,6$-Tetrachlorophenol

2,3,4,6-Tetrachlorophen
3-Methylchol anthrene

Dilution Factor

Extraction (Allowed) Hold Time

Analytical (Allowed) Hold rime

$9-11-92$

\begin{tabular}{|c|c|}
\hline $\begin{array}{r}\text { CFA } \\
\text { 02-LF2-11 } \\
\text { UPGRADIENT WELL } \\
\text { CFA22010 } \\
\text { GROUND WATER } \\
\text { U9/L } \\
\text { CFA2001A }\end{array}$ & $\begin{array}{r}\text { CFA } \\
\text { O2-LF2-11 } \\
\text { UPGRADIENT WELL } \\
\text { CFAZ202D } \\
\text { GROUND WATER } \\
\text { Ug/L } \\
\text { CFA2001A }\end{array}$ \\
\hline $\begin{array}{l}10 \text { UR } \\
10 \text { UR } \\
10 \text { UR } \\
10 \text { UR } \\
20 \text { UXR }\end{array}$ & $\begin{array}{l}10 \text { UR } \\
10 \text { UR } \\
10 \text { UR } \\
10 \text { UR } \\
20 \text { UXR }\end{array}$ \\
\hline $\begin{array}{l}10 \text { UXR } \\
10 \text { UXR }\end{array}$ & $\begin{array}{l}10 \text { UXR } \\
10 \text { UXR }\end{array}$ \\
\hline $\begin{array}{c}1.000 \\
13(7) d^{\star} \\
10(40) d\end{array}$ & $\begin{array}{c}1.000 \\
13(7) d^{\star} \\
10(40) d\end{array}$ \\
\hline
\end{tabular}

10 UR

10 UR

$10 \underset{10}{\text { UXR }}$

1.000

$10(40) d$ 
CFA Landfills 11 and 111 fY89 Hells - 3rd Quarter October 1990 S\&A Data Document

TABLE -. CFA LANDFILLS II AND III FYB9 WELLS - SEMIVOLATILE APPENDIX IX ORGANIC FIELD BLANK DATA

AREA LOCATION

TYPE OF LOCATION

SAMPLE NUMBER

MEDIA

SOG NUMBER

\section{TARGET COMPOUNDS}

Phenol

2-Chlorophenol

2-Methylphenol

2-Nitrophenol

2.4-Dimethylphenol

Naphthal ene

2-Methylnaphthalene

2,4,6-Trichloropheno

$2,4,5$ - Trichloropheno

2-Chloronaph that en

Acenaphthene

2,4-D initrophenol

4-Nitrophenol

Fluorene

4,6-D initro-2-methylphenol

Pentachlorophenol

Phenanthrene

Anthracene

Fluoranthene

Pyrene
Benzo(a) anthracene

\section{Chrysene}

Benzo(b)fluoranthene

Benzo(k)fluoranthene

indeno(1,2,3-cd)pyrene

Dibenz $(a, h)$ anthracene

Benzo(s, $h$, i)perylene

3-Methyl phenol

2,6-0ichloropheno

1,3-Dini trobenzene

2,3,4,6-Tetrachlorophenol

-Methylchol anthrene

Dilution Factor

Extraction (Allowed) Hold Time
Analytical (Allowed) Hold Time

9-11-92
CFA

ASTM TYPEII H2O

CFAZOOIA

10 Us

Us

10 UJ

$10 \mathrm{UJ}$

10 UJ

10 UJ

$10 \mathrm{UJ}$

10 UJ

10 us

10 UJ

$10 \mathrm{UJ}$

50 UJ

50 UJ

$10 \mathrm{UJ}$

50 UJ

$50 \mathrm{UJ}$

10 UJ

10 UJ

10

10 UJ

$10 \mathrm{UJ}$

$10 \mathrm{UJ}$

$10 \mathrm{UJ}$

10 UJ

$10 \mathrm{UJ}$

10 us

$10 \mathrm{UJ}$

10 UJ

$10 \mathrm{UJ}$

10 UXJ

1.000

$12(7) d^{*}$
$14(40) d$ 
CFA Landfills II and III FY89 Mells - 3rd Quarter October 1990 S\&A Data Document

TABLE _-:- CFA LANDFILLS II AND 111 FYBO WELLS - IMORGANIC DATA

Page 1 of 2

\begin{tabular}{|c|c|c|c|c|c|c|}
\hline $\begin{array}{l}\text { AREA } \\
\text { LOCATION }\end{array}$ & $\begin{array}{r}\text { CFA } \\
03-L F 3-8\end{array}$ & $\begin{array}{r}\text { CFA } \\
03-L F 3-8\end{array}$ & $\begin{array}{r}\text { CFA } \\
02-1: 2-8\end{array}$ & $\begin{array}{r}\text { CFA } \\
02-L F 2-8\end{array}$ & $\begin{array}{r}\text { CFA } \\
02-L F 2-9\end{array}$ & $\begin{array}{r}\text { CFA } \\
02-L F 2-9\end{array}$ \\
\hline $\begin{array}{l}\text { TYPE OF LOCATION } \\
\text { SAMPLE NUMBER }\end{array}$ & $\begin{array}{l}\text { DOWN GRAD HELL } \\
\text { CFA1901H }\end{array}$ & $\begin{array}{r}\text { DOWN GRAD WELL } \\
\text { CFA1901M }\end{array}$ & $\begin{array}{l}\text { DOUN GRAD WELL } \\
\text { CFA2001H }\end{array}$ & $\begin{array}{l}\text { DOWN GRAD MELL } \\
\text { CFAZOOIM }\end{array}$ & $\begin{array}{r}\text { DOWN GRAD WELL } \\
\text { CFA2101H }\end{array}$ & $\begin{array}{l}\text { DOWH GRAD WELL } \\
\text { CFA2101M }\end{array}$ \\
\hline MEDIA & GROUND WATER & GROUND WATER & GROUND WATER & GROUND MATER & GROUND MATER & GROUND MATER \\
\hline $\begin{array}{l}\text { UNITS } \\
\text { SDG NUMBER } \\
\end{array}$ & $\begin{array}{r}\text { ug/L } \\
\text { CFA2001A }\end{array}$ & $\begin{array}{r}\text { Ug/L } \\
\text { CFA2001A }\end{array}$ & $\begin{array}{r}u g / L \\
\text { CFA2001A }\end{array}$ & $\begin{array}{r}u g / L \\
\text { CFA2001A }\end{array}$ & $\begin{array}{r}u g / L \\
\text { CFA2001A }\end{array}$ & $\begin{array}{r}\text { Ug/L } \\
\text { CFA2001A }\end{array}$ \\
\hline \multicolumn{7}{|l|}{ FIELD MEASUREMENTS } \\
\hline$\overline{\mathrm{pH}}$ & 8.3 & 8.3 & 9.1 & 9.1 & 8.9 & 8.9 \\
\hline Conductivity (US) & 0.5 & 0.5 & 0.6 & 0.6 & 0.6 & 0.6 \\
\hline Temperature (C) & 11.7 & 11.7 & 11.7 & 11.7 & 11.5 & 11.5 \\
\hline \multicolumn{7}{|l|}{ ANALYTES } \\
\hline$\overline{\text { Arsenic }}$ & -. &.-- & $\ldots$ & $\cdots$ &.-- &.-- \\
\hline Barium & $103 \mathrm{~B}$ & $94.0 \mathrm{~B}$ & $154 \mathrm{~B}$ & $130 \mathrm{~B}$ & $167 \mathrm{~B}$ & $162 \mathrm{~B}$ \\
\hline Cadnium & -- & $\ldots$ & $\ldots$ & $\ldots$ & $\ldots$ & $\ldots$ \\
\hline Chromium & 409 & $\cdots$ & 776 & 12.0 & 30.0 & $9.0 \mathrm{~B}$ \\
\hline Iron & 5590 & $57.0 \mathrm{~B}$ & 3990 & $49.0 \mathrm{~B}$ & 2250 & 103 \\
\hline Lead & $\cdots$ & $\cdots$ & ... & $\cdots$ & -.. & $\ldots$ \\
\hline Manganese & 54.0 & $10.0 \mathrm{~B}$ & 72.0 & $6.0 \mathrm{~B}$ & $9.0 \mathrm{~B}$ & $5.0 \mathrm{~B}$ \\
\hline Mercury & 0.34 & $\cdots$ & 0.37 & $0.12 \mathrm{~B}$ & $\cdots$ & $\cdots$ \\
\hline Selenium & $3.1 \mathrm{BHJ}$ & 3.4 BHJ & -.. & $\cdots$ & $\cdots$ &.- \\
\hline Silver & -- & $\cdots$ & $\cdots$ & $\cdots$ & $\cdots$ & $\cdots$ \\
\hline Sodium & 39700 & 38600 & 40000 & 39500 & 41700 & 42900 \\
\hline $\begin{array}{l}\text { Total (Allowed) Hold Time } \\
\text { Total (Allowed) Hold Time } \\
\text { Total (All owed) Hold Time }\end{array}$ & $\begin{array}{l}42(180) d \\
20(26) d \\
42(180) d\end{array}$ & $\begin{array}{l}42(180) d \\
20(26) d \\
42(180) d\end{array}$ & $\begin{array}{l}43(180) d \\
21(26) d \\
43(180) d\end{array}$ & $\begin{array}{r}43(180) d \\
21(26) d \\
43(180) d\end{array}$ & $\begin{array}{l}42(180) d \\
20(26) d \\
42(180) d\end{array}$ & $\begin{array}{l}42(180) d \\
20(26) d \\
42(180) d\end{array}$ \\
\hline
\end{tabular}
a. ICP
b. CVAAS
c. GFAAS
9-11-92 
CFA Landfills II and III FY89 Mells - 3rd Quarter October 1990 S\&A Data Document

TABLE _--:- CFA LANDFILLS 11 AND III FY89 WELLS - INORGANIC DATA (Continued)

Page 2 of 2

\begin{tabular}{|c|c|c|c|c|c|c|}
\hline $\begin{array}{l}\text { AREA } \\
\text { LOCATION } \\
\text { TYPE OF LOCATION } \\
\text { SAMPLE NUMBER } \\
\text { MEDIA } \\
\text { UNITS } \\
\text { SDG NUMBER }\end{array}$ & $\begin{array}{r}\text { CFA } \\
\text { O2-LF2-9 } \\
\text { DOWN GRAD WELL } \\
\text { CFA2102H } \\
\text { GROUND HATER } \\
\text { Ug/L } \\
\text { CFA2001A } \\
\end{array}$ & $\begin{array}{r}\text { CFA } \\
\text { O2-LF2-9 } \\
\text { DOWN GRAD WELL } \\
\text { CFA2102M } \\
\text { GROUND WATER } \\
\text { Ug/L } \\
\text { CFA2001A } \\
\end{array}$ & $\begin{array}{r}\text { CFA } \\
\text { O2-LF2-11 } \\
\text { UPGRADIENT UELL } \\
\text { CFA2201H } \\
\text { GROUND WATER } \\
\text { Ug/L } \\
\text { CFA2001A } \\
\end{array}$ & $\begin{array}{r}\text { CFA } \\
\text { O2-LF2-11 } \\
\text { UPGRADIENT LELL } \\
\text { CFA2201M } \\
\text { GROUND WATER } \\
\text { Ug/L } \\
\text { CFA2001A } \\
\end{array}$ & $\begin{array}{r}\text { CFA } \\
\text { O2-LF2-11 } \\
\text { UPGRADIENT WELL } \\
\text { CFA2202H } \\
\text { GROUND WATER } \\
\text { Ug/L } \\
\text { CFA2001A } \\
\end{array}$ & $\begin{array}{r}\text { CFA } \\
\text { O2-LF2-11 } \\
\text { UPGRADIENT MELL } \\
\text { CFA2202M } \\
\text { GROUND UATER } \\
\text { Ug/L } \\
\text { CFA2001A } \\
\end{array}$ \\
\hline $\begin{array}{l}\text { FIELD MEASUREMENTS } \\
\text { pH } \\
\text { Conduct ivity (uS) } \\
\text { Temperature (C) }\end{array}$ & $\begin{array}{r}8.9 \\
0.6 \\
11.5\end{array}$ & $\begin{array}{r}8.9 \\
0.6 \\
11.5\end{array}$ & $\begin{array}{r}8.0 \\
0.7 \\
11.0\end{array}$ & $\begin{array}{r}8.0 \\
0.7 \\
11.0\end{array}$ & $\begin{array}{r}8.0 \\
0.7 \\
11.0\end{array}$ & $\begin{array}{r}8.0 \\
0.7 \\
11.0\end{array}$ \\
\hline $\begin{array}{l}\text { ANALYIES } \\
\text { Arsenic } \\
\text { Barium } \\
\text { Cadmium } \\
\text { Chromium } \\
\text { Iron }\end{array}$ & $\begin{array}{c}157 \text { B } \\
206 \\
2220\end{array}$ & $\begin{array}{c}\ldots- \\
151 \mathrm{~B} \\
\ldots- \\
\ldots 9.0 \mathrm{~B}\end{array}$ & $\begin{array}{l}\cdots \\
108 \text { B } \\
\cdots \\
169 \\
7510\end{array}$ & $\begin{array}{l}2.0 \text { UWJ } \\
103 \mathrm{~B} \\
\cdots- \\
\ldots 8.0 \\
48.0 \mathrm{~B}\end{array}$ & $\begin{array}{ll}2.0 & 8 \\
114 & 8 \\
\cdots-. \\
202 \\
9520\end{array}$ & $\begin{array}{c}98.0 \text { B } \\
\cdots \\
\cdots \\
132\end{array}$ \\
\hline $\begin{array}{l}\text { Lead } \\
\text { Manganese } \\
\text { Mercury } \\
\text { Seleniun } \\
\text { Silver }\end{array}$ & $\begin{array}{c}-.- \\
24.0 \\
0.32 \\
\cdots \\
\cdots\end{array}$ & 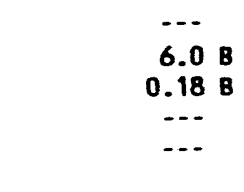 & $\begin{array}{c}64.0 \\
\cdots \\
\cdots\end{array}$ & $\begin{array}{l}\cdots .- \\
24.0 \\
\cdots 3.8 B \\
\cdots\end{array}$ & $\begin{array}{c}\cdots \\
69.0 \\
\cdots \\
\cdots \\
\cdots\end{array}$ & $\begin{array}{l}1.9 \text { BHJ } \\
24.0 \\
0.10 \text { B } \\
3.0 \text { BHJ } \\
\ldots\end{array}$ \\
\hline Sodium & 42600 & 43100 & 44000 & 45500 & 43200 & 44900 \\
\hline $\begin{array}{l}\text { Total (Allowed) Hold Time } \\
\text { Total (Allowed) Hold Time } \\
\text { Total (All owed) Hold Time }\end{array}$ & $\begin{array}{l}42(180) d \\
20(26) d \\
42(180) d\end{array}$ & $\begin{array}{l}42(180) d \\
20(26) d \\
42(180) d\end{array}$ & $\begin{array}{l}41(180) d \\
19(26) d \\
41(180) d\end{array}$ & $\begin{array}{l}41(180) d \\
19(26) d \\
41(180) d\end{array}$ & $\begin{array}{l}41(180) d \\
19(26) d \\
41(180) d\end{array}$ & $\begin{array}{l}41(180) d \\
19(26) d \\
41(180) d\end{array}$ \\
\hline
\end{tabular}
a. ICP
b. CVAAS
c. GFAAS
9-11-92 
CFA Landfills II and III FY89 Wells - 3rd Quarter October 1990 s\&A Data Document

TABLE _--- CFA LANDFILLS II AND III FY89 WELLS - INORGANIC FIELD BLANK DATA

\begin{tabular}{l} 
AREA \\
LOCATION \\
IYPE OF LOCATION \\
SAMPLE NUMBER \\
MEDIA \\
UNITS \\
SDG NUMBER \\
\hline
\end{tabular}

CFA
ASTM TYPEII H2O ASTM TYPEII H2O
OO-FIELD BLANK CFA2701H

SOG NUMBER

ASTM TYPEII H2O

ASTM TYPEII H2O
OO-FIELD BLANK

CFA2701M

ANALYTES

Arsenic

Barium

Cadmium

Chromium

Iron

Lead

Manganese

Mercury

Selenium

Silver

Sodium

Total (Allowed) Hold Time

Total (Allowed) Hold Time

Total (Allowed) Hold Time
a. ICP
b. CVAAS
c. GFAAS
9-11-92 WATER

CFA2001A

$\begin{array}{r}\text { HATER } \\ \text { Ug/L } \\ \hline\end{array}$

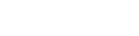

\begin{tabular}{|c|c|}
\hline & ... \\
\hline - - & -.. \\
\hline$\cdots$ & $\cdots$ \\
\hline & \\
\hline
\end{tabular}

$1.4 \mathrm{~B}$

$3.0 \mathrm{~B}$

$0.14 \mathrm{~B}$

$2.0 \mathrm{WJ}$

$+\cdot-$

0.138

-..

161 B 388 в

$43(180) d \quad 43(180) d$

21(26)d 21(26)d

$43(180) d \quad 43(180) d$
Page 1 of 1 
CFA Landfills 11 and III FY89 Wells - 3rd Quarter October 1990 S\&A Data Document - Method Validation Level A

TABLE _... CFA LANDFILls II AND III FY89 wELls - NON-METALS DATA

Page 1 of 1

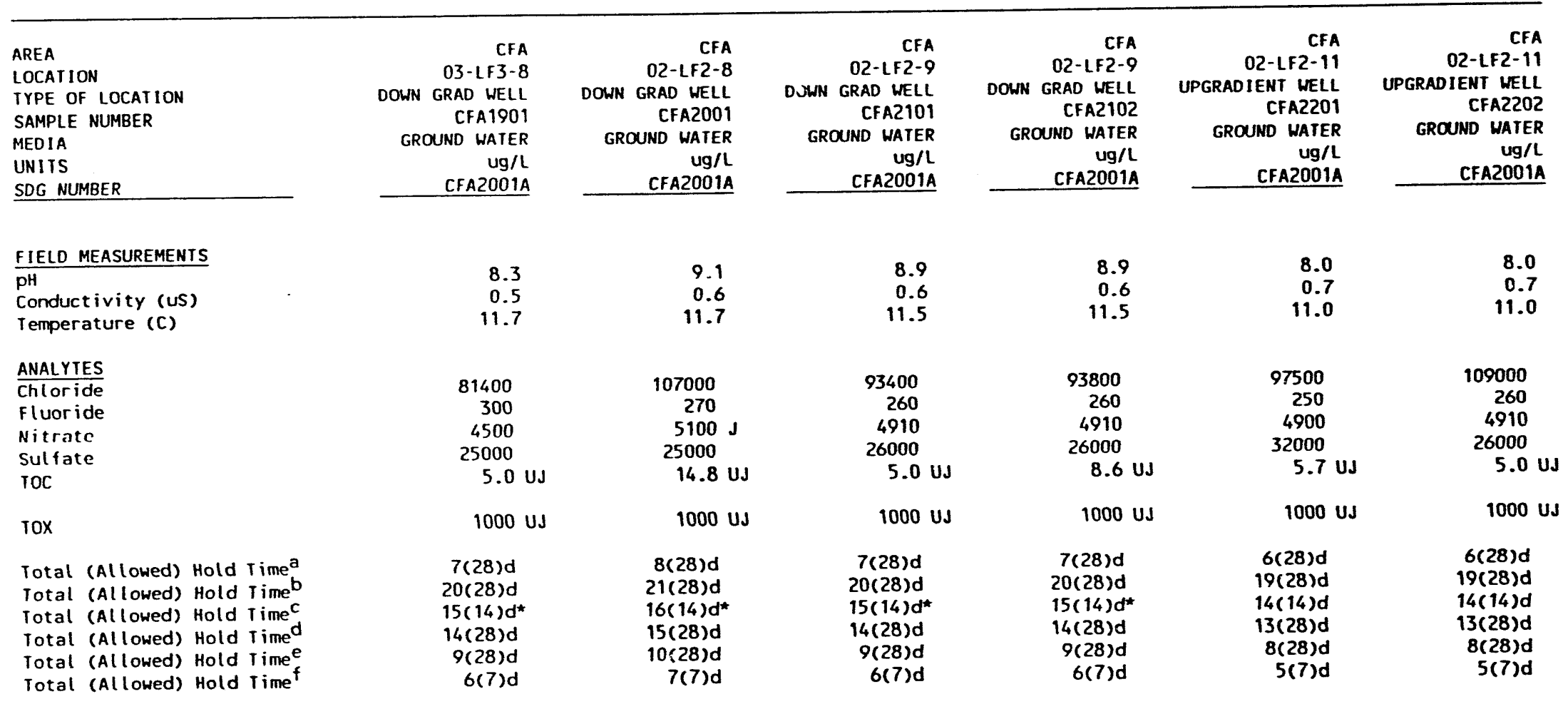

\footnotetext{
a. Chloride - Method 9251

b. Fluoride - Method 340.3

c. Nitrate - Method 353.1

d. Sulfate - Method 9036

e. TOC - Method 9060

f. TOX - Method 9020

$9-22-92$
} 
CFA Landfills 11 and III FY89 Wells - 3rd Quarter October 1990 S8A Data Document - Method Validation Level A

TABLE _. CFA LANDFILLS II AND III FY89 WELLS - NON-METALS FIELD BLANK DATA

\begin{tabular}{|c|c|}
\hline AREA & \\
\hline LOCATION & ASTM TYPEII HZO \\
\hline TYPE OF LOCATION & OO-FIELD BLANK \\
\hline SAMPLE NUMBER & CFA2701 \\
\hline MEDIA & WATER \\
\hline UNITS & $u g / L$ \\
\hline SDG NUMBER & CFA2001A \\
\hline ANALYTES & \\
\hline Chloride & $\cdots$ \\
\hline Fluoride & -.. \\
\hline Nitrate & $10.0 \mathrm{~L}$ \\
\hline Sulfate & 5000 \\
\hline TOC & $11.1 \mathrm{~L}$ \\
\hline TOX & $1000 \mathrm{c}$ \\
\hline Total (Allowed) Hold Timca & $8(28) d$ \\
\hline Total (Allowed) Hold Timeb & $21(28) d$ \\
\hline Total (Allowed) Hold Timec & $16(14) d^{*}$ \\
\hline Total (Allowed) Hold Timed & $15(28) d$ \\
\hline Total (Allowed) Hold Time & $10(28) d$ \\
\hline Total (Allowed) Hold Time & $7(7) d$ \\
\hline
\end{tabular}
a. Chloride - Method 9251
c. Nitrate - Method 353.1
c. Nitrate - Method 353.1
d. Sulfate - Method 9036
e. TOC - Method 9060
f. Tox - Method 9020

$9-22-92$

5000

$11.1 \mathrm{UJ}$

1000 UJ

$(28) d$

$6(14) d^{\star}$

$0(28) \mathrm{d}$

$7(7) d$ 
CFA Landfills II and III FY89 Wells - 3rd Quarter October 1990 S\&A Data Document

TABLE CFA LANDFILLS II AND III FYBG WELLS - ORGANOCHLORINE PESTICIDE APPENDIX IX DATA

Page 1 of 1

\begin{tabular}{|c|c|c|c|c|c|c|}
\hline $\begin{array}{l}\text { AREA } \\
\text { LOCATION } \\
\text { TYPE OF LOCATION } \\
\text { SAMPLE MUMBER } \\
\text { MEDIA } \\
\text { UNITS } \\
\text { SDG NUMBER } \\
\end{array}$ & $\begin{array}{r}\text { CFA } \\
\text { O3-LF3-8 } \\
\text { DOWN GRAD WELL } \\
\text { CFA1901G } \\
\text { GROUND WATER } \\
\text { Ug/L } \\
\text { CFA2001A }\end{array}$ & $\begin{array}{r}\text { CFA } \\
\text { O2-LF2-8 } \\
\text { DOWN GRAD WELL } \\
\text { CFA2001G } \\
\text { GROUND WATER } \\
\text { Ug/L } \\
\text { CFAZOOIA } \\
\end{array}$ & $\begin{array}{r}\text { CFA } \\
\text { O2-LF2-9 } \\
\text { DOWN GRAD WELL } \\
\text { CFA2101G } \\
\text { GROUND WATER } \\
\text { Ug/L } \\
\text { CFA2001A } \\
\end{array}$ & $\begin{array}{r}\text { CFA } \\
\text { O2-LF2-9 } \\
\text { DOWN GRAD WELL } \\
\text { CFA2102G } \\
\text { GROUND HATER } \\
\text { Ug/L } \\
\text { CFAZO01A } \\
\end{array}$ & $\begin{array}{r}\text { CFA } \\
\text { O2-LF2-11 } \\
\text { UPGRADIENT WELL } \\
\text { CFA2201G } \\
\text { GROUND WATER } \\
\text { Ug/L } \\
\text { CFA2001A } \\
\end{array}$ & $\begin{array}{r}\text { CFA } \\
\text { O2-LF2-11 } \\
\text { UPGRADIENT WELL } \\
\text { CFAZ202G } \\
\text { GROUND WATER } \\
\text { Ug/L } \\
\text { CFA2001A } \\
\end{array}$ \\
\hline $\begin{array}{l}\text { FIELD MEASUREMENIS } \\
\text { pH } \\
\text { Conduct ivity (uS) } \\
\text { Temperature (C) }\end{array}$ & $\begin{array}{r}8.3 \\
0.5 \\
11.7\end{array}$ & $\begin{array}{r}9.1 \\
0.6 \\
11.7\end{array}$ & $\begin{array}{r}8.9 \\
0.6 \\
11.5\end{array}$ & $\begin{array}{r}8.9 \\
0.6 \\
11.5\end{array}$ & $\begin{array}{r}8.0 \\
0.7 \\
11.0\end{array}$ & $\begin{array}{r}8.0 \\
0.7 \\
11.0\end{array}$ \\
\hline $\begin{array}{l}\text { TARGET COMPOUMDS } \\
\text { gamma-BHC (Lindane) } \\
\text { Endrin } \\
\text { Methoxychlor } \\
\text { Toxaphene } \\
\text { Aroclor-1016 }\end{array}$ & $\begin{array}{l}0.04 \text { UJ } \\
0.06 \text { UJ } \\
1.80 \text { UJ } \\
2.40 \text { UJ } \\
0.50 \text { UJ }\end{array}$ & $\begin{array}{l}0.04 \mathrm{UJ} \\
0.06 \mathrm{UJ} \\
1.80 \mathrm{UJ} \\
2.40 \mathrm{UJ} \\
0.50 \mathrm{UJ}\end{array}$ & $\begin{array}{l}0.04 \mathrm{UJ} \\
0.06 \mathrm{UJ} \\
1.80 \mathrm{UJ} \\
2.40 \mathrm{UJ} \\
0.50 \mathrm{UJ}\end{array}$ & $\begin{array}{l}0.04 \text { UJ } \\
0.06 \text { UJ } \\
1.80 \text { UJ } \\
2.40 \mathrm{UJ} \\
0.50 \mathrm{UJ}\end{array}$ & $\begin{array}{l}0.04 \mathrm{UJ} \\
0.06 \mathrm{UJ} \\
1.80 \mathrm{UJ} \\
2.40 \mathrm{UJ} \\
0.50 \mathrm{UJ}\end{array}$ & $\begin{array}{l}0.04 \mathrm{UJ} \\
0.06 \mathrm{UJ} \\
1.80 \mathrm{UJ} \\
2.40 \mathrm{UJ} \\
0.50 \mathrm{UJ}\end{array}$ \\
\hline $\begin{array}{l}\text { Aroclor }-1221 \\
\text { Aroclor-1232 } \\
\text { Aroclor }-1242 \\
\text { Aroclor }-1248 \\
\text { Aroclor }-1254\end{array}$ & $\begin{array}{l}0.50 \text { UJ } \\
0.50 \text { UJ } \\
0.65 \text { UJ } \\
0.50 \text { UJ } \\
1 \text { UJ }\end{array}$ & $\begin{array}{l}0.50 \text { UJ } \\
0.50 \text { UJ } \\
0.65 \text { UJ } \\
0.50 \text { UJ } \\
1 \text { UJ }\end{array}$ & $\begin{array}{l}0.50 \text { UJ } \\
0.50 \text { UJ } \\
0.65 \text { UJ } \\
0.50 \text { UJ } \\
1 \text { UJ }\end{array}$ & $\begin{array}{l}0.50 \mathrm{UJ} \\
0.50 \mathrm{UJ} \\
0.65 \mathrm{UJ} \\
0.50 \mathrm{UJ} \\
1 \mathrm{UJ}\end{array}$ & $\begin{array}{l}0.50 \text { UJ } \\
0.50 \text { UJ } \\
0.65 \text { UJ } \\
0.50 \text { UJ } \\
1 \text { UJ }\end{array}$ & $\begin{array}{l}0.50 \mathrm{UJ} \\
0.50 \mathrm{UJ} \\
0.65 \mathrm{UJ} \\
0.50 \mathrm{UJ} \\
1 \mathrm{UJ}\end{array}$ \\
\hline $\begin{array}{l}\text { Dilution Factor } \\
\text { Extraction (Allowed) Hold Time } \\
\text { Analytical (All lowed) Hold Time }\end{array}$ & $\begin{array}{c}1.0 \\
10(7) d^{\star} \\
30(40) d\end{array}$ & $\begin{array}{l}1.0 \\
12(7) \mathrm{d}^{\star} \\
30(40) \mathrm{d}\end{array}$ & $\begin{array}{c}1.0 \\
10(7) d^{*} \\
30(40) d\end{array}$ & $\begin{array}{c}1.0 \\
10(7) d^{*} \\
30(40) d\end{array}$ & $\begin{array}{c}1.0 \\
12(7) \mathrm{d}^{*} \\
28(40) \mathrm{d}\end{array}$ & $\begin{array}{c}1.0 \\
12(7) d^{*} \\
28(40) d\end{array}$ \\
\hline
\end{tabular}

9-14-92 
CFA Landfills II and III FY89 Wells - 3rd Quarter October 1990 S\&A Data Document

TABLE

Page 1 of 1

\begin{tabular}{|c|c|}
\hline $\begin{array}{l}\text { AREA } \\
\text { LOCATION } \\
\text { TYPE OF LOCATION } \\
\text { SAMPLE NUMBER } \\
\text { MEDIA } \\
\text { UNITS } \\
\text { SDG NUMBER } \\
\end{array}$ & $\begin{array}{r}\text { CFA } \\
\text { ASTM IYPEII H20 } \\
\text { O0-FIELD BLANK } \\
\text { CFA2701G } \\
\text { WATER } \\
\text { Ug/L } \\
\text { CFA2001A } \\
\end{array}$ \\
\hline $\begin{array}{l}\text { IARGET C.WPOUNDS } \\
\text { gamma-BHC (L indane) } \\
\text { Endrin } \\
\text { Methoxychlor } \\
\text { Toxaphene } \\
\text { Aroclor-1016 }\end{array}$ & $\begin{array}{l}0.04 \mathrm{UJ} \\
0.06 \mathrm{UJ} \\
1.80 \mathrm{UJ} \\
2.40 \mathrm{UJ} \\
0.50 \mathrm{UJ}\end{array}$ \\
\hline $\begin{array}{l}\text { Aroclor }-1221 \\
\text { Aroclor }-1232 \\
\text { Aroclor }-1242 \\
\text { Aroclor }-1248 \\
\text { Aroclor }-1254\end{array}$ & $\begin{array}{r}0.50 \text { UJ } \\
0.50 \text { UJ } \\
0.65 \text { UJ } \\
0.50 \text { UJ } \\
1 \text { UJ }\end{array}$ \\
\hline $\begin{array}{l}\text { Dilution Factor } \\
\text { Extraction (Allowed) Hold Time } \\
\text { Analytical (Allowed) Hold Time }\end{array}$ & $\begin{array}{c}1.0 \\
12(7) d^{\star} \\
30(40) d\end{array}$ \\
\hline
\end{tabular}

Analytical (Allowed) Hold I ime 30(40)d

$9-14-92$ 
CFA Landfills 11 and III FYB9 Wells - 3rd Quarter October 1990 S\&A Data Document

TABLE _._- CFA LANDFILLS 11 AND 111 FY89 WELLS - ORGANOCHLORINE HERBICIDE APPENDIX IX DATA

Page 1 of 1

AREA

SAMPLE NUMBER

MEDIA

SDG NUMBER

FIELD MEASUREMENIS

pH

Conduct ivity (uS)

Temperature (C)

TARGET COMPOUNDS

2,4-D

Silvex

$2,4,5-1$

Dilution Factor

Exiraction (Allowed) Hold Time

Analytical (Allowed) Hold rime

$9-14-92$
TYPE OF LOCATION

\begin{tabular}{r} 
CFA \\
O3-LF3-8 \\
DOWN GRAD WELL \\
CFA19012 \\
GROUND HATER \\
Ug/L \\
CFA2001A \\
\hline \\
\\
8.3 \\
0.5 \\
11.7 \\
$12 \mathrm{UJ}$ \\
$1.70 \mathrm{UJ}$ \\
$2 \mathrm{UJ}$ \\
1.0 \\
$6(7) \mathrm{d}$ \\
$42(40) \mathrm{d}^{\star}$
\end{tabular}

\begin{tabular}{rr} 
CFA & CFA \\
O2-LF2-8 & O2-LF2-9 \\
DOWN GRAD HELL & DOWN GRAD HELL \\
CFA20012 & CFA21012 \\
GROUND WATER & GROUND WATER \\
UG/L & Ug/L \\
CFA2001A & CFA2001A \\
\hline
\end{tabular}

$\begin{array}{r}\text { CFA } \\ \text { O2-LF2-9 } \\ \text { DOWN GRAD WELL } \\ \text { CFA2102Z } \\ \text { GROUND WATER } \\ \text { UG/L } \\ \text { CFAZO01A } \\ \hline\end{array}$

9.1
0.6
11.7

$12 \mathrm{UJ}$

$1.70 \mathrm{UJ}$
$2 \mathrm{UJ}$

1.0

$8(7) d^{*}$

$42(40) d^{\star}$
8.9
0.6

0.6
11.5

12 UJ

$.70 \mathrm{UJ}$
$2 \mathrm{UJ}$

1.0

$6(7) \mathrm{d}$

$42(40) d^{*}$

$\begin{array}{rr}\text { CFA } & \text { CFA } \\ \text { O2-LF2-11 } & \text { O2-LF2-11 } \\ \text { UPGRADIENT HELL } & \text { UPGRADIENT HELL } \\ \text { CFA22012 } & \text { CFA22022 }\end{array}$

GROUND HATER GROUND WATER

CFA2001A

Ug/L
CFAZOO1A 
CFA Landfills II and III FY89 Wells - 3rd Quarter October 1990 S\&A Data Document

IABLE -. CFA LANDFILLS II AND III FY89 WELLS - ORGANOCHLORINE HERBICIDE APPENDIX IX FIELD BLANK DATA

AREA

LOCATION

TYPE OF LOCATION

SAMPLE NUMBER

MNIIS

SOG MUMBER

TARGET COMPOUNDS

2,4-D

Silvex

$2,4,5-1$

Dilution Factor

Extraction (Allowed) Hold Time
Analytical (Allowed) Hold rime

$\begin{array}{r}\text { CFA } \\ \text { ASIM TYPEII H2O } \\ \text { O0-FIELO BLANK } \\ \text { CFA27012 } \\ \text { WATER } \\ \text { Ug/L } \\ \text { CFAZ001A } \\ \hline\end{array}$

9-14-92

\section{AFA}

A2001A

$12 \mathrm{UJ}$

$1.70 \mathrm{UJ}$

20

$8(7) \mathrm{d}^{*}$

$8(7) \mathrm{d}^{\star}$ 
CFA Landfills II and III FY89 Wells - 3rd Quarter October 1990 S\&A Data Document - Method Validation Level C

TABLE _-_- CFA LANDFILLS II AND III FY89 WELLS - ANALYSIS RESULTS FOR GAMMA-EMITTING RADIONUCLIDES

Page 1 of 1

Date: $\underline{01 / 24 / 91}$

Lab Name: EGGRML

Report No.: SICS00391
Case No.:

SDG No.: CFA19012

\begin{tabular}{|c|c|c|c|c|c|c|c|c|c|c|c|c|c|c|}
\hline $\begin{array}{c}\text { Field } \\
\text { Sample No. }\end{array}$ & Location & $\begin{array}{c}\text { Lab } \\
\text { Sample ID }\end{array}$ & $\begin{array}{l}\text { Sample } \\
\text { Matrix }\end{array}$ & $\begin{array}{l}\text { Anal } \\
\text { Type }\end{array}$ & $\begin{array}{l}\text { Sample } \\
\text { Value }\end{array}$ & $\begin{array}{l}\text { Sample } \\
\text { Error }\end{array}$ & Units & $\begin{array}{l}\text { Anal } \\
\text { Date }\end{array}$ & $\begin{array}{c}\text { Sample } \\
\text { Date }\end{array}$ & $\begin{array}{l}\text { Sample } \\
\text { Size }\end{array}$ & rield & $\begin{array}{c}\text { Detector } \\
\text { ID }\end{array}$ & DQF & ASL \\
\hline CFA19012 & 03-LF3-8 & A6102390042 & WATER & GAMMA & ND & & $\mathrm{pCi} / \mathrm{mL}$ & $10 / 23 / 90$ & $10 / 23 / 90$ & 510.0000 & 0.0 & RML A6 & & \\
\hline CFA20012 & 02-LF2-8 & A5 102290045 & WATER & GAMMA & ND & & $\mathrm{pCi} / \mathrm{mL}$ & $10 / 22 / 90$ & $10 / 22 / 90$ & 540.0000 & 0.0 & RML A5 & & \\
\hline CFA21012 & 02-LF2-9 & C5102390043 & WAIER & GAMMA & ND & & $\mathrm{pCi} / \mathrm{mL}$ & $10 / 23 / 90$ & $10 / 23 / 90$ & 530.0000 & 0.0 & RML C5 & & \\
\hline CFA21022 & 02-LF2-9 & A5 102390041 & WATER & GAMMA & ND & & $\mathrm{pCi} / \mathrm{mL}$ & $10 / 23 / 90$ & $10 / 23 / 90$ & 540.0000 & 0.0 & RML A5 & & \\
\hline CFA22012 & $02-L F 2-11$ & A5 102490037 & WATER & GAMMA & ND & & $p c i / m L$ & $10 / 24 / 90$ & $10 / 24 / 90$ & 540.0000 & 0.0 & RML A5 & & \\
\hline CFA22022 & $02-L F 2-11$ & A6102490038 & WATER & GAMMA & ND & & $\mathrm{pCi} / \mathrm{mL}$ & $10 / 24 / 90$ & $10 / 24 / 90$ & 540.0000 & 0.0 & RML A6 & & \\
\hline CFA27012 & O0-FIELD BLANK & A4 102290044 & WATER & GAMMA & ND & & $\mathrm{pCi} / \mathrm{mL}$ & $10 / 22 / 90$ & $10 / 22 / 90$ & 540.0000 & 0.0 & RML A4 & & \\
\hline
\end{tabular}

$9-20-92$ 
CFA Landfills II and III FY89 Wells - 3rd Quarter October 1990 S\&A Data Document Method Validation Level C

TABLE _-_-_ CFA LANDFILLS II AND III FYBO WELLS - ANALYSIS RESULTS FOR GROSS ALPHA

Page 1 of 1

Date: $11 / 07 / 90$

Lab Name: EGGRML

Case No.:

Report No.: CFA19011

SDG NO.: $\quad$ CFA19011

\begin{tabular}{|c|c|c|c|c|c|c|c|c|c|c|c|c|c|c|}
\hline $\begin{array}{c}\text { Field } \\
\text { Sample No. }\end{array}$ & Location & $\begin{array}{l}\text { Lab } \\
\text { Sample ID }\end{array}$ & $\begin{array}{l}\text { Sample } \\
\text { Matrix }\end{array}$ & $\begin{array}{l}\text { Anal } \\
\text { Type }\end{array}$ & $\begin{array}{l}\text { Sample } \\
\text { Value }\end{array}$ & $\begin{array}{l}\text { Sample } \\
\text { Error }\end{array}$ & Units & $\begin{array}{l}\text { Anal } \\
\text { Date }\end{array}$ & $\begin{array}{l}\text { Sample } \\
\text { Date }\end{array}$ & $\begin{array}{l}\text { Sample } \\
\text { size }\end{array}$ & Yield & $\begin{array}{l}\text { Detector } \\
\text { ID }\end{array}$ & DOF & ASL \\
\hline CFA19011 & $03-L F 3-8$ & B & WATER & ALPHA & $0.00 E-03$ & $1.20 \mathrm{E}-03$ & $\mathrm{pCi} / \mathrm{mL}$ & $10 / 29 / 90$ & $10 / 23 / 00$ & NP & 0.0 & & & \\
\hline CFA20011 & $02-L F 2-8$ & 0 & WATER & ALPHA & $0.00 E-03$ & $1.30 E-03$ & $\mathrm{pCi} / \mathrm{mL}$ & $10 / 29 / 90$ & $10 / 22 / 90$ & NP & 0.0 & & & \\
\hline CFA21011 & $02-$ LF2-9 & A & WATER & ALPHA & $2.00 \mathrm{E}-03$ & $1.40 E-03$ & $\mathrm{pCi} / \mathrm{mL}$ & $10 / 29 / 90$ & $10 / 23 / 90$ & NP & 0.0 & & & \\
\hline CFA21021 & 02-LF2-9 & $F$ & WATER & ALPHA & $0.00 E-03$ & $1.20 E-03$ & $\mathrm{pCi} / \mathrm{mL}$ & $10 / 29 / 90$ & $10 / 23 / 90$ & NP & 0.0 & & & \\
\hline CFA22011 & $02-L F 2-11$ & $E$ & WATER & ALPHA & $0.00 E-03$ & $1.20 \mathrm{E}-03$ & $\mathrm{pCi} / \mathrm{mL}$ & $10 / 29 / 90$ & $10 / 24 / 92$ & NP & 0.0 & & & \\
\hline CFA22021 & $02-$ LF2-11 & c & WATER & ALPHA & $2.00 \mathrm{E}-04$ & $9.00 E-04$ & $\mathrm{pCi} / \mathrm{mL}$ & $10 / 29 / 90$ & $10 / 24 / 90$ & NP & 0.0 & & & \\
\hline CFA27011 & OO-FIELD BLANK & $\underline{G}$ & WATER & ALPHA & $0.00 E-04$ & $9.00 E-04$ & $\mathrm{pCi} / \mathrm{mL}$ & $10 / 29 / 90$ & $10 / 23 / 90$ & NP & 0.0 & & & \\
\hline
\end{tabular}

NP - Information Not Provided by Laboratory

9-20-92 
CFA Landfills 11 and III FY89 Hells - 3rd Quarter October 1990 S\&A Data Document Method Validation Level C

TABLE _.-- CFA LANDFILLS II AND III FY89 WELLS - ANALYSIS RESULTS FOR GROSS BETA

Page 1 of 1

Date: $11 / 07 / 90$

Lab Name: EGGRML

Report No.: CFA19011
Case No.:

SDG No.: CFA19011

\begin{tabular}{|c|c|c|c|c|c|c|c|c|c|c|c|c|c|c|}
\hline $\begin{array}{c}\text { Field } \\
\text { Sample No. }\end{array}$ & Location & $\begin{array}{l}\text { Lab } \\
\text { Sample ID }\end{array}$ & $\begin{array}{l}\text { Sample } \\
\text { Matrix }\end{array}$ & $\begin{array}{l}\text { Anal } \\
\text { Type }\end{array}$ & $\begin{array}{l}\text { Sample } \\
\text { Value }\end{array}$ & $\begin{array}{l}\text { Sample } \\
\text { Error }\end{array}$ & Units & $\begin{array}{l}\text { Anal } \\
\text { Date }\end{array}$ & $\begin{array}{c}\text { Sample } \\
\text { Date }\end{array}$ & $\begin{array}{l}\text { Sample } \\
\text { Size }\end{array}$ & Yield & $\begin{array}{c}\text { Detector } \\
\text { ID }\end{array}$ & DQF & ASL \\
\hline CFA19011 & 03-LF3-8 & B & WATER & BETA & $1.00 \mathrm{E}-02$ & $0.30 E-02$ & $\mathrm{pCi} / \mathrm{mL}$ & $10 / 29 / 90$ & $10 / 23 / 00$ & NP & 0.0 & & & \\
\hline CFAZ20011 & $02-$ LF2-8 & $\underline{D}$ & WATER & BETA & $1.00 E-02$ & $0.20 E-02$ & $\mathrm{pCi} / \mathrm{mL}$ & $10 / 29 / 90$ & $10 / 22 / 90$ & NP & 0.0 & & & \\
\hline CFA21011 & $02-L F 2-9$ & $A$ & WATER & BETA & $1.50 E-02$ & $0.40 E-02$ & $\mathrm{pCi} / \mathrm{mL}$ & $10 / 29 / 90$ & $10 / 23 / 90$ & NP & 0.0 & & & \\
\hline CFA21021 & $02-L F 2-9$ & $\mathbf{F}$ & WATER & BETA & $1.50 \mathrm{E}-02$ & $0.40 E-02$ & $\mathrm{pCi} / \mathrm{mL}$ & $10 / 29 / 90$ & $10 / 23 / 90$ & NP & 0.0 & & & \\
\hline CFA22011 & $02-L F 2-11$ & $\underline{E}$ & WATER & BETA & $1.30 \mathrm{E}-03$ & $0.40 E-03$ & $\mathrm{pCi} / \mathrm{mL}$ & $10 / 29 / 90$ & $10 / 24 / 92$ & $\mathrm{NP}$ & 0.0 & & & \\
\hline CFA22021 & $02-1 F 2-11$ & c & WATER & BETA & $5.00 E-03$ & $2.00 E-03$ & $\mathrm{pCi} / \mathrm{mL}$ & $10 / 29 / 90$ & $10 / 24 / 90$ & NP & 0.0 & & & \\
\hline CFA27011 & OO-FIELD BLANK & $\underline{G}$ & WATER & BETA & $2.00 E-03$ & $2.00 E-03$ & $\mathrm{pCi} / \mathrm{mL}$ & $10 / 29 / 90$ & $10 / 22 / 90$ & NP & 0.0 & & & \\
\hline
\end{tabular}

NP - Information Not Provided by Laboratory

9-20-92 
CFA Landfills II and III FY89 Wells - 3rd Quarter October 1990 S\&A Data Document Method Validation Level C

TABLE --:- CFA LANDFILLS II AND III FY89 WELLS - ANALYSIS RESULTS FOR TRITIUM

Page 1 of 1

Date: $11 / 07 / 90$

Lab Name: EGGRML

Report No.: CFA19015
Case No.:

SDG NO.: CFA19015

\begin{tabular}{|c|c|c|c|c|c|c|c|c|c|c|c|c|c|c|}
\hline $\begin{array}{l}\text { Field } \\
\text { Sample No. }\end{array}$ & Location & $\begin{array}{l}\text { Lab } \\
\text { Sample ID }\end{array}$ & $\left|\begin{array}{l}\text { Sample } \\
\text { Matrix }\end{array}\right|$ & $\begin{array}{l}\text { Anal } \\
\text { Type }\end{array}$ & $\begin{array}{l}\text { Sample } \\
\text { value }\end{array}$ & $\begin{array}{l}\text { Sample } \\
\text { Error }\end{array}$ & Units & $\begin{array}{l}\text { Anal } \\
\text { Date }\end{array}$ & $\begin{array}{l}\text { Sample } \\
\text { Date }\end{array}$ & $\begin{array}{l}\text { Samp!e } \\
\text { Size }\end{array}$ & Yield & $\begin{array}{l}\text { Detector } \\
\text { ID }\end{array}$ & DOF & ASL \\
\hline CFA19015 & $03-L F 3-8$ & 4 & HATER & IRITIUM & $3.90 \mathrm{E}+01$ & $0.20 E+01$ & $\mathrm{pCi} / \mathrm{mL}$ & $10 / 27 / 90$ & $10 / 23 / 00$ & 10.0000 & 0.0 & & - & - \\
\hline CFA20015 & $02-$ LF2-8 & 1 & WATER & IRITIUM & $3.60 E+01$ & $0.20 E+01$ & $\mathrm{pCi} / \mathrm{mL}$ & $10 / 27 / 90$ & $10 / 22 / 90$ & 10.0000 & 0.0 & & & - \\
\hline CFA21015 & $02-$ LF2-9 & 5 & WATER & IRITIUM & $4.00 E+01$ & $0.20 E+01$ & $p C i / m L$ & $10 / 27 / 90$ & $10 / 23 / 90$ & 10.0000 & 0.0 & & & \\
\hline CFA21025 & 02-LF2-9 & $\underline{2}$ & HATER & IRITIUM & $4.10 E+01$ & $0.20 E+01$ & $\mathrm{pci} / \mathrm{mL}$ & $10 / 27 / 90$ & $10 / 23 / 90$ & 10.0000 & 0.0 & & & \\
\hline CFA22015 & $02-L F 2-11$ & 7 & HATER & IRITIUM & $4.30 E+01$ & $0.20 E+01$ & $\mathrm{pCi} / \mathrm{mL}$ & $10 / 27 / 90$ & $10 / 24 / 92$ & 10.0000 & 0.0 & & & - \\
\hline CFA22025 & $02-$ LF2-11 & 6 & WATER & IRITIUM & $4.10 E+01$ & $0.20 E+01$ & $\mathrm{pCi} / \mathrm{mL}$ & $10 / 27 / 90$ & $10 / 24 / 90$ & 10.0000 & 0.0 & & & - \\
\hline CFA27015 & OO-FIELD BLANK & 3 & HATER & IRITIUM & $8.00 E-02$ & $1.20 \mathrm{E}-02$ & $\mathrm{pCi} / \mathrm{mL}$ & $10 / 27 / 90$ & $10 / 22 / 90$ & 10.0000 & 0.0 & & & \\
\hline & & & & & & & & & & & & & & \\
\hline
\end{tabular}

$9-20-52$ 
Appendix C.4

Validated Groundwater Quality Data for CFA Landfills II and III

April 1991 

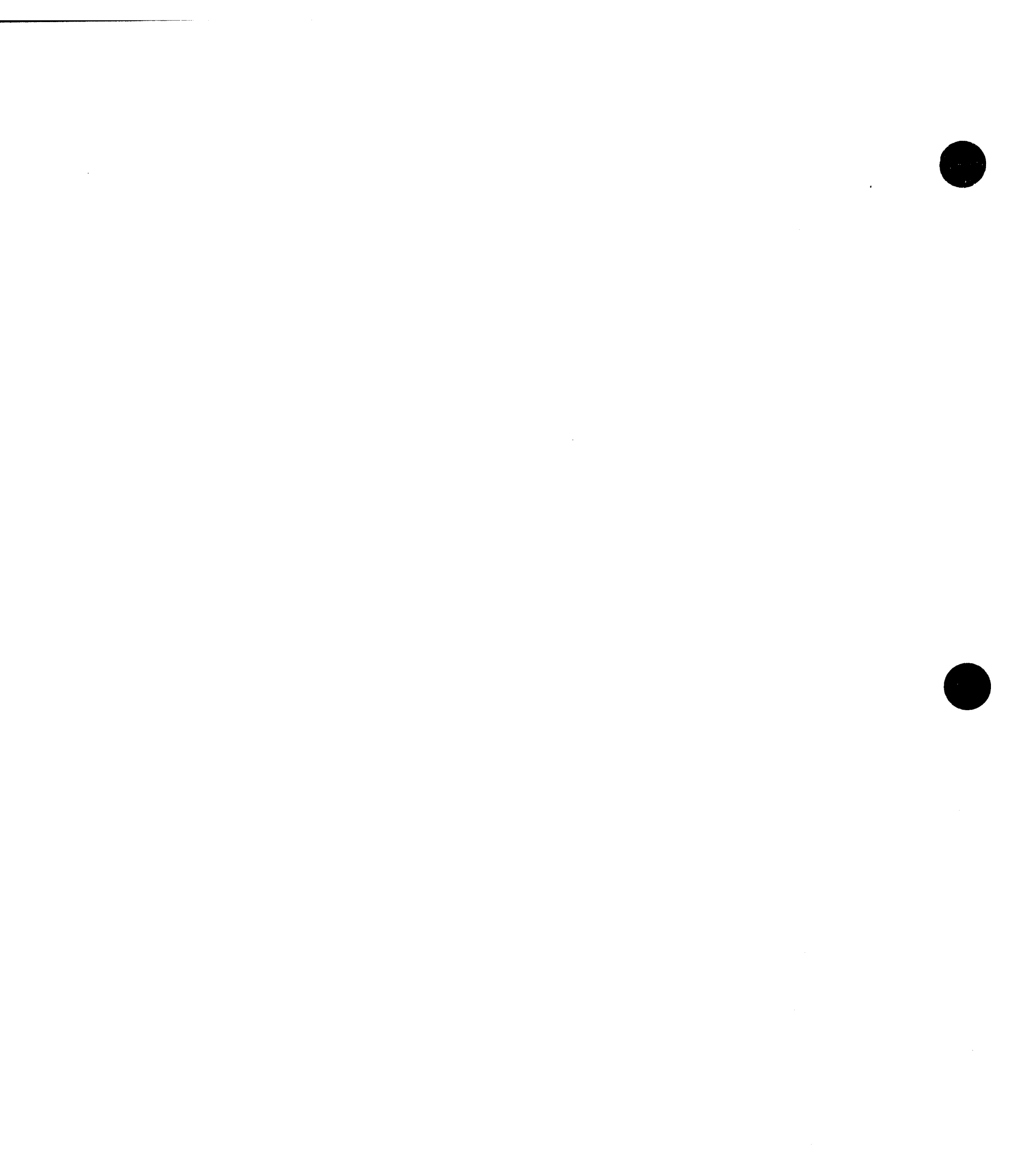

$$
\text { C.4-2 }
$$

\section{C.4-2}




\section{DATA QUALIFIER DEFINITIONS}

\section{Organic Analysis Data Flags}
A - Indicates that a tentatively identified compound is a suspected aldol-condensation product.
B - Used when the analyte is found in the associated blank as well as in the sample.
C. - Applies to pesticide results where the identification has been confirmed by GC/MS.
D - Identifies all compounds identified in an analysis at a secondary dilution factor.
E - Identifies compounds which have concentrations that exceed the calibration range of the GC/MS instrument for that specific analysis.
J - Indicates as estimated value.
$\mathbf{U}$ - Indicates compound was analyzed for but not detected.

\section{Organic Validation Data Qualifiers}

J - The analyte was positively identified in the sample, but the associated numerical value may not be an accurate representation of the amount actually present in the environmental sample. The data should be seriously considered for decision making and are usable for many purposes.

N - Presumptive evidence of the presence of the material.

NJ - Presumptive evidence of the presence of the material at an estimated quantity.

$\mathbf{R}$ - The data are unusable (may or may not be present). Resampling and reanalysis is necessary for verification.

U - The material was analyzed for but was not detected. The associated numerical value is the sample quantitation limit.

UJ - The material was analyzed for, but was not detected. The sample quantitation limit is an estimated quantity.

Reference: Environmental Restoration Department, Sample Management Office Standard Operating Procedures 12.1.4 and 12.1.5 (EG\&G Idaho). 


\section{DATA QUALIFIER DEFINITIONS}

Inorganic Analysis Data Flags

B - Value is less than the CRDL, but greater than the IDL.

E - Value is estimated because of the presence of interference.

N - Spiked sample recovery not within control limits.

NR - Analyte was not required to be analyzed.

S - Value was determined by the method of standard additions (MSA).

U - Analyte was analyzed for but not detected.

W - Post digestion spike for Furnace AA analysis is out of control limits (85\% to $115 \%$ ), while sample absorbance is less than $50 \%$ of spike absorbance.

* - Duplicate analysis not within control limits.

Methods

A - Flame AA.

CV - Manual Cold Vapor AA.

F - Furnace AA.

NR - Not run, analyte not required to be analyzed.

P - Inductively Coupled Plasma - Atomic Emission Spectrometry.

\section{Inorganic Validation Data Qualifiers}

J - The analyte was analyzed for and was positively identified, but the associated numerical value may not be consistent with the amount actually present in the environmental sample.

R - The data are unusable.

U - The material was analyzed for, but was not detected above the level of the associated value. The associated value is either the sample quantitation limit or the sample detection limit.

UJ - The material was analyzed for, $r$ t was not detected. The associated value is an estimate and may not accurateig reflect the instrument detection limit in the sample matrix. 
CFA Landfills II and III FY89 Wells - 4th Quarter April 1991 S\&A Data Document

TABLE _-_- CFA LANDFILLS II AND III FY89 WELLS - 524.2 VOLATILE ORGANIC DATA

Page 1 of 2

\begin{tabular}{|c|c|c|c|c|c|}
\hline $\begin{array}{l}\text { AREA } \\
\text { LOCATION } \\
\text { TYPE OF LOCATION } \\
\text { SAMPLE NUMBER } \\
\text { MEDIA } \\
\text { UNITS } \\
\text { SDG MUMBER } \\
\end{array}$ & $\begin{array}{r}\text { CFA } \\
\text { O3-LF3-8 } \\
\text { DOWN GRAD WELL } \\
\text { CFA2801A } \\
\text { GRCUND HATER } \\
\text { Ug/L } \\
\text { CFA2801A } \\
\end{array}$ & $\begin{array}{r}\text { CFA } \\
\text { O2-LF2-8 } \\
\text { DOWN GRAD WELL } \\
\text { CFA2901A } \\
\text { GROUND HATER } \\
\text { Ug/L } \\
\text { CFA2801A } \\
\end{array}$ & $\begin{array}{r}\text { CFA } \\
\text { O2-LF2-9 } \\
\text { DOWN GRAD HELL } \\
\text { CFA3001A } \\
\text { GROUND HATER } \\
\text { Ug/L } \\
\text { CFA2801A } \\
\end{array}$ & $\begin{array}{r}\text { CFA } \\
\text { O2-LF2-9 } \\
\text { DOWN GRAD HELL } \\
\text { CFA3002A } \\
\text { GROUND WATER } \\
\text { Ug/L } \\
\text { CFA2801A }\end{array}$ & $\begin{array}{r}\text { CFA } \\
\text { O2-LF2-11 } \\
\text { UPGRADIENT WELL } \\
\text { CFA3101A } \\
\text { GROUND WATER } \\
\text { Ug/L } \\
\text { CFA2801A } \\
\end{array}$ \\
\hline $\begin{array}{l}\text { FIELD MEASUREMENTS } \\
\text { pH } \\
\text { Conductivity (uS) } \\
\text { Temperature (C) }\end{array}$ & $\begin{array}{r}7.9 \\
0.6 \\
11.0\end{array}$ & $\begin{array}{r}8.4 \\
0.6 \\
12.2\end{array}$ & $\begin{array}{r}8.4 \\
0.6 \\
11.7\end{array}$ & $\begin{array}{r}8.4 \\
0.6 \\
11.7\end{array}$ & $\begin{array}{r}7.6 \\
0.7 \\
12.8\end{array}$ \\
\hline $\begin{array}{l}\text { TARGET COMPOUNDS } \\
\text { Chloromethane } \\
\text { Methylene Chloride } \\
\text { Chloroform } \\
1,1,1-\text { Trichloroethane } \\
1,2-\text { Dichloroethane }\end{array}$ & $\begin{array}{l}1.8 \\
0.4 \mathrm{~J} \\
0.4 \mathrm{~J} \\
0.6\end{array}$ & $\begin{array}{l}0.2 \mathrm{~J} \\
0.7 \\
0.5 \\
0.7\end{array}$ & $\begin{array}{l}\cdots \\
0.4 \mathrm{~J} \\
0.5 \mathrm{~J} \\
0.5\end{array}$ & $\begin{array}{l}\cdots \\
0.5 \\
0.5 \mathrm{~J} \\
0.6\end{array}$ & $\begin{array}{ll}\cdots \\
\cdots \\
\cdots \\
\cdots\end{array}$ \\
\hline $\begin{array}{l}\text { Trichloroethene } \\
\text { Toluene } \\
\text { Xylene (total meta \& para) } \\
\text { 1,2-Dibromo-3-chloropropane } \\
\text { Naph thalene }\end{array}$ & $\begin{array}{l}0.2 \mathrm{~J} \\
0.5 \mathrm{uJ} \\
\cdots\end{array}$ & $\begin{array}{l}0.6 \\
0.5 \mathrm{~J} \\
0.2 \mathrm{~J} \\
0.5 \mathrm{UJ} \\
0.4 \mathrm{~J}\end{array}$ & $\begin{array}{l}0.2 \mathrm{~J} \\
\cdots \\
0.5 \mathrm{UJ} \\
0.3 \mathrm{~J}\end{array}$ & $\begin{array}{l}\cdots \\
\cdots \\
0.5 \text { UJ } \\
\cdots-\end{array}$ & $\begin{array}{l}\cdots \\
\cdots \\
\cdots .- \\
0 .-\end{array}$ \\
\hline $\begin{array}{l}\text { Dilution Factor } \\
\text { Total (Allowed) Hold Time }\end{array}$ & $\begin{array}{r}1.000 \\
12(14) d\end{array}$ & $\begin{array}{r}1.000 \\
13(14) d\end{array}$ & $\begin{array}{r}1.000 \\
12(14) d\end{array}$ & $\begin{array}{r}1.000 \\
13(14) d\end{array}$ & $\begin{array}{r}1.000 \\
13(14) \mathrm{d}\end{array}$ \\
\hline
\end{tabular}

rotal (Allowed) Hold Time

$12(14) d$

$12(14) \mathrm{d}$

1.000
$13(14) d$

9-13-92 
CFA Landfills II and III FY89 Wells - 4th Quarter April 1991 S\&A Data Document

TABLE

CFA LANDFILLS II AND III FY89 WELLS - 524.2 VOLATILE ORGANIC DATA (Continued)

Page 2 of 2

AREA

LOCATION

TYPE OF LOCATION

SAMPLE MUMBER

MEDIA

SDG MUMBER

CFA

02-LF2-11

CFA3102A

GROUND HATER

Ug/L
CFA2801A

FIELD MEASUREMENTS

PH

Conductivity (uS)

Temperature (C)

Depth $(f t)$

IARGET COMPOUNDS

Chloromethane

Methylene chloride

Chloroform

1,1,1-Trichloroethane

1,1,1-Trichloroethan

Trichloroethene

Toluene

ylene (total meta \& para)

1,2-Dibromo-3-chloropropane

Naphthalene

7.6
0.7
12.8

Dilution Factor

Total (Allowed) Hold Time

12.8

$-$.

$0.5^{\mathrm{J}}$

-..

-..

$0.5 \mathrm{uJ}$

$.5 \mathrm{UJ}$

1.000

$13(14) d$

9-13-92 
CFA Landfills II and III FY89 Hells - 4th Quarter April 1991 S\&A Data Document

TABLE - - - CFA LANDFILLS II AND III FY89 WELLS - 524.2 VOLATILE ORGANIC FIELD BLANK DATA

AREA

LOCATION

TYPE OF LOCATION

TYPE OF LOCATI

SAMPLE

UMITS

SDG NUMBER

IARGET COMPOUNDS

Methylene Chloríde

Chloroform

1,2-Dichloroethane

1,2-Dichloroethane

Toluene

1,2-D ibromo-3-chlor:sropane

Trichlorof luoromentane

CFA

OC
DO-FIELD BLANK

CFA3601A

WATER

Ug/L
CFA2801A

$\Omega$ Dilution Factor

is Total (Allowed) Hold Time

9-13-92

1.8
40
0.9
$0.5 \mathrm{~J}$
$0.3 \mathrm{~J}$
$0.5 \mathrm{JJ}$
$0.8 \mathrm{~J}$
1.000
$12(14) \mathrm{d}$

- $-13-92$ 


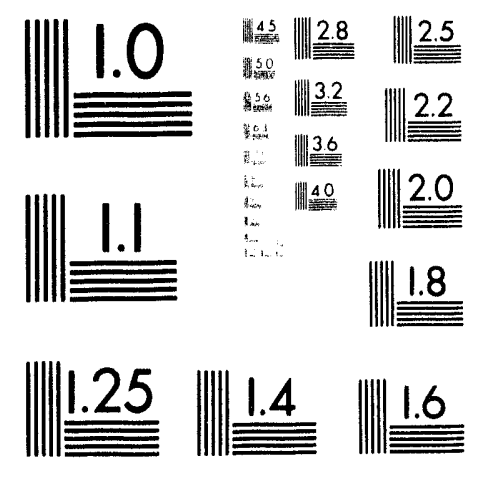



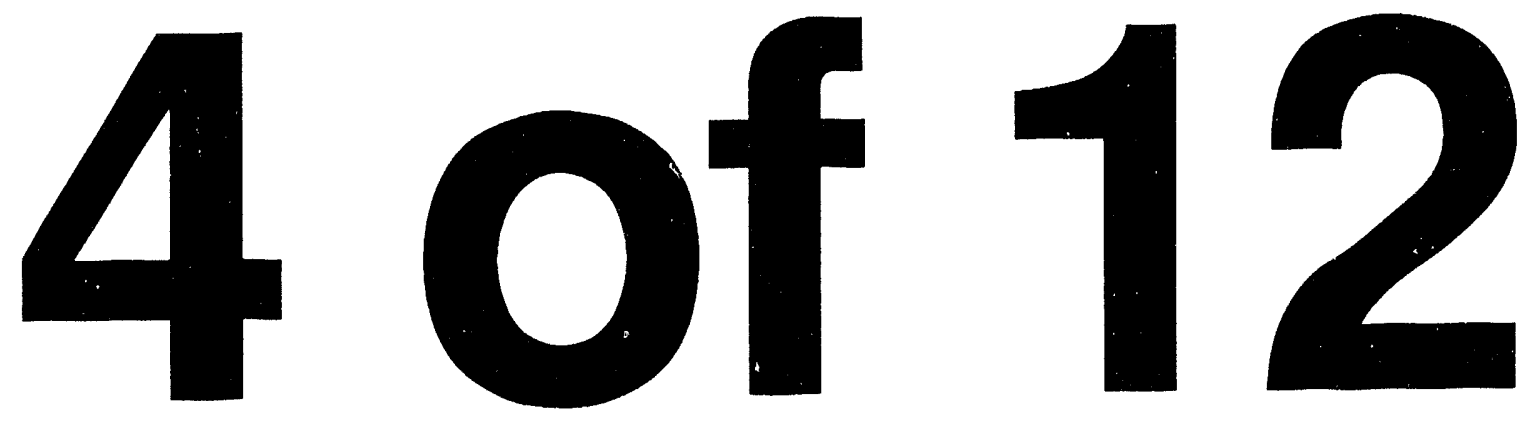
CFA Landfills II and III FY89 Hells - 4th Quarter April 1991 S\&A Dsta Document

TABLE

CFA LANDFILLS II AND III FY89 WELLS - 524.2 VOLATILE ORGANIC TRIP BLANK DATA

AREA

IOCATIOH

TYPE OF LOCATION

SAMPLE NUMBER

MEDIA

SDG NUMBER

TARGET COMPOUNDS

Methylene Chloride

trans-1,2-Dichloroethene

Chloroform

Carbon Tetrachloride

1,1-Dichloropropene

\section{Benzene}

1,2-Dichloroethane

Trichloroethene

Bromodichloromethane

Toluene

1,1,2-Trichloroethane

Tetrachloroethene

Chlorobenzene

Xylene (total meta \& para)

1,4-Dichlorobenzene

\section{1,2-Dichlorobenzene}

1,2-0 ibromo-3-chloropropane

Hexach lor obutadiene

Naphthal ene

1,2,3-Trichlorobenzene

Irichlorofluoromethane

Dilution Factor
Total (Allowed) Hold Time

9-13-92

\begin{tabular}{|c|c|c|}
\hline $\begin{array}{r}\text { CFA } \\
\text { OC } \\
\text { O0-TRIP BLANK } \\
\text { CFA3201A } \\
\text { HATER } \\
\text { Ug/L } \\
\text { CFA2801A } \\
\end{array}$ & $\begin{array}{r}\text { CFA } \\
\text { OC } \\
\text { O0-TRIP BLANK } \\
\text { CFA3201ARE } \\
\text { WATER } \\
\text { Ug/L } \\
\text { CFA2801A } \\
\end{array}$ & $\begin{array}{r}\text { CFA } \\
\text { OC } \\
\text { O0-TRIP BLANK } \\
\text { CFA3401A } \\
\text { WAER } \\
\text { Ug/L } \\
\text { CFA2801A } \\
\end{array}$ \\
\hline $\begin{array}{l}1.1 \\
0.2 \mathrm{~J} \\
0.2 \mathrm{~J} \\
0 .-\end{array}$ & $\begin{array}{c}1.4 \\
\cdots \\
\cdots \\
\cdots\end{array}$ & $\begin{array}{c}1 \\
36 \\
0.4 \mathrm{~J}\end{array}$ \\
\hline $\begin{array}{l}0.2 \mathrm{~J} \\
0.3 \mathrm{~J} \\
0.3 \mathrm{~J}\end{array}$ & $\begin{array}{l}\cdots \\
\cdots \\
\cdots \\
\cdots\end{array}$ & $\begin{array}{c}\cdots \\
\cdots \\
0.5 \\
0.5\end{array}$ \\
\hline $0.3 \mathrm{~J}$ & $\cdots$ & $\ldots$ \\
\hline $\begin{array}{ll}0.2 & \mathrm{~J} \\
0.2 & \mathrm{~J} \\
0.2 & \mathrm{~J} \\
0.3 \mathrm{~J} & \\
0.2 & \mathrm{~J}\end{array}$ & $\begin{array}{l}\cdots \\
\cdots \\
\cdots \\
\cdots \\
\cdots\end{array}$ & $\begin{array}{l}\cdots \\
\cdots \\
\cdots \\
\cdots\end{array}$ \\
\hline $\begin{array}{l}0.2 \mathrm{~J} \\
0.5 \mathrm{UJ} \\
0.2 \mathrm{~J} \\
1.2 \mathrm{~J} \\
0.3 \mathrm{~J}\end{array}$ & $\begin{array}{l}\ldots . . \\
0.5 \mathrm{UJ} \\
0.2 \mathrm{~J}\end{array}$ & $\begin{array}{l}0.5 \text { UJ } \\
\cdots \\
\cdots \\
\cdots\end{array}$ \\
\hline$\cdots$ & ... & 1.4 \\
\hline $\begin{array}{r}13.000 \\
13(14) d\end{array}$ & $\begin{array}{r}1.000 \\
14(14) d\end{array}$ & $\begin{array}{r}1.000 \\
12(14) \mathrm{d}\end{array}$ \\
\hline
\end{tabular}


CFA Landfills II and III FY89 Wells - 4th Quarter April 1991 S\&A Data Document

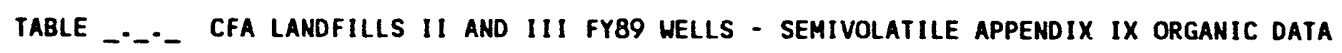

Page 1 of 4

\begin{tabular}{|c|c|c|c|c|c|}
\hline $\begin{array}{l}\text { AREA } \\
\text { LOCATION } \\
\text { TYPE OF LOCATION } \\
\text { SAMPLE NUMBER } \\
\text { MED IA } \\
\text { UNI IS } \\
\text { SDG NUMBER }\end{array}$ & $\begin{array}{r}\text { CFA } \\
03-L F 3-8 \\
\text { DOWN GRAD HELL } \\
\text { CFA2801D } \\
\text { GROUND WATER } \\
\text { Ug/L } \\
\text { CFA2801A }\end{array}$ & $\begin{array}{r}\text { CFA } \\
\text { O3-LF3-8 } \\
\text { DOWN GRAD HELL } \\
\text { CFA2801G } \\
\text { GROUND WATER } \\
\text { Ug/L } \\
\text { CFA2801A }\end{array}$ & $\begin{array}{r}\text { CFA } \\
\text { O2-LF2-8 } \\
\text { DOWN GRAD WELL } \\
\text { CFA2901D } \\
\text { GROUND WATER } \\
\text { Ug/L } \\
\text { CFA2801A }\end{array}$ & $\begin{array}{r}\text { CFA } \\
\text { O2-LF2-9 } \\
\text { DOWN GRAD HELL } \\
\text { CFA3001D } \\
\text { GROUND HATER } \\
\text { Ug/L } \\
\text { CFA2801A }\end{array}$ & $\begin{array}{r}\text { CFA } \\
\text { O2-LF2-9 } \\
\text { DOWN GRAD WELL } \\
\text { CFA3002D } \\
\text { GROUND WATER } \\
\text { Ug/L } \\
\text { CFA2801A }\end{array}$ \\
\hline
\end{tabular}

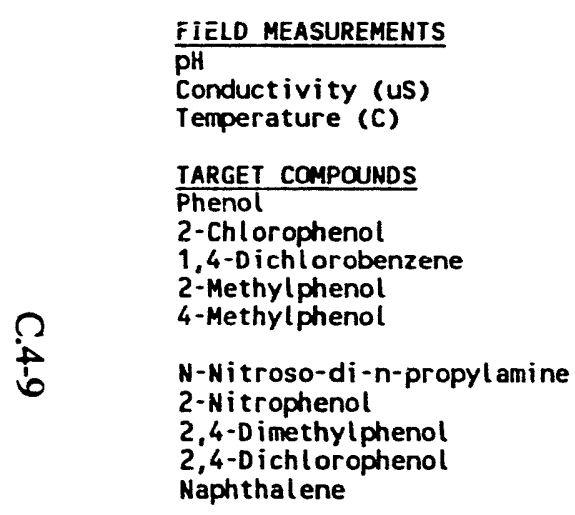

Hexachlorobutadiene

4-Chloro-3-methylphenol

2-Methylnaph thalene

Hexachlorocyclopentadi ene

2,4,6-Trichlorophenol

2,4,5-Trichlorophenol

2-Chloronaph thal ene

Acenaph thylene

Acenaph thene
2,4-Dinitrophenol

4-Nitrophenol

Fluorene

4,6-Dinitro-2-methylpheno

N-Nitrosodiphenyl amine (1)

Pentachlorophenol

Phenanthrene

Anthracene

Fluoranthene

Pyrene

Benzo(a)anthracene

9-13-92

$\begin{array}{rr}7.9 & 7.9 \\ 0.6 & 0.6 \\ 11.0 & 11.0 \\ & \\ 20 \mathrm{UJ} & \\ 20 \mathrm{UJ} & 20 \mathrm{UJ} \\ 20 \mathrm{UJ} & 20 \mathrm{UJ} \\ 20 \mathrm{UJ} & 20 \mathrm{UJ} \\ 20 \mathrm{UJ} & 20 \mathrm{UJ} \\ 20 \mathrm{UJ} & 20 \mathrm{UJ} \\ 20 \mathrm{UJ} & 20 \mathrm{UJ} \\ 20 \mathrm{UJ} & 20 \mathrm{UJ} \\ 20 \mathrm{UJ} & 20 \mathrm{UJ} \\ 20 \mathrm{UJ} & 20 \mathrm{UJ} \\ 20 \mathrm{UJ} & 20 \mathrm{UJ} \\ 20 \mathrm{UJ} & 20 \mathrm{UJ} \\ 20 \mathrm{UJ} & 20 \mathrm{UJ} \\ 20 \mathrm{UJ} & 20 \mathrm{UJ} \\ 20 \mathrm{UJ} & 20 \mathrm{UJ} \\ 100 \mathrm{UJ} & 20 \mathrm{UJ} \\ 20 \mathrm{UJ} & 100 \mathrm{UJ} \\ 20 \mathrm{UJ} & 20 \mathrm{UJ} \\ 20 \mathrm{UJ} & 20 \mathrm{UJ} \\ 100 \mathrm{UJ} & 20 \mathrm{UJ} \\ 100 \mathrm{UJ} & 100 \mathrm{UJ} \\ 20 \mathrm{UJ} & 100 \mathrm{UJ} \\ 100 \mathrm{UJ} & 20 \mathrm{UJ} \\ 20 \mathrm{UJ} & 100 \mathrm{UJ} \\ 100 \mathrm{UJ} & 20 \mathrm{UJ} \\ 20 \mathrm{UJ} & 100 \mathrm{UJ} \\ 20 \mathrm{UJ} & 20 \mathrm{UJ} \\ 20 \mathrm{UJ} & 20 \mathrm{UJ} \\ 20 \mathrm{UJ} & 20 \mathrm{UJ} \\ 20 \mathrm{UJ} & 20 \mathrm{UJ} \\ & 20 \mathrm{UJ} \\ & \\ & \end{array}$

8.4
0.6
12.2
$20 \mathrm{UJ}$
$20 \mathrm{UJ}$
$20 \mathrm{UJ}$
$20 \mathrm{UJ}$
$20 \mathrm{UJ}$
$20 \mathrm{UJ}$
$20 \mathrm{UJ}$
$20 \mathrm{UJ}$
$20 \mathrm{UJ}$
$20 \mathrm{UJ}$
$20 \mathrm{UJ}$
$20 \mathrm{UJ}$
$20 \mathrm{UJ}$
$20 \mathrm{UJ}$
$20 \mathrm{UJ}$
$100 \mathrm{UJ}$
$20 \mathrm{UJ}$
$20 \mathrm{UJ}$
$20 \mathrm{UJ}$
$100 \mathrm{UJ}$
$100 \mathrm{UJ}$
$20 \mathrm{UJ}$
$100 \mathrm{UJ}$
$20 \mathrm{UJ}$
$100 \mathrm{UJ}$
$20 \mathrm{UJ}$
$20 \mathrm{UJ}$
$20 \mathrm{UJ}$
$20 \mathrm{UJ}$
$20 \mathrm{UJ}$

8.4
0.6

8.4

7.9
0.6

8.4

11.7

0.6
11.7

$00 \mathrm{UJ}$

20 UJ

20 UJ

20 UJ

$20 \mathrm{UJ}$

20 UJ

$20 \mathrm{UJ}$

20 UJ

20 UJ

20 UJ

20 UJ

20 UJ

20 UJ

20 UJ

$20 \mathrm{UJ}$

$20 \mathrm{UJ}$

$20 \mathrm{UJ}$

20 UJ

20 UJ

$20 \mathrm{UJ}$

20 UJ

20 UJ

20 UJ

$20 \mathrm{UJ}$

$20 \mathrm{UJ}$

$20 \mathrm{UJ}$

$20 \mathrm{UJ}$

20 UJ

$100 \mathrm{UJ}$

$20 \mathrm{UJ}$

$20 \mathrm{UJ}$

100 UJ

$100 \mathrm{UJ}$

20 UJ

20 UJ

20 UJ

$20 \mathrm{UJ}$

$100 \mathrm{UJ}$

$100 \mathrm{UJ}$

$20 \mathrm{UJ}$

$100 \mathrm{UJ}$

$20 \mathrm{UJ}$

$100 \mathrm{UJ}$

$100 \mathrm{UJ}$

100

$100 \mathrm{UJ}$

20 UJ

$20 \mathrm{UJ}$

20 UJ

$20 \mathrm{UJ}$

20 UJ

$100 \mathrm{UJ}$

$20 \mathrm{UJ}$

$20 \mathrm{UJ}$

20 UJ

$20 \mathrm{UJ}$

20 UJ

$20 \mathrm{UJ}$ 
CFA Landfills II and III FY89 Wells - 4th Quarter April 1991 S\&A Data Document

TABLE _-_- CFA LANDFILLS II AND III FY89 hELLS - SEMIVOLATILE APPENDIX IX ORGANIC DATA (Cont inued)

Page 2 of 4

\begin{tabular}{|c|c|c|c|c|c|}
\hline $\begin{array}{l}\text { AREA } \\
\text { LOCATION } \\
\text { TYPE OF LOCATION } \\
\text { SAMPLE NUMBER } \\
\text { MEDIA } \\
\text { UNITS } \\
\text { SDG NUMBER } \\
\end{array}$ & $\begin{array}{r}\text { CFA } \\
\text { 03-LF3-8 } \\
\text { DOWN GRAD WELL } \\
\text { CFA2801D } \\
\text { GROUND WATER } \\
\text { Ug/L } \\
\text { CFA2801A }\end{array}$ & $\begin{array}{r}\text { CFA } \\
\text { O3-LF3-8 } \\
\text { DOWN GRAD WELL } \\
\text { CFA2801G } \\
\text { GROUND WATER } \\
\text { Ug/L } \\
\text { CFA2801A } \\
\end{array}$ & $\begin{array}{r}\text { CFA } \\
\text { O2-LF2-8 } \\
\text { DOWN GRAD WELL } \\
\text { CFA29010 } \\
\text { GROUND WATER } \\
\text { Ug/L } \\
\text { CFA2801A } \\
\end{array}$ & $\begin{array}{r}\text { CFA } \\
\text { O2-LF2-9 } \\
\text { DOWN GRAD WELL } \\
\text { CFA3001D } \\
\text { GROUND WATER } \\
\text { Ug/L } \\
\text { CFA2801A } \\
\end{array}$ & $\begin{array}{r}\text { CFA } \\
\text { O2-LF2-9 } \\
\text { DOWN GRAD WELL } \\
\text { CFA3002D } \\
\text { GROUND WATER } \\
\text { Ug/L } \\
\text { CFA2801A } \\
\end{array}$ \\
\hline $\begin{array}{l}\text { Chrysene } \\
\text { Di-n-octyl phthal ate } \\
\text { Benzo(b)f fuoranthene } \\
\text { Benzo(k)fluoranthene } \\
\text { Benzo(a)pyrene }\end{array}$ & $\begin{array}{l}20 \mathrm{UJ} \\
9 \mathrm{JJ} \\
20 \mathrm{UJ} \\
20 \mathrm{UJ} \\
20 \mathrm{UJ}\end{array}$ & $\begin{array}{l}20 \text { UJ } \\
20 \text { UJ } \\
20 \text { UJ } \\
20 \text { UJ } \\
20 \text { UJ }\end{array}$ & $\begin{array}{l}20 \text { UJ } \\
20 \text { UJ } \\
20 \text { UJ } \\
20 \text { UJ } \\
20 \text { UJ }\end{array}$ & $\begin{array}{l}20 \mathrm{UJ} \\
48 \mathrm{JJ} \\
20 \mathrm{UJ} \\
20 \mathrm{UJ} \\
20 \mathrm{UJ}\end{array}$ & $\begin{array}{l}20 \mathrm{UJ} \\
20 \mathrm{UJ} \\
20 \mathrm{UJ} \\
20 \mathrm{UJ} \\
20 \mathrm{UJ}\end{array}$ \\
\hline $\begin{array}{l}\text { Indeno }(1,2,3-c d) \text { pyrene } \\
\text { Dibenz }(a, h) \text { anthracene } \\
\text { Benzo(g, } h, i) \text { perylene } \\
\text {-Methylphenol } \\
\text { 2,6-Dichlorophenol }\end{array}$ & $\begin{array}{l}20 \text { UJ } \\
20 \text { UJ } \\
20 \text { UJ } \\
20 \text { UJ } \\
20 \text { UJ }\end{array}$ & $\begin{array}{l}20 \text { UJ } \\
20 \text { UJ } \\
20 \text { UJ } \\
20 \text { UJ } \\
20 \text { UJ }\end{array}$ & $\begin{array}{l}20 \mathrm{UJ} \\
20 \mathrm{UJ} \\
20 \mathrm{UJ} \\
20 \mathrm{UJ} \\
20 \mathrm{UJ}\end{array}$ & $\begin{array}{l}20 \text { UJ } \\
20 \text { UJ } \\
20 \text { UJ } \\
20 \text { UJ } \\
20 \text { UJ }\end{array}$ & $\begin{array}{l}20 \mathrm{UJ} \\
20 \mathrm{UJ} \\
20 \mathrm{UJ} \\
20 \mathrm{UJ} \\
20 \mathrm{UJ}\end{array}$ \\
\hline $\begin{array}{l}\text { 1,3-Dini trobenzene } \\
2,3,4,6 \text {-Tetrachlorophenol } \\
\text { 3-Methylchol anthrene }\end{array}$ & $\begin{array}{l}40 \mathrm{UJ} \\
20 \mathrm{UJ} \\
20 \mathrm{UJ}\end{array}$ & $\begin{array}{l}40 \mathrm{UJ} \\
20 \mathrm{UJ} \\
20 \mathrm{UJ}\end{array}$ & $\begin{array}{l}40 \mathrm{UJ} \\
20 \mathrm{UJ} \\
20 \mathrm{UJ}\end{array}$ & $\begin{array}{l}40 \text { UJ } \\
20 \text { UJ } \\
20 \text { UJ }\end{array}$ & $\begin{array}{l}40 \text { UJ } \\
20 \text { UJ } \\
20 \text { UJ }\end{array}$ \\
\hline $\begin{array}{l}\text { Dilution Factor } \\
\text { Extraction (Allowed) Hold rime } \\
\text { Analytical (Allowed) Hold Time }\end{array}$ & $\begin{array}{l}2.000 \\
4(7) d \\
9(40) d\end{array}$ & $\begin{array}{l}2.000 \\
4(7) d \\
9(40) d\end{array}$ & $\begin{array}{l}2.000 \\
8(7) d^{\star} \\
11(40) d\end{array}$ & $\begin{array}{l}2.000 \\
8(7) d^{j *} \\
10(40) d\end{array}$ & $\begin{array}{l}2.000 \\
8(7) d^{\star} \\
10(40) d\end{array}$ \\
\hline
\end{tabular}

9-13-92 
CFA Landfills II and III FY89 Hells - 4th Quarter April 1991 S\&A Data Document

TABLE _--- CFA LANDFILLS II AND III FY89 wells - SEMIVOLATILE APPENDIX IX ORGANIC DATA (Continued)

\begin{tabular}{l} 
AREA \\
LOCATION \\
TYPE OF LOCATION \\
SAMPIE NUMBER \\
MEDIA \\
UNITS \\
SDG NUMBER \\
\hline
\end{tabular}

\section{FIELD MEASUREMENTS}

Conductivity (uS)

Temperature (C)

TARGET COMPOUNDS

2-Chlorophenol

1.4-Dichlorobenzene

2-Methylphenol

$\stackrel{\Omega}{A}$

N-Nitroso-di-n-propylamine

2-Nitrophenol

\begin{tabular}{|c|c|}
\hline $\begin{array}{r}\text { CFA } \\
\text { O2-LF2-11 } \\
\text { UPGRADIENT WELL } \\
\text { CFA31010 } \\
\text { GROUND WATER } \\
\text { Ug/L } \\
\text { CFA2801A } \\
\end{array}$ & $\begin{array}{r}\text { CFA } \\
\text { O2-LF2-11 } \\
\text { UPGRADIENT WELL } \\
\text { CFA3102D } \\
\text { GROUND WATER } \\
\text { Ug } / L \\
\text { CFA2801A } \\
\end{array}$ \\
\hline $\begin{array}{r}7.6 \\
0.7 \\
12.8\end{array}$ & $\begin{array}{r}7.6 \\
0.7 \\
12.8\end{array}$ \\
\hline $\begin{array}{l}20 \mathrm{UJ} \\
20 \mathrm{UJ} \\
20 \mathrm{UJ} \\
20 \mathrm{UJ} \\
20 \mathrm{UJ}\end{array}$ & $\begin{array}{l}20 \mathrm{UJ} \\
20 \mathrm{UJ} \\
20 \mathrm{UJ} \\
20 \mathrm{UJ} \\
20 \mathrm{UJ}\end{array}$ \\
\hline $\begin{array}{l}20 \text { UJ } \\
20 \text { UJ } \\
20 \text { UJ } \\
20 \text { UJ } \\
20 \text { UJ }\end{array}$ & $\begin{array}{l}20 \mathrm{UJ} \\
20 \mathrm{UJ} \\
20 \mathrm{UJ} \\
20 \mathrm{UJ} \\
20 \mathrm{UJ}\end{array}$ \\
\hline $\begin{array}{l}20 \text { UJ } \\
20 \text { UJ } \\
20 \text { UJ } \\
20 \text { UJ } \\
20 \text { UJ }\end{array}$ & $\begin{array}{l}20 \mathrm{UJ} \\
20 \mathrm{UJ} \\
20 \mathrm{UJ} \\
20 \mathrm{UJ} \\
20 \mathrm{UJ}\end{array}$ \\
\hline $\begin{array}{r}100 \mathrm{UJ} \\
20 \mathrm{UJ} \\
20 \mathrm{UJ} \\
20 \mathrm{UJ} \\
100 \mathrm{UJ}\end{array}$ & $\begin{array}{r}100 \text { UJ } \\
20 \text { UJ } \\
20 \text { UJ } \\
20 \text { UJ } \\
100 \text { UJ }\end{array}$ \\
\hline $\begin{array}{r}100 \text { UJ } \\
20 \text { UJ } \\
100 \text { UJ } \\
20 \text { UJ } \\
100 \text { UJ }\end{array}$ & $\begin{array}{r}100 \mathrm{UJ} \\
20 \mathrm{UJ} \\
100 \mathrm{UJ} \\
20 \mathrm{UJ} \\
100 \mathrm{UJ}\end{array}$ \\
\hline $\begin{array}{l}20 \mathrm{UJ} \\
20 \mathrm{UJ} \\
20 \mathrm{UJ} \\
20 \mathrm{UJ} \\
20 \mathrm{UJ}\end{array}$ & $\begin{array}{l}20 \mathrm{UJ} \\
20 \mathrm{UJ} \\
20 \mathrm{UJ} \\
20 \mathrm{UJ} \\
20 \mathrm{UJ}\end{array}$ \\
\hline
\end{tabular}

2,4-D imethylphenol

2,4-Dichlorophenol

Naphthalene

Hexachlorobutadiene

4-Chloro-3-methylphenol

2-Methylnaphthalene

Hexachlor ocyclopentadien

ophenol

2,4,5-Trichlorophenol

2-Chloronaph thalene

Acenaphthylene

Acenaphthene

2,4-Dinitrophenol

4-Nitrophenol

Fluorene

4,6-Dinitro-2-methylphenol

$\mathrm{N}-\mathrm{Ni}$ trosodiphenylamine (1)

Pentachlorophenol

Phenanthrene

Anthracene

Fluoranthene

Pyrene

Benzo(a)anthracene

9-13-92 
CFA Landfills II and III FY89 Wells - 4th Quarter April 1991 S\&A Data Document

TABLE -. CFA LANDFILLS II AND III FY89 WELLS - SEMIVOLATILE APPENDIX IX ORGANIC DRTA (Continued)

\begin{tabular}{|c|c|c|}
\hline $\begin{array}{l}\text { AREA } \\
\text { LOCATION } \\
\text { TYPE OF LOCATION } \\
\text { SAMPLE NUMBER } \\
\text { MEDIA } \\
\text { UNITS } \\
\text { SDG MUMBER } \\
\end{array}$ & $\begin{array}{r}\text { CFA } \\
\text { O2-LF2-11 } \\
\text { UPGRADIENT HELL } \\
\text { CFA31010 } \\
\text { GROUND WATER } \\
\text { Ug/L } \\
\text { CFA2801A } \\
\end{array}$ & $\begin{array}{r}\text { CFA } \\
\text { O2-LF2-11 } \\
\text { UPGRADIENT HELL } \\
\text { CFA3102D } \\
\text { GROUND HATER } \\
\text { Ug } / L \\
\text { CFA2801A }\end{array}$ \\
\hline $\begin{array}{l}\text { Chrysene } \\
\text { Di-n-octylphthalate } \\
\text { Benzo(b)fluoranthene } \\
\text { Benzo(k)fluoranthene } \\
\text { Benzo(a)pyrene }\end{array}$ & $\begin{array}{l}20 \mathrm{UJ} \\
4 \mathrm{~J} \\
20 \mathrm{UJ} \\
20 \mathrm{UJ} \\
20 \mathrm{UJ}\end{array}$ & $\begin{array}{l}20 \mathrm{UJ} \\
20 \mathrm{UJ} \\
20 \mathrm{UJ} \\
20 \mathrm{UJ} \\
20 \mathrm{UJ}\end{array}$ \\
\hline $\begin{array}{l}\text { Indeno }(1,2,3-c d) \text { pyrene } \\
\text { Dibenz }(a, h) \text { anthracene } \\
\text { Benzo(g,h,i)perylene } \\
\text { 3-Methy (phenol } \\
\text { 2,6-Dichlorophenol }\end{array}$ & $\begin{array}{l}20 \mathrm{UJ} \\
20 \mathrm{UJ} \\
20 \mathrm{UJ} \\
20 \mathrm{UJ} \\
20 \mathrm{UJ}\end{array}$ & $\begin{array}{l}20 \mathrm{UJ} \\
20 \mathrm{UJ} \\
20 \mathrm{UJ} \\
20 \mathrm{UJ} \\
20 \mathrm{UJ}\end{array}$ \\
\hline $\begin{array}{l}\text { 1,3-Dini trobenzene } \\
2,3,4,6-\text { Tetrachlorophenol } \\
\text { 3-Methylchol anthrene }\end{array}$ & $\begin{array}{l}40 \text { UJ } \\
20 \text { UJ } \\
20 \text { UJ }\end{array}$ & $\begin{array}{l}40 \mathrm{UJ} \\
20 \mathrm{UJ} \\
20 \mathrm{UJ}\end{array}$ \\
\hline $\begin{array}{l}\text { Dilution Factor } \\
\text { Extraction (Allowed) Hold Time } \\
\text { Analytical (Allowed) Hold Time }\end{array}$ & $\begin{array}{c}2.000 \\
8(7) d^{\star} \\
10(40) d^{d}\end{array}$ & $\begin{array}{c}2.000 \\
8(7) d^{\star} \\
10(40) d\end{array}$ \\
\hline
\end{tabular}

9-13-92 
CFA Landíills II and III fY89 Hells - 4th Quarter April 1991 : AA Data Document

TABLE _. CFA LANDFILLS II AND III FY89 WELLS - SEMIVOLATILE APPENDIX IX ORGANIC FIELD BLANK DATA

\section{AREA}

LOCATION

TYPE OF LOCATION

SAMPLE NUMBER

MEDIA

SDG NUMBER

CFA

OC

OO-FIELD BLANK

CFA36010

WATER

Ug/L
CFA2801A

TARGET COMPOUNDS

henol

-Chlorophenol

1,4-Dichl orobenzene

2-Methylphenol

20 UJ

20 Us

20 UJ

$20 \mathrm{UJ}$

N-Nitroso-di-n-propylamine

N-Nitroso-di-
2-Nitrophenol

$20 \mathrm{UJ}$

20 UJ

20 UJ

$20 \mathrm{UJ}$

2,4-Dimethylphenol

2,4-Dichtorophenol

20 UJ

Hexachl orobutadiene

$20 \mathrm{UJ}$

20 UJ

20 UJ

20 UJ

4-Chloro-3-methylphenol

2-Methylnaphthal ene

Hexachl or ocycl opentadiene

$20 \mathrm{UJ}$

2,4,5-Trichlorophenol

100 us

$200 \mathrm{UJ}$

$20 \mathrm{UJ}$

$20 \mathrm{UJ}$

$100 \mathrm{UJ}$

Acenaph thylene

Acenaph thene

2,4-Dini trophenol

100 Us

$20 \mathrm{UJ}$

20 UJ

$20 \mathrm{UJ}$

$100 \mathrm{UJ}$

Fluorene

4,6-Dinitro-2-methylphenol
$N$-Nitrosodiphenylamine (1)

Pentachlorophenol

$20 \mathrm{UJ}$

Phenanthrene

Anthracene

Fluoranthene

Pyrene

Byrene Benzo(a)anthracene

$20 \mathrm{UJ}$

$20 \mathrm{UJ}$

20 UJ

$50 \mathrm{us}$

Chrysene

50 UJ

$3 \mathrm{~J} \mathrm{Ju}$

20 UJ

Di-nzo(b)f phoranthe

Denzo(b)fluoranthene

20 UJ

Benzo(a)pyrene

9-13-92 
CFA Landfills II and III FY89 Wells - 4th Quarter April 1991 S\&A Data Document

TABLE

CFA LANDFILLS II AND III FY89 WELLS - SEMIVOLATILE APPENDIX IX ORGANIC FIELD BLANK DATA (Cont inued)

Page 2 of 2

AREA

LOCATION

TYPE OF LOCATION

SAMPLE NUMBER

CFA

SDG NLIMBE

CFA2801A

Indeno $(1,2,3-c d)$ pyrene

Dibenz $(a, h)$ anthracene

Benzo( $g, h, i)$ perylene

2,6-Dichlorophenol

1,3-Dinitrobenzene

2,3,4,6-Tetrachlorophenol

$20 \mathrm{UJ}$

$20 \mathrm{uJ}$

$20 \mathrm{LJ}$

20 UJ

$40 \mathrm{us}$

$20 \mathrm{UJ}$

$20 \mathrm{UJ}$

3-Methyl chol anthren

2.000

Analytical (Allowed) Hold Time $\quad 9(40) d$

$\because \quad$ Analytical (Allowed) Hold Time

9-13-92 


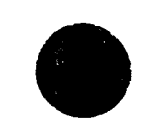

CFA Landfills II and III FY89 Wells - 4th Quarter April 1991 S\&A Data Document

TABLE _-_- C CFA LANDFILLS II AND III FY89 WELLS - INORGANIC DATA

Page 1 of 3

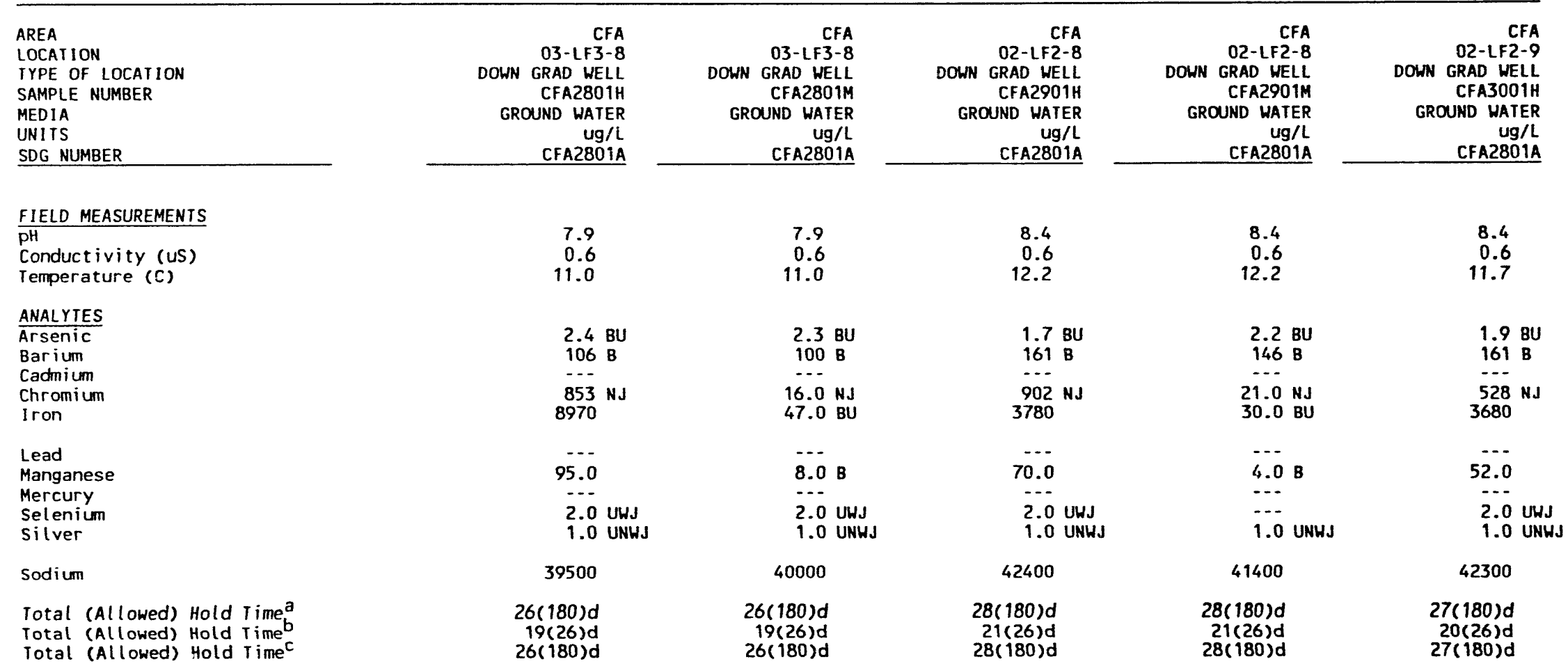
a. ICP
b. CVAAS
b. GFAAS
9-13-92 
CFA Landfills II and III FY89 wells - 4th Quarter April 1991 S\&A Data Document

TABLE -. CFA LANDFILlS II AND 111 fYBo hells - INORgANIC dATA (Continued)

Page 2 of 3

\begin{tabular}{|c|c|c|c|c|c|}
\hline $\begin{array}{l}\text { AREA } \\
\text { LOCATION } \\
\text { TYPE OF LOCATION } \\
\text { SAMPLE NUMBER } \\
\text { MEDIA } \\
\text { UNITS } \\
\text { SDG NUMBER } \\
\end{array}$ & $\begin{array}{r}\text { CFA } \\
\text { O2-LF2-9 } \\
\text { DOWN GRAD WELL } \\
\text { CFA3O01M } \\
\text { GROUND WATER } \\
\text { Ug/L } \\
\text { CFAZ801A } \\
\end{array}$ & $\begin{array}{r}\text { CFA } \\
\text { O2-LF2-9 } \\
\text { DOWN GRAD HELL } \\
\text { CFA3O02H } \\
\text { GROUND HATER } \\
\text { Ug/L } \\
\text { CFAZ801A } \\
\end{array}$ & $\begin{array}{r}\text { CFA } \\
\text { O2-LF2-9 } \\
\text { DOWN GRAD WELL } \\
\text { CFA3002M } \\
\text { GROUHD WATER } \\
\text { Ug/L } \\
\text { CFA2801A } \\
\end{array}$ & $\begin{array}{r}\text { CFA } \\
\text { O2-LF2-11 } \\
\text { UPGRADIENT WELL } \\
\text { CFA3101H } \\
\text { GROUND WATER } \\
\text { Ug/L } \\
\text { CFA2801A } \\
\end{array}$ & $\begin{array}{r}\text { CFA } \\
\text { O2-LF2-11 } \\
\text { UPGRADIENT WELL } \\
\text { CFA3101M } \\
\text { GROUND WATER } \\
\text { Ug/L } \\
\text { CFA2801A } \\
\end{array}$ \\
\hline $\begin{array}{l}\text { FIELD MEASUREMENIS } \\
\text { pH } \\
\text { Conductivity (uS) } \\
\text { Temperature (C) }\end{array}$ & $\begin{array}{r}8.4 \\
0.6 \\
11.7\end{array}$ & $\begin{array}{r}8.4 \\
0.6 \\
11.7\end{array}$ & $\begin{array}{r}8.4 \\
0.6 \\
11.7\end{array}$ & $\begin{array}{r}7.6 \\
0.7 \\
12.8\end{array}$ & $\begin{array}{r}7.6 \\
0.7 \\
12.8\end{array}$ \\
\hline $\begin{array}{l}\text { ANALYTES } \\
\text { Arsenic } \\
\text { Barium } \\
\text { Cadmium } \\
\text { Chromium } \\
\text { Iron }\end{array}$ & $\begin{array}{c}43.6 \\
154 \mathrm{~B} \\
\ldots .0 \\
26.0 \mathrm{NJ}\end{array}$ & $\begin{array}{l}2.7 \mathrm{BU} \\
164 \mathrm{~B} \\
-.- \\
753 \mathrm{NJ} \\
5260\end{array}$ & $\begin{array}{c}5.0 \mathrm{BU} \\
159 \mathrm{~B} \\
\ldots- \\
28.0 \mathrm{NJ} \\
\ldots\end{array}$ & $\begin{aligned} 4.0 \mathrm{BU} \\
140 \mathrm{~B} \\
-. \\
663 \mathrm{NJ} \\
19600\end{aligned}$ & $\begin{aligned} 2.6 \mathrm{BU} \\
125 \mathrm{~B} \\
3.0 \mathrm{~B} \\
10.0 \mathrm{UN} \\
34.0 \mathrm{BU}\end{aligned}$ \\
\hline $\begin{array}{l}\text { Lead } \\
\text { Manganese } \\
\text { Mercury } \\
\text { Selenium } \\
\text { Silver }\end{array}$ & $\begin{array}{l}8.0 \text { B } \\
\cdots \\
1.0 \\
1.0 \text { UNWJ }\end{array}$ & $\begin{array}{l}73.0 \\
\overline{-.} \\
2.0 \\
1.0 \text { UNJ }\end{array}$ & $\begin{array}{l}7.0 \\
7.0 \text { B } \\
2.0 \text { UWJ } \\
1.0 \text { UNWJ }\end{array}$ & $\begin{array}{l}1.0 \text { BWJ } \\
147 \\
-2.0 \text { US } \\
3.1 \text { BNWJ }\end{array}$ & $\begin{array}{l}27.0 \\
\overline{2.3} \text { BWJ } \\
1.0 \text { UNHJ }\end{array}$ \\
\hline Sodium & 42200 & 44400 & 43600 & 49000 & 47100 \\
\hline $\begin{array}{l}\text { Total (Allowed) Hold Time } \\
\text { Total (Allowed) Hold Time } \\
\text { Total (All owed) Hold Time }\end{array}$ & $\begin{array}{l}27(180) d \\
20(26) d \\
27(180) d\end{array}$ & $\begin{array}{l}27(180) d \\
20(26) d \\
27(180) d\end{array}$ & $\begin{array}{l}27(180) d \\
20(26) d \\
27(180) d\end{array}$ & $\begin{array}{l}27(180) d \\
20(26) d \\
27(180) d\end{array}$ & $\begin{array}{l}27(180) d \\
20(26) d \\
27(180) d\end{array}$ \\
\hline
\end{tabular}
a. ICP
c. GFAAS
$9-13-92$ 
CFA Landfills II and III FY89 Wells - 4th Ouarter April 1991 S8A Data Document

TABLE _-.- CFA LANDFILLS II AND III FYBO WELLS - INORGANIC DATA (Cont inued)

Page 3 of 3

\begin{tabular}{|c|c|c|}
\hline $\begin{array}{l}\text { AREA } \\
\text { LOCATION } \\
\text { TYPE OF LOCATION } \\
\text { SAMPLE NUMBER } \\
\text { MEDIA } \\
\text { UNITS } \\
\text { SDG NUMBER } \\
\end{array}$ & $\begin{array}{r}\text { CFA } \\
\text { O2-LF2-11 } \\
\text { UPGRAD IENT WELL } \\
\text { CFA3102H } \\
\text { GROUND WATER } \\
\text { Ug } / L \\
\text { CFA2801A }\end{array}$ & $\begin{array}{r}\text { CFA } \\
\text { O2-LF2-11 } \\
\text { UPGRADIENT WELL } \\
\text { CFA310ZM } \\
\text { GROUND WATER } \\
\text { Ug } / L \\
\text { CFA2801A } \\
\end{array}$ \\
\hline $\begin{array}{l}\text { FIELD MEASUREMENIS } \\
\text { pH } \\
\text { Conductivity (uS) } \\
\text { Temperature (C) }\end{array}$ & $\begin{array}{r}7.6 \\
0.7 \\
12.8\end{array}$ & $\begin{array}{r}7.6 \\
0.7 \\
12.8\end{array}$ \\
\hline $\begin{array}{l}\text { ANALYIES } \\
\text { Arsenic } \\
\text { Barium } \\
\text { Cadmium } \\
\text { Chromium } \\
\text { Iron } \\
\text { Lead } \\
\text { Manganese } \\
\text { Mercury } \\
\text { Selenium } \\
\text { Silver } \\
\text { Sodium }\end{array}$ & $\begin{array}{c}143 \text { B } \\
\ldots 8 \\
489 \text { NJ } \\
19100 \\
\cdots . . \\
130 \\
\cdots- \\
2.0 \text { UWJ } \\
1.0 \text { UNWJ } \\
48100\end{array}$ & $\begin{array}{c}1.9 \mathrm{BU} \\
129 \mathrm{~B} \\
-- \\
15.0 \mathrm{NJ} \\
88.0 \mathrm{BU} \\
--- \\
26.0 \\
-.- \\
\overline{1.0} \\
47200\end{array}$ \\
\hline $\begin{array}{l}\text { Total (Allowed) Hold Timea } \\
\text { Total (Allowed) Hold Time } \\
\text { Total (Allowed) Hold Time }\end{array}$ & $\begin{array}{l}27(180) d \\
20(26) d \\
27(180) d\end{array}$ & $\begin{array}{l}27(180) d \\
20(26) d \\
27(180) d\end{array}$ \\
\hline
\end{tabular}

a. ICP

b. CVAAS

$9-13-92$ 
CFA Landfills II and III FY89 Wells - 4th Quarter April 1991 S\&A Data Document

TABLE -. CFA LANDFILLS II AND III FY89 WELLS - INORGANIC FIELD BLANK DATA

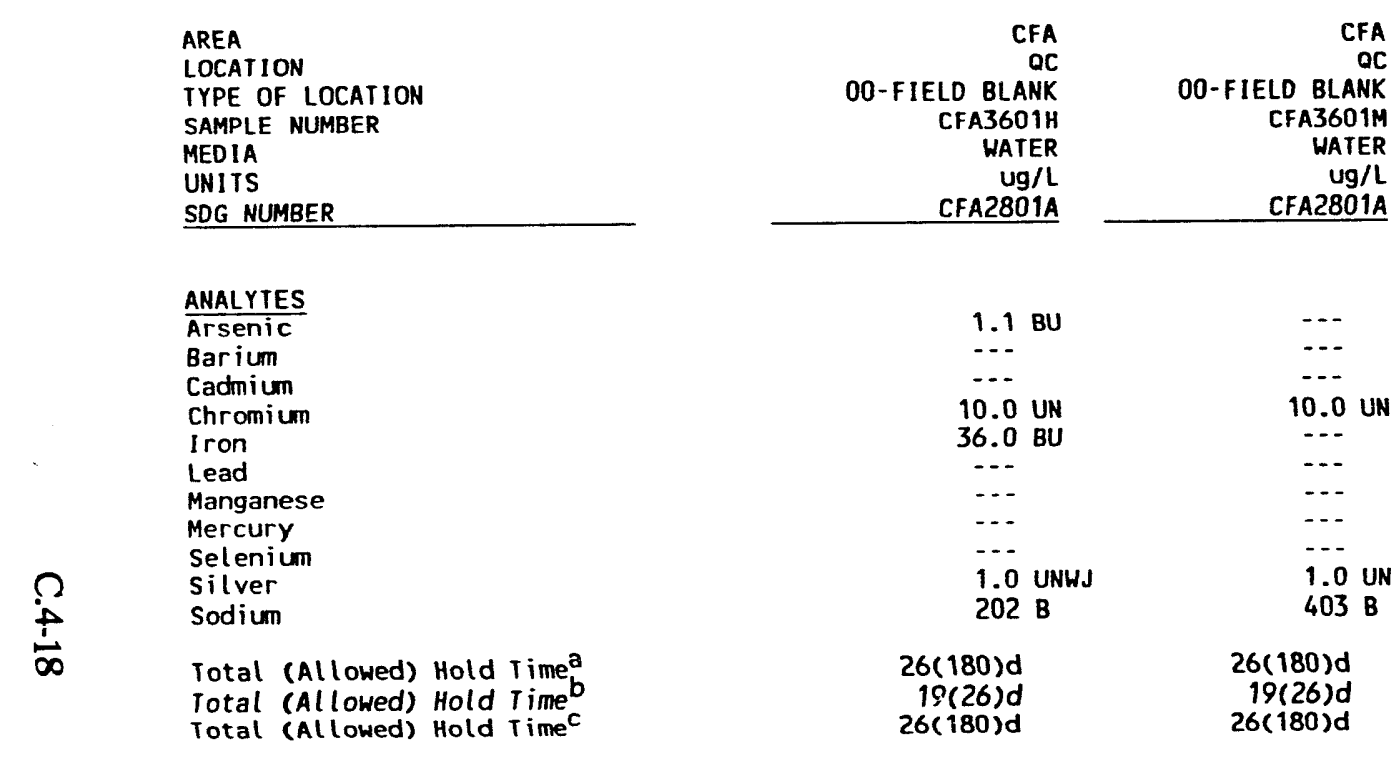
a. ICP
CVAAS
c. GFAAS
9-13-92

\section{ACA}

BTONK

(1)

A2801A 

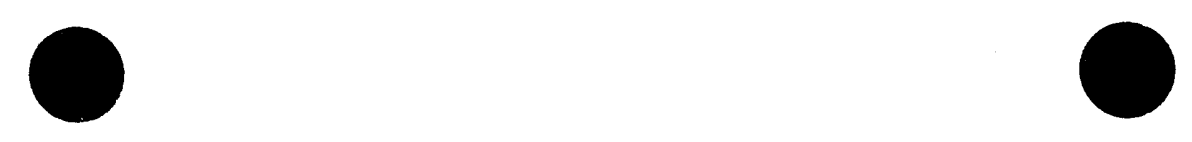

CFA Landfills II and III FY89 Wells - 4th Ouarter April 1991 S\&A Data Document - Method Validation Level A

TABLE _-_-_ CFA LANDFILLS 11 AND 111 FY89 WELLS - NON-METALS DATA

Page 1 of 3

AREA

LOCATION

TYPE OF LOCATION

SAMPLE NUMBER

MEDIA

SOG NUMBER

\section{FIELD MEASUREMENTS}

$\mathrm{pH}$

PH

Temperature (C)

ANALYTES

Fluoride

Fluoride

Sulfate

TOC

IOX

Total (Allowed) Hold Time a

Iotal (Allowed) Hold Time

Iotal (Allowed) Hold Time

rotal (Allowed) Hold Timed

rotal (Allowed) Hold Time

Total (Allowed) Hold Time

a. Chloride - Method 9251

b. Fluoride - Method 340.3

c. Nitrate - Method 353.1

d. Sulfate - Method 9036

e. IOC - Method 9060

f. IOX - Method 9020

\begin{tabular}{rr} 
CFA & CFA \\
03-LF3-8 & O2-LF2-8 \\
DOWN GRAD WELL & DOWN GRAD WELL \\
CFA2801 & CFA2901 \\
GROUND WATER & GROUND WATER \\
Ug/L & Ug/L \\
CFA2801A & CFA2801A \\
\hline
\end{tabular}

CFA
O2-LF2-9

DOWN GRAD HELL

CFA3001

GROUND WATER

CFA2801A

\section{4}

0.6
12.2

0.6
11.0

89800

110
4290

4290
27000

$1000 \mathrm{UJ}$

$32.3:$

$5(28) d$

26(28)d

$15(28) d$

$5(28) d$

$7(7) \mathrm{d}$
117000

270
4620

4620
25000

$1300 \mathrm{~J}$

$7.0 \mathrm{~J}$

$7(28) d$

$28(28) \mathrm{d}$

4(28)d

$7(28) d$

$9(7) d^{\star}$
8.5

8.5
0.6
11.7

108000

280
4600

4600
28000

$7700 \mathrm{~J}$

$7.8 \mathrm{~J}$

$6(28) \mathrm{d}$

$27(28) d$

$3(28) d$

$16(28) d$

$6(28) d$

$8(7) d^{\star}$
CFA

02-LF2-11

UPGRADIENT WELL
CFA3101
GROUND WATER

O2-LF2-9
DOWN GPAD WELL

CFA3002

GROUND WATER

CFA2801A

CFA2801A

7.6

0.7
12.8

0.6
11.7

109000

108000

260
4700

28000

1000 UJ

$33.2 \mathrm{~J}$

4790

23000

$20700 \mathrm{~J}$

$5.1 \mathrm{~J}$

\section{$6(28) d$}

$27(28) d$
$3(28) d$

$16(28) d$

$6(28) d$

$8(7) d^{\star}$ 6(28)d

$27(28) d$

$3(28) d$

$16(28) d$

6(28)d

$8(7) d^{\star}$ 
CFA Landfills 11 and 111 FY89 Wells - 4th Quarter April 1991 S\&A Data Document - Method Validation Level A

TABLE _-:- CFA LANDFILLS 11 AND 111 FY89 WELLS - NON-METALS DATA (Cont inued)

Page 2 of 3

\begin{tabular}{|c|c|c|c|c|c|}
\hline $\begin{array}{l}\text { AREA } \\
\text { LOCAIION } \\
\text { TYPE OF LOCATION } \\
\text { SAMPLE NUMBER } \\
\text { MEDIA } \\
\text { UNIIS } \\
\text { SOG NUMBER } \\
\end{array}$ & $\begin{array}{r}\text { CFA } \\
\text { O2-LF2-11 } \\
\text { UPGRADIENT WELL } \\
\text { CFA3102 } \\
\text { GROUND WATER } \\
\text { Ug } / L \\
\text { CFA2801A } \\
\end{array}$ & $\begin{array}{r}\text { CFA } \\
\text { O2-LF2-11 } \\
\text { UPGRADIENT WELL } \\
\text { CFA3103 } \\
\text { GROUND WATER } \\
\text { Ug/L } \\
\text { CFA2801A } \\
\end{array}$ & $\begin{array}{r}\text { CFA } \\
\text { O2-LF2-11 } \\
\text { UPGRADIENT WELL } \\
\text { CFA3104 } \\
\text { GROUND WATER } \\
\text { Ug/L } \\
\text { CFA2801A } \\
\end{array}$ & $\begin{array}{r}\text { CFA } \\
\text { O2-LF2-11 } \\
\text { UPGRADIENT WELL } \\
\text { CFA3105 } \\
\text { GROUND WATER } \\
\text { Ug/L } \\
\text { CFA2801A } \\
\end{array}$ & $\begin{array}{r}\text { CFA } \\
\text { 02-LF2-11 } \\
\text { UPGRADIENT WELL } \\
\text { CFA3106 } \\
\text { GROUND WATER } \\
\text { Ug/L } \\
\text { CFA2801A } \\
\end{array}$ \\
\hline $\begin{array}{l}\text { FIELD MEASUREMENTS } \\
\mathrm{pH} \\
\text { Conductivity (uS) } \\
\text { lemperature (c) }\end{array}$ & $\begin{array}{r}7.6 \\
0.7 \\
12.8\end{array}$ & $\begin{array}{r}7.6 \\
0.7 \\
12.8\end{array}$ & $\begin{array}{r}7.6 \\
0.7 \\
12.8\end{array}$ & $\begin{array}{r}7.6 \\
0.7 \\
12.8\end{array}$ & $\begin{array}{r}7.6 \\
0.7 \\
12.8\end{array}$ \\
\hline $\begin{array}{l}\text { ANALYTES } \\
\text { Chloride } \\
\text { Fluoride } \\
\text { Nitrate } \\
\text { Sulfate } \\
\text { rOC }\end{array}$ & $\begin{array}{r}110000 \\
270 \\
4840 \\
28000 \\
1000 \mathrm{uJ}\end{array}$ & & & & \\
\hline TOX & $7.8 \mathrm{~J}$ & $8.2 \mathrm{~J}$ & $5.0 \mathrm{UJ}$ & $11.3 \mathrm{~J}$ & $44.0 \mathrm{~J}$ \\
\hline $\begin{array}{l}\text { Total (Allowed) Hold Timea } \\
\text { Jotal (Allowed) Hold Timeb } \\
\text { Total (All owed) Hold Timec } \\
\text { Total (All owed) Hold Timed } \\
\text { Total (All owed) Hold Time } \\
\text { Total (All owed) Hold Time }\end{array}$ & $\begin{array}{l}6(28) d \\
27(28) d \\
3(28) d \\
16(28) d \\
6(28) d \\
8(7) d^{\star}\end{array}$ & $8(7) d^{*}$ & $8(7) d^{\star \star}$ & $8(7) d^{\star}$ & $8(7) d^{\star}$ \\
\hline $\begin{array}{l}\text { a. Chloride - Method } 9251 \\
\text { b. Fluoride - Method } 340.3 \\
\text { c. Nitrate - Method } 353.1 \\
\text { d. Sulfate - Method } 9036 \\
\text { e. TOC - Method } 9060 \\
\text { f. TOX - Method } 9020\end{array}$ & & & & & \\
\hline
\end{tabular}


CFA Landfills 11 and 111 FY89 Wells - 4th Quarter April 1991 S\&A Data Document - Method Validation Level A

TABLE _.- CFA LANDFILLS II AND III FY89 WELLS - NON-METALS DATA (Continued)

AREA

LOCATION

SAMPLE NUMBER

MEDIA

SDG NUMBER

$\begin{array}{rr}\text { CFA } & \text { CFA } \\ \text { O2-LF2-11 } & \text { O2-LF2-11 } \\ \text { UPGRADIENT HELL } & \text { UPGRADIENT WELL } \\ \text { CFA3107 } & \text { CFA3108 } \\ \text { GROUND WATER } & \text { GROUND WATER } \\ \text { Ug/L } & \text { Ug/L } \\ \text { CFA2801A } & \text { CFA2801A }\end{array}$

\section{FIELD MEASUREMENTS}

pH

Conductivity (uS)

\section{ANALYTES}

Chloride

Fluoride

Nitrate
Sulfate

$\Omega$ roc

Total (Allowed) Hold Timea

Total (Allowed) Hold Time

Total (Allowed) Hold Time

Iotal (Allowed) Hold Time

rotal (Allowed) Hold Time

12.8

$30.1 \mathrm{~J}$

$20.9 \mathrm{~J}$

a. Chloride - Method 9251

b. Fluoride - Method 340.3

c. Nitrate - Method 353.1

e. IOC - Method 9060

e. TOC - Method 9060

\section{WELL}


CFA Landfills II and III FY89 Wells - 4th Quarter April 199: S\&A Data Document - Method Validation Level A

TABLE _._-_ CFA LANDFILLS II AND 111 FYBg WELLS - NON-METALS FIELD BLANK DATA

Page 1 of 1

\section{AREA}

LOCATION

TYPE OF LOCATION

SAMPLE NUMBER

MEDIA

UNITS
SDG NUMBER

CFA

O0-FIELD BLANK

CFA3601

WATER

Ug/L
CFA2801A

\section{ANALYTES}

Chloride

Fluoride

Nitrate

Sulfate

roc

rox

300

-...

$1000 \mathrm{UJ}$

Total (Allowed) Hold Timea

rotal (Allowed) Hold Time

rotal (Allowed) Hold Timec

Total (Allowed) Hold Timed

Total (Allowed) Hold Time

rotal (Allowed) Hold Timef

$40.4 \mathrm{~J}$

a. Chloride - Method 9251

b. Fluoride - Method 340 .

c. Nitrate - Method 353.1

d. Sulfate - Method 9036

e. TOC - Method 9060

f. Tox - Method 9020

9-22-92

$5(28) d$

$26(28) d$

$5(28) d$

$15(28) d$

$5(28) d$

$7(7) d$ 
CFA Landfills II and III FY89 Wells - 4th Quarter April 1991 - S\&A Data Document

TABLE _--: CFA LANDFILLS II AND III FY89 WELLS - ORGANOCHLORINE PESTICIDE APPENDIX IX DATA

\begin{tabular}{l} 
AREA \\
LOCATION \\
TYPE OF LOCATION \\
SAMPLE NUMBER \\
MEDIA \\
UNITS \\
SDG MUMBER \\
\hline
\end{tabular}

$\begin{array}{r}\text { CFA } \\ \text { O2-LF2-8 } \\ \text { DOWN GRAD WELL } \\ \text { CFA2901G } \\ \text { GROUND WATER } \\ \text { Ug/L } \\ \text { CFA28O1A } \\ \hline\end{array}$

$\begin{array}{r}\text { CFA } \\ \text { O2-LF2-9 } \\ \text { DOWN GRAD WELL } \\ \text { CFA3001G } \\ \text { GROUND HATER } \\ \text { Ug/L } \\ \text { CFA2801A } \\ \hline\end{array}$

$\begin{array}{r}\text { CFA } \\ \text { O2-LF2-9 } \\ \text { DOWN GRAD HELL } \\ \text { CFA3002G } \\ \text { GROUND HATER } \\ \text { Ug/L } \\ \text { CFA2801A } \\ \hline\end{array}$

\begin{tabular}{rr} 
CFA & CFA \\
O2-LF2-11 & 02-LF2-11 \\
UPGRADIENT HELL & UPGRADIENT HELL \\
CFA3101G & CFA3102G \\
GROUND WATER & GROUND WATER \\
Ug/L & Ug/L \\
CFA2801A & CFA2801A \\
\hline
\end{tabular}

FIELD MEASUREMENTS

Conductivitiy (uS)

Temperature

8.4
0.6

0.6
12.2

8.4
0.6

0.6
11.7

8.4

IARGET COMPOUNDS

None Detected

Dilution Factor

Extraction (Allowed) Hold Time

Analytical (Allowed) Hold TIme

1.0
$4(7) d$
$3(40) d$

1.0
$3(7) d$
$3(40) d$

1.0
$3(7) d$

$3(7) d$
$3(40) d$

1.0
$4(7) \mathrm{d}$

$4(7) d$
$3(40) d$

1.0
$4(7) \mathrm{d}$

$3(40) d$ 
CFA Landfills II and III FY89 Hells - 4th Quarter April 1991 - S\&A Data Document

TABLE _-_-_ CFA LANDFILLS II AND III FY89 WELLS - ORGANOCHLORINE PESTICIDE APPENDIX IX FIELD BLANK DATA

AREA

LOCATION

TYPE OF LOCATION

SAMPLE NUMBER

MEDIA

UNITS
SDG NUMBER

MUMBER

$\begin{array}{r}\text { CFA } \\ \text { QC } \\ \text { O0-FIELD BLANK } \\ \text { CFA3601G } \\ \text { HATER } \\ \text { Ug/L } \\ \text { CFA2801A } \\ \hline\end{array}$

TARGET COMPOUNDS

None Detected

Dilution Factor

Extraction (Allowed) Hold Time

Analytical (Allowed) Hold Time

1.0
$0(7) d$

$0(7) d$
$3(40) d$ 
CFA Landfills 11 and III FY89 Wells - 4th Quarter April 1991 S\&A Data Document

TABLE _-:- CFA LANDFILLS II AND III FY89 WELLS - ORGANOCHLORINE HERBICIDE APPENDIX IX DATA

Page 1 of 1

\begin{tabular}{|c|c|c|c|c|c|c|}
\hline $\begin{array}{l}\text { AREA } \\
\text { LOCATION } \\
\text { TYPE OF LOCATION } \\
\text { SAMPLE NUMBER } \\
\text { MEDIA } \\
\text { UNITS } \\
\text { SDG NUMBER } \\
\end{array}$ & $\begin{array}{r}\text { CFA } \\
\text { 03-LF3-8 } \\
\text { DOWN GRAD HELL } \\
\text { CFA28012 } \\
\text { GROUND HATER } \\
\text { Ug/L } \\
\text { CFAZ801A } \\
\end{array}$ & $\begin{array}{r}\text { CFA } \\
\text { O2-LF2-8 } \\
\text { DOWN GRAD HELL } \\
\text { CFA29012 } \\
\text { GROUND WATER } \\
\text { Ug/L } \\
\text { CFA2801A } \\
\end{array}$ & $\begin{array}{r}\text { CFA } \\
02-L F 2-9 \\
\text { DOWN GRAD WELL } \\
\text { CFA30012 } \\
\text { GROUND WATER } \\
\text { Ug/L } \\
\text { CFA2BO1A } \\
\end{array}$ & $\begin{array}{r}\text { CFA } \\
\text { O2-LF2-9 } \\
\text { DOWN GRAD WELL } \\
\text { CFA3002Z } \\
\text { GROUND WATER } \\
\text { Ug/L } \\
\text { CFA2801A } \\
\end{array}$ & $\begin{array}{r}\text { CFA } \\
\text { O2-LF2-11 } \\
\text { UPGRADIENT WELL } \\
\text { CFA3101Z } \\
\text { GROUND WATER } \\
\text { Ug } / L \\
\text { CFA2801A } \\
\end{array}$ & $\begin{array}{r}\text { CFA } \\
\text { O2-LF2-11 } \\
\text { UPGRADIENT WELL } \\
\text { CFA3102Z } \\
\text { GROUND WATER } \\
\text { Ug/L } \\
\text { CFA2801A } \\
\end{array}$ \\
\hline $\begin{array}{l}\text { FIELD MEASUREMENIS } \\
\mathrm{pH} \\
\text { Conductivity (uS) } \\
\text { Temperature }\end{array}$ & $\begin{array}{r}7.9 \\
0.6 \\
11.0\end{array}$ & $\begin{array}{r}8.4 \\
0.6 \\
12.2\end{array}$ & $\begin{array}{r}8.4 \\
0.6 \\
11.7\end{array}$ & $\begin{array}{r}8.4 \\
0.6 \\
11.7\end{array}$ & $\begin{array}{r}7.6 \\
0.7 \\
12.8\end{array}$ & $\begin{array}{r}7.6 \\
0.7 \\
12.8\end{array}$ \\
\hline \multicolumn{7}{|l|}{ IARGET COMPOUNDS } \\
\hline \multicolumn{7}{|l|}{ None Detected } \\
\hline $\begin{array}{l}\text { Dilution Factor } \\
\text { Extraction (Allowed) Hold Time } \\
\text { Analytical (Allowed) Hold TIme }\end{array}$ & $\begin{array}{r}1.0 \\
1(7) d \\
3(40) d\end{array}$ & $\begin{array}{r}1.0 \\
4(7) d \\
4(40) d\end{array}$ & $\begin{array}{r}1.0 \\
4(7) d \\
4(40) d\end{array}$ & $\begin{array}{r}1.0 \\
4(7) d \\
4(40) d\end{array}$ & $\begin{array}{r}1.0 \\
4(7) d \\
4(40) d\end{array}$ & $\begin{array}{r}1.0 \\
4(7) d \\
4(40) d\end{array}$ \\
\hline
\end{tabular}


CFA Landfills 11 and 111 FYB9 Wells - 4th Quarter April 1991 S\&A Data Document

TABLE - CFA LANDFILLS 11 AND 111 FY89 WELLS - ORGANOCHLORINE HERBICIDE APFENDIX IX FIELD BLANK DATA

\begin{tabular}{lr} 
AREA & CFA \\
LOCATION & OC \\
TYPE OF LOCATION & OO-FIELD BLANK \\
SAMPLE NUMBER & CFA36012 \\
MEDIA & WATER \\
UNITS & Ug/L \\
SDG NUMBER & CFA2801A \\
\hline
\end{tabular}

TARGET COMPOUNDS

None Detected

Dilution Factor

Extraction (Allowed) Hold Time

Hold Time

1.0

$1(7) d$
$3(40) d$

9-13-92 
CFA Landfills II and III FY89 Wells - 4th Quarter April 1991 S\&A Data Document - Method Validation Level C

TABLE _-_-_ CFA LANDFILLS II AND III FY89 WELLS - ANALYSIS RESULTS FOR GAMMA-EMITTING RADIONUCLIDES

Page 1 of 1

Date: $07 / 17 / 91$

Lab Name: RML

Report No.: STPHY91031
Case No.:

SDG No.: CFA028012

\begin{tabular}{|c|c|c|c|c|c|c|c|c|c|c|c|c|c|c|}
\hline $\begin{array}{c}\text { Field } \\
\text { Sample No. }\end{array}$ & Location & $\begin{array}{l}\text { Lab } \\
\text { Sample ID }\end{array}$ & $\begin{array}{l}\text { Sample } \\
\text { Matrix }\end{array}$ & $\begin{array}{l}\text { Anal } \\
\text { Type }\end{array}$ & $\begin{array}{l}\text { Sample } \\
\text { Value }\end{array}$ & $\begin{array}{l}\text { Sample } \\
\text { Error }\end{array}$ & Units & $\begin{array}{l}\text { Anal } \\
\text { Date }\end{array}$ & $\begin{array}{l}\text { Sample } \\
\text { Date }\end{array}$ & $\begin{array}{l}\text { Sample } \\
\text { Size }\end{array}$ & Yield & $\begin{array}{c}\text { Detector } \\
\text { ID }\end{array}$ & DOF & ASL \\
\hline CFA028012 & $03-L F 3-8$ & A5042691045 & WATER & GAMMA & ND & & $\mathrm{pCi} / \mathrm{L}$ & $04 / 26 / 91$ & $04 / 24,91$ & 0.5400 & 0.0 & RML A5/PG-1 & & \\
\hline CFAO2028012D & $03-L F 3-8$ & A6051591038 & WATER & GAMMA & ND & & $\mathrm{pCi} / \mathrm{L}$ & $05 / 15 / 91$ & $04 / 24 / 9 !$ & 0.5400 & 0.0 & RML A6/PG-13 & & \\
\hline CFA029012 & $02-$ LF2-8 & A5042291041 & WATER & GAMMA & ND & & $\mathrm{pCi} / \mathrm{L}$ & $04 / 22 / 91$ & $04 / 22 / 91$ & 0.5400 & 0.0 & RML A5/PG-1 & & \\
\hline CFA0 30012 & 02-LF2-9 & A6042391029 & WATER & GAMMA & ND & & $\mathrm{pCi} / \mathrm{L}$ & $04 / 23 / 91$ & $04 / 23 / 91$ & 0.5400 & 0.0 & RML $\quad$ A6/PG -13 & & \\
\hline CFA030022 & 02-LF2-9 & A5042391028 & WATER & GAMMA & ND & & pCi/L & $04 / 23 / 91$ & $04 / 23 / 91$ & 0.5400 & 0.0 & RML A5/PG-1 & & \\
\hline CFA031012 & $02-L F 2-11$ & A6042491037 & WATER & GAMMA & ND & & $p C i / L$ & $04 / 24 / 91$ & $04 / 23 / 91$ & 0.5400 & 0.0 & RML $\quad$ A6/PG -13 & & \\
\hline CFA031022 & $02-L F 2-11$ & A5042491036 & WATER & GAMMA & ND & & $\mathrm{pCi} / \mathrm{L}$ & $04 / 24 / 91$ & $04 / 23 / 91$ & 0.5400 & 0.0 & RML A5/PG-1 & & \\
\hline CFA036012 & OO-FIELD BLANK & A5051591037 & IIATER & GAMMA & ND & & $\mathrm{pCi} / \mathrm{L}$ & $05 / 15 / 91$ & $04 / 24 / 91$ & 0.5400 & 0.0 & RML A5/PG-1 & & \\
\hline CFA0 360120 & O0-FIELD BLANK & A5052291033 & WATER & GAMMA & ND & & $\mathrm{pCi} / \mathrm{L}$ & $05 / 22 / 91$ & $04 / 24 / 91$ & 0.5400 & 0.0 & RML $A 5 / P G-1$ & & \\
\hline
\end{tabular}

9-18-92 
CFA Landfills II and III FY90 Wells - 1st Quarter April 1991 S\&A Data Document

TABLE _-_-_ CFA LANDFILLS II AND III FY90 WELLS - 524.2 VOLATILE ORGANIC DATA

Page 1 of 2

\begin{tabular}{|c|c|c|c|c|c|}
\hline $\begin{array}{l}\text { AREA } \\
\text { LOCATION } \\
\text { TYPE OF LOCATION } \\
\text { SAMPLE NUMBER } \\
\text { MEDIA } \\
\text { UNITS } \\
\text { SDG MUMBER } \\
\end{array}$ & $\begin{array}{r}\text { CFA } \\
\text { 02-LF2-12 } \\
\text { DOWN GRAD WELL } \\
\text { CFA03701ARE } \\
\text { GROUND WATER } \\
\text { Ug/L } \\
\text { CFA03301A } \\
\end{array}$ & $\begin{array}{r}\text { CFA } \\
\text { O3-LF3-9 } \\
\text { DOWN GRAD WELL } \\
\text { CFA03801A } \\
\text { GROUND WATER } \\
\text { Ug/L } \\
\text { CFA03301A } \\
\end{array}$ & $\begin{array}{r}\text { CFA } \\
\text { 03-LF3-10 } \\
\text { DOWN GRAD WELL } \\
\text { CFA03901A } \\
\text { GROUND WATER } \\
\text { Ug/L } \\
\text { CFA03301A } \\
\end{array}$ & $\begin{array}{r}\text { CFA } \\
\text { O3-LF3-10 } \\
\text { DOWN GRAD WELL } \\
\text { CFAO3902A } \\
\text { GROUND WATER } \\
\text { Ug/L } \\
\text { CFA03301A } \\
\end{array}$ & $\begin{array}{r}\text { CFA } \\
\text { O3-LF3-11 } \\
\text { UPGRADIENT WELL } \\
\text { CFAO4001A } \\
\text { GROUND WATER } \\
\text { Ug/L } \\
\text { CFA03301A } \\
\end{array}$ \\
\hline $\begin{array}{l}\text { FIELD MEASUREMENTS } \\
\text { PH } \\
\text { Conductivity (uS) } \\
\text { Temperature ( } \mathrm{C} \text { ) }\end{array}$ & $\begin{array}{r}7.8 \\
0.6 \\
11.2\end{array}$ & $\begin{array}{r}7.7 \\
0.6 \\
12.6\end{array}$ & $\begin{array}{r}7.7 \\
0.6 \\
11.2\end{array}$ & $\begin{array}{r}7.7 \\
0.6 \\
11.2\end{array}$ & $\begin{array}{r}7.8 \\
0.5 \\
11.4\end{array}$ \\
\hline $\begin{array}{l}\text { TARGET COMPOUNDS } \\
\text { Dichlorodifluoromethane } \\
\text { Chloromethane } \\
1,1 \text {-Dichloroethene } \\
\text { Methylene Chloride } \\
\text { Chloroform }\end{array}$ & $\begin{array}{l}0 \mathrm{R} \\
-- \\
-- \\
0.1 \mathrm{JB} \\
0.3 \mathrm{~J}\end{array}$ & $\begin{array}{l}0 . \mathrm{R}^{2} \\
0.7 \\
0.2 \mathrm{JB} \\
0.1 \mathrm{~J}\end{array}$ & $\begin{array}{c}0 \mathrm{R} \\
0.6 \\
\cdots \\
\cdots--\end{array}$ & $\begin{array}{c}0 R \\
0.5 \\
\cdots \\
\cdots \\
\cdots\end{array}$ & $\begin{array}{l}0 \mathrm{R} \\
0.7^{2} \\
0.5 \mathrm{~J} \\
\cdots \\
\cdots\end{array}$ \\
\hline $\begin{array}{l}\text { 1,1,1-Trichloroethane } \\
\text { Carbon Tetrachloride } \\
1,2-D i c h \text { loroethane } \\
\text { Irichloroethene } \\
\text { Trans-1,3-Dichloropropene }\end{array}$ & $\begin{array}{l}0.8 \\
0.6 \\
0.6 \\
0.1 \mathrm{~J} \\
\text { NP }\end{array}$ & $\begin{array}{l}0.5 \\
0.5 \\
0.1 \\
0.1 \\
\text { NP }\end{array}$ & $\begin{array}{l}\cdots \\
\cdots .- \\
0.5 \\
0.1 \mathrm{~J} \\
\text { NP }\end{array}$ & $\begin{array}{l}0.4 \mathrm{~J} \\
-. . \\
0.6 \\
\cdots \\
\text { NP }\end{array}$ & $\begin{array}{l}0.5 \\
0.6 \\
0.6 \\
0.5 \mathrm{~J} \\
\text { NP }\end{array}$ \\
\hline $\begin{array}{l}\text { Toluene } \\
\text { cis-1,3-Dichloropropene } \\
\text { Tetrachloroethene } \\
\text { Trichlor of luoromethane }\end{array}$ & $\begin{array}{l}0.3 \mathrm{~J} \\
\mathrm{NP} \\
1.4\end{array}$ & $\begin{array}{l}0.2 \mathrm{~J} \\
\mathrm{NP} \\
1.0\end{array}$ & $\begin{array}{l}0.4 \mathrm{~J} \\
\mathrm{NP} \\
1.2\end{array}$ & $\begin{array}{l}0.3 \mathrm{~J} \\
\text { NP } \\
0.2 \mathrm{~J} \\
0.7\end{array}$ & $\begin{array}{c}\cdots \\
N P \\
-1.1\end{array}$ \\
\hline $\begin{array}{l}\text { Dilution Factor } \\
\text { Total (Allowed) Hold Time }\end{array}$ & $\begin{array}{r}1.000 \\
11(14) d\end{array}$ & $\begin{array}{r}1.000 \\
8(14) d\end{array}$ & $\begin{array}{r}1.000 \\
8(14) d\end{array}$ & $\begin{array}{r}1.000 \\
9(14) d\end{array}$ & $\begin{array}{r}1.000 \\
13(14) d\end{array}$ \\
\hline
\end{tabular}

NP - Information Not Provided by Laboratory

9-13-92 


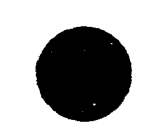

CFA Landfills II and III FY90 Wells - 1st Quarter April 1991 S\&A Data Document - April 1991

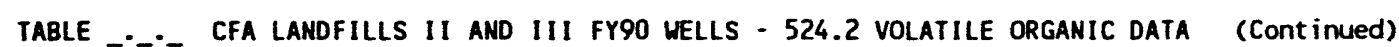

AREA

LOCATION

LOCATION TYPE OF LOCATION

SAMPLE NUMBER

MED IA

SDG NUMBER

CFA
O3-LF3-11
UPGRADIENT WELL
CFAO4002A
GROUND WATER
Ug/L
CFA03301A

\section{FIELD MEASUREMENTS}

PH

Conductivity (US)

\section{TARGET COMPOUNDS}

Dichlorodifluoromethane

Chloromethane
1,1-Dichloroethene

Methylene Chloride

Chloroform

$\Omega \quad$ Carbon Tetrachloride

1,2-Dichloroethane

Irichloroethene
Irans-1,3-Dichloropropene

Toluene

cis-1,3-D ichloropropene

Tetrachl oroethene

irichlor of luorome thane

Dilution Factor

Total (Allowed) Hold Time

0.7 R

0.7

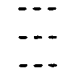

0.7
$0.8 \mathrm{~J}$

$0.5 \mathrm{~J}$

0.5

0.5

1.0

1.000

$13(14) \mathrm{d}$

NP - Information Not Provided by Laboratory
$9-13-92$ 
CFA Landfills II and III FY90 Wells - 1st Quarter April 1991 S\&A Data Document

TABLE _--: CFA LANDFILLS II AND III FY90 WELLS - 524.2 VOLATILE ORGANIC FIELD BLANK DATA

\begin{tabular}{|c|c|}
\hline $\begin{array}{l}\text { AREA } \\
\text { LOCATION } \\
\text { TYPE OF LOCATION } \\
\text { SAMPLE NUMBER } \\
\text { MEDIA } \\
\text { UNITS } \\
\text { SDG NUMBER } \\
\end{array}$ & $\begin{array}{r}\text { CF } \\
0 \\
\text { 00-FIELD BLAN } \\
\text { CFA04501 } \\
\text { WATE } \\
\text { Ug/ } \\
\text { CFA03301 }\end{array}$ \\
\hline $\begin{array}{l}\text { TARGET COMPQUNDS } \\
\text { Dichlorodifluoromethane } \\
\text { Chloromethane } \\
\text { Methyl ene Chloride } \\
1,1 \text {-Dichloroethane } \\
\text { chloroform }\end{array}$ & $\begin{array}{c}0 R \\
1 \\
0.6 \mathrm{~J} \\
34\end{array}$ \\
\hline $\begin{array}{l}1,1,1-\text { Trichloroethane } \\
1,2-D \text { ichloroethane } \\
\text { Trans-1,3-D ichloropropene } \\
\text { Toluene } \\
\text { cis-1,3-Dichloropropene }\end{array}$ & $\begin{array}{l}0.6 \mathrm{~J} \\
0.7 \\
\text { NP } \\
0.4 \mathrm{~J} \\
\text { NP }\end{array}$ \\
\hline $\begin{array}{l}\text { Tetrachloroethene } \\
\text { Dibromochloromethane } \\
1,2,4-\text { Trichlorobenzene } \\
\text { Naphthal ene } \\
\text { Trichlorof tuorome thane }\end{array}$ & $\begin{array}{l}0.3 \mathrm{~J} \\
0.8 \mathrm{~J} \\
0.5 \\
0.6 \mathrm{~J} \\
0.8\end{array}$ \\
\hline $\begin{array}{l}\text { Dilution Factor } \\
\text { Total (Allowed) Hold Time }\end{array}$ & $\begin{array}{l}1.000 \\
9(14) d\end{array}$ \\
\hline
\end{tabular}

NP - Information Not Provided by Laboratory
$9-13-92$ 
CFA Landfills II and III FY90 Hells - 1st Quarter April 1991 S\&A Data Document

TABLE _-_-_ CFA LANDFILLS II AND III FY90 WELLS - 524.2 VOLATILE ORGANIC IRIP BLANK DATA

\begin{tabular}{|c|c|c|}
\hline $\begin{array}{l}\text { AREA } \\
\text { LOCATION } \\
\text { TYPE OF LOCATION } \\
\text { SAMPLE NUMBER } \\
\text { MEDIA } \\
\text { UNITS } \\
\text { SDG NUMBER } \\
\end{array}$ & $\begin{array}{r}\text { CFA } \\
\text { OC } \\
\text { OO-TRIP BLANK } \\
\text { CFA03301A } \\
\text { HATER } \\
\text { Ug/L } \\
\text { CFA03301A } \\
\end{array}$ & $\begin{array}{r}\text { CFA } \\
\text { OC } \\
\text { O0-TRIP BLANK } \\
\text { CFA04101A } \\
\text { WATER } \\
\text { Ug/L } \\
\text { CFA03301A }\end{array}$ \\
\hline $\begin{array}{l}\text { TARGET COMPOUNDS } \\
\text { Dichlorodifluoromethane } \\
\text { Chloromethane } \\
\text { Methylene Chloride } \\
1,1 \text {-D ichloroethane } \\
\text { Chloroform }\end{array}$ & $\begin{array}{l}0 \mathrm{R} \\
0.6^{-} \\
0.9 \mathrm{~J} \\
\cdots \\
\cdots\end{array}$ & $\begin{array}{c}\text { OR R } \\
\cdots \\
0.1 \mathrm{~J} \\
44\end{array}$ \\
\hline $\begin{array}{l}\text { Bromodichloromethane } \\
\text { Trans-1,3-D ichl oropropene } \\
\text { Toluene } \\
\text { cis-1,3-Dichloropropene } \\
\text { Dibromochlorome thane }\end{array}$ & $\begin{array}{l}\text { HP } \\
0.4 \mathrm{NP} \\
\mathrm{NP} \\
\cdots\end{array}$ & $\begin{array}{l}0.6 \\
\text { NP } \\
-\cdots \\
\text { NP } \\
0.1 \mathrm{~J}\end{array}$ \\
\hline Trichlorofluoromethane & $0.2 \mathrm{~J}$ & $0.4 \mathrm{~J}$ \\
\hline $\begin{array}{l}\text { Dilution Factor } \\
\text { Total (Allowed) Hold Time }\end{array}$ & $\begin{array}{r}1.000 \\
13(14) d\end{array}$ & $\begin{array}{r}1.000 \\
10(14) d\end{array}$ \\
\hline
\end{tabular}

MP - Information Not Provided by Laboratory 9-13-92 
CFA Landfills II and III FY90 Wells - 1st Quarter April 1991 S\&A Data Document

TABLE _-_- CFA LANDFILLS II AND III FY90 WELLS - SEMIVOLATILE ORGANIC DATA

Page 1 of 1

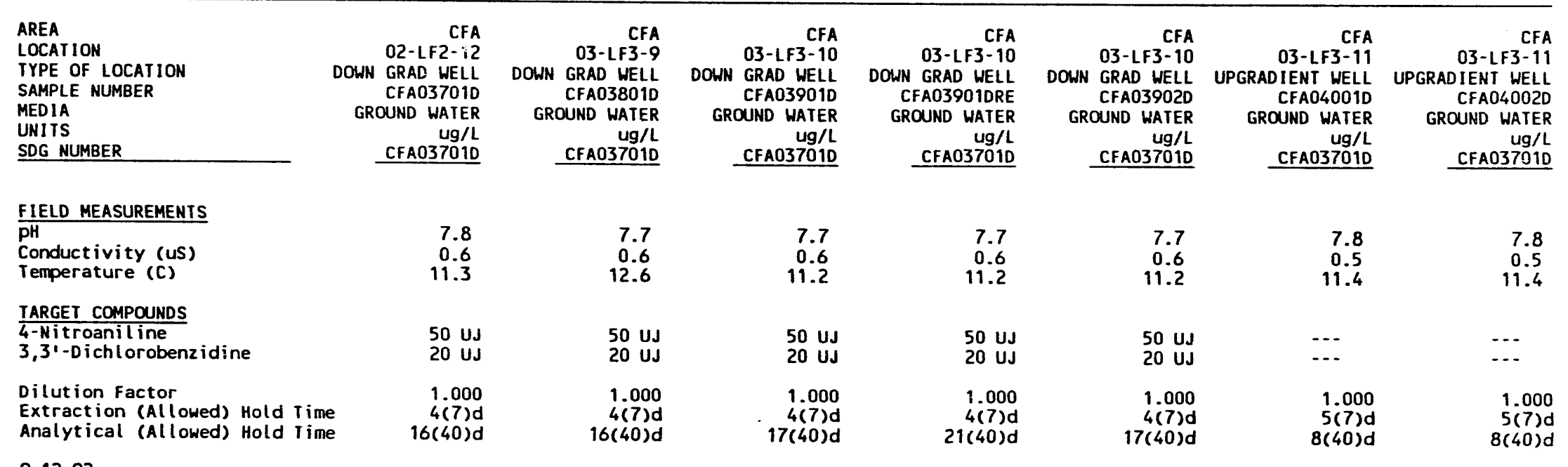

9-12-92 


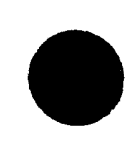

CFA Landfills II and III FY90 Wells - 1st Quarter April 1991 S\&A Data Document

TABLE _--:- CFA LANDFILLS II AND III FY9O WELLS - SEMIVOLATILE ORGANIC FIELD BLANK DATA

\begin{tabular}{lr} 
AREA & CFA \\
LOCATION & OC \\
TYPE OF LOCATION & OO-FIELD BLANK \\
SAMPLE NUMBER & CFAO4501D \\
MEDIA & WATER \\
UNITS & Ug/L \\
SDG NUMBER & CFA03701D \\
\hline
\end{tabular}

\section{TARGET COMPOUNDS}

$4-N i$ troaniline

3,3'-Dichlorobenzidine

50 UJ

Dilution Factor

Extraction (Allowed) Hold Time

Analytical (Allowed) Hold time

20 UJ

1.000

$4(7) d$

9-12-92

OC

ANK

TER

$17(40) d$

W 
CFA Landfills II and III FY90 Wells - 1st Quarter April 1991 S\&A Data Document

TABLE .. CFA LANDFILLS II AND III FY9O WELLS - INORGANIC DATA

Page 1 of 3

\begin{tabular}{|c|c|c|c|c|c|}
\hline $\begin{array}{l}\text { AREA } \\
\text { LOCATION } \\
\text { TYPE OF LOCATION } \\
\text { SAMPLE NUMBER } \\
\text { MEDIA } \\
\text { UNIIS } \\
\text { SDG NUMBER } \\
\end{array}$ & $\begin{array}{r}\text { CFA } \\
\text { O2-LF2-12 } \\
\text { DOWN GRAD WELL } \\
\text { CFA03701H } \\
\text { GROUND WATER } \\
\text { Ug/L } \\
\text { CFA03701 } \\
\end{array}$ & $\begin{array}{r}\text { CFA } \\
\text { O2-LF2-12 } \\
\text { DOWN GRAD WELL } \\
\text { CFA03701M } \\
\text { GROUND WATER } \\
\text { Ug/L } \\
\text { CFA03701 } \\
\end{array}$ & $\begin{array}{r}\text { CFA } \\
\text { 03-LF3-9 } \\
\text { DOWN GRAD WELL } \\
\text { CFA03801H } \\
\text { GROUND WATER } \\
\text { Ug/L } \\
\text { CFA03701 } \\
\end{array}$ & $\begin{array}{r}\text { CFA } \\
\text { O3-LF3-9 } \\
\text { DOWN GRAD WELL } \\
\text { CFA03801M } \\
\text { GROUND WATER } \\
\text { Ug/L } \\
\text { CFA03701 } \\
\end{array}$ & $\begin{array}{r}\text { CFA } \\
\text { 03-LF3-10 } \\
\text { DOWN GRAD WELL } \\
\text { CFA03901H } \\
\text { GROUND WATER } \\
\text { Ug/L } \\
\text { CFA03701 } \\
\end{array}$ \\
\hline $\begin{array}{l}\text { FIELD MEASUREMENTS } \\
\text { pH } \\
\text { Conductivity (uS) } \\
\text { Temperature (C) }\end{array}$ & $\begin{array}{r}7.8 \\
0.6 \\
11.3\end{array}$ & $\begin{array}{r}7.8 \\
0.6 \\
11.3\end{array}$ & $\begin{array}{r}7.7 \\
0.6 \\
12.6\end{array}$ & $\begin{array}{r}7.7 \\
0.6 \\
12.6\end{array}$ & $\begin{array}{r}7.7 \\
0.6 \\
11.2\end{array}$ \\
\hline $\begin{array}{l}\text { ANALYTES } \\
\text { Arsenic } \\
\text { Barium } \\
\text { Cadmium } \\
\text { Chromium } \\
\text { Iron }\end{array}$ & $\begin{array}{c}1.4 \mathrm{~B} \\
166 \mathrm{~B} \\
\cdots-. \\
24.0 \\
234\end{array}$ & $\begin{array}{l}1.3 \mathrm{~B} \\
118 \mathrm{~B} \\
-.- \\
7.0 \mathrm{~B} \\
\cdots-.\end{array}$ & $\begin{array}{c}2.2 \mathrm{~B} \\
149 \mathrm{~B} \\
-15.0 \\
455\end{array}$ & $\begin{array}{c}1.1 \text { B } \\
98.0 \text { B } \\
\ldots . . \\
\cdots\end{array}$ & $\begin{array}{c}117 \text { B } \\
25.0 \\
404\end{array}$ \\
\hline $\begin{array}{l}\text { Lead } \\
\text { Magnesium } \\
\text { Mercury } \\
\text { Selenium } \\
\text { Silver }\end{array}$ & $\begin{array}{c}4.5 \mathrm{H} \\
15700 \mathrm{UJ} \\
2.0 \mathrm{UJ} \\
2.0 \mathrm{UJ} \\
-.-\end{array}$ & \begin{tabular}{l}
3.9 \\
$15500 \mathrm{~J}$ \\
\hdashline 2.0 HNUJ \\
$\ldots .$.
\end{tabular} & $\begin{array}{r}2.0 \mathrm{BW} \\
18400 \mathrm{WJ} \\
2.0 \mathrm{UJ} \\
2.0 \mathrm{WJ} \\
\ldots\end{array}$ & $\begin{array}{l}2.7 \text { BH } \\
16400 \\
\ldots . . \\
2.0 \text { WNUJ } \\
\ldots . .\end{array}$ & $\begin{array}{c}5.0 \\
14900 \\
\ldots .0 \\
2.0 \mathrm{WJ} \\
\ldots . .\end{array}$ \\
\hline Sodium & 39000 & $39700 \mathrm{~J}$ & 34400 & 32900 & 35000 \\
\hline $\begin{array}{l}\text { Total (Allowed) Hold Timea } \\
\text { Total (Allowed) Hold Time } \\
\text { Total (All owed) Hold Timec }\end{array}$ & $\begin{array}{l}28(180) d \\
35(26) d^{\star} \\
28(180) d\end{array}$ & $\begin{array}{l}37(180) d \\
38(26) d^{\star} \\
37(180) d\end{array}$ & $\begin{array}{l}28(180) d \\
35(26) d^{\star} \\
28(180) d\end{array}$ & $\begin{array}{l}37(180) d \\
38(26) d^{\star} \\
37(180) d\end{array}$ & $\begin{array}{l}36(180) d \\
37(26) d^{\star} \\
36(180) d\end{array}$ \\
\hline
\end{tabular}
a. ICP
b. CVAAS
c. GFAAS
9-12-92 
CFA Landfills II and III FY90 Wells - 1st Ouarter April 1991 S\&A Data Document

TABLE _--: CFA LANDFILLS II AND III FY90 WELLS - INORGANIC DATA (Cont inued)

Page 2 of 3

\begin{tabular}{|c|c|c|c|c|c|}
\hline $\begin{array}{l}\text { AREA } \\
\text { LOCATION } \\
\text { TYPE OF LOCATION } \\
\text { SAMPLE NUMBER } \\
\text { MEDIA } \\
\text { UNITS } \\
\text { SDG NUMBER } \\
\end{array}$ & $\begin{array}{r}\text { CFA } \\
\text { 03-LF3-10 } \\
\text { DOUN GRAD HELL } \\
\text { CFA03901M } \\
\text { GROUND WATER } \\
\text { Ug/L } \\
\text { CFA03701 } \\
\end{array}$ & $\begin{array}{r}\text { CFA } \\
\text { 03-LF3-10 } \\
\text { DOWN GRAD HELL } \\
\text { CFA03902H } \\
\text { GROUND HATER } \\
\text { Ug/L } \\
\text { CFA03701 } \\
\end{array}$ & $\begin{array}{r}\text { CFA } \\
\text { 03-LF3-10 } \\
\text { DOWN GRAD HELL } \\
\text { CFA03902M } \\
\text { GROUND HATER } \\
\text { Ug/L } \\
\text { CFA03701 } \\
\end{array}$ & $\begin{array}{r}\text { CFA } \\
\text { O3-LF3-11 } \\
\text { UPGRADIENT WELL } \\
\text { CFAO4001H } \\
\text { GROUND WATER } \\
\text { Ug/L } \\
\text { CFA03701 } \\
\end{array}$ & $\begin{array}{r}\text { CFA } \\
\text { O3-LF3-11 } \\
\text { UPGRADIENT WELL } \\
\text { CFAO4001M } \\
\text { GROUND WATER } \\
\text { Ug/L } \\
\text { CFA03701 } \\
\end{array}$ \\
\hline $\begin{array}{l}\text { FIELD MEASUREMENTS } \\
\mathrm{pH} \\
\text { Conduct ivity (uS) } \\
\text { Temperature (C) }\end{array}$ & $\begin{array}{r}7.7 \\
0.6 \\
11.2\end{array}$ & $\begin{array}{r}7.7 \\
0.6 \\
11.2\end{array}$ & $\begin{array}{r}7.7 \\
0.6 \\
11.2\end{array}$ & $\begin{array}{r}7.8 \\
0.5 \\
11.4\end{array}$ & $\begin{array}{r}7.8 \\
0.5 \\
11.4\end{array}$ \\
\hline $\begin{array}{l}\text { ANALYTES } \\
\text { Arsenic } \\
\text { Barium } \\
\text { Cadmium } \\
\text { Chromium } \\
\text { Iron }\end{array}$ & $\begin{array}{l}\cdots \\
151 \text { B } \\
\cdots \\
\cdots \\
\cdots-\end{array}$ & $\begin{array}{c}1.9 \mathrm{~B} \\
158 \mathrm{~B} \\
\cdots- \\
51.0 \\
527\end{array}$ & $\begin{array}{c}11.0 \\
\ldots \text { B } \\
\cdots- \\
\cdots\end{array}$ & \begin{tabular}{c}
$1.4 \mathrm{BH}$ \\
$150 \mathrm{~B}$ \\
\hdashline.- \\
29.0 \\
410
\end{tabular} & $\begin{array}{l}\cdots \\
111 \text { в } \\
\cdots- \\
\cdots \\
\cdots\end{array}$ \\
\hline $\begin{array}{l}\text { Lead } \\
\text { Magnesium } \\
\text { Mercury } \\
\text { Selenium } \\
\text { Silver }\end{array}$ & $\begin{array}{rl}2.0 & \mathrm{BW} \\
15600 & \\
2.0 \mathrm{UJ} \\
2.0 \mathrm{NUJ} \\
\ldots .-\end{array}$ & $\begin{array}{c}8.6 \mathrm{~W} \\
15600 \\
2.0 \mathrm{UJ} \\
2.0 \mathrm{UJ} \\
\ldots . .\end{array}$ & $\begin{array}{c}1.5 \mathrm{~B} \\
14900 \\
\cdots \\
2.0 \mathrm{WNUJ} \\
\cdots\end{array}$ & $\begin{array}{c}3.7 \mathrm{~W} \\
13900 \\
0.20 \mathrm{UN} \\
2.0 \mathrm{WJ} \\
\ldots\end{array}$ & $\begin{array}{c}2.2 \mathrm{BH} \\
13400 \\
-\cdots \\
2.0 \mathrm{NUJ} \\
-\cdots\end{array}$ \\
\hline Sodium & 35100 & 35400 & 35400 & 25800 & 26600 \\
\hline $\begin{array}{l}\text { Total (Allowed) Hold Timea } \\
\text { Total (Allowed) Hold Time } \\
\text { Total (All owed) Hold Timec }\end{array}$ & $\begin{array}{l}27(180) d \\
34(26) d^{\star} \\
27(180) d\end{array}$ & $\begin{array}{l}27(180) d \\
34(26) d^{\star} \\
27(180) d\end{array}$ & $\begin{array}{l}36(180) d \\
37(26) d^{\star} \\
36(180) d\end{array}$ & $\begin{array}{l}14(180) d \\
29(26) d^{\star} \\
14(180) d\end{array}$ & $\begin{array}{l}42(180) d \\
43(26) d^{\star} \\
42(180) d\end{array}$ \\
\hline
\end{tabular}
a. ICP
b. CVAAS
c. GFAAS
9-12-92 
CFA Landfills II and III FY90 Hells - 1st Quarter April 1991 S\&A Data Document

TABLE -. CFA LANDFILLS II AND 111 FY9O WELLS - INORGANIC DATA (Continued)

\begin{tabular}{|c|c|c|}
\hline $\begin{array}{l}\text { AREA } \\
\text { LOCATION } \\
\text { TYPE OF LOCATION } \\
\text { SAMPLE NUMBER } \\
\text { MEDIA } \\
\text { UNITS } \\
\text { SDG NUMBER }\end{array}$ & $\begin{array}{r}\text { CFA } \\
\text { O3-LF3-11 } \\
\text { UPGRADIENT WELL } \\
\text { CFAO4002H } \\
\text { GROUND WATER } \\
\text { Ug/L } \\
\text { CFA03701 }\end{array}$ & $\begin{array}{r}\text { CFA } \\
\text { 03-LF3-11 } \\
\text { UPGRADIENT WELL } \\
\text { CFAO4002M } \\
\text { GROUND WATER } \\
\text { Ug/L } \\
\text { CFA03701 } \\
\end{array}$ \\
\hline $\begin{array}{l}\text { FIELD MEASUREMENTS } \\
\text { pH } \\
\text { Conduct ivity (US) } \\
\text { Temperature (C) }\end{array}$ & $\begin{array}{r}7.8 \\
0.5 \\
11.4\end{array}$ & $\begin{array}{r}7.8 \\
0.5 \\
11.4\end{array}$ \\
\hline $\begin{array}{l}\text { ANALYTES } \\
\text { Arsenic } \\
\text { Barium } \\
\text { Cadmium } \\
\text { Chromium } \\
\text { Iron }\end{array}$ & \begin{tabular}{l}
$1.3 \mathrm{BH}$ \\
$149 \mathrm{~B}$ \\
\hdashline $120 \mathrm{~J}$ \\
$1010 \mathrm{~J}$
\end{tabular} & $\begin{array}{l}1.3 \mathrm{~B} \\
109 \mathrm{~B} \\
\cdots \cdots \\
\cdots-\end{array}$ \\
\hline $\begin{array}{l}\text { Lead } \\
\text { Magnesium } \\
\text { Mercury } \\
\text { Selenium } \\
\text { Sitver }\end{array}$ & $\begin{array}{c}2.2 \mathrm{~B} \\
14300 \mathrm{~J} \\
0.20 \text { UN } \\
2.0 \text { WUJ } \\
\ldots .-\end{array}$ & \begin{tabular}{c}
3.7 \\
13500 \\
\hdashline 3.5 BUNJ \\
$\ldots .$.
\end{tabular} \\
\hline Sodium & $26800 \mathrm{~J}$ & 26200 \\
\hline $\begin{array}{l}\text { Total (Allowed) Hold Time } \\
\text { Total (Allowed) Hold Time } \\
\text { Total (Allowed) Hold Time }\end{array}$ & $\begin{array}{l}14(180) d \\
29(26) d^{\star} \\
14(180) d\end{array}$ & $\begin{array}{l}42(180) d \\
29(26) d^{\star} \\
42(180) d\end{array}$ \\
\hline
\end{tabular}

a. ICP

b. CVAAS

c. GFAAS

9-12-92 
CFA Landfills II and III FY90 Wells -1st Quarter April 1991 S\&A Data Document

TABLE --_- CFA LANDFILLS II AND III FY9O WELLS - INORGANIC FIELD BLANK DATA

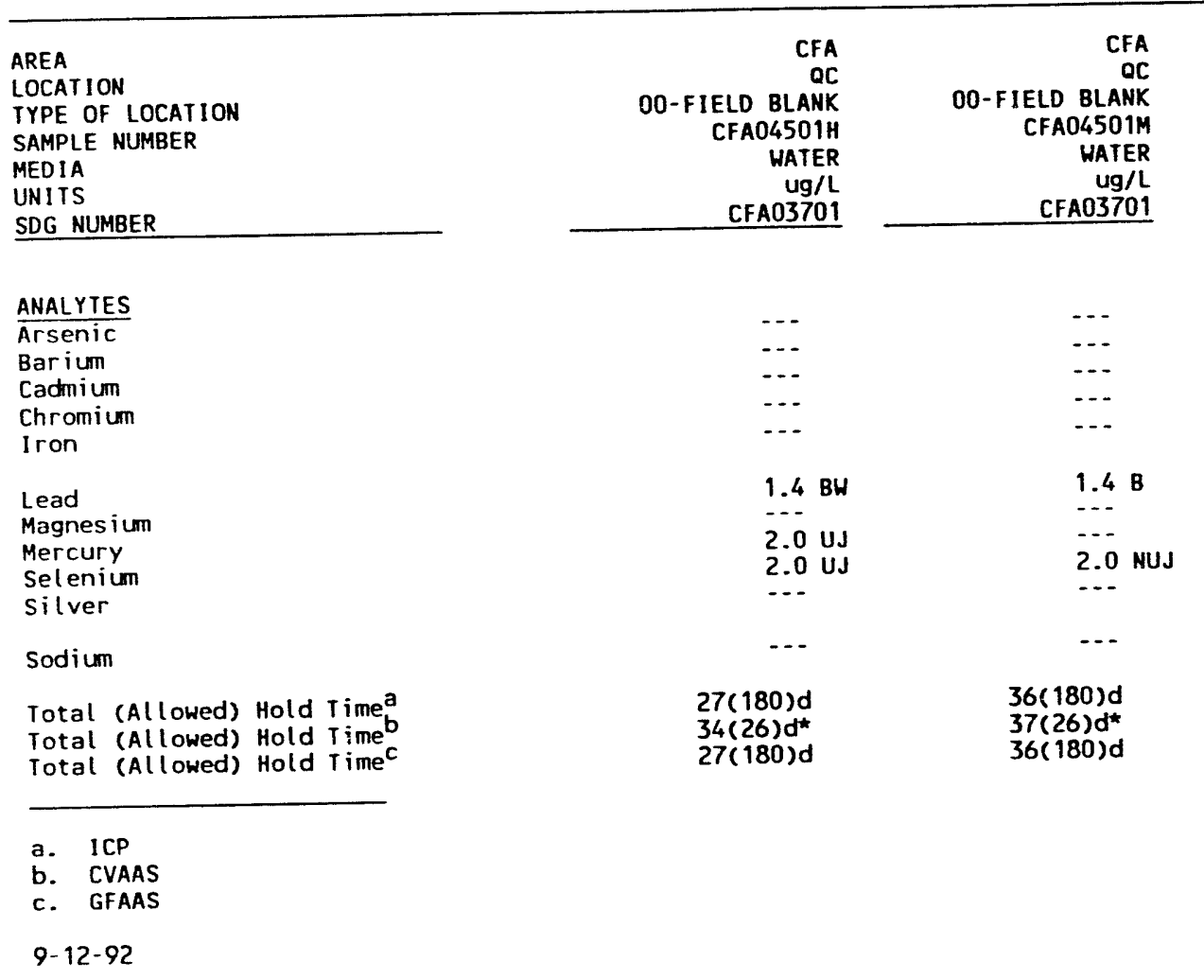


CFA Landfills 11 and III FY90 Wells - 1st Quarter April 1991 S\&A Data Document - Method Validation Level A

TABLE

CFA LANDFILLS II AND III FYOO WELLS - NON-METALS DATA

Page 1 of 3

\section{AREA}

LOCATION

TYPE OF LOCATION

SAMPLE NUMBER

MEDIA

SDG NUMBER

\section{FIELD MEASUREMENTS}

pH

Conductivity (uS)

Temperature (C)

\section{ANALYTES}

Fluoride

Nitrate

Sulfate

IOC

TOX

$\Omega$

in Total (Allowed) Hold Timea

Total (Allowed) Hold Time

Toral (Allowed) Hold I ime

Total (Allowed) Hold Time

Total (Allowed) Hold Time

Total (Allowed) Hold Time

a. Chloride - Method 9252

b. Fluoride - Method 340.2

c. Nitrate - Method 353.1
d. Sulfate - Method 9038

e. IOC - Method 9060

f. Tox - Method 9020

9-22-92
CFA

$02-L F 2-12$

CWN GRAD WELL

GOUND HATER

$\mathrm{mg} / \mathrm{L}$
CFA03701

DOWN GRAD LF3-9

CFAO3801

GROUND WATER

$\mathrm{mg} / \mathrm{L}$
CFA03701

$\begin{array}{r}\text { CFA } \\ \text { O3-LF3-10 } \\ \text { DOWN GRAD WELL } \\ \text { CFAO3901 } \\ \text { GROUND WATER } \\ \text { mg/L } \\ \text { CFA03701 } \\ \hline\end{array}$

CFA
03-LF3-10
DOWN GRAD WELL
CFA03902

GROUND WATER

CFA03701

UPRADIENT WELL

CFA04009

CFA04001

$\mathrm{mg} / \mathrm{L}$

CFA03701

$\begin{array}{ll}7.8 & 7.7 \\ 0.6 & 0.6\end{array}$

0.6
11.3

7.7
0.6

0.6
11.2

7.7

0.6
11.2

7.8

7.7
0.6
12.6

90.2

$86.7 \mathrm{~J}$

40.0

561
$37.2 \mathrm{~J}$
4.1

$83.8 \mathrm{~J}$

40.0

698

$33.9 \mathrm{~J}$
3.3

17.0

22.0

40.0

642
$29.7 \mathrm{~J}$

3.6

20.0

$77.0 \mathrm{~J}$

40.0

$36.0 \mathrm{~J}$

$36.0 \mathrm{~J}$
7.4

11.0

11.4

$9(28) d$

$24(28) d$

$10(28) d$

(28)d

$9(28) d$
$9(7) d^{\star}$

$9(28) d$
$24(28) d$

$24(28) d$
$10(28) d$

$10(28) d$

$9(28) d$

$8(28) d$
$23(28) d$
$9(28) d$
$9(28) d$
$9(28) d$
$8(7) d \star$

$8(28) d$
$33(28) d$

$9(28) d$

$9(28) d$

$8(7) d^{\star}$ 8(28)d

$23(28) d$

$9(28) d$

$9(28) d$

$8(7) d^{\star}$
$91.9 \mathrm{~J}$

40.0

$36.6 \mathrm{~J}$

3.6

56.0

$12(28) d$

$29(28) \mathrm{d}$

$14(28) \mathrm{d}$

$12(28) \mathrm{d}$

$8(7) d^{\star}$ 
CFA Landfills 11 and III FY90 Wells - 1st Quarter April 1991 S\&A Data Document - Method Validation Level A

TABLE _-_-_ CFA LANDFILLS II AND III FYgO WELLS - NON-METALS DATA

Page 2 of 3

\begin{tabular}{|c|c|c|c|c|c|}
\hline $\begin{array}{l}\text { AREA } \\
\text { LOCATION } \\
\text { TYPE OF LOCATION } \\
\text { SAMPLE NUMBER } \\
\text { MEDIA } \\
\text { UNITS } \\
\text { SDG NUMBER } \\
\end{array}$ & $\begin{array}{r}\text { CFA } \\
\text { O3-LF3-11 } \\
\text { UPGRADIENI WELL } \\
\text { CFAO4002 } \\
\text { GROUND WATER } \\
\mathrm{mg} / \mathrm{L} \\
\text { CFA03701 } \\
\end{array}$ & $\begin{array}{r}\text { CFA } \\
\text { O3-LF3-11 } \\
\text { UPGRADIENI WELL } \\
\text { CFAO4003 } \\
\text { GROUND WATER } \\
\mathrm{mg} / \mathrm{L} \\
\text { CFA03701 } \\
\end{array}$ & $\begin{array}{r}\text { CFA } \\
\text { O3-LF3-11 } \\
\text { UPGRADIENT WELL } \\
\text { CFAO4004 } \\
\text { GROUND WATER } \\
\text { mg/L } \\
\text { CFA03701 } \\
\end{array}$ & $\begin{array}{r}\text { CFA } \\
\text { O3-LF3-11 } \\
\text { UPGRADIENT WELL } \\
\text { CFAO4005 } \\
\text { GROUND WATER } \\
\text { mg/L } \\
\text { CFA03701 } \\
\end{array}$ & $\begin{array}{r}\text { CFA } \\
\text { 03-LF3-11 } \\
\text { UPGRADIENT WELL } \\
\text { CFAO4006 } \\
\text { GROUND WATER } \\
\mathrm{mg} / \mathrm{L} \\
\text { CFA03701 } \\
\end{array}$ \\
\hline $\begin{array}{l}\text { FIELD MEASUREMENTS } \\
\text { pH } \\
\text { Conductivity (uS) } \\
\text { Temperature (C) }\end{array}$ & $\begin{array}{r}7.8 \\
0.5 \\
11.4\end{array}$ & $\begin{array}{r}7.8 \\
0.5 \\
11.4\end{array}$ & $\begin{array}{r}7.8 \\
0.5 \\
11.4\end{array}$ & $\begin{array}{r}7.8 \\
0.5 \\
11.4\end{array}$ & $\begin{array}{r}7.8 \\
0.5 \\
11.4\end{array}$ \\
\hline $\begin{array}{l}\text { ANALYYES } \\
\text { Chloride } \\
\text { Fluoride } \\
\text { Nitrate } \\
\text { Sul fate } \\
\text { TOC }\end{array}$ & $\begin{array}{c}77.6 \mathrm{~J} \\
40.0 \\
582 \\
32.6 \mathrm{~J} \\
3.2\end{array}$ & 3.7 & 5.1 & 1.6 & 3.5 \\
\hline Tox & 71.0 & 36.0 & 49.0 & 60.0 & 46.0 \\
\hline $\begin{array}{l}\text { Total (Allowed) Hold Time } \\
\text { Total (Allowed) Hold Time } \\
\text { Total (Allowed) Hold Time } \\
\text { Total (All owed) Hold Timed } \\
\text { Total (All lowed) Hold Time } \\
\text { Total (Allowed) Hold Time }\end{array}$ & $\begin{array}{l}12(28) d \\
14(28) d \\
12(28) d \\
13(28) d \\
8(7) d^{\star}\end{array}$ & $\begin{array}{l}13(28) d \\
8(7) d^{*}\end{array}$ & $\begin{array}{l}13(28) d \\
8(7) d^{\star}\end{array}$ & $\begin{array}{c}13(28) d \\
8(7) d^{\star}\end{array}$ & $\begin{array}{l}13(28) d \\
8(7) d^{\star}\end{array}$ \\
\hline $\begin{array}{l}\text { a. Chloride - Method } 9252 \\
\text { b. Fluoride - Method } 340.2 \\
\text { c. Nitrate - Method } 353.1 \\
\text { d. Sulfate - Method } 9038 \\
\text { e. TOC - Method } 9060 \\
\text { f. Tox - Method } 9020\end{array}$ & & & & & \\
\hline
\end{tabular}


CFA Landfills II and III FY90 Wells - 1st Quarter April 1991 S\&A Data Document - Method Validation Level A

TABLE _-.- CFA LANDFILLS 11 AND III FY90 WELLS - NON-METALS DATA

$\begin{array}{lrr}\text { AREA } & \text { CFA } & \text { CFA } \\ \text { LOCATION } & \text { O3-LF3-11 } & \text { O3-LF3-11 } \\ \text { TYPE OF LOCATION } & \text { UPGRADIENT WELL } & \text { UPGRADIENT WELL } \\ \text { SAMPLE NUMBER } & \text { CFAO4007 } & \text { CFAO4008 } \\ \text { MEDIA } & \text { GROUND WATER } & \text { GROUND WATER } \\ \text { UNITS } & \text { mg/L } & \text { mg/L } \\ \text { SDG NUMBER } & \text { CFA03701 } & \text { CFA03701 }\end{array}$

SDG NUMBER

CFA03701

$\frac{\text { FIELD MEASUREMENTS }}{\text { pH }}$

Temperature (C)

7.8
0.5

0.5
11.4

7.8

ANAL YTES

Chloride

Fluoride

Nitrate

Sulfate

TOC

f Total (Allowed) Hold Timea

Total (Allowed) Hold Timeb

rotal (Allowed) Hold Timec

Total (Allowed) Hold Timed

Total (Allowed) Hold Time
Total (Allowed) Hold Timef

$13(28) d$

$8(7) d^{\star}$

$13(28) d$

. Chloride - Method 9252

b. Fluoride - Method 340.2

c. Nitrate - Method 353.1

d. Sulfate - Method 9038

e. TOC - Method 9060

f. Tox - Method 9020

9-22-92 


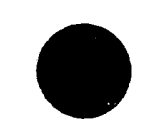

CFA Landfills 11 and III FY90 Wells - 1st Quarter April 1091 S\&A Data Document - Method Validation Level A

TABLE .. CFA LANDFILLS II AND 111 FY90 WELLS - NON-METALS FIELD BLANK DATA

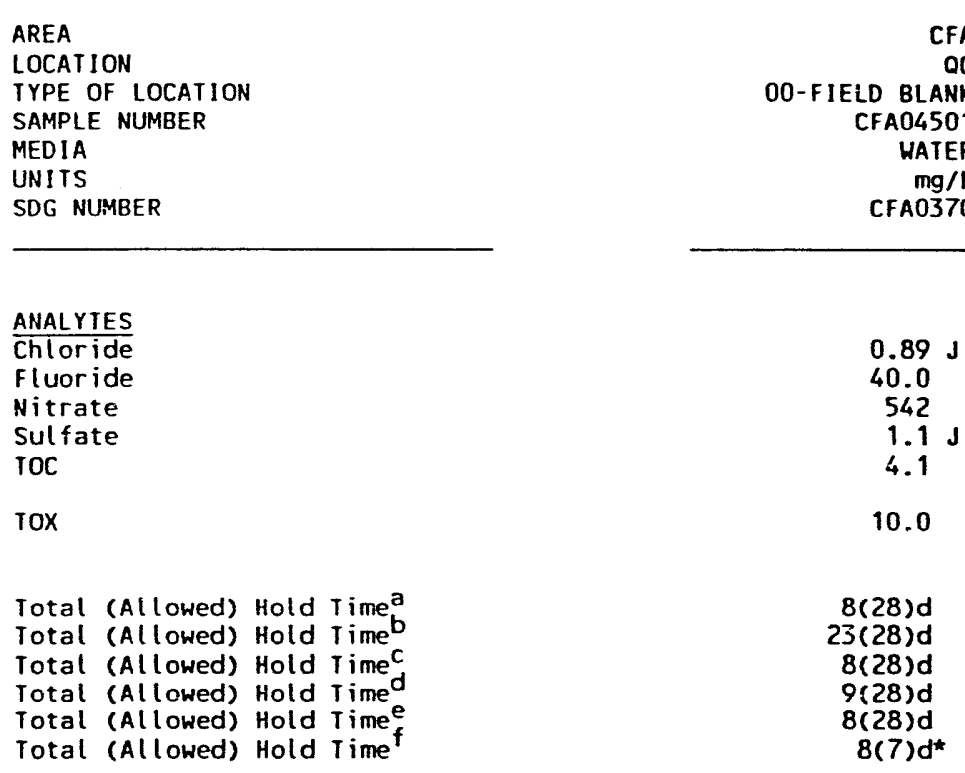
a. Chloride - Method 9252
b. Fluoride - Method 340.2
b. Fluoride - Method 340.2
c. Nitrate - Method 353.1
e. TOC - Method 9060
f. TOX - Method 9020
$9-22-92$

g/L

$0.89 \mathrm{~J}$

$1.1 \mathrm{~J}$

10.0

$8(28) d$

$8(28) d$

(28)d

$8(7) d^{*}$ 
CFR Landfills II and III fY90 Wells - 1st Quarter April 1991 S\&A Data Document

TABLE -. CFA LANDFILLS II AND III FY90 WELLS - ORgANOCHLORINE PESTICIDE APPENDIX IX DATA

Page 1 of 1

\begin{tabular}{l} 
AREA \\
LOCATION \\
TYPE OF LOCATION \\
SAMPLE NUMBER \\
MEDIA \\
UNITS \\
SDG NUMBER \\
\hline
\end{tabular}

FIELD MEASUREMENTS

$\mathrm{pH}$

Conductivity (uS)

Temperature (C)

\begin{tabular}{rrr} 
CFA & CFA & CFA \\
O2-LF2-12 & O3-LF3-9 & 03-LF3-10 \\
GRAD HELL & DOWN GRAD WELL & DOWN GRAD WELL \\
CFAO3701G & CFA03801G & CFA03901G \\
GROUND HATER & GROUND WATER & GROUND WATER \\
Ug/L & Ug/L & Ug/L \\
CFAO3701G & CFA03701G & CFA03701G \\
\hline
\end{tabular}

$\begin{array}{r}\text { CFA } \\ \text { O3-LF3-10 } \\ \text { DOWN GRAD WELL } \\ \text { CFA03902G } \\ \text { GROUND WATER } \\ \text { Ug/L } \\ \text { CFAO3701G } \\ \hline\end{array}$

CFA
O3-LF3-11
UPGRADIENT WELL
CFA04001G

TARGET COMPOUNDS

\section{None Detected}

Dilution Factor

Extraction (Allowed) Hold Time

Analytical (Allowed) Hold Time

7.8
0.6

0.6
11.3

7.7
0.6

7.7
0.6

$\begin{array}{r}\text { Ug/L } \\ \text { CFA03701G } \\ \hline\end{array}$

03-LF3-11

GRADIENT WELL
CFA04002G

GROUND WATER

$\mathrm{ug} / \mathrm{L}$
CFA037016

9-13-92

1.0

$4(7) d$
$15(40) d$

1.0

$4(7) d$
$15(40) d$

1.0
$4(7) d$

$4(7) d$
$15(40) d$

1.0
$4(7) d$
$15(40) d$

7.8

0.5
11.4

7.8
0.5

7.7

0.6
11.2

0.5

$\overbrace{i}^{A}$ 
CFA Landfills II and III FY90 Wells - ist Quarter April 1991 S\&A Data Document

TABLE _-_-_ CFA LANDFILLS II AND III FY90 WELLS - ORGANOCHLORINE PESTICIDE APPENDIX IX FIELD BLANK DATA

AREA

LOCATION

TYPE OF LOCATION

SAMPLE NUMBER

MEDIA

\begin{tabular}{l} 
UNITS \\
SOG MUMBER \\
\hline
\end{tabular}

CFA

OC
OO-FIELD BLANK

CFA04501G

GROUND WATER

TARGET COMPOUNDS

None Detected

Dilution Factor

Extraction (Allowed) Hold Time

Analytical (Allowed) Hold Time

1.0
$4(7) d$

9-13-92

$16(40) d$ 
CFA Landfills II and III FY90 Wells - 1st Quarter April 1991 S\&A Data Document

TABLE _.-- CFA LANDFILLS II AND III FY9O WELLS - ORGANOCHLORINE HERBICIDE APPENDIX IX DATA

Page 1 of 1

\begin{tabular}{|c|c|c|c|c|c|c|}
\hline $\begin{array}{l}\text { AREA } \\
\text { LOCATION } \\
\text { TYPE OF LOCATION } \\
\text { SAMPLE NUMBER }\end{array}$ & $\begin{array}{r}\text { CFA } \\
\text { 02-LF2-12 } \\
\text { DOWN GRAD WELL } \\
\text { CFA037012 }\end{array}$ & $\begin{array}{r}\text { CFA } \\
\text { 03-LF3-9 } \\
\text { DOWN GRAD WELL } \\
\text { CFA038012 }\end{array}$ & $\begin{array}{r}\text { CFA } \\
\text { 03-LF3-10 } \\
\text { DOWN GRAD WELL } \\
\text { CFA039012 }\end{array}$ & $\begin{array}{r}\text { CFA } \\
\text { 03-LF3-10 } \\
\text { DOWN GRAD WELL } \\
\text { CFA039022 }\end{array}$ & $\begin{array}{r}\text { CFA } \\
\text { O3-LF3-11 } \\
\text { UPGRADIENT WELL } \\
\text { CFAO4001Z }\end{array}$ & $\begin{array}{r}\text { CFA } \\
\text { O3-LF3-11 } \\
\text { UPGRADIENT WELL } \\
\text { CFA04002Z }\end{array}$ \\
\hline $\begin{array}{l}\text { MEDIA } \\
\text { UNITS } \\
\text { SDG NUMBER } \\
\end{array}$ & $\begin{array}{r}\text { GROUND WATER } \\
\text { Ug/L } \\
\text { CFA037012 } \\
\end{array}$ & $\begin{array}{r}\text { GROUND HATER } \\
\text { ug } / L \\
\text { CFA037012 } \\
\end{array}$ & $\begin{array}{r}\text { GROUND MATER } \\
\text { Ug } / L \\
\text { CFA037012 } \\
\end{array}$ & $\begin{array}{r}\text { GROUND WATER } \\
\text { Ug/L } \\
\text { CFA037012 } \\
\end{array}$ & $\begin{array}{r}\text { GROUND WATER } \\
\text { Ug } / \mathrm{L} \\
\text { CFA037012 } \\
\end{array}$ & $\begin{array}{r}\text { GROUND WATER } \\
\text { Ug/L } \\
\text { CFA037012 } \\
\end{array}$ \\
\hline $\begin{array}{l}\text { FIELD MEASUREMENTS } \\
\text { pH } \\
\text { Conductivity (uS) } \\
\text { Temperature (C) }\end{array}$ & $\begin{array}{r}7.8 \\
0.6 \\
11.2\end{array}$ & $\begin{array}{r}7.7 \\
0.6 \\
12.6\end{array}$ & $\begin{array}{r}7.7 \\
0.6 \\
11.2\end{array}$ & $\begin{array}{r}7.7 \\
0.6 \\
11.2\end{array}$ & $\begin{array}{r}7.8 \\
0.5 \\
11.4\end{array}$ & $\begin{array}{r}7.8 \\
0.5 \\
11.4\end{array}$ \\
\hline $\begin{array}{l}\text { TARGET COMPOUNDS } \\
2,4-D \\
\text { Silvex } \\
2,4,5-T\end{array}$ & $\begin{array}{rl}1 & \mathrm{UJ} \\
0.50 \mathrm{UJ} & \\
0 \mathrm{R}\end{array}$ & $\begin{array}{rl}1 & \mathrm{UJ} \\
0.50 \mathrm{UJ} \\
0 \mathrm{R}\end{array}$ & $\begin{array}{rl}1 & \mathrm{UJ} \\
0.50 \mathrm{UJ} & \\
0 \mathrm{R}\end{array}$ & $\begin{array}{l}1 \mathrm{UJ} \\
0.50 \mathrm{UJ} \\
0 \mathrm{R}\end{array}$ & $\begin{array}{rl}1 & \text { UJ } \\
0.50 & \mathrm{UJ} \\
0 \mathrm{R}\end{array}$ & $\begin{array}{rl}1 & U J \\
0.50 & U J \\
0 & R\end{array}$ \\
\hline $\begin{array}{l}\text { Dilution Factor } \\
\text { Extraction (Allowed) Hold Time } \\
\text { Analytical (Allowed) Hold Time }\end{array}$ & $\begin{array}{l}7(7) d \\
7(40) d\end{array}$ & $\begin{array}{r}7(7) d \\
7(40) d\end{array}$ & $\begin{array}{r}7(7) d \\
7(40) d\end{array}$ & $\begin{array}{r}7(7) d \\
10(40) d\end{array}$ & $\begin{array}{r}6(7) d \\
14(40) d\end{array}$ & $\begin{array}{r}6(7) d \\
14(40) d\end{array}$ \\
\hline
\end{tabular}

9-12-92 


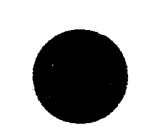

CFA Landfills II and III FY90 Wells - 1st Quarter April 1991 S\&A Data Document

TABLE _-_-_ CFA LANDFILLS II AND III FY90 WELLS - ORGANOCHLORINE HERBICIDE APPENDIX IX FIELD BLANK DATA

AREA

LOCATION

TYPE OF LOCATION

SAMPLE NUMBER

MEDIA

SDG NUMBER

\section{TARGET COMPOUNDS}

2,4-D

Silvex
$2,4,5-T$

Dilution Factor

Extraction (Allowed) Hold Time

Analytical (Allowed) Hold Time

9-12-92
CFA

OC
O0-FIELD BLANK

CFA045012

HATER

$\begin{array}{r}\text { ug/L } \\ 037012 \\ \hline\end{array}$

CFA037012

1 UJ
0.50 UJ

$O R$

$7(7) d$
$20(40) d$ 
CFA Landfills II and III FY90 Wells - Ist Quarter April 1991 S\&A Data Document - Method Validation Level C

TABLE CFA LANDFILLS II AND III FY9O WELLS - ANALYSIS RESULTS FOR GAMMA-EMITTING RADIONUCLIDES

Page 1 of 1

Date: $\underline{07 / 17 / 91}$

Lab Name: RML

Case No.:

Report No.: STPHY91031

SDG NO.: CFA037012

\begin{tabular}{|c|c|c|c|c|c|c|c|c|c|c|c|c|c|c|}
\hline $\begin{array}{c}\text { Field } \\
\text { Sample No. }\end{array}$ & Location & $\begin{array}{l}\text { Lab } \\
\text { Sample } 10\end{array}$ & $\begin{array}{l}\text { Sample } \\
\text { Matrix }\end{array}$ & $\begin{array}{l}\text { Anal } \\
\text { Type }\end{array}$ & $\begin{array}{l}\text { Sample } \\
\text { Value }\end{array}$ & $\begin{array}{l}\text { Sample } \\
\text { Error }\end{array}$ & Units & $\begin{array}{l}\text { Anal } \\
\text { Date }\end{array}$ & $\begin{array}{c}\text { Sample } \\
\text { Date }\end{array}$ & $\begin{array}{l}\text { Sample } \\
\text { Size }\end{array}$ & Yield & $\begin{array}{c}\text { Detector } \\
\text { ID }\end{array}$ & DQF & ASL \\
\hline CFA037012 & $02-$ LF2- 12 & A5050291038 & WATER & GAMMA & ND & & $\mathrm{pCi} / \mathrm{L}$ & $05 / 02 / 91$ & $04 / 29 / 91$ & 0.5400 & 0.0 & RML A5/PG-1 & & \\
\hline CFA038012 & 03-LF3-9 & C5050191043 & WATER & GAMMA & ND & & $\mathrm{pCi} / \mathrm{L}$ & $05 / 01 / 91$ & $04 / 30 / 91$ & 0.5400 & 0.0 & RML C5/PG-20 & & \\
\hline CFA039012 & $03-L F \geq-10$ & A6050191042 & WATER & GAMMA & ND & & $p C i / L$ & $05 / 01 / 91$ & $05 / 01 / 91$ & 0.5400 & 0.0 & RML $A 6 / P G-13$ & & \\
\hline CFA039022 & $03-L F 3-10$ & A5050191041 & WATER & GAMMA & ND & & $p C i / L$ & $05 / 01 / 91$ & $05 / 01 / 91$ & 0.5400 & 0.0 & RML A5/PG-1 & & \\
\hline CFA040012 & 03-LF3-11 & A6042691046 & WATER & GAMMA & ND & & $\mathrm{pCi} / \mathrm{L}$ & $04 / 26 / 91$ & $04 / 25 / 91$ & 0.5400 & 0.0 & RML A6/PG-13 & & \\
\hline CFA040022 & 03-LF3-11 & $A 6051391043$ & WATER & GAMMA & ND & & $p C i / L$ & $05 / 13 / 91$ & $04 / 25 / 91$ & 0.5400 & 0.0 & RML A6/PG-13 & & \\
\hline CFA045012 & O0-FIELD BLANK & A6050291039 & WATER & GAMMA & ND & & $\mathrm{pCi} / \mathrm{L}$ & $05 / 02 / 91$ & $05 / 01 / 91$ & 0.5400 & 0.0 & RML A6/PG-13 & & \\
\hline
\end{tabular}

9-18-92 
Appendix C.5

Validated Groundwater Quality Data for CFA Landfills II and III

\author{
July 1991
}



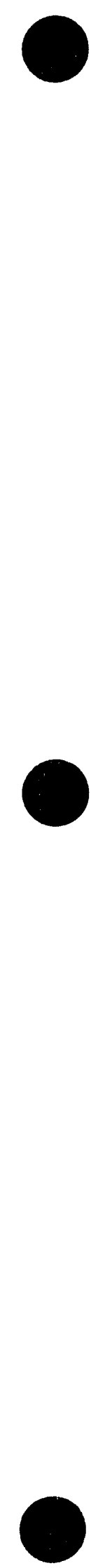

C.5-2 


\section{DATA QUALIFIER DEFINITIONS}

\section{Organic Analysis Data Flags}
A - Indicates that a tentatively identified compound is a suspected aldol-condensation product.
B - Used when the analyte is found in the associated blank as well as in the sample.
C - Applies to pesticide results where the identification has been confirmed by GC/MS.
D - Identifies all compounds identified in an analysis at a secondary dilution factor.
E - Identifies compounds which have concentrations that exceed the calibration range of the GC/MS instrument for that specific analysis.
J - Indicates as estimated value.
$\mathbf{U}$ - Indicates compound was analyzed for but not detected.

\section{Organic Validation Data Qualifiers}

J - The analyte was positively identified in the sample, but the associated numerical value may not be an accurate representation of the amount actually present in the environmental sample. The data should be seriously considered for decision making and are usable for many purposes.

N - Presumptive evidence of the presence of the material.

NJ - Presumptive evidence of the presence of the material at an estimated quantity.

R - The data are unusable (may or may not be present). Resampling and reanalysis is necessary for verification.

$\mathbf{U}$ - The material was analyzed for but was not detected. The associated numerical value is the sample quantitation limit.

UJ - The material was analyzed for, but was not detected. The sample quantitation limit is an estimated quantity.

Reference: Environmental Restoration Department, Sample Management Office Standard Operating Procedures 12.1.4 and 12.1.5 (EG\&G Idaho). 


\section{DATA QUALIFIER DEFINITIONS}

Inorganic Analysis Data Flags

B - Value is less than the CRDL, but greater than the IDL.

E - Value is estimated because of the presence of interference.

N - Spiked sample recovery not within control limits.

NR - Analyte was not required to be analyzed.

S - Value was determined by the method of standard additions (MSA).

U - Analyte was analyzed for but not detected.

W - Post digestion spike for Furnace AA analysis is out of control limits (85\% to $115 \%$ ), while sample absorbance is less than $50 \%$ of spike absorbance.

* - Duplicate analysis not within control limits.

\section{Methods}

A - Flame AA.

CV - Manual Cold Vapor AA.

F - Furnace AA.

NR - Not run, analyte not required to be analyzed.

P - Inductively Coupled Plasma - Atomic Emission Spectrometry.

Inorganic Validation Data Qualifiers

J - The analyte was analyzed for and was positively identified, but the associated numerical value may not be consistent with the amount actually present in the environmental sample.

R - The data are unusable.

U - The material was analyzed for, but was not detected above the level of the associated value. The associated value is either the sample quantitation limit or the sample detection limit.

UJ - The material was analyzed for, but was not detected. The associated value is an estimate and may not accurately reflect the instrument detection limit in the sample matrix. 
CFA Landfills 11 and 111 FY90 - 2nd Quarter July 1991 S\&A Data Document

TABLE _-_- CFA LANDFILLS 11 AND 111 FY90 WELLS - 524.2 VOLATILE ORGANIC DATA

Page 1 of 4

\begin{tabular}{|c|c|c|c|c|c|}
\hline $\begin{array}{l}\text { AREA } \\
\text { LOCATION } \\
\text { TYPE OF LOCATION } \\
\text { SAMPLE NUMBER } \\
\text { MEDIA }\end{array}$ & $\begin{array}{r}\text { CFA } \\
\text { O2-LF2-12 } \\
\text { DOWN GRAD WELL } \\
\text { CFA04601A } \\
\text { GROUND WATER }\end{array}$ & $\begin{array}{r}\text { CFA } \\
\text { 03-LF3-9 } \\
\text { DOWN GRAD WELL } \\
\text { CFA04701A } \\
\text { GROUND WATER }\end{array}$ & $\begin{array}{r}\text { CFA } \\
\text { O3-LF3-10 } \\
\text { DOWN GRAD WELL } \\
\text { CFA04801A } \\
\text { GROUND WATER }\end{array}$ & $\begin{array}{r}\text { CFA } \\
\text { 03-LF3-10 } \\
\text { DOWN GRAD WELL } \\
\text { CFA04802A } \\
\text { GROUND WATER }\end{array}$ & $\begin{array}{r}\text { CFA } \\
\text { 03-LF3-11 } \\
\text { UPGRADIENT WELL } \\
\text { CFA04901A } \\
\text { GROUND WATER }\end{array}$ \\
\hline $\begin{array}{l}\text { UNITS } \\
\text { SDG NUMBER }\end{array}$ & $\begin{array}{r}u g / L \\
\text { CFA04701A }\end{array}$ & $\begin{array}{r}\text { Ug/L } \\
\text { CFA04701A }\end{array}$ & $\begin{array}{r}\mathrm{ug} / \mathrm{L} \\
\text { CFA04701A }\end{array}$ & $\begin{array}{r}u g / L \\
\text { CFA04701A }\end{array}$ & $\begin{array}{r}U \mathrm{U} / \mathrm{L} \\
\text { CFA04701A }\end{array}$ \\
\hline
\end{tabular}

SDG NUMBER

CFA04701A

CFAO4701A

\section{FIELD MEASUREMENTS}

Conduct ivity (US)

Temperature (C)

TARGET COMPOUNDS

Dichlorodifluorome thane

Chloromethane

Vinyl Chloride

Bromethane

1,1-Dichloroethene

Methylene Chloride

trans-1,2-Dichloroethene

trans-1,2-Dichloroeth

2,2-Dichloropropan

cis-1,2-Dichloroethene

Chloroform

Bromoch loromethane

1,1,1-Trichloroethan

Carbon Tetrachloride

1,1-D ichloropropene

Benzene

Trichloroethethe

1,2-Dichloropropane

Bromodichloromethane

Dibromomethane

Trans-1,3-Dichloropropene

Tol uene

cis-1,3-Dichloropropene

1,1,2-Trichloroethane

Tetrachloroethene

1,3-Dichloropropane

Dibromochloromethane

1,2-D ibromoethane

7.8
0.7

0.7
14.6

\subsection{6}

0.6
15.7

7.5
0.6

0.6
13.5

7.5

0.6
13.5

8.4
0.6

NP

NP
NP
NP
NP

NP

NP

NP

NP

NP

NP
NP
NP
NP

NP

NP

NP

NP.

NP

NP

NP

NP
NP
NP

NP

NP

NP
NP
NP

NP
NP
NP

NP

NP

NP

NP

NP

9-11-92 
CFA Landfills II and III FY90 - 2nd Quarter July 1991 S\&A Data Document

TABLE _-_- CFA LANDFILLS II AND III FY90 WELLS - 524.2 VOLATILE ORGANIC DATA (Cont inued)

Page 2 of 4

AREA

LOCATION

TYPE OF LOCATION

SAMPLE NUMBER

MEDIA

SDG NUMBER

Chlorobenzene

1,1,1,2-Tetrachloroethane

Ethylbenzene

Xylene (total meta \& para)

Xylene (ortho)

Styrene

1,1,2,2-Tetrachloroethane

Bromobenzene

1,2,3-Trichloropropane

n-Propylbenzene

1,3 5-Trimethylbenzene

4-chlorotoluene

tert-Butylbenzene

1,2,4-Irimethylbenzene

sec-Butylbenzene

1,3-Dichlorobenzene

1,4-Dichlorobenzene

n-Butylbenzene

1,2-Dichlorobenzene

1,2-D ibromo-3-chloropropane

1,2,4-Trichlorobenzene

Hexachlorobutadiene

Naphthalene

1,2,3-Trichlorobenzene

Dilution Factor

Total (Allowed) Hold Time

9-11-92

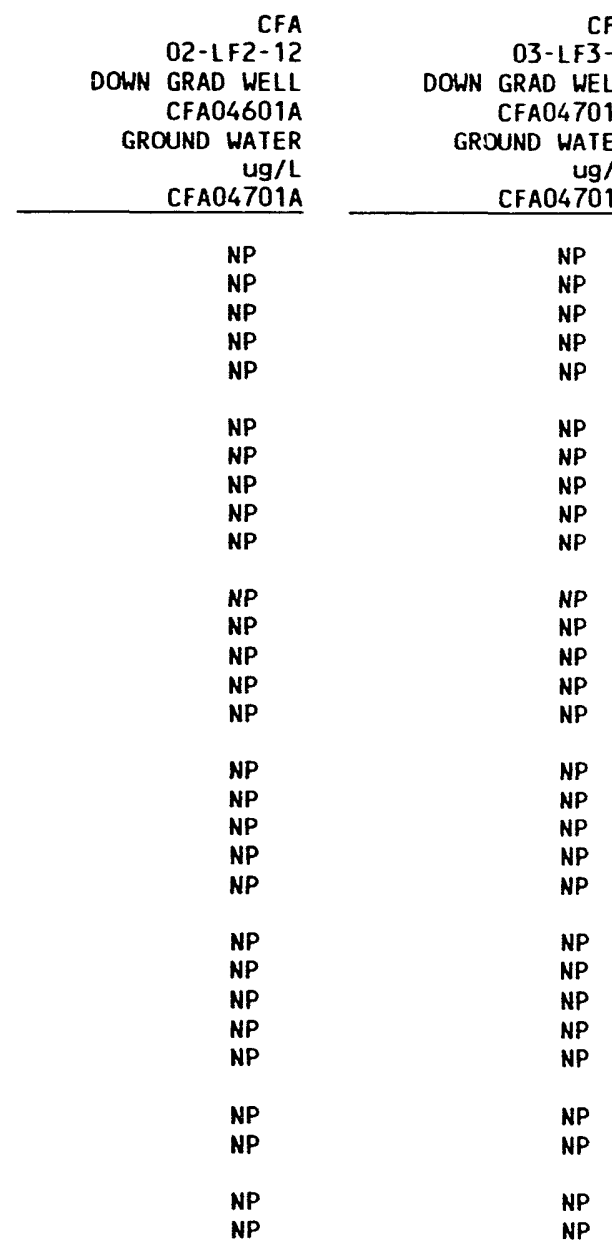

$\begin{array}{r}\text { CFA } \\ \text { O3-LF3-10 } \\ \text { DOWN GRAD WELL } \\ \text { CFA04801A } \\ \text { GROUND WATER } \\ \text { UG/L } \\ \text { CFAO4701A } \\ \hline\end{array}$

CFA
03-LF3-10
DOWN GRAD WELL
CFA04802A
GROUND WATER
Ug/L
CFA04701A
CFA
03-LF3- 11
IENT WELL
CFA0 $4901 A$
GROUND WATER
Ug/L

\begin{tabular}{|c|c|c|c|}
\hline NP & NP & NP & $\cdots$ \\
\hline NP & NP & NP & $\ldots$ \\
\hline NP & NP & NP & $\cdots$ \\
\hline NP & NP & NP & $\ldots$ \\
\hline NP & NP & NP & $\ldots$ \\
\hline NP & NP & NP & $\ldots$ \\
\hline NP & NP & NP &.- \\
\hline NP & NP & NP & $\ldots$ \\
\hline NP & NP & NP & $\ldots$ \\
\hline NP & NP & NP & $\cdots$ \\
\hline NP & NP & NP & $\ldots$ \\
\hline NP & NP & NP & ... \\
\hline NP & NP & NP & $\ldots$ \\
\hline NP & NP & NP & $\ldots$ \\
\hline NP & NP & NP & $\ldots$ \\
\hline NP & NP & NP & -. \\
\hline NP & NP & NP & $\ldots$ \\
\hline NP & NP & NP & $\ldots$ \\
\hline NP & NP & NP & $\ldots$ \\
\hline NP & NP & NP & - - \\
\hline NP & NP & NP & - . \\
\hline NP & NP & NP & $\ldots$ \\
\hline NP & NP & NP & $1 R$ \\
\hline NP & NP & NP & \\
\hline NP & NP & NP & $\cdots$ \\
\hline NP & NP & NP & - - \\
\hline NP & NP & NP & $\ldots$ \\
\hline NP & NP & NP & 1.000 \\
\hline NP & $12(14) d$ & NP & $11(14) d$ \\
\hline
\end{tabular}

- 
CFA Landfills II and III FY90 - 2nd Quarter July 1991 S\&A Data Document

TABLE _--- CFA LANDFILLS 11 AND III FY90 WELLS - 524.2 VOLATILE ORGANIC DATA (Continued)

Page 3 of 4

AREA

TYPE OF LOCATION

SAMPLE NUMBER

MEDIA

CFA

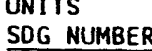

CFA
03-LF3-11
UPGRADIENT WELL
CFA04902A
GROUND WATER
UG/L
CFA04701A

FIELD MEASUREMENTS

Conduct ivity (uS)

Temperature (C)

Depth ( $f t$ )

TARGEI COMPOUNDS

Dichlorodif luorome thane

Chl oromethane

vinyl Chloride

Bromome thane

NP

$\cdots$

-..

1 us

Methylene Chloride

trans-1,2-Dichloroethene

1,1-Dichloroethane

2,2-Dichloropropane

cis-1,2-Dichloroethene

Chloroform

Bromochlorome thane

1,1,1-Trichloroethane

Carbon Tetrachloride

1,1-Dichloropropene

Benz-Dichloroethane

Irichloroethene

1,2-Dichloropropane

Bromodichlorome thane

Dibromome thane

Irans-1,3-0 ichloropropene

Toluene

cis-1,3-Dichloropropene

1,1,2-Trichloroethane

Tetrachloroethene

1,3-Dichl oropropane

D ibromochl orome than

1,2-Dibromoethane

9-11-92

AFA

WATER
$U g / L$ 
CFA Landfills II and III FY90 - 2nd Quarter July 1991 S\&A Data Document

TABLE

CFA LANDFILLS II AND III FY90 WELLS - 524.2 VOLATILE ORGANIC DATA (Continued)

Page 4 of 4

AREA

LOCATION

TYPE OF LOCATION

SAMPLE NUMBER

MEDIA

UNITS

SDG NUMBER

Chlorobenzene

1,1,1,2-Tetrachloroethane

Ethylbenzene

Xylene (total meta \& para)

xylene (ortho)

\section{Styrene}

sopropylbenzene

$1,1,2,2$-Tetrachloroethane Bromobenzene

1,2,3-Trichloropropane

$\begin{array}{ll}n \text { n-Propylbenzene } \\ \text { in } & \text { 2-chlorotoluene } \\ \infty & \text { 4-Chlorotoluene }\end{array}$

ì 1,3,5-Trimethyl

tert-Butylbenzene

1,2,4-Trimethylbenzene

sec-Butylbenzene

1,3-Dichlorobenzene

1,4-Dichlorobenzene

n-Butylbenzene

1,2-Dichloroberizene

1,2-Dibromo-3-chloropropane

1,2,4-Trichl orobenzene

Hexach l or obut adi ene

\section{Naphthal ene}

1,2,3-Trichlorobenzene

Dilution Factor

Total (Allowed) Hold Time

9-11-92
CFA
$03-L F 3-11$

UPGRADIENT WELL

CFA04902A

GROUND WATER

CFA04701A

- -

$\cdots$

$\cdots$

…

$\cdots$

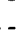

$\cdots$

.

$1 \mathrm{R}$

...

...

1.000

$11(14) d$

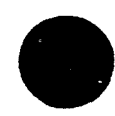


CFA Landfills II and III FY90 - 2nd Quarter July 1991 S\&A Data Document

IABLE _-_- CFA LANDFILLS II AND 111 FY90 WELLS - 524.2 VOLATILE ORGANIC FIELD BLANK DATA

\begin{tabular}{l} 
AREA \\
LOCATION \\
IYPE OF LOCATION \\
SAMPLE NUMBER \\
MEDIA \\
UNIIS \\
SDG NUMBER \\
\hline
\end{tabular}

\section{TARGET COMPOUNDS}

Dichlorodifluorome thane

Chl oromethane

Vinyl Chlorid

Bromome thane

\section{1,1-Dichloroethene}

Methylene Chloride

I-Dich Dichloroethene

1. 1 -Dichloroethane

cis-1,2-Dichloroethen

Chloroform

Bromochloromethane
1,1,1-Trichloroethane

Carbon Tetrachloride

1,1-Dichloropropene

Benzene

1,2-Dichloroethane

Trichloroethene

1,2-Dichloropropane

\section{Bromodichlorome thane}

Dibromome thane
Trans-1,3-Dichloropropene

Tol wene
cis-1,3-D ichloropropene

1,1,2-Trichloroethane

retrachloroethene

1,3-Dichloropropane

Dibromochlorome thane

1,2-D ibromoethane

9-11-92
CFA

OD-FIELD BLANK

CFA05401A

WATER

Ug/L
CFA04701A

$$
\begin{array}{ll}
\text { NP } \\
1 & \text { UJ } \\
1 & \text { UJ } \\
1 & \text { UJ } \\
1 & \text { UJ } \\
1 & \text { UJ } \\
0.8 & J \\
1 & \text { UJ } \\
1 & \text { UJ } \\
1 & \text { UJ } \\
1 & \text { UJ } \\
43.8 & J \\
1 & \text { UJ } \\
1 & \text { UJ } \\
1 & \text { UJ } \\
1 & \text { UJ } \\
1 & \text { UJ } \\
1 & \text { UJ } \\
1 & \text { UJ } \\
1 & \text { UJ }
\end{array}
$$

$0.5 \mathrm{~J}$

1 UJ

1 UJ

1 UJ

1 UJ

U.

$1 \mathrm{R}$

1 UJ 
CFA Landfills 11 and 111 FY90 - 2nd Quarter July 1991 S\&A Data Document

TABLE CFA LANDFILLS $I 1$ AND III FY90 WELLS - 524.2 VOLATILE ORGANIC FIELD BLANK DATA (Cont inued)

\section{AREA}

LOCATION

TYPE OF LOCATION

SAMPLE NUMBER

MEDIA

SDG NUMBER

Chlorobenzene

1,1,1,2-Tetrachloroethane

Ethylbenzene

xylene (total meta \& para)

xylene (or tho)

\section{Styrene}

Bromoform

Isopropylbenzene

1,1,2,2-Tetrachloroethane

Bromobenzene

1,2,3-Trichloropropane

$\Omega \quad$ 2-Propylbenzene

in 1,3,5-Trimethylbenzene

\section{tert-Butyibenzene}

1,2,4-Trimethylbenzene

sec-Butylbenzene

1,3-Dichlorobenzene

1,4-Dichlorobenzene

\section{n-Butylbenzene}

1,2-Dichlorobenzene

1,2-Dibromo-3-chloropropane

1,2,4-Trichlorobenzene

Hexach lor obutadiene

Naphthal ene
1,2,3-Trichlorobenzene

Dilution Factor

Total (Allowed) Hold Time

9-11-92
CFA

OO-FIELD BLANK

CFA05 $401 A$

WATER

Ug/L
A

1 us

1 UJ

$\begin{array}{lll}1 & \mathrm{UJ} \\ 0.5 & \mathrm{UJ}\end{array}$

0.5 UJ

1 Us

1 UJ

1 UJ

1 UJ

$\begin{array}{lll}1 & \text { UJ } \\ 1 & \text { UJ }\end{array}$

$\begin{array}{lll}1 & \text { UJ } \\ 1 & \text { UJ }\end{array}$

1 UJ

$1 \mathrm{UJ}$

1 U.

1 US

$1 \mathrm{UJ}$

$1 \mathrm{uJ}$

1 US

$1 \mathrm{R}$

1 UJ

$1 \mathrm{uJ}$

1 UJ

1.000

$13(14) d$ 
CFA Landfills II and III FY90 - 2nd Quarter July 1991 S\&A Data Document

TABLE _-.- CFA LANDFILLS II AND 11 I FY90 WELLS - 524.2 VOLATILE ORGANIC TRIP BLANK DATA

Page 1 of 1

AREA
LOCATION
TYPE OF LOCATION
SAMPLE NUMBER
MEDIA
UNIIS
SDG NUMBER

\section{TARGET COMPOUNDS}

Dichlorodifluoromethane

Methylene Chloride

1,1,1-Trichloroethane

1,3-Dichloropropane

1,2-Dibromo-3-chloropropane

Dilution Factor

Total (All owed) Hold Time

\begin{tabular}{rrr} 
CFA & CFA & CFA \\
OC & OC & OC \\
00-TRIP BLANK & O0-TRIP BLANK & O0-TRIP BLANK \\
CFA05001A & CFA05101A & CFA05201A \\
WATER & WATER & WAIER \\
Ug/L & Ug/L & Ug/L \\
CFA04701A & CFA04701A & CFA04701A \\
\hline
\end{tabular}

$\begin{array}{rrr}\text { NP } & \text { NP } & \text { NP } \\ 1 & U J & 0.5 \mathrm{~J} \\ 0.1 \mathrm{~J} & 0.6 \mathrm{~J} & -1 \mathrm{R} \\ 1 \mathrm{R} & 1 \mathrm{R} & 1 \mathrm{R} \\ 1 \mathrm{R} & 1 \mathrm{R} & 1.000 \\ 11.000 & 1.000 & 12(14) \mathrm{d}\end{array}$


CFA Landfills 11 and III FY90 Wells - 2nd Quarter July 1991 S\&A Data Document

TARLE _-_- CFA LANDFILLS $\|$ AND $\| 11$ FY9O WELLS - SEMIVOLATILE PRIORITY POLLUANT ORGANIC DATA

Page 1 of 1

\begin{tabular}{l} 
AREA \\
LOCATION \\
TYPE OF LOCATION \\
SAMPLE NUMBER \\
MEDIA \\
UNITS \\
SDG NUMBER \\
\hline
\end{tabular}

\section{FIELD MEASUREMENTS}

PH

Temperature $(C)$

TARGET COMPOUNDS

None Detected

Dilution Factor

Extraction (Allowed) Hold Time Analytical (Allowed) Hold Time

\begin{tabular}{rr} 
CFA & CFA \\
O2-LF2-12 & 03-LF3-9 \\
DOWN GRAD WELL & DOWN GRAD WELL \\
CFA04601D & CFA04701D \\
GROUND WATER & GROUND WATER \\
Ug/L & Ug/L \\
CFA04701D & CFA04701D \\
\hline
\end{tabular}

$\begin{array}{r}\text { CFA } \\ \text { O3-LF3-10 } \\ \text { DOWN GRAD WELLL DOWN } \\ \text { CFAO } 8010 \\ \text { GROUND WATER } \\ \text { Ug/L } \\ \text { CFA047010 } \\ \hline\end{array}$

CFA
03-LF3-10

CFA
03-LF3-10 03-LF3-11

UPGRADIENT WELL UPGRADIENT LE- 11

CFA04802D CFA04901D CFAOLO02D

GROUND WATER

CFA047010

GROUND WAIER

Ug/L

CFA04902D

GROUND WATER

CFA047010

$\begin{array}{ll}7.8 & 7.6 \\ 0.7 & 0.6\end{array}$

0.7
14.6

7.6
0.6
15.7

7.5

0.6
13.5

7.5
0.6

0.6
13.5

$\begin{array}{r}\text { Ug/L } \\ \text { CFA047010 } \\ \hline\end{array}$

9-10-92
1.000
$3(7) d$

$3(7) d$
$24(40) d$
$3(7) d$
$23(40) d$

$24(40) d$
1.000
$3(7) d$

$3(7) d$
$24(40) d$
8.4
0.6
15.2

8.4

0.6
15.2
$3(7) d$
$23(40) d$

1.000
$3(7) d$
$24(40) d$

1.000

$3(7) d$
$24(40) d$ 
CFA Landfills II and III FY90 Wells - 2nd Quarter July 1991 S\&A Data Document

TABLE _-_- CFA LANDFILLS 11 AND 111 FY90 WELLS - SEMIVOLATILE PRIORITY POLLUTANT ORGANIC FIELD BLANK DATA

\begin{tabular}{lr} 
AREA & CFA \\
LOCATION & OC \\
TYPE OF LOCATION & OO-FIELD BLANK \\
SAMPLE NUMBER & CFAO5401D \\
MEDIA & WATER \\
UNITS & Ug/L \\
SDG NUMBER & CFA04701D \\
\hline
\end{tabular}

\section{TARGET COMPOUNDS}

None Detected

Dilution Factor

Extraction (Allowed) Hold Time

$3(7) d$

Analytical (Allowed) Hold Time

9-10-92 
CFA Landfills II and III FY90 Wells - 2nd Quarter July 1991 S\&A Data Document - Method Validation Level A

TABLE _. CFA LANDFILLS II AND III FY9O WELLS - NON-METALS DATA

Page 1 of 3

\begin{tabular}{|c|c|c|c|c|c|}
\hline $\begin{array}{l}\text { AREA } \\
\text { LOCATION } \\
\text { TYPE OF LOCATION } \\
\text { SAMPLE NUMBER } \\
\text { MEDIA } \\
\text { UNITS } \\
\text { SDG NUMBER } \\
\end{array}$ & $\begin{array}{r}\text { CFA } \\
\text { O2-LF2-12 } \\
\text { DOWN GRAD WELL } \\
\text { CFA04601 } \\
\text { GROUND WATER } \\
\text { mg/L } \\
\text { CFA04701 } \\
\end{array}$ & $\begin{array}{r}\text { CFA } \\
03-L F 3-9 \\
\text { DOWN GRAD WELL } \\
\text { CFAO4701 } \\
\text { GROUND WATER } \\
\mathrm{mg} / \mathrm{L} \\
\text { CFA04701 } \\
\end{array}$ & $\begin{array}{r}\text { CFA } \\
\text { O3-LF3-10 } \\
\text { DOWN GRAD WELL } \\
\text { CFA04801 } \\
\text { GROUND WATER } \\
\text { mg/ll } \\
\text { CFA04701 } \\
\end{array}$ & $\begin{array}{r}\text { CFA } \\
\text { O3-LF3-10 } \\
\text { DOWN GRAD WELL } \\
\text { CFA04802 } \\
\text { GROUND HATER } \\
\mathrm{mg} / \mathrm{L} \\
\text { CFA04701 } \\
\end{array}$ & $\begin{array}{r}\text { CFA } \\
\text { O3-LF3-11 } \\
\text { UPGRADIENT WELL } \\
\text { CFAD4901 } \\
\text { GROUND WATER } \\
\mathrm{mg} / L \\
\text { CFA04701 } \\
\end{array}$ \\
\hline $\begin{array}{l}\text { FIELD MEASUREMENTS } \\
\mathrm{pH} \\
\text { Conductivity (uS) } \\
\text { Temperature (C) }\end{array}$ & $\begin{array}{r}7.8 \\
0.7 \\
14.6\end{array}$ & $\begin{array}{r}7.6 \\
0.6 \\
15.7\end{array}$ & $\begin{array}{r}7.5 \\
0.6 \\
13.5\end{array}$ & $\begin{array}{r}7.5 \\
0.6 \\
13.5\end{array}$ & $\begin{array}{r}8.4 \\
0.6 \\
15.2\end{array}$ \\
\hline $\begin{array}{l}\text { ANALYTES } \\
\text { Chloride } \\
\text { Fluoride } \\
\text { Nitrate } \\
\text { Sulfate } \\
\text { TOC }\end{array}$ & $\begin{array}{r}93.8 \\
0.37 \\
9.0 \\
43.0 \\
-.-\end{array}$ & $\begin{array}{r}75.0 \\
0.37 \\
9.0 \\
38.0 \\
-. .\end{array}$ & $\begin{array}{r}102 \\
0.39 \\
6.7 \\
45.2 \\
\cdots . .\end{array}$ & $\begin{array}{r}83.4 \\
0.36 \\
8.9 \\
42.8 \\
-.-\end{array}$ & $\begin{array}{r}69.5 \\
0.47 \\
4.6 \\
36.0\end{array}$ \\
\hline Tox & $70.0 \mathrm{~J}$ & $50.0 \mathrm{~J}$ & 53.0 & 85.0 & $25.0 \mathrm{~J}$ \\
\hline $\begin{array}{l}\text { Total (Allowed) Hold Time } \\
\text { Total (Allowed) Hold Time } \\
\text { Total (Allowed) Hold Time } \\
\text { Total (Allowed) Hold Timed } \\
\text { Total (Allowed) Hold Time } \\
\text { Total (Allowed) Hold Time }\end{array}$ & $\begin{array}{l}12(28) d \\
14(28) d \\
8(28) d \\
9(28) d \\
9(28) d \\
8(7) d^{\star}\end{array}$ & $\begin{array}{l}14(28) d \\
16(28) d \\
11(28) d \\
11(28) d \\
11(28) d \\
9(7) d^{\star}\end{array}$ & $\begin{array}{l}11(28) d \\
13(28) d \\
7(28) d \\
8(28) d \\
8(28) d \\
7(7) d\end{array}$ & $\begin{array}{l}11(28) d \\
13(28) d \\
7(28) d \\
8(28) d \\
8(28) d \\
7(7) d\end{array}$ & $\begin{array}{l}13(28) d \\
15(28) d \\
10(28) d \\
10(28) d \\
10(28) d \\
8(7) d^{\star}\end{array}$ \\
\hline
\end{tabular}

a. Chloride - Method 9252

b. Fluoride - Method 340.2

c. Nitrate - Method 353.3
d. Sulfate - Method 375.4

e. TOC - Method 9060

f. TOX - Method 9020

$9-22-92$ 


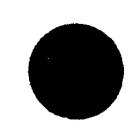

TABLE _-_- CFA LANDFILLS II AND III FYgO WELLS - NON-METALS DATA (Cont inued)

Page 2 of 3

\begin{tabular}{l} 
AREA \\
LOCAIION \\
IYPE OF LOCATION \\
SAMPLE NUMBER \\
MEDIA \\
UNITS \\
SDG NUMBER \\
\hline
\end{tabular}

FIELD MEASUREMENTS

Conductivity (uS)

Temperature (C)

ANALYTES

Chloride

Fluoride

Nitrate

Sulfate

TOC

Total (Allowed) Hold Timea

rotal (Allowed) Hold rime

Total (Allowed) Hold Time

rotal (Allowed) Hold Time

Total (Allowed) Hold Time

rotal (Allowed) Hold Time
a. Chloride - Method 9252
b. Fluoride - Method 340.2
c. Nitrate - Method 353.3
d. Sulfate - Method 375.4
e. TOC - Method 9060
f. Tox - Method 9020
9-22-92

\begin{tabular}{|c|c|c|c|c|}
\hline $\begin{array}{r}\text { CFA } \\
\text { 03-LF3-11 } \\
\text { UPGRADIENT WELL } \\
\text { CFAO4902 } \\
\text { GROUND WATER } \\
\mathrm{mg} / \mathrm{L} \\
\text { CFA04701 } \\
\end{array}$ & $\begin{array}{r}\text { CFA } \\
\text { 03-LF3-11 } \\
\text { UPGRADIENT WELL } \\
\text { CFA04903 } \\
\text { GROUND WATER } \\
\mathrm{mg} / \mathrm{L} \\
\text { CFAO4701 } \\
\end{array}$ & $\begin{array}{r}\text { CFA } \\
\text { O3-LF3-11 } \\
\text { UPGRADIENT WELL } \\
\text { CFAO4904 } \\
\text { GROUND WATER } \\
\mathrm{mg} / \mathrm{L} \\
\text { CFAO4701 } \\
\end{array}$ & $\begin{array}{r}\text { CFA } \\
\text { O3-LF3-11 } \\
\text { UPGRADIENT WELL } \\
\text { CFAO4905 } \\
\text { GROUND WATER } \\
\text { mg/l } \\
\text { CFA04701 } \\
\end{array}$ & $\begin{array}{r}\text { CFA } \\
\text { 03-LF3-11 } \\
\text { UPGRADIENT WELL } \\
\text { CFA04906 } \\
\text { GROUND WATER } \\
\text { mg/L } \\
\text { CFA04701 } \\
\end{array}$ \\
\hline $\begin{array}{r}8.4 \\
0.6 \\
15.2\end{array}$ & $\begin{array}{r}8.4 \\
0.6 \\
15.2\end{array}$ & $\begin{array}{r}8.4 \\
0.6 \\
15.2\end{array}$ & $\begin{array}{r}8.4 \\
0.6 \\
15.2\end{array}$ & $\begin{array}{r}8.4 \\
0.6 \\
15.2\end{array}$ \\
\hline $\begin{array}{r}59.0 \\
0.36 \\
6.4 \\
40.0\end{array}$ & & & & \\
\hline $7.0 \mathrm{~J}$ & $19.0 \mathrm{~J}$ & $26.0 \mathrm{~J}$ & $20.0 \mathrm{~J}$ & $296 \mathrm{~J}$ \\
\hline $\begin{array}{l}13(28) d \\
15(28) d \\
10(28) d \\
10(28) d \\
10(28) d \\
8(7) d^{\star}\end{array}$ & $\begin{array}{c}10(28) d \\
8(7) d^{\star}\end{array}$ & $\begin{array}{c}10(28) d \\
8(7) d^{\star}\end{array}$ & $\begin{array}{l}10(28) d \\
8(7) d^{\star}\end{array}$ & $\begin{array}{c}10(28) d \\
8(7) d^{\star}\end{array}$ \\
\hline
\end{tabular}


CFA Landfills II and III fY90 Wells - 2nd Quarter July 1991 S\&A Data Document - Method Validation Level A

TABLE _-_- CFA LANDFILLS II AND 111 FY90 WELLS - NON-METALS DATA (Cont inued)

Page 3 of 3

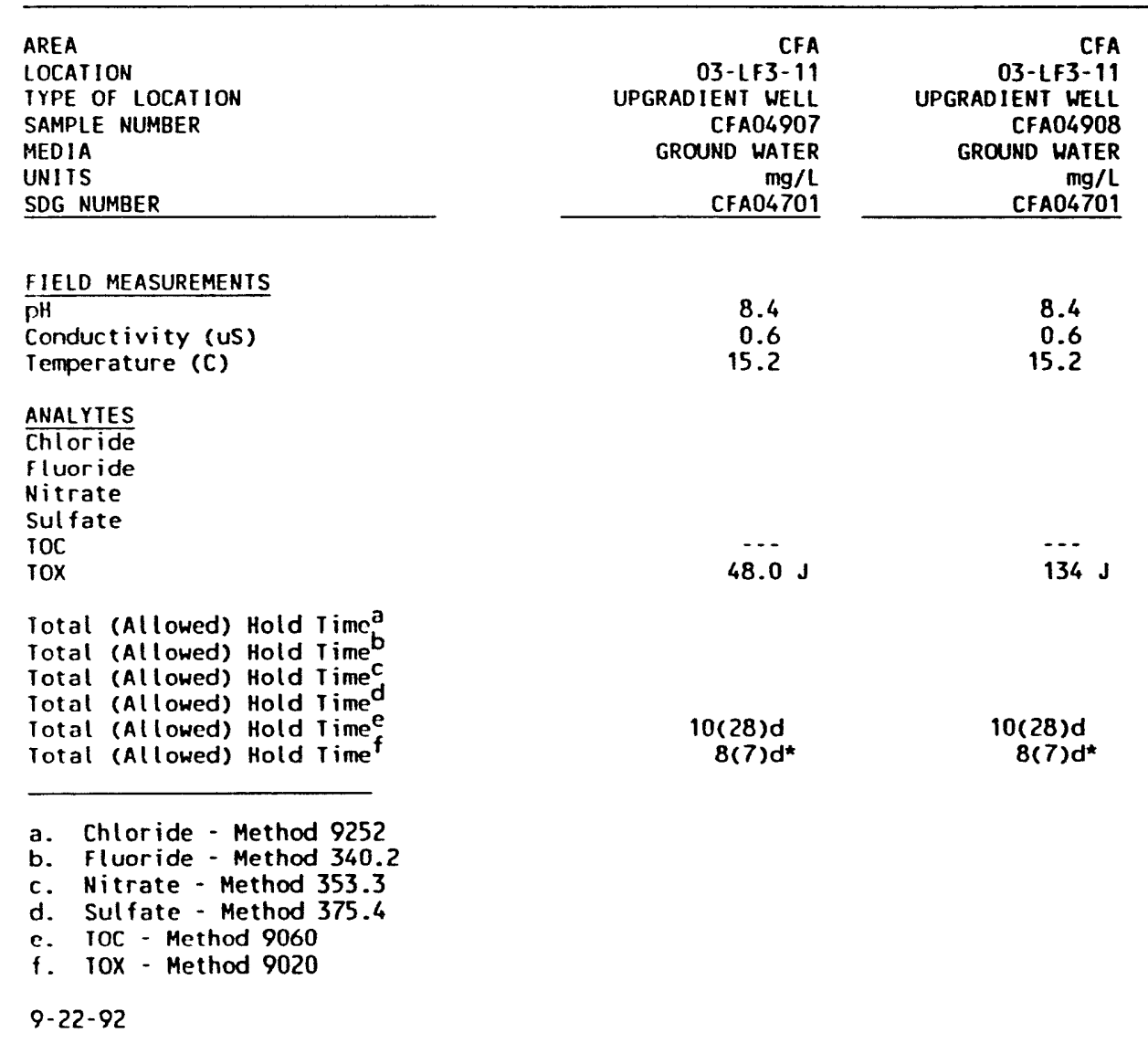


CFA Landfills II and III FY90 Wells - 2nd Quarter July 1991 S\&A Data Document - Method Validation Level A

TABLE -. CFA LANDFILLS II AND 11 FY90 WELLS - NON-METALS FIELD BLANK DATA

AREA

LOCATION

TYPE OF LOCATION

SAMPLE NUMBER

MEDIA

SDG NUMBER

\section{ANALYTES}

Chloride

Fluoride

Nitrate

TOC

Tox

Total (Allowed) Hold Timea

Total (Allowed) Hold Time

Total (Allowed) Hold Time

rotal (Allowed) Hold rime

rotal (Allowed) Hold rime

a. Chloride - Method 9252

a. Chloride - Method 9252

b. Fluoride - Method 340.2

c. Nitrate - Method 353.3

d. SuC - Method 9060

e. TOC - Method 9060
f. TOX - Method 9020

$9-22-92$
00-FIELD BLANK

CFA05401

HATER

WATER

$\mathrm{mg} / \mathrm{L}$
CFA04701

1.7
0.29

0.29

1.1
-1.00

47.0

47.0

$12(28) d$
$14(28) d$

$14(28) d$
$8(28) d$

$8(28) d$
$9(28) d$

$9(28) d$

$9(28) d$
$8(7) d^{\star}$ 
CFA Landfills $1 /$ and III FY90 Wells - 2nd Quarter July 1991 S\&A Data Document

TABLE --:- CFA LANDFILLS II AND III FY90 WELLS - ORGANOCHLORINE PESTICIDE APPENDIX IX DATA

Page 1 of 1

\begin{tabular}{|c|c|c|c|c|c|c|}
\hline $\begin{array}{l}\text { AREA } \\
\text { LOCATION } \\
\text { TYPE OF LOCATION } \\
\text { SAMPLE NUMBER } \\
\text { MEDIA } \\
\text { UNITS } \\
\text { SDG NUMBER } \\
\end{array}$ & $\begin{array}{r}\text { CFA } \\
\text { O2-LF2-12 } \\
\text { DOWN GRAD WELL } \\
\text { CFA04601G } \\
\text { GROUND WATER } \\
\text { Ug/L } \\
\text { CFA04701G } \\
\end{array}$ & $\begin{array}{r}\text { CFA } \\
\text { O3-LF3-9 } \\
\text { DOWN GRAD WELL } \\
\text { CFA04701G } \\
\text { GROUND HATER } \\
\text { Ug/L } \\
\text { CFA04701G } \\
\end{array}$ & $\begin{array}{r}\text { CFA } \\
\text { 03-LF3-10 } \\
\text { DOWN GRAD WELL } \\
\text { CFA04801G } \\
\text { GROUND HATER } \\
\text { Ug/L } \\
\text { CFA04701G } \\
\end{array}$ & $\begin{array}{r}\text { CFA } \\
\text { O3-LF3-10 } \\
\text { DOWN GRAD HELL } \\
\text { CFA04802G } \\
\text { GROUND HATER } \\
\text { Ug/L } \\
\text { CFA04701G } \\
\end{array}$ & $\begin{array}{r}\text { CFA } \\
\text { 03-LF3-11 } \\
\text { UPGRADIENT WELL } \\
\text { CFA04901G } \\
\text { GROUND WATER } \\
\text { Ug } / \mathrm{L} \\
\text { CFA04701G } \\
\end{array}$ & $\begin{array}{r}\text { CFA } \\
\text { 03-LF3-11 } \\
\text { UPGRADIENT WELL } \\
\text { CFA04902G } \\
\text { GROUND WATER } \\
\text { Ug/L } \\
\text { CFA.04701G } \\
\end{array}$ \\
\hline \multicolumn{7}{|l|}{ FIELD MEASUREMENTS } \\
\hline $\begin{array}{l}\text { pH } \\
\text { Conductivity (uS) } \\
\text { Temperature (C) }\end{array}$ & $\begin{array}{r}7.8 \\
0.7 \\
14.6\end{array}$ & $\begin{array}{r}7.6 \\
0.6 \\
15.7\end{array}$ & $\begin{array}{r}7.5 \\
0.6 \\
13.5\end{array}$ & $\begin{array}{r}7.5 \\
0.6 \\
13.5\end{array}$ & $\begin{array}{r}8.4 \\
0.6 \\
15.2\end{array}$ & $\begin{array}{r}8.4 \\
0.6 \\
15.2\end{array}$ \\
\hline \multicolumn{7}{|l|}{ IARGET COMPOUNDS } \\
\hline Mone Detected & & & & & & \\
\hline $\begin{array}{l}\text { Dilution Factor } \\
\text { Extraction (Allowed) Hold Time } \\
\text { Analytical (All lowed) Hold Time }\end{array}$ & $\begin{array}{r}1.0 \\
3(7) d \\
10(40) d\end{array}$ & $\begin{array}{r}1.0 \\
3(7) d \\
11(40) d\end{array}$ & $\begin{array}{r}1.0 \\
3(7) \mathrm{d} \\
10(40) \mathrm{d}\end{array}$ & $\begin{array}{r}1.0 \\
3(7) \mathrm{d} \\
10(40) \mathrm{d}\end{array}$ & $\begin{array}{r}1.0 \\
3(7) d \\
11(40) d\end{array}$ & $\begin{array}{r}1.0 \\
3(7) \mathrm{d} \\
11(40) \mathrm{d}\end{array}$ \\
\hline
\end{tabular}

9-12-92 
CFA Landfills II and III FY90 Wells - 2nd Quarter July 1991 S\&A Data Document

TABLE -. CFA LANDFILLS II AND III FY90 WELLS - ORGANOCHLORINE PESTICIDE APPENDIX IX FIELD BLANK DATA

\begin{tabular}{|c|c|}
\hline $\begin{array}{l}\text { AREA } \\
\text { LOCATION } \\
\text { TYPE OF LOCATION } \\
\text { SAMPLE NUMBER } \\
\text { MEDIA } \\
\text { UNITS } \\
\text { SDG NUMBER } \\
\end{array}$ & $\begin{array}{r}\text { CFA } \\
\text { OC } \\
\text { 00-FIELD BLANK } \\
\text { CFA05401G } \\
\text { WATER } \\
\text { Ug/L } \\
\text { CFA04701G } \\
\end{array}$ \\
\hline $\begin{array}{l}\text { FIELD MEASUREMENTS } \\
\text { pH } \\
\text { Conduct ivity (uS) } \\
\text { Temperature (C) }\end{array}$ & $\begin{array}{r}0.0 \\
0 \\
0.0\end{array}$ \\
\hline TARGET COMPOUNDS & \\
\hline None Detected & \\
\hline $\begin{array}{l}\text { Dilution Factor } \\
\text { Extraction (Allowed) Hold time } \\
\text { Analytical (All owed) Hold time }\end{array}$ & $\begin{array}{r}1.0 \\
3(7) d \\
10(40) d\end{array}$ \\
\hline
\end{tabular}

9-12-92

(10

0.0

0
0.0

1.0

10(40) 
CFA Landfills II and III FY90 Wells - 2nd Quarter July 1991 S\&A Data Document

TABLE _-_-_ CFA LANDFILLS II AND III FY9O WELLS - ORGANOCHLORINE HERBICIDE APPENDIX IX DATA

Page 1 of 1

\section{AREA}

TYPE OF LOCATION

SAMPLE NUMBER

MEDIA

SDG NUMBER

FIELD MEASUREMENTS

$\mathrm{pH}$

Temperature (C)

TARGEI COMPOUNDS

2,4-D

Sil vex

Dilution Factor

Extraction (Allowed) Hold Time

Analytical (Allowed) Hold Time

9-11-92
Conductivity (uS)

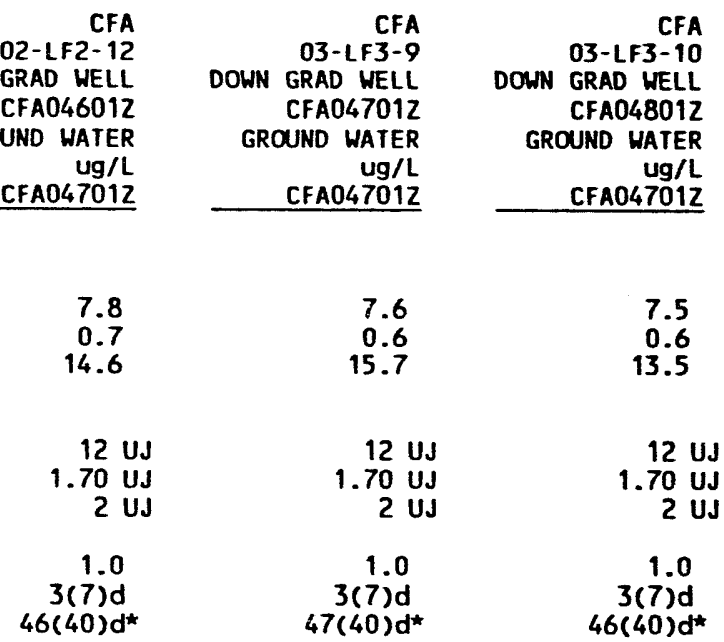

DOWN

CFA
O3-LF3-10
GRAD WELL

CFA048022
GROUND HATER

Ug/L
CFAOG7012

CFA
03-LF3-11

UPGRADIENT HELL

CFA049012

GROUND WATER

Ug/L
CFA047012

CFA
03-LF3-11

UPGRADIENT WELL

GROUND UATER

GROUND WATER

CFA047012

Ug/L
CFA047012

7.5
0.6
13.5

$12 \mathrm{UJ}$
$1.70 \mathrm{UJ}$
$2 \mathrm{UJ}$
1.0
$3(7) \mathrm{d}$
$(40) \mathrm{d}^{*}$

7.5
0.6

\section{4}

0.6
15.2

8.4

0.6
15.2

12 UJ

12 UJ
1.70 UJ
2 UJ

$12 \mathrm{UJ}$

$1.70 \mathrm{UJ}$
$2 \mathrm{UJ}$

1.0
$3(7) d$

$3(7) d$

1.0

$3(7) d$
$47(40) d^{*}$

$47(40) d^{\star}$ 
CFA Landfi!ls 11 and III FY90 Wells - 2nd Quarter July 1991 S\&A Data Document

TABLE -. CFA LANDFILLS II AND 111 FY90 WELLS - ORGANOCHLORINE HERBICIDE APPENDIX IX FIELD BLANK DATA

AREA

LOCATION

TYPE OF LOCATION

SAMPLE NUMBER

MEDIA

SDG NUMBER

TARGET COMPOUNDS

2,4-D

Silvex

$2,4,5-T$

Dilution Factor

Extraction (Allowed) Hold Time

Analytical (Allowed) Hold Time

9-11-92
CFA

DO-FIELD BLANK

CFA054012

WATER

$U 9 / L$
CFA047012

$$
\begin{gathered}
12 \text { UJ } \\
1.70 \text { UJ } \\
2 \text { UJ } \\
1.0 \\
3(7) d \\
47(40) d^{\star}
\end{gathered}
$$


Lab Name: EGGRML

Report No.: STPHY91043
Date: $09 / 20 / 91$

Case No.:

SOG No.: CFAO4601

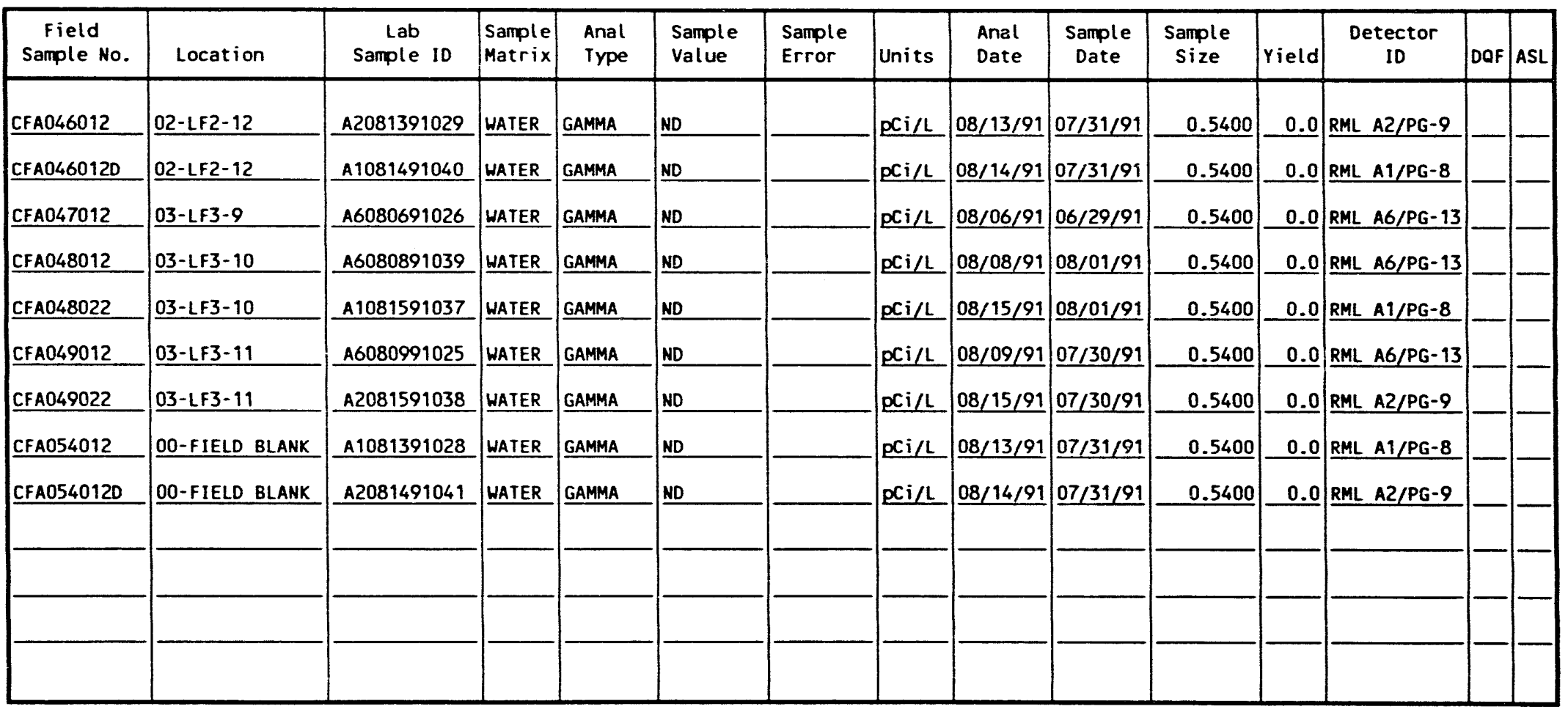

9-18-92 


\section{Appendix D}

End-of-Well Reports for Landfills II and III

Borehole Logs (SAIC) at CFA Landfills II and III With Sample Collection Depths and Soil Descriptions

Borehole Completion Diagrams with Instrument Locations 
EG\&G Idaho, Ine.

FORM EGG-2631"

(Rev. 01-92)

\section{ENGINEERING DESIGN FILE}

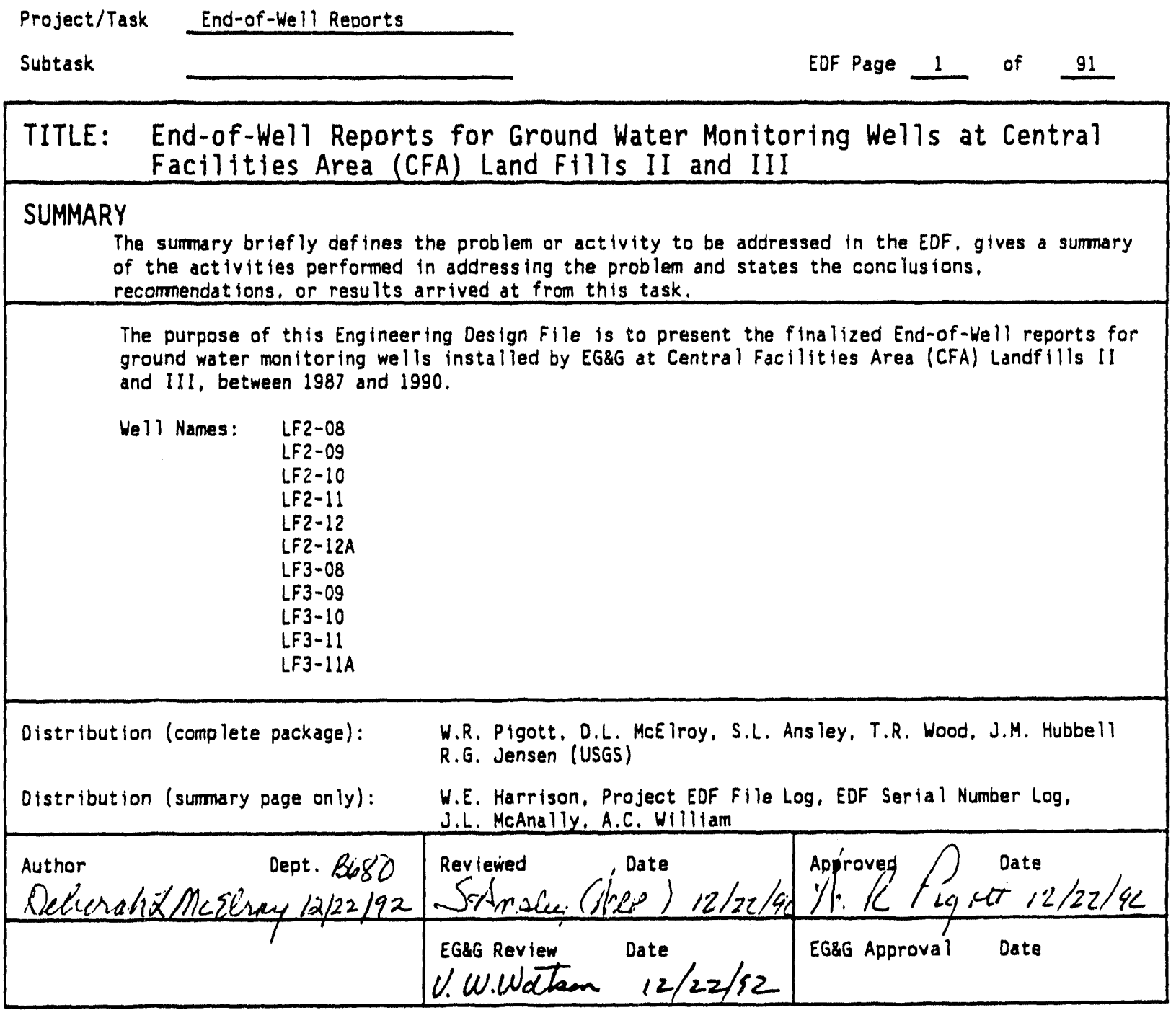


END-OF-WELL REPORT

CENTRAL FACILITIES LANDFILL AREA

WELL LF2-08 
I. GENERAL INFORMATION

A. Project Name: Hydrogeologic Characterization Study for CFA Landfills

B. Well Name/Number: LF2-08

C. Well Location: INEL, Central Facilities Area Landfill II Northing: $682877.71 \mathrm{ft}$ Easting: $294356.74 \mathrm{ft}$ Land Surface Elevation: 4932.88 (bolt in cement pad) NRTS datum

D. Plans:

Survey Date: December 1990, MK-Ferguson

1) Hydrogeologic Characterization Study for CFA Landfills II and III, Idaho National Engineering Laboratory, Technical Work Plan, submitted to EG\&G Idaho, Inc. by Science Applications International Corporation, Apri1 15, 1988.

2) Hydrogeologic Characterization for CFA Landfills II and III, Idaho National Engineering Laboratory, Technical Work Plan, EGG-ER-8670, August 1989 .

3) Statement of Work for Groundwater Monitoring Well Construction at the CFA Landfills II and III, EG\&G Idaho, Inc.

4) Synopsis of Work, Hydrogeologic Characterization Study for CFA Landfills II and III, 31 January 1989 letter report, John Jaacks, Science Applications International Corporation.

5) Hydrogeologic Characterization Study at CFA Landfills II and III Health and Safety Plan, EGG-ER-8445, February 1989.

E. Logbooks used:

1) Science Applications International Corporation Logbooks, submitted to EG\&G Idaho, Inc., $04 / 25 / 88$ to $07 / 13 / 88$.

2) Field Team Leader's Daily Drilling Logbook, ERP Logbook CFA-001.

3) Field Team Leader's Daily Drilling Logbook, ERP Logbook CFA-003.

\section{DRILLING AND COMPLETION OBSERVATIONS}

A. Drilling Company: Denning Well Drilling (drilling)

Drillers: Duane Denning, Jody Denning

On-Site Geologist: John Jaacks, Tom Dube Project Manager: Larry Hull

Rig type: 650 Chicago Pneumatic

Bit type: Tricone bit 
B. Summary of Drilling Activities:

\begin{tabular}{|c|c|c|}
\hline DATE & ACTIVITY & MATERIALS USED \\
\hline $25-A p r-88$ & $\begin{array}{l}\text { Surficial sediments were cased off using } \\
12 \text { l/4-inch ID carbon steel casing (see } \\
\text { caliper log on page } 6 \text { of this section). } \\
\text { The logbooks do not directly state the } \\
\text { type of drilling that was used, but } \\
\text { occasional references to a compressor, } \\
\text { water truck, and foam in the cuttings } \\
\text { suggest the drilling method was air } \\
\text { rotary and that water and/or foam were } \\
\text { added as needed to lubricate the bit or } \\
\text { lift cuttings to the surface. Basalt } \\
\text { was encountered at } 27.5 \mathrm{ft} \text { below land } \\
\text { surface (bls) and the surface casing was } \\
\text { pushed to about } 28.5 \mathrm{ft} \text { bls. }\end{array}$ & $\begin{array}{l}2 \text { bags bentonite, } \\
30 \mathrm{ft} \text { of } 12 \\
1 / 4-\text { inch ID carbon } \\
\text { steel surface } \\
\text { casing }\end{array}$ \\
\hline 26-Apr-88 & $\begin{array}{l}\text { Surficial sediments were cleaned out of } \\
\text { the surface casing, using an air rotary } \\
\text { method with a } 12-\text { inch minimum ID tricone } \\
\text { bit. Drilled to } 76 \mathrm{ft} \text { bls and grouted } \\
\text { up to } 42 \mathrm{ft} \text { bls. }\end{array}$ & \\
\hline $27-A p r-88$ & $\begin{array}{l}\text { Drilled out cement grout and into basalt } \\
\text { to } 110 \mathrm{ft} \text { bls. }\end{array}$ & \\
\hline 28-Apr-88 & Drilled from 110 to $204 \mathrm{ft}$ bls. & \\
\hline 29-Apr-88 & Drilled from 204 to $306 \mathrm{ft}$ bls. & \\
\hline $02-$ May-88 & Drilled from 306 to $406 \mathrm{ft}$ bls. & \\
\hline $03-$ May -88 & $\begin{array}{l}\text { Drilled to } 416 \mathrm{ft} \text { bls and lost } \\
\text { circulation. Grouted the borehole. }\end{array}$ & $2 \mathrm{yd}^{3}$ cement \\
\hline $04-$ May-88 & $\begin{array}{l}\text { Top of cement tagged at } 340 \mathrm{ft} \text { bls. } \\
\text { Drilled through cement and continued } \\
\text { drilling to } 445 \mathrm{ft} \text { bls. }\end{array}$ & \\
\hline $05-$ May -88 & $\begin{array}{l}\text { Drilled from } 445 \mathrm{ft} \text { bls to a total depth } \\
\text { of } 526 \mathrm{ft} \text { bls. }\end{array}$ & \\
\hline $06-$ May -88 & $\begin{array}{l}\text { USGS logged borehole: TV-video, natural } \\
\text { gamma, and caliper logs were conducted. } \\
\text { Water was measured at } 480 \mathrm{ft} \text { bls. }\end{array}$ & \\
\hline
\end{tabular}


C. Summary of Well Construction Procedures:

\begin{tabular}{|c|c|c|}
\hline DATE & ACTIVITY & MATERIALS USED \\
\hline $06-J u 7 y-88$ & $\begin{array}{l}\text { Installed well screen and well casing to } \\
495 \text { ft bls, with } 1.5 \text { ft remaining above } \\
\text { land surface. Descriptions of the } \\
\text { casing and screen are a 'best guess' of } \\
\text { what was placed in LF2-08. Casing and } \\
\text { screen sizes were not recorded in the } \\
\text { logbooks. The casing above ground } \\
\text { surface was field checked and is } \\
\text { accurate. The remainder of the casing } \\
\text { and screen descriptions were found in } \\
\text { well construction plans (see Section } \\
\text { I-D), and construction diagrams (filled } \\
\text { out after actual construction was } \\
\text { finished). } \\
\text { Installed 6-inch ID Type } 304 \text { stainless } \\
\text { steel well screen, wire-wrapped (0.02- } \\
\text { inch slot size) with an endplug, below } \\
\text { the water table. The well screen was } \\
\text { joined to 6-inch nominal pipe size, } \\
\text { Type } 304 \text { stainless steel well casing. } \\
\text { The stainless steel well casing was } \\
\text { joined to 6-inch nominal pipe size } \\
\text { (6.625-inch oD) carbon steel well casing } \\
\text { with a dielectric coupling at } 435 \text { ft } \\
\text { bls. Three centralizers were installed } \\
\text { (depths are not known). }\end{array}$ & $\begin{array}{l}10 \mathrm{ft} \text { of } 6 \text {-inch ID } \\
\text { stainless steel } \\
\text { well screen, } 40 \mathrm{ft} \\
\text { of } 6 \text { inch nominal } \\
\text { pipe size } \\
\text { stainless steel } \\
\text { well casing, } 446.5 \\
\text { ft of } 6 \text { inch } \\
\text { nominal pipe size } \\
\text { carbon steel well } \\
\text { casing }\end{array}$ \\
\hline $\begin{array}{l}11-J u 7 y-88 \\
\text { to } \\
13-J u 7 y-88\end{array}$ & $\begin{array}{l}\text { Installed filter pack consisting of } 1 / 4 \\
\text { inch pea gravel. A bentonite seal was } \\
\text { installed above the filter pack and the } \\
\text { remaining annular space was filled with } \\
\text { expanding cement to the surface. A } \\
\text { tremie pipe was used to install the } \\
\text { filter pack and bentonite and cement } \\
\text { seals. }\end{array}$ & \\
\hline 15-Aug-88 & $\begin{array}{l}\text { Installed surface cement pad. Surface } \\
\text { completion included a locking aluminum } \\
\text { well cover, cement pad, and impingement } \\
\text { posts (impingement posts placed in } \\
\text { November 1989). }\end{array}$ & \\
\hline
\end{tabular}


D. Summary of We11 Development Procedures:

\begin{tabular}{|c|c|c|}
\hline DATE & ACTIVITY & MATERIALS USED \\
\hline 15-Sept-89 & $\begin{array}{l}\text { Hawley Brothers Drilling performed the } \\
\text { well development. A wire brush was } \\
\text { lowered down the borehole to } 440 \mathrm{ft} \text { bls. } \\
\text { The brush was retrieved, and a Grundfos } \\
\text { pump was placed down the well, to } 3.3 \mathrm{ft} \\
\text { off the bottom. The bottom of the well } \\
\text { is } 490 \text { to } 491 \mathrm{ft} \text { bls, } 5 \mathrm{ft} \text { shorter than } \\
\text { SAIC logbooks had recorded. Two pump } \\
\text { and recovery cycles were completed, } \\
\text { pumping for } 1 \text { minute each time, and } \\
\text { purging a total of } 33 \text { gallons of water. }\end{array}$ & \\
\hline 18-Sept-89 & $\begin{array}{l}\text { Four pump and recovery cycles were } \\
\text { performed. Water was pumped for two to } \\
\text { three minute intervals, at } 18 \mathrm{gpm} \text {, and } \\
147 \text { gallons of water was purged. } \\
\text { The pump was retrieved and a surge block } \\
\text { was placed down the well. The we17 was } \\
\text { surged for } 27 \text { minutes at } 477 \text { to } 485 \mathrm{ft} \\
\text { bls. }\end{array}$ & \\
\hline 19-Sept-89 & $\begin{array}{l}\text { The same interval ( } 477 \text { to } 485 \mathrm{ft} \text { bls) } \\
\text { was again surged for } 35 \text { minutes. The } \\
\text { surge block was pulled, and a pump } \\
\text { installed for further well development. } \\
\text { The well was pumped in pump and recovery } \\
\text { intervals at approximately } 18 \mathrm{gpm}, \\
\text { taking parameter measurements } \\
\text { concurrently (pH, temperature, percent } \\
\text { dissolved oxygen, specific conductance). } \\
\text { A total of } 413 \text { gallons was pumped in } \\
\text { this sequence. }\end{array}$ & \\
\hline 1989 & $\begin{array}{l}\text { Hydrostar piston pump was placed down } \\
\text { the well. The planned intake depth was } \\
486 \mathrm{ft} \text { bls (actual intake depth not } \\
\text { recorded in logbooks). }\end{array}$ & \\
\hline
\end{tabular}

\section{GEOLOGICAL OBSERVATIONS}

A. Geological log, geophysical logs, stratigraphic column, and well construction diagram are attached.

B. Samples collected. No samples were collected. 


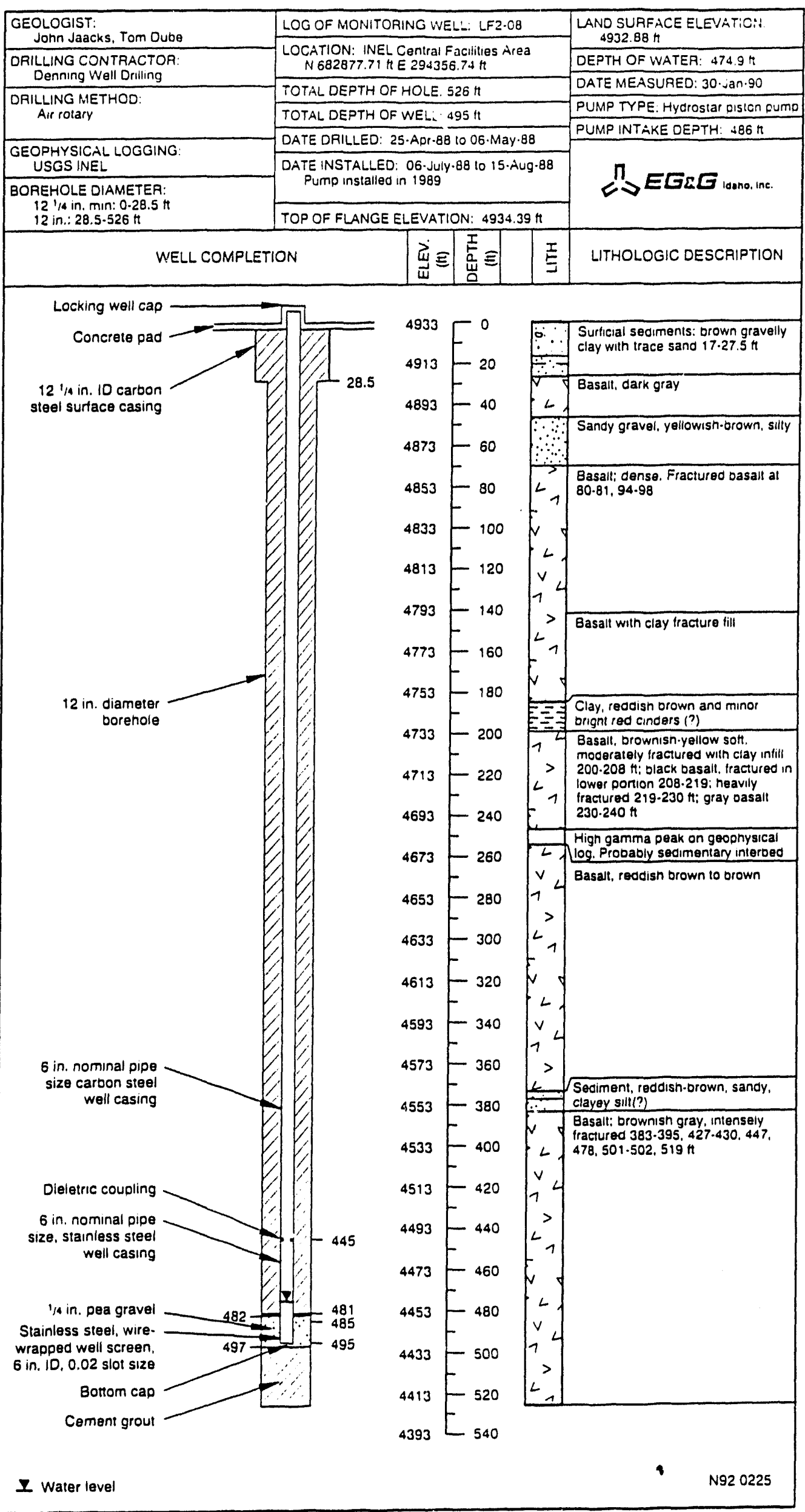



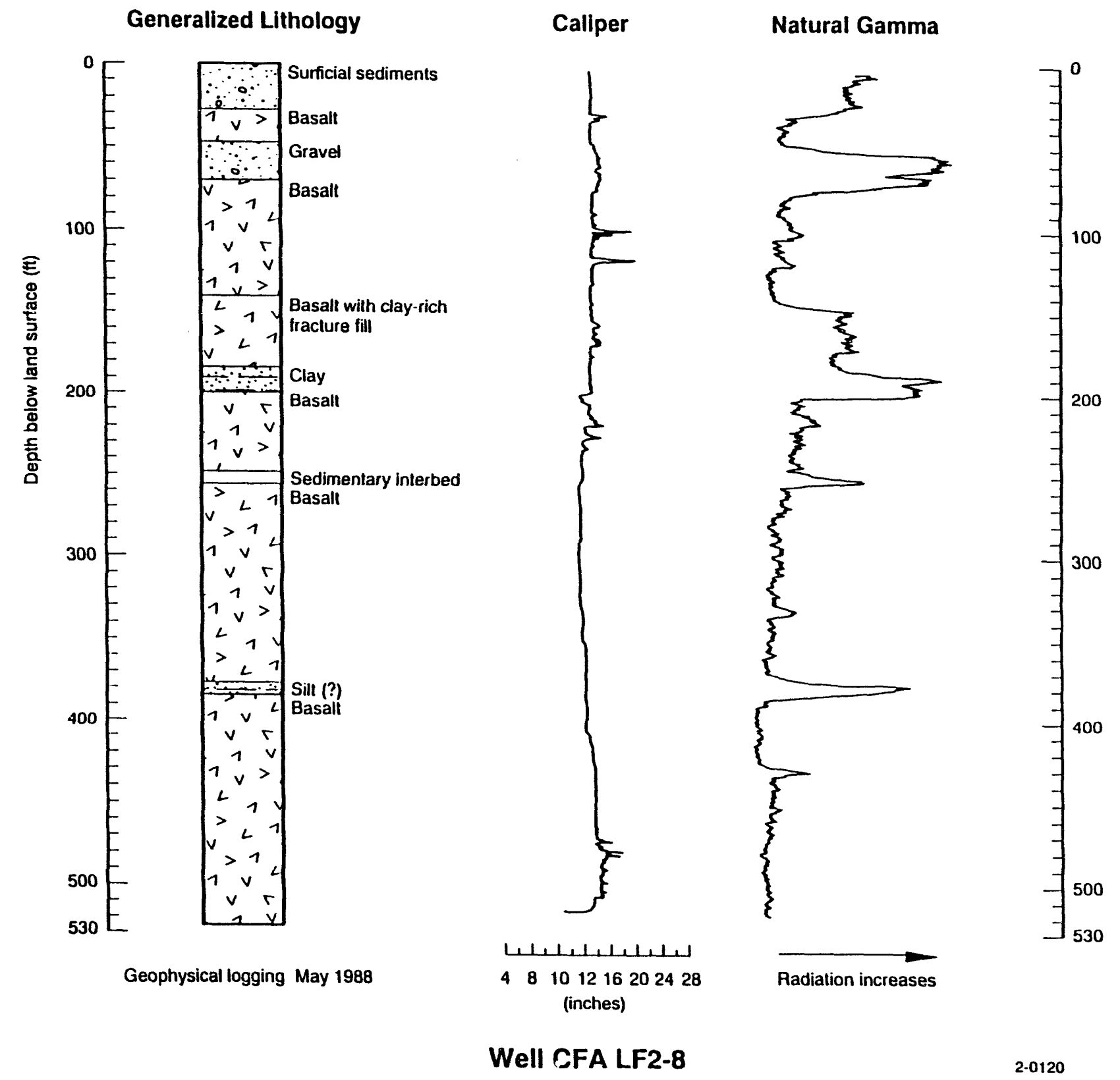

Well CFA LF2-8 
END-OF-WELL REPORT

CENTRAL FACILITIES LANDFILL AREA

WELL LF2-09 
I. GENERAL INFORMATION

A. Project Name: Hydrogeologic Characterization Study for CFA Landfills

B. We11 Name/Number: LF2-09

C. Well Location: INEL, Central Facilities Area Landfill II Northing: $682898.62 \mathrm{ft}$ Easting: $294194.66 \mathrm{ft}$ Land Surface Elevation: $4933.37 \mathrm{ft}$ (brass marker) NRTS datum

D. Plans: Survey Date: December 1990, MK-Ferguson

1) Hydrogeologic Characterization Study For CFA Landfills II and III, Idaho National Engineering Laboratory Technical Work Plan, submitted to EG\&G Idaho, Inc. by Science Applications International Corporation, April 15, 1988.

2) Statement of Work for Groundwater Monitoring Well Construction at the CFA Landfills II and III, EG\&G Idaho, Inc.

3) Synopsis of Work, Hydrogeologic Characterization Study for CFA Landfills II and III, 31 January 1989 letter report, John Jaacks, Science Applications International Corporation.

4) Hydrogeologic Characterization Study for CFA Landfills II and III, Idaho National Engineering Laboratory, Technical Workplan, EGG-ER-8670, August 1989.

5) Hydrogeologic Characterization Study at CFA Landfills II and III, Health and Safety Plan, EGG-ER-8445, February 1989.

E. Logbooks used:

1) Well Drilling, Installation, and Development Logbook, No. CFA-2, EG\&G Idaho, Inc.

2) Science Applications International Corporation Logbooks, submitted to EG\&G Idaho, Inc., 04/18/88 through $11 / 08 / 88$.

3) Field Team Leader's Daily Logbook, ERP Logbook No. CFA-001.

\section{DRILLING AND COMPLETION OBSERVATIONS}

A. Drilling Company: Denning Well Drilling Drillers: D. Denning

On-Site Geologist: J. Jaacks/S. Ansley

Project Manager: L.C. Hul1/M.H. Doornbos

Rig type: 670 Chicago Pneumatic

Bit type: Tricone bit 
B. Summary of Drilling Activities:

\begin{tabular}{|c|c|c|}
\hline DATE & ACTIVITY & MATERIALS USED \\
\hline 18-Apri1-88 & $\begin{array}{l}\text { Set up at drill site. Drove surface } \\
\text { casing to basalt ( } 32 \mathrm{ft} \text { bls) and } \\
\text { bentonite was placed in annular space. } \\
\text { Cleaned sediments out of casing. } \\
\text { Drilled into basalt with } 12-1 / 4 \text { inch } \\
\text { tricone bit, using air rotary. Foam was } \\
\text { used occasionally, when circulation } \\
\text { returns were poor. }\end{array}$ & $\begin{array}{l}34 \mathrm{ft} \text { of } 12-1 / 4 \text { in } \\
\text { ID carbon steel } \\
\text { surface casing; } 2 \\
\text { bags bentonite, } \\
\text { foam }\end{array}$ \\
\hline 19-Apri1-88 & $\begin{array}{l}\text { Drilled to } 130 \mathrm{ft} \text { bls. Hole caving } \\
\text { occurred at approximately } 49-60 \mathrm{ft} \text { bls. } \\
\text { Water used down hole to lubricate bit. }\end{array}$ & \\
\hline 20-Apri1-88 & $\begin{array}{l}\text { Because of caving problems, the hole was } \\
\text { backfilled with sand from } 130 \text { to } 76 \mathrm{ft} \\
\text { bls and filled with cement from } 76 \text { to } 25 \\
\mathrm{ft} \text { bls. }\end{array}$ & $\begin{array}{l}2 y d^{3} \text { sand; } 2 y^{3} \\
\text { Port } 1 \text { and Type I } \\
\text { cement with sand } \\
\text { and } 5 \% \text { bentonite }\end{array}$ \\
\hline $21-A p r i 1-88$ & $\begin{array}{l}\text { Cement and backfill sand drilled out. } \\
\text { Drilled into basalt to } 190 \mathrm{ft} \text { bls. }\end{array}$ & \\
\hline 22-Apri1-88 & Drilled from 180 to $310 \mathrm{ft}$ bls. & \\
\hline 25-Apri1-88 & $\begin{array}{l}\text { Drilled from } 310 \text { to } 410 \mathrm{ft} \text { bls. } \\
\text { Borehole caving from sedimentary layer } \\
\text { encountered at approximately } 377 \text { to } \\
385 \mathrm{ft} \text { bls. }\end{array}$ & \\
\hline 26-Apri1-88 & $\begin{array}{l}\text { Hole had } 20 \mathrm{ft} \text { of cave-in. Drilled and } \\
\text { cleaned out borehole, and poured in } \\
\text { cement to } 345 \mathrm{ft} \text { bls to seal off caving } \\
\text { layer. }\end{array}$ & $\begin{array}{l}2 \mathrm{yd}^{3} \text { expansive } \\
\text { cement with sand } \\
\text { and } 5 \% \text { bentonite }\end{array}$ \\
\hline 27-Apri1-88 & $\begin{array}{l}\text { Drilled out cement ( } 345 \text { to } 410 \mathrm{ft} \text { bls) } \\
\text { and continued drilling to } 470 \mathrm{ft} \text { bls. }\end{array}$ & \\
\hline 28-Apri1-88 & Drilled from $470 \mathrm{ft}$ to $590 \mathrm{ft}$ bls. & \\
\hline 29-Apri1-88 & $\begin{array}{l}\text { Drilled from } 590 \text { to } 676 \mathrm{ft} \text { bls. } \\
\text { Encountered a clay layer at } 630 \mathrm{ft} \text {, } \\
\text { which was caving in. }\end{array}$ & \\
\hline $02-$ May-88 & $\begin{array}{l}\text { Performed geophysical logging: natural } \\
\text { gamma, neutron, and caliper. }\end{array}$ & \\
\hline $03-$ May -88 & $\begin{array}{l}\text { Completed downhole logging: TV log and } \\
\text { gamma log. Water level at } 483 \mathrm{ft} \text { bls. }\end{array}$ & \\
\hline
\end{tabular}




\begin{tabular}{|c|c|c|}
\hline DATE & ACTIVITY & MATERIALS USED \\
\hline $07-J u T y-88$ & $\begin{array}{l}6 \text {-inch casing and well screen lowered to } \\
613 \mathrm{ft} \text { bls. Borehole caving prevented } \\
\text { lowering the well screen to the desired } \\
632 \text { to } 642 \mathrm{ft} \text { interval. }\end{array}$ & $\begin{array}{l}160 \mathrm{ft} \text { of } 6 \text { inch } \\
\text { ID stainless } \\
\text { steel casing; } 453 \\
\text { ft of } 6 \text { inch ID } \\
\text { carbon steel } \\
\text { casing }\end{array}$ \\
\hline $03-A u g-88$ & $\begin{array}{l}\text { 6-inch casing pulled. Tried to clean } \\
\text { out borehole, using foam, but not } \\
\text { successful. No circulation. }\end{array}$ & 2 barrels of foam \\
\hline 04 -Aug-88 & $\begin{array}{l}\text { Borehoie was cleaned to } 638 \mathrm{ft} \text { bls using } \\
\text { foam. Developed hole for } 7.5 \text { hours to } \\
\text { try and clean out the sand. }\end{array}$ & 6 barrels of foam \\
\hline 05 -Aug-88 & $\begin{array}{l}\text { Sand level at } 590 \mathrm{ft} \text { bls. Used rig } \\
\text { compressors to blow sand out to } 638 \mathrm{ft} \\
\text { bls. Developed hole for } 10 \mathrm{hrs} \text {. }\end{array}$ & 3 buckets of foam \\
\hline 08 -Aug-88 & $\begin{array}{l}\text { Sand level at } 558 \mathrm{ft} \text { bls. Borehole } \\
\text { cleaned to } 630 \mathrm{ft} \text { bls and developed. }\end{array}$ & $\begin{array}{l}6 \text { buckets ( } 5 \mathrm{gal} \\
\text { ea.) of EZ-Foam }\end{array}$ \\
\hline 09 -Aug-88 & $\begin{array}{l}\text { Developed hole for } 3 \text { hrs but sand still } \\
\text { heaving into borehole. }\end{array}$ & EZ-Foam \\
\hline 10 -Aug-88 & $\begin{array}{l}\text { USGS conducted a resistivity log to look } \\
\text { for water-bearing fractures, none found. }\end{array}$ & \\
\hline 11-Aug-88 & $\begin{array}{l}\text { Top of well casing was sealed with a } \\
\text { welded plate and completion of hole was } \\
\text { deferred until } 1989 \text {. }\end{array}$ & \\
\hline
\end{tabular}

C. Summary of Well Construction Procedures:

\begin{tabular}{|c|c|c|}
\hline DATE & ACTIVITY & MATERIALS USED \\
\hline 20-Sept-89 & $\begin{array}{l}\text { Cut top plate of casing. Deconned } \\
\text { tremie pipe, casing, set up rig over } \\
\text { borehole. }\end{array}$ & \\
\hline $21-$ Sept-89 & $\begin{array}{l}\text { Began lowering tremie pipe down the } \\
\text { borehole. Tagged bottom at } 615.05 \mathrm{ft} \\
\text { bls, and found sand in bottom of } \\
\text { borehole. Water level at } 478.9 \mathrm{ft} \text { bls. } \\
\text { Sand plugged up tremie pipe, so water } \\
\text { was run through the pipe in an attempt } \\
\text { to unplug the tremie. Cement grout was } \\
\text { found down the tremie and water was } \\
\text { added because it was so thick. }\end{array}$ & $\begin{array}{l}3 \text { yd }^{3} \text { cement grout } \\
\text { comprising } 45 \% \\
\text { sand, } 50 \% \text { cement, } \\
\text { and } 5 \% \text { bentonite, } \\
60 \text { gallons water } \\
\text { (extra) }\end{array}$ \\
\hline
\end{tabular}




\begin{tabular}{|c|c|c|}
\hline DATE & ACTIVITY & MATERIALS USED \\
\hline 22-Sept-89 & $\begin{array}{l}\text { Tagged top of grout at } 526.25 \mathrm{ft} \text { bls. } \\
\text { Decided to shovel grout down the } \\
\text { borehole to bring the level up to the } \\
\text { completion zone. }\end{array}$ & $\begin{array}{l}.25 \mathrm{yd}^{3} \text { Port land } \\
\text { Type I and II } \\
\text { cement with sand } \\
\text { and bentonite } \\
\text { added }\end{array}$ \\
\hline $25-\operatorname{Sep} t-89$ & $\begin{array}{l}\text { Tagged top of cement at } 512.7 \mathrm{ft} \text { bls. } \\
\text { Poured bentonite pellets down the } \\
\text { tremie, but they bridged. Pulled tremie } \\
\text { to replace with a larger ( } 2.98 \mathrm{NX} \text { ) } \\
\text { tremie. Lost sounder down the borehole. }\end{array}$ & Bentonite pellets \\
\hline 26-Sept-89 & $\begin{array}{l}\text { Added bentonite down the tremie pipe and } \\
\text { flushed with water. Bentonite plugged } \\
\text { tremie pipe; then pulled the tremie out } \\
\text { of the borehole. Put new tremie ( } 1.5 \\
\text { inch) down the borehole. }\end{array}$ & \\
\hline $\begin{array}{l}27-\text { Sept }-89 \\
\text { to } \\
02-\text { Oct }-89\end{array}$ & 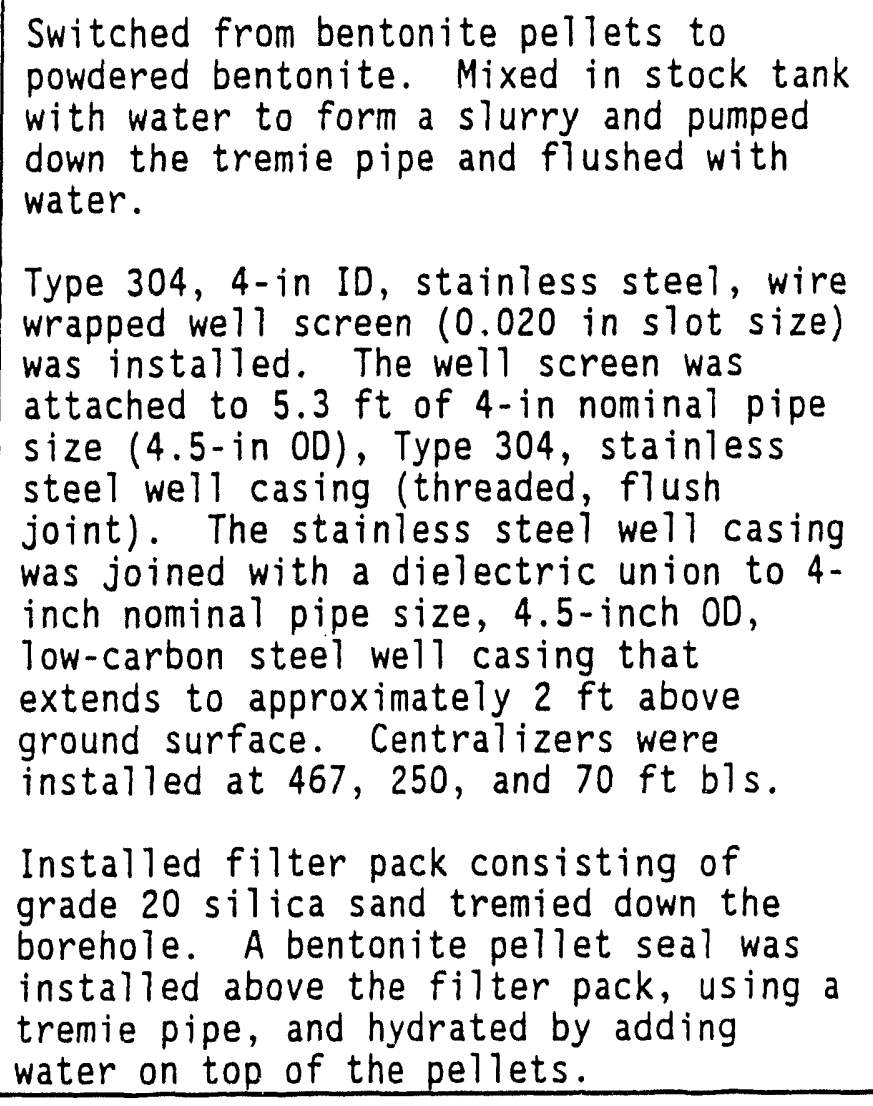 & $\begin{array}{l}55 \text { gallons } \\
\text { bentonite slurry, } \\
70+\text { gallons water, } \\
470 \mathrm{ft} \text { of carbon } \\
\text { steel well casing, } \\
0.5 \mathrm{ft} \text { dielectric } \\
\text { joint, } 5 \mathrm{ft} \\
\text { stainless steel } \\
\text { well casing, } 25 \mathrm{ft} \\
\text { stainless steel } \\
\text { well screen, } 1 \text { end } \\
\text { plug, } 34 \text { (100 } \\
\text { pound) bags grade } \\
20 \text { silica sand, } 5 \\
\text { buckets bentonite } \\
\text { pellets }\end{array}$ \\
\hline $\begin{array}{l}03-0 c t-89 \\
\text { to } \\
09-0 c t-89\end{array}$ & $\begin{array}{l}\text { Grouted the annular space to the surface } \\
\text { using a tremie pipe with } 20 \mathrm{ft} \text { of } \\
\text { annular space. }\end{array}$ & $\begin{array}{l}20.2 \text { yd cement } \\
\text { grout comprising } \\
50 \% \text { Type I and II } \\
\text { cement, } 45 \% \text { sand, } \\
\text { and } 5 \% \text { bentonite }\end{array}$ \\
\hline
\end{tabular}




\begin{tabular}{|c|c|c|}
\hline DATE & ACTIVITY & MATERIALS USED \\
\hline $09-N o v-89$ & $\begin{array}{l}\text { Surface pad and impingement posts } \\
\text { installed. }\end{array}$ & \\
\hline $16-$ Nov- 89 & $\begin{array}{l}\text { Hydrostar piston pump installed, intake } \\
\text { depth set at } 486.5 \mathrm{ft} \text { bls. }\end{array}$ & \\
\hline 1989 & $\begin{array}{l}\text { The } 121 / 4 \text {-inch ID surface casing was } \\
\text { cut off to near ground surface. The } \\
4.5-\text { inch } 00 \text { well casing extends } 2 \mathrm{ft} \\
\text { above ground level and is topped with an } \\
\text { aluminum locking box to protect the } \\
\text { wellhead. }\end{array}$ & \\
\hline
\end{tabular}

C. Summary of Well Development Procedures:

\begin{tabular}{|c|c|c|}
\hline DATE & ACTIVITY & MATERIALS USED \\
\hline $09-0 c t-89$ & $\begin{array}{l}\text { Placed Grundfos pump down the well and } \\
\text { pumped } 1,770 \text { gallons out of the well, } \\
\text { until the water was clear. }\end{array}$ & \\
\hline $10-0 c t-89$ & $\begin{array}{l}\text { Pulled pump and put surge block down the } \\
\text { well. Surged screened interval in } 5 \mathrm{ft} \\
\text { sections, } 10 \text { to } 15 \text { minutes each. Pulled } \\
\text { surge block. }\end{array}$ & \\
\hline $11-0 c t-89$ & $\begin{array}{l}\text { Replaced pump down the we 11, purged for } \\
88 \text { minutes at approximately } 18.5 \mathrm{gpm} \\
\text { until well parameters (temperature, } \mathrm{pH} \text {, } \\
\text { specific conductance, percent dissolved } \\
\text { oxygen) stabilized. See attached well } \\
\text { development data on page } 6 \text { of this } \\
\text { section }\end{array}$ & \\
\hline $12-0 c t-89$ & Pulled the pump. & \\
\hline
\end{tabular}

\section{GEOLOGICAL OBSERVATIONS} A. Geological log, geophysical logs, stratigraphic column, and well
construction diagram are attached.

B. Samples collected. No samples were collected. 


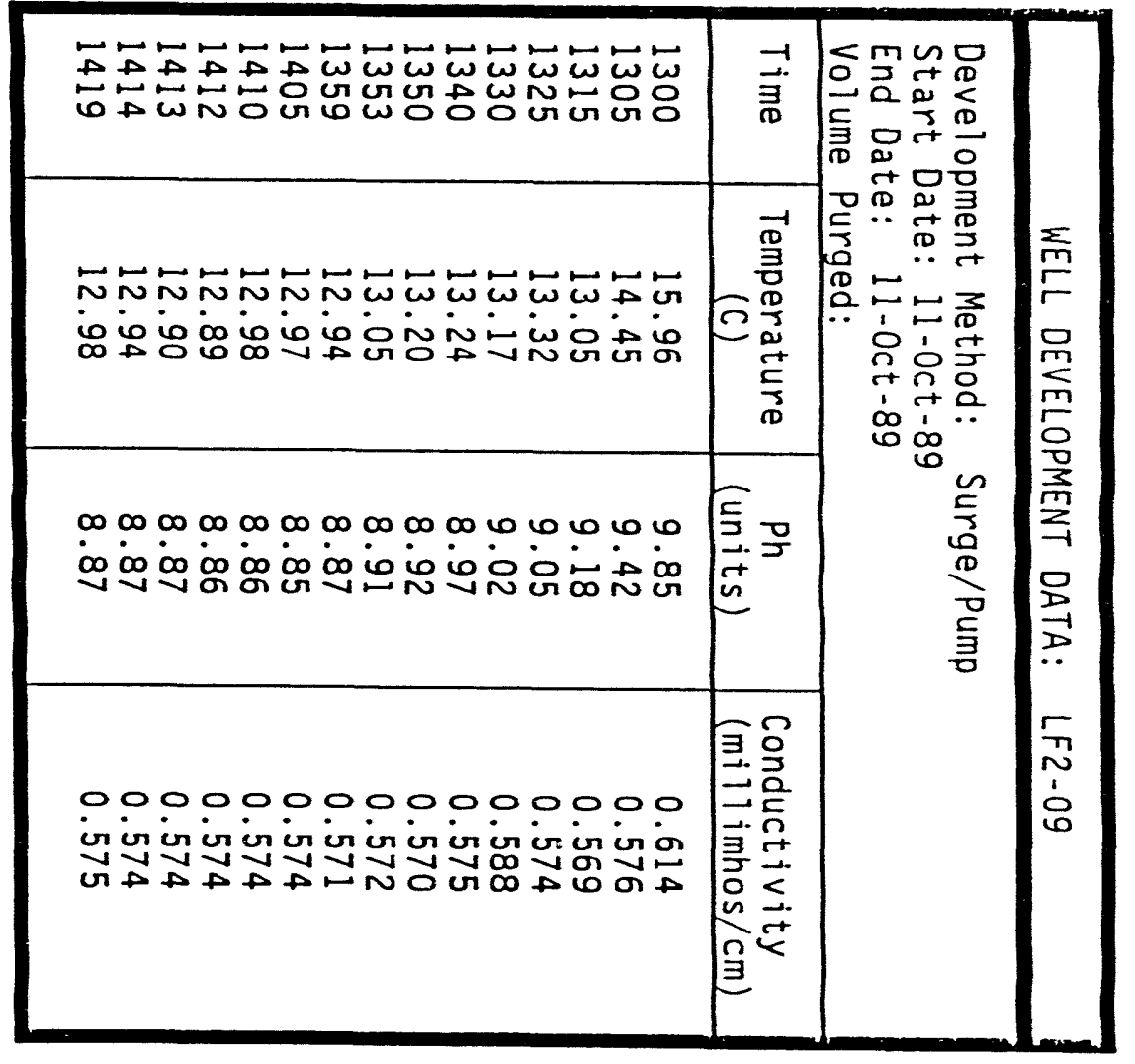




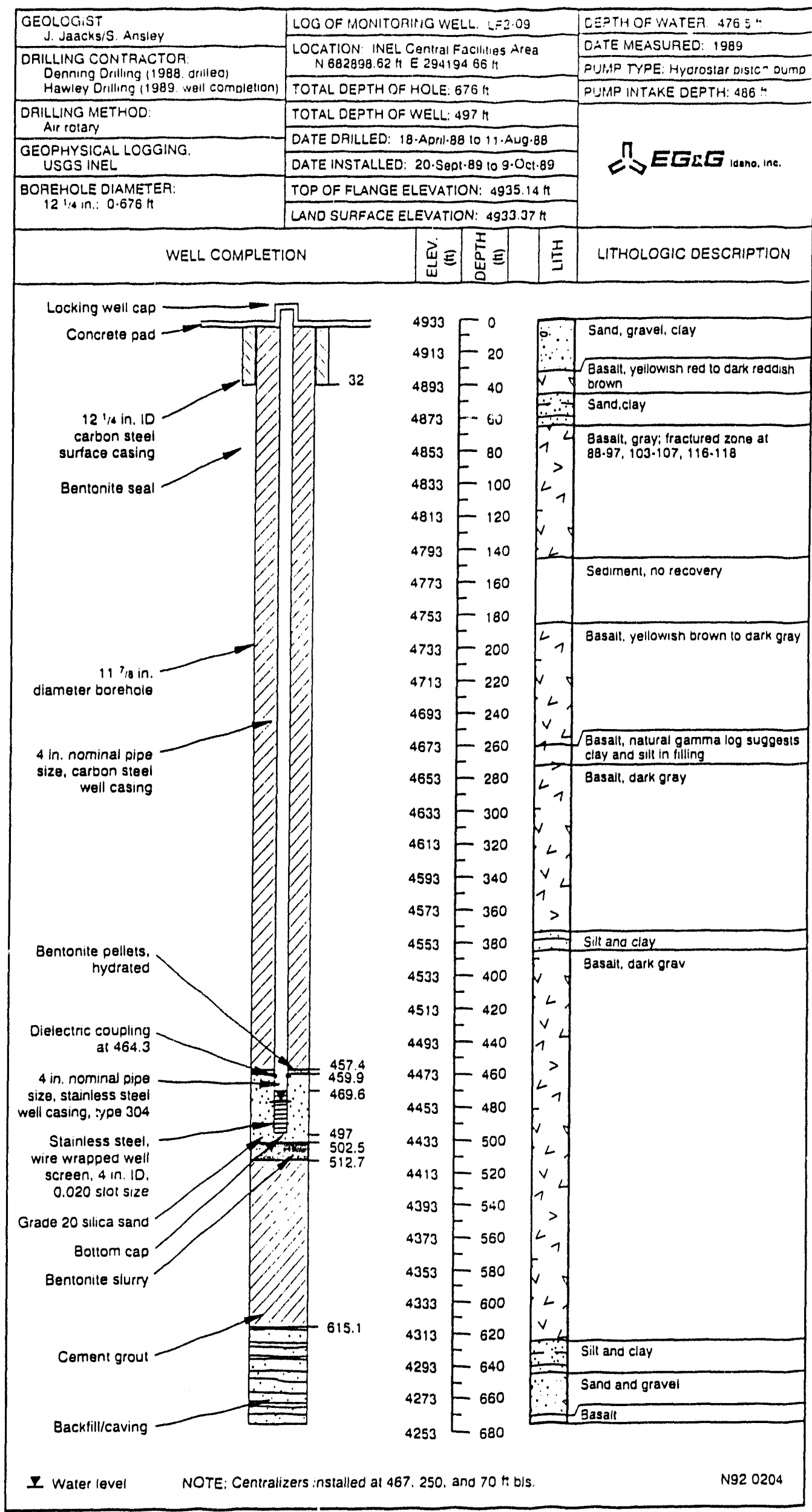



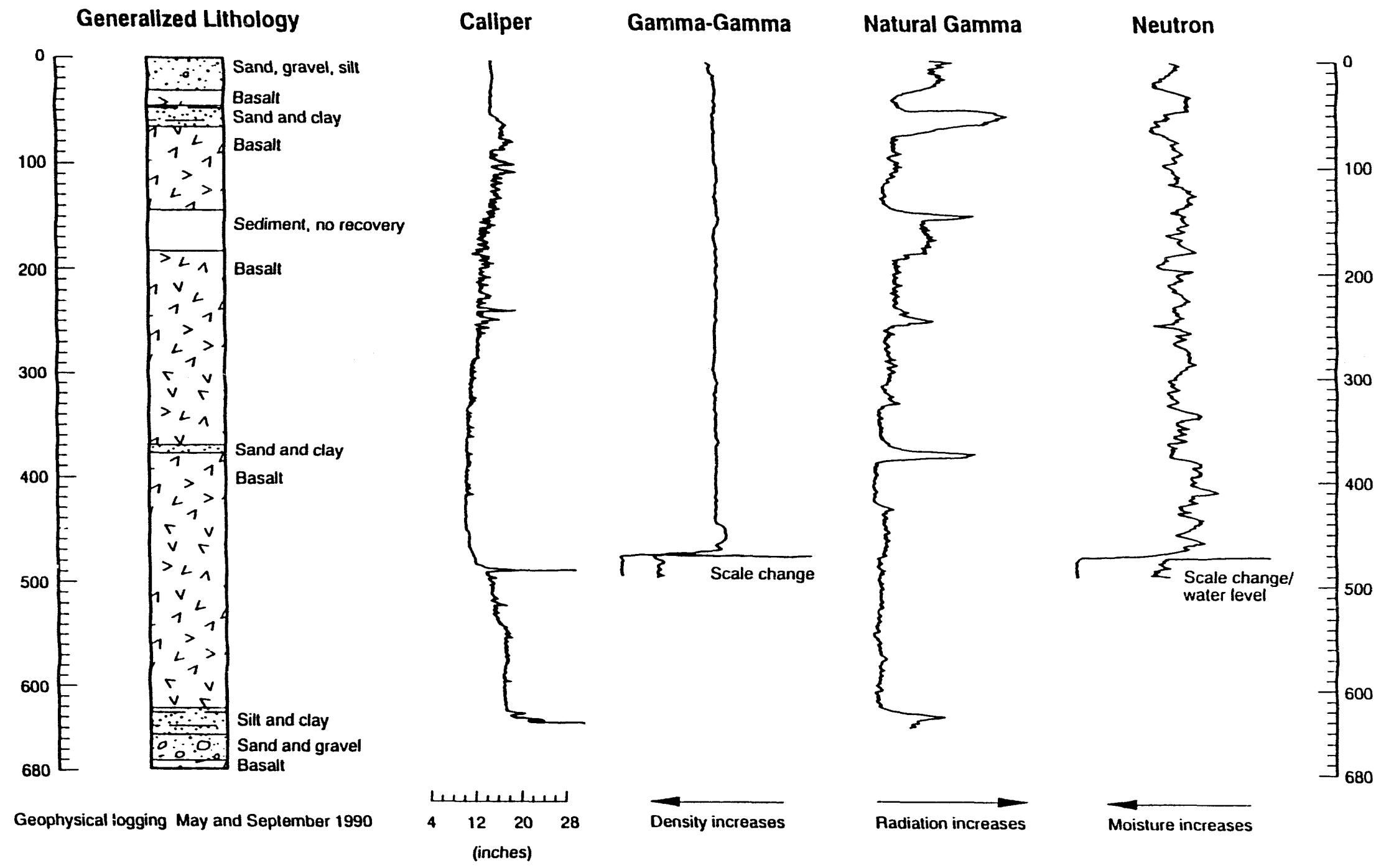

Well CFA LF2-09 
END-OF-WELL REPORT

CENTRAL FACILITIES LANDFILL AREA

WELL LF2-10 and LF2-07B 
I. GENERAL INFORMATION

A. Project Name: Hydrogeologic Characterization Study for CFA Landfills

B. Well Name/Number: LF2-10 $\left(2-7^{1}\right)$ and LF2-07B (shallow twin)

C. Well Location: INEL, Central Facilities Area Landfill II Northing: $682827.91 \mathrm{ft}$ Easting: $294266.84 \mathrm{ft}$ Land Surface Elevation: $4933.39 \mathrm{ft}$ (NRTS datum)

D. Plans: Survey Date: Not known

1) Hydrogeologic Characterization Study for CFA Landfills II and III, Idaho National Engineering Laboratory, Technical Work Plan, submitted to EG\&G Idaho, Inc. by Science Applications International Corporation, Aprit 15, 1988.

2) Synopsis of Work, Hydrogeologic Characterization Study for CFA Landfills II and III, 31 January 1989 letter report, John Jaacks, Science Applications International Corporation.

3) Hydrogeologic Characterization Study at CFA Landfills II and III, Health and Safety Plan, EGG-ER-8445, February 1989.

E. Logbooks used:

1) Science Applications International Corporation Logbooks, submitted to EG\&G Idaho, Inc., 03/May/88 to 09/Sept/Aug/88.

F. Other references:

1) Bishop, Carolyn B., 1992, LF2-10 Pumping Test Performed on August 17, 1992, Memo Number CWB-28-92.

II. DRILLING AND COMPLETION OBSERVATIONS

A. Drilling Company: Denning Well Drilling

Drillers: Dan Denning

On-Site Geologist: John Jaacks

Project Manager: Larry Hull

Rig type: 650 Chicago Pneumatic

Bit type: Unknown

1. Borehole LF2-10 is referred to as both LF2-10 and LF2-7 in the logbooks. 
B. Summary of Drilling Activities:

\begin{tabular}{|c|c|c|}
\hline DATE & ACTIVITY & MATERIALS USED \\
\hline $03-$ May-88 & $\begin{array}{l}\text { Drilled from } 0 \text { to } 3 \mathrm{ft} \text { bls. Set surface } \\
\text { casing to } 26 \mathrm{ft} \text { bls (according to } \\
\text { caliper log) then began to drill out } \\
\text { surface casing. Logbooks do not } \\
\text { directly record the type of drilling } \\
\text { that was used, but occasional references } \\
\text { to a compressor, water truck, and foam } \\
\text { in the cuttings suggest the drilling } \\
\text { method was air rotary and that water } \\
\text { and/or foam were added as needed to } \\
\text { lubricate the bit or lift cuttings to } \\
\text { the surface. Encountered basalt at } 28 \\
\text { to } 29 \text { ft bls. Drilled to } 51 \text { ft bls. } \\
\text { Encountered an interbed and stopped } \\
\text { drilling to rig up for sampling. }\end{array}$ & $\begin{array}{l}123 / 4 \text {-inch carbon } \\
\text { steel surface } \\
\text { casing }\end{array}$ \\
\hline $04-$ May-88 & $\begin{array}{l}\text { Began coring for samples. Lexan liner } \\
\text { used to retain core collapsed during } \\
\text { coring. Coring halted to investigate } \\
\text { sampling problems. }\end{array}$ & \\
\hline $05-$ May-88 & $\begin{array}{l}\text { Began HXB wireline coring. Cored from } \\
51 \text { to } 65 \mathrm{ft} \text { bls in } 5 \mathrm{ft} \text { increments, } \\
\text { al though recovery was poor. One sample } \\
\text { was collected, near } 64 \mathrm{ft} \text { bls. }\end{array}$ & \\
\hline 06-May-88 & $\begin{array}{l}\text { Reamed from } 51 \text { to } 65 \mathrm{ft} \text { bls and drilled } \\
\text { to } 72 \mathrm{ft} \text { bls. Grouted in borehole. }\end{array}$ & $1 \mathrm{yd}^{3}$ cement \\
\hline $09-$ May-88 & $\begin{array}{l}\text { Drilled out cement from } 43 \text { to } 69 \mathrm{ft} \text { bls, } \\
\text { drilled into basalt to } 160 \mathrm{ft} \text { bls. }\end{array}$ & \\
\hline 10-May-88 & Drilled from 160 to $245 \mathrm{ft}$ bls. & \\
\hline $11-$ May-88 & Orilled from 245 to $350 \mathrm{ft}$ bls. & \\
\hline 12-May-88 & $\begin{array}{l}\text { Drilled from } 350 \text { to } 376 \mathrm{ft} \text { bls. At } 376 \\
\mathrm{ft} \text { bls, began coring sedimentary layer. } \\
\text { No recovery. }\end{array}$ & \\
\hline 13-May-88 & $\begin{array}{l}\text { Detected organic vapor in borehole, } \\
\text { greater than } 110 \mathrm{ppm} \text { with Organic Vapor } \\
\text { Analyzer. Continued core sampling of } \\
\text { the interbed below } 376 \mathrm{ft} \text {. Three of the } \\
\text { samples retained sediment (SS10, SS11, } \\
\text { SS12). }\end{array}$ & \\
\hline
\end{tabular}




\begin{tabular}{|c|c|c|}
\hline DATE & ACTIVITY & MATERIALS USED \\
\hline 16-May-88 & $\begin{array}{l}\text { Continue core sampling of interbed. } \\
\text { Collect } 2 \mathrm{ft} \text { of sample from } 368 \text { to } 370 \\
\mathrm{ft} \text { bls. Reaned borehole and drilled to } \\
395 \mathrm{ft} \text { bls. The borehole was grouted } \\
\text { from } 395 \text { to } 365 \mathrm{ft} \text { bls. }\end{array}$ & $1 y d^{3}$ cement \\
\hline 18-May-88 & $\begin{array}{l}\text { Drilled out grout and drilled into } \\
\text { basalt to } 470 \mathrm{ft} \text { bls. }\end{array}$ & \\
\hline 19-May-88 & Drilled from 470 to $580 \mathrm{ft}$ bls. & \\
\hline 20-May- 88 & Drilled from 580 to $627 \mathrm{ft}$ bls. & \\
\hline 23-May-88 & $\begin{array}{l}\text { Encountered a sedimentary interbed at } \\
627 \mathrm{ft} \text {. Began coring interbed. } \\
\end{array}$ & \\
\hline $24-$ May -88 & $\begin{array}{l}\text { Heaving sands and gravel in the borehole } \\
\text { made coring/sampling difficult. Removed } \\
\text { pipe from borehole and found portions of } \\
21 / 2 \text { drill stems clogged. Litill bit } \\
\text { was ruined by drilling without } \\
\text { compressed air or water. }\end{array}$ & \\
\hline 25-May-88 & $\begin{array}{l}\text { Borehole has caved } 4 \text { to } 5 \mathrm{ft} \text { overnight. } \\
\text { Begin drilling with a } 12-\text { inch drill bit } \\
\text { to clean out the borehole to } 632 \mathrm{ft} \text { bls, } \\
\text { and then grouted the borehole. New twin } \\
\text { borehole (LF2-7B) is started about } 20 \mathrm{ft} \\
\text { east of LF2-10, to collect samples from } \\
\text { the upper sedimentary interbed. Surface } \\
\text { casing is driven to refusal and borehole } \\
\text { LF2-7B is drilled to about } 50 \mathrm{ft} \text { bls.. }\end{array}$ & $\begin{array}{l}3 \mathrm{yd}^{3} \text { cement, ? ft } \\
\text { of carbon stee] } \\
\text { surface casing }\end{array}$ \\
\hline 26-May-88 & $\begin{array}{l}\text { Collected core samples from LF2-7B from } \\
50 \text { to } 70 \mathrm{ft} \text { bIs. Borehole LF2-7B is } \\
\text { grouted on } 07-J u 1 y-88 \text { and } 13-J u 1 y-88 \text {, } \\
\text { and completely filled to the surface for } \\
\text { abandonment. }\end{array}$ & \\
\hline $27-$ Мау -88 & $\begin{array}{l}\text { Returned to drilling out grout on } \\
\text { LF2-10. Several cement bridges were } \\
\text { encountered in the borehole before } \\
\text { reaching the top of the cement at } 580 \\
\text { ft. The cement did not grout to the } \\
\text { bottom of the heaving sand layer. Made } \\
\text { an unsuccessful attempt to collect a } \\
\text { core sample. }\end{array}$ & \\
\hline 31-May-88 & $\begin{array}{l}\text { Recovered one sample from the interbed } \\
\text { below the } 630-\mathrm{ft} \text { depth. No recovery was } \\
\text { made on the three other sample attempts. }\end{array}$ & \\
\hline
\end{tabular}




\begin{tabular}{|c|c|c|}
\hline DATE & ACTIVITY & MATERIALS USED \\
\hline 01 -June-88 & $\begin{array}{l}\text { Replaced rock bit with an auger bit and } \\
40 \mathrm{ft} \text { of welded auger flights to sample } \\
\text { in the heaving sands. Recovered only } \\
\text { one sample from } 5 \text { attempts. No } \\
\text { description of the sample is recorded. }\end{array}$ & \\
\hline $02-J u n e-88$ & $\begin{array}{l}\text { Modified rubber catch on sampler and } \\
\text { recovered two samples. Problems were } \\
\text { encountered with core stem plugging up, } \\
\text { had to pump the heaving sands out of the } \\
\text { core stem using foam. }\end{array}$ & \\
\hline 03 -June-88 & $\begin{array}{l}\text { Collected three samples, however only } \\
\text { one had any recovery. Pumped cement } \\
\text { grout down the borehole to cement off } \\
\text { the sand/gravel interbed. }\end{array}$ & $6 \mathrm{yd}^{3}$ cement \\
\hline 06 -June- 88 & $\begin{array}{l}\text { Top of cement at } 628 \mathrm{ft} \text { bls, drilled } \\
\text { through at about } 633 \mathrm{ft} \text { bls. Drilled to } \\
650 \mathrm{ft} \text { and pulled out stem. The } \\
\text { borehole caved to about } 638 \mathrm{ft} \text { bls. } \\
\text { Grouted borehole. }\end{array}$ & $\begin{array}{l}4 \mathrm{yd}^{3} \text { cement } \\
\text { (bentonite/sand } \\
\text { mix) }\end{array}$ \\
\hline 07 -June- 88 & $\begin{array}{l}\text { Top of cement at } 580 \mathrm{ft} \text { bls. Drilled } \\
\text { down to basalt at } 663 \mathrm{ft} \text { bls and then } \\
\text { grouted the borehole. }\end{array}$ & ? $y d^{3}$ cement \\
\hline 08 -June-88 & $\begin{array}{l}\text { Top of cement at } 630 \mathrm{ft} \text { bls. Drilled } \\
\text { through cement and into basalt to } 670 \mathrm{ft} \\
\text { bls. Cement sidewalls appear to extend } \\
\text { to about } 650 \mathrm{ft} \text { bls. }\end{array}$ & \\
\hline $\begin{array}{l}09-\text { June }-88 \\
\text { to } \\
19-\text { June- } 88\end{array}$ & $\begin{array}{l}\text { Because of poor success with drilling } \\
\text { and grouting LF2-10, it was decided to } \\
\text { case off the heaving sand interbed with } \\
8 \text {-inch casing. This time period was } \\
\text { spent preparing cost estimates and } \\
\text { specifications for the casing } \\
\text { installation. }\end{array}$ & \\
\hline
\end{tabular}




\begin{tabular}{|c|c|c|}
\hline DATE & ACTIVITY & MATERIALS USED \\
\hline $\begin{array}{l}20-\text { June }-88 \\
\text { to } \\
23-\text { June }-88\end{array}$ & $\begin{array}{l}\text { Installed } 8 \text {-inch casing. A drive shoe } \\
\text { welded to lower opening of the casing } \\
\text { and stop blocks were welded onto the } \\
\text { outer wall of the casing to prevent the } \\
\text { casing from slipping past the elevators. } \\
\text { After the casing joints were welded, the } \\
\text { stop blocks were torched off and two } \\
\text { diamond plates were welded and then } \\
\text { pounded flush against the casing wal1. } \\
\text { The } 8 \text {-inch casing was driven to } 672 \mathrm{ft} \text {, } \\
\text { with } 1.3 \mathrm{ft} \text { above ground surface, but } \\
\text { slippage occurred later. }\end{array}$ & $\begin{array}{l}673.23 \text { ft of } 8- \\
\text { inch casing, in } 20 \\
\text { ft lengths }\end{array}$ \\
\hline $23-J u n e-88$ & $\begin{array}{l}\text { Began drilling at } 640 \mathrm{ft} \text { bls, with a } \\
77 / 8 \text {-inch tricone bit. The casing } \\
\text { stick-up slipped down into the borehole. }\end{array}$ & \\
\hline $24-J u n e-88$ & $\begin{array}{l}\text { Evacuated dirt from around the } 8 \text {-inch } \\
\text { casing and welded additional } 8 \text {-inch } \\
\text { casing to the casing string. Pounded } \\
\text { casing to refusal and cut off excess } \\
\text { casing. }\end{array}$ & \\
\hline 27 -June- 88 & $\begin{array}{l}\text { Begin drilling but casing is still } \\
\text { dropping with footage gained. Welded } \\
\text { more } 8 \text {-inch casing on to the casing } \\
\text { string to reach a total depth for the 8- } \\
\text { inch casing of } 676 \mathrm{ft} \text { bls. }\end{array}$ & \\
\hline $28-J u n e-88$ & $\begin{array}{l}\text { Drilled from } 740 \text { to } 816 \mathrm{ft} \text { b7s. } \\
\text { Borehole is caving at } 816 \mathrm{ft} \text { bis. }\end{array}$ & \\
\hline $30-J u n e-88$ & $\begin{array}{l}\text { USGS performed geophysical logging of } \\
\text { LF2-10: TV, caliper, gamma-gamma, and } \\
\text { natural gamma logs were conducted. } \\
\text { Water level was measured at } 482 \mathrm{ft} \text { bls. } \\
\text { TV log data recorded in the logbook } \\
\text { lists a total depth at } 833 \mathrm{ft} \text { bls along } \\
\text { with lithologic descriptions below the } \\
816-\mathrm{ft} \text { depth. The } 833-\mathrm{ft} \text { total depth is } \\
\text { inconsistent with the total depth } \\
\text { recorded by the geologist for June } 28 \text {, } \\
1992 \text { (of } 816 \mathrm{ft} \text { bls) but the } \\
\text { inconsistency has not been reconciled. }\end{array}$ & \\
\hline $\begin{array}{l}26-\text { June-91 } \\
\text { and } \\
\text { Sept-92 }\end{array}$ & $\begin{array}{l}\text { A Grundfos } 25550-265 \mathrm{hp} \text { pump was } \\
\text { installed by USGS for temporary use, } \\
\text { with an intake level set at } 547 \mathrm{ft} \text { bls. } \\
\text { The pump was removed in September } 1992 \text {. }\end{array}$ & \\
\hline
\end{tabular}


C. Summary of Well Construction Procedures:

\begin{tabular}{|c|c|c|}
\hline DATE & ACTIVITY & MATERIALS USED \\
\hline $07-J u 1 y-88$ & Began grouting bottom of borehole. & $1 / 2 \mathrm{yd}^{3}$ cement \\
\hline $12-J u 7 y-88$ & $\begin{array}{l}\text { Measured bottom of borehole at } 830 \mathrm{ft} \\
\text { bls. }\end{array}$ & \\
\hline $13-J u 1 y-88$ & Tremied cement down borehole LF2-10. & ? $y d^{3}$ cement \\
\hline $14-J u 7 y-88$ & $\begin{array}{l}\text { Measured bottom of borehole at } 820 \mathrm{ft} \\
\text { bls. Poured cement into annular space } \\
\text { between the } 12 \text {-and } 8 \text {-inch casing. }\end{array}$ & $<1 \mathrm{yd}^{3}$ cement \\
\hline 20-JuTy-88 & $\begin{array}{l}\text { Poured } 9 \text { buckets of bentonite down the } \\
\text { borehole. }\end{array}$ & $\begin{array}{l}9 \text { buckets } \\
\text { bentonite }\end{array}$ \\
\hline $21-J u 7 y-88$ & $\begin{array}{l}\text { Measured bottom of borehole at } 780 \mathrm{ft} \\
\text { bls. Poured down } 6 \text { buckets of bentonite } \\
\text { and } 1 \text { bucket of gravel. Began } \\
\text { installation of } 6-\text { inch stainless steel } \\
\text { well screen and well casing. }\end{array}$ & $\begin{array}{l}6 \text { buckets } \\
\text { bentonite, } 1 \\
\text { bucket of gravel, } \\
10 \mathrm{ft} \text { of } 6 \text {-inch ID } \\
\text { stainless steel } \\
\text { well screen, } 300 \\
\text { ft of } 6 \text {-inch } \\
\text { nominal pipe size } \\
\text { stainless steel } \\
\text { well casing }\end{array}$ \\
\hline $\begin{array}{l}22-J u\rceil y-88 \\
\text { to } \\
25-J u\rceil y-88\end{array}$ & $\begin{array}{l}\text { Completed installation of well screen } \\
\text { and well casing to } 765 \text { ft bls. The } \\
\text { stainless steel well casing was joined } \\
\text { to } 6 \text {-inch nominal pipe size ( } 6 \text {-inch ID, } \\
\text { field measurement) carbon steel well } \\
\text { casing with a dielectric coupling at } 445 \\
\text { ft bls. Three centralizers were } \\
\text { installed, depths are not known. The } \\
\text { stainless steel well screen and well } \\
\text { casing had flush-threaded joints and the } \\
\text { carbon steel casing had welded joints. } \\
\text { Began installation of a filter pack } \\
\text { consisting of l/4-inch pea gravel and } \\
\text { placed a bentonite seal above the filter } \\
\text { pack. The gravel was vibrated down the } \\
\text { borehole, because of bridging between } \\
\text { the } 8 \text { - and } 6 \text {-inch casings. }\end{array}$ & $\begin{array}{l}450 \text { ft of } 6 \text {-inch } \\
\text { nominal pipe size } \\
\text { carbon steel well } \\
\text { casing, } 5 \text { buckets } \\
\text { of gravel }\end{array}$ \\
\hline $26-J u 7 y-88$ & $\begin{array}{l}\text { USGS arrived and performed a gamma-gamma } \\
\text { log of the borehole. }\end{array}$ & \\
\hline
\end{tabular}




\begin{tabular}{|c|c|c|}
\hline DATE & ACTIVITY & MATERIALS USED \\
\hline $28-J u l y-88$ & $\begin{array}{l}\text { Poured fine sand down the borehole, } \\
\text { using a jackhammer to vibrate borehole. } \\
\text { The vibration was used because material } \\
\text { was bridging in the casing annular } \\
\text { space. Poured } 7 \mathrm{yd}^{3} \text { cement down the } \\
\text { borehole, between the } 8 \text { - and } 12 \text {-inch } \\
\text { casing. }\end{array}$ & $1 / 2$ bag fine sand \\
\hline $29-J u T y-88$ & $\begin{array}{l}\text { Poured cement down the borehole, but it } \\
\text { bridged. Tried banging, plunging with a } \\
\text { wire, moving the } 6 \text {-inch casing, and } \\
\text { jackhammering the casing with no } \\
\text { success. } \\
\text { USGS onsite to a neutron and gamma-gamma } \\
\text { log. The rest of the cement was poured } \\
\text { into the borehole in the } 6 \text { - to } 8 \text {-inch } \\
\text { and } 8 \text { - to } 12 \text {-inch annular spaces. } \\
\text { USGS ran another gamma-gamma log. Three } \\
\text { buckets of bentonite were poured into } \\
\text { the } 8 \text { - to } 12 \text {-inch annular space. } \\
\text { Because of cement bridging, a decision } \\
\text { was made to check into perforation of } \\
\text { the casing. }\end{array}$ & $\begin{array}{l}5 \mathrm{yd}^{3} \text { cement, } 3 \\
\text { buckets ( } 5 \text { gallons } \\
\text { each) of bentonite }\end{array}$ \\
\hline
\end{tabular}




\begin{tabular}{|c|c|c|}
\hline DATE & ACTIVITY & MATERIALS USED \\
\hline $\begin{array}{l}08-\text { Sept }-88 \\
\text { to } \\
09-\text { Sept }-88\end{array}$ & $\begin{array}{l}\text { Haliburton Inc. and } 0 \text { il Well Perforators } \\
\text { arrived onsite to do casing perforation } \\
\text { and cementing. The plan was to } \\
\text { perforate and grout the borehole from } \\
755 \text { to } 700 \text { ft bls, and perforate and } \\
\text { complete the well from } 680 \text { to } 695 \text { ft } \\
\text { bls. The casing was perforated from } 745 \\
\text { to } 755 \text { ft. UsG performed a TV log, but } \\
\text { were unable to view results because of } \\
\text { blasting powder residue. } \\
\text { Completed two sessions of pressurized } \\
\text { grouting into the } 6 \text {-inch casing. The } \\
\text { cement appeared to be infilling a lower } \\
\text { fracture. Temperature logs were run and } \\
\text { disclosed the cement level was at } 652 \text { ft } \\
\text { bls. } \\
\text { A head of water was placed in the wel1, } \\
\text { and when the water disappeared, it was } \\
\text { concluded that the fracture was not yet } \\
\text { sealed and that the perforations were } \\
\text { open. A decision was made to add } \\
\text { calcium chloride and bentonite to the } \\
\text { cement mix. Cement was again pressure } \\
\text { grouted down the borehole. Temperature } \\
\text { log results indicated the cement had } \\
\text { gone into the fracture at } 752 \text { ft. } \\
\text { A second perforation was performed at } \\
725 \text { to } 735 \text { ft bls. The geologist } \\
\text { logbook does not disclose whether other } \\
\text { sections of the casing were perforated } \\
\text { or if further cementing efforts were } \\
\text { made. Later diagrams and completion } \\
\text { plans show } 700 \text { to } 715 \text { ft bls as the } \\
\text { perforation interval. However, grouting } \\
\text { attempts were curtailed on September } 9 \text {, } \\
\text { l988 because the cement was sinking into } \\
\text { formation, and a large fracture was } \\
\text { suspected to be causing the sink. } \\
\text { Therefore, the well was sealed shut with } \\
\text { a welded cap and further attempts to } \\
\text { complete or seal the well were deferred. }\end{array}$ & $\begin{array}{l}69 \text { sacks of } \\
\text { cement, } 3 \text { sacks of } \\
\text { calcium chloride, } \\
2 \text { buckets } \\
\text { bentonite }\end{array}$ \\
\hline
\end{tabular}




\begin{tabular}{|c|c|c|}
\hline DATE & ACTIVITY & MATERIALS USED \\
\hline August 1989 & $\begin{array}{l}\text { Colog Inc. performed density and caliper } \\
\text { logging of the borehole to determine } \\
\text { grout and gravel/sand depths between the } \\
6 \text { - and } 8 \text {-inch casing ( } 109 \text { copy is } \\
\text { attached). Based on their } \\
\text { interpretation, grout had bridged in the } \\
\text { annulus from land surface to } 78 \mathrm{ft} \text { bls. } \\
\text { A casing restriction (possibly a break } \\
\text { in the stainless steel casing) was noted } \\
\text { at } 655 \mathrm{ft} \text { bls. Pipe ID was logged as } \\
6.35 \text { inches from } 0 \text { to } 452 \mathrm{ft} \text { bls and } 6.4 \\
\text { inches below } 452 \mathrm{ft} \text {. However, field } \\
\text { measurement of exposed surface pipe } \\
\text { determined a } 6 \text {-inch ID for the carbon } \\
\text { steel casing. The sand pack bridged } \\
\text { frequent7y in the } 6 \text { to } 8 \text {-inch casing } \\
\text { annular space, from } 78 \text { to } 634 \mathrm{ft} \mathrm{bls.}\end{array}$ & \\
\hline 17-Aug - 92 & $\begin{array}{l}\text { A single-well, short duration pumping } \\
\text { test was performed. A slight drawdown } \\
\text { followed by a rapid recovery indicated } \\
\text { the well was in communication with the } \\
\text { aquifer. }\end{array}$ & \\
\hline 1989 & $\begin{array}{l}\text { Installed surface cement pad. Surface } \\
\text { completion included a locked casing, } \\
\text { cement pad, and impingement posts. }\end{array}$ & \\
\hline
\end{tabular}

D. Summary of Well Development Procedures: None recorded.

\section{GEOLOGICAL OBSERVATIONS}

A. Geological log, geophysical logs, stratigraphic column, and well construction diagram are attached.

B. Samples collected.

Sample Number

Comment

Borehole LF2-10:

LF2-10-SS10

13-May-88, collected from interbed at $376 \mathrm{ft} \mathrm{bls.} \mathrm{Recovered} 10$ inches from $5 \mathrm{ft}$ of coring. 
Sample Number

LF2-10-SS11

LF2-10-SS12

Unknown

Unknown

Unknown

LF2-7-SS1

Unknown

LF2-7-SS3

Unknown

Unknown

Unknown

Unknown

Unknown

\section{Comment}

13-May-88, collected 6 inches of sample from $5 \mathrm{ft}$ of coring, $377 \mathrm{ft}$ bls.

13-May-88, collected 12 inches of sample from 377 to $378 \mathrm{ft}$ bls.

13-May-88, collected $2 \mathrm{ft}$ of sample from 368 to $370 \mathrm{ft}$ bls (muddy, silty sand).

23-May-88, collected 60 inches of sample from 628 to $633 \mathrm{ft}$ bls.

23-May-88, collected 54 inches of sample from 629 to $633 \mathrm{ft}$ bls.

23-May-88, collected 8 inches of sample from $629 \mathrm{ft}$ bls (brownish-red semi-indurated sand to silty sand with minor fine pebbles).

23-May-88, collected 15 inches of sediment at $634 \mathrm{ft}$ bls.

23-May-88, collected 24 inches of sample from 634 to $636 \mathrm{ft}$ bls (red medium to coarse unconsolidated sand underlain by 8-10 inches of pebbles/gravel).

24-May-88, collected 53 inches of sediment from 629 to $634 \mathrm{ft}$ bls (heaved sand).

05-31-88, collected sample drilling 6 inches into the interbed, depth of drilling unknown (fine to medium sand above a gravel layer).

01-June-88, collected 49 inches of sample from 636 to $641 \mathrm{ft}$ ols.

02-June-88, collected 6-inch sample from 641 to $646 \mathrm{ft}$ bls (clay).

02-June-88, collected 37 inches of sample from 641 to $646 \mathrm{ft}$ bls (fine to medium sand with a gravel base?) 
Sample Number

Unknown

Iwin borehole LF2-07B:

Unknown

Unknown

Unknown

Unknown

Unknown

Unknown
Comment

03-June-88, collected 36 inches of sample from 656 to $661 \mathrm{ft}$ bis (reddish-brown gravel).

26-May-88, twin borehole LF2-07B:

collected 6 inches of sample at 50 ft bls (brown silty fine sand).

26-May-88, twin borehole LF2-07B: collected 36 inches of sample from 50 to $53 \mathrm{ft}$ bls (orange-brown silty clay)

26-May-88, twin borehole LF2-07B: collected 60 inches of sample from 53 to $58 \mathrm{ft}$ bis (granular to pebbly brown silty sand from 53-54.5 ft, brown, slightly sandy, silty clay from 54.4-58 ft).

26-May-88, twin borehole LF2-07B: collected 29 inches of sample from 58 to $60 \mathrm{ft}$ bls.

26-May-88, twin borehole LF2-07B: collected 58 inches of sample from 60 to $65 \mathrm{ft}$ bls (grayish-yellow, dense, indurated clay).

26-May-88, twin borehole LF2-07B: collected 60 inches of sample from 65 to $70 \mathrm{ft}$ bls (12 inches of yellow-brown dense clay underlain by $4 \mathrm{ft}$ of black vesicular basalt). 


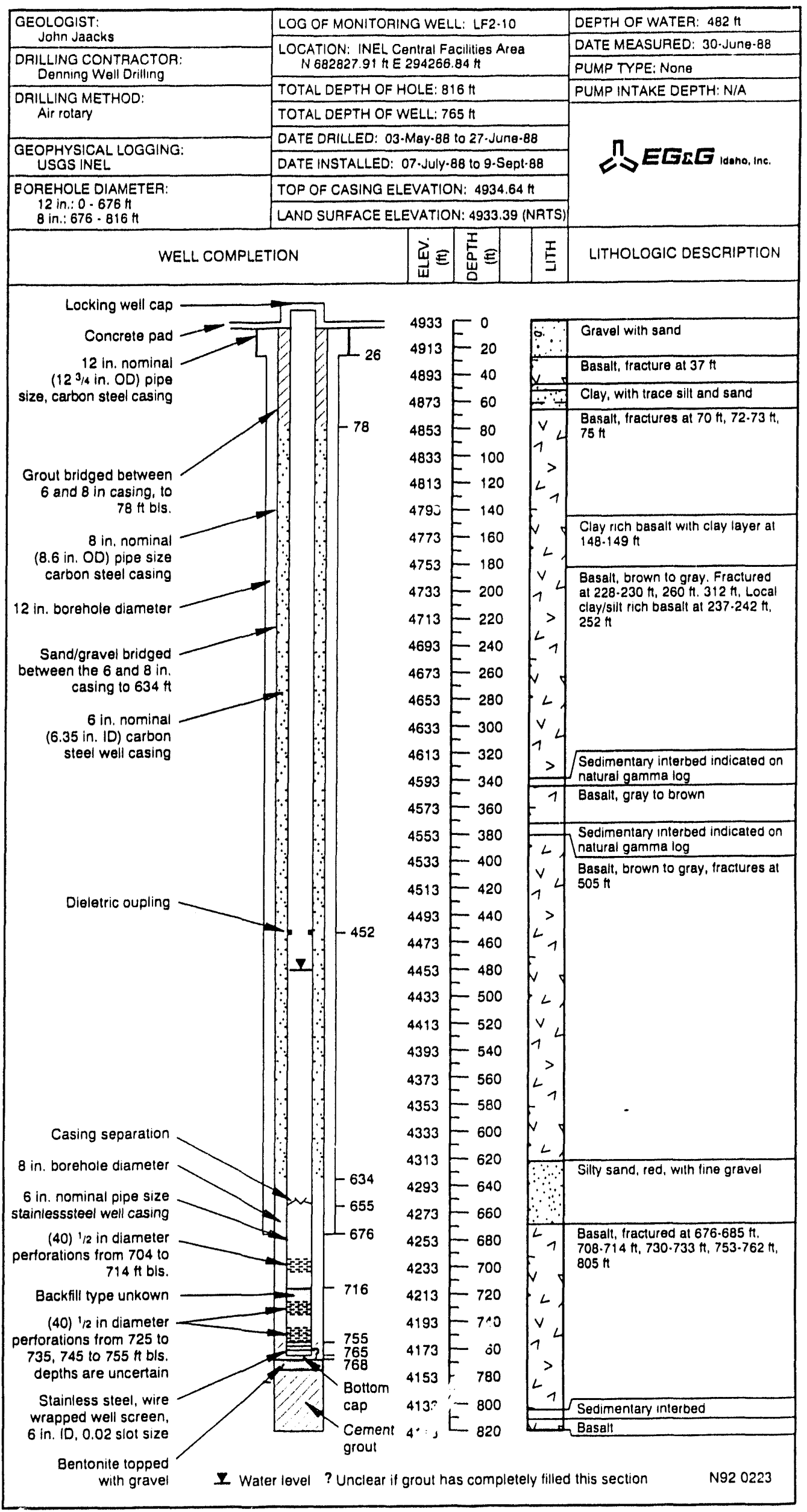



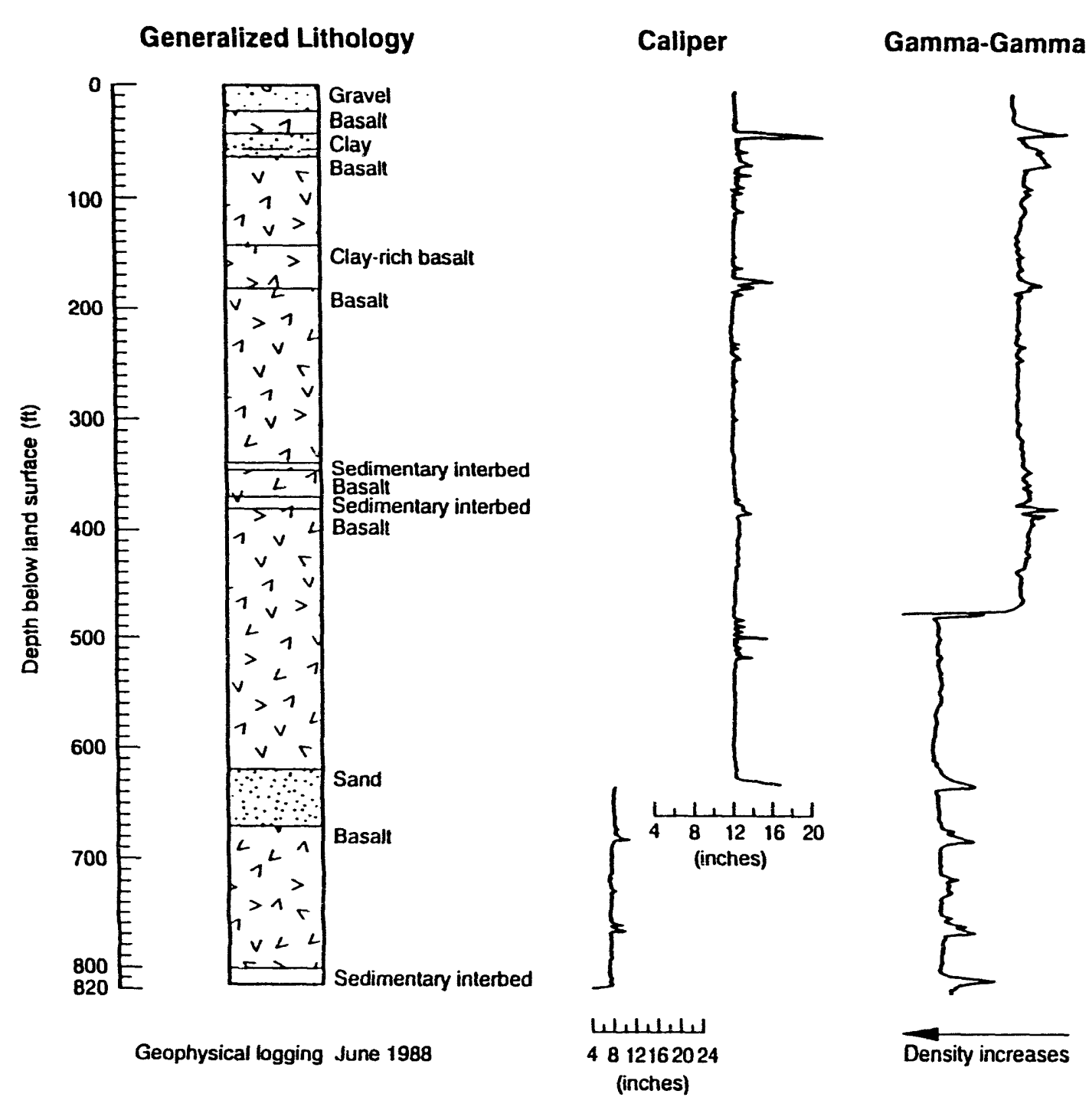

Natural Gamma

Neutron
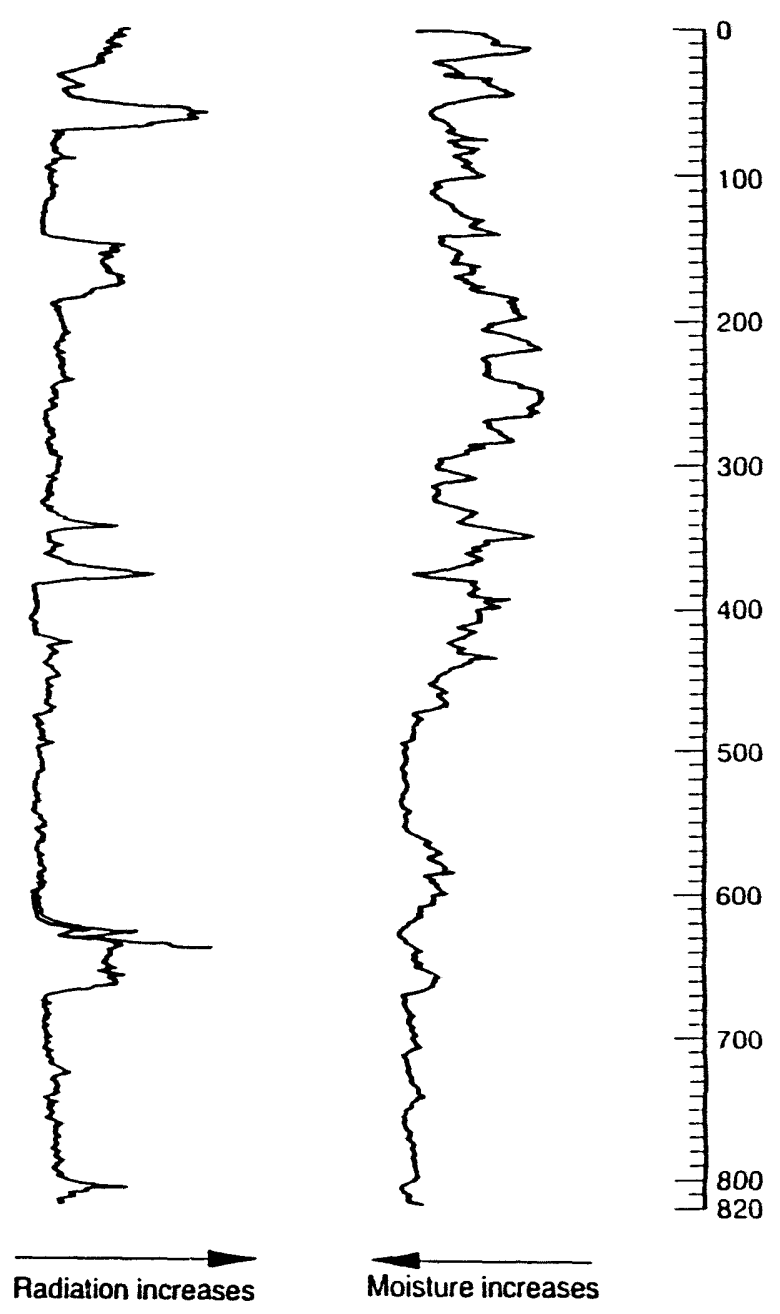

Well CFA LF2-10 
END-OF-WELL REPORT

CENTRAL FACILITIES LANDFILL AREA

WELL LF2-11 
I. GENERAL INFORMATION

A. Project Name: Hydrogeologic Characterization Study for CFA Landfills

B. Well Name/Number: LF2-11

C. Well Location: INEL, Central Facilities Area Landfill II Northing: 684290.72 Easting: 295460.16 Land Surface Elevation: $4929.52 \mathrm{ft}$ (brass marker)

D. Plans:

Survey Date: December 1990, MK-Ferguson

1) Hydrogeologic Characterization for CFA Landfills II and III, Idaho National Engineering Laboratory, Technical Work P7an, EGG-ER-8670, August 1989.

2) Hydrogeologic Characterization Study at CFA Landfills II and III, Health and Safety Plan, EGG-ER-8445, February 1989.

E. Logbooks used:

1) Well Drilling, Installation, and Development Logbook, CFA-2

2) Field Team Leaders Daily Logbook, CFA-002, 10/17/89 to 10/20/89.

3) Field Team Leaders Daily Logbook, CFA-003, 10/20/89 to $12 / 06 / 89$.

4) Field Team Leaders Daily Logbook, CFA-004, 12/06/89 to $12 / 11 / 89$.

5) Field Team Leaders Daily Logbook, CFA-005, 12/12/89 to $12 / 18 / 89$.

\section{DRILLING AND COMPLETION OBSERVATIONS}

A. Drilling Company: Hawley Brothers Drilling

Drillers: Mortier Barrus, Anthony Firkins

On-Site Geologist: Shannon Ansley, Brian Higgs

Project Manager: Martin Doornbos

Rig type: Chicago Pneumatic, model unknown

Bit type: Tricone

B. Summary of Drilling Activities:

\begin{tabular}{||l|l|l||}
\hline DATE & \multicolumn{1}{|c||}{ ACTIVITY } & MATERIAI.S USED \\
\hline 17-0ct-89 & $\begin{array}{l}\text { Set up at drill site. Using an air } \\
\text { rotary drilling method with a tricone } \\
\text { bit, a pilot hole (diameter not recorded } \\
\text { in iogbook) was drilled to about 34 ft } \\
\text { but the hole caved in before casing was } \\
\text { driven. }\end{array}$ & \\
\hline 18-0ct-89 & $\begin{array}{l}\text { Re-rigging cables and hammer hook-up, } \\
\text { did not meet safety and contract } \\
\text { specifications. }\end{array}$ & \\
\hline
\end{tabular}




$$
\begin{aligned}
& \text { 震 }
\end{aligned}
$$

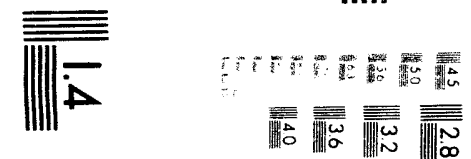

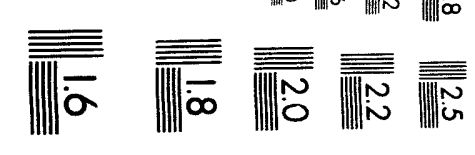



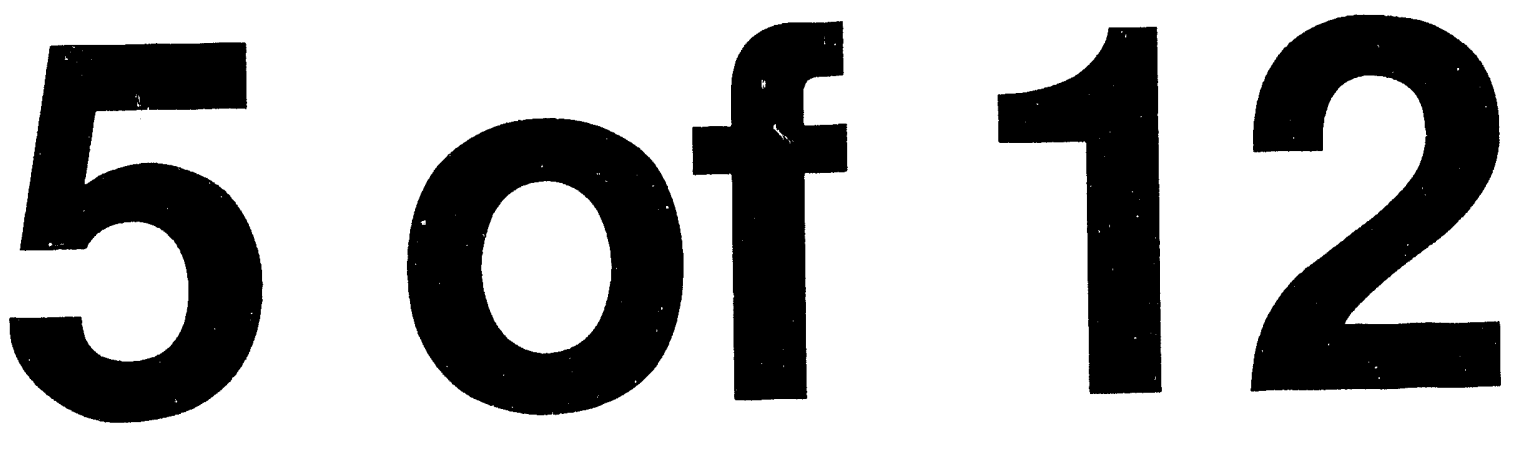


\begin{tabular}{|c|c|c|}
\hline DATE & ACTIVITY & MATERIALS USED \\
\hline $19-0 c t-89$ & $\begin{array}{l}\text { Borehole opened again. Hammered in 12- } \\
\text { inch casing to } 24 \mathrm{ft} \text { bls, leaving } 10 \\
\text { inches of stick-up. }\end{array}$ & $\begin{array}{l}25 \mathrm{ft} \text { of } 12 \text {-inch } \\
\text { nominal pipe size } \\
\text { carbon steel } \\
\text { surface casing }\end{array}$ \\
\hline $20-0 c t-89$ & Cleaned out casing to $25 \mathrm{ft}$. & \\
\hline $23-0 c t-89$ & $\begin{array}{l}\text { Drilled } 5 \mathrm{ft} \text { into basalt to } 32 \mathrm{ft} \text { bls. } \\
\text { Top of basalt at } 25.3 \mathrm{ft} \text { bls. Installed } \\
10 \text {-inch casing to } 30.3 \mathrm{ft} \text { bls and filled } \\
\text { bottom of borehole and the annulus } \\
\text { between the } 10 \text { - and } 12 \text {-inch casings. }\end{array}$ & $\begin{array}{l}30 \mathrm{ft} \text { of } 10 \text {-inch } \\
\text { nominal pipe size } \\
\text { carbon steel } \\
\text { surface casing, } \\
2 \text { bags cement, } \\
30 \text { shovels sand, } \\
1 \text { shovel bentonite }\end{array}$ \\
\hline $25-0 c t-89$ & $\begin{array}{l}\text { Cut } 12 \text {-inch casing stick-up to just } \\
\text { below ground level. Drilled out } 30 \mathrm{ft} \\
\text { of cement grout with } 9 / 8 \text {-inch bit. } \\
\text { Most of day spent correcting equipment } \\
\text { problems (equipment did not meet } \\
\text { specifications of the drilling } \\
\text { contract). }\end{array}$ & \\
\hline $26-0 c t-89$ & $\begin{array}{l}\text { Drilled into basalt and encountered an } \\
\text { interbed at } 42.4 \mathrm{ft} \text { bls. Removed } \\
\text { tricone bit to replace with coring } \\
\text { assembly. Cored one sample. }\end{array}$ & \\
\hline $27-0 c t-89$ & $\begin{array}{l}\text { Reamed hole with } 97 / 8 \text {-inch bit. } \\
\text { Continued drilling through basalt to } \\
130 \mathrm{ft} \text { bls. }\end{array}$ & \\
\hline $30-0 c t-89$ & Drilled to $170 \mathrm{ft}$ bls. & \\
\hline $31-0 c t-89$ & Drilled to $270 \mathrm{ft}$ bls. & \\
\hline $01-$ Nov -89 & Drilled to $322 \mathrm{ft}$ bls. & \\
\hline $02-N o v-89$ & $\begin{array}{l}\text { Driller's helper never showed up to } \\
\text { work. }\end{array}$ & \\
\hline $03-$ Nov -89 & $\begin{array}{l}\text { Drilled to about } 376 \mathrm{ft} \text { bls; about } 18 \mathrm{ft} \\
\text { into an interbed when drill string } \\
\text { became stuck in the borehole. Lost all } \\
\text { hydraulic fluid from the rig onto the } \\
\text { ground. Rest of day spent in cleanup of } \\
\text { hydraulic fluid spill. }\end{array}$ & \\
\hline $06-N o v-89$ & No drillers showed up. & \\
\hline
\end{tabular}




\begin{tabular}{|c|c|c|c|c|c|c|c|c|c|c|}
\hline 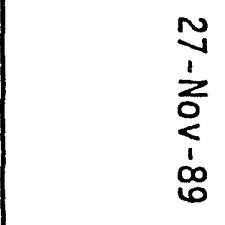 & $\begin{array}{l}\tilde{N} \\
1 \\
z_{0} \\
0 \\
1 \\
0 \\
0\end{array}$ & 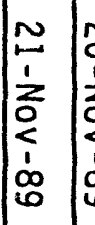 & $\mid \begin{array}{l}0 \\
0 \\
1 \\
\vdots \\
0 \\
1 \\
1 \\
0 \\
0\end{array}$ & $\begin{array}{l}\breve{w} \\
\vdots \\
z_{0} \\
\vdots \\
0 \\
0 \\
o\end{array}$ & 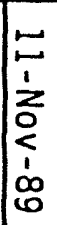 & 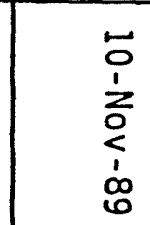 & 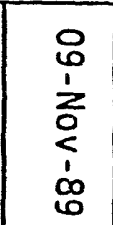 & $\begin{array}{l}0 \\
0 \\
1 \\
z \\
0 \\
1 \\
1 \\
0 \\
0\end{array}$ & $\begin{array}{l}0 \\
1 \\
1 \\
2 \\
0 \\
1 \\
0 \\
0 \\
0\end{array}$ & \\
\hline 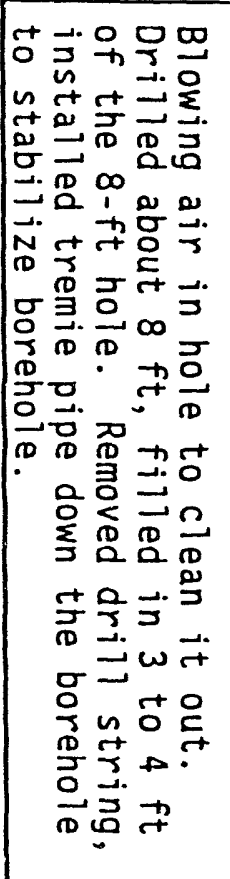 & 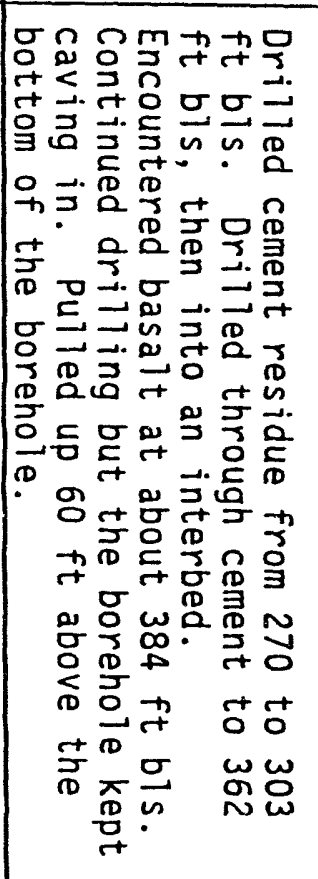 & 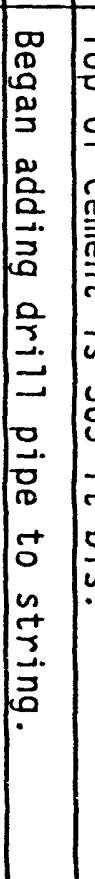 & 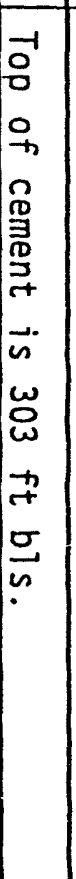 & 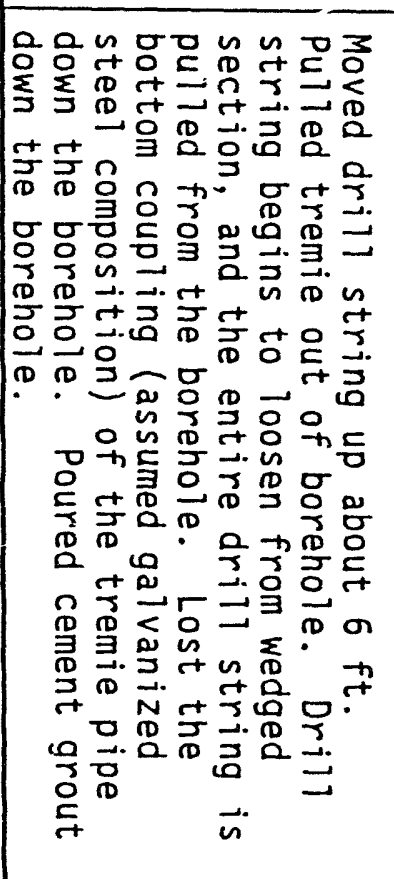 & 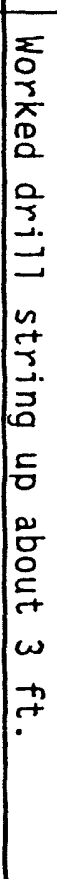 & 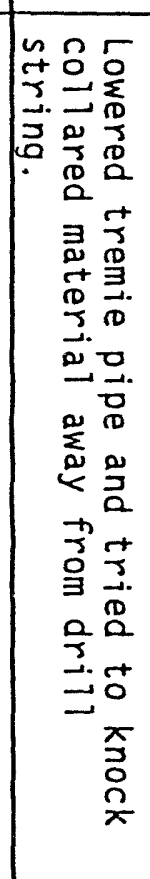 & 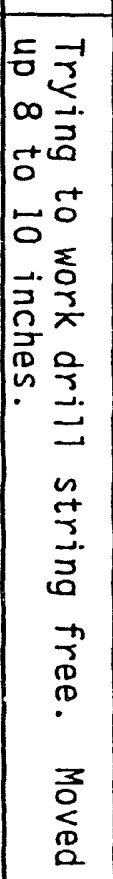 & 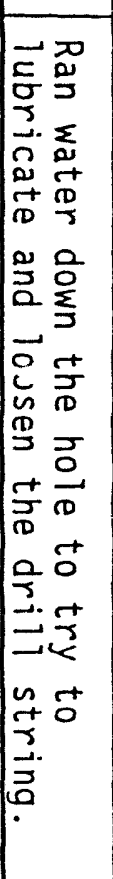 & 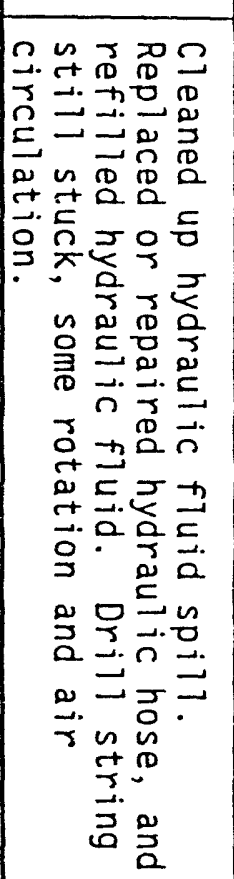 & 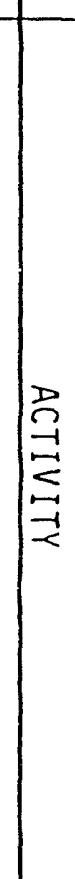 \\
\hline & & & & 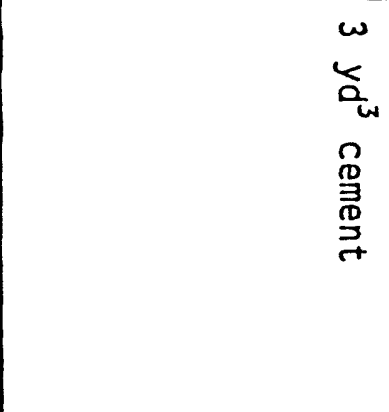 & & & & 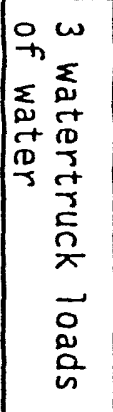 & & 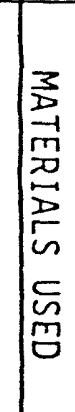 \\
\hline
\end{tabular}




\begin{tabular}{|c|c|c|}
\hline DATE & ACTIVITY & MATERIALS USED \\
\hline $28-$ Nov -89 & $\begin{array}{l}\text { Tagged top of borehole at } 374.55 \mathrm{ft} \text { bls. } \\
\text { Added cement grout to borehole. Tagged } \\
\text { top of wet cement at } 367.9 \mathrm{ft} \text { bls. } \\
\text { Ordered more cement }\left(5 \mathrm{yd}^{3}\right) \text {. Poured } 3.5 \\
\text { yds into the borehole and tagged wet } \\
\text { cement at } 320 \mathrm{ft} \text { bls. Put } 293 \mathrm{ft} \text { of } \\
\text { drill steel down the borehole. }\end{array}$ & $\begin{array}{l}3 \text { yd }^{3} \text { cement with } \\
54 \text { pounds calcium } \\
\text { chloride and } 300 \\
\text { pounds bentonite, } \\
3.5 \text { yd }^{3} \text { cement } \\
\text { with } 350 \text { pounds of } \\
\text { bentonite (not } \\
\text { known if calcium } \\
\text { chloride was } \\
\text { added) }\end{array}$ \\
\hline 29-Nov-89 & $\begin{array}{l}\text { Drilled through cement to } 374 \mathrm{ft} \text { bls, } \\
\text { then into the interbed to } 384 \mathrm{ft} \text { bls. } \\
\text { Grouted borehole. }\end{array}$ & $\begin{array}{l}3 y^{3} \text { cement with } \\
5 \% \text { bentonite and } \\
1 \% \text { calcium } \\
\text { chloride }\end{array}$ \\
\hline $30-$ Nov -89 & $\begin{array}{l}\text { Tagged top of cement at } 249.8 \mathrm{ft} \text { bls. } \\
\text { Cement was still green so drilling was } \\
\text { terminated for the day to let the cement } \\
\text { cure. }\end{array}$ & \\
\hline $01-D e c-89$ & $\begin{array}{l}\text { Drilled out cement and continued } \\
\text { drilling to } 410 \mathrm{ft} \text { bls. Grouted bottom } \\
\text { of borehole with cement. }\end{array}$ & $\begin{array}{l}1 \mathrm{yd}^{3} \text { cement with } \\
100 \text { pounds } \\
\text { bentonite and } 1 \% \\
\text { calcium chloride }\end{array}$ \\
\hline $04-D e c-89$ & $\begin{array}{l}\text { Tagged top of cement at } 379 \mathrm{ft} \text { bls. } \\
\text { Drilled out grout, bottom at about } 409 \\
\mathrm{ft} \text { bls. Drilled into basalt to } 454 \mathrm{ft} \\
\text { bls. }\end{array}$ & \\
\hline $05-D e c-89$ & $\begin{array}{l}\text { Drilled from } 454 \text { to a total depth of } \\
510.4 \mathrm{ft} \text { bls. Used water for } \\
\text { circulation }(5 \mathrm{gpm}) \text { below } 483 \mathrm{ft} \text {. }\end{array}$ & \\
\hline 06-Dec-89 & $\begin{array}{l}\text { USGS onsite to perform borehole logging. } \\
\text { Water table measured at } 470.2 \mathrm{ft} \text { bis. }\end{array}$ & \\
\hline \multirow[t]{2}{*}{ 13-Dec-89 } & $\begin{array}{l}\text { Set Hydrostar piston pump in well with } \\
\text { intake at } 480 \mathrm{ft} \text { bls. }\end{array}$ & \\
\hline & $\begin{array}{l}\text { Installed locking cap. Surface } \\
\text { completion included concrete pad and } \\
\text { impingement posts. }\end{array}$ & \\
\hline
\end{tabular}


C. Summary of Well Construction Procedures:

\begin{tabular}{|c|c|c|}
\hline DATE & ACTIVITY & MATERIALS USED \\
\hline $06-$ Dec-89 & Poured bentonite into the borehole. & $\begin{array}{l}4 \text { buckets } \\
\text { bentonite }\end{array}$ \\
\hline $07-$ Dec -89 & $\begin{array}{l}\text { Installed } 4 \text {-inch ID, Type } 304 \text {, stainless } \\
\text { steel, wire-wrapped well screen }(0.02- \\
\text { inch slot size) below the water table } \\
\text { with an endplug attached to the bottom } \\
\text { of the screen. Joined the well screen } \\
\text { to } 4 \text {-inch nominal pipe size, stainless } \\
\text { steel well casing and joired the } \\
\text { stainless steel well casing to the } \\
4 \text {-inch nominal pipe size carbon steel } \\
\text { well casing with a dielectric union. A } \\
\text { centralizer was placed at } 459,300 \text {, and } \\
\text { lo0 ft bls. The } 4 \text {-inch casing was } \\
\text { welded to the } 10-\text {-inch casing with } 2 \\
\text { angles, and the } 4 \text {-inch casing was cut } \\
\text { off to allow } 2 \text { ft of stick-up. } \\
\text { Installed filter pack consisting of } \\
\text { grade-20 silica sand, free falling from } \\
\text { land surface. A bentonite seal was } \\
\text { installed above the filter pack, using a } \\
\text { tremie pipe. }\end{array}$ & $\begin{array}{l}33 \mathrm{ft} \text { stainless } \\
\text { steel screen, } 1 \\
\text { end plug, } 5 \mathrm{ft} \\
\text { stainless steel } \\
\text { casing, } 0.5 \mathrm{ft} \\
\text { dielectric joint, } \\
460 \mathrm{ft} \text { of carbon } \\
\text { steel casing, } 17 \\
\text { bags silica sand, } \\
6 \text { buckets } \\
\text { bentonite }\end{array}$ \\
\hline 08-Dec-89 & $\begin{array}{l}\text { Began grouting well annulus with cement. } \\
\text { Tagged top of cement at } 450 \mathrm{ft} \text { bls. }\end{array}$ & $\begin{array}{l}70 \text { gallons cement } \\
\text { with sand and } \\
\text { bentonite added }\end{array}$ \\
\hline $09-D e c-89$ & $\begin{array}{l}\text { Poured } 4 \mathrm{yds}^{3} \text { cement into borehole using } \\
\text { a tremie pipe. }\end{array}$ & $\begin{array}{l}4 \mathrm{yd}^{3} \text { cement with } \\
\text { sand, bentonite, } \\
\text { and calcium } \\
\text { chloride }\end{array}$ \\
\hline 11-Dec-89 & $\begin{array}{l}\text { Continued grouting annular space in } \\
\text { borehole. }\end{array}$ & $5 \mathrm{yd}^{3}$ cement \\
\hline
\end{tabular}

D. Summary of Well Development Procedures:

\begin{tabular}{|c|c|c|}
\hline DATE & ACTIVITY & MATERIALS USED \\
\hline $12-\operatorname{Dec}-89$ & $\begin{array}{l}\text { Developed well using a surge block. } \\
\text { Surged three } 10 \mathrm{ft} \text { sections of the well } \\
\text { screen for about } 20 \text { minutes each. } \\
\text { Placed Grundfos } 5 \text { hp pump down the well } \\
\text { and pumped water at } 20 \text { gallons per } \\
\text { minute until parameters stabilized ( } 30 \\
\text { minutes). Pulled the pump. }\end{array}$ & \\
\hline
\end{tabular}


A. Geological log, geophysical logs, stratigraphic column, and well construction diagram are attached.

B. Samples collected.

Sample Number

Unknown

\section{Comments}

$42.4-47 \mathrm{ft}$, basalt plug in bottom of tube; tube collapsed.

Samples are stored in the INEL Core Library. 


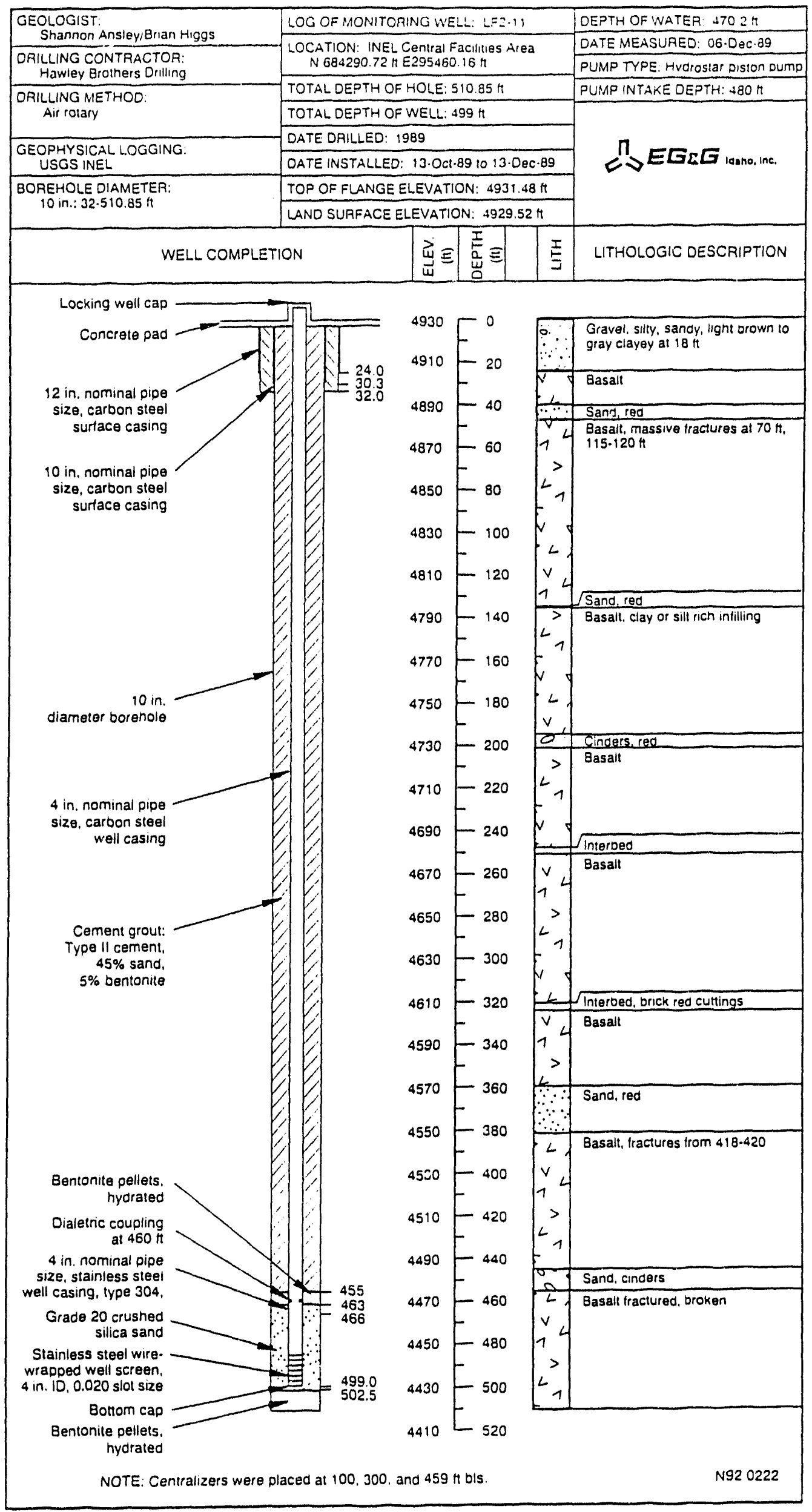




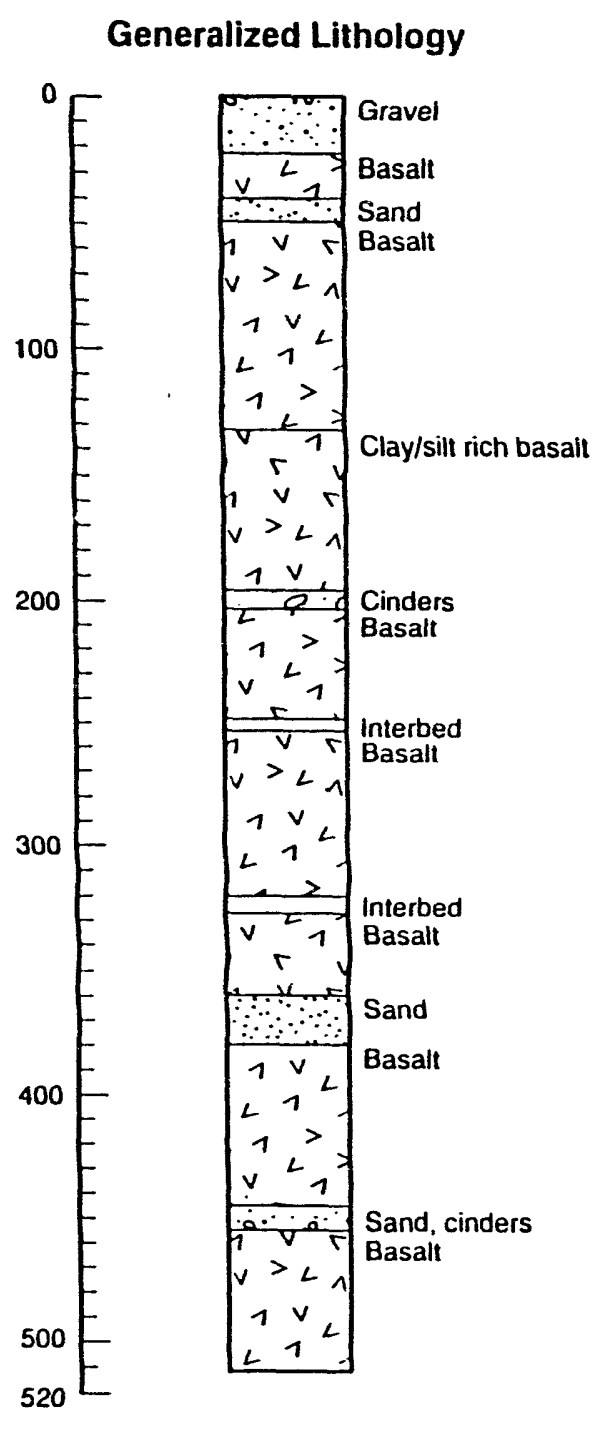

Geophysical logging December 1989

\section{Caliper}

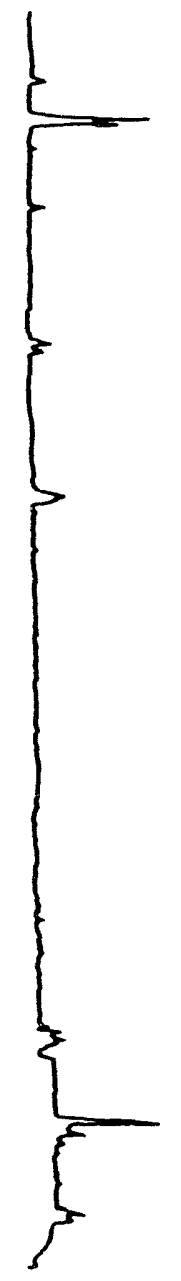

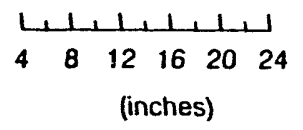

Gamma-Gamma

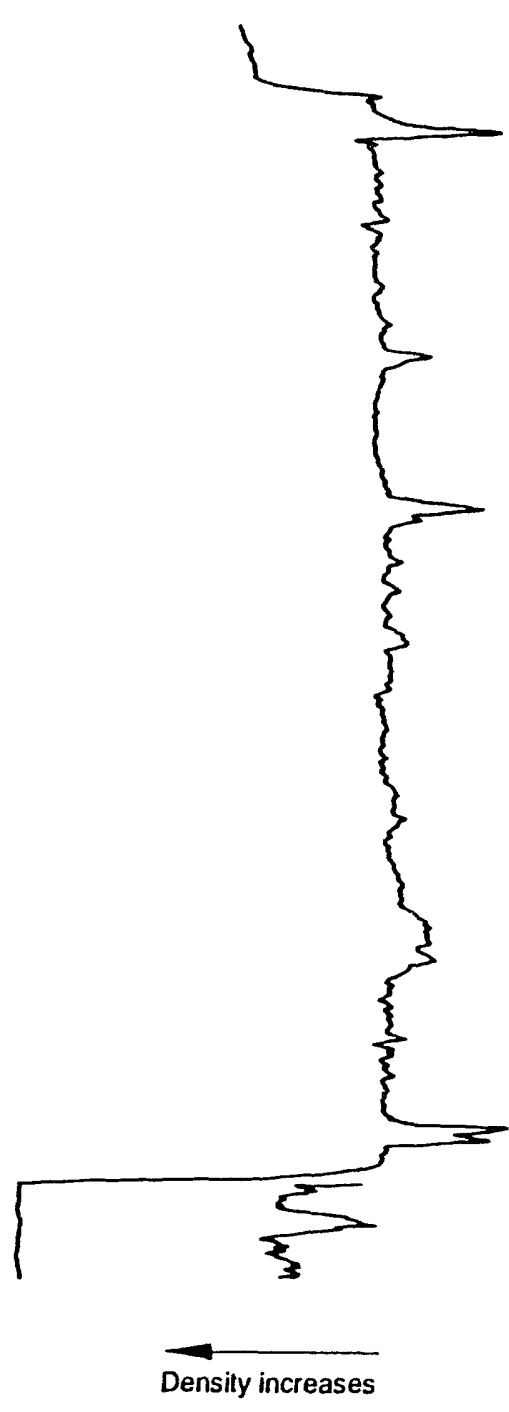

Densily increases
Natural Gamma

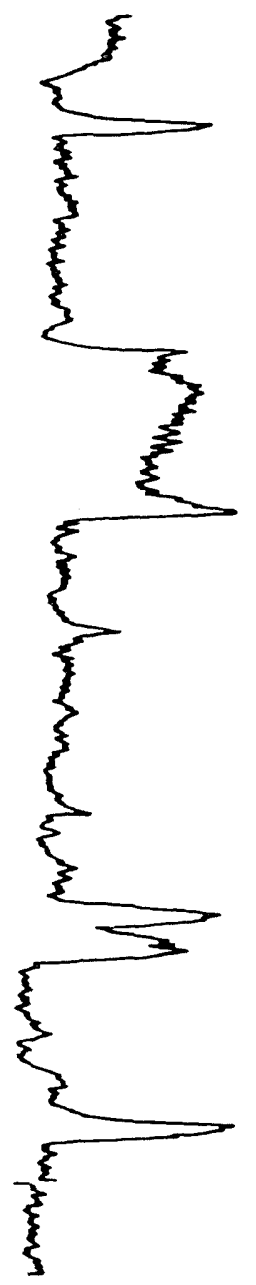

Radiation increases

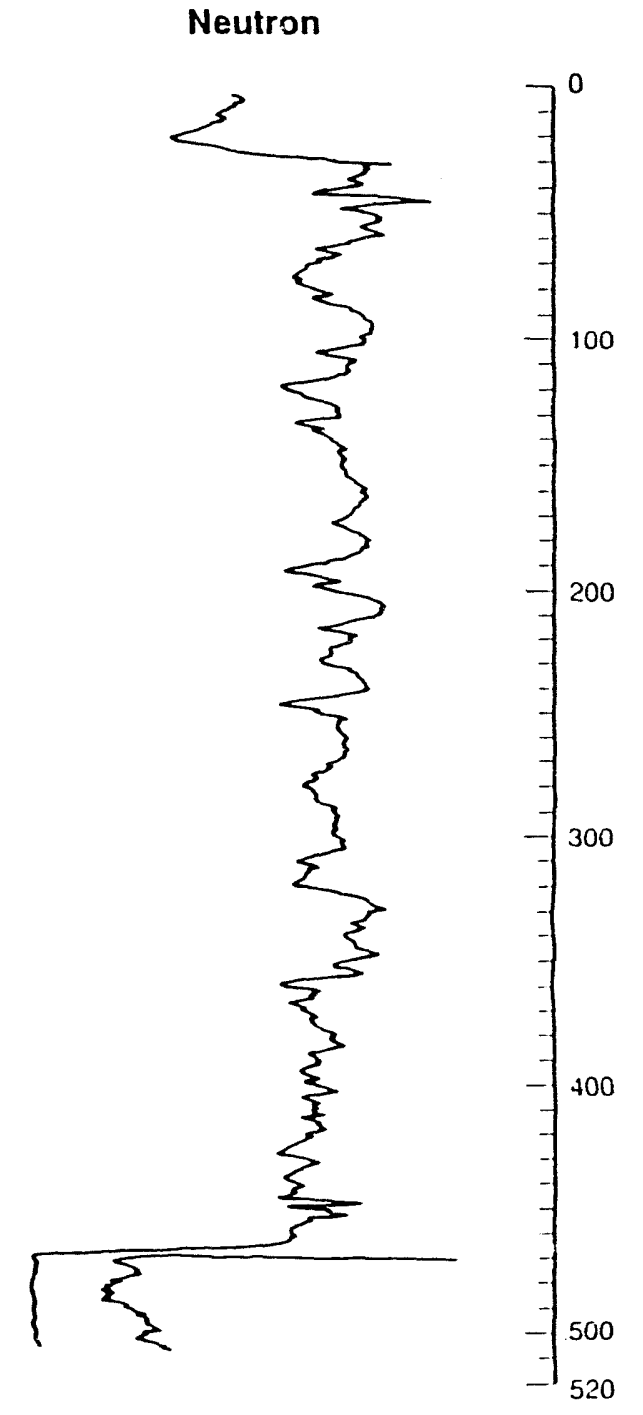

Moisture increases

Well CFA LF2-11 
END-OF-WELL REPORT

CENTRAL FACILITIES LANDFILL AREA

WELL LF2-12 
I. GENERAL INFORMATION

A. Project Name: Hydrogeologic Characterization Study for CFA Landfills

B. Well Name/Number: LF2-12

C. Well Location: INEL, Central Facilities Area Landfill II Northing: $682924.45 \mathrm{ft}$ Easting: $294018.71 \mathrm{ft}$ Land Surface Elevation: $4933.87 \mathrm{ft}$ (brass marker) NRTS datum

D. Plans:

Survey Date: December 1990, MK-Ferguson

1) Technical Work Plan for the Hydrogeologic Characterization of CFA Landfills II and III, EGG-WM-9119, JuTy 6, 1990.

2) Health and Safety Plan for Operations Performed for the Environmental Restoration Program, Well Installation and Groundwater

- Sampling at CFA Landfills II and III, EGG-WM-9105, May 1990.

E. Logbooks used:

1) Field Team Leader's Daily Logbook, ERP-29-90

2) Wel1 Drilling, Installation, and Development Logbook, ERP-146-90

\section{DRILLING AND COMPLETION OBSERVATIONS}

A. Drilling Company: PC Exploration

Drillers: Mack Stillwe11, Paul Harmon

On-Site Geologist: David Burgess

Project Manager: Shannon Ansley

Rig type: Reichdri11-700

Bit type: Tricone bit for surficial sediments, air hammer bit for basalt

B. Summary of Drilling Activities:

\begin{tabular}{|c|c|c|}
\hline DATE & ACTIVITY & MATERIALS USED \\
\hline $02-0 c t-90$ & $\begin{array}{l}\text { Drilled through surficial sediments and } \\
\text { into basalt with } 15 \text {-inch tricone bit. } \\
\text { Hit basalt at approximately } 23 \mathrm{ft} \text { bis. } \\
\text { TD for day was } 30 \mathrm{ft} \text { bls. }\end{array}$ & \\
\hline $03-0 c t-90$ & $\begin{array}{l}\text { Pushed 12-inch surface casing down to } 28 \\
\mathrm{ft} \text { bls. could not push to bottom of } \\
\text { hole, but is } 5 \text { to } 6 \mathrm{ft} \text { into the basalt }\end{array}$ & $\begin{array}{l}32 \mathrm{ft} \text { of } 12 \text {-inch } \\
\text { ID carbon steel } \\
\text { surface casing }\end{array}$ \\
\hline $04-0 c t-90$ & $\begin{array}{l}\text { Drilled with } 5.5-\text { inch hammer bit to top } \\
\text { of interbed at } 46 \text { ft bls. Collected } \\
\text { interbed samples (\#CF0090105, } \\
\text { CF00100105, and CF00110205) from } 46 \text { to } \\
50.5 \mathrm{ft} \text { bls. After interbed samples } \\
\text { were collected, the borehole was reamed } \\
\text { and drilled with an 11 } 7 / 8 \text {-inch bit to } \\
65 \mathrm{ft} \text { bls. Cement grouted the borehole. }\end{array}$ & $\begin{array}{l}2 \text { yd }^{3} \text { cement, with } \\
\text { bentonite, sand } \\
\text { and calcium } \\
\text { chloride added }\end{array}$ \\
\hline
\end{tabular}




\begin{tabular}{|c|c|c|}
\hline DATE & ACTIVITY & MATERIALS USED \\
\hline $05-0 c t-90$ & $\begin{array}{l}\text { Top of cement tagged at } 25 \mathrm{ft} \text { bls. } \\
\text { Drilled through cement and into basalt, } \\
\text { to } 100 \mathrm{ft} \text { bls. The borehole was } \\
\text { subsequently grouted. }\end{array}$ & $\begin{array}{l}4 y^{3} \text { cement, with } \\
\text { bentonite, sand, } \\
\text { and calcium } \\
\text { chloride added }\end{array}$ \\
\hline $08-0 c t-90$ & $\begin{array}{l}\text { Top of cement tagged at } 50 \mathrm{ft} \text { bls. } \\
\text { Drilled to } 257 \mathrm{ft} \text { bls. Hit interbed at } \\
\text { approximate y } 225 \mathrm{ft} \text { bls, back in basalt } \\
\text { at } 235 \mathrm{ft} \text { bls. Adding water spray to } \\
\text { circulation to keep down dust. Grouted } \\
\text { the borehole to } 120 \mathrm{ft} \text { bls. }\end{array}$ & $\begin{array}{l}5.5 \text { yd }^{3} \text { cement, } \\
\text { with bentonite, } \\
\text { sand, and calcium } \\
\text { chloride added }\end{array}$ \\
\hline $09-0 c t-90$ & $\begin{array}{l}\text { Drilled to } 337 \mathrm{ft} \text { bls. Took a deviation } \\
\text { measurement of } 1 \text { degree from vertical, } \\
\text { at } 200 \mathrm{ft} \text { bls then grouted the borehole. }\end{array}$ & $\begin{array}{l}4 \text { yd }^{3} \text { cement, with } \\
\text { sand, bentonite, } \\
\text { and calcium } \\
\text { chloride }\end{array}$ \\
\hline $10-0 c t-90$ & $\begin{array}{l}\text { Tagged the top of the grout at } 243 \mathrm{ft} \\
\text { bls and drilled to } 374 \mathrm{ft} \text { bls. Had } \\
\text { trouble with the borehole sloughing in } \\
\text { from an interbed/cinder zone at } 374 \text { to } \\
380 \mathrm{ft} \text {. }\end{array}$ & \\
\hline $11-0 c t-90$ & $\begin{array}{l}\text { Cleaned out hole, drilled to } 377 \mathrm{ft} \mathrm{bls,} \\
\text { and grouted. }\end{array}$ & $\begin{array}{l}3.5 \mathrm{yd}^{3} \text { cement, } \\
\text { with sand, } \\
\text { bentonite, and } \\
\text { calcium chloride } \\
\text { added }\end{array}$ \\
\hline $12-0 c t-90$ & $\begin{array}{l}\text { Tagged top of grout at } 289 \mathrm{ft} \mathrm{bls.} \\
\text { Drilled to } 437 \mathrm{ft} \text { bls. Grouted } \\
\text { borehole. }\end{array}$ & $\begin{array}{l}? \text { yd }^{3} \text { cement with } \\
\text { sand, bentonite, } \\
\text { and calcium } \\
\text { chloride added }\end{array}$ \\
\hline $15-0 c t-90$ & $\begin{array}{l}\text { Tagged top of grout at } 327 \mathrm{ft} \text { bls, } \\
\text { cement was still wet. Drilled through } \\
\text { cement and took a deviation measurement } \\
\text { at } 400 \mathrm{ft} \text { bls, which was } 1.5 \text { degrees } \\
\text { from vertical. Drilled to a total depth } \\
\text { of } 517 \mathrm{ft} \text { bls and measured the water } \\
\text { table at } 489 \mathrm{ft} \text { bls. }\end{array}$ & \\
\hline $16-0 c t-90$ & $\begin{array}{l}\text { Before the circulating air process } \\
\text { began, the water level was measured at } \\
475.5 \mathrm{ft} \text { bls. Caving had occurred from } \\
517 \text { to } 509 \mathrm{ft} \text { bis. Some bridging or } \\
\text { obstruction was noticed in borehole at } \\
384 \mathrm{ft} \text { bls. USGS sent a camera down the } \\
\text { hole for a TV log. Log showed a slight } \\
\text { offset in the hole at approximately } 380 \\
\text { ft bls. Cement had not held well. }\end{array}$ & \\
\hline
\end{tabular}


C. Summary of Wel1 Construction Procedures:

\begin{tabular}{|c|c|c|}
\hline DATE & ACTIVITY & MATERIALS USED \\
\hline $17-0 c t-90$ & $\begin{array}{l}\text { Installed } 4 \text {-inch ID, passivated, Type } \\
304 \text {, stainless steel, wire wrapped well } \\
\text { screen }(0.02 \text {-inch slot size) below water } \\
\text { table with a threaded, passivated } \\
\text { endplug attached to the bottom of the } \\
\text { screen. Joined the well screen to } 4- \\
\text { inch nominal pipe size }(4.5-\text { inch } 00) \text {, } \\
\text { Schedule } 105 \text {, Type } 304 \text {, flush threaded, } \\
\text { passivated, stainless steel well casing. } \\
\text { Installed centralizers at } 469 \mathrm{ft}, 334 \\
\text { ft, and } 163 \mathrm{ft} \text { bls. Began installing } \\
\text { filter pack, using the tremie pipe. } \\
\text { Installed } 8-12 \text { grade and } 6-9 \text { grade sand } \\
\text { to } 492 \mathrm{ft} \text { bls, then stopped for the day. }\end{array}$ & $\begin{array}{l}22 \mathrm{ft} \text { well screen; } \\
470 \mathrm{ft} \text { well } \\
\text { casing; top and } \\
\text { bottom caps; three } \\
\text { centralizers; } 7 \\
\text { sacks silica sand } \\
\text { ( } 8-12 \text { grade); } 10 \\
\text { sacks silica sand } \\
\text { (6-9 grade) }\end{array}$ \\
\hline $18-0 c t-90$ & $\begin{array}{l}\text { Completed installation of filter pack } \\
\text { consisting of } 6-9 \text { grade silica sand, } \\
10-20 \text { grade silica sand. A bentonite } \\
\text { seal was installed above the filter } \\
\text { pack. Water was added to the bentonite }\end{array}$ & $\begin{array}{l}24 \text { sacks silica } \\
\text { sand }(6-9 \text { grade }) \\
1 \text { sack silica sand } \\
\text { (10-20 grade); } 25 \\
\text { gallons bentonite } \\
\text { pellets, hydrated }\end{array}$ \\
\hline $\begin{array}{l}19-0 c t-90 \\
\text { to } \\
23-0 c t-90\end{array}$ & $\begin{array}{l}\text { Grouted well annulus with cement mix, } \\
\text { using tremie pipe. }\end{array}$ & $\begin{array}{l}12 \text { ( } 94 \text { pound) } \\
\text { sacks cement; } 16 \\
\text { ( } 70 \text { pound) sacks } \\
\text { sand; } 22 \text { pounds of } \\
\text { bentonite; } 14 \text { yd }^{3} \\
\text { cement with sand } \\
\text { and bentonite } \\
\text { added }\end{array}$ \\
\hline $24-0 c t-90$ & $\begin{array}{l}\text { Cut } 12 \text {-inch casing off at ground level, } \\
\text { installed } 6 \text {-inch ID, locking, carbon } \\
\text { steel, protective casing. Surface } \\
\text { completion included concrete pad and } \\
\text { impingement posts. }\end{array}$ & $\begin{array}{l}6 \mathrm{ft} \text { of } 6 \text {-inch ID } \\
\text { carbon steel } \\
\text { protective casing }\end{array}$ \\
\hline $16-N o v-90$ & $\begin{array}{l}\text { Installed Hydrostar piston pump. Pump } \\
\text { intake set at } 481.36 \mathrm{ft} \text { b1 s. }\end{array}$ & \\
\hline
\end{tabular}


B. Summary of Well Development Procedures:

\begin{tabular}{|c|c|c|}
\hline DATE & ACTIVITY & MATERIALS USED \\
\hline $16-N o v-90$ & $\begin{array}{l}\text { Surged well for } 60 \text { minutes using a surge } \\
\text { block. Lowered a submersible pump into } \\
\text { the well and began evacuating water. } \\
\text { Measured specific conductance, } \\
\text { temperature, pH, and dissolved oxygen of } \\
\text { the development water. Pumped } \\
\text { approximately } 300 \text { gallons, until } \\
\text { parameters stabilized. }\end{array}$ & \\
\hline
\end{tabular}

\section{GEOLOGICAL OBSERVATIONS}

A. Geological log, geophysical logs, stratigraphic column, and well construction diagram are attached.

B. Samples collected.

Sample Depth

$46-50.5 \mathrm{ft}$
Comments

Sample numbers are \#CF0090105, \#CF0010105, \#CF00110205

Samples are stored in the INEL Core Library

NOTE: The borehole number LF2-12A was recorded in the logbooks throughout the drilling and completion of this borehole. The number was changed to LF2-12 before the December 1990 surveying of the boreholes. 


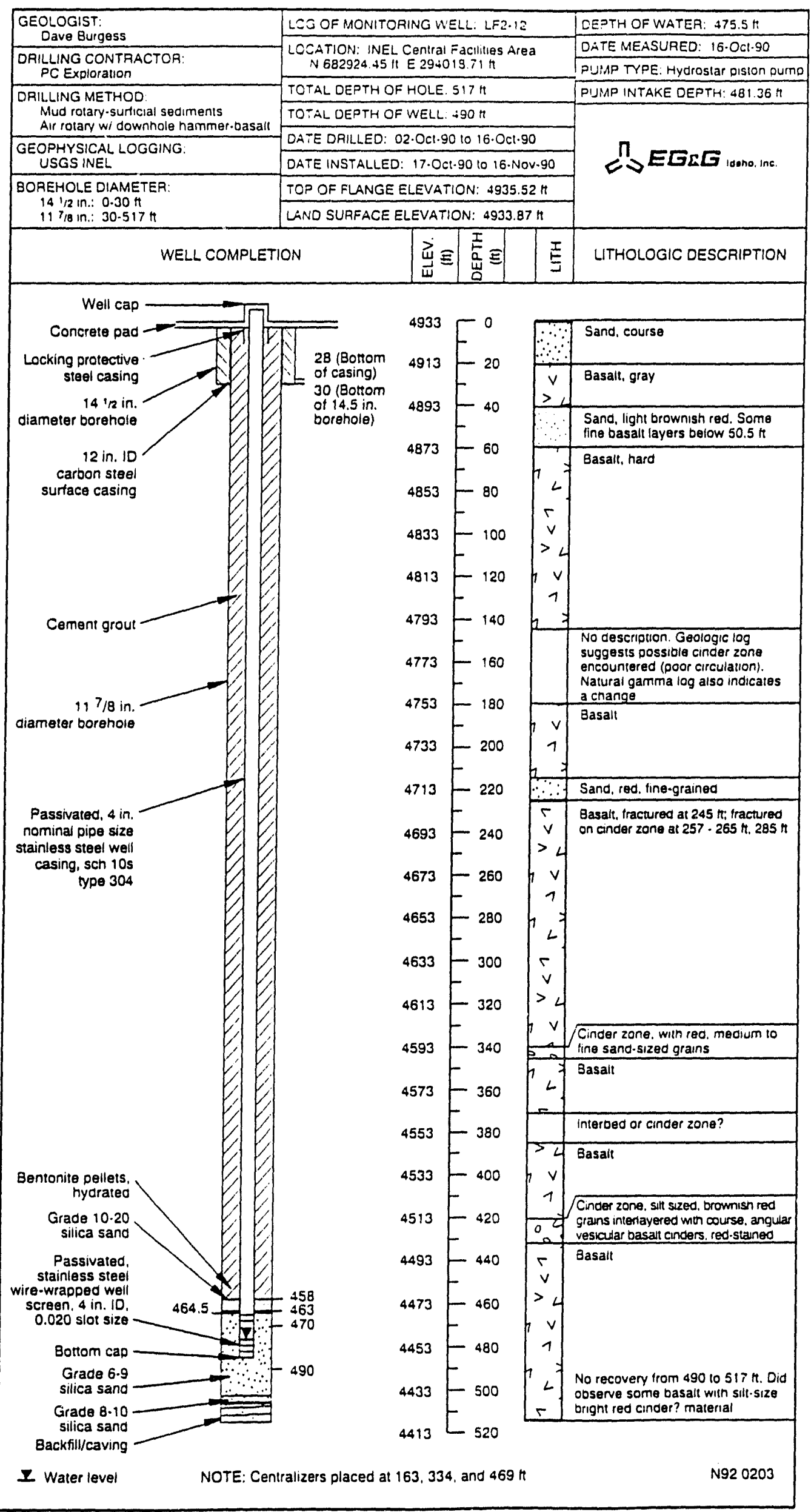



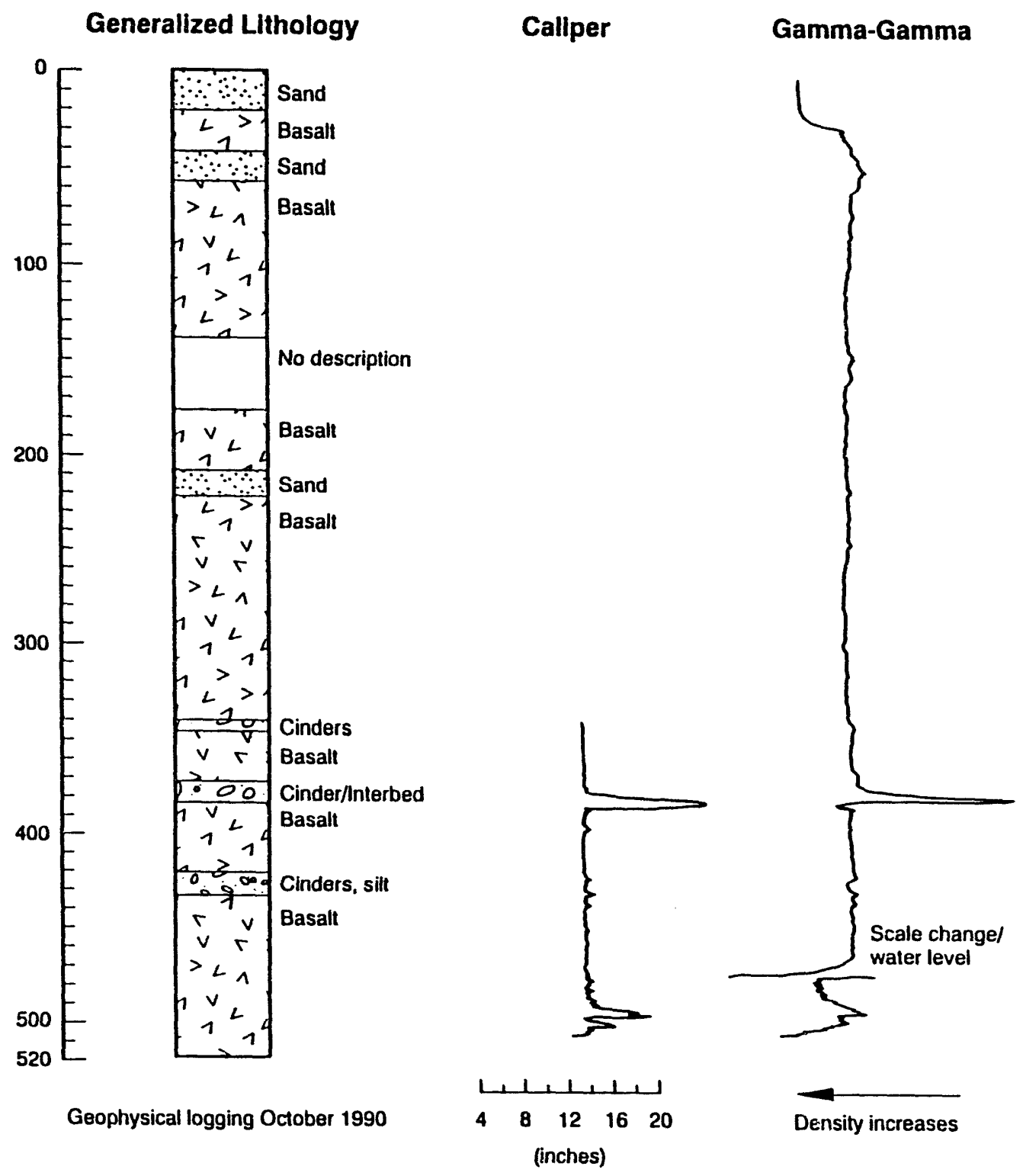

Natural Gamma

Well CFA LF2-12

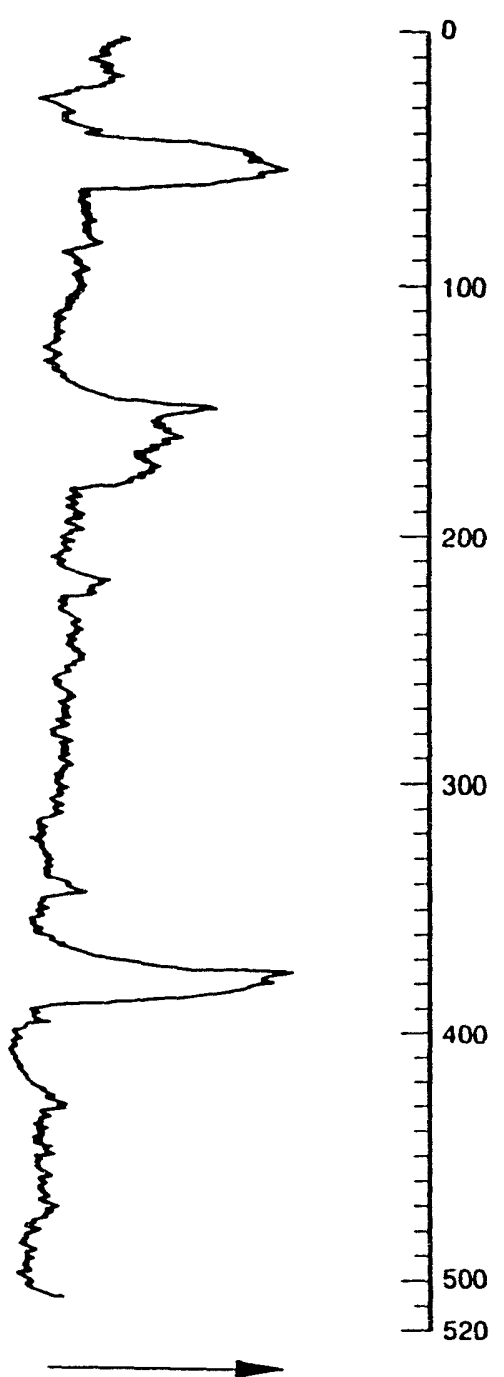

Radiation increases 
END-OF-WELL REPORT

CENTRAL FACILITIES LANOFILL AREA

WELL LF2-12A 
I. GENERAL INFORMATION

A. Project Name: Hydrogeologic Characterization Study for CFA Landfi $11 \mathrm{~s}$

B. Well Name/Number: LF2-12A

C. Well Location: INEL, Central Facilities Area Landfill II Northing: $683203.14 \mathrm{ft}$ Easting: $294037.19 \mathrm{ft}$ Land Surface Elevation: $4933.7 \mathrm{ft}$ (NRTS datum)

D. Plans: Survey Date: December 1990, MK-Ferguson

1) Technical Work Plan for the Hydrogeologic Characterization of CFA Landfills II and III, EGG-WM-9119, July 6, 1990.

2) Health and Safety Plan for Operations Performed for the Environmental Restoration Program, Well Installation and Groundwater Sampling at CFA Landfills II and III, EGG-WM-9105, May 1990.

E. Logbooks used:

1) Field Team Leader's Daily Logbook, ERP-26-90

2) Well Drilling, Installation, and Development Logbook, ERP-146-90

\section{DRILLING AND COMPLETION OBSERVATIONS}

A. Drilling Company: PC Exploration

Drillers: Gregg Hickman, Bill Pavlock / Mack Stillwell, Paul Harmon

On-Site Geologist: John Ruth, Shannon Ansley, Deborah McElroy

Project Manager: Shannon Ansley

Rig type: Reichdrill-700

Bit type: Tricone bit for surficial sediments, air hammer bit for basalt

\section{B. Summary of Drilling Activities:}

\begin{tabular}{||l|l|l||}
\hline DATE & \multicolumn{1}{|c|}{ ACTIVITY } & MATERIALS USED \\
\hline $11-J u T y-90$ & Decon equipment, take equipment blank. & \\
\hline 12-July-90 & $\begin{array}{l}\text { Used mud rotary to drill through } \\
\text { surficial sediments with 14.5-inch } \\
\text { diameter drill bit. Hit basalt at 25 to } \\
26 \mathrm{ft} \mathrm{bls.} \mathrm{TD} \mathrm{for} \mathrm{day} \mathrm{was} \mathrm{26.5} \mathrm{ft.}\end{array}$ & $\begin{array}{l}\text { Hi-yield bentonite } \\
\text { and water }\end{array}$ \\
\hline $13-J u i y-90$ & $\begin{array}{l}\text { Drilled to 30.9 ft bls. Surface casing } \\
\text { (12-inch) pushed to 29.9 ft bls, 1 ft } \\
\text { short of total depth. Could not push } \\
\text { any further. Pressure grouted annulus. }\end{array}$ & $\begin{array}{l}\text { 2 yd Type I and } \\
\text { with bentonite; 33 } \\
\text { ft of 12-inch ID } \\
\text { carbon steel } \\
\text { surface casing }\end{array}$ \\
\hline
\end{tabular}




\begin{tabular}{|c|c|c|}
\hline DFTE & ACTIVITY & MATERIALS USED \\
\hline $18-J u T y-90$ & $\begin{array}{l}\text { Planned to use } 5.5 \text {-inch bit to drill } \\
\text { through basalt to first interbed, where } \\
\text { samples will be collected. A larger } 11 \\
7 / 8 \text {-inch bit is used after sample } \\
\text { collection is completed. Drilled to } 48 \\
\text { ft with } 5.5 \text {-inch bit and reached first } \\
\text { interbed. }\end{array}$ & \\
\hline 19-JuTy-90 & $\begin{array}{l}\text { Collected shelby tube sample from } 48 \text { to } \\
50.3 \mathrm{ft} \mathrm{bls.}\end{array}$ & \\
\hline $20-J u 1 y-90$ & $\begin{array}{l}\text { Reamed shelby tube hole to } 50 \mathrm{ft} \text { bls, } \\
\text { with } 117 / 8 \text {-inch bit. Collected a } \\
\text { shelby tube sample from } 50.3 \text { to } 52.6 \mathrm{ft} \\
\text { bls, } 54.0 \text { to } 55.8 \mathrm{ft} \text { bls, } 55.3 \text { to } 57.6 \\
\mathrm{ft} \text { bls, } 57.1 \text { to } 59.4 \mathrm{ft} \text { bls. Hit solid } \\
\text { basalt at } 59.6 \mathrm{ft} \mathrm{bls,} \mathrm{and} \mathrm{drilled} \mathrm{to} 70 \\
\mathrm{ft} \text { bls. }\end{array}$ & \\
\hline $23-J u T y-90$ & $\begin{array}{l}\text { Began reaming borehole with } 117 / 8 \text {-inch } \\
\text { hammer bit. Stopped reaming at } 19.1 \mathrm{ft} \\
\text { bls because surface casing had slipped } \\
\text { down about } 0.5 \text { ft. Surface casing is no } \\
\text { longer sealed. Crew welded an extra } 1 \\
\text { ft of surface casing on to the existing } \\
\text { surface casing. }\end{array}$ & \\
\hline 24-July-90 & Reamed to $69 \mathrm{ft}$ bls. & \\
\hline $25-J u 7 y-90$ & $\begin{array}{l}\text { Took a deviation measurement at } \\
\text { approximately } 94 \mathrm{ft} \text { bls, which was } 2 \\
\text { degrees from vertical. Drilled to } 114.5 \\
\text { ft bls. Using some water spray in hole. }\end{array}$ & \\
\hline $26-J u 1 y-90$ & Drilled to $171.7 \mathrm{ft}$ bls. & \\
\hline $27-J u 7 y-90$ & $\begin{array}{l}\text { Drilled to } 200 \mathrm{ft} \text { bls. Took deviation } \\
\text { measurement at } 180 \mathrm{ft} \text { bls, which was } 5 \\
\text { degrees from vertical. }\end{array}$ & \\
\hline $30-J u 1 y-90$ & $\begin{array}{l}\text { Repeated deviation measurements; } 0.5 \\
\text { degrees from vertical at } 100 \mathrm{ft} \text { bls, } 0 \\
\text { degrees from vertical at } 200 \mathrm{ft} \text { bls. } \\
\text { Survey instrument was set in hammer and } \\
\text { appeared to work better. Drilled to } 226 \\
\mathrm{ft} \text { bls. }\end{array}$ & \\
\hline $31-J u 7 y-90$ & $\begin{array}{l}\text { Took a deviation measurement at } 295 \mathrm{ft} \\
\text { bls, which was } 1 \text { degree from vertical. } \\
\text { Drilled to } 375 \mathrm{ft} \text { bls. Drill string } \\
\text { became stuck in borehole; can rotate bit } \\
\text { but cannot pull up. }\end{array}$ & \\
\hline
\end{tabular}




\begin{tabular}{|l|l|c|}
\hline \multicolumn{1}{|c|}{ DATE } & \multicolumn{1}{|c|}{ ACTIVITY } & MATERIALS USED \\
\hline 01-Aug-90 & Working to pull drill string. Finally & \\
to & we were able to pull out the drill pipe & \\
04-Sept-90 & $\begin{array}{l}\text { and collar but the drill bit unscrewed } \\
\text { and remained in the borehole USGS ran }\end{array}$ & \\
& TV log and observed a water ievel at & \\
& 317 ft bls, but this was attributed to & \\
& drilling fluid and dissipated the & \\
& following day. Drillers tried fishing & \\
& for the drill bit, but attempts were & \\
& unsuccessfuT. Decision was made to & \\
& abandon the borehole. & \\
\hline
\end{tabular}

\section{Summary of Construction/Abandonment Procedures:}

On 13-Dec-1990, the bottom of the borehole was tagged at $357 \mathrm{ft} \mathrm{bls.}$ The borehole was then backfilled with cement to $60 \mathrm{ft}$ bls, using 18.5 $\mathrm{yd}^{3}$ of cement. On 14-Dec-1990, the surface casing was cut to 6 inches bls and the borehole was grouted to $5 \mathrm{ft}$ bls using $16 \mathrm{yd}^{3}$ of cement. The borehole was later backfilled with cement to the surface, and a cement pad was poured over the top of the well casing. A brass marker was placed in the grout for identification and survey use.

The logbooks did not document the entire abandonment procedure for this borehole. The information not documented in the logbook was verbally communicated by Shannon Ansley (Project Manager during the drilling) on September 1, 1990.

NOTE: The borehole number LF2-12 was used in the logbooks, to record the drilling of the borehole. The number was changed to LF2-12A before the December 1990 location survey, in order to designate the borehole as abandoned $(A)$.

\section{GEOLOGICAL OBSERVATIONS}

A. Geological log, stratigraphic column, and well construction/abandonment diagram are attached.

B. Samples collected. Core samples are stored in the INEL Core l.ibrary.

$$
\begin{aligned}
& \text { Sample Depth } \\
& \hline 48-50.3 \mathrm{ft} \\
& 50.3-52.6 \mathrm{ft} \\
& 54.0-55.8 \mathrm{ft} \\
& 55.3-57.6 \mathrm{ft} \\
& 57.1-59.4 \mathrm{ft}
\end{aligned}
$$

Comment

$1 \mathrm{ft}$ of recovery $(48-49 \mathrm{ft}$ bls $)$

$2.3 \mathrm{ft}$ of undisturbed sample. Top $0.5 \mathrm{ft}$ is $\mathrm{fi} 11,54-55.8 \mathrm{ft}$ is undisturbed sediment.

$0.8 \mathrm{ft}$ of $\mathrm{fill}$ on top, $1.5 \mathrm{ft}$ undisturbed core

$0.6 \mathrm{ft}$ of $\mathrm{fill}$ on top, $1.7 \mathrm{ft}$ undisturbed core 
Rinsate sample (Equipment Blank):

\#61071190-01

\#61071190-02A

$\# 61071190-02 \mathrm{~B}$

\#61071190-03
TCCP metals

VOA

VOA

VOA-trip blank 


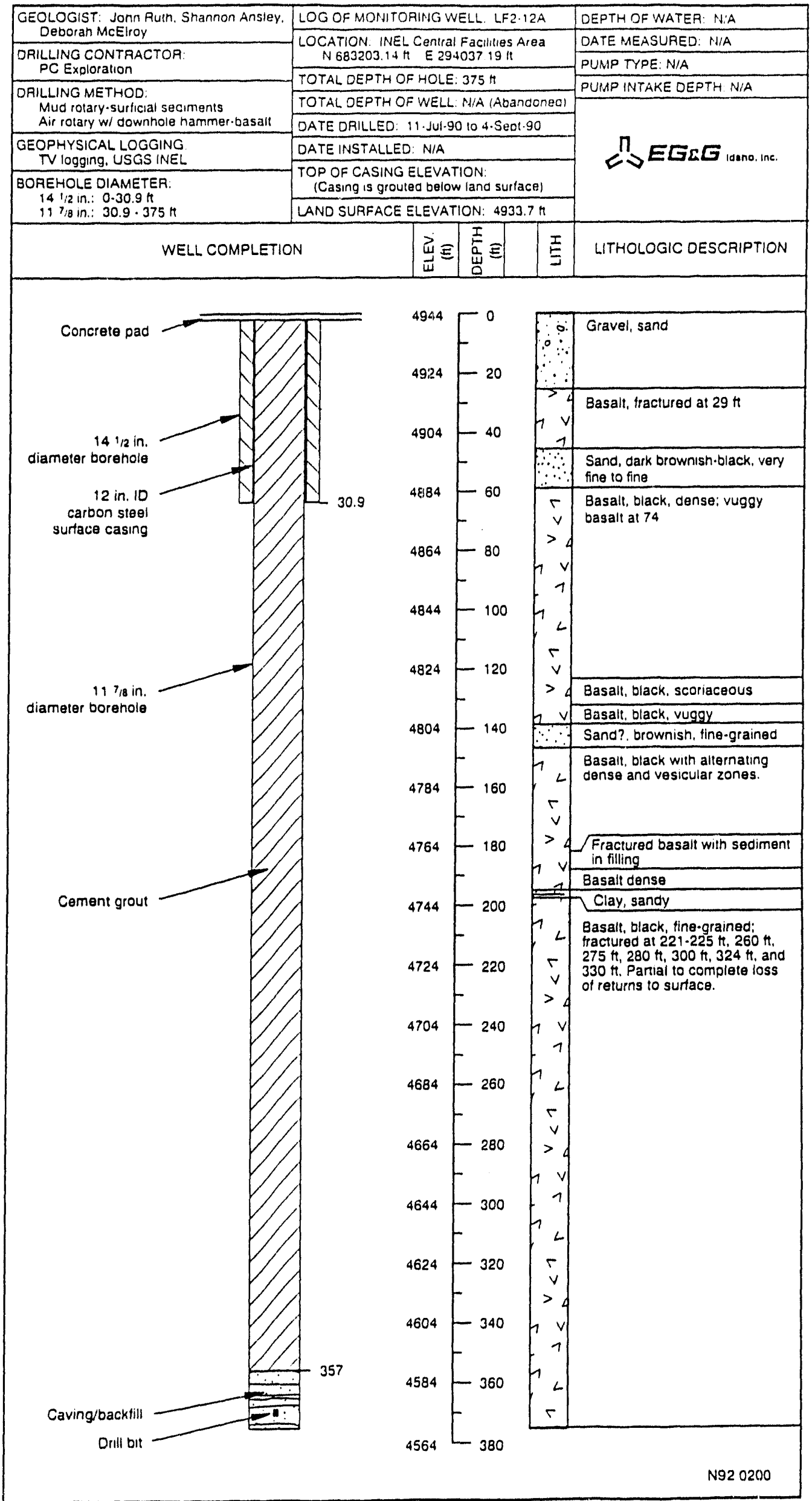


END-OF-WELL REPORT

CENTRAL FACILITIES LANDFILL AREA WELL LF3-08 
I. GENERAL INFORMATION
A. Project Name:
Hydrogeologic Characterization Study for CFA

B. Well Name/Number: LF3-08

C. Well Location: INEL, Central Facilities Area Landfill III Northing: $683111.08 \mathrm{ft}$ Easting: $291537.83 \mathrm{ft}$
Land Surface Elevation: $4941.43 \mathrm{ft}$ (brass marker)
Survey Date: December 1990, MK-Ferguson

D. Plans:

1) Hydrogeologic Characterization Study for CFA Landfills II and III, Idaho National Engineering Laboratory, Technical Work Plan, submitted to EG\&G Idaho, Inc. by Science Applications International Corporation, Apri1 15, 1988.

2) Hydrogeologic Characterization for CFA Landfills II and III, Idaho National Engineering Laboratory, Technical Work Plan, EGG-ER-8670, August 1989.

3) Statement of Work for Groundwater Monitoring Well Construction at the CFA Landfills II and III, EG\&G Idaho, Inc.

4) Synopsis of Work, Hydrogeologic Characterization Study for CFA Landfills II and III, 31 January 1989 letter report, John Jaacks, Science Applications International Corporation.

5) Hydrogeologic Characterization Study at CFA Landfills II and III, Health and Safety Plan, EGG-ER-8445, February 1989.

E. Logbooks used:

1) Science Applications International Corporation Logbooks, submitted to EG\&G Idaho, Inc., 08/May/88 to $15 /$ Aug/88.

2) Field Team Leader's Daily Logbook, ERP Logbook CFA-003.

3) Field Team Leader's Daily Logbook, ERP Logbook CFA-001.

\section{DRILLING AND COMPLETION OBSERVATIONS}

A. Drilling Company: Denning Well Drilling

Drillers: Jody Denning

On-Site Geologist: John Jaacks

Project Manager: Larry Hull

Rig type: 650 Chicago Pneumatic

Bit type: Unknown

B. Summary of Drilling Activities:

\begin{tabular}{|c|l|c|}
\hline DATE & \multicolumn{1}{|c|}{ ACTIVITY } & MATERIALS USED \\
\hline 08-May-88 & $\begin{array}{l}\text { Surface casing was driven to refusal, 17 } \\
\mathrm{ft} \text { bls. }\end{array}$ & \\
\hline
\end{tabular}




\begin{tabular}{|c|c|c|}
\hline DATE & ACTIVITY & MATERIALS USED \\
\hline $09-$ May -88 & $\begin{array}{l}\text { The logbooks do not directly state the } \\
\text { type of drilling that was used, but } \\
\text { occasional references to a compressor, } \\
\text { water truck, and foam in the cuttings } \\
\text { suggest the drilling method was air } \\
\text { rotary and that water and/or foam were } \\
\text { added as needed to lubricate the bit or } \\
\text { lift cuttings to the surface. We } \\
\text { drilled to } 57 \text { ft bls and then attempted } \\
\text { to core to } 70 \mathrm{ft} \text { bls. Coring of samples } \\
\text { was unsuccessful, so the collection } \\
\text { method was changed to split spoon } \\
\text { sampling. Only two samples were } \\
\text { recovered from the } 57 \text { to } 70 \text { ft interval. }\end{array}$ & \\
\hline $10-$ May-88 & $\begin{array}{l}\text { Cement grouted the sedimentary interval. } \\
\text { Top of grout was at } 40 \mathrm{ft} \text { bls. }\end{array}$ & $\begin{array}{l}1 \mathrm{yd}^{3} \text { expanding } \\
\text { cement }\end{array}$ \\
\hline $11-$ May -88 & $\begin{array}{l}\text { Drilled through the grout to } 70 \mathrm{ft} \text { bls. } \\
\text { Drilled into basalt to } 166 \mathrm{ft} \text { bls. }\end{array}$ & \\
\hline $12-$ May-88 & Drilled from 166 to $220 \mathrm{ft}$ bls. & \\
\hline $13-$ May -88 & Drilled from 220 to $306 \mathrm{ft}$ bls. & \\
\hline $16-M a y-88$ & Drilled from 306 to $386 \mathrm{ft}$ bls. & \\
\hline $17-$ May -88 & Drilled from 386 to $459 \mathrm{ft}$ bls. & \\
\hline $23-$ May-88 & $\begin{array}{l}\text { Drilled to a total depth of } 526 \mathrm{ft} \text { bls. } \\
\text { Water was encountered at } 486 \mathrm{ft} \mathrm{b} \mathrm{b} s .\end{array}$ & \\
\hline 25-May-88 & $\begin{array}{l}\text { USGS arrived onsite to perform borehole } \\
\text { logging. Performed TV, natural gamma, } \\
\text { and caliper logs. }\end{array}$ & \\
\hline 19-Sept - 88 & $\begin{array}{l}\text { USGS performed neutron and gamma-gamma } \\
\text { logs of the borehole. }\end{array}$ & \\
\hline
\end{tabular}


C. Summary of Well Construction Procedures:

\begin{tabular}{|c|c|c|}
\hline DATE & ACTIVITY & MATERIALS USED \\
\hline $11-J u 1 y-88$ & $\begin{array}{l}\text { Installed well screen and well casing to } \\
510 \text { ft bls, with } 1.5 \text { ft of casing above } \\
\text { ground level. Casing and screen } \\
\text { descriptions are a best guess' of what } \\
\text { was placed in LF3-08. Casing and screen } \\
\text { sizes were not recorded in the logbooks. } \\
\text { Casing and screen descriptions were } \\
\text { found in well construction plans (see } \\
\text { Section I-D) and construction diagrams } \\
\text { (filled out after actual construction } \\
\text { was finished). } \\
\text { Installed } 6 \text { inch ID Type } 304 \text { stainless } \\
\text { steel well screen, wire-wrapped (0.02- } \\
\text { inch slot size) with an endplug, below } \\
\text { the water table. The well screen was } \\
\text { joined to } 6 \text { inch nominal pipe size, Type } \\
304 \text { stainless steel well casing. The } \\
\text { stainless steel well casing was joined } \\
\text { to } 6 \text { inch carbon steel well casing with } \\
\text { a dielectric coupling at } 460 \text { ft bls. } \\
\text { Three centralizers were installed, } \\
\text { depths are not known. Construction } \\
\text { plans called for flush-threaded well } \\
\text { screen, well casing, and a } \\
\text { flush-threaded endplug. }\end{array}$ & $\begin{array}{l}10 \mathrm{ft} \text { of } 6 \text {-inch ID } \\
\text { stainless steel } \\
\text { well screen, } 40 \mathrm{ft} \\
\text { of } 6 \text { inch nominal } \\
\text { pipe size } \\
\text { stainless steel } \\
\text { well casing, } \\
464 \mathrm{ft} \text { of } 6 \text { inch } \\
\text { nominal pipe size } \\
\text { carbon steel well } \\
\text { casing }\end{array}$ \\
\hline $\begin{array}{l}18-J u T y-88 \\
\text { to } \\
19-\text { July-88 }\end{array}$ & $\begin{array}{l}\text { Grouted the borehole from total depth to } \\
512 \mathrm{ft} \text { bls. Installed a filter pack } \\
\text { consisting of } 1 / 4 \text { inch pea gravel. A } \\
\text { bentonite seal was installed above the } \\
\text { filter pack and the remaining annular } \\
\text { space was filled with expanding cement } \\
\text { to the surface. A tremie pipe was used } \\
\text { to install the filter pack and bentonite } \\
\text { and cement seals. }\end{array}$ & $\begin{array}{l}13 \mathrm{yd}^{3} \text { expanding } \\
\text { cement, } 1 / 4 \mathrm{yd}^{3} \\
\text { pea gravel, } 4 \\
\text { buckets of } \\
\text { bentonite }\end{array}$ \\
\hline 15-Aug-88 & $\begin{array}{l}\text { Installed surface cement pad. Surface } \\
\text { completion included a locking aluminum } \\
\text { well cover, cement pad, and impingement } \\
\text { posts. }\end{array}$ & \\
\hline $17-$ Nov -89 & $\begin{array}{l}\text { Hydrostar piston pump was lowered into } \\
\text { the well, with the pump intake placed at } \\
503 \mathrm{ft} \text { bis. }\end{array}$ & \\
\hline
\end{tabular}


D. Summary of Well Development Procedures:

\begin{tabular}{|c|c|c|}
\hline DATE & ACTIVITY & MATERIALS USED \\
\hline $12-$ Sept-89 & $\begin{array}{l}\text { Placed Grundfos pump, Type } 25 \mathrm{~S} \text {, downhole } \\
\text { for wel1 development. Pumped ground } \\
\text { water at } 4.1 \mathrm{gpm} \text { for } 6 \text { minutes. }\end{array}$ & \\
\hline 13-Sept-89 & 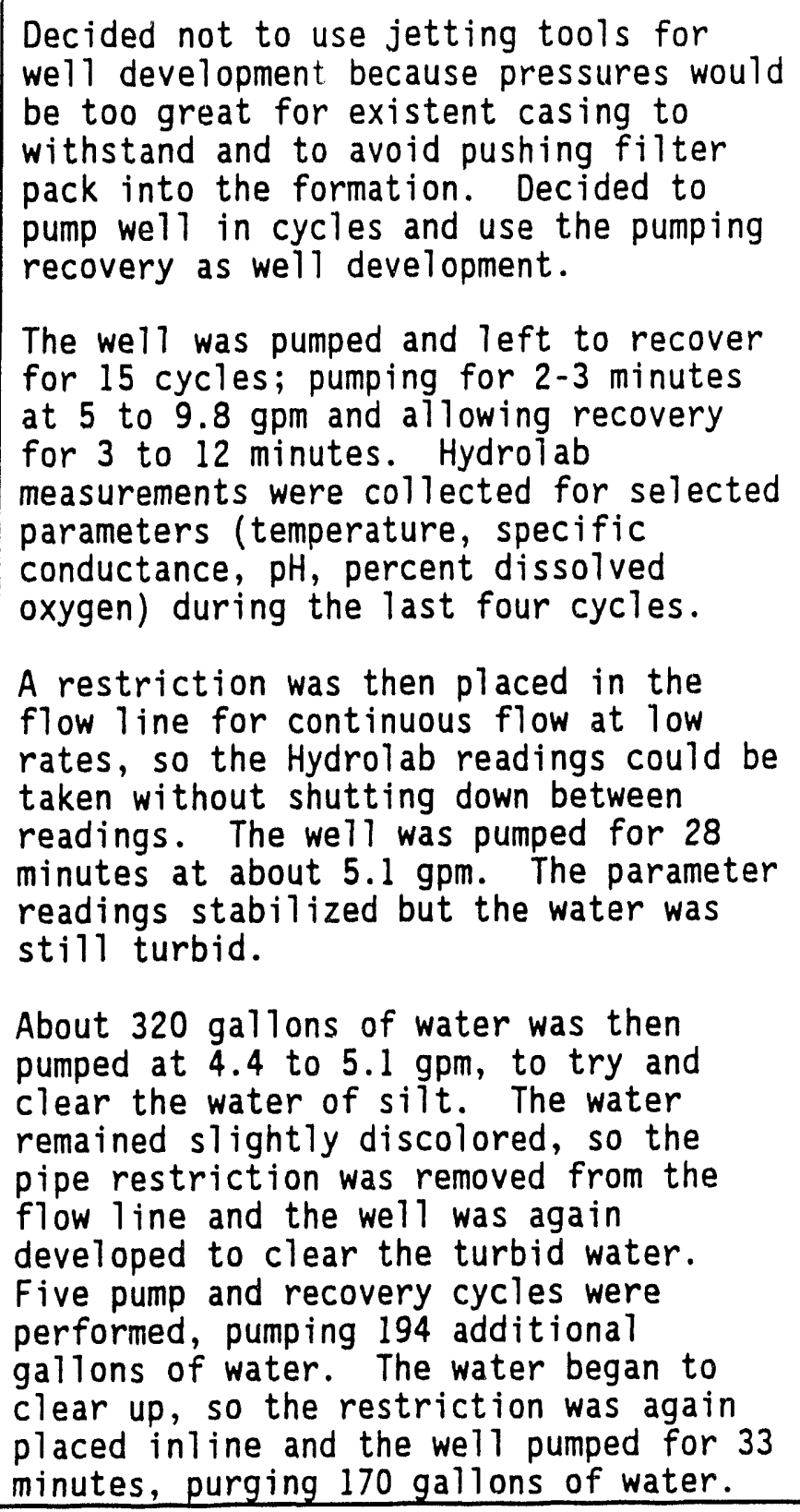 & \\
\hline 14-Sept-89 & $\begin{array}{l}\text { Pulled Grundfos pump. The bottom of the } \\
\text { pump had sludge on it, silty with some } \\
\text { sand. This suggests that the lower } 5 \mathrm{ft} \\
\text { of well may have filled in with silt. }\end{array}$ & \\
\hline
\end{tabular}




\section{GEOLOGICAL OBSERVATIONS}

A. Geological log, geophysical logs, stratigraphic column, and well construction diagram are attached.

B. Samples collected. Two samples were collected, the location and ultimate use of the samples were not recorded. 


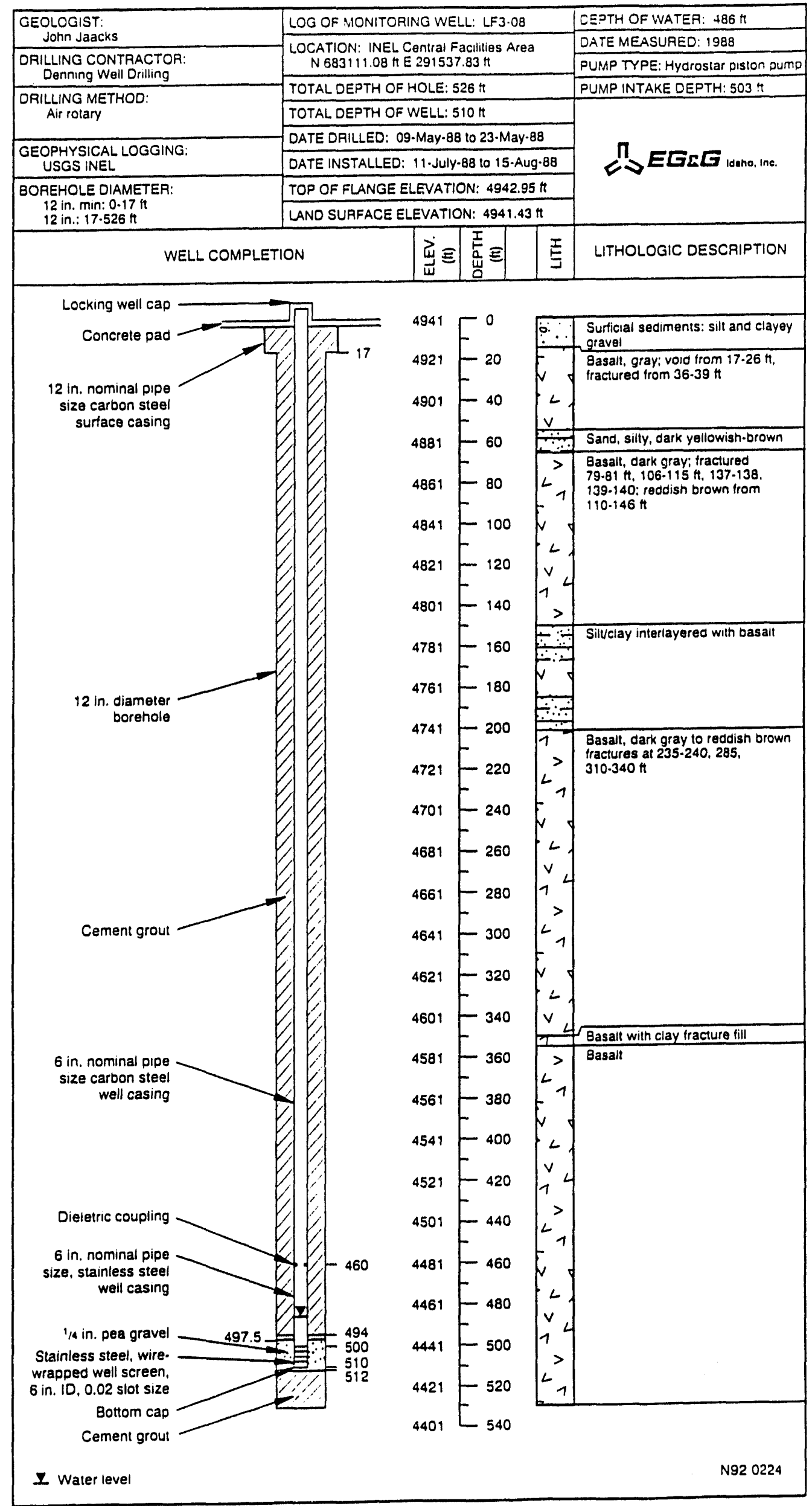



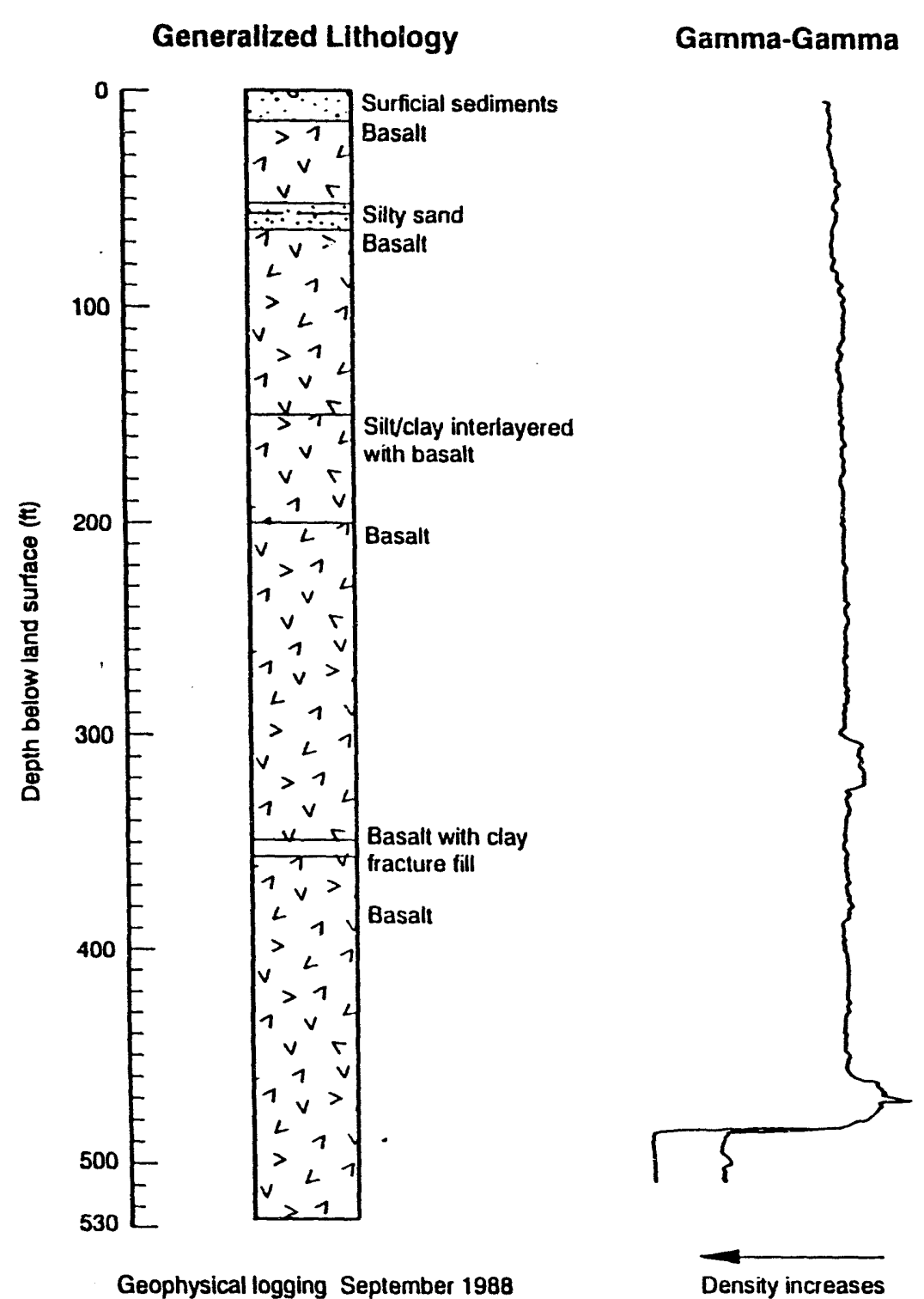

Natural Gamma

Neutron
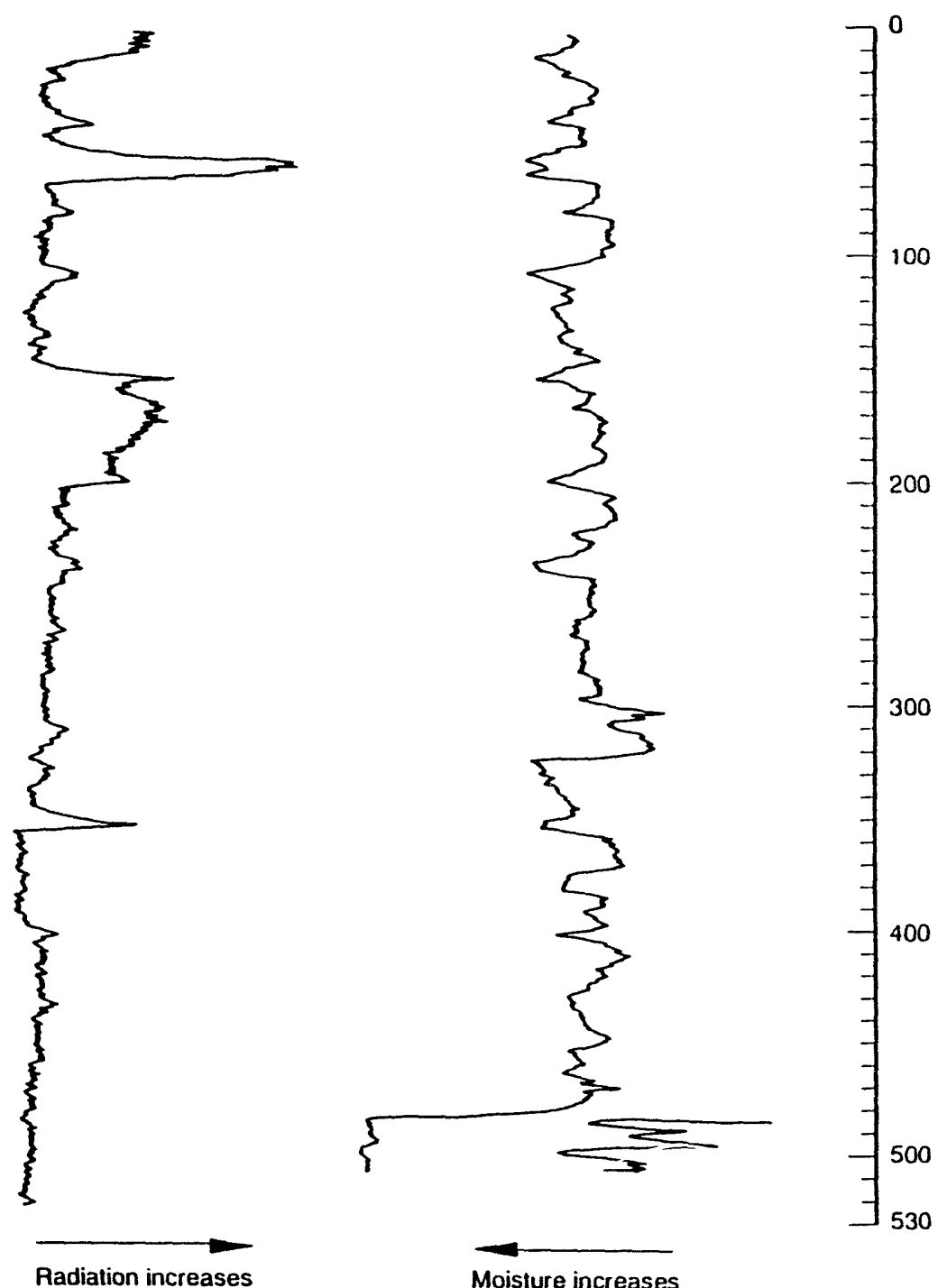

Radiation increases 


\section{END-OF-WELL REPORT}

CENTRAL FACILITIES LANDFILL AREA

WELL LF3-09 
I. GENERAL INFORMATION

A. Project Name: Hydrogeologic Characterization Study for CFA Landfills

B. Well Name/Number: LF3-09

C. Well Location: INEL, Central Facilities Area Landfill III Northing: $682822.69 \mathrm{ft}$ Easting: $291512.30 \mathrm{ft}$ Land Surface Elevation: $4942.33 \mathrm{ft}$ (brass marker) NRTS datum

D. Plans:

Survey Date: December 1990, MK-Ferguson

1) Technical Work Plan for the Hydrogeologic Characterization of CFA Landfi7ls II and III, EGG-WM-9119, July 6, 1990.

2) Health and Safety Plan for Operations Performed for the Environmental Restoration Program, Well Installation and Groundwater Sampling at CFA Landfills II and III, EGG-WM-9105, May 1990.

E. Logbooks used:

1) Field Team Leader's Daily Logbook, ERP-347-90

2) Well Drilling, Installation, and Development Logbook, ERP-353-90

\section{DRILLING AND COMPLETION OBSERVATIONS}

A. Drilling Company: PC Exploration

Drillers: Mack Stillwe11, Paul Harmon

On-Site Geologist: Kenneth Manchester, Brian Higgs

Project Manager: Shannon Ansley

Rig type: Reichdri11-700

Bit type: Tricone bit for surficial sediments, air hammer bit for basalt

B. Summary of Drilling Activities:

\begin{tabular}{|c|c|c|}
\hline DATE & ACTIVITY & MATERIALS USED \\
\hline $18-0 c t-90$ & $\begin{array}{l}\text { Drilled } 17.5 \mathrm{ft} \text { with mud rotary and a } \\
14.5-\text { inch tricone bit through surficial } \\
\text { sediments and into basalt. Pushed in } \\
\text { and grouted the } 12-\text { inch ID surface } \\
\text { casing. Could not push to total depth; } \\
\text { pushed to } 14.48 \mathrm{ft} \text { bls (approximately } \\
4.5 \mathrm{ft} \text { into the basalt) } \\
\end{array}$ & $\begin{array}{l}\text { Grout mix: } 70 \\
\text { gallons water, } 10 \\
\text { bags Portland } \\
\text { Cement, } 20 \text { pounds } \\
\text { bentonite, 20 } \\
\text { pounds caicium } \\
\text { chloride }\end{array}$ \\
\hline $19-0 c t-90$ & $\begin{array}{l}\text { Drilled with } 5.5 \text {-inch air hammer bit to } \\
62 \text { ft bls. Hit a soft zone at } 51 \text { to } 62 \\
\text { ft bls, but did not collect shelby tube } \\
\text { samples because of poor drilling } \\
\text { conditions. Circulation was poor to } \\
\text { nonexistent and there appeared to be a } \\
\text { rubble zone. }\end{array}$ & \\
\hline
\end{tabular}




\begin{tabular}{|c|c|c|}
\hline DATE & ACTIVITY & MATERIALS USED \\
\hline $22-0 c t-90$ & $\begin{array}{l}\text { Reamed borehole with } 117 / 8 \text {-inch hammer } \\
\text { bit to } 62 \mathrm{ft} \text { bls, then drilled to } 64 \mathrm{ft} \\
\text { bls. The borehole was then grouted to } 5 \\
\mathrm{ft} \text { bls to stabilize the hole. }\end{array}$ & $\begin{array}{l}2.5 \text { yd }^{3} \text { cement } \\
\text { grout with } \\
\text { bentonite, sand, } \\
\text { cement, and } \\
\text { calcium chloride } \\
\text { added }\end{array}$ \\
\hline $23-0 c t-90$ & $\begin{array}{l}\text { Drilled out grout with } 5.5 \text {-inch hammer } \\
\text { bit and continued drilling to } 67 \mathrm{ft} \text { bls. } \\
\text { Stopped drilling to collect shelby tube } \\
\text { interbed samples, however, hole had } \\
\text { filled in } 2.6 \mathrm{ft} \text {. Cleaned out hole and } \\
\text { drilled to } 68 \mathrm{ft} \text { bls. Borehole filled } \\
\text { in } 4.0 \mathrm{ft} \text { while waiting to get sampling } \\
\text { tube down the borehole. A decision was } \\
\text { made to terminate collection of an } \\
\text { interbed sample, because of the poor } \\
\text { drilling conditions. Reamed out } \\
\text { borehole with ll } 7 / 8 \text {-inch bit, and then } \\
\text { drilled to } 76 \mathrm{ft} \text { bls. The borehole was } \\
\text { grouted and approximately } 6 \text { ft of } \\
\text { backfill was in the bottom of the } \\
\text { borehole prior to grouting. }\end{array}$ & $\begin{array}{l}2 \text { yd }^{3} \text { grout with } \\
\text { sand, bentonite, } \\
\text { and calcium } \\
\text { chloride added }\end{array}$ \\
\hline $24-0 c t-90$ & $\begin{array}{l}\text { Tagged grout at } 31 \mathrm{ft} \text { bls. Drilled out } \\
\text { grout with } 117 / 8-i n c h \text { bit. Drilled } \\
\text { through basalt to } 125 \mathrm{ft} \text { bis. Grouted } \\
\text { the borehole. }\end{array}$ & $\begin{array}{l}3 \text { yd }^{3} \text { cement grout } \\
\text { with sand, } \\
\text { bentonite, and } \\
\text { calcium chloride } \\
\text { added }\end{array}$ \\
\hline $25-0 c t-90$ & $\begin{array}{l}\text { Top of grout encountered at } 95 \mathrm{ft} \text { bls. } \\
\text { Drilled to } 136 \mathrm{ft} \text { bls and took a } \\
\text { deviation measurement }(0.25 \text { degrees from } \\
\text { the vertical). Had trouble keeping the } \\
\text { borehole clean after drilling through } \\
\text { the grout. Continued drilling to } 165 \mathrm{ft} \\
\text { bls. Grouted the borehole. }\end{array}$ & $\begin{array}{l}5 \text { yd }^{3} \text { cement grout } \\
\text { with sand, } \\
\text { bentonite, and } \\
\text { calcium chloride } \\
\text { added }\end{array}$ \\
\hline $26-0 c t-90$ & $\begin{array}{l}\text { Tagged top of grout at } 50 \mathrm{ft} \text { bls. } \\
\text { Drilled through grout (bottom of grout } \\
\text { at approximately } 59 \mathrm{ft} \text { bls) and found } \\
\text { approximately } 6 \mathrm{ft} \text { of } \mathrm{fill} \text { beneath the } \\
\text { grout. Drilled to } 196 \mathrm{ft} \text { bls. Grouted } \\
\text { the borehole. }\end{array}$ & $\begin{array}{l}2.5 \mathrm{yd}^{3} \text { cement } \\
\text { with bentonite, } \\
\text { sand, and calcium } \\
\text { chloride added }\end{array}$ \\
\hline $29-0 c t-90$ & $\begin{array}{l}\text { Drilled through grout and into basalt. } \\
\text { Total depth for the day was } 243 \mathrm{ft} \text { bls. } \\
\text { Grouted the borehole. }\end{array}$ & $\begin{array}{l}3 \text { yd }^{3} \text { cement with } \\
\text { bentonite, sand, } \\
\text { and calcium } \\
\text { chloride added }\end{array}$ \\
\hline
\end{tabular}




\begin{tabular}{|c|c|c|}
\hline DATE & ACTIVITY & MATERIALS USED \\
\hline $30-0 c t-90$ & $\begin{array}{l}\text { Tagged top of cement at } 196 \mathrm{ft} \text { bls. } \\
\text { Drilled through cement and basalt to } 276 \\
\text { ft bls. Grouted the borehole. }\end{array}$ & $\begin{array}{l}3 \text { yd }^{3} \text { cement with } \\
\text { bentonite, sand, } \\
\text { and calcium } \\
\text { chloride added }\end{array}$ \\
\hline $31-0 c t-90$ & $\begin{array}{l}\text { Tagged top of cement at } 206 \mathrm{ft} \mathrm{bls} \text {. } \\
\text { Drilled through cement grout. Drilled } \\
\text { to } 321 \mathrm{ft} \text { bls. Grouted the borehole. }\end{array}$ & $\begin{array}{l}3 y^{3} \text { cement with } \\
\text { bentonite, sand, } \\
\text { and calcium } \\
\text { chloride added }\end{array}$ \\
\hline $01-$ Nov -90 & $\begin{array}{l}\text { Tagged top of cement at approximately } \\
246 \mathrm{ft} \text { bls. Drilled to } 395.5 \mathrm{ft} \mathrm{bls} \text {. } \\
\text { Took a deviation shot at } 300 \mathrm{ft}(1.5 \\
\text { degrees from vertical). Grouted the } \\
\text { borehole. }\end{array}$ & $\begin{array}{l}5 y^{3} \text { cement with } \\
\text { bentonite, sand, } \\
\text { and calcium } \\
\text { chloride added }\end{array}$ \\
\hline $02-$ Nov -90 & $\begin{array}{l}\text { Top of cement grout is at about } 284 \mathrm{ft} \\
\text { bls. Drilled to } 437 \mathrm{ft} \mathrm{bls.} \mathrm{Grouted} \\
\text { the borehole. }\end{array}$ & $\begin{array}{l}2.5 \mathrm{yd}^{3} \text { cement } \\
\text { with bentonite, } \\
\text { sand, and calcium } \\
\text { chloride added }\end{array}$ \\
\hline $05-N o v-90$ & $\begin{array}{l}\text { Top of cement grout is at } 367 \mathrm{ft} \text { bls. } \\
\text { Drilled to } 470 \mathrm{ft} \text { bls, total for the } \\
\text { day. Took a deviation shot at } 400 \mathrm{ft} \text {, } \\
\text { which showed the borehole was } 2 \text { degrees } \\
\text { from vertical. Bottom of grout was at } \\
429 \mathrm{ft} \text { bls, and } 8 \mathrm{ft} \text { of fill material } \\
\text { was below the grout. }\end{array}$ & \\
\hline $06-$ Nov-90 & $\begin{array}{l}\text { Drilled to } 517 \mathrm{ft} \text { bls. Water level } \\
\text { measured at } 484.5 \mathrm{ft} \text { bls. Total depth } \\
\text { for borehole is } 517 \mathrm{ft} \text { bis. }\end{array}$ & \\
\hline $07-$ Nov -90 & USGS began logging borehole. & \\
\hline
\end{tabular}

C. Summary of Well Construction Procedures:

\begin{tabular}{|c|c|c|}
\hline DATE & ACTIVITY & MATERIALS USED \\
\hline $07-$ Nov -90 & $\begin{array}{l}\text { Began installing well screen and well } \\
\text { casing in the borehole. Installed } \\
4-\text { inch ID, passivated, stainless steel } \\
\text { well screen, wire-wrapped }(0.020 \text {-inch } \\
\text { slot size) and flush threaded with an } \\
\text { endplug threaded to the bottom of the } \\
\text { screen. Installed } 4 \text {-inch nominal pipe } \\
\text { size (4.5-inch 0D), passivated, } \\
\text { stainless steel well casing, schedule } \\
\text { los, Type } 304 \text {, flush threaded, down the } \\
\text { borehole. Installed three stainless } \\
\text { steel centralizers. }\end{array}$ & $\begin{array}{l}20 \mathrm{ft} \text { of well } \\
\text { screen, } 480 \mathrm{ft} \text { of } \\
\text { well casing, } \\
\text { endplug, } 3 \\
\text { centralizers }\end{array}$ \\
\hline
\end{tabular}




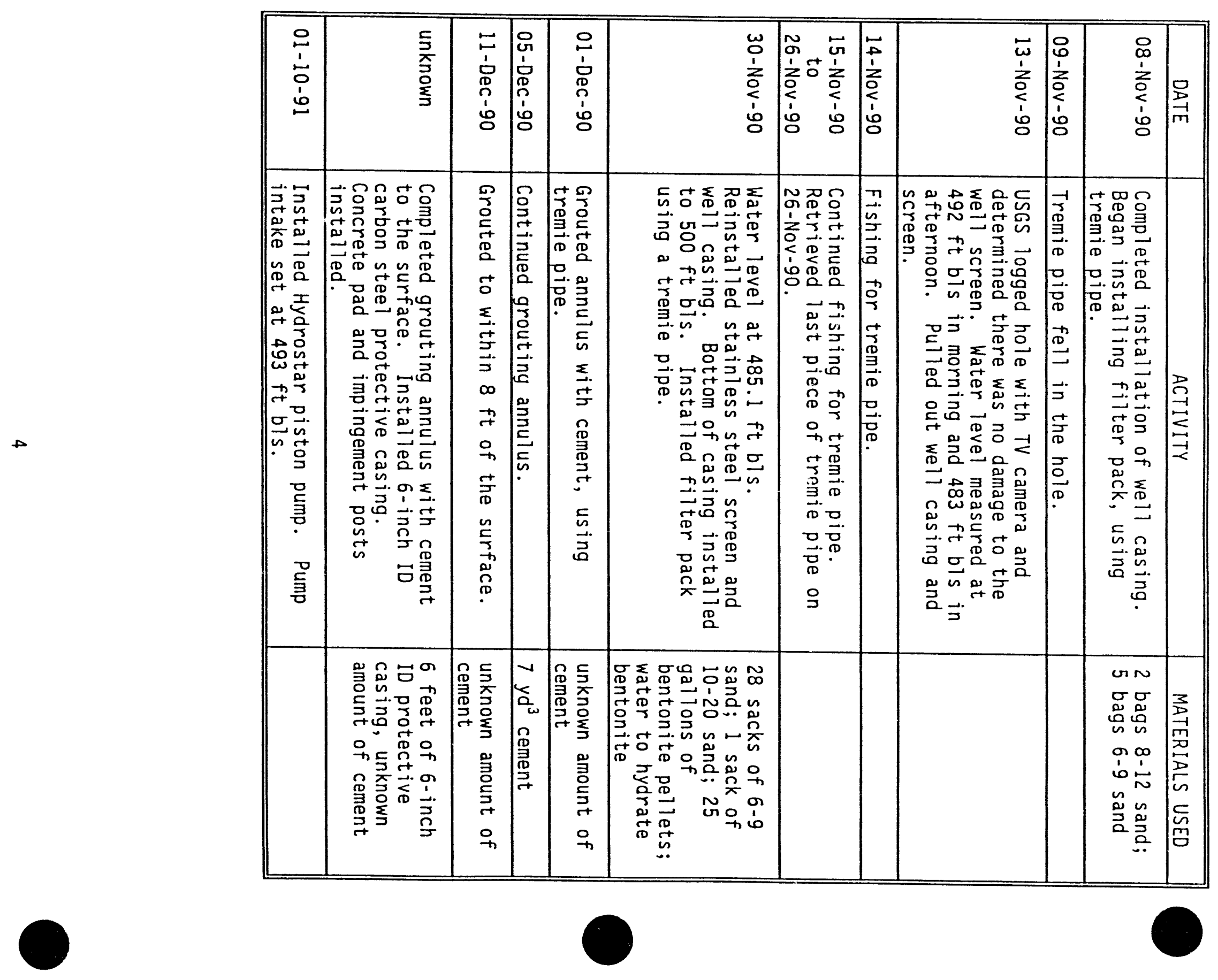


C. Summary of Well Development Procedures:

\begin{tabular}{||l|l|c||}
\hline \multicolumn{1}{|c|}{ DATE } & \multicolumn{1}{|c||}{ ACTIVITY } & MATERIALS USED \\
\hline $12-$ Dec-90 & Installed surge block. & \\
\hline 13-Dec-90 & $\begin{array}{l}\text { Surged well with surge block for 40 } \\
\text { minutes. Lowered a submersible pump } \\
\text { into the well and pumped about 300 } \\
\text { gallons of water out of the we1l, unti1 } \\
\text { water was clear and measured parameters } \\
\text { stabilized. }\end{array}$ & \\
\hline
\end{tabular}

\section{GEOLOGICAL OBSERVATIONS}

A. Geological log, geophysical logs, stratigraphic column, and well construction diagram are attached.

B. Samples collected. No samples were collected. 


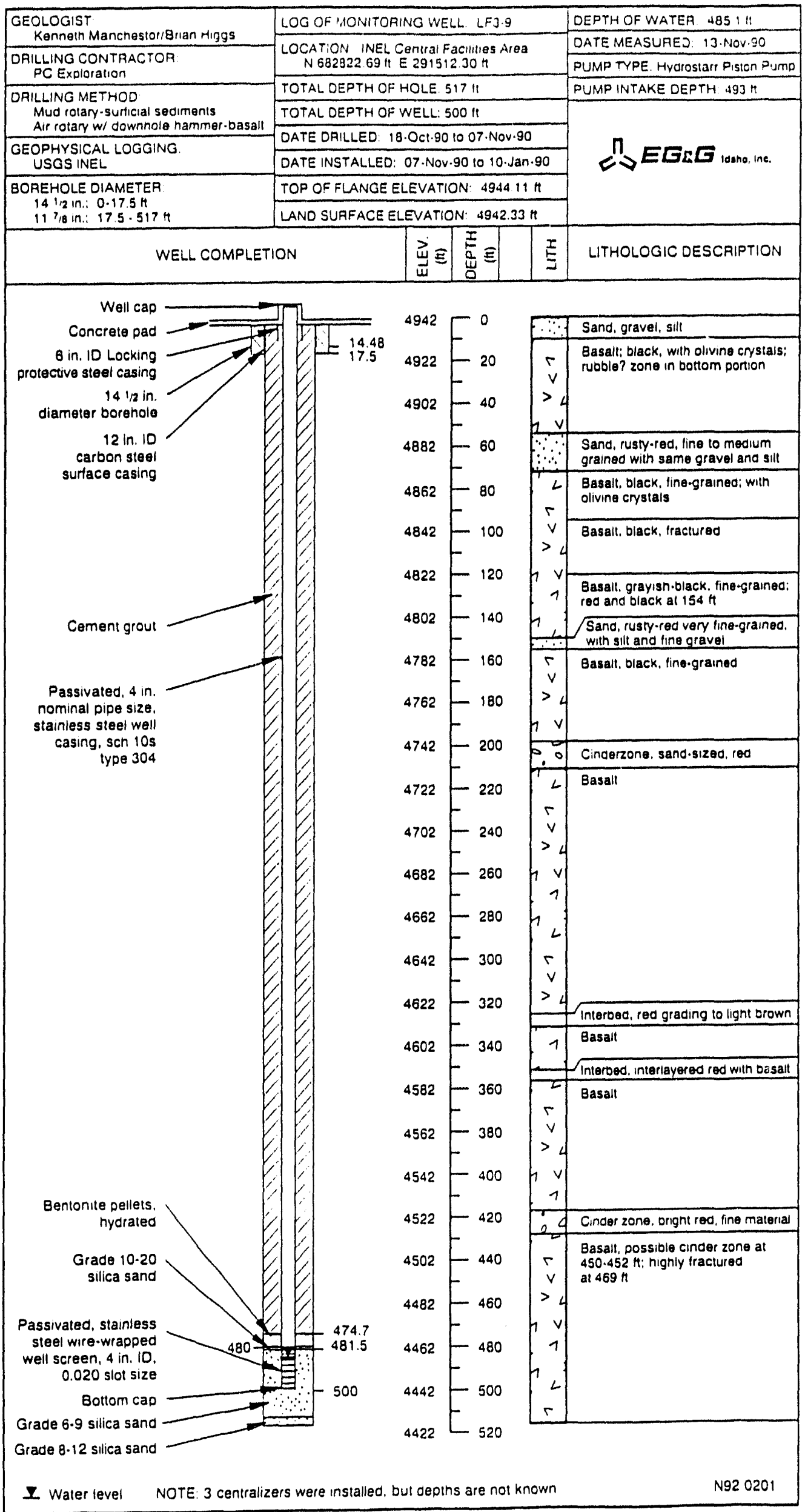




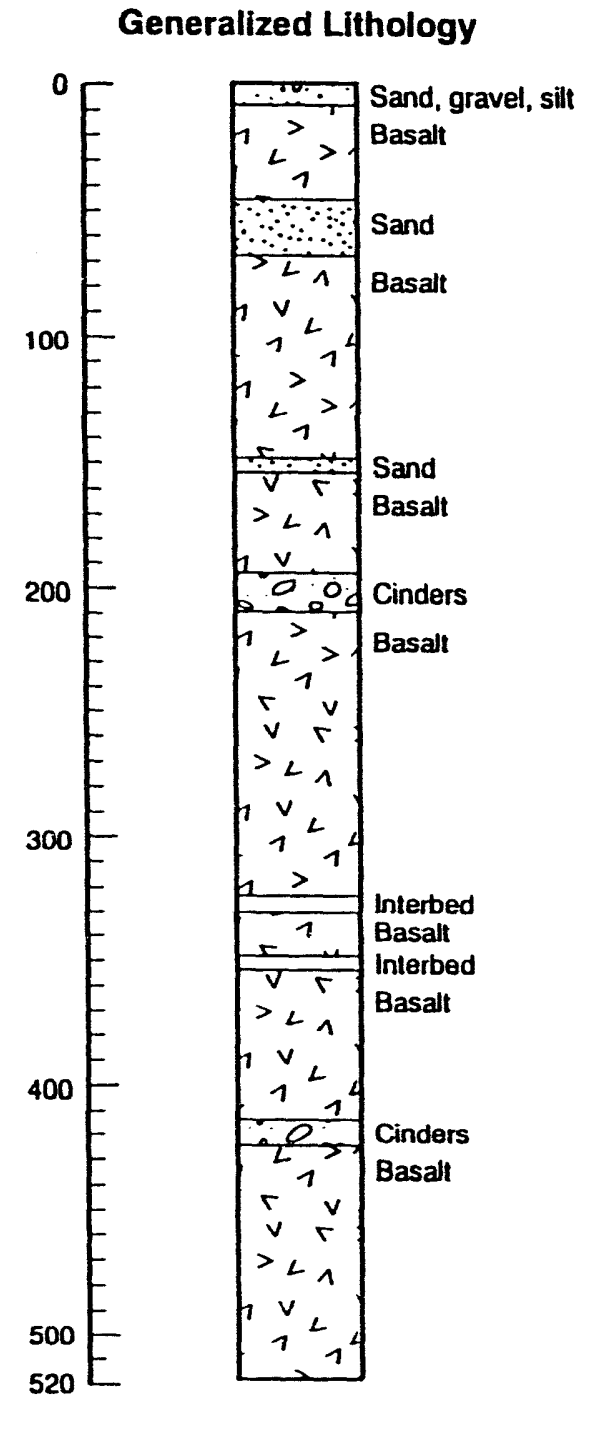

Geophysical logging November 1990
Callper

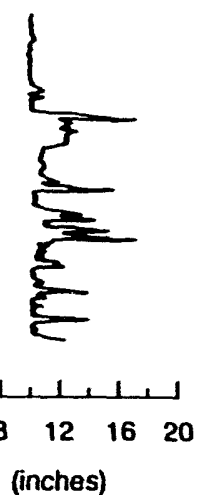

(inches)
Gamma-Gamma

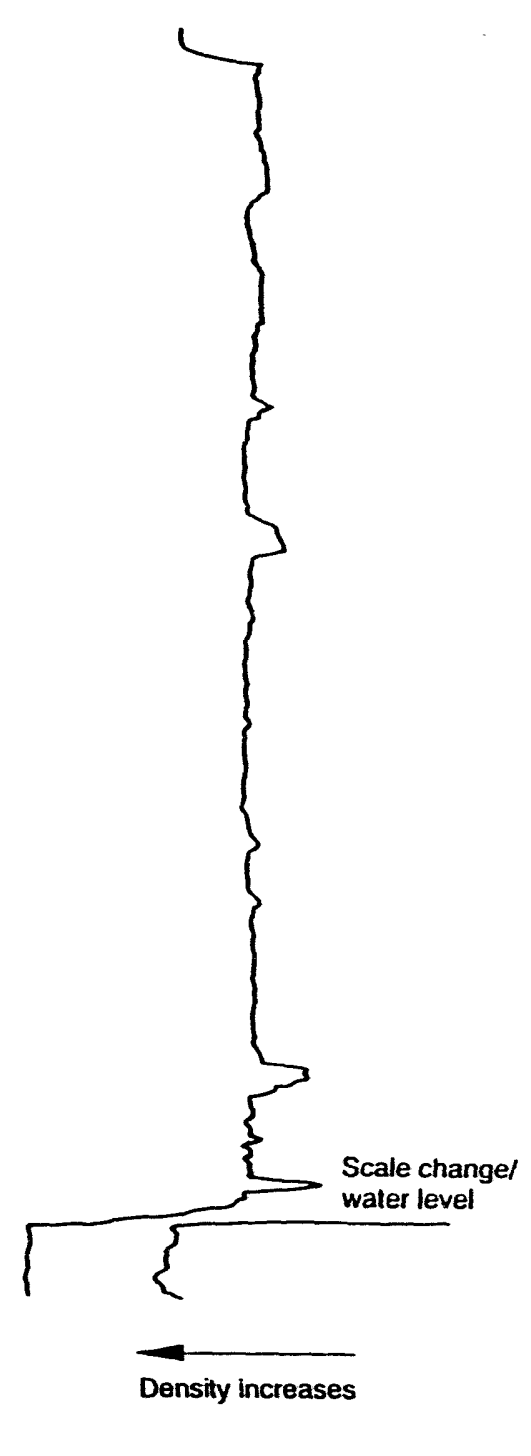

Natural Gamma

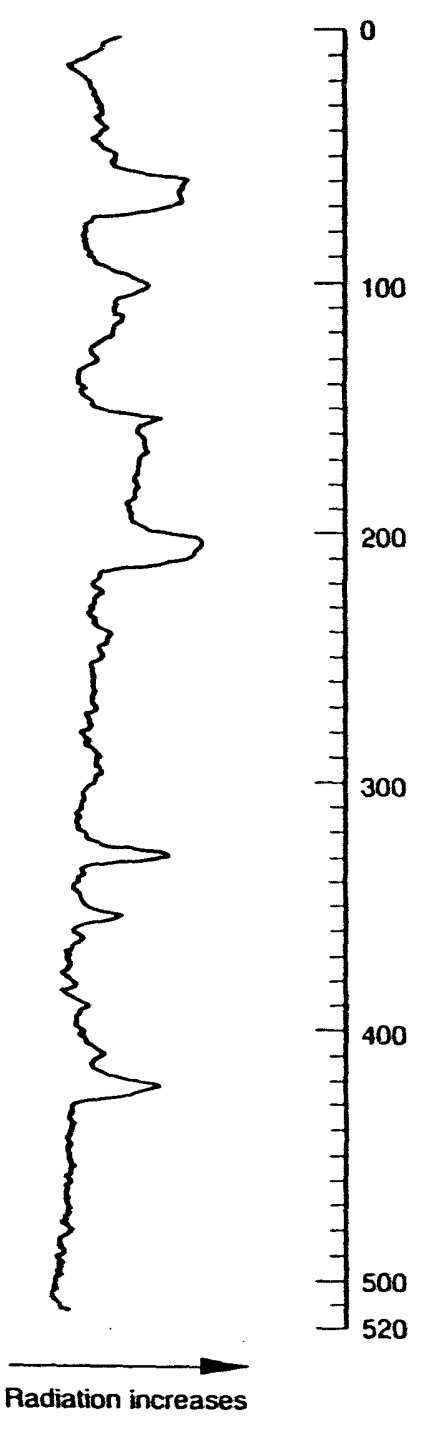

Well CFA LF3-9 
END-OF-WELL REPORT

CENTRAL FACILITIES LANDFILL AREA

WELL LF3-10 
I. GENERAL INFORMATION

A. Project Name: Hydrogeologic Characterization Study for CFA Land $i 11 \mathrm{~s}$

B. Well Name/Number: LF3-10

C. Well Location: INEL, Central Facilities Area Landfill III Northing: $683528.93 \mathrm{ft}$ Easting: $290875.41 \mathrm{ft}$ Land Surface Elevation: $4943.87 \mathrm{ft}$ (brass marker)

D. Plans:

Survey Date: December 1990, MK-Ferguson

1) Technical Work Plan for the Hydrogeologic Characterization of CFA Landfills II and III, EGG-WM-9119, July 6, 1990.

2) Health and Safety Plan for Operations Performed for the Environmental Restoration Program, Well Installation and Groundwater Sampling at CFA Landfills II and III, EGG-WM-9105, May 1990.

E. Logbooks used:

1) Well Drilling, Installation, and Development Logbook, ERP-31-90

2) Field Team Leader's Daily Logbook, ERP-26-90

3) Field Team Leader's Daily Logbook, ERP-347-90

4) Field Team Leader's Daily Logbook, ERP-29-90

\section{DRILLING AND COMPLETION OBSERVATIONS}

A. Drilling Company: PC Exploration

Drillers: Mack Stillwell, Paul Harmon

On-Site Geologist: David Burgess

Project Manager: Shannon Ansley

Rig type: Reichdri17-700

Bit type: Tricone bit for surficial sediments, air hammer bit for basalt

B. Summary of Drilling Activities:

\begin{tabular}{|c|c|c|}
\hline DATE & ACTIVITY & MATERIALS USED \\
\hline 05-Sept-90 & Set up at drill site. & \\
\hline $06-$ Sept-90 & $\begin{array}{l}\text { Began drilling into surficial sediment } \\
\text { with a } 14.5 \text {-inch tricone bit using the } \\
\text { mud rotary method. Drilled to } 25 \mathrm{ft} \\
\text { bls. }\end{array}$ & \\
\hline $07-$ Sept-90 & $\begin{array}{l}\text { Continued mud rotary drilling, hit } \\
\text { basalt at about } 28-29 \mathrm{ft} \text { bls. Stopped } \\
\text { at } 33 \mathrm{ft} \text { bls. Pushed surface casing } \\
\text { into the borehole, grouted using tremie } \\
\text { pipe. }\end{array}$ & $\begin{array}{l}35 \mathrm{ft} \text { of } 12 \text {-inch } \\
\text { carbon steel } \\
\text { surface casing; } \\
\text { cement with sand } \\
\text { and bentonite } \\
\text { added. }\end{array}$ \\
\hline
\end{tabular}




\begin{tabular}{|c|c|c|}
\hline DATE & ACTIVITY & MATERIALS USED \\
\hline $10-\operatorname{Sept}-90$ & $\begin{array}{l}\text { Deconned } 117 / 8 \text {-inch hammer bit and } \\
\text { collar. }\end{array}$ & \\
\hline $11-$ Sept-90 & $\begin{array}{l}\text { Deconned pipe, set up for drilling } \\
\text { through the first interbed with air } \\
\text { hammer }(5.5 \text {-inch bit). Drilled from } 33 \\
\text { to } 61 \mathrm{ft} \text { bls. Collected } 0.8 \mathrm{ft} \text { of } \\
\text { interbed soil sample with shelby tube } \\
\text { from } 60.2 \text { to } 61.0 \mathrm{ft} \text { bls. }\end{array}$ & \\
\hline 12-Sept-90 & $\begin{array}{l}\text { Cleaned hole to } 61.2 \mathrm{ft} \text { bls. Collected } \\
\text { shelby tube samples from } 61.2 \text { to } 65.15 \\
\mathrm{ft} \text { bls, recovered } 1.6 \mathrm{ft} \text { of core. } \\
\text { Borehole was sloughing, therefore, } \\
\text { drilled/reamed to } 65.15 \mathrm{ft} \text { bls with } \\
117 / 8 \text {-inch bit and grouted hole with } \\
\text { cement. }\end{array}$ & $\begin{array}{l}2 y^{3} \text { cement with } \\
\text { sand and bentonite }\end{array}$ \\
\hline 13-Sept-90 & $\begin{array}{l}\text { Drilled out cement and collected shelby } \\
\text { tube sample from } 65.5 \text { to } 67.25 \mathrm{ft} \text { bls } \\
\text { with } 1.25 \mathrm{ft} \text { of } \mathrm{fi} 11 \text { on top; } 67.65 \text { to } \\
69.0 \mathrm{ft} \text { bls with } 2.65 \mathrm{ft} \text { of fill on top. } \\
\text { Again, the borehole was caving in and } \\
\text { further attempts to collect samples were } \\
\text { unsuccessful. Reamed to } 70 \mathrm{ft} \text { bis with } \\
\text { the } 117 / 8 \text {-inch bit. }\end{array}$ & \\
\hline 14-Sept-90 & $\begin{array}{l}\text { Drilled with } 117 / 8 \text {-inch bit through the } \\
\text { remainder of interbed and into the } \\
\text { basalt to } 83 \mathrm{ft} \text { bls. Grouted borehole } \\
\text { to } 62 \mathrm{ft} \text { bls to prevent further } \\
\text { sloughing. }\end{array}$ & $\begin{array}{l}2 \mathrm{yd}^{3} \text { cement with } \\
\text { sand and bentonite }\end{array}$ \\
\hline 17-Sept-90 & $\begin{array}{l}\text { Drilled out cement from } 62 \text { to } 83 \mathrm{ft} \text { bls } \\
\text { and cont inued drilling to } 220 \mathrm{ft} \text { bl s. } \\
\text { Performed deviation measurements at } 100 \\
\text { and } 200 \mathrm{ft} \text { bls. Grouted borehole. }\end{array}$ & $\begin{array}{l}3.5 y^{3} \text { cement } \\
\text { with sand, } \\
\text { bentonite, and } 2 \% \\
\text { calcium chloride }\end{array}$ \\
\hline 18-Sept-90 & $\begin{array}{l}\text { Drilled out cement grout from } 116 \mathrm{ft} \text { bls } \\
\text { to } 200 \mathrm{ft} \text { bls. The interbed was open } \\
\text { (not grouted) from } 83 \text { and } 116 \mathrm{ft} \mathrm{b} / \mathrm{s} \text {. } \\
\text { Therefore, it was grouted to prevent } \\
\text { sloughing. }\end{array}$ & $\begin{array}{l}5 \text { yd }^{3} \text { cement with } \\
\text { sand, bentonite, } \\
\text { and calcium } \\
\text { chloride }\end{array}$ \\
\hline 19-Sept-90 & $\begin{array}{l}\text { Drilled out cement grout from } 60.0 \text { to } \\
220 \mathrm{ft} \text { bls and continued drilling to } 330 \\
\mathrm{ft} \text { bls. Took deviation measurement at } \\
300 \mathrm{ft} \text { bls }(0.25 \text { degrees from vertical). } \\
\text { Grouted borehole. }\end{array}$ & $\begin{array}{l}5 \text { yd }^{3} \text { cement with } \\
\text { sand, bentonite, } \\
\text { and calcium } \\
\text { chloride }\end{array}$ \\
\hline
\end{tabular}




\begin{tabular}{|c|c|c|}
\hline DATE & ACTIVITY & MATERIALS USED \\
\hline 20-Sept-90 & $\begin{array}{l}\text { Drilled out cement from } 205 \text { to } 320 \mathrm{ft} \\
\text { bls and continued drilling to } 380 \mathrm{ft} \\
\text { bls, where drill began to lose } \\
\text { circulation. Grouted hole. }\end{array}$ & $\begin{array}{l}5 \text { yd }^{3} \text { cement with } \\
\text { sand, bentonite, } \\
\text { and calcium } \\
\text { chloride }\end{array}$ \\
\hline 21-Sept-90 & $\begin{array}{l}\text { Drilled out cement from } 290 \text { to } 375 \mathrm{ft} \\
\text { bls, then drilled to } 437 \mathrm{ft} \text { bls and } \\
\text { grouted the borehole. }\end{array}$ & $\begin{array}{l}5.5 \mathrm{yd}^{3} \text { cement } \\
\text { with sand, } \\
\text { bentonite, calcium } \\
\text { chloride, and } \\
\text { minor gravel }\end{array}$ \\
\hline 24-Sept-90 & $\begin{array}{l}\text { Drilled out cement grout from } 297 \text { to } 437 \\
\mathrm{ft} \text { bls. Took deviation measurement at } \\
400 \mathrm{ft} \text { bls }(0.25 \text { to } 0.50 \text { degrees from } \\
\text { vertical). }\end{array}$ & \\
\hline 25-Sept-90 & $\begin{array}{l}\text { Drilled from } 437 \text { to } 510 \mathrm{ft} \text { bls. Water } \\
\text { level measured at } 485.8 \mathrm{ft} \text { bls. } \\
\text { Performed geophysical logging: natural } \\
\text { gamma, gamma-gamma, caliper, and TV } \\
\text { logs. }\end{array}$ & \\
\hline 26-Sept-90 & $\begin{array}{l}\text { Depth of hole is } 496.5 \mathrm{ft} \text { bls, filled in } \\
13.5 \mathrm{ft} \text {. Drilled from } 510 \text { to a total } \\
\text { depth of } 530 \mathrm{ft} \text { bls. }\end{array}$ & \\
\hline
\end{tabular}

C. Summary of Well Construction Procedures:

\begin{tabular}{|c|c|c|}
\hline DATE & ACTIVITY & MATERIALS \\
\hline 27-Sept-90 & $\begin{array}{l}\text { Tremie pipe was deconned. Tagged bottom } \\
\text { of hole at } 522 \mathrm{ft} \text { bls. Installed } 4 \text {-inch } \\
\text { ID, passivated, stainless steel well } \\
\text { screen, wire-wrapped }(0.020 \text {-inch slot } \\
\text { size) and flush threaded with an endplug } \\
\text { threaded to the bottom of the screen. } \\
\text { Installed } 4 \text {-inch nominal pipe size } \\
\text { (4.26-inch ID), passivated, stainless } \\
\text { steel well casing, schedule IOS, type } \\
304 \text {, flush threaded, down the borehole } \\
\text { with } 2 \text { ft of stick-up. Installed three } \\
\text { stainless steel centralizers. }\end{array}$ & $\begin{array}{l}500 \mathrm{ft} \text { of well } \\
\text { casing; } 20 \mathrm{ft} \text { of } \\
\text { wel1 screen; } \\
\text { endplug, } 3 \\
\text { centralizers }\end{array}$ \\
\hline 28-Sept-90 & $\begin{array}{l}\text { Measured the water level at } 486 \mathrm{ft} \text { bls, } \\
\text { Installed filter pack consisting of } 6-9 \\
\text { grade sand and topped with } 1.5 \mathrm{ft} \text { of } 10- \\
20 \text { grade sand, using a tremie pipe. } \\
\text { Installed bentonite pellet seal above } \\
\text { the filter pack using a tremie pipe. } \\
\text { Water was added to the hole during the } \\
\text { sand and bentonite placement. }\end{array}$ & $\begin{array}{l}314 \text { gallons of } 6-9 \\
\text { grade sand, } 5 \\
\text { gallons } 10-20 \\
\text { grade sand, } 25 \\
\text { gallons bentonite } \\
\text { pellets, } 445 \\
\text { gallons water }\end{array}$ \\
\hline
\end{tabular}




\begin{tabular}{||l|l|l||}
\hline 02-0ct-90 & $\begin{array}{l}\text { Grouted the well annulus using a tremie } \\
\text { pipe. }\end{array}$ & $\begin{array}{l}6 \text { sacks cement, } \\
11 \text { pounds } \\
\text { bentonite, 5.5 } \\
\text { sacks sand }\end{array}$ \\
\hline 09-0ct-90 & Continued grouting the well annulus. & \\
\hline $10-0 c t-90$ & Continued grouting the well annulus. & $\begin{array}{l}6 \text { yd }^{3} \text { cement with } \\
\text { bentonite and sand } \\
\text { added }\end{array}$ \\
\hline $11-0$ ct-90 & $\begin{array}{l}\text { Continued grouting the well annulus. } \\
\text { 23-0ct-90 }\end{array}$ & $\begin{array}{l}\text { 3 yd cement with } \\
\text { bentonite and sand } \\
\text { added }\end{array}$ \\
\hline $\begin{array}{l}\text { Completed grouting annulus with cement } \\
\text { from 5 ft bls to 7and surface. } \\
\text { Installed 6-inch ID carbon steel } \\
\text { protective casing. Concrete pad and } \\
\text { impingement posts installed. }\end{array}$ & $\begin{array}{l}6 \text { feet of 6-inch } \\
\text { ID protective } \\
\text { casing, cement } \\
\text { with sand and } \\
\text { bentonite added }\end{array}$ \\
\hline 01-Nov-90 & $\begin{array}{l}\text { Installed Hydrostar piston pump. Pump } \\
\text { intake set at 493.75 bls. Installed } \\
\text { locking protective box over pump head at } \\
\text { surface. }\end{array}$ & \multicolumn{2}{|l}{} \\
\hline
\end{tabular}

D. Summary of Well Development Procedures:

\begin{tabular}{||l|l||}
\hline DATE & \multicolumn{1}{|c|}{ ACTIVITY } \\
\hline 31-0ct-90 & $\begin{array}{l}\text { Surged well in 5-ft intervals using a surge block, for total } \\
\text { of } 80 \text { minutes. Water level (1 hour later) measured at 486 ft } \\
\text { bls. Lowered a submersible pump into the well and began }\end{array}$ \\
& $\begin{array}{l}\text { evacuating water. Measured the specific conductance, } \\
\text { temperature, pH, and dissolved oxygen of the development } \\
\text { water. Purged about } 300 \text { gallons of water. }\end{array}$ \\
\hline
\end{tabular}




\section{GEOLOGICAL OBSERVATIONS}

A. Geological log, geophysical logs, stratigraphic column, and well construction diagram are attached.

B. Samples collected. Samples are stored in the INEL Core Library.

Sample Depth

$60.2-61.0 \mathrm{ft}$

$62.3-63.15 \mathrm{ft}$

$64.4-65.15 \mathrm{ft}$

$65.5-67.25 \mathrm{ft}$

$67.65-69.0 \mathrm{ft}$

\section{Comments}

$0.8 \mathrm{ft}$ of core

$1.75 \mathrm{ft}$ of undisturbed core with $1.25 \mathrm{ft}$ of $\mathrm{fil1}$ on top

$1.35 \mathrm{ft}$ of undisturbed core with $2.65 \mathrm{ft}$ of $\mathrm{fill}$ on top 


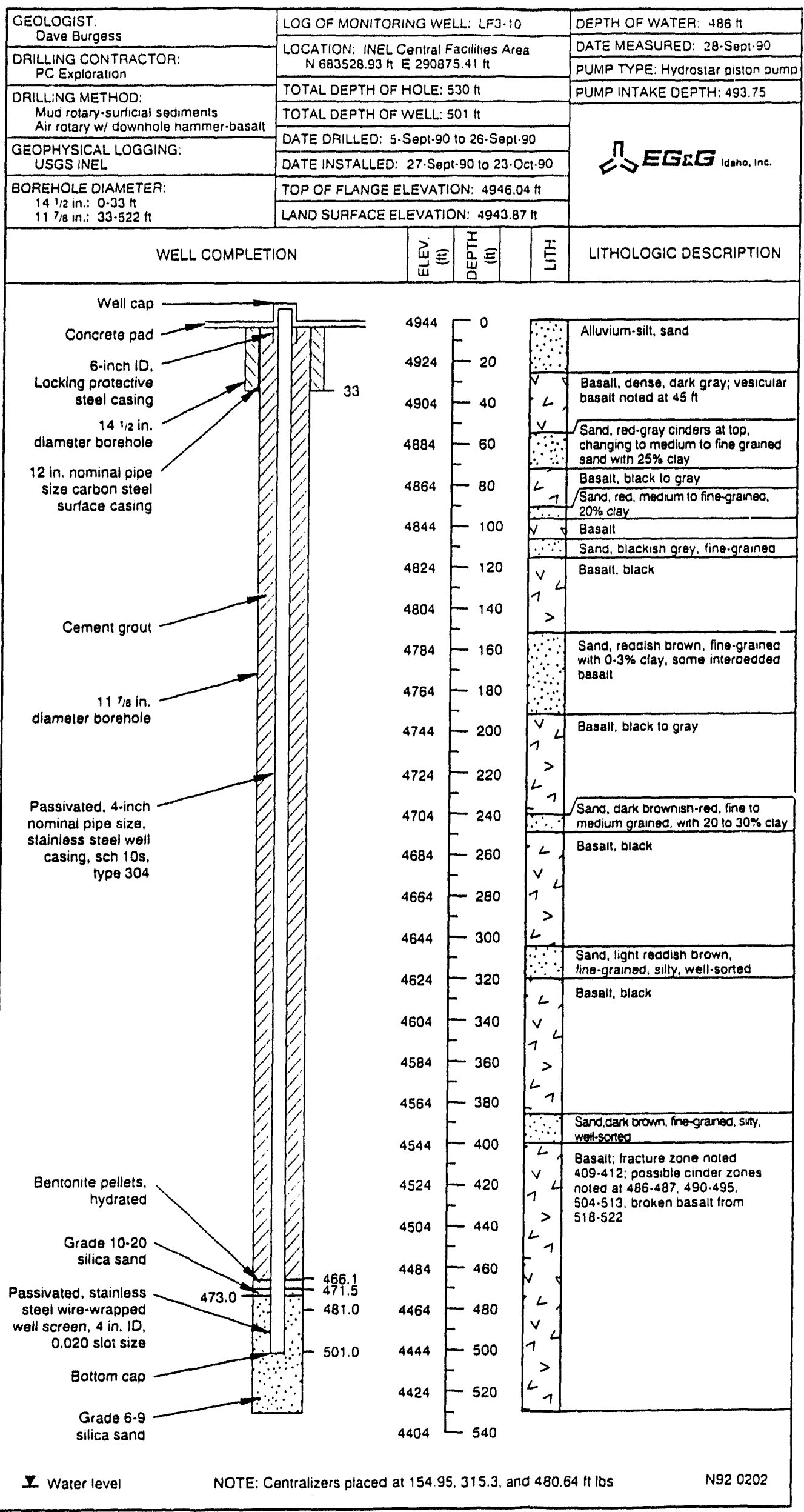



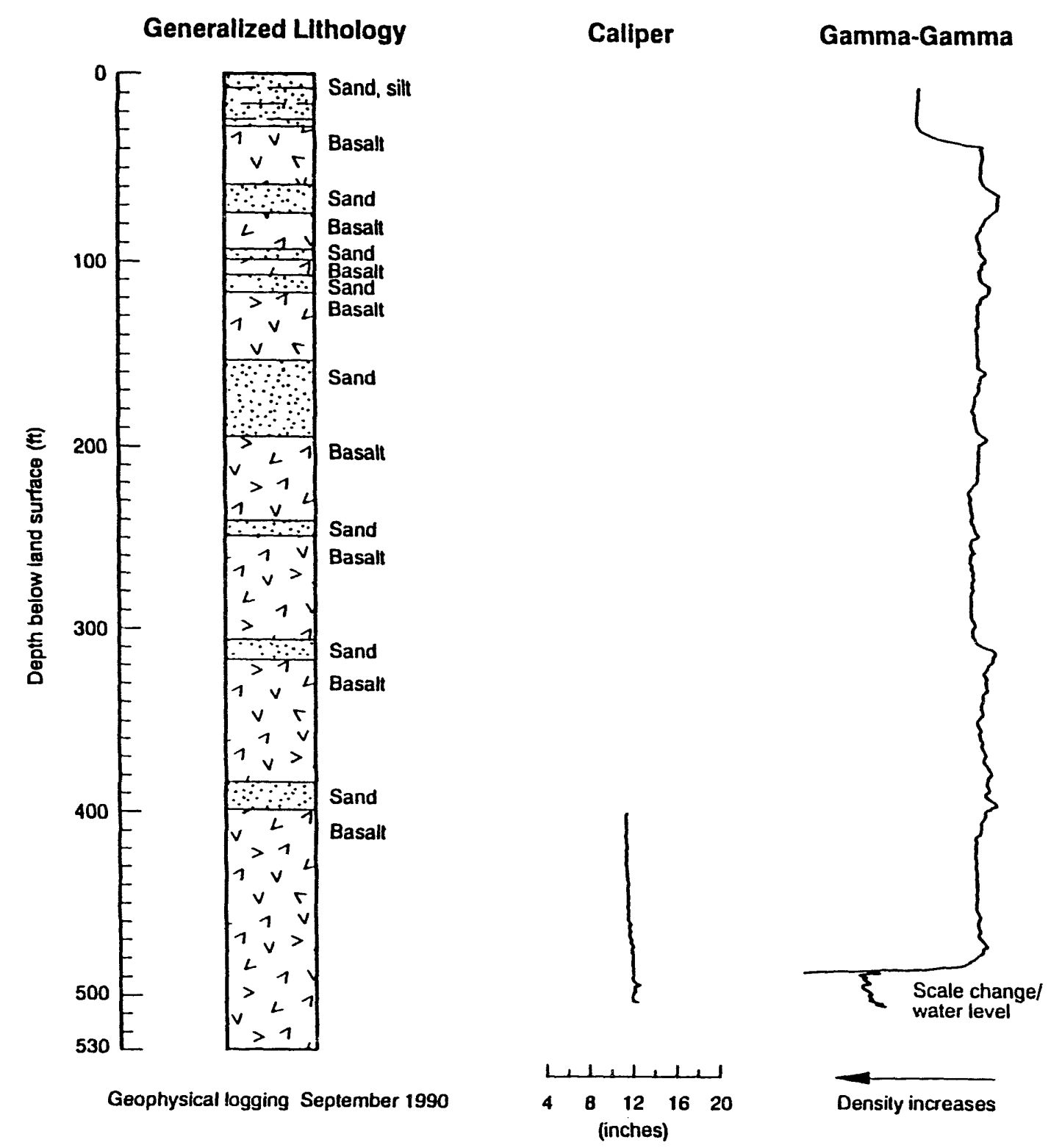

Natural Gamma

Well CFA LF3-10 
END-OF-WELL REPORT

CENTRAL FACILITIES LANDFILL AREA

WELL LF3-11 
I. GENERAL INFORMATION

A. Project Name: Hydrogeologic Characterization Study for CFA Landfills

B. Well Name/Number: LF3-11

C. Well Location: INEL, Central Facilities Area Landfill III Northing: $686243.56 \mathrm{ft}$ Easting: $292682.55 \mathrm{ft}$ Land Surface Elevation: 4935.44 (brass marker) NRTS datum

D. Plans:

Survey Date: December 1990, MK-Ferguson

1) Technical Work Plan for the Hydrogeologic Characterization of CFA Landfills II and III, EGG-WM-9119, July 6, 1990.

2) Health and Safety Plan for Operations Performed for the Environmental Restoration Program, Well Installation and Groundwater Sampling At CFA Landfills II and III, EGG-WM-9105, May 1990.

E. Logbooks used:

1) Field Team Leader's Daily Logbook, ERP-27-90

2) Field Team Leader's Daily Logbook, ERP-347-90

3) Field Team Leader's Daily Logbook, ERP-29-90

4) Well Drilling, Installation, and Development Logbook, ERP-30-90

II. DRILLING AND COMPLETION OBSERVATIONS

A. Drilling Company: PC Exploration

Drillers: Scott McNinch, Wade Schott

On-Site Geologist: Kenny Manchester

Project Manager: Shannon Ansley

Rig type: Reichdril1-700

Bit type: Tricone bit for surficial sediments, air-hammer, button bit for basalt

B. Summary of Drilling Activities:

\begin{tabular}{||c|l|c||}
\hline DATE & \multicolumn{1}{|c||}{ ACTIVITY } & MATERIALS USED \\
\hline 11 -Sept-90 & Deconned dril1 rig. & \\
\hline 12 -Sept-90 & $\begin{array}{l}\text { Began mud rotary drilling in the } \\
\text { surficial sediments with a } 143 / 4-\text { inch } \\
\text { tricone bit. Hit basalt at } 21.5 \mathrm{ft} \mathrm{b} \mathrm{s}, \\
\text { drilled to } 29.5 \mathrm{ft} \text { bls. }\end{array}$ & \\
\hline
\end{tabular}




\begin{tabular}{|c|c|c|}
\hline DATE & ACTIVITY & MATERIALS USED \\
\hline 13-Sept-90 & $\begin{array}{l}\text { Set surface casing at } 29.5 \mathrm{ft} \text { bls and } \\
\text { grouted with a cement mix containing } 2 \% \\
\text { bentonite. }\end{array}$ & $\begin{array}{l}30 \mathrm{ft} \text { of } 12-\text { inch } \\
\text { ID carbon steel } \\
\text { surface casing, } 14 \\
\text { sacks cement, } 140 \\
\text { gallons water, } 2 / 3 \\
\text { bag of bentonite }\end{array}$ \\
\hline $14-$ Sept -90 & $\begin{array}{l}\text { Tagged cement at about } 15 \mathrm{ft} \text { bls. } \\
\text { Drilled with a } 5.5-\text { inch button bit and a } \\
\text { downhole air hammer. A switch was made } \\
\text { to a } 117 / 8 \text {-inch hammer bit after sample } \\
\text { collection in the first interbed. } \\
\text { Drilled to } 36 \mathrm{ft} \text { bls, where bit } \\
\text { collared; may have encountered the } \\
\text { interbed at } 35 \mathrm{ft} \text { bls. Circulation was } \\
\text { lost, so pulled out of the borehole. }\end{array}$ & \\
\hline 17-Sept-90 & $\begin{array}{l}\text { Drilled to } 45 \mathrm{ft} \text { bls, no interbed } \\
\text { encountered from } 37 \text { to } 45 \mathrm{ft} \text { bls. } \\
\text { Reamed borehole with } 117 / 8 \text {-inch hammer } \\
\text { bit to } 38 \mathrm{ft} \text { bls. }\end{array}$ & \\
\hline 18-Sept-90 & Grouted borehole up to $20 \mathrm{ft}$ bls. & $\begin{array}{l}165 \text { gallons cement } \\
\text { mix ( } 15 \text { sacks } \\
\text { cement, } 30 \text { pounds } \\
\text { calcium chloride) }\end{array}$ \\
\hline $19-$ Sept-90 & $\begin{array}{l}\text { Drilled out grout from } 20 \mathrm{ft} \text { to } 38 \mathrm{ft} \\
\text { bls. Drilled borehole to } 58 \mathrm{ft} \mathrm{bls.} \\
\text { Grouted the borehole. }\end{array}$ & $1.5 \mathrm{yd}^{3}$ cement \\
\hline 20-Sept-90 & $\begin{array}{l}\text { Tagged grout at } 35 \mathrm{ft} \text { bls. Drilled out } \\
\text { grout and continued drilling to } 138 \mathrm{ft} \\
\text { bls. Grouted the borehole. }\end{array}$ & $\begin{array}{l}4 \text { yd }^{3} \text { cement with } \\
\text { bentonite, sand, } \\
\text { and calcium } \\
\text { chloride added }\end{array}$ \\
\hline $21-\operatorname{Sept}-90$ & $\begin{array}{l}\text { Tagged cement grout at about } 30 \mathrm{ft} \text { bls. } \\
\text { Drilled out the grout to } 125 \mathrm{ft} \text { bls. } \\
\text { The borehole contained backfill from } 125 \\
\text { to } 138 \mathrm{ft} \text { bls. Took a deviation } \\
\text { measurement at } 100 \mathrm{ft} \text { bls ( } 0.5 \text { degrees } \\
\text { from vertical). Drilled new hole from } \\
138 \text { to } 199.5 \mathrm{ft} \text { bls. }\end{array}$ & \\
\hline $24-$ Sept-90 & $\begin{array}{l}\text { Drilled to } 238.3 \mathrm{ft} \text { bls and grouted in } \\
\text { the borehole. }\end{array}$ & $\begin{array}{l}5 \mathrm{yd}^{3} \text { cement with } \\
\text { sand and bentonite } \\
\text { added }\end{array}$ \\
\hline
\end{tabular}




\begin{tabular}{|c|c|c|}
\hline DATE & ACTIVITY & MATERIALS USED \\
\hline $25-\operatorname{Sept}-90$ & $\begin{array}{l}\text { Tagged cement at about } 108 \mathrm{ft} \text { bls. } \\
\text { Drilled out cement to } 236 \mathrm{ft} \text { bls ( } 2 \mathrm{ft} \\
\text { of backfill below cement). Drilled to } \\
238 \text { and took a deviation measurement at } \\
200 \mathrm{ft} \text { ( } 1 \text { degree from vertical). } \\
\text { Drilled to } 338 \mathrm{ft} \text { bls. Grouted the } \\
\text { borehole. }\end{array}$ & $\begin{array}{l}5 \mathrm{yd}^{3} \text { cement with } \\
\text { bentonite and sand } \\
\text { added }\end{array}$ \\
\hline 26-Sept-90 & $\begin{array}{l}\text { Drilled out cement to } 338 \text { and continued } \\
\text { to drill to } 340 \mathrm{ft} \text { bls. Took a } \\
\text { deviation shot }(0.5 \mathrm{degrees} \text { from } \\
\text { vertical) at } 300 \mathrm{ft} \text { bls. Drilled to } 385 \\
\mathrm{ft} \text { bls and grouted the borehole. }\end{array}$ & $\begin{array}{l}2 \mathrm{yd}^{3} \text { cement with } \\
\text { bentonite and sand }\end{array}$ \\
\hline 27-Sept-90 & $\begin{array}{l}\text { Tagged cement at } 325 \mathrm{ft} \text {, drilled to } \\
\text { about } 350 \mathrm{ft} \text {, but cement appeared wet. } \\
\text { Tripped out drill string to let cement } \\
\text { grout set up. }\end{array}$ & \\
\hline 28-Sept-90 & Drilled to $397 \mathrm{ft} \mathrm{bls.}$ & \\
\hline 29-Sept-90 & Grouted the borehole. & $\begin{array}{l}\text { Cement mixed with } \\
\text { calcium chloride, } \\
\text { bentonite, and } \\
\text { sand }\end{array}$ \\
\hline $01-0 c t-90$ & $\begin{array}{l}\text { Lowered drill string to } 185 \mathrm{ft} \text { bls, but } \\
\text { still did not encounter cement grout. } \\
\text { It is suspected the grout filled a } \\
\text { fracture void. Decided to pull out of } \\
\text { hole and regrout, because of lost } \\
\text { circulation and poor grouting. }\end{array}$ & $\begin{array}{l}5 \mathrm{yd}^{3} \text { cement with } \\
\text { bentonite and sand } \\
\text { added }\end{array}$ \\
\hline $02-0 c t-90$ & $\begin{array}{l}\text { Drilled out grout. Continued drilling } \\
\text { into basait to } 431 \mathrm{ft} \text { bls. Took a } \\
\text { deviation shot at } 400 \mathrm{ft} \text { bls ( } 1 \text { degree } \\
\text { from vertical). Poured grout in } \\
\text { borehole. }\end{array}$ & $\begin{array}{l}2 \text { yd }^{3} \text { cement with } \\
\text { sand, bentonite, } \\
\text { and calcium } \\
\text { chloride added }\end{array}$ \\
\hline $03-0 c t-90$ & $\begin{array}{l}\text { Tagged grout at } 394 \mathrm{ft} \text { bls and drilled } \\
\text { out to } 431 \mathrm{ft} \text { bls. Drilled new hole to } \\
532 \mathrm{ft} \text {, total depth for this borehole. }\end{array}$ & \\
\hline $04-0 c t-90$ & $\begin{array}{l}\text { Depth to water was } 477 \mathrm{ft} \text { bls. USGS } \\
\text { logged borehole, taking video, gamma- } \\
\text { gamma, natural gamma, and caliper logs. } \\
\text { Logs were zeroed at the top of the } 12 \text { - } \\
\text { inch surface casing }(0.75 \mathrm{ft} \text { above land } \\
\text { surface). }\end{array}$ & \\
\hline
\end{tabular}


C. Summary of Well Construction Procedures:

\begin{tabular}{|c|c|c|}
\hline DATE & ACTIVITY & MATERIALS USED \\
\hline $04-0 c t-90$ & $\begin{array}{l}\text { Installed } 4 \text {-inch ID, passivated, } \\
\text { stainless steel weli screen, wire- } \\
\text { wrapped ( } 0.02 \text {-inch slot size) and flush } \\
\text { threaded with an endplug threaded to the } \\
\text { bottom of the screen. Began } \\
\text { installation of } 4 \text {-inch nominal pipe size } \\
(4.5-\text { inch OD), passivated, stainless } \\
\text { steel well casing, schedule los, type } \\
304 \text {, flush threaded, down the borehole } \\
\text { with } 2 \text { ft of stick-up. Installed three } \\
\text { stainless steel centralizers. }\end{array}$ & $\begin{array}{l}20 \mathrm{ft} \text { of } 4 \text {-inch ID } \\
\text { wel1 screen, } 480 \\
\mathrm{ft} \text { of } 4 \text {-inch well } \\
\text { casing, } 3 \\
\text { centralizers. }\end{array}$ \\
\hline $05-0 c t-90$ & $\begin{array}{l}\text { Completed installation of we } 11 \text { casing. } \\
\text { Measured water level at } 480.5 \mathrm{ft} \mathrm{bls} \text {. } \\
\end{array}$ & \\
\hline $08-0 c t-90$ & $\begin{array}{l}\text { Measured water level at } 477.7 \mathrm{ft} \text { bls. } \\
\text { Assumed this measurement was the most } \\
\text { stable measurement. Installed filter } \\
\text { pack consisting of } 6-9 \text { grade sand and } \\
\text { topped with } 1.5 \mathrm{ft} \text { of } 10-20 \text { grade sand, } \\
\text { using a tremie pipe. Installed } \\
\text { bentonite pellet seal above the filter } \\
\text { pack using a tremie pipe. Water was } \\
\text { added to the hole during the sand and } \\
\text { bentonite placement. }\end{array}$ & $\begin{array}{l}50 \text { sacks of } 6-9 \\
\text { grade silica sand, } \\
1 \text { bag of } 10-20 \\
\text { grade silica sand, } \\
25 \text { gallons } \\
\text { Volclay/Pure Gold } \\
\text { bentonite pellets, } \\
75 \text { gallons water }\end{array}$ \\
\hline $10-0 c t-90$ & $\begin{array}{l}\text { Began grouting the well annulus using a } \\
\text { tremie pipe. }\end{array}$ & $\begin{array}{l}\text { First cement grout } \\
\text { lift: } 50 \text { gallons } \\
\text { water, } 11 \text { pounds } \\
\text { bentonite, } 6 \text { bags } \\
\text { cement, } 5.5 \text { bags } \\
\text { sand; } 1 \text { ift \#2: } \\
5.5 \mathrm{yd}^{3} \text { cement } \\
\text { with sand and } \\
\text { bentonite added }\end{array}$ \\
\hline $11-0 c t-90$ & $\begin{array}{l}\text { Continued grouting well annulus. } \\
\text { Appeared to be filling a void, because } \\
\text { lifts were small. }\end{array}$ & $\begin{array}{l}1 \mathrm{yd}^{3} \text { cement with } \\
\text { sand and bentonite } \\
\text { added }\end{array}$ \\
\hline $12-0 c t-90$ & Continued grouting we 11 annulus. & $\begin{array}{l}6 \mathrm{yd}^{3} \text { cement with } \\
\text { sand and bentonite } \\
\text { added }\end{array}$ \\
\hline $15-0 c t-90$ & Continued grouting we 11 annulus. & $\begin{array}{l}6 \mathrm{yd}^{3} \text { cement with } \\
\text { sand and bentonite } \\
\text { added }\end{array}$ \\
\hline
\end{tabular}




\begin{tabular}{||c|l|l||}
\hline DATE & \multicolumn{1}{|c|}{ ACTIVITY } & MATERIALS USED \\
\hline $\begin{array}{c}\text { 16-0ct-90 } \\
\text { to }\end{array}$ & $\begin{array}{l}\text { Completed grouting well annulus to } \\
\text { surface. Installed 6-inch ID carbon } \\
\text { steel protective casing. Concrete pad } \\
\text { and impingement posts were installed. }\end{array}$ & $\begin{array}{l}6 \mathrm{ft} \text { of 6-in ID } \\
\text { protective casing, } \\
\text { unknown amount of } \\
\text { cement }\end{array}$ \\
\hline 05-Nov-90 & $\begin{array}{l}\text { Installed a Hydrostar piston pump. Pump } \\
\text { intake was set at 485.06 ft bls. Water } \\
\text { level was 477.7 ft bls. }\end{array}$ & \\
\hline
\end{tabular}

D. Summary of Well Development Procedures:

\begin{tabular}{||l|l|c|}
\hline DATE & \multicolumn{1}{|c|}{ ACTIVITY } & MATERIALS USED \\
\hline 02-Nov-90 & $\begin{array}{l}\text { Surged well for a total of } 55 \text { minutes } \\
\text { using a surge block. Lowered a }\end{array}$ & \\
& $\begin{array}{l}\text { submersible pump into the wel1 and began } \\
\text { evacuating water Purged about }\end{array}$ & \\
& $\begin{array}{l}\text { 300 gallons untij water was clear and } \\
\text { parameters (i.e., temperature, pH, } \\
\text { conductivity, dissolved oxygen) } \\
\text { stabilized. }\end{array}$ & \\
\hline
\end{tabular}

\section{GEOLOGICAL OBSERVATIONS}

A. Geological log, geophysical logs, stratigraphic column, and well construction diagram are attached.

B. Samples collected. No samples were collected.

Note: Borehole number LF3-11A was used in logbook ERP-27-90 to record the drilling of this borehole, which was the second attempt to drill borehole LF311. The borehole number was changed to LF3-11 and is referred to as LF3-11 in logbooks ERP-347-90 and ERP-29-90. 


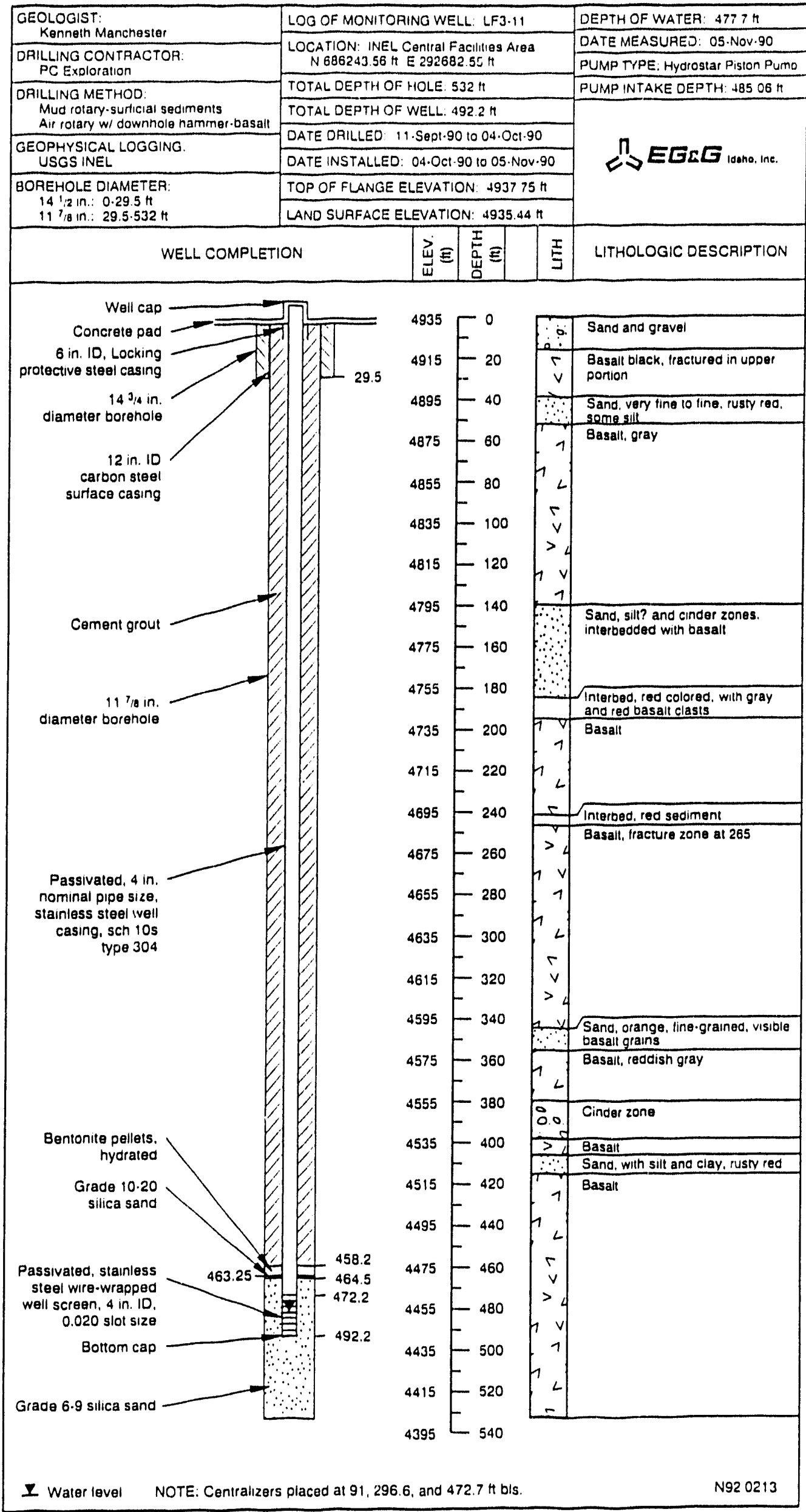




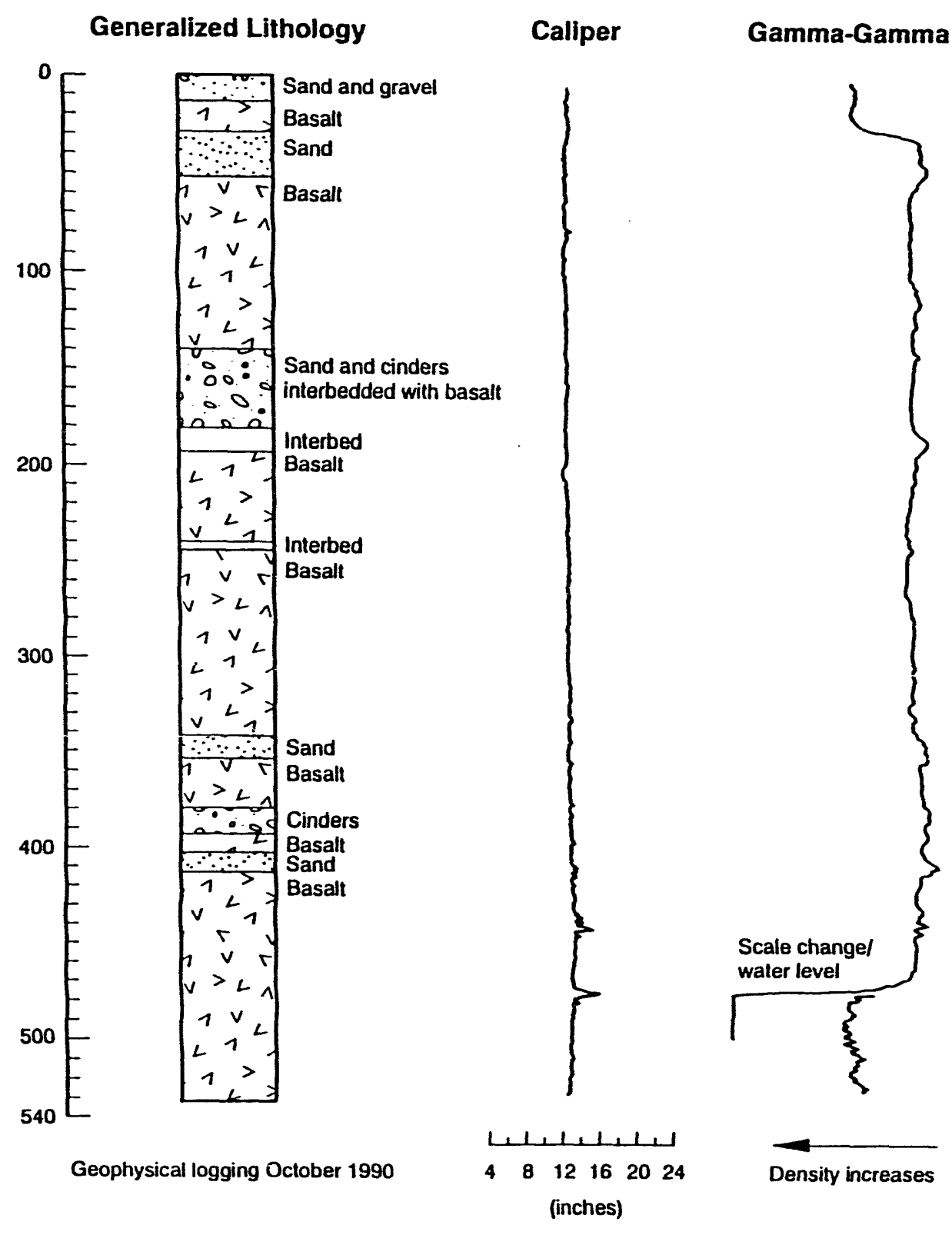

Natural Gamma
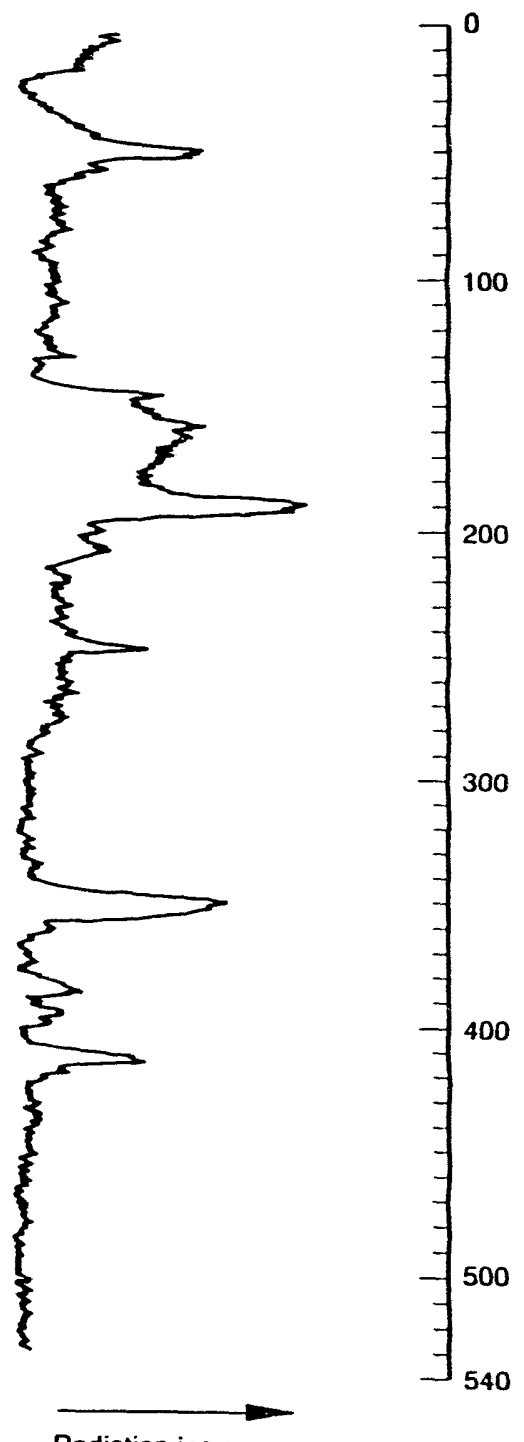

200

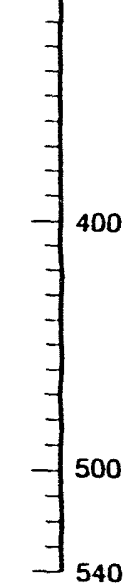

Well CFA LF3-11 
END-OF-WELL REPORT

CENTRAL FACILITIES LANDFILL AREA

WELL LF3-11A 
I. GENERAL INFORMATION

A. Project Name: Hydrogeologic Characterization Study for CFA Landf $i 11 \mathrm{~s}$

B. Wel1 Name/Number: LF3-11A

C. Well Location: INEL, Central Facilities Area Landfill III Northing: $686412.70 \mathrm{ft}$ Easting: $292601.45 \mathrm{ft}$ Land Surface Elevation: $4936.0 \mathrm{ft}$ (NRTS datum)

D. Plans: Survey Date: December 1990, MK-Ferguson

1) Technical Work Plan for the Hydrogeologic Characterization of CFA Landfi77s II and III, EGG-WM-9119, July 6, 1990.

2) Health and Safety Plan for Operations Performed for the Environmental Restoration Program, Well Installation and Groundwater Sampling at CFA Landfills II and III, EGG-WM-9105, May 1990.

E. Logbooks used:

1) Field Team Leader's Daily Logbook, ERP-27-90

2) Field Team Leader's Daily Logbook, ERP-347-90

3) Well Drilling, Installation, and Development Logbook, CFA-3

\section{DRILLING AND COMPLETION OBSERVATIONS}

A. Drilling Company: PC Exploration

Drillers: Mack Stillwe11, Paul Harmon, Scott McNinch, Wade Schott On-Site Geologist: David Burgess

Project Manager: Shannon Ansley

Rig type: Reichdril1-700

Bit type: Tricone bit for surficial sediments, air hammer bit for basalt.

B. Summary of Drilling Activities:

\begin{tabular}{|c|c|c|}
\hline DATE & ACTIVITY & MATERIALS USED \\
\hline 06 -Aug-90 & Drill rig and pipe deconned. & \\
\hline $07-$ Aug-90 & Casing deconned. & \\
\hline 10 -Aug-90 & $\begin{array}{l}\text { Deconned mud pump, bit, and hose. } \\
\text { Drilled approximately } 5 \text { ft bls with } \\
15-\text { inch tricone bit, using mud rotary } \\
\text { method. }\end{array}$ & \\
\hline
\end{tabular}




\begin{tabular}{|c|c|c|}
\hline DATE & ACTIVITY & MATERIALS USED \\
\hline 13 -Aug- 90 & $\begin{array}{l}\text { Drilled through surficial sediments and } \\
\text { into basalt. Drilled to } 35 \mathrm{ft} \text { bls and } \\
\text { basalt was encountered at approximately } \\
28 \mathrm{ft} \text { bls. Surface casing was pushed to } \\
32.8 \mathrm{ft} \text { bls and grouted. }\end{array}$ & $\begin{array}{l}37 \mathrm{ft} \text { of } \\
121 / 4 \text {-inch ID } \\
\text { carbon steel } \\
\text { surface casing; } 20 \\
\text { sacks of cement } \\
\text { with } 2 \% \text { bentonite, } \\
140 \text { gallons of } \\
\text { water }\end{array}$ \\
\hline 15-Aug-90 & $\begin{array}{l}\text { Drilled with } 5.5 \text {-inch hammer bit to } \\
\text { approximately } 100 \mathrm{ft} \text { bls. Sample } \\
\text { collection was attempted at } 69 \mathrm{ft} \text { bls, } \\
\text { but either the interbed was nonexistent } \\
\text { or very thin at that depth. }\end{array}$ & \\
\hline 16 -Aug-90 & $\begin{array}{l}\text { Reamed with } 117 / 8 \text {-inch hammer bit to } \\
65 \mathrm{ft} \mathrm{bls.}\end{array}$ & \\
\hline 17 -Aug- 90 & Continued reaming borehole. & \\
\hline 20-Aug-90 & $\begin{array}{l}\text { Took a deviation measurement at } 100 \mathrm{ft} \text {, } \\
\text { which was } 11 / 4 \mathrm{degree} \text { from vertical. } \\
\text { Drilled to } 117 \mathrm{ft} \text { bls. Cable clamps } \\
\text { broke/slipped and bit, causing hammer } \\
\text { and } 3 \text { drill pipes to fall down hole. }\end{array}$ & \\
\hline 21-Aug-90 & Worked to fish drill string out of hole. & \\
\hline 22-Aug-90 & $\begin{array}{l}\text { Fished drill string out of hole with an } \\
\text { overshot assembly. Drilled to } 133 \mathrm{ft} \\
\text { bis. }\end{array}$ & \\
\hline 23-Aug-90 & Drilled to $157 \mathrm{ft}$ bls. & \\
\hline 24-Aug- 90 & Drilled to $215 \mathrm{ft}$ bls. & \\
\hline 25-Aug-90 & Grouted the borehole. & $\begin{array}{l}\text { Unknown amount of } \\
\text { cement }\end{array}$ \\
\hline 27 - Aug -90 & $\begin{array}{l}\text { Tagged top of cement at } 78 \mathrm{ft} \text { bl s. } \\
\text { Drilled out } 40 \mathrm{ft} \text { of cement and then } \\
\text { decided cement was too wet to continue } \\
\text { drilling. }\end{array}$ & \\
\hline 28-Aug-90 & $\begin{array}{l}\text { Drilled out cement to } 215 \mathrm{ft} \text { bls. } \\
\text { Continued drilling in basalt, to } 220 \mathrm{ft} \\
\text { bls. Took a deviation shot ( } 5 \text { degrees } \\
\text { from vertical). There may have been a } \\
\text { problem with the deviation tool. } \\
\text { Grouted the borehole. }\end{array}$ & $2 \mathrm{yd}^{3}$ cement \\
\hline
\end{tabular}




\begin{tabular}{|c|c|c|}
\hline DATE & ACTIVITY & MATERIALS USED \\
\hline 29-Aug-90 & $\begin{array}{l}\text { Tagged top of cement at } 202 \mathrm{ft} \text { bls. } \\
\text { Drilled to } 300 \mathrm{ft} \text { bls and took deviation } \\
\text { shot at } 300 \mathrm{ft} \text { bls, }(0.75 \text { degrees from } \\
\text { vertical). Drilled to } 339 \mathrm{ft} \text { bls. }\end{array}$ & \\
\hline 30-Aug-90 & $\begin{array}{l}\text { Drilled to } 379 \mathrm{ft} \text { bls. Grouted the } \\
\text { borehole. }\end{array}$ & $3 \mathrm{yd}^{3}$ cement \\
\hline 31-Aug-90 & $\begin{array}{l}\text { Tagged grout at } 310 \mathrm{ft} \text { bls. Drilled out } \\
\text { grout to } 320 \mathrm{ft} \text { bls, but it seemed wet } \\
\text { so work was stopped. }\end{array}$ & \\
\hline $04-$ Sept-90 & $\begin{array}{l}\text { Drilled through the grout to } 370 \mathrm{ft} \text { bls. } \\
\text { Took a deviation measurement at } 385 \mathrm{ft} \\
\text { bls (0.5 degrees from vertical) and then } \\
\text { drilled to } 497 \mathrm{ft} \text { bls. Pulled the } \\
\text { drilistring up } 100 \mathrm{ft} \text { from the bottom. }\end{array}$ & \\
\hline $\begin{array}{l}05-\text { Sept }-90 \\
\text { to } \\
07-\text { Sept-90 }\end{array}$ & $\begin{array}{l}\text { In the morning drillers were unable to } \\
\text { pull the drilistring up. Rigged up a } \\
\text { tremie pipe to blow air around the bit } \\
\text { to try and free the drill string. Lost } \\
\text { one section of tremie down the borehole. }\end{array}$ & \\
\hline $10-$ Sept-90 & $\begin{array}{l}\text { Were able to retrieve the drill pipe, } \\
\text { but the collar and hammer remained in } \\
\text { the borehole. USGS ran a camera down } \\
\text { the borehole, and found the borehole was } \\
\text { bridged at } 315 \mathrm{ft} \text { bls. }\end{array}$ & \\
\hline 11 -Sept-90 & $\begin{array}{l}\text { Decided to abandon the borehole. The } \\
\text { borehole was renamed LF3-11A } \\
\text { (A=abandoned). }\end{array}$ & \\
\hline 14-Dec-90 & $\begin{array}{l}\text { Began tripping in the tremie pipe to } \\
\text { grout up the borehole. Tagged collar } \\
\text { and hammer at } 337 \mathrm{ft} \text { bls. Poured } \\
\text { bentonite down the borehole, using the } \\
\text { tremie pipe (to } 318 \mathrm{ft} \text { bls). }\end{array}$ & $\begin{array}{l}100 \text { gallons } \\
\text { bentonite hydrated } \\
\text { with } 70 \text { gallons of } \\
\text { water }\end{array}$ \\
\hline $17-$ Dec- 90 & $\begin{array}{l}\text { Poured cement grout to about } 268 \mathrm{ft} \text { bls, } \\
\text { using tremie pipe. }\end{array}$ & $\begin{array}{l}2 y^{3} \text { cement with } \\
\text { bentonite and sand } \\
\text { added }\end{array}$ \\
\hline 18-Dec-90 & $\begin{array}{l}\text { Poured cement grout to about } 100 \mathrm{ft} \text { bls, } \\
\text { using tremie pipe. Another cement grout } \\
\text { lift was poured, but depth and amount is } \\
\text { not known. }\end{array}$ & $\begin{array}{l}8 \text { yd }^{3} \text { cement with } \\
\text { bentonite and sand } \\
\text { added }\end{array}$ \\
\hline
\end{tabular}




\begin{tabular}{||l|l|l|}
\hline DATE & \multicolumn{1}{|c|}{ ACTIVITY } & MATERIALS USED \\
\hline unknown & $\begin{array}{l}\text { Borehole abandonment completed. Surface } \\
\text { casing was cut 6 inches bls, and a } \\
\text { square cement pad was instalied with a } \\
\text { brass survey marker over the borehole } \\
\text { grout. }\end{array}$ & $\begin{array}{l}\text { Cement with } \\
\text { bentonite and sand } \\
\text { added }\end{array}$ \\
\hline
\end{tabular}

C. Summary of Well Construction Procedures:

Borehole was abandoned.

D. Summary of Well Development Procedures:

Borehole was abandoned.

\section{GEOLOGICAL OBSERVATIONS}
A. Geological $\log$ is attached.
B. Samples collected. No samples were collected.

NOTE: The borehole number LF3-11 was recorded in the logbooks during the drilling of the borehole. The number was changed to LF3-1IA before the December 1990 location survey, in order to designate the borehole as abandoned $(A)$. 


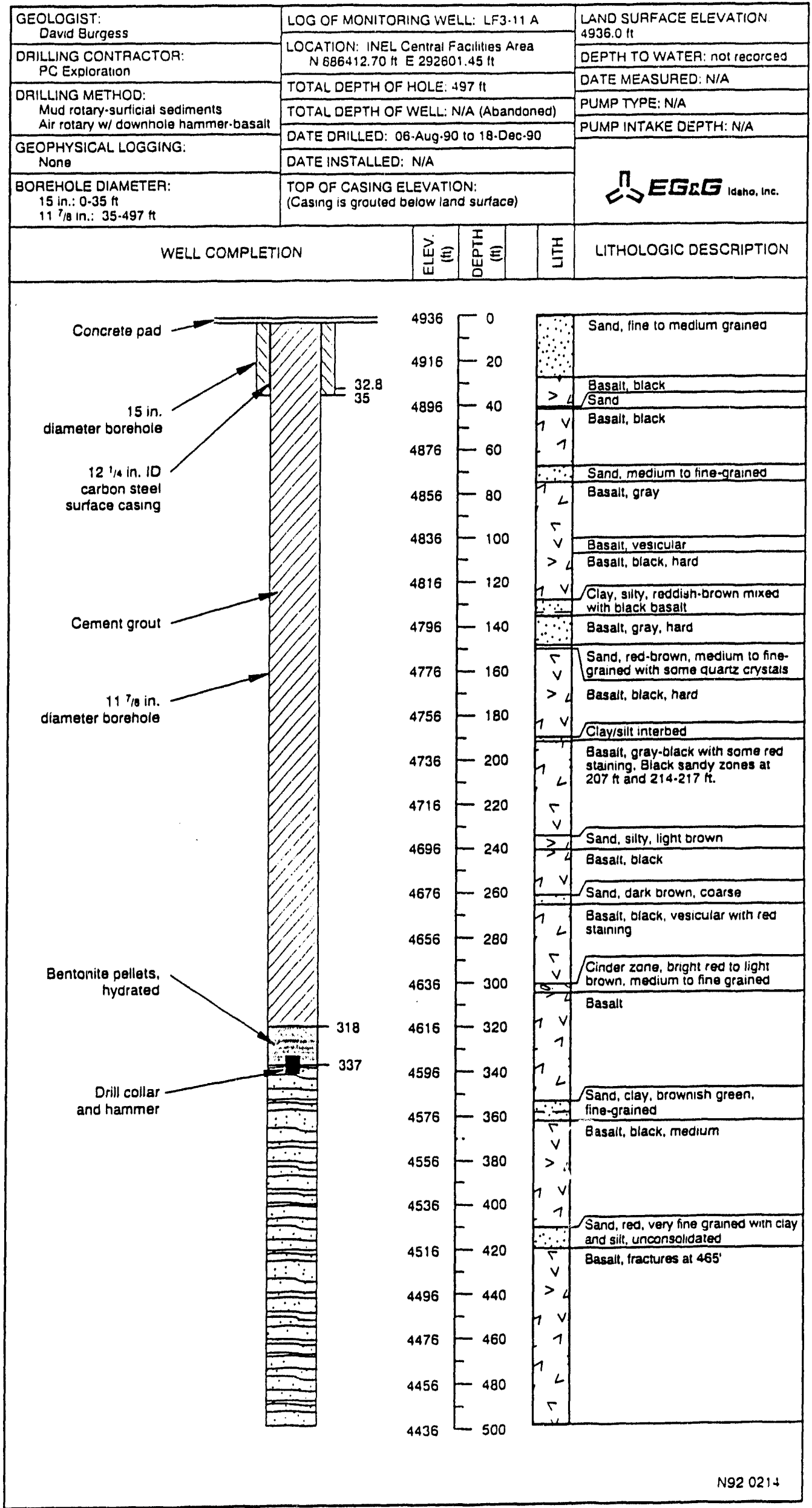


END-OF-WELL REPORT

WELL USGS 85 
Well Name: USGS 085 (1/C)

Composite confidence level: C

Coordinates

TRS: T3N-R29E-36bcbI $(1 / C)$
Northing: 685921.96
Easting: $291433.91 \quad(16 / C)$
Latitude: 433246.14
Longitude: $1125711.91 \quad(16 / C)$
Elevation Information
Elevation (ft.): $4940 \quad(1 / C)$
Datum: Mean sea level
(94/E)

Well Category

Well type: Observation ( $1 / C)$

Well status: Active (124/E)

Well instaliation type: single (1/C)

Drilling Information

Driller's name: Hopkins ( $1 / C)$

Year: 1962 ( $1 / \mathrm{C})$

Driling method: Cable tooled (1/C)

Work-over year: NF (115/)

Depth

Bore hole total depth BLS (ft.): 637 (1/C)

Well total depth BLS (ft.): 637 (1/C)

Water Information

Initial water level BLS (ft.): 481.83 (1/C)

Date: $10 / 25 / 62$ ( $1 / C)$
Water quality sampling frequency: Semi-annual (124/E)

Pump Information

Pump type: submersible $(124 / E)$

Depth BLS (ft.): 522 (124/E)

Discharge rate (gpm): 22 (124/E)

Water level access type: Line (124/E)

Available Information

Construction diagram: $Y$

Lithologic log: $Y$

Natural gamma log: Y

T.V. camera/photo log: $N$

Fiuid conductivity log: $N$
Gamma gamma log: N Caliper log: Y Driller's log: $Y$ Neutron log: $N$ Geologist's log: $N$ Electric log: N site schedule: $Y$ Field book: N 
Well: USGS 085 (continued)

Construction Information

Borehole Dimensions:

\begin{tabular}{|c|c|c|}
\hline Top/Bottom & $\operatorname{BLS}(\mathrm{ft.})(I / C)$ & Diameter (in.) (1/C) \\
\hline & $\begin{array}{c}0 / 68 \\
68 / 522 \\
22 / 637\end{array}$ & $\begin{array}{l}12 \\
10 \\
6\end{array}$ \\
\hline
\end{tabular}

Surface/Protective Casing:

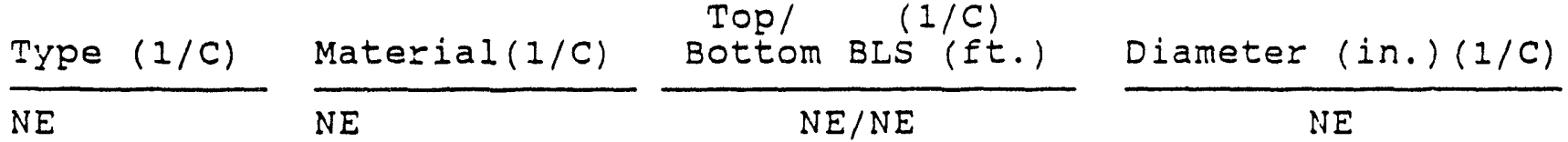

Well Casing:

$\frac{\text { Top/Bottom BLS (ft.)(1/C) }}{-2.3 / 522} \frac{\text { Diameter (in.)(1/C) }}{6.0} \frac{\text { Material(1/C) }}{\text { Steel }}$

Cement/Fill:

$\frac{\text { Top/Bottom BLS (ft.)(1/C) }}{\text { NF/NF }} \frac{\text { Type }(1 / C)}{N F}$

Completion Zone:

$\frac{\text { Type (I/C) }}{\text { open hole }} \frac{\text { Top/Bottom BLS (ft.)(1/C) }}{522 / 637} \frac{(\mathrm{in.})(I / C)}{6.0}$

$\frac{\text { Material }(114 /)}{\text { NA }} \frac{\text { Slot Size (114/) }}{\text { NA }} \quad \frac{\text { Filter Pack Type }(114 /)}{\text { NA }}$

Concrete Pad: NE (124/E)

Locking Cap: Yes (124/E)

Comments (115) 


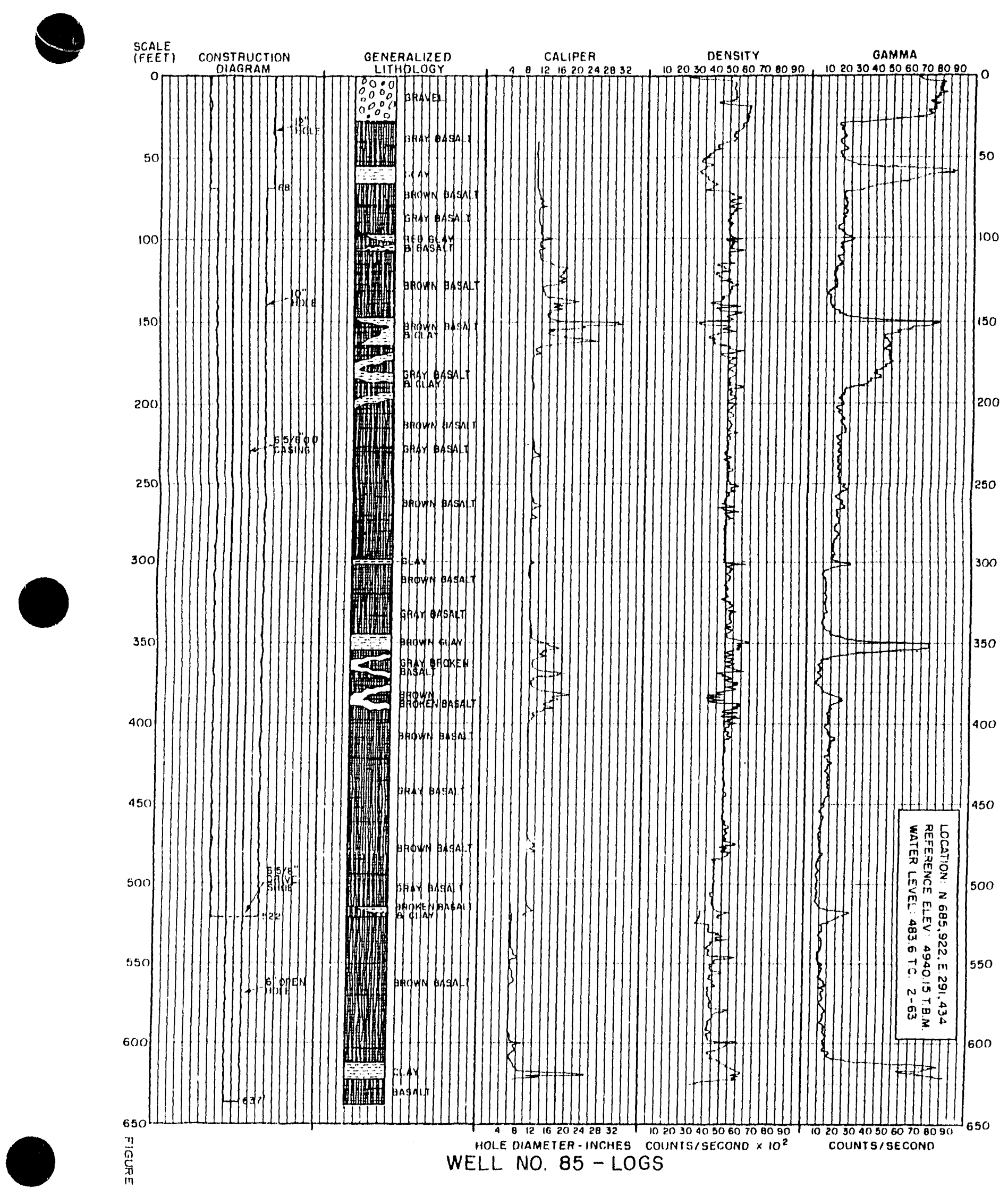

Page 3 
Borehole Logs (SAIC) at CFA Landfills II and III With Sample Collection Depths and Soil Descriptions 


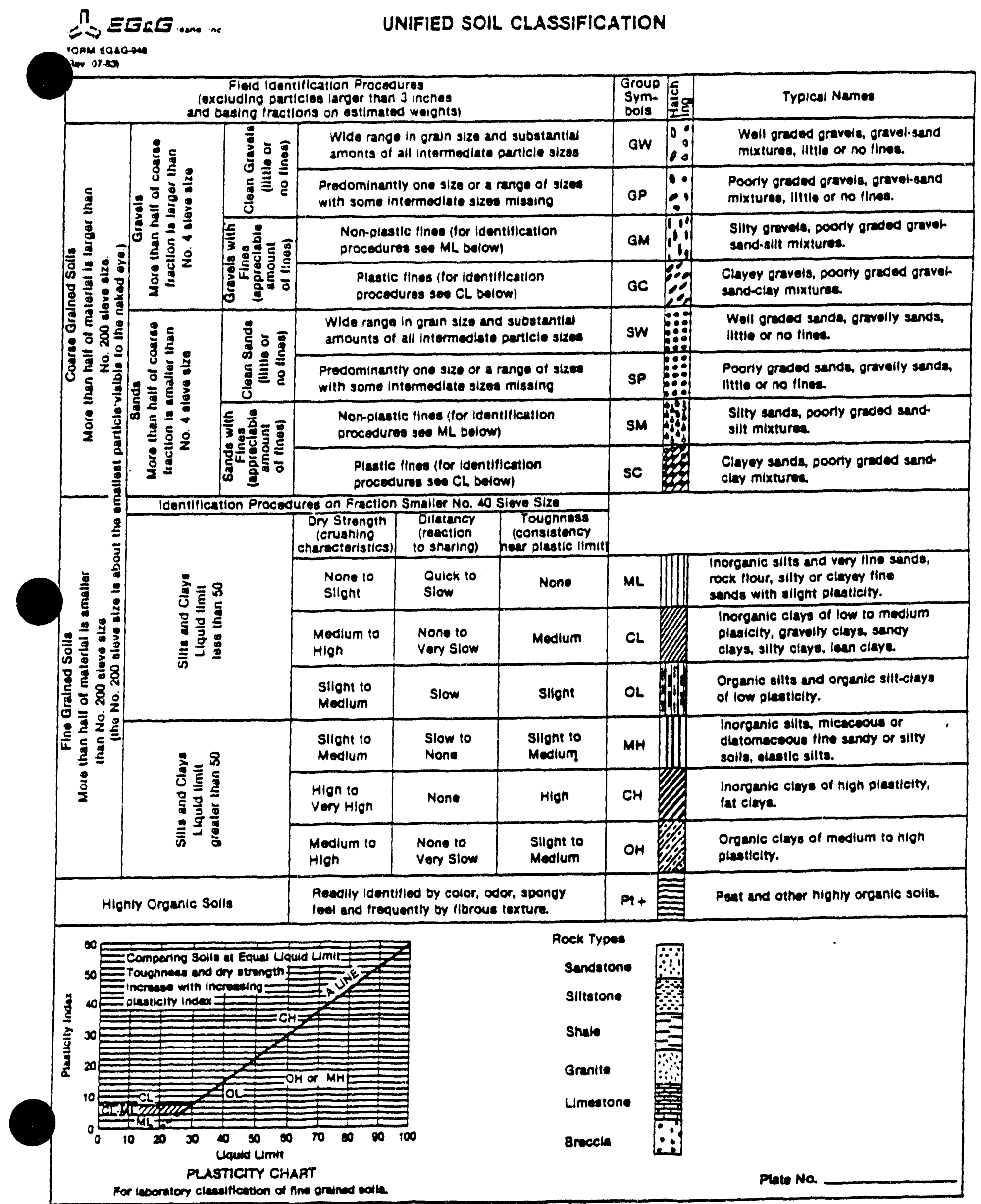




\section{LF $2-1$}

PRO JECT NUMBER $2-817-07-667-00$

PROJECT NAME EG\&G/INEL LandF11Is - CFA

LOCATION CFA LandFIII II

DRILLINC COMPANY Denning Well Drilling, Inc.

RIC TYPE \& NUMBER

ORILLINC METHOD 10" HOLlOW Stem AUger

WEATHER Cold, snowing

FIELD PARTY Jody and Dan Denning

CEOLOCIST John Jaacks, III and Brett Freier

DATE BECUN $12 / 16 / 87$ DATE COMPLETED 12/16/87

FIELO BOOK NO.

TOTAL DEPTH $27.13 \mathrm{ft}$.

SHEET 1 OF 2

\begin{tabular}{|c|c|c|}
\hline \multicolumn{3}{|c|}{ CROUNOWATER TABLE } \\
\hline WD=While & Drilling & $A B=A$ lter Boring \\
\hline $\operatorname{Depth}(f()$ & & \\
\hline Time & & \\
\hline Date & & \\
\hline
\end{tabular}

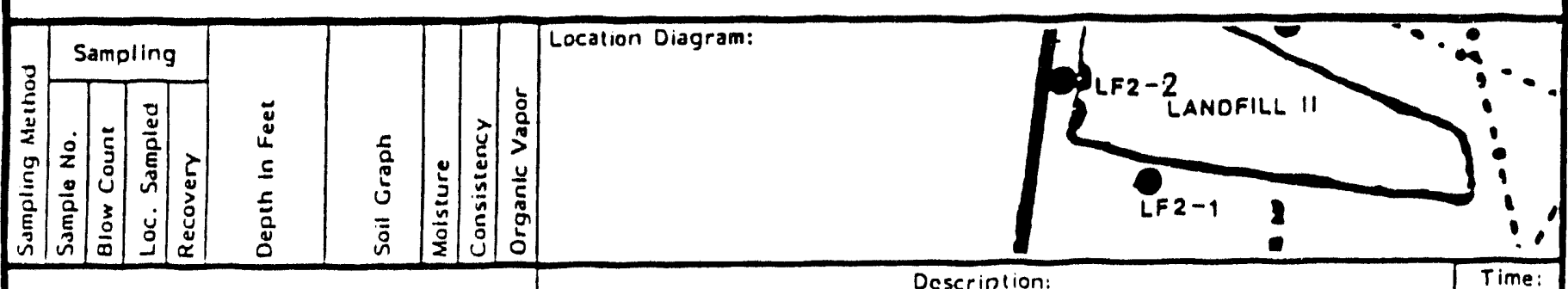

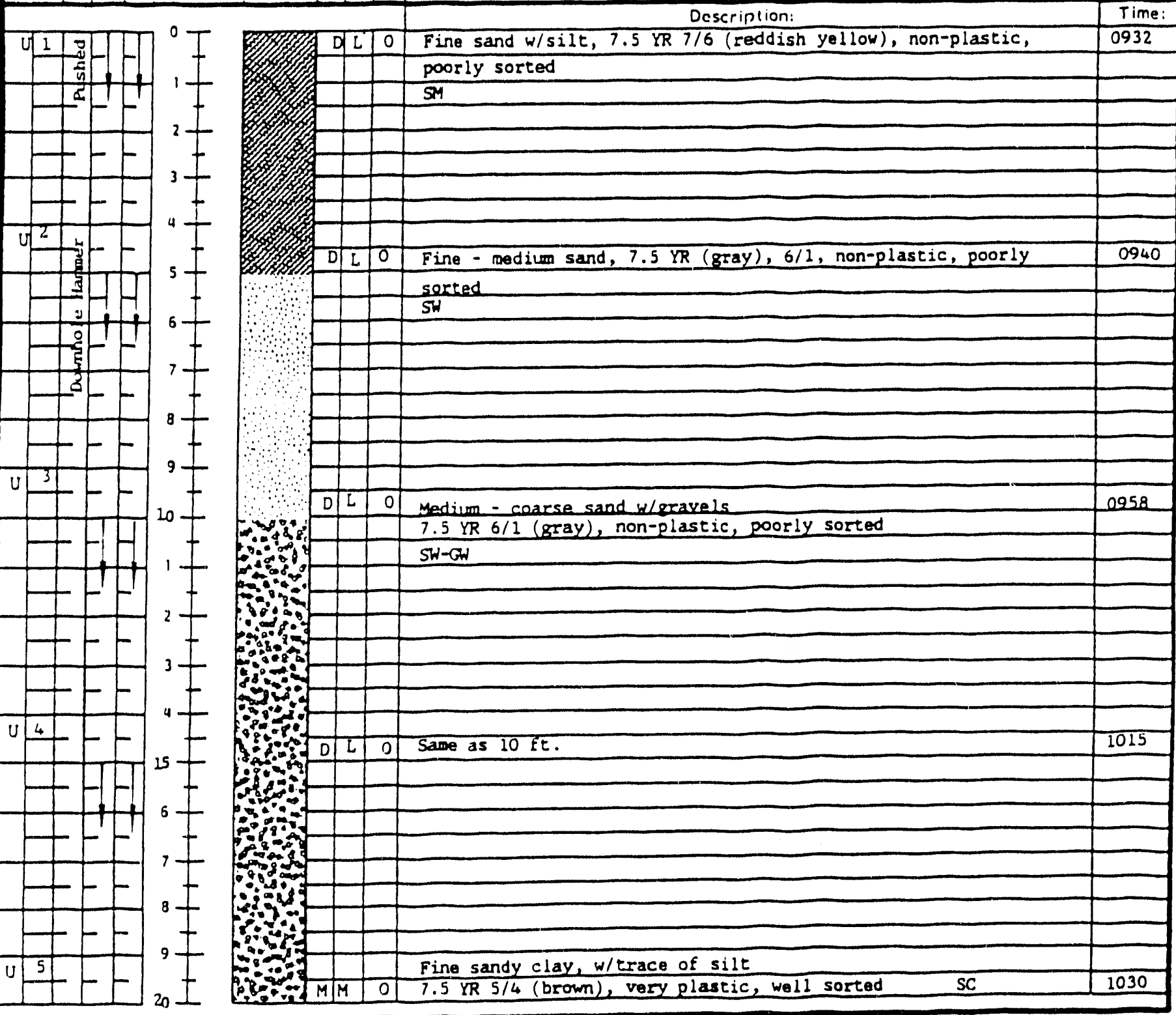




\section{gAIE.}

PROJECT NUMBER $\quad 3-817-07-667-00$

PROJECT NAME EGSG/INEL LandEIII - CFA

LOCATION CEA Landfill II

DRILLINC COMPANY

RIC TYPE \& NUMBER

DRILLINC METHOD 10" HOLlOW Scem Auger

WEATHER Cold, snowing

FIELD PARTY Jody and Dan Denning

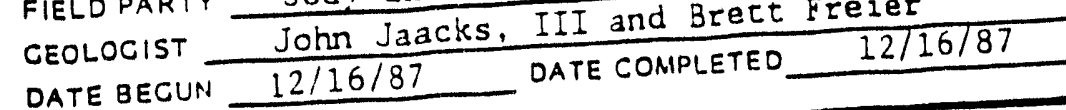

BOREHOLE NUMBER:

LF 2-1

FIELD BOOK NO. TOTAL DEPTH $27.13 \mathrm{EE}$. SHEET 2 OF 2

\begin{tabular}{|l|l|l|l|}
\hline \multicolumn{3}{|c|}{ CROUNOWATER TABLE } \\
\hline WD=While Drilling $A B=A$ fter Boring \\
\hline Depen(ft) & & & \\
\hline Time & & & \\
\hline Date & & & \\
\hline
\end{tabular}
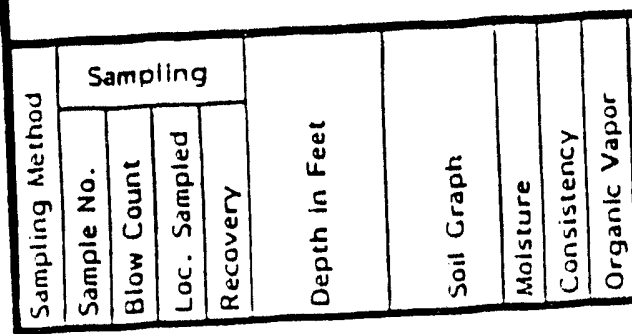

Location Diagram:

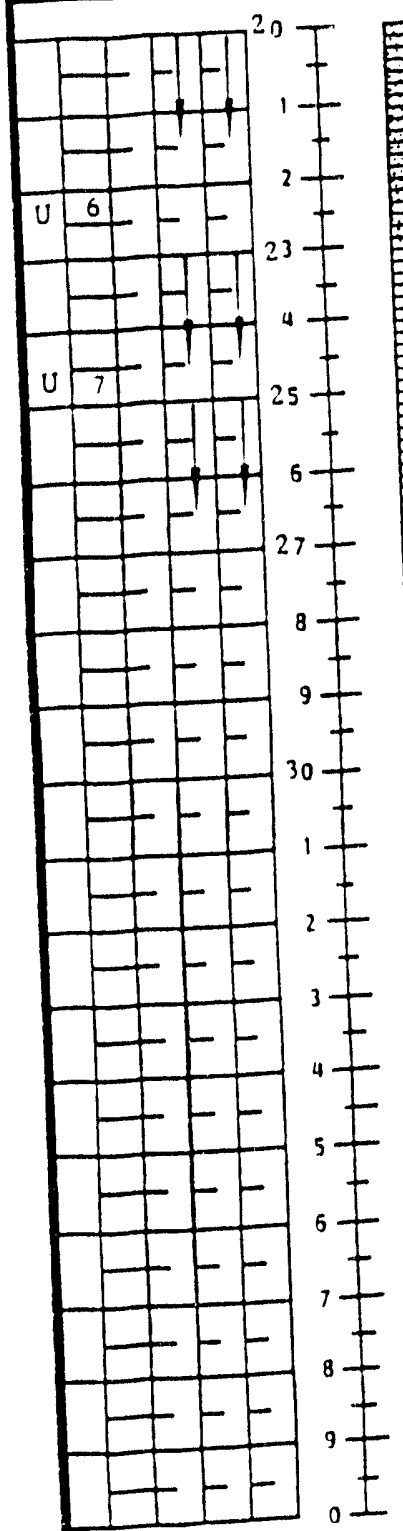

Description:

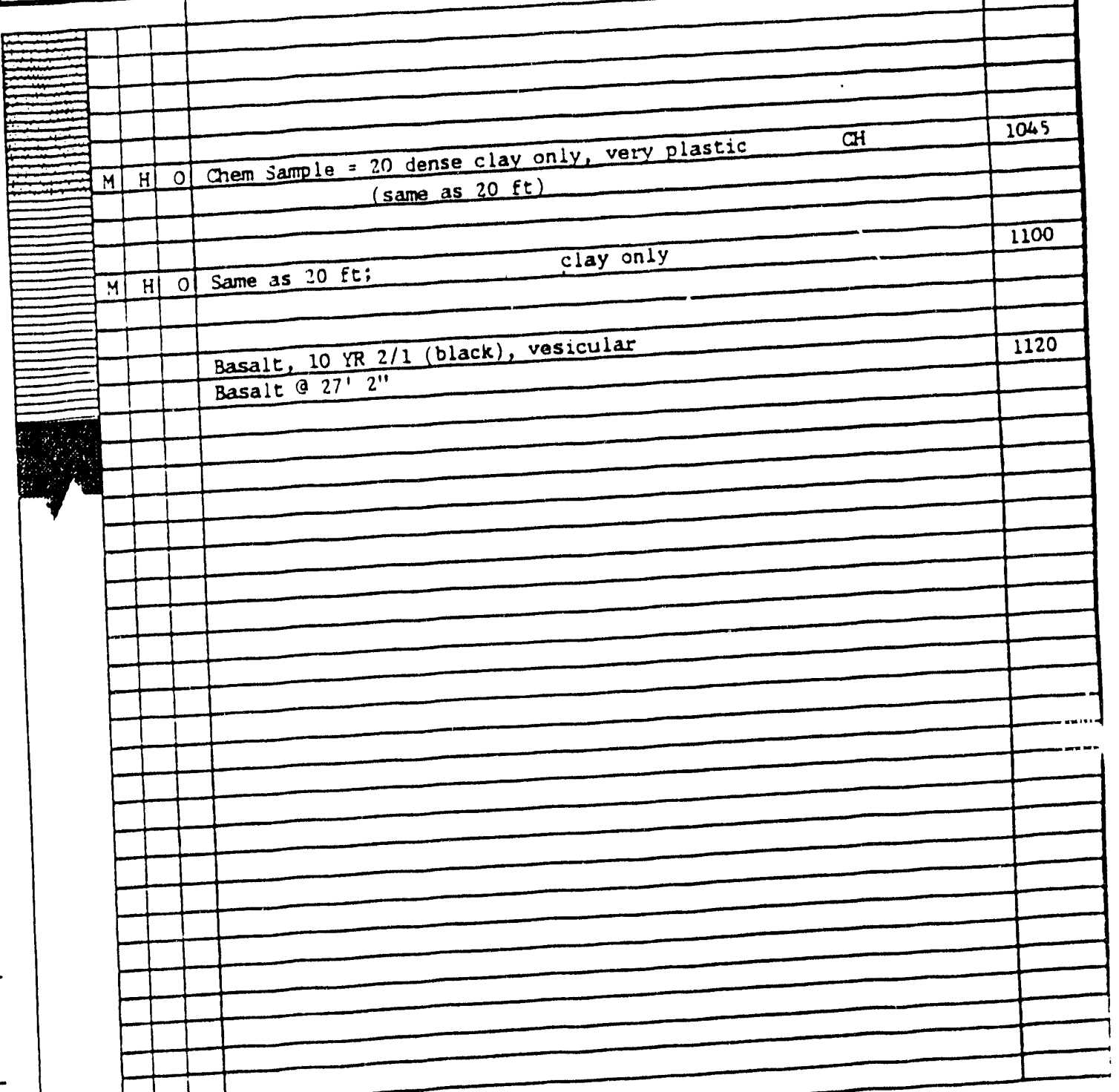




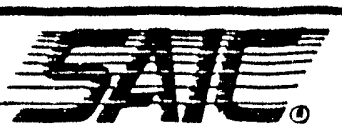

FIELD BOREHOLE LOC

BOREHOLE NUMBER:

LF2-3( $\begin{aligned} & 2-2 \text { ACCORDING } \\ & \text { TO EGCG })\end{aligned}$

PROJECT NUMBER 2-817-07-667-00

PROJECT NAME EG\&G/INEL CFA Landfills

LOCATION CFA LandFIII II

ORILLINC COMPANY Denning Well Drilling, InC.

RIC TYPE \& NUMBER

DRILLINC METHOD 10" Hollow Stem Auger

WEATHER Snowing, very cold, very poor visibility

FIELO PARTY Jody and Dan Denning

CEOLOCIST John Jaacks, III and Brett Freier

DATE BECUN $12 / 17 / 87$ DATE COMPLETED $12 / 17 / 87$

FIELO BOOK NO.

TOTAL DEPTH $31 \mathrm{ft}$.

SHEET 1 OF 2

\begin{tabular}{|c|c|c|}
\hline \multicolumn{3}{|c|}{ GROUNDWATER TABLE } \\
\hline WD $=$ While & Drilling & $A B=$ Afler Boring \\
\hline $\operatorname{Depth}(f t)$ & & \\
\hline Time & & \\
\hline Date & & \\
\hline
\end{tabular}

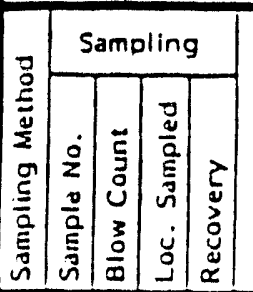
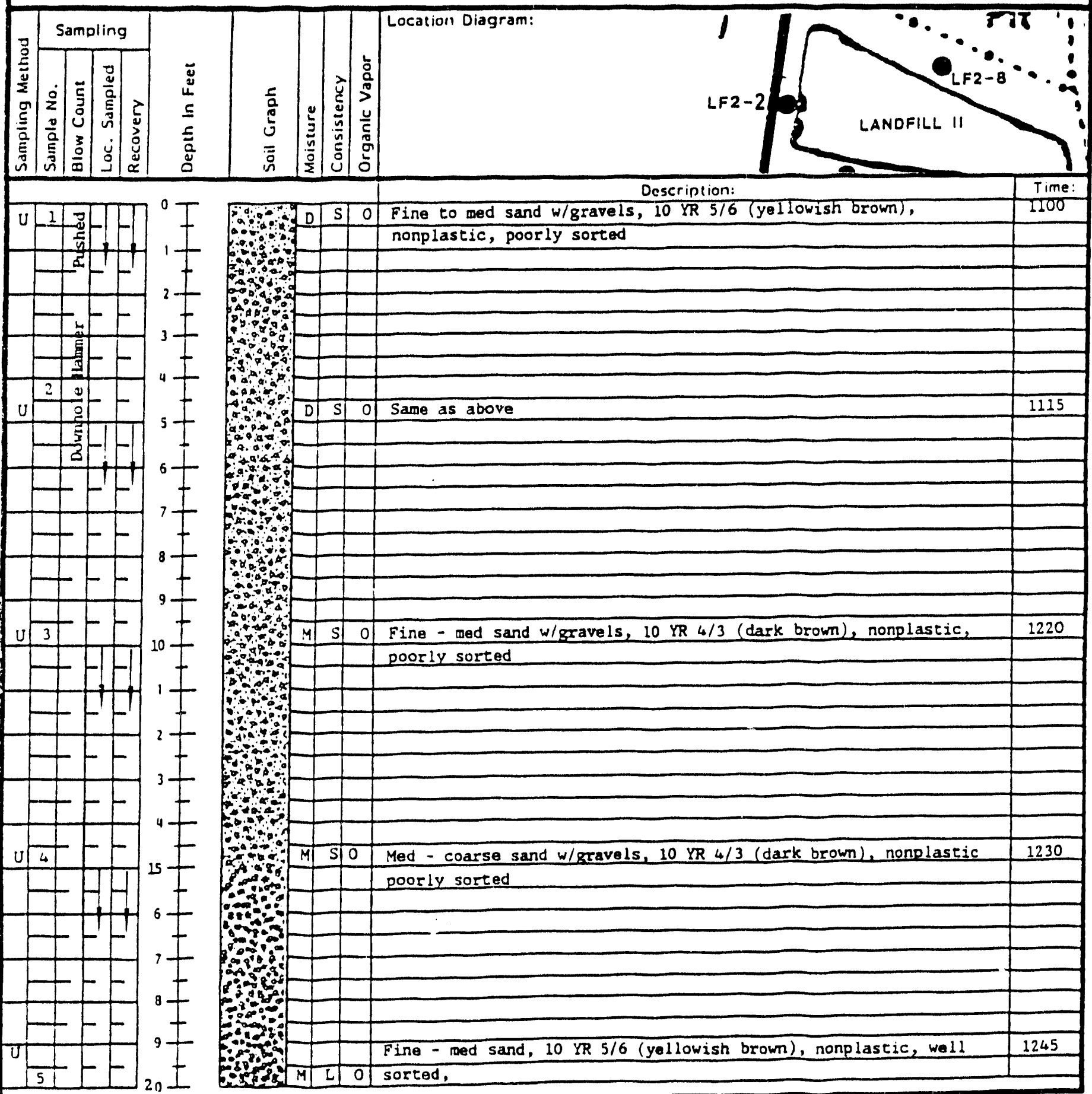

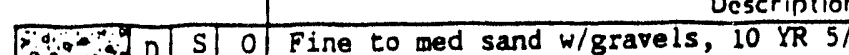

6 (yellowish brown),

nonplastic, poorly sorted

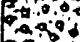

00.40.

Paing

8 a

and

4.2. 0 D $S$ S 0 Same as above

$0 \%$

a. 0.0 .0

Exise

$4 \div-4-4$

P.i.

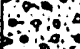

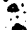

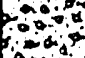

Fine - med sand w/gravels, 10 YR $4 / 3$ (dark brown), nonplastic

poorly surted

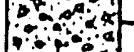

$\rightarrow 0$

ais.

40

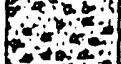

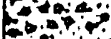

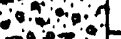

$\rightarrow-\infty$ StO Med - coarse sand w/gravels, $10 \mathrm{YR} 4 / 3$ (dark brown) nonplastic

poorly sorted

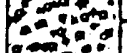

ind

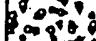

ing
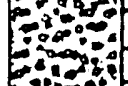
PROJECT NUMBER 2-817-07-567-00

PROJECT NAME EG\&G/INEL CFA Landfill

LOCATION CFA LandFIII II

ORILLINC COMPANY Denning Well Drilling, Inc.

RIC TYPE \& NUMBER

ORILLINC METHOD 10" HOLlOW Stem Auger

WEATHER Sowing very cold very por visibility

FIELD PARTY - Jody and Dan Denning

CEOLOCIST John Jaacks, III and Brett Freier

DATE BECUN $12 / 17 / 87$ DATE COMPLETED $12 / 17 / 87$
FIELD BOOK NO.

TOTAL OEPTH $31 \mathrm{ft}$.

SHEET 2 OF 2

\begin{tabular}{|l|l|l|l|}
\hline \multicolumn{3}{|c|}{ CROUNOWATER TABLE } \\
\hline WD=While Drilling & $A B=A$ fter Boring \\
\hline Depth(ft) & & & \\
\hline Time & & & \\
\hline Date & & & \\
\hline
\end{tabular}
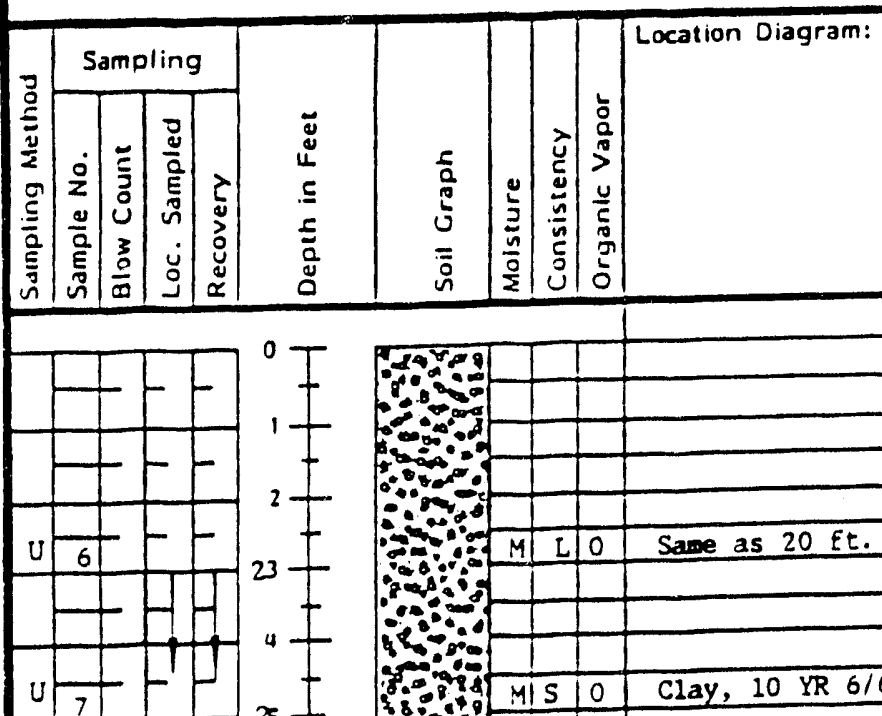

Description:

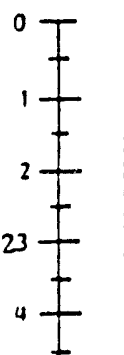

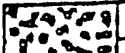
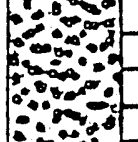

\begin{tabular}{lllll} 
& & & \\
\hline
\end{tabular}

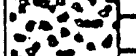

is:-

(

다

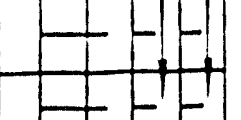

$x+$

$6+$

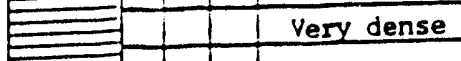

7

$8+$

$9+$

$30 t$

$\begin{array}{lll}49 & -11 \\ 31 & \end{array}$

$2+$

${ }_{3}+$

4

$s+$

$6+$

7

8

f

$0 工$

\begin{tabular}{|c|c|c|c|}
\hline \multicolumn{4}{|l|}{$\approx$} \\
\hline \multirow{2}{*}{\multicolumn{4}{|c|}{$E$}} \\
\hline & & & \\
\hline \multicolumn{4}{|l|}{$\sum$} \\
\hline \multirow{2}{*}{\multicolumn{4}{|c|}{$\equiv$}} \\
\hline & \multicolumn{3}{|c|}{$E$} \\
\hline \multirow{2}{*}{ 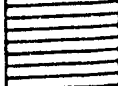 } & & & 1340 \\
\hline & $\square$ & Same as $25 \mathrm{ft}$ & 10 \\
\hline \multirow{2}{*}{$\equiv$} & & & 1355 \\
\hline & 0 & Basalt, 10 YR $2 / 1$ (black), vesicular & \\
\hline$\because$ & & & \\
\hline$\because$ & & & \\
\hline & & & \\
\hline & & & \\
\hline & & & \\
\hline & & & \\
\hline & & & \\
\hline & & & \\
\hline & & & \\
\hline & & & \\
\hline & & & \\
\hline & & & \\
\hline & & & \\
\hline & & & \\
\hline & & & \\
\hline
\end{tabular}




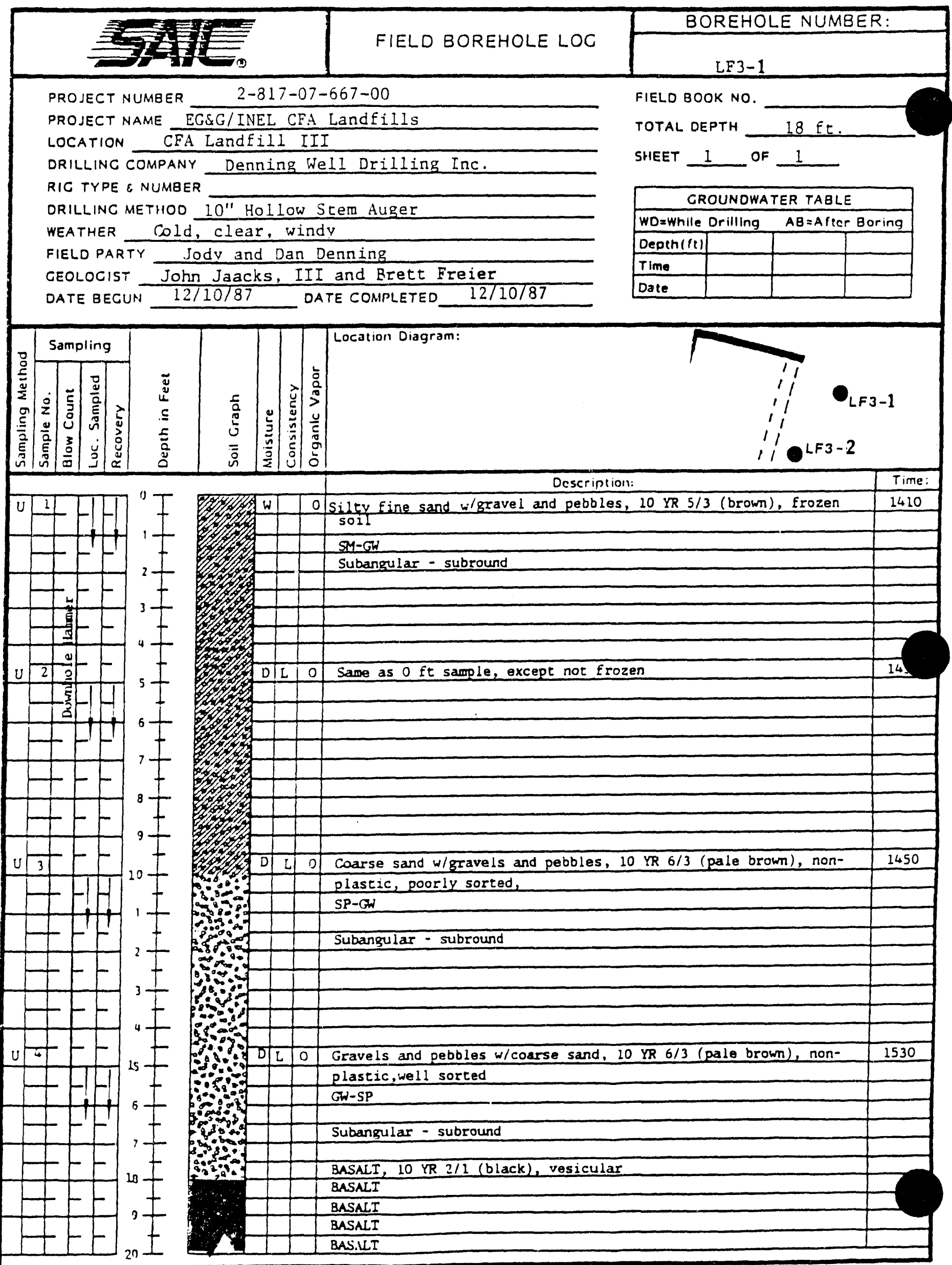




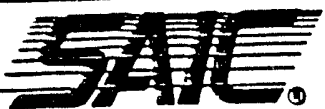

FIELD BOREHOLE LOC

ROJECT NUMBER

$2-817-07-667-00$

PRo JeCT NAME EGSG/INEL CFA Landfills

LOCATION CFA Landfill III

ORILLINC COMPANY Denning Well Drilling InC.

RIC TYPE \& NUMBER

ORILLING METHOD 10" Hollow Stem Auger

WEATHER Cold, clear, windy

FIELD PARTY Jody and Dan Denning

CEOLOCIST John Jaacks, III and Brett Freier

DATE BECUN $12 / 11 / 87$ DATE COMPLETED $12 / 11 / 87$
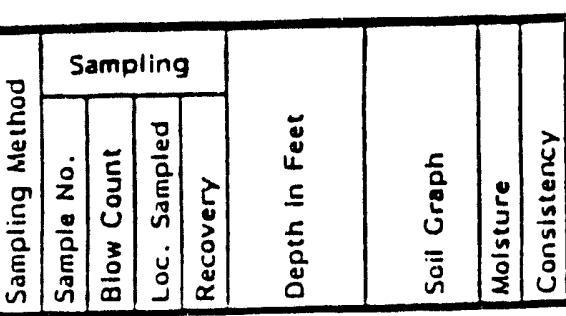

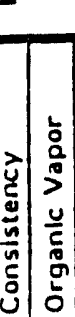

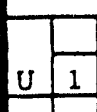
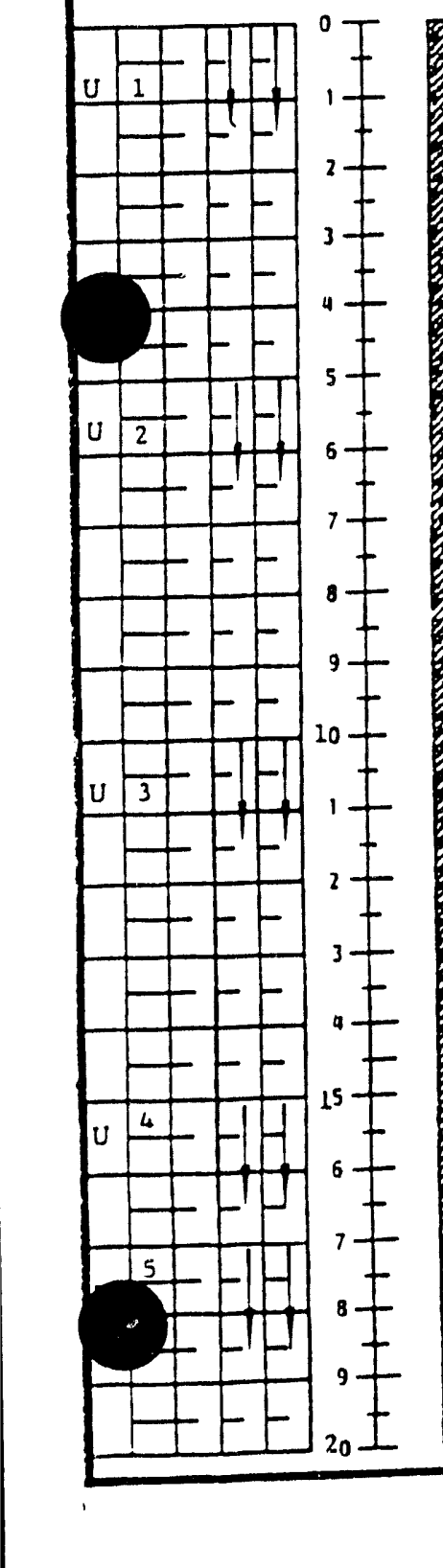
Location Diagram:

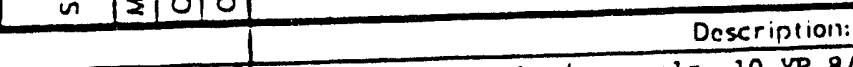

BOREHOLE NUMBER:

$$
\text { LF 3-2 }
$$

FIELD BOOK NO. TOTAL DEPTH $26.5 \mathrm{ft}$. SHEET 1 OF

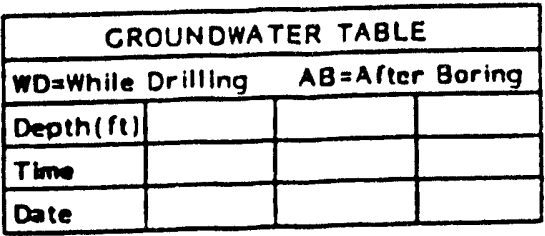

Description:
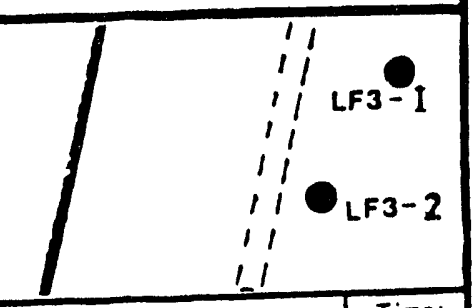

\begin{tabular}{|c|c|c|c|c|c|}
\hline \multicolumn{4}{|c|}{ 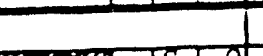 } & \multicolumn{2}{|l|}{ Description: } \\
\hline Oegiege & MTS & & & Silty, fine sand $w /$ gravels, 10 YR $8 / 4$ (very pale brown), non- & 1220 \\
\hline 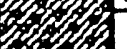 & & & & plastic, poorly sorted, subangular - subrounded & \\
\hline 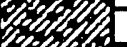 & & & & 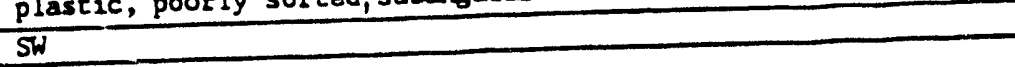 & \\
\hline yosese & & & & & \\
\hline Pse & & & & & \\
\hline & & & & & \\
\hline & & & & & \\
\hline 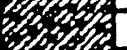 & & & & & \\
\hline segse & $\bar{D}$ & 5 & 0 & Silty fine - medium sand $w /$ gravels, 10 YR $5 / 3$ (brown), non- & 1245 \\
\hline 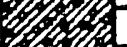 & & & & $\begin{array}{l}\text { Silty fine - medium sand w/gravels, } \\
\text { plastic, poorly sorted }\end{array}$ & \\
\hline ses & & & & $\begin{array}{l}\text { Plastic, poorly sorted } \\
\text { SM-GW }\end{array}$ & \\
\hline Yeges & & & & & \\
\hline 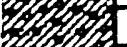 & & & & Subangular - subrounded & \\
\hline 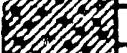 & & & & & \\
\hline 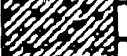 & & & & & \\
\hline Sos & & & & & \\
\hline 03 & & & & & \\
\hline 3 & & & & & \\
\hline ysta & D & $\underline{S}$ & 0 & Silcy fine - medium sand and grave $1 \mathrm{~s}, 10 \mathrm{YR} 5 / 9$ (brown), non- & 1300 \\
\hline 30 & & & & 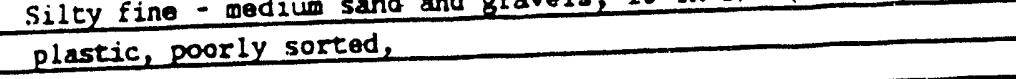 & \\
\hline ereot & & & & $\begin{array}{l}\text { plastic, poorly sorted, } \\
\text { Sy-Gid }\end{array}$ & \\
\hline ose & & & & & \\
\hline Plest & & & & Subangular - subrounded & \\
\hline & & & & & \\
\hline Yyes & & & & & \\
\hline Cosest & & & & & \\
\hline egsesy & DI & $\underline{s}$ & 요 & Same as above & 1320 \\
\hline eresta & & & & & \\
\hline 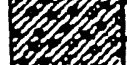 & & & & CHDM SNPLE: Siley fine sand $w /$ clay and gravels & \\
\hline 18. & & & & CHDM SNMPE: Siley fine sand $w /$ clay and gravels & 1349 \\
\hline 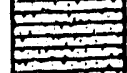 & & & & 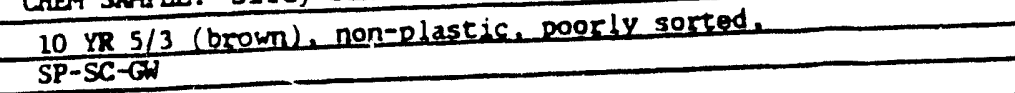 & \\
\hline 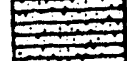 & & & & SP-SC-GN & \\
\hline & & & & Subanzular - subrounded & \\
\hline & & & & (mom) & \\
\hline & & & & ay, very plastic 10 YR $4 / 6$ (very yollowish brown) & \\
\hline
\end{tabular}




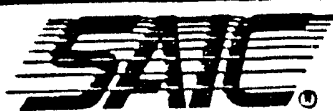

FIELD BOREHOLE LOC

PROJECT NUMBER

$2-817-07-667-00$

PROJECT NAME EG\&G/INEL Landfi11 - CFA

LOCATION CEA Iandfil] IIT

DRILLINC COMPANY Denning Wel! Drilling, InC.

RIC TYPE \& NUMBER

DRILLINC METHOD 10" HOLlOW Stem Auger

WEATHER Cold, windy, clear

FIELD PARTY Jody and Dan Denning

CEOLOCIST John Jaacks, III and Brett Freier

DATE BECUN $12 / 10 / 87$ DATE COMPLETED $12 / 10 / 87$
BOREHOLE NUMBER:

LF 3-2

FIELD BOOK NO.

TOTAL OEPTH

SHEET 2 OF 2

CROUNDWATER TABLE

$W D=$ While Drilling $A B=A$ fter Boring

Uepth(ft)

Time

Date

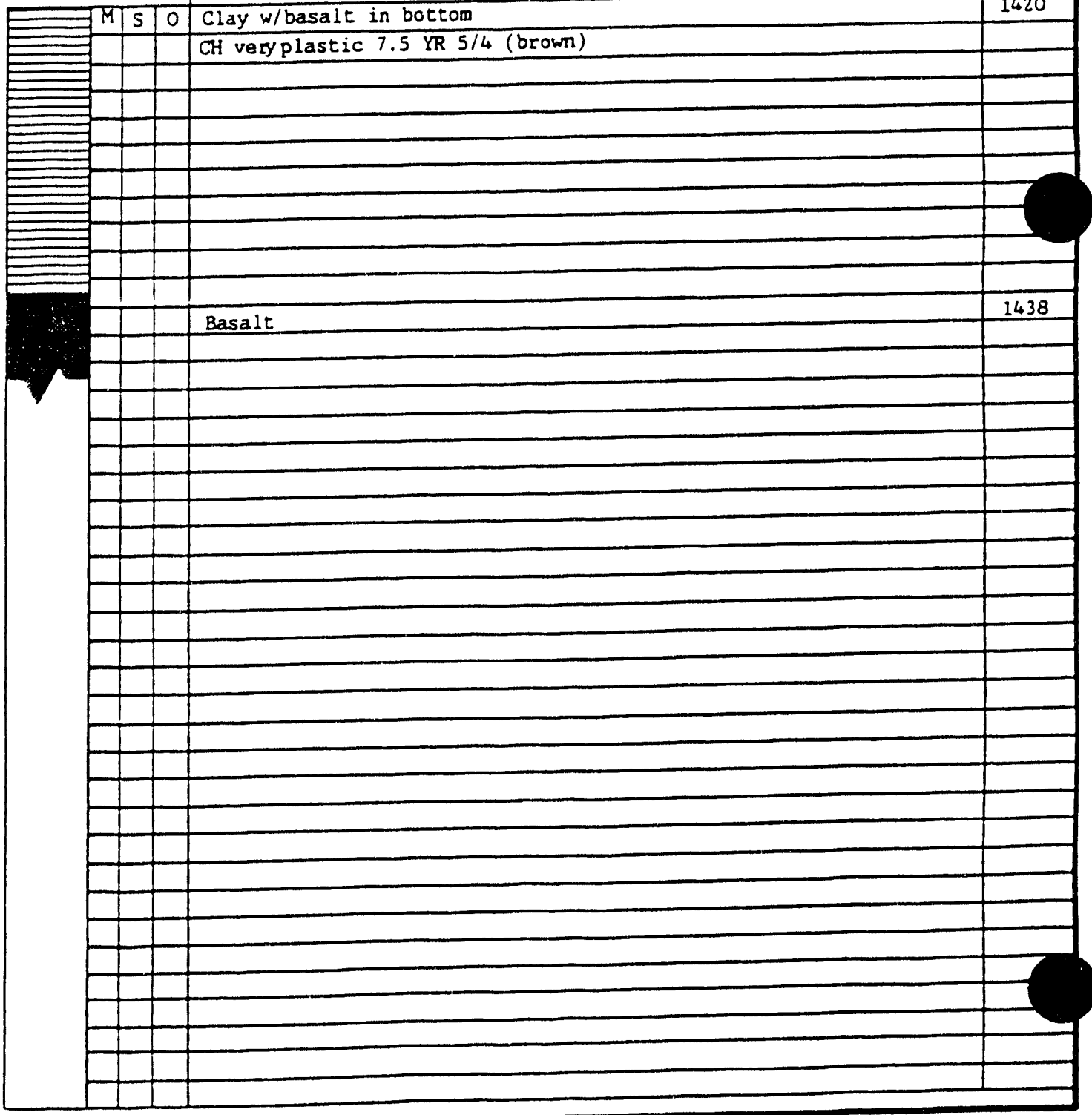


PROJECT NUMBER

$2-817-07-667-00$

PROJECT NAME EG\&G/INEL Landfills-CFA

LOCATION CFA LandFIII III

DRILLINC COMPANY Denning Well Drilling InC.

RIC TYPE ¿ NUMBER

ORILLINC METHOD 10" Hollow Stem Auger

WEATHER ibry cold, clear wiady

FIELD PARTY Jody and Dan Denning

GEOLOCIST John Jaacks and Brett Freier

DATE BECUN $12 / 12 / 87$ DATE COMPLETED
BOREHOLE NUMBER:

LF3-4

FIELD BOOK NO.

TOTAL DEPTH

$11.5 \mathrm{ft}$.

SHEET 1 OF 1

\begin{tabular}{|l|l|l|l|}
\hline \multicolumn{3}{|c|}{ CROUNOWATER TABLE } \\
\hline WO=While Drilling & $A B=$ After Boring \\
\hline DeDth(ri) & & & \\
\hline Time & & & \\
\hline Date & & & \\
\hline
\end{tabular}

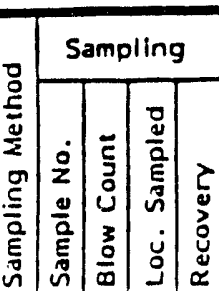

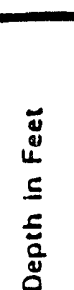

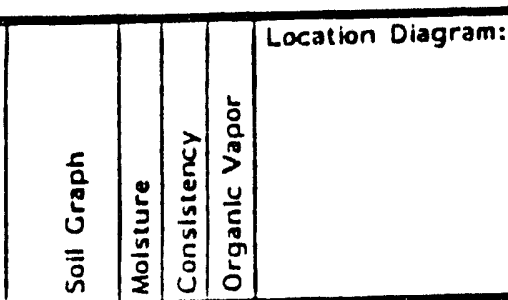

$12 / 12 / 87$

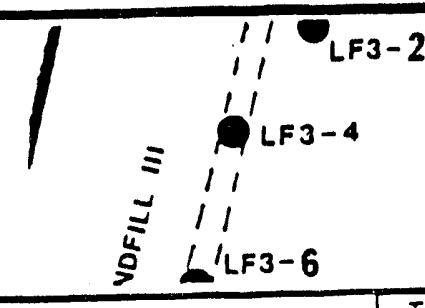

Description:

Silty clay w/gravels and pebbles

10 YR 7/4 (very pale brown), slightly plastic, well sorted angular to subrounded/subprismoidal to subdiscoidal $M L-C L-G W$

ans - subrounded

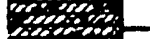

$1+$

(very pale

brom 10" sample sleeve, nonplastic, well sorted

SM-GN angular to subrounded

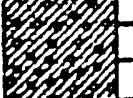

CHOMICAL SAMPLE

fine - coarse sand w/gravels 10 YR $5 / 2$ (grayish brown), non-

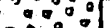

plastic, poorly sorted, angular to subrounded

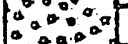

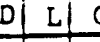

BASALT, 10 YR $2 / 1$ (black), vesicular 


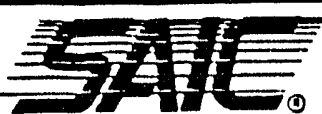

FIELD BOREHOLE LOC

PROJECT NUMBER

$2-817-07-667-00$

PROJECT NAME EGSG/INEL Landfill-CFA

LOCATION CFA LandFIII III

DRILLINC COMPANY Denning Well Drilling, Inc.

RIC TYPE \& NUMBER

DRILLINC METHOD 10" Hollow Stem Auger

WEATHER Very cold, clear, very windy

FIELD PARTY Jody and Dan Denning

CEOLOCIST John Jaacks and Brett Freier

DATE BECUN 12/14/87 DATE COMPLETED

$12 / 14 / 87$
BOREHOLE NUMBER:

LF 3-6

FIELD BOOK NO.

TOTAL DEPTH $17 \mathrm{ft}$. SHEET 1 OF 1

\begin{tabular}{|l|l|l|l|}
\hline \multicolumn{4}{|c|}{ CROUNOWATER TABLE N/A } \\
\hline WO=While Drilling & AB=After Boring \\
\hline Depth(fet) & & & \\
\hline Time & & & \\
\hline Date & & & \\
\hline
\end{tabular}

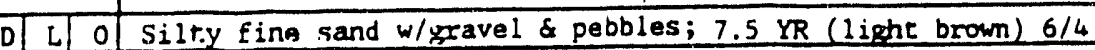
nonplastic, poorly sorted, subaneular to subrounded.

SMA-GW

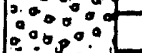

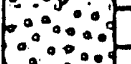

$\therefore \therefore$

$\therefore 0$

$\therefore \therefore-1$

$\begin{array}{lllll} & \therefore\end{array}$

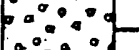

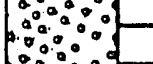

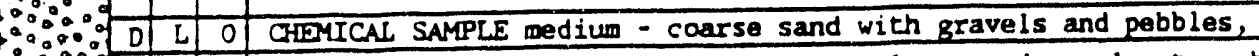

10 YR $4 / 1$ (dark gray), nonplastic, poorly sorted angular to sub-

rounded

SM-GW

$\because \because$

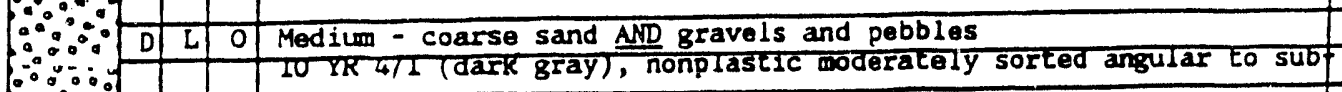

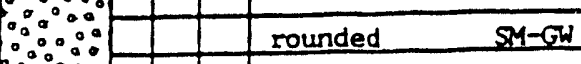

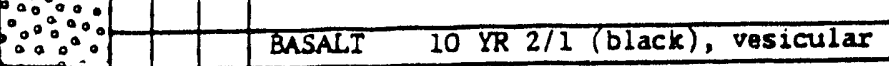

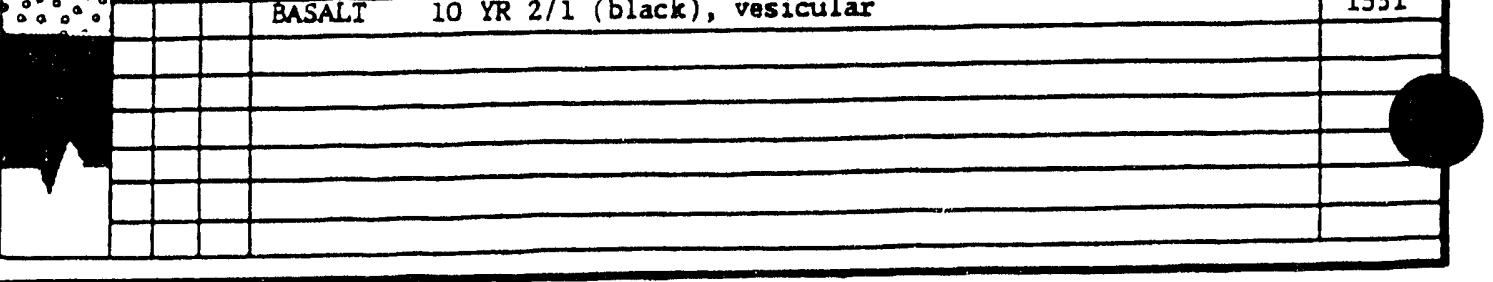




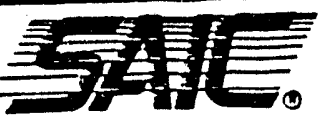

PROJECT NUMBER 2-817-07-667-00

FIELD BOREHOLE LOC

LOCATION CFA Landfill III, 2200' Line

DRILLINC COMPANY Denning Well Drilling, Inc.

RIC TYPE $\&$ NUMBER

ORILLINC METHOD $10^{\prime \prime}$ Hollow Stem Auger w/2.5" DEM Type U

WEATHER $20^{\circ} \mathrm{F}$, windy, snow Split Spoon

FIELD PARTY Jody Denning, Dan Denning

CEOLOCIST John Jaacks, III \& Brett Freier

DATE BECUN 12/10/87 DATE COMPLETED 12/10/87
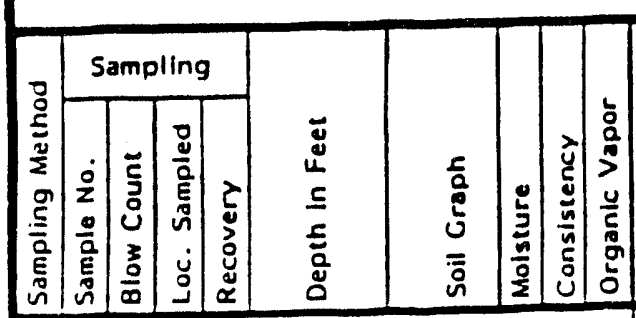

Location Diagram:

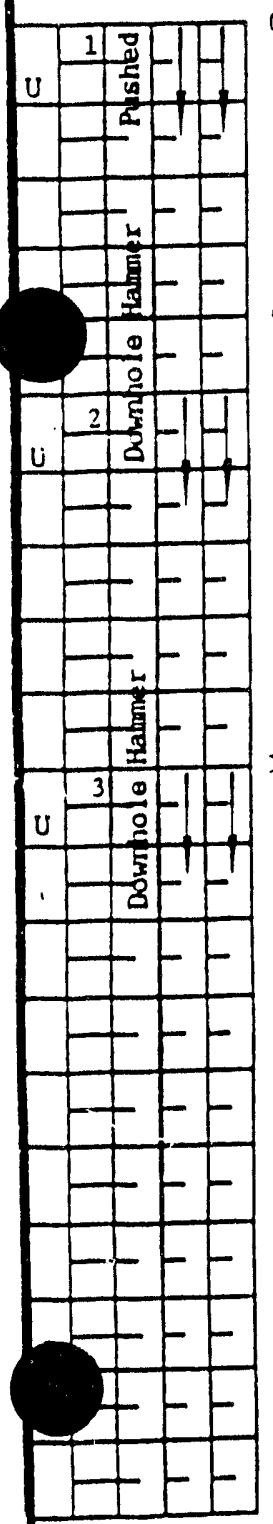

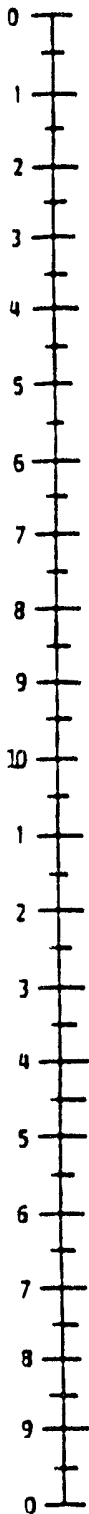

BOREHOLE NUMBER:

LF 3-7

FIELD BOOK NO.

1

$12.2 \mathrm{ft}$

SHEET 1 OF 1

\begin{tabular}{|l|l|l|l|}
\hline \multicolumn{4}{|c|}{ CROUNDWATER TABLE } \\
\hline WD=while Drilling & AB = Alter Boring \\
\hline Depth(f() & N/A & & \\
\hline Time & & & \\
\hline Date & & & \\
\hline
\end{tabular}

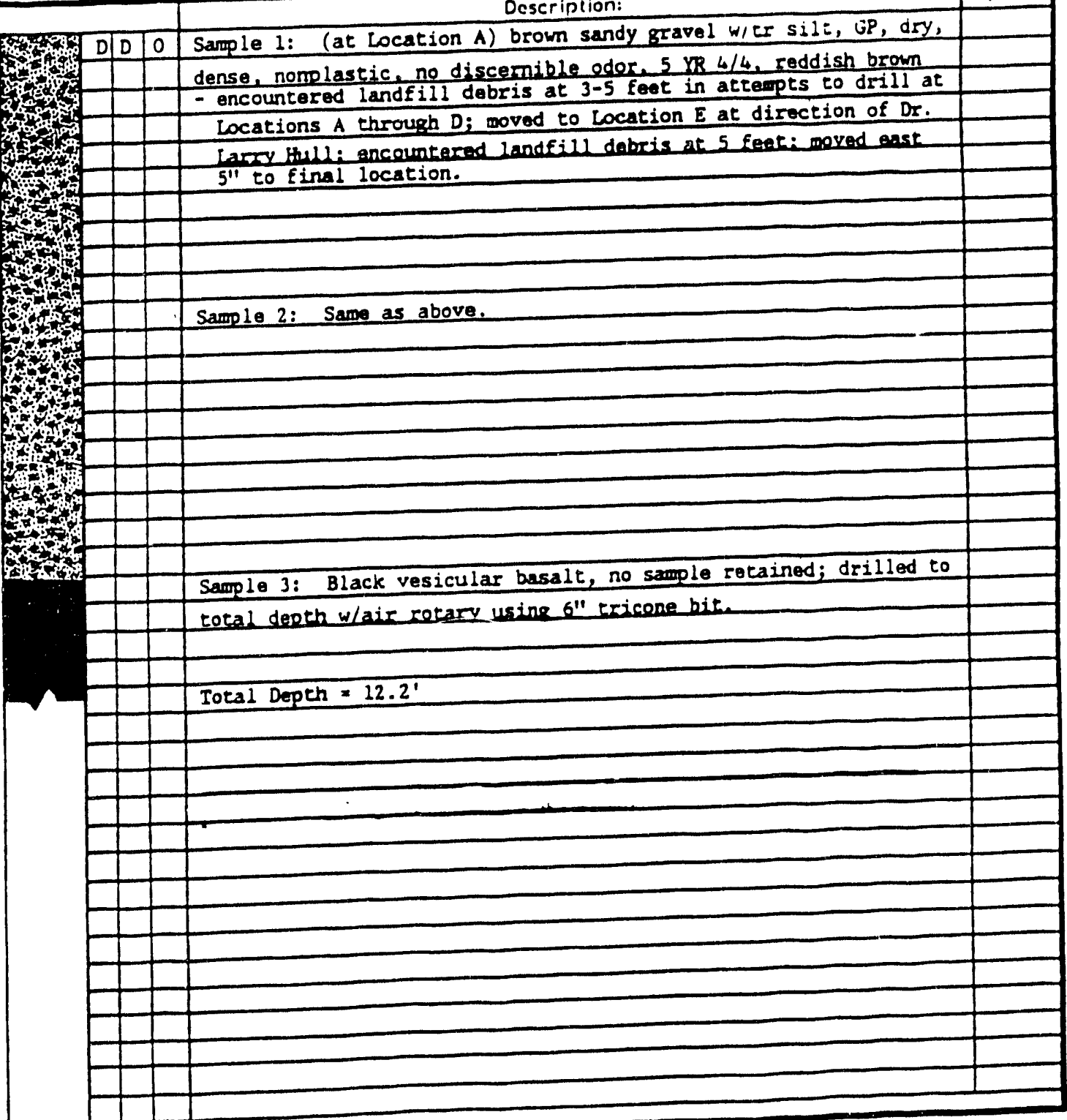


Borehole Completion Diagrams with Instrument Locations 


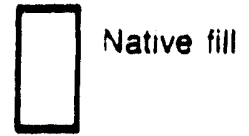

Silica flour

咩

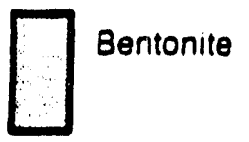

- H343 Heat dissipation block

$\Delta \quad \$ 11632$ Salinity probe

Eas sampling port

8.1524 


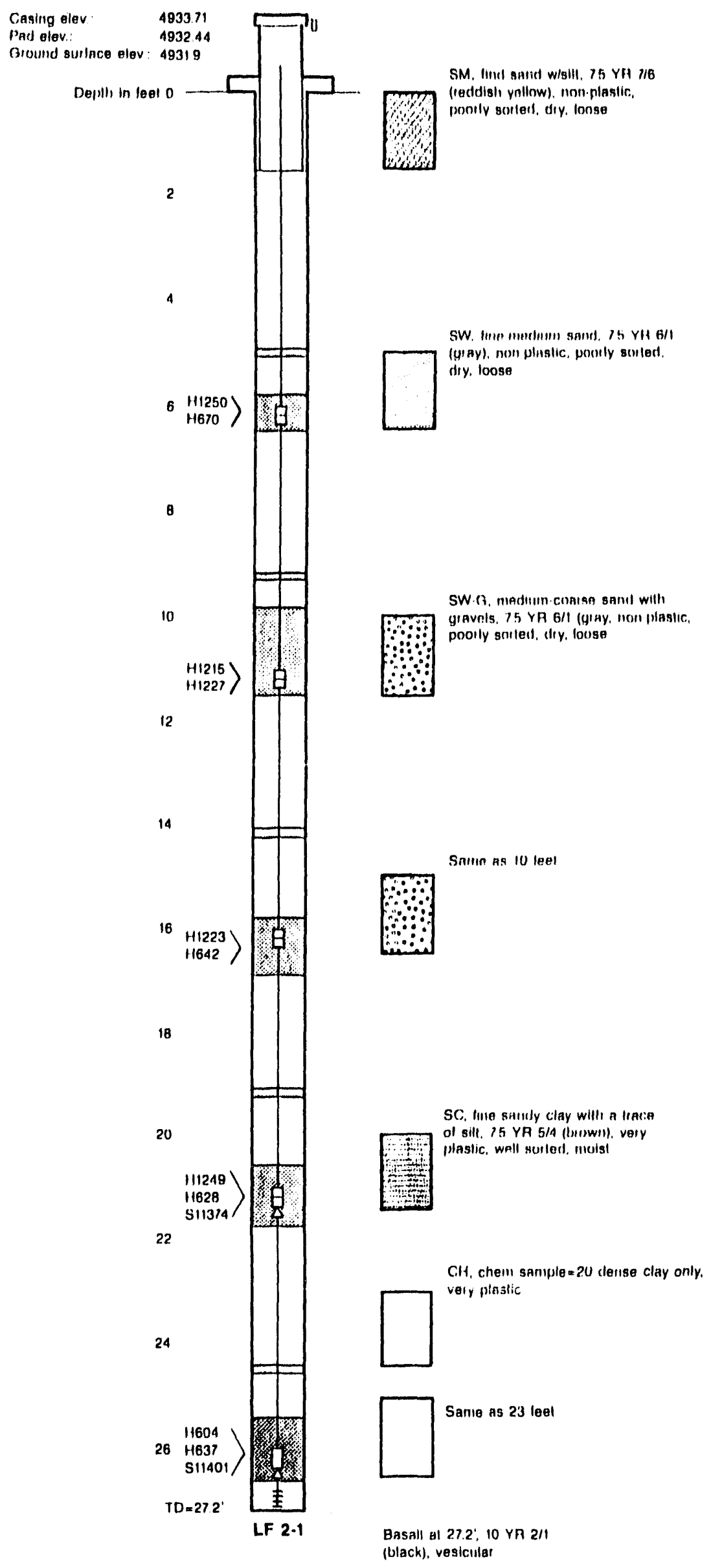

(black), vesicular

Figure D-1. Instrumentation for borehole LF2-1. 


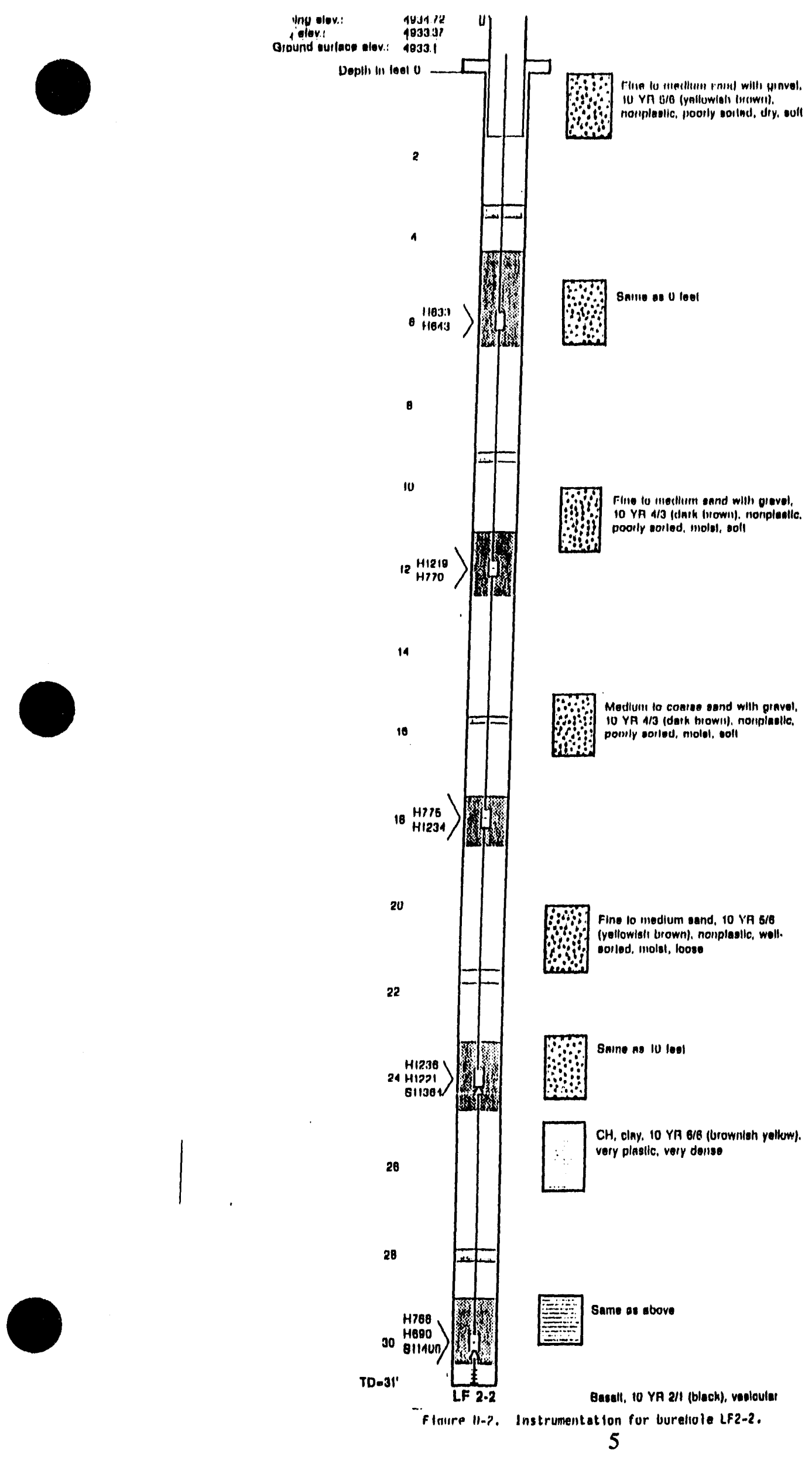




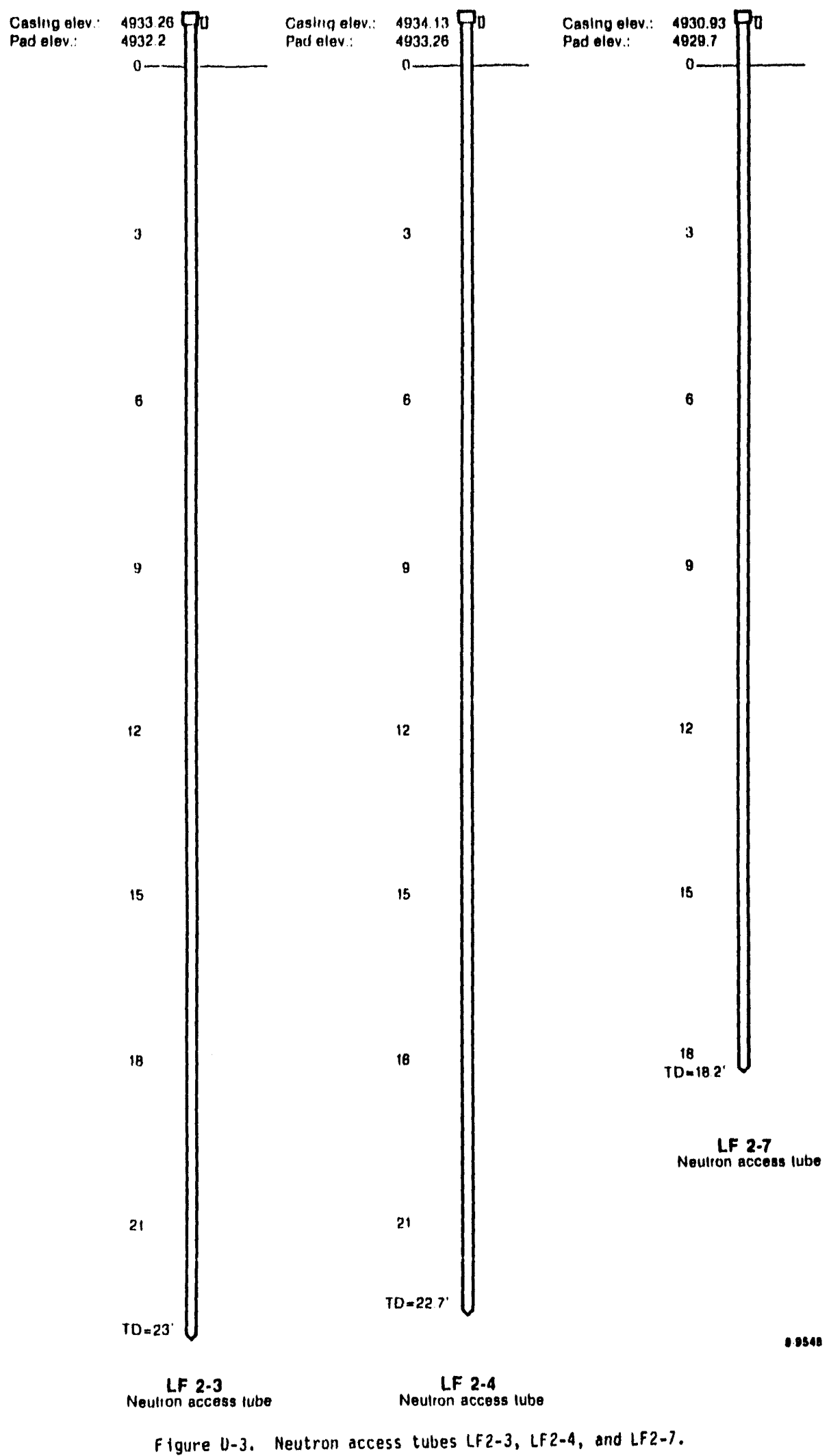




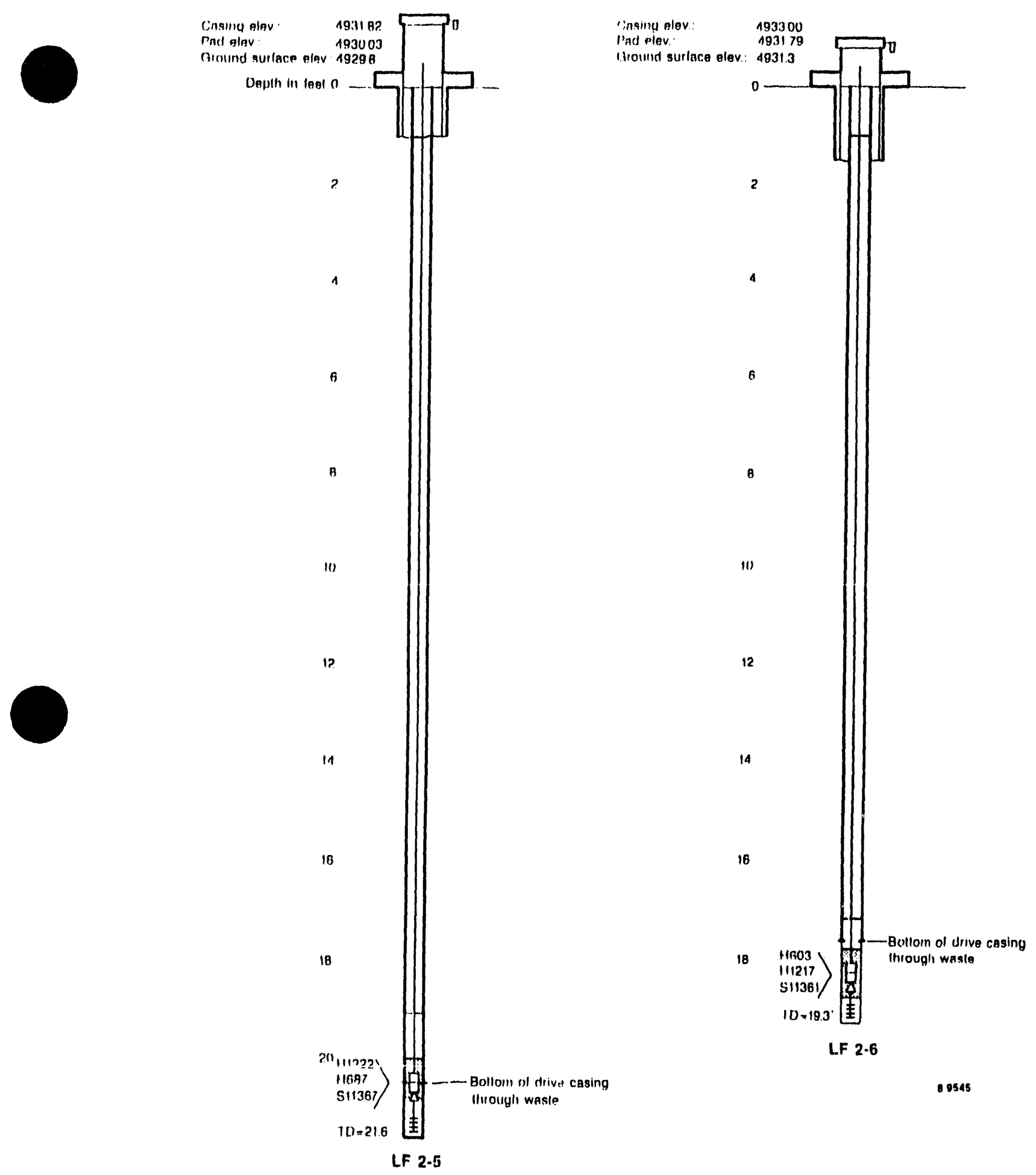

Figure 0-4. Bottoms of drive casings through waste for boreholes LF2-5 and LF2-6. 


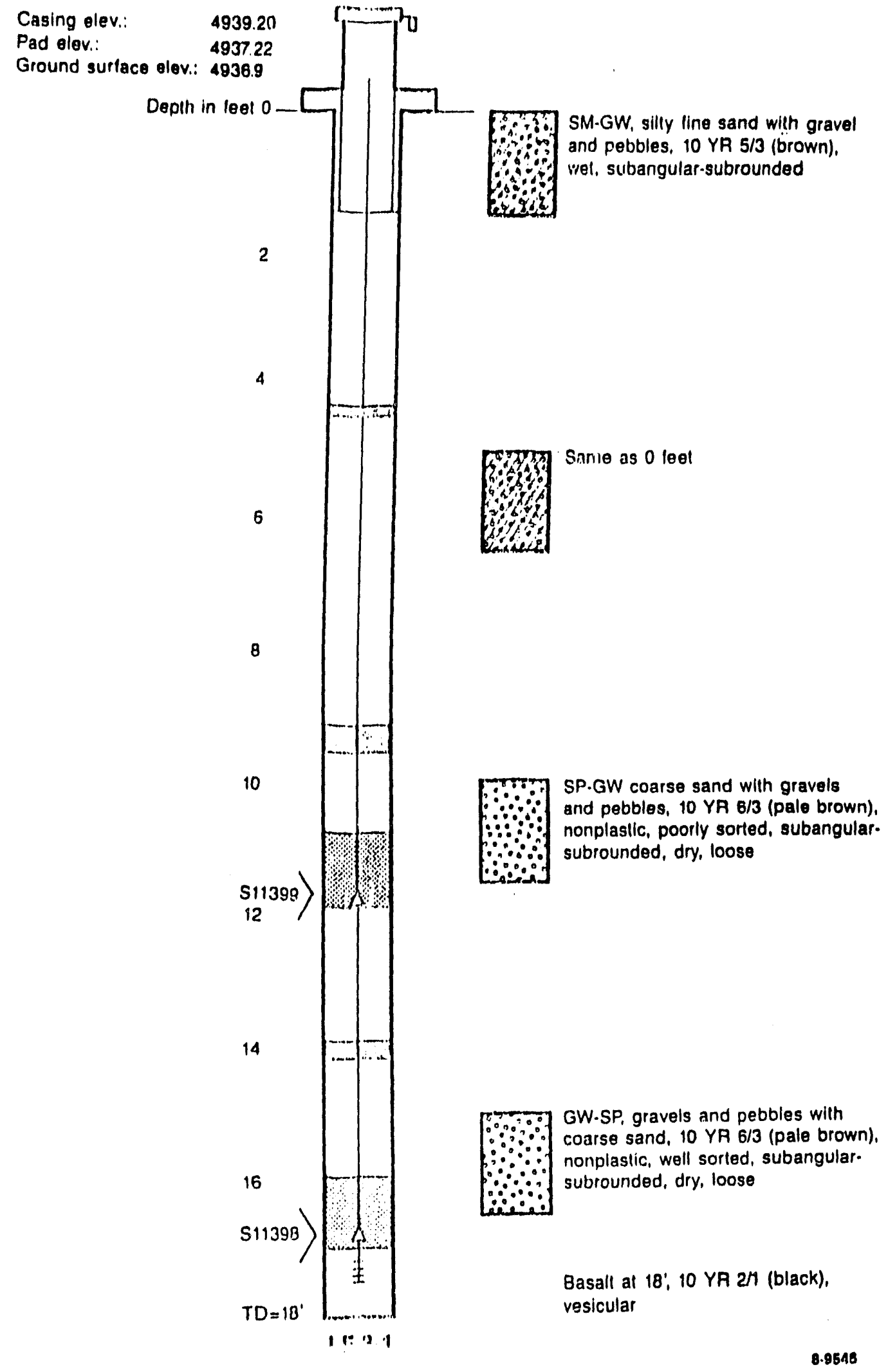

Figure 0-6. Instrumentation for borehole LF 3-1. 


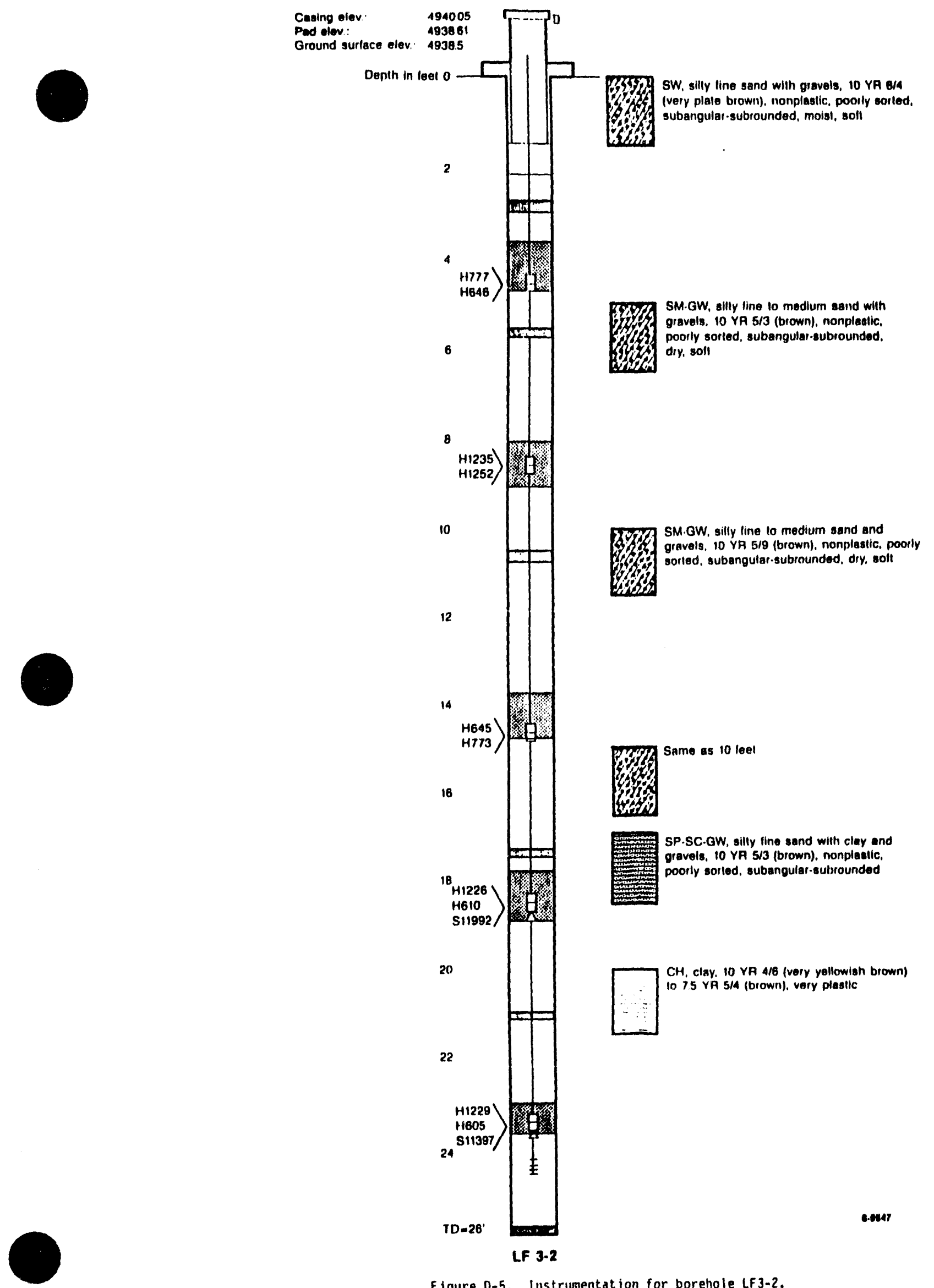

Figure D-5. Instrumentation for borehole LF3-2. 


\section{Casing e!ev.: \\ Pad elev.: \\ Ground surlace elev.: 4940.1}

Depin in leet 0

6

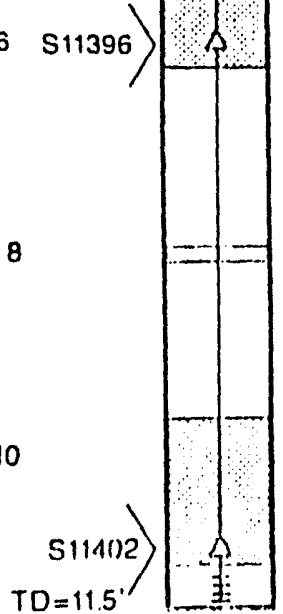

LF 3-4
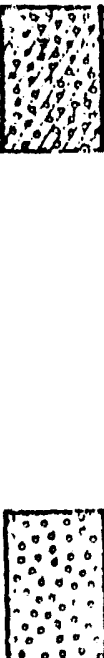

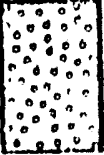

Fine to coarse sand with gravel. 10 YR 5/2 (grayish brown), nonplastic. poorly sorted, angular to subrounded. dry. loose

Basall al 11.5: 10 YR $2 / 1$ (black), vesicular
SM-GW, silty line to medium to coarse sand with gravels, 10 YR 7/4 (very pale brown, nonplastic, well sorted. angular to subrounded, dry, loose
ML.CL.GW, silly clay with gravels and pebbles, 10 YR 7/4 (very pale brown. slightly plastic, well sorted. angular to subrounded/subprismoidal to subdiscoidal, wel, solt

Figure U-7. Instrumentation for borehole LF 3-4. 


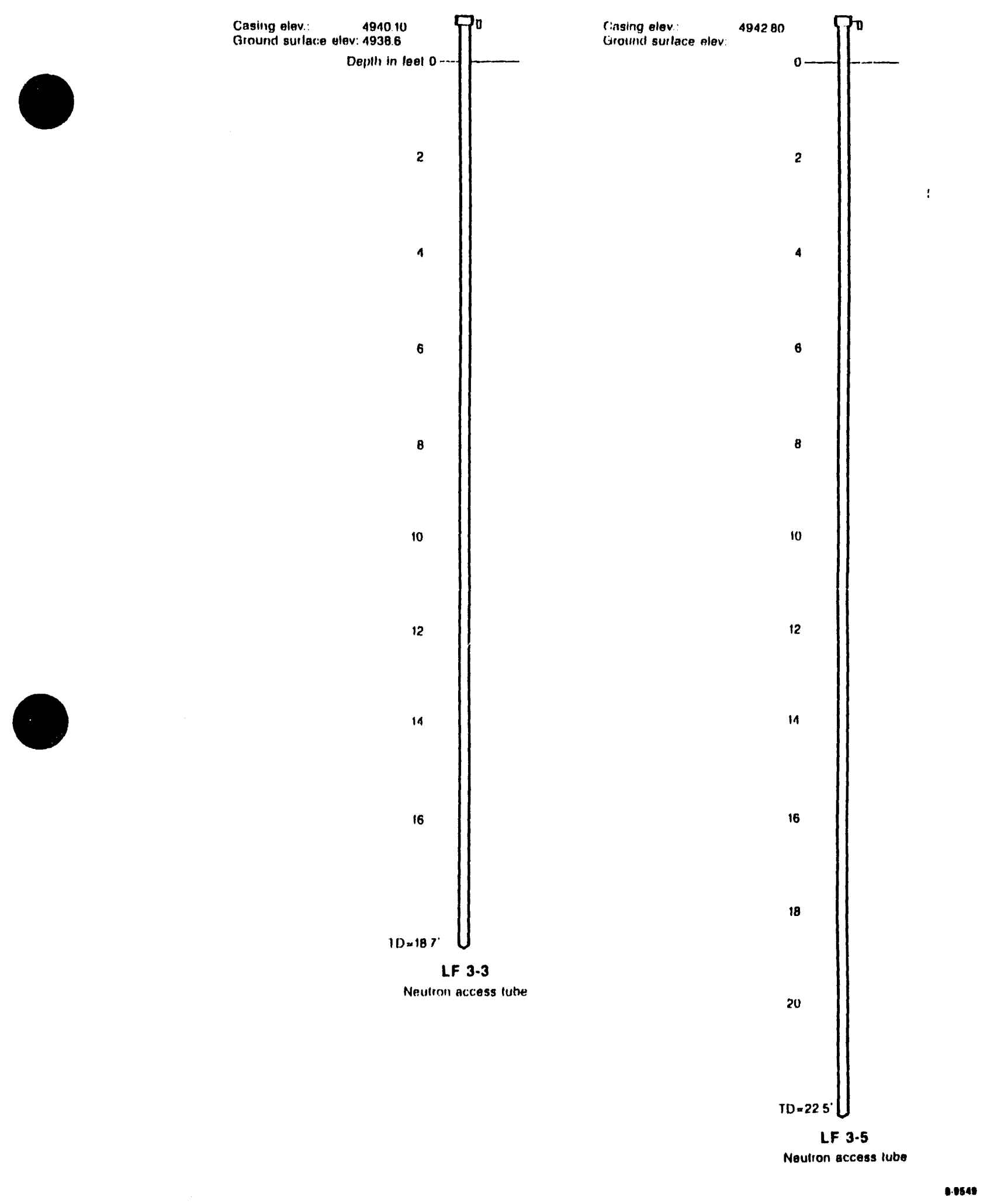

Figure D-8. Neutron access tubes LF 3-3 and LF 3-5. 


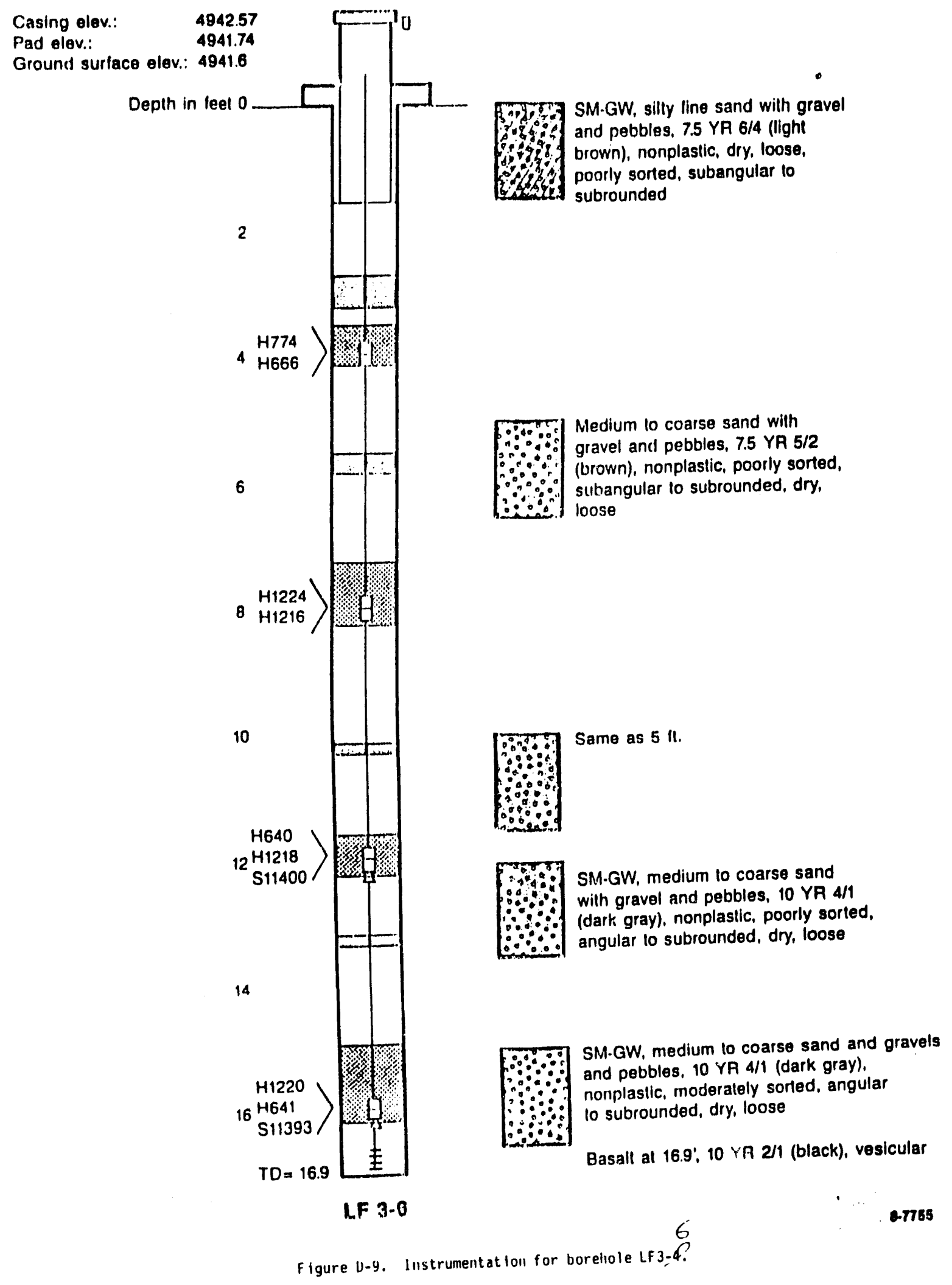




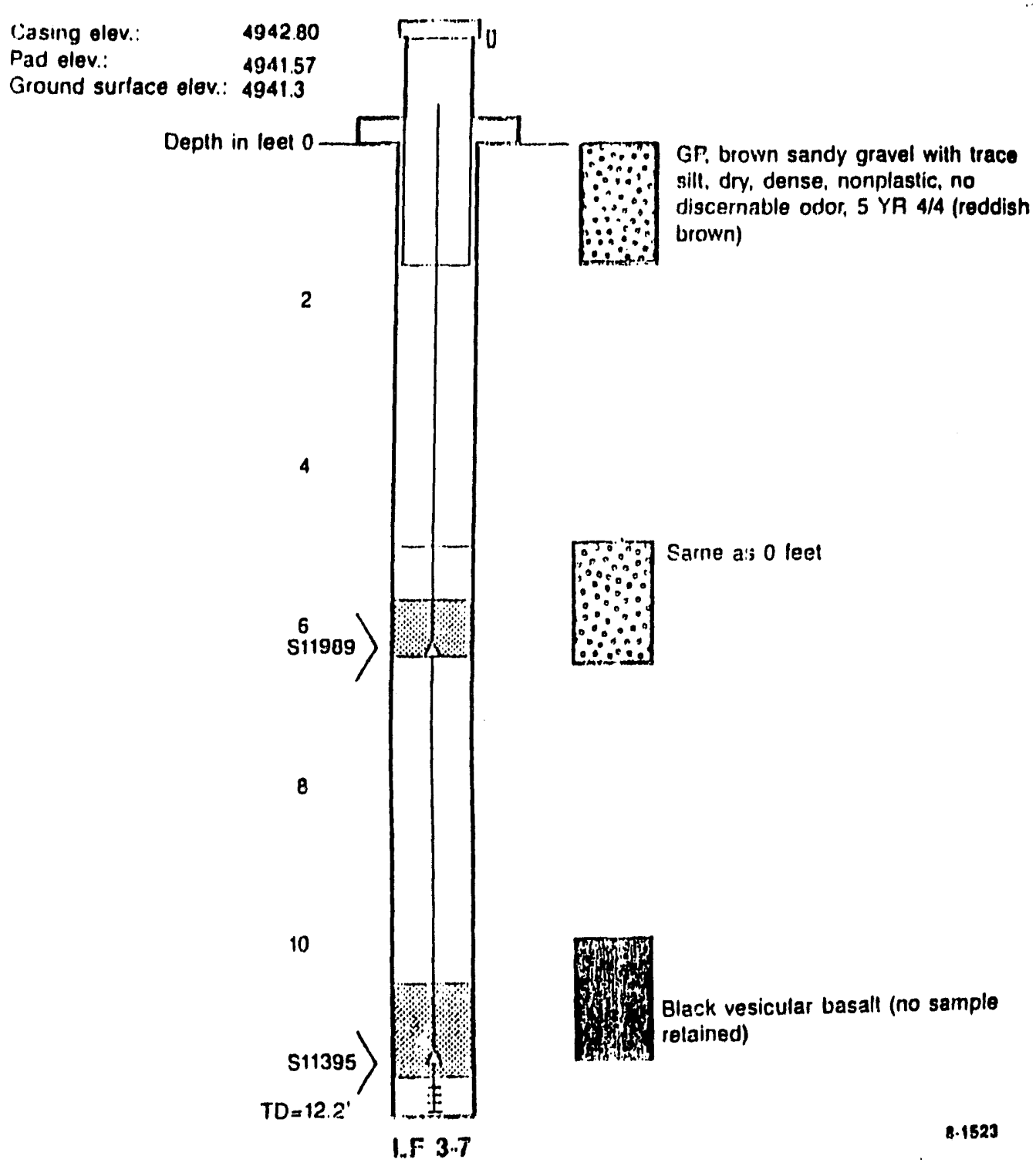

Figure U-10. Instrumentation for bureliole LF 3-7. 


\section{Appendix E}

Baseline and Background Concentrations of Trace Elements in Surface Soils 


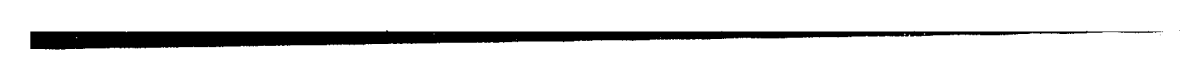

.

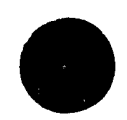

E-2 


\section{Appendix E \\ Baseline and Background Concentrations of Trace Elements in Surface Soils"}

\section{E.1 GENERAL INFORMATION ABOUT THE SURFACE SOIL CHARACTERIZATION EFFORT}

In 1989, surface soils at several active EG\&G Idaho facilities were samples to establish baseline concentrations of various analytes (Martin et al., 1990). Composited samples were collected at gridded areas at Power Burst Facility (PBF), Central Facilities Area (CFA), Test Reactor Area (TRA), and Test Area North (TAN). A total of 12 samples were also collected offsite (three at each of four sites), in undisturbed or "background" locations. These included two sites in undeveloped sagebrush ecosystems in Bingham County, a river bed near Arco, and a playa east of Howe. [Specific sampling locations on- and offsite may be found in Martin et al. (1990.] The Bingham County sites approximated soil types found near PBF and southen CFA (at the southen end of the Idaho National Engineering Laboratory) where the landscape is dominated by loess-covered lava flows. The Arco site was representative of soils found amid the Big Lost River plain, namely TRA and most of CFA. The Howe site contained soil similar to that found around TAN.

The data from the 1989 effort were collected to establish baseline concentrations adjacent to active EG\&G Idaho facilities. They could be used to estimate probable background levels at the facilities. Technically, the sites are not considered background because they are too close to areas of disturbance.

Tables E-1 and E-2 contain data for inductively-coupled plasma (ICP) -sensitive elements and mercury. (Mercury was analyzed for using the conventional cold vapor technique.) ICP is not the preferred analytical method for background levels of antimony, arsenic, selenium, silver, and thallium, and ICP data for those elements should be interpreted with extreme caution. Because of this, the data for these same elements were put in separate table (Table E-3) with atomic absorption (AA) data for these same elements. The AA data were taken from Spry et al. (1990, Rev. 1). Table E-4 lists waste area groups (WAGs) associated with each of the facility areas and the number of samples collected at each site.

a. Source: Track 1 Sites: Guidance for Assessing Low Probability Hazard Sites at the INEL, DOE/ID 10340(92), July 1992, Revision 1, Appendix F. 
Table E-1. ICP measurements of trace elements (ppm) in INEL surface soils.

Background

$\mathrm{n}=12 \quad \mathrm{k}=2.706$

\begin{tabular}{|c|c|c|c|c|c|}
\hline & Median & Mean & $\begin{array}{l}\text { Standard } \\
\text { deviation }\end{array}$ & $\begin{array}{c}\text { Upper }^{3} \\
\text { tolerance limit }\end{array}$ & $\begin{array}{c}90 \text { th } \\
\text { percentile }\end{array}$ \\
\hline $\mathrm{Al}$ & 12850 & 11946 & 4646 & 24520 & 16200 \\
\hline $\mathrm{Ba}$ & 184 & 181 & 45 & 303 & 233 \\
\hline $\mathrm{Be}$ & 1.35 & 1.26 & 0.27 & 2.00 & 1.50 \\
\hline $\mathrm{Cd}$ & 275 & 2.91 & 1.82 & 7.83 & 5.3 \\
\hline $\mathrm{Ca}$ & 10650 & 9163 & 4338 & 20903 & 15000 \\
\hline $\mathrm{Cr}$ & 19.85 & 22.86 & 8.73 & 46.48 & 35.1 \\
\hline Co & 6.15 & 6.43 & 3.33 & 15.45 & 10.8 \\
\hline $\mathrm{Cu}$ & 44.65 & 45.92 & 12.91 & 80.85 & 59.9 \\
\hline $\mathrm{Fe}$ & 17050 & 17717 & 5097 & 31512 & 23900 \\
\hline $\mathrm{Pb}$ & 44 & 45 & 9 & 68 & 57 \\
\hline $\mathrm{Hg}^{\mathrm{b}}$ & 0.05 & 0.05 & 0.01 & 0.07 & 0.06 \\
\hline $\mathrm{Mg}$ & 6075 & 6743 & 2814 & 14359 & 11000 \\
\hline $\mathrm{Mn}$ & 502.5 & 442.8 & 212.7 & 1018.5 & 636 \\
\hline $\mathrm{Ni}$ & 16.8 & 21.7 & 11.0 & 51.4 & 36.5 \\
\hline $\mathrm{K}$ & 3445 & 3283 & 1704 & 7896 & 5180 \\
\hline $\mathrm{Na}$ & 115.5 & 126.4 & 53.4 & 270.8 & 192 \\
\hline V & 28.5 & 30.2 & 4.8 & 43.1 & 37.1 \\
\hline $\mathrm{Zn}$ & 68.1 & 96.4 & 56.7 & 249.9 & 184 \\
\hline \multicolumn{6}{|c|}{$\begin{array}{l}\text { a. Upper tolerance limit (action level) }=\bar{x}+k(s) \\
\bar{x}=\text { mean } s=\text { standard deviation } \\
k=\text { coefficient for } 95 \text { th percentile with } 95 \% \text { confidence }\end{array}$} \\
\hline
\end{tabular}


Table E-2. ICP measurements of trace elcments (ppm) in INEL surface soils.

\section{Central Facilities Area}

$$
n=11 \quad k=2.782
$$

\begin{tabular}{|c|c|c|c|c|c|}
\hline & Median & Mean & $\begin{array}{l}\text { Standard } \\
\text { deviation }\end{array}$ & $\begin{array}{c}\text { Upper }^{3} \\
\text { tolerance limit }\end{array}$ & $\begin{array}{c}90 \text { th } \\
\text { percentile }\end{array}$ \\
\hline Al & 9340 & 9293 & 2002 & 14864 & 11500 \\
\hline $\mathrm{Ba}$ & 170 & 177 & 26 & 249 & 207 \\
\hline $\mathrm{Be}$ & 0.76 & 0.92 & 0.39 & 1.99 & 1.40 \\
\hline $\mathrm{Cd}$ & 3.2 & 2.8 & 1.2 & 6.0 & 4.1 \\
\hline $\mathrm{Ca}$ & 22000 & 22055 & 6094 & 39010 & 29800 \\
\hline $\mathrm{Cr}$ & 19.7 & 21.2 & 2.7 & 28.7 & 24 \\
\hline Co & 3 & 5 & 4 & 17 & 9 \\
\hline $\mathrm{Cu}$ & 12.3 & 12.9 & 4.0 & 24.2 & 17 \\
\hline $\mathrm{Fe}$ & 15400 & 15827 & 3121 & 24511 & 19100 \\
\hline $\mathrm{Pb}$ & 20.7 & 27.3 & 19.2 & 53.3 & 54.4 \\
\hline $\mathrm{Hg}^{\mathrm{b}}$ & 0.05 & 0.05 & 0.01 & 0.08 & 0.07 \\
\hline $\mathrm{Mg}$ & 5840 & 5825 & 603 & 7504 & 6530 \\
\hline $\mathrm{Mn}$ & 274 & 285 & 88 & 530 & 404 \\
\hline $\mathrm{Ni}$ & 19.7 & 21.1 & 5.3 & 35.9 & 26.9 \\
\hline $\mathrm{K}$ & 2270 & 2294 & 781 & 4466 & 3500 \\
\hline $\mathrm{Na}$ & 144 & 153 & 45 & 279 & 197 \\
\hline V & 27.3 & 29.5 & 5.5 & 44.9 & 36.8 \\
\hline $\mathrm{Zn}$ & 85 & 90 & 14 & 128 & 111 \\
\hline a. $\begin{array}{l}U \\
\bar{x} \\
k\end{array}$ & $\begin{array}{l}\text { lerance limi } \\
\text { n } s=\text { stand } \\
\text { ficient for } 9\end{array}$ & $\begin{array}{l}\text { detion leve } \\
\text { deviation } \\
\text { percentile }\end{array}$ & $\begin{array}{l}\bar{x}+k(s) \\
95 \% \text { con }\end{array}$ & & \\
\hline
\end{tabular}


Table E-3. ICP and AA measurements of trace elements (ppm) in INEL surface soils.

\begin{tabular}{|c|c|c|c|c|c|}
\hline \multicolumn{6}{|c|}{$\begin{array}{l}\text { Power Burst Facility } \\
n=69 \quad k=1.986\end{array}$} \\
\hline & Median & Mean & $\begin{array}{l}\text { Standard } \\
\text { deviation }\end{array}$ & $\begin{array}{c}\text { Upper }^{3} \\
\text { tolerance limit }\end{array}$ & $\begin{array}{c}90 \text { th } \\
\text { percentile }\end{array}$ \\
\hline $\mathrm{Al}$ & 9180 & 9362 & 1889 & 13114 & 11550 \\
\hline $\mathrm{Ba}$ & 149 & 155 & 26 & 205 & 178 \\
\hline $\mathrm{Be}$ & 0.31 & 0.38 & 0.56 & 1.49 & 0.33 \\
\hline $\mathrm{Cd}$ & 0.82 & 1.05 & 0.43 & 1.90 & 1.60 \\
\hline $\mathrm{Ca}$ & 14000 & 15444 & 8532 & 32385 & 28050 \\
\hline $\mathrm{Cr}$ & 17 & 18 & 6 & 30 & 24 \\
\hline Co & 5.5 & 6.4 & 4.3 & 15.0 & 14.1 \\
\hline $\mathrm{Cu}$ & 9.5 & 19.4 & 44.7 & 108.2 & 16.5 \\
\hline $\mathrm{Fe}$ & 14400 & 14959 & 3356 & 21622 & 17100 \\
\hline $\mathrm{Pb}$ & 21.7 & 24.7 & 11.0 & 46.5 & 38.7 \\
\hline $\mathrm{Hg}^{\mathrm{b}}$ & 0.05 & 0.05 & 0.01 & 0.07 & 0.05 \\
\hline $\mathrm{Mg}$ & 5290 & 5216 & 1751 & 8693 & 7035 \\
\hline $\mathrm{Mn}$ & 265 & 277 & 68 & 413 & 343 \\
\hline $\mathrm{Ni}$ & 16.5 & 16.1 & 5.0 & 26.1 & 21.8 \\
\hline $\mathrm{K}$ & 2430 & 2470 & 464 & 3392 & 2980 \\
\hline $\mathrm{Na}$ & 129 & 130 & 75 & 279 & 214 \\
\hline $\mathrm{V}$ & 19.1 & 27.9 & 11.1 & 50.0 & 26 \\
\hline $\mathrm{Zn}$ & 65.1 & 73.5 & 42.4 & 157.7 & 83.7 \\
\hline a. $\begin{array}{l}U \\
\bar{x} \\
k\end{array}$ & $\begin{array}{l}\text { lerance li } \\
\text { in } s=\text { sta } \\
\text { fficient fo }\end{array}$ & $\begin{array}{l}\text { action level } \\
\text { d deviation } \\
\text { a percentile }\end{array}$ & $\begin{array}{l}=\bar{x}+k(s) \\
\text { ith } 95 \% \text { conf }\end{array}$ & & \\
\hline
\end{tabular}


Table E-4. Sample numbers from each of the sampled sites.

\section{Test Area North}

$n=54 \quad k=2.040$

\begin{tabular}{|c|c|c|c|c|c|}
\hline & Median & Mean & $\begin{array}{l}\text { Standard } \\
\text { deviation }\end{array}$ & $\begin{array}{c}\text { Upper }^{2} \\
\text { tolerance limit }\end{array}$ & $\begin{array}{c}90 \text { th } \\
\text { percentile }\end{array}$ \\
\hline Al & 14200 & 14223 & 4892 & 24206 & 20800 \\
\hline $\mathrm{Ba}$ & 193.5 & 182.8 & 61.4 & 308.0 & 254 \\
\hline $\mathrm{Be}$ & 1.1 & 1.1 & 0.4 & 1.8 & 1.5 \\
\hline $\mathrm{Cd}$ & 2.1 & 24 & 1.2 & 5.0 & 4.6 \\
\hline $\mathrm{Ca}$ & 79800 & 83480 & 26034 & 136602 & 121000 \\
\hline $\mathrm{Cr}$ & 28.65 & 28.85 & 7.65 & 44.45 & 38.90 \\
\hline Co & 8.3 & 8.1 & 3.9 & 16.1 & 13.3 \\
\hline $\mathrm{Cu}$ & 18.9 & 18.5 & 9.1 & 36.9 & 27.4 \\
\hline $\mathrm{Fe}$ & 19350 & 18839 & 5797 & 30668 & 27000 \\
\hline $\mathrm{Pb}$ & 38.8 & 35.9 & 19.9 & 76.5 & 55.6 \\
\hline $\mathrm{Hg}^{\mathrm{b}}$ & 0.05 & 0.08 & 0.12 & 0.32 & 0.06 \\
\hline $\mathrm{Mg}$ & 12900 & 12539 & 2180 & 16987 & 14300 \\
\hline Mn & 373 & 366 & 109 & 588 & 490 \\
\hline $\mathrm{Ni}$ & 31.8 & 31.2 & 10.1 & 51.7 & 42.5 \\
\hline $\mathrm{K}$ & 3585 & 3641 & 1322 & 6338 & 5480 \\
\hline $\mathrm{Na}$ & 288.5 & 327.3 & 179.8 & 694.1 & 522 \\
\hline V & 42.15 & 41.44 & 11.02 & 63.93 & 53.70 \\
\hline $\mathrm{Zn}$ & 115 & 131 & 69 & 272 & 182 \\
\hline a. $\begin{array}{l}U \\
\bar{x} \\
k\end{array}$ & $\begin{array}{l}\text { lerance li } \\
\text { in } s=\text { sta } \\
\text { fficient fo }\end{array}$ & $\begin{array}{l}\text { ction leve } \\
\text { deviation } \\
\text { percentile }\end{array}$ & $\begin{array}{l}=\bar{x}+k(s) \\
\text { vith } 95 \% \text { con }\end{array}$ & & \\
\hline
\end{tabular}

E-7 


\section{E.2 REFERENCES}

Martin, K. L., C. J. Barnard, A. L. Freeman, M. R. Groh, K. T. Kissell, S. J. Lord, G. L. Olson, P. D. Randolph, R. N. Wihelmsen, 1990, Preliminary Assessment of Surface Soils at Active EG\&G Idaho Facilities Data Document, EG\&G-ESQ-9225, September 1990.

Spry, M. J. and S. N. Stanisich, 1990, Closure Plan for the CFA Motor Pool Pcnd, EGG-WM-8997, May 1990. 


\section{Appendix F}

Resolutions of

Idaho Department of Health and Welfare and

Environmental Protection Agency

Review Comments of the

Draft Remedial Investigation/Feasibility Study

Work Plan and Addenda for Operable Unit 4-12:

Central Facilities Area Landfills II and III at the Idaho National Engineering Laboratory

January 1993 


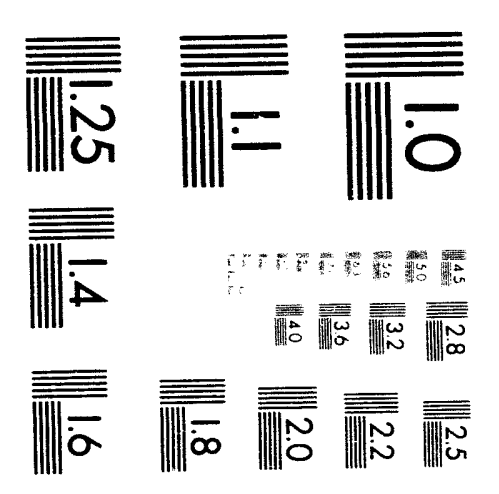



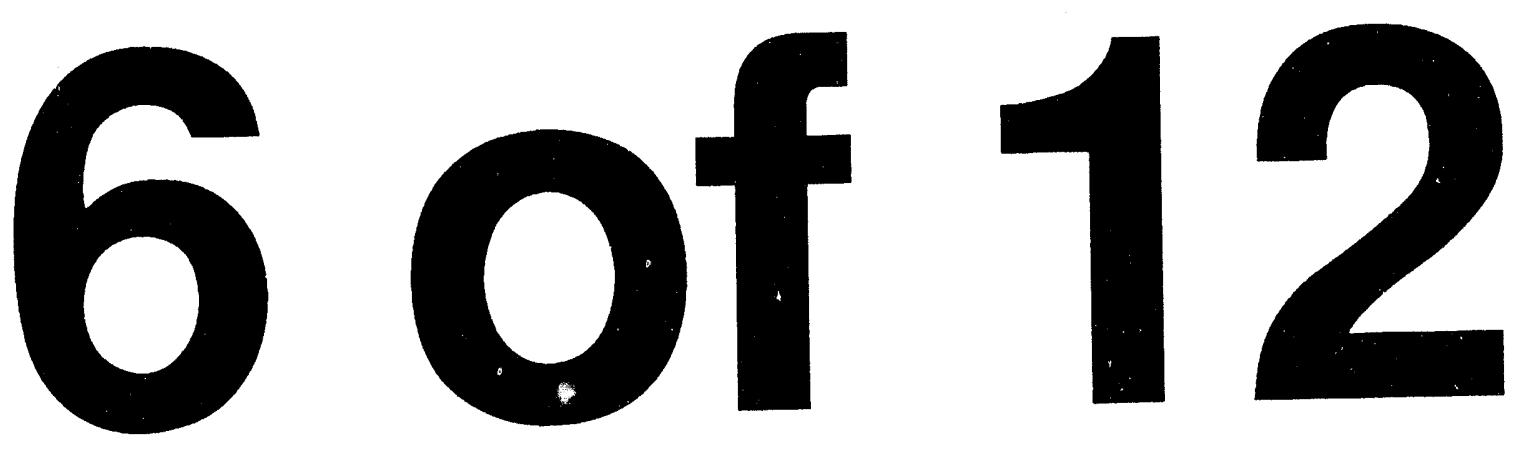


\section{ACRONYMS}

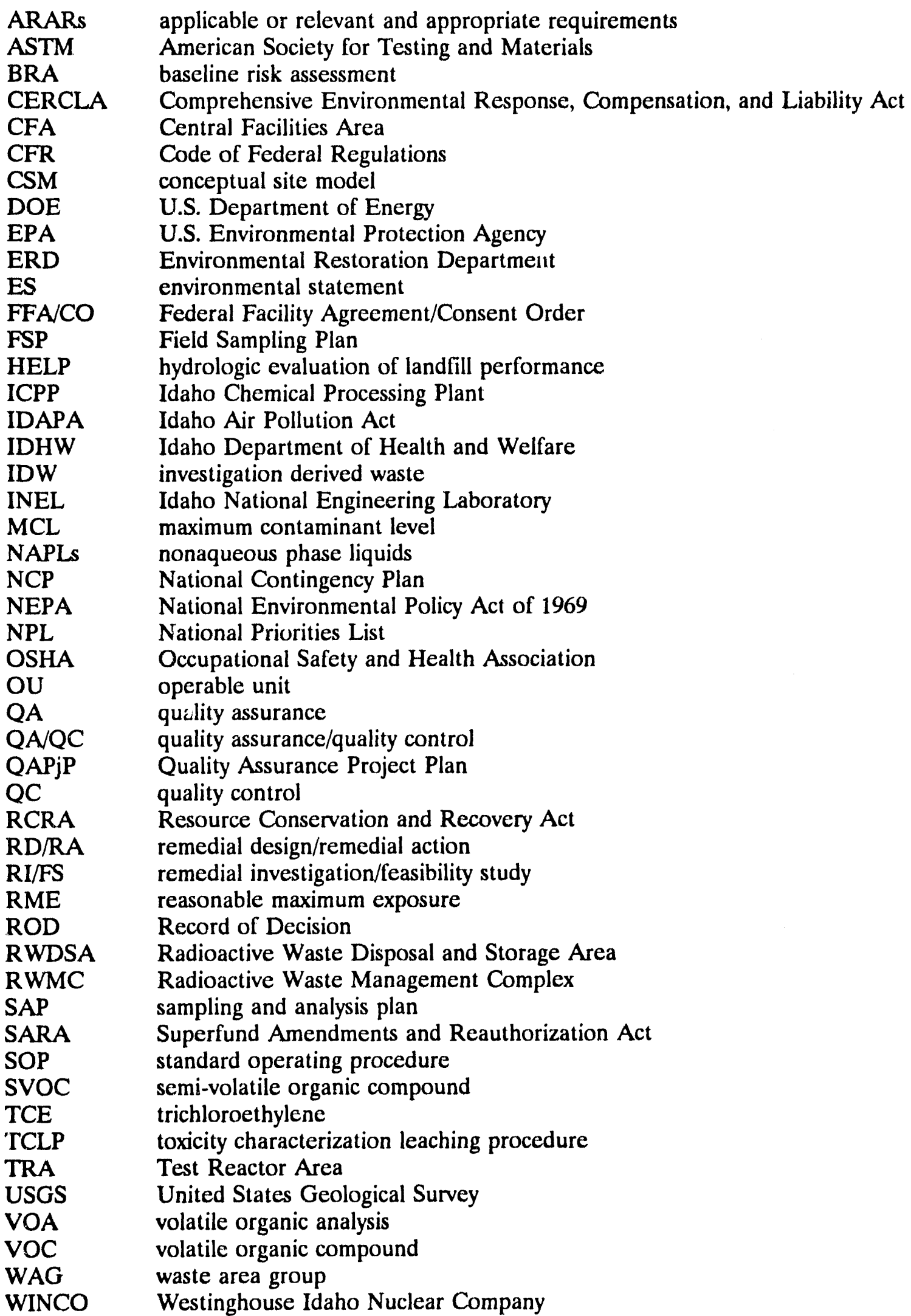




\section{IDAHO DEPARTMENT OF HEALTH AND WELFARE REVIEW COMMENTS AND RESOLUTIONS}

\section{General Comments}

1. The phrase "municipal landfill" is generally reserved for those landfills which serve a municipality and primarily receive household waste. The INEL is a industrial site, therefore the CFA landfills readily fall under the definition of a "industrial landfill." Tables 2-3 and 2-4 suggest that much of the waste disposed in landfills II and III was categorized as "trash" or cafeieria garbage, which is somewhat similar to household waste; however, to avoid potential confusion resulting from the term "municipal," IDHW recommends deleting this word in the document unless it is used to reference EPA guidance or to describe the general approach for this remedial investigation.

Resolution: Reference to CFA landfills as municipal has been deleted.

2. The text repeatedly references "principal and low-level threats," as defined by the NCP. Prior to completion of the remedial investigation, it is premature to determine whether the CFA landfills pose a principal or low-level threat. This designation is also somewhat subjective, as approximately $54,000 \mathrm{~m}^{3}$ of liquid wastes (i.e. mobile contaminants) were disposed in CFA Landfill I. Some remedial project managers may consider these wastes a principal threat. Due to the ambiguous definition of these terms, IDHW recommends deleting them from the text.

Resolution: Reference to CFA landfills presenting a "low-level threat rather than a principal threat" has been deleted.

3. The work plan briefly discusses the investigation of Landfill II which was conducted by ICF Technology, Inc. (1990), but doesn't adequately summarize the findings of this study. IDHW recommends reiterating the conclusions of the geophysical survey, and including key figures from that report which summarize the data collection effort. Furthermore, the ICF Technology study and the Landfill II soil gas survey should be compared to the data quality objectives developed for this remedial investigation to ensure that additional work at Landfill II is not required.

Resolution: A copy of the ICF Technology, Inc. report entitled "Innovative Technology Demonstration Idaho National Engineering Laboratory (INEL) CFA Landfill II Idaho Falls, Idaho" has been placed in the Administrative Record. As stated in the Work Flan the ICF Technology, Inc. study was an innovative research demonstration study. The study has been reviewed by a qualified geophysicist. It is felt the Work Plan text adequately summarizes the conclusive results of this study that are relevant to the RI/FS. The geophysical study identified the boundaries of the landfill and determined that metal was scattered throughout the landfill. Since these objectives of the work plan have been met for the geophysical survey it is not recommended at this time to perform additional geophysical surveys at CFA landfill II. However, the QA/QC procedures of sampling and analysis for the soil gas sampling task are not available. The data are not reliable for quantitative assessments. Therefore, 
additional shallow soil gas sampling has been identified for CFA landfill II to fulfill the objectives outlined in this Work Plan.

4. The work plan and the field sampling plan would benefit greatly from a expanded section on the data needs and data gaps for this remedial investigation. In some sections, the document refers to data needs which are not considered in the current scope for this investigation (for example see comment 48), and the rationale for not addressing these items should be discussed. IDHW has included a partial list of data needs as an addendum to these comments, and suggests that a expanded section(s) on data needs be included. A useful exercise would be to summarize in the text the input parameters required to run the proposed models (e.g., HELP) and list how each parameter will be determined or estimated. Examples can be found in Section 1 of the QAPjP for Operable Unit 7-08 (Anderson and Lugar, 1992) and Section 4 of the Work Plan for Operable Unit 7-08 (Chatwin et al., 1992).

Resolution: Sections 4.0 and 5.0 have been expanded as appropriate and identifies the existing data for both landfills, data needs and gaps for both landfills, and input parameters to be used.

5. Trichloroethene and 1,1,1-trichloroethane were detected in ground-water samples collected from the CFA production wells in April 1991 (Barresh and Marts, 1991). The CFA landfills appear to be a possible source of this contamination. For this reason, and also to take advantage of opportunistic sampling, IDHW recommends expanding the scope of the work plan to include the following:

a. Incorporation of the CFA production wells into the ground-water investigation.

Resolution: CFA production wells \#1 and \#2 will be added to the groundwater investigation.

b. Add soil gas sampling locations around the perimeter of CFA Landfill III to evaluate the horizontal extent of the vapor plume. Existing data for Landfill II should be examined to determine if additional sampling locations are required.

Resolution: Soil gas samples will be collected from around the perimeter of CFA landfill III. QA/QC data is not available for the existing shallow soil gas data for Landfill II; therefore, additional shallow soil gas samples will be collected from landfill II.

c. Seep/leachate sampling along the toe of the slope on the northeast section of landfill II. This can be easily accomplished by conducting periodic visual inspections (e.g., weekly) to determine if any seeps are present. If so, a sample can be collected with minimal effort. This will provide baseline data on the types and relative mobility of contaminants placed in the landfill.

Resolution: Periodic visual inspection of seep/leachate along the toe of the slope on the northeast section of landfill II will be made and if present will be collected for VOC, metal, and SVOC analyses. 
d. Installation of leachate wells and/or suction lysimeters in the surficial sediments. Optimum locations for these devices can be evaluated by using the lithologic logs from the existing borings at the landfills, geophysical data, and the results of the soil gas surveys to determine:

1. Saturated areas.

2. Potential areas for "ponding" in the subsurface (i.e. low points in the clay layer and sediment/basalt interface).

3. Preferential migration paths.

4. Hot spots.

These data, coupled with analytical data collected from the samples of pore fluid, can be used to evaluate source term, contaminant mobility, and the requirement/feasibility of a leachate collection system. In addition, leachate wells and suction lysimeters can be incorporated into the monitoring program if a containment option is selected.

Resolution: Past drilling activities at the CFA landfills have not indicated the presence of leachate and the moisture monitoring data does not suggest sufficient amounts of infiltration to warrant this investigation. However, per recent scoping meetings between EPA, IDHW, and DOE, it has been agreed that seven borings will be augured and sampled through Landfill II. Soil samples (and leachate if present) will be collected and analyzed for VOCs, SVOCs, and metals. The objective of this task is to gather information on leachable contaminants for input to fate and transport modeling, and to reduce uncertainties associated with the Landfill II waste inventory records.

e. IDHW recommends a meeting early in the investigation to evaluate the results of the monthly water-level measurements to determine if additional ground-water monitoring wells are required.

Resolution: A meeting will be scheduled early in the investigation phase to evaluate results of monthly-water level measurements to determine if additional ground-water monitoring wells are required.

\section{Specific Comments}

1. Page iii - Recommend including full reference for the NCP (National Oil and Hazardous Substances Pollution Contingency Plan; 40 CFK 300) and citing specific "DOE orders, directives and policies" applicable to this work plan and/or remedial investigation.

Resolution: Full reference for NCP has been added to the text. The reference to DOE orders, directives, and policies is a general statement. Where appropriate specific titles are provided. DOE orders are listed with number and title in the discussion of ARARs (Scction 3.5). 
2. Page v, Paragraph 1 - The work plan does not "assign authorities" for RI/FS activities. This was done under the Federal Facilities Agreement/Consent Order signed by the DOE, EPA, and Idaho Department of Health and Welfare (IDHW).

Resolution: "Assign authorities" has been deleted from the text.

3. Page v, Paragraph 4 - The text should also state that the portion of Landfill III not addressed in this operable unit will be investigated under the Comprehensive RI for the Central Facilities Area (operable unit 4-13).

Resolution: The text has been revised as appropriate.

4. Page vii, Paragraph 2 - "Hot-spot removal" has also been identified as a preliminary remedial activity for the CFA landfills.

Resolution: Hot-spot removal, groundwater monitoring, and leachate collection/monitoring were added to the text.

5. Page 1-2, Paragraph 1 - This paragraph should indicate that the risk assessment will also estimate potential future risk to human health. Also see comment \#2.

Resolution: "Current/future" has been added to the text.

6. Page 1-2, Paragraph 2 - Define specifically how "The RI/FS process will be supplemented as needed to meet DOE policy requirements for assessment of environmental impacts." Also indicate which DOE policy pertains to the assessment of environmental impacts, and how this will be incorporated into ". . a single, integrated set of documents. .."

Resolution: These statements have been revised to eliminate the inference to NEPA (see EPA comment \#1 Section 1.0, EPA's position is that CERCLA meets the substantive requirements of NEPA).

7. Page 1-4, Paragraph 3 - Expand the text to explain how Landfill I has been addressed as a Track 2 investigation under the FFA/CO and the mechanism for assessing the "active" portion of Landfill III. Also see comment \#3.

Resolution: The following text has been added to the Work Plan. "Landfill I has been addressed as a Track 2 investigation under the FFA/CO and the active portion of Landfill III will be addressed under a separate investigation after closure (approximately 30 years from now)."

8. Page 2-1, Paragraph 3 - The hazardous substances listed in tables $2-5$ and $2-6$ should also be discussed in this paragraph. Furthermore, given the mission of the INEL (nuclear reactor and spent fuel research), methods used for radionuclide screening of wastes taken to the landfills should also be discussed in the work plan. 
Resolution: The following text has been added to the Work Plan. "To a lesser extent hazardous wastes; such as, waste oil, solvents, and paint were also disposed of at the landfill." Text has also been added to Section 2.0 describing the screening of waste material prior to disposal at the CFA landfill by a Radiological Control Technician to confirm the requirement of nonradioactive waste disposal at the landfill.

9. Page 2-1, Paragraph 4 - Reference to "nonradiological and nonhazardous" should be deleted. Instead the text can reference tables $2-5$ and 2-6. A section summarizing the waste disposal at the Radioactive Waste Management Complex would be useful to indicate the intended uses of the CFA landfills.

Resolution: "Nonradiological and nonhazardous" has been deleted from the text. The RWMC is outside the scope of this Work Plan and it does not seem appropriate to provide information on this operable unit.

10. Page 2-5, Figure 2-4 - Please indicate what grid system is used for this figure, and also show t'e actual survey points used to develop the topographic map. In this figure, some of the contour lines cross and some bifurcate, therefore revising the contour interval to $0.5 \mathrm{ft}$ may be appropriate.

Resolution: The figure provides the raference report which details the survey grid used to develop this map. The grid consisted of over 191 survey points and a figure of these actual grid locations is provided in the referenced report. The figure will be cleaned up so that lines do not cross.

11. Page 2-12, Figure 2-7 - Indicate if the depths shown are in feet or meters (it appears to be feet).

Resolution: The depths are in feet. The figure has been deleted from the document upon revision of this section based on other comments to provide more specific geology pertinent to the CFA landfills opposed to general statements applicable to the INEL as a whole.

12. Page 2-13, Paragraph 2 - The text repeatedly states that the interbeds are composed of "finegrained materials (silt and clay)"; however, this assertion is not supported by the well logs in Appendix D. For example, the only continuous interbed beneath the landfills, which occurs at a depth of 50 to $70 \mathrm{ft}$ (see Figure 2-9) is described as a sandy gravel in the geologist's log for LF2-08. In fact, only LF2-09 and LF2-10 suggest the presence of clay, all other logs state that sand is the primary component. Please clarify. Furthermore, due to the potential impact of this interbed on contaminant migration, it would be useful to include lithofacies and isopach maps of this unit.

Resolution: The text has been changed to reflect the variable composition of the interbeds encountered during the drilling at the landfills and tables have been added listing at what depths clay was encountered. Interbed thickness and extent are controlled largely by the irregular surface of the basalt flow on which it was deposited; therefore, it is difficult to estimate or correlate between available horizontal control points. 
The interbed at a depth of 600 to $650 \mathrm{ft}$ is a "heaving sand and gravel" according to the logs for LF2-09 and LF2-10. This material is not a likely candidate for isolating the upper part of the Snake River Plain aquifer from the deeper sections, as stated in the text. Note that page 2-26 suggests the difference in hydraulic head across this interbed is attributed to a deviation from vertical in well LF2-10.

Resolution: This statement will be deleted from the text. However, it is important to note that the logs for these wells also indicate the presence of clay in these interbeds.

13. Page 2-13, Paragraph 3 - The lithologic logs (and/or geophysical data) used to estimate the thickness and extent of the clay layer should be included in the report slong with an isopach map. This would simplify evaluation of the potential impact of the clay layer on contaminant migration.

Resolution: Lithologic logs for the wells and boreholes have been provided in Appendix D as supporting information. A table has also been added to the Work Plan listing the clay encountered during drilling and the corresponding depths. See resolution to comment \#12 concerning isopach map.

14. Page 2-16, Paragraph 1 - See comment \#12.

Resolution: See resolution to comment \#12.

15. Page 2-16, Paragraph 4 - Define what is meant by the phrase "recent times" (e.g., 1984).

Resolution: The phrase "recent times" has been deleted and replaced with "and does not flow onto the INEL."

16. Page 2-19, Paragraph 2 - See comment \#12.

Resolution: See resolution to comment \#12.

17. Page 2-22, Figure 2-11 - Reference the date and source of the data used to construct this water table map.

Resolution: The date May 1989 and the source of the data from Environmental Restoration Information System have been provided with the Figure.

18. Page 2-28, Section 2.2.1 - The text states that finer-grained material was used for the cover material. Rather than making this general statement, summarize the cover characterization study conducted at Landfill II by the University of Idaho (1990).

Resolution: The phrase "finer-grained" has been deleted from the text and the following summary information from the referenced report has been added. "Cover material thickness ranges from 6 in to over $3 \mathrm{ft}$ and consists predominantly of sand and gravel, with measured saturated hydraulic conductivities ranging from 0.002 to $0.0025 \mathrm{~cm} / \mathrm{s}$." 
This section would also benefit from a summary of disposal methods for liquid wastes (c.g., was the material in drums or dumped into the landfill). Specific data on the chemical composition of these wastes would also be useful, if available. Also, did the interviews with site personnel indicate the potential presence of "hot-spots"?

Resolution: There is not sufficient information available to provide additional text to the Work Plan to adequately address the above. There is no specific data available on the chemical composition of these wastes and no "hot-spots" have been identified. However, additional source term information will be pursued and evaluated throughout the RI.

19. Page 2-30, Paragraph 3 - The following should be added to the source term information:

a. Radioactively contaminated soil taken to Landfill II from the Idaho Chemical Processing Plant (see page 6; WINCO, 1992).

Resolution: The above referenced report states the soil was disposed of at the INEL Central Radioactive Waste Disposal and Storage Area (RWDSA). This is more commonly referred to as the Radioactive Waste Management Complex (RWMC), not to be confused with Central Facilities Area landfills. Therefore, the above statement was not added to the text as it does not apply to CFA landfills.

b. The 120 drums of trichlorocthene which may have been disposed in Landfill II (page 2-1; ICF Technology, 1990).

Resolution: This statement was conjecture on the part of ICF to demonstrate the potential for using high risk geophysical surveys at the landfills to detect potential contaminant migration. The waste inventory does not indicate the disposal of 120 drums of TCE. While this statement has not been added to the text, it will be further investigated and pertinent information will be transmitted to IDHW and EPA.

20. Page 2-31, Figure 2-14 - This figure should be revised to reflect the current location of the "active" landfill operation. This will illustrate the potential influence of contaminant migration from that area on the data generated in this remedial investigation.

Resolution: A figure illustrating the current location of the active landfill has been incorporated. Filled vs. unfilled portions of the landfill are not presented due to the active changing status of the landfill.

21. Page 2-34, Table 2-5 - Is any additional information known about the "waste oils" (e.g., motor oil, transformer oil, etc).

Resolution: No additional information pertaining to the phrase "waste oils" is available.

22. Page 3-7, Paragraph 3 - IDHW feels the work plan should summarize all existing data pertinent to this operable unit. Therefore, the soil gas data collected by EG\&G Idaho in 1988 and 1989 should be presented in the work plan, along with the reason(s) why the data are not considered reliable. 
Resolution: The data will be provided in Appendix A of the Work Plan. The data is not presented here because it has not been validated and the QA/QC procedures of sampling and analysis are not available. The data are not reliable for quantitative assessments. Therefore, conclusions with regard to health risks for volatile organics can not be made because the reliability of the data are suspect. For purposes of this Work Plan, the above data will be used for project planning only.

23. Page 3-8, Paragraph 4 - Delete or quantify the word "limited." For example, some individuals may interpret the detection of solvents at a depth of 450 feet as indicative of extensive contaminant migration.

Resolution: The text has been deleted from the Work Plan. Table 3-2 contained some erroneous data that has been removed, i.e., sample depth of $450 \mathrm{ft}$. This data presents the soil gas data collected from the instrumented shallow boreholes. The table has been revised accordingly.

24. Page 3-8, Section 3.1.2 - The stratigraphic logs for these borings should be included in the work plan to allow for a review of trends resulting from geologic influences (i.e. contamination concentrated above a clay layer). Furthermore, it would be beneficial to know if any leachate was encountered in these borings.

Resolution: The stratigraphic logs for the boreholes have been added to Appendix D. No leachate was encountered during this boring and sampling effort. The text has been revised to clearly state this fact.

25. Page 3-13, Section 3.1.5 - The results of the geophysical surveys (i.e. maps) should be included in the work plan along with a discussion of whether they meet the data quality objectives.

Resolution: A copy of the ICF technology, Inc., report entitled "Innovative Technology Demonstration Idaho National Engineering Laboratory (INEL) CFA Landfill II Idaho Falls, Idaho" has been placed in the Administrative Record. See general comment resolution \#3.

26. Page 3-13, Paragraph 2 - There is a possibility that additional scoping of ground-water contamination at the INEL will be conducted. Therefore, recommend revising the text to state that the ground-water contamination from the TRA and ICPP will be addressed "in other remedial investigations at the INEL."

Resolution: The text has been revised as suggested.

27. Page 3-14, Section 3.3 - The brief site characterization in section 3.1 is not adequate to address preliminary public health and environmental impacts. Additional characterization and modeling of source term information would need to be compared to species-specific contaminant concentration data.

Resolution: The first sentence of this paragraph has been deleted. 
28. Page 3-15, Figure 3-3 -

a. If an INEL worker could be exposed via dermal contact with soil, it seems soil ingestion would also be a potential exposure route.

Resolution: Incidental soil ingestion has been added for the industrial scenario.

b. The vegetative cover at the landfills is somewhat sparse, consequently an explanation why the conceptual site model does not include inhalation of particulates is required.

Particularly since page 2-28 states that finer-grained overburden material was used in the cover.

Resolution: Incidental soil ingestion and inhalation of dust have been added to the conceptual site model.

c. The exposure point for some of the pathways may be within the operable unit boundary, depending on the needs of the project managers to make an informed remedial decision. Recommend deleting exposure points from the conceptual site model.

Resolution: The column "exposure point" has been deleted from the figure.

d. If this CSM is only for office, construction, and sanitary wastes as indicated under "Contaminant Source," an additional CSM should be prepared for the hazardous substances listed in Tables 2-5 and 2-6 (e.g., chromates, solvents, mercury, etc).

Resolution: Hazardous waste has been added to the figure under contaminant source column.

29. Page 3-16, Paragraph 1 - The CFA landfills may be the source for the organic solvents detected in the CFA production wells. Therefore, the analytical data from these wells should be included in the work plan.

Resolution: The data is provided in a report published by Barresh and Marts, 1991 and the report has been referenced in the Work Plan. Therefore the data will not be incorporated into the Work Plan. Future sampling of these wells under this RI will result in the analytical data being incorporated into the RI report.

30. Page 3-17, Paragraph 1 - Additional preliminary remedial action alternatives include: 1) hotspot removal, 2) ground-water monitoring, 3) leachate collection/monitoring, and 4) deed restriction. A combination of two or more alternatives may also be appropriate.

Resolution: The above mentioned preliminary remedial action alternatives have been added to the text.

31. Page 3-20, Second Paragraph - In the paragraph that contains four bullet items for a State requirement to be considered an $\mathrm{ARAR}$, delete the third bullet item, "not result in an in-State ban on land disposal of hazardous waste." Under CERCLA section 121(d), a State-wide ban 
prohibiting land disposal of hazardous substances may be an ARAR if the state requirement is of general applicability and was adopted by formal means on the basis of hydrologic, geologic, or other relevant considerations and for reasons to protect human and the environment, and the State provides arrangements and pays for incremental costs from using a hazardous waste disposal facility.

Resolution: This bullet has been deleted from the text.

32. Page 3-22, Table 3-7 - When a state has been granted authorization for operating a Federal program, the requirements of the state program are considered as stringent or more stringent than those requirements of the parallel Federal law or regulation. In such cases, the authorized state requirement is documented as the potential ARAR in the identification and communication of State ARARs. The State of Idaho has federal authorization for enforcement of RCRA. Please revise this table accordingly.

Resolution: Since the State does not have more stringent requirements under the RCRA program the minimum requirements that must be met are the RCRA requirements listed in the table. However, additional State ARARs have been added to Table 3-9.

33. Page 3-24, Table 3-9 - Add to the list of potential State ARARs:

Hazardous Waste Management Act of 1983 (Idaho Code \$39-4401 to 4432);

Hazardous Waste Management System: General (IDAPA \$16.01.5004);

Identification and Listing of Hazardous Wastes (IDAPA \$16.01.5005);

Land Disposal Restrictions (IDAPA \$16.01.5011).

The citation to the Solid Waste Management Regulations: General should be "IDAPA $\$ 16.01 .6004,01$." $^{\prime \prime}$

Resolution: The above potential State ARARs have been added to the text.

34. Page 4-1, Paragraph 2 - It is unlikely that the limited sampling of the soil cover proposed in this work plan will quantify the "extent and magnitude of contamination in surface soils." Please explain.

Resolution: The word "extent" has been replaced with "presence."

35. Page 4-3, Cover Investigation -

a. Recommend revising text to state this phase will evaluate potential for infiltration rather than "evaluate the geologic unit governing transport of contaminants."

Resolution: The phrase "and evaluate potential for infiltration" has been added to the text. However, the phrase "evaluate geologic unit governing transport of contaminants" 
will remain in the text, because an additional objective of the cover investigation is to determine soil properties that may influence the transport of soil gas through the cover to the surface.

b. The task of identifying potential borrow-sources for a cover is more appropriate for the remedial design phase.

Resolution: This task has been deleted from the text.

36. Page 4-4, Table 4-1 - The moisture monitoring data discussed in section 3.1 .3 was collected during a drought (1988-1991). The current snowpack in southeast Idaho is more representative of "normal" conditions, therefore IDHW recommends additional moisture data be collected during the RI (1993). A summary of the locations and depths of the moisturemonitoring equipment already in place would also be useful (see section 3.1.3).

Resolution: Moisture monitoring with the neutron probe will be conducted on the five existing neutron access holes on a monthly basis during the RI field activities. A summary of moisture-monitoring equipment for existing boreholes will be provided in of the Work Plan.

37. Page 4-6, Table 4-3 - The surface soil samples for volatile organic compounds and metals would seem to correspond to analytical level IV as defined on page 4-5. Furthermore, since waste oils were disposed in the landfill, it would seem that samples for semivolatile organic compounds should be collected.

Resolution: The table has been corrected to reflect analytical level IV data for soil analyses. Semivolatile organic analysis of the soils will be added to the Work Plan.

38. Page 4-7, Table 4-3 - The water balance equation will be useful to determine the potential for leachate, but it is unlikely that it will be sufficient to determine if the existing cover is adequate due to other factors such as surface runon, hydraulic conductivity of the cover material, etc.

Resolution: The text has been rephrased to indicate the water balance equation will be used to determine if the existing cover is adequate for maximizing evapotranspiration.

39. Page 5-3, Section 5.3.1 - Please define the criteria which will be used to establish "additional survey locations." Also, state the accuracy of the survey (e.g., vertical control within $0.1 \mathrm{ft}$ and horizontal control within $3 \mathrm{ft}$ ). Also indicate the grid which will be used.

Resolution: The text has been revised to incorporate the requested information.

40. Page 5-4, Section 5.3.3.1 - Recommend collecting soil gas samples along the perimeter of the landfill to evaluate horizontal extent of vapor migration.

Resolution: Soil gas samples will be collected around the perimeter of the landfills as recommended. 
41. Page 5-9, Paragraph 1 - Indicate the specific method which will be used in the "statistical sampling design" to determine number and locations of flux chamber sampling points.

Resolution: The text has been revised to indicate a minimum of eight samples will be collected from each landfill based on the shallow soil gas data results. If the soil gas data is not available at the time of sampling then the sites will be chosen randomly.

42. Page 5-9, Paragraph 2 -

a. The text states that the surface of the landfill will be examined for visual signs of staining or stressed vegetation, but does not indicate whether this information will be used to determine sample locations. Furthermore, this task should be scheduled such that the data collected from the soil gas survey can also be used to determine sampling locations.

Resolution: The text has been revised to reflect the use of this information in sample site selection. Due to the limited time period allocated for RI field investigations and the turn around time for the laboratory analyses and data validation this task must proceed without the aid of the soil gas data in sample site selection.

b. Background sampling locations should be indicated on a site map, or, at a minimum, the text should state the criteria which will be used to determine whether the sampling location is "outside the influence of the landfills." Furthermore, two samples will probably not be adequate to address the variability of metals concentrations in soils. It may be preferable to use an existing reference (e.g., Martin et al, 1990; Spry et al, 1992).

Resolution: References such as the one mentioned above will be used to provide information on background metal concentrations at CFA.

43. Page 5-10, Paragraph 1 - See comment \#35.

Resolution: The text has been revised to include "to evaluate the potential for infiltration."

44. Page 5-10, Paragraph 4 - Does "intact" mean undisturbed?

Resolution: "Intact" has been replaced with the word "undisturbed" for clarity.

45. Page 5-11, Paragraph 1 - ASTM D-421 is the preparation method for particle-size analysis, ASTM D-422 is the method number for the analyses.

Resolution: The correct method ASTM D-422 has replaced the preparation method.

46. Page 5-12, Paragraph 4 -

a. Volatile organic compounds may be the most mobile compound disposed in the landfills; therefore, it is unlikely that analysis for VOCs will be discontinued. Furthermore, if VOCs are detected in groundwater, continued monitoring will be conducted to evaluate seasonal trends regardless of if concentrations are below maximum contaminant levels. 
Resolution: Per the 3/1/93 scoping meeting between DOE, EPA, and IDHW, the groundwater sampling section has been revised to reflect that two rounds of groundwater samples will be collected for use in the BRA and the third round will be used for confirmation.

b. A variety of hazardous wastes were placed in the CFA landfills, therefore the text should indicate why analyses will only be conducted for VOCs, metals, and common anions. For example, waste oils were placed in the landfills so it would seem prudent to sample for semivolatile compounds. Given the variety of wastes typically placed in industrial landfills, it may be appropriate to perform Appendix IV analyses for the first sampling event.

Resolution: An examination of five quarters of existing data (Appendix C) shows no SVOCs other than a few detections for phthalate, which may be attributable to lab contamination. Analytical results for herbicides and pesticides also showed no detection of any constituents. Given that SVOCs, herbicides, and pesticides have not been detected and the fact that VOCs are more mobile, sampling for these compound classes (SVOCs, pesticides, and herbicides) will not be incorporated into the sampling scheme for the RI.

c. The CFA production wells are located downgradient of the landfills, therefore these wells should be included in the sampling events and collection of water level data associated with this $\mathrm{RI}$.

Resolution: The CFA production wells have been added to the groundwater monitoring program. The wells have dedicated pumps which prohibit the measurement of water levels.

47. Page 5-16, Section 5.6.2 -

a. Contaminant transport modeling will also utilize available inventory information to assess risks posed by this operable unit.

Resolution: The text has been revised to include "existing waste inventory information."

b. The text states that the following migration routes will be modeled "if necessary":

- gas phase migration to the atmosphere.

- gas phase migration to the aquifer.

- liquid phase migration to the aquifer.

Explain how the use of existing data, as well as data collected during this investigation, will determine if modeling of these migration routes is necessary. For example, since solvent vapors were detected at a depth of $450 \mathrm{ft}$ (Table 3-2), it would appear that gas phase migration is occurring. Also, since approximately 54,000 cubic meters of liquid waste was disposed in Landfill II (Table 2-5), it would seem that liquid phase migration should be evaluated. 
Resolution: "If necessary" has been deleted from the text. Text has also been revised to explain conditions under which gas phase migration to the aquifer and liquid phase migration to the aquifer will be modeled and the modeling approach to be used. In addition, the units on the liquid wastes in Table 2-5 were incorrectly stated as $\mathrm{m}^{3}$ and should have been liters. This reduces the volume by a factor of 1000 , and decreases the migration potential. The data indicating solvent vapors were detected at a depth of 450 $\mathrm{ft}$ is incorrect and has been deleted from Table 3-2.

48. Page 5-16, Section 5.6.2.1 - The text states that release rate of contaminants is controlled by "... moisture content near the waste, solubility of the chemicals, and other factors." Explain how these data needs will be addressed in this RI. See general comment \#4.

Resolution: The text has been revised to better address data needs and modeling inputs.

49. Page 5-18, Paragraph 3 - The organic solvents disposed at the landfills are not high viscosity compounds. Furthermore, the statement that movement of nonaqueous phase liquids could only occur by immiscible displacement assumes saturated conditions, and therefore is not applicable to the vadose zone. The text should indicate which specific activities planned for this remedial investigation will indicate if NAPLs are present in the landfills and have migrated into the underlying basalt. Also, what is the significance of the evidence that at Landfill II, contaminant migration has reached the underlying sedimentary interbed in one location and possibly three others (p. 4-5; ICF Technology, 1989).

Resolution: Discussion of the nonaqueous phase liquids has been revised as noted in the comment. The presence of NAPLs will be evaluated during the subsurface soil sampling activity. There is no evidence that contamination has reached the underlying interbed as reported in the ICF Technology report (p. 4-5). In response to this statement on page 4-5, it is felt the data in the report (and their own discussion) does not conclusively support this statement. Additionally, the one-dimensional model assumptions made for this interpretation were violated. For instance, the model assumes infinitely horizontal layers whereas the landfill is three-dimensional and second the assumption that greater time equates with greater depth is true for normal soils with low conductivity but is violated at the landfill were there is a large concentration of high conductivity metal. See resolution to comment \#3.

50. Page 5-26, Paragraph 3 - See comment \#27.

Resolution: The text has been revised.

51. Section 6 - Many of the document submittal dates listed in this schedule have different target dates than the schedule presented in the Final Scope of Work for Operable Unit 4-12 (Keck and Stormberg, 1992). These schedule changes represent a modification of a final document, and must be conducted in accordance with Section 8.22 of the Federal Facilities Agreement/Consent Order.

Resolution: The schedule has been revised to be consistent. 


\section{Sampling and Analysis Plan}

52. Page 2-5, Section 2.2.1. - The topographic survey should also address the adequacy of the existing cover for controlling surface-water "runon," which could result in increased infiltration rates.

Resolution: Runon has been added to the text.

53. Page 2-6, Paragraph 1 - The lithologic logs and construction diagrams for the shallow boreholes should be included in the work plan.

Resolution: The logs and construction diagrams for the shallow boreholes will be provided in Appendix D.

54. Page 2-8, Paragraph 1 - The text states that ground-water sampling will occur in April, July, and October; however, the schedule provided in Section 6 indicates that samples will be collected in April, June, and July. Please clarify this discrepancy.

Resolution: The schedule in Section 6 was incorrect. This schedule has been revised to be consistent with the text and proposed seasonal sampling.

55. Page 3-1, Section 3.1 - There is no evidence presented in the work plan to indicate that different types of soils were used as cover material for the landfills. Furthermore, minor variations in the composition of cover material would probably not be distinguishable by visual observation. Therefore, IDHW recommends selecting the sampling locations using a random number generator or similar method. If a sufficient number of samples are collected, this method should be adequate to address spatial variations. To ensure representative samples are collected, this method should also be used for the surface soil samples which will be used to characterize contaminant concentrations (Section 3.2). A contingency should be included for collecting additional samples based on stressed vegetation and information from other RI activities (e.g., geophysical and soil gas surveys).

Resolution: The text has been revised to indicate the samples will be collected randomly. However, due to the limited time allowed for the RI field autivities it will not be possible to wait for previous task results before proceeding with this task.

56. Page 3-4, Table 3-3 -

a. Collecting four replicates from the ten wells in the sampling network corresponds to $40 \%$ QA/QC samples. Please provide the rationale for this as it would seem a bit excessive. Note that page 3-20 of the FSP states that "Field replicates will be collected at a minimum frequency of 1 per 20 samples or 1 per sampling round for each sample medium." Additionally, IDHW recommends randomly selecting the well(s) at which replicate samples will be collected. This will reduce the potential for biasing analytical results. 
Resolution: QA/QC sample quantity (replicates) has been reduced and replicates will be collected randomly as requested.

b. To further prevent cross-contamination, the upgradient wells, which are presumably "cleaner" than downgradient wells, should be sampled first. Furthermore, the text states that the rinsate blank will be collected after sampling the first well. Collecting a rinsate after sampling a supposedly "clean" well may not adequately evaluate the effectiveness of the decontamination procedure. Although the analytical data in Appendix C.1 does not seem to indicate any clear variations between upgradient and downgradient wells, IDHW recommends sampling upgradient wells first, and also collecting the rinsate after sampling downgradient wells.

Resolution: Well sampling order will be determined based on existing data and the concentrations of chromium in the various wells. As stated, there are no clear variations between up and down gradient wells and therefore the best approach to prevent or minimize cross contamination is to sample least contaminated wells first and progress to the more contaminated wells. As suggested a rinsate will be collected after sampling the downgradient wells.

c. IDHW feels the two primary routes for sample contamination in this investigation are: 1) organic contamination from volatilization of solvents in the landfill, and 2) metals contamination from wind-blown dust. Therefore, it may be advantageous to collect the field blank near midday, since volatilization rates and wind speeds typically increase as the air temperature rises.

Resolution: The objective of the field blank is to monitor the conditions during sampling and not to target specific environmental conditions. However, if samples are collected in the afternoon, then a field blank will be collected at that time.

d. State the rationale for only replicating one surface soil sample (one of twenty-seven) rather than the minimum of 1 per 20 recommended on page 3-20.

Resolution: The text has been revised to indicate that 2 replicate samples will be taken per the recommended minimum of 1 per 20.

57. Page 3-15, Paragraph 3 - DOE should identify the percolation ponds which they believe may influence regional flow. A location map would also be useful.

Resolution: The text has been revised to clearly state the percolation ponds are at ICPP that may be influencing groundwater flow at CFA.

58. Page 3-20, Section 3.7 - Reference the document which states that "The desired number of field quality control samples is at least $20 \%$ of the total number of field samples taken." Furthermore, the text recommends that rinsate blanks, field blanks, and field replicates be collected at a minimum frequency of 1 per 20 , for a total of 3 per 20 or $1.5 \%$. Please clarify.

Resolution: The text has been revised to consistently follow all QA/QC protocols. 
59. Page 3-21 - Appears to be some missing text.

Resolution: The following text has been inserted: "of field activities."

60. Page 5-4, Paragraph 1 - Please explain why 3-4 days was the time period selected for gravitational drainage. How will the volume of water required to wet the soil column be determined (e.g., what porosity value will be assumed or how will this parameter be determined)? Also see comment \#55 regarding the selection of sampling locations.

Resolution: The text has been revised in the following manner in answer to the above questions. In-situ field capacity is defined as the amount of water remaining in a soil two or three days after having been wetted and after free drainage is negligible. The volume of water required for the test will be estimated based on the soil porosity, which in turn will be estimated by knowing the bulk density of the soil, which will be obtained using the neutron densometer and the particle density (assumed to be $2.65 \mathrm{~g} / \mathrm{cm}^{3}$ for mineral soils).

61. Page 5-6, Paragraph 1 - IHDW recommends using a few soil borings to verify the accuracy of the ground penetrating radar study in determining the thickness of the cover material.

Resolution: The text has been revised to indicate a few soil borings will be taken for confirmation of the geophysical survey determination of soil cover thickness.

62. Page 5-6, Section 5.5.1 - It would be beneficial to also collect replicate samples of the soil gas to evaluate the accuracy of the analytical laboratory.

Resolution: The text has been revised to indicate replicate samples of soil gas samples will be taken as part of the QA/QC.

63. Page 5-9, Section 5.7.2 -

a. Please indicate what method will be used to collect the ground-water samples (e.g., submersible pump). Also clarify in Section 5.7.1 if a minimum of three well volumes will be purged prior to sampling.

Resolution: The text has been revised as requested.

b. See comment \#46.

Resolution: See resolution to comment \#46.

64. Page 5-11, Section 5.8 - In the handling and disposition of investigation derived waste (IDW), two options are mentioned for handling the 60,000 gallons of purge water. If the first option is used (i.e., removal of chromium by a portable treatment unit), please discuss options for the final disposition of the removed chromium and of the treated water (which may contain tritium). 
In the second option mentioned (i.e., discharge to the TRA warm waste pond), reference should be made to the new lined evaporation pond being constructed for operations at the TRA facility, if appropriate. Is this a viable option, considering the timing for completion of the new TRA evaporation pond? Clarify what types of constituents will be discharged to the new TRA evaporation pond from TRA operations and whether the contaminants from the CFA purge water would be consistent. Would evaporation of the purge water in the TRA pond have any significant added effect to air quality over the permitted pond discharges from operations?

It is suggested that both options be considered together. In other words, if the new TRA pond does not receive chromium contaminated water, then the purge water may be treated to remove the chromium prior to discharge to the TRA evaporation pond.

Please discuss options for the handling and disposition of the estimated 300 gallons of decontamination water.

Resolution: A detailed IDW plan has been added to the text.

\section{Community Relations Plan}

65. Page 1-1, Second Paragraph - This review is pursuant to the FFA/CO and is not to be construed as a review or approval of this deliverable as a NEPA document.

Resolution: Comment noted, no action.

66. Page 3-1, Fourth Paragraph - Last sentence inaccurately states that the "NCP amends existing provisions and adds new major authorities to CERCLA." More accurately, the Superfund Amendments and Reauthorization Act (SARA), passed by Congress in 1986 revised and added new major authorities to CERCLA. One of the modifications made by SARA was a requirement for the NCP to be revised in order to implement the regulatory changes required by SARA. Recommend combining the last two sentences to state "The investigation and remedial activities being conducted under the FFA/CO will be conducted in accordance with the revised NCP, which implements CERCLA, as amended by SARA."

Resolution: The text has been revised as stated above.

67. Page 3-2, First Paragraph - Removal actions are more accurately actions taken to respond promptly to an urgent need to protect public health or welfare or the environment from a release or threatened release of a hazardous substance. Remedial actions are more permanent remedies taken to prevent or minimize the release or threat of release of hazardous substances that present a current/future danger to public health, welfare, or the environment. Please revise this paragraph accordingly.

Resolution: The text has been revised as suggested above. 
The end objective of the RI/FS for the CFA landfills, as described in section 4.1 of the Work Plan, is to evaluate the risk presented by the CFA Landfills to human health and the environment and evaluate remedial action alternatives if unacceptable risks are identified. Please revise this paragraph accordingly.

Resolution: The text has been revised as suggested above.

68. Page 3-4 - This review is pursuant to the FFA/CO and is not to be construed as a review or approval of this deliverable as a NEPA document.

Resolution: Comment noted, no action.

69. Page 5-2, Last Paragraph - Statement that "the following types of documents ... are contained in the information repositories" is repeated again at the top of page 5-3.

Resolution: The repeated phrase was deleted.

The schedule in Table 5-1 is inconsistent with that presented in Table 6-1 of the Work Plan. Specifically, see dates for placement of the Final RI/FS Report in the Administrative Record, public comment periods on the RI/FS Report and Proposed Plan, date for placement of the Final ROD in the Administrative Record and public notice of availability of the ROD. Also see comment \#51.

Resolution: The schedule will be revised to be consistent with RI/FS schedules and schedules outlined in the text of the Work Plan.

70. Page 5-4, Second Paragraph - Statement that "the ROD will be issued following EPA or State-of-Idaho approval, and will include the summary" should be more accurately stated as "the ROD, which includes the responsiveness summary, is issued following EPA approval and concurrence by IDHW."

Resolution: The text has been revised as suggested above.

71. Appendix A, List of Contacts - Delete entry of John Ledger as a contact for State of Idaho Division of Environmental Quality; Revise/update entry for Shawn Rosenberger as follows:

Shawn Rosenberger, Remediation Technical Supervisor Idaho Department of Health and Welfare, Division of Environmental Quality 900 North Skyline

Idaho Falls, Idaho 83402

(208) $528-2650$

Resolution: The text has been revised as suggested above. 


\section{References}

Anderson, I. R., and Lugar, R. M., 1992, Sampling and Analysis Plan for the Organic Contamination in the Vadose Zone, Operable Unit 7-08 Focused RI/FS, EGG-WM-10175, Vol. I.

Barresh, W., and Marts, K, 1991, Duplicate Sampling of Ground Water Collected in the Area Between the Idaho Chemical Processing Plant and the Central Facilities Area, INEL, 1991, INEL, Oversight Program Technical Report 91-02.

Chatwin, T. D. et al., 1992, Final Work Plan for the Organic Contamination in the Vadose Zone, Operable Unit 7-08 Focused RI/FS, EGG-WM-10049.

ICF Technology, Inc., 1989, Innovative Technology Demonstration, Idaho National Engineering Laboratory, CFA Landfill II, Idaho Falls, Idaho.

Martin, K. L., and others, 1990, Preliminary Assessment of Surface Soils at active EG\&G Facilities, EGG-ESQ-9225.

Spry, M. J., and others, 1992, Final Remedial Investigation Report for the CFA Motor Pool Pond (Operable Unit 4-11) at the Idaho National Engineering Laboratory, EGG-WM-9973.

WINCO, 1992, Draft Idaho Chemical Processing Plant Sampling and Analysis Plan for Operable Unit 3-07, Tank Farm Area. 


\section{ENVIRONMENTAL PROTECTION AGENCY REVIEW COMMENTS AND RESOLUTIONS}

\section{General Comments}

1. Comments are based on review of the Draft Work Plan (EGG-ERD-10453), published January 1993, and received February 1, 1993.

Resolution: No action.

2. This document generally conforms with the December 1992 Scope of Work for the RI/FS at this OU, but needs some refinement in order to provide the needed level of confidence in the results of the investigation and baseline risk assessment. While the process of developing a Conceptual Site Model (CSM), identification of data requirements and data gaps, and development of a Sampling and Analysis Plan (SAP) which addresses the data gaps has been presented, there are a number of points in the document where significant concerns have not been adequately addressed. Some specific points are identified in the comments below.

Resolution: The document will be revised per the following comments to provide a more detailed document.

3. Investigation of this OU is complicated by the fact that both the source and the site geology are non-homogeneous, making it questionable whether sample results are representative. The SAP should incorporate a significant degree of flexibility in the form of "if/then" decisions which can be made as sampling progresses. This will allow collection of the greatest amount of usable data for the resources expended. This has been incorporated to scme extent, as with the concept of limiting analysis if low probability contaminants are not found in the first round analysis, but could be expanded. For example, if early in the remedial investigation results indicate that the 110 -foot interbed is competent across the site and is a competent barrier to the migration of contaminants, further investigation of the basalt aquifer inay not be necessary because contamination from the site would not migrate to it. However, investigation of the perched water table that may manifest itself on top of the interbed could be required. This constant feedback and data review process will optimize the remedial investigation process and ensure that only necessary data are collected.

Resolution: Due to the limited time available for RI field investigations to meet FFA/CO schedules this may not be possible. When "if/then" decisions are appropriate to the field program they have been provided in the text.

4. The detection limit for each particular analyte should be specified in all tables containing chemical results rather than the term "ND." Many of the data summary tables for chemical analysis (e.g., Table 3-2) list "ND" to indicate that an analyte was not detected. These tables should be modified to indicate "less than."

Resolution: Table 3-2 has been revised to include the detection limit for all non-detects. 
5. All historical data that are to be used in the baseline risk assessment must be of sufficient quality to be included. The work plan proposes using soil-gas samples to characterize and evaluate volatile inhalation. It is not appropriate to use these results to calculate an RME in a baseline risk assessment, although they may be appropriately referenced as qualitative data.

Resolution: The soil gas samples collected will be of quality level III data which is sufficient for baseline risk assessments. However, it is important to note that the data will be used in the contaminant fate and transport modeling to support a qualitative risk assessment by estimating exposure concentrations for the inhalation pathway.

\section{Specific Comments}

1. Page v: In the second paragraph, there is no reason at this time to conclude that these landfills pose only a low-level threat. If the investigation reveals that there are significant hot spots for example, these could be remediated as a principal threat with the remainder handled by engineering controls (i.e. containment), as outlined in the NCP. See

40 CFR 300.430(a)(1)(iii)(C). It is also more accurate to say the agencies decided that these landfills "...should be investigated and remediated as necessary through the CERCLA-driven RI/FS process."

Resolution: Reference to CFA landfills posing a "low-level threat" has been deleted from the text.

In the fourth paragraph, clarify that the active portion of Landfill III is not part of the Operable Unit being investigated under this RI/FS, but it will be addressed under another OU in WAG 4.

Resolution: The text has been revised as appropriate.

2. Page vi: As noted on page 2-26 of the Work Plan, the ICPP and TRA are both up-gradient of the CFA landfills; this is essential information which should be included here, along with a general statement comparing contaminant concentrations found in up- and down-gradient wells. There is no clear evidence that the sampling pumps contribute to the chromium levels in the samples, therefore the statement about the pumps should be revised to indicate that they are a possible source which will be investigated during this RI.

Resolution: "Two upgradient facilities" has been added to the text for clarity. There is supporting evidence to suggest the pumps may also be contributing to the chromium concentrations found in the wells due to the large difference between concentrations from filtered and non-filtered samples, where in many instances the filtered samples are considerably lower than non-filtered samples suggesting the presence of particulate chromium.

3. Review the ES to ensure acronyms have all been spelled out once before being used, and that are correctly defined. [e.g., "ARARs", National Priorities List (NPL)]. Suggest a review of Acronyms and definitions (e.g., OSHA) 
Resolution: The document will be reviewed and the necessary changes to the text will be made.

4. Page 1-2: In the first paragraph, clarify that the risk assessment evaluates both current conditions and modeled future conditions.

Resolution: "Current/future" has been added to the text.

In the last paragraph, reference to "assessment of environmental impacts" sounds more like NEPA than CERCLA. The EPA position on this issue is that CERCLA meet the substantive requirements of NEPA, and there should be no impact on CERCLA schedules or activities because of NEPA.

Resolution: The text has been revised and reference to NEPA has been deleted.

5. Page 2-1: State whether LF II is currently fenced.

Resolution: The text has been revised to indicate Landfill II is fenced.

Identify/3xplain measures taken by DOE to ensure that radioactive waste was not disposed in this landfill.

Resolution: Text has been added to section 2.0 describing the required screening of waste prior to disposal at the CFA landfill by a Radiological Control Technician to confirm the waste meets the nonradioactive waste disposal requirement.

6. Page 2-6: Clarify that at the time of closure the LF III cover was compacted to a uniform depth of $2 \mathrm{ft}$ and state whether there has been an further cover maintenance since closure.

Resolution: The text has been revised as suggested above and no further cover maintenance has been performed on the cover since that time.

7. Page 2-7: Explain why the 1956-1961 data was used to compute relative humidity. If this is the most current local data available, so state.

Resolution: The text has been revised to indicate this six-year period of data represents the data period of record available for this site.

8. Page 2-8: The geologic description in part 2.1.2 is mostly general background of the site. Since the next section of the report is the "Initial Evaluation", conditions which are of significance to this specific OU should be presented. This part of the report should address the geologic structure that would influence the migration of contaminants from the landfills. A discussion of cross-cutting features in the CFA area, such as faults and flow interior discontinuities such as vesicular zones and joints within the entablature and colonnade would establish a framework for understanding the information present in subsequent sections. 
Resolution: The text has been revised to present the site specific geology at the landfills. The structure and geology of the vadose zone and aquifer are extremely complex as suggested in the text. Modelers usually make gross simplifying assumptions that tend to homogenize the actual site complexity.

9. Page 2-13: The discussion of the "clay layer", in the last full paragraph on this page, borders on deceptive. While it is of some value to know that this layer is not present under LF III, there is nothing to indicate whether does exist under LF II. If this layer is not found under either of the two landfills being investigated under this RI, the fact that "...where present, it would significantly impede the migration of any leachate to the water table" has no bearing on the RI. (Strongly recommend that any core samples from site boreholes which are available in the USGS INEL Core Storage Library be examined directly, rather than having to rely on field boring logs)

Resolution: The text has been revised and evidence of clay layers in or around Landfill II will be detailed in the text with supporting lithologic data provided in Appendix D.

The nomenclature used to describe the stratigraphy of the basalt flow group should be briefly presented. For example, the designation "DE-3" on Figure 2-9 is unclear and should be explained.

Resolution: The individual basalt flow designations, i.e., "DE-3" have been removed from the figure.

10. Page 2-16: The effects of geologic features, specifically aquifer anisotropy and nonhomogeneity, need to be discussed in part 2.1.3. An understanding of the mechanics of contaminant migration in groundwater through the fractured basalt underlying the site is incomplete without such discussions; this is a critical aspect of both the investigation of existing contamination and any predictive fate and transport modeling for evaluation future risks at the site.

Resolution: The text has been revised to indicate the groundwater flow is controlled by the fractured basalt and interbeds. Simplified models cannot account for these aquifer heterogeneities.

Either provide a more definitive statement on when the Little Lost River flows last reached the INEL, or eliminate the discussion entirely, since the statement has already been made that such flows "...would have no effect on the CFA landfills.."

Resolution: The text has been revised to clearly state the Little Lost River does not flow onto the INEL.

11. Page 2-21: Hydraulic conductivity should be used to analyze contaminant migration, and typical values should be presented in addition to the transmissivity values. If a groundwater problem has three-dimensional overtones (as the INEL site aquifer system does), it is best to use hydraulic conductivity in that analysis (Freeze and Cherry 1979). The wide range of transmissivity values discussed in the paragraph illustrates the reason for also presenting the 
hydraulic conductivity of the aquifer. Unlike transmissivity, hydraulic conductivity is not a function of the length of the open interval.

Resclution: Hydraulic conductivity values have been added to Table 2-2.

12. Page 2-25: The discussion of the observed variations in hydraulic head is of interest, and may be necessary in this part in order to explain why they are not being taken at "face value" when evaluating the environmental setting and developing the investigative approach for this OU. However, it seems that developing an "explanation" for data anomalies in this section has led to a presumption that the borehole deviation logging will resolve the issue, and there is no provision for further investigation in the discussion of RI/FS Tasks (Section 5). The first full paragraph on this page begins with a statement that "...positioning of the open interval does not appear to be a problem", then concludes the discussion of the apparent high head at USGS-85 by noting that the completion interval for this well does not cross the water table as do most other well in the area, so this well "could be reflecting higher heads within the aquifer." At best this is poor organization of the presentation, and at worst at complete contradiction within a single paragraph.

A fourth explanation for the variation in hydraulic head is also possible. Data presented in Table 2-2 indicate a wide (4 orders of magnitude) variation in transmissivities measured at wells located near each other. Hydraulic gradients and water levels are affected by variations in transmissivity. Since, according to the text, well plumbness is the primary cause of the apparent area of low hydraulic head (third paragraph), the text should discuss the reason that the anomalously low hydraulic head was not observed at other U.S. Geological Survey wells near the landfills (e.g., 34 through 40). All wells have presumably been installed in a similar manner.

Resolution: Not all wells have been installed in a similar manner. In general, USGS wells that are numbered less than 100 have been drilled using a cable tool rig and wells numbered greater than 100 have been drilled using a rotary rig. Typically, wells drilled with a cable tool rig tend to be more plumb or straighter. Variations in $\mathrm{T}$ can change the gradient but can't create a closed low (gradient reversal). The text will be revised to more clearly present possibilities for the apparent closed low.

13. Page 2-26: The last sentence in the first paragraph presents a "conclusion" based on the assumptions about the well alignments made in the same paragraph. This conclusion needs to be qualified, and until "proved", should not be presented as the only possible explanation.

Resolution: The sentence has been deleted from the text.

It would be appropriate to include in this section some indication of the magnitude of the sources at ICPP and TRA. When the billions of gallons of water discharged at these two sites are considered, it helps put in perspective the potential of these relatively small landfills as a source of the contamination currently found in the aquifer 
Resolution: The text has been revised to indicate the amounts of tritium and chromium disposed to injection wells and infiltration ponds from past disposal practices at ICPP and TRA.

14. Page 2-30: The idea that an SOP approved 1986 "may approximate the previous operational practices" needs to be further developed (if deemed significant for the RI/FS) or dropped completely, as it represents too large a "leap of faith."

Resolution: The paragraph was deleted from the text.

15. Tables 2-3 thru 2-6: There needs to be a discussion of the reliability of the information reported in these tables, as well as an indication of how much research has been done and is planned to further refine the data. It is recommended, for example, that any time the disposal of barrels or other containers is recorded, the records be searched to find their source, condition and contents. The fact that the reporting system may have required the disposal reported in Table $2-5$ to be recorded in cubic meters rather than liquid measure may have resulted in reporting errors. If the numbers are accurate, these quantities are rather large and information on disposal methods should be developed as input for fate and transport models.

Resolution: The tables have been revised and expanded as possible. Proper units have been included. The information in the tables are useful for providing information on the general types of wastes most commonly disposed of to the landfills. However, the quantities may be over or under the actual volume of waste disposed to the landfills as they were not generally weighed prior to disposal. Attempts to verify this information have not been successful, but will be continued throughout the RI.

16. Significant information in Table 3-1 needs to be discussed further in the text. For example, item V. C. indicates that the uppermost interbed was sampled, but samples were not analyzed. This interbed is a critical element of the subsurface investigation. If the samples were lost/discarded, or have an uncertain chain of custody, they may be of little value. However, if the samples are still exist they may still be usable. Also, data collected from drilling and sampling wastes (item VI in the table) are referenced in the table but are not discussed in the text. These data should be presented and discussed.

Resolution: The samples have been discarded and therefore will be of little or no value. Drilling and sampling wastes were collected for IDW disposition and analyzed using TCLP methods. All wastes were found to be nonhazardous and in compliance with RCRA requirements for land disposal.

17. Page 3-7: OHSA standards can be of value when discussing relative risk concepts, and may help screen contaminants of concern at a site, but they cannot and will not be used as a "stand-alone" substitute for CERCLA risk assessments. 
Resolution: This preliminary evaluation is a review of existing information to demonstrate the potential for risks to human health and the environment. The intent of the evaluation is not to be used as a stand-alone substitute for CERCLA risk assessment. The RI field activities proposed in this Work Plan will support data requirements for the CERCLA risk assessment to be performed in the RI.

18. Page 3-9: The sentence starting with "Table 3-3..." at the top of the page is a duplication of the previous sentence.

Resolution: The text has been deleted.

The statement that "It is interesting to note that five of the above mentioned compounds were found in one borehole..." leads to the question of what are the implications of this information, and what action does it indicate should be taken during the investigation.

Resolution: The implication of this statement is that additional surface soil sampling is required at this particular location and the Work Plan identifies the need for additional surface soil sampling at both landfills. Additionally, subsurface samples will also be collected at Landfill II per recent scoping meeting.

In the last paragraph, recommend replacing the term "safe risk-based levels", as many people interpret "safe" as zero risk. The discussion of chromium would be more complete if either a bounding condition of $100 \%$ chromium VI, or some other (conservative) assumed speciation was evaluated. This may also allow a conclusion to be reached about the cumulative risk, as well as the risk from individual contaminants.

Resolution: The word "safe" has been deleted from the text. A conservative assumption of $100 \% \mathrm{Cr}^{6+}$ will be made for this presentation.

19. Page 3-11: Because the recent weather patterns in the INEL area have been atypical, and the 1993 snowmelt may present a more nearly normal condition at the site, this part should include a discussion of how significant the differences would be. It is likely that such an analysis would lead to additional data collection, which would need to take place in tine very near future. This will probably provide more useful information than the analysis of the old data which was collected under atypical conditions (as discussed on page 3-12).

Resolution: RI activities have been revised to include the collection of additional moisture monitoring data from existing neutron access tubes, as well as evaluating existing data.

Table 3-4 presents risk-based screening concentrations for contaminants detected in soil. The table states that no health data are available for 4-methylphenol. Appendix II of the Region 10 supplemental risk assessment guidance for Superfund presents a risk-based concentration for 4-methylphenol of $1,000 \mathrm{mg} / \mathrm{kg}$, corresponding to a hazard quotient of 0.1 . This discrepancy should be corrected.

Resolution: Table II-2 has been revised according to the new health data available for this compound. 
20. Page 3-13: There is a very limited rationale presented for suspecting the sampling pumps are a significant source of particulate chromium. This should be viewed as an incidental portion of the overall investigation, with more emphasis given to the last full paragraph on this page.

Resolution: The text will be revised to indicate that the pumps may be an additional yet probably subordinate source of chromium.

21. Page 3-14: Delete "the presence" from last sentence of the first full paragraph.

Also, confirm that Arco is closer to this site than Atomic City.

Resolution: The phrase "the presence" has been deleted from the text. Atomic city $7 \mathrm{mi}$ southeast of CFA has also been added to the text.

22. Page 3-15: This table presents the preliminary conceptual model for the CFA landfills II and III. INEL worker exposure to soil via ingestion should be included in the model. Residential exposure to groundwater via dermal exposure is also a consideration, although dermal exposure for most contaminants will be of minor consequence when compared with ingestion. In addition, based on surface soil results (and the sparse cover), potential inhalation of fugitive dust may also need to be considered.

Resolution: Incidental soil ingestion and inhalation have been added to the industrial scenario.

23. Page 3-16: The fact that tritium has been found in the CFA drinking water above the MCL is a issue that should be closed out in this document. A statement which explains how this concern is being addressed/investigated is needed.

Resolution: The tritium plume in the groundwater is due to past disposal practices at TRA and ICPP and is not considered part of this operable unit as stated in the text. Due to the complexity of groundwater flow and extent of the plume this problem will be further investigated and resolved under the INEL site-wide WAG-10.

24. Table 3-8: Confirm that DOE Order 5480.1B is correctly identified as having two different titles.

Resolution: The table has been revised to correctly identify the DOE orders 5480.B, 5482.1B, and 5480.10.

25. General: The rationale for the performance of the RI/FS should include clearly explain how data gaps are being addressed. If data gaps are not resolved, additional activities or the scope of planned activities need to be expanded. For example, past sampling indicates that methylene chloride may have been released to landfill soils, and the extent of this contamination needs to be addressed. The reliability of the sedimentary interbed (thought to be approximately 110 feet below the landfills) preventing downward contaminant migration needs to be further evaluated. Data only suggest that this interbed is present beneath the landfills (page 2-16 of the work plan). This evaluation may be especially important for non- 
aqueous phase liquids (NAPL) that are suspected to have been placed in the landfills. (some bounding calculations could reduce the significance of the NAPLs as contaminants of concern) The monitoring well network downgradient of the landfills ends at approximately each landfill's boundary. Since leachate and NAPLs from the landfills may move both horizontally and vertically through the vadose zone, depending upon the distribution of sedimentary interbeds beneath the release point, there needs to be an explanation of why these possibilities are not being investigated under this RI.

Resolution: Text has been incorporated to include data gaps and needs.

26. Table 4.1: Apparently the correct entry for the first "Action" for the Geophysical Survey of Landfill III includes "magnetics."

Resolution: The text has been revised using the correct spelling.

The "Action" to "Survey local areas for appropriate material" should be limited until there is more evidence that there will be a need to design and construct a cover. (Note that there is support for the added efficiency of collecting opportunity samples where there is a reasonable likelihood they will be necessary)

Resolution: This action has been deleted from the Work Plan.

27. Table 4-3: This table presents the data quality objectives. No semivolatile organic compound (SVOC) analyses are proposed for the surface soil or groundwater samples. SVOC (benzoic acid and 4-methylphenol) were detected in previous subsurface soil samples. Also, according to the work plan, approximately 14 million gallons of waste including waste oil, solvents, chemicals and paint were disposed of in the landfills. It is therefore appropriate to analyze for SVOC in groundwater and surface soils.

Resolution: Semivolatile organic analyses of the surface soil will be added to the Work Plan. A review of five quarters of existing groundwater data (Appendix C) shows no SVOCs other than a few detections of phthalate, which may be attributable to laboratory contamination. Based on scoping meetings, it has been agreed that groundwater samples for metals, VOCs, common anions and alkalinity will be collected for analysis.

28. Page 4-4: As with the second comment for Table 4-1, the EDA data collection should be limited during the RI.

Resolution: The data collection for EDA is limited during the RI; however if a task fulfills several objectives including EDA it will be noted.

29. General: The descriptions of RI/FS tasks need to include the data quality requirements of the end use(s) of the data and the way in which the RI/FS will meet those requirements. It is essential that the collected data be compatible with its ultimate use including level of accuracy and precision. Data collected during this RI/FS will mainly be used to model contaminant migration. The chcice of hydrogeologic models that will be used must be made early enough in the RI/FS to allow for any change(s) to the data acquisition activities. Depending on the 
chosen model, may also require parameter estimates of unsaturated hydraulic conductivity, dispersivity, and sorptive properties. Data acquisition activities related to these parameters are not presently addressed in the RI/FS work plan.

Resolution: The analytical levels and data quality requirements of the identified RI tasks are provided in Section 4.0. Input parameters to potential models will rely on the RI collected data, other data collected specifically for the INEL, and/or the literature.

There are also numerous instances of statements such as "sites will be selected" for sampling, or "the borehole and cuttings will be surveyed periodically..."; there should be specific criteria provided within the work plan for such decisions.

Resolution: When appropriate the text will be revised to be more specific.

30. Page 5-3: Units of measurement are expressed in the Metric system in this section and in the English system in other sections. The type of unit should be used consistently throughout the work plan.

Resolution: The units expressed in text will be consistent throughout the document and will be presented as follows: English unit (metric unit).

31. Page 5-9: The discussion of background sample locations needs to be expanded. If the background levels established in the Track 1 Guidance document are not going to be used, the location of the sample points will require consideration of other potential impacts from site operations, and can not be limited to the "influence of the landfills."

Resolution: Background levels established in the Track 1 guidance will be used.

32. Page 5-10: The locations of the soil cover borings or the rationale for selecting the locations in the field should be discussed in this section. Details for field personnel should be placed in the FSP, and the person(s) making the location decisions should be identified.

Resolution: A total of 12 samples will be collected at a distribution of one per acre. A visual inspection of the surface soil will be made for evidence of soil differences. If differences are found a sample will be obtained from the different soils. If no differences are found, samples will be collected randomly. Determination of differences will be made by soil scientist from Applied Geosciences Unit.

33. Page 5-11: In the second paragraph, the text notes that soil cover samples will be analyzed for saturated hydraulic conductivity. Flow may occur under unsaturated conditions, and the computer model used to determine contaminant transport may require an estimate of unsaturated hydraulic conductivity. Since the model input requirements are unknown at this time, unsaturated hydraulic conductivity estimates should also be determined. This comment also applies to deeper soil horizons. 
Resolution: The HELP code, which will be used to evaluate soil cover performance, requires saturated hydraulic conductivity values (not unsaturated hydraulic conductivity values) as input. The hydraulic conductivity-moisture content (or capillary head) relationship can be obtained from the soil capillary pressure-moisture content relationship which will be determined for the soil cover samples. This is a common technique to obtain unsaturated hydraulic conductivity-moisture content relationships.

34. Page 5-12: The discussion of groundwater sampling should be revised to reflect the results of the meeting between the WAG managers and the DOE consultants on 3/1/93.

Recommend adding the CFA production wells to the network of wells which will be sampled.

Resolution: The text will be revised as suggested.

35. Page 5-17: The text indicates that the release rate of leachable contaminants $1:$ controlled by a number of factors and processes. Site data related to several of the processes ( $\%$.g., diffusion, dispersion, sorption) are not discussed in the work plan, and plans to obtain those data are not presented. This apparent data gap should be addressed.

Resolution: The test has been revised to indicate that GW screen will be used to model leachable contaminants and that RI collected data, existing information and standard default parameters will be used. The amount of water (from infiltration) reaching the waste and generating leachate will be determined from soil cover inodeling using the HELP code. Data used as input to the HELP code will be collected during the RI field investigation.

Describing (modeling) the leachate as it moves downward out of the landfill and into the underlying basalt is difficult due to the heterogeneity of the basalt. Due to the heterogeneity of the basalt, it is doubtful that laboratory studies to measure these parameters would be relevant (particularly regarding scale of experimental cores versus field processes). Factors and processes relevant to modeling contaminant migration through basalt will be based on past observation of contaminant migration at the INEL and other sites and on data in the literature (i.e., Track 1 Guidance Document).

36. Page 5-18: Revise the conditional "could" to "will", in the last sentence of part 5.6.2.3. The text notes that NAPLs may have been disposed of at the landfills and that data obtained from the RI/FS will be used to determine their presence. No chemical analysis of soils is planned for the RI/FS, but the NAPLs would be detectable in soils before they are detectable in the water table. The data that will be used should be explained.

Resolution: Soil samples will be collected from the cover and subsurface for volatile organics, semivolatile organics, and metal analyses.

37. Page 5-27: See comment \#1 for Section 1, regarding NEPA.

In the last sentence of the first paragraph under 5.12, change the term "public relations" to "public involvement" or a similar term. Community relations under CERCLA is more interactive than is usually implied by "public relations." 
Resolution: The text has been revised as suggested above.

Recommend clarifying the roles of all three agencies in the RD/RA process, rather than using the vague "in accordance with the FFA/CO."

Resolution: The text has been revised.

38. Update the schedule to reflect the 3/1/93 discussion of ground water sampling.

Resolution: The schedule has been updated to reflect the above mentioned discussion and for consistency with the text.

39. Identify Primary and Secondary documents, and enforceable deadline, if any. (Am not certain of any value in predicting a schedule beyond the submittal date for the draft ROD)

Resolution: The schedule will be revised as suggested.

\section{Field Sampling Plan}

40. In general the FSP is well prepared and presents the necessary information for the performance of data acquisition activities. However, many of the inadequacies of the work plan discussed above have been incorporated into the FSP and should be addressed in both, as necessary.

Resolution: The text will be revised accordingly based on previous comments.

41. The method used by field personnel to select a sampling location is not adequately described. Also, the person or persons who will select the sampling locations need to be identified. SOPs in the FSP provide some guidance (e.g., Project Geophysicist for geophysical surveys), but this information is lacking for the soil sampling activities. An SOP for soil sampling was not included in the FSP.

Resolution: The field team leader is responsible for sample selection under the direct guidance of the soil scientist. The SOPs will be included in the FSP.

42. Section 2.2.4, page 2-6, second paragraph - The paragraph discusses the information to be generated from the geophysical surveys. Any information on the distribution of the perched aquifer in the landfill should be added to this section.

Resolution: There is no indication of perched water at the landfills.

43. Section 2.2.5, page 2-6, last paragraph - The last sentence of the paragraph states that the soil-gas survey will be used to provide information for the baseline risk assessment. This information is not usable to compute an RME in the baseline risk assessment, and the text should be clarified. 
Resolution: The text has been revised to state the data will be used to provide information for the contaminant fate and transport in support of the baseline risk assessment.

44. Section 3.1, page 3-1, fourth paragraph; Section 5.2.2, page 5-3, second paragraph - The text should describe the decision process field personnel will used to randomly select sampling stations and identify the person(s) who will select sampling locations.

Resolution: Text has been added to provide more information on sampling requirements and responsible personnel.

45. Section 3.1, page 3-1, last paragraph - The paragraph discusses the soil investigation of the landfill cover material on Landfill III. Some discussion of acceptable criteria for cover material should be included.

Resolution: Text has been added to provide more details on sample site selection requirements.

46. Section 3.7, page 3-20, first bullet - The text indicates that trip blanks will be collected at a minimum frequency of one per cooler when volatile organic compound samples are being collected. The quality assurance project plan (Section 11.2) indicates that the blanks will be collected a minimum frequency of one per sampling episode. This inconsistency should be explained or corrected.

Resolution: Trip blanks will be collected at a rate of one per cooler for VOAs and the text will be revised to clearly state this.

47. Section 3.7, page 3-21, last paragraph - The last sentence of this paragraph is incomplete and should be corrected.

Resolution: The error has been corrected.

48. Section 5.2.1, page 5-2, first paragraph - The text indicates that the 12 soil cover sampling stations have been randomly selected. The tentative locations of these stations should be shown on a map. This statement conflicts with sections 3.1 of the FSP and 5.3.5.1 of the work plan, which indicate the stations will be selected in the future. The inconsistency should be explained or corrected.

Resolution: The text has been revised to indicate "the samples will be randomly selected" and provide consistency throughout the document.

49. Section 5.7.1, page 5-9, second paragraph - The text indicates that color will be used as a parameter to evaluate sufficient well development. SOP 11.8 indicates that dissolved oxygen will be used as a parameter. This inconsistency needs to be explained or corrected. Turbidity is measurable in the field and is a good indicator of well development. It is recommended that this parameter be used instead of color or dissolved oxygen because it is more reliably measured (EPA 1986a). 
Resolution: The text has been revised to be consistent with the procedures outlined in the SOP. Groundwater collected beneath the INEL is very low in turbidity, therefore dissolved oxygen is better indicator of well development.

50. Section 5.8, page 5-11 - Disposal of personal protection equipment is not discussed in the section and should be included. The rationale for selecting a portable treatment unit or discharge to the waste pond should be described. The text should explain the procedure for disposing of tritium-contaminated water that cannot be treated. An SOP for the operation of the treatment unit, including instructions for testing to ensure proper operation, should be provided.

Resolution: A detailed IDW plan has been added to the work plan.

51. Section 6.2, page 6-7, Table 6-2 - Shelby tubes should be capped and sealed on each end to prevent loss of moisture and sample material and to minimize disturbance of the sample. These details should be included in an SOP.

Resolution: Intact soil core samples will be collected using a core soil sampler (not Shelby tube) following ASTM D-1587 which covers preparation (sealing) of core sample for shipment. A copy of this standard is included in Appendix B of the FSP.

52. Appendix B - SOPs for collecting surface and cover soil samples and for performing borehole deviation logging are missing and should be included.

Resolution: These SOPs will be added to the document.

\section{References}

EPA, 1986a, RCRA Groundwater Monitoring Technical Enforcement Guidance Document, OSWER-9950-1, U.S. Environmental Protection Agency, September 1986.

EPA, 1986b, Quality Criteria for Water, Office of Water Regulations and Standards, EPA 440/586-001, U.S. Environmental Protection Agency, May 1986.

EPA, 1989, Risk Assessment Guidance for Superfund, Volume 1, Human Health Evaluation Manual (Part A), EPA/540/1-89/002, U.S. Environmental Protection Agency, December 1989.

EPA, 1991, Supplemental Risk Assessment Guidance for Superfund, U.S. Environmental Protection Agency, August 1991.

EPA Region 10, 1991, Supplemental Guidance for Superfund Risk Assessments in Region 10, ES-098, U.S. Environmental Protection Agency, August 1991.

EPA, 1992, Final Guidance for Data Useability in Risk Assessment (Part A), OSWER Directive 9285.7-09A, U.S. Environmental Protection Agency, April 1992.

Freeze, R.A. and J.A. Cherry, 1979, Groundwater, Prentice-Hall, Inc., Englewood Cliffs, New Jersey. 


\title{
Remedial Investigation/Feasibility Study Addenda for Operable Unit 4-12: Central Facilities Area Landfills II and III at the Idaho National Engineering Laboratory
}

Published July 1993

\author{
Idaho National Engineering Laboratory \\ EG\&G Idaho, Inc. \\ Idaho Falls, Idaho 83415
}

Prepared for the

U.S. Department of Energy

Assistant Secretary for Environmental Restoration and Waste Management Under DOE Idaho Operations Office

Contract DE-AC07-76ID01570 


\section{CONTENTS}

RI SAMPLING AND ANALYSIS PLAN

Part I-Field Sampling Plan

Part II-Quality Assurance Project Plan

HEALTH AND SAFETY PLAN

COMMUNITY RELATIONS PLAN 
Remedial Investigation Sampling and Analysis Plan Part I-Field Sampling Plan for Operable Unit 4-12: Central Facilities Area Landfills II and III at the Idaho National Engineering Laboratory

Rev. 0

July 1993

Idaho National Engineering Laboratory

EG\&G Idaho, Inc.

Idaho Falls, Idaho 83415

Prepared for the

U.S. Department of Energy

Assistant Secretary for Environmental Restoration and Waste Management Under DOE Idaho Operations Office

Contract DE-AC07-76ID01570 


\section{Remedial Investigation Sampling and Analysis Plan \\ Part I-Field Sampling Plan \\ for Operable Unit 4-12: \\ Central Facilities Area Landfills II and III \\ at the Idaho National Engineering Laboratory}

Rev. 0

July 1993

Reviewed by:

$\frac{\text { Shea }}{\text { J.J.Shea, Chairman, ER Independent Review Committee }}$
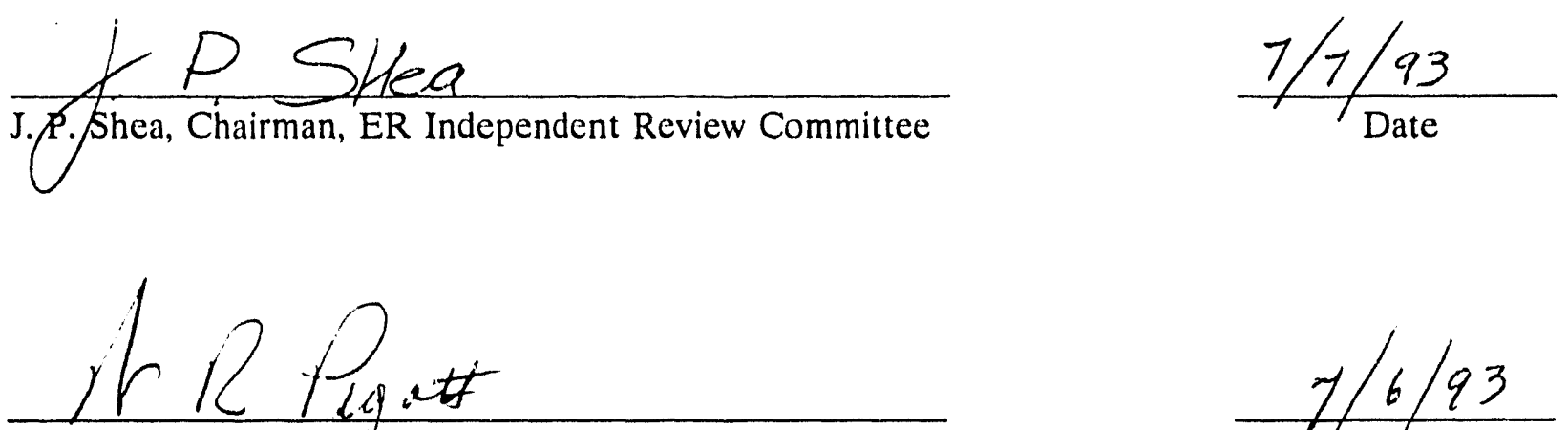

W. R. Pigott, Manager. WAG 4

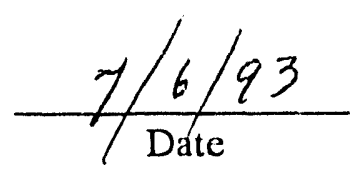

KP Shlea for W.N. Wyland

W. N. Wyland, Landlord, Central Facilities Area

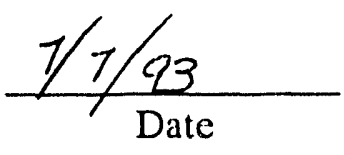

Approved by:

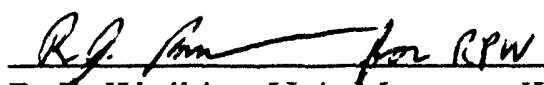

R. P. Wadkins, Unit Manager, WAGs 1, 4, and 5
$7 / 6 / 93$

Date 


\section{CONTENTS}

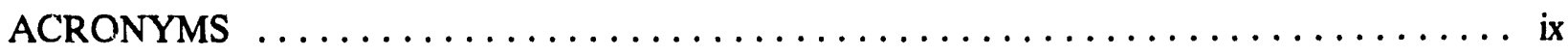

1. INTRODUCTION AND BACKGROUND $\ldots \ldots \ldots \ldots \ldots \ldots \ldots \ldots \ldots \ldots \ldots$

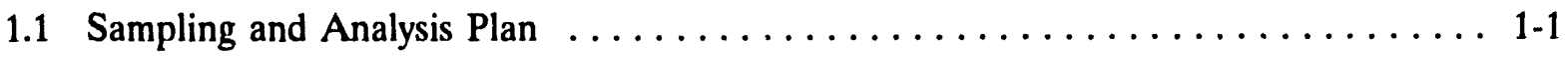

1.2 Project Area Background and Location $\ldots \ldots \ldots \ldots \ldots \ldots \ldots \ldots \ldots \ldots \ldots \ldots \ldots \ldots$

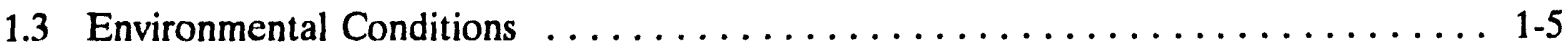

1.4 Project Organization and Responsibility $\ldots \ldots \ldots \ldots \ldots \ldots \ldots \ldots \ldots \ldots \ldots$

2. SAMPLING OBJECTIVES AND MEDIA SPECIFIC RATIONALE $\ldots \ldots \ldots \ldots . \ldots 2-1$

2.1 Data Needs and Data Objectives $\ldots \ldots \ldots \ldots \ldots \ldots \ldots \ldots \ldots \ldots \ldots \ldots \ldots \ldots \ldots \ldots \ldots$

2.1.1 Data Needs .............................. 2-1

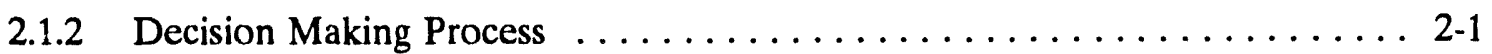

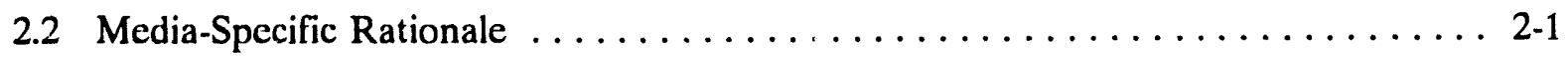

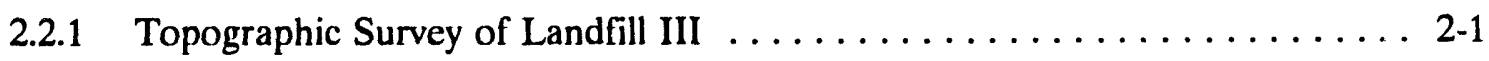

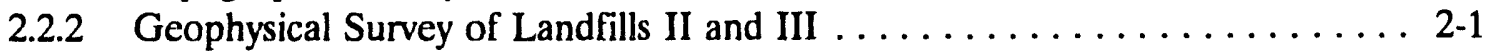

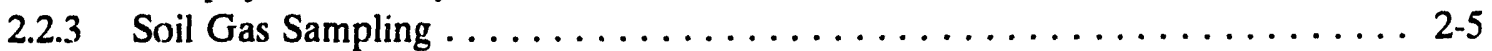

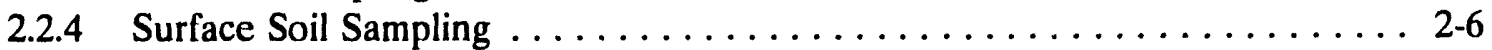

2.2.5 Cover Characterization of Landfill III $\ldots \ldots \ldots \ldots \ldots \ldots \ldots \ldots \ldots \ldots \ldots$

2.2.6 Water Level Measurements and Borehole Deviation Logging . . . . . . . 2-6

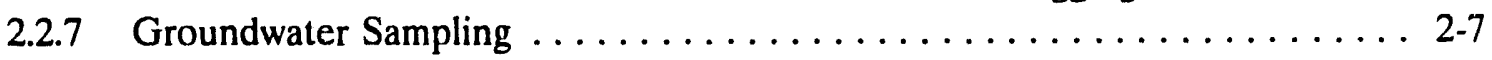

2.2.8 Subsurface Soil Sampling of Landfill II $\ldots \ldots \ldots \ldots \ldots \ldots \ldots \ldots \ldots \ldots$

3. SAMPLING LOCATION, FREQUENCY, AND SAMPLING/

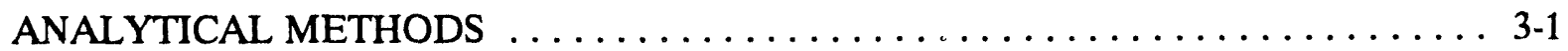

3.1 Topographic Survey of Landfill III $\ldots \ldots \ldots \ldots \ldots \ldots \ldots \ldots \ldots \ldots \ldots \ldots \ldots \ldots \ldots$

3.2 Geophysical Survey of Landfills II and III $\ldots \ldots \ldots \ldots \ldots \ldots \ldots \ldots \ldots \ldots \ldots \ldots$

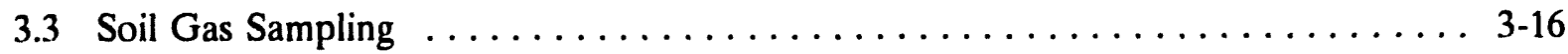

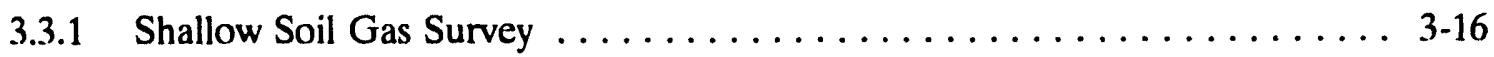

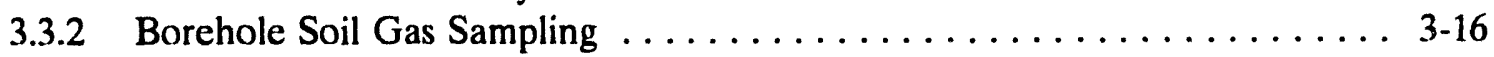

3.3.3 Surface-Flux Chamber Sampling $\ldots \ldots \ldots \ldots \ldots \ldots \ldots \ldots \ldots \ldots \ldots . \ldots \ldots$

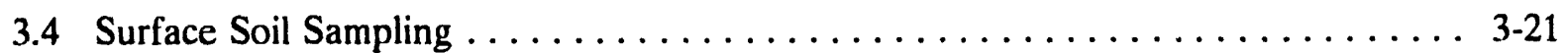


3.5 Cover Characterization of Landfill III $\ldots \ldots \ldots \ldots \ldots \ldots \ldots \ldots \ldots \ldots \ldots \ldots \ldots \ldots$

3.6 Water Level Measurements and Borehole Deviation Logging . . . . . . . . . 3-22

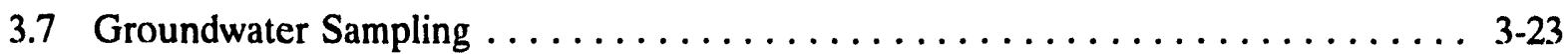

3.8 Subsurface Soil Sampling of Landfill II $\ldots \ldots \ldots \ldots \ldots \ldots \ldots \ldots \ldots \ldots \ldots \ldots \ldots$

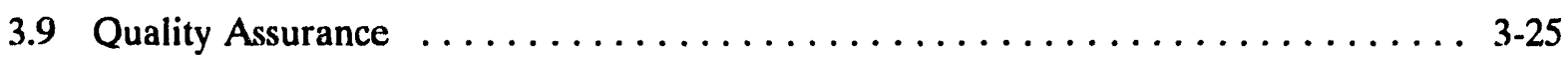

4. SAMPLE IDENTIFICATION SYSTEM $\ldots \ldots \ldots \ldots \ldots \ldots \ldots \ldots \ldots \ldots \ldots \ldots$

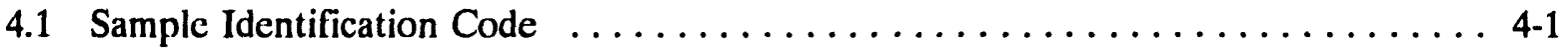

4.2 Sampling and Analysis Plan Table/Data Base $\ldots \ldots \ldots \ldots \ldots \ldots \ldots \ldots \ldots \ldots \ldots$

4.2.1 General . . . . . . . . . . . .

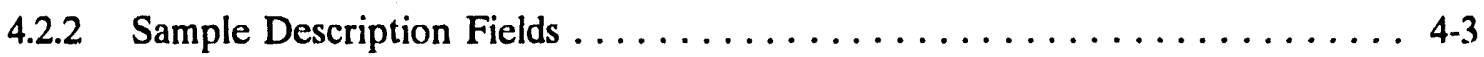

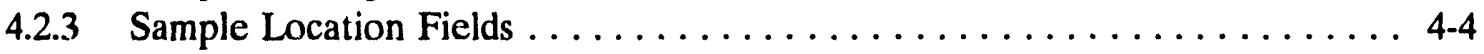

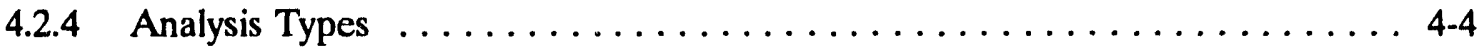

5. SAMPLING EQUIPMENT AND PROCEDURES $\ldots \ldots \ldots \ldots \ldots \ldots \ldots \ldots \ldots$.1

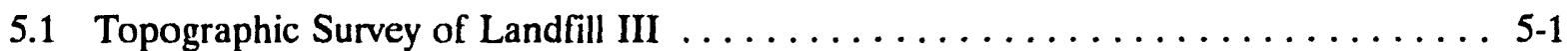

5.2 Geophysical Survey of Landfills II and III ............... 5 .1

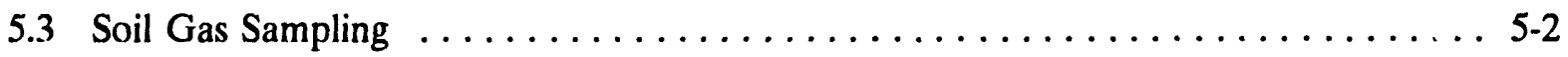

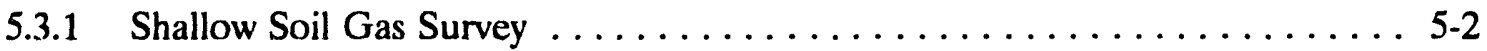

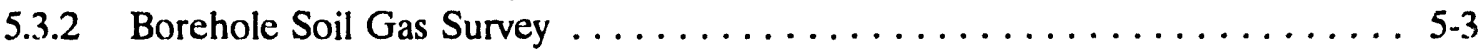

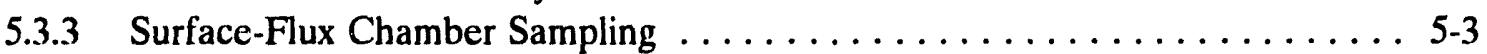

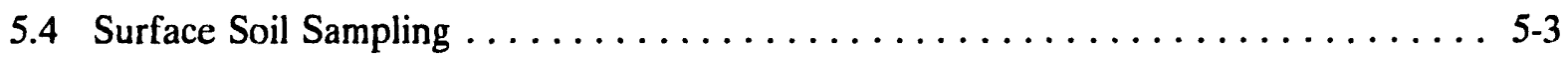

5.5 Cover Characterization of Landfill III $\ldots \ldots \ldots \ldots \ldots \ldots \ldots \ldots \ldots \ldots \ldots \ldots \ldots$

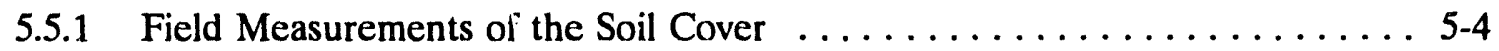

5.5.2 Collection of Soil Samples for Laboratory Analysis . . . . . . . . . 5-5

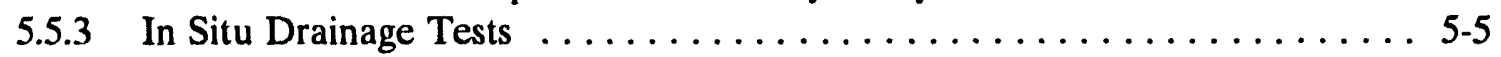

5.6 Water Level Measurements and Borehole Deviation Logging . . . . . . . . . 5-6

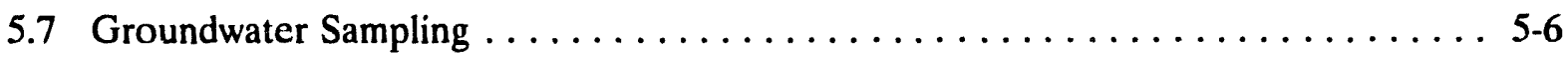

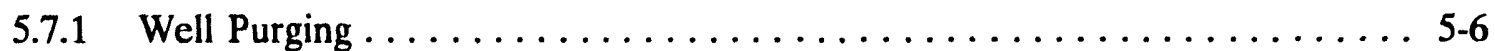

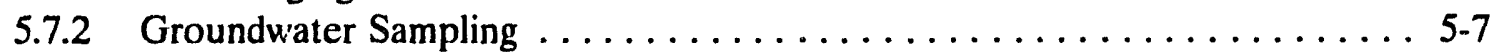


5.8 Subsurface Sampling of Landfill II $\ldots \ldots \ldots \ldots \ldots \ldots \ldots \ldots \ldots \ldots \ldots$

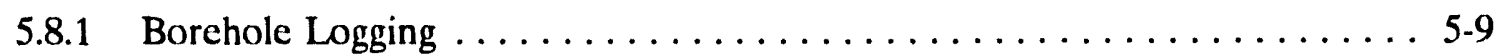

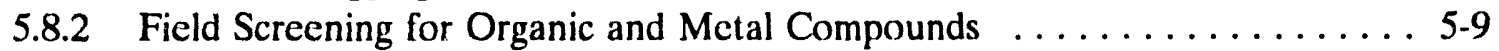

5.8 .3 Soil Sampling for Organic and Metal Compounds ............. 5-12

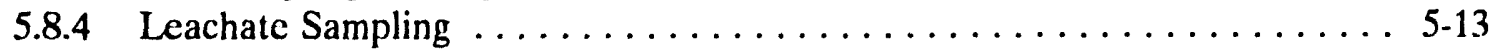

5.8 .5 Leachate Wells ... . . . . . . . . . . . . . . . . . . 5-13

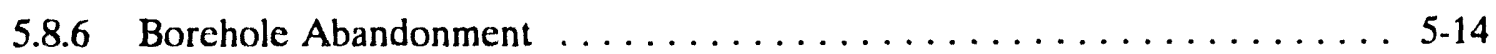

5.9 Handling and Disposition of Investigation-Derived Waste $\ldots \ldots \ldots \ldots \ldots \ldots$.14

6. DOCUMENT MANAGEMENT AND SAMPLE CONTROL $\ldots \ldots \ldots \ldots \ldots \ldots \ldots$ 6-1

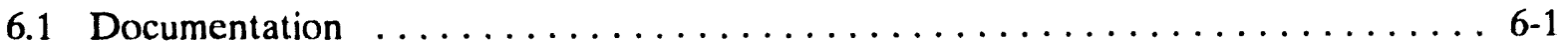

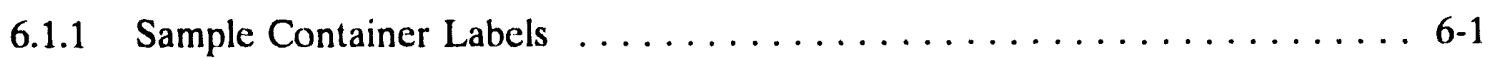

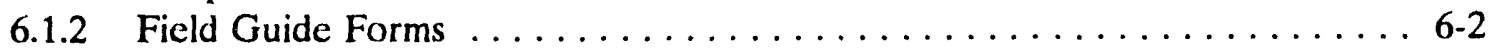

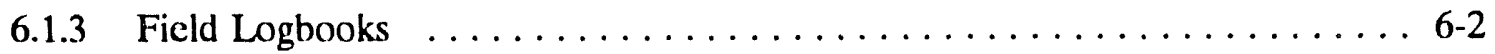

6.1 .4 Data Sheets ......................... 6-2

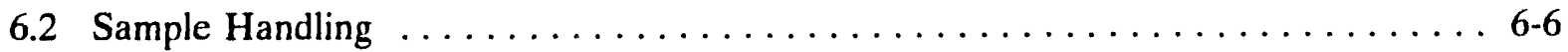

6.2.1 Sample Preservation . . . . . . . .

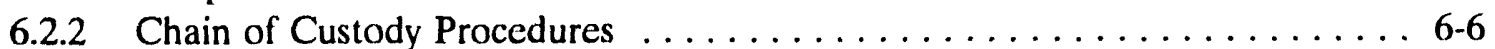

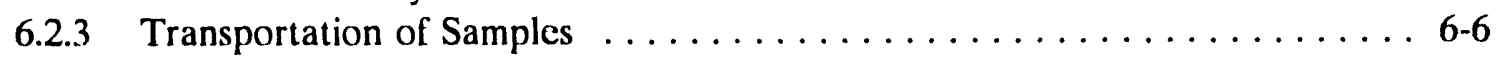

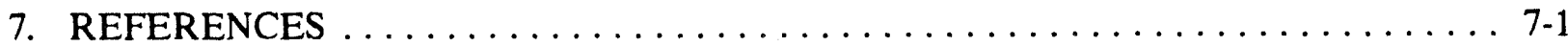

\section{FIGURES}

1-1. Map of Idaho showing the location of the INEL, Snake River Plain, and generalized groundwater flow lines of the Snake River Plain Aquifer $\ldots \ldots \ldots \ldots \ldots \ldots \ldots \ldots$

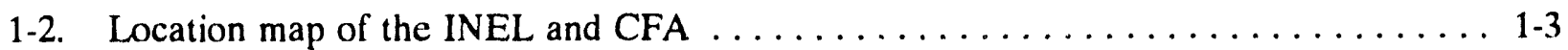

1-3. Location map of CFA Landfills II and III $\ldots \ldots \ldots \ldots \ldots \ldots \ldots \ldots \ldots \ldots \ldots \ldots \ldots$

1-4. Organization chart for the CFA Landfills II and III Operable Unit $\ldots \ldots \ldots \ldots$

3-1. Geophysical survey grids for (a) seismic refraction, (b) induced electromagnetic measurements, and (c) magnetic field measurements $\ldots \ldots \ldots \ldots \ldots \ldots \ldots \ldots$

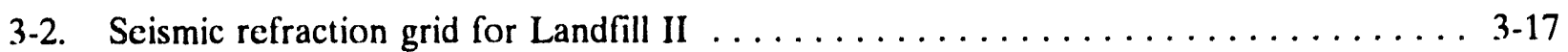

3-3. Shallow soil gas sample locations for Landfill II $\ldots \ldots \ldots \ldots \ldots \ldots \ldots \ldots$

3-4. Shallow soil gas sample locations for Landfill II . . . . . . . . . . . . 3-19 
3-5. Location of boreholes to be used in borehole gas sampling .

3-6. Locations of CFA monitoring wells for water level measurements and groundwater

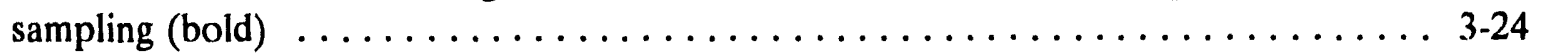

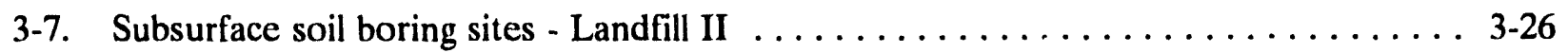

5-1. Schematic of the subsurface sampling program $\ldots \ldots \ldots \ldots \ldots \ldots \ldots \ldots \ldots \ldots$

5-2. Generalized logic for VOC analysis sampling $\ldots \ldots \ldots \ldots \ldots \ldots \ldots \ldots \ldots \ldots$

6-1. Sample container label $\ldots \ldots \ldots \ldots \ldots \ldots \ldots \ldots \ldots \ldots \ldots \ldots \ldots \ldots \ldots \ldots \ldots$

6-2. Example of a sample field guide form $\ldots \ldots \ldots \ldots \ldots \ldots \ldots \ldots \ldots \ldots \ldots$

6-3. Example of a lithologic $\log \ldots \ldots \ldots \ldots \ldots \ldots \ldots \ldots \ldots \ldots \ldots \ldots \ldots \ldots$

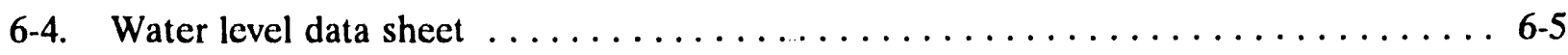

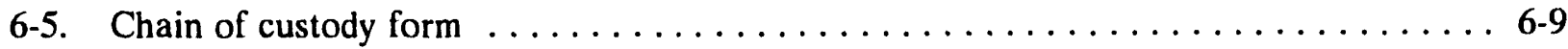

6-6. Packaging of environmental samples for shipment to the analytical laboratory ..... 6-10 TABLES

2-1. Data quality objectives summary for CFA Landfills II and III RI/FS $\ldots \ldots \ldots \ldots$

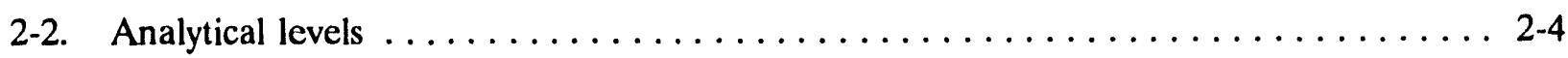

3-1. Summary of location, media, sample type, and analysis--soil cover investigation . . . . 3-2

3-2. Summary of location, sample type, and analysis - groundwater, soil and soil gas . . . 3-3

3-3. QA/QC samples for groundwater, surface soil, and soil gas $\ldots \ldots \ldots \ldots \ldots \ldots$

3-4. Laboratory analytical protocols/chemical analytes for groundwater samples . . . . . 3-6

3-5. EPA Method 601 target analyte list and method detection limits $\ldots \ldots \ldots \ldots .7$

3-6. Contract required quantitation limits for volatile organics $\ldots \ldots \ldots \ldots \ldots \ldots \ldots$

3-7. Contract required quantitation limits for semivolatile organics $\ldots \ldots \ldots \ldots \ldots \ldots$

3-8. Contract required detection limits for metals $\ldots \ldots \ldots \ldots \ldots \ldots \ldots \ldots \ldots \ldots$

3-9. EPA Method 524.2 (Rev. 3.0) target analyte list and method detection limits ... . . 3-14 
3-10. Wells to be measured on a monthly basis for water level data

3-11. Monitoring wells to be sampled

3-12. Approximate coordinate locations of the landfill boreholes

4-1. Example of a sampling and analysis plan table for chemical analysis

5-1. Preliminary screening for OU 4-12 soil sample investigation-derived waste

5-2. Preliminary screening for SRPA well purge and decontamination water investigation-

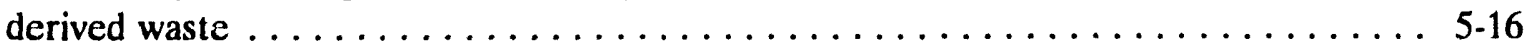

5-3. Preliminary screening for OU 4-12 water sample investigation-derived waste $\ldots \ldots 5-18$

6-1. Typical sample requirements-aqueous samples $\ldots \ldots \ldots \ldots \ldots \ldots \ldots \ldots \ldots$ 6-7

6-2. Typical sample requirements-chemical, physical, and hydraulic propertics-soil samples . 6-8 
$\bullet$ 


\title{
ACRONYMS
}

\author{
ARDC Administrative Records and Document Control \\ ASTM Amcrican Socicty for Testing and Matcrials \\ bls below land surface \\ CERCLA Comprehensive Environmental Response, Compensation, and Liability Act \\ CFA Central Facilitics Area \\ CFR Code of Federal Regulations \\ CLP Contract Laboratory Program \\ COC Chain of Custody \\ DMP Data Management Plan \\ DOE U.S. Department of Energy \\ DOT U.S. Department of Transportation \\ DQOs data quality objectives \\ ECD electron capture detector \\ EDA Engineering Design of Alternatives \\ EPA U.S. Environmental Protection Agency \\ ERAA evaluations of remedial action alternatives \\ ER Environmental Restoration \\ ESRP Eastern Snake River Plain \\ FFA/CO Federal Facility Agreement/Consent Order \\ FID flame ionization detector \\ FSP Ficld Sampling Plan \\ GC gas chromatograph \\ $\mathrm{HNu}$ photoionization detector \\ ICPP Idaho Chemical Processing Plant \\ IDHW Idaho Department of Health and Welfare \\ IDW investigation-derived waste \\ INEL Idaho National Enginecring Laboratory \\ MAC maximum allowable concentration \\ NCP National Contingency Plan
}


OSHA Occupational Safety and Health Association

OU operable unit

PD program directive

QAPjP Quality Assurance Project Plan

QA/QC quality assurance/quality control

QC quality control

RA remedial action

RI remedial investigation

RI/FS remedial investigation/feasibility study

SAP Sampling and Analysis Plan

SC site characterization

SOP standard operating procedure

SOW statement of work

SVOC semivolatile organic compound

TRA Test Reactor Area

USGS United States Geological Survey

VOA volatile organics analysis

VOC volatile organic compound

WAG waste area group 


\section{Remedial Investigation Sampling and Analysis Plan Part I-Field Sampling Plan for Operable Unit 4-12: Central Facilities Area Landfills II and III at the Idaho National Engineering Laboratory}

\section{INTRODUCTION AND BACKGROUND}

\subsection{Sampling and Analysis Plan}

The purpose of this Sampling and Analysis Plan is to guide the collection and analysis of samples for the remedial investigation of Central Facilities Area (CFA) Landfills II and III. This investigation has been chosen to facilitate data development at this operable unit pursuant to a Federal Facility Agreement/Consent Order (FFA/CO) between the Department of Energy (DOE), the Environmental Protection Agency (EPA), and the State of Idaho, which will guide the overall Comprehensive Environmental Response, Compensation, and Liability Act (CERCLA) response at the Idaho National Engineering Laboratory (INEL).

The Sampling and Analysis Plan consists of three parts: the Field Sampling Plan (FSP), the Quality Assurance Project Plan (Q.APjP), and the Data Management Plan (DMP). These plans have been prepared pursuant to the National Contingency Plan (NCP) (EPA 1990) and guidance from the EPA on the preparation of sampling and analysis plans. The FSP describes the field activities that will occur as part of the remedial investigation, and the QAPjP describes the processes and programs that will be used to ensure the data generated will be suitable for their intended uses. The guiding DMP for the RI/FS will be the Data Management Plan for the EG\&G Idaho Environmental Restoration Department (EG\&G Idaho 1992) and is incorporated herein by reference.

\subsection{Project Area Background and Location}

The INEL is located approximately $42 \mathrm{mi}(68 \mathrm{~km})$ west of Idaho Falls, Idaho, and occupies $890 \mathrm{mi}^{2}\left(2,304 \mathrm{~km}^{2}\right.$ ) (Figure 1-1). The work to be performed will be conducted at the Central Facilities Area Landfills II and III, located in the south-central portion of the INEL (Figures 1-2 and 1-3). CFA landfills are located approximately $0.5 \mathrm{mi}(0.8 \mathrm{~km})$ from CFA proper. The landfills are to be remediated through the CERCLA-driven remedial investigation/feasibility study (RI/FS) process.

Landfill II, operational from 1970 to 1982 , covers approximately 14 acres (5.6 hectares) and has a maximum depth of $15 \mathrm{ft}(5 \mathrm{~m})$. Landfill III, opened in 1982 when Landfill II shut down, comprises approximately 11 acres (4 hectares) and is approximately $12 \mathrm{ft}(4 \mathrm{~m})$ deep. The western portion of Landfill III is still operational but will not be considered in the scope of this FSP. The landfills are CERCLA landfills that are primarily composed of cafeteria, office, and construction wastes, and to a lesser extent hazardous wastes. The major types of waste accepted 


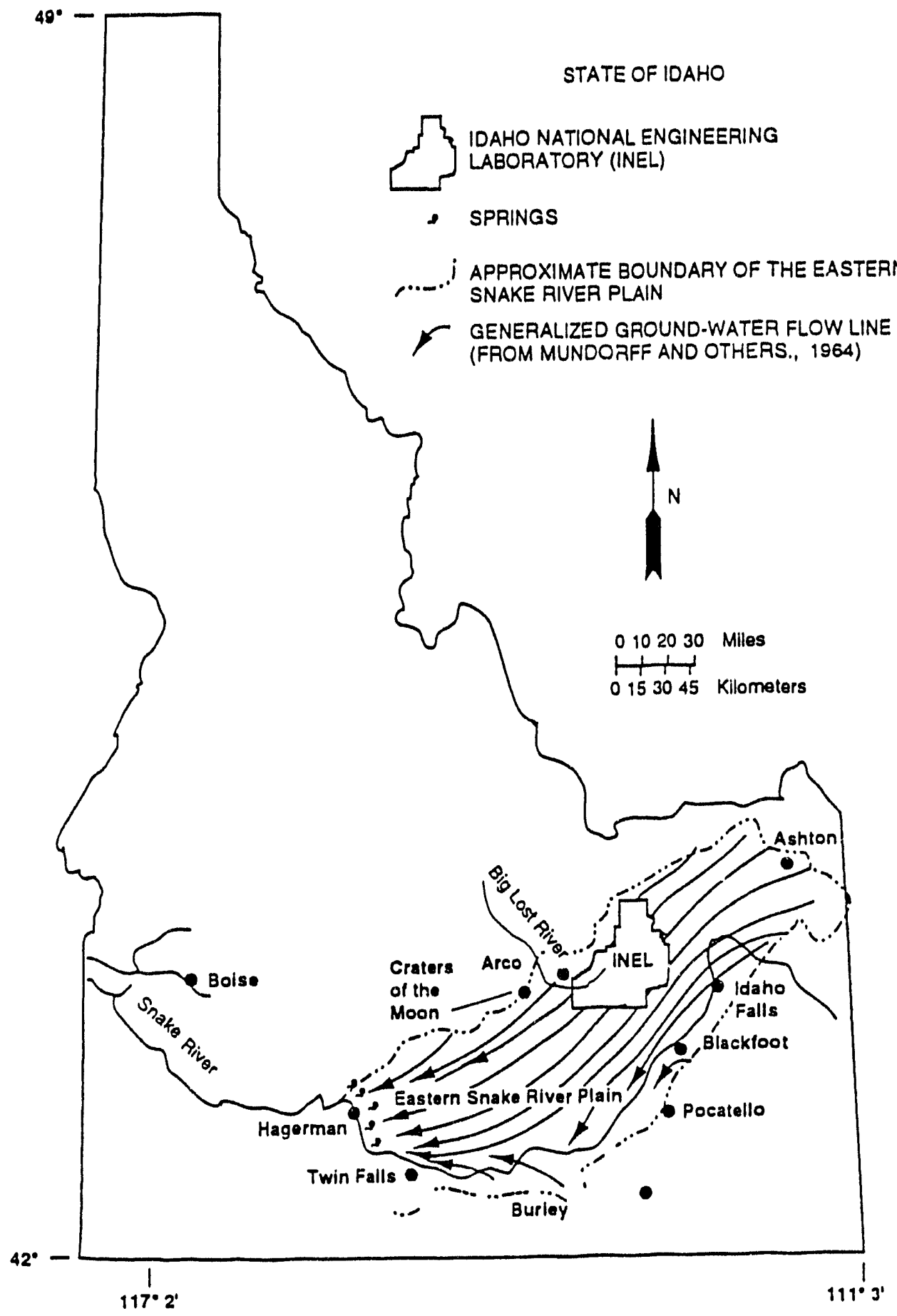

Figure 1-1. Map of Idaho showing the location of the INEL, Snake River Plain, and generalized groundwater flow lines of the Snake River Plain Aquifer. 


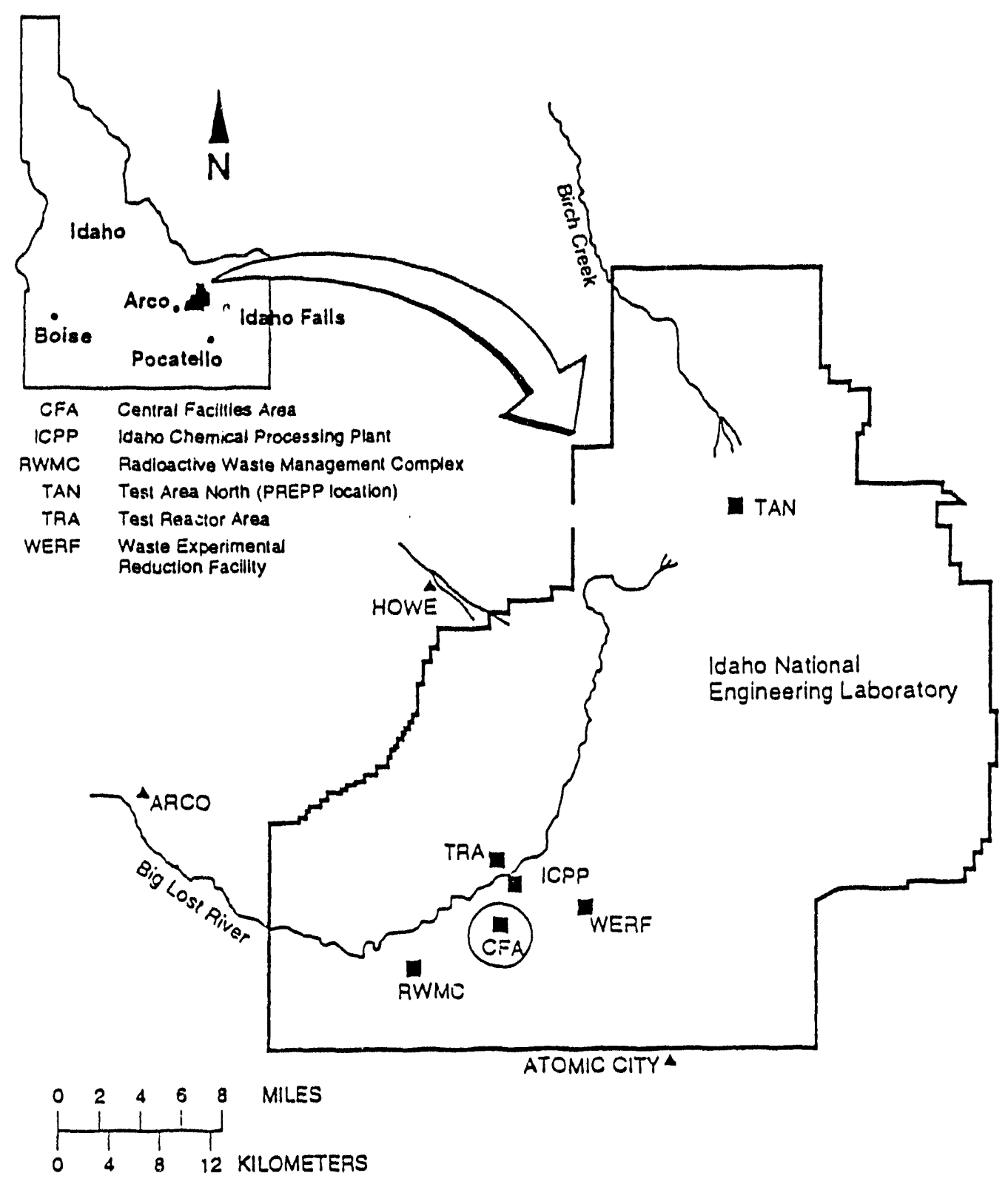

R92 1390

Figure 1-2. Location map of the INEL and CFA. 


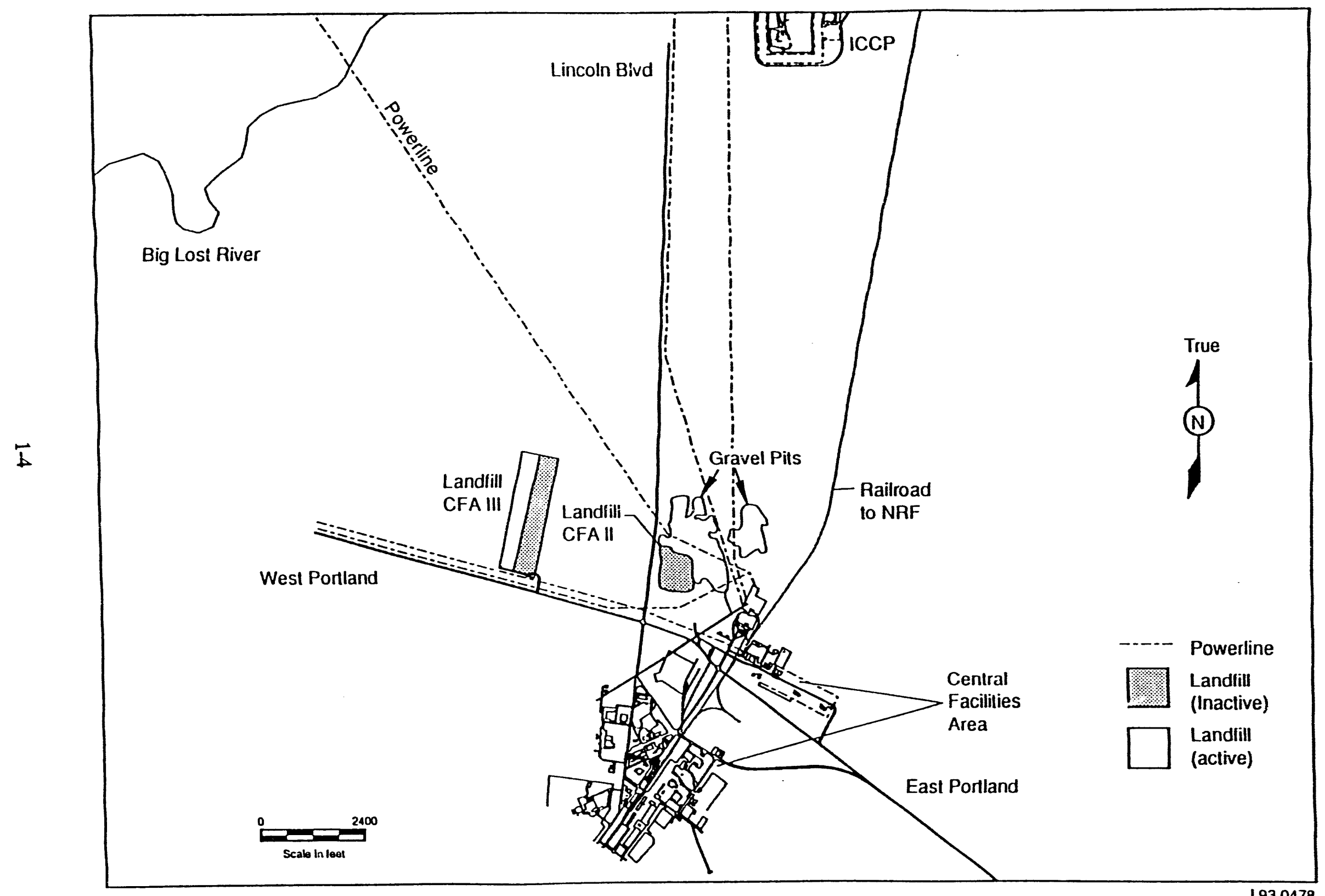

Figure 1-3. Location map of CFA Landfills II and III. 
at the landfills include trash, cafeteria garbage, wood and scrap lumber, masonry concrete, scrap metal, weeds and grass, dirt and gravel, asphalt, and asbestos. To a much lesser extent "other" wastes include waste oil, solvents, chemicals, and paint. Both landfills are covered with generally coarse soil materials that have been revegetated with crested wheatgrass. For a more thorough discussion of the landfills, see Section 2 of the Work Plan.

\subsection{Environmental Conditions}

The climate on the INEL is typical of a desert environment with average temperatures ranging from $16.1^{\circ} \mathrm{F}\left(-8.8^{\circ} \mathrm{C}\right)$ in January to $68^{\circ} \mathrm{F}\left(20^{\circ} \mathrm{C}\right)$ in July and average annual precipitation of 8.71 in. $(22.12 \mathrm{~cm})$. Winds at CFA generally originate in the southwest and travel to the northeast at average speeds between 5 and $9 \mathrm{mph}$ ( 8 and $14 \mathrm{kph})$. See Section 2.1 of the Work Plan for more information on the meteorology of the INEL.

Geology of CFA is characteristic of the Eastern Snake River Plain (ESRP), typically, basalt flows separated by sedimentary interbeds. Surficial sediments are composed of sands and gravels up to $40 \mathrm{ft}(12 \mathrm{~m})$ thick and a $8-\mathrm{ft}(2.4-\mathrm{m})$ thick clay layer, which is not continuous beneath the landfills.

Groundwater on the INEL flows from the northeast to the southwest. Depth to groundwater varies across the INEL from $200 \mathrm{ft}(61 \mathrm{~m})$ in the northeast to more than $1,000 \mathrm{ft}$ $(305 \mathrm{~m})$ in the southeast. Depth to groundwater beneath the CFA landfills is approximately $480 \mathrm{ft}(146 \mathrm{~m})$. A more detailed discussion of the geology and hydrology of the INEL is contained in Sections 2.1.2 through 2.1.3 of the Work Plan.

\subsection{Project Organization and Responsibility}

Several organizations will be directly involved in performing and reviewing this project. The project documentation receives internal review, which is outlined in Environmental Restoration (ER) Program Directive (PD) 2.2, Internal and Independent Review of Documents. An organizational chart for sampling and analysis activities is shown in Figure 1-4. 


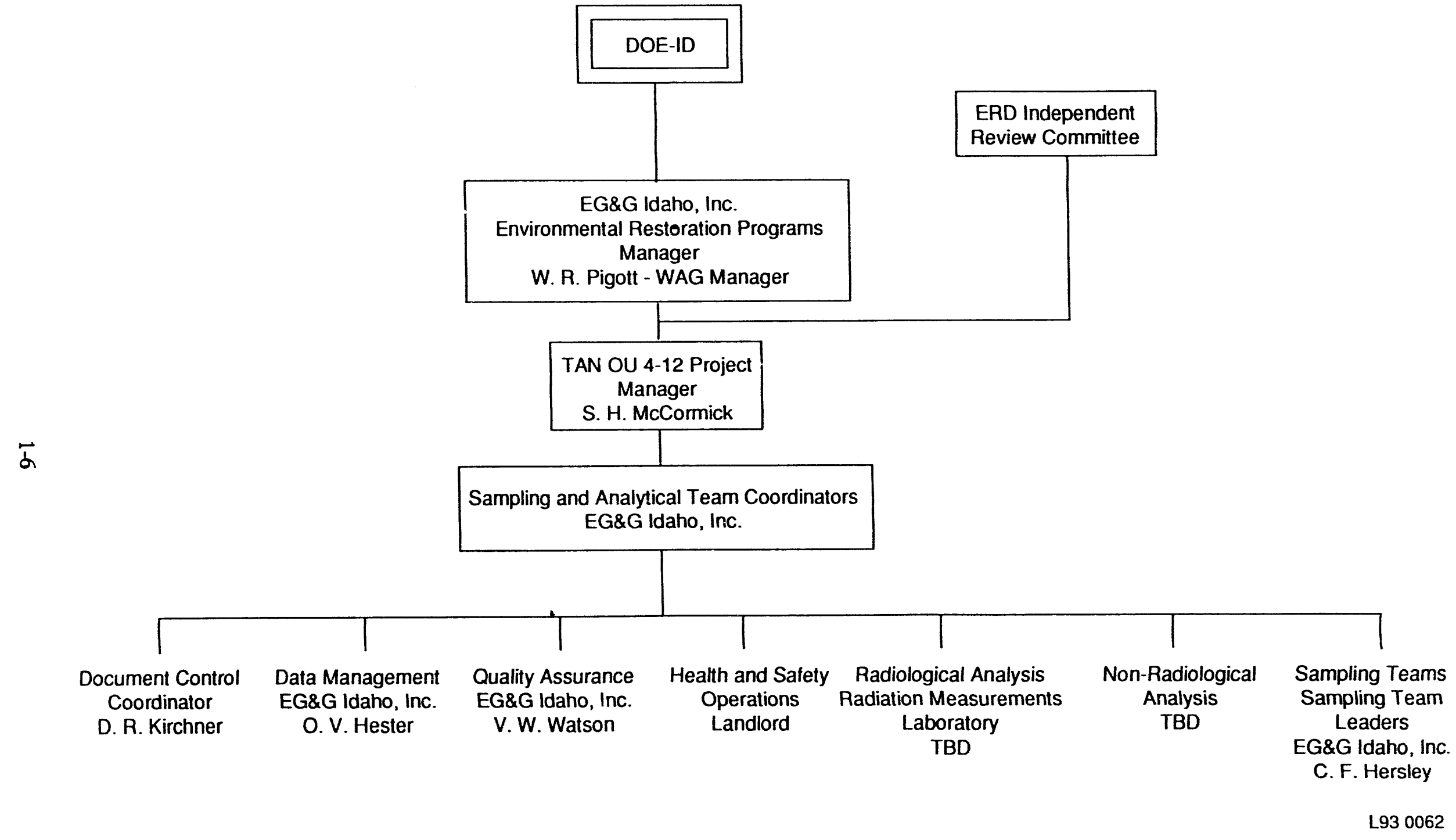

Figure 1-4. Organization chart for the CFA Landfills II and III Operable Unit. 


\section{SAMPLING OBJECTIVES AND MEDIA SPECIFIC RATIONALE}

\subsection{Data Needs and Data Objectives}

This section presents an identification of the data needs required for completing the remedial investigation for the CFA Landfills II and III RI/FS. Data needs and the data quality objectives (DQOs) are defined for each medium of concern.

\subsubsection{Data Needs}

Data needs have been identified by evaluating existing data and determining what additional data are necessary for site characterization, risk assessment, fate and transport modeling, and the development of remedial action alternatives. The data needs, sampling objectives, and measurement approach to meet the data objectives for the RI are provided in Table 2-1.

\subsubsection{Decision Making Process}

The data quality objectives identified in Table 2-1 specify the quality of data required to support decisions during the CFA Operable Unit 4-12 remedial response process. A variety of analytical methods are available to provide the necessary data. The analytical levels in Table 2-1 were identified to meet the intended use and Table 2-2 defines the five analytical support levels that will be used for this investigation. The analytical levels relate to the location in which the analysis is done (lab vs. field), the sophistication of the analytical method (internal standard operating procedure, EPA approved, or modified approved procedure), and the level of detail with which Quality Assurance/Quality Control (QA/QC) is specified in the SOW to the laboratory and documented in the data package. Statements of accuracy and precision for the analytical methods identified in Table 2-1 can be found in the QAPjP.

\subsection{Media-Specific Rationale}

\subsubsection{Topographic Survey of Landfill III}

A topographic map of Landfill III will be prepared to (1) identify topographic anomalies, (2) document erosional features, and (3) determine the adequacy of existing landfill cover for controlling erosion from surface runon/runoff.

\subsubsection{Geophysical Survey of Landfills II and III}

A combination of geophysical surveys will be performed at Landfill III. The objectives of the surveys are to (1) determine the perimeter boundary of Landfill III and the boundaries of the trenches in the landfill, (2) determine the depth and topography of the underlying basalt, (3) define areas containing high concentrations of metal, and (4) determine overburden cover thickness. The depth and topography of the underlying basalt at Landfill II will also be determined. Seismic refraction methods will be used to determine the thickness of surficial sediments and the general topography of the underlying basalt at Landfills II and III. Induced 
Table 2-1. Data quality objectives summary for CFA Landfills II and III RI/FS.

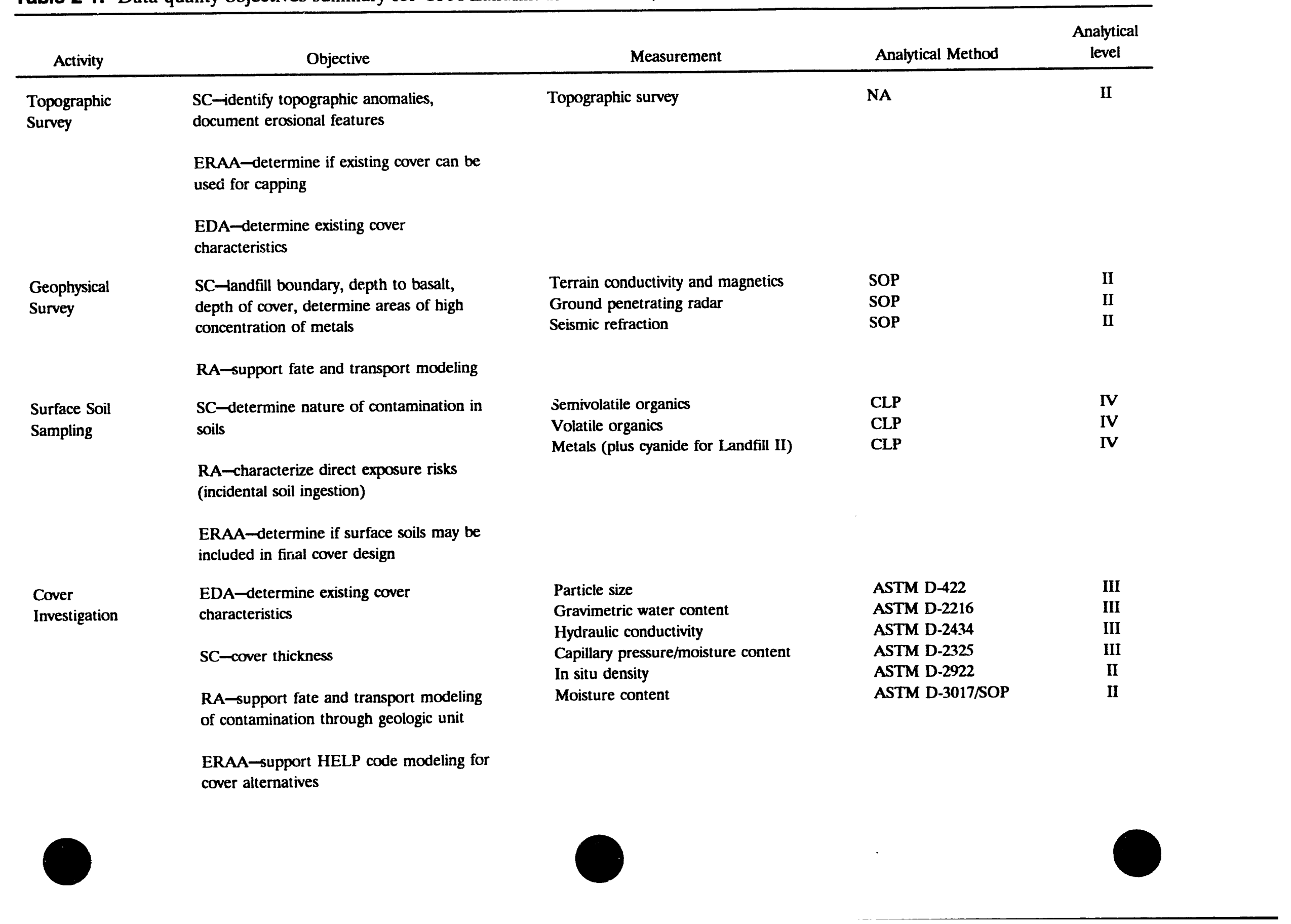


Table 2-1. (continued).

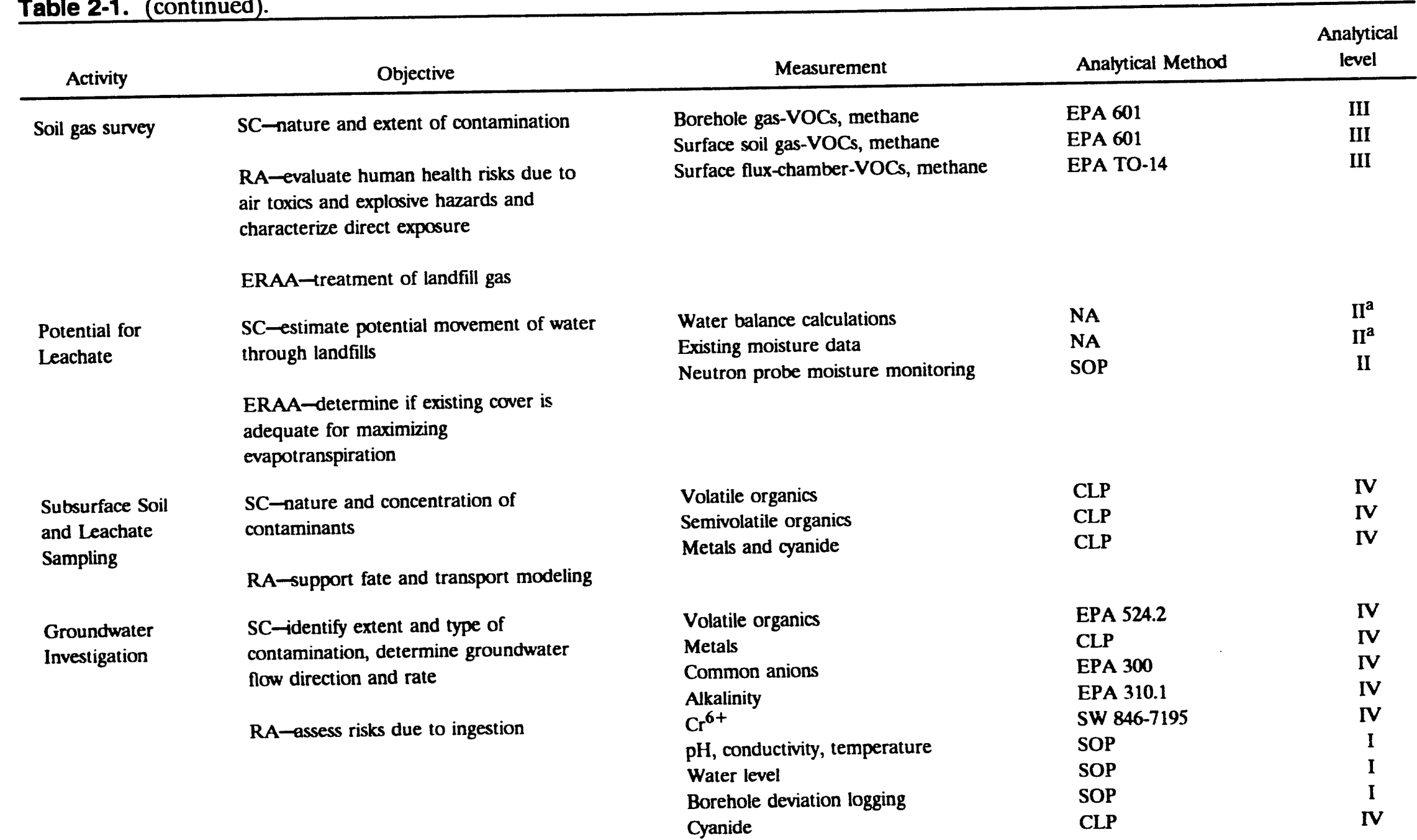

a. Based on previously collected data from neutron probe, salinity, and heat dissipation blocks.

SOP - Standard operating procedure (see Appendix B of the Field Sampling Plan) 
Table 2-2. Analytical levels. ${ }^{a}$

Level I Analyses done by on-site instrumentation primarily used for monitoring air for health and safety purposes (e.g., organic vapor monitoring instruments). Limited quantitative information can be gathered along with limited qualitative information (e.g., presence of volatile organics, not which compound is present).

Level II Analyses done by field instrumentation or in a mobile laboratory that provides qualitative as well as quantitative results (e.g., portable x-ray fluorescence or gas chromatograph). Data from these analyses can be used for site characterization and monitoring during remedial activities.

Level III Analyses done by any approved laboratory procedure [i.e., approved by American Society for Testing and Materials (ASTM), EPA, the ER Independent Review Committee (ER IRC), the USGS, etc.]. Data from these analyses can be used to confirm analyses performed by Level II techniques, evaluate engineering design, etc.

Level IV Chemical analyses done by any EPA-approved method or any radiological analyses by methods as specified by the Radiological Statement of Work. The laboratory deliverables consist of an EPA Contract Laboratory Program (CLP) type data package or the data package for radiological analyses specified by the Statement of Work for radiological analyses.

Level V Analyses done by modified approved methods. The EPA Special Analytical Services and ER IRC-approved methods for experimental analyses in unusual matrices are examples of Level $V$ support. These data are also used for decisions requiring the highest level of confidence in the data.

a. EG\&G Idaho, Environmental Restoration. 
electromagnetic measurements will be used to map concentrations of conductive materials such as steel drums, scrap metal, and areas of elevated soil moisture at Landfill III. Magnetic field measurements will be used to map the distribution of buried ferrous objects at Landfill II. Ground penetrating radar will be used to determine the overburden cover thickness at Landfill III. Several random boreholes will be augered through the cover material to confirm the results of the ground penetrating radar.

Data from the geophysical surveys will be used to generate maps showing the landfill boundarics, topography of the basalt surface, the distribution of electrically conductive bodics, the distribution of buried magnetic material, and the thickness of overburden cover. These data will be used to confirm the historical landfill boundaries, as input data to the fate and transport modeling studies, and for comparison to soil gas and soil contaminant data.

\subsubsection{Soil Gas Sampling}

A combination of soil gas sampling techniques will be used at the landfills to (1) identify areas within the landfill containing high concentrations of explosive or toxic landfill gas to perform an assessment of human health risks duc to air toxics and explosive hazards, (2) evaluate the feasibility of gas collection and treatment, (3) evaluate remedial actions, and (4) estimate concentrations of selected volatile organic compounds (VOCs) being emitted to the atmosphere to characterize direct exposure risks by inhalation. This will be accomplished through a shallow soil gas survey, soil gas collection from the instrumented boreholes, and by sampling with a surface-flux chamber.

The objectives of the shallow soil gas survey at Landfills II and III are to (1) define potential source areas for volatile organics, (2) determine the concentration and distribution of methane, and (3) delineate vapor phase contaminant migration. The results of this survey and existing data obtained from the 1989 shallow soil gas survey at Landfill II will be used to provide information for contaminant fate and transport modeling in support of a qualitative baseline risk assessment for the inhalation pathway.

A total of nine shallow boreholes instrumented with gas ports have been installed in Landfills II and III (sce Appendix D of the Work Plan for borehole logs). In May 1988, soil gas samples were collected from these boreholes and several volatile organics were detected at low to moderate concentrations. Methane was detected in several boreholes above the lower explosive

limit in Landfill II. During the RI, borchole soil gas samples will be collected from these existing instrumented boreholes to (1) determine the concentration of vapor phase volatile organics and methane in the subsurface soils, (2) evaluate the feasibility of gas collection and treatment, and (3) evaluate potential remedial actions. Data collected will be used for fate and transport modeling and the baseline risk assessment.

VOC emission rates from the surface of the Landfills II and III will be determined using an emission flux chamber (EPA 1986). The chamber is placed on the soil surface and air of known purity is swept through the chamber at a known, constant rate. Air samples are collected at the exit of the chamber and VOC concentrations determined by gas chromatography. 


\subsubsection{Surface Soil Sampling}

Surface soil will be collected from each landfill for analysis of volatile organics, semivolatile organics, and metals (including cyanide for Landfill II samples). These data will be collected to (1) determine the distribution and concentration of contamination potential in surface soils

(2) determine if the surficial soils should be included in the source control actions for the landfills, and (3) characterize direct exposure risks for the landfills.

\subsubsection{Cover Characterization of Landfill III}

Because the existing soil cover may be used as a component of the final cover system, further investigation of the cover material is warranted. The objectives of the landfill cover characterization are to (1) determine the permeability of the existing cover, (2) determine the thickness of the cover, (3) evaluate the physical properties of the geologic unit governing the transport of contaminants, (4) evaluate remedial alternatives, and (5) cvaluate potential for infiltration. The cover material on Landfill II was investigated in 1989 (Miller et al. 1990).

To accomplish the above objectives, in-situ measurements for density, moisture content, and cover thickness (if the geophysical survey is unsuccessful) will be collected across the landfill. In-situ drainage tests will be performed at the same sites chosen for collection of samples for laboratory analysis of soil physical and hydraulic properties. Samples will also be collected and submitted for laboratory testing for moisture content, particle size analysis, porosity, saturated hydraulic conductivity, and capillary pressure/moisture content relationships.

Moisture monitoring data will be collected from existing neutron access tubes at Landfills II and III to delineate soil moisture with depth. This data, along with existing moisture monitoring information, will then be used to assess the potential for contaminant leaching. Additionally, the toe slope of Landfill II will be examined weekly for the presence of leachate. If leachate is present, samples will be collected for volatile organic, semivolatile organic, and metals (plus cyanide) analysis.

\subsubsection{Water Level Measurements and Borehole Deviation Logging}

The regional groundwater flow at CFA is in a south-to-southwest direction. However, regional flow may be affected by infiltration from percolation ponds and aquifer heterogeneities. These effects could have a direct bearing on the direction and rate of contaminant migration. Thus, it is important to understand the groundwater flow system at CFA. Monthly water level measurements will be collected from available wells within approximatcly a $1-\mathrm{mi}(1.6-\mathrm{km})$ radius of the CFA landfills. Water level measurements will begin in May 1993 and continue through December 1993.

Borehole deviation logs will be run by the USGS on the landfill wells (LF designator), and if possible, the USGS wells identified in Table 3-10. Information obtained from the logging effort and water level measurements will be used to correct the measured depth to water for borehole deviation effects and to confirm the upgradient and downgradient locations of existing monitoring wells. 


\subsubsection{Groundwater Sampling}

The objectives of groundwater sampling at CFA Landfills II and III are to (1) determine whether or not the particulate chromium in the up and down gradient wells near the landfills is from the sampling pumps (Hydrostar model), (2) provide verification of existing water quality data, which indicates that the landfills are not a source of groundwater contamination, and (3) characterize ingestion exposure risks.

Three rounds of groundwater samples will be collected and analyzed from a network of 10 monitoring wells located both upgradient and downgradient from CFA Landfills II and III and from two production wells used for drinking water at CFA. The first sampling event will take place in the May-June time frame, with the second and third events targeted for July and October, respectively. The results of each sampling event will be evaluated by DOE, EPA, and IDHW in order to determine the scope and/or need of each subsequent event. The analyses to be performed and the desired analytical support level are presented in Section 3 and in Table 5-1 of the QAPjP. The groundwater quality data will be used for fate and transport modeling and the risk assessment evaluation.

\subsubsection{Subsurface Soil Sampling of Landfill II}

Subsurface soil samples will be collected for analysis of volatile organics, semivolatile organics, and metals (plus cyanide). The data will be collected to (1) determine the nature and concentration of leachable contaminants within and below the waste unit, and (2) determine if leachate is present in or below the landfill. The results of this investigation will be used as input to fate and transport modeling and to reduce the uncertainty associated with the source term information for Landfill II. If leachate is encountered during subsurface soil sampling, leachate samples will be collected for the above mentioned analyses and the boreholes will be completed as leachate monitoring wells. The need for subsurface soil sampling at Landfill III will be evaluated by DOE, EPA, and IDHW based on surface soil and soil gas sampling results from Landfill III. 


\section{SAMPLING LOCATION, FREQUENCY, AND SAMPLING/ANALYTICAL METHODS}

The material presented in this section is intended to support the data quality objectives summarized in Section 2. Tables 3-1 and 3-2 provide a summary of the sampling plan and include sample type, media, location, and analyses. Samples will be collected in laboratory cleaned bottles and packaged according to ASTM or EPA-recommended procedures (see Section 6.2). Quality assurance samples will be included to satisfy the quality assurance requirements for the field operation (sec Quality Assurance Project Plan for project QA/QC goals), and qualified (ERapproved) analytical and testing laboratories will analyze all samples. Ficld guide forms (see Figure 6-2 in Section 6.1.2) outlining sample collection location, sample numbers, and analysis requested will be provided for each sample location. The forms will be generated from the sampling plan data base, which will ensure unique sample numbers.

Sample and Analysis Plan tables for the surface soil, groundwater, soil gas, subsurface soil, and geotechnical analyses are provided in Appendix A of the FSP. QA/QC samples to be collected are also identified (also see Table 3-3). These tables will aid in the sample labelling and tracking. All sample locations will be staked and surveyed.

All activitics outlined in this FSP will be carried out in accordance with applicable health and safety regulations and requirements as discussed in the Health and Safety Plan addendum for this project.

\subsection{Topographic Survey of Landfill III}

A topographic map of Landfill III will be prepared. The field survey will use the sample locations from the shallow soil gas investigation (see Figure 3-3) as the initial survey grid. Horizontal control for the survey will be class 3 [distance accurate to $0.007 \mathrm{ft}(0.002 \mathrm{~m})$ ], and the vertical control will be $3 \mathrm{rd}$ order $[0.05 \mathrm{ft}(0.015 \mathrm{~m})]$. If this survey grid does not provide adequate horizontal and vertical control to compile a topographic map with $1 \mathrm{ft}$ contour intervals at a scale of 1 in. $(2.54 \mathrm{~cm})$ equals $100 \mathrm{ft}(30.6 \mathrm{~m})$, then additional survey points will be necessary to further define the topography of the site. Locations for additional survey points will be determined in the field based on the surveyor's judgement. A topographic map covering the 11 acres ( 4 hectares) of the site and immediate surrounding area will be prepared consisting of contour intervals not to excecd $1 \mathrm{ft}(0.3 \mathrm{~m})$ and with a scale of $1 \mathrm{in} .(2.54 \mathrm{~cm})$ equals $100 \mathrm{ft}(30.6 \mathrm{~m})$. A map of the surface-water drainage system in this area will also be prepared.

\subsection{Geophysical Survey of Landfills II and III}

A combination of geophysical surveys will be performed at Landfill III. Scismic refraction methods will be used to determine the thickness of surficial sediments and the general topography of the underlying basalt. The data will be collected on profiles across the short dimension of the landfill and will provide depth estimates every $16.4 \mathrm{ft}(5 \mathrm{~m})$ along each profile with $98 \mathrm{ft}(30 \mathrm{~m})$ between profiles (Figure 3-1a). Induced electromagnetic measurements will be used to map concentrations of conductive materials such as steel drums, scrap metal, and areas of elevated soil moisture. These measurements will be made on a regular grid of $3 \times 13 \mathrm{ft}(1 \times 4 \mathrm{~m})$ over the 
Table 3-1. Summary of location, media, sample type, and analysis--soil cover investigation.

\begin{tabular}{|c|c|c|c|c|c|c|c|c|c|}
\hline \multirow[b]{2}{*}{ Location } & \multirow[b]{2}{*}{ Media } & \multirow[b]{2}{*}{ Sample type } & \multicolumn{3}{|c|}{ Field measurements } & \multicolumn{3}{|c|}{ Lahoratory analysis } & \multirow[b]{2}{*}{$\begin{array}{l}\text { Capillary pressure - } \\
\text { moisture content } \\
\text { (ASTM D-2325) }\end{array}$} \\
\hline & & & In situ moisture & $\begin{array}{l}\text { In situ density }{ }^{a} \\
\text { (ASTM D-2922) }\end{array}$ & $\begin{array}{c}\text { Cover }^{b} \\
\text { thickness }\end{array}$ & $\begin{array}{l}\text { Gravimetric water } \\
\text { content }^{c} \\
\text { (ASTM D-2216) }\end{array}$ & $\begin{array}{c}\text { Particle size } \\
\text { (ASTM D-422) }\end{array}$ & $\begin{array}{l}\text { Constant head } \\
\text { permeability } \\
\text { (ASTM D-2434) }\end{array}$ & \\
\hline \multirow[t]{2}{*}{ Landfill III } & Soil & Grab & $24^{d}$ & 24 & - & 16 & 16 & - & - \\
\hline & & & - & - & - & $16^{e}$ & - & - & - \\
\hline & & & $2^{f}$ & - & - & - & - & - & - \\
\hline . & Soil & Augering & - & - & 12 & - & - & - & - \\
\hline & Soil & Core & - & - & - & - & - & 8 & 8 \\
\hline Landfill II & Soil & Grab & 36 & - & - & - & - & - & - \\
\hline Total & - & - & 37 & 24 & 12 & 16 & 16 & 8 & 8 \\
\hline a. Measuren & ents are pla & inned at 4 -in. a & id 8-in. depths at ea & ch of the 12 sample $s$ & tes. & & & & \\
\hline b. Will only & performe & d if the geophy & sical investigation is & unsuccessful in deter & mining cover & less. & & & \\
\hline c. Samples v & ill be collec & ted at $1-\mathrm{ft}$ incr & ments; sample num & xer is based on collec & ing two samp & ar site. & & & \\
\hline 1. Measuren & ents are pla & anned at 4-in. a & ad 8-in. depths at $e$ & ch of the 12 sample & ites following & U D-3017. & & & \\
\hline Measuren & ents are pla & anned at each o & f the 8 sample sites & for in situ drainage te & sts; sample nu & is based on collecting & two samples per s & & \\
\hline Measurem & ents are pla & inned at 6-in. $d$ & epth increments in & ach of the two existin & neutron pro & cess tubes following $S$ & & & \\
\hline
\end{tabular}


Table 3-2. Summary of location, sample type, and analysis - groundwater, soil and soil gas. ${ }^{\mathrm{a}}$

\begin{tabular}{|c|c|c|c|c|c|c|c|c|c|}
\hline Location & Sample type & $\begin{array}{l}\text { Level IV } \\
\text { volatiles } \\
(524.2)^{\mathrm{c}, \mathrm{f}}\end{array}$ & $\begin{array}{c}\text { Level IV } \\
\text { CLP inorganics } \\
\text { metals }^{c}\end{array}$ & $\begin{array}{l}\text { Level IV } \\
\mathrm{Cr}^{6+c}\end{array}$ & $\begin{array}{l}\text { Additional } \\
\text { water quality } \\
\text { analytes }^{c}\end{array}$ & $\begin{array}{l}\text { Level III } \\
\text { volatiles } \\
\text { and methane } \\
(\text { EPA 601) }\end{array}$ & $\begin{array}{c}\text { Level IV } \\
\text { CLP metals }\end{array}$ & $\begin{array}{l}\text { Level IV } \\
\text { volatile } \\
\text { organics } \\
(\mathrm{CLP})^{\mathrm{e}}\end{array}$ & $\begin{array}{c}\text { Level IV } \\
\text { Semivolatile } \\
\text { organics } \\
\text { (CLP) }^{e}\end{array}$ \\
\hline Existing landfill wells ${ }^{b}$ & Grab & 12 & 12 & 12 & 12 & - & - & - & - \\
\hline Shallow soil gas & Grab & - & - & - & - & 165 & - & - & - \\
\hline Borehole soil gas & Grab & - & - & - & - & 9 & - & - & - \\
\hline Surface flux chamber & Grab & - & - & - & - & $16^{g}$ & - & - & - \\
\hline Subsurface soil & Grab & - & - & - & - & - & 14 & 14 & 14 \\
\hline Surface soil & Grab & - & - & - & - & - & 26 & 26 & 26 \\
\hline Total & - & 12 & 12 & 12 & 12 & 190 & 40 & 40 & 40 \\
\hline
\end{tabular}

a. QA/QC samples are provided in Table 3-3 for groundwater, surface and subsurface soil, and soil gas samples.

b. Three rounds of sampling are planned (May-June, July, and October).

c. See Table 3-4 for water quality analyses to be performed.

d. EPA 601 analyte list is provided in Table 3-5. Shallow soil gas samples include 84 sites at Landfill III and 81 sites at Landfill II.

e. EPA CLP analyte lists for volatile organics, semivolatile organics, and metals are provided in Tables 3-6, 3-7, and 3-8, respectively.

f. EPA 524.2 target analyte list is provided in Table 3-9.

g. EPA TO-14 will be used for surface flux soil gas analysis. 
Table 3-3. QA/QC samples for groundwater, surface soil, and soil gas (also see Sampling and Analysis Plan tables in Appendix A).

\begin{tabular}{|c|c|c|c|}
\hline Well & Type & Quantity & Comment \\
\hline \multirow[t]{4}{*}{$\begin{array}{l}\text { Existing } \\
\text { wells }\end{array}$} & Replicates & 2 & $\begin{array}{l}\text { Replicates will be collected at random from one upgradient } \\
\text { and one downgradient well. }\end{array}$ \\
\hline & Rinsate & 1 & $\begin{array}{l}\text { One rinsate sample will be collected from both the pump and } \\
\text { bailer for analysis after sampling the downgradient wells. }\end{array}$ \\
\hline & Field blank & 1 & $\begin{array}{l}\text { One field blank will be collected at the beginning of the } \\
\text { sampling event. }\end{array}$ \\
\hline & Trip blanks & TBD & $\begin{array}{l}\text { Trip blanks will be prepared as necessary to accompany each } \\
\text { sample cooler for VOC analysis. }\end{array}$ \\
\hline \multirow[t]{3}{*}{$\begin{array}{l}\text { Soil gas } \\
\text { survey }\end{array}$} & Replicates & 18 & $\begin{array}{l}\text { Field replicates will be collected at a frequency of } 1 \text { per } 10 \\
\text { samples. }\end{array}$ \\
\hline & $\begin{array}{l}\text { Field control } \\
\text { samples }\end{array}$ & 26 & $\begin{array}{l}\text { Field control samples will be collected as discussed in } \\
\text { Section } 5.3 .1 \text { at the following times: } \\
\text { - Prior to each days activities } \\
\text { - After every } 20 \text { th sample per day. } \\
\text { - At the end of each sampling day. } \\
\text { These samples will be analyzed similar to the actual field } \\
\text { samples. }\end{array}$ \\
\hline & $\begin{array}{l}\text { Analytical } \\
\text { quality control }\end{array}$ & 18 & $\begin{array}{l}\text { Duplicate analysis will be run after every tenth sample } \\
\text { analyzed. }\end{array}$ \\
\hline \multirow{2}{*}{$\begin{array}{l}\text { Soil gas } \\
\text { surface flux } \\
\text { chamber }\end{array}$} & Field control & 2 & Two field blanks will be collected at random (one per landfill). \\
\hline & Replicates & 2 & Two replicates will be collected at random (one per landfill). \\
\hline \multirow[t]{2}{*}{ Surface soil } & Replicates & 2 & Two replicates will be collected at random (one per landfill). \\
\hline & Rinsate & 2 & Two rinsates will be collected at random (one per landfill). \\
\hline \multirow{3}{*}{$\begin{array}{l}\text { Subsurface } \\
\text { soil }\end{array}$} & Replicates & 1 & One replicate will be collected at random. \\
\hline & Rinsate & 1 & One rinsate will be collected at random. \\
\hline & $\begin{array}{l}\text { Site } \\
\text { comparison } \\
\text { samples }\end{array}$ & 4 & $\begin{array}{l}\text { Four soil samples spiked by the EPA in April } 1992 \text { will be } \\
\text { analyzed for semivolatile and metal analytes to evaluate } \\
\text { laboratory precision and accuracy. }\end{array}$ \\
\hline \multicolumn{4}{|c|}{$\begin{array}{l}\text { F. Field control samples are anticipated to be collected over six days and would include six prior to the day's activities, } \\
\text { eight samples during sampling, and six at the end of the day for the shallow soil gas survey performed at Landfills II and } \\
\text { III. Also collected will be one sample prior to, one during, and one at the end of the day for the borehole soil gas } \\
\text { sampling performed at each landfill. }\end{array}$} \\
\hline
\end{tabular}




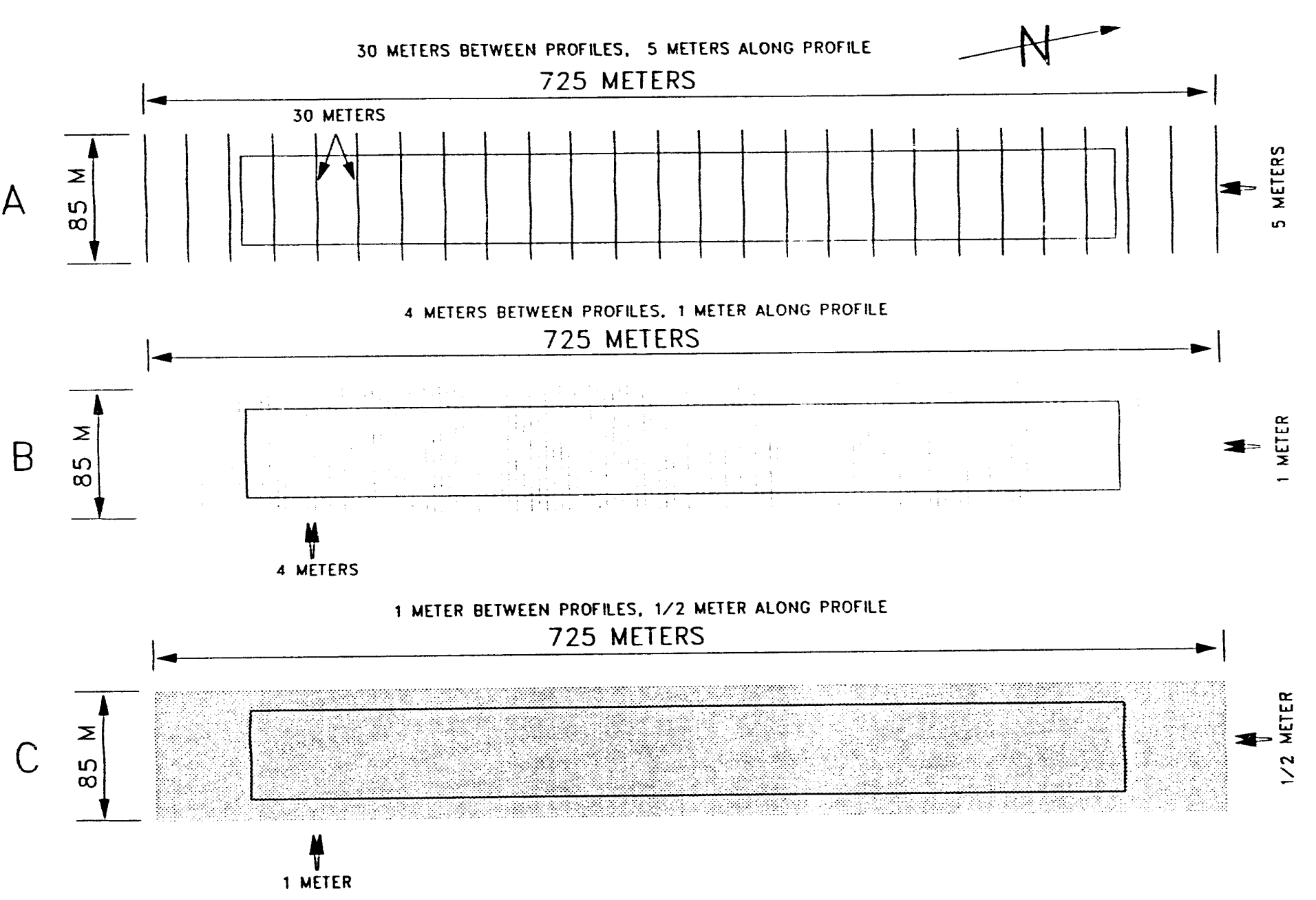

Figure 3-1. Geophysical survey grids at Landfill III for (a) seismic refraction, (b) induced electromagnetic measurements, and (c) magnetic field measurements. 
Table 3-4. Laboratory analytical protocols/chemical analytes for groundwater samples.

Measurement Parameter

Volatiles

EPA $524.2^{\mathrm{a}}$
Comments

Specify target analyte list to laboratory in statement of work (SOW). Tentatively identified compounds specified in the analytical SOW will be reported.

Sulfates

EPA 300

Chloride

EPA 300

Fluoride

EPA 300

Alkalinity

EPA 310.1

Nitratc/Nitrite

EPA 300

CLP Mctals

EPA CLP ${ }^{b}$

Hexavalent Chromium

SW 846-7195

Gamma scan

Cyanide

EG\&G RML-SOP D-11 (7/89)

EPA CLP ${ }^{\mathrm{h}}$

a. Methods for the Determination of Organic Compounds in Drinking Water (U.S.

Environmental Monitoring Systems Laboratory, Cincinnati, OH, December 1988, EPA 600/4-88/039).

b. EPA Contract Lab Program Statement of Work for Inorganics Analysis, Multimedia, Multiconcentration, (SOW No. 788, incl. Rev. 2/89 and 6/89). 
Table 3-5. EPA Method 601 target analyte list and method detection limits.

\begin{tabular}{|c|c|c|c|}
\hline \multirow{2}{*}{ Parameler } & \multicolumn{2}{|c|}{ Relention ume (min) } & \multirow{2}{*}{$\begin{array}{l}\text { Helhod delection } \\
\text { limil }(\mu g / L)\end{array}$} \\
\hline & Colvinn 1 & Column 2 & \\
\hline Chloromethane & 1.50 & 5.28 & 0.08 \\
\hline 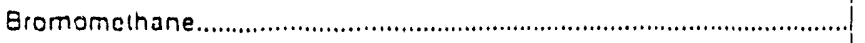 & 2.17 & 7.05 & 1.18 \\
\hline 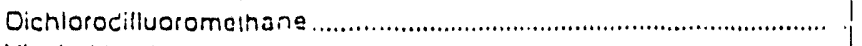 & 2.62 & nd & 1.81 \\
\hline 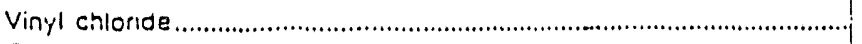 & 2.67 & 5.28 & 0.18 \\
\hline 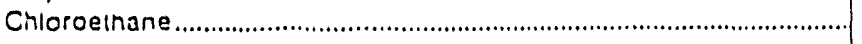 & 3.33 & 8.68 & 0.52 \\
\hline 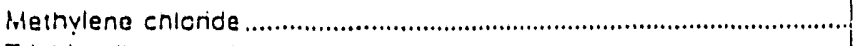 & 5.25 & 10.1 & 0.25 \\
\hline 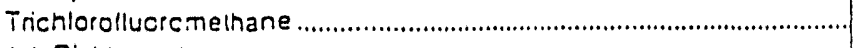 & 7.18 & nd & nd \\
\hline 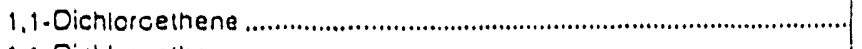 & 7.93 & 7.72 & 0.13 \\
\hline 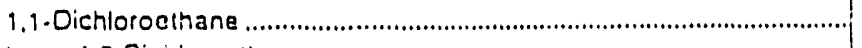 & 9.20 & 12.5 & 0.07 \\
\hline 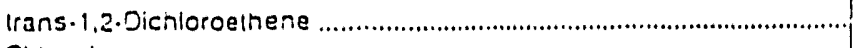 & 10.1 & 9.38 & 0.10 \\
\hline Chlorolorm & 10.7 & 12.1 & 0.05 \\
\hline 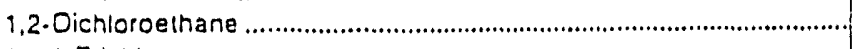 & 11.4 & 15.4 & 0.03 \\
\hline 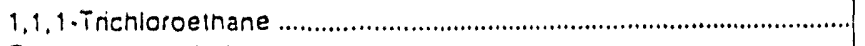 & 12.6 & 13.1 & 0.03 \\
\hline 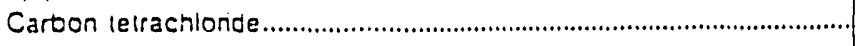 & 13.0 & 14,4 & 0.12 \\
\hline 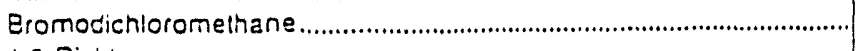 & 13.7 & 14.6 & 0.10 \\
\hline 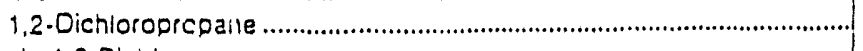 & 14.9 & 16.5 & 0.04 \\
\hline 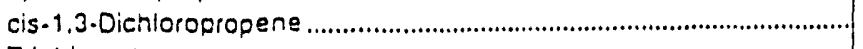 & 15.2 & 16.5 & 0.34 \\
\hline 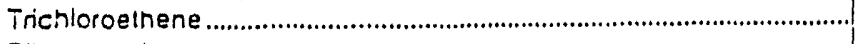 & 15.8 & 13.1 & 0.12 \\
\hline 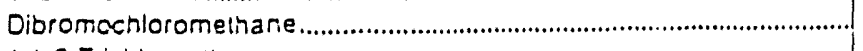 & 16.5 & 16.6 & 0.09 \\
\hline 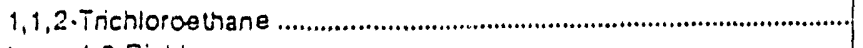 & 16.5 & 18.1 & 0.02 \\
\hline 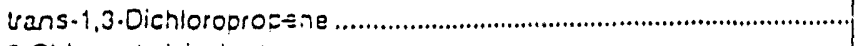 & 16.5 & 18.0 & 0.20 \\
\hline 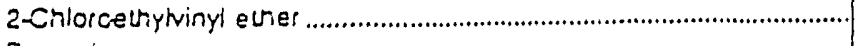 & 18.0 & nd & 0.13 \\
\hline 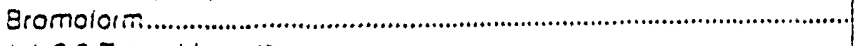 & 19.2 & 19.2 & 0.20 \\
\hline 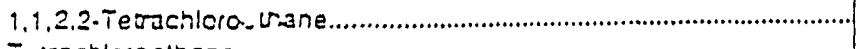 & 21.6 & rod & 2.03 \\
\hline 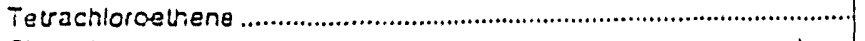 & 21.7 & $\vdots \vdots 0$ & 0.03 \\
\hline 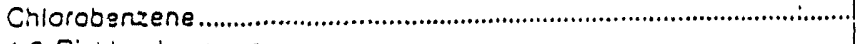 & 24.2 & 18.8 & 0.25 \\
\hline 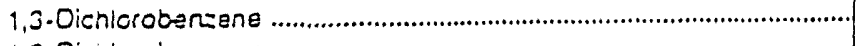 & 24.0 & 224 & 0.32 \\
\hline 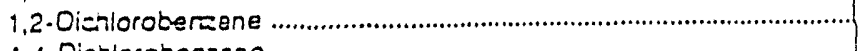 & 34.9 & $\approx .5$ & 0.15 \\
\hline 1,4-Dichlcrobenzene & 3.4 .4 & 22.3 & 0.24 \\
\hline
\end{tabular}

Column 1 concitions: Carcopack. 5 (60:50 mesh) caled with 1\% SP-iC00 packed in an a $11 \times 0.1$ in. 10 stainiess slesl or glass calumn with helium camer gas al 40 'mL/min fiew rale. Column lemperalure held at $45^{\circ} \mathrm{C}$ lor 3 min inen programmed at 8. E'miri $10220^{\circ} \mathrm{C}$ and held for is min.

Culumn 2 conditions: Porsil-C (100/120 mesh) coaled with n-oc:ane Dacked in a $6.4 \times 0.1$ in. 10 stainless siegl or glass column with helium carrier gas al $40 \mathrm{~mL} / \mathrm{min}$ llow rala. Column lemperalure held al so $\mathrm{C}$ lor 3 min then programmed al $\sigma^{\circ} \mathrm{C} /$ $\min 10170^{\circ} \mathrm{C}$ and held lor $4 \mathrm{~min}$.

nd $=$ nol delemined.

Nole: Acetonc, benzene, and methane will be annlized according to EPA Method 601 using a flame ionization detector. The method delection linuit for these three constiluents is $1.0 \mu \mathrm{g} /$. 
Table 3-6. Contract required quantitacion limits for volatile organics. ${ }^{a}$

\begin{tabular}{|c|c|c|c|}
\hline \multirow[b]{2}{*}{ Volatiles } & \multirow[b]{2}{*}{ CAS number } & \multicolumn{2}{|c|}{ Quantitation Limits ${ }^{b}$} \\
\hline & & $\begin{array}{l}\text { Water } \\
\text { (ug/L) }\end{array}$ & $\begin{array}{c}\text { Low Soil/sediment } \\
(\mathrm{ug} / \mathrm{Kg})\end{array}$ \\
\hline 1. Chloromethane & $74-87-3$ & 10 & 10 \\
\hline 2. Bromomethane & $74-83-9$ & 10 & 10 \\
\hline 3. Vinyl Chloride & $75-01-4$ & 10 & 10 \\
\hline 4. Chloroethane & $75-00-3$ & 10 & 10 \\
\hline 5. Methylene Chloride & $75-09-2$ & 5 & 5 \\
\hline 6. Acetone & $67-64-1$ & 10 & 10 \\
\hline 7. Carbon Disulfide & $75-15-0$ & 5 & 5 \\
\hline 8. 1,1-Dichloroetbene & $75-35-4$ & 5 & 5 \\
\hline 9. 1,1-Dichloroethane & $75-34-3$ & 5 & 5 \\
\hline 10. 1,2-Dichloroethene (total) & $540-39-0$ & 5 & 5 \\
\hline 11. Chloroform & $67-66-3$ & 5 & 5 \\
\hline 12. 1, 2-Dichloroethane & $107-06-2$ & 5 & 5 \\
\hline 13. 2-Butanone & $78-93-3$ & 10 & 10 \\
\hline 14. 1, 1, 1-Trichloroethane & $71-55-6$ & 5 & 5 \\
\hline 15. Carbon Tetrachloride & $56-23-5$ & 5 & 5 \\
\hline 16. Vinyl Acetate & $108-05-4$ & 10 & 10 \\
\hline 17. Bromodichloromethane & $75-27-4$ & 5 & 5 \\
\hline 18. 1, 2-Dichloropropane & $78-87-5$ & 5 & 5 \\
\hline 19. cis-1, 3-Dichloropropene & $10061-01-5$ & 5 & 5 \\
\hline 20. Trichloroethene & $79-01-6$ & 5 & 5 \\
\hline 21. Dibromochloromethane & $124-48-1$ & 5 & 5 \\
\hline 22. 1, 1,2-Trichloroethane & $79-00-5$ & 5 & 5 \\
\hline 23. Benzene & $71-43-2$ & 5 & 5 \\
\hline 24. trans-1, 3-Dichloropropene & $10061-02-6$ & 5 & 5 \\
\hline 25. Bromoform & $75-25-2$ & 5 & 5 \\
\hline 26. 4-Methyl-2-pentanone & $108-10-1$ & 10 & 10 \\
\hline 27. 2-Hexanone & $591-78-6$ & 10 & 10 \\
\hline
\end{tabular}


Table 3-6. (continued).

\begin{tabular}{lrcc}
\multicolumn{1}{c}{ Volatiles } & \multicolumn{2}{c}{ Quantitation Limits $^{b}$} \\
\cline { 3 - 4 } & CAS number & $\begin{array}{c}\text { Water } \\
(\mathrm{ug} / \mathrm{L})\end{array}$ & $\begin{array}{c}\text { Low Soil/sediment } \\
(\mathrm{ug} / \mathrm{Kg})\end{array}$ \\
\hline 28. Tetrachloroethene & $127-18-4$ & 5 & 5 \\
29. Toluene & $108-88-3$ & 5 & 5 \\
30. 1 1, 2, 2-Tetrachloroethene & $79-34-5$ & 5 & 5 \\
31. Chlorobenzene & $108-90-7$ & 5 & 5 \\
32. Ethyl Benzene & $100-41-4$ & 5 & 5 \\
33. Styrene & $100-42-5$ & 5 & 5 \\
34. Xylenes (Total) & $1330-20-7$ & 5 & 5 \\
\hline
\end{tabular}

a. Specific quantitation limits are highly matrix dependent. The quantitation limits listed herein are provided for guidance and may not always be achievable.

b. Quantitation limits listed for soil/sediment are based on net weight. The quantitation limits calculated by the laboratory for soil/sediment, calculated on dry weight basis as required by the contract, will be higher.

c. Medium Soil/Sediment Contract Required Quantitation Limits (CRQL) for Volatile TCL Compounds are 125 times the individual Low Soil/Sediment CRQL. 
Table 3-7. Contract required quantitation limits for semivolatile organics.

\begin{tabular}{|c|c|c|c|c|}
\hline \multirow{3}{*}{\multicolumn{2}{|c|}{ Semivolatiles }} & \multirow[b]{3}{*}{ CAS Number } & \multicolumn{2}{|c|}{ Quantitation Limits $^{\mathrm{a}}$} \\
\hline & & & Water & $\begin{array}{c}\text { Low } \\
\text { Soil/Sediment }\end{array}$ \\
\hline & & & $u g / L$ & $\mathrm{ug} / \mathrm{Kg}$ \\
\hline 1. & Phenol & $108-95-2$ & 10 & 330 \\
\hline 2. & bis(2-Chloroethyl) ether & $111-44-4$ & 10 & 330 \\
\hline 3. & 2-Chlorophenol & $95-57-8$ & 10 & 330 \\
\hline 4. & 1,3-Dichlorobenzene & $541-73-1$ & 10 & 330 \\
\hline 5. & 1,4-Dichlorobenzene & $106-46-7$ & 10 & 330 \\
\hline 6. & Benzyl alcohol & $100-51-6$ & 10 & 330 \\
\hline 7. & 1,2-Dichlorobenzene & $95-50-1$ & 10 & 330 \\
\hline 8. & 2-Methylphenol & $95-48-7$ & 10 & 330 \\
\hline 9. & bis(2-Chloroisopropyl) ether & $108-60-1$ & 10 & 330 \\
\hline 10. & 4-Methylphenol & $106-44-5$ & 10 & 330 \\
\hline 11. & N-Nitroso-di-n-dipropylamine & $621-64-7$ & 10 & 330 \\
\hline 12. & Hexachloroethane & $67-72-1$ & 10 & 330 \\
\hline 13. & Nitrobenzene & $98-95-3$ & 10 & 330 \\
\hline 14. & Isophorone & $78-59-1$ & 10 & 330 \\
\hline 15. & 2-Nitrophenol & $88-75-5$ & 10 & 330 \\
\hline 16. & 2,4-Dimethylphenol & $105-67-9$ & 10 & 330 \\
\hline 17. & Benzoic acid & $65-85-0$ & 50 & 1600 \\
\hline 18. & bis(2-Chloroethoxy) methane & $111-91-1$ & 10 & 330 \\
\hline 19. & 2,4-Dichlorophenol & $120-83-2$ & 10 & 330 \\
\hline 20. & 1,2,4-Trichlorobenzene & $120-82-1$ & 10 & 330 \\
\hline 21. & Naphthalene & $91-20-3$ & 10 & 330 \\
\hline 22. & 4-Chloroaniline & $106-47-8$ & 10 & 330 \\
\hline 23. & Hexachlorobutadiene & $87-68-3$ & 10 & 330 \\
\hline 24. & $\begin{array}{l}\text { 4-Chloro-3-methylphenol } \\
\text { (para-chloro-meta-cresol) }\end{array}$ & $59-50-7$ & 10 & 330 \\
\hline
\end{tabular}


Table 3-7. (continued).

\begin{tabular}{|c|c|c|c|c|}
\hline & & & Qus & n Limits \\
\hline & & & Water & $\begin{array}{c}\text { Low } \\
\text { Soil/Sediment }^{b}\end{array}$ \\
\hline & Semivolatiles & CAS Number & $\mathrm{ug} / \mathrm{L}$ & $\mathbf{u g} / \mathbf{K g}$ \\
\hline 25. & 2-Methylnaphthalene & $91-57-6$ & 10 & 330 \\
\hline 26. & Hexachlorocyclopentadiene & $77-47-4$ & 10 & 330 \\
\hline 27. & 2,4,6-Trichlorophenol & $88-06-2$ & 10 & 330 \\
\hline 28. & 2,4,5-Trichlorophenol & $95-95-4$ & 50 & 1600 \\
\hline 29. & 2-Chloronaphthalene & $91-58-7$ & 10 & 330 \\
\hline 30. & 2-Nitroaniline & $88-74-4$ & 50 & 1600 \\
\hline 31. & Dimethylphthalate & $131-11-3$ & 10 & 330 \\
\hline 32. & Acenaphthylene & $208-96-8$ & 10 & 330 \\
\hline 33. & 2,6-Dinitrotoluene & $606-20-2$ & 10 & 330 \\
\hline 34. & 3-Nitroaniline & $99-09-2$ & 50 & 1600 \\
\hline 35. & Acenaphthene & $83-32-9$ & 10 & 330 \\
\hline 36. & 2,4-Dinitrophenol & $51-28-5$ & 50 & 1600 \\
\hline 37. & 4-Nitrophenol & $100-02-7$ & 50 & 1600 \\
\hline 38. & Dibenzofuran & $132-64-9$ & 10 & 330 \\
\hline 39. & 2,4-Dinitrotoluene & $121-14-2$ & 10 & 330 \\
\hline 40. & Diethylphthalate & $84-66-2$ & 10 & 330 \\
\hline 41. & 4-Chlorophenyl-phenyl ether & $7005-72-3$ & 10 & 330 \\
\hline 42. & Fluorene & $86-73-7$ & 10 & 330 \\
\hline 43. & 4-Nitroaniline & $100-01-6$ & 50 & 1600 \\
\hline 44. & 4,6-Dinitro-2-methylphenol & $534-52-1$ & 50 & 1600 \\
\hline 45. & $\mathrm{~N}$-nitrosodiphenylamine & $86-30-6$ & 10 & 330 \\
\hline 46. & 4-Bromophenyl-phenylether & $101-55-3$ & 10 & 330 \\
\hline 47. & Hexachlorobenzene & $118-74-1$ & 10 & 330 \\
\hline 48. & Pentachlorophenol & $87-86-5$ & 50 & 1600 \\
\hline
\end{tabular}


Table 3-7. (continued).

\begin{tabular}{|c|c|c|c|c|}
\hline & & & Qual & n Limits ${ }^{\mathrm{a}}$ \\
\hline & & & Water & $\begin{array}{c}\text { Low } \\
\text { Soil/Sediment }{ }^{b}\end{array}$ \\
\hline & Semivolatiles & CAS Number & $\mathrm{ug} / \mathrm{L}$ & $\mathrm{ug} / \mathrm{Kg}$ \\
\hline 49. & Phenanthrene & $85-01-8$ & 10 & 330 \\
\hline 50. & Anthracene & $120-12-7$ & 10 & 330 \\
\hline 51. & Di-n-butylphthalate & $84-74-2$ & 10 & 330 \\
\hline 52. & Fluoranthene & $206-44-0$ & 10 & 330 \\
\hline 53. & Pyrene & $129-00-0$ & 10 & 330 \\
\hline 54. & Butylbenzylphthalate & $85-68-7$ & 10 & 330 \\
\hline 55. & 3,3'-Dichlorobenzidine & $91-94-1$ & 20 & 660 \\
\hline 56. & Benzo(a) anthracene & $56-55-3$ & 10 & 330 \\
\hline 57. & Chrysene & 218-01-9 & 10 & 330 \\
\hline 58. & bis(2-Ethylhexyl)phthalate & $117-81-7$ & 10 & 330 \\
\hline 59. & Di-n-octylphthalate & $117-84-0$ & 10 & 330 \\
\hline 60. & Benzo(b)fluoranthene & $205-99-2$ & 10 & 330 \\
\hline 61. & Benzo(k)fluoranthene & 207-08-9 & 10 & 330 \\
\hline 62. & Benzo(a)pyrene & $50-32-8$ & 10 & 330 \\
\hline 63. & Indeno(1,2,3-cd)pyrene & $193-39-5$ & 10 & 330 \\
\hline & $\operatorname{Dibenz}(\mathrm{a}, \mathrm{h})$ anthracene & $53-70-3$ & 10 & 330 \\
\hline 65. & Benzo(g,h,i)perylene & $191-24-2$ & 10 & 330 \\
\hline
\end{tabular}

a. Quantitation limits listed for soil/sediment are based on wet weight. The quantitation limits calculated by the laboratory for soil/sediment, calculated on dry weight basis as required by the contract, will be higher.

b. Medium Soil/Sediment Contract Required Quantitation Limits (CRQL) for SemiVolatile TCL Compounds are 60 times the individual Low Soil/Sediment CRQL. 
Table 3-8. Contract required detection limits for metals.

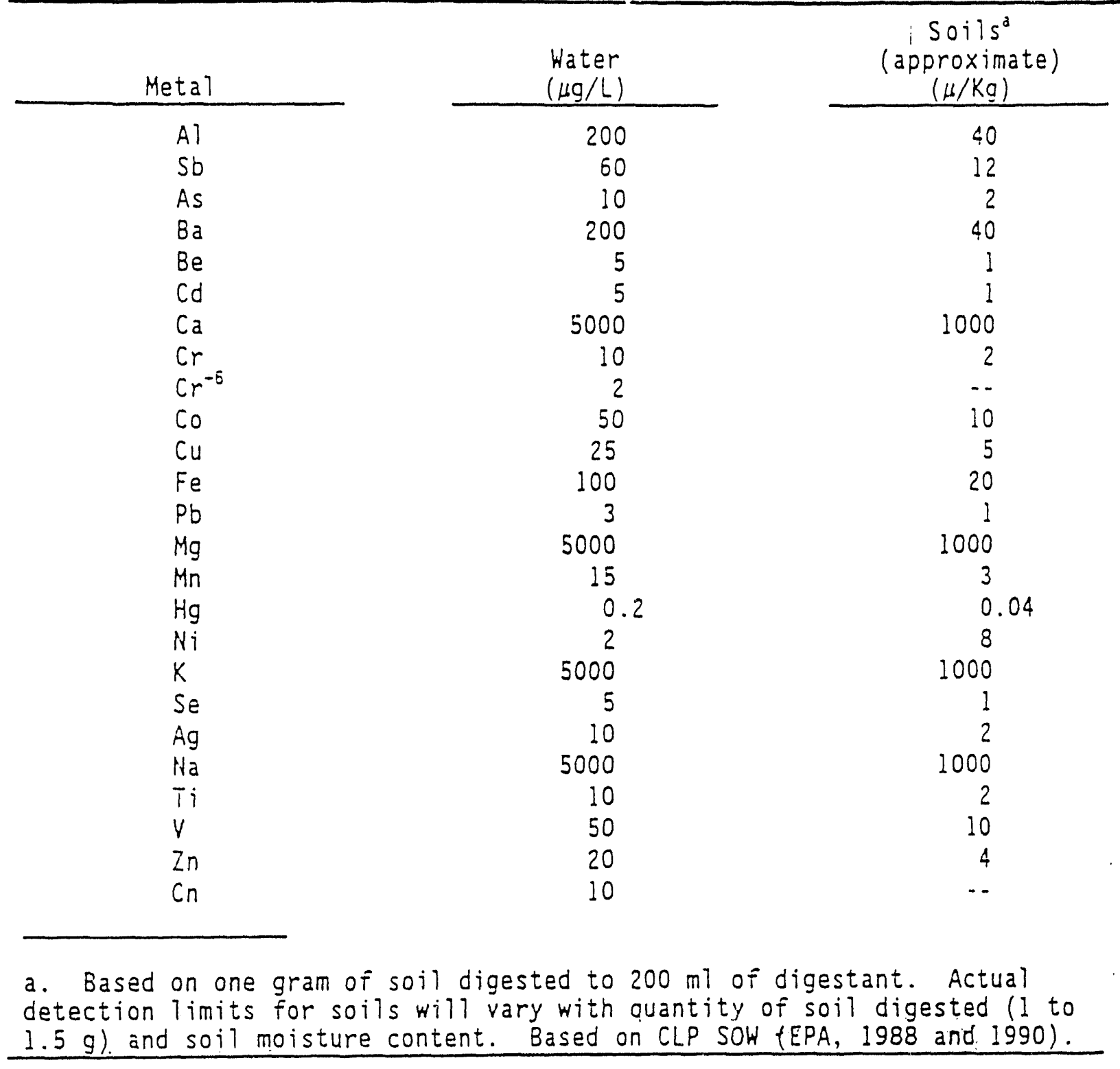


Table 3-9. EPA Method 524.2 (Rev. 3.0) target analyte iist and method detection limits.

\begin{tabular}{|c|c|c|c|}
\hline \multirow[b]{2}{*}{ Volatiles } & \multirow[b]{2}{*}{ CAS number } & \multicolumn{2}{|c|}{ Method detection limits ${ }^{\mathbf{a}, \mathbf{b}}$} \\
\hline & & $\begin{array}{l}\text { Wide bore } \\
\text { column } \\
(\mu g / L)\end{array}$ & $\begin{array}{c}\text { Narrow bore } \\
\text { column } \\
(\mu \mathrm{g} / \mathrm{L})\end{array}$ \\
\hline $\begin{array}{l}\text { 1. Dichlorodifluoromethane } \\
\text { 2. Chloromethane } \\
\text { 3. Vinyl Chloride } \\
\text { 4. Bromomethane } \\
\text { 5. Chloroethane }\end{array}$ & $\begin{array}{l}75-71-8 \\
74-87-3 \\
75-01-4 \\
74-83-9 \\
75-00-3\end{array}$ & $\begin{array}{l}0.10 \\
0.13 \\
0.17 \\
0.11 \\
0.10\end{array}$ & $\begin{array}{l}0.11 \\
0.05 \\
0.04 \\
0.06 \\
0.02\end{array}$ \\
\hline $\begin{array}{l}\text { 6. 1,1-Dichloroethene } \\
\text { 7. Methylene Chloride } \\
\text { 8. trans-1,2-Dichloroethene } \\
\text { 9. 1,1-Dichiorcethane } \\
\text { 10. 2,2-Dichloropropane }\end{array}$ & $\begin{array}{r}75-35-4 \\
75-04-2 \\
150-60-5 \\
75-34-3 \\
590-20-7\end{array}$ & $\begin{array}{l}0.12 \\
0.03 \\
0.06 \\
0.04 \\
0.35\end{array}$ & $\begin{array}{l}0.05 \\
0.09 \\
0.03 \\
0.03 \\
0.05\end{array}$ \\
\hline $\begin{array}{l}\text { 11. cis-1,2-Dichloroethene } \\
\text { 12. Chloroform } \\
\text { 13. Bromochloromethane } \\
\text { 14. 1,1,1-Trichloroethane } \\
\text { 15. Carbon Tetrachloride }\end{array}$ & $\begin{array}{r}156-69-4 \\
67-66-3 \\
74-97-5 \\
71-55-6 \\
50-23-5\end{array}$ & $\begin{array}{l}0.12 \\
0.03 \\
0.04 \\
0.08 \\
0.21\end{array}$ & $\begin{array}{l}0.06 \\
0.02 \\
0.07 \\
0.04 \\
0.08\end{array}$ \\
\hline $\begin{array}{l}\text { 16. 1,1-Dichloropropene } \\
\text { 17. Eenzene } \\
\text { 18. 1,2-Dichloroethane } \\
\text { 19. Trichloroethene } \\
\text { 20. 1,2-Dichloropropane }\end{array}$ & $\begin{array}{l}563-58-6 \\
71-43-2 \\
107-06-2 \\
79-01-6 \\
78-87-58\end{array}$ & $\begin{array}{l}0.10 \\
0.04 \\
0.06 \\
0.19 \\
0.04\end{array}$ & $\begin{array}{l}0.02 \\
0.03 \\
0.02 \\
0.02 \\
0.02\end{array}$ \\
\hline $\begin{array}{l}\text { 21. Bromodichloromethane } \\
\text { 22. Dibromomethane } \\
\text { 23. trans-1,3-Dichloropropene } \\
\text { 24. Toluene } \\
\text { 25. cis-1,3-Dichloropropene }\end{array}$ & $\begin{array}{r}75-27-4 \\
74-95-3 \\
10061-02-6 \\
108-88-3 \\
10061-01-5\end{array}$ & $\begin{array}{l}0.08 \\
0.24 \\
N D \\
0.11 \\
\text { ND }\end{array}$ & $\begin{array}{l}0.03 \\
0.03 \\
N D \\
0.08 \\
N D\end{array}$ \\
\hline $\begin{array}{l}\text { 26. 1,1,2-Trichloroethane } \\
\text { 27. Tetrachloroethene } \\
\text { 28. 2,3-Dichloropropane } \\
\text { 29. Dibromochloromethane } \\
\text { 30. 1,2-Dibromoethane }\end{array}$ & $\begin{array}{l}79-00-5 \\
127-18-4 \\
142-28-9 \\
124-48-1 \\
106-93-4\end{array}$ & $\begin{array}{l}0.10 \\
0.14 \\
0.04 \\
0.05 \\
0.06\end{array}$ & $\begin{array}{l}0.03 \\
0.05 \\
0.04 \\
0.07 \\
0.02\end{array}$ \\
\hline $\begin{array}{l}\text { 31. Chlorobenzene } \\
\text { 32. 1,1,2,2-Tetrachloroethane } \\
\text { 33. Ethylbenzene } \\
\text { 34. Xylene (total meta \& para) } \\
\text { 35. Xylene (ortho) }\end{array}$ & $\begin{array}{r}108-90-7 \\
630-20-6 \\
100-41-4 \\
1330-20-7 \\
95-47-6\end{array}$ & $\begin{array}{l}0.04 \\
0.05 \\
0.06 \\
0.13 \\
0.11\end{array}$ & $\begin{array}{l}0.03 \\
0.04 \\
0.03 \\
0.06 \\
0.06\end{array}$ \\
\hline
\end{tabular}




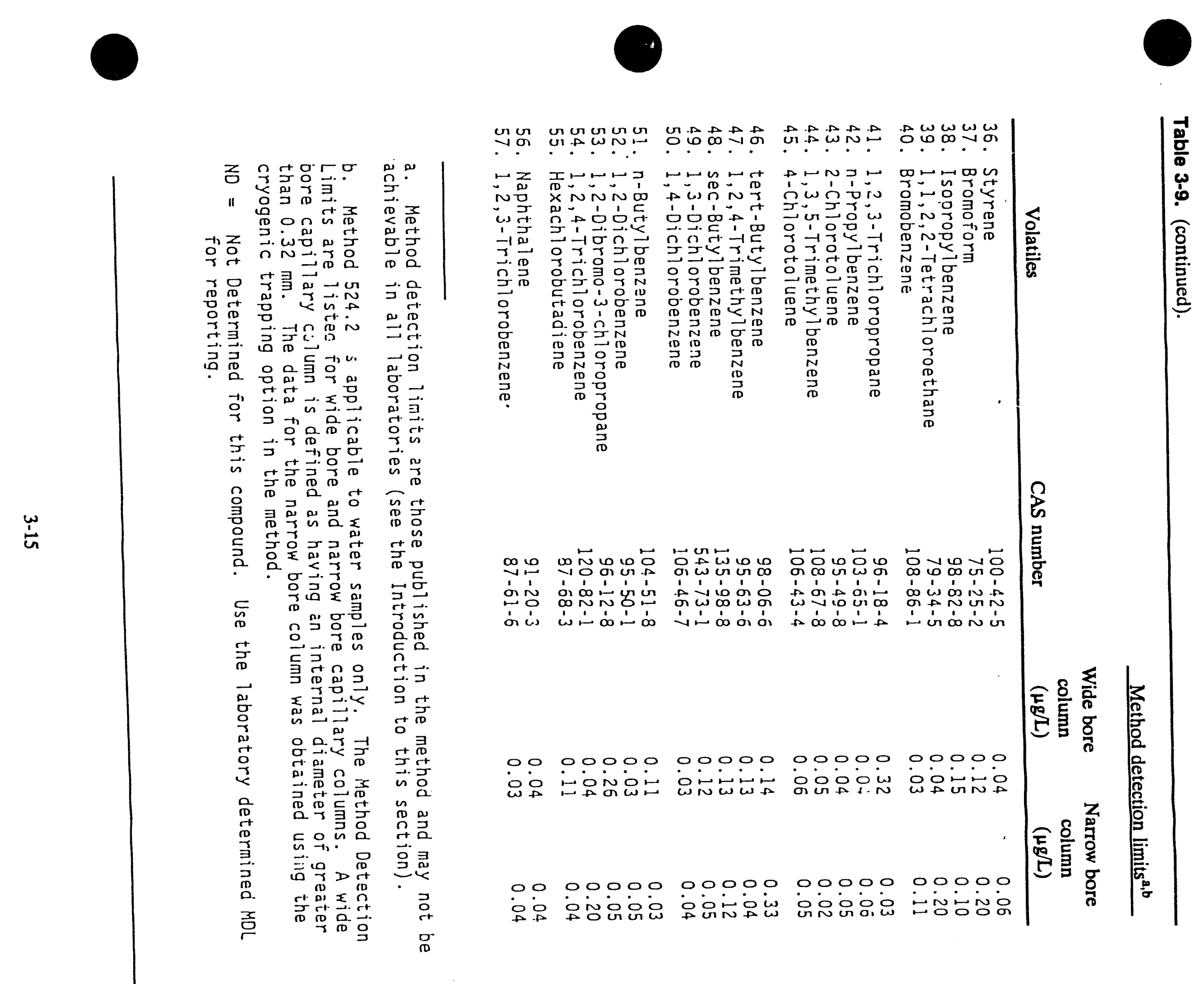


entire landfill area (Figure 3-1b). Magnetic field measurements will be used to map the distribution of buried ferrous objects. These measurements will be made on a regular grid of 1.6 $\times 3 \mathrm{ft}(0.5 \times 1 \mathrm{~m})$ over the entire survey area (Figure 3-1c). These sampling grids should allow for the clear definition of the landfill area and the individual trenches making up Landfill III. Ground penetrating radar will be used to determine the overburden cover thickness and random borings will be drilled to confirm the results. The depth and topography of the underlying basalt at Landfill II will also be determined using seismic refraction. Depth estimates will be collected every $16.4 \mathrm{ft}(5 \mathrm{~m})$ along each profile with $100 \mathrm{ft}(30.6 \mathrm{~m})$ between profiles (Figure 3-2).

\subsection{Soil Gas Sampling}

\subsubsection{Shallow Soil Gas Survey}

A shallow soil gas survey [less than $5 \mathrm{ft}(1.5 \mathrm{~m})$ deep] will be carried out across Landfills II and III. A regularly spaced sampling grid on $100-\mathrm{ft}(30.6-\mathrm{m})$ centers will be used over an area completely enclosing landfill III and along the perimeter of the landfill (Figure 3-3). The regularly spaced grid and the grid size used for the soil gas sampling is based on the nature of the landfill with regularly spaced trenches and no known hot spots (EPA 1991). A regularly spaced sampling grid on $100-\mathrm{ft}(30.6-\mathrm{m})$ centers will also be used over an area completely enclosing Landfill II (Figure 3-4) (EPA 1991). All samples will be analyzed according to EPA Method 601 (see Table 3-5) using a gas chromatograph equipped with either an electron capture detector (GC/ECD) or a flame ionization detector (GC/FID). Specific analytes standardized for the GC/FID analysis will include methane, benzene, and acctone.

QA/QC samples are presented in Table 3-3. Analytical support level III data will be acquired for all samples and will be used in the fate and transport modeling efforts in support of a qualitative evaluation of the air pathway for the baseline risk assessment. Procedures for the soil gas investigation are provided in Section 5.3.

\subsubsection{Borehole Soil Gas Sampling}

Borehole soil gas samples will be collected from the nine existing, instrumented boreholes at both Landfill II and III (Figure 3-5) to provide information on the concentrations of vapor phase volatile organics and methane in the subsurface soils. Sample collection, analysis, and $\mathrm{QA} / \mathrm{QC}$ will be as described for the shallow soil gas survey.

\subsubsection{Surface-Flux Chamber Sampling}

VOC cmission rates from the landfill surface will be determined using an emission flux chamber (EPA 1986). The chamber is placed on the soil surface and air of known purity is swept through the chamber at a known, constant rate. Air samples are collected at the exit of the chamber and VOC concentrations are determined by gas chromatography. The VOC emission rate is calculated as: 

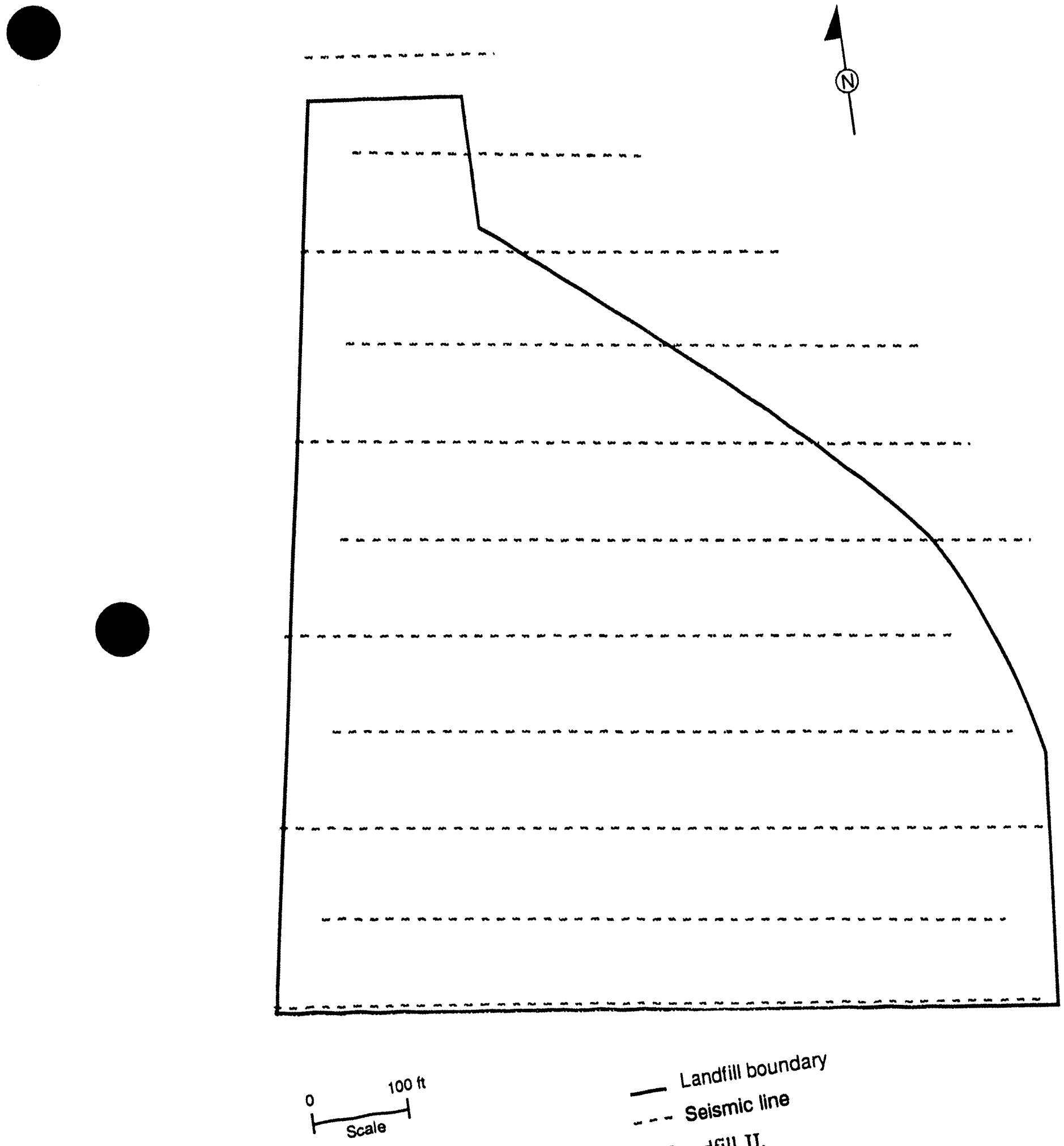

Figure Seismic refraction grid for Landfill II.

$3-17$ 


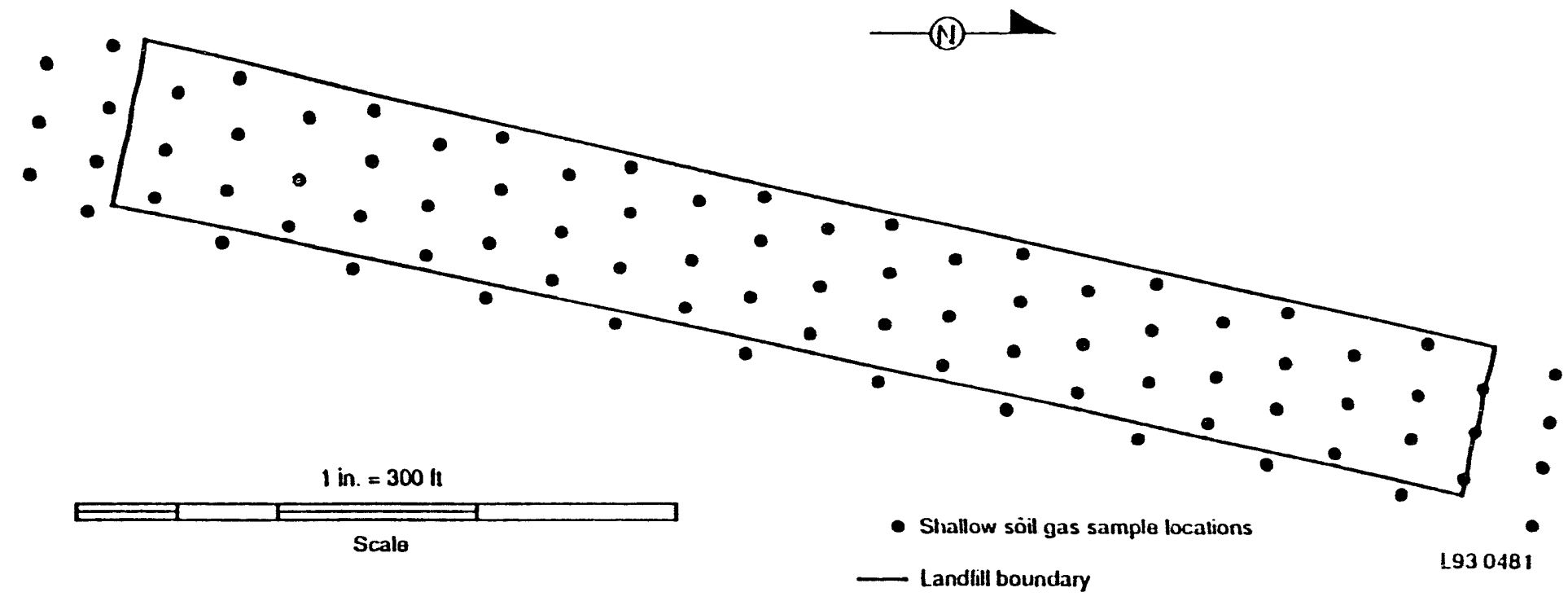

Figure 3-3. Shallow soil gas sample locations for Landfill III. 


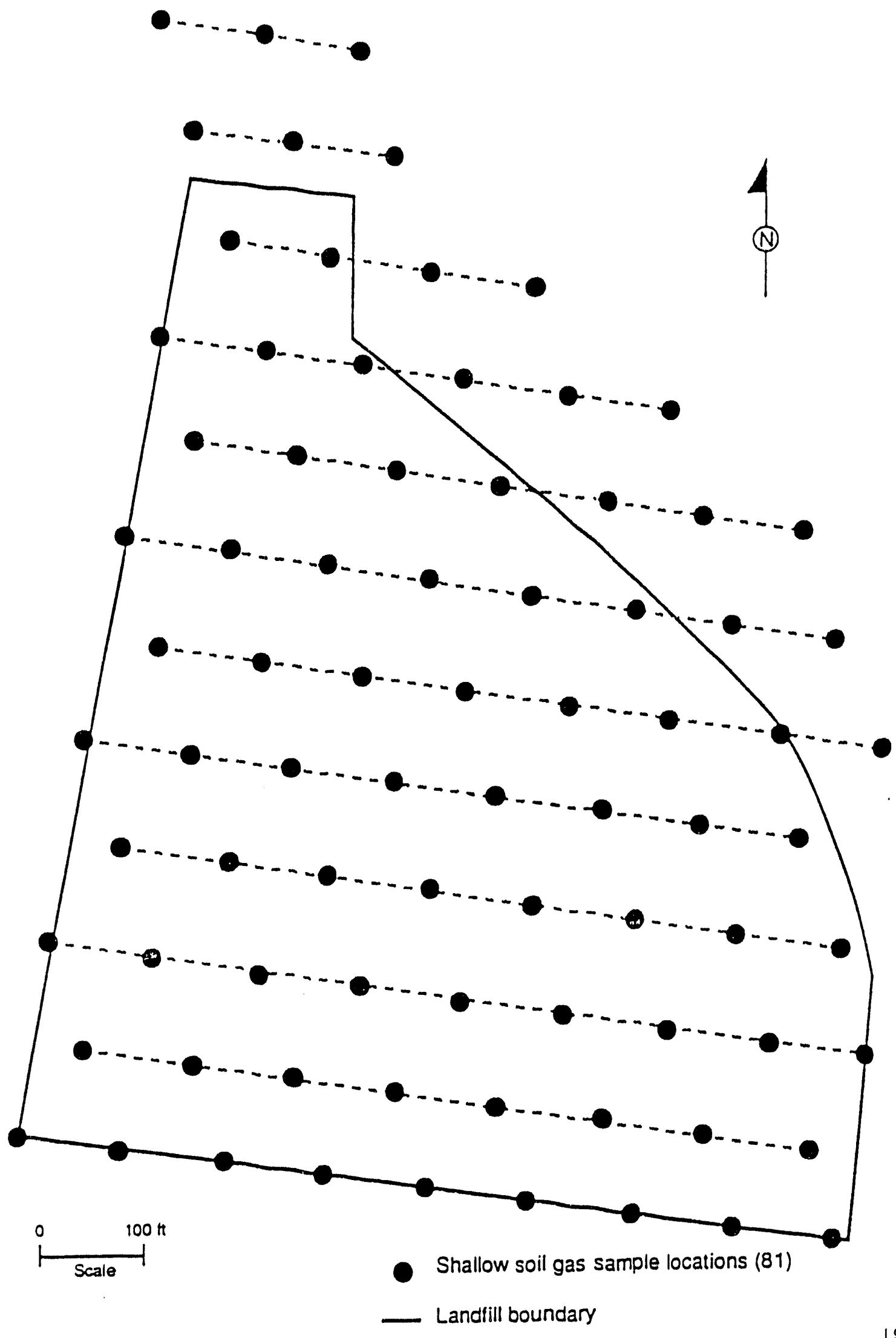

L93 0480

Figure 3-4. Shallow soil gas sample locations for Landfill II. 

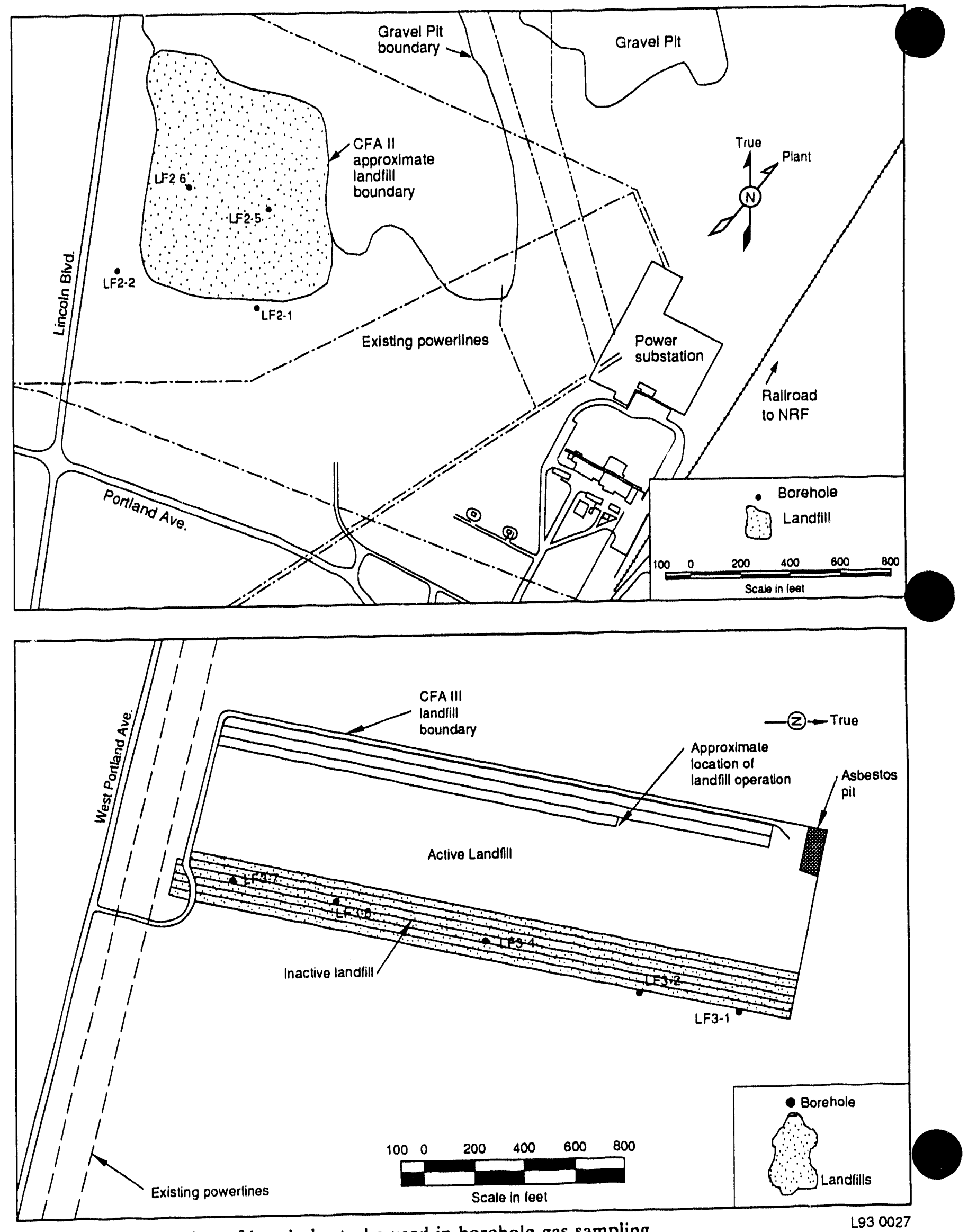

Figure 3-5. Location of boreholes to be used in borehole gas sampling. 


$$
E_{i}=\frac{Q C_{i}}{A}
$$

where

$\mathrm{E}_{\mathrm{i}}=$ emission rate of compound $\mathrm{i}, \mu \mathrm{g} / \mathrm{m}^{2}-\mathrm{s}$

$\mathrm{C}_{\mathrm{i}}=$ concentration of compound $\mathrm{i}$ in exhaust gas, $\mu \mathrm{g} / \mathrm{m}^{3}$

$\mathrm{Q}=$ volumetric air flow rate, $\mathrm{m}^{3} / \mathrm{s}$

$\mathrm{A}=$ chamber surface area, $\mathrm{m}^{2}$.

A minimum of eight sampling sites per landfill will be selected. Sampling locations will be selected based on any hot spots identified during the shallow soil gas survey. If the shallow soil gas data are not available at the time of sampling or hot spots are not identified, sample locations will be randomly selected. All samples will be analyzed according to EPA Method TO-14 (EPA 1988).

\subsection{Surface Soil Sampling}

Surface soil will be collected from the soil cover of each landfill for analysis of volatile organics, semivolatile organics, and metals. At least one sample per acre will be collected for analysis (EPA 1991). The actual sampling locations will correspond to randomly selected grid points from among the points that were established for the shallow soil gas survey. Before sampling is initiated, the soils exposed at the surface will be examined visually by a soil scientist for evidence of staining, signs of vegetation stress, and differences in soil type to aid in sample site selection. The geophysical surveys may also provide data on anomalies or zones of surficial soil that warrant investigation. The results from soil gas collected in FY-88 from the instrumented boreholes and from the shallow soil gas survey performed on CFA Landfill II in FY-89 will also be used to select sample sites.

Samples for CLP metals (including cyanide for Landfill II samples) will be collected from the 0- to 6-in. (0- to $15-\mathrm{cm})$ depth to characterize direct exposure risks. Samples for volatile and semivolatile organics will be collected from the 18- to 24-in. (46- $1061-\mathrm{cm}$ ) depth because these compounds tend to volatilize from the soil at shallower depths.

\subsection{Cover Characterization of Landfill III}

The soil cover material on Landfill III will be sampled for physical and hydraulic properties testing, and to determine cover material thickness, if the ground penetrating radar survey was unsuccessful in determining cover thickness (Table 3-1). A total of 12 random sampling sites will be selected based on the collection of at least one sample per acre (EPA 1991). Actual sampling sites will be the same as those identified for the surface soil sampling (see Section 3.4). In situ measurements of the soil cover at each of the 12 sites will include density (ASTM D-2922), moisture content (ASTM D-3017), and cover thickness. Measurements will be taken at 4- and 8 -in. (10- and 20-cm) depths. Samples from 8 (to be chosen randomly) of the 12 sites will also be collected at $1-\mathrm{ft}(0.3-\mathrm{m})$ depth increments for laboratory analysis of gravimetric water content (ASTM D-2216) and particle size analysis (ASTM D-422). 
Undisturbed soil samples will be collected at eight of the 12 sites (to be chosen randomly) for analysis of constant-head permeability (ASTM D-2434), capillary pressure-moisture content relationships (ASTM D-2325), and porosity. Samples will be collected by driving a thin-walled sampler (ASTM D-1587) a minimum of 8 in. $(20 \mathrm{~cm})$ into the ground. In situ drainage tests will also be performed at the eight sites. Samples collected during the in situ drainage tests will be analyzed for gravimetric water content (ASTM D-2216). Detailed procedures for the soil cover investigation are provided in Section 5.5.

The moisture content of the soil cover and the landfill soils will be monitored over time using a neutron probe moisture gauge. Neutron probe measurements (SOP) of soil moisture will be made monthly during the RI field activities at 6 -in. $(15-\mathrm{cm})$ depth intervals using the existing neutron probe access tubes at Landfill II (LF2-03, LF2-04, and LF2-07) and at Landfill III (LF3-03 and LF3-05). Additionally, the toe slope at Landfill II will be inspected weekly. If seepage/leachate is observed, samples will be collected for volatile organic compound (VOC), semivolatile organic compound (SVOC), and metals analysis.

\subsection{Water Level Measurements and Borehole Deviation Logging}

The regional groundwater flow at CFA is in a south-to-southwest direction. However, regional flow may be affected by infiltration from percolation ponds at ICPP and aquifer heterogeneities. These effects could have a direct bearing on the direction and rate of contaminant migration. Thus, it is important to understand the groundwater flow system at CFA. Monthly water level measurements will be collected from available wells (Table 3-10 and Figure 3-6), within approximately a $1-\mathrm{mi}(1.6-\mathrm{km})$ radius of the CFA landfills. Monthly water level measurements will begin in May 1993 and continue through December 1993.

Borehole deviation logs will be run by the USGS on the landfill wells (LF designator), and if possible, the USGS wells identified in Table 3-10. Prior to conducting deviation surveys, the dedicated sampling pumps installed in the wells will be removed. Information obtained from the logging effort will be used to correct the measured depth to water for borehole deviation effects.

Table 3-10. Wells to be measured on a monthly basis for water level data.

$\begin{array}{lll}\text { LF } 2-08 & \text { USGS-020 } & \text { USGS-082 } \\ \text { LF 2-09 } & \text { USGS-034 } & \text { USGS-085 } \\ \text { LF 2-10 } & \text { USGS-035 } & \text { USGS-111 } \\ \text { LF 2-11 } & \text { USGS-036 } & \text { USGS-112 } \\ \text { LF 2-12 } & \text { USGS-037 } & \text { USGS-113 } \\ \text { LF 3-08 } & \text { USGS-038 } & \text { USGS-114 } \\ \text { LF 3-09 } & \text { USGS-039 } & \text { USGS-115 } \\ \text { LF 3-10 } & \text { USGS-057 } & \text { USGS-116 } \\ \text { LF 3-11 } & \text { USGS-077 } & \end{array}$




\subsection{Groundwater Sampling}

Three rounds of groundwater samples will be collected and analyzed from a network of 10 monitoring wells located both upgradient and downgradient from CFA Landfills II and III and from two production wells used for drinking water at CFA (sce Table 3-11 and Figure 3-6). The first sampling event will take place in the May-June time frame, with the second and third events targeted for July and October, respectively. The results of each sampling event will be evaluated by DOE, EPA, and IDHW in order to determine the scope and/or need of each subsequent event. Analytical results from the first two rounds of sampling will be used in the baseline risk assessment, and the results from the third sampling event will be used for confirmation.

Existing landfill monitoring wells will be sampled for volatile organics (EPA 524.2), CLP metals and hexavalent chromium (SW 846-7195) (unfiltered and filtered), cyanide, nitrate, sulfate, chloride, fluoride, and alkalinity. A sample from each well will also be collected for analysis of gamma-emitting radionuclides. These sampler will be submitted to the Radiation Measurements Laboratory at the INEL for analysis, with subsequent data used to establish shipping procedures for samples being sent to offsite laboratories. QA/QC samples to be collected and analyses to be performed are presented in Tables 3-3 and 3-4, respectively. Target analyte lists for metals and volatile organics are provided in Tables $3-8$ and $3-9$, respectively.

\subsection{Subsurface Soil Sampling of Landfill II}

Seven auger holes (B-1 through B-7) will be drilled and continuously sampled (with a splitspoon sampler) from land surface to the top of basalt (Figure 3-7). Borehole sites were selected primarily from the results of the 1989 shallow soil gas survey and also to provide spatial coverage across the landfill. The approximate coordinates of the seven boreholes are provided in Table 3-12. Actual borchole locations will be surveyed after they are completed. Each split spoon sample will be field screened ( $\mathrm{HNu}$ ) and visually examined for signs of staining and discoloration. Samples will be selected for laboratory analysis based on field screcning results (see Section 5.8). However, a minimum of two samples per borehole will be submitted for analysis: one from within the waste unit and one from below the waste unit. The soil samples will be analyzed for CLP volatile organics, semivolatile organics, and metals (including cyanide).

If leachate is encountered in any of the boreholes during augering, samples will be collected and submitted for CLP volatile organic, semivolatile organic, and metals analysis. Leachate wells will be installed in these boreholes for long-term leachate monitoring purposes. 
Table 3-11. Monitoring wells to be sampled.

Landfill II:

Landfill III:

Production Wells:
Downgradient - LF 2-8, LF 2-9, LF 2-10, LF 2-12 Upgradient - LF 2-11

Downgradient - LF 3-8, LF 3-9, LF 3-10

Upgradient - USGS-85, LF 3-11

CFA-1, CFA-2

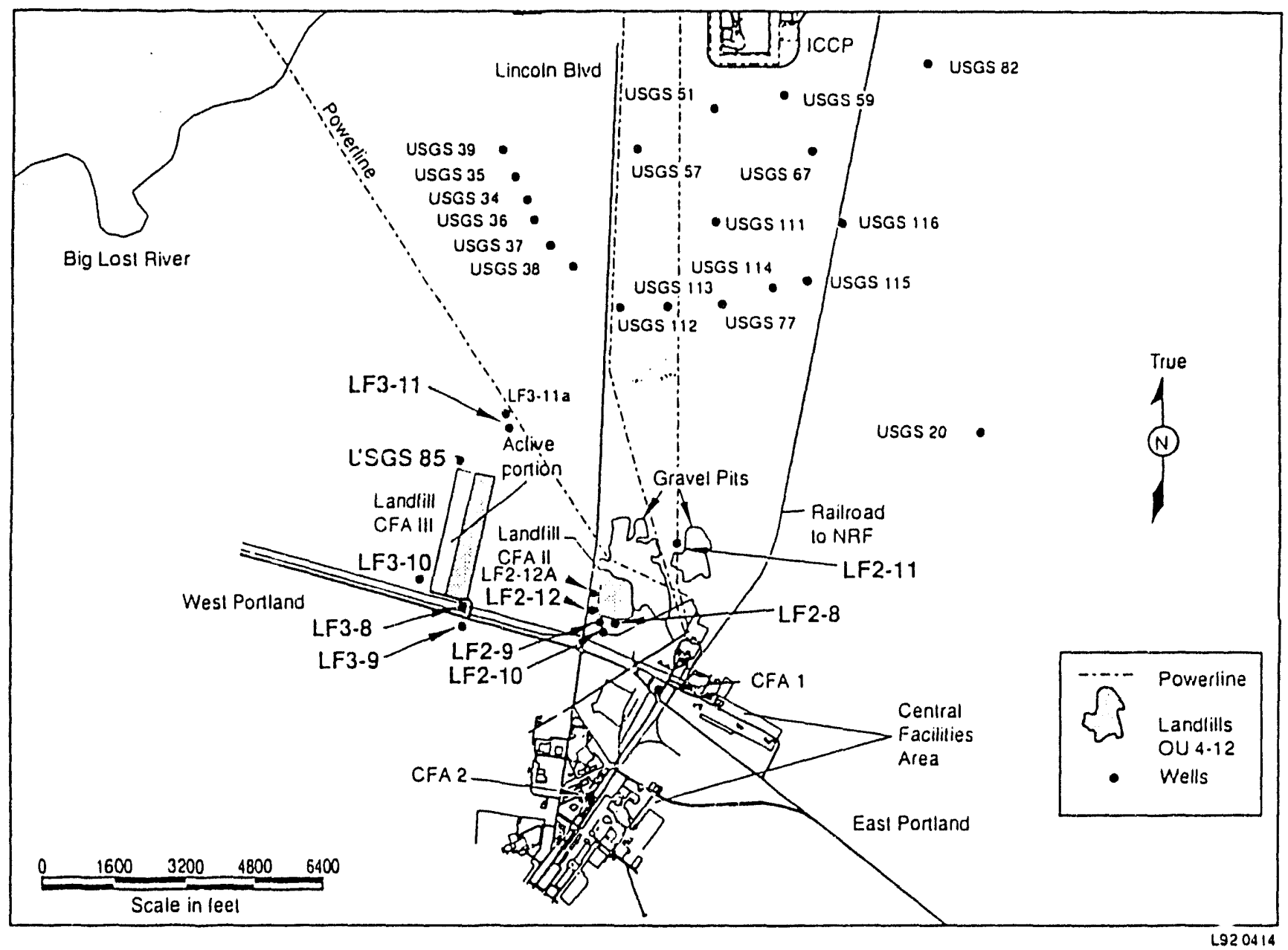

Figure 3-6. Locations of CFA monitoring wells for water level measurements and groundwater sampling (bold). 
Table 3-12. Approximate coordinate locations of the landfill boreholes.

\begin{tabular}{ccc}
\hline Borehole & Northing & Easting \\
\hline B-1 & 683900 & 294300 \\
B-2 & 683625 & 294200 \\
B-3 & 683575 & 294450 \\
B-4 & 683450 & 294500 \\
B-5 & 683300 & 294250 \\
B-6 & 683300 & 294450 \\
B-7 & 683275 & 294700 \\
\hline
\end{tabular}

\subsection{Quality Assurance}

Internal quality control $(\mathrm{QC})$ checks are established by submitting $\mathrm{QC}$ samples to the analytical laboratory. The types and frequency of collection for field quality control samples are discussed in the QAPjP and Table 3-3 of the FSP.

The sampling and analysis plan tables (Appendix A) provide the anticipated number, type, and analysis of all samples including the field QA/QC samples. The actual number and type of samples collected will be presented in the remedial investigation report following the completion of field activities. 


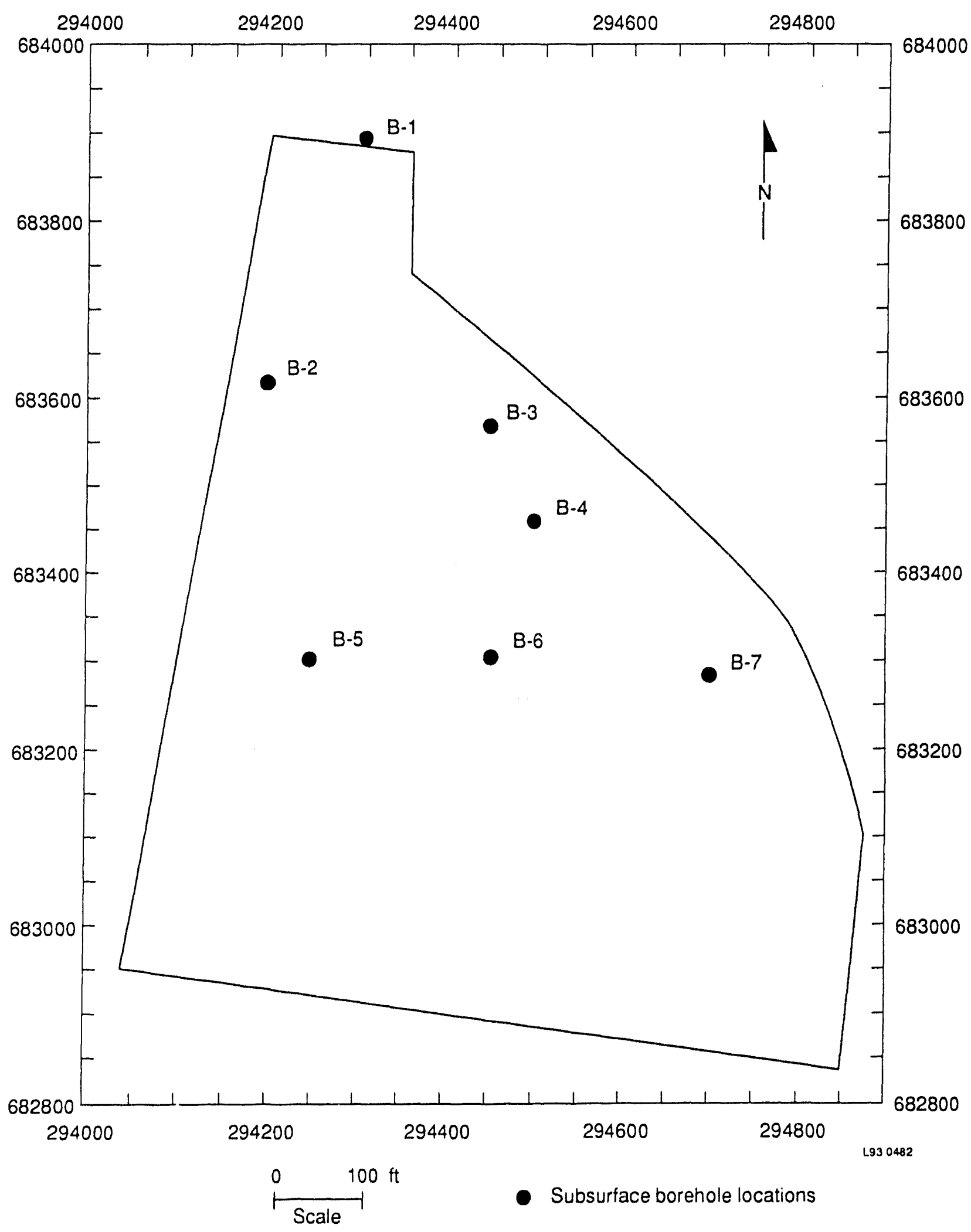

Figure 3-7. Subsurface soil boring sites - Landfill II. 


\section{SAMPLE IDENTIFICATION SYSTEM}

\subsection{Sample Identification Code}

A systematic character sample identification code will be used to uniquely identify all samples. The uniqueness of the number is required for maintaining consistency and ensuring no two samples are assigned the same identification code.

The first two designators of the code, $4 \mathrm{C}$, refers to the waste area group (WAG 4) and INEL site location (CFA). The third designator, $Q$, refers to the sample type or location. The next three numbers designate the sequential sample number for the project. A two character set (i.e., 01,02 , etc.) will be used next to designate replicate samples. The last two characters refer to a particular analysis (and bottle type). Refer to Section 6 (Tables 6-1 and 6-2) for specific aliquot/bottle code designations.

For examrle, a groundwater sample collected from the aquifer might be designated as 4 CQ00101DV where (from left to right):

- $\quad 4 \mathrm{C}$ designates the waste area group as CFA

- $\quad$ Q designates that it is an aquifer sample

- 001 designates the sequential sample number

- 01 designates type of sample (i.e., replicate)

- DV designates VOC analysis by method 524.2.

A sampling and analysis plan table/data base will be used to record all pertinent information (well designation, borehole number, depth, media, date, etc.) associated with each sample identification code. Sampling and analysis plan tables for the OU 4-12 RI/FS are presented in Appendix A of this FSP. The following section describes the table/data base.

\subsection{Sampling and Analysis Plan Table/Data Base}

\subsubsection{General}

The sampling and analysis plan (SAP) table/data base format was developed to facilitate the reporting of SAP information to the data management group. The table is used as a planning tool for sample tracking, and as a data base when analyses are received. An example of the SAP table for the CFA Landfill II and III remedial investigation effort is shown in Table 4-1. The following sections describe the information found in the SAP table/data base. 
Table 4-1. Example of a sampling and analysis plan table for chemical analysis (also see Appendix A).

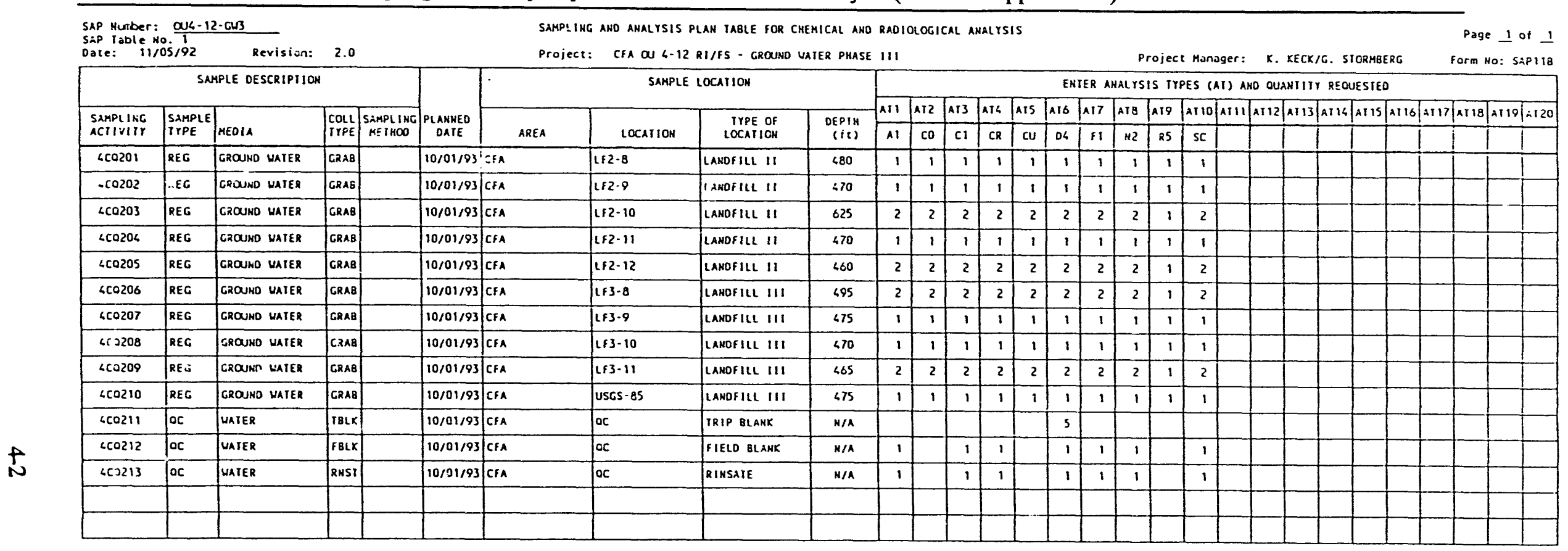

Enter the appropriate analysis type code in the boxes between the double lines under "ENTER AMALYSSIS Trpes". Refer to SAP Table 2. Sampling And Analysis Plan Table - Codes 1 Descriptions.

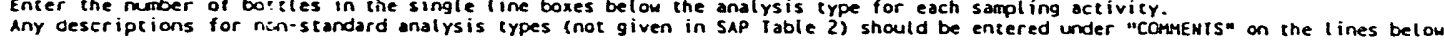

COMAEXIS
AII: Alkalinity
AI2: CLP Melals - Filleered
133: cLP metals - Unfilitered
114: Chromium vI $(C r \cdot 6)$ - Unfiltered
ATS: Chromiun vi $(\mathrm{Cr} \cdot 6)$ - Filleerec
A16: Orinking yater wians (526.2) iv
A17: Fluoride
A18: Nicrace/nitrice
A19: Camb screen
Al10: Sulface/chloride

AIII:

AI12:

AI13: 


\subsubsection{Sample Description Fields}

The sample description fields contain information relating individual sample characteristics.

\section{Sample Number}

The sample number is used to link information from other sources (field data, analytical data, etc.) to the information in the SAP table for data reporting, sample tracking, and completeness reporting. The sample number is also used by the analytical lab to track and report analytical results.

\section{Sample Type}

Sample type is a four-character field. Data in this field will be selected from the following:

REG for a regular sample

QC for a quality control sample

Media

Media is a 15-character field. Data in this field will be selected from the following:

SOIL

SUBSURFACE SOIL

GROUNDWATER

SOIL GAS

WATER for other waters (e.g., rinsates, field blanks, trip blanks)

\section{Collection Type}

Collection type is a four-character field. Data in this field will be selected from the following:

$\begin{array}{ll}\text { GRAB } & \text { for grab } \\ \text { COMP } & \text { for composite } \\ \text { TBLK } & \text { for trip blanks } \\ \text { RNST } & \text { for equipment rinsates } \\ \text { FBLK } & \text { for field blanks }\end{array}$

Sampling Method

Information in the sampling method field identifies a standard sampling method by number. If the sampling method is nonstandard, this field should reference the comment field where a brief explanation of the sampling method should be entered. 


\section{Planned Date}

The date or an eight character event identifier relating to the planned collection of the sample (i.e., 04/01/93) should be entered here.

\subsubsection{Sample Location Fields}

This grcup of fields identifies the exact location for the sample in three-dimensional space, starting with the general AREA and narrowing the focus to an exact location geographically, then specifying the depth in the DEPTH field.

\section{Area}

The AREA field is a 15-character field that identifies the general sample collection area. This field should contain the standard identifier for the INEL area being sampled. For this investigation all samples are being collected in the Central Facilities Area and thus the area identifier will be "CFA".

\section{$\underline{\text { Location }}$}

This is a 15-character descriptive field that may contain geographical coordinates, $x-y$ coordinates, building numbers, or other location identifying details. It also contains program specific information such as a borehole or well number. Data in this field will normally be subordinated to the AREA. This information is included on the labels generated by data management to aid sampling personnel.

\section{Type of Location}

The type of location field is a 15-character field containing information descriptive of the exact sample location. Information in this field may overlap that in the location field, but it is intended to add detail to the location.

\section{Depth}

The depth of a sample location is the distance in feet from surface level or a range in feet from the surface. The depth field is 10 characters wide.

\subsubsection{Analysis Types}

\section{AT1-AT10, etc.}

These fields are used to indicate analysis types (radiological, chemical, hydrological, etc.). Space is provided at the bottom of the form to clearly identify each type. A standard abbreviation should also be provided if possible. 


\section{SAMPLING EQUIPMENT AND PROCEDURES}

This section addresses the sampling equipment and procedures to be used for the planned field investigations, which were identified during the scoping process to fill identified data gaps. These field investigations can be grouped into the following:

- Topographic survey of Landfill III

- Geophysical survey of Landfills II and III

- Soil gas surveys of Landfills II and III

- Surface soil sampling of Landfills II and III

- Cover characterization of Landfill III

- Water level measurements and borehole deviation logging

- Groundwater sampling

- Subsurface soil sampling of Landfill II.

The following text describes the planned field investigations. All sample locations will be staked and surveyed. Standard Operating Procedures (SOPs) for the individual field tasks can be found in Appendix B.

\subsection{Topographic Survey of Landfill III}

A topographic map of Landfill III will be prepared. The field survey will use the sample locations from the shallow soil gas investigation as the initial survey grid. Horizontal control for the survey will be class 3 [distance accurate to $0.007 \mathrm{ft}(0.002 \mathrm{~m})$ ], and the vertical control will be $3 \mathrm{rd}$ order $[0.05 \mathrm{ft}(0.015 \mathrm{~m})]$. If this survey grid does not provide adequate horizontal and vertical control to compile a topographic map with $1 \mathrm{ft}$ contour intervals at a scale of 1 in. $(2.54 \mathrm{~cm})$ equals $100 \mathrm{ft}(30.6 \mathrm{~m})$, then additional survey points will be necessary to further define the topography of the site. Locations for additional survey points will be determined in the field based on the surveyor's judgement. A topographic map covering the 11 acres ( 4 hectares) of the site and immediate surrounding area will be prepared consisting of contour intervals not to exceed $1 \mathrm{ft}(0.3 \mathrm{~m})$ and with a scale of 1 in. $(2.54 \mathrm{~cm})$ equals $100 \mathrm{ft}(30.6 \mathrm{~m})$. A map of the surfacewater drainage system in this area will also be prepared. The topographic survey will be performed using the standard operating procedures of the survey subcontractor (MK Ferguson of Idaho).

\subsection{Geophysical Survey of Landfills II and III}

Seismic refraction methods will be used following the standard operating procedure (SOP 11.14.2) provided in Appendix B of the FSP to determine the thickness of surficial sediments and the general topography of the underlying basalt at both landfills. Induced electromagnetic measurements will be used following the standard operating procedure (SOP 11.14.1) provided in Appendix B of the FSP to map concentrations of conductive materials such as steel drums, scrap metal and areas of elevated soil moisture at Landfill III. Magnetic field measurements will be used following the standard operating procedure (SOP 11.14.3) provided in Appendix B of the FSP to map the distribution of buried ferrous objects at Landfill III. Ground penetrating radar will be used to map the overburden cover soil materials indicating depth of 
cover soils at Landfill III. Confirmation horings will also be taken to confirm the ground penetrating radar results. If ground penetrating radar is not successful then soil borings will be taken to determine the depth of cover material.

Data from the geophysical surveys will be used to generate maps showing the topography of the cover soil materials, the basalt surlace, the distribution of electrically conductive bodies and buried magnetic material, and overburden cover thickness.

\subsection{Soil Gas Sampling}

\subsubsection{Shallow Soil Gas Survey}

Each soil gas sample will be collected in a $1 / 2-$ to $1-\mathrm{in} .(1.3-$ to $2.5-\mathrm{cm})$ hole driven to a depth of approximately $4 \mathrm{ft}(1.2 \mathrm{~m})$ using a drive rod or electric hammer drill. The entire sampling system will then be purged with ambient air drawn through a dust and organic vapor cartridge. A stainless steel probe will be inserted to the full depth of the hole and sealed off from the atmosphere. A sample of in situ soil gas will be withdrawn through the probe to purge ambient air from the sampling system. A second sample of soil gas will then be withdrawn through the probe and trapped in a pre-evacuated glass vial at two atmospheres of pressure. The self-sealing vial will be detached from the sampling system, packaged, and sent for laboratory analysis.

Procedures for assuring sample quality are as follows:

1. Prior to each day's activities, all sampling equipment, drive rods and probes will be decontaminated as described in SOP 11.5 for field decontamination of sampling equipment (Appendix B). The only exceptions to this SOP are (a) isopropanol will not be used and (b) the internal probe surfaces will be flushed dry with prepurified nitrogen.

2. The sample probe and apparatus including tubing, valves, and injection needles will be purged with a volume of filtered ambient air approximately 100 times the void space volume of the sampling system prior to each sample extraction.

3. Sampling apparatus is then purged with in situ soil gas approximately 25 void space volumes of the sampling system. This in situ soil gas is used to purge the filtered air from the sampling equipment.

4. Samples are then collected, packaged, and shipped to the laboratory for analysis. Chain of custody and documentation precedures, as discussed in Section 6, will be followed.

Field control samples will be collected at the beginning of each day's activities, after every twentieth soil gas sample per day, and at the end of each day's field activities (see Table 3-3). These samples are obtained by drawing prepurified nitrogen or filtered ambient air through the sampling apparatus with the sample probe attached. The field control samples are labeled and analyzed in the same manner as the actual fiel $/$ samples and are visually indistinguishable from them. Analytical quality control will be asser ed using carrier gas blanks, standards, and duplicate 
analyses after every tenth sample analyzed. Field replicate samples will also be collected at a frequency of 1 per 10 sampling sites.

All samples will be analyzed according to EPA Method 601 using a gas chromatograph equipped with either an ECD or a FID. Specific analytes standardized for FID analysis include methane, benzene, and acetone.

\subsubsection{Borehole Soil Gas Survey}

Borehole soil gas samples will be collected and analyzed as described above for the shallow soil gas survey. The depths of the gas ports are provided in the "Sampling and Analysis Plan Tables (Appendix A).

\subsubsection{Surface-Flux Chamber Sampling}

VOC emission rates from the surface of Landfills II and III will be determined using an emission flux chamber (EPA 1986). The chamber is placed on the soil surface and air of known purity is swept through the chamber at a known, constant rate for at least four residence time periods. Residence time is defined as chamber volume/air flow rate. For example, for a standard $30 \mathrm{~L}$ chamber and a typical sweep air flow rate of $5.0 \mathrm{~L} /$ minute, the chamber will be swept for at least 24 minutes prior to sampling. Air samples are collected at the exit of the chamber in stainless steel evacuated canisters and VOC concentrations determined by gas chromatography/mass spectrometry using analytical method EPA TO-14 (EPA 1988). Field QA/QC samples will include one field blank and one replicate sample collected from each landfill. The field blank sample will be collected using the same procedures discussed for the actual samples, except that the chamber will be placed on a sheet of Teflon. The replicate sample will be collected by repeating the actual sampling procedure for one randomly chosen sampling point.

\subsection{Surface Soil Sampling}

Surface soil will be collected from the soil cover of each landfill for volatile organics, semivolatile organics, and metals (including cyanide for Landfill II samples) analyses. A lithologic description of each sample will be noted in the sample logbook. Samples for CLP metals will be collected from the 0 - to 6 -in. $(0-$ to $15-\mathrm{cm})$ depth to characterize direct exposure risks. Samples for VOCs and SVOCs will be collected from the 18- to 24-in. (46- to 61-cm) depth because these compounds tend to volatilize from the soil at shallower depths. Surface soil samples will be collected using stainless steel trowels or shovels, hand augers, or soil sampling tubes.

Samples collected for volatile organic and semivolatile organic analysis will not be homogenized and will be transferred directly from the sampling device to a clean sample container (see Table 6-1). Sample containers will be filled leaving as little headspace as possible. Care should be taken to minimize disturbance to the sample during the transfer from the sampling device to the container. Preservatives are not required for these samples. Sample material collected for metals analysis will be placed in a clean stainless steel pan or glass bowl and thoroughly mixed to homogenize the sample. The sample will then be transferred to a clean sample container (see Table 6-1) and prepared for shipment to the analytical laboratory. Preservatives are not required for these samples. 
Samples will be collected and packaged according to the standard operating procedures for surface soil sampling (see Appendix B). All sample equipment will be decontaminated between each sample site according to the standard operating procedure (SOP 11.5) provided in Appendix B of the FSP. Sample material will be surveyed with field instruments (HNu, Ludlum $2 \mathrm{~A}$ ) upon retrieval and readings will be noted in the sample logbook. Excess sample material will be returned to the borehole/sample site.

\subsection{Cover Characterization of Landfill III}

\subsubsection{Field Measurements of the Soil Cover}

Ficld measurements of the soil cover will include the following:

- In situ density and moisture content using a nuclear density gauge (neutron densimeter) following ASTM D-2922 and ASTM D-3017. A standard count will be performed at the EG\&G Materials Testing Laboratory. Measurements will be taken at 4- and 8-in. (10- and $20-\mathrm{cm})$ depths. In the event that conditions prevent a measurement at the 8in. $(20-\mathrm{cm})$ depth, readings at 6 in. $(15 \mathrm{~cm})$ will be collected. In situ moisture content will also be measured using a down-hole neutron moisture meter per SOP. Neutron probe measurements will be collected monthly. Measurements will be taken at 6 -in. $(15 \mathrm{~cm})$ depth intervals using existing neutron access lubes at Landfills II and III.

- Cover thickness will be determined using gasoline-powered and hand augers if the geophysical survey was unsuccessful in determining the cover depth. Augering will continue until either waste is encountered or the auger cannot be advanced due to hole conditions. The borehole and soil cuttings will be surveyed periodically by an Industrial Hygienist. Wastes encountered will not be removed from the borehole and each hole will be back filled with a mixture of native soil and bentonite. Cover material thickness at each site will be recorded and presented graphically in a plan view map of Landfill III.

Procedures to determine the cover thickness are summarized below:

1. The power auger is used to open a hole into the surface of the cover. A new location within $3 \mathrm{ft}(0.9 \mathrm{~m})$ of the sample location marker is chosen if concrete, asphalt, or rocks are stuck at a relatively shallow depth, preventing further auger advance.

2. The hand auger is used to clean the hole and auger decper until waste is encountered or the hole becomes blocked.

3. The depth is recorded at which wastes are encountered or at which augering cannot continue. The reason for stopping augering at the recorded depth shall be noted in the field logbook (i.e., encountered waste, hole caved-in could not continue).

4. The hole is back-filled with a mixture of native cover material and bentonite. 


\subsubsection{Collection of Soll Samples for Laboratory Analysis}

Soil samples will be collected for subsequent laboratory testing for moisture content and particle size analysis. Samples will be collected at approximately $1-\mathrm{ft}(0.3-\mathrm{m})$ depth increments using a $3.5-\mathrm{in} .(8.9-\mathrm{cm})$ bucket auger. Augering will continue until either waste is encountered or the hand auger can no longer be advanced due to hole conditions. Soil material removed from the borchole will be surveyed by an $\mathrm{IH}$ and inspected for waste prior to being transferred to the sample bag. Sampling procedures are summarized as follows:

1. Augering will be initiated at the center of the pad constructed and used for nuclear gauge readings. Material recovered in the auger will be transferred to a ziploc sample bag. The bag will be closed during periods when additional materials are being augered to reduce evaporation losses.

2. At the completion of each $1-\mathrm{ft}(0.3-\mathrm{m})$ interval, the sample volume is mixed and a random 11- to $18-\mathrm{oz}(300-$ to $500-\mathrm{g})$ subsample is collected in a glass or plastic jar. The jar is scaled, bagged, and placed in a portable cooler for gravimetric water content analysis in accordance with ASTM D-2216. The remaining sample is then sealed and double-bagged for particle size analysis according to ASTM D-422.

Intact (undisturbed) soil samples will be collected with 3-in. (7.6-cm) O.D. carbon-steel, thin-walled tubing, in accordance with ASTM D-1587. The upper 3 to 4 in. $(7.6$ to $10.2 \mathrm{~cm}$ ) of dry, cohesionless soil will be removed and the thin-walled tube driven a minimum of 8 in. $(20 \mathrm{~cm})$ into the ground. The tube will be retrieved by excavating around the tube. The samples will be sealed and shipped to the laboratory for analysis of saturated hydraulic conductivity using constant-head permeability techniques (ASTM D-2434), capillary pressure-moisture content relationships (ASTM D-2325) and porosity.

\subsubsection{In Situ Drainage Tests}

In situ drainage tests will be conducted to estimate the total in situ field capacity of the cover materials at Landfill III. In situ field capacity is defined as the amount of water remaining in a soil two or three days after having been wetted and after free drainage is negligible. The goal is to provide an upper limit on the amount of water stored in the soil after saturation and a subsequent drainage period. Each test will be performed by adding a volume of water sufficient to wet the cover material to a depth of approximately 1 to $2 \mathrm{ft}(0.3$ to $0.6 \mathrm{~m})$. The volume of water required will be estimated based on the soil porosity, which in turn will be estimated by knowing the bulk density of the soil, which will be obtained using the neutron densimeter and the particle density (assumed to be $2.65 \mathrm{~g} / \mathrm{cm}^{3}$ for mineral soils). Two to three days will be allowed for gravitational drainage. Cover material will then be hand augered with a $3.5-\mathrm{in} .(8.9-\mathrm{cm})$ bucket auger, and samples will be collected at approximately $1-\mathrm{ft}(0.3-\mathrm{m})$ increments through the wetted depth. Sampling procedures are summarized below:

1. A site within $3 \mathrm{ft}(0.9 \mathrm{~m})$ of the previous augering will be cleared of plants, debris, and the upper 3 to 4 in. (7.6 to $10.2 \mathrm{~cm}$ ) of soil. A 5-gal (19-L) bucket with the bottom removed will be seated into the soil cover and loose soil material will be piled around the base. The bucket will be filled with water and monitored for seepage, with more 


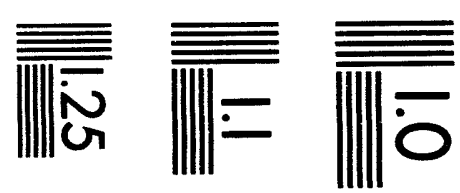

$$
\begin{aligned}
& \text { E } \\
& \text { REE }
\end{aligned}
$$

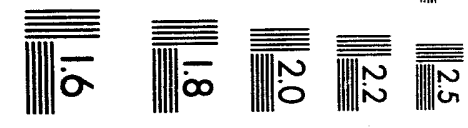



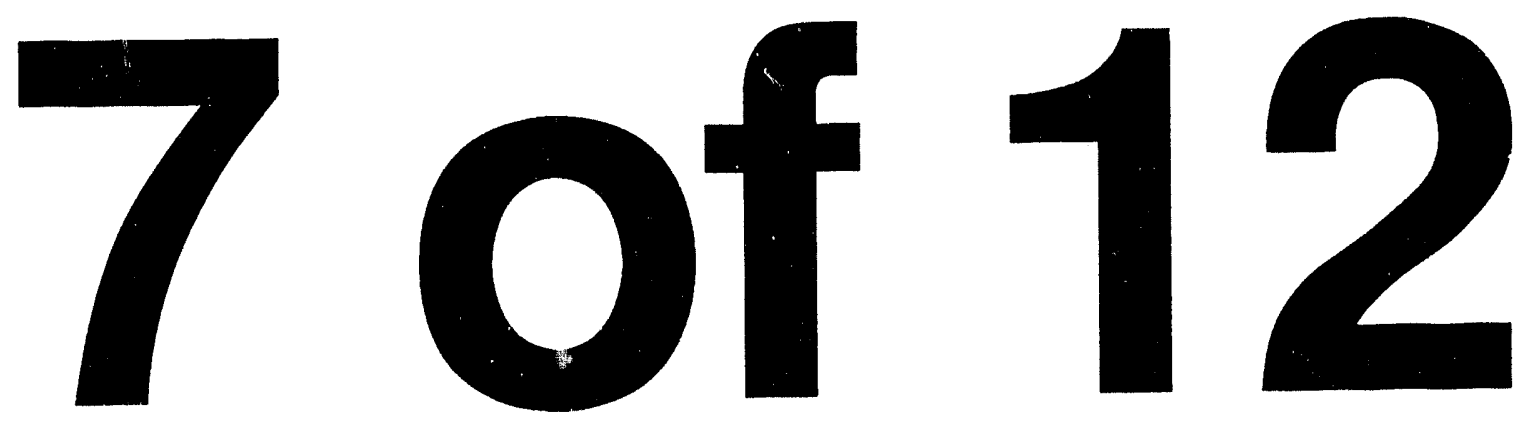
material added around the base (if necessary) to prevent water leakage. The water will be allowed to infiltrate for 1 to 1.5 hours, after which time the bucket will be refilled and its top covered with plastic to prevent evaporative losses and other weather related phenomena (precipitation) from affecting the test.

2. After 2 to 3 days, the bucket will be removed and the cover material will be hand augered and two samples will be collected at $0-1 \mathrm{ft}(0.3-\mathrm{m})$ and $1-2 \mathrm{ft}(0.3-0.6 \mathrm{~m})$ intervals. The Ziploc bag will be closed during periods when additional materials are being augered to reduce evaporative losses.

3. An 11 to $18-\mathrm{oz}(300-500 \mathrm{~g})$ sample from each interval will be placed in a glass or plastic jar. Samples will then be packaged and shipped to the laboratory for gravimetric water content analysis in accordance with ASTM D-2216.

4. The augered hole will be backfilled with a mixture of native soil and bentonite.

\subsection{Water Level Measurements and Borehole Deviation Logging}

A second-order survey of all wells on the INEL is being conducted to establish a common datum for INEL wells. The wells identified in Table 3-8 will be included in this survey to ensure that all wells used in this investigation reference the same datum.

Borehole deviation logs will be run by the USGS on the landfill wells, and if possible, the USGS wells identified in Table 3-10. The deviation logging will be carried out using USGS procedures. Prior to conducting deviation surveys, the dedicated sampling pumps installed in the wells will be removed. Information obtained from the logging effort will be used to correct the measured depth to water for borehole deviation effects.

Water levels will be measured monthly (May-December) in the wells within approximately a 1-mi (1.6-km) radius of the CFA landfills (see Table 3-10 and Figure 3-6). The measurements will be made using either an electronic measuring tape (solinst) or a steel tape measure as described in SOP 11.9 (see Appendix B).

\subsection{Groundwater Sampling}

\subsubsection{Well Purging}

All wells (except the CFA production wells) will be purged prior to sample collection using a submersible pump. The CFA production wells will be purged using the existing dedicated production pumps. During the purging operation, a Hydrolab (see Appendix B) or equivalent will be used to measure specific conductance, $\mathrm{pH}$, dissolved oxygen, and temperature. Well purging procedures are provided in SOP 11.8 (Appendix B). After a minimum three well casing volumes of water have been purged from the well and when three consecutive Hydrolab readings are within the limits listed below, a sample for water quality analysis can be collected. 
pH: \pm 0.1 standard units

temperature: $\pm 0.5^{\circ} \mathrm{C}$

specific conductance: $\pm 10 \mathrm{mmhos} / \mathrm{cm}$

dissolved oxygen: $\pm 1 \mathrm{mg} / \mathrm{L}$

\subsubsection{Groundwater Sampling}

Prior to sampling, all sampling equipment that comes in contact with the water sample will be cleaned following the SOP for decontaminating sampling equipment (see SOP 11.5 in Appendix B).

Additionally, the dedicated Hydrostar sampling pumps will be removed from the wells, the water level in the well will be measured, and using a submersible pump, each well will be purged a minimum of three well-casing volumes until the $\mathrm{pH}$, temperature, specific conductance, and dissolved oxygen of the purge water have stabilized (not to exceed five well-casing volumes) as defined above and in the standard operating procedures found in Appendix B. The production wells will be sampled from a sampling port on the existing production well pumps.

Groundwater samples will be collected for analysis of volatile organics, CLP metals, and hexavalent chromium, cyanide, nitrate, sulfate, chloride, fluoride, and alkalinity. Samples collected for metals and $\mathrm{Cr}^{6+}$ analysis will include both filtered and unfiltered samples. The analyses to be performed and the desired analytical support level are presented in Tables 3-2 and 3-4. The requirements for containers, preservation methods, sample volumes, and holding times for the analyses listed above are provided in Table 6-1. Sample bottles for liquid inorganic analyses will be filled to approximately $90-95 \%$ of capacity to allow for content expansion or preservation. A separate aliquot of the same volume for VOA samples will be collected to determine the correct amount of preservative and will be tested for $\mathrm{pH}$. The 0.01 -gal $(40-\mathrm{mL})$ glass VOA vials will be filled completely with no headspace or air bubbles. Hydrochloric acid preservative will be introduced into the VOA vials prior to sample collection and tested for $\mathrm{pH}<2$ after sample collection. One 0.13 -gal $(500-\mathrm{mL})$ sample will be collected for analysis of nitrate/nitrite and preserved with concentrated sulfuric acid $\left(\mathrm{H}_{2} \mathrm{SO}_{4}\right)$ to a $\mathrm{pH}<2$. An additional 0.13-gal $(500-\mathrm{mL})$ sample will also be collected for nitrate/nitrite analysis. This second sample will not be preserved with $\mathrm{H}_{2} \mathrm{SO}_{4}$ and will be used in lieu of the preserved sample if holding times are met. One 0.13 -gal $(500-\mathrm{mL})$ sample will be collected for sulfate and chloride analysis, and one 0.07 -gal $(250-\mathrm{mL})$ sample will be collected for alkalinity analysis. Two 0.26 -gal $(1-\mathrm{L})$ sample will be collected for fluoride analysis. Two 0.26-gal (1-L) samples will be collected for metals analysis and will be acidified to a $\mathrm{pH}<2$ using $10 \%$ ultrapure nitric acid (certified metals free). Two 0.13-gal $(500-\mathrm{mL})$ samples will be collected for hexavalent chromium analysis. One of the metals

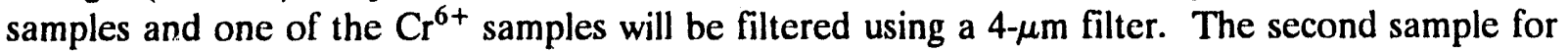
metals and $\mathrm{Cr}^{6+}$ will be collected unfiltered. One 0.26-gal (1-L) sample will be collected for cyanide analysis and will be preserved using $\mathrm{NaOH}$ to a $\mathrm{pH}>12$. One 0.14 -gal $(540-\mathrm{mL})$ sample will be collected and preserved with nitric acid to a $\mathrm{pH}<2$ for gross gamma analysis for shipping purposes. In addition to the above preservation requirements, all samples with the exception of fluoride, metals, and gamma, require cooling to $39^{\circ} \mathrm{F}\left(4^{\circ} \mathrm{C}\right)$. The preferred order for sample collection is as follows:

- Temperature, $\mathrm{pH}$, specific conductance, and dissolved oxygen (during purging) 
- Volatile organics [collected at flow rates $<5 \mathrm{gal} / \mathrm{min}(19 \mathrm{~L} / \mathrm{min})$ or with a bailer; production wells will be sampled from a sample port at $<5 \mathrm{gal} / \mathrm{min}(19 \mathrm{~L} / \mathrm{min})]$

- Total and dissolved metals, $\mathrm{Cr}^{6+}$ and cyanide

- Inorganics (sulfate, chloride, fluoride, nitrate/nitrite)

- Alkalinity

- Gamma.

\subsection{Subsurface Sampling of Landfill II}

Subsurface soil samples will be collected from seven boreholes borings using a drill rig equipped with hollow-stem augers from land surface to the top of basalt. Sampling will be conducted for lithologic logging and chemical analyses. A split-barrel (split-spoon) sampler will be used to continuously sample in $2-\mathrm{ft}(0.6-\mathrm{m})$ increments.

A minimum of two samples per borehole will be collected and shipped for laboratory analysis: one from within the waste unit and one from below the waste. The sample from below the waste unit will always be collected and will be from sediment material just above the basal clay (if present), or just above the sediment/basalt interface if the basal clay is not present. For boreholes where basal clay is encountered (and leachate is not present), the borehole will be advanced to the top of basalt. Boreholes without basal clay will be backfilled with $2 \mathrm{ft}$ of bentonite followed by auger cuttings. Boreholes where clay is encountered will be backfilled with bentonite to the top of the clay layer followed by auger cuttings to land surface. Sampling from within the waste unit will begin at a depth of $3 \mathrm{ft}$ and samples will be selected for analysis using the following criteria:

1. Any sample exhibiting a VOC headspace result of $>20 \mathrm{ppm}$ will be collected for VOC and SVOC analysis.

2. If headspace analyses for a given borehole are less than $20 \mathrm{ppm}$ but greater than zero, the sample with the highest reading will be sent for VOC and SVOC analysis.

3. If no samples show a positive ( $>0 \mathrm{ppm}$ ) VOC headspace reading, samples for VOC and SVOC analysis will be collected using the following randomly-selected default depths:
B-1 $\quad 13-15 \mathrm{ft}$
B-2 $\quad 13-15 \mathrm{ft}$
B-3 8-10 ft
B-4 13-15 ft
B-5 3-5 ft
B-6 $\quad 3-5 \mathrm{ft}$
B-7 $\quad 13-15 \mathrm{ft}$
B-8 $\quad 8-10 \mathrm{ft}$

4. Samples for metals (and cyanide) analysis will be selected based on visual examination of the split spoon samples (staining/discoloration). If discrete zones of discoloration are 
not encountered, or if discoloration is prevalent throughout the borehole, samples for metals analysis will be collected at the same depths as for the VOCs and SVOCs.

5. In addition to headspace results, samples exhibiting nonaqueous saturation or staining by oil will be submitted for SVOC analysis.

As shown on Figure 5-1, the bottom portion of each 2-ft $(0.6-\mathrm{m})$ split-barrel sample will be retained for potential VOC analysis, and the next portion will be retained for VOC headspace screening. The third portion of the split-barrel will be retained for SVOC analysis. The fourth portion of the split-barrel sample will be composited for potential metals (and cyanide) analysis and the uppermost portion will be retained for radiation screening.

Field screening for VOCs will be conducted using an HNu. The action level for total VOCs is $20 \mathrm{ppm}$. This level has been chosen to be consistent with other INEL investigations.

Figure 5-2 shows the general VOC field screening and sampling approach. The same logic shown in Figure 5-2 will be followed for SVOCs. All soil samples will be submitted to the Radiation Measurements Laboratory (RML) for a 20 -minute gamma scan before they are shipped. The same-day 20-minute gamma scan is designed so that VOC or SVOC holding times are not exceeded.

\subsubsection{Borehole Logging}

Borehole logs will be generated during drilling to $\log$ the material/soil encountered. The borehole log will follow the procedures in ER SOP 11.15, "Geologic Logging."

\subsubsection{Field Screening for Organic and Metal Compounds}

Soil samples will be collected from each split-barrel sample and field screened for VOCs using the headspace method. The procedure for VOC field screening is as follows:

1. Obtain a soil sample aliquot from the split-barrel sampler, disturbing the soil sample as little as possible.

2. Immediately place the sample in a $250-\mathrm{mL}$ wide-mouth glass sample container, leaving a headspace of approximately 1 in. $(2.54 \mathrm{~cm})$.

3. Cap the container with a piece of aluminum foil (inside of roll towards sample), and secure with a rubber band.

4. Place the container in a $70^{\circ} \mathrm{F}$ water bath for 20 minutes.

5. After the sample has warmed and released any potential VOCs into the headspace, puncture the aluminum foil with an $\mathrm{HNu}$ probe and take immediate measurements of VOCs.

6. Record results in the EG\&G Idaho sample logbook and the boring log. 


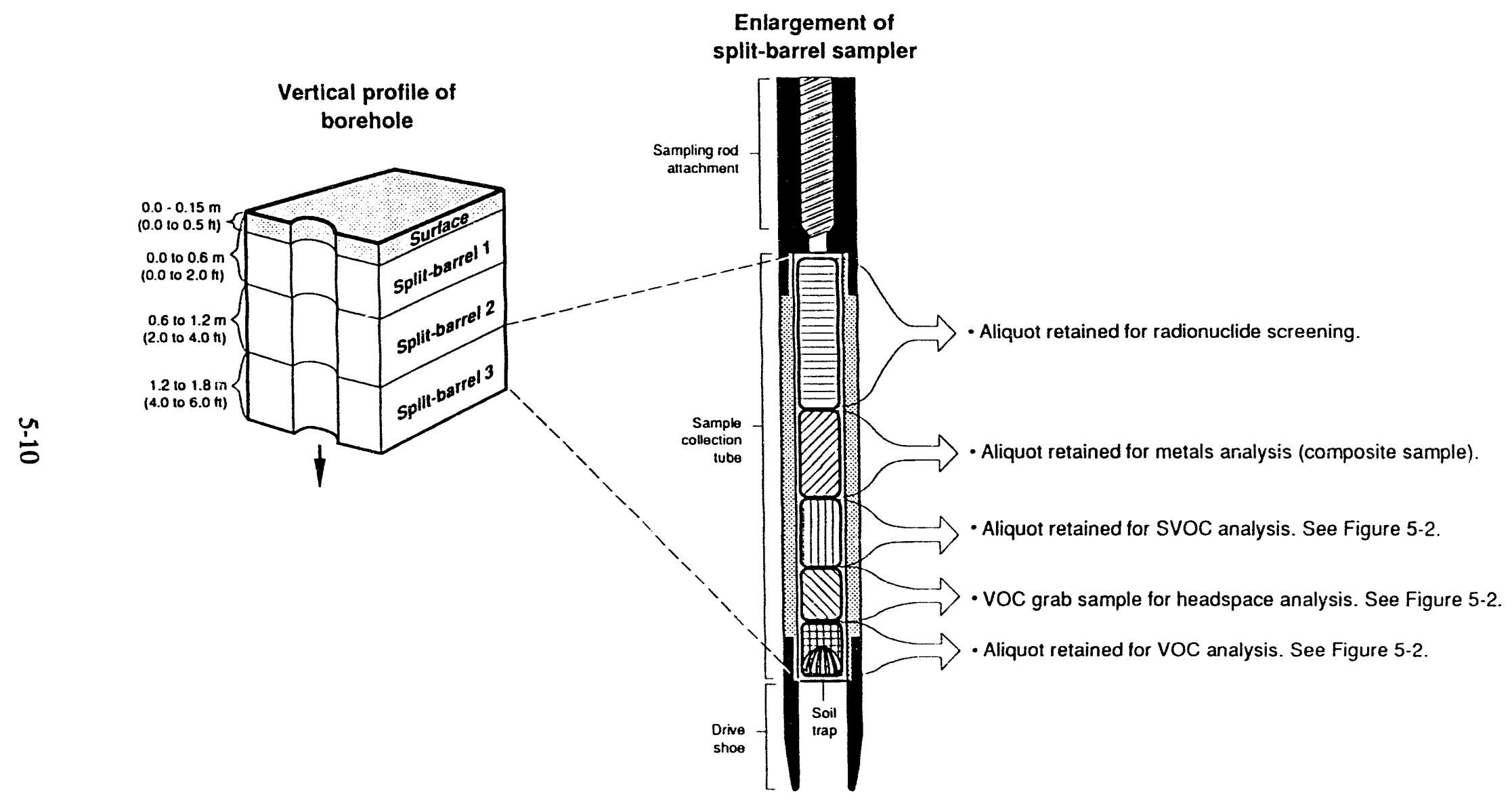

Figure 5-1. Schematic of the subsurface sampling program. 


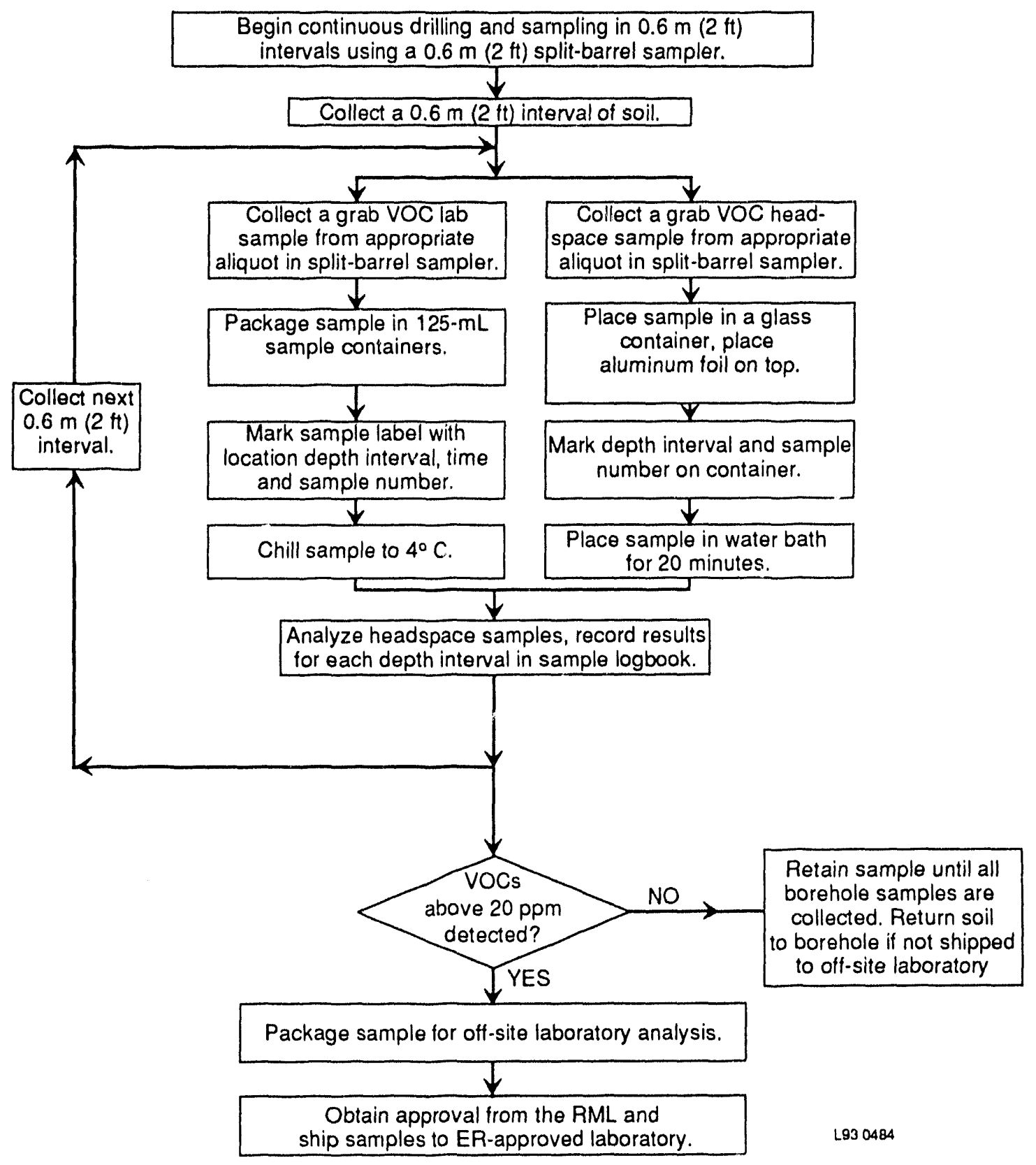

Figure 5-2. Generalized logic for VOC analysis sampling. 


\subsubsection{Soil Sampling for Organic and Metal Compounds}

Grab samples for VOCs and SVOCs will be collected according to the following procedure:

1. Use a clean sampling spoon or spatula to extract a sample from the base of the decontaminated split-barrel sampler (the end closest to the drive shoe), disturbing the soil sample as little as possible.

2. Quickly remove coarse fragments. Immediately place the sample in a $125-\mathrm{mL}$, clear, wide-mouth glass sample container.

3. Cap the container, affix a label marked with the depth interval, sample location and number, analysis, time, sampler's name, and date. Record all sample information in the EG\&G Idaho sample logbook.

4. Use a clean sampling spoon or spatula to extract an SVOC sample from the midsection of the decontaminated split-barrel sampler, disturbing the soil sample as little as possible.

5. Quickly remove coarse fragments. Immediately place the sample in a $125-\mathrm{mL}$, clear, wide-mouth glass sample container.

6. Cap the container, affix a label marked with the depth interval, sample location and number, analysis, time, sampler's name, and date. Record all sample information in the EG\&G Idaho sample logbook.

7. Place the samples in Ziploc bags.

8. Chill to $4^{\circ} \mathrm{C}$ quickly by placing the samples in an ice-filled cooler.

9. Package samples and complete the Chain-of-Custody/Request for Analysis Record per ER SOP 5.7, "Chain-of-Custody Record."

10. Transport the samples for shipment to the analytical laboratory.

Samples for metals analysis will be collected according to the following procedure:

1. Use a clean sampling spoon or spatula to extract a sample from the decontaminated split-barrel sampler, and place the soil in a Pyrex glass bowl (or aluminum pan).

2. Thoroughly mix the sample and remove coarse fragments and organic material from the Pyrex bowl.

3. Scrape the sample in the bowl from the sides and bottom to the middle of the bowl and mix the soil well.

4. Quarter the sample and move the quarters to the four quadrants of the bowl. 
5. Mix each quarter of the sample individually.

6. Roll each quarter to the center of the bowl and mix the entire sample again.

7. Repeat this procedure to ensure that all parts of the sample are mixed and that the sample is as homogeneous as possible.

8. Place the sample in a $250-\mathrm{mL}(8-\mathrm{oz})$ wide-mouth glass container.

9. Cap the container, affix a label marked with the depth interval, sample location and number, analysis, time, sampler's name, and date. Record all sample information in the EG\&G Idaho sample logbook.

10. Place the samples in Ziploc bags.

11. Chill to $4^{\circ} \mathrm{C}$ quickly by placing the samples in an ice-filled cooler.

12. Package samples and complete the Chain-of-Custody/Request for Analysis Record per ER SOP 5.7, "Chain-of-Custody Record."

13. Transport the samples for shipment to the analytical laboratory.

\subsubsection{Leachate Sampling}

If leachate is encountered during augering and soil sampling, samples will be collected for VOC, SVOC, and metals (including cyanide) analysis. Upon encountering the free liquid, augering will be stopped and the borehole will be allowed to stand open for $1 / 2$ to 1 hour. A clean bailer will be lowered through the hollow stem of the augers to collect the samples. Volatile organics will be collected in 0.01-gal $(40-\mathrm{mL})$ glass vials (with no headspace) and preserved with hydrochloric acid to a $\mathrm{pH}$ less than 2 . Semivolatile organics will be collected in 0.26-gal (1-L) amber-glass jars. No preservative is required other than cooling to $4^{\circ} \mathrm{C}$. Metals will be collected in a 0.26 -gal (1-L) HDPE narrow-mouth bottle and preserved with nitric acid to a $\mathrm{pH}$ less than 2. Cyanide will be collected in a 0.26-gal (1-L) HDPE narrow-mouth bottle and preserved with $\mathrm{NaOH}$ to a $\mathrm{pH}>12$.

\subsubsection{Leachate Wells}

If leachate is encountered in any of the boreholes, leachate collection wells will be installed after augering and soil sampling are completed. However, augering and soil sampling in these boreholes will not progress through the clay layer above the basalt (if present). The wells will be constructed through the hollow-stem augers as follows:

1. Place a 1 to $2-\mathrm{ft}(0.3$ to $0.6-\mathrm{m})$ plug of bentonite pellets in the bottom of the borehole.

2. Install 2-in. (5-cm) stainless steel casing with 5 to $10 \mathrm{ft}(1.5$ to $3 \mathrm{~m})$ of 0.010 -in. $(0.03-\mathrm{cm})$ wired-wrapped screen. 
3. Install a quartz sand filter pack to $2 \mathrm{ft}(0.6 \mathrm{~m})$ above the top of the screen.

4. Backfill the remainder of the borehole with bentonite to within $2 \mathrm{ft}(0.6 \mathrm{~m})$ of land surface, followed by a cement bentonite cap.

5. Place a locking 2 -in. $(5-\mathrm{cm})$ stainless steel cap on the well casing.

\subsubsection{Borehole Abandonment}

Upon reaching the top of basalt, dry boreholes will be abandoned as follows:

1. Insert a bentonite plug to the top of the clay layer (if present) or to a height of $2 \mathrm{ft}$ $(0.6 \mathrm{~m})$ above the basalt surface if clay is not present.

2. Backfill the remainder of the borehole with the borehole cutting material in the order they were removed from the borehole during cutting.

3. Establish the coordinates of each borehole site by staking the site and surveying it.

\subsection{Handling and Disposition of Investigation-Derived Waste}

Investigation-derived wastes (IDW) will be generated at OU 4-12 as a result of remedial investigation activities discussed in Chapter 5 of the work plan. The types and quantities of IDW that will be generated are shown in Tables 5-1 through 5-3.

The disposition and handling of IDW at OU 4-12 will be consistent with Guide to Management of Investigation-Derived Wastes. Activities generating IDW include sampling of the surface soil and the cover of Landfills II and III, sampling of the subsurface soil at Landfill II, and sampling of groundwater wells near OU 4-12. The amounts of IDW for each activity are shown in Tables 5-1 through 5-3.

\section{Soil Sampling}

IDW from sampling of the surface soil of the Landfills II and III and the subsurface soils at Landfill II will consist of the unused sample portion that may be returned to INEL from the analytical laboratory and drill cuttings from boreholes. This IDW will be soil samples from the site and water samples used in the CA/QC process. Those water samples, which will have been preserved with nitric acid as part of the sample preparation process, will be neutralized by the laboratory before they are returned to INEL. The total amount of IDW from this activity is estimated at $37 \mathrm{Kg}$ (see Table 5-1). This estimate assumes the analytical laboratory will use 50\% of the sample and return the remaining portion to INEL for disposal at OU 4-12. Unused surface soil and water samples will be returned to the surface cover of the Landfill from which the sample was collected. Drill cuttings from boreholes will be segregated as they are brought to the surface and separated from the surface soil of the landfill. Upon completion of sampling and drilling the cuttings will be placed back into the borehole. Excess drill cuttings that will not fit into the borehole will be held at the site until analysis of samples from that hole are complete. 
Table 5-1. Preliminary screening for OU 4-12 soil sample investigation derived waste.

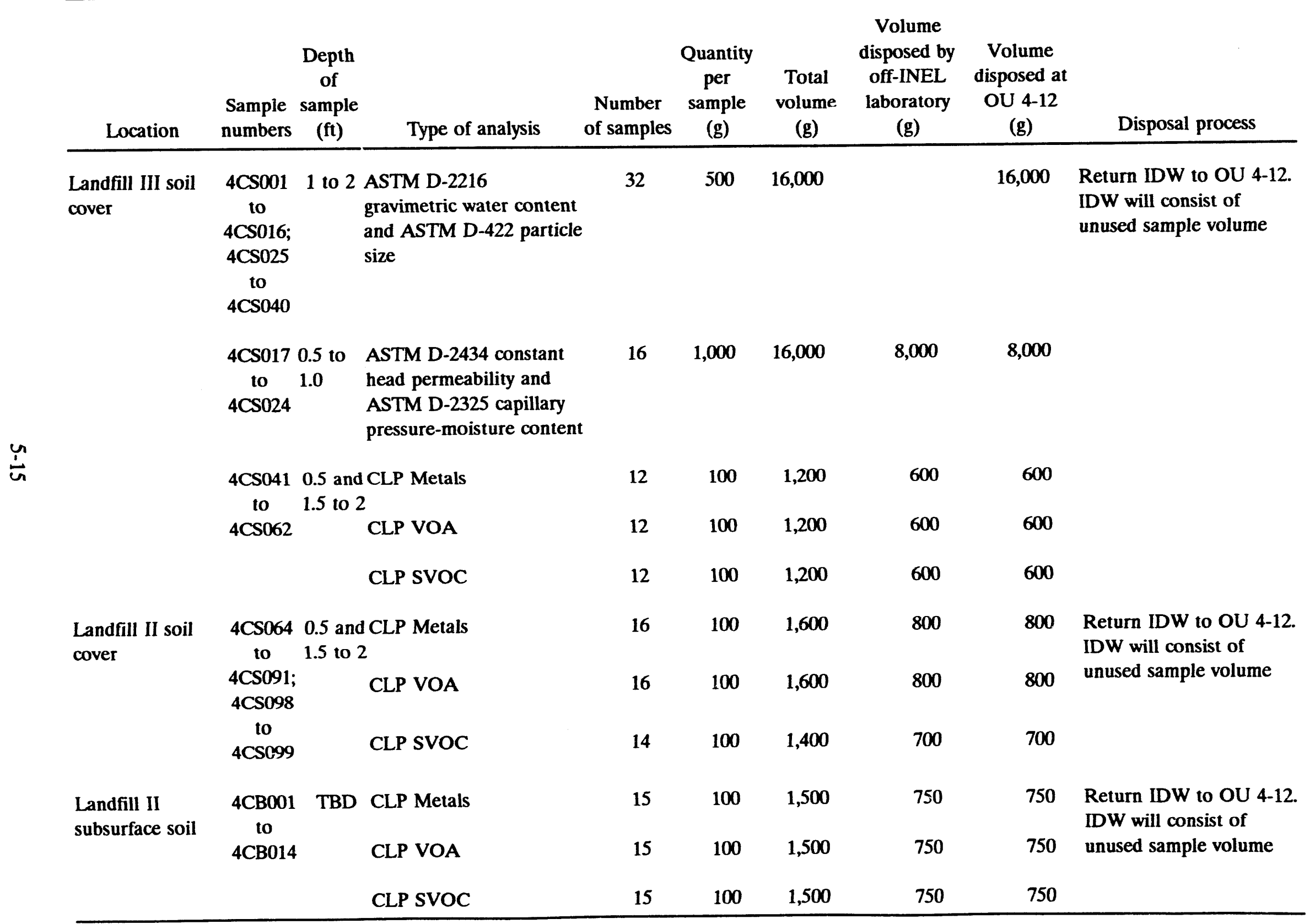


Table 5-2. Preliminary screening for SRPA well purge and decontamination water investigationderived waste.

\begin{tabular}{|c|c|c|c|c|}
\hline Location & $\begin{array}{l}\text { Casing radius } \\
\text { (in) }\end{array}$ & $\begin{array}{l}\text { Water depth in casing } \\
(\mathrm{ft})\end{array}$ & $\begin{array}{l}\text { Estimated } \\
\text { purge } \\
\text { volume }^{\mathrm{a}} \\
\text { (gal) } \\
\text { (L) }\end{array}$ & $\begin{array}{c}\text { Process knowledge } \\
(\mu \mathrm{g} / \mathrm{L}-\mathrm{Mo} / \mathrm{Yr}) \\
(\mathrm{pCi} / \mathrm{mL} \mathrm{RAD})\end{array}$ \\
\hline LF2-8 & 3 & 20 & $\begin{array}{l}147 \mathrm{gal} \\
667 \mathrm{~L}\end{array}$ & $\begin{array}{l}\text { Chromium- } \mu \mathrm{g} / \mathrm{L} \\
206-2 / 90 \\
285-5 / 90 \\
776-10 / 90 \\
902-4 / 91 \\
\text { Tritium } \mathrm{pCi} / \mathrm{ml} \\
42-3 / 90 \\
39-5 / 90 \\
36-11 / 90\end{array}$ \\
\hline LF2-9 & 2 & 21 & $\begin{array}{l}69 \mathrm{gal} \\
313 \mathrm{~L}\end{array}$ & $\begin{array}{l}\text { Chromium } \mu \mathrm{g} / \mathrm{L} \\
379-2 / 90 \\
567-5 / 90 \\
206-10 / 90 \\
753-4 / 91 \\
\text { Tritium } \mathrm{pCi} / \mathrm{mL} \\
44-3 / 90 \\
44-6 / 90 \\
41-11 / 90\end{array}$ \\
\hline LF2-10 & 3 & 283 & $\begin{array}{l}2,075 \mathrm{gal} \\
9,420 \mathrm{~L}\end{array}$ & $\begin{array}{l}\text { Chromium } \mu \mathrm{g} / \mathrm{L} \\
\text { No data } \\
\text { Tritium } \mathrm{pCi} / \mathrm{m} \text { ! } \\
\text { No data }\end{array}$ \\
\hline LF2-11 & 2 & 29 & $\begin{array}{l}95 \mathrm{gal} \\
431 \mathrm{~L}\end{array}$ & $\begin{array}{l}\text { Chromium } \mu \mathrm{g} / \mathrm{L} \\
379-2 / 90 \\
216-5 / 90 \\
202-10 / 90 \\
663-4 / 91 \\
\text { Tritium pCi/ml } \\
48-3 / 90 \\
48-5 / 90 \\
43-11 / 90\end{array}$ \\
\hline LF2-12 & 2 & 15 & $\begin{array}{l}50 \mathrm{gal} \\
227 \mathrm{~L}\end{array}$ & $\begin{array}{l}\text { Chromium } \mu \mathrm{g} / \mathrm{L} \\
24-4 / 91\end{array}$ \\
\hline
\end{tabular}


Table 5-2. (continued).

\begin{tabular}{|c|c|c|c|c|}
\hline Location & $\begin{array}{l}\text { Casing radius } \\
\text { (in) }\end{array}$ & $\begin{array}{l}\text { Water depth in casing } \\
\text { (ft) }\end{array}$ & $\begin{array}{l}\text { Estimated } \\
\text { purge } \\
\text { volume }^{\text {a }} \\
\text { (gal) } \\
\text { (L) }\end{array}$ & $\begin{array}{c}\text { Process knowledge } \\
\text { ( } \mu \mathrm{g} / \mathrm{L}-\mathrm{Mo} / \mathrm{Yr}) \\
(\mathrm{pCi} / \mathrm{mL} \mathrm{RAD})\end{array}$ \\
\hline LF3-8 & 3 & 24 & $\begin{array}{l}176 \mathrm{gal} \\
799 \mathrm{~L}\end{array}$ & $\begin{array}{l}\text { Chromium } \mu g / L \\
409-4 / 91 \\
853-4 / 91\end{array}$ \\
\hline LF3-9 & 2 & 15 & $\begin{array}{l}49 \mathrm{gal} \\
222 \mathrm{~L}\end{array}$ & $\begin{array}{l}\text { Chromium } \mu \mathrm{g} / \mathrm{L} \\
45-4 / 91\end{array}$ \\
\hline LF3-10 & 2 & 15 & $\begin{array}{l}49 \mathrm{gal} \\
222 \mathrm{~L}\end{array}$ & $\begin{array}{l}\text { Chromium } \mu \mathrm{g} / \mathrm{L} \\
51-4 / 91\end{array}$ \\
\hline LF3-11 & 2 & 15 & $\begin{array}{l}49 \mathrm{gal} \\
222 \mathrm{~L}\end{array}$ & $\begin{array}{l}\text { Chromium } \mu \mathrm{g} / \mathrm{L} \\
120-4 / 91\end{array}$ \\
\hline USGS 85 & 3 & 156 & $\begin{array}{l}1,144 \mathrm{gal} \\
5,194 \mathrm{~L}\end{array}$ & $\begin{array}{l}\text { Chromium } \mu \mathrm{g} / \mathrm{L} \\
1-9 / 77 \\
20-9 / 77 \\
1-10 / 84 \\
20-10 / 84\end{array}$ \\
\hline
\end{tabular}

a. Purge volume computed from ER\&WM Department SOP $11.8 \mathrm{~V}=\left(\mathrm{h}_{1}-\mathrm{h}_{2}\right) \mathrm{r}^{2}(0.163)$, where V $=$ static well volume in gallons, $h_{1}=$ depth of well in feet, $h_{2}=$ depth to water in feet, $r=$ inside radius of well casing in inches. Volumes are based on a maximum of 5 well casing volumes. 
Table 5-3. Preliminary screening for OU 4-12 water sample investigation-derived waste.

\begin{tabular}{|c|c|c|c|c|c|c|c|c|c|}
\hline Location & $\begin{array}{c}\text { Sample } \\
\text { numbers } \\
\text { for } 3 \\
\text { phases }\end{array}$ & $\begin{array}{c}\text { Depth } \\
\text { of } \\
\text { sample } \\
\text { (ft) }\end{array}$ & $\begin{array}{l}\text { Type of } \\
\text { analysis }\end{array}$ & $\begin{array}{l}\text { Number } \\
\text { samples } \\
\text { for } 3 \\
\text { phases }\end{array}$ & $\begin{array}{l}\text { Volume } \\
\text { per } \\
\text { sample } \\
\text { (L) }\end{array}$ & $\begin{array}{c}\text { Total } \\
\text { volume } \\
\text { (L) }\end{array}$ & $\begin{array}{l}\text { Volume } \\
\text { disposed } \\
\text { off-INEL } \\
\text { laboratory } \\
\text { (L) }\end{array}$ & $\begin{array}{c}\text { Volume } \\
\text { disposed } \\
\text { at OU 4-12 } \\
\text { (L) }\end{array}$ & Disposal process \\
\hline \multirow{18}{*}{$\begin{array}{l}\text { Groundwater } \\
\text { Samples } \\
\text { LF 2-8 } \\
\text { LF 2-9 } \\
\text { LF 2-10 } \\
\text { LF 2-11 } \\
\text { LF 2-12 } \\
\text { LF 3-8 } \\
\text { LF 3-9 } \\
\text { LF 3-10 } \\
\text { LF 3-11 } \\
\text { USGS 85 } \\
\text { CFA-1 } \\
\text { CFA-2 } \\
\text { Trip blank } \\
\text { Field blank } \\
\text { Rinsate }\end{array}$} & Phase 1 & 460 & Alkalinity & 36 & 0.25 & 9.0 & 4.5 & 4.5 & \multirow{20}{*}{$\begin{array}{l}\text { Lse Best Management Practice. If } \\
\text { data indicate contaminant levels are } \\
\text { above MAC levels discharge to an } \\
\text { area of similar contamination (TRA } \\
\text { Warm Waste Pond-Cell 52). } \\
\text { If data indicates contaminant levels } \\
\text { are below MAC levels discharge } \\
\text { water samples to ground in the } \\
\text { vicinity of the well. }\end{array}$} \\
\hline & $\begin{array}{c}\text { 4CQ001 } \\
\text { to }\end{array}$ & $\begin{array}{c}\text { to } \\
625\end{array}$ & CLP metals/F & 36 & 1.0 & 36 & 18 & 18 & \\
\hline & $4 \mathrm{CQ} 012$ & & CLP metals & 36 & 1.0 & 36 & 18 & 18 & \\
\hline & Phase 2 & & Chromium ${ }^{+6} \mathrm{~F}$ & 36 & 0.5 & 18 & 9 & 9 & \\
\hline & $\begin{array}{l}\text { 4CQ101 } \\
\text { to }\end{array}$ & & VOA 524.2 & 36 & 0.8 & 29 & 14.5 & 14.5 & \\
\hline & $4 C Q 112$ & & Fluoride & 36 & 1.0 & 36 & 18 & 18 & \\
\hline & & & Nitrate/ite & 36 & 0.5 & 18 & 9 & 9 & \\
\hline & $\begin{array}{l}\text { Phase } 3 \\
4 C S 201\end{array}$ & & Gamma & 36 & 0.54 & 19 & $19^{a}$ & & \\
\hline & $\begin{array}{c}\text { to } \\
4 \mathrm{CS} 212\end{array}$ & & Sulfate/chloride & 36 & 0.5 & 18 & 9 & 9 & \\
\hline & $4 C Q 013$ & NA & VOA 524.2 & 15 & 0.8 & 12 & 6 & 6 & \\
\hline & $\begin{array}{l}4 C Q 113 \\
4 C Q 213\end{array}$ & & & & & & & & \\
\hline & $\begin{array}{c}\text { 4CQ014 \& } \\
\text { 4CQ015 }\end{array}$ & NA & Alkalinity & 6 & 0.25 & 1.5 & 0.75 & 0.75 & \\
\hline & & & CLP metals & 6 & 1.0 & 6.0 & 3.0 & 3.0 & \\
\hline & 4CQ114 \& & & Chromium ${ }^{+6}$ & 6 & 0.5 & 3.0 & 1.5 & 1.5 & \\
\hline & 4CQ11J & & VOA 524.2 & 6 & 0.8 & 4.8 & 2.4 & 2.4 & \\
\hline & & & Fluoride & 6 & 1.0 & 6.0 & 3.0 & 3.0 & \\
\hline & 4CQ214 \& & & Nitrate/ite & 6 & 0.5 & 3.0 & 1.5 & 1.5 & \\
\hline & $4 C Q 215$ & & Sulfate/chloride & 6 & 0.5 & 3.0 & 1.5 & 1.5 & \\
\hline \multirow[t]{2}{*}{ Purge water } & $4 \mathrm{CQ016}$ & INA & CLP metals & 3 & 1.0 & 3.0 & 1.5 & 1.5 & \\
\hline & & & VOA 524.2 & 3 & 0.8 & 2.4 & 1.2 & 1.2 & \\
\hline
\end{tabular}

a. Disposed by Radiological Measurements Laboratory at TRA. 
If laboratory results indicate that the samples from that hole are not hazardous, the cuttings will be disposed in the active INEL landfill. If laboratory results indicate that the samples from that hole are hazardous, the cuttings will be containerized and held pending the treatment decision for Landfill II. Large pieces of waste such as metal, plastic, wood, etc., will be disposed in the active INEL landfill.

\section{Groundwater Sampling}

IDW from groundwater sampling will consist of purge water from the Snake River Plain Aquifer that has been pumped from the wells listed in Table 5-2 and the unused sample portion returned by the analytical laboratory for disposal. Purge water will be generated before sample collection in accordance with SOP 11.8 to remove standing water from the well casing. The SOP requires 3 to 5 well volumes be removed from the well and other water quality parameters be met before samples are collected. The estimated amount of purge water generated from each well for each of the three phases of groundwater sampling is shown in Table 5-1. Process knowledge from past sampling of the monitoring wells indicates the presence of tritium and possibly chromium. The source of tritium is believed to be processes associated with ICPP and TRA. The source of chromium is believed to be the processes associated with TRA and possibly shavings from dedicated sampling pumps located in some of the wells. Purging and sampling will be performed after the dedicated pumps are removed.

Purge water from aquifer wells will be handled in a manner that does not pose unacceptable risk to human health. Existing data from previous groundwater investigations was used to determine the potential risk to human health from well purging operations. The maximum concentrations for chromium and tritium from historical data indicate potential concentrations of chromium at $902 \mu \mathrm{g} / \mathrm{L}$ and of tritium at $48,000 \mathrm{pCi} / \mathrm{L}$. Risk calculations were performed for ingestion and inhalation pathways in residential and occupational scenarios assuming purge water was distributed over an area of $6 \times 6 \mathrm{~m}$ and depth of $1 \mathrm{~m}$. Maximum concentrations from existing data for chromium and tritium were used, which represent the worst case for potential risk.

This worst-case potential risk posed by purge water containing chromium and/or tritium is 1.5E-10 for carcinogenic effect (tritium, soil ingestion, occupational scenario) and a hazard quotient of 0.0001 (chromium VI, soil ingestion, residential scenario). Because the potential risk is well below EPA's recommended target risk range identified in the NCP of $10^{-6}$ to $10^{-4}$ for carcinogens or a hazard index of 1 for non-carcinogenic contaminants, the purge water could be safely disposed directly to the ground during well purging operations. However, due to the potential for changes in contaminant concentration levels or appearance of new contaminants, the purge water will be containerized pending receipt of sample results. This containerization represents a departure from DOE's IDW strategy, but is being performed on this OU due to schedule constraints.

If sample results indicate concentrations are at or below the historical maximum levels cited above, then the purge water will be disposed directly to the ground. If the concentrations are above said levels, then a risk calculation such as that used above will be performed to determine appropriate disposition of the purge water.

There is potential for freezing of the third-round (October, 1993) purge water while waiting for sample results. The WAG-4 managers (EPA/IDHW/DOE) may determine the risk acceptable based on sample results from the first two rounds. If so, the water may be disposed to the ground 
to prevent freezing of the purge water tank. Purge water will be discharged at least 200 feet from the landfills.

\section{Decontamination}

IDW generated from decontamination activities will be collected in a tank, sampled and disposed to the ground if the data indicate that constituent concentrations levels are less than historic levels cited above.

\section{Personal Protective Equipment}

Personal Protective Equipment used at OU 4-12 may be generated and will be decontaminated, if necessary, and disposed in the active INEL landfill. 


\section{DOCUMENT MANAGEMENT AND SAMPLE CONTROL}

Section 6.1 summarizes document management and sample control. Documentation includes all field documents used to record field data and document sampling procedures. Sampling documents include chain of custody forms and sample container labels. Section 6.2 outlines the sample containers and preservatives that will be used and discusses chain of custody, radioactivity screening, and sample packaging for shipment to the analytical laboratories.

\subsection{Documentation}

The Field Team Leader is responsible for controlling and maintaining all field documents and records, and for ensuring that all required documents are submitted to ER Administrative Records and Document Control (ARDC). All entries will be made in permanent ink. All errors will be corrected by drawing a single line through the error and entering the correct information. All corrections will be initialed and dated. The serial or ID number and disposition of all controlled documents (e.g., chain of custody forms) will be recorded in the document control logbook. If any documents are lost, a new document will be completed. The loss of a document and an explanation of how the loss was rectified will be recorded in the document control logbook, which is maintained by ARDC. The serial number and disposition of all damaged or destroyed field documents will also be recorded. All voided and completed documents will be maintained with ARDC.

\subsubsection{Sample Container Labels}

Waterproof, gummed labels will be used that show information concerning the sample ID number, the name of the project, sample location, and analysis type. In the field, labels will be completed and placed on the containers before collecting the sample. Information concerning sample date, time, preservative used, field measurements of hazards, and the sampler's initials will be filled out during field sampling. Figure 6-1 shows an example of a sample container label.

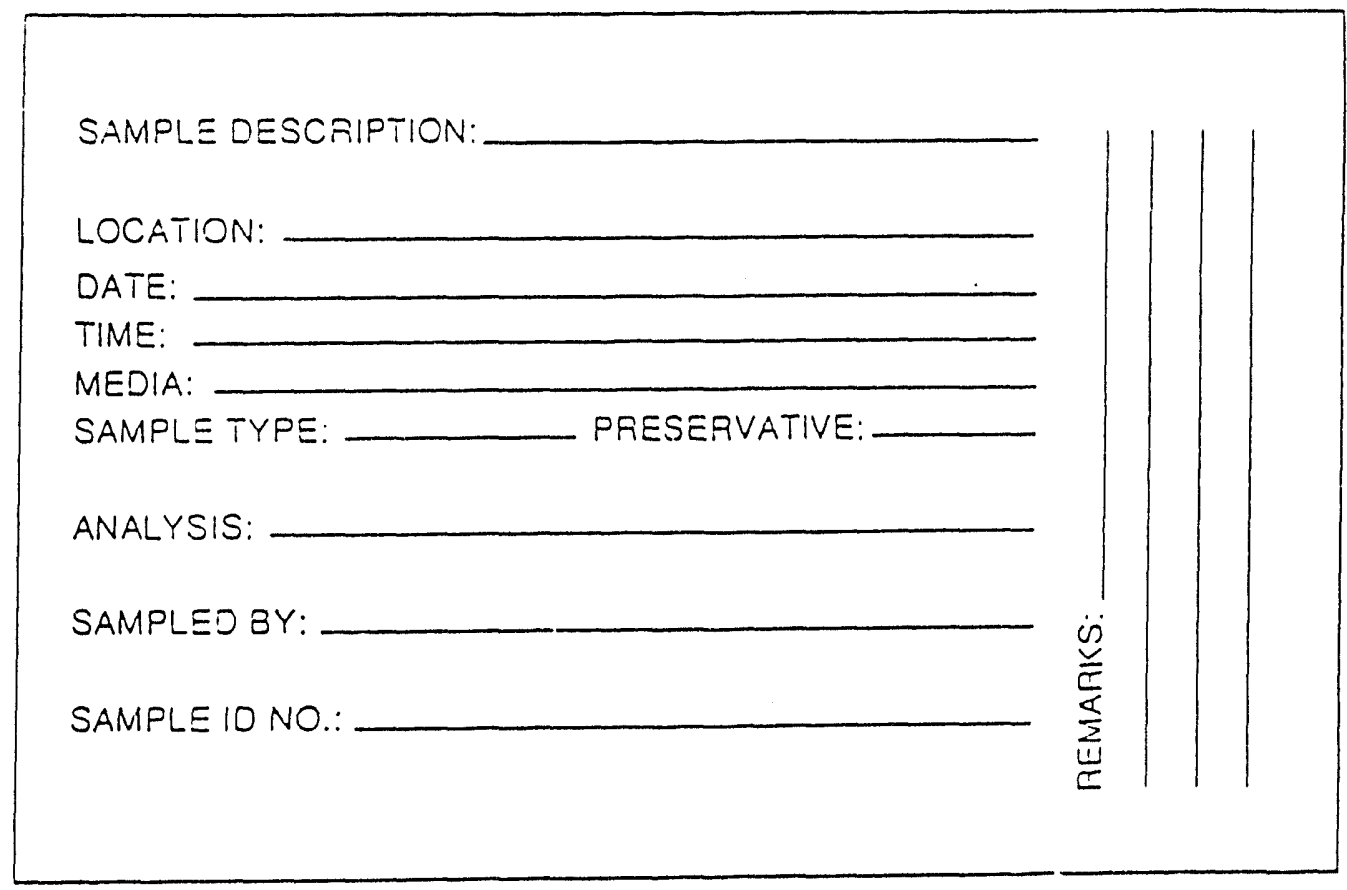

Figure 6-1. Sample container label. 


\subsubsection{Field Guide Forms}

Field guide forms are used to facilitate sample container documentation and organization of field activities. Field guide forms contain information on the sample request number, sample ID numbers, sample location, aliquot ID, analysis type, container size and type, and sample preservation. An example of a field guide form is shown in Figure 6-2.

\subsubsection{Field Logbooks}

Field logbooks, in accordance with ARDC format, will be used to record information necessary to interpret the analytical data. All field information pertaining to the sampling team activities will be entered in the loghooks. Entries will be dated and signed by the individual making the entry. All logbooks will be QC checked for accuracy and completeness by the Field Team Leader or an appointed designee. The person responsible for QC checks will sign his or her name in the appropriate space, indicating the above information is true and correct. All field logbooks will be controlled and managed according to EG\&G Idaho ER PD 4.2.

6.1.3.1 Sample Logbooks. Sample logbooks will be used by the field teams. Each sample logbook will contain copies of forms to record specific sample collection information. Appendix C contains examples of the sheets contained in this book. Soil cover, soil gas, groundwater, and all QC samples will be recorded in the sample logbook.

6.1.3.2 Field Team Leader's Daily Logbook. A project logbook will be maintained by the Field Team Leader. This logbook will contain a daily summary of all the team activities, problems encountered, deviations from the sampling and analysis plan, visitor log, and list of site contacts. This logbook will be signed and dated at the end of each day's sampling activities.

6.1.3.3 Field Instrument Calibration/Standardization Logbook. Each piece of equipment requiring periodic calibration or standardization will have a logbook to record equipment calibration data. This logbook will contain logsheets to record the date, time, method of calibration, and instrument identification number (see Appendix C).

6.1.3.4 Sample Shipping Logbook. This logbook will be used to record the sample ID number, collection date, shipping date, cooler ID number, destination, date shipped, chain-of-custody number, sample shipping classification, name of shipper, and QA check (see Appendix C).

6.1.3.5 Geologic Logbook. This logbook will be used to record the lithology of the sediment and waste during the subsurface sampling investigation at Landfill II. Information to be recorded includes grain size, sorting, color, texture, moisture, etc. Figure 6-3 is an example of a lithologic log.

\subsubsection{Data Sheets}

In addition to field logbooks, data sheets will be used to record field information. A water level data sheet will be used to record pertinent information when measuring the water level in wells. A data sheet will also be used to record soil cover thickness. Figure 6-4 is an example of a data sheet. 


\section{ENVIRONMENTAL PROBLEM NUMBER:}

Sampling Team:

Account Code: CFA

Sample Request: 4C001

Sample Number(s): 4CQ001 through 4CQ003

Media: Water

Sample Type: Grab

Sampling Method: SOP 11.1.1

Suspected Sampling Hazard: Metals

Sample Location(s): CFA OU4-12, LF2-8

ANALYSES VOA-524.2, Metals - CLP

RAD: NA

NON-RAD: 524.2 Organics, CLP Inorganics,

FIELD: Rad, Organic Vapors

Sample Containers, Volumes, and Preservatives:

\begin{tabular}{clll}
$\begin{array}{c}\text { Aliquot ID } \\
\left(10^{\text {th }} \text { char. }\right)\end{array}$ & $\begin{array}{l}\text { Analytical } \\
\text { Parameter }\end{array}$ & $\begin{array}{l}\text { Container Volume } \\
\text { or Type }\end{array}$ & Preservative \\
\hline 2 & VOA & $40-\mathrm{mL} \mathrm{vial}$ & $4^{\circ} \mathrm{C} / \mathrm{HCL}$ \\
1 & CLP Inorganics & $1000-\mathrm{mL} \mathrm{WM} \mathrm{glass}$ & $4^{\circ} \mathrm{C} / \mathrm{HNO}_{3}$
\end{tabular}

Figure 6-2. Example of a sample field guide form. 


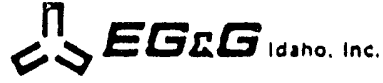

FORM EGSG.020

(Rev. 0783)

Surface Elevation (feet)

Geological
Classification
Description

GEOSCIENCES

FIELD LOG OF DRILL HOLE:

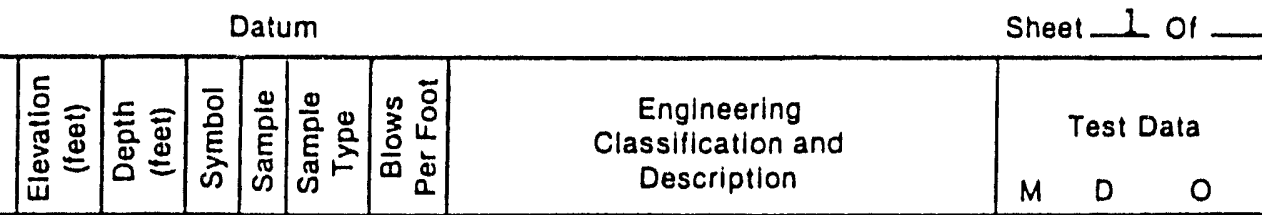




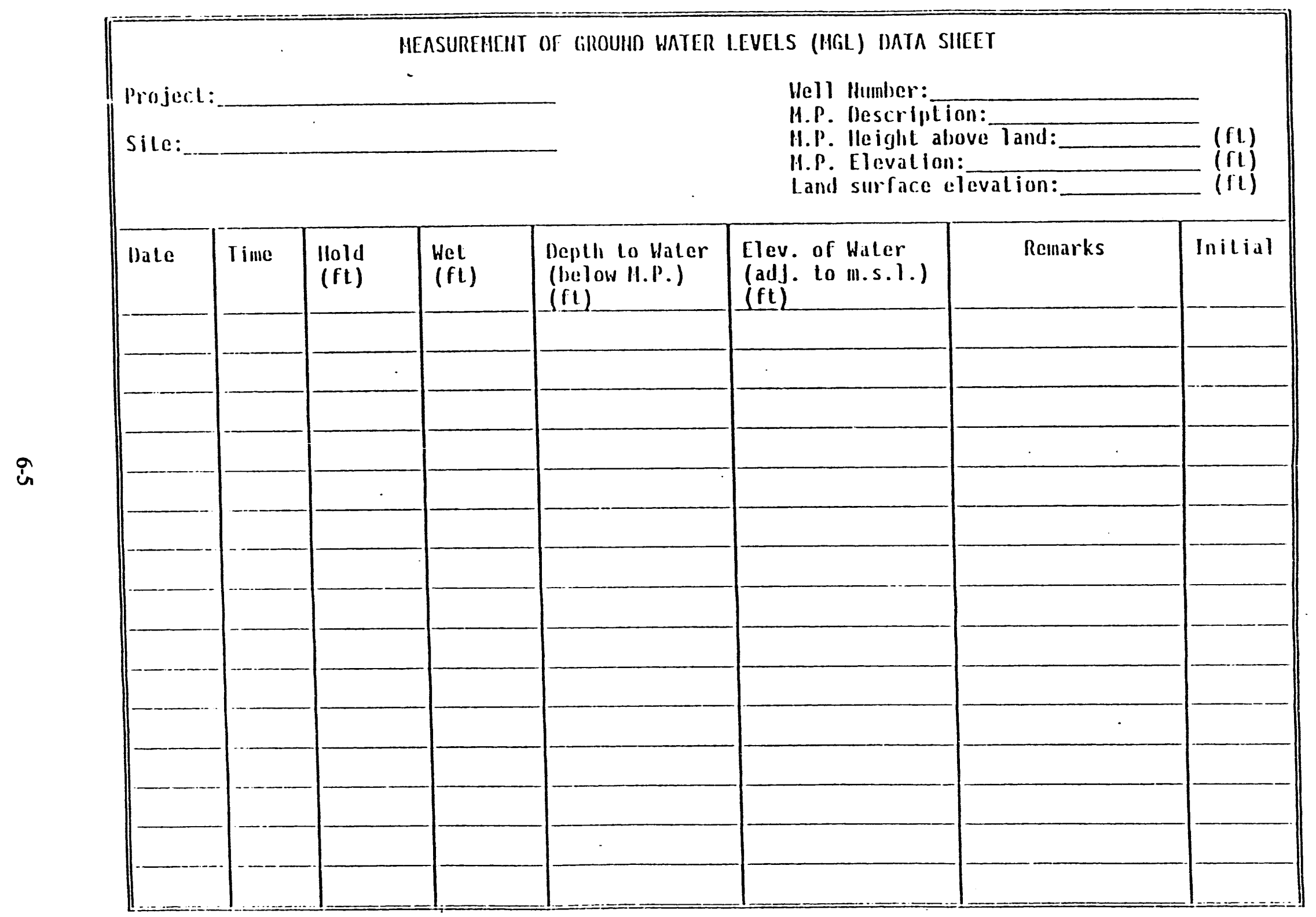

Figure 6-4. Water level data sheet. 


\subsection{Sample Handling}

Tables 6-1 and 6-2 outline generic requirements for containers, preservation methods, sample volumes, and holding times for solid and aqueous samples. Specific requirements pertaining to the types of samples and analytes involved in this project will be followed as prescribed in the tables.

\subsubsection{Sample Preservation}

Preservation of groundwater samples will be performed immediately upon sample collection (see Table 6-1). The temperature will be checked periodically prior to shipment to ensure adequate preservation for those samples requiring temperatures at $39^{\circ} \mathrm{F}\left(4^{\circ} \mathrm{C}\right)$ for preservation. Each field team will be equipped with field sample preservation kits, which may include nitric acid, sulfuric acid, hydrochloric acid, and $\mathrm{pH}$ indicator paper. Ice chests (coolers) containing frozen blue ice will be used to chill samples, if required, in the field after sample collection.

\subsubsection{Chain of Custody Procedures}

To maintain and document sample possession, chain of custody $(\mathrm{COC})$ procedures will be followed per ER PD 5.7 and the QAPJP. The purpose of COC is to document the identity of a sample and its handling from the point of collection until laboratory analysis is complete. The COC record will be a multiple-copy form, which serves as a written record of the sample handling. When a sample changes custody, the person(s) relinquishing and receiving the sample will sign a $\mathrm{COC}$ record. Each change of possession will be documented. Thus, a written record tracking sample handling will be established.

COC procedures will begin immediately after sample collection. COC forms (Figure 6-5) will be initiated the day of sample collection. Sample bottles will be stored in a secured area accessible only to the field team members.

\subsubsection{Transportation of Samples}

All short-holding-time samples will be shipped "priority one/overnight" via Federal Express through the Federal Express Office, in accordance with the regulations issued by the Department of Transportation (DOT) (49 CFR Parts 171 through 178) and EPA sample handling, packaging, and shipping methods (40 CFR 261.C.3C.3).

All samples will be packaged and transported in a manner that protects the integrity of the sample and prevents sample leakage. Packaging procedures will vary, depending on the suspected sample concentrations and DOT hazard class. Figure 6-6 depicts a properly packaged and labeled cooler containing nonradioactive environmental samples for shipment to the analytical laboratory. The temperature of each batch of coolers (i.e., those arriving at the laboratory at the same time) will be checked. One cooler per batch will be opened, a thermometer will be placed inside and allowed to equilibrate, and the temperature will be recorded in a logbook by personnel at the analytical laboratory. The laboratory will communicate these temperatures to the field to ensure adequate coolant is used to cool the samples during shipment. 
Table 6-1. Typical sample requirements-aqueous samples.

\begin{tabular}{|c|c|c|c|c|c|}
\hline \multirow[b]{2}{*}{ Analvtical parameter } & \multicolumn{2}{|c|}{ Container } & \multirow[b]{2}{*}{ Preservative } & \multirow[b]{2}{*}{ Holding time $^{a}$} & \multirow{2}{*}{$\begin{array}{c}\text { Sample volume } \\
(\mathrm{mL})^{b}\end{array}$} \\
\hline & $(\mathrm{mL})$ & Tvpe & & & \\
\hline Volatile organics & 40 & $\begin{array}{l}\text { Glass vial w/ } \\
\text { teflon septa }\end{array}$ & $\begin{array}{l}4^{\circ} \mathrm{C} \text { and } 1: 1 \\
\mathrm{HCL} \text { to } \mathrm{pH}<2\end{array}$ & 14 days & $\begin{array}{l}80 / 2-40 \\
(6 \times 40 \mathrm{~mL} \text { for } \\
\text { full } \mathrm{QC})\end{array}$ \\
\hline CLP metals & 1000 & HDPE (NM) & $\mathrm{pH}<2, \mathrm{HNO}_{3}$ & $\begin{array}{l}6 \text { months, } \\
\text { Hg } 28 \text { days }\end{array}$ & 1,000 \\
\hline Hexavalent chromium & 500 & HDPE (NM) & $4^{\circ} \mathrm{C}$ & $24 \mathrm{hrs}$ & 500 \\
\hline Alkalinity & 250 & Glass & $4^{\circ} \mathrm{C}$ & $\begin{array}{l}\text { ASAP (none } \\
\text { given) }\end{array}$ & 50 \\
\hline Fluoride & 1000 & HDPE & - & 48 hours & 50 \\
\hline Sulfate/chloride & 500 & Glass & $4^{\circ} \mathrm{C}$ & 28 days & 50 \\
\hline Nitrate/nitrite & 500 & Glass & $\begin{array}{l}2 \mathrm{~mL} \mathrm{H}_{2} \mathrm{SO}_{4} / \mathrm{L} \\
\text { sample as } \\
\text { specified if } \\
\text { H.T. > } 24 \mathrm{hrs}\end{array}$ & $\begin{array}{l}\text { ASAP } \\
\text { must be preserved }\end{array}$ & 250 \\
\hline Gamma & 540 & Plastic & $\mathrm{pH}<2, \mathrm{HNO}_{3}$ & Screen immediately & 500 \\
\hline Cyanide & 1000 & HDPE & $\mathrm{pH}>12 \mathrm{NaOH}$ & 14 days & 500 \\
\hline
\end{tabular}

a. Holding times are from date of collection as referred to in Federal Register Vol. 49, No. 209, October 26, 1984.

b. Samples will be collected unfittered except for metals which will have both filtered and unfiltered samples collected. 
Table 6-2. Typical sample requirements-chemical, physical, and hydraulic properties-soil samples.

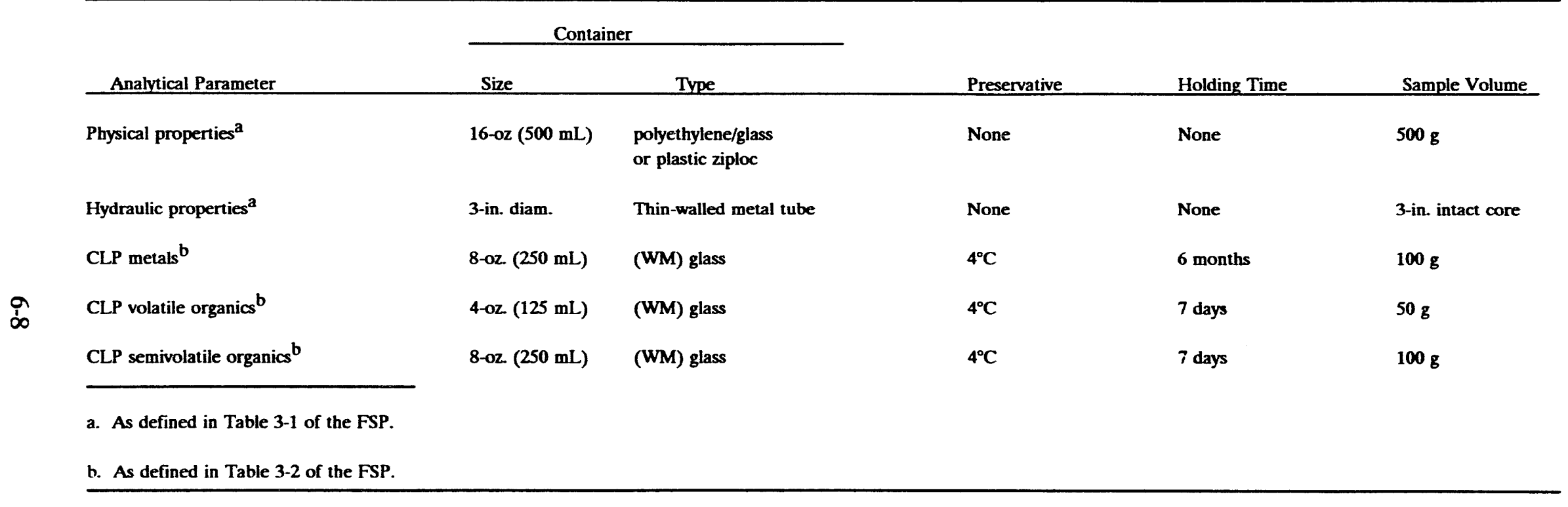




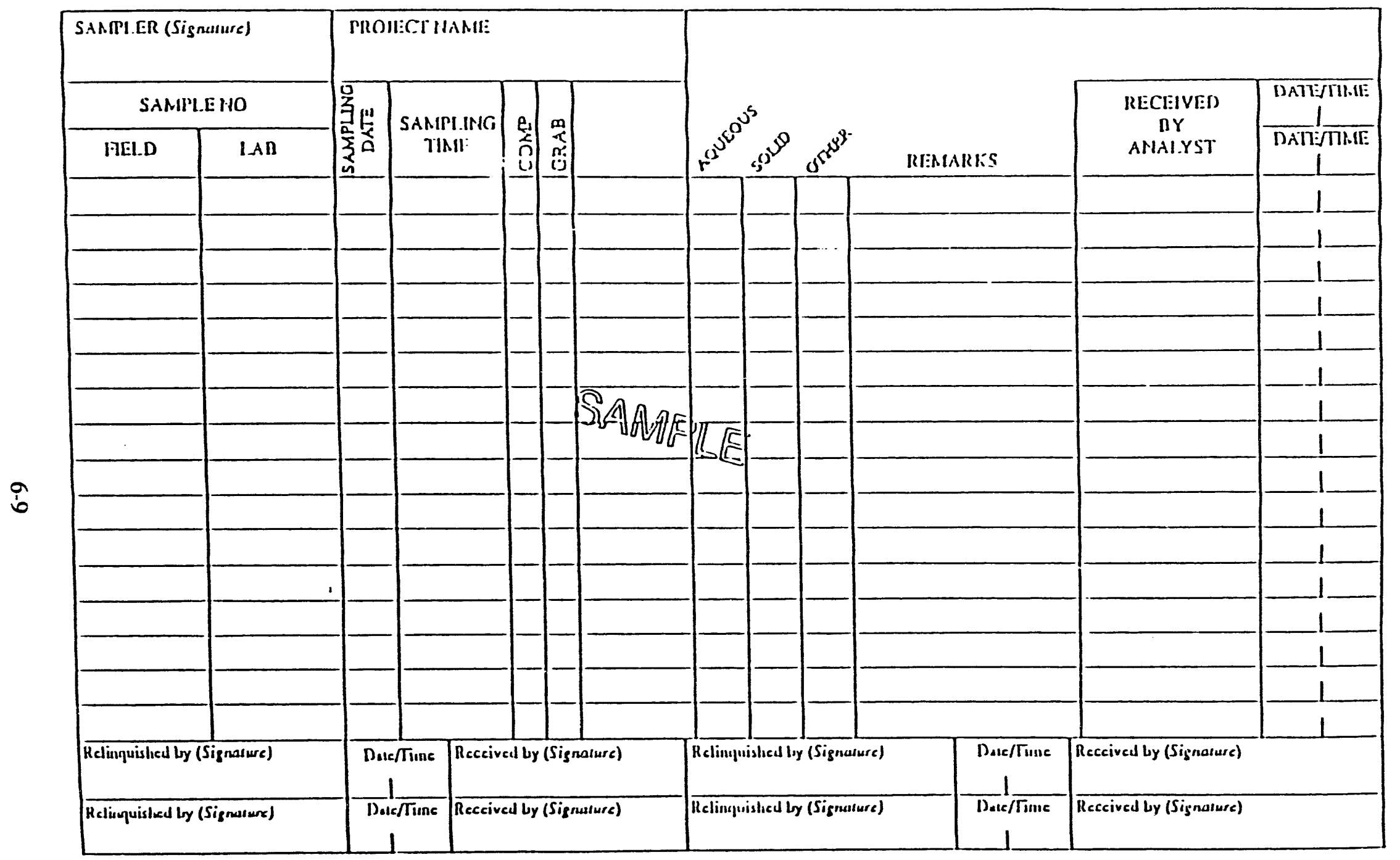

Figure 6-5. Chain of custody form. 


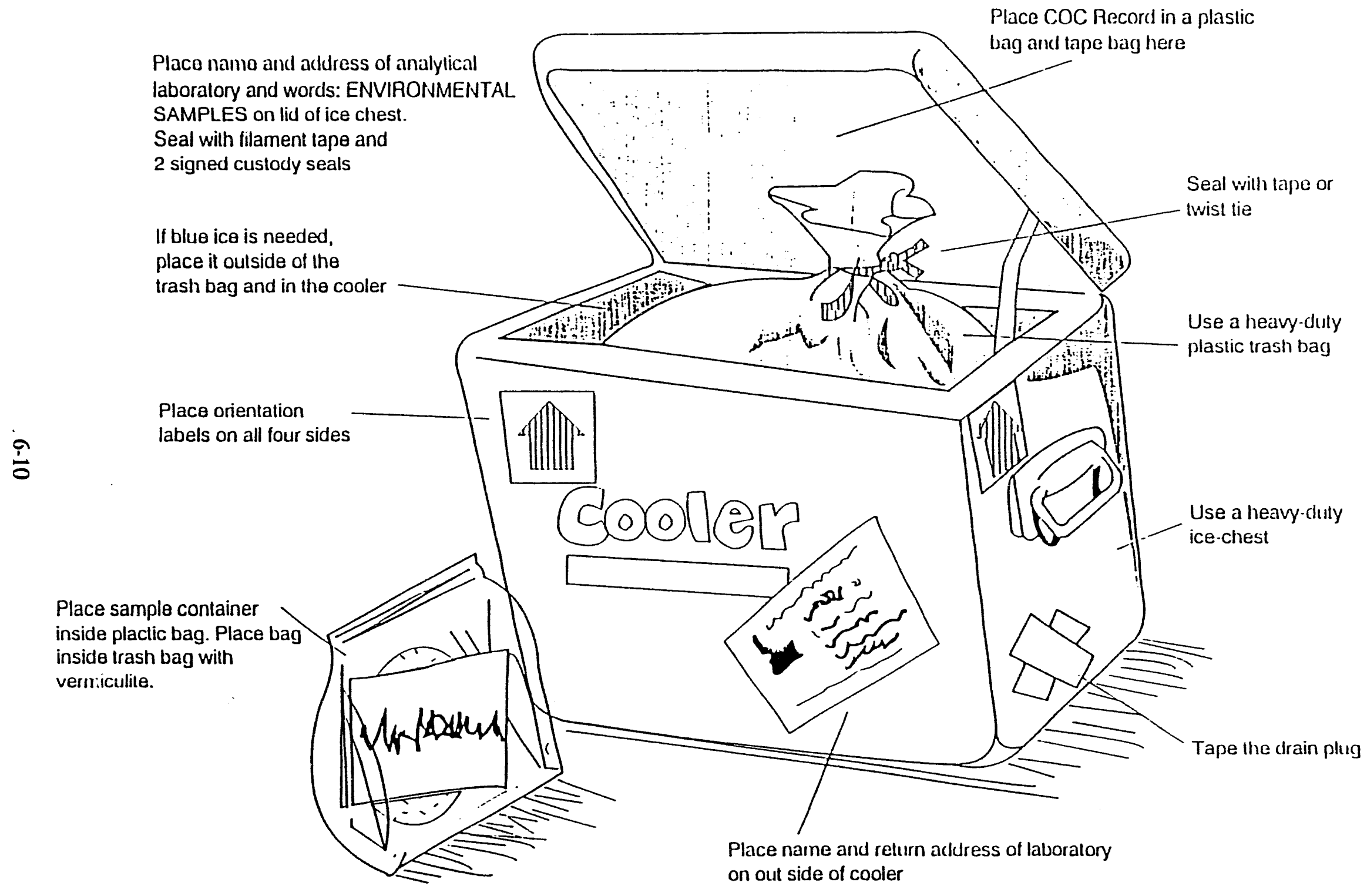

Figure 6-6. Packaging of environmental samples for shipment to the analytical laboratory. 
6.2.3.1 Field Radiation Screening and Green Tagging. All samples and field equipment will be surveyed by the Health Physics Technician for radiological contamination for shipping purposes. If the soil and soil gas samples are determined to be nonradioactive (betagamma activity is less than $100 \mathrm{cpm}$ above background and the health physics technician determines no alpha activity), the health physics technician will release the samples so they may leave the site. Samples will be collected during groundwater sampling for a gamma screen. This information, along with surveys by a health physics technician, will be used to determine shipping requirements. It should be noted that "real time" monitoring for tritium in the groundwater is not available. Historical data will be used to determine shipping requirements with respect to tritium. For soil samples that do not meet these criteria, an aliquot of the sample will be sent to the radiation measurement laboratory at the Test Reactor Area for a gamma scan characterization. Based on this characterization, samples will be packaged accordingly.

6.2.3.2 Custody Seals. Custody seals will be placed on all shipping containers. Clear, plastic tape will be placed over the seals to ensure that the seals are not accidentally broken during shipment.

6.2.3.3 Onsite and Offsite Shipping. An onsite shipment is any transfer of material within the perimeter of the INEL. Site-specific requirements for transporting of samples within Site boundaries and those required by the shipping/receiving department will be followed. Shipments within INEL boundaries will conform to DOT requirements, as stated in 49 CFR. Offsite sample shipment will be coordinated with EG\&G shipping personnel and will conform to all applicable DOT requirements. 


\section{REFERENCES}

EG\&G Idaho, 1992, Data Management Plan for the EG\&G Idaho Environmental Restoration Department, O. V. Hester, C. S. Watkins, and D. J. Yurman, EGG-ERD-10227, Revision 0, June 1992.

EPA, 1986, Measurement of Gaseous Emission Rates from Land Surfaces Using an Emission Isolation Flux Chamber, EPA/600/8-86/008, Environmental Monitoring Systems Laboratory, Office of Research and Development, U.S. Environmental Protection Agency, Las Vegas, NV 89114.

EPA, 1988, Compedium of Methods for the Determination of Toxic Organic Compounds in Ambient Air, U.S. Environmental Protection Agency, Atmospheric Research and Exposure Assessment Laboratory, Office of Research and Development, U.S. Environmental Protection Agency, Research Triangle Park, NC 27711.

EPA, 1990, "National Oil and Hazardous Substances Pollution Contingency Plan," Federal Register, Volume 55, No. 46, pp. 8,813-65, 1990.

EPA, 1991, Conducting Remedial Investigations/Feasibility Studies for CERCLA Municipal Landfill Sites, EPA/540/P-91/001, Office of Emergency Remedial Response, Washington D.C.

Miller, S. M., J. E. Hammel, and L. F. Hall, 1990, Characterization of Soil Cover and Estimation of Water Infiltration of Central Facilities Area Landfill II, Idaho National Engineering Laboratory (INEL), Idaho Water Resources Research Institute, University of Idaho, Moscow, ID. 


\section{Appendix A}

\section{Sampling and Analysis Plan Tables}


Sap Munter: aps-12-sc

SAHPLING AND AMALYSIS PLAN TABLE FOR CHERICAL AND RADIOLOGICAL MMALYSIS SAP Table Ho. The Revision: 3.0 Project: CFA OU 4-12 RI/FS - SOIL COVER

\begin{tabular}{|c|c|c|}
\hline $\begin{array}{l}\text { SAMPLING } \\
\text { ACrIVIIIY }\end{array}$ & $\mid \begin{array}{c}\text { SAMPLE } \\
\text { TYPEE }\end{array}$ & MEOIA \\
\hline $4 c 5001$ & REG & soll \\
\hline ecs002 & REG & solt \\
\hline \&cs003 & REG & soll \\
\hline scsoos & REG & soll \\
\hline scsoos & REG & so:L \\
\hline sesoos & REG & solt \\
\hline $6 \mathrm{Cs} 5007$ & REG & SOIL \\
\hline $4 \mathrm{Cs008}$ & REG & solt \\
\hline $4 C 5009$ & REG & soll \\
\hline $8 C 5010$ & REG & SOIL \\
\hline scsol1 & REG & SOIL \\
\hline scso12 & REG & soll \\
\hline 4CSO13 & REG & soll \\
\hline 455016 & REG & soll \\
\hline $4 c 5015$ & REGG & soIt \\
\hline
\end{tabular}

3.0

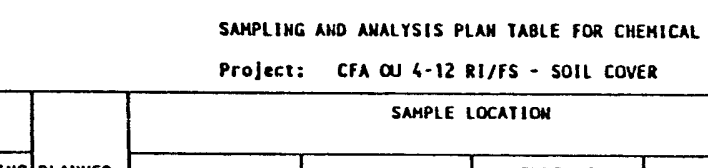

Projecs Manager: K. KECK/G. STORKBERG
ENTER ANALYSIS IYPES (AI) AND OUAKTIIY REOUESTED

Page 1 of 2 Form No: SAP118

\begin{tabular}{|c|c|c|c|c|c|c|c|c|c|c|c|c|c|c|c|c|c|c|c|c|c|c|}
\hline \multirow{3}{*}{\multicolumn{2}{|c|}{\begin{tabular}{|l|l|}
\multicolumn{2}{|l|}{} \\
COLL \\
IYPE & SMELLING \\
\end{tabular}}} & \multirow{3}{*}{$\begin{array}{l}\text { PLAMEED } \\
\text { DAIE }\end{array}$} & \multicolumn{4}{|c|}{ SAMPLE LOCATION } & \multicolumn{16}{|c|}{ ENTER ANALYSIS IYPES (AI) AND OUAKTITY REOUESTED } \\
\hline & & & \multirow[b]{2}{*}{ AREA } & \multirow[b]{2}{*}{ LOCAIION } & \multirow{2}{*}{$\begin{array}{l}\text { TYPE OF } \\
\text { LOCAIIOW }\end{array}$} & \multirow{2}{*}{$\begin{array}{c}\text { DEPIM } \\
(\mathrm{ft})\end{array}$} & AII & \begin{tabular}{|l|l|}
12 & $A$ \\
\end{tabular} & A.3 & AT4 & Ars & AT6 - & A17 & ATB & A19 & al10/, & NIIIAT12 & AII3 & A116 & A715 $\mid$ Ar16 & \begin{tabular}{|l|l|l|l|l|} 
AT17 & AIIB \\
\end{tabular} & $A 119 \mid A 120$ \\
\hline & & & & & & & ow & $\mathbf{P A}$ & & & & & & & & & & & & & & \\
\hline GRAB & & $06 / 01 / 93$ & CFA & SC-TBD-1 & LAMOFILL III & 1 & 1 & 1 & & & & & & & & & & & & & & \\
\hline GRAB & & $00 / 01 / 93] \mathrm{c}$ & CFA & $5 c-180-1$ & LAMDFILL :II & 2 & 1 & 1 & & & & & & & & & & & & & & \\
\hline GRAB & & $06 / 01 / 93$ & CFA & $S C-T B O-2$ & LAMDFILL III & 1 & 1 & 1 & & & & & & & & & & & & & & \\
\hline GRAB & & 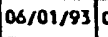 & CFA & $5 C-180-2$ & LANOFILL III & 2 & 1 & 1 & & & & & & & & & & & & & & \\
\hline GRAB & & 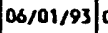 & CFA & SC- TBO-3 & LAMDFILL III & 1 & 1 & 1 & & & & & & & & & & & & & & \\
\hline GRAB & & $06 / 01 / 93$ & cra & SC-TBD-3 & LaNDFILL "II & 2 & 1 & 1 & & & & & & & & & & & & & & \\
\hline GRAB & & $06 ; 01 / 93$ & CFA & $5 C-780-4$ & LANOFILL III & 1 & 1 & 1 & & & & & & & & & & & & & & \\
\hline GRAB & & $\infty \alpha / 01 / 93$ & CFA & $5 C-180-6$ & LAKOFILL III & 2 & 1 & 1 & & & & & & ' & & & & & & & & \\
\hline GRAB & & $06 / 01 / 93$ & CFA & SC-TBO-S & LANOFILL III & 1 & 1 & 1 & & & & & & & & & & & & & & \\
\hline GRAB & & -6/01/93 & CFA & SC-TBO-S & LANDFILL III & 2 & 1 & 1 & & & & & & & & & & & & & & \\
\hline GRAB & & $\infty 6 / 01 / 93$ & CFA & $5 C-180-6$ & LaNDFILL & 1 & 1 & 1 & & & & & & & & & & & & & & \\
\hline CRAB & & $|06 / 01 / 93|$ & CFA & SC-180-6 & LANDFIIL III & 2 & 1 & 1 & & & & & & & & & & & & & & \\
\hline$\therefore A B$ & & $|06 / 01 / 93|$ & CFA & $5 C-180-7$ & LANOFILL "II & 1 & 1 & 1 & & & & & & & & & & & & & & \\
\hline GRAB & & $06 / 01 / 93$ & CFA & SC- $8 B D-7$ & LAMOFILL III & 2 & 1 & 1 & & & & & & & & & & & & & & \\
\hline CRAB & & $\mid 06 / 01 / 93$ & CFA & $5 C-180-8$ & LANDFILL III & 1 & 1 & 1 & & & & & & & & & & & & & & \\
\hline
\end{tabular}

Enter the appropriate analysis type code in the boxes between the double lines under "ENIER ANAtrSIS TYPES". Refer to SAP Iable 2, Sampling And Analysis Plan Table - Codes t Descriptions.

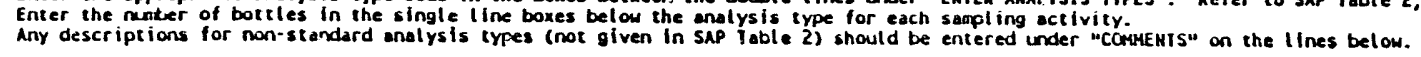
All: Grevimetric vater Content (ASin 0-2216
A12: Particle Size Analysis (ASIH D-622)
AI3:
A14:
Ars:
Al6:
A18:
A.r:
AI10:

AIII:

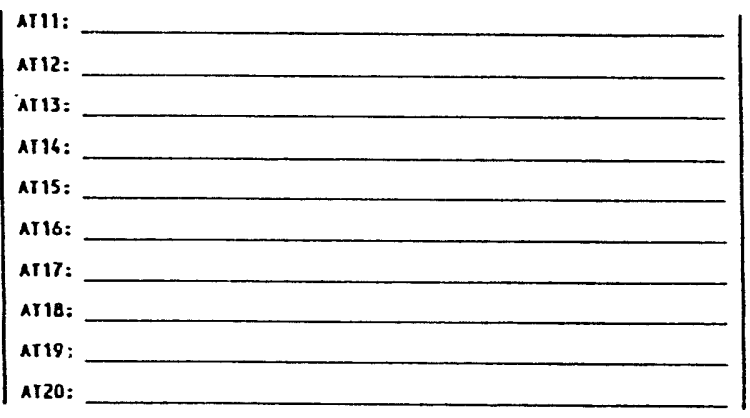

COHAENS

SC-rBD-1 = (S)oll (C)over - Location (I) (B)e (D)etermined - Site (1)_elc. Specific stmple locations uill be determined in the field $-2$ 
SAP Number: $\frac{\text { ax }-12-S C}{1}$ Date: 06/07/93 Revision: 3.0

SAMPLING AND aHALYSIS PLAN TABLE FOR ChEMICAL ANO RNDIOLOGICAL aMALYSIS Project: CFA OU 6-12 RI/FS - SOIL COVER

Page 2 of 2 SAMPLE LOCATION

Project Manager: K. KECK/G. STORHBERG Form Ho: SAP118 EhTER AHALYSIS TYPES (AT) AND OUANIIIY REQUESTED

\begin{tabular}{|c|c|c|c|c|c|c|c|c|c|c|c|c|c|c|c|c|c|c|c|c|c|c|c|c|c|c|c|c|}
\hline & & & & & & & & & & & & & & & & & & & & - & & & 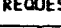 & . & & & & \\
\hline $\begin{array}{l}\text { SAMPLING } \\
\text { ACIIVIIYY }\end{array}$ & \begin{tabular}{|c|} 
SAMPLE \\
IYPEE
\end{tabular} & MEOIA & $\mid \begin{array}{l}\text { COLL } \\
\text { IYPE }\end{array}$ & $\begin{array}{c}\text { SAMPL ING } \\
\text { MEIHCD }\end{array}$ & $\begin{array}{l}\text { PLANHED } \\
\text { DATE }\end{array}$ & & & IYPE of & DEPIM & ari & Ar2 & AI3 & A16 & Ars & A16 & Ant & A18 & A19 & & AIIII & I:12 A & Nri3 Ar & $114 / \mathrm{Ar}$ & AIIS/ 1 & $116 / 11$ & $117 \mid A 118$ & AI19 & 120 \\
\hline 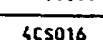 & IREG & to & & & & AREA & Locarton & LOCATIOH & $(t t)$ & GN & PA & & & & & & & & & & & & & & & & & \\
\hline 825010 & pete & 50011 & GRAB & & $06 / 01 / 93$ & CFA & $\mid 5 C-7 B D-8$ & LANDFILL III & 2 & 1 & 1 & & & & & & & & & & & & & & & & & \\
\hline & & & & $\ldots$ & & & & & & & & & & & & & & & & & & & & & & & & \\
\hline & & & & & & & & & & & & & & & & & & & & & & & & & & & & \\
\hline & & & & & & & & & & & & & & & & & & & & & & & & & & & & \\
\hline & & & & & & & & & & & & & & & & & & & & & & & & & & & & \\
\hline & & & & & & & & & & & & & & & & & & & & & & & & & & & & \\
\hline & & & & & & & & & & & & & & & & & & & & & & & & & & & & \\
\hline & & & & & & & & & & & & & & & & & . & & & & & & & & & & & \\
\hline & & & & & & & & & & & & & & & & & - & & & & & & & & & & & \\
\hline & & & & & & & & & & & & & & & & & & & & & & & & & & & & \\
\hline & & & & & & & & & & & & & & & & & & & & & & & & & & & & \\
\hline & & & & & & & & & & & & & & & & & & & & & & & & & & & & \\
\hline & & & & & & & & & & & & & & & & & & & & & & & & & & & & \\
\hline & & & & & & & & & & & & & & & & & & & & & & & & & & & & \\
\hline & & & & & & & & & & & & & & & & & & & & & & & & & & & & \\
\hline & & & & & & & & & & & & & & & & & & & & & & & & & & & & \\
\hline
\end{tabular}

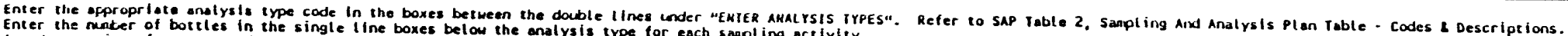
Any descriptions for non-standard analysis types (not given in SAP Yable 2) should be entered inder "ConMENIS" on the lines below.

AI1: Gravimetric Water Content (ASTH D-2216)

A12: Particle Size Analysls (ASIM D-62?)

A13:

A16:

A15:

AIs:

A17:

A18:

A19.

AI10:

10:
Ar11: AT12:

AII3:

AT16:

A117:

Arse:

Ar19:

AT20:
COHAEN IS

SC- IBO-1 = (S)Oil (C)over - Location (I) O (B)e (O)etermined - site (1), etc. Specific sample locations will the deterained in the field

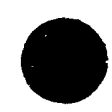


SAP Number: aKf-12-1SOI

SAMPLING AND ANALYSIS PIAN TABLE FOR CHEMICAL AND RADIOLOGICAL AMALYSIS

Date: 06/16/93 Revision: 3.0

Prolect: CFA OU 4-12 RI/FS - IM-SIIU DRAIHAGE IEST/IXIACT SHELBY TUBE

Project Hanager: K. KECK/G. SIORHBERG

Page 1 of $\underline{2}$

\begin{tabular}{|c|c|c|}
\hline $\begin{array}{l}\text { SAMPLING } \\
\text { ACrIVIIIY }\end{array}$ & $\begin{array}{c}\text { SAMPLE } \\
\text { TYPE }\end{array}$ & MEDIA \\
\hline scsoly & REG & soll \\
\hline 625018 & REG & sole \\
\hline ecso19 & REG & solt \\
\hline $4 \mathrm{CSO20}$ & REG & soll \\
\hline \&c5021 & REG & solt \\
\hline $6 c 5022$ & REG & 5011 \\
\hline $6 \mathrm{CSO} 023$ & REG & soll \\
\hline \&c5026 & REG & solt \\
\hline $6 \mathrm{cs025}$ & REG & soll \\
\hline $6 c \leq 028$ & REG & solt \\
\hline \&es027 & REG & soll \\
\hline ¿CsO28 & |REG & solt \\
\hline 625029 & REG & solt \\
\hline $4 \operatorname{cs} 030$ & REG & solt \\
\hline scso31 & REG & solt \\
\hline
\end{tabular}

\begin{tabular}{|c|c|}
\hline $\begin{array}{l}\text { COLL } \\
\text { IYPE }\end{array}$ & SAMPII \\
\hline GRAB & \\
\hline GRAB & \\
\hline GRAB & \\
\hline GRAB & \\
\hline GRAB & \\
\hline GRAB & \\
\hline GRAB & \\
\hline GRAB & \\
\hline $\cos P$ & \\
\hline $\operatorname{CONP}$ & \\
\hline COMP & \\
\hline COMP & \\
\hline COAP & \\
\hline COMP & \\
\hline & \\
\hline
\end{tabular}

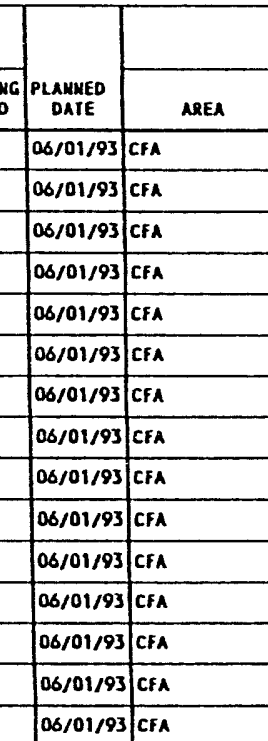
SAMPLE LOCATIOH

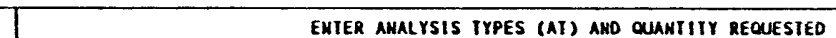

Foria No: SAP11B

\begin{tabular}{|c|c|c|c|c|c|c|c|c|c|c|c|c|c|c|c|c|c|}
\hline & & & AII & A12 & ars & 116 & AIS & AT6 & AI7 & A18 & AT9 & Atio|aril & Ar12|ATl3 & ArI6|aris & ar16|ariz & AI18 $\mid$ ar19 & ar20 \\
\hline Locallow & LOCAILOW & (It) & CH & ct & GN & & & & & & & & & & & & \\
\hline $5 C-18 D-1$ & LANOFILL III & .67 & 1 & 1 & & & & & & & & & & & & & \\
\hline SC- $180-2$ & LAMDFILL III & .07 & 1 & 1 & & & & & & & & & & & & & \\
\hline $5 C-180-3$ & LaMOFIIL III & .67 & 1 & 1 & & & & & & & & & & & & & \\
\hline $5 C-580-6$ & Lanoflll III & .67 & 1 & 1 & & & & & & & & & & & & & \\
\hline $5 C-180-5$ & (АNOFILL & .67 & 1 & 1 & & & & & & & & & & & & & \\
\hline $5 C-180.6$ & LANDFILL III & .67 & 1 & 1 & & & & & & & & & & & & & \\
\hline SC-TBO-7 & LLAMOFILL III & .67 & 1 & 1 & & & & & & & & & & & & & \\
\hline $5 C+180-8$ & LaMDFILL III & .67 & 1 & 1 & & & & & & $\cdot$ & & & & & & & \\
\hline $5 C-180-1$ & LAADFILL III & 0.1 & & & 1 & & & & & & & & & & & & \\
\hline$S C-\mathrm{SBD}-2$ & LANDFILL III & 0.1 & & & 1 & & & & & & & & & & & & \\
\hline SC- TBO-3 & LAROFILL III & 0.1 & & & 1 & & & & & & & & & & & & \\
\hline $5 C-180-6$ & LaADFILL III & $0-1$ & & & 1 & & & & & & & & & & & & \\
\hline SC- r8D-5 & LAHOFILL II & 0.1 & & & 1 & & & & & & & & & & & & \\
\hline $5 C-180 \cdot 6$ & LANOFILL II & 0.1 & & & 1 & & & & & & & & & & & & \\
\hline $5 C-160-7$ & LANDFILL II & $0-1$ & & & 1 & & & & & & & & & & & & \\
\hline
\end{tabular}

Enter the appropriate analysls type code in the boxez between the double lines under "ENIER AMALYSIS IYPES". Refer to SAP Teble 2, Sampling And Analysis Plan Table - Codes 2 Descriptions.

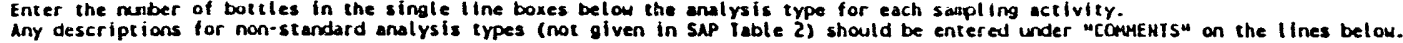

Al1: Constant kead Permesbillity (Astin 0-2636)

A12: Capillary Pressure-Molsture Content (ASIM 0.2325)

AI3: Gravimetric Hoter Content (ASIM 0-2216)

AT6:

AIS:

A16:

art:

AI8:

A19:

Ar10:
COMAENTS

SC-IBD-1 = (S20il (C)over - Location (T) $)$ (B)e (D)etermined - Sile (11) etc. Specific sample locations will be determined in the field

Samples analyzed for Constent Head Permeability (ASIM 0-2634) and Capill ary Pressure - Moisture Content ASIM 0-2325 will be collected by Sheltby rube, Collection Hethod Asin 0-1587. 
SAP Mutiter: $046-12-1$ SOI
SAP Table Mo

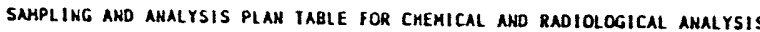

Project: CFA OU 4-12 R1/FS - IN-SITU ORAIMAGE TEST/IMTACT SHELGY TUBE SAHPLE LOCATIOH

Page $\underline{2}$ of $\underline{2}$

\begin{tabular}{|c|c|c|c|c|c|c|c|c|c|c|c|c|c|c|c|c|c|c|c|c|c|c|c|c|c|c|c|c|c|}
\hline & & MPLE DE & & & & & SAMPL & Locariou & & & & & & & & & & & & & & & & & & & & & \\
\hline & & & & & & & & & & & & & & & & & & & & AT) $A N$ & & & $Y$ REOU & UESTEO & & & & & \\
\hline SAMPL ING & SAMPLE & J MERU & cots & SAMPLING & PLAMMEd & & & IYPE OF & DEPIH & Ari & Al2 & AIs & A16 & A15 & ATt & A17 & 118 & Al9 & Al10 & Anil & Ar12 & $A T 13$ & Al16 & AT15 & AII 16 & Arin & Aris & Al19. A & A 120 \\
\hline AcIivity & IrPE & JEDIA & |IYPE & MEI HOO & DAIE & AREA & LOCATIOM & LOCATIOH & (ft) & $\mathrm{CH}$ & $a$ & cou & & & & & & & & & & & & & & & & & \\
\hline ses032 & REG & solt & Comp & & |06/01/93 & CFA & $5 c-180-8$ & LAMDFILL III & $0-1$ & & & 1 & & & & & & & & & & & & & & & & & \\
\hline 625033 & REG & solt & comp & & $|06 / 01 / 93|$ & CFA & sc- T80-1 & LАRDFIL III & $1-2$ & & & 1 & & & 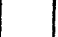 & & & & & & & & & & & & & & \\
\hline 655036 & REG & solt & comp & & | $6 / 01 / 93$ & CFA & |sc- & LaNDFILL III & $1-2$ & & & 1 & & & & & & & & & & & & & & & & & \\
\hline 6eso3s & REG & SOIL & cosp & & $|06 / 01 / 93|$ & cFa & $5 C-180-3$ & LandFILL III & $1-2$ & & & 1 & & & & & & & & & & & & & & & & & \\
\hline $4 c 5036$ & REG & solt & COHP & & $|06 / 01 / 93|$ & CFA & $5 c-580-4$ & LaNDFILL III & $1-2$ & & & 1 & & & 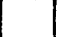 & & & & & & & & & & & & & & \\
\hline 4cso37 & REG & solt & comp & & $06 / 01 / 93$ & CFA & $5 C-180-5$ & LANOFILL III & $1-2$ & & & 1 & & & & & & & & & & & & & & & & & \\
\hline $6 c 5038$ & REG & soll & consp & & $06 / 01 / 93$ & cra & $5 C-180-6$ & LANDFILL III & $1-2$ & & & 1 & & & & & & & & & & & & & & & & & \\
\hline $6 \operatorname{cs} 039$ & REG & solt & consp & & $06 / 01 / 93$ & CfA & $5 C-180-7$ & LANOFILL III & $1-2$ & & & 1 & & & & & . & & & & & & & & & & & & \\
\hline $6 c 50 \div 0$ & REG & solt & COMP & & $06 / 01 / 93$ & cra & $5 C-I B O-8$ & LANOFILL III & $1-2$ & & & 1 & & & & & & & & & & & & & & & & & \\
\hline & & & & & & & & & & & & & & & & & & & & & & & & & & & & & \\
\hline & & & & & & & & & & & & & & & & & & & & & & & & & & & & & \\
\hline & & & & & & & & & & & & & & & & & & & & & & & & & & & & & \\
\hline & & & & & & & & & & & & & & & & & & & & & & & & & & & & & \\
\hline & & & & & & & & & & & & & & & & & & & & & & & & & & & & & \\
\hline & & & & & & & & & & & & & & & & & & & & & & & & & & & & & \\
\hline
\end{tabular}

Enter the appropt iate analysis type cade in the boxes between the dowble tines under "EHIER AMALYSIS IYPES". Refer to SAP Table 2, Sampl ing And Analysis Plen Table - Codes 2 Descriptions.

Any descriptions for non-standard analysis iypes (not given in SAP Table 2) should be entered under "contents" on the lines below.

All: Conslant Head Permeability (ASIK 0-2436)

Aril:

C O H HE T T

A12: Capillary Pressure-Molsture Content (ASIM 0-2325)

SC-raD-1 $=$ (S)OIl (C) over - Location (T)O (B)e (O)etermined - Site (11), etc -

AI3: Gravimetric Water content (ASIM D-2216)

AIs:

Al13:

Ais:

AIIL:

AIb:

AT15:

AI7:

Ar16:

A18:

AI17:

A19:

AI 18:

Ar10:

AT19:

A120:

Specific sample locations will be determined in the field

Somples analyzed for Constant hiead Permeabilitix (ASIN 0.2636) and Copillary Pressure - Moisture content ASIM 0-2325 will be collected by Shelby lutre.

Collecrion Hethod AStM D-1587. 


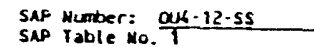

SAMPLING AND AMALYSIS PLAN TABLE For CHEMICAL AND RADIOLOGICAL AMALYSIS Date: $07 / 07 / 93$ Revision: 5.0 Project: CFA OU -12 RI/FS - SURFACE SOIL SMMPLES

\begin{tabular}{|c|c|c|}
\hline $\begin{array}{l}\text { SAMPLING } \\
\text { ACTIVIIYY }\end{array}$ & \begin{tabular}{|c|} 
SLMPLE \\
IYPEE
\end{tabular} & MEOIA \\
\hline sesosi & REG & soll \\
\hline $4 c 50<2$ & REG & solL \\
\hline \&cso6s & REG & Soll \\
\hline $8 c 5066$ & REG & soIL \\
\hline scsoss & REG & soll \\
\hline $4 c 5046$ & REG & solk \\
\hline $8 \mathrm{cs} a<t$ & REG & soll \\
\hline$\angle C 50<B$ & REG & solt \\
\hline $\operatorname{scs} 049$ & REG & SOIL \\
\hline scsoso & REG & soll \\
\hline $4 \operatorname{cscos} 1$ & REG & soll \\
\hline $4 c \operatorname{sos} 2$ & REG & SOIL \\
\hline $6 \cos 03$ & REG & SOIL \\
\hline $6 \operatorname{csos} 6$ & REG & soil \\
\hline $4 \operatorname{cscoss}$ & REG & SOIL \\
\hline
\end{tabular}

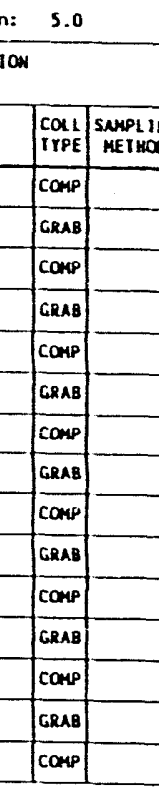

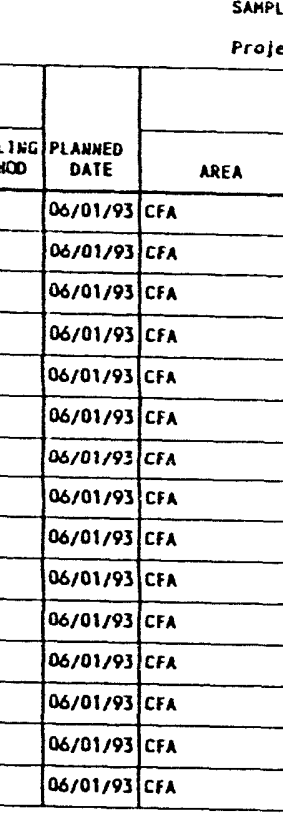

\section{SAMPLE LOCAHIOW}

$$
\text { T. }
$$

Project Munager: K. XECX/G. STORMBERG

Page 1 of $\leq$ Project Munager: K. KECX/G. STORMBERG FOrm KO: SAPIII ENTER ANAL YSIS IYPES (AI) AMD OUNAIIIY REQUESTED

\begin{tabular}{|c|c|c|c|c|c|c|c|c|c|c|c|c|c|c|c|c|c|c|c|c|c|}
\hline Lacho & IYPE of & & DEPIH & ArI & A12 & Al3 & ar6 & ATS & 116 & A17 & A18 & Ars & AI10a & ariila & Ar12/A & ATI3|AT16 & Aris, & Ar16, & $A+17 \sqrt{A}$ & $\operatorname{Ar} 18 \mid A$ & Ario arz \\
\hline Location & Locarion & & (ft) & a & $\mathrm{cv}$ & os & & & & & & & & & & & & & & & \\
\hline $55-180-1$ & LANOFILI II & & .5 & 1 & & & & & & & & & & & & & & & & & \\
\hline $5 S-$ TAD- 1 & LAMDFILL I: & & $1-5-2.0$ & & 1 & 1 & & & & & & & & & & & & & & & \\
\hline$S S-T B D-2$ & LANOFSIL "I & & .5 & 1 & & & & & & & & & & & & & & & & & \\
\hline$S S-1 B 0-2$ & LANDF ILL II & 111 & $1.5 \cdot 2.0$ & & 1 & 1 & & & & & & & & & & & & & & & \\
\hline $5 S-T B O-3$ & LandFilL & 111 & .5 & 1 & & & & & & & & & & & & & & & & & \\
\hline $55-180-3$ & LandFILL II & $n i$ & $1.5-2.0$ & & 1 & 1 & & & & & & & & & & & & & & & \\
\hline $5 S-180-6$ & LANOFILL "I & 111 & .5 & 1 & & & & & & & & & & & & & & & & & \\
\hline SS- TBD- 6 & LAMDFILL "I & 111 & $1.5-2.0$ & & 1 & 1 & & & & & & & & & & & & & & & \\
\hline SS-180-5 & LANDFILL II & 111 & .5 & 1 & & & & & & & & & & & & & & & & & \\
\hline $5 S-180-5$ & LaNDFill "II & 111 & $1.5-2.0$ & & 1 & 1 & & & & & & & & & & & & & & & \\
\hline SS-TBO. & LANOFILL II & 111 & .5 & 2 & & & & & & & & & & & & & & & & & \\
\hline $55-180-6$ & LAKDFHL II & 111 & $1.5-2.0$ & & 3 & 3 & & & & & & & & & & & & & & & \\
\hline $5 S-180-7$ & LANDFIL $\|$ & 111 & .5 & & & & & & & & & & & & & & & & & & \\
\hline SS- IBQ-7 & LANDFILI 11 & 111 & $1.5-2.0$ & & & & & & & & & & & & & & & & & & \\
\hline $5 S-T B D \cdot B$ & LANDFILL II & 111 & .5 & 1 & 1 & 1 & & & & & & & & & & & & & & & \\
\hline & & & & & & & & & & & & & & & & & & & & & \\
\hline
\end{tabular}

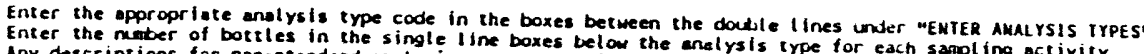

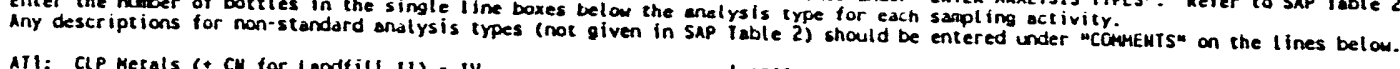

All: CLP ketals (+ CM for Landfill II) - IY

Aril:

A12: Clp volaclles - iv

AI3: CLP Senivolatiles - IV

A14:

ATS:

AT6:

a17:

AIs:

A110:

Art2:

AT13:

Ar16:

An15:

Ar16:

A118:

AI19:

AT20:
CONAENIS

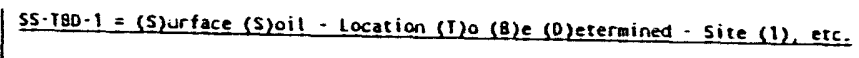

Specific sample locations will be deterained in the field.$$
\text { - }
$$ 
SAP Number: Ous-12-ss

$\begin{array}{ll}\text { SAP Yable Ho. } & \\ \text { Date: } 07 / 07 / 93 & \text { Revision: } 5.0\end{array}$

SAMPLING AND AMALYSIS PLAN tABLE fOR CHEMICAL aMD RADIOLOGICAL amalySIS

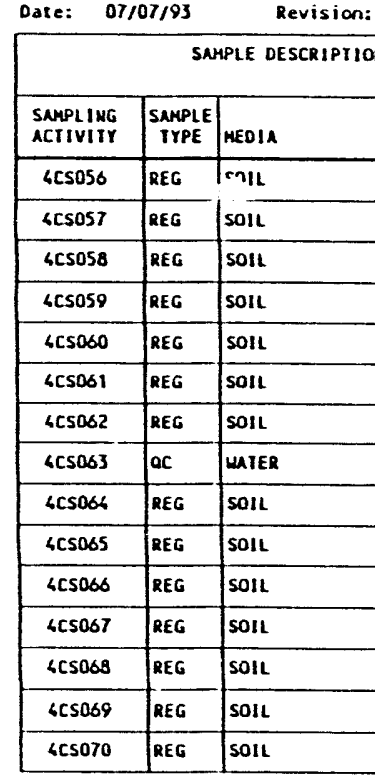

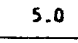

ProjeCr. CFA OU $4-12$ R1/FS - SURFACE SOIL SAMPLES
Page $\underline{2}$ of $\underline{4}$

Project Manager: K. KECK/G. STORHBERG

ENTER ANALYSIS IYPES (AT) AND QUANTITY REQUESTED

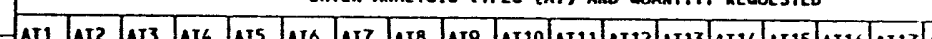

\begin{tabular}{|c|c|c|c|c|c|c|c|c|c|c|c|c|c|c|c|c|c|c|c|c|c|}
\hline \multicolumn{2}{|l|}{ 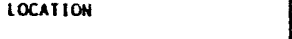 } & \multicolumn{20}{|c|}{ ENTER ANALYSIS IYPES (AT) AMD DUANTITY REOUESTED } \\
\hline & & AI1 & AI2 & AI3 & AT6 & ATS & A16 & A17 & Ar8 & At9 & An10 & ATII & AT12 & AII3 & AI1 & ATIS & A116 & 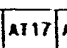 & $1+18 \theta^{A}$ & $\operatorname{arng}$ & Aโ20 \\
\hline LOCAILOW & $(\mathrm{ft})$ & c1 & $\mathbf{c v}$ & os & & & & & & & & & & & & & & & & & \\
\hline LAMOFILL III & 1.5-2.0 & & 1 & 1 & & & & & & & & & & & & & & & & & \\
\hline LAMOFILL III & .5 & 1 & & & & & & & & & & & & & & & & & & & \\
\hline LAMDFILL III & $1.5 \cdot 2.0$ & & 1 & 1 & & & & & & & & & & & & & & & & & \\
\hline LAKDFILL III & .5 & 1 & & & & & & & & & & & & & & & & & & & \\
\hline LANDFILL "II & $1.5-2.0$ & & 1 & 1 & & & & & & & & & & & & & & & & & \\
\hline LANDFILL III & .5 & 1 & & & & & & & & & & & & & & & & & & & \\
\hline LAMOFILL III & $1.5-2.0$ & & 1 & 1 & & & & & & & & & & & & & & & & & \\
\hline RIMSATE & N/A & 1 & 1 & 1 & & & & & & & & & & & & & & & & & \\
\hline LAHOFILL $\|$ & .5 & 2 & & & & & & & & & & & & & & & & & & & \\
\hline LANDFILL 11 & 1.5-2.0 & & 2 & 2 & & & & & & & & & & & & & & & & & \\
\hline LANDFILL "I & .5 & 1 & & & & & & & & & & & & & & & & & & & \\
\hline LAMOF ILL " & $1.5-2.0$ & & 1 & 1 & & & & & & & & & & & & & & & & & \\
\hline :AMOFILL "I & .5 & 1 & & & & & & & & & & & & & & & & & & & \\
\hline LANDFIIL UI & $1.5-2.0$ & & 1 & 1 & & & & & & & & & & & & & & & & & \\
\hline LANDFILL II & .5 & 1 & & & & & & & & & & & & & & & & & & & \\
\hline
\end{tabular}

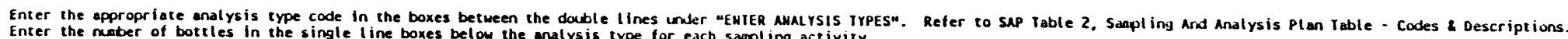

Any descriptions for non-standard analysis types (not given in SAP Toble 2) should be entered under "Conitents" on the llines belou.

AII: CIP Metals ( + CN for Landfill II) - IY

\begin{tabular}{|c|c|c|c|c|}
\hline COLt & $\begin{array}{c}\text { SAMPL LNG } \\
\text { HEI HNO }\end{array}$ & $\begin{array}{c}\text { PLAMAED } \\
\text { DAIE }\end{array}$ & AREA & LOCAIIOA \\
\hline CRAB & & $06 / 01 / 93$ & CFA & SS-TBD-8 \\
\hline comp & & $06 / 01 / 93$ & CFA & $5 S-7 B D-9$ \\
\hline GRAB & & $06 / 01 / 93$ & CFA & $55-180-9$ \\
\hline COMP & & |06/01/93 & CFA & $S S-18 B-10$ \\
\hline CRAB & & |06/01/93| & CFA & $\mid s S-1 B D-10$ \\
\hline comp & & |06/01/93 & cra & $5 S-180-11$ \\
\hline GRAB & & 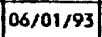 & CFA & $55-180-11$ \\
\hline RMST & & |05/01/93 & cra & oc \\
\hline comp & & $|06 / 01 / 93|$ & cFa & $5 S-1 B D-1$ \\
\hline GRAB & & as/01/93 & CFA & SS-TBD-1 \\
\hline cons & & |06/01/93 & CFa & $55-180-2$ \\
\hline GRAB & & $06 / 01 / 93$ & CFA & SS-TBO-2 \\
\hline $\operatorname{comp}$ & & $06 / 01 / 93$ & CFA & $55-180-3$ \\
\hline GRAB & & $06 / 01 / 93$ & CFA & $5 S-180-3$ \\
\hline comp & & $06 / 01 / 93$ & CFA & $55-180-6$ \\
\hline
\end{tabular}

MENTS

A12: cLp volotiles - IV

A13: CIP Semivolatlles - iv

A14:

ATS:

Art2:

AI13:

AI 13:

AT6:

AI7:

Ar16:

A18:

Als:

At 10:

AI18:

Al18:
AI19:

A120:

$\$ S-I B D-1=(S)$ urface (S)oil - Location (I) 0 (B)e (D)eternined - Site (1), etc. Specific sanple locarions will be determined in the field

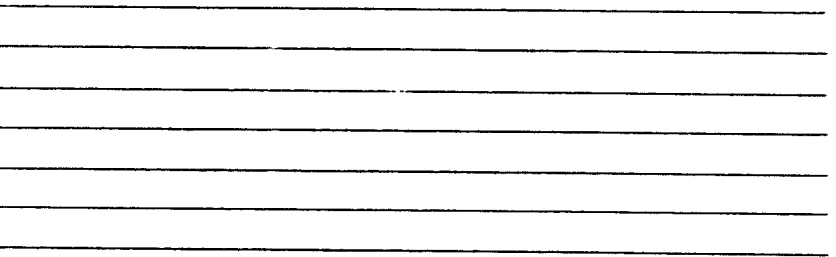


SAP Muaber: $\frac{\alpha \mu-12-S S}{1}$ SAP Table Mo Th
Date: $07 / 07 / 93 \quad$ Revision: 5.0

SAMPLING ANO ANALYSIS PLAN TABLE FOR CHEMICAL ANO RROIOOOGICAL AMULYSIS

Project: CFA OU 4-12 RI/FS - SURFACE SOIL SMMPLES

Page 3 of 6 Date: $07 / 07 / 93 \quad$ Revision:

Project Manager: K. KECK/G. STORHBERG Form Mo: SAPI18 SAMPLE LOCAIION

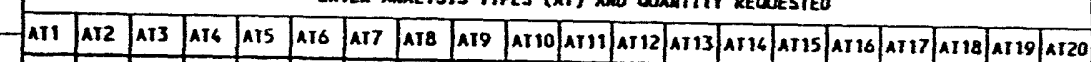

\begin{tabular}{|c|c|c|}
\hline $\begin{array}{l}\text { AAPPLING } \\
\text { ACTIVITY }\end{array}$ & $\begin{array}{l}\text { SNMPLE } \\
\text { TPPE }\end{array}$ & MEDIA \\
\hline $6 c 5071$ & REG & solt \\
\hline $6<5072$ & REG & SOIL \\
\hline $6 \mathrm{csc} 3$ & REG & solt \\
\hline 6C5074 & REG & solt \\
\hline \&cs075 & REG & soll \\
\hline \&CSO7b & REG & solt \\
\hline $4 c 507$ & |REG & solt \\
\hline SCSOrg & REG & solt \\
\hline $6 C 5079$ & REG & solt \\
\hline \&Cs080 & REG & solt \\
\hline ¿CsOa1 & REG & solt \\
\hline $4 c 5082$ & REG & soll \\
\hline $8 c 508\}$ & REG & soll \\
\hline$\angle \mathrm{CsOB} 6$ & REG & soll \\
\hline \&cs08s & REG & solt \\
\hline
\end{tabular}

\begin{tabular}{|c|c|}
\hline col, & $\begin{array}{l}\text { SAMPL IV } \\
\text { METHOO }\end{array}$ \\
\hline CRAB] & \\
\hline $\cos p$ & \\
\hline GReA] & \\
\hline comp & \\
\hline GRAB & \\
\hline COMP & \\
\hline GRAB & \\
\hline $\operatorname{cosp}$ & \\
\hline GRAs] & \\
\hline $\cos p$ & \\
\hline GRAB] & \\
\hline comp & \\
\hline GRRA & \\
\hline $\cos p$ & \\
\hline CRAB] & \\
\hline
\end{tabular}

\begin{tabular}{|c|c|c|}
\hline $\begin{array}{l}\text { PLAMUED } \\
\text { DAIE }\end{array}$ & AREA & LOCATIOA \\
\hline $06 / 01 / 93$ & CFA & SS-TBD-4 \\
\hline $06 / 01 / 93$ & CFA & ss- Tso-5 \\
\hline os/01/93 & CFA & SS-TBO-S \\
\hline $06 / 01 / 93$ & CFA & $5 S-180-6$ \\
\hline $06 / 01 / 93$ & CFA & $\mid 55-180-6$ \\
\hline $06 / 01 / 93$ & cra & $5 S-180-7$ \\
\hline $06 / 01 / 93$ & CFA & $55-180-7$ \\
\hline $06 / 01 / 93$ & CFA & $55-180-8$ \\
\hline $06 / 01 / 93$ & CrA & SS-TBO-8 \\
\hline $06 / 01 / 93$ & CFA & SS- TBD-9 \\
\hline $00 / 01 / 93$ & CFA & SS-TBD-9 \\
\hline $06 / 01 / 93$ & Cra & $5 S-180-10$ \\
\hline $06 / 01 / 93$ & CFA & SS-TBD-10 \\
\hline $06 / 01 / 93$ & CFA & SS-TED-11 \\
\hline $05 / 01 / 93$ & CFA & $5 S-T B D-11$ \\
\hline
\end{tabular}

\begin{tabular}{|c|c|c|c|c|c|c|c|c|c|c|c|c|c|c|c|c|c|c|c|c|}
\hline TRPE OF & & Al1 & A12 & A13 & A16 & Ars & 1916 & Aา7 & 118 & A19 & Arto & AIII/A & AI12/A & \begin{tabular}{l|l} 
AII3/A \\
,
\end{tabular} & 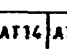 & $A T 15 / A$ & \begin{tabular}{ll|l}
$116 \mid A 1$ &
\end{tabular} & IIIT|AI18 & Ar19/a & Aा20 \\
\hline LOCAIIOW & (ft) & cl & $\mathrm{cr}$ & os & & & & & & & & & & & & & & & & \\
\hline LAMDFILL "I & $1.5-2.0$ & & 1 & 1 & & & & & & & & & & & & & & & & \\
\hline LAMDFILL II & .5 & 1 & & & & & & & & & & & & & & & & & & \\
\hline LANDFILI II & $1.5-2.0$ & & 1 & 1 & & & & & & & & & & & & & & & & \\
\hline LANDFIIL "I & .5 & 1 & & & & & & & & & & & & & & & & & & \\
\hline LaHDFILL "II & $1.5-2.0$ & & 1 & 1 & & & & & & & & & & & & & & & & \\
\hline LANDFIIL "II & 5 & 1 & & & & & & & & & & & & & & & & & & \\
\hline LAMDFIIL II & & & & & & & & & & & & & & & & & & & & \\
\hline Waverill in & $1.5-2.0$ & & 1 & 1 & & & & & & & & & & & & & & & & \\
\hline LaNDFILL II & .5 & 1 & & & & & & & & & & & & & & & & & & \\
\hline LANDFILL II & $1.5-2.0$ & & 1 & 1 & & & & & & & & & & & & & & & & \\
\hline LAMDEFILL 11 & .5 & 1 & & & & & & & & & & & & & & & & & & \\
\hline LandF ILL "II & $1.5-2.0$ & & 1 & 1 & & & & & & & & & & & & & & & & \\
\hline LAMOFILL "I & .5 & 1 & & & & & & & & & & & & & & & & & & \\
\hline LANDFILL II & $1.5-2.0$ & & 1 & 1 & & & & & & & & & & & & & & & & \\
\hline LAMDFILL "I & .5 & 1 & & & & & & & & & & & & & & & & & & \\
\hline LaMOFILL "I & $1.5-2.0$ & & 1 & 1 & & & & & & & & & & & & & & & & \\
\hline
\end{tabular}

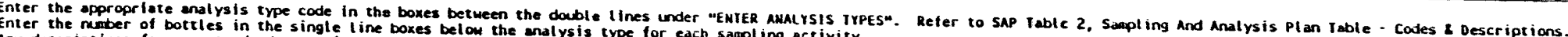
Any descriptions for non-stendard analysis types (not given in SAP lable 2) should be entered under "CCriatentS" on the tines below.

Al1: Cip Merals (o CM for Landfill i1) - Iy

A12: cLp volatiles: IV

13: Cip Seqivolatiles - iv

AT6:

Ars:

ATb:

A17:

AT8:

AT9:

Ar10:
AIII: AI12:

AI13:

Ar16:

Al15:

AIs6:

An17:

AI18:

AI19:

arzo:
COMAENIS

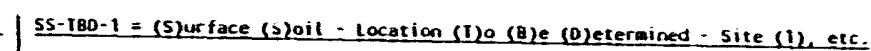
Specific somple locations will be determined in the field. 


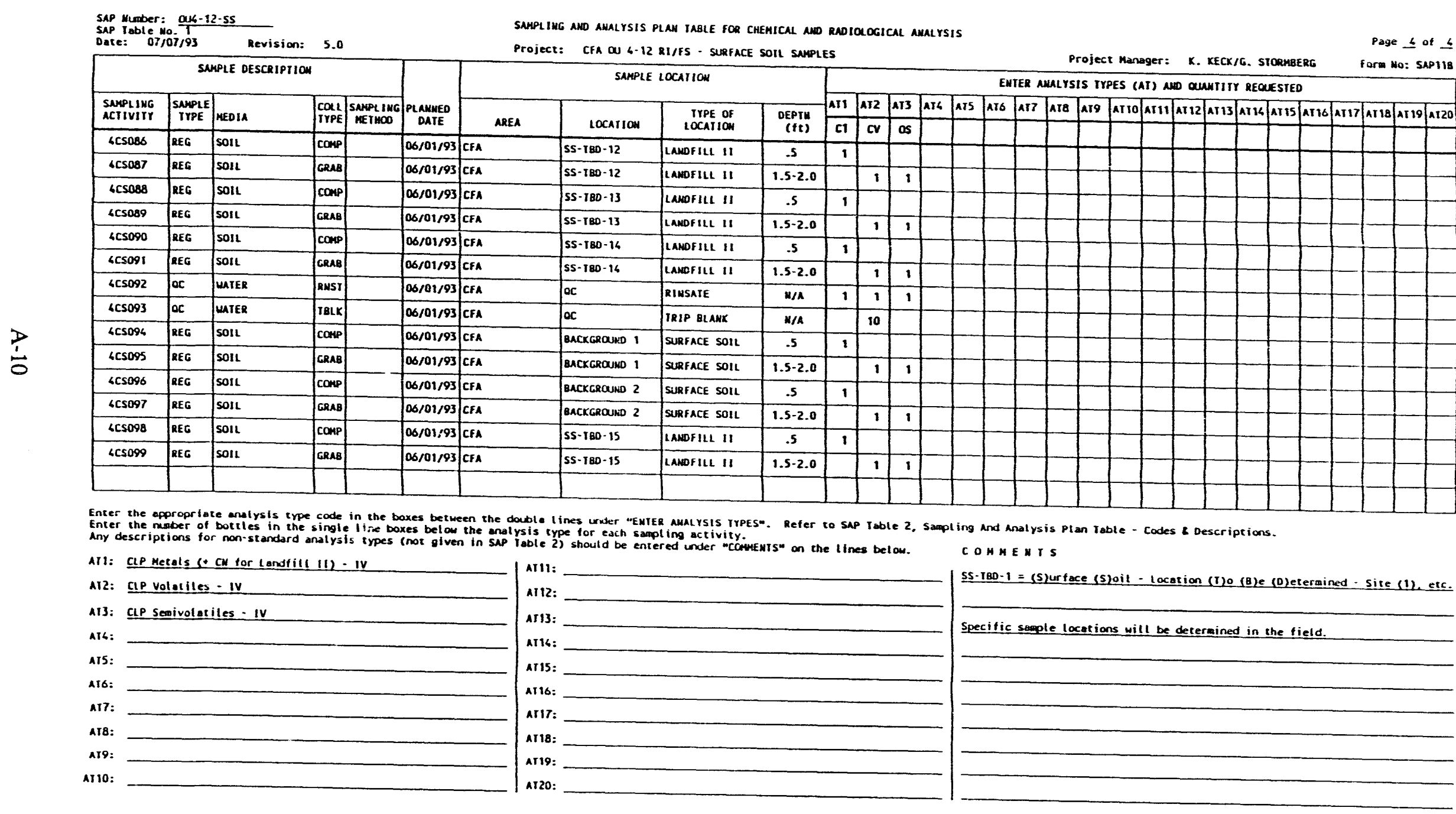


SAP Mumber: aus-12-SSG

SAMPLING AMD AMALYSIS PLAN TABIE FOR CHEMICAL ND RNOTOROGICAL AMALYSIS

\begin{tabular}{|c|c|c|c|}
\hline \multicolumn{4}{|c|}{ SMMPLE DESCRIPIIO } \\
\hline $\begin{array}{l}\text { SAMPLING } \\
\text { ACTIVITY }\end{array}$ & $\left|\begin{array}{c}\text { SAMPLE } \\
\text { TYPE }\end{array}\right|$ & MEOLA & \\
\hline $6 \mathrm{cta00}$ & REG & solt a & cas \\
\hline $4 c 6002$ & REG & soll a & GAS \\
\hline $4[6003$ & REG & soll 6 & Gas \\
\hline sc6006 & REG & soll G & GAS \\
\hline $6 c 6005$ & REG & solk a & ans \\
\hline $8 \mathrm{CCO006}$ & REG & solt a & Gas \\
\hline sec007 & REG & soll G & GAS \\
\hline $6 c 6008$ & REG & soll a & GAS \\
\hline \&c6009 & REG & solt a & GAS \\
\hline 406010 & REG & soll a & \\
\hline $6 \mathrm{ccol1}$ & REG & soIt G & \\
\hline $6 c 6012$ & REG & soth a & \\
\hline $4 \mathrm{cgOa3}$ & REG & sont G & GSS \\
\hline $6 \mathrm{CGO16}$ & REG & soll a & \\
\hline \&CGO15 & REG & solt a & Gas \\
\hline
\end{tabular}

\begin{tabular}{|c|c|c|c|c|}
\hline & & & & $G$ AND AKALYSIS \\
\hline 6.0 & & & & CFA OU $6-12$ \\
\hline & & & & SMMPLE \\
\hline $\mid$\begin{tabular}{c|}
$\mathrm{COO} 2$ \\
$\mathrm{TYPE}$
\end{tabular} & $\begin{array}{c}\text { SAMPL ING } \\
\text { MEI HOD }\end{array}$ & $\begin{array}{c}\text { PLAMUES } \\
\text { OATE }\end{array}$ & AREA & LOCAIIOW \\
\hline GRAB & & $06 / 25 / 93$ & CFA & $56-1$ \\
\hline GrAB & & $06 / 25 / 93$ & CFA & sot-2 \\
\hline GRAB & & $\infty 6 / 25 / 93$ & CFA & so-3 \\
\hline cras & & $06 / 25 / 93$ & CFA & $5 c-6$ \\
\hline Gras & & $06 / 25 / 93$ & $\operatorname{cin}$ & $56-5$ \\
\hline GRAB & & $|0 s / 25 / 93|$ & CFA & sc-6 \\
\hline GRAB & & $06 / 25 / 93$ & CFA & $5 \mathrm{~s}-7$ \\
\hline GRAB & & $00 / 25 / 93$ & CFA & $5 G-8$ \\
\hline GRAB & & $06 / 25 / 93$ & CFA & $56-9$ \\
\hline GRAB & & $06 / 25 ; 93$ & CFA & $56-10$ \\
\hline GRAB & & $06 / 25 / 93$ & CFA & $S G \cdot 11$ \\
\hline GraB & & $06 / 25 / 93$ & CFA & $s G-12$ \\
\hline GRAB & & $06 / 25 / 93$ & CFA & $\mathrm{sg}-13$ \\
\hline Cras & & $\infty 6 / 25 / 93$ & CFA & $56-16$ \\
\hline |GRAB & & $06 / 25 / 93$ & CFA & $5 G-15$ \\
\hline
\end{tabular}

6-12 RI/FS - SHALLOH SNIL GAS

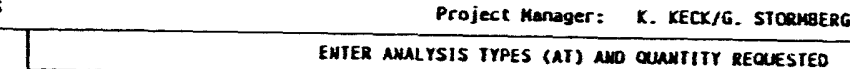

Page 1 ot 12

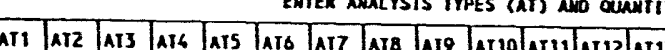

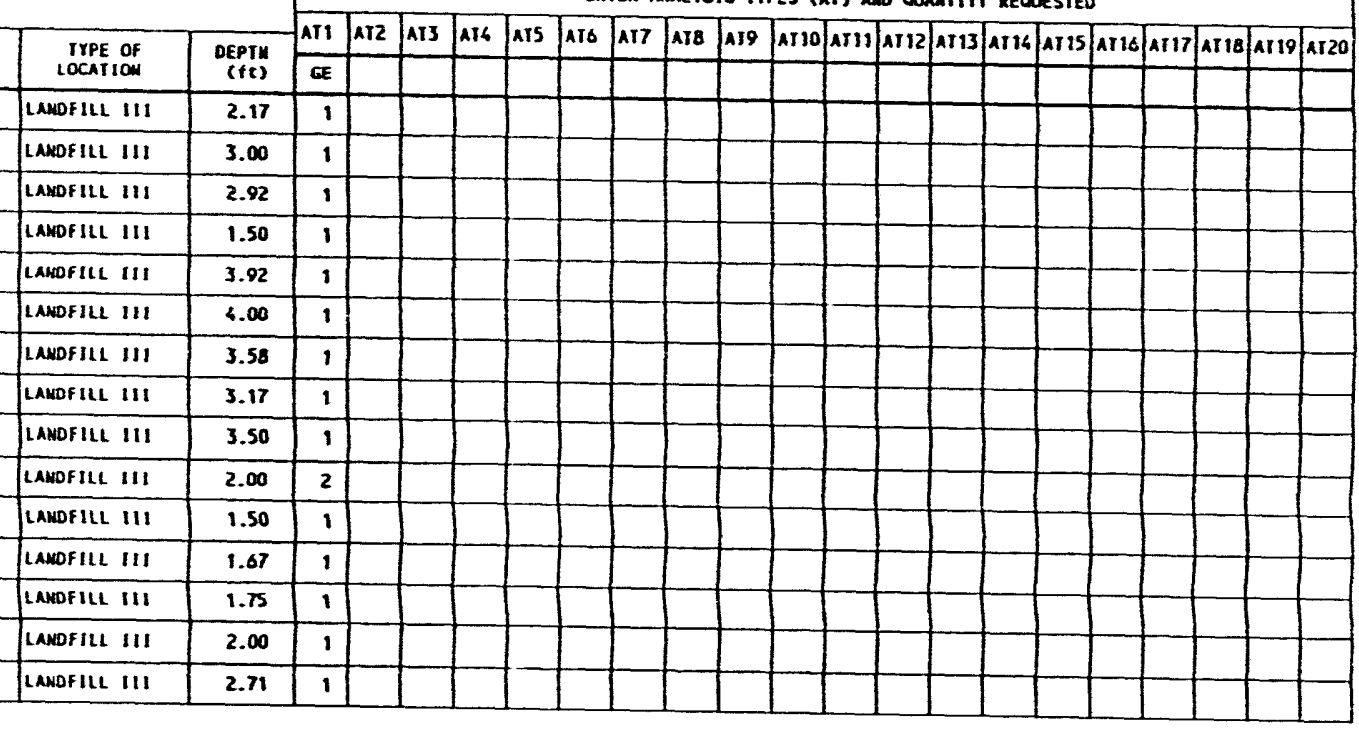

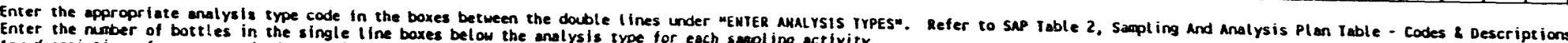

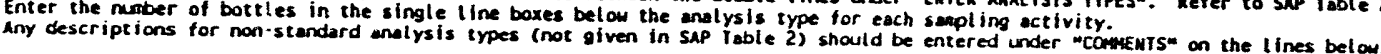

A11: EPA Method SOI GC/ECO/FIO

A13:

A16:

AIS:

A16:

A17:

AI8:

A19:

A110:
ArII:

AI12:

ATIS:

ArI6:

AI15:

AI16:

AI17:

Ar18:

AI19:

A119:
CONME IS

fCIL = Field control sample

sG-1 = (5)oil (G) as - site (1). etc 


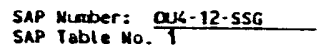

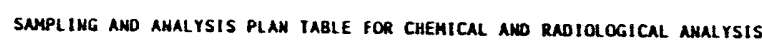
Dete: 07/07/93 Revision: 6.0 Project: CFA OU 4-12 RI/FS - SHALLOU SOIL GAS

Page 2 of $\underline{12}$

\begin{tabular}{|c|c|c|c|c|c|c|c|c|c|c|c|c|c|c|c|c|c|c|c|c|c|c|c|c|c|c|c|}
\hline & & MPLE OESCR & & & & & sмmp: & 10 a & & & & & & & & & & & & & & & & & & & \\
\hline & & & & & & & & & & & & & & & & & & & tes (A) & i) aro & D OUAMT & IIITY REOU & XUESTED & & & & \\
\hline $\begin{array}{l}\text { SAMPL ING } \\
\text { ACTIVIIY }\end{array}$ & SMAPLE & MED1A & $\left|\begin{array}{c}\mathrm{col} L \\
\text { Trye }\end{array}\right|$ & SMAPLIMG & PLaMké & & & TYPE OF & DEPTM & Ant & A12 & AT3 & 116 & A15 & A16 & a17 & A18 & AT9 & AT 10|A & AIII/A & ATI2|AT & {$[13 \mid A+16$} & AIISA & Ar16 A & Ariv & AI18 & \begin{tabular}{|l|l|l|}
$\Lambda 119$ & $\wedge 120$ \\
\end{tabular} \\
\hline & & Jedisa & |ire & METHOD & DATE & RREA & LOCATIOW & Location & (ft) & GE & & & & & & & & & & & & & & & & & \\
\hline SCGO16 & REG & SOIL GAS & Gras & & $06 / 25 / 93$ & CFA & SG-16 & LANDFILL III & 1.42 & 1 & & & & & & & & & & & & & & & & & \\
\hline 4CG017 & REG & soll GAS & Grab & & $06 / 25 / 93$ & CFA & $56-17$ & LaNOFILL III & 1.25 & 1 & & & & & & & & & & & & & & & & & \\
\hline $6 \mathrm{CGO18}$ & REG & SOIL GAS & Grab & & $06 / 25 / 93$ & CFA & $5 G-18$ & LARDFILL III & 1.42 & 1 & & & & & & & & & & & & & & & & & \\
\hline sc6019 & REG & SOIL GAS & GRAB & & $00 / 25 / 93$ & CFA & $56-19$ & LANDFIIL III & 1.92 & 1 & & & & & & & & & & & & & & & & & \\
\hline $4 C 6020$ & REG & SOIL EAS & GRAe & & $06 / 25 / 93$ & Cra & $5 G-20$ & LANDFILL III & 1.58 & 2 & & & & & & & & & & & & & & & & & \\
\hline scG021 & REG & SOIL GAS & GRAB & & $06 / 25 / 93$ & Cra & $56-21$ & LANOFILL III & 1.75 & 1 & & & & & & & & & & & & & & & & & \\
\hline $6 c 6022$ & REG & SOIL GAS & GRAB & & $06 / 25 / 93$ & CFA & $56-22$ & LAMOFILL III & 2.00 & 1 & & & & & & & & & & & & & & & & & \\
\hline 866023 & REG & SOIL GAS & GRAB & & $06 / 25 / 93$ & CFA & $56-23$ & LANOFILL III & 2.42 & 1 & & & & & & & & & & & & & & & & & \\
\hline 666026 & REG & SOIL GAS & GRAB & & $|06 / 25 / 93|$ & CFA & $56-24$ & LAMOFILL III & 3.17 & 1 & & & & & & & & & & & & & & & & & \\
\hline $8 \mathrm{CGO25}$ & REG & SOIL GAS & GRAB & & $06 / 25 / 93$ & CFA & $56-25$ & LAMDFILL III & 2.08 & 1 & & & & & & & & & & & & & & & & & \\
\hline 460026 & REG & SOIL GAS & GRAB & & $00 / 25 / 93$ & CFA & $56-26$ & LANOFILL III & 1.92 & 1 & & & & & & & & & & & & & & & & & \\
\hline scG027 & REG & SOIL GAS & Grab & & $06 / 25 / 93$ & CFA & $56-27$ & LANOFILL III & 1.50 & 1 & & & & & & & & & & & & & & & & & \\
\hline $4 \mathrm{cGO28}$ & REG & soll als & GRAB & & $00 / 25 / 93$ & CFA & $5 \varepsilon-28$ & LAMDFILL III & 4.00 & 1 & & & & & & & & & & & & & & & & & \\
\hline 806029 & |REG & solt GAS & GRAB & & $\infty 6 / 25 / 93$ & CFA & $56-29$ & LAMOFILL III & 1.58 & 1 & & & & & & & & & & & & & & & & & \\
\hline $4 \operatorname{ccos} 0$ & REG & SOIL GAS & GRAB & & $\infty 08 / 25 / 93$ & CFA & $56-30$ & LAMOFILL III & 1.58 & 2 & & & & & & & & & & & & & & & & & \\
\hline
\end{tabular}

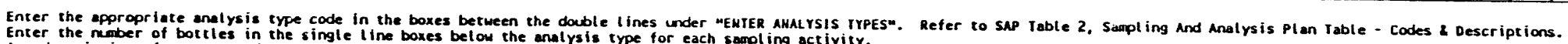

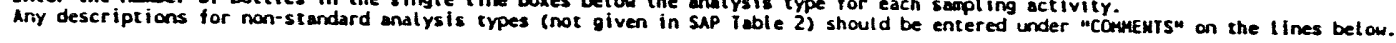

AI1: EPA Method SOI GC/ECD/FID

AI2:

AI3:

AI6:

A15:

Ais:

A17:

AT8:

AT9:

At10:
AII1:

AI12:

AT13:

Ar16:

Ar15:

AI16:

AI17:

AI 18:

AI 19:

Ar20:
COMHEN IS

FCTL = Field Control Sample

SG-1 = (s)oil (G)as - site (1), et

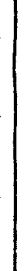




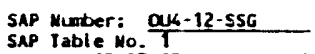

SAMPLING AKD ANALYSIS PLAH IABLE FOR CHEMICAL AND RNDIOLOGICAL ANALYSIS

\begin{tabular}{|c|c|c|c|}
\hline \multicolumn{4}{|c|}{ SAMPLE DESCRIPIIO } \\
\hline $\begin{array}{l}\text { SAMPLIAG } \\
\text { ACIIVITY }\end{array}$ & \begin{tabular}{|c|} 
SAMPLIE \\
TYPE
\end{tabular} & MEDIA & \\
\hline $6 \mathrm{cc031}$ & REG & soll & \\
\hline $4 c \cos 2$ & REG & soll & GAS \\
\hline $6 c 6033$ & REG & solt & Gas \\
\hline sccosi & REG & solt & GAS \\
\hline «cG035 & REG & solt & aAs \\
\hline $4 c \cos 6$ & REG & solt & GAS \\
\hline 466037 & REG & son & GAS \\
\hline $6 \operatorname{ccos} 8$ & REG & soll & GAS \\
\hline $6 c \cos \theta$ & REG & SOIL & GAS \\
\hline 466040 & REG & solt & GAS \\
\hline $4 c 6061$ & REG & solt & Gas \\
\hline $4 c 6042$ & REG & sol1 & Gas \\
\hline $6 c 6043$ & REG & solt & GAS \\
\hline $4 \mathrm{CGO44}$ & REG & solt & GAS \\
\hline sccoss & REG & soll & GAS \\
\hline
\end{tabular}

\begin{tabular}{|c|c|c|c|c|}
\hline & & & & \\
\hline & & & & \\
\hline & METHOO & DAIE & AREA & LOCASIOW \\
\hline GRAB & & $06 / 28 / 93$ & CrA & $5 G-31$ \\
\hline GRAB & & $06 / 28 / 93$ & CFA & sG-32 \\
\hline GRAB & & $06 / 28 / 93$ & CFA & $56-33$ \\
\hline | CRAB & & $\mid \alpha \delta / 28 / 93$ & CFA & $5 G-34$ \\
\hline GRAB & & $06 / 28 / 93$ & CFA & sG-35 \\
\hline GRAB & & $06 / 28 / 93$ & CFA & $56-36$ \\
\hline GRAB & & $06 / 28 / 93$ & CFA & $15 G-37$ \\
\hline GRAB & & $06 / 28 / 93$ & CFA & $5 G-38$ \\
\hline CRAB & & $06 / 28 / 93$ & CFA & sc-39 \\
\hline GRAB & & $06 / 28 / 93$ & CFA & $56-60$ \\
\hline GRAB & & $06 / 28 / 93$ & CFA & $56-61$ \\
\hline Grab & & |06/28/93 & cfa & $\mid 56-42$ \\
\hline GRAB & & 06/28/93 & CFA & $\mid s G-43$ \\
\hline Gras & & $\mid 06 / 28 / 93$ & CFA & 150.66 \\
\hline GRAB & & $\mid 06 / 28 / 9]$ & crs & sG-65 \\
\hline
\end{tabular}
4-12 R1/FS - SHALLOH SOIL GAS

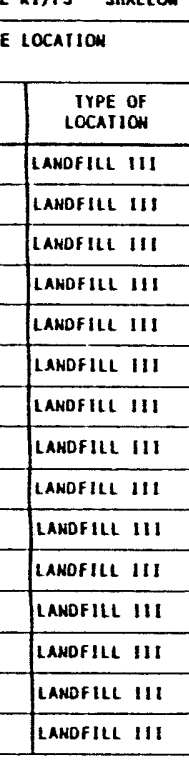

\section{T} Project Manager: K. KECK/G. STORHBERG
EMTER AMALYSIS TYPES (AI) ANO QUANIIIY REOUESTED

Page $\underline{3}$ of $\underline{12}$

Form No: SAP11B

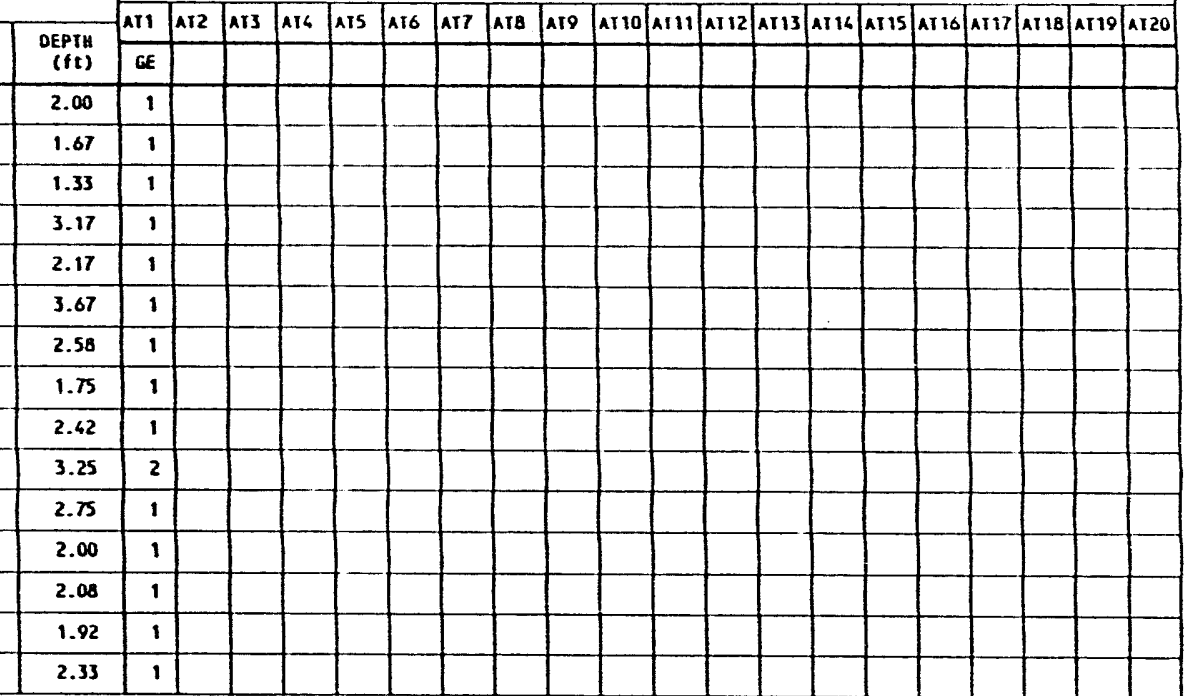

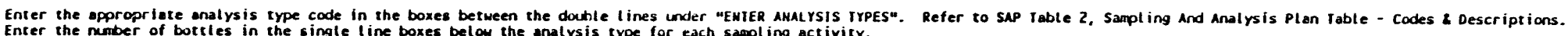

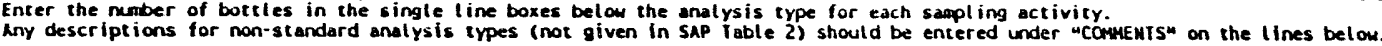

AI1: EPA Method 601 GC/ECD/FIO

A12:

A13:

A14:

ATS:

AI6:

A17:

A18:

Al9:

Aril:

Ar12:

AI13:

AT16:

AI15:

AI16:

A117:

Ar10:

Ar1s:

arlo:

Ar20:

fCIL = Field Control Sample

sG-1 = (s)oil (G)as - site (1), etc. 
SAP Mumber: OUG-12-SSG
SAP Table No.

SAMPLING AND AMALYSIS PLAN IABLE FOR CHEMICAL AMO RADTOLOGICAL AMALYSIS

\begin{tabular}{|c|c|c|}
\hline $\begin{array}{l}\text { SAMPLING } \\
\text { ACrIVIITY }\end{array}$ & \begin{tabular}{|c|} 
SAMPLE \\
TYPE
\end{tabular} & MEOIA \\
\hline $4 c 6066$ & REG & soll GAS \\
\hline $4 C G 067$ & REG & SOIL GAS \\
\hline $4 C 6048$ & REG & SOIL GAS \\
\hline $4 c 6049$ & REG & SOIL GAS \\
\hline $4 c \cos 0$ & REG & SOIL GAS \\
\hline $6 c 6051$ & REG & SOIL GAS \\
\hline $4 c \cos 2$ & REG & soll GAS \\
\hline sccos3 & REG & soll oas \\
\hline $4 c 6056$ & REG & Soll GAS \\
\hline $6 c \cos 5$ & REG & SOIL GAS \\
\hline $8 c \cos \theta$ & REG & SOIL GAS \\
\hline $6 c \cos 7$ & REG & soll Gas \\
\hline $8 c \cos \theta$ & REG & soIL GAS \\
\hline$<\cos \theta$ & REG & SOIL GAS \\
\hline $4 c 6000$ & REG & SOIL GAS \\
\hline
\end{tabular}

Project: CFA OU 6-12 RI/FS - SHALLON SOIL GAS

Project Manager: K. KECK/G. STORMBERG

Page 6 of 12

Form Mo: SAP11B EMTER AMALYSIS IYPES (AT) AMD QUAMIITY REQUESTED

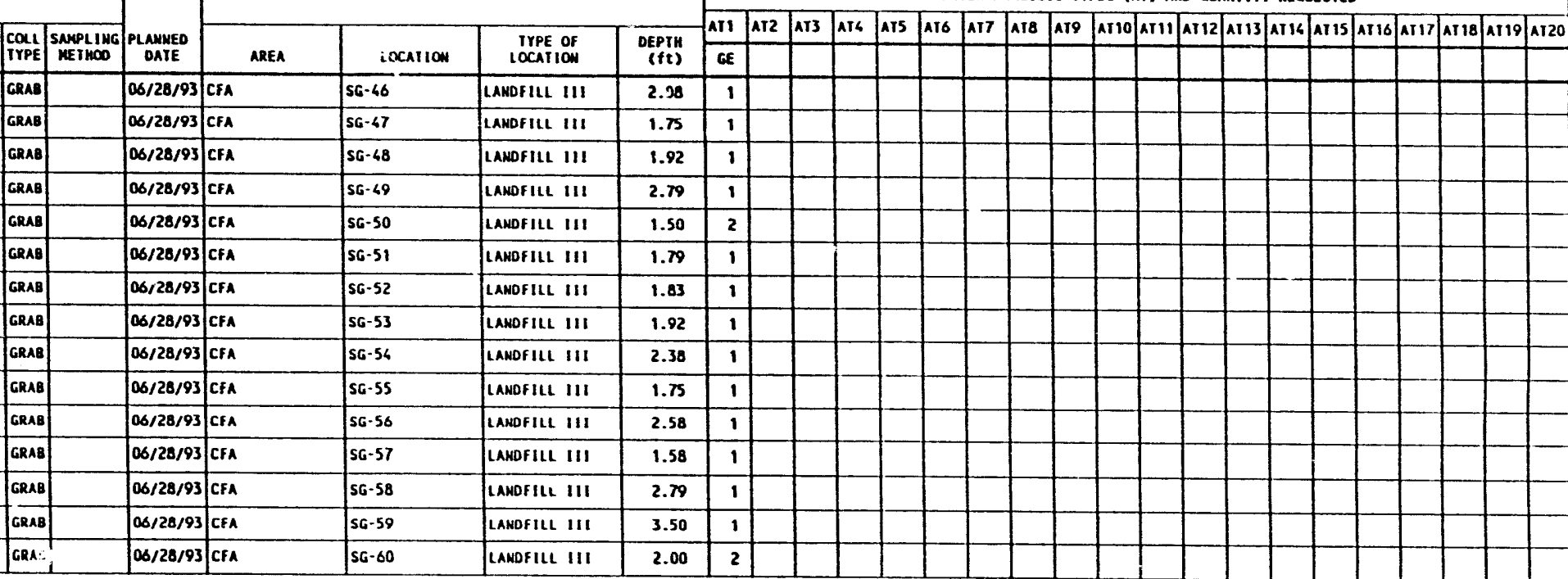

Enter the appropriate analysis type code in the boxes between the dobble lines under "ENIER ANALYSIS IYPES". Reter to SAP Table 2, Sampling And Analysis Plan Jable - Codes 20 Descriptions.

any descriptions for non-standard analysis types (not given in SAP Table 2) should be entered under "CoNyEWIS" on the llnes belou.

Al1: EPA Method 601 GC/ECO/FID

Ar11:

An12:

Al2:

AI3:

A16:

AI5:

A16:

A17:

A18:

A19:

Ar10:
Ar13:

Ar14:

A.16:

Ar17:

An18:

ar19:

ar20:
C OMMEN IS

ECIL = Field control Sample

sG-1 = (S)oil (G)as - site (1), etc.

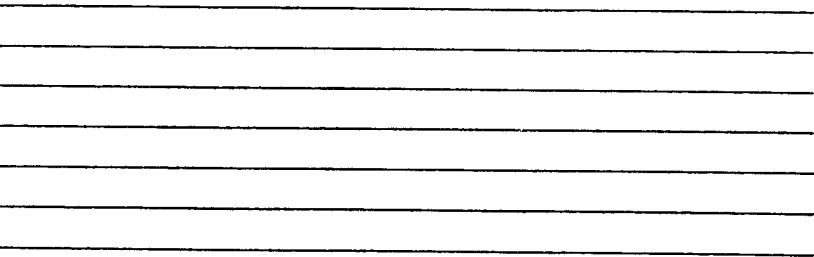




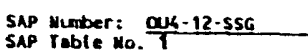

Date: 07/07/93 Revision: 6.0

\begin{tabular}{|c|c|c|}
\hline \multicolumn{3}{|c|}{ SNAPLE DESCRIPIIOK } \\
\hline $\begin{array}{l}\text { SANPLINGG } \\
\text { ACIIVIITY }\end{array}$ & $\mid$\begin{tabular}{|c|} 
SAMPLE \\
TYPE
\end{tabular} & MEDIA \\
\hline $6 c \cos 1$ & REG & soll GAS \\
\hline $4 c 6062$ & REG & soll GAS \\
\hline $6 c 6063$ & REG & SOIL GAS \\
\hline $4 c 6064$ & REG & Soll GAS \\
\hline 4c606s & REG & solt aAs \\
\hline $4 c 6066$ & REG & SOIL GAS \\
\hline $6 C 6067$ & REG & SOIL GAS \\
\hline 4cross & REG & SOIL GAS \\
\hline$<66069$ & REG & soll GAS \\
\hline 666070 & REG & soll GAS \\
\hline \&c6071 & REG & SOIL GAS \\
\hline sc6072 & REG & SOIL GAS \\
\hline $66 \mathrm{con} 3$ & REG & soll GAS \\
\hline $4 c 6074$ & REG & solt GAS \\
\hline sccors & REG & soll GAS \\
\hline
\end{tabular}

SMMPLIMG AND AMALYSIS PLAN tABLE FOR CHEALCAL AND RNDIOLOGICAL akALYSIS

Project: CFA OU 6-12 R1/FS - SHALLOW SOIL GAS

$$
\text { SAMPLE LOCAIIOW }
$$

Project Manager: $\quad$ K. KECK/G. STORHBERG
ENTER AMALYSIS TYPES (AT) AND QUAMTIIY REOUESTED

Page $\underline{5}$ of $\underline{12}$ Form No: SAP118

\begin{tabular}{|c|c|c|}
\hline $\begin{array}{l}\text { PLAMMED } \\
\text { DAITE }\end{array}$ & AREA & LOCATION \\
\hline $06 / 28 / 93$ & CFA & SG-61 \\
\hline $06 / 28 / 93$ & cra & S6-62 \\
\hline $06 / 20 / 93$ & CFA & SG- -63 \\
\hline $06 / 28 / 93$ & CFA & $56-66$ \\
\hline $06 / 28 / 93$ & CrA & $56-65$ \\
\hline $06 / 28 / 93$ & CFA & $56-66$ \\
\hline $06 / 28 / 93$ & CFA & $56-67$ \\
\hline $06 / 29 / 93$ & CFA & $56-68$ \\
\hline $06 / 29 / 93$ & CFA & sG-69 \\
\hline $06 / 29 / 93$ & CFA & sG-70 \\
\hline $06 / 29 / 93$ & cra & $56-71$ \\
\hline $06 / 29 / 93$ & cfa & $56-72$ \\
\hline $00 / 29 / 93$ & CFA & $\mid 56-73$ \\
\hline $06 / 29 / 93$ & CFA & $56-74$ \\
\hline |06/29/93 & CFA & $s a-75$ \\
\hline
\end{tabular}

\begin{tabular}{|c|c|c|c|c|c|c|c|c|c|c|c|c|c|c|c|c|c|c|c|c|c|}
\hline & & ArI & $A 12$ & AT3 & AT4 & ATS & AI6 & AI7 & ATS & A19 & Arro & AriI & \begin{tabular}{|l|}
$A 112$ \\
\end{tabular} & AT13 & Ar16, & AT15 & Ar16/a & AII7/A & AIIBA & Atrofa & $A>20$ \\
\hline LOCATION & $(\mathrm{ft})$ & GE & & & & & & & & & & & & & & & & & & & \\
\hline LANDFILL $" 11$ & 3.67 & 1 & & & & & & & & & & & & & & & & & & & \\
\hline LAMDFILL "II & 2.00 & 1 & & & & & & & & & & & & & & & & & & & \\
\hline tandF ILL & 1.92 & 1 & & & & & & & & & & & & & & & & & & & \\
\hline [LAMDFIIL It & 1.75 & 1 & & & & & & & & & & & & & & & & & & & \\
\hline LANDFILL II & 1.50 & 1 & & & & & & & & & & & & & & & & & & & \\
\hline LAKDFILL II & 10.00 & 1 & & & & & & & & & & & & & & & & & & & \\
\hline LANDFILL "II & 3.50 & 1 & & & & & & & & & & & & & & & & & & & \\
\hline LANDFILL "I & 2.25 & 1 & & & & & & & & & & & & & & & & & & & \\
\hline LANDFILL II & 2.50 & 1 & & & & & & & & & & & & & & & & & & & \\
\hline LANOFILL II & 2.00 & 2 & & & & & & & & & & & & & & & & & & & \\
\hline LaMdflll II & 1.38 & 1 & & & & & & & & & & & & & & & & & & & \\
\hline LANDFHL II & 2.00 & 1 & & & & & & & & & & & & & & & & & & & \\
\hline LAMPFILL & 2.00 & 1 & & & & & & & & & & & & & & & & & & & \\
\hline LAMOFILL "I & 3.42 & 1 & & & & & & & & & & & & & & & & & & & \\
\hline LaNDFILL II & 2.25 & $t$ & & & & & & & & & & & & & & & & & & & \\
\hline
\end{tabular}

Enter the appropriate enalysis type code in the boxes between the double lines under "EMIER AMALYSIS irpES". Refer to SAP rable 2, Sampling And Analysis Plan Table - Codes 2 Descriptions.

Any descriptions for non-siandard analysis types (not given in SAP Table 2) should be entered under "cowiugwis" on the lines below.

A11: EPA Methad SOI GC/ECO/FIO

AI2:

A13:

AT6:

Ats:

AI6:

ar7:

AI8:

A19:

Allo: aril:

AI12:

AII3:

AI16:

Al15:

ar16:

Al17:

A118:

Ar19:

ar10:
COHAENIS

FCIL = field control sample

SG-1 = (S)oil (G)as - site $(12$, etc 
SAP Munber: OQK-12-SSG

\begin{tabular}{|c|c|c|}
\hline \multicolumn{3}{|c|}{ SNMPLE DESCRIPIIO } \\
\hline $\begin{array}{l}\text { SAMPLIMG } \\
\text { ACriviry }\end{array}$ & $\begin{array}{l}\text { SAMPLE } \\
\text { IYPEE }\end{array}$ & MEDIA \\
\hline $4 c 6076$ & REG & soll GAs \\
\hline $4 \mathrm{ccort}$ & REG & soll GAs \\
\hline $4 c 6078$ & REG & soll as: \\
\hline ८с6079 & REG & soll as: \\
\hline$\angle C 6080$ & oc & soll ans \\
\hline sccosi & loc & soll Gas \\
\hline 6c6082 & oc & soll Gas: \\
\hline \&ecoss & REG & soll on: \\
\hline secoss & REG & soll an: \\
\hline $4 c 6085$ & REG & solt GA \\
\hline $4 \mathrm{CCOBO}$ & REG & soll an \\
\hline $4 C 6087$ & REG & soll on \\
\hline sccose & REG & solt on \\
\hline $4 c 6089$ & REG & soll on \\
\hline ८c6090 & REG & soll an \\
\hline
\end{tabular}

\begin{abstract}
6.0
\end{abstract}
SAMPLIMG AND AMALYSIS PLAN TABLE FOR CHEMICAL AMD RMOIOLOGICAL AMALYSIS

Project: CFA OU 4-12 Ri/fFS - SHALLOU SOLL GAS

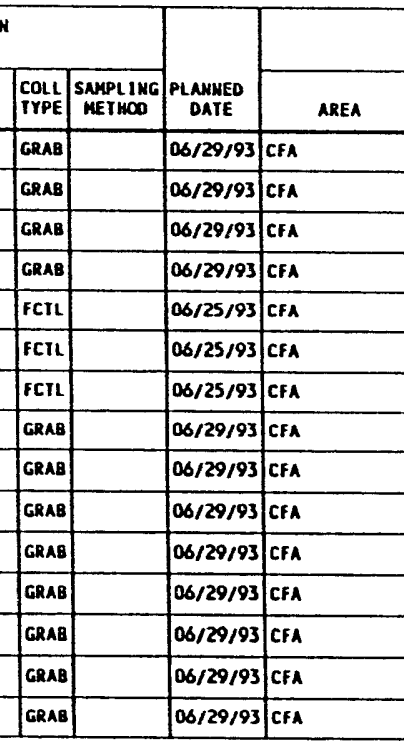

Project Manager: K. KECK/G. STORMBERG SARPLE LOCAIIOH EMTER AMALYSIS TYPES (AT) NOD QUMUIUTY REOUESTED
Page of of 12

Form Ho: SAP118

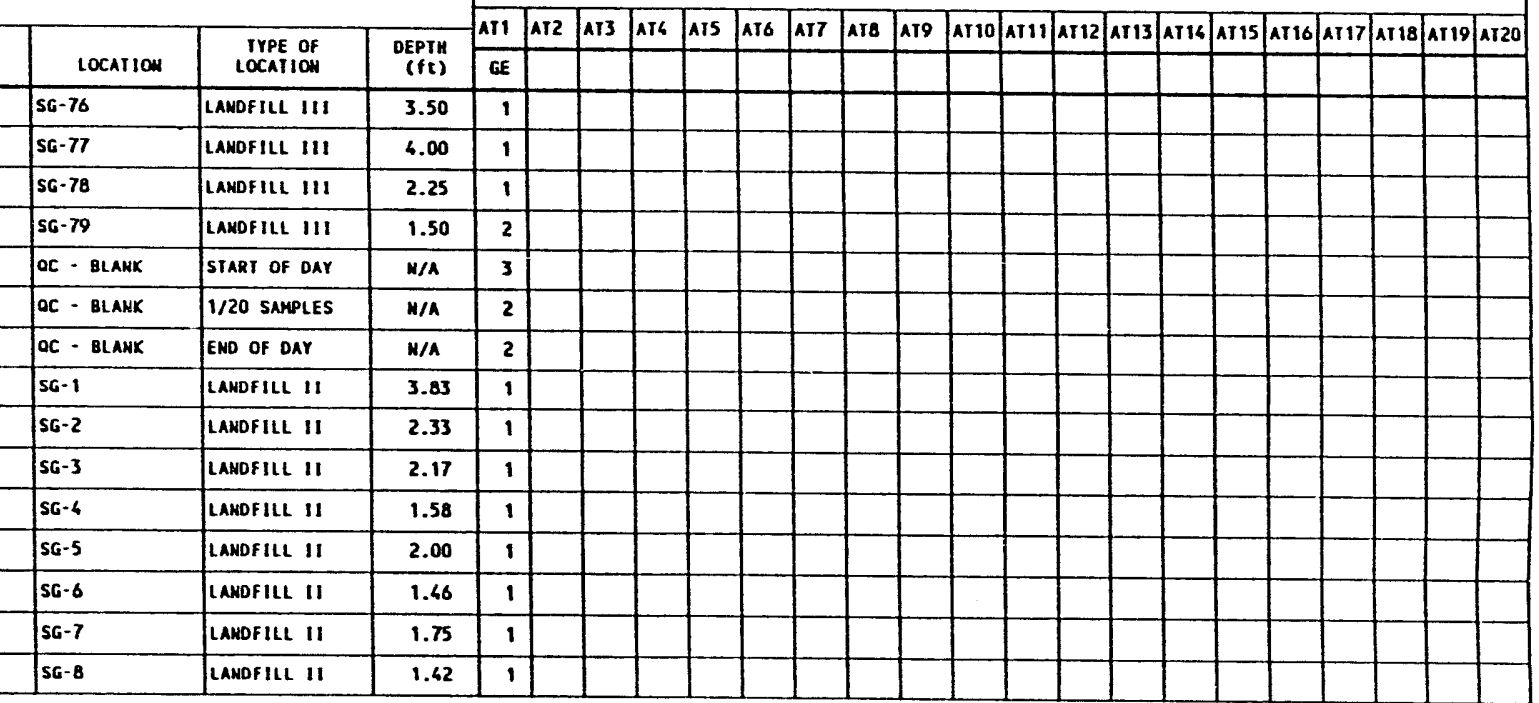

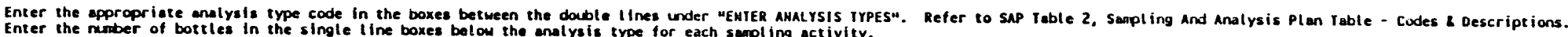

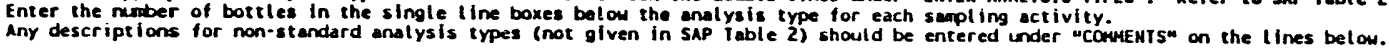

COMNEN TS

All: EPA Method 601 GC/ECO/FIO

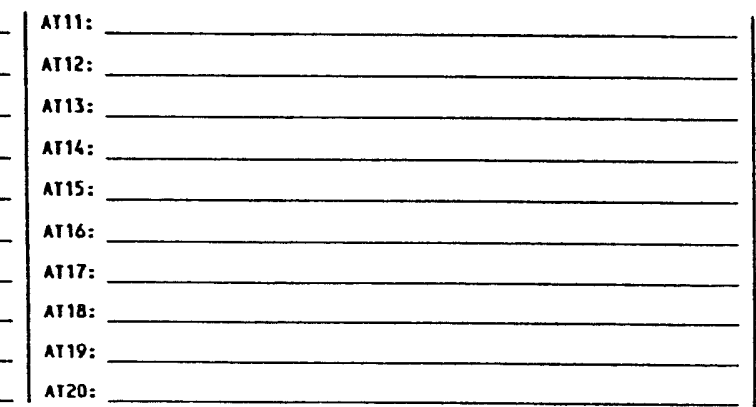

FCIL = field control sample
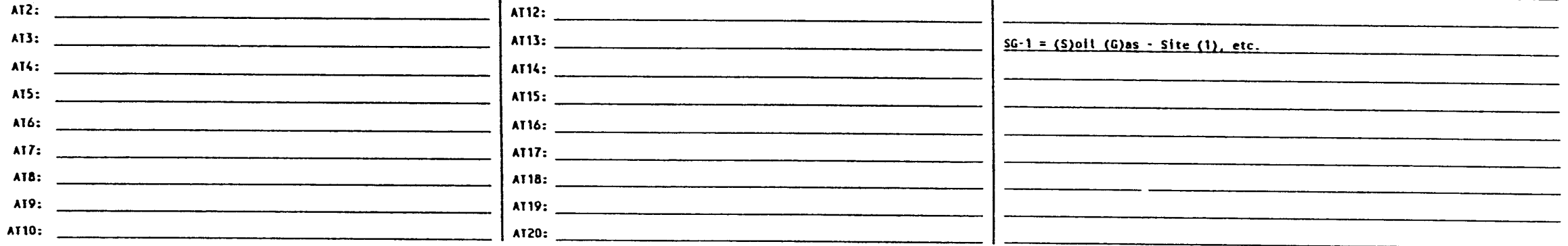


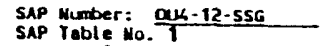

SAMPLING RMO ANALYSIS PLAN TABLE GOR CHEMICAL AMO RADIOLOGICAL AMALYSIS

\begin{tabular}{|c|c|c|}
\hline \multicolumn{3}{|c|}{ SAMPLE DESCRIPITO } \\
\hline $\begin{array}{l}\text { SAMPLING } \\
\text { ACIIVIIT }\end{array}$ & $\begin{array}{l}\text { SMMPLE } \\
\text { TYPPE }\end{array}$ & KEDIA \\
\hline \&c6091 & REG & soll Ga: \\
\hline sc6092 & REG & soll an \\
\hline 6cG093 & REG & soll GA \\
\hline 4c6094 & REG & soll Ge \\
\hline 4c6095 & REG & soll GA \\
\hline 6C6096 & REG & SOIL GA \\
\hline 466097 & |REG & SOIL GA \\
\hline \&CG098 & REG & soll ar \\
\hline «c6099 & REG & SOIL GA \\
\hline $46 G 100$ & REG & soll GA \\
\hline \&cGtol & REG & soll GA \\
\hline $4 C 6102$ & REG & soll Gs. \\
\hline 4CG 103 & REG & so:l as \\
\hline SEG106 & REG & SOIL GA \\
\hline $4 \mathrm{CG} 105$ & TREG & sort Ga \\
\hline
\end{tabular}

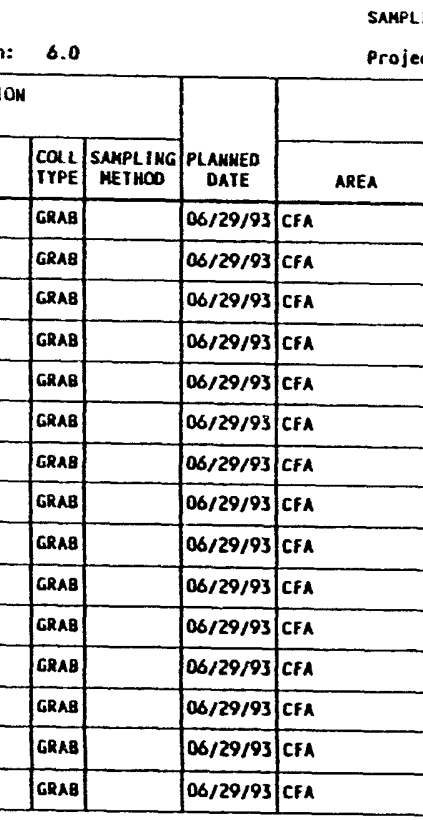

Project: CFA OU 4-12 RL/FS - SHALLOU SOIL GAS

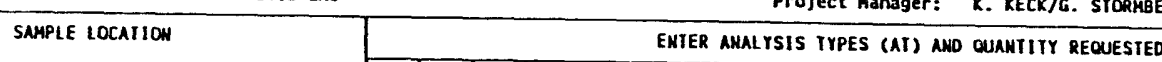

Project Manager: K. KECK/G. STORMBERG

Page 7 of 12

Form No: SAP11B

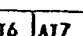

Enter the appropriate anslysis type code in the boxes between the double lines under Any descriptions for non-stondard analysis types (not given in SAP lable 2) should be entered under "Cowiments" on the LInes betou.

AII: EPA Method 601 GC/ECO/FIO

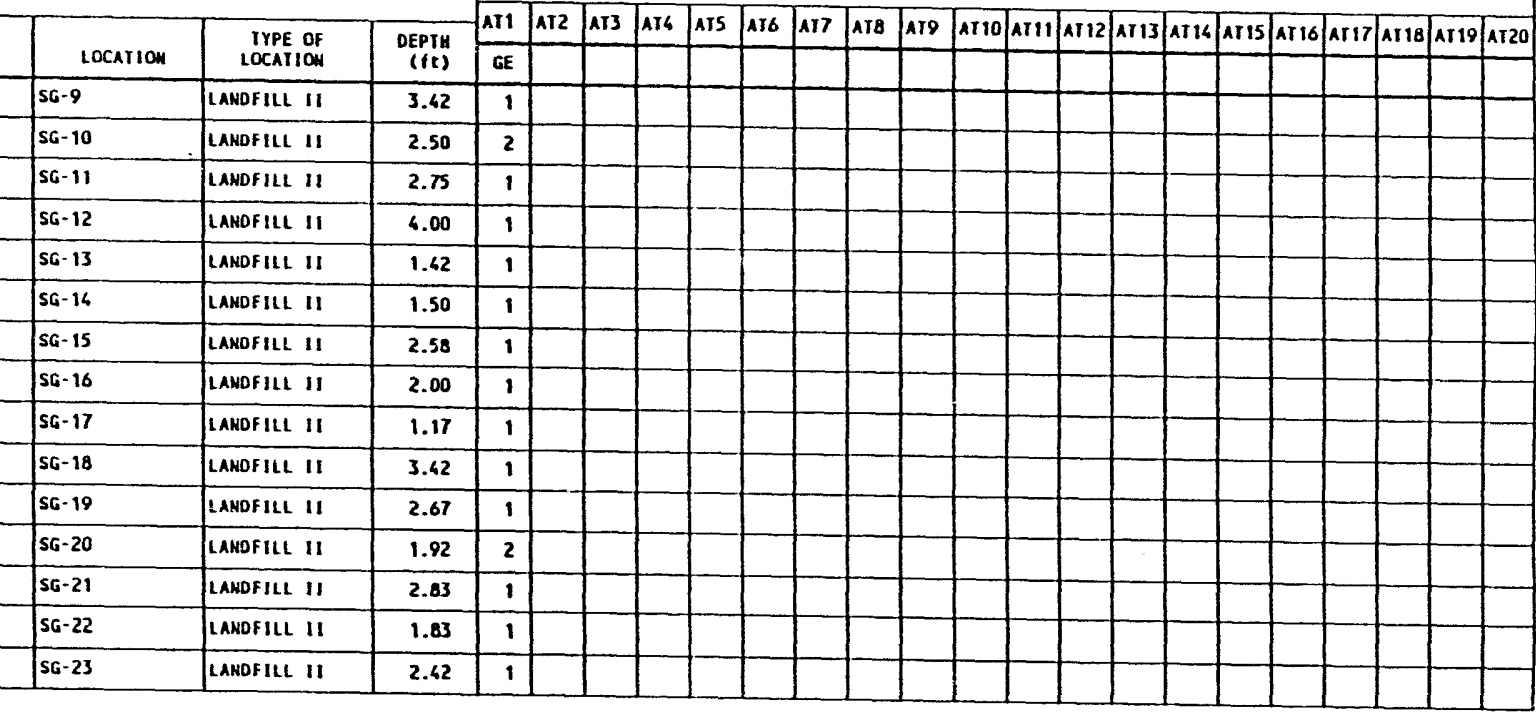

AII:

AT3:

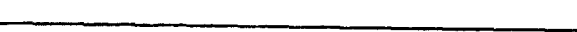

ATS

AI6:

AI7:

ATB:

AI9:

Ar 10: ar11: AII2:

AT13:

AT14:

AT15:

Al16:

Ar17:

AI18:

Ar 19:

ar20:
CONAEN IS

FCIL = field Contral Sampie

$s G-1=(5)$ ail (G)as - site (1) etc. 
SAP Munber: OK-12-5sG

SAMPLING ANO AMALYSIS PLAN TABLE FOR CHEMICAL AMO RROJOLOGICAL ANALYSIS

\begin{tabular}{|c|c|c|}
\hline \multicolumn{3}{|c|}{ SAMPLE DESCRIPIIO } \\
\hline $\begin{array}{l}\text { SAMPLING } \\
\text { ACTIVIIIY }\end{array}$ & SAMPLE & MEDIA \\
\hline 406106 & REG & soll an \\
\hline $4[G 107$ & REG & soll an \\
\hline $6 \mathrm{CG} 108$ & REG & solt aA \\
\hline 4C6109 & REG & soll an \\
\hline $4 c 6110$ & REG & SOIL GA \\
\hline «cGi11 & REG & soll an \\
\hline $4 \mathrm{Cg} 112$ & REG & solt an \\
\hline $4[6,113$ & REG & soll aA \\
\hline 6CG114 & REG & soll an \\
\hline $4 C G 115$ & REG & soll an \\
\hline 6CG116 & REG & soll an \\
\hline $4 C G 117$ & REG & solt GA \\
\hline $4 C G 118$ & REG & soll at \\
\hline $46 G 119$ & REG & soll an \\
\hline & & \\
\hline
\end{tabular}

6.0

Project: CFA OU 4-12 RI/FS - SHALLOU SOIL GAS

\begin{tabular}{|c|c|c|c|}
\hline $\begin{array}{l}\text { COL } \\
\text { IYPE }\end{array}$ & $\begin{array}{l}\text { SMMPLIMG } \\
\text { MET HOO }\end{array}$ & $\begin{array}{c}\text { PL A ANHED } \\
\text { DATE }\end{array}$ & AREA \\
\hline GRAB & & $06 / 29 / 93$ & CFA \\
\hline GRAB & & $06 / 29 / 93$ & CFA \\
\hline GRAB] & & $06 / 29 / 93$ & CFA \\
\hline GRA日 & & $06 / 29 / 93$ & cra \\
\hline GRAB & & $06 / 29 / 93$ & CFA \\
\hline GRAB & & $06 / 30 / 93$ & CFA \\
\hline GRAB & & $06 / 30 / 93$ & CFA \\
\hline GRAB & & $06 / 30 / 93$ & CFA \\
\hline GRAB & & $\infty</ 30 / 93$ & CFA \\
\hline GRAB & & $06 / 30 / 93$ & CFA \\
\hline GRAB & & $00 / 30 / 93$ & CFA \\
\hline GRAB & & $06 / 30 / 93$ & CFA \\
\hline GRAB & & $106 / 30 / 93$ & CFA \\
\hline GRAB & & $00 / 30 / 93$ & CFA \\
\hline GRAB & & $\infty 6 / 30 / 93$ & CFA \\
\hline
\end{tabular}

SAMPLE LOCAIION

$$
\text { SAMPLE LOCAIION }
$$

T

Proiect Manager: $x$ Page 8 of 12 enter amalysis types (at) ano ouahtitr kequested

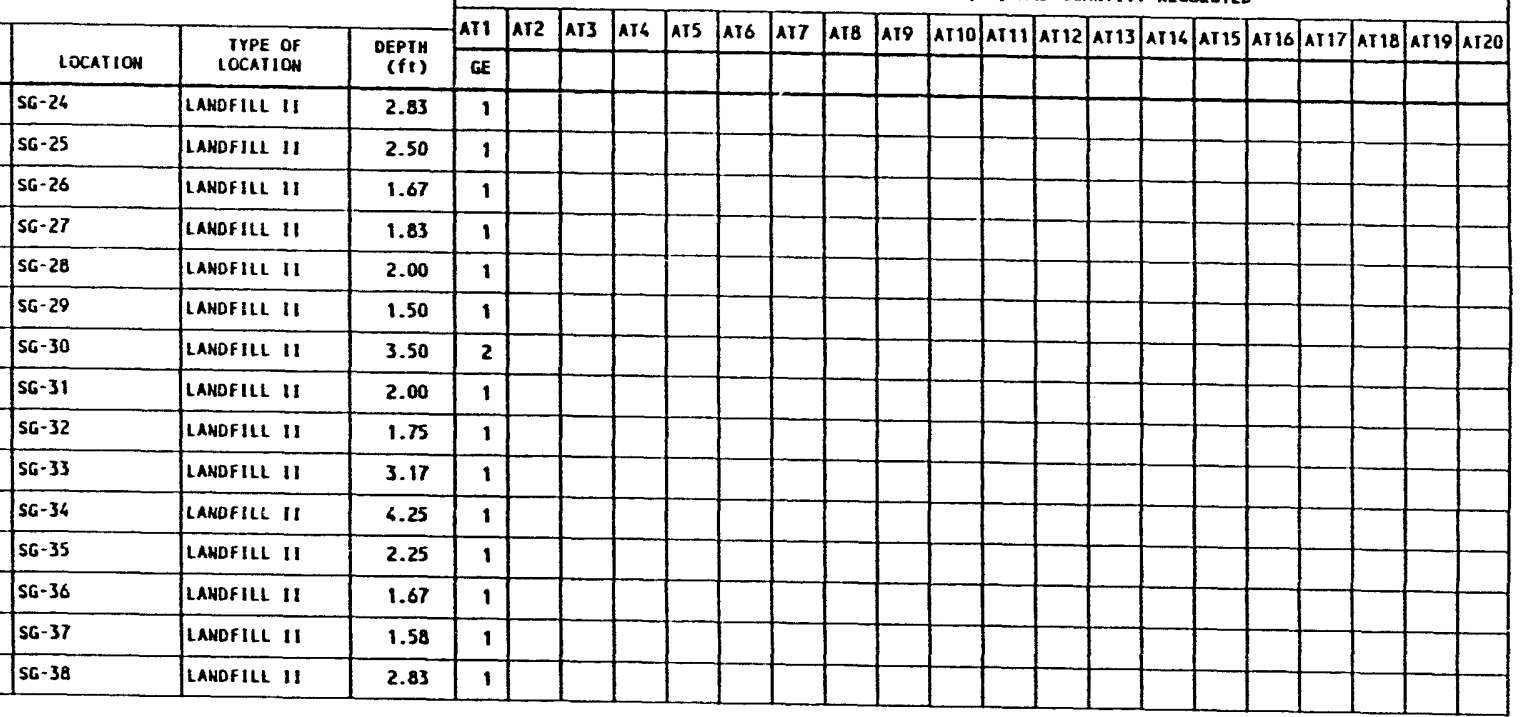

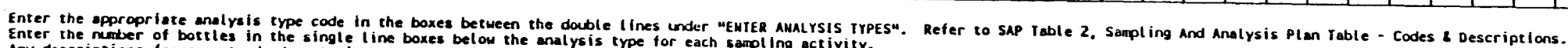

Any descriptions for non-standard analysis iypes (not given in SAP Yable 2) should be entered under "coniments" on the lines below.
Ar1: EPA Method 601 GC/ECO/FIO

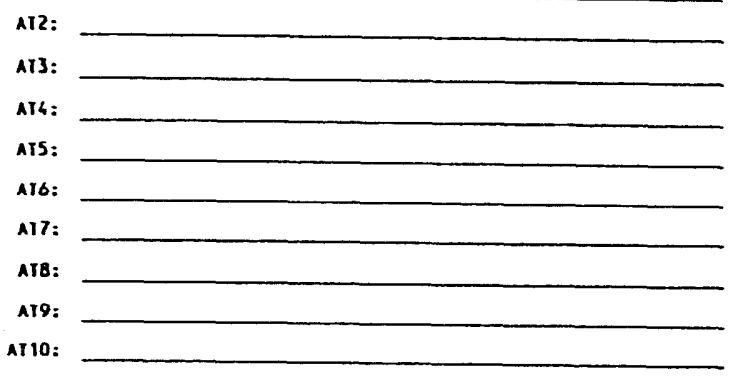
AI 10:

AIII:

Ar12:

AI13:

Ar14:

AI15:

Ar16:

Ar17:

AI18:

AT 19:
AI20:

CONAEN IS

FCIL = Field control sample

sG-1 $=$ (s)oil (G)as - site (1), etc. 
SAp Number: Ouk-12-ssc

SAMPLING AMO AHALYSIS PLAM TABLE FOR CHEMICAL ANO RADIOLOGICAL ANALYSIS

Page $\underline{\text { g of }} 12$ Date: 07/707/93 Revision: 6.0 Project: CFA OU 6-12 RI/FS - SHALLOU SOIL GAS

\begin{tabular}{|c|c|c|}
\hline $\begin{array}{l}\text { SNAPLING } \\
\text { ACIIVIIIY }\end{array}$ & \begin{tabular}{|l|} 
SAMPPEE \\
IYPE
\end{tabular} & MEOIA \\
\hline $6 C G 121$ & REG & SOIL GAS \\
\hline $4 c G 122$ & REG & SOIL GAS \\
\hline 606123 & REG & SOIL GAS \\
\hline \&CG126 & REG & SOIL GAS \\
\hline 6CG125 & REG & SOIL GAS \\
\hline $4 C G 126$ & REG & soll Gas \\
\hline $4 C 6127$ & REG & SOIL GAS \\
\hline $6 C G 128$ & REG & SOIL GAS \\
\hline $60 G 129$ & REG & SOIL GAS \\
\hline $6 C G 130$ & REG & SOIL GAS \\
\hline $4 C G 131$ & REG & soIL GAS \\
\hline $60 G 132$ & REG & SOIL GAS \\
\hline $4 C G 133$ & REG & SOIL GAS \\
\hline $60 \mathrm{CG} 136$ & REG & SOIL GAS \\
\hline 406135 & REG & soll GAS \\
\hline
\end{tabular}

\begin{tabular}{|c|c|}
\hline COA & SAMPL INA \\
TrPE & METHCO \\
\hline GRAB & \\
\hline GRAB & \\
\hline GRAB & \\
\hline GRAB & \\
\hline GRAB & \\
\hline GRAB & \\
\hline GRAB & \\
\hline GRAB & \\
\hline GRAB & \\
\hline GRAB & \\
\hline GRAB & \\
\hline GRAB & \\
\hline GRAB & \\
\hline GRAB & \\
\hline GRAB & \\
\hline
\end{tabular}

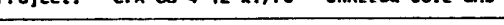

Project Manager: K. KECK/G. STORHBERG Form No: SAP11B

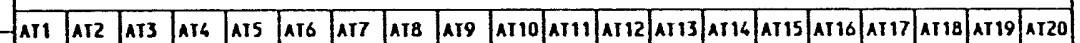

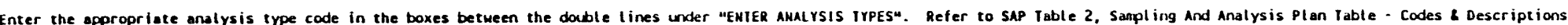

Enter the mater of bottles in the single line boxes below the analysis type for each sappling activity.
Any descriptions for non-standard analysis types (not given in SAP Iable 2) should be entered under "COMHENIS" on the lines below.

COHAENTS
All: EPA Method 601 GC/ECD/FID
All1:
AI2:
AI4:
Ars:
A17:
AT8:
A19:
AI10:
A114:
AT15:
AI 16:
AI17:
AT 18:
Ar19:
AI20:

\begin{tabular}{|c|c|c|c|c|c|c|c|c|c|c|c|c|c|c|c|c|c|c|}
\hline & & AII & A 12 A & AI3 1 & \begin{tabular}{l|l} 
AT4 & 1
\end{tabular} & AIS 1 & A16 1 A & $\begin{array}{lll}A 77 & A \\
\end{array}$ & \begin{tabular}{l|l}
$A T B$ & $A 1$ \\
\end{tabular} & AI9 & AIIOAIII & AI12 & \begin{tabular}{|l|l|l|}
$A 13$ & $A 114$ \\
\end{tabular} & AIIS & \begin{tabular}{|l|l|l|} 
A116 & AI17 \\
\end{tabular} & $A$ AIB $A$ & \begin{tabular}{|l|l|l|} 
AT19 & Al \\
\end{tabular} & AI20 \\
\hline $\begin{array}{l}\text { IYPE OF } \\
\text { LOCAIIOW }\end{array}$ & $\begin{array}{l}\text { DEPH } \\
\text { (ft) }\end{array}$ & EE & & & & & & & & & & & & & & & & \\
\hline LAMDFILL $\|$ & 3.17 & 1 & & & & & & & & & & & & & & & & \\
\hline LANDFILL "I & 1.75 & 2 & & & & & & & & & & & & & & & & \\
\hline LANDFILL "II & 3.00 & 1 & & & & & & & & & & & & & & & & \\
\hline LANDFILL "I & 1.50 & 1 & & & & & & & & & & & & & & & & \\
\hline LANOFILL "I & 1.83 & 1 & & & & & & & & & & & & & & & & \\
\hline LANDFILL "I & 3.25 & 1 & & & & & & & & & & & & & & & & \\
\hline LAHDFILL II & 1.67 & 1 & & & & & & & & & & & & & & & & \\
\hline LAMOFILL "I & 2.67 & 1 & & & & & & & & & & & & & & & & \\
\hline LANDFILL II & 2.08 & 1 & & & & & & & & & & & & & & & & \\
\hline LAMDFILL II & 6.08 & 1 & & & & & & & & & & & & & & & & \\
\hline LANDFILL "I & 2.50 & 1 & & & & & & & & & & & & & & & & \\
\hline LAMOFILL II & 1.67 & 2 & & & & & & & & & & & & & & & & \\
\hline LANOF ILL II & 1.92 & 1 & & & & & & & & & & & & & & & & \\
\hline LANDFILL II & 1.83 & 1 & & & & & & & & & & & & & & & & \\
\hline LANDFIIL "I & 1.92 & 1 & & & & & & & & & & & & & & & & \\
\hline
\end{tabular}

FCTL = field control sample

G-1 = (5)oll (G)as - site (1) etc 


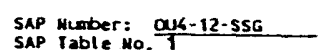
Date: $07 / 07 / 93$ Revision: 0.0

SAMPLING ANO ANALYSIS PLAN IABLE FOR CHEHICAL AND RADTOLOGICAL AMALYSIS

\begin{tabular}{|c|c|c|}
\hline $\begin{array}{l}\text { SAMPIVG } \\
\text { ACTIVIII }\end{array}$ & $\begin{array}{l}\text { SAMPLE } \\
\text { TYPE }\end{array}$ & MEDIA \\
\hline 6CG136 & REG & SOIL GAS \\
\hline 6CG137 & REG & soll GAS \\
\hline $6 C 6138$ & REG & SOIL GAS \\
\hline 6CG139 & REG & SOIL GAS \\
\hline ¿CG160 & REG & SOIL GAS \\
\hline $6 \mathrm{CG} 161$ & REG & SOIL GAS \\
\hline $4 C 6162$ & REG & SOIL GAS \\
\hline $6 C 6163$ & REG & SOIL GAS \\
\hline $6 C 6146$ & REG & SOIL GAS \\
\hline $6 C G 165$ & REG & SOIL GAS \\
\hline $6 c 6166$ & REG & soll aAs \\
\hline 6CG167 & REG & Soll GAS \\
\hline $4 C G 1<8$ & REG & SOIL GAS \\
\hline 406169 & REG & soll Gas \\
\hline SCG150 & REG & SOl1 GAS \\
\hline
\end{tabular}

\begin{tabular}{|c|c|c|c|c|}
\hline $\begin{array}{l}\text { COLL } \\
\text { ITPE }\end{array}$ & $\begin{array}{l}\text { SMMPLING } \\
\text { METHOO }\end{array}$ & $\begin{array}{c}\text { PLAMUED } \\
\text { DATE }\end{array}$ & AREA & LOCAIIOW \\
\hline GRAB & & OS/30/93 & cra & $5 G-56$ \\
\hline GRAB & & $06 / 30 / 93$ & CFA & sG. 55 \\
\hline GRAB & & $06 / 30 / 93$ & cra & sG-56 \\
\hline GRAB & & $06 / 30 / 93$ & CrA & $5 G-57$ \\
\hline GraB & & $\mid 06 / 30 / 93$ & CFA & sG-58 \\
\hline GRAB & & $\infty 6 / 30 / 93$ & CFA & 56.59 \\
\hline GRAB & & $00 / 30 / 93$ & cFa & sc-60 \\
\hline GRAB & & $00 / 30 / 93$ & CFA & $5 G-61$ \\
\hline GrAB & & $|06 / 30 / 93|$ & cra & $56-62$ \\
\hline GRAB & & $06 / 30 / 93$ & CFA & $56-63$ \\
\hline GRAB & & $06 / 30 / 93$ & CFA & $56-64$ \\
\hline GRAB & & $06 / 30 / 93$ & CFA & $5 G .65$ \\
\hline GRAB & & $\mid 06 / 30 / 93$ & CFA & $56-66$ \\
\hline GrAB & & $06 / 30 / 93$ & CFA & $56-67$ \\
\hline GRAB & & $\mid 06 / 30 / 93$ & CFA & $56-68$ \\
\hline
\end{tabular}

S - SHALLOU SOlL GaS Project Manager: K. XECX/G. STORHBE
EHTER AMALYSIS TYPES (AT) AND OUANTIIY REOUESTED

Page 10 of 12 Foral No: SAP118

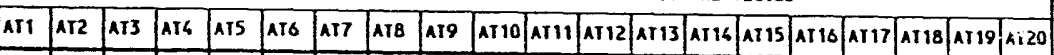

\begin{tabular}{|c|c|}
\hline $\begin{array}{l}\text { IYPE OF } \\
\text { LOCAIIOH }\end{array}$ & $\begin{array}{c}\text { DEPTH } \\
\text { (ft) }\end{array}$ \\
\hline "ANDFILI & 2.00 \\
\hline LaHDFILL "I & 2.50 \\
\hline LAMOFILL II & 3.42 \\
\hline LANDFHLL "I & 4.00 \\
\hline LAMDFILL "II & 2.17 \\
\hline LAMDFILL "I & 2.00 \\
\hline LANDFILL II & 2.42 \\
\hline LAMDFILL II & 2.42 \\
\hline LANOFILL II & 3.00 \\
\hline LANDFILL "II & 2.50 \\
\hline LANDFLLL 11 & 1.58 \\
\hline LARDFILL & 2.33 \\
\hline LAMDFILL "I & 1.50 \\
\hline LaNDFILL "I & 1.83 \\
\hline
\end{tabular}

Enter the appropriate analysis type code in the boxes beived

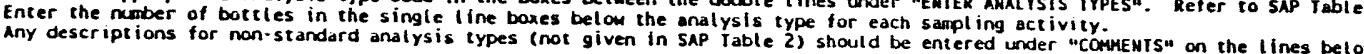

All: EPA Method 601 GC/ECO/AID

AI3:

Ats:

AI6:

A17:

A18:

A19:

Al10:
AIII:

A112:

Ar13:

Arr6:

Ar15:

Arlo:

arriz:

A118:

Arlo:

A120:
CONMENIS

fCIL = Field control Sample

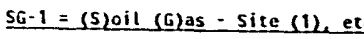


SAP Munber: aK-12-5SG

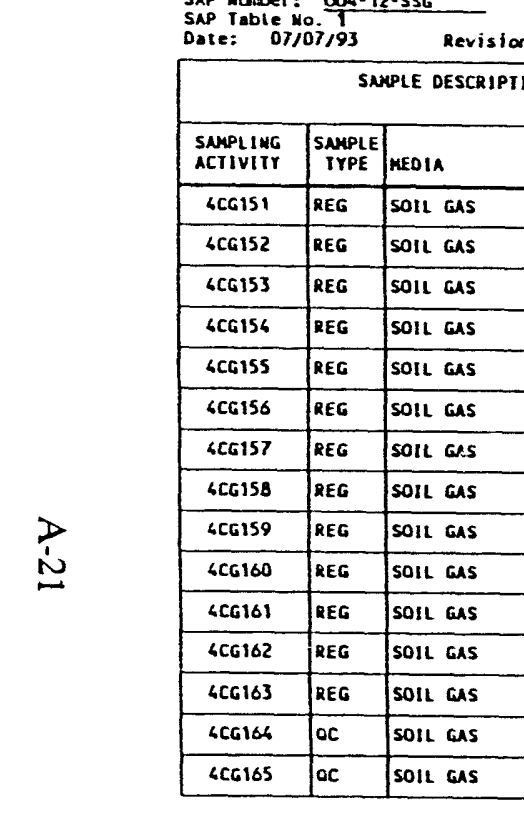

\begin{tabular}{|c|c|c|c|c|}
\hline & & & & I6 AND ANALYSIS \\
\hline 6.0 & & & & CfA ou $4-12$ \\
\hline & & & & SAMPLE \\
\hline $\begin{array}{c}\mathrm{COL} \\
\mathrm{TYPE}\end{array}$ & $\begin{array}{c}\text { SAMPLIMG } \\
\text { METHOO }\end{array}$ & $\begin{array}{l}\text { PLANMED } \\
\text { DAIE }\end{array}$ & AREA & LOCATIOW \\
\hline GRAB & & $07 / 01 / 93$ & CFA & $56-69$ \\
\hline GRAB & & $07 / 01 / 93$ & CFA & $56-70$ \\
\hline GRAB & & $07 / 01 / 93$ & CFA & $56-71$ \\
\hline GRAB] & & $07 / 01 / 93$ & CFA & $5 G-72$ \\
\hline GRAB & & $07 / 01 / 93$ & CFA & $56-73$ \\
\hline GRAB & & |07/01/93 & CFA & $5 G-74$ \\
\hline GRAB & & |07/01/93 & CFA & $56-75$ \\
\hline GRAB & & $07 / 01 / 93$ & CFA & s6-76 \\
\hline GRAB & & $07 / 01 / 93$ & CFA & $56-77$ \\
\hline GRAB & & |07/01/93 & CFA & sG-78 \\
\hline GRAB & & $07 / 01 / 93$ & CFA & $56-79$ \\
\hline GRAB & & [07/01/93 & Cra & $56-80$ \\
\hline GRAB & & |07/01/93 & Cra & SG-81 \\
\hline fCrL & & $06 / 30 / 93$ & CFA & OC - ВI АMK \\
\hline FCit & & $\mid \infty 8 / 29 / 93$ & CFA & OC - BLANK \\
\hline
\end{tabular}

ANAYSIS PLAN TABLE FOR CHEMICAL ANO RNOIOCOGICAL AMNYSIS FCil $200 / 29 / 93$ CFA

\begin{tabular}{l|l}
\hline OC - blakx & Start of dar \\
\hline
\end{tabular}
4.12 RI/FS - SHALLON SOIL GAS

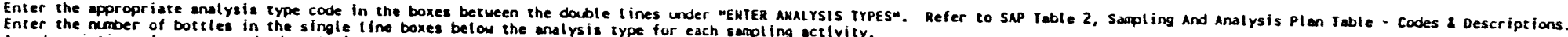

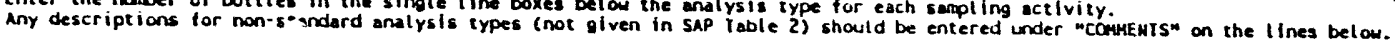

AI1: EPA Methad 601 CC/ECO/FID

AT2:

AT3:

A16: $\longrightarrow$ AI16:

AIS: $\longrightarrow$ AI15:

AT6: - AI16:

A17:

A18:

A19: — AI19:

Al10:
CONAENIS

FCIL = Field Control Sample

$\underline{\text { SG-1 }} \equiv$ (s)oil (G)as - site (1) etc. 


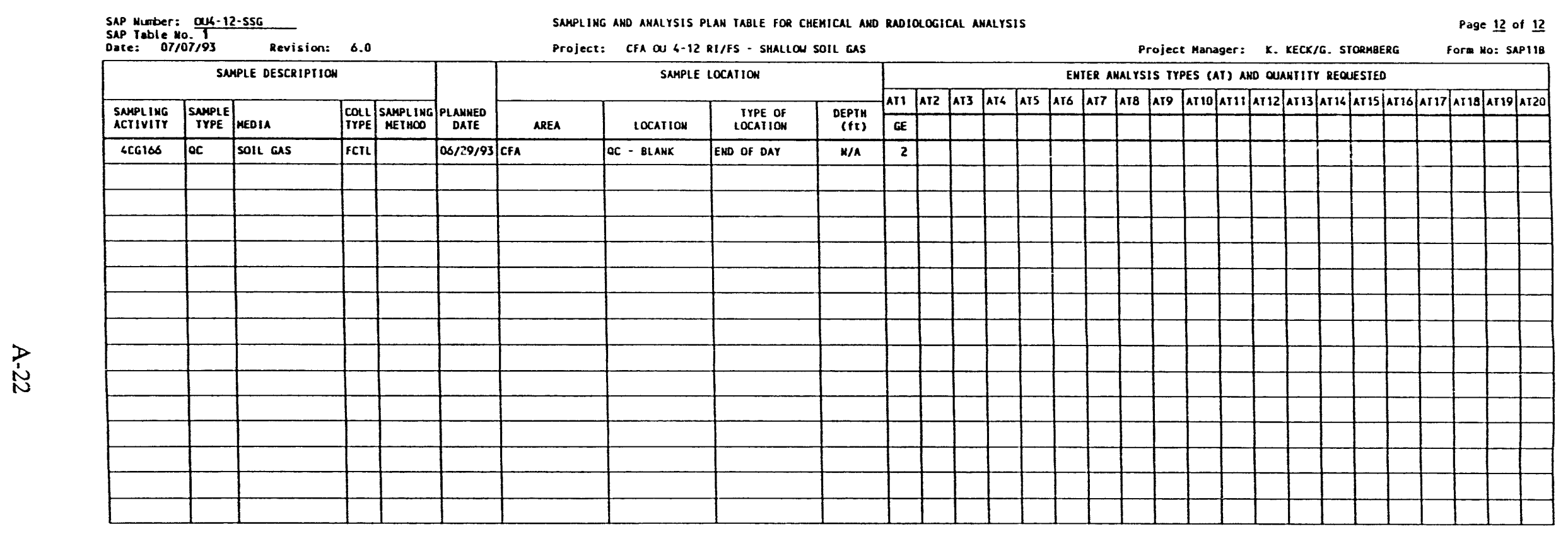

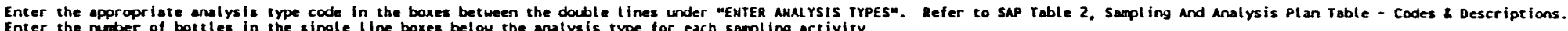

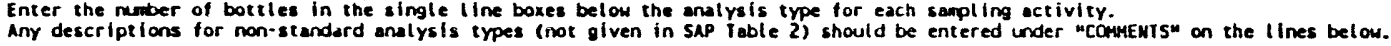

AI1: EPA Method $\Delta 01$ GC/ECD/FID

A12:

AT3:

AIs:

ATS:

AT6:

A17:

Ar8:

A19:

Ar10:
AI11:

A112:

at13:

AT15:

Ar16:

AI17:

A118:

AI19:

Aт20:
COMHEN IS

FCIL = Field control sample

sq-1 $=(s)$ oil $(G) a s-$ site $(1)$ etc.

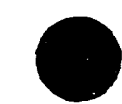


SAP Number: OW-12-8SG

Revision: 5.0

SAMPLING ANO ANALYSIS PLAN TABLE FOR CHEMICAL AND RNDIOLOGICAL AMALYSIS

SAP Table Mo: $07 / 07 / 93$

Revision:

Project: CFA OU 6-12 91/FS - BOREMOLE SOIL CAS

Project Manager: K. KECK/G. STORMEERG

Page 1 of 1

\begin{tabular}{|c|c|c|c|c|c|c|c|c|c|c|c|c|c|c|c|c|c|c|c|c|c|c|c|c|c|c|c|}
\hline \multirow{4}{*}{\begin{tabular}{|l|} 
\\
$\begin{array}{l}\text { SurPLING } \\
\text { ACTIVIIY }\end{array}$ \\
\end{tabular}} & \multicolumn{4}{|c|}{ SMPLE DESCRIPTIOW } & \multirow{4}{*}{$\begin{array}{l}\text { PLAMMED } \\
\text { DATE }\end{array}$} & \multirow{2}{*}{\multicolumn{4}{|c|}{ SAMPLE LOCATIOM }} & \multirow{2}{*}{\multicolumn{18}{|c|}{ EMTER ANALYSIS IYPES (AI) AND OUNHITY RECUESTEO }} \\
\hline & & & & & & & & & & & & & & & & & & & & & & & & & & & \\
\hline & $\begin{array}{l}\text { SMMPLE } \\
\text { TYPE }\end{array}$ & MEOH & coal & SMMPL ING & & \multirow{2}{*}{ AREA } & \multirow[b]{2}{*}{ LOCAIION } & \multirow{2}{*}{$\begin{array}{l}\text { TYPE OF } \\
\text { LOCAIIOW }\end{array}$} & \multirow{2}{*}{$\begin{array}{l}\text { DEPTI } \\
\text { (ft) }\end{array}$} & AII & A12 & AI3 & AT6 & ats & A16 1 & AI7 $\Lambda$ & \begin{tabular}{r|r}
18 & $\operatorname{ATS}$ \\
\end{tabular} & AI10 & Sarin/a & \begin{tabular}{ll|l}
$A T 12$ & $A T$ \\
\end{tabular} & III]At & \begin{tabular}{r|r|}
116 & $A 11$ \\
\end{tabular} & $15 \mid a 11$ & 16) & 7 anis & Aris & A120 \\
\hline & & Then & & | NeTHor & & & & & & GE & & & & & & & & & & & & & & & & & \\
\hline SLutor & REG & soll cas & GRAB & & $07 / 01 / 93$ & CFA & LF2-1 & LaNDFILL "I & 27 & 1 & & & & & & & & & & & & & & & & & \\
\hline $60 G 168$ & REG & sol1 Gas & Gene & & $07 / 01 / 93$ c & CFA & $152-2$ & LAMDFILL 11 & 31 & $t$ & & & & & & & & & & & & & & & & & \\
\hline 606169 & REG & SOIL GAS & ceess & & $07 / 01 / 93 \mid \mathrm{C}$ & CFA & 152.6 & LANDFILL II & 19 & 1 & & & & & & & & & & & & & & & & & \\
\hline $66 G 170$ & REG & SoIt GAS & GRAB & & $07 / 01 / 93$ & CFA & LF2-5 & LaMOF ILL II & 21 & 2 & & & & & & & & & & & & & & & & & \\
\hline scain & oc & soll Gas & FCII & & $007 / 01 / 93 \mid \mathrm{c}$ & CFA LF U] & $O C-8 L A M K-1 F 2-5$ & $1 / 20$ SAMPIES & N/A & 1 & & & & & & & & & & & & & & & & & \\
\hline 406176 & $R E G$ & soll Gas & CRAB & & $07 / 01 / 93$; & CFA & LF3-1 & LAMDFILL III & 17.5 & 1 & & & & & & & & & & & & & & & & & \\
\hline 606175 & REG & SOIL GAS & Geas & & $07 / 01 / 93]^{c}$ & CFA & $153-2$ & LANDFIIL $\| I$ & 25 & 1 & & & & & & & & & & & & & & & & & \\
\hline 606176 & REG & SOLL CAS & CRAB & & |07/01/93]c & CFA & $183-6$ & LandFlll "II & 11.5 & 2 & & & & & & & & & & & & & & & & & \\
\hline 6 cain & REG & SOIL GAS & |GRAB & & $07 / 01 / 93$ & CFA & L.5.6 & LANOFILL III & 16.5 & 1 & & & & & & & & & & & & & & & & & \\
\hline 606178 & REG & SOIL GAS & Gres & & $07 / 01 / 93$ & CFA & LEF3-7 $>$ > & LANDFILL III & 12 & 1 & & & & & & & & & & & & & & & & & \\
\hline 606180 & $\infty c$ & SOIL GAS & fere & & $07 / 01 / 93$ & CFA LF III & 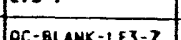 & & & 1 & & & & & & & & & & & & & & & & & \\
\hline LCG181 & oc & SOIL GAS & FCTI & & $07 / 01 / 93$ & CrA Lf III & 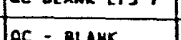 & 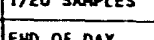 & $m / 1 / 4$ & 1 & & & & & & & & & & & & & & & & & \\
\hline & & & & & & & De - DaAk & FWD OF DAY & $m / n$ & 1 & & & & & & & & & & & & & & & & & \\
\hline & & & & & & & & & & & & & & & & & & & & & & & & & & & \\
\hline & & & & & & & & & & & & & & & & & & & & & & & & & & & \\
\hline & & & & & & & & & & & & & & & & & & & & & & & & & & & \\
\hline
\end{tabular}

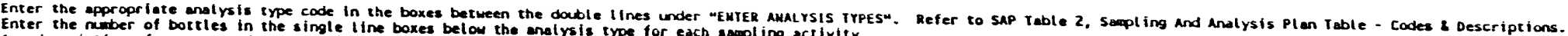

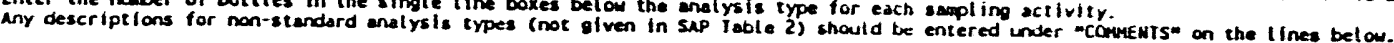

AI1: EPA Method bO1 CC/EED/EID

A12:

A13:

ATS:

A16:

air

Are:

AI10: ari1:

al12:

AI13:

Ar16:

AT 15:

Aก17:

ar18:

AT19:

AT20:
FCIL = Field Control Sample

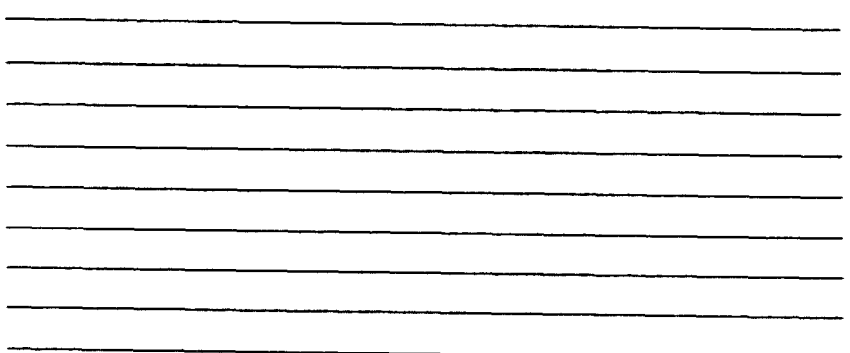


SAP Mumber: OUS-12-SF
SAP Table Wo. Th

SAMPLIMG AND ANALYSIS PLAM TABLE FOR CHEMICAL ANO RNDIOLOGICAL AMALYSIS

Date: $07 / 07 / 93$ Revision: 3.0

Project: IFA OU 4-12 RI/FS - SURFACE FIUX CHAHBER

Page 1 of $\underline{2}$ SAMPLE LOCATION

Project Hanager: X. KECK/G. SIORHBERG

Form Ho: SAP118 ENTER AMAI YSIS TYPES (AT) ANO OUMNIIIT REGUESTED

\begin{tabular}{|c|c|c|}
\hline $\begin{array}{l}\text { SAMPLIIMG } \\
\text { ACtriviII }\end{array}$ & \begin{tabular}{|c|} 
SAMPLE \\
TPPE
\end{tabular} & MEDIA \\
\hline $4 C G 182$ & REG & solt GAS \\
\hline $6[G 183$ & REG & soth Gas \\
\hline 6CG186 & REG & solt aAs \\
\hline $6 C G 185$ & REG & SOIL GAS \\
\hline 6CG188 & REG & soll Gas \\
\hline 6CG187 & RES & SOIL GAS \\
\hline $6 C G 188$ & REG & Soll Gas \\
\hline $6 C G 189$ & REG & SOIL GAS \\
\hline $4 C 6190$ & REG & SOIL GAS \\
\hline ४CG191 & REG & SOIL GAS \\
\hline $4 C G 192$ & REG & soll GAS \\
\hline 4CG193 & REG & soll Gas \\
\hline 6CG196 & REG & SOIL GAS \\
\hline 6CG195 & REG & soIL GAS \\
\hline sec19s & REG & SOIL GAS \\
\hline
\end{tabular}

\begin{tabular}{|c|c|c|c|}
\hline \begin{tabular}{|l|}
$\mathrm{COL}$ \\
$\mathrm{IYPE}$
\end{tabular} & \begin{tabular}{|c|} 
SAMPL ING \\
KETHOO
\end{tabular} & $\begin{array}{c}\text { PL ANMED } \\
\text { DAIE }\end{array}$ & AREA \\
\hline GRAB & & $\mid \infty 8 / 01 / 93$ & CFA \\
\hline GRAB & & $00 / 01 / 93$ & Cra \\
\hline GRAB & & $06 / 01 / 93$ & CFA \\
\hline GRAB & & $06 / 01 / 93$ & cFA \\
\hline GRAB & & $06 / 01 / 93$ & CFA \\
\hline GRAB & & Q6/01/93 & CFA \\
\hline GRAB & & $\mid \infty 6 / 01 / 93$ & CFA \\
\hline GRAB & & $06 / 01 / 93$ & CrA \\
\hline GRAB & & $06 / 01 / 93$ & cra \\
\hline GRAB & & $0</ 01 / 93$ & CFA \\
\hline GRAB & & $|\infty 6 / 01 / 93|$ & CFA \\
\hline GRAB & & O6/01/93 & CFA \\
\hline GRAB & & $06 / 01 / 93$ & CFA \\
\hline GRAB & & $06 / 01 / 93$ & CFA \\
\hline Ggab & & & CFA \\
\hline
\end{tabular}

\begin{tabular}{|c|}
\hline LOCATIO \\
\hline SGF-TBD-1 \\
\hline SGF-TBD - 2 \\
\hline SGF - TBD-3 \\
\hline SGF-TBD-6 \\
\hline SGF-TBO-5 \\
\hline SGF-TBO-6 \\
\hline $5 G F-180-7$ \\
\hline $5 G F-J B O-8$ \\
\hline SGF-TBD-1 \\
\hline SGF-TBO-2 \\
\hline SGF - TED - 3 \\
\hline SGF- $-1 B D-4$ \\
\hline SGF- TBD - 5 \\
\hline SGF-TBD-6 \\
\hline
\end{tabular}

\begin{tabular}{|c|c|c|c|c|c|c|c|c|c|c|c|c|c|c|c|c|c|c|}
\hline \multirow{2}{*}{$\begin{array}{c}\text { IYPE OF } \\
\text { LOCAIIOW } \\
\end{array}$} & \multirow{2}{*}{$\begin{array}{c}\text { OEPIH } \\
\text { (It) }\end{array}$} & \multicolumn{2}{|c|}{\begin{tabular}{|l|l|} 
A11 & A12 \\
\end{tabular}} & \multirow[t]{2}{*}{ Ar3 } & \multirow[t]{2}{*}{ A16 } & \multirow[t]{2}{*}{ A15 } & \multirow[t]{2}{*}{ A16 } & \multirow[t]{2}{*}{ ant } & \multirow[t]{2}{*}{ A18 } & \multirow[t]{2}{*}{ A19 } & \multirow[t]{2}{*}{ AI10/10 } & \begin{tabular}{|l|l|} 
AII2 & AII13 \\
\end{tabular} & An & Aris & \begin{tabular}{|l|l|l|l|} 
An17 \\
\end{tabular} & AI & \begin{tabular}{|l|l|l|}
1 & \\
\end{tabular} & 1120 \\
\hline & & vi & & & & & & & & & & & & & & & & \\
\hline LAMOFILL 11 & W/A & 1 & & & & & & & & & & & & & & & & \\
\hline LAMDFILL "I & N/A & 1 & & & & & & & & & & & & & & & & \\
\hline LANDFILL II & 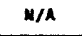 & 1 & & & & & & & & & & & & & & & & \\
\hline LANOFILL II & W/A & 1 & & & & & & & & & & & & & & & & \\
\hline LANOFIIL II & w/A & 1 & & & & & & & & & & & & & & & & \\
\hline LanOFILL "I & W/A & 1 & & & & & & & & & & & & & & & & \\
\hline LAMOFILL "I & N/A & 1 & & & & & & & & & & & & & & & & \\
\hline LANOFILL " & N/A & 1 & & & & & & & & & & & & & & & & \\
\hline LAMOFSIL III & w/A & 1 & & & & & & & & & & & & & & & & \\
\hline LAMDFILL III & $w / A$ & 1 & & & & & & & & & & & & & & & & \\
\hline LAMOF IIL "II & $\mathrm{H} / \mathrm{A}$ & 1 & & & & & & & & & & & & & & & & \\
\hline LAMOFILL "II & $N / A$ & 1 & & & & & & & & & & & & & & & & \\
\hline LAMDFILL III & W/A & 1 & & & & & & & & & & & & & & & & \\
\hline LAMOFILL III & $w / \AA$ & 1 & & & & & & & & & & & & & & & & \\
\hline LANOFILL III & $\omega / A$ & 1 & & & & & & & & & & & & & & & & \\
\hline
\end{tabular}

Enter the appropriate analysis type code in the boxes between the double lines under "EHIER AMALYSIS TYPES". Refer to SAP Tabie 2, Sampling And Analysis Ptan Table - Cudes 2 Descriptions.

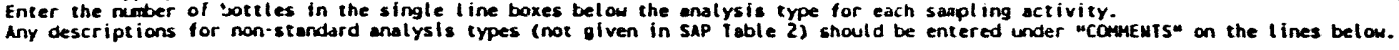

AII: vacs (rod. 10-16)

A12:

AI3:

A16:

Ars:

AI6:

AI7:

A18:

Arro:
Aril:

Al12:

Ar13:

AI16:

AT15:

A116:

Ar17:

AT18:

A119:

arzo:
CONAE I IS

SGF-1BD-1 - (S)urface (G)as (F)lux - Location (T)O (B)e (D)eterwined site (1), etc.

Specific sample locatlons uill be deternined in the field.

One field replicate will be collected randonly at each landfill

Qne field blank will be collected randouly at each landtill 
SAP Mumber: Table No $\frac{\text { OK-12-SF }}{1}$

SMMPL ING ANO ANALYSIS PLAN tABLE FOR CHEHICAL AND RADJOLOGICAL AMALYSIS

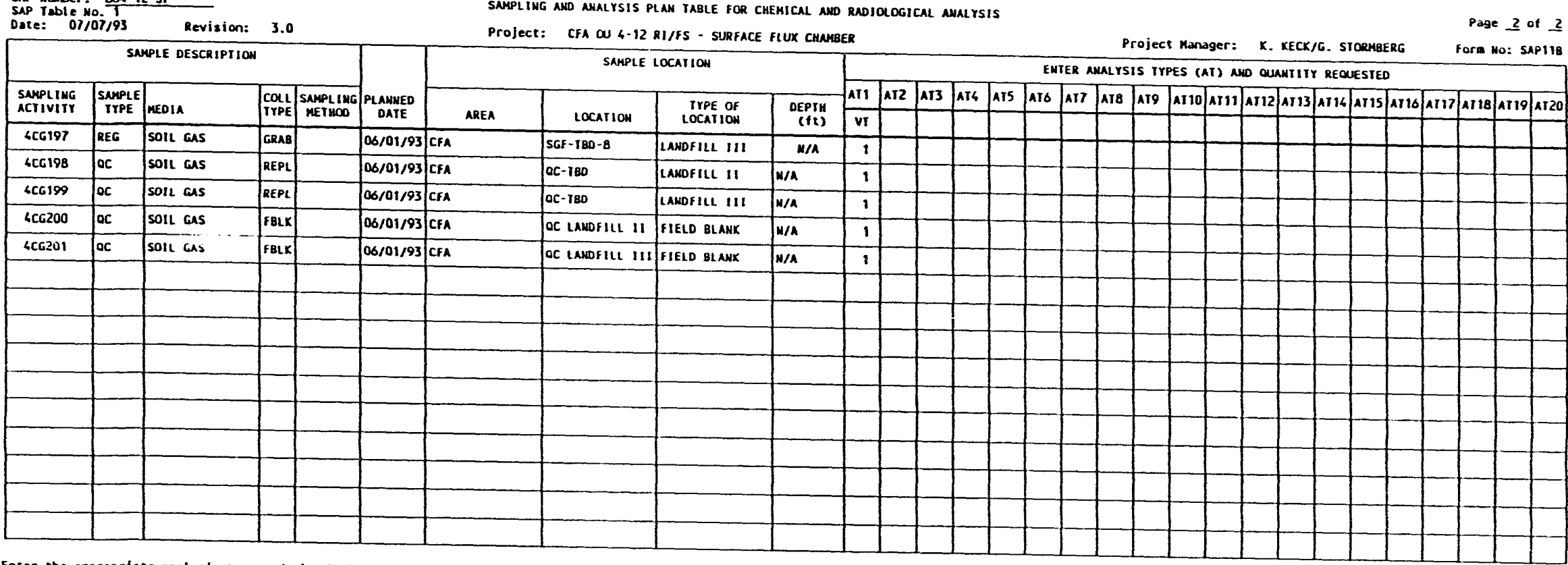

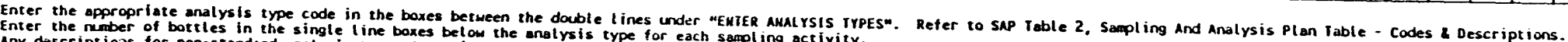

Any descriptions for non-standard analysis ippes (not given in SAP Yable 2) should be entered under "CormenIS" on the tines betow.

AI1: vocs (Mad. 10-16)

AI2:

AIs:

A16:

ATs:

A16:

A18:

A19:
Aril:

Ari2:

ATIS:

AI14:

AI 15:

AI16:

A117:

Ar18:

Al19:

AI20:
CONMENTS

SGF-JBD-1 - (S)urface (G)as (F)lux - Location (T)o (B)e (0)etermined sire (1). etc.

Specific sample locations will be deterained in the field.

ane field repl icate will be collected randomily at each landt il

One field blank will be collected randuaty at each tandfill 
SAP Mumber: QKK-12-Guil

Date: $07 / 07 / 93$ Revision:

Revision: 9.0

SAMPLING AND AMALYSIS PLAM table fOR CHEHICAL and RnOTOLOGICAL AHALYSIS

Project: CFA QU 6-12 R1/FS - GROWNO MATER PBASE I

\begin{tabular}{|c|c|c|c|c|c|c|c|c|c|c|c|c|c|c|c|c|c|c|c|c|c|c|c|c|c|c|c|c|}
\hline & & & & & & & & & & & & & & & & & & Mana: & & K. $\mathbf{K E}$ & /G. & $c r 004$ & & & & & & \\
\hline & & & & & & & & & & & & & & & & IER N & WaLYS & is ir & PES (A & (I) AMO & D OUAN & InTITY & REOUE: & ESTED & & & & \\
\hline $\begin{array}{l}\text { SAMPLING } \\
\text { ACtIVIIY }\end{array}$ & $\mid$ & MEDIA & $\mid$ & $\begin{array}{c}\text { SMMPL ING } \\
\text { METHOD }\end{array}$ & SNMPLE DATE & AREA & sorino" & IYPE of & DEPTH & AnI & $1 \sqrt{2}$ & A13 & AT6 & ATS & Ars & AI7 & ar8 & AI9 & Ar10 & AIII/A & $\operatorname{li12/A}$ & \begin{tabular}{|l|l|} 
Ar13A \\
\end{tabular} & 1214 /A & $\operatorname{Ar} 15 \mid A$ & \begin{tabular}{l|l}
$A$ & 16
\end{tabular} & $117 \mid A T$ & \begin{tabular}{l|l}
1118 & $A 119$
\end{tabular} & 19 arzo \\
\hline 400001 & REG & GROANO UNTER & GaAB & & $06 / 07 / 93$ & cFa & 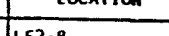 & LOCANIOW & (ft) & A1 & co & c1 & CR & a & 06 & 07 & FI & N2 & 25 & sc & & & & & & & & \\
\hline 600002 & REG & Groumo UAIER & GRAB & & nenter & & |Lr<-8 & LANOFILL "II & 488 & 1 & 1 & 1 & 1 & 1 & 1 & 1 & 1 & 1 & 1 & 1 & & & & & & & & \\
\hline $4 c 0003$ & REG & GROUMD MATER & cent & & $00 / 0 / / 93$ & CFA & LF2-9 & LANDFIIL II & 690 & 1 & 1 & 1 & 1 & 1 & 1 & 3 & 1 & 1 & 1 & 1 & & & & & & & & \\
\hline $4 c 0006$ & REG & GROUNO UATER & |loxas & & $06 / 09 / 93$ & CFA & LF2-10 & LANDFTLL II & 500 & 1 & 1 & 1 & 1 & 1 & 1 & 1 & 1 & 1 & 1 & 1 & & & & & & & & \\
\hline $4 \operatorname{cooss}$ & REG & GROANOD WATER & |Gras & & $08 / 02 / 93$ & CFA & LF2-11 & LANDFHLL 11 & 685 & 1 & 1 & 1 & 1 & 1 & 1 & 1 & 1 & 1 & 1 & 1 & & & & & & & & \\
\hline 400006 & |REG & GROUND UATER & GRAB & & $5 / 20<6 / 01 / 93$ & CFA & LF2-12 & LANDFILL II & 485 & 1 & 1 & 1 & 1 & 1 & 1 & & 1 & 1 & 1 & 1 & & & & & & & & \\
\hline 400007 & REG & GROUND MAIER & GRAB & & $06 / 07 / 93$ & CFA & LFS-8 & LANDFILL III & 500 & 1 & 1 & 1 & 1 & 1 & 1 & 1 & 1 & 1 & 1 & 1 & & & & & & & & \\
\hline 4c0007 & REG & GroUnO MATEX & GRAB & & $5 / 10 \leq 5 / 20 / 93$ & CFA & $153-9$ & LANDFILL IIII & 495 & 1 & 1 & 1 & 1 & 1 & 1 & & 1 & 1 & 1 & 1 & & & & & & & & \\
\hline sc0008 & REG & GroUnd UATER & GRAB & & $06 / 02 / 93$ & CFA & LFS-10 & LANDFILL III & 495 & 1 & 1 & 1 & 1 & 1 & 1 & & 1 & 1 & 1 & 1 & & & & & & & & \\
\hline 400009 & REG & GROUND UAIER & GRAB & & $00 / 02 / 93$ & CFA & LF3-11 & LANOFILL III & 487 & 1 & 1 & 1 & 1 & 1 & 1 & & 1 & 1 & 1 & 1 & & & & & & & & \\
\hline 600010 & REG & Grolmo MAIER & GrAB & & $06 / 01 / 93$ & CFA & USGS- BS & LANOFILL III & 488 & 1 & 1 & 1 & 1 & 1 & 1 & & 1 & 1 & 1 & 1 & & & & & & & & \\
\hline $4 c 0011$ & REG & GROUND MATER & GRAB & & $08 / 08 / 93$ & CFA & CFA-1 & LAMDFILL II & 488 & 1 & 1 & 1 & 1 & 1 & 1 & & 1 & 1 & 1 & 1 & & & & & & & & \\
\hline 600012 & REG & Grouno WAJER & GRAB & & $06 / 08 / 93$ & CFA & CFA-2 & LANDFIIL "II & 471 & 1 & 1 & 1 & 1 & 1 & 1 & & 1 & 1 & 1 & 1 & & & & & & & & \\
\hline 400013 & lac & JATER & resx & & $5 / 10-6 / 09 / 93$ & CFA & ac & IRIP BLANK & M/A & & & & & & 15 & & & & & & & & & & & & & \\
\hline $4 c 0016$ & oc & WATER & FBLX & & $06 / 01 / 93$ & CFA & LF3-10 & OC-FIELD BLANK & n/A & 1 & & 1 & 1 & & 1 & & 1 & 1 & & 1 & & & & & & & & \\
\hline 400015 & loc & WAJER & RHST & & $05 / 10 / 93$ & CFA & PUMPP - BEF ORE & OC - RIMSAIE & $N / A$ & 1 & & 1 & 1 & & 1 & & 1 & 1 & 1 & 1 & & & & & & & & \\
\hline
\end{tabular}

Enter the appropriate analysis type code in the boxes between the double 1 ines under "ENIER ANALYSIS ITPES"

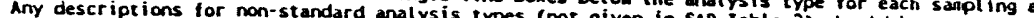

AI1: Alkaliniux

COMAENTS

Ai2: CLP Hetels - Filitered

A13: CLe Hetals-Unfiltered

Al6: Chromiun ve $(C r+6)$ - Unfiltered

Aril: Sulfate/chloride

AIS: Chromiun VI (Cr+6) - Filtered

AI12:

AI6: Drinking Hater voAs (524.2) IV - (Bailer)

Ar13:

A17: Rrinking Hater voAs (524.2) IV - (Pump)

Al8: Elworide

A19: Hitrote/mitrite

AI 10: Gompe Screen

Ar16:

AI15:

AT16:

AI17:

AT18:

AI19:
The field team leader yill determine three random locations at which s ser of replicate samples each will be collected.

References to "Bafler" and "Pump" for analysis còdes "DS" and D7" corinking Hater voAs (526.2) 1V2 pertain to well samoles only.

Sample depths 1 isted for USGS-85, CFA-1, and CFA-2 represent depths to water (depth of pmp sertings is unknomin. Samples collected for analysis cade "D6" at these wells applies to "Pump" samples since no ports are avail able tor 
SAMPLING AND AMALYSIS PLAN TABLE FOR CHEMICAL AMD RRDIOLOGICAL amaLYSIS

SAP Mumber: QQW-12-GUI

\begin{tabular}{lll} 
SAP Table Mo & Rate: $07 / 07 / 93 \quad$ Revision: 9.0 \\
\hline
\end{tabular} Project: CFA OU 6-12 RI/FS - GROUND HAIER PHASE I

\begin{tabular}{|c|c|c|c|c|c|c|c|c|c|c|c|c|c|c|c|c|c|c|c|c|c|c|c|c|c|c|c|c|}
\hline Dare: 07 & $07 / 93$ & Revision: & 9.0 & & & Project: & FA OU $6-12$ RI/FS & GROUND MAIER & SE I & & & & & & & & ject & Manag & & K. $\mathrm{KE}$ & $E C K / G$ & sto & SRMBERG & & & form & Mo: SA & P118 \\
\hline & & MPLE DESCRIPIIO & & & & & SAMPLE & Locarion & & & & & & & & TER AN & HALYSI & IS TYP & PES (A) & (1) AND & D OUAH & IntIIYY & REQUE & ESIED & & & & \\
\hline & & & & & & & & & & AII & A12 & ars & AT4 & A.5 & A16 & A17 & ATB & Ar9 & Arto & AIII) & AI12 & A.13 & \begin{tabular}{|l|l|l|} 
A &
\end{tabular} & $A T 15$ & AI16 & A.17 aris & $3 \mid \operatorname{arrig}$ & Aโ20 \\
\hline acrivity & TrPE & MEDIA & TYPE & MEIHOO & SAMPLE DATE & AREA & LOCATION & LOCATIOH & (ft) & $\mathbf{A 1}$ & co & c1 & $C R$ & cu & 06 & 07 & F1 & 42 & R5 & sc & & & & & & & & \\
\hline 400016 & oc & GROWO MATER & REPL & & $06 / 02 / 93$ & CFA & $\mid 162-11$ & LaNDFILL II & 685 & 1 & 1 & 1 & 1 & 1 & 1 & & 1 & 1 & & 1 & & & & & & & & \\
\hline 400017 & oc & GROUNO MATER & REPL & & $06 / 09 / 93$ & CFA & $1 F 2-10$ & LANDFILL " & 500 & 1 & 1 & 1 & 1 & 1 & 1 & & 1 & 1 & & 1 & & & & & & & & \\
\hline 800018 & foc & GROUND MAIER & REPL & & $06 / 08 / 93$ & CFA & CFA-1 & LANDFILL II & 468 & 1 & 1 & 1 & 1 & 1 & 1 & & 1 & 1 & & 1 & & & & & & & & \\
\hline 600019 & oc & | UATER & |RKST & & $05 / 10 / 93$ & CFA & BAILER & OC - RINSAIE & N/A & 1 & & 1 & 1 & & 1 & & 1 & 1 & 1 & 1 & & & & & & & & \\
\hline $6 \mathrm{co020}$ & oc & WAYER & RHST & & $06 / 07 / 93$ & CFA & PUAMP - After & OC - RL HSATE & M/A & 1 & & 1 & 1 & & 1 & & 1 & 1 & 1 & 1 & & & & & & & & \\
\hline & & & & & & & & & & & & & & & & & & & & & & & & & & & & \\
\hline & & & & & & & & & & & & & & & & & & & & & & & & & & & & \\
\hline & & & & & & & 1 & & & & & & & & & & & & & & & & & & & & & \\
\hline & & & & & & & & & & & & & & & & & & & & & & & & & & & & \\
\hline & & & & - & & & & & & & & & & & & & & & & & & & & & & & & \\
\hline & & & & & & & & & & & & & & & & & & & & & & & & & & & & \\
\hline & & & & & & & & & & & & & & & & & & & & & & & & & & & & \\
\hline & & & & & & & & & & & & & & & & & & & & & & & & & & & & \\
\hline & & & & & & & & & & & & & & & & & & & & & & & & & & & & \\
\hline & & & & & & & & & & & & & & & & & & & & & & & & & & & & \\
\hline
\end{tabular}

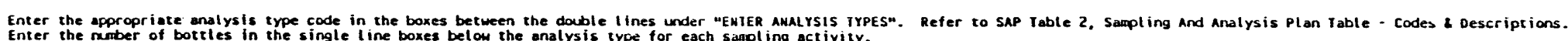

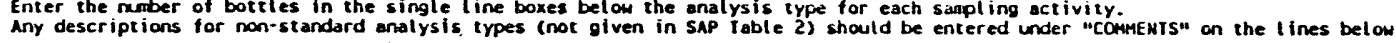

Al1: Alkallinity

AIII: Sulfase/chloride

AT2: CLP Helals - Filtered

AT12:

AI3: SLP Hetels - Unfliltered

Ar13:

AT6: Chroniun vi $(C r+6)$ - Unfiltered

Ar13:

ATS: Shroaiun bI (crob) - Filltered

Ar15:

A16: Drinking yater vass (524.2) IV - (Bailer)

AI7: Drinking Hater vous (526 2) iY - (PPme)

AI16:

AIB: Iluoride

Ar9: Mitrate/Mitrice

Al10: Gams screen

AI19:

CONMENTS

The field team leader will determine inree fandom locat tuns st which a set of replicate samples each will is collecred.

References to "gailer" and "Pump" for analys is codes "p L" and D?" sorinking vater voas (s.54.2) iv) pertain to well samples only.

Sample depths IIsted for USGS-85. CFA-1, and CFA- 2 represent depths to vater (depth of pump setrings is unknoun). Samples collected for analys is code "DS" at these wells applies to "puapu sumples since no ports are available for

butting. 
SAP Mumber: OUS-12-GU2

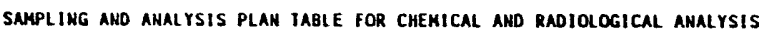

Oate: 07/07/93 Revision: 7.0

Project: CFA OU 4-12 RI/FS - GROUNO MATER PHASE II

Page 1 of $\underline{2}$

\begin{tabular}{|c|c|c|c|c|c|c|c|c|c|c|c|c|c|c|c|c|c|c|c|c|c|c|c|c|c|c|c|c|c|}
\hline te: 07 & & Revision: & 7.0 & & & & CFA OS 4-1 & RI/FS - GROUNO & R PHASE & & & & & & & & & roject & Manag & & & $\mathrm{KECK} / \mathrm{C}$ & G. Sic & IORMBER & & & form $\mathrm{N}$ & KO: SAS & \\
\hline & & MPLE DESCRIPIIO & & & & & SAMPL & LOCATION & & & & & & & & TER Al & WeCrs! & is irp & DES ( & ii) $A N$ & to aus & mitir & REQ & JESTED & & & & & \\
\hline SAMPLIMG & SMMPLE & & |coul & | SAMPLING] & & & & & & AII & A12 & A13 & A14 & AI5 & a16 & AI? & ATB & A19 & AT10 & Arin & Ar12 $A$ & AII3 & Ar14 & At 15 & AI16 & $A 117$ & ar18 & AI19 & $A 120$ \\
\hline Acrivitir & TYPE & MEDIA & IYPE & METHOO & DAIE & AREA & LOCAIION & Location & (fit) & A1 & co & c1 & c2 & CR & cu & D4 & 07 & F1 & 42 & RS & sc & & & & & & & & \\
\hline 600101 & REG & GROWD WATER & GRAB & & $07 / 26 / 93]$ & CFA & LF2-8 & LAMDFILL "I & 488 & 1 & 1 & 1 & 1 & 1 & 1 & 1 & 1 & 1 & 1 & 1 & 1 & & & & & & & & \\
\hline 400102 & REG & GROUNO UATER & GRAB & & $07 / 26 / 93$ & EFA & LF2-9 & СаNOFILL "I & 690 & 1 & 1 & 1 & 1 & 1 & 1 & 1 & 1 & 1 & 1 & 1 & 1 & & & & & & & & \\
\hline 400103 & REG & GROUMO UATER & Gras & & $\mid 07 / 26 / 93$ & CFA & |LF2-10 & LANDFILL " & 500 & 1 & 1 & 1 & 1 & 1 & 1 & 1 & 1 & 1 & 1 & 1 & 1 & & & & & & & & \\
\hline 600106 & REG & GROUMD UAIER & GRAB & & $007 / 26 / 93$ & CFA & LF2-11 & LANDFILL "I & 685 & 1 & 1 & 1 & 1 & 1 & 1 & 1 & 1 & 1 & 1 & 1 & 1 & & & & & & & & \\
\hline 400105 & REG & GROUND MAIER & GRAB & & $|07 / 26 / 93|$ & CFA & $142-12$ & I ANDFIIL "I & 485 & 1 & 1 & 1 & 1 & 1 & 1 & 1 & & 1 & 1 & 1 & 1 & & & & & & & & \\
\hline 600106 & REG & GROMHO MAIER & GRAB & & $07 / 26 / 93$ & CFA & LFS-8 & LLANOFILL III & 500 & 1 & 1 & 1 & 1 & 1 & 1 & 1 & 1 & 1 & 1 & 1 & 1 & & & & & & & & \\
\hline 400107 & REG & GroNSD MATER & GRAB & & $|07 / 26 / 93|$ & CFA & |LF3-9 & LAMDFILL III & 495 & 1 & 1 & 1 & 1 & 1 & 1 & 1 & & 1 & 1 & 1 & 1 & & & & & & & & \\
\hline $4 c 0108$ & REG & Gromo miter & CRAB & & $07 / 26 / 93$ & CFA & $153-10$ & LaMOFILL "II & 495 & 1 & 1 & 1 & 1 & 1 & 1 & 1 & & 1 & 1 & 1 & 1 & & & & & & & & \\
\hline 800109 & REG & GROUND WAIER & GraB & & $|07 / 26 / 93|$ & CFA & (LF3-1) & LANDFILL III & 487 & 1 & 1 & 1 & 1 & 1 & 1 & 1 & & 1 & 1 & 1 & 1 & & & & & & & & \\
\hline 620110 & REG & GROWND WAIER & GRAB & & $|07 / 26 / 93|$ & CFA & |usGs-85 & JLANDFILL III & 488 & 1 & 1 & 1 & 1 & 1 & 1 & & 1 & 1 & 1 & 1 & 1 & & & & & & & & \\
\hline 4call1 & REG & GROUND MATER & GRAB & & $\mid 07 / 26 / 93$ & CFA & CFA-1 & LandF ILL " & 668 & 1 & 1 & 1 & 1 & 1 & 1 & & 1 & 1 & 1 & 1 & 1 & & & & & & & & \\
\hline $4 c 0112$ & REG & GrowNo mater & GRAB & & $07 / 26 / 93$ & CFA & CFA-2 & LaNofILL "II & 471 & 1 & 1 & 1 & 1 & 1 & 1 & & 1 & 1 & 1 & 1 & 1 & & & & & & & & \\
\hline 400113 & oc & fuATE?. & roLx & & $07 / 26 / 93$ & CFA & oc & IRIP BLANK & N/A & & & & & & & 15 & & & & & & & & & & & & & \\
\hline 400116 & oc & $\begin{array}{l}\text { JuIER } \\
\end{array}$ & Fatx & & $|07 / 26 / 93|$ & CFA & oc & FIELD BLANK & $w / A$ & 1 & & 1 & 1 & 1 & & 1 & & 1 & 1 & & 1 & & & & & & & & \\
\hline 600115 & loc & GROAND MAIER & REPL & & $|07 / 26 / 93|$ & CFA & OC - TBO & REPLICAIE & IBD & 1 & 1 & 1 & 1 & 1 & 1 & 1 & & 1 & 1 & & 1 & & & & & & & & \\
\hline
\end{tabular}

Enter the appropriate analyais type code In the boxes between the double IInes under "EHTER AMALYSIS TYPES". Refer to SAP Table 2, Sanpling And Analysis Ptan Table - Codes 2 Descriptions.

Enter the number of bottles in the single line boxes below the analysis type for each sampling activity.
Any descriptions for non-stendard analysis types (not given in SAP Yable 2) should be entered under "Cormewis" on the lines betow.

All: Alkalinity

AT2: CLP Merels - Filitered

Arl1: Gamma Sereen

ar12: Sulfate/chloride

AI3: ClP Metols - Unfllitered

Ar13:

A16: Cyanide

AI16:

AIS: Chroaium vI (crob) - Unfilltered

Alb: Chroaiun VI $(\mathrm{C} r+6)$ - Filtered

A17: Orinking water vous (526.2) iv - (Baller)

A18: Orinking weter voAs (526.2) iv - (Pump)

A19: Fluoride

An10: Nitrate/mitrite

Ar15:

Ar16:

AI17:

AI18:

AT 19:

ar20:
CONAEN IS

The field tean leader will detergine two random locations at which a set of replicate samples each will be collected.

References to "Bailer" ond Pump" for analysis codes "04" and "07" (0rinking water vans (524.2) iv) pertain to well samples only.

$\bar{\square}$


SAP Number: ouk-12-GU2

SAMPLIMG AND AMALYSIS PLAN TABLE FOR CHEHICAL AMD RRDIOLOGICAL AMALYSIS SAP Table Mo
Date: $07 / 07 / 93$
Revision: 7.0 Project: CFA QU 6-12 RI/FS - GROUMO MATER PHASE "I

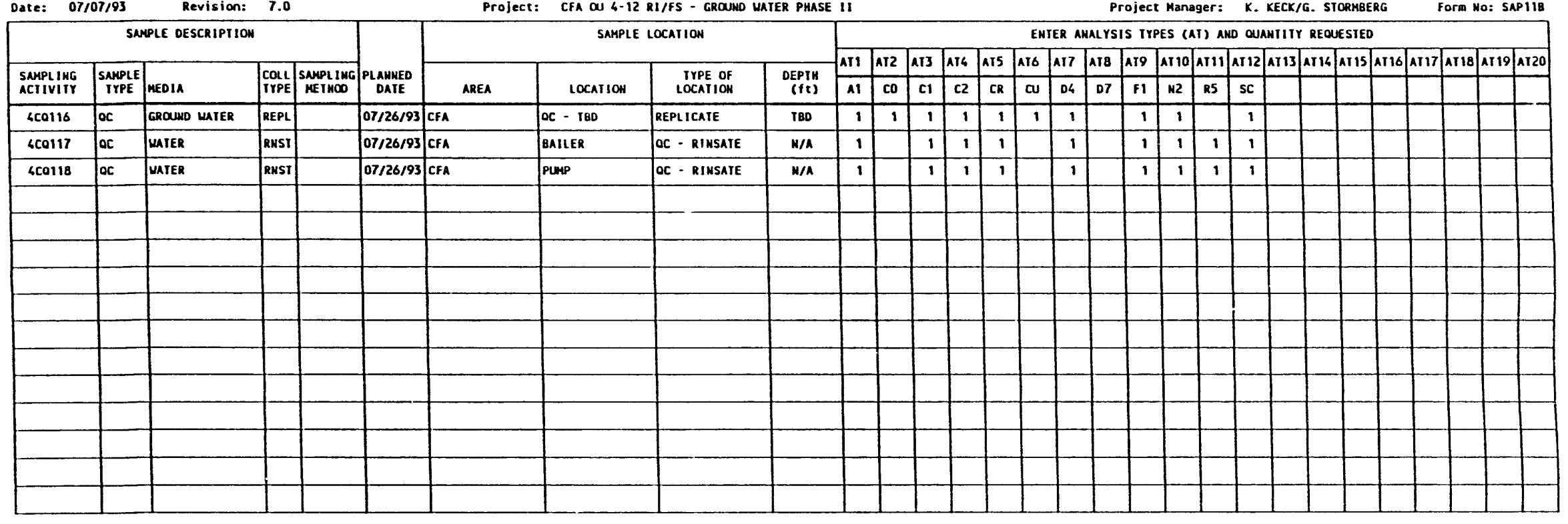

Enter the approprlate analysis type code in the boxes between the double lifnes under "EMTER AMALYsis irpes". Refer to SAP Table 2, Sampl ing And Analysis Plan Table - Codes 4 Descriptions. Any descriptions for non-standard analysis ippes (not given in SAP Yable 2) should be entered under "Cownemis" on the tines below.

Al1: Alkalinity

AI2: CLP Metsis - fillered

AI3: CIP Metnls - unfiliered

A14: Cyanide

ATS: Chromiun VI (Cr+b) - Unfiltered

AI6: Chromiun VI (Cr+6) - filltered

AT7: Orinking Uater vaAs (S226.2) IV - (Bailer)

A18: prinking water vons (526.2) iv - (Pump)

A19: Fluoride

Al 10: Mitrate/mitrite
AIII: Ganma Screen

Ar12: Sulfate/chioride

AI 13:

AT14:

AI15:

A116:

AI17:

Aก18:

At 19:

AT20:
COMNENTS

The field team leader will determine two randon locations at which a set of replicate samples each will be collected.

References to "Bailer" and Pump" for analysis codes "D6" and "07" sorinking Water vols (524.2) IV) pertain to well sauples only. 
SAP Mumber: ous-12-av3

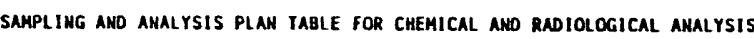
Date: $07 / 07 / 93$ Revision: 7.0 Project: CFA OW 4-12 RI/FS - GROUHD UATER PHASE HII

Page 1 of 2

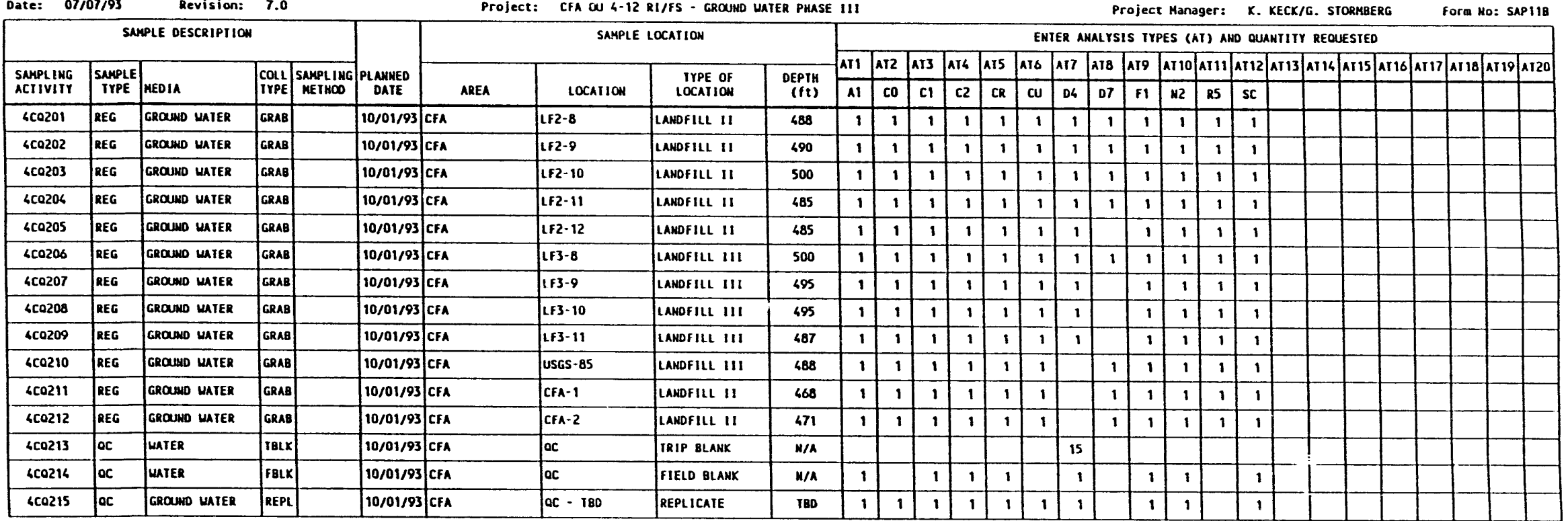

Enter the appropriate analysis type code in the boxes between the double lines under "EMTER ANALYSIS TYPES". Refer to SAP Table 2, Sampling And Analysis Plan Table - Codes 2 Descriptions. Any descriptions for non-standard analysis types (not given in SAP Yable 2) should be entered under "Cominewis" on the lines belou.

AII: Alkalinity

Al2: CLP Metals - Filtered

AlII: Gamma Screen

Al12: Sulfate/chloride

Ais: clp Merals - Unfillered

A16: Cyanide

Ar13:

ATS: Chromium vI (Cr.b) - Untiltered

AI6: Chromiun VI (Cr+6) - Filtiered

A17: Drinking water voas (524.2) iv - (Bailer)

A18: Drinking vater VOAs (526.2) iV - (Pump)

A19: Flworide

Al 30: Mitrate/Hitrite

Al14:

Ar15:

AI16:

AI17:

AT18:

Ar19:

A120:
COM HE IT

The field team leader will determine two random locations at which a set of replicate samples each will be collected.

References to "Baller" and "Pung" for analysis codes "04" and "07" Gerinking water voas (526.2) IV) pertain to yell samples oniy. 
SAP Mumber: ous-12-GU3 SAP Table Mo. $\frac{1}{1}$ Revision: 7.0
Date: $07 / 07 / 93 \quad$ SMPPLE OESCRIPIIOM
SAMPLING AND ANALYSIS PLAN IABLE FOR CHEMICAL AMD RNDIOLOGICAL AMALYSIS Project: CFA OU 4-12 RI/FS - GROUND HATER PHASE III

\begin{tabular}{|c|c|c|c|c|c|c|c|c|c|c|c|c|c|c|c|c|c|c|c|c|c|c|c|c|c|c|c|c|}
\hline \multicolumn{5}{|c|}{ SARPLE OESCRIPTIOW } & \multicolumn{5}{|c|}{ SAHPLE TOCAIION } & \multicolumn{19}{|c|}{ 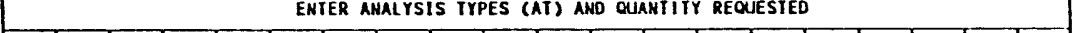 } \\
\hline & & & $\operatorname{con} 1$ & SAMPLING & & & & IYPE OF & DEPTH & AII & 실 & AIs & A14 & A15 & A16 & A17 & A18 & A19 & AI10 & AIII & \begin{tabular}{|l|l|}
112 & 1 \\
\end{tabular} & AII3 & AI14/A & AI15 A & aI16 & \begin{tabular}{|l|l|l|}
$A 17$ & $A I 18$ \\
\end{tabular} & $A+19$ & $\Lambda \geq 20$ \\
\hline Acriviti & TYPE & MEDIA & TYFE & HETHOO & DATE & AREA & LOCAIIOW & LOCAIIOW & (ft) & A1 & co & c1 & c2 & $C R$ & cu & D6 & 07 & F1 & M2 & R5 & sc & & & & & & & \\
\hline 620216 & oc & GROUND UAIER & REPL & & 10/01/93 C & CFA & ac- $\mathbf{t B D}$ & REPLICATE & TBO & 1 & 1 & 1 & 1 & 1 & 1 & 1 & & 1 & 1 & & 1 & & & & & & & \\
\hline 400217 & oc & JATER & RNST & & $10 / 01 / 93$ C & CFA & BAILER & oc - RINSATE & $N / \AA$ & 1 & & 1 & 1 & 1 & & 1 & & 1 & 1 & 1 & 1 & & & & & & & \\
\hline 600218 & oc & JATER & RUSI & & $|10 / 01 / 93| \mathrm{c}$ & cFA & pump & OC - RINSAIE & N/A & 1 & & 1 & 1 & 1 & & 1 & & 1 & 1 & 1 & 1 & & & & & & & \\
\hline & & & & & & & & & & & & & & & & & & & & & & & & & & & & \\
\hline & & & & & & & & & & & & & & & & & & & & & & & & & & & & \\
\hline & & & & & & & & & & & & & & & & & & & & & & & & & & 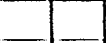 & 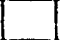 & \\
\hline & & & & & & & & & & & & & & & & & & & & & & & & & & & & \\
\hline & & & & & & & & & & & & & & & & & & & & & & & & & & & & \\
\hline & & & & & & & & & & & & & & & & & & & & & & & & & & & & \\
\hline & & & & & & & & & & & & & & & & & & & & & & & & & & & & \\
\hline & & & & & & & & & & & & & & & & & & & & & & & & & & & & \\
\hline & & & & & & & & & & & & & & & & & & & & & & & & & & & & \\
\hline & & & & & & & & & & & & & & & & & & & & & & & & & & & & \\
\hline & & & & & & & & & & & & & & & & ( & & & & & & & & & & & & \\
\hline & & & & & & & & & & & & & & & & & & & & & & & & & & & & \\
\hline
\end{tabular}

Páge $\underline{2}$ of $\underline{2}$ FOrm Mo: SAP11B

Enter the appropriate analysis type code in the boxes between the double lines under "EMTER AMALYSIS IYPES". Refer to SAP Ioble 2. Sampl ing And Analysis Plan Table - Codes 2 Descriptions.

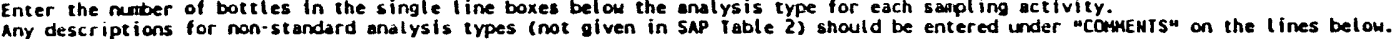

Arl: Alkalinity

Ar2: GLP Metals - Filtered

A13: Cip Metals - Untiltered

A16: Cyanide

A15: Chroalun VI (cr+6)-Unfiltered

AI6: Chromiun VI $(C r \cdot 6)$ - Filltered

A37: Orinking yater voas (526.2) IV - (Bailer)

AI8: Drinking water voAs (524.2) iv - (Pume)

A18: Fluoride

Ar10: Nitrate/Mitrite

Ar11: Gamma Screen

Ar12: Sulfate/chloride

Ar13:

Ar14:

AI15:

AI15:

AI17:

Al18:

Ar19:

AT20:
COHAENIS

The field team leader will determine two randon locations at which a set of replicate samples each vill be collected.

References to "Bailer" and "Punp" for analysis codes "DS" and "07" corinking Water voas (524.2) IV) pertain to well samples only.

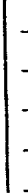


SAP Mumber: OU6-12-SBS

SAP Table Mo.
Date: $07 / 07 / 93$

SAMPLING ANO ANALYSIS PLAN TABIE FOR CHEMICAL ANO RaDtological amaLYSIS

\begin{tabular}{|c|c|c|c|c|c|c|c|c|c|c|c|c|c|c|c|c|c|c|c|c|c|c|c|c|c|c|c|c|}
\hline & & & & & & & & & & & & & & & & & & oject & Manag & ger: & K. $\times E$ C & $c K / G .51$ & STORMBE & & & form No & : SAP 1 & \\
\hline & & & & & & & SAKPL & Locarion & & & & & & & & ER ANA & IALYsis & $S$ TYPE & ES (AT & 1) AND & ouanr & IIY REQ & DUESTED & & & & & \\
\hline $\begin{array}{l}\text { SAMPLING } \\
\text { ACTIVIIY }\end{array}$ & $\begin{array}{c}\text { SMMPLE } \\
\text { TYPE }\end{array}$ & MEOIA & \begin{tabular}{|l|l|} 
COA \\
IYPEE
\end{tabular} & SNMPL ING & PLAMUED & APEA & socer & TYPE OF & DEPTM & ari & $\Lambda \sqrt{2}$ & $A 13$ & \begin{tabular}{|l|l|} 
AI6 \\
\end{tabular} & AIS & \begin{tabular}{l|l} 
AT6 & a \\
\end{tabular} & $\operatorname{arp/1}$ & \begin{tabular}{|l|l|}
18 & $A 1$ \\
\end{tabular} & A19 1 & $A n 10 \mid A 1$ & AIII/AT & \begin{tabular}{l|l} 
TI2 & $A T$ \\
\end{tabular} & 13 AI16 & 6) & Ario & \begin{tabular}{|l|l|} 
Arita &
\end{tabular} & \begin{tabular}{|l|l|l|}
$A T 18$ & $A$ \\
\end{tabular} & \begin{tabular}{ll|l}
$\mathrm{AI} 19$ & $\mathrm{AT}$ \\
\end{tabular} & 220 \\
\hline ८сBO01 & REG & soll & Gan & & DAIE & AREA & IOCATIOW & LOCAIIOM & (fti) & c1 & cv & os & R5 & & & & & & & & & & & & & & & \\
\hline \&C8002 & REG & soul & (congen & & $00 / 21 / 93$ & CFA & $\theta-1$ & Lamdfill & 180 & 1 & 1 & 1 & 1 & & & & & & & & & & & & & & & \\
\hline 609003 & & & [oxas & & $06 / 21 / 93$ & CFA & $8-1$ & Lamoftll II & IBD & 1 & 1 & 1 & 1 & & & & & & & & & & & & & & & \\
\hline${ }_{6}^{8 C 8003}$ & |REG & sol1 & GRAB & & $\infty s / 21 / 93$ & CrA & $8-2$ & LaMDFIIL It & 580 & 1 & 1 & 1 & 1 & & & & & & & & & & & & & & & \\
\hline sc8006 & REG & soit & cores & & $08 / 21 / 93$ & CFA & $8-2$ & LAMOFIL II & I80 & 1 & 1 & 1 & 1 & & 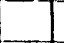 & & & & & & & & & & & & & \\
\hline $6 \mathrm{CBO0S}$ & REG & soll & Gre日 & & $00 / 21 / 93$ & CFA & $8-3$ & Lanoflil 11 & TBD & 1 & 1 & 1 & 1 & & & & & & & & & & & & & & & \\
\hline$<28000$ & REG & soll & GRAB & & $00 / 21 / 93$ & CFA & $8-3$ & IANDFILI II & IBO & 1 & 1 & 1 & 1 & & 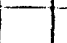 & & & & & & & & & & & & & \\
\hline $6 \mathrm{CB007}$ & REG & solt & GRAB & & $008 / 21 / 93$ & Cra & $8-4$ & LAMOFILL "I & 580 & 1 & 1 & 1 & $T$ & & & & & & & & & & & & & & & \\
\hline $6 \mathrm{co000}$ & REG & soll & CRAB & & $008 / 21 / 93$ & cra & $8-6$ & LAMDFILL II & TRD & $T$ & 1 & 1 & 1 & & & & & & & & & & & & & & & \\
\hline 6c8009 & REG & soll & GRAB & & $06 / 21 / 93$ & Cra & 8-5 & LaNDFILL "I & TBD & 1 & 1 & 1 & 1 & & & & & & & & & & & & & & & \\
\hline $6 c 8010$ & ReG & solt & GRAB & & $\mid \infty \infty / 21 / 93$ & CFA & B-5 & LANOFILL II & TBD & 2 & 2 & 3 & 2 & & & & & & & & & & & & & & & \\
\hline ¿c8011 & REG & soll & GRAB & & $108 / 21 / 93$ & cra & $8-6$ & LaNof 4 & 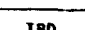 & 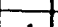 & & & - & & & & & & & & & & & & & & & \\
\hline $6 \mathrm{CBO12}$ & REG & solt & GRAB & & $\infty 6 / 21 / 93$ & cra & | $8-6$ & Tanotill & wa & 1 & 1 & 1 & 1 & & & & & & & & & & & & & & & \\
\hline 6CBO13 & REG & solt & GRAB & & $06 / 21 / 93$ & CFA & B-7 & LANDFILL II & FBO & 1 & 1 & 1 & 1 & & & & & & & & & & & & & & & \\
\hline $6 \mathrm{CBO16}$ & REG & soll & GRAB & & $06 / 21 / 93$ & CFA & |8-7 $>$ & LAMOFILL II & T80 & 1 & 1 & 1 & 1 & & & & & & & & & & & & & & & \\
\hline $6 C 8015$ & oc & | WATER & RMSI & & $06 / 21 / 93$ & cra & oc & LANDFIIL II & 180 & 1 & 1 & 1 & 1 & & & & & & & & & & & & & & & \\
\hline & & & & & & & & RINSAIE & N/A & 1 & 1 & 1 & 11 & & & & & & & & & & & & & & & \\
\hline
\end{tabular}

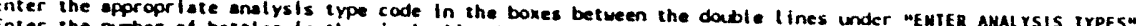

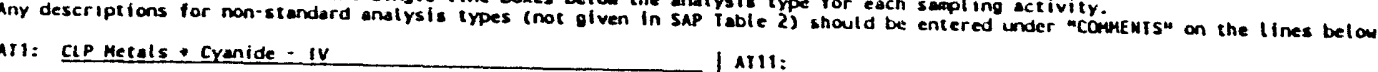

AI2: CIP volutiles - IY

An11:

AIS: CIP Seaivolatiles - iv

Al6: Gamme screen

A15:

AIS:

AI7:

ar8:

A19:

Anto

$$
\text { A113: }
$$

AI16:

Ar15:

Ar16:

AI17:

Ar18:

Ar19.

Ar20:

COMNENIS

B-1 = (B)orehole - (1) etc.

Specific sample depths will be deterained in the field

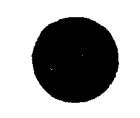


SAP Number: OUA-12-SBS

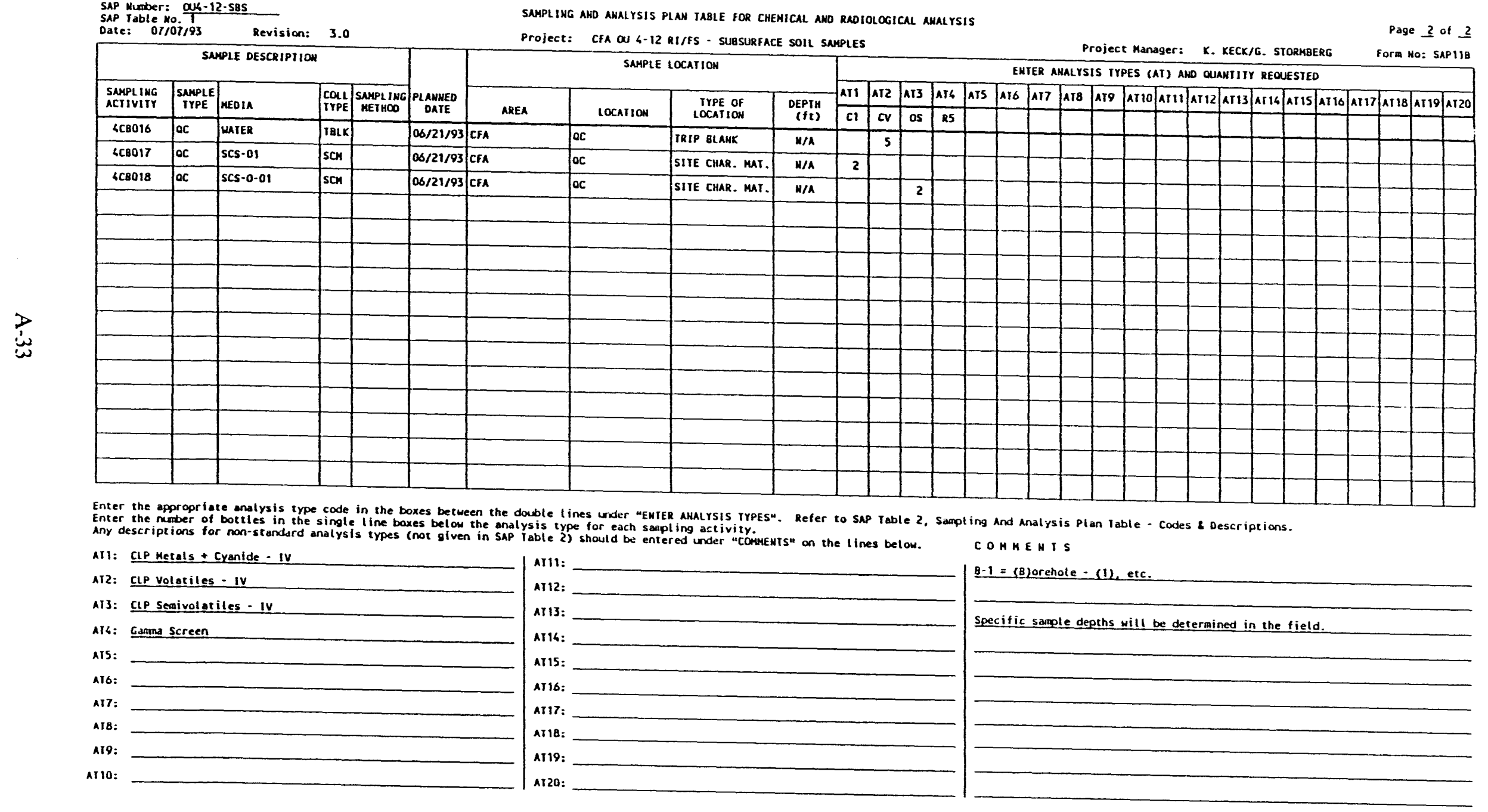

SAMPLIMG AND AMALYSIS PLAN TABLE FOR CHEHICAL ANO RNDTOLOGICAL AMALYSIS 
SAP Number: aus-12-GLO

SAMPLING AND ANALYSIS PLAN TABLE FOR CHEMTCAL AND RADIOLOGICAL AMALYSIS Date: $07 / 07 / 93$ Revision: 0.0 Project: CFA OU 4-12 Grouno HATER DISPOSAL - PHASES I, II, AND III

Page 1 of 2

\begin{tabular}{|c|c|c|c|c|c|c|c|c|c|c|c|c|c|c|c|c|c|c|c|c|c|c|c|c|c|c|c|c|c|}
\hline & & & & & \multirow{2}{*}{\multicolumn{5}{|c|}{ SAMPLE LOCATION }} & \multirow{2}{*}{\multicolumn{20}{|c|}{ EHTER AHALYSIS IYPES (AT) AND OUANIITY REOUESTED }} \\
\hline \multirow{3}{*}{\begin{tabular}{|l|} 
\\
$\begin{array}{l}\text { SAMPLING } \\
\text { ACIIVIII }\end{array}$
\end{tabular}} & \multicolumn{4}{|c|}{ SAMPLE OESCRIPIION } & \multirow{3}{*}{$\begin{array}{c}\text { PLANMED } \\
\text { DAIE }\end{array}$} & & & & & & & & & & & & & & & & & & & & & & & & \\
\hline & SAMPLE] & & conts: & SAMPLIMG & & \multirow[b]{2}{*}{ AREA } & \multirow[b]{2}{*}{ LOCATLON } & \multirow{2}{*}{$\begin{array}{l}\text { TYPE of } \\
\text { LOCAIIOW }\end{array}$} & \multirow{2}{*}{$\begin{array}{c}\text { DEPIH } \\
\text { (fe) }\end{array}$} & \multirow{2}{*}{\begin{tabular}{|c|}
$A I 1$ \\
$R 8$ \\
\end{tabular}} & \multirow{2}{*}{ A12 } & \multirow[t]{2}{*}{ A13 } & \multirow{2}{*}{114} & \multirow{2}{*}{ Ars } & \multirow{2}{*}{ A16 } & \multirow{2}{*}{ A17 } & AI & \multirow[t]{2}{*}{5} & \multirow[t]{2}{*}{ aric } & \multirow[t]{2}{*}{$11 / 1$} & \multirow{2}{*}{112 A } & \multirow[t]{2}{*}{$113 \mid A$} & \multirow[t]{2}{*}{ Aris } & \multirow[t]{2}{*}{$A+15$} & $\operatorname{Ar16}$ & Ar17 & AT 18 |A & arig & A120 \\
\hline & IYPE & MED IA & IYYPE & METHOO & & & & & & & & & & & & & & & & & & & & & & & & & \\
\hline $4 c 0001$ & REG & GroUMD YATER & CRAB & & $07 / 09 / 93$ & CFA & LF2-8 & LAMOFILL II & CONIAIMER & 1 & & & & & & & & & & & & & & & & & & & \\
\hline \&CD002 & REG & GROUND HAIER & GRAB & & $07 / 09 / 93$ & CFA & LF2-9 & LAMOFILL UI & CONTAINER & 1 & & & & & & & & & & & & & & & & & & & \\
\hline $6 \mathrm{CDO03}$ & REG & GROUND UAIER & CRAB & & 07/09/93 & CFA & LF2-10 & LAMDFILL UI & CONIAINER & 1 & & & & & & & & & & & & & & & & & & & \\
\hline $6 \mathrm{c0006}$ & REG & GROUND MAIER & GRAB & & $07 / 09 / 93$ & CFA & LF2-11 & LAMDFILL "I & CONTAINER & 1 & & & & & & & & & & & & & & & & & & & \\
\hline $4 C 0005$ & REG & GROUND HAIER & GRAB & & $07 / 09 / 93$ & CFA & $1 F 2-12$ & LANDFILL II & COMTAIMER & 1 & & & & & & & & & & & & & & & & & & & \\
\hline 400006 & REG & GROUNO UATER & GRAQ & & $07 / 09 / 93$ & Cra & LFS-8 & LAMOF ILL III & CONtainer & 1 & & & & & & & & & & & & & & & & & & & \\
\hline $4 C 0007$ & REG & GROUND MAIER & GRAB & & $07 / 09 / 93$ C & CFA & $153-9$ & LANDFILL III & COMIAIMER & 1 & & & & & & & & & & & & & & & & & & & \\
\hline 600008 & REG & GROUMD WAIER & GRAB & & $07 / 09 / 93$ & CFA & LF $3-10$ & LaNOFILL III & CONIAINER & 1 & & & & & & & & & & & & & & & & & & & \\
\hline 600009 & REG & GROUMD WATER & GRAB & & $07 / 09 / 93$ & CFA & LF3-11 & LANOFILL III & COW'AIMER & 1 & & & & & & & & & & & & & & & & & & & \\
\hline 400010 & REG & GROUND UATER & GRAB & & $07 / 09 / 93$ & CFa & USGS - 85 & LAMDFILL III & COWTAIMER & 1 & & & & & & & & & & & & & & & & & & & \\
\hline 400011 & REG & GROUHO MAIER & GRAB & & $07 / 26 / 93$ & CFA & LF2-8 & LANOFILI II & 488 & 1 & & & & & & & & & & & & & & & & & & & \\
\hline 600012 & REG & GROUND HATER & GRAB & & $07 / 26 / 93$ & CFA & LF2-9 & LAMDFIIL $" 1$ & 490 & 1 & & & & & & & & & & & & & & & & & & & \\
\hline $4 \operatorname{co0} 13$ & REG & GROUKO WAIER & CRAB & & $07 / 26 / 93$ & CFA & LF2-10 & LANDFILL II & 500 & 1 & & & & & & & & & & & & & & & & & & & \\
\hline 400016 & REG & GROUNO MAIER & GRAB & & $07 / 26 / 93$ & CFA & LF2-11 & LAMOFILL "II & 485 & 1 & & & & & & & & & & & & & & & & & & & \\
\hline 400015 & REG & GROUND MATER & GRAB & & |07/26/93 & CFA & $182-12$ & LandFILL II & 485 & 1 & & & & & & & & & & & & & & & & & & & \\
\hline
\end{tabular}

Enter the appropriate analysis type code in the boxes between the double lines under "ENIER AMALYSIS TYPES". Refer to SAP Table 2, Sampling And Analysis Plan Table - Codes \& Descriptions.

Any descriptions for non-standard analysis types (not given in SAP Tabte 2) should be entered under "COMments" on the lines below.

All: Iririun

A12:

A13:

A14:

A15:

A16:

arg:

AI 10:

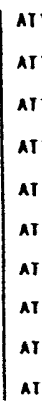

C OMMEN IS

AI11:
AI12:
AI13:
AI16:
AI15:
AI16:
AI17:
AI18:
AI19:
AT20:

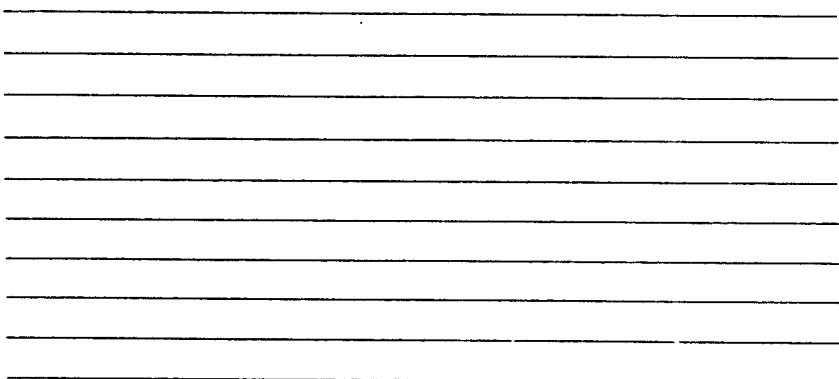


SAP Number: OUK-12-GWO

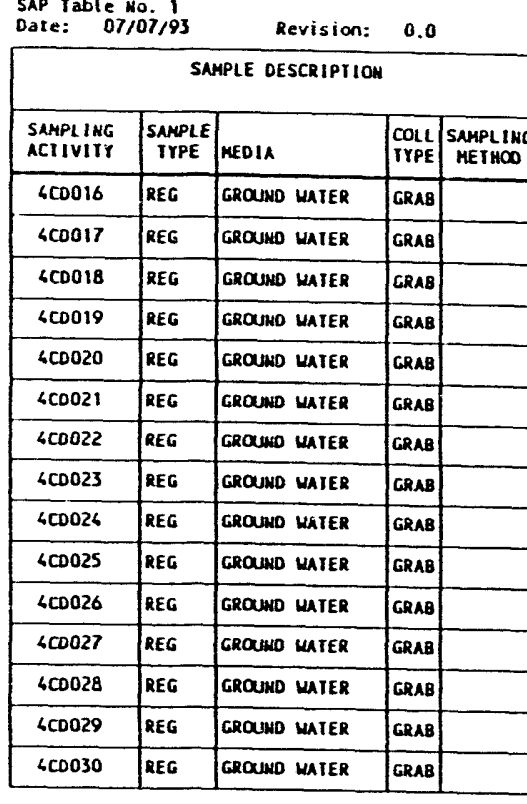

Samplimg akd akalysis plan table for Chemical ako radological analysis

Project: CFA OU 4-12 Growmo Hater dISPOSAL - PHASES 1, II, AND III

Project Manager: K. KECK/G. STORMBERG

Page $\underline{2}$ of $\underline{2}$ SAMPLE LOCATION Form No: SAP118

\begin{tabular}{|c|c|c|c|c|c|c|c|c|c|c|c|c|c|c|c|c|c|c|c|c|c|c|c|c|}
\hline \multirow{3}{*}{$\begin{array}{c}\text { PLANNED } \\
\text { DAIE }\end{array}$} & & & & & \multicolumn{20}{|c|}{ EMIER AMALYSIS IYPES (AT) AND OUAMTHIT REOUESTED } \\
\hline & AOFe & & TYPE OF & & All & ar2 & 113 & A16 & A1s & AT6 & A17 & ATB & Arg & Ar 10 & Aln & AIi2 & aIIs] & aI14/ & AII5/A & AI 16/A & AT17/A & AII8]A & $A \pi 19$ & A120 \\
\hline & AREA & LOCAIION & LOCATION & $(\mathrm{tt})$ & R8 & & & & & & & & & & & & & & & & & & & \\
\hline $07 / 26 / 93$ & cFa & LF3-8 & LAabrtLL "I & 500 & 1 & & & & & & & & & & & & & & & & & & & \\
\hline |07/26/93 & CFA & LF\}-9 & LAMOFIL & 105 & S & & & & & & & & & & & & & & & & & & & \\
\hline $07 / 26 / 93$ & CFA & |LF3-10 10 - & LLANDFILL & 105 & 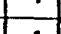 & & & & & & & & & & & & & & & & & & & \\
\hline 07/26/93 & CFA & LF3-11 & 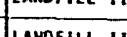 & 695 & 1 & & & & & & & & & & & & & & & & & & & \\
\hline 07126103 & CFA & posin & LANDFILL $\|$ & 487 & 1 & & & & & & & & & & & & & & & & & & & \\
\hline $0 / 2 / 20 / 45$ & cra & |usas - 8s & LANDFILL "I & 488 & 1 & & & & & & & & & & & & & & & & & & & \\
\hline 10/01/93 & CFA & $452-8$ & LANOFHL "I & 488 & 1 & & & & & & & & & & & & & & & & & & & \\
\hline 10/01/93 & CFA & LF2-9 & LANOFILL "I & 490 & 1 & & & & & & & & & & & & & & & & & & & \\
\hline 10/01/93 & CFA & LF2-10 & LANOFILL II & 500 & 1 & & & & & & & & & & & & & & & & & & & \\
\hline 10/01/93 & CFA & LF2-11 & LAMDFILL II & 485 & 1 & & & & & & & & & & & & & & & & & & & \\
\hline 10/01/93 & cra & $\mid 1 F 2-12$ & LANDFILL "I & 485 & 1 & & & & & & & & & & & & & & & & & & & \\
\hline 10/01/93 & CFA & LF3-B & LaNDFILL II & 500 & 1 & & & & & & & & & & & & & & & & & & & \\
\hline $10 / 01 / 93$ & CFA & LF3-9 & LANOFILL II & 495 & 1 & & & & & & & & & & & & & & & & & & & \\
\hline 10/01/93 & CFA & $\angle f 3-10$ & LANDFILL II & 495 & 1 & & & & & & & & & & & & & & & & & & & \\
\hline 10/01/93 & CFA & $153-11$ & LAMDFILL II & 687 & 1 & & & & & & & & & & & & & & & & & & & \\
\hline $10 / 01 / 93$ & CFA & USCS-8S & LAMOFILL il & 488 & 1 & & & & & & & & & & & & & & & & & & & \\
\hline
\end{tabular}

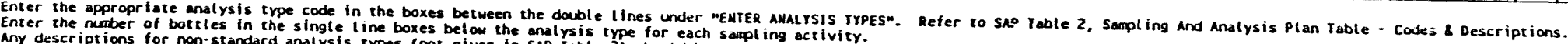

Any descriptions for non-standard analysis types (not given in SAP Table 2) should be entered under "corwewrs" on the lines below.

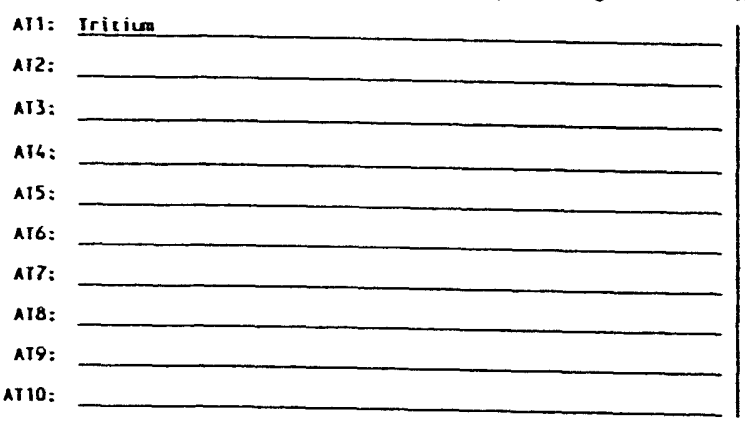

Ar11:
Ar12:
Ar13:
Ar14:
Ar15:
Ar16:
Ar17:
Ar18:
Ar19:
AI20:

COMNENIS

$$
\text { 1 }
$$


Appendix B

\section{Standard Operating Procedures}




\section{Appendix B}

\section{Standard Operating and Test Procedures}

The following standard operating procedures pertain to sample collection and field measurements. This appendix contains the following methods:

- Field Decontamination of Sampling Equipment

- Groundwater Sampling

- Measurement of Groundwater Levels

- Electromagnetic Terrain Conductivity Surveys

- $\quad$ Seismic Land Refraction Survey

- Land Magnetic Survey

- $\quad$ Soil Sampling

- Hydrolab Operation

- Operation of Neutron Moisture Probe

- $\quad$ Field Sampling Method for Well Deviation Logging

- $\quad$ ASTM-D2922, Standard Test Methods for Density of Soil and Soil-Aggregate in Place by Nuclear Methods (Shallow Depth)

- $\quad$ ASTM-D2325, Standard Test Method for Capillary-Moisture Relationships for Coarse- and Medium-Textured Soils by Porous-Plate Apparatus

- $\quad$ ASTM-D2434, Standard Test Method for Permeability of Granular Soils (Constant Head)

- $\quad$ ASTM-D2216, Standard Method for Laboratory Determination of Water (Moisture) Content of Soil, Rock, and Soil-Aggregate Mixtures

- $\quad$ ASTM-D422, Standard Method for Particle-Size Analysis of Soils

- $\quad$ ASTM-D1587, Standard Practice for Thin-Walled Tube Sampling of Soils

- $\quad$ ASTM-D3017, Standard Test Method for Moisture Content of Soil and SoilAggregate in Place by Nuclear Methods (Shallow Depth) 
$\because$. .

INFORMATION ONLY

ENVIRONMENTAL RESTORATION DEPARTMENT

ENVIRONMENTAL STANDARD OPERATING PROCEDURE

COVER SHEET

SOP Number: $\quad$ SOP-11.5

Revision:

0

Title: STANDARD OPERATING PROCEDURE FOR FIELD DECONTAMINATION OF SAMPLING EQUPMENT

Prepared by:

KN. Xech

K. N. Keck, Sr. Scientist

Date: $\underline{02 / 26 / 92}$

Geosciences Group

Reviewed by: $\frac{1}{\text { Fo Shea, Chairman }}$

Date: $\underline{0 z / 27 / 92}$

Approved by:

Tuis 6 Tan Viene

L. C. VanDeusen, Manager

Site Remediation Group

Approved by:

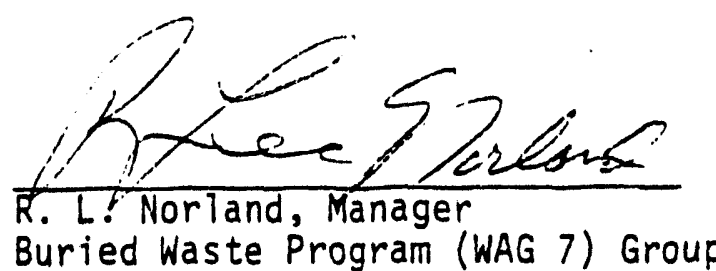

Date: $2 / 28 / 92$

Date:

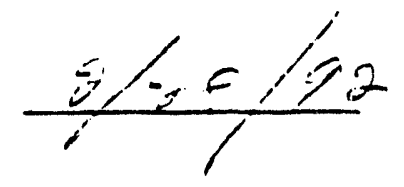


ENVIRONMENTAL

STANDARD OPERATING

PROCEDURES MANUAL
TITLE: FIELD DECOKTAMINATION OF SAMPLING EQUIPMENT

NUMBER: 11.5

ISSUE DATE: 02/28/92

\subsection{PURPOSE AIN SCOPE}

To provide general instructions for field decontamination of sampling equipment used to support environmental investigations. Thorough decontamination is required to prevent cross contamination between samples and sampling sites.

\subsection{PROCEDURE}

2.1 All sampling equipment (i.e. Teflon bailers, split-spoon samplers, funnels, spoons, pans) is decontaminated before sampling activities begin, before moving sampling activities to another location/well, and after sampling activities are completed. If several samples are being collected from a single location, such as with split-spoon sampling at depth, the sampling equipment is thoroughly decontaminated between samples. However, full decontamination of sampling equipment between locations is not required when collecting subsamples that will constitute a single composite sample. For composite samples, the equipment used between subsample collection need only be brushed or wiped off to remove any large chunks of soll adhering to the equipment.

2.2 Establish a central decontamination location away from the immediate sampling site.

\subsection{Material and Equipment Needs:}

a. Non-phosphate detergent (i.e., Microclean)

b. Wire brush/bottle brush

c. Water:
a. Organic-free water
b. Deionized tap water
c. Uncontaminated/potable tap water

d. Isopropanol

e. Carboy and/or 55-gal drums (poly) for storage of tap water used in steam cleaning/decontamination, as appropriate

f. Waste water collection system (may include):
a. Plastic sheeting
b. Containers for waste water collection (separate containers for water, solids, and solvents) 
g. Steam cleaner and generator (optional)

h. Personal protective equipment (PPE) as stated in the Health and Safety PIan

i. Stainless steel pans with lids

j. Maislan wipes

k. Blotter paper

1. Spray bottles

m. Sponges

2.4 Field Decontamination Procedure for Sampling Equipment:

a. Physically remove any bulk material adhering to the item that requires decontamination by using a wire brush or scraper.

Note: Wire brushes should not be used on nop-metal equipment.

b. Remove gross contamination with tap water and rinse, using pressurized or gravity flow tap water. Scrub brushes or wire brushes may help in removing material.

c. Wash and scrub the equipment with a non-phosphate detergent and tap water.

d. Rinse thoroughly with tap water. Steam clean the equipment if a steam cleaner is available and the equipment is not heat and steam sensitive.

Note: Steam cleaning is not acceptable if radiological contamination is present, due to the potential for creating airborne contamination problems.

e. Check for adhered soils; use a brush to dislodge any particles.

f. Double rinse with organic-free water.

g. Spray-rinse all surfaces with isopropanol from an approved wash bottle.

h. Collect the isopropanol in a container for appropriate disposal (see Section 2.5). One effective collection technique is to $p$ lace a large glass or stainless steel funnel below the tools during insing. Allow waste to flow into appropriately sized bottles for later disposal. Use a 
ENVIRONMENTAL STANDARD CPERATINE PROCEDUREES MANUAL
TITLE: FIELD DECONTAMINATION OF SAMPLING EQUIPMENT

NUMBER: 11.5

ISSUE DATE: $02 / 28 / 92$

stainless steel mixing bowl as a collection vessel. The bowl is the last item cleaned in the sequence of operations.

i. If a rinsate sample is required for $Q A$, make an additional final rinse of the equipment, using ASTM Type II or HPCL grade water, and collect it in the appropriate sample bottles.

j. If the equipment is known to be or suspected of being radioactively contaminated, have the Health Physics Technician collect a swipe (100 $\mathrm{cm}^{2}$ smear) sample for immediate radionuclide analysis.

k. The item is considered clean and suitable for use if there are $<20 \mathrm{dpm} / 100 \mathrm{~cm}^{2}$ (beta, gamma) or $<200 \mathrm{dpm} / 100 \mathrm{~cm}^{2}$ (beta, gamma) above background levels and no detectable alpha.

1. If the radiological limits are not met, discuss with the Health Physics Technician the possibility of using a special decontamination solution for radionuclides and/or disposing and replacing the item.

m. Allow sampling equipment to completely dry prior to re-use.

n. Wrap and store sampling equipment. Aluminum foil is recommended for equipment used in the sample collection for organic analysis. Use plastic wrap or bags if equipment is used for sample collection intended for inorganic analysis. Attach a label to the wrapping or bag indicating the date of decontamination and the initials of the person who performed the decontamiantion.

2.5 The final disposition of rinse water and material dislodged from equipment will be specified in the Sampling and Analysis Plan and the Investigative Derived Waste Plan. The ERP Project Manager, Field Team Leader, Health Physics Technician and/or Safety officer, and Environemntal Coordinator for the facility, and the facility engineer determine appropriate disposal to decontaminated wash water. All solvents used during decontamination are collected for appropriate disposal.

\subsection{DEFINITIONS}

No terms cited in this procedure require special explanation. 


\subsection{REFERENCES}

U.S. DOE, 1989. The Environmental Survey Manual, 2nd edition, Appendix G-Decontamination Guidance, Section G2.4, sampling Equipment, $p$. $G-6$.

NIOSH/OSHA/USCG/EPA, 1985. Decontamiantion of the Occupational Safety and Health Guidance Manual for Hazardous Waste Site Activities, Chapter 10. U.S. Printing Office, October, 1985.

U.S. EPA, 1985. Decontamination Techniques for Mobile Response Equipment Used at Waste Sites (State-of-the-Art Survey), EPA/600/52-85/105. 
INFORMATION ONLY

ENVIRONMENTAL RESTORATION DEPARTMENT

DOCUMENT APPROVAL COVER SHEET

Document Number: SOP -11.8

Revision:

Title: ERD Standard Operating Procedure - Ground Water Sampling

Prepared by: Karen X Keek

Date: $3-9-92$

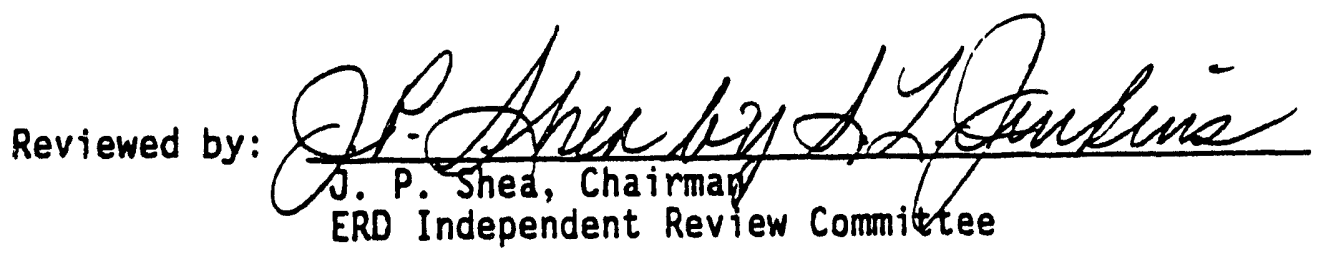

Date: $3-9-92$

ERD Independent Review Committee

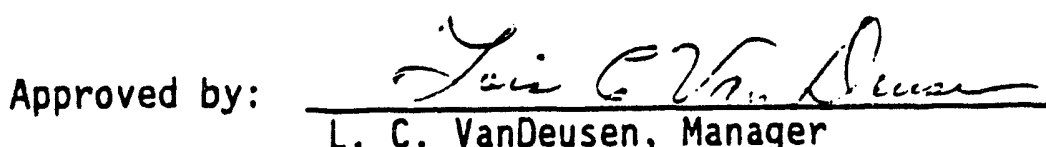

Date: $2-36-42$ Site Remediation Group

Approved by:

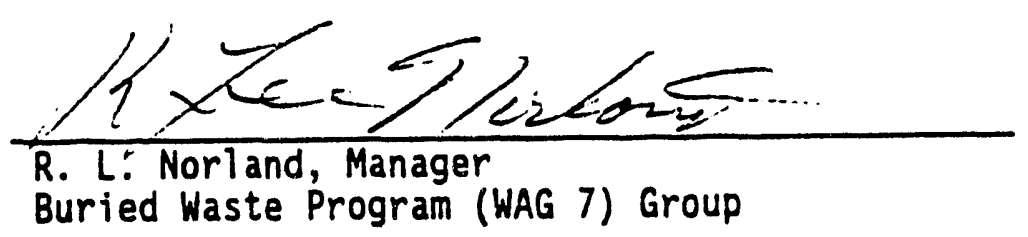

Date: $3-27-92$ Buried Waste Program (WAG 7) Group 
ENVIRONHENTAL

STANDARD OPERATING

PROCEDURES MANUAL
TITIE: GROUND WATER SAMPLING

NUMBER: 11.8
ISSUE DATE: $03 / 09 / 92$

APPROVED:

Manager

\subsection{PURPOSE AND SCOPE}

This procedure provides general instructions and requirements for the sampling of ground water. Ground water sampling entails coliecting ground water for geochemical and contaminant chemistry analyses for ground water adjacent to the well screen. Often the investigator will be evaluating contaminants at the parts per million (ppm) or parts per billion (ppb) concentration levels. Consequently, the possibilities of errors in data collection are enlarged. Therefore, extreme care and quality control must be used when obtaining samples.

Implement this procedure in conjunction with the statement of work (SOW) for the analytical laboratory. The laboratory should be contacted through the ERD SMO prior to sampling to obtain the proper samplehandling specifications.

\subsection{PROCEDURE}

\subsection{Quality Assurance}

Activities conducted according to this procedure will be in compliance with an investigation-specific Quality Assurance Project Plan (QAPJP) or other project-level plan as applicable.

\subsection{Health and Safety}

Activities conducted according to this procedure will be in compliance with an investigation-specific Health and Safety Plan and/or Safe Work Permit, as required.

\subsection{Training}

All personnel training relative to the use of this procedure shall be conducted in compliance with Section 2.0 of QPP-149 (EG\&G, 1991a) or other applicable EG\&G QPPs at the direction of the Project Manager.

\subsection{Field Equipment}

A list of necessary and recommended equipment is included in Table 1. Sampling equipment will be decontaminated prior to use in the field and after use according to ERP-SOP-11.5 Field

Decontamination of Sampling Equipment. Clean sampling equipment should not be placed directiy on the ground or other contaminated surfaces prior to insertion into the well. Non-dedicated pumps and tubing must be thoroughly decontaminated between well sampling sites. 
ENVIRONMENTAL STANDARD OPERATING PROCEDURES MANUAL

\section{TITLE: GROUND WATER SAMPLING}

NUMBER: 11.8
ISSUE DATE: 03/09/92

\subsection{Measurement of Static Water Level}

Prior to bailing, purging and sampling of the well, the static water level in the well must be measured. Water levels are measured from the surveyed reference marker and recorded to the nearest $0.01 \mathrm{ft}$. Procedures for taking static water level measurements are outlined in ERP-SOP-11.9 Measurement of Ground Water Levels. Repeat the ground water level measurements again after sample collection.

\subsection{Purging the Well}

The water standing in a well prior to sampling may not be representative of in-situ ground-water quality. The standing water in the well and filter pack should be removed so that formation water replaces the stagnant water. When purging standing water in the casing, typically three to five times the calculated volume of water in the well is removed in an effort to obtain a representative sample from the aquifer. The actual number of volumes to be removed are specified in the Sampling and Analysis PIan (SAP). To calculate the volume of standing water in a well, the following generalized equation may be used:

$V=\left(h_{2}-h_{2}\right) r^{2}(0.163)$

where: $V=$ static well volume in gallons

$h_{1}=$ depth of the well in feet, from the top of the casing

$h_{2}=$ depth to water, in feet, from the top of the casing

$r^{2}=$ inside radius of well casing in inches

Well purging continues until the volume specified in the SAP is removed and certain indicator parameters (i.e., pH, specific conductance, dissolved oxygen and temperature) are stabilized. Take measurements periodically during purging and again after sample collection to check the stability of the water sampled over time. Stabilization of the indicator parameters is satisfied when successive readings indicate the following criteria are met:

a. $\mathrm{pH}: \pm 0.1$ standard units

b. Specific conductance: $\pm 10 \mathrm{micromhos} / \mathrm{cm}$

c. Temperature: $\pm 0.5^{\circ} \mathrm{C}$

c. Dissolved oxygen: $\pm 1 \mathrm{mg} / \mathrm{L}$

Document the readings of the indicator parameters on the well purging field measurements data sheet (Figure 1). After purging the wel1, record the amount of water removed on the data sheet. 
ENVIROHHENTAL

STANDARD OPERATING

PROCEDURES MANUAL
TITLE: GROUND WATER SAMPLING

NUMBER: 11.8
ISSUE DATE: $03 / 09 / 92$

\subsection{Low-Yield Formations}

When purging a low-yield well (a well that is incapable of yielding three casing volumes), evacuate the well to dryness once. As soon as the well recovers sufficiently (ample water for collection), the first sample should be tested for $\mathrm{pH}$, specific conductance, dissolved oxygen and temperature. Samples should then be collected and containerized in the order of the parameters' volatilization sensitivity. 'In the event the well has very 7 imited production it may be possible to collect smaller volumes depending on the analysis required and after consultation with the analytical laboratory and ERD SMO. Retest the well after the samples have been collected for $\mathrm{pH}$, specific conductance, dissolved oxygen, and temperature as a measure of purging efficiency and as a check on the stability of the water samples over time.

\subsection{Disposal of Purge Water}

Refer to the site specific SAP and Investigation Derived Waste Plan for the proper handling of purge water.

\subsection{Selecting Equipment for Collecting the Water Sample}

Select sampling equipment so that disturbance of the actual concentrations of the chemical constituents of interest is minimized. To remove water from the well, bailers, low-volume suction pumps, and submersible pumps may be used. Use of dedicated bailers or pumps for each well is desirable, where feasible, to avoid cross contamination.

\subsubsection{Dedicated Pumps}

Many of the production wells at the INEL have dedicated high capacity turbine pumps. The advantage of having dedicated pumps at a well include: avoiding cross-contamination between wells, water samples are readily available, and provides an efficient manner for sample collection. However, the high flowrates may impact the volatiles present in the water due to the agitation of the water.

\subsubsection{Bailer}

A bottom-filling bailer constructed of Teflon ${ }^{\mathrm{m}}$, or stainiess steel can be used to remove the stagnant water in monitoring wells and obtain samples. The bailer is preferred when volatile stripping is of concern or the well casing diameter is too narrow to accept a submersible pump. However, this method can be very time-consuming and is recommended for shallow wells only. The bailer should not come in contact with any materials outside of the well casing. Wear clean disposable gloves during sampling and 
changed between each well sampling. Keep the bailer cord (teflon coated) clean and change the cord after each well sampling. Sample from 5 to 10 feet below water level or as the SAP specifies. Lower the bailer slowly until it contacts the water surface and allow the bailer to sink and fill with a minimum of surface disturbance. Slowly raise the bailer to the surface. Tip the bailer to allow slow discharge from the top of the bailer to the sample bottle, allowing the water sample to flow gently down the side of the sample bottle with minimum entry disturbance.

\subsubsection{Electric Submersible Pumps}

Submersible pumps are used for both the purging and collection of samples from depths which often exceed the limitations of conventional sampling equipment and can be used to sample several monitoring wells in a brief period of time. Before lowering into the well, the discharge tubing is rolled out and cleaned using a cloth and non-phosphate detergent followed by a rinse with distilled water. Then the pump is slowly lowered into the well with the safety line. All tubing and cord is gently wiped clean with cloth as the pump is lowered. Ideally the pump is set just below the dynamic water level and above the screened section of the well. The pump should not be set on the bottom.

\subsubsection{Positive Displacement Pumps}

Positive displacement pumps work by blowing compressed air or an inert gas into a sample chamber. The gas displaces the water in the chamber and forces it up an excavation tube. The gas is blown intermittently, using a pressure-controlled regulator, to allow, for recovery. Water returns to the sample chamber from the well through the bottom of the sampler, and is then prevented from leaving the bottom by a ball check-valve. Although the sampler is in contact with compressed air or inert gas, there is no violent introduction of gas into the sample, so the sample water is unaltered. All downhole parts must be assembled and cleaned with a non-phosphate detergent and rinsed before use in each well.

\subsubsection{Air-lift Pumps}

Air-lift pumps are useful for evacuation of the well or as skimmers, separating liquid from solid, but not for sampling. The violent introduction of air into the water changes its chemical characteristics. These pumps may be used when samples are to be analyzed for constituents that are not volatile, are not effectad by aeration, and are not effected by changes in $\mathrm{pH}$.

\subsubsection{Lysimeters}

Lysimeters are used for sampling water in the unsaturated zone. They induce the collection of soil moisture through negative 
pressure. A vacuum is put on the chamber, which is buried in the unsaturated zone, and moisture is drawn into the sample chamber through a porous-filter intake. Depending on soil texture and moisture content, as much as several hours or days under vacuum may be required.

\subsection{Filling the Containers}

Inspect the containers first to ensure they are the right type and number and are certifiably pre-cleaned. Wear clean gloves to prevent skin oils, dust particles or other contaminants from contaminating the sample. Gloves may also serve to protect the sampler from direct skin contact with the sample material, when potential contaminants are present. Affix the waterproof gummed Tabels containing information concerning the sample iD number, name of project area/well, type of analysis, date, and time to the containers at the time of collection. Place clear plastic tape over the label to protect it from damage. Transfer samples in the field from the sampling equipment directly into the container that is specifically prepared for that analysis. It is not an acceptable practice for samples to be composited in a common container in the field and then split in the laboratory, or poured first into a wide mouth container and then transferred into smaller containers. Pour the samples carefully into the containers, avoiding agitation or turbulence, which might result in loss of volatile organics and/or excessive oxygenation of the samples. Fill the bottles to the neck, except for volatiles, which require no headspace to minimize the possibility of volatilization of organics. Be careful to avoid breakage and to el iminate the entry of, or contact with, any substance other than the water sample being collected. Do not remove caps until, the actual sampling time and then just long enough to fill the container.

Samples should be collected and containerized in the order of the volatilization sensitivity of the parameters of interest. A preferred collection for some common ground-water parameters is as follows:
a. Volatile organics (VOA)
b. Purgeable organic carbon (POC)
c. Purgeable organic halogens (POX)
d. Total organic halogens (TOX)
e. Total organic carocn (TOC)
f. Extractable organics 
g. Total metals

h. Dissolved metals

i. Phenols

j. Cyanide

k. Sulfate and chloride

1. Turbidity

m. Nitrate and ammonia

n. Radionuclides

\subsection{Filtration}

Prior to preservation, water samples for dissolved metals analysis are filtered through a 0.45 micron pore-size filter to remove suspended particulate matter. Some radionuclides require filtration except certain radionuclides (i.e., tritium, carbon 14, and radioiodines). Usually the majority of radioactivity is in the solid phase and dissolved isotopes have an affinity for adsorption on solid particles in the sample, sampling material, and sample container walls, necessitating filtration. The SAP should specify if filtration is necessary for samples. Filtration should be done as soon as possible after a water sample is obtained, preferably simultaneously with the production of the water. Where possible, the standard procedure should be to use an in-line flow-through filter. Refer to the SAP for direction as to whether the metals and/or radionuclides need to be filtered.

\subsection{Sample Preservation and Handling}

Sample preservation is required for many of the chemical constituents and physiochemical parameters that are not chemically stable but are measured or evaluated in a ground water sampling program. Methods of sample preservation are generally intended to retard biological action, retard hydrolysis, and reduce sorption effects. Preservation methods usually include pH control, chemical addition, refrigeration, and protection from $1 \mathrm{ight}$. Specific preservation methods for each constituent are found in the sow for the analytical services. A summary list of appropriate sample container types and sample preservation is found in Table 2. Appropriate chemical preservation is performed in the field for the various analytical parameters at the time of sampling. Indicate the type and amount of preservation used in the field logbook.

Samples should be preserved at approximately $4^{\circ} \mathrm{C}$ in the dark 
ENVIRONMENTAL

STANDARD OPERATING

PROCEDURES MANUAL
TITLE: GROUND WATER SAMPLING

NUMBER: 11.8
ISSUE DATE: $03 / 09 / 92$

during transport to the laboratory for analysis, excepting samples for metals and radionuclide analysis.

A documented chain-of-custody program shall be used to identify and trace all samples, from the point of collection to final analysis. The procedures for following this chain-of-custody and proper sample handling and packaging is outlined in ERP-SOP-11.3 Chain-of-Custody, and ERP-SOP-11.3.I Sample Handling, Packaging and Shipping.

\subsection{Field Quality Control Samples}

The SAP should provide for the routine collection and analys is of the following field QC samples: trip blanks, field blanks, equipment blanks, and duplicate samples. A trip blank is used for purgeable organic compounds only. Trip blanks are typicaliy prepared by the analytical lab sent to the project site and stored with precleaned sample containers, taken to sampling location and treated like a sample from that point on and travel with the collected VOA samples. Trip blanks are not opened and are returned and analyzed with the project samples. A field blank is prepared in the field with organic-free water. Fill a vial with organic-free water and follow all other sampling and handling practices. The sample accompanies the project samples to the laboratory and are analyzed for specific chemical parameters unique to the site at which they are prepared. The equipment blank is collected from the field equipment rinsate as a check for decontamination thoroughness. Pour organic-free water through or over the cleaned equipment and collect water in sample bottle and return to laboratory for analysis. Duplicates are collected as "second samples" from a selected well. They are collected as either split samples (collected from the same bailer volume or pumping discharge) or as second-run samples (separate bailer volumes or different pumping discharges) from the same well.

\subsection{Transportation of Samples}

Make prior arrangements for timely delivery of the samples to the analytical laboratory. All on-site and off-site shipments must follow DOT 49 CFR shipping requirements. EG\&G Form 176 "Request for Shipment of Materials" will be filled out for off-site shipments and will accompany the shipment to its fina? destination. If the total activity level of the sample is above EG\&G and DOT 49. CFR standards $(0.002 \mathrm{pC} \mathrm{i} / \mathrm{L})$, procedures for shipping radioactive materials will be implemented. DOE-ID form 5480 will be filled out prior to removing the sample from the site. Requirements regarding transportation of samples of potentially hazardous material, on the INEL, are detailed in EG\&G Company Procedure 14.1 Onsite Transportation of Hazarcous Materiail. 
ENVIRONMENTAL STANDARD OPERATING PROCEDURES MANUAL
TITLE: GROUND WATER SAMPLING

NUMBER: 11.8

Samples collected from a controlled radioactive area must be surveyed by an HP technician and if clean a release tag will be issued prior to removing from the site.

\subsection{Departure from the Site}

When leaving the site ensure the well cap is replaced and locked, the area is policed for trash, and the pump and power is off. Return keys or any other plant property and inform contacts of any unusual circumstances.

\subsection{DEFINITIONS}

Duplicates/replicates-are collected as "second samples" from a selected well and/or project site. They are collected as either split samples (collected from the same bailer voiume or pumping discharge) or as second-run samples (separate bailer volumes or different pumping discharges) from the same well.

Equipment blanks-are collected from the field equipment rinsate as a check for decontamination thoroughness.

Field Blanks-are prepared in the field with organic-free water. These samples accompany the project samples to the laboratory and are analyzed for specific chemical parameters unique to the site at which they were prepared.

Trip blanks-are used for purgeable organic compounds only. They are sent to the project site and travel with the collected samples. Trip blanks are not opened and are returned and analyzed with the project samples.

\subsection{REFERENCES}

DOE, 1989. DOE Environmental Survey Manual, Appendix E, "Field Sampling Protocols and Guidance." DOE Office of Environmental Audit.

EG\&G, 1991a, Quality Plan for the Environmental Restoration Program, QPP-149, EG\&G Idaho, Inc., Idaho Falls, Idaho.

EG\&G, 1991, Environmental Standard Operating Procedures, ERP-SOP-11.2 Field Logbooks, EG\&G Idaho, Inc., Idaho Falls, Idaho.

EG\&G, 1991, Environmental Standard Operating Procedures, ERP-SOP-11.3 Chain-of-Custody, EGaG Idaho, Inc., Idaho Falls, Idaho.

EG\&G, 1991, Environmental Standard Operating Procedures, ERP-SOP-11.3.1 Sample Handling, Packaging, and Shipping, EG\&G Idaho, Inc., Idaho Falls, Idaho. 
ENYIRONMENTAL

STANDARD OPERATING

PROCEDURES MANUAL
TITLE: GROUND WATER SAMPLING

NUMBER: 11.8

EG\&G, 1991, Environmental Standard Operating Procedures, ERP-SOP-11.5 Field Decontamination of Sampling Equipment, EG\&G Idaho, Inc., Idaho Falls, Idaho.

EG\&G, 1991, Environmental Standard Operating Procedures, ERP-SOP-11.9 Measurement of Ground Water Levels, EG\&G Idaho, Inc., Idaho Falls, Idaho.

EG\&G, 1991, EG\&G Company Procedure 14.1 Onsite Transportation of Hazardous Material, EG\&G Idaho, Inc., Idaho Falls, Idaho.

United States Environmental Protection Agency, 1986, RCRA Ground-Water Monitoring Technical Enforcement Guidance Document, Office of Waste Programs Enforcement, Office of Solid Waste and Emergency Response, Washington DC. 
ENVIRONMENTAL

STANDARD OPERATING

PROCEDURES MANUAL
TITLE: GROUND WATER SAMPLING

NUMBER: 11.8

Table 1. Field Equipment List.

Appropriate field logbooks

Data Forms

Pencils, pens, permanent markers

Key to unlock wellhead

Watch

Electronic water-level measuring device or

Weighted steel tape marked

in hundredths of $\mathrm{ft}$

Chalk

Safety equipment specified in

Health and Safety Plan

Flashlight

Mirror

Pump, bailer, bailer line

Purge hosing

Bucket

Specific conductance, dissolved oxygen,

$\mathrm{pH}$, and temperature sensing devices, calibrated buffer solutions

Sampling Manifold

Sample bottles, preservatives

Pipette or eye dropper for

dispensing preservatives

Reagent grade water

Tape- clear tape for bottles, parafilm, strapping tape and duct tape
Vermiculite

Chain-of-Custody Forms

Custody Seals

This-side-up Arrows

Address labels for coolers

Coolers

Blue Ice

Ziploc baggies

Plastic trash bags

Tools

Appropriate containers

for purge water, as applicable

Scissors, knife

Shipping papers, forms 
Tabı.... Typical ground water sample requirements. ${ }^{c}$

\begin{tabular}{|c|c|c|c|c|c|c|}
\hline Analyt ical Parameter & \multicolumn{2}{|c|}{$\begin{array}{r}\text { Conta iner } \\
\text { Type } \\
\end{array}$} & \multirow{2}{*}{$\frac{\text { Preservative }}{4 \text { drops } \mathrm{HCL}^{\circ} \mathrm{C}}$} & \multirow{2}{*}{$\frac{\text { Holding Time }}{14 \text { days }}$} & \multirow{2}{*}{$\begin{array}{l}\text { Samp le Volume } \\
80 \mathrm{ml} / 2 \times 40 \mathrm{ml} \\
(6 \times 40 \mathrm{ml} \text { for full } \\
0 \mathrm{C})^{\mathrm{b}}\end{array}$} & \multirow{3}{*}{ 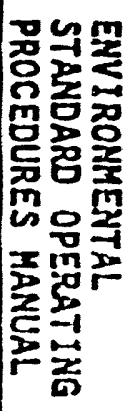 } \\
\hline Volatile oryanics & $40 \mathrm{ml}$ & $\begin{array}{c}\text { amber glass } \\
\text { vlal }\end{array}$ & & & & \\
\hline $\begin{array}{l}\text { Semivolatile organics } \\
\text { PCBs/pest icides } \\
\text { organophosphorus pesticides/ } \\
\text { organochlorine herbicides }\end{array}$ & $\begin{array}{l}1 \mathrm{~L} \\
\text { per } \\
\text { ana lys is }\end{array}$ & $\begin{array}{c}\text { amber glass } \\
\text { Jugs }\end{array}$ & $4^{\circ} \mathrm{C}$ & $\begin{array}{l}\text { extract } 7 \text { days. } \\
\text { analyze } 40 \text { days }\end{array}$ & $\begin{array}{l}1 \mathrm{~L} \text { per analysis } \\
\text { (pest.. herb., } \\
\text { etc.) } \\
3 x_{\mathrm{b}} 1 \mathrm{~L} \text { (for full } \\
\mathrm{QC} \text { ) }\end{array}$ & \\
\hline Nitrate & $1000 \mathrm{ml}$ & HDPE & $\begin{array}{l}4^{\circ} \mathrm{C} \\
\mathrm{pH}<2 \mathrm{H}_{2} \mathrm{SO}_{6}\end{array}$ & 14 days & $1000 \mathrm{ml}$ & 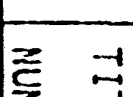 \\
\hline Antions & $125 \mathrm{ml}$ & HDPE (NM) & $4^{\circ} \mathrm{C}$ & $\begin{array}{l}28 \text { days } \\
48 \mathrm{hrs} \mathrm{NO}_{3} . \mathrm{PO}^{2}\end{array}$ & $125 \mathrm{ml}$ & $m_{0}^{\infty}$ \\
\hline All metals/cations & $1000 \mathrm{ml}$ & HDPE (MM) & $\mathrm{pH}_{2} \mathrm{HNO}_{3}$ & $\begin{array}{c}6 \text { months } \\
\mathrm{Hg} 28 \text { days } \\
\end{array}$ & $1 \mathrm{~L}$ & $\because \quad$ 妿 \\
\hline $\mathrm{Cr}^{6+}$ & $500 \mathrm{ml}$ & HDPE (NM) & $4^{\circ} \mathrm{C}$ & 24 hrs & $500 \mathrm{ml}$ & ${ }^{\infty}$ \\
\hline Cyanide & $1000 \mathrm{ml}$ & HDPE (NM) & $\begin{array}{l}\mathrm{pH}>12 \mathrm{NaOH} \\
6 \mathrm{~g} \text { ascorbic acid }\end{array}$ & 14 days & $\begin{array}{l}2 \times 1 \mathrm{~L} \\
(\text { for full OC) }\end{array}$ & $\frac{\pi}{m}$ \\
\hline Sulf ide & $500 \mathrm{ml}$ & glass (NM) & $\begin{array}{l}\mathrm{pH}>9 \mathrm{MaOH} / 2 \text { inc } \\
\text { acetate }\end{array}$ & 1 days & $\begin{array}{l}3 \times 500 \mathrm{ml} \\
(\text { for full } Q \mathrm{C})^{b}\end{array}$ & 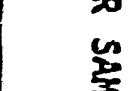 \\
\hline Alkalinity & $500 \mathrm{ml}$ & HDPE (MM) & $4^{\circ} \mathrm{C}$ & 14 days & $500 \mathrm{ml}$ & $\overline{0}$ \\
\hline Suspended particles & $500 \mathrm{ml}$ & HDPE (WH) & $4^{\circ} \mathrm{C}$ & 7 tays & $500 \mathrm{ml}$ & $\ddot{n}$ z \\
\hline Gross alpha, beta screen & $125 \mathrm{ml}$ & HDPE (NM) & $\mathrm{pH}<2 \mathrm{INO}_{3}$ & $\begin{array}{c}\text { screen } \\
\text { inmediately }\end{array}$ & $100 \mathrm{ml}$ & m \\
\hline Gamma ana lys is or screen & $540 \mathrm{ml}$ & plastic & $\mathrm{pH}<2 \mathrm{IMO}_{3}$ & 1 year & $500 \mathrm{ml}$ & 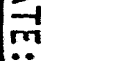 \\
\hline Rad. analysis/Total U & $2-1 / 2 \mathrm{gal}$ & plast ic & $\mathrm{pH}<2 \mathrm{HMO}_{3}$ & 1 year & $4 L$ & 0 \\
\hline $5 r-90$ & $1000 \mathrm{ml}$ & HDPE (MH) & $\mathrm{pH}<2 \mathrm{INO}_{3}$ & - & $1000 \mathrm{ml}$ & 8 \\
\hline Tritium & $125 \mathrm{ml}$ & HDPE (NH) & none & 1 year & $100 \mathrm{mI}$ & $\operatorname{lin}_{10}$ \\
\hline
\end{tabular}


ENVIRONMENTAL

STANDARD OPERATING

PROCEDURES MANUAL

\section{TITLE: GROUND WATER SAMPLING}

NUMBER: 11.8

Figure 1. Well Purging Field Measurement Data Sheet.

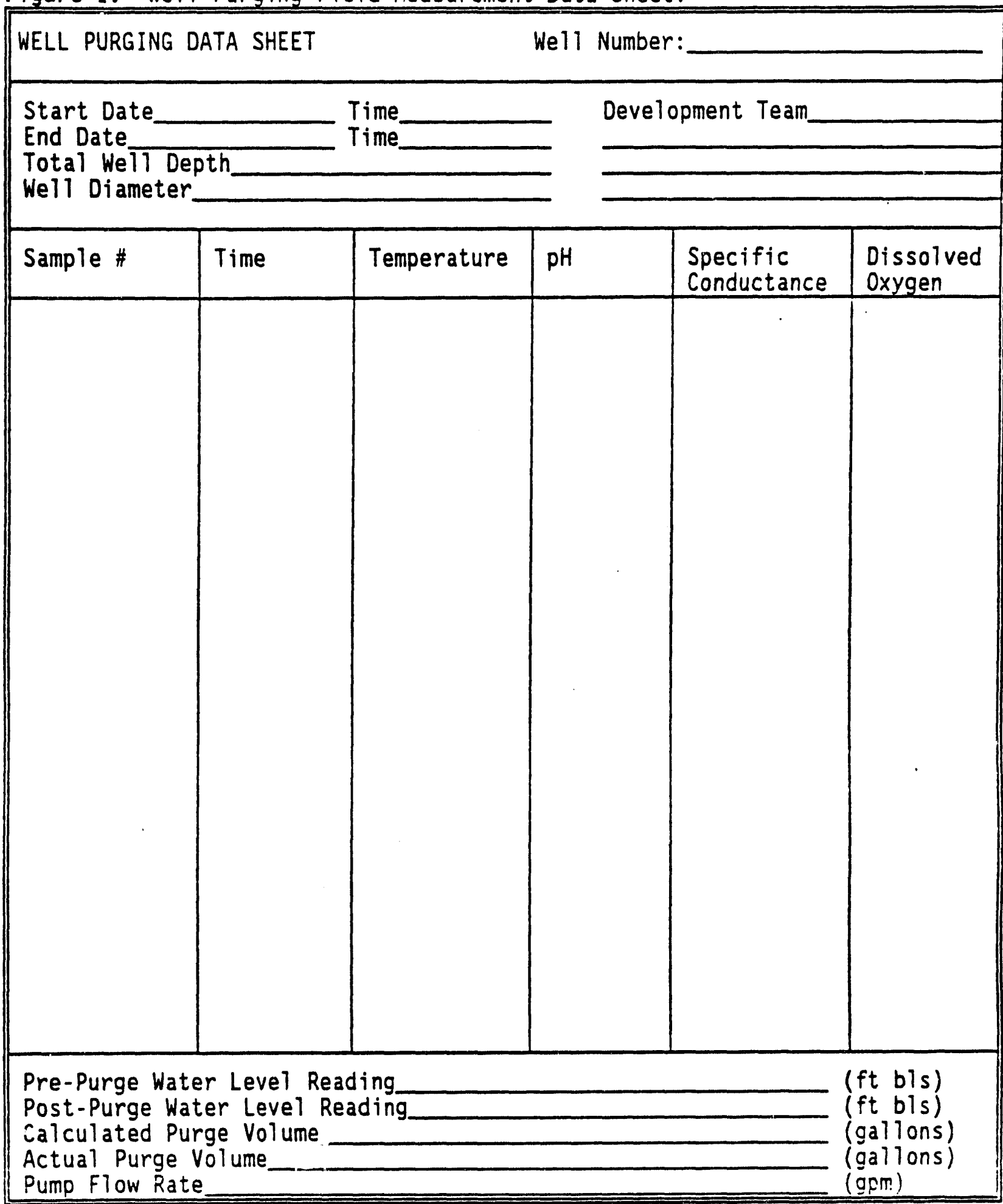


ENVIRONMENTAL RESTORATION DEPARTMENT

INFORMATION ONLY

ENVIRONMENTAL STANDARD OFERATING PROCEDURE

COVER SHEET

SOP Number: SOP-11.9

Revision:

0

Title: STANDARD OPERATING PROCEDURE FOR MEASUREMENT OF GROUND WATER LEVELS

Prepared by: $\frac{\text { K. N. Keck }}{\text { K. N. Keck, Sr. Scien }}$

Geosciences Group

Reviewed by:

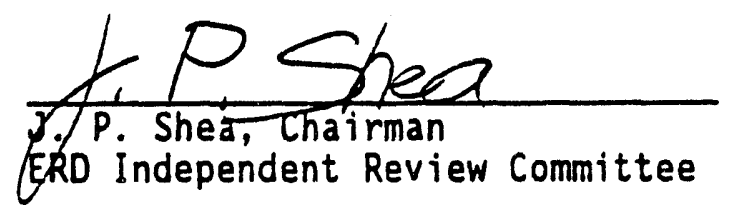

Date: $\underline{02 / 26 / 92}$

Date: $\underline{02 / 27 / 92}$

Approved by: Soin 6 Zien Dime

Date: $2 / 28 / 9 \geq$

L. C. VanDeusen, Manager

Site Remediation Group

Approved by:
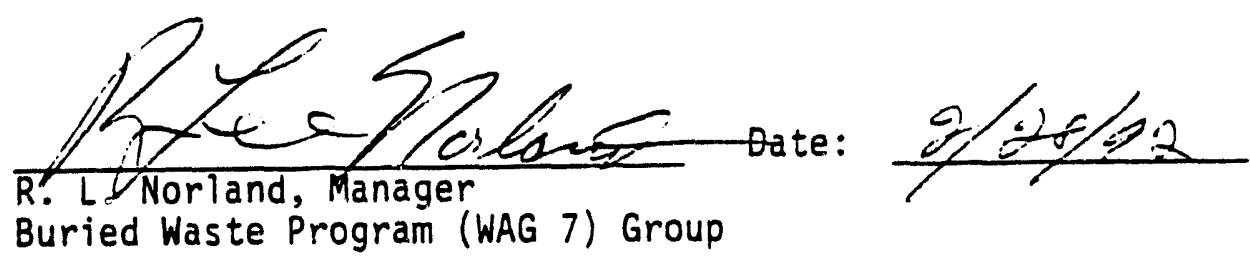

Buried Waste Program (WAG 7) Group 
EHYIRONHENTAL

STANDARD OPERATING

PROCEDURES HAMUAL
TITLE: MEASUREHENT OF GROUHD WATER LEYELS

NUNBER: 11.9
ISSUE DATE: $02 / 28 / 92$

\section{PURPOSE AND SCOPE}

To provide general instructions for Field Personnel to measure ground water levels in wells intercepting both regional and perched water systems. This information may be used to determine the regional groundwater flow direction and to determine water level fluctuations. Also, prior to bailing, purging, and/or sampling, calculate the static water level in the well and the volume of standing water in the well.

\section{PROCEDURE}

2.1 Each well should have a permanent, easily identified measuring point from which its water level measurement is taken. The measuring point is established to the nearest 0.01 foot by a licensed surveyor in relation to an established National Geodetic Vertical Datum (NGVD). In remote areas, a temporary benchmark is established to facilitate resurveying.

2.2 The measurement will be taken to 0.01 foot. The device used to detect the water level surface is sufficientiy sensitive so that a measurement to \pm 0.01 foot is obtained reliably. A weighted water level steel or fiberglass measuring tape, electronic water level indicator, or transducer will suffice.

2.3 As a field calibration check, all new or newly repaired electronic water level indicator are checked against a weighted measuring tape in at least one well, prior to use.

2.4 Whenever nondedicated equipment is used, procedures as out lined in ERP-SOP-11.5, "Field Decontamination of Sampling Equipment" are instituted where wells are suspected or known to be contaminated.

2.5 At contaminated sites fumes and gases may be present, requiring both radiologic and hazardous ronstituent monitoring equipment. Refer to the site-specific Health and Safety PI an and/or safe work permit, for the proper personal protective equipment (PPE) required.

2.6 Material and equipment to perform groundwater level measurements include:
a. Black or dark colored pen or permanent non-smearable marker of a color that will copy.
b. Appropriate "Measurement of Groundwater Levels" (MGL) For $s$ (see Figure 1).
c. Keys and/or combinations for all well head protective casings and/or continuous recorder housing locks. 
ENVIRONMENTAL STANDARD OPERATING PROCEDURES MANUAL
TITLE: MEASUREMENT OF GROUND WATER LEYELS

NUMBER: 11.9
ISSUE DATE: $02 / 28 / 92$

d. Weighted water level measuring tape with length greater than the anticipated water depth, or electronic water level indicator, or continuous recorder.

e. Carpenters' chalk (if using weighted measuring tape).

f. Portable computer and cable assembly for downloading water level measurements to magnetic disks and/or drum charts (as appropriate for continuous recorder)

2.7 If a weighted measuring tape is used, the water-level measurement should proceed in the following order:

a. Rinse the first ten feet of the measuring tape with detergent solution, then with distilled water and dried with a clean cloth. If previous measurements have been made at this well refer to them to estimate where to hold the measuring tape.

b. Chalk the lower segment (3-5 ft) of the tape by carefully drawing the tape across a piece of carpenter's chalk. Chalk need not be used on stainless-steel tapes.

c. Lower the weighted tape slowly down the center of the casing or riser pipe until the tape penetrates the water surface.

d. After water is encountered in the well, hold the tape at the closest even foot marker at the measuring point (typically top of well casing on north side). Make a note in field logbook and MGL form of measuring point location. If a measuring point is not identified on the well casing or apron, mark the measuring point where the tape is held at the top of the casing, so that successive measurements are taken from the same point. Record the "hold" measurement in feet on the MGL Form (see Figure 1).

Note: If a measuring point is not marked on the well contact the area landlord or appropriate personnel to have a measuring point permanently marked on the well and recorded in the INEL Comprehensive Well Survey Database.

e. Pull or reel the measuring tape out of the well.

f. Record the measurement to the nearest $0.01 \mathrm{ft}$ where the tape became wet on the MGL form.

g. Depth to water (DTW) is found by subtracting the "wet" measurement from the "nold" measurement. Record deptn to water to the nearest $0.01 \mathrm{ft}$ on the MGL form. 
ENVIRONMENTAL

STANDARD OPEPATING

PROCEDURES MANUAL
TITLE: MEASUREMENT OF GROUND WATER LEYELS

NUMBER: 11.9
ISSUE DATE: $02 / 28 / 92$

h. Water-level elevation relative to mean sea level is found by subtracting the depth to water from the measuring point elevation.

i. Repeat the measurement and compare the DTW measurements with past measurements at this well, if available. If the value is inconsistent with past values or if this is the first measurement at this well, repeat the measurement and record the results in the MGL form.

j. Repeat step 2.7a.

2.8 If an electronic water-level indicator is used, the water-level measurement proceeds as follows:

a. Check battery condition and continuity as recommended in the owners' manual. The continuity cell car be tested by placing it in water and observing the audio or visual signal.

b. Measurement markers on the wire may slip or move out of place. Periodically, check that the measurement markers on the wire have not shifted with a tape measure.

c. Clean the first ten feet of the electric tape with detergent solution; rinse with distilled water; and dry it with a clean cloth.

d. Slowly lower the probe into the center of the casing until a contact with the water surface is indicated. Raise and lower the probe several times to ascertain surface water level. Use caution so that the electric tape is not cut by a sharp casing edge. Record the measurement to the nearest $0.01 \mathrm{ft}$ on the MGL form; the reading represents DTW.

Note: If the tape is not incremented in $0.01 \mathrm{ft}$, measure (using folding ruler or tape measure with $0.01 \mathrm{ft}$ increments) the distance from the "hold" mark to the nearest tape band or marker and add or subtract to the band or marker reading. Repeat the reading before pulling out the electronic waterlevel indicator. Record all measurements on the MGL form.

e. Reel the probe out of the well.

f. Compare the DTW measurement with past measurements at this well, if available. If the value is inconsistent with past values or if this is the first measurement at this well, repeat the measurement and record the results in the $M G L$ form. 
g. Water-level elevation relative to mean sea level is found by subtracting the depth to water from the measuring point elevation.

h. Repeat step 2.8c.

2.9 If a continuous recorder is used, the water-level measurement proceeds as follows:

a. Cheek batteries prior to use in the field to ensure an adequate power supply for the recorder. Reset (or set, if an initial installation) the downhole float or the transducer, as appropriate in compliance with the manufacturers' operating manual.

b. For initial installation, install the recorder at the wellhead and fit the supplied weatherproof housing as specified in the owners' manual. Install a new chart (for drum recorders) or initiate the data recording function as necessary. Initial and date the chart or disk and specify well number. Recheck all operating functions.

c. For routine maintenance, change the chart or download data onto magnetic disks, as appropriate for the type of recorder. Initial and date the chart or disk and specify well number.

d. Water-level elevation relative to mean sea level is found by subtracting the depth to water from the measuring point elevation.

\section{DEFINITIONS}

Depth to Water (DTW) - is the depth from the MP to the water level intercept point.

Land Surface Datum (1.s.d.) - is a surveyed benchmark indicating the true elevation at the land surface, generally identified by a brass marker set in the concrete surrounding the well.

Measurement Point (MP) - is a fixed, clearly marked point of reference at the top of the well riser casing or on the apron, and where applicable, the protective casing; from which the depth to groundwater is measured. 
ENVIRONMEPTAL

STANDARD OPERATING

TITLE: MEASUREMENT OF GROUND WATER LEVELS

PROCEDURES MANUAL

NUMGER: 11.9

ISSUE DATE: $02 / 28 / 92$

\section{REFERENCES}

U.S. EPA, 1986. RCRA Groundwater Monitoring Technical Enforcement Guide (TEGD).

ERP Environmental Standard Operating Procedures Manual, 1992. ERP-SOP11.5, Field Decontamination of Sampling Equipment. 
MEASUREMENT OF GROUND WATER LEVELS (MGL) DATA SIIEET

Project:

Sile:
Hell Number:

M.P. Description:

M.P. Ileight above 1 and:

M.P. Elevation:

Land surface elevation:

\begin{tabular}{|c|c|c|c|c|c|c|c|}
\hline Date & Time & $\begin{array}{l}\text { llold } \\
\text { (ft) }\end{array}$ & $\begin{array}{l}\text { Wet } \\
\text { (ft) }\end{array}$ & $\begin{array}{l}\text { Depth to Water } \\
\text { (below M.P.) } \\
\text { (ft) }\end{array}$ & $\begin{array}{l}\text { Elev. of Water } \\
\text { (adj. to m.s.l.) } \\
\text { (ft) }\end{array}$ & Remarks & Initial \\
\hline & & & & & & & \\
\hline & & & & & & & \\
\hline & & & & & & & \\
\hline & & & & & & & \\
\hline & & & & & & & \\
\hline & & & & & & & \\
\hline & & & & & & & \\
\hline & & & & & & & \\
\hline & & & & & & & \\
\hline & & & & & & & \\
\hline & & & & & & & \\
\hline & & & & & & & \\
\hline & & & & & & & \\
\hline & & & & . & & & \\
\hline & & & & & & & \\
\hline
\end{tabular}

Figure 1. Measurement of Ground Water Levels lata Sheet.

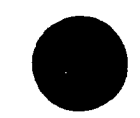


ENVIRONMENTAL RESTORATION DEPARTMENT

ENVIRONMENTAL STANDARD OPERATING PROCEDURE

COVER SHEET

SOP Number: $\quad$ SOP-11.14.1

Revision:

0

Title: STANDARD OPERATING PROCEDURE FOR ELECTROMAGHETIC TERRAIN CONDUCTIVITY SURVEYS

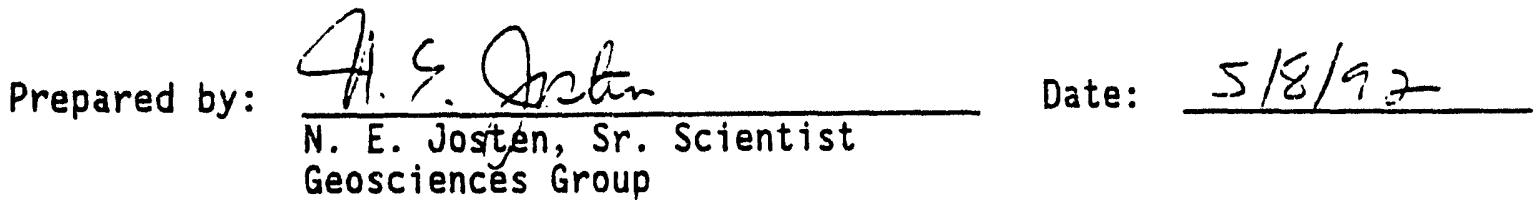

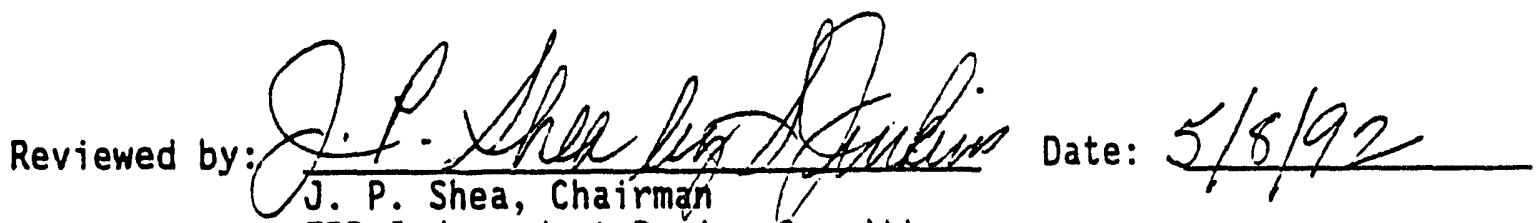
ERD Independent Review Committee

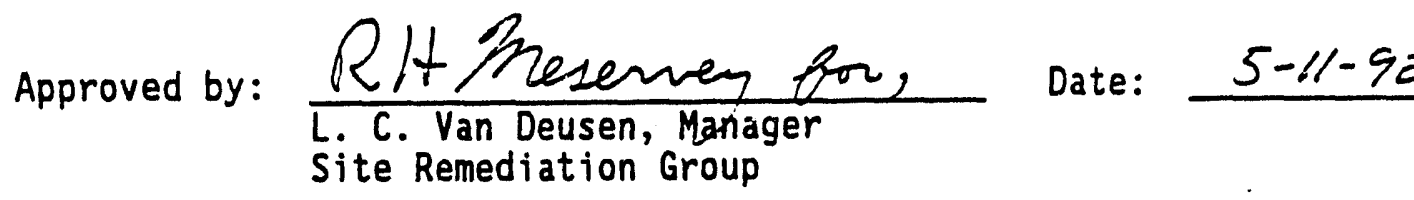
Approved by:

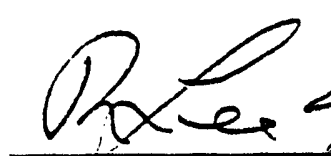
R. K. Norland Manager Buried Waste Program (WAG 7) Group

Date: $5 / 11 / 92$

Field Changes Authorized by DRR No.: 
ENVIRONMENTAL

STANDARD OPERATING

PROCEDURES MANUAL
TITLE: ELECTROMAGNETIC TERRAIN CONDUCTIVITY SURVEYS

NUMGER: 11.14 .1
ISSUE DATE: $5 / 8 / 92$

\section{PURPOSE AND SCOPE}

This procedure provides general instructions for Field Personnel under the direct supervision of a Project Geophysicist to perform electromagnetic terrain conductivity (EM) surveys. The purpose of an EM survey is to record variations in electrical conductivity caused by natural or manmade features of the subsurface. The recorded data may be used for subsequent geologic interpretations and/or detection of buried materials.

2. PROCEDURE

\subsection{Quality Assurance}

Activities conducted according to this procedure will be in compliance with an investigation-specific Quality Assurance Project Plan (QAPP) or other project level plan as applicable.

\subsection{Health and Safety}

Activities conducted according to this procedure will be in compliance with an investigation-specific Health and Safety Plan and Safe Work Permits as required.

\subsection{Training}

The Project Geophysicist supervising the land magnetic survey must have relevant experience in the acquisition, processing and interpretation of geophysical data.

Field Technicians must be trained to properly operate the specific geophysical equipment that is used for the survey. This training may be provided by the qualified Project Geophysicist.

\subsection{General}

Electromagnetic terrain conductivity measurements are made at discrete station locations using a noncontacting terrain conductivity meter. The conductivity meter may be operated in a vertical or horizontal dipole mode, and both inphase and quadrature components of the induced electromagnetic field may be measured.

Measurements are normally made along linear profiles, with station spacing dependent on the estimated depth to targets. Normally, the maximum distance between stations should be from one-half to one-fourth the estimated target depth.

Areal coverage is produced by making measurements along additional profiles, usually parallel to each other. Profile spacing will 
depend on the nature of the targets, including the depth of the targets and their expected distribution in the subsurface.

Interpretations of the electromagnetic data are based on the identification of anomalous features of the induced electromagnetic field. The shape and amplitude of anomalies, and the ratio of the quadrature response to the inphase response may be used to help identify the causative body. The ratio of the vertical dipole response to the horizontal dipole response may be used to investigate the depth to the causative body. Independent information such as geology, other geophysical data, and cultural feature naps are used to constrain the interpretation of the electromagnetic data.

\subsection{Equipment}

\subsubsection{Description of Equipment}

Terrain conductivity meters covered by this Procedure are coil-type frequency domain electromagnetic induction sensors. A high frequency primary electromagnetic field is transmitted into the earth and the resulting secondary electromagnetic field, produced by induced eddy currents, is measured. The strength of the secondary field is a function of the inter-coil spacing, operating frequency, and subsurface conductivity. For relatively homogeneous rock or soils, the ratio of the secondary to the primary magnetic field is directly proportional to the ground conductivity. When complex geology or strong conductors are present, the measured values are complex functions of the subsurface conductivity configuration.

Investigation depth is related to coil spacing and coil mode. The two coil modes used are the horizontal and vertical dipole modes, denoted HDM and VDM, respectively. HDM measurements are performed with the transmitter and receiver coils placed in a horizontal, coplanar position. VDM measurements are performed with vertical, coplanar coils. Depending upon coil spacing and orientation, investigative depths of up to about 30 feet can be achieved with commonly available instruments.

The principal components of a conductivity meter are a transmitter coil, a receiver coil, and an electronic control unit. Each is described briefly below.

\section{a. Transmitter coil}

The transmitter coil is a self-contained dipole transmitter. It op ates continuously at a frequency of about $10 \mathrm{kHz} \mathrm{fo}^{\prime}$ typical instruments. 


\section{ENVIRONMENTAL} STANDARD OPERATING PROCEDURES MANUAL
TITLE: ELECTROMAGNETIC TERRAIN CONDUCTIVITY SURVEYS

NUMBER: 11.14 .1
ISSUE DATE: $5 / 8 / 92$

\section{b. Receiver coil}

The receiver coil is a self-contained dipole receiver. It is fixed at a calibrated distance from the transmitter coil. The receiver coil detects induced secondary fields, which are transmitted to the control unit as a time varying voltage.

c. Control unit

The control unit separates the receiver coil output voltage into two components. The inphase component is inphase with the primary field. The quadrature component is 90 degrees out of phase with the primary field. The component to be measured is selected by the control unit settings. Inphase readings are displayed as the ratio of secondary to primary field strength. Quadrature readings are displayed directly as apparent ground conductivity values in millimhos per meter. Data are displayed as deflections on an analog meter. Switches on the control unit are used to set measurement modes, calibration modes, and meter reading ranges.

\subsubsection{Preparation of Equipment}

The equipment functional checks and adjustments described below shall be performed daily prior to data acquisition. Completion of these checks shall be documented in the field notebook or on the data sheets. Absolute calibration is not required if the data are interpreted in terms of relative variations only.

a. Assemble the conductivity meter as per the instructions in the instrument's operating manual.

b. Check that batteries are adequately charged and charge or replace as necessary.

c. Calibrate the transmitter coil output as per the instructions in the instrument's operating manual.

Note: The following checks should be conducted, if possible, in an area free of metallic cultural features.

d. Set the instrument for inphase component measurement and calibrate the receiver coil output by nulling the output reading as per instructions in the instrument's operating manual. 
e. If an area with known, uniform conductivity is available, an absolute calibration of the ouadrature component readings can be performed as per instructions in the operating manual.

\subsection{Field Procedure}

\subsubsection{Location of Electromagnetic Survey Lines}

a. The Project Geophysicist shall determine the proposed locations of electromagnetic survey lines. Locations shall be noted on a line location map.

b. The accuracy and precision of the line location survey will depend on the objectives for the electromagnetic survey. Locations may be determined by optical surveying, or by taped measurements. The survey should be tied to at least one known point, which should be identified in the field notebook. Firal station coordinates should be reported using a standard US Geological Survey map projection.

c. If lines are to be optically surveyed, the line location map shall be furnished to the licensed surveyor. Upon completion of surveying, the surveyor will provide the Project Geophysicist with a map showing the locations and stationing of all electromagnetic survey lines. If required, the surveyor will place non-metalitic stakes or flagging to identify station locations.

d. If line locations are not optically surveyed, they shall be determined by the Field Technician using taped measurements. The method for laying out the electromagnetic survey lines shall be noted in the field notebook. Non-metallic stakes and flagging will be placed as required.

e. As work progresses, additional lines may be specified by the Project Geophysicist. If specified, the additional lines should be $i$ isted or added to the line location map. Any changes to the field operation based on field conditions shall be approved by the Project Geophysicist and shall be documented in the field notebook.

\subsubsection{Data Acquisition}

a. The Project Geophysicist shall verify that the EM survey lines have beer: located as specified, and that 
ENVIRONMENTAL

STANDARD OPERATING

PROCEDURES MANUAL
TITLE: ELECTROMAGNETIC TERRAIN CONDUCTIVITY SURVEYS

NUMBER: 11.14 .1
ISSUE DATE: $5 / 8 / 92$

field conditions are accurately noted in the field notebook.

b. Data shall be collected along each traverse either continuously, using a chart recorder, or at intervals specified by the Project Geophysicist using a digital data logger, or by manually recording meter deflections on data sheets. All measurements shall be made with the coil boom oriented in the same direction.

c. Measurement of different phase components, using different coil boom orientations, or using different dipole orientations, are made by repeating step $b$. The survey configuration, including boom orientation, dipole orientation, and phase component shall be annotated on all data sheets.

d. When a digital data logger is used for data acquisition during the survey, data shall be transferred from the data logger to a personal computer at the conclusion of the field day. After the data has been transferred, the data shall be stored on both a hard disk and floppy diskette, and a hardcopy listing of the data shall be produced.

\subsubsection{Documentation on Field Records}

The following information shall be documented in at least one field notebook entry:

1. project

2. project location

3. instrument type and identification number.

The following information shall be documented in the field notebook on a daily basis:

1. date

2. type of survey (dipole mode, boom orientation, phase component, etc.)

3. line numbers completed

4. station numbers completed

5. diskette numbers if applicable 


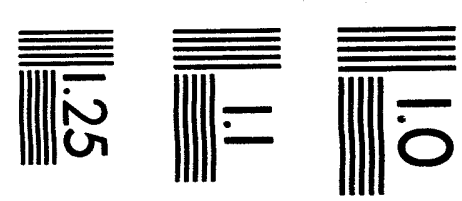

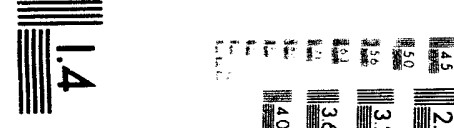

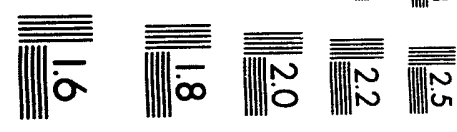



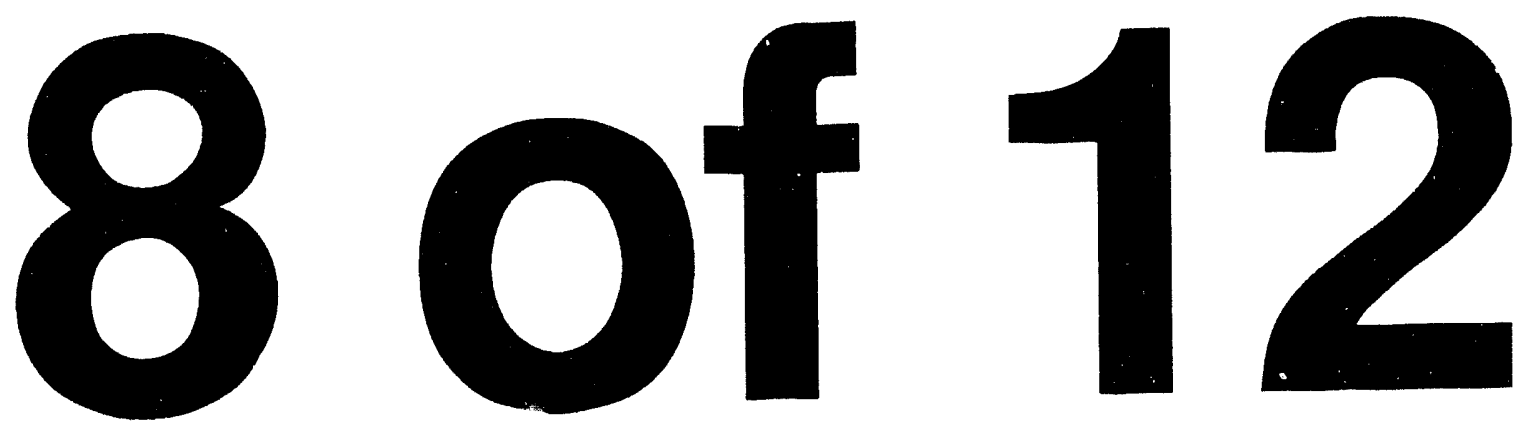
6. locations of cultural features or other debris observed during data acquisition that are suspected to affect the EM readings collected during daily operations

7. weather conditions.

\subsection{Data Reduction}

EM data require no standard data reduction operations.

\subsection{Data Processing}

\subsubsection{Analog Data Processing}

a. Conductivity values from chart recordings shall be transferred to a standardized data sheet.

b. Conductivity profiles or a conductivity contour map shall then be prepared from data listed on data sheets. Profiles shall be generated using distance along each survey traverse plotted on the $x$-axis and apparent conductivity (quadrature component) or Secondary Field / Primary field ratio (inphase component) on the $y$-axis. Contour maps shall be generated by transferring conductivity values onto an appropriate plan map (or overlay of a plan map), and then contouring the data at appropriate contour intervals.

\subsubsection{Digital Data Processing}

Digital processing may be performed on data acquired using either digital or analog recording methods. If analog data are to be processed digitally, the analog data shall be entered into a personal computer. The data may then $b$ = profiled and/or contoured.

Profiles shall be produced using commercially available software. Contour maps of the EM data shall be produced using commercially available software. Other techniques for processing and displaying digital EM data may be used as necessary.

\subsection{Data Interpretation}

\subsubsection{General}

Interpretation of the EM data shall be performed by or under the direct supervision of the Project Geophysicist. 
ENVIRONMENTAL STANDARD OPERATING

PIOCEDURES MANUAL
TITLE: ELECTROMAGNETIC TERRAIN CONDUCTIVITY SURVEYS

NUMBER: 11.14 .1

ISSUE DATE: $5 / 8 / 92$

\subsubsection{Plotiing of Known EM Sources}

Known sources of anomalous EM readings shall be noted on the profiles, contour maps, or contour-map overlays. These sources may include cultural features and debris observed during the data acquisition, and geologic features such as rock outcrops, faults or changes in soil type.

\subsubsection{Identification of Anomalous Trends}

EM profiles shall be aligned to identify possible trends that may be indicative of buried metal or other targets of interest. EM inphase and quadrature contour maps shall be analyzed to identify zones of anomalous ground conductivity and the presence of metallic conductors.

A comparison of EM survey results with cultural features and geologic features should be performed to evaluate obvious causes for the observed magnetic trends. The EM survey data should also be compared with any other available geologic or geophysical data to aid in determining the causes for other observed magnetic trends.

\subsection{Documentation of Results}

Results of the EM data interpretation shall be compiled in a report. The form and scope of the report will depend on the purpose of the EM survey. The report shall include maps and profiles of the EM data, and a hardcopy listing of the data.

The report, maps, profiles and hardcopy listing shall be delivered to the Field Data Coordinator for the Environmental Restoration Department. If required, diskettes of the digital data sinall also be provided to the Data Coordinator. The information provided to the Data Coordinator shall be complete and suitable for loading into a data base.

\subsection{DEFINITIONS}

Project Geophysicist - qualified geophysicist having final responsibility for the survey design, data acquisition, interpretation, and reporting for the geophysical survey. Qualifications are defined in section 2.3 above.

Field Technician - personnel performing various tasks during the geophysical survey under the direct supervision of the Project Geophysicist. Qualifications are defined in section 2.3 above. 
Cultural features - manmade structures such as fences, pipes, powerlines, buildings etc. that occur in the vicinity of a survey site and which may effect the recorded geophysical data.

\subsection{REFERENCES}

The following references provide general discussions of magnetometers, magnetic surveying and magnetic data interpretation:

Benson, R., R. A. Glaccum, and M. R. Noel, 1988, Geophysical Techniques for Sensing Buried Wastes and Waste Miaration, Dublin, Ohio: National Water Well Association.

Dobrin, M. B., 1976, Introduction to Geophysical Prospecting, New York: McGraw-Hill, Inc.

U. S. Environmental Protection Agency, 1987, A Compendium of Superfund Field Operations Methods, U. S. Environmental Protection Agency Document PB88-181557.

McNeil1, J. D., 1980, Electromagnetic Terrain Conductivity Measurement at Low Induction Numbers, Ontario, Canada: Geonics Limited Technical Note TN-6.

Telford, W. M., L. P. Geldart, R. E. Sheriff, D. A. Keys, 1976, Applied Geophvsics, New York: Cambridge University Press. 


\section{INFORMATION ONLY}

ENVIRONMENTAL RESTORATION DEPARTMENT

ENVIRONMENTAL STANDARD OPERATING PROCEDURE

COVER SHEET

SOP Number: SOP-11.14.2 Revision:

Title: STANDARD OPERATIHG PROCEDURE FOR SEISMIC LAND REFRACTION SURVEY

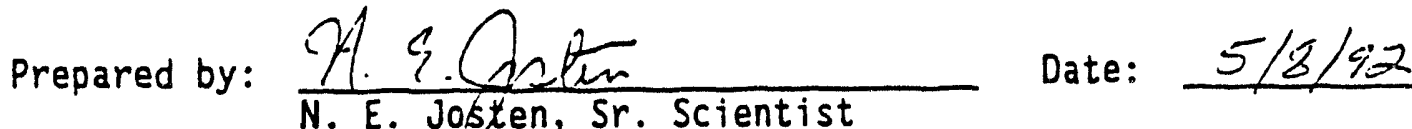
Geosciences Group

Reviewed by:

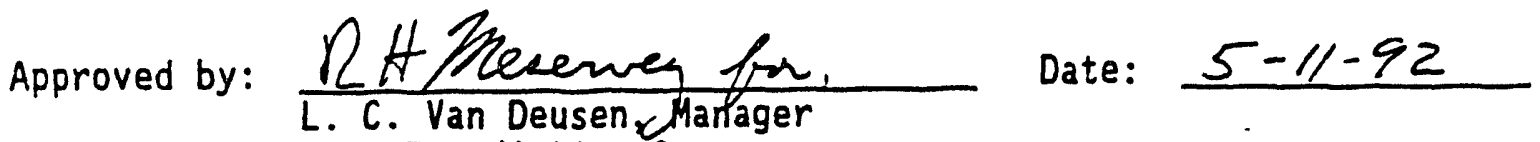
Site Remediation Group

Approved by:
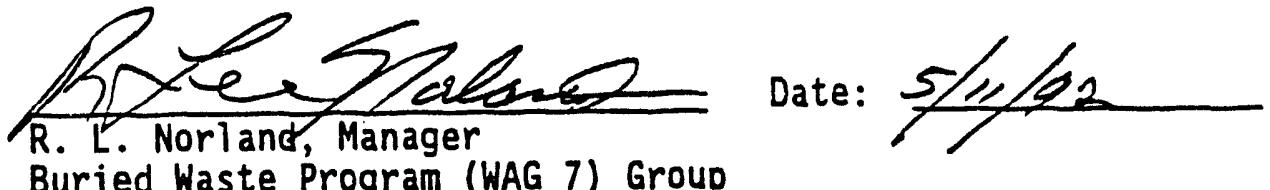

Field Changes Authorized by DRR No.: 
ENVIRONMENTAL STANDARD OPERATING

PROCEDURES MANUAL

\section{TITLE: SEISMIC LAND REFRACTION SURVEY}

NUMBER: 11.14 .2

\section{PURPOSE AND SCOPE}

This procedure provides general instructions for field personnel under the direct supervision of a Project Geophysicist to perform seismic 1 and refraction surveys. Land refraction surveys are commoniy used to determine depth to bedrock and/or thickness of geologic layers overlying bedrock. Refraction methods can also be used to classify various subsurface materials based on their seismic velocities.

\section{PROCEDURE}

\subsection{Quality Assurance}

Activities conducted according to this procedure will be 'in compliance with an investigation-specific Quality Assurance Project PIan (QAPP) or other project level plan as applicable.

\subsection{Health and Safety}

Activities conducted according to this procedure will be in compliance with an investigation-specific Health and Safety Plan and Safe Work Permits as required.

\subsection{Training}

The Project Geophysicist supervising the refraction seismic survey must have relevant experience in the acquisition, processing and interpretation of geophysical data.

Field Technicians must be trained to properly operate the specific geophysical equipment that is used for the survey. This training may be provided by the qualified Project Geophysicist.

\subsection{General}

Measurements are made by the continuous profiling method using a 12 or 24-channel seismic refraction digital data acquisition system. Measurements utilize nonexplosive seismic energy sources.

Continuous profiling is accomplished by having an end shot of one spread coincident with an end shot of the succeeding spread. The length of each spread is determined by the required depth of penetration. The deeper the required penetration, the longer the spread must be.

The number of recordings made for each spread may vary, depending upon the detail needed for a given survey. Four recordings are usually made for each 12-trace spread and five recordings are usually made for each 24-trace spread. 
Interpretations are based on the measurement of the time required for the seismic waves to travel through the ground to a series of geophones (vibration sensitive devices).

\subsection{Equipment}

\subsubsection{Description of Equipment}

Seismic signals are introduced into the ground by a signal generator, which is usually a mechanical impact device. Seismic waves travel through the ground and are detected by a series of up to 24 geophones that are physically coupled to the ground. The geophones convert vibratory ground motions to electrical signals. These signals travel through insulated signal cables to a multi-channel data acquisition system. The data acquisition system digitizes, amplifies and filters incoming signals from each geophone simultaneously. The signals for each of the channels are recorded on a photographic record and/or an internal mass storage device.

\section{a. Data Acquisition System}

The seismograph consists of front end analog high and low cut filters, programmable variable gain amplifiers, 16-bit digitizers, 32-bit stacking memory, system trigger mechanism, and internal mass memory for data storage. The programmable recording parameters such as sample rate, record length, and filter settings will be selected in the field as conditions dictate. The programmable parameters are digitally stored with each record.

A roll switch is sometimes used for acquisition of long data lines, when more than one seismic cable is laid out. The roll switch is a device that allows the user to select any 24 channels from the 48 available input channels.

\section{b. Signat Cables}

The signal cables consist of 24 pairs of conducting wires in an insulating casing. The wire pairs, one pair for each data channel, may be accessed from either end of the cable. Takeouts for connecting geophones are a waterproof variety and are located at either 2, 5, or 180 meter spacings. The selection of the takeout spacing will be made by the Project Geophysicist and will be noted in the Project Task Plan. 
ENVIRONMENTAL STANDARD OPERATING PROCEDURES MANUAL
TITLE: SEISMIC LAND REFRACTION SURVEY

NUMBER: 11.14 .2

\section{c. Geophones}

The geophones can be one of many types, but are generally moving coil transducers. The natural period and axis orientation of the geophones may vary depending on the application. The geophone type selection will be made by the Project Geophysicist and will be noted in the field notebook.

\section{d. Energy Source}

Seismic energy is normally generated by a weight impact system. The selection of the energy source will be made by the Project Geophysicist and will be noted in the field notebook.

\section{e. Trigger Cable}

The trigger cable sends a pulse from the energy source to the seismograph to initiate recording of seismic signals traveling through the signal cable. The trigger pulse also serves as a uniform time reference for data on all recorded channels.

\subsubsection{Preparation of Equipment}

The following equipment checks and calibration shall be performed prior to data acquisition.

a. The initial time break and clock of the recording system will be checked by the input of a 10 millisecond 1 volt square pulse into the trigger circuit and data channel one, with all filters "out". A visual check of the paper record will be made to verify the pulse duration.

b. The data acquisition system (geophones, signal cabies and recording system) shall be subjected to an operational check to verify that all channels respond in a similar manner when the geophones are subjected to a simultaneous common input signal.

\subsection{Field Procedure}

\subsubsection{Location of Refraction Lines and Shot Points}

a. The Project Geophysicist shall determine the proposed locations of seismic refraction lines, proposed locations of shots along each line, and the cable length and system (12 or 24 trace) to be used. Locations shall be noted on a line location map. 
ENVIRONMENTAL STANDARD OPERATING PROCEDURES MANUAL

\section{TITLE: SEISMIC LAND REFRACTION SURVEY}

NUMBER: 11.14 .2

b. The accuracy and precision of the line location survey will depend on the objectives for the seismic refraction survey. Locations may be determined by optical surveying, or by taped measurements. The survey should be tied to at least one known point, which should be identified in the field notebook. Final station coordinates should be reported using a standard US Geological Survey map projection.

c. If lines are to be optically surveyed, the line location map shall be furnished to the licensed surveyor. Upon completion of surveying, the surveyor will provide the Project Geophysicist with a map showing the locations and stationing of all seismic refraction lines. If required, the surveyor will place stakes or flagging to identify station locations.

d. If line locations are not optically surveyed, they shall be determined by the Field Technician using taped measurements. The method for laying out the seismic refraction survey lines shall be noted in the field notebook. Stakes and flagging will be placed as required.

e. As work progresses, cross-check lines may be specified by the Project Geophysicist for additional data acquisition. If specified, the cross-check lines should be listed or added to the line location map. Any changes to the field operation based on field conditions shall be approved by the Project Geophysicist and shall be documented in the field notebook.

\subsubsection{Data Acquisition}

a. The Project Geophysicist shall verify that the seismic refraction lines have been located as specified, and that field conditions have been accurately noted in the field notebook.

b. Seismic refraction cables shall be laid out along the lines of investigation, with shot points and geophones located along the cable with reference to pre-marked points on the cables themselves, or as measured by a tape in the field.

c. Geophones, fitted with spiked bases to provide good ground contact (except where spiked bases are not feasible, such as on pavements, bare rock exposures, etc.), shall be emplaced at their specified locations. 
ENVIRONMENTAL STANDARD OPERATING PROCEDURES MANUAL
TITLE: SEISHIC LAND REFRACTION SURVEY

NUMBER: 11.14 .2

d. Amplifier settings shall be adjusted to optimize the expected signal to noise ratio.

e. After checking the energy source and the safety of personnel around the weight impact system, recording may begin.

f. Each record shall be printed and inspected. All information pertaining to a particular data record shall be noted in the field notebook.

g. Data shall be checked as they are acquired to assure that the data are of an acceptable quality. If necessary, data acquisition shall be repeated.

\subsubsection{Documentation of Field records}

The following information shall be documented in at least one field notebook entry:

1. project name

2. project iscation

3. seismic equipment type and identification number

The following information shall be documented in the field notebook on a daily basis:

1. date

2. survey configuration (shot spacing, geophone spacing, energy source, etc.)

3. Tine numbers completed

4. shots completed

5. diskette numbers if applicable

6. locations of cultural features or other features observed during data acquisition that are suspected to affect the seismic refraction data collected during daily operations

7. weather conditions.

The following information shall be noted on each seismic record: 


\begin{tabular}{|l|lrl|}
\hline $\begin{array}{l}\text { ENVIRONMENTAL } \\
\text { STANDARD OPERATING } \\
\text { PROCEDURES MANUAL }\end{array}$ & TITLE: & SEISMIC LAND REFRACTION SURVEY & \\
NUMBER: 11.14 .2 & ISSUE DATE: $5 / 8 / 92$ \\
\hline
\end{tabular}

1. location of shot (including offset distance)

2. energy source type

3. depth of shot (if applicable)

4. time of shot

5. Tocation of spread

6. length of spread

7. line number

8. record number

\subsection{Data Reduction}

Seismic land refraction data require no standard data reduction operations.

\subsection{Data Processing}

\subsubsection{Preliminary Travel-Time Readings}

In order to maintain a continuous check on data quality and field conditions, the Field Technician should read as many of the field recordings as possible while the work is in progress.

\subsubsection{Time Distance Plots}

a. The field recordings shall be down loaded from the seismograph as per the manual instructions.

b. Time-distance plots shall then be prepared for each seismic refraction line. Time shall be plotted on the "y" (vertical) axis. Distance shall be plotted on the " $x$ " (horizontal) axis. Alternate scales may be used. Scales shall be noted on each roll of timedistance plots.

\subsection{Data Interpretation}

\subsubsection{General}

Interpretation of the seismic refraction data shall be performed by or under the direct supervision of the Project Geophysicist.

2.9.2 Construction and Interpretation of Velocity Profiles 


\section{ENVIRONMENTAL STANDARD OPERATING PROCEDURES MANUAL}

\section{TITLE: SEISMIC LAND REFRACTION SURVEY}

NUMBEP: 11.14 .2
ISSUE DATE: $5 / 8 / 92$

a. Immediately prior tu data interpretation, the Project Geophysicist shall examine the time-distance plots for completeness and acequacy of the seismic refraction coverage. Recommendations for additions and/or deletions in the field program can be made at any stage of data processing, depending upon the purpose of the project or conditions in the fieid.

b. Interpretation of the time-distance plots shall consist of spot-checking the accuracy of the traveltime readings and the resulting time-distance plot, and drawing lines-of-best-fit to the data. This allows seismic velocity values to be measured and depths to jeismic velocity interfaces to be calculated from the basic seismic critical distance formula (Dobrin ,1976):

$$
D=\frac{X}{2} \sqrt{\left(\frac{V_{2}-V_{1}}{V_{2}+V_{1}}\right)}
$$

where $X_{c}$ (the critical distance) is that distance on the surface measured from the shot point to that point where the refracted seismic wave returns to the surface as a first arrival; $V_{1}$ and $V_{2}$ are the velocity values of two (homogeneous) layers where $V_{2}$ is greater than $V_{1}$; and $D$ is the depth to the top of the underlying refracting medium.

c. The time intercept method may also be used (Dobrin, 1976). $T_{1}$ is the intercept time of the line defining the slope of the refracting layer:

$$
\text { Depth }-V_{2} * V_{2} /\left(V_{2}^{2}-V_{1}^{2}\right) \cdot 5 * T_{1} / 2
$$

When dipping layers are evident, equations for dipping layers will be used. Ensure all depth calculations appear with the appropriate set of travel-time data.

d. If necessary, commercial software may be used to perform generalized reciprocal method (GRM) interpretations of the seismic refraction data.

e. The seismic refraction data shall be plotted as velocity profiles for final presentation. Seismic 
velocity layers, thus defined, shall be compared with any other geologic or geophysical data to aid in interpreting the cause of velocity anomalies.

\subsection{Documentation of Results}

Results of the seismic refraction data interpretation shall be compiled in a report. The form and scope of the report will depend on the purpose of the seismic refraction survey. The report shall include a line location map, time-distance plots, velocity profiles, and copies of seismic records.

The report, maps, profiles and records shall be delivered to the Field Data Coordinator for the Environmental Restoration Department. If required, diskettes of the digital data shall also be provided to the Data Coordinator. The information provided to the Data Coordinator shall be complete and suitable for loading into a data base.

\section{DEFINITIONS}

Project Geophysicist - qualified geophysicist having final responsibility for the survey design, data acquisition, interpretation, and reporting for the geophysical survey. Qualifications are defined in section 2.3 above.

Field Technician - personnel performing various tasks during the geophysical survey under the direct supervision of the Project Geophysicist. Qualifications are defined in section 2.3 above.

Geophone - vibration sensitive device.

$P$ wave (compressional) - a seismic wave used in seismic land refraction survey for depth determination and material classification. This wave is transmitted through earth materials as a series of compressions and rarefactions. As a "p" wavefront passes a point in the earth, the point moves forward and backward in the direction of wave propagation, giving rise to its alternate designation as a "longitudinal" wave. The "p" wave is transmitted to subsurface strata, and is refracted back through the uppermost layers to the detectors on the ground surface.

Seismic spraad - geophones spaced at known intervals along a cable on the ground surface with end shots.

Shot - a point source inducing "p" waves.

Generalized reciprocal method - a seismic interpretation technique that is useful for interpreting seismic data from rapidly varying interfaces. 
ENVIRONMENTAL

STANDARD OPERATING

PROCEDURES MANUAL

\section{TITLE: SEISMIC LAMD REFRACTION SURVEY}

NUMBER: 11.14 .2

\section{REFERENCES}

The following references provide general discussions of magnetometers, magnetic surveying and magnetic data interpretation.

Benson, R., and J. E. Rocchio, 1990, Geophysical Techniques for Sensing Buried Wastes and Waste Miaration, Dublin, Ohio: National Water Well Association.

Dobrin, M. B., 1976, Introduction to Geophysical Prosoecting, New York: McGraw-Hi11, Inc.

U. S. Environmental Protection Agency, 1987, A Compendium of Superfund Field Operations Methods, U. S. Environmenta? Protection Agency Document PB88-181557.

Telford, W. M., L. P. Geldart, R. E. Sheriff, D. A. Keys, 1976, Applied Geophysics, New York: Cambridge University Press. 
INFORMATION ONE:

ENVIRONMENTAL RESTORATION DEPARTMENT

ENVIRONMENTAL STANDARD OPERATING PROCEDURE COVER SHEET

SOP Number: $\quad$ SOP -11.14.3

Revision:

0

Title: STANDARD OPERATING PROCEDURE FOR LAND MAGNETIC SURVEYS

Prepared by: $\frac{7 .}{\text { C. Queen }}$

N. E. Jouster, Sr. Scientist Geosciences Group
Date: $5 / 2 / 92$

Date: $5 / 8 / 92$

ERD Independent Review Committee

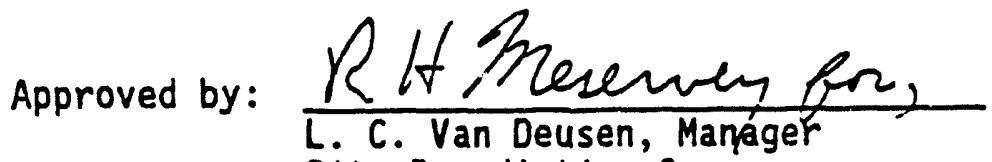

Date: $5-11-92$ Site Remediation Group

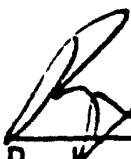

R. K. Norland, Manager

Buried Waste Program (WAG 7) Group
Approved by:

Date: $5 / 11 / 22$

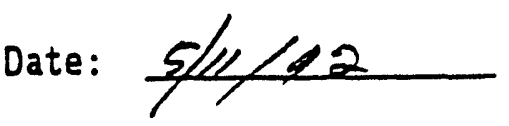

Field Changes Authorized by DRR No.: 


\begin{tabular}{|l|lr|}
\hline $\begin{array}{l}\text { ENVIRONMENTAL } \\
\text { STANDARD OPERATING } \\
\text { PROCEDURES MANUAL }\end{array}$ & TITLE: LAND MAGNETIC SURVEYS \\
\hline
\end{tabular}

\subsection{PURPOSE AND SCOPE}

This procedure provides general instructions for field personnel under the direct supervision of a Project Geophysicist to perform 1 and magnetic surveys. The purpose of a land magnetic survey is to record variations in the intensity of the earth's magnetic field. The recorded data may be used for subsequent geologic interpretations and/or detection of buried ferrous metal.

\subsection{PROCEDURE}

\subsection{Quality Assurance}

Activities conducted according to this procedure will be in compliance with an investigation-specific Quality Assurance Project Plan (QAPP) or other project level plan as applicable.

\subsection{Health and Safety}

Activities conducted according to this procedure will be in compliance with an investigation-specific Health and Safety Plan and Safe Work Permits as required.

\subsection{Training}

The Project Geophysicist supervising the 1 and magnetic survey must have relevant experience in the acquisition, processing and interpretation of geophysical data.

Field Technicians must be trained to properly operate the specific geophysical equipment that is used for the survey. This training may be provided by the qualified Project Geophysicist.

\subsection{General}

Measurements of the earth's magnetic field are made at discrete station locations using a portable magnetometer. The total magnetic fieid and/or the vertical gradient of the total field may be measured.

Measurements are normally made along linear profiles, with station spacing dependent on the estimated depth to targets. Normally, the maximum distance between stations should be from one-half to one-fourth the estimated target depth.

Areal coverage is produced by making measurements along additional profiles, usually parallel to each other. Profile spacing will depend on the nature of the targets, including the depth of the targets and their expected distribution in the subsurface. 
ENVIRONMENTAL

STANDARD OPERATING

PROCEDURES MANUAL
TITLE: LAND MAGNETIC SURVEYS

NUMBER: 11.14 .3
ISSUE DATE: $\quad 5 / 8 / 92$

Interpretations of the magnetic data are based on the identification of anomalous features in the measured magnetic data. The shape, amplitude, and steepness of magnetic anomalies are features that may be used to help identify the causative body. Independent information such as geology, other geophysical data, and cultural feature maps are used to constrain the interpretation of the magnetic data.

\subsection{Equipment}

\subsubsection{Description of Equipment}

The magnetic sensors used most often for environmental work are proton precession magnetometers and magnetic gradiometers. Proton precession instruments measure the total magnitude of the earth's magnetic field at one or two points, corresponding with the sensor element location(s). A proton precession magnetometer is sensitive and stable to with in \pm 1 gamma over the range of 20,000 to 90,000 gammas. The measurement technique is based on the fact that protons will precess about an external magnetic field at a frequency that is proportional to the external field strength. The precession frequency of the protons is related to the magnetic field strength through the proton gyromagnetic ratio, which is a fundamental atomic constant. Measuring the precession frequency of the protons allows the total magnetic field to be determined simply by dividing the observed precession frequency by the proton gyromagnetic ratio. Vertical gradient data is produced by computing the difference between the total field at the two sensor elements, and dividing by the distance between the elements.

\section{a. Sensor unit}

The sensor unit consists of two proton precession sensors spaced a fixed distance apart. Each sensor outputs an oscillating electric current that is transmitted to the control unit by means of a signal cable. The sensor unit is mounted on a non-ferrous aluminum rod. Sections may be added to the rod to vary the height of the sensors above the ground. The sensor unit is marked with a North arrow so that it can be oriented for maximum signal to noise ratio.

\section{b. Signal cable}

The signal cable is a durable, insulated conducting cable. For some magnetometers, the length of this cable is calibrated and changing the cable length will adversely affect data quality. 
ENYIRONMENTAL

STANDARD OPEPATING

PROCEDURES MANUAL
TITLE: LAND MAGNETIC SURVEYS

NUMBER: 11.14 .3
ISSUE DATE: $5 / 8 / 92$

\section{c. Control unit}

The control unit contains the electronics that analyze the sensor outputs, compute total magnetic field values for each sensor, and generate statistical information on the accuracy of the data. The control unit also contains a keyboard for programming the magnetometer function, a display for monitoring the data, and an internal memory for storing data values. An output port on the control unit is used to transfer data to a digital computer.

\subsubsection{Preparation of Equipment}

The following equipment checks and set-up procedures shall be performed prior to data acquisition. These checks shall be documented in the field notebook.

a. The control unit, sensors, and connecting cable shall be assembled per the instructions in the instrumerit's operating manual.

b. Check that batteries are adequately charged and charge or replace as required

c. The magnetometer shall be programmed for operation in total field or vertical gradient mode, and for internal recording of location coordinates, as per the instructions in the instrument's operating manual.

d. The sensitivity of the land magnetometers is preset by the manufacturer. The measurement technique of the magnetometers is based upon the principle of proton precession and no calibration is required.

e. In order to adjust the magnetometer to the magnetic field range at the survey area, tuning shall be performed prior to data acquisition on the first day of the survey. Tuning shall be performed in accordance with the instructions in the instrument's operating manual.

\subsection{Field Procedure}

\subsubsection{Location of Magnetic Survey Lines}

a. The Project Geophysicist shall determine the proposed locations of magnetic survey lines. Locations shall be noted on a line location map.

b. The accuracy and precision of the line location survey 
ENVIRONMENTAL

STANDARD OPERATING

PROCEDURES MANUAL
TITLE: LAND MAGNETIC SURVEYS

NUMBER: 11.14 .3

will depend on the objectives for the magnetic survey. Locations may be determined by optical surveying, or by taped measurements. The survey should be tied to at least one known point, which should be identified in the field notebook. Final station coordinates should be reported using a standard US Geological Survey map projection.

c. If lines are to be optically surveyed, the line location map shall be furnished to the licensed surveyor. Upon completion of surveying, the surveyor will provide the Project Geophysicist with a map showing the locations and stationing of all magnetic survey lines. If required, the surveyor will place non-metallic stakes or flagging to identify station locations.

d. If line locations are not optically surveyed, they shall be determined by the Field Technician using taped measurements. The method for laying out the magnetic survey 1 ines shall be noted in the field notebook. Non-metallic stakes and flagging will be placed as required.

e. As work progresses, additional lines may be specified by the Project Geophysicist. If specified, the additional lines should be listed or added to the line location map. Any changes to the field operation based on field conditions shall be approved by the Project Geophysicist and shall be documented in the field notebook.

\subsubsection{Data Acquisition}

a. The Project Geophysicist shall verify that the magnetic survey lines have been located as specified, and that field conditions have been noted in the field notebook.

b. A base station should be chosen within the area of the magnetometer operations. The base station location should be free from the influence of conditions which might affect readings (e.g. fences, metallic utilities or powerlines). Base station readings shall be used to assess the effect of diurnal variations in the earth's magnetic field. Such readings shall be taken throughout the day at sufficient intervals (normally not exceeding 2 hours).

c. Field stations shall be established along survey lines at intervals dependent upon the purpose of the survey. 


\begin{tabular}{|l|lrl|}
\hline $\begin{array}{l}\text { ENVIRONMENTAL } \\
\text { STANDARD OPERATING } \\
\text { PROCEDURES MANUAL }\end{array}$ & TITLE: LAND MAGMETIC SURVEYS & \\
NUMBER: & 11.14 .3 & ISSUE DATE: & $5 / 8 / 92$ \\
\hline
\end{tabular}

d. Readings shall be taken at each station. To assure optimum results, sensors are marked with an arrow or the letter "N". This mark should be pointed approximately toward magnetic north.

e. Data obtained with the magnetometer shall be stored in the instrument's internal memory and transferred to a personal computer at the conclusion of the field day. After transfer to a computer, data shall be stored on both hard disk and floppy diskette (if available), and a hardcopy listing of the data shall be produced.

\subsubsection{Documentation of Field Records}

The following information shall be documented in at least one field notebook entry:

1. project name

2. project location

3. magnetometer type and identification number.

The following information shall be documented in the field notebook on a daily basis:

1. date

2. type of survey (total field or gradiometer)

3. Tine numbers completed

4. station numbers completed

5. base station location and times of reoccupation

6. diskette numbers if applicable

7. locations of cultural features or other debris observed during data acquisition that are suspected to affect the magnetic readings collected during daily operations

8. weather conditions. 
ENVIRONMENTAL

STANDARD OPERATING

PROCEDURES MANUAL
TITLE: LAND MAGNETIC SURVEYS

NUMBER: 11.14 .3
ISSUE DATE: $5 / 8 / 92$

\subsection{Data Reduction}

\subsubsection{General}

Since field readings taken during land magnetic surveys are already expressed in magnetic total field units (gammas), the only data reduction required is the application of diurnal corrections, when necessary. Diurnal corrections are not required for vertical gradient surveys.

\subsubsection{Diurnal Corrections}

Base station readings shall be compiled and plotted so that the need for diurnal correction can be determined. This determination will depend upon the purpose(s) of the project.

If diurnal corrections are necessary, a convenient reference value within the range of base station readings for survey lines shall be selected. Using this reference value, all station readings for survey lines shall be adjusted to correct for the diurnal variations.

a. If two successive base station values are above the reference value, a linear trend shall be determined for the corresponding time interval. All stations completed during that time interval shall be corrected by subtracting the difference between the linear trend value and the reference value, based on the time of the station reading.

b. If two successive base station values are below the reference value, a linear trend shall be determined for the corresponding time interval. All stations completed during that time interval shall be corrected by adding the difference between the linear trend value and the reference value, based on the time of the station reading.

\subsection{Data Processing}

\subsubsection{Analog Data Processing}

Final magnetic values may be plotted as profiles or contour maps. Profiles shall be generated using distance along each survey traverse plotted on the $x$-axis and magnetic field strength (gamma) or vertical gradient (gamma/meter) on the $y$-axis. Contour maps shall be generated by transferring magnetic values onto an appropriate plan map (or overlay of a plan map), and then contouring the data at an appropriate contour level. 
ENVIRONMENTAL STANDARD OPERATING PROCEDURES MANUAL
TITLE: LAND MAGNETIC SURVEYS

NUMBER: 11.14 .3

Locations of surficial or other known sources of anomalous magnetic fields (e.g. power lines, pipes, fences) shall be noted on the magnecic profiles or contour maps.

Alternatively these may be shown on an overlay of the plan map.

\subsubsection{Digital Data Processing}

Digital processing may be performed on data acquired using either digital or analog recording methods. If analog data are to be processed digitally, the analog data shall be entered into a personal computer. The data may then be profiled and/or contoured.

Profiles shall be produced using commercially available software. Contour maps of the magnetic data shall be produced using commercially available software. other techniques for processing and displaying digital magnetic data may be used as necessary.

\subsection{Data Interpretation}

\subsubsection{Genera1}

Interpretation of the magnetic data shall be performed by, or under the direct supervision of the Project Geophysicist.

\subsubsection{Plotting of Known Magnetic Sources}

Known sources of anomalous magnetic values shall be noted on the profiles, contour maps, or contour-map overlays. These sources may include cultural features and geologic features such as rock outcrops and faults.

\subsubsection{Identification of Anomalous Trends}

Magnetic profiles shall be aligned to identify possible trends that may be indicative of buried metal or other targets of interest. Magnetic contour maps shall be analyzed to identify zones of anomalous magnetic field strength.

A comparison of magnetic survey results with cultural features and geologic features should be performed to evaluate obvious causes for the observed magnetic trends. The magnetic survey data should al so be compared with any other available geologic or geophysical data to aid in determining the causes for other observed magnetic trends. 
ENVIRONMENTAL STANDARD OPERATING PROCEDURES MANUAL
TITLE: LAND MAGNETIC SURVEYS

NUMBER: 11.14 .3
ISSUE DATE: $5 / 8 / 92$

\subsection{Documentation of Results}

Results of the magnetic data interpretation shall be compiled in a report. The form and scope of the report will depend on the purpose of the magnetic survey. The report shall include maps and profiles of the magnetic data, and a hardcopy listing of the data.

The report, maps, profiles and hardcopy listing shall be delivered to the Field Data Coordinator for the Environmental Restoration Department. If required, diskettes of the digital data shall also be provided to the Data Coordinator. The information provided to the Data Coordinator shall be complete and suitable for loading into a data base.

\subsection{DEFINITIONS}

Project Geophysicist - qualified geophysicist having final responsibility for the survey design, data acquisition, interpretation, and reporting for the geophysical survey. Qualifications are defined in section 2.3 above.

Field Technician - personnel performing various tasks during the geophysical survey under the direct supervision of the Project Geophysicist. Qualifications are defined in section 2.3 above.

Total Field Magnetometer - an instrument used to measure and record the earth's magnetic field intensity.

Total Field Gradiometer - an instrument composed of two magnetometers that measures and records the gradient of the earth's magnetic field intensity.

Cultural features - manmade structures such as fences, pipes, powerlines, buildings etc. that occur in the vicinity of a survey site and which may effect the recorded geophysical data.

\subsection{REFERENCES}

The following references provide general discussions of magnetometers, magnetic surveying and magnetic data interpretation.

Benson, R., R. A. Glaccum, and M. R. Noel, 1988, Geophysical Iechniques for Sensing Buried Wastes and Waste Miaration, Dublin, Ohio: National Water Well Association.

Breiner, S., 1973, Applications Manual for Portable Magnetometers, Sunnyvale, California: Geometrics.

Dobrin, M. B., 1976, Introduction to Geophysical Prospecting, New York: McGraw-Hi11, Inc. 
ENVIRCNMENTAL

STANDARD OPERATING

PROCEDURES MANUAL
TITLE: LAND MAGNETIC SURVEYS

NUMBER: 11.14 .3

U. S. Environmental Protection Agency, 1987, $\dot{E}$ Compendium of Superfund Field Ooerations Methods, U. S. Environmental Protection Agency Document PB88-181557.

Telford, W. M., L. P. Geldart, R. E. Sheriff, D. A. Keys, 1976, Applied Geophysics, New York: Cambridge University Press. 


\section{ENVIRONMENTAL RESTORATION DEPARTMENT}

DOCUMENT APPROVAL COVER SHEET

Document Number: SOP - 11.12

Title: SOIL SAMPLING

Prepared by:

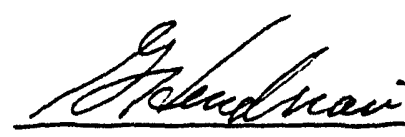

Date: $4-28-92$

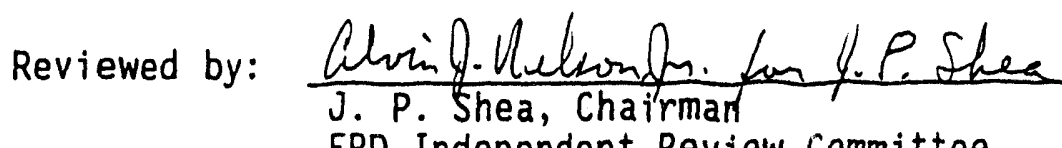

Date: $5 / 5192$

ERD Independent Review Committee

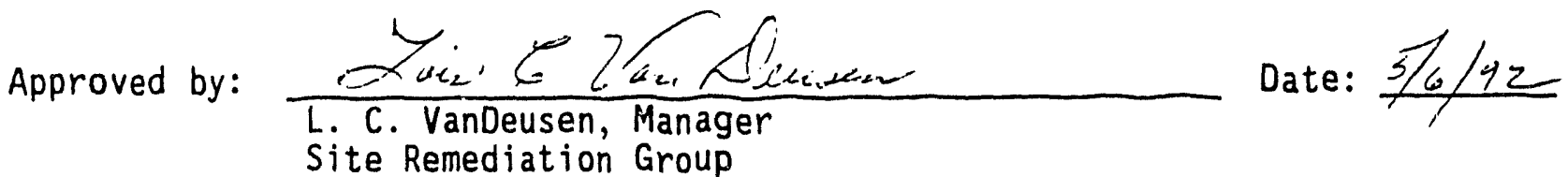

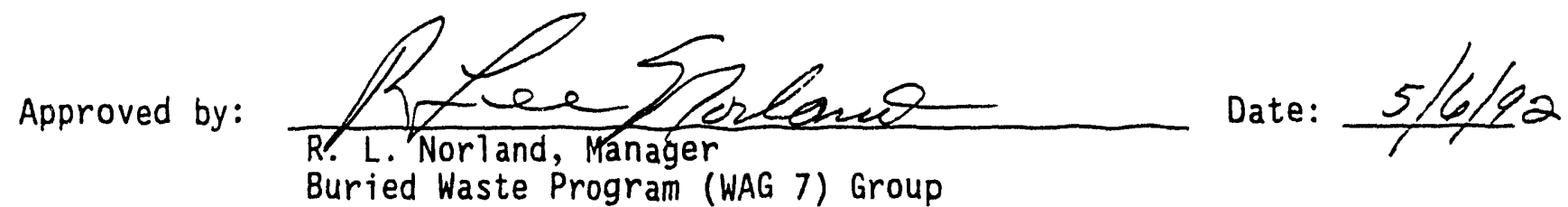

Field Changes Authorized by DRR No. 


\section{ENVIRONMENTAL STANDARD OPERATING} PROCEDURES MANUAL
TITLE: SOIL SAMPLING

NUMBER: 11.12
ISSUE DATE: $04 / 27 / 92$

\section{PURPOSE AND SCOPE}

This procedure establishes requirements for the acquisition of soil samples from surface or subsurface soils for chemical and/or radiological analyses in support of environmental investigations at the Idaho National Engineering Laboratory (INEL).

\section{PROCEDURE}

\subsection{Prerequisites}

No prerequisites other than those described in Sections 2.2 through 2.5 apply to this SOP.

\subsection{Quality Assurance}

This procedure will normally be invoked in the context of an investigation-specific Quality Assurance Project Plan (QAPjP) or other project-level $\mathrm{plan}$, and may be subject to periodic systems audits in compliance with the procedures referenced therein. Activities conducted in compliance with this procedure may also be audited as part of quality program audits performed under the auspices of the ERD Quality Program PIan, QPP-149 (EG\&G 1991a). Modifications of this procedure that may be required to suit the needs of a particular project or in response to unforseen field conditions shall be processed as a temporary Document Revision Request (DRR) in compliance with Section 2.4 of Standard Operating Procedure (SOP) 11.1, "Preparation of Environmenta1 Standard Operating Procedures" (EG\&G, 1991C). Permanent changes shall be processed in compliance with Section 2.3 of SOP 11.1 .

\subsection{Health and Safety}

All activities conducted in compliance with this procedure are subject to the applicable controls of investigation-specific Health and Safety Plans (HASPs) and Sampling and Analys is Plans (SAPs); safe work permits are required on a daily or weekly basis, depending on the safety hazards associated with activity.

\subsection{Training}

All personnel training relative to the use of this procedure shall be conducted in compliance with section QP-2 of QPP-149 (EG\&G 1991a) and Program Directive (PD) 1.3, "Employee Training" (EG\&G, 1991b) at the direction of the Project Manager. 
Page 2

ENVIRONMENTAL STANDARD OPERATING PROCEDURES MANUAL
TITLE: SOIL SAMPLING

NUMBER: 11.12
ISSUE DATE: $04 / 27 / 92$

\subsection{Precautions/Limitations}

If samples are acquired in pits, no personnel shall enter an unshored pit greater than four feet deep; the pit atmosphere must be sampled for toxic vapors and oxygen content in compliance with ERD SOP 11.10.2, "Occupational Health Monitoring" (EG\&G, 1992c) and cleared prior to entry.

\subsection{Materials and Equipment}

Materials and equipment necessary to complete the activities addressed by this procedure may include:

a. appropriate field monitoring instruments (e.g., radiation survey meter, combustible gas monitor, or organic vapor analyzer) as required by the applicable HASP, SAP, QAPJP, or cognizant health and safety representative or health physicist;

b. trowels, thief samplers, sampling tiers, hand augers, or shovels, for surface sampling;

c. Lexan plastic drive tubes, plastic end caps, and duct tape, if required by governing project plans (Note: all other downhole sampling equipment is normally provided by the drilling subcontractor);

d. hacksaw and spare blades, for trimming Lexan tubes;

e. stainless steel mixing bowl spoons, and spatulas, for sample homogenization;

f. pocket tape, in $0.1 \mathrm{ft}$ increments;

g. sample containers and preservatives, as specified in Table 1 , for the parameters of interest specified in applicable project plans;

h. sample labels, custody seals, chain of custody forms, sample analysis request forms, sample coolers, packaging materials, and shipping documents as required by ERD SOP 11.3, "Sample Chain of Custody, Packaging, and Handling" (EG\&G, 1992a);

i. personal protective equipment, as specified for potential site conditions by the site HASP; 
ENVIRONMENTAL STANDARD OPERATING PROCEDURES MANUAL
TITLE: SOIL SAMPLING

NUMBER: 11.12
ISSUE DATE: $04 / 27 / 92$

j. decontamination waste storage containers, labels, and forms as described in ERD SOP 11.1.10, "Collection, Handling, and Disposal of Waste Materials from Environmental Drilling and Sampling Operations" (EG\&G, 1992b); and

k. copies of applicable field sampling plans, SAPs, HASPs, QAPjPs included in the SAP, or other project plans, as well as field log books (see Section 2.10), blank safe work permit forms (if available), daily field activity report forms, organized in a weather-resistant portable file box; and

1. portable hand held gasoline powered auger.

\subsection{Calibration}

Measuring and test equipment used in the implementation of this procedure that requires calibration and maintenance in compliance with Section QP-12 of QPP-149 (EG\&G, 1991a) includes combustible gas monitors and OVAs/OVMs; field calibration by users shall also be required for such equipment as specified by ERD SOP 11.10.2, "Occupational Health Monitoring" (EG\&G, 1992c). All sampling equipment, bowls, spoons, spatulas and other equipment that could potentially contact sample materials shall be decontaminated after each use in compliance with ERD SOP 11.8, "Field Decontamination of Sampling Equipment" (EG\&G, 1991d), or the decontamination procedures applicable to the facility in which the work is being performed.

\subsection{Transportation}

Transportation of equipment will normally require the use of a cargo van, pickup, light or meduim-duty flatbed truck, or other vehicle. Final selection will be determined by the type and amount of equipment that is required in the field.

\subsection{Procedure}

\subsubsection{Decontamination Requirements}

All sampling equipment and ancillary items that may come in direct contact with the sample shall be decontaminated by the assigned Field Team Member prior to use in compliance with ERD SOP 11.8, "Field Decontamination of Sampling Equipment" (EG\&G, 1991d). A11 decontamination wastes should be collected and characterized for disposal as required by ERD SOP 11.1.10, "Collection, Handling, and Disposal, of 
ENVIRONMENTAL STANDARD OPERATING PROCEDURES MANUAL
TITLE: SOIL SAMPLING

NUMBER: 11.12
ISSUE DATE: $\quad 04 / 27 / 92$

\subsubsection{Sample Quantities, Types, and Documentation Requirements}

Soil samples shall be collected in the quantities and types specified in site-specific project plans. A chronological record of all sampling activities shall be maintained in compliance with ERD SOP 11.2, "Field Log Books" (EG\&G, 1992e). All samples shall be transferred to the analytical laboratory specified in governing QAPJPs or other sitespecific project plans under the formal chain of custody controls specified in ERD SOP 11.3, "Chain of Custody, Packaging, and Handling" (EG\&G, 1992a). The required analyses shall be specified on the chain of custody form and/or the sample analysis request form provided by the analytical laboratory, as noted in ERD SOP 11.3.

\subsubsection{Sample Container Selection}

Table 1 provides guidance on the type and minimum size of the sample container, preservation requirements, storage and handling requirements, and maximum holding times for the most common analyses performed on soil samples from environmental investigations at the INEL. All sample containers must be properly cleaned and prepared by the analytical laboratory or container supplier; container packages or prepared shuttles from the laboratory should be received onsite with custody seals intact. Cleanliness certifications for factory-prepared containers shall be identifiable to the shipping lot received, and shall be forwarded to the project $Q A$ records in compliance with governing QPP or QAPJP requirements.

\subsubsection{General Requirements for Subsurface Sampling}

A11 subsurface drilling-based sampling shall be conducted in conjunction with the requirements of ERD SOP 11.6, "Drilling and Installation of Monitoring Wel1s" (EG\&G, 199le) and/or ERD SOP 11.3.1, "Test Borehole Drilling and Geologic Logging" (EG\&G, 1992e), as applicable to the sampling activity. Sampling shall start at the ground surface and continue at depth intervals specified in the site-specific project plan. For auger drilling rigs, drive tube/split barrel/split spoon samples shall be collected sequentially at 5 foot depth intervals or as specified in the applicable site specific SAP or other project plan. For wireline or other coring arrangements, interbed samples shall be collected with a decontaminated core sampler as appropriate based on stratigraphy and as directed by the Field Team Leader. When any sampler is removed from the borehole, care 
ENVIRONMENTAL STANDARD OPERATING PROCEDURES MANUAL

\section{TITLE: SOIL SAMPLING}

NUMBER: 11.12
ISSUE DATE: $04 / 27 / 92$

shall be exercised when handling the sampler or breaking the drill rods, as such jarring may result in loss of all or a portion of the sample. Procedures for managing poor recovery shall be reviewed by the Field Team Leader with the drilling supervisor prior to initiating drilling and sampling activities. If any sample is lost or $\leq 50 \%$ recovery is realized, the following minimum steps shall be taken.

1. The Field Team Member shall confirm that the appropriate sample catcher are in operating order.

2. The boring shall be advanced to the bottom of last sample interval and a second sample attempt made after considering adjustments to the sampling method, which may include varying the frequency of blows used to advance the sampler, in combination with the following options:

a. if split tube or drive tube samplers are being used, letting the sampler "rest" after being driven to the appropriate sample interval; note that in all cases requiring the use of a splittube or California sampler, the sampler shall be routinely driven 18 inches from the bottom of the specified sampling interval, with blow counts recorded for each 6 -inch section;

b. advancing the sampler hydraulically, rather than driving with a hammer;

c. varying the sample catcher design; or

d. replacing the sampler drive shoe.

3. Adjust the rate or volume of circulation air; high pressures may wash away weak, soft, or unconsolidated material, preventing it from entering the core barrel. Discharge at the top of the casing should be observed for evidence of destruction to the core. Observation of rock chips instead of powdered rock indicates washing and excessive bit pressure.

If poor recovery continues, the Field Team Leader and the drilling supervisor shall contact the Project Manager for direction or refer to the direction provided in applicable site-specific project plans. 
ENVIRONMENTAL STANDARD OPERATING PROCEDURES MANUAL

\section{TITLE: SOIL SAMPLING}

NUMBER: 11.12
ISSUE DATE: $04 / 27 / 92$

All samples shall be inspected and logged by the Field Team Member. The soil shall be classified according to procedures specified in the governing site-specific project plan and recorded on the appropriate geologic log as specified in ERD SOP 11.3.1, "Test Borehole Drilling and Geologic Logging" (EG\&G, 1992e). Sample-specific characteristics such as odor, staining, or oily sheen should be noted on the geologic log. When specified by the sitespecific HASP and applicable project plan, collected samples shall be surveyed by Health Physics (HP) personnel for alpha and gross beta-gamma activity, and by appropriate volatile organics monitoring devices in compliance with ERD SOP 11.10.2, "Occupational Health Monitoring" (EG\&G, 1992C) if volatile organics are part of the parameters of interest in the investigation. All readings shall be documented in the field $\log$ book in compliance with ERD SOP 11.2, "Field Log Books" (EG\&G, 1992d) and on the associated geologic log at the corresponding depth.

If radiological survey readings are $\geq 100 \mathrm{cpm}$ above background (beta-gamma) or if any alpha is detected with field instrumentation, appropriate radiological controls shall apply to the sample handling and shipment process as described in the site-specific HASP, governing project plans, and ERD SOP 11.3, "Chain of Custody, Sample Handling, and Packaging" (EG\&G, 1992a).

Sample material shall be extracted from the sampler and transferred to the appropriate containers, which are specified by analytical category in Table 1 . Chain of custody controls shall be implemented immediately upon transfer of sample materials to the containers, in compliance with ERD SOP 11.3 (EG\&G, 1992a). Holding times shall be considered to have begun when each sample container is closed; sample numbers corresponding to those entered on the chain of custody forms shall be entered into the field $\log$ book.

\subsubsection{Subsurface Soil Sample Acquisition Methods}

Subsurface soil sample acquisition methods shall be as specified by applicable project plans or by the Project Manager, and shall be implemented by the Field Team Leader and/or Field Team Member, as specified therein. Several routine methods are described below: 
ENVIRONMENTAL

STANDARD OPERATING PROCEDURES MANUAL

\section{TITLE: SOIL SAMPLING}

NUMBER: 11.12
ISSUE DATE: $04 / 27 / 92$

a. Split-Tube Sampling Method: The sampling horizon may be exposed by any drilling technique that will produce suitable wall clearance for insertion of the sampler. Depth to the sample horizon shall be measured using the combined lengths of the downhole tools, drill rod or auger flight lengths, and amount of stickup above the drill collar. Particular attention must be paid to the calculated depth to ensure that the sampler (see Figure 1) is resting on the desired sample interval. Sampler diameter selection shall be based on geologic logging observations. 2 -inch diameter samplers are appropriate for nonlithified clays, silts, sands and fine gravels; 4 - or 6-inch samplers shall be selected for zones with coarse gravels and cobbles, or when larger sample volumes are required. Samplers shall be driven to the depth specified in the project specific plan or 18 inches with the drive weight (noting the weight of the hammer being used); blow counts for the first 18 inches of penetration shall be recorded in 6 -inch increments, counted, and recorded on the Borehole Log. The downhole bit or auger, the split tube sampler, liners and the spatulas or spoons shall be decontaminated prior to use in compliance with ERD SOP 11.8, "Field Decontamination of Sampling Equipment" (EG\&G, 1991d).

b. Thin-Walled ("Shelby") Tube Sampling Method: This method is normally used to obtain undisturbed samples of cohesive soils or sludges. Any drilling technique is acceptable to expose the sampling horizon provided that sufficient clearance is present to permit insertion of the sampling equipment. Depth measurements shall be based on cumulative measurements of drill rods or auger flights, downhole tool length, and amount of stick up at the drill collar. Particular attention must be paid to the depth calculations to ensure that the sampler is resting on the desired sample interval. The sample tube is attached to an appropriate Shelby head subassembly, which is then connected to the drill rods or auger flights, and inserted to a maximum of 15 tube diameters by steady pressure with no rotation. Depending upon soil conditions, the tube shall be left in place for 5-10 minutes to dissipate negative pressure prior to withdrawal of the tube. Depending on project-specific requirements, the sample tube may be capped, labeled, sealed, and routed to cognizant 
EG\&G shipping authority and the laboratory in compliance with SOP 11.3, "Chain of Custody, Sample Handling, and Shipping" (EG\&G, 1992a). Alternately, the sample may be exposed at the surface, removed with a stainless steel spatula and transferred to a suitable container after visual examination. The downhole drilling tool, insertion tool, sample tube, and stainless spatula shall be decontaminated prior to use in compliance with ERD SOP 11.8, "Field Decontamination of Sampling Equipment" (EG\&G, 1991d).

c. Drive Tube (Ring-lined Barrel or "California") Sampling Method: This method may be used to obtain environmental samples, and is especially appropriate when volatile organic compounds are among the analytes of interest. The sampling horizon may be exposed by any drilling method that will produce suitable wall clearance for insertion of the sampler (see Figure 2). Depth to the sample horizon shall be measured using the combined lengths of the downhole tools, drill rod or auger flight lengths, minus the amount of stick up above the drill collar. Particular attention must be paid to the depth calculations to ensure that the sampler is resting on the desired sample interval. Samplers shall be driven to the interval specified in the SAP or 18 inches (if not specified) with a surface or downhole drive weight assembly (note the weight of the hammer being used in the field $10 \mathrm{~g}$ ). If required by project-specific requirements, blow counts of the first 18 inches of penetrations shall be counted and recorded in 6 -inch increments on the borehole $10 \mathrm{~g}$. The sampler shall be retrieved and carefully disassembled. Trim the soil flush with the sampling barrel with the stainless steel spatula, and remove the sample-filled rings. Place each ring in a suitable container and cap and seal with clean plastic caps at both ends. Downhole samplers and stainless steel spatulas shall be decontaminated prior to use; excess sample material shall be collected and disposed of in compliance with ERD SOP 11.1.10, "Collection, Handling, and Disposal of Waste Materials from Environmental Drilling and Sampling Operations" (EG\&G, 1992b).

d. Wireline and Lined Double-Wall Core Barrel Method: Wireline methods are preferred for the acquisition of samples in the sediment interbeds commonly found 
ENVIRONMENTAL STANDARD OPERATING PROCEDURES MANUAL

\section{TITLE： SOIL SAMPLING}

NUMBER: 11.12
ISSUE DATE: $04 / 27 / 92$

d. Wireline and Lined Double-Wall Core Barrel Method: Wireline methods are preferred for the acquisition of samples in the sediment interbeds commonly found between basalt flows at the INEL. The sediment horizon shall be exposed using the coring methods described in ERD SOP 11.3.1, "Test Borehole Drilling and Geologic Logging" (EG\&G, 1992e), and the driller shall be instructed to conduct a sampling run. Interbed samples shall be collected with a decontaminated lined dual-wall core sampler with Lexan or steel liner as specified by the site-specific project plan, or as directed by the Project Manager or Field Team Leader. If a Lexan inner barrel liner is used, it may not be necessary to remove the sample material for transfer to a sample container. If permitted by the site-specific project plans, the sample may be left undisturbed, the Lexan tube trimmed with a hacksaw, and the ends sealed with plastic caps and duct tape. Samples so prepared may be subcored if potential contamination of the sample is a primary concern, or if special atmospheres or sample transfer situations are necessary. If Lexan liners are not used, the sample material shall be removed using stainless steel spoons or a stainless steel spatula and transferred to a suitable container after visual examination. The downhole drilling tool, insertion tool, sample tube, and stainless steel spatula shall be decontaminated prior to use in compliance with ERD SOP 11.5, "Field Decontamination of Sampling Equipment" (EG\&G, 1991d).

e. Other methods may be outlined in project specific documentation.

\subsubsection{Acquisition of Surface Samples}

Surface samples may be acquired from the ground surface, from containers of cuttings, or from test pits exposed with backhoes or shovels. Sample acquisition methods to be implemented by the Field Team Member and/or Field Engineer may be selected from the following, as appropriate, for the soils being sampled and as specifically directed by sitespecific project plans or the Project Manager:

a. Thief Sampler: The thief sampler (see Figure 3) consists of two slotted concentric stainless steel tubes with pointed tip; the inner tube may be rotated 
ENVIRONMENTAL STANDARD OPERATING PROCEDURES MANUAL
TITLE: SOIL SAMPLING

NUMBER: 11.12
ISSUE DATE: $04 / 27 / 92$

its slots. To take a sample, close the sampler and insert it into the soil to the desired sampling interval. Rotate the inner tube to open the sampler, and tap to introduce a flow of soil into the interior. Close the sampler, withdraw it from the soil, and lay it horizontally with the slots facing upward. Remove the inner tube and transfer the sample to the appropriate container.

b. Sampling Trier: The sampling trier (see Figure 4) should be used in sticky solid soils with a particle size less than one half of the trier diameter; insert the trier into the sampling interval at an approximately 45 degree angle, and rotate the handle 360 degrees to cut a core of material. Withdraw the trier with the concave side up, and transfer the sample material to an appropriate container.

c. Hand Auger: Hand augers should be used in hard packed soils or sediments. Insert the auger through the catchpan at the desired sampling location and auger to the required interval. Withdraw the auger and transfer the sample material in the catchpan (or that may have adhered to the auger surface) to an appropriate container.

d. Grab Samples: When permitted by site-specific project plans, grab samples may be taken with a decontaminated shovel or trowel and directly transferred to an appropriate container.

\subsection{Data Reduction, Calculations, and Reporting}

The Field Team Leader is responsible for completion of all documentation and for final delivery to the ERD ARDC. Records required as the result of soil sampling activities include the following:

a. bound field $\log$ books providing a chronological record of all drill site monitoring activities, prepared in compliance with ERD SOP 11.2, "Field Log Books" (EG\&G, 1992e);

b. chain of custody forms, completed in compliance with the requirements of ERD SOP 11.3, "Chain of Custody, Sample Handling, and Packaging" (EG\&G, 1992a); and 
ENVIRONMENTAL STANDARD OPERATING PROCEDURES MANUAL

\section{TITLE: SOIL SAMPLING}

NUMBER: 11.12
ISSUE DATE: $04 / 27 / 92$

c. copies of all safe work permits.

All records shall be routed to the project $Q A$ records files as specified by governing QAPJPS or QPPS.

\section{DEFINITIONS}

Surface sample refers to a sample taken from the land surface down to a depth of 6 inches, unless otherwise defined in governing SAPs or other project plans.

\section{REFERENCES}

ASTM, 1991; Annual Book of ASTM Standards; American Society for Testing and Materials, Philadelphia, Pennsylvania.

EG\&G, 1991a; Quality Program Plan for the Environmental Restoration Program, QPP-149; EG\&G Idaho, Inc., Idaho Falls, Idaho.

EG\&G 1991b; Program Directive 1.3, "Employee Training"; EG\&G Idaho, Inc., Idaho Falls, Idaho.

EG\&G, 1991c; Standard Operating Procedure 11.1, "Preparation of Environmental Standard Operating Procedures"; EG\&G Idaho, Inc., Idaho Falls, Idaho.

EG\&G, 1991d; Standard Operating Procedure 11.5, "Field Decontamination of Sampling Equipment"; EG\&G Idaho, Inc., Idaho Falls, Idaho.

EG\&G, 1991e; Standard Operating Procedure 11.6, "Drilling and Installation of Monitoring Wells"; EG\&G Idaho, Inc., Idaho Falls, Idaho.

EG\&G, 1992a; Standard Operating Procedure 11.3, "Chain of Custody, Sample Handling, and Packaging"; EG\&G Idaho, Inc., Idaho Falls, Idaho.

EG\&G, 1992b; Standard Operating Procedure 11.1.10, "Collection, Handling, and Disposal of Waste Materials from Environmental Drilling and Sampling Operations"; EG\&G Idaho, Inc., Idaho Falls, Idaho.

EG\&G, 1992c; Standard Operating Procedure 11.10.2, "Occupational Health Monitoring"; EG\&G Idaho, Inc., Idaho Falls, Idaho.

EG\&G, 1992d; Standard Operating Procedure 11.2, "Field Log Books"; EG\&G Idaho, Inc., Idaho Falls, Idaho. 
Page 12

ENVIRONMENTAL

STANDARD OPERATING

PROCEDURES MANUAL
TITLE: SOIL SAMPLING

NUMBER: 11.12

EG\&G, 1992e; Standard Operating Procedure 11.3.1, "Test Borehole Drilling and Geologic Logging"; EG\&G Idaho, Inc., Idaho Falls, Idaho.

EPA, 1986; SW-846, Test Methods for Evaluating Solid Waste; U.S.

Environmental Protection Agency, Washington, D.C.

EPA, 1986; Soil Sampling Quality Assurance Users Guide; U.S.

Environmental Protection Agency, Washington, D.C.

DOE, 1989; The Environmental Survey Manual, Appendix E, Field Sampling Protocols and Guidance, U.S. Department Of Energy, Office of Environmental Audits, Washington, D.C. 
ENVIRONMENTAL STANDARD OPERATING PROCEDURES MANUAL
TITLE： SOIL SAMPLING

NUMBER: 11.12

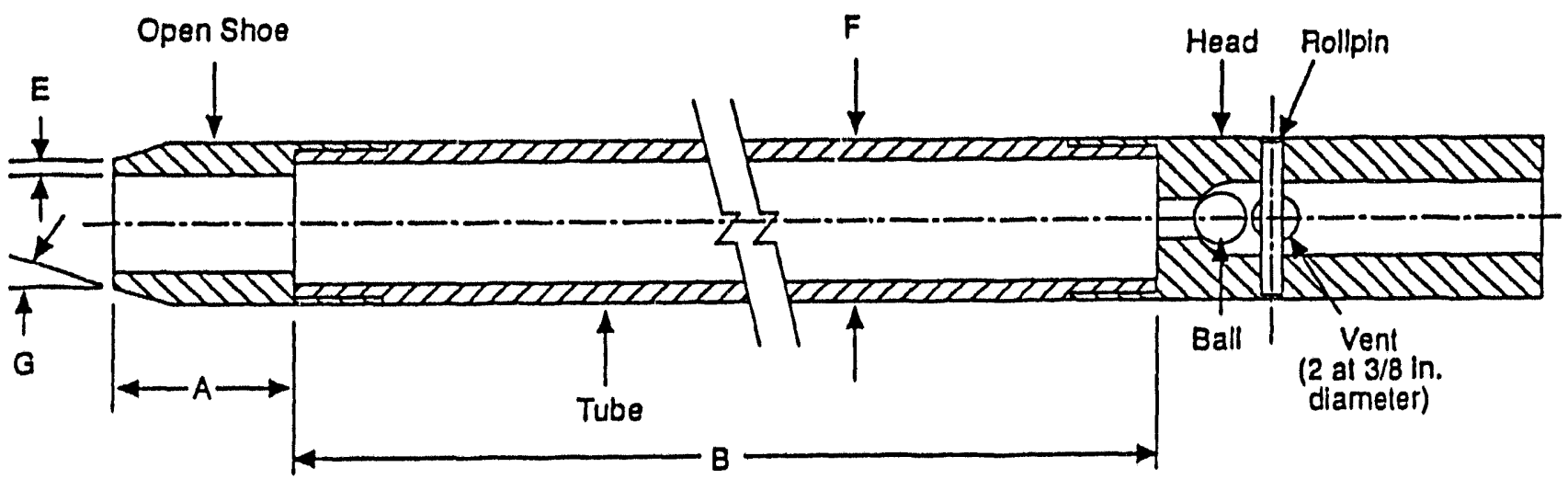

Notes:

$A=1.0102 .0 \mathrm{in} .(251050 \mathrm{~mm})$

$B=18.0$ to 30.0 in. $(0.457$ to $0.762 \mathrm{~m})$

$C=1.375 \pm 0.005-0.00$ in. $(34.93 \pm 0.13 \mathrm{~mm})$

$D=1.50 \pm 0.05 \cdot 0.00 \mathrm{ln} .(38.1 \pm 1.3 \cdot 0.0 \mathrm{~mm})$

$E=0.10 \pm 0.02 \mathrm{in} .(2.54 \pm 0.25 \mathrm{~mm})$

$F=2.00 \pm 0.05 \cdot 0.00 \mathrm{in} .(50.8 \pm 1.3 \cdot 0.0 \mathrm{~mm})$

$\mathrm{G}=16.0^{\circ} 1023.0^{\circ}$

The $11 / 2 \mathrm{in.} \mathrm{(38} \mathrm{mm)} \mathrm{inside} \mathrm{diameler} \mathrm{split} \mathrm{barrel} \mathrm{may} \mathrm{be} \mathrm{used} \mathrm{wilh} \mathrm{a} \mathrm{16-gage} \mathrm{wall} \mathrm{thickness} \mathrm{split}$ liner. The penetrating end of the drive shoe may be slighlly rounded. Metal or plastic retainers may be used to relain soil samples.

Source: ASTM D-1586 (ASTM, 1991) 
ENVIRONMENTAL

STANDARD OPERATING

PROCEDURES MANUAL
TITLE: SOIL SAMPLING

NUMBER: 11.12

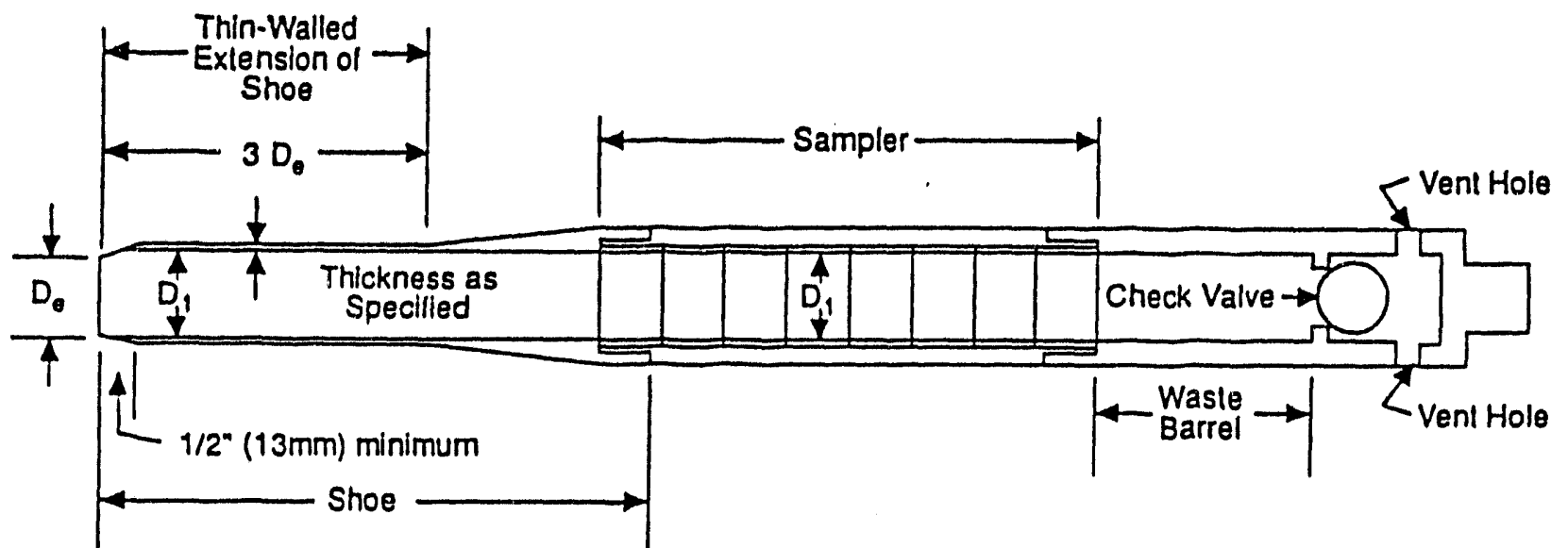

Noles:

1. Inside clearance ratio $=\left(D_{1}-D_{\theta}\right) / D_{\theta}$

2. Dimensional tolerance of $D_{1}= \pm 0.003$ in $( \pm 0.08 \mathrm{~mm}$ )

Source: ASTM D-3550 (ASTM, 1991)

FIGURE 2

RING LINED BARREL ("CALIFORNIA") SAMPLER 
Page 15

ENVIRONMENTAL

STANDARD OPERATING

PROCEDURES MANUAL.
TITLE: SOIL SAMPLING

NUMBER: 11.12

ISSUE DATE: $04 / 27 / 92$

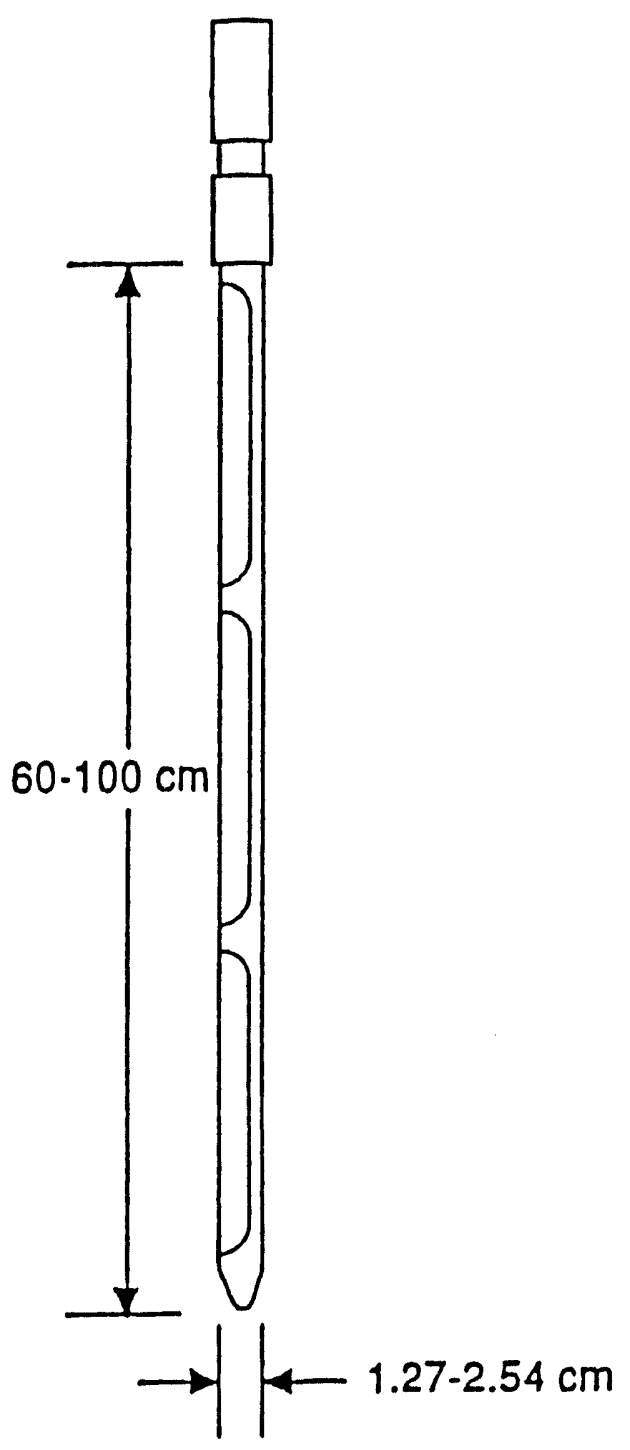

Source: SW-846 (EPA, 1986)

FIGURE 3

THIEF SAMPLER 
Page 16

ENVIRONMENTAL

STANDARD OPERATING

PROCEDURES MANUAL
TITLE: SOIL SAMPLING

NUMBER: 11.12

ISSUE DATE: $04 / 27 / 92$
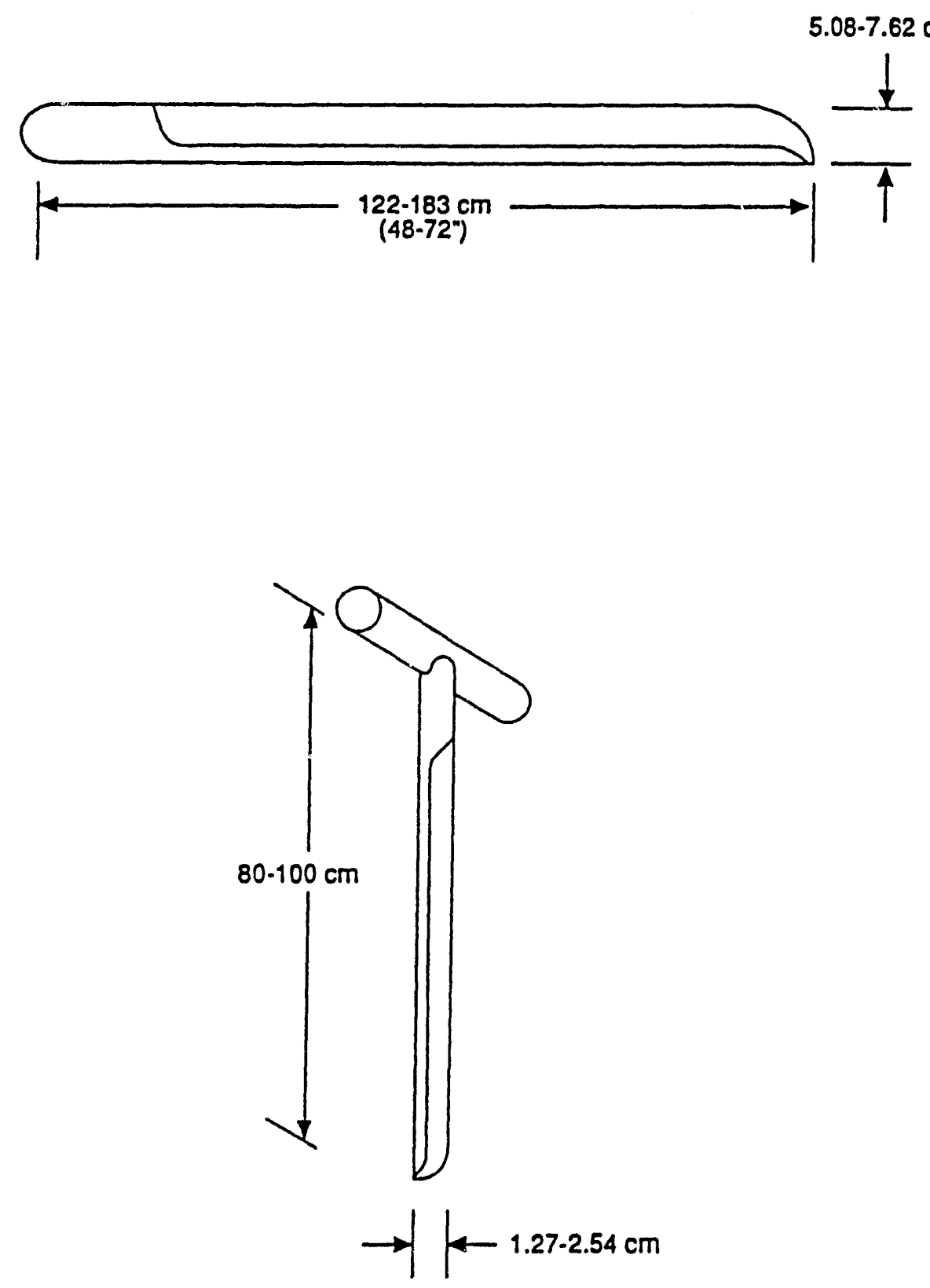

Sonrce: SW-846 (BPA, 1986)

FIGURE 4

SAMPLING TRIER 
ENVIRONMENTAL STANDARD OPERATING PROCEDURES MANUAL
TITLE: SOIL SAMPLING

NUMBER: 11.12

ISSUE DATE: $04 / 27 / 92$

\section{TABLE 1}

SAMPLE CONTAINERS, PRESERVATION REQUIREMENTS, AND BOLDING TIMES FOR ENVIROMMENTAL SOIL SAMPLES

\begin{tabular}{|c|c|c|c|}
\hline ANALYSIS & CONTAINER & PRESERVATION & HOLDING TIME ${ }^{2}$ \\
\hline $\begin{array}{l}\text { Anions, water } \\
\text { extractable } \\
\text { (Sulfate, N1trate, } \\
\text { Nitrite, Bromide, } \\
\text { Fluoride, Phosphate) }\end{array}$ & $40 z ., P$ & $\begin{array}{l}\text { Cool, } 4^{\circ} \mathrm{C} \text {, do not } \\
\text { Ireeze }\end{array}$ & $\begin{array}{l}28 \text { days except nitrate and } \\
\text { nitrite, which are } 14 \text { days }\end{array}$ \\
\hline Ammonia as $\mathrm{N}$ & $40 z ., P$ & $\begin{array}{l}\text { Cool, } 4^{\circ} \mathrm{C} \text {, do not } \\
\text { Ereeze }\end{array}$ & 28 days \\
\hline sulfide & $402,, P$ & $\begin{array}{l}\text { Cool, } 4^{\circ} \mathrm{C} \text {, do not } \\
\text { freeze }\end{array}$ & 28 days \\
\hline Cyanide & $40 z . . P$ & $\begin{array}{l}\text { Cool, } 4^{\circ} \mathrm{c} \text {, do not } \\
\text { ereeze }\end{array}$ & 14 days \\
\hline $\begin{array}{l}\text { O1I and grease (freon } \\
\text { extractable) }\end{array}$ & $402 \ldots, 6$ & $\begin{array}{l}\text { Cool, } 4^{\circ} \mathrm{C} \text {, do not } \\
\text { freaze }\end{array}$ & 28 days \\
\hline Total Organic Carbon & $40 z ., G$ & $\mathrm{Cool}, 4^{\circ} \mathrm{C}$ & 28 days \\
\hline $\begin{array}{l}\text { Metals except mercury } \\
\text { and chromiun vI }\end{array}$ & $40 z, P$ & none required & 6 wonths \\
\hline $\begin{array}{l}\text { Chromium VI (water } \\
\text { extractable) }\end{array}$ & 402,2 & none required & $\begin{array}{l}6 \text { months before extraction, } \\
24 \text { hours after extraction }\end{array}$ \\
\hline Mercury & $402, ?$ & none required & 28 days \\
\hline volatile organics & $\begin{array}{l}\text { Two } 2 \text { oz., G, teflon- } \\
\text { lined cap, wtth } \\
\text { minimum headspace }\end{array}$ & $\begin{array}{l}\text { Cool, } 4^{\circ} \mathrm{C} \text {, do not } \\
\text { freeze }\end{array}$ & 7 days \\
\hline Semivolatile organics & $\begin{array}{l}8 \text { oz, G, teflon I1ned } \\
\text { cap }\end{array}$ & $\begin{array}{l}\text { Cool, } 4^{\circ} \mathrm{C} \text {, do not } \\
\text { freeze }\end{array}$ & $\begin{array}{l}7 \text { days until extraction, } 40 \\
\text { days to analys } 1 \mathrm{~s}\end{array}$ \\
\hline Pest1c1de/PCBg & $\begin{array}{l}8 \mathrm{oz}, \mathrm{G}, \text { tetlon I1ned } \\
\text { cap }\end{array}$ & $\begin{array}{l}\text { Cool, } 4^{\circ} \mathrm{C} \text {, do not } \\
\text { freeze }\end{array}$ & $\begin{array}{l}7 \text { days until extraction, } 40 \\
\text { days to analys } 18\end{array}$ \\
\hline Berbicides & $\begin{array}{l}8 \mathrm{oz}, \mathrm{G}, \text { teflon I1ned } \\
\text { cap }\end{array}$ & $\begin{array}{l}\text { Cool, } 4^{\circ} \mathrm{C} \text {, do not } \\
\text { Ereeze }\end{array}$ & $\begin{array}{l}7 \text { days unt } 11 \text { extraction, } 40 \\
\text { days to analysis }\end{array}$ \\
\hline Phosphate Pesticides & $\begin{array}{l}8 \circ z, G, \text { teflon 11ned } \\
\text { cap }\end{array}$ & $\begin{array}{l}\text { Cool, } 4^{\circ} \mathrm{C} \text {, do not } \\
\text { freeze }\end{array}$ & $\begin{array}{l}7 \text { days until extraction, } 40 \\
\text { days to analysis }\end{array}$ \\
\hline D1oxins/Furans & $\begin{array}{l}\text { o oz, G, teflon lined } \\
\text { cap }\end{array}$ & $\begin{array}{l}\text { Cool, } 4^{\circ} \mathrm{C} \text {, do not } \\
\text { Ereeze }\end{array}$ & $\begin{array}{l}7 \text { days unt1l extraction, } 40 \\
\text { days to analys } 1 \mathrm{~s}\end{array}$ \\
\hline Rad1onucl1des & $802, P$ & none required & $\begin{array}{l}6 \text { months of } 5 \text { half-lives, } \\
\text { whlchever is less }\end{array}$ \\
\hline
\end{tabular}

P = Polyethylene

G = Glass

Sources: Test Methods for Evaluating Solid Waste (SW-846) (EPA, 1986) and 40 CFR 136.3, Table II. 


\section{Standard Operating Procedure}

\section{Hydrolab Operation}

Procedure: Calibration and Operation of the Hydrolab Surveyor II for $\mathrm{pH}$, temperature, and conductivity measurements.

References: Operating Manual, Hydrolab Surveyor II, Hydrolab Corporation, 1986

Description:

Types of Data Collected: Information obtained from this procedure includes measurements of aqueous samples for $\mathrm{pH}$, temperature, and conductivity. Additional procedures included in the Hydrolab Operating Manual provide the means for measuring dissolved oxygen, Oxidation-Reduction Potential, and depth.

Principle of Operation: After calibration procedures are completed, water samples are collected from the well head (or other water source) and measurements taken for $\mathrm{pH}$, temperature, and conductivity. If dissolved oxygen and/or ORP measurements are necessary, they are also carried out assuming proper calibration procedures have been completed for these parameters. Field measurements for $\mathrm{pH}$, temperature and conductivity will be collected during both well development and well purging activities to monitor groundwater quality.

Limitations: There are generally no limitations for the Hydrolab other than instrument design limitations, if proper calibration, operation and handling are observed. For additional information, see the Operation Manual for the Hydrolab.

Equipment Needs:

1) System parts: There are five main components to the basic Surveyor II. These are the display unit, the data cable, the sonde, the sample circulator, and the battery pack (see operator's manual for component function).

2) Calibration equipment: The following materials and equipment will always be needed to carry out the calibration operations:

a. The CALIBRATION CUP and CALIBRATION CUP SOFT COVER.

b. A supply of DEIONIZED WATER.

c. A supply of GRANULAR POTASSIUM CHLORIDE REAGENT.

d. The USE of an ANALYTICAL BALANCE.

e. A small supply of QUINHYDRONE REAGENT. 
f. BUFFER STANDARDS of $\mathrm{pH} 4.0,7.0,10.0$

g. DISSOLVED OXYGEN SENSOR MEMBRANES

h. SCISSORS

i. SOFT PAPER WIPERS (Kleenex)

j. A 1000-MILLILITER VOLUMETRIC FLASK

k. A 500-MILLILITER VOLUMETRIC FLASK

1. A 100-MILLILITER VOLUMETRIC FLASK

m. A VISE (CLAMP) for holding the Sonde included with Surveyor II.

The mechanical items that you will need continuously during calibrations are the VISE (clamp) and the CALIBRATION CUP and COVER. The vise or clamp stand should be sturdily mounted to the benchtop near your deionized water supply and preferably near a sink. 
Precautions:

\section{Safety Considerations:}

1. If the sample media is suspected of or known to contain hazardous and/or radiologic contaminants, appropriate personal protective equipment and monitoring will be implemented as outlined in the site specific Health and Safety Plan.

2. After measurements have been carried out, sample water will be disposed of properly.

3. All equipment should be decontaminated after each use.

\section{Transportation Considerations: Initial calibrations are often}

completed in the laboratory prior to leaving for the field site. In this case, excessive shock or vibration should be avoided as it can cause cumulative damage to sensitive system components, as well as negate calibrations. Cushion the instrument from vibrations.

\section{Special Training Requirements: None}

\section{Additional Precautions:}

1. Do not let the sensors dry out. This can be avoided by keeping the storage cap, containing a little water, in place on the sonde.

2. Don't allow the instrument to lie in direct sunshine or in such places as the closed trunk of a car, particularly in summer, for long periods because the temperatures inside the sealed Display Unit and Sonde rise to very high levels. This will cause no permanent damage but the operation of the system may be temporarily impaired (stability, calibration). Keeping the instrument in ventilated shade will avoid the heat problem.

3. Very low temperatures should also be avoided. Try to keep the temperatures of the Display Unit and Sonde higher than $-10^{\circ} \mathrm{C}$. Temperatures below $-50^{\circ} \mathrm{C}$ might cause damage to vulnerable parts such as the liquid crystal display of the Display Unit.

Calibration/Standardization:

It is not necessary to wait until you arrive at the field site to calibrate; however, wherever calibrations are performed, record values in the field log book. It is much easier to do 
careful, accurate calibrations under laboratory conditions and the stability of the Surveyor II is such that it will readily maintain calibration during transportation. If you feel better checking calibration in the field, do so of course but treat the findings as an intermediate post-calibration: record the results but do not adjust the system. Adjusting the instrument in the field destroys the value of the reliable laboratory post-calibration and may lead to loss of data quality control.

Note: Do not turn the Surveyor II off between calibration steps or your calibration factors will not be saved. Instructions for saving calibration factors are discussed below.

Frequency: The Surveyor II should be calibrated each time it is taken into the field. It is, however, not necessary to calibrate the Hydrolab between each sample.

Who Performs: Field personnel familiar with the calibration and operation of the Hydrolab.

\section{Criteria for Timing:}

\section{Procedures:}

Conductivity: The most precise data will be obtained if the Surveyor II conductivity system is calibrated to fit the characteristics of the waters being investigated. That is, if measurements are to be made in fresh water, the low conductivity range of the instrument should be calibrated and the mid and high ranges ignored. Or, if it is seawater that is being surveyed, the high conductivity range should be calibrated and the low and mid ranges ignored. (NOTE: The Surveyor II automatically switches to the range appropriate to the conductive values currently being measured, so you may have the feeling that there is only ONE range. Actually there are three: $0-1.5 \mathrm{mmhos} / \mathrm{cm}, 1.5-15 \mathrm{mmhos} / \mathrm{cm}$, and $15-150 \mathrm{mmhos} / \mathrm{cm}$ with the standard cell; and 0-0.3 mmhos $/ \mathrm{cm}, 0.3-3 \mathrm{mmhos} / \mathrm{cm}$, and 3-30 mmhos $/ \mathrm{cm}$ with the D-5 cell attachment in place. If the D-5 cell is required, consult the operating manual.

Standard solutions of potassium chloride salt are used for calibrating the conductive system of the Surveyor II. For maximum accuracy as well as convenience, it is best to 
make up concentrated stock solutions of the salt (1 molar is suggested) and then to dilute to the concentrations desired. If the stock solution is always made the same, the weighings are always the same with less chance for number errors. And most important, there is no necessity for weighing out tiny amounts of $\mathrm{KC1}$ in order to achieve low concentrations. The conductivity ranges of the Surveyor II instrument have been so selected that simply by diluting a 1 molar $\mathrm{KC} 1$ solution by multiples of 2 and 10, two benchmark solutions can be prepared for each of the low, mid, and high ranges. For example, the low conductivity range can be calibrated at 1.413 millimhos/cm using a 0.01 molar $\mathrm{KC} 1$ solution. To achieve that concentration, dilute the 1.0 molar stock solution by 10 TWICE. If desired, check the low range at 0.718 millimhos $/ \mathrm{cm}$ by using the solution resulting after diluting the $1.413 \mathrm{millimhos} / \mathrm{cm}$ solution by two.

A slightly different example would be preparing for seawater measurements. Seawater conductivities, of course, run in the low 50's millimhos/cm, so it would be best to calibrate the high range NOT at 111.9 millimhos $/ \mathrm{cm}$ but at $58.64 \mathrm{millimhos} / \mathrm{cm}$ using the 0.5 molar solution obtained by diluting the stock solution by 2 .

Similarly, if you knew that your measurements would most probably lie in the neighborhood of 0.400 millimhos/cm, the low range would be better calibrated at 0.718 instead of 1.413 millimhos/cm.

In other words, try to calibrate above but as near as possible to the expected data range. However, if your field data should take an unexpected turn and fall into an uncalibrated range, don't worry about it. Go ahead and take your readings, calibrate the range later (post-cal), and correct the readings if required. 
LOW RANGE

0

0.718

$1.413 \mathrm{mmho} / \mathrm{cm}$

0

$0.005 \mathrm{M}$

$0.01 \mathrm{M}$ conc.

MID RANGE

0

6.668

$12.900 \mathrm{mmho} / \mathrm{cm}$

0

$0.05 \mathrm{M}$

O.1M conc.

HIGH RANGE

0

58.640

$111.900 \mathrm{mmho} / \mathrm{cm}$

0

$0.5 \mathrm{M}$

$1 . \mathrm{M}$ conc.

To make the stock solution, weigh out carefully 74.557 grams of reagent grade $\mathrm{KC1}$.

Wash the $\mathrm{KC} 1$ into a $1000 \mathrm{ml}$ volumetric flask and fill to mark with deionized water.

Be sure that the $\mathrm{KC} 1$ dissolves completely before use. The conductivity of the stock solution will be 111.900 millimhos/cm.

TO DILUTE BY 2: Fill the $500 \mathrm{ml}$ volumetric flask to the mark with the solution to be diluted. Transfer the $500 \mathrm{ml}$ into the clean $1000 \mathrm{ml}$ flask. Fill to the mark with deionized water.

TO DILUTE BY 10: Fill the $100 \mathrm{ml}$ flask to the mark with the solution to be diluted. Transfer the contents of the $100 \mathrm{ml}$ flask to the clean $1000 \mathrm{ml}$ flask and fill to the mark with deionized water.

It is safe to keep the stock solution for a few weeks in a tightly sealed, all-glass container, but evaporation and contamination should be kept in mind. if there is any doubt, make a fresh stock solution.

The critical items in the conductivity calibration are TEMPERATURE and KCL CONCENTRATION. Begin as follows:

1. Prepare the standard $\mathrm{KC1}$ solution whose conductivity will lie near the top of the range of your measurements. 
2. Without removing the calibration cup, rinse the inside of the cup and sensor array several times with deionized water. If your deionized water comes from a tap, remove the sonde from the vise and hold the calibration cup under the tap.

3. Fill the calibration cup about $2 / 3$ with your standard. Cover the cup and shake the Sonde to flush the sensors thoroughly with the standard. Discard the standard in the calibration cup and repeat. Discard the second portion of standard. This procedure is to insure that the concentration of the standard in the cup is what you want it to be.

5. Put the Sonde in the vise, cup up.

6. Fill the cup to within about a centimeter of the top with standard. Be sure that no bubbles are trapped in the bores of the sensor.

7. Switch to TEMPerature. You should find it quite stable by now.

8. Switch to CONDuctivity. Use the SLOPE control to adjust the displayed reading to the value of your standard.

9. The conductivity system is calibrated.

$\mathrm{pH}$ calibration: Except in certain special situations, $\mathrm{pH}$ buffer solutions are the universal standards for $\mathrm{pH}$ calibration. There are buffers available with values over virtually the whole $\mathrm{pH}$ range, but the ones used the most have (at $25^{\circ} \mathrm{C}$ ) the $\mathrm{pH}$ values 4.0, 7.0, and 10.0. Supplied in powder form, buffers should be prepared with deionized water according to the instructions provided. Buffer solutions will keep for a few weeks at room temperature and so can be made up and stored for calibration purposes.

For best precision, you should calibrate for the range of $\mathrm{pH}$ values expected in the field. That is, for high $\mathrm{pH}$ waters calibrate at 7.0 and 10.0. For acid water, calibrate at 7.0 and 4.0.

Begin the $\mathrm{pH}$ system calibration as follows:

1. With the calibration cup screwed into place, flush the cup and sensors thoroughly with deionized water. Secure the Sonde in the vise, cup up.

2. Fill the cup with $\mathrm{pH} 7.0$ buffer sufficient to cover the membrane of the DO sensor. This is IMPORTANT because the DO sensor plays an indirect but essential part in the $\mathrm{pH}$ measurement. 
3. Allow a minute or two for thermal equilibration. The importance of thermal equilibrium to measurements can hardly be overstated, not only during calibration but also during measurements in the field.

4. Switch to $\mathrm{pH}$. Now use the ZERO CONTROL to set the displayed value to 7 . CAUTION: Be sure to use the ZERO control. The slope control will not have the correct effect.

5. Pour out the 7.0 buffer and flush the sensors with deionized water. CAUTION: It is hard to resist saving the buffer solutions used for calibration and if very careful, they can be retained and used a couple of times without causing errors. But dilution and cross-contamination should be kept in mind as sources of trouble. Whenever in doubt, mix fresh buffers.

6. Fill the cup with $\mathrm{pH} 4.0$ (or $\mathrm{pH} 10$.) buffer (cover the DO sensor) and allow a couple of minutes for equilibration.

7. Use the SLOPE CONTROL to adjust the displayed reading to 4.0 (or 10.0 ).

8. Remove the 4.0 (or 10.0 ) buffer, flush the sensors, and check the reading with the 7.0 buffer once more.

9. The reading at 7.0 should have changed little if at all. If it did change slightly, repeat steps (5) through (8).

10. That completes the $\mathrm{pH}$ calibration.

Saving Calibration Settings: Now that the Surveyor II has been given all of the calibration information via the ZERO and SLOPE CONTROLS, it is necessary to tell the instrument to store the information in its permanent memory for use during all of the measurements to follow. Again, do not turn the instrument OFF until the calibration factors have been saved. To do this:

FIRST SET THE FUNCTION SWITCH TO 'BATT', THEN

PUSH BOTH CONTROL SWITCHES, SIMULTANEOUSLY, AWAY FROM THE DISPLAY (TOWARD YOU).

\section{WAIT UNTIL THE WORD 'SAVE' APPEARS IN THE DISPLAY}

This SAVING operation MUST be done at the end of the calibration procedure, everytime. If it is not done, the instrument will use old calibration information from the last SAVE, introducing systematic errors into data that would otherwise be correct.

Calibration of the Surveyor II is finished. 
2. Remove the calibration cup from the Sonde, half fill the storage cup with deionized water or tap water and screw it onto the Sonde (snugly so that it won't leak).

Installation: None

Operating Procedures:

Moving to the Field: In transporting the Surveyor II, there are three things to be concerned about:

Drying-out of the sensors

Extreme temperatures

Vibration and shock

The first of these won't happen if care is taken to keep the storage cup, containing a little water, in place on the Sonde.

Don't allow the instrument to lie in direct sunshine or in such places as the closed trunk of a car, particularly in summer, for long periods because the temperatures inside the sealed Display Unit and Sonde rise to very high levels. This will cause no permanent damage but the operation of the system may be temporarily impaired (stability, calibration). Keeping the instrument in ventilated shade will avoid the heat problem.

Very low temperatures should also be avoided. Try to keep the temperatures of the Display Unit and sonde higher than $-10^{\circ} \mathrm{C}$. Temperatures below $-50^{\circ} \mathrm{C}$ might cause damage to vulnerable parts such as the liquid crystal display of the Display Unit.

Excessive shock and vibration can cause cumulative damage to the parts of the system. Never, for instance, carry the instrument loose in the bed of a truck or van. Similarly, nodes of extreme vibration sometimes develop at places in small boats powered by outboard engines. Cushion the instrument from these vibrations.

Field Operations: When you are to the point where you are ready to begin measurements. 
1. Remove the storage cup from the Sonde and screw on the circulator in its place.

2. Connect the Circulator to the socket of the data cable.

3. Collect water sample.

4. Place the Sonde in the water, set the Display Unit switch to the desired parameter.

As far as the Surveyor II goes, there is only one item that you have to really concentrate on during field measurements: THERMAL EQUILIBRIUM. With the exception of DEPTH and BATT, all of the measurements are functions of temperature. These functions are stored in the Display Unit, and it is programmed to correct for them before displaying the data. The corrections are based upon the assumption that all of the sensors are at the same temperature as that of the water. If that equilibrium condition is not met, errors will creep into the displayed data values.

Thermal equilibrium is always lost for a brief time whenever the environs of the Sonde change. This will be true when the Sonde is first put into the water and whenever it is moved into cooler or warmer regions of the water column. All that you have to do to avoid errors from this cause is to wait for equilibrium to return before taking your reading. You can judge this by watching the display. In general, the DISSOLVED OXYGEN sensor is the slowest to match its temperature to that of the water, so watch the DO reading. When it has just about stopped changing (changing very slowly) it's time to read all of the parameter values. (If you are not sure that you are allowing enough time for equilibration, take readings at the same location both as the Sonde is lowered and again as it is recovered. If the descending and ascending readings at corresponding depths differ consistently, you are most likely reading quicker than conditions allow.)

If it becomes necessary to verify calibration in the field, check each parameter by measuring appropriate standards just as you did during the laboratory calibration. But DO NOT USE THE CALIBRATION CONTROLS TO ADJUST THE

INSTRUMENT. Just note down the readings vs the standards used. If something has indeed changed, use these readings to make corrections later. But waiting for the results of the post-calibration before disturbing instrument settings avoids confusion and helps with overall data quality control. 
It sometimes happens, with conductivity, for example, that values actually encountered in the field unexpectedly fall outside of the range that was calibrated for initially. That is nothing to worry about. Go ahead with the readings and, during post-calibration, use an additional standard to extend the range to include the unexpected readings. Correct the data as required.

When measurements are complete or are to be interrupted for any reason, disconnect and remove the circulator. Screw the storage cup $1 / 2$ filled with clean water onto the Sonde to avoid dryout of the sensors.

\section{Post Calibration:}

1. Do post-calibration before doing any sensor maintenance.

2. Follow the same procedure used in the initial calibration preferably using the same equipment and standard solutions, but do not adjust the instrument using the calibration controls.

3. Simply write down the final readings of the standards and make them part of the data documents.

The value of the post-calibration results lies in what they can tell you about what happened to the sensors since the initial calibration. If the post-cal readings duplicate the values of the standards, you can be confident that the sensors were responding properly to conditions in the water. On the other hand, if one or more of the post-cal readings is out of tolerance, suspect:

1. An error in either the initial or post-calibration. Such things as having forgotten to SAVE the calibration information, using contaminated or deteriorated standards, or failure to allow for thermal equilibration could be the trouble.

2. Sensor fouling. Check the appearance of the sensors for stains or coatings: oils, clays, precipitates, fibrous material, biological material, etc.

3. Instrument malfunction. Check battery voltage. Check for sensor damage. See Malfunctions, Section 4.

Common Problems and Solutions: See Operator's Manual

Special Maintenance: 
Servicing the Sensors: In the interests of efficiency, you will want

to spend a minimum of time servicing sensors. But at the same time, the hours spent in the field have to produce high quality data. What, then, is the right amount of servicing effort? Try deciding upon the basis of a careful visual examination of the sensors, the results of the post-calibration, and the behavior of the instrument in the field.

If the instrument is responsive and stable in the field, the post-cal measurements are all in tolerance, and visual inspection (made after quick cleanup) reveals no problems, then the quick cleaning described below is all that is required.

On the other hand, irregular behavior in the measurement of any parameter in the field, out-of-tolerance post-cal measurements, or visible trouble signs will help you decide exactly where and what additional work is needed. Information on servicing the instrument can be found in the Operations Manual.

QUICK CLEANUP: Always begin sensor servicing simply by cleaning away silt, oils and greases, and soluble compounds. To do that quickly:

1. Fill the storage cup $1 / 2$ with warm detergent solution and screw it onto the sonde.

2. Shake the sonde vigorously to wash the sensors in the detergent solution.

3. Use a cotton-tipped swab and detergent solution to dislodge remaining foreign matter. Be sure that the threaded area and rubber sealing ring of the sonde endcap are free of grit.

4. Rinse the sensors and sonde endcap with tap or deionized water.

5. Visually check the sensor array for any of the following signs of trouble:

(a) Slack, wrinkled, or perforated DO sensor membrane

(b) Bubbles in the electrolyte under the DO membrane

(c) Obstructions (other than the electrodes) inside the bores of the conductivity cell block

(d) Evidence of coatings or precipitates on $\mathrm{pH}$, reference, ORP, or DO sensors, conductivity electrodes

6. If this is to conclude servicing (field performance, post-cal, visual all satisfactory), half fill the storage cup with water and screw it onto the sonde.

7. To do further servicing, see the appropriate sections in the operator's manual. 
CAUTION: The glass pH electrode is VERY vulnerable to accidental damage while other sensors or parts of the system are being worked on. Make it a habit always to protect the electrode by slipping a piece of flexible, thick-walled tubing (Tygon) over it during servicing operations.

Data Recorded in the Field: Field data recorded as part of this procedure includes:

Calibration information for temperature, $\mathrm{pH}$, and conductivity (if done in field), including manufacturer and lot number of all calibration standards.

Date and time of calibration

Date and time of field measurements

Values measured for $\mathrm{pH}$, temperature, and conductivity

Post calibration readings

Sampling site identification and pertinent sampling information (i.e. volume of purge water for which sample was collected, etc.).

Data Reduction: None if field measurements fall within calibration limits. If field values are outside calibration limits, connections to the data are made after post calibration procedures are completed.

\section{Data Reporting:}

Units: $\quad \mathrm{pH}: \quad$ standard units

Temperature: degrees $\mathrm{C}$

Conductivity: mmhos/cm

Dissolved oxygen: $\mathrm{mg} / \mathrm{I}$

Precision: see operators manual

Accuracy: see operators manual

Comments: see operators manual

General QA/QC comments: None 
ENVIRONMENTAL RESTORATION DEPARTMENT

DOCUMENT APPROVAL COVER SHEET

Document Number:

WAG 7-SOP-13.2.1

Revision:

0

Title: OPERATION OF NEUTRON MOISTURE PROBE

Prepared by:

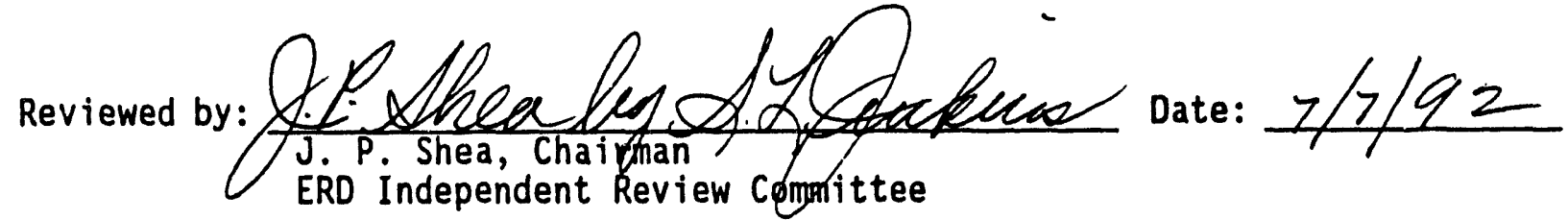

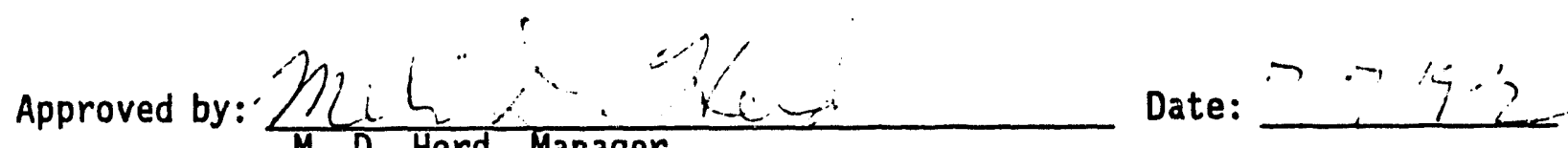

M. D. Herd, Manager

TRU Rod Unit

Approved by: Buried Waste Program (WAG 7) Group

Field Changes Authorized by DRR No. 


\begin{tabular}{l|lrl}
$\begin{array}{l}\text { ENVIRONMENTAL } \\
\text { STANDARD OPERATING }\end{array}$ & TITLE: OPERATION OF NEUTRON MOISTURE PROBE \\
PROCEDURES MANUAL & NUMBER: WAG 7-SOP-13.2.1 & ISSUE DATE: $07 / 06 / 92$ \\
\hline
\end{tabular}

\subsection{PURPOSE AND SCOPE}

This procedure provides general instructions to operate the Campbell Pacific Hydroprobe neutron moisture probe (hydroprobe) used to measure soil moisture profiles.

The hydroprobe depends on the ability of hydrogen to slow fast neutrons. A fast neutron source (50 mCi Americium-241/Beryllium) and a slow neutron detector are lowered down a pre-drilled and cased hole. The fast neutrons are slowed by the hydrogen in the soil water, and the resultant slow neutrons are counted by the detector. The count is then correlated with soil moisture content.

\subsection{PROCEDURE}

\subsection{Prerequisites}

Training is required before using or operating the hydroprobe. This training is explained in section 2.4 .

Hydroprobe access tubes must be installed in the soil prior to hydroprobe use.

\subsection{Quality Assurance/Quality Control}

There are no specific quality assurance/quality control (QA/QC) requirements except for obtaining the standard count which is described in section 2.7 and Table 1 .

\subsection{Health and Safety Requirements}

Activities conducted according to this procedure shall be in compliance with an investigation-specific Health and Safety Plan and Safe Work Permit, as required.

The hydroprobe contains a sealed man made radioactive source (Americium241/Beryllium); therefore, the person using this instrument is required to wear a direct reading dosimeter (DRD) and a thermoluminescent dosimeter (TLD) with an albedo.

The hydroprobe must be leak tested every six months by the Radioactive Waste Management Complex (RWMC) source custodian to ensure the source is adequately sealed.

The hydroprobe must be stored in its proper case when not in use and stored in a secured limited access radioactive storage area with placards on the entrance designating radiation. The storage area will be approved by an RWMC health physics representative. 
ENVIRONMENTAL STANDARD OPERATING

PROCEDURES MANUAL
TITLE: OPERATION OF NEUTRON MOISTURE PROBE

NUMBER: WAG 7-SOP-13.2.1

ISSUE DATE: $07 / 06 / 92$

\subsection{Training}

All personnel must complete a manufacturer's training course or equivalent course designed to permit and teach a hydroprobe operator to use the hydroprobe safely, in accordance with applicable legal restrictions, and accurately obtain the desired field results.

\subsection{Precautions/Limitations}

If the hydroprobe shows any indication of leakage from the source, a Health Physics Technician (HPT) shall be called immediately to survey the source.

In the event of the hydroprobe becoming lodged in a hole, no effort shall be made to forcibly dislodge it as this may shear the cable.

Sliould the radioactive source be lost down a well so it cannot be retrieved, the Nuclear Regulatory Commission (NRC), the United States Geological Survey (USGS) retrieval experts, Radioactive Waste Management Complex (RWMC) Operations, and RWMC Health Physics shall be contacted to decide on acceptable well cementing or capping procedures.

If the hydroprobe is stolen, the person(s) responsible for the source at that time shall immediately call the Warning Communications Center (WCC), the EG\&G Hydrology Unit Manager, the RWMC Operations Manager, the NRC, the RWMC Health Physics work leader, and the Idaho Department of Health and Welfare INEL Oversight Program Administrator. A list of appropriate telephone numbers is included in Appendix B.

Other precautions and limitations are covered in the manufacturer's manual (see Appendix $C$ ) and in sections 2.3, 2.8, 2.9 and Table 2, Step 2 of this SOP.

\subsection{Materials and Equipment}

In addition to the hydroprobe, casing (aluminum, PVC, or iron) for the soil hole is required. A slotted screwdriver is also necessary for calibration of the hydroprobe.

\subsection{Calibration/Standardization}

Calibration of the hydroprobe will be in accordance with the manufacturer's manual (see Appendix C). Calibration shall be performed using the same type of access tube as used in the field.

Before collecting data, a new "standard count" should be performed and the updated value entered into the hydroprobe memory. Table l provides step-by-step instructions for recalculating and updating the standard count. 
\begin{tabular}{|l|l|}
\hline ENVIRONMENTAL & TITLE: OPERATION OF NEUTRON MOISTURE PROBE
\end{tabular}

STANDARD OPERATING

PROCEDURES MANUAL

NUMBER: WAG 7-SOP-13.2.1

ISSUE DATE: $07 / 06 / 92$

\subsection{Transportation}

The packaging and transport of the source shall be done in accordance with Department of Transportation (DOT) Regulations. Requirements for package labeling are in Subpart E of 49 CFR 172 of the DOT regulations. General requirements for shipping and packaging radioactive material are in Subpart I of 49 CFR 173 of the DOT regulations. The following procedures shall be followed when transporting the source.

1. Transport the source in its own DOT-7A container.

2. Lock the source in an enclosed area when not in use.

3. Label contents properly on the source container.

\subsection{Procedure Description/Installation}

The procedure for operation of the Campbell Pacific Hydroprobe is described in detail in the manufacturer's manual (see Appendix C). The operator shall read this manual carefully. The major topics discussed in the manual are use of personnel monitoring, instructions for use of the hydroprobe, proper storage of the hydroprobe, instructions for transporting the hydroprobe, leak testing methodology, and emergency procedures.

Table 2 provides general instructions for taking moisture readings with the hydroprobe. Appendix A provides an example data worksheet for recording soil moisture readings with the hydroprobe.

\subsection{Data Reduction, Calculations, and Reporting}

Hydroprobe count readings will be converted to water content values using the appropriate calibration curve.

\subsection{DEFINITIONS}

No terms cited in this procedure require special explanation.

\subsection{REFERENCES}

Campbell Pacific Nuclear Corp., 1984, Hydroprobe Moisture Depth Gauge Manual, CPN corp., 2830 Howe Road, Martinez, California. 
ENVIRONMENTAL STANDARD OPERATING PROCEDURES MANUAL
TITLE: OPERATION OF NEUTRON MOISTURE PROBE

ISSUE DATE: 07/06/92

Table 1. Calculating and updating the standard count.

\begin{tabular}{|c|c|c|}
\hline STEP & ACTION & COMMENTS \\
\hline 1 & $\begin{array}{l}\text { Transport hydroprobe to field } \\
\text { location. }\end{array}$ & Note Section 2.8 in SOP. \\
\hline 2 & Remove adaptor ring. & $\begin{array}{l}\text { Use a slotted screwdriver to } \\
\text { loosen the set screw at the } \\
\text { bottom of the hydroprobe--slide } \\
\text { the metal adaptor ring out and } \\
\text { set aside. }\end{array}$ \\
\hline 3 & $\begin{array}{l}\text { Place hydroprobe on top of } \\
\text { closed case. }\end{array}$ & $\begin{array}{l}\text { Locate the hydroprobe on the } \\
\text { indented metal plate attached } \\
\text { to the case for this purpose. } \\
\text { If "READY" is not displayed on } \\
\text { the LCD press the "CLEAR" } \\
\text { button until "READY" appears. }\end{array}$ \\
\hline 4 & Press "STD" button. & $\begin{array}{l}\text { The current standard count is } \\
\text { shown on the LCD. }\end{array}$ \\
\hline 5 & Press "STEP" button. & $\begin{array}{l}\text { The current "P" value is shown } \\
\text { on the LCD. }\end{array}$ \\
\hline 6 & Press "STEP" button. & $\begin{array}{l}\text { The current "CHI SQ" value is } \\
\text { shown on the LCD. }\end{array}$ \\
\hline 7 & Press "STEP" button. & "NEW STANDARD??" is displayed. \\
\hline 8 & Press "ENTER" button. & $\begin{array}{l}\text { "COUNTING" is displayed--walk } \\
\text { away ( } 10 \mathrm{ft} \text { or more) until the } \\
\text { count is complete. }\end{array}$ \\
\hline 9 & Record new "STD" count. & $\begin{array}{l}\text { Display reads "MS" then the new } \\
\text { count, i.e. MS } 5487 \text {. }\end{array}$ \\
\hline 10 & $\begin{array}{l}\text { Press "STEP" button then record } \\
\text { new "p" value. }\end{array}$ & $\begin{array}{l}\text { The new "P" value is shown on } \\
\text { the LCD. }\end{array}$ \\
\hline 11 & $\begin{array}{l}\text { Press "STEP" button then record } \\
\text { new "CHI SQ" value. }\end{array}$ & $\begin{array}{l}\text { The new "CHI SQ" value is shown } \\
\text { on the } L C D \text {. }\end{array}$ \\
\hline 12 & Press "STEP" button. & New standard is redisplayed. ${ }^{c}$ \\
\hline 13 & Press "ENTER" button. & $\begin{array}{l}\text { Updates the current standard to } \\
\text { "new" standard. }\end{array}$ \\
\hline 14 & Replace adaptor ring. & Insert ring into set screw. \\
\hline 15 & $\begin{array}{l}\text { The hydroprobe is now ready to } \\
\text { take soil moisture readings. }\end{array}$ & \\
\hline
\end{tabular}

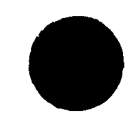


ENVIRONMENTAL STANDARD OPERATING

PROCEDURES MANUAL
TITLE: OPERATION OF NEUTRON MOISTURE PROBE

NUMBER: WAG 7-SOP-13.2.1

a. A standard count should be conducted more than $10 \mathrm{ft}$ from hydrogen sources. The case should be placed on the bare ground when possible and away from vehicles.

b. Counting is indicated by audible "beeps" as the new standard is calculated. The process requires about 6 minutes.

c. The LCD display is in a loop at this point, showing STD, P, and CHI SQ values as the STEP button is pressed. 
ENVIRONMENTAL

STANDARD OPERATING

PROCEDURES MANUAL
TITLE: OPERATION OF NEUTRON MOISTURE PROBE

NUMBER: WAG 7-SOP-13.2.1

ISSUE DATE: $07 / 06 / 92$

Table 2. Instructions for taking Hydroprobe soil moisture readings.

\begin{tabular}{|c|c|c|}
\hline STEP & ACTION & COMMENTS \\
\hline 1 & $\begin{array}{l}\text { Calculate and update } \\
\text { standard count. }\end{array}$ & See Table 1 \\
\hline 2 & Insert dummy probe. & $\begin{array}{l}\text { Before each use the access } \\
\text { tube shall be tested for } \\
\text { water and obstructions by } \\
\text { running the dummy probe to } \\
\text { full depth. The hydroprobe } \\
\text { shail be used in a dry } \\
\text { access tube. }\end{array}$ \\
\hline 3 & $\begin{array}{l}\text { Place Hydroprobe on access } \\
\text { tube. }\end{array}$ & $\begin{array}{l}\text { If "READY" is not displayed } \\
\text { on the LCD press "CLEAR" } \\
\text { untif "READY" is shown. }\end{array}$ \\
\hline 4 & $\begin{array}{l}\text { Lower Probe to bottom of } \\
\text { tube. }\end{array}$ & \\
\hline 5 & Press "START" button. & $\begin{array}{l}\text { "COUNTING" is displayed on } \\
\text { the LCD. Reading is } \\
\text { obtained in less than one } \\
\text { minute. }\end{array}$ \\
\hline 6 & Record reading. & \\
\hline 7 & Raise probe to next depth." & \\
\hline 8 & Press "START" button. & \\
\hline 9 & Record reading. & \\
\hline 10 & Repeat steps 7 through 9. & $\begin{array}{l}\text { Until all depths are } \\
\text { recorded. }\end{array}$ \\
\hline 11 & $\begin{array}{l}\text { Return probe to gauge } \\
\text { housing. }\end{array}$ & $\begin{array}{l}\text { Gently pull the probe into } \\
\text { the locking position. }\end{array}$ \\
\hline
\end{tabular}

a. Depths are marked in increments using metal markers attached to the probe cable. The disk shaped markers slip into a slot at the top of the gauge and support the probe at that depth. Care should be taken to keep track of each depth recorded and ensure the probe is raised to a new marker after each count. Before each use, a check should be conducted to ensure that screws holding the markers in place do not come loose, causing the probe to become lodged in the access tube.

b. Problems can occur when pulling the probe back into the gauge housing--Do NOT FORCE IT. While applying light pressure on the cable readjust the gauge on the access tube so the holes line up exactly. The probe should slide smoothly into the housing. Press the disk to pull the probe into locking position. It should click when it locks. 
ENVIRONMENTAL

STANDARD OPERATING

PROCEDURES MANUAL
TITLE: OPERATION OF NEUTRON MOISTURE PROBE

NUMBER: WAG 7-SOP-13.2.1

\section{APPENDIX A}

NEUTRON MOISTURE PROBE DATA SHEET

DATE

OPERATOR

ACCESS TUBE \#

TIME

STOP $\underset{\text { (units }=\text { DEPTH }}{\text { COUNT }}$

1
2
3
4
5
6
7
8
9

10

11

12

13

14

15

16

17

18

19

20

21

22

23

24

25

26

27

28

29

30
STD

CHI SO

P

DEPTH OF ACCESS TUBE

COUNTING TIME INTERVAL 
ENVIRONMENTAL

STANDARD OPERATING

PROCEDURES MANUAL
TITLE: OPERATION OF NEUTRON MOISTURE PROBE

NUMBER: WAG 7-SOP-13.2.1

ISSUE DATE: $07 / 06 / 92$

\section{APPENDIX B}

TELEPHONE LIST
Campbell Pacific Nuclear Corp. (manufacturer)

EG\&G Idaho Hydrology Unit Manager (Tom Wood)

Idaho Department of Heal th and Welfare INEL Oversight Program

NRC Nuclear Materials Licensing Section (William Fisher)

Hydroprobe Radiation Safety Officer (Joel Hubbell, EG\&G Idaho)

RWMC Health Physics

RWMC Operations ( $T$. Cline)

USGS Idaho Office (Larry Mann/Roger Jensen)

Warning Communications Center (WCC)
(510) $228-9770$

(208) $526-1293$

(208) $334-0498$

(817) $860-8100$

(208) $526-1747$

(208) $526-2710$

(208) $526-2767$

(208) $526-2158$

(208) $526-1515$ 
ENVIRONMENTAL

STANDARD OPERATING

PROCEDURES MANUAL
TITLE: OPERATION OF NEUTRON MOISTURE PROBE NUMBER: WAG 7-SOP-13.2.1

\section{APPENDIX C}

503DR HYDROPROBE MOISTURE DEPTH GAUGE MANUAL

CAMPBELL PACIFIC NUCLEAR CORPORATION 


\section{MANUAL CHANGE NOTICE 503DR}

\section{VERSION 503A-230}

To take a standard count press: STD, step till the gauge displays "NEW STD?" and press ENTER.

In Format the gauge now allows a record to contain 0 to 99 keydatas and 0 to 99 readings.

Selftest has been removed from the Menu.

While taking a count the display shows "COUNTING".

While taking a reading in Log Mode, the display shows "COUNT $N "$, where " $N$ " is the number of the reading as defined in iormat and counts down to "1".

The displayed units are: CNT, RAT, PCF, GCC, \%V, IPF, AND CPC.

There are now provisions for 16 calibrations. A calibration may be selected by pressing STEP until the number is displayed and pressing ENTER as described previously or by selecting directly. e.g. press CALIB, 12, ENTER to select the 12th calibration.

The Format command now displays the number of records of storage after the "SET FMT?" display. Press ENTER to complete formatting the gauge.

The version display now includes the model number. e.g. "503A-230".

The print dump format has been changed. The first line header include the new version number. In PRINT LP a top of form command is issued atter the header and calibration information and before starting the date print out. 


\section{WARRANTY}

CPN products are guaranteed against defective material and workmanship for a period of eighteen (18) months from the date of receipt by customer, or a maximum of twenty-four (24) months from the date of manufacture, whichever comes first. Detector tubes are guaranteed for (12) months from the date of shipment from CPN. Fuses and batteries are guaranteed for (6) months from the date of shipment from CPN.

Upon their prepaid retum, CPN will replace free of charge any part found to be defective within these warranty periods. CPN reserves the right to repair all defective parts at our factory, or authorized CPN service facility.

This warranty is void if inspection shows evidence of abuse, misuse, or unauthorized repair. This warranty covers only replacement of defective materials and workmanship. This warranty does not cover damage caused by exposure to excessive moisture.

If, for any reason, this unit must be retumed to our factory for warranty service, please contact us for return authorization and shipping instructions. Include with the shipment: customer purchase order number, CPN Company invoice number and date, serial number of gauge and reason for retum. 
WHITE SECTION: OPERATING INSTRUCTIONS

PINK SECTION: RADIATION SAFETY PRECAUTION

GREEN SECTION: OPERATOR SERVICE

YELLOW SECTION: READIATION SIMPLIFIED

LT. GREEN SECTION: TRAININC

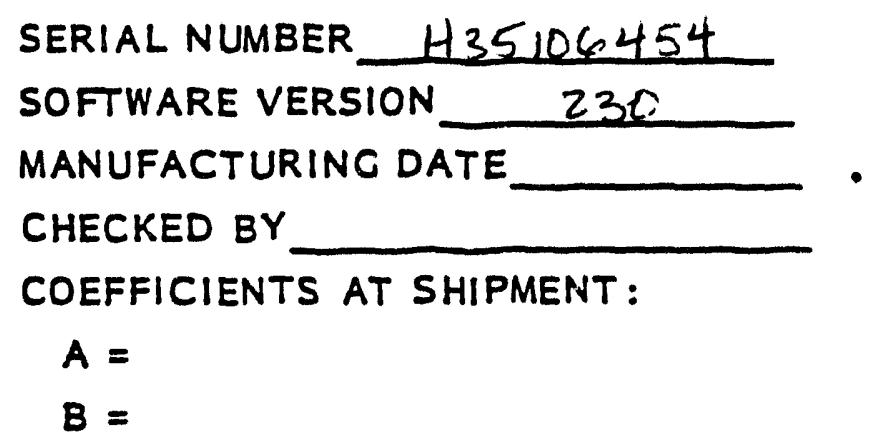


INSTRUCTIONS

TABLE OF CONTENTS

(White Section)

GENERAL
9.1 INTRODUCTION
1.2 SPECIFICATIONS
1.3 INSTALLATION
1.3 .1 GETTING STARTED
1.3.2 CABLE STOPS
9.3 .3 TUEE ADAPTERS
1.4 OPERATION CAUTIONS

\subsection{OPERATION}

2.1 CONTROLS AND DISPLAY

2.2 KEYBOARD LAYOUT

2.3 KEYBOARD OPERATION

2.4 BATTERY

2.5 OPERATING PROCEDURES

2.5 .9 TAKING A READING

2.5 .2 TO LOG A READING

2.6 STANDARD COUNT

2.7 PRINT

.2 .8 REMOTE OPERATION

2.9 RS232C SERIAL LINK (HARDWARE DESCRIPTION)

.0 SPECIAL

3.1 CALIBRATION

3.2 ERROR MESSAGES

$\begin{array}{ll} & \text { APPENDIX } \\ \text { A } & \text { COUNTING STATISTICS } \\ B & \text { DRDUMP PROGRAMS } \\ \text { C CABLE WIRING DIAGRAMS }\end{array}$

SOFTWARE VERSION 226, MaY 9, 1984 


\subsection{GENERAL}

\subsection{INTRODUCTION}

The MOdel 503DR HYDROPROBE, NEUTRON DEPTH MOISTURE GAGE measures the sub-surface moisture in soil and other materials by use of a probe containing a source of high energy neutrons and a slow (thermal) neutron detector. The probe is lowered into a pre-drilled and cased hole 19.5 or 2 inch diameter). Hydrogen as present in the water in the soil slows the neutrons down for detection. The moisture data is displayed directly in units of interest on an above surface electronic assembly which is integral to the source shield assembly.

The gage is supplied with a 12 foot cable and five adjustable cable stops. Adfitional stops and longer sable lengths are available.

Upon retraction of the probe into the shield, the probe locks automatically in place for transportation.

The complete assembly is supplied with a plastic shipping and carrying container which contains; accessory items, cable, instruction manual, charger, and other materials which the operator may wish to carry. 


\subsection{SPECIFICATIONS}

UNCTION:

NGE:

PRECISION:

COUNT TIME:

TEMP:

POWER:

BATTERY LIFE:

CONSUMPTION:

RECHARGE:

DISPLAY:

DATA STORAGE:

DATA OUTPUT:

CALIBRATION:

UNITS:

NEUTRON SOURCE:

ENCAPSULATION:

HIELDING:

SHIPPING:

SPECIAL FORM

APPROVAL:

CONSTRUCTION:

SURFACE UNIT:

PROBE: MOdel -2

Model -1.5

CARRYING \&

SHIPPING:
Sut-surface moisture measurements.

0 to $\equiv 2 \% \mathrm{vol},(0.32 \mathrm{gm} / \mathrm{cc}),(20 \mathrm{pef})$, (3.84 in/ft).

$0.24 \%$ at $24 \%$ vol at one minute.

$1,4,16,32,64$, and 256 sec.

0 TO $700 C$ operating.

AA NICAD batier pack $(0.5$ Ah) 8 cells.

500-1000 Charge-discharge cycles.

$6.5 \mathrm{~mA}$ avg. (allows more than

3000 each 16 sec counts).

14 hours at $c / 10$ via wall charger.

8 character alpha/numerical Liquid-

Crystal Display. Easily

readable in direct sunlight.

3072 cells of; counts, identifiers,

or keyboard entry of auxiliary values.

Format operator programmable.

$0-15$ key and $0-15$ count per record

RS232C serial dump to external printer,

computer, CRT or coupler.

8 ea user programmed (linear).

User selectable:

in/ft, pef, $\mathrm{gm} / \mathrm{cc}, \mathrm{z}$ vol, $\mathrm{cm} / 30 \mathrm{~cm}$,

count and count ratio.

$50 \mathrm{mc}$ i Americium-241/Be

Double Sealed Capsule, CPN-139

Silicon based paraffin

Radioactive Material,

Special Form, N.O.S., UN2974

Transport Index 0.9

Yellow II Label

USA DOT TA, Type A Package

USA/0195/S

Aluminum with epoxy paint

or hard-anodize finish.

stainless steel wear parts

15.7\$, 6.8"Wx7.0"D×14.0"H

$(7.12),(172.7 \times 977.8 \times 355.6)$

$2.3 \#, 9.865$ " dia $\times 12.7$ "L

$(9.043),(47.4$ dia $\times 322.6)$

$1.7 \$, 1.500 "$ dia $\times 12.7 " L$

$(0.779),(38.1$ dia $\times 322.6)$

$36.5 \mathrm{H}, 24 \mathrm{WW} \times 13$ "D $\times 10 " \mathrm{H}$

$(16.56),(609.6 \times 330.2 \times 254)$ 


\subsection{INSTALLATION}

\subsubsection{GETTING STARTED}

The stand-by power drain of the gage is less than the self-discharge of the NICAD cells thus eliminating the need for a power switch. The DR is always $O N$. When first received it is recommended that the gage be placed on charge overnight to insure starting with a full charge.

You will have to set the gage to a configuration to meet your field conditions. To assist in understanding the gage initially, it is shipped from the factory in the following configuration:

$\begin{array}{ll}\text { UNITS } & \text { Inches per foot } \\ \text { TIME } & 1 \text { second } \\ \text { CALIB } & \# 1 \text { Factory calibration in saturated and dry sand. } \\ & \text { A (slope) approx } 2.5 \text { in/ft per countratio. } \\ & \text { and B (intercept) approx }-.06 \text { in/ft } \\ \text { STD } & \text { Standard count apDrox } 10000 \\ \text { FMT } & 1 \text { ID, } 1 \text { Keydata and } 3 \text { Depths allowing } 279 \text { records }\end{array}$

With the gage sitting on the nameplate on the shipping case press START. The gage should take a one second count and display the equivalent moisture of the wax in the shield. It should be approximately 2.4 inches per foot.

Most. of the commands are READ/STEP/WRITE. That is, when first called up you read the display to see the current value, step to a new value and then write (enter) the new value into memory. Try this by using the following keystrokes to change the time from 1 to 16 seconds.

\#PRESS \#\#

$\begin{array}{ll} & \text { READY } \\ \text { TIME } & \text { TIME } 1 \\ \text { STEP } & \text { TIME 4 } \\ \text { STEP } & \text { TIME 16 } \\ \text { ENTER } & \text { READY }\end{array}$

\section{(Read the current value)}

(Step to a new value)

(Write it to memory)

Do the same for Units, changing from Inches per foot to : Volume.

$\begin{array}{ll}\star \star P R E S S & \text { \#DISPLAY } \\ & \text { READY } \\ \text { UNITS } & \text { UNIT IF } \\ \text { STEP } & \text { UNIT CC } \\ \text { STEP } & \text { UNIT ZV } \\ \text { ENTER } & \text { READY }\end{array}$

Take another count by pressing START. The measured result should be the same as above except that the count should take 16 seconds and the display should be approximately $20.0 \%$ moisture by volume (which is equivalent to 2.4 in per foot in the reading above). 
Please read the remainder of this manual

Section 2.5.1 is task oriented and describes the steps to use the gage to take readings. When you are ready to take it further and log readings, read section 2.5 .2 .

Sections 2.1, 2.2, and 2.3 are function oriented describing what each key does.

Sections 2.9 contain the necessary information to hardware interface the gage to a computer or printer.

Sections 2.7 and 2.8 cover the sottware interface information.

\section{3 .2 CABLE STOPS}

The gage is supplied with five each clamp on cable stops. This will allow taking measurements at one foot increments in a root zone up to five feet deep. For a deeper root zone or for smaller increments order more stops. Fis $1.3 .2-1$ shows a cross-section of the gage. Use it to position the first stop so that the meaurement point on the probe (as indicated oy the band) is in the middle of the top foot of the root zone. Its actual location will depend upon how high the access tubes stick out of the soil. Install all tubes the same distance.

For example, if the base of the gage is 5.D" above the soil and you want to take the first measurement at 6", Dlace the stop at $5.35+5.0+6.0=16.35^{\prime \prime}$ above the stop reference line.

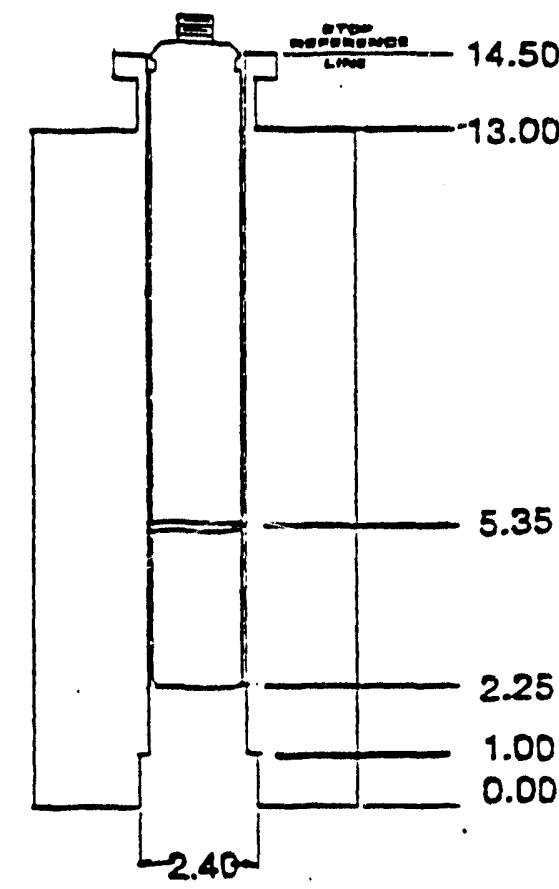

\subsubsection{TUBE ADAPTER RING}

FIG 1.32-1

The bottom of the gage contains an oversize hole to allow inserting an adapter ring with a diameter to match the type of access tubing being used. The ring is secured by a serew through the front of the casting. Unless specified otherwise at time of order, an adapter ring for aluminum tubing will be supplied. Adapter rings for other type (e.g. diameter) are available from (PN Corp. or can be constructed locally. 


\subsection{OPERATION}

\subsection{CONTROLS AND DISPLAY}

Most functions are directly entered by pressing the appropriate key. Options are reviewed by STEPing, and selected with ENTER.

\begin{tabular}{|c|c|}
\hline Key & Function \\
\hline $\begin{array}{l}\text { D. WET } \\
\text { DODRY } \\
\text { WATER }\end{array}$ & $\begin{array}{l}\text { Display latest wet density reading }(501 \text { only) } \\
\text { Display latest dry density reading }(501 \text { only) } \\
\text { Display latest moisture reading }\end{array}$ \\
\hline $\begin{array}{l}\text { UNITS } \\
\text { TIME } \\
\text { CALIB } \\
\quad \text { COEFF } \\
\quad \text { SLFCAL }\end{array}$ & $\begin{array}{l}\text { Select measurement units (CT,RO,PF, IF, GC,CC, ZV) } \\
\text { Select counting time }(1,4,16,32,64,256) \text { sec } \\
\text { Select calibration }(1 . .8) \text {, and optionally: } \\
\text { Enter coefficients directly } \\
\text { Semi-automatic calibration }\end{array}$ \\
\hline $\begin{array}{l}\text { LOG } \\
\text { RCL } \\
\text { PRINT } \\
\quad \text { PRINT CD } \\
\text { PRINT LP }\end{array}$ & $\begin{array}{l}\text { Log a tube site record } \\
\text { Recall a record for review } \\
\text { Dump records to external device } \\
\text { Dump to an active device (Computer Device) } \\
\text { Dump to a passive device (Line Printer) }\end{array}$ \\
\hline $\begin{array}{l}\text { MENU } \\
\text { SELFTEST } \\
\text { BAUDRATE } \\
\text { ATTRIB } \\
\text { SERNO } \\
\text { VERSION } \\
\text { STD } \\
\text { FMT }\end{array}$ & $\begin{array}{l}\text { Seleet mise function: } \\
\text { Self test of circuits (destructive RAM test) } \\
\text { Select baudrate }(110,300,1200,2400,4800,9600) \\
\text { Set attributes for the Print LP dump. } 3 \text { ea } \\
\text { Prefix, } 3 \text { ea suffix, and a TOp of Form. } \\
\text { Display/Enter a four digit serial number } \\
\text { Display software version } \\
\text { Display/update standard count summary } \\
\text { Set record format, clear records }\end{array}$ \\
\hline $\begin{array}{l}\text { START } \\
\text { CLEAR (NO) } \\
\text { STEP } \\
\text { ENTER (YES) }\end{array}$ & $\begin{array}{l}\text { Take a reading } \\
\text { Clear, abort, "NO" } \\
\text { Next, skip, toggle } \\
\text { Enter data, make selection, "YES" }\end{array}$ \\
\hline $\begin{array}{l}\text { STEP \& CLEAR } \\
\text { Display }\end{array}$ & $\begin{array}{l}\text { Master Reset (Hold STEP down and press CLEAR) } \\
\text { Function }\end{array}$ \\
\hline $\begin{array}{l}\text { "READY" } \\
\text { "." } \\
\text { "I" }\end{array}$ & $\begin{array}{l}\text { Gage is ready for operation. } \\
\text { Decimal point in lower left indicates } \\
\text { low battery condition. } \\
\text { Bar in left character with no } \\
\text { keyboard response indicates } \\
\text { cutoff condition. }\end{array}$ \\
\hline
\end{tabular}




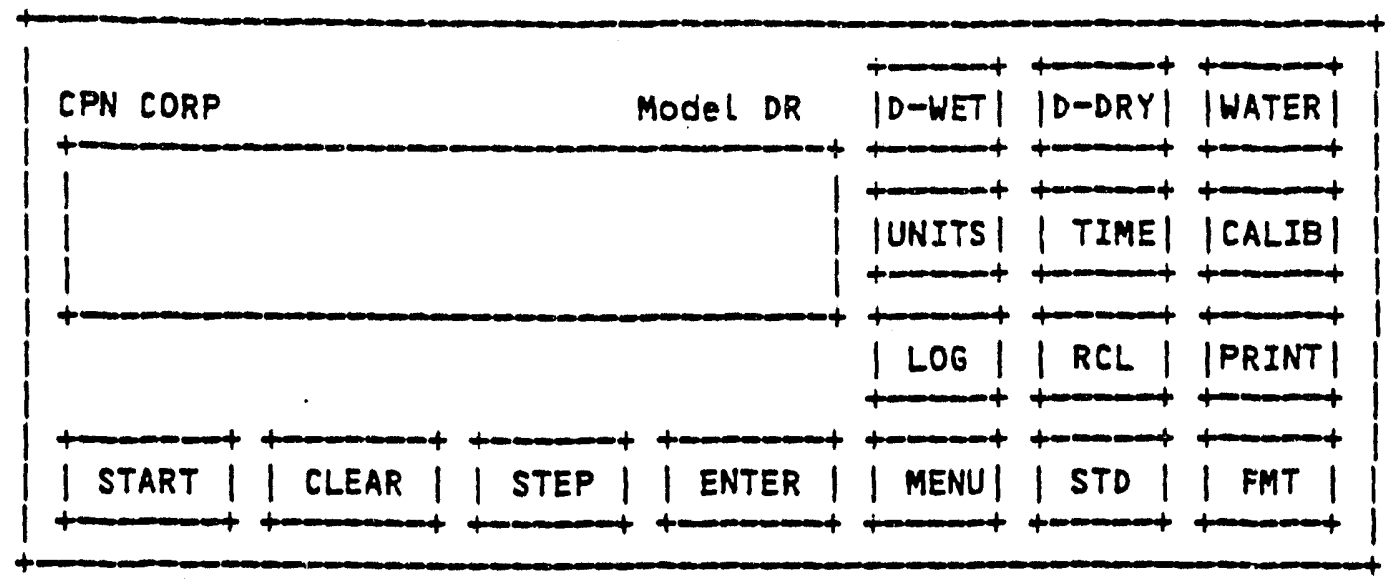

\subsection{KEYBOARD OPERATION}

D-WET (501 ONLY)

Displays wet density using eurrently selected calibration and units

(On a 503 returns to "READY").

D-DRY (501 ONLY)

Displays dry density using currently selected calibration and units

con a 503 returns to "READY").

WATER

Displays the most recent moisture reading in current units using current calibration $\mathrm{e.9.} \mathrm{"9234} \mathrm{CT".} \mathrm{If} \mathrm{number} \mathrm{to} \mathrm{be} \mathrm{displayed} \mathrm{is}$ undefined (e.g. exceedes the display size) then "*.* CT" will be displayed.

UNITS

Display/Select measurement unit. Displays surrent unit e.g. "UNIT IF". To seleet new unit, STEP to desired unit and pless ENTER. If CLEAR or another selection key is pressed, unit remains unchanged.

\begin{tabular}{lll} 
Mne & Unit & Conversion \\
\cline { 3 - 4 } CT & counts/unit-time(16 sec disply) & $\mathrm{n} / \mathrm{a}$ \\
RO & ratio (count/stdcount) & $\mathrm{n} / \mathrm{a}$ \\
PF & Lbs of water/cubic foot of soil 62.428 \\
GC & gms of water/cubic cm of soil & 1.0 \\
IF & inches of water/ft of soil & 12.0 \\
CC & em of water/30 cm of soil & 30.0 \\
IV & percent water by volume & 100.0
\end{tabular}

The conversion numbers are shown for reference only. The gage performs the conversion internally depending upon your selection of units. $\epsilon . g$. a readings in $\mathrm{gm} / \mathrm{cc}$ is divided by 1.0 and multiplied by 12 to get an equivalent reading in inches per foot. 
CALIB

Display/Select calibration (1 thru 8) and/or review/change its' coefficients.

Before pressing CALIB, select the appropiate units and time via the UNITS and TIME keys. If counts (CT) or ratio (RO) is the selected unit the gage will display "SET UNIT". If SLFCAL is to be used to take a count, select a time of 256 seconds for maximum precision.

\section{TO SELECT A CALIBRATION}

Active calibration is displayed on entry e.g. "CALIB 4". To select another calibration, STEP to the desired number and press ENTER. When the prompt "COEFFs ?" appears press CLEAR to return to "READY".

\section{TO ENTER/CHANGE COEFFICIENTS WHEN THE COEFFICIENTS ARE KNOWN}

Select the calibration number as above. When "COEFF ?" is displayed press ENTER. Coefficient " $A$ " is the slope, " $B$ " is the intercept in an expression of the form:

$$
\text { Display }=A \times(\text { ratio })+B
$$

Where ratio is count divided by standard count

Enter $A$ and $B$ using the same units as previously selected by the UNITS key.

The eurrent "A" coefficient will be displayed. Press ENTER to accept it or change it via the numeral keys and press ENTER. Same for "B". Returns to "READY" after last coefficient entered.

TO ENTER/CHANGE COEFFICIENTS WHEN THE COUNTS ARE KNOWN OR TO BE DETERMINED

Select the calibration number as above. Press STEP till "SLFCAL ?" is displayed. Press ENTER.

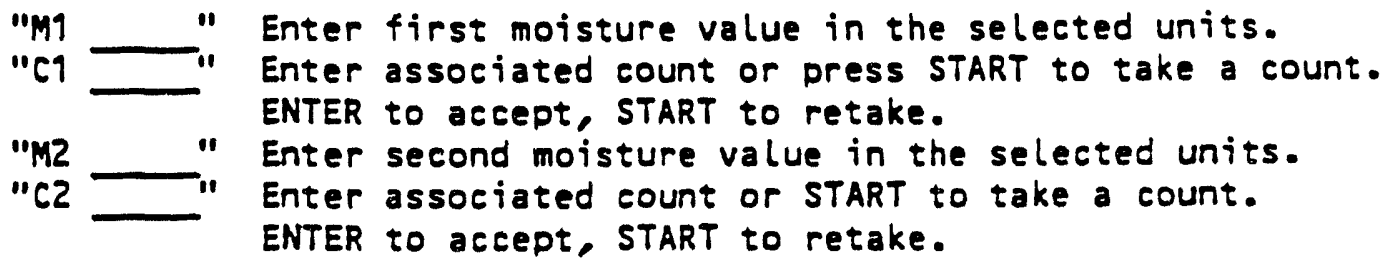

Either the low or high data pair may be entered first. When taking a count place the probe in the appropiate moisture standard before pressing START. After coefficients are computed and stored, display returns to "READY". To review the coefficients use "COEFFS ?". 
Arms the storage mechanism to $\log$ a tube site record. As defined presiously by the FMT key, each RECORD consists of 1 each ID, $D$ TO 15 keypad entries, and 0 to 15 depths. ID number, keypad entries and depths start at the highest value and down count to 1 . When LOG is first pressed the current record number is displayed. Press ENTER to use it as the default ID number or key in a meaningfull record number. The record number decrements from the maximum to 1 , each time a new record is logged. The record number is thus an indicator of how many additional records may be logged.

Keypad fields are read/modify/write. i.e. it will first display what is stored in that location (normally blank for a new (ocation). Key in a value and press ENTER to store the new value. Press CLEAR to abort a wrong key entry. A keypad field may be skipped by pressing STEP.

Moisture fields are stored the same except that value is from a count initiated by pressing START. It may be retaken but will only be stored and advanced to the next field when you press ENTER.

A record is not stored in the log until the prompt "DATA OK?" appears and ENTER is pressed. If at that time you press STEP instead, the display will step around to the beginning of the record sillowing it to be viewed and edited. To accept an existing keydata or moisture value press ENTER or STEP. To change the data, write over the keydata field followed by ENTER or press START for a new count followed by ENTER. The corrected record is finally stored when you press ENTER while "DATA OK?" is displayed.

REL

To review the record log. On entry displays ID number of last record logged. STEP back thru the log to the ID number of desired record and press ENTER, or enter ID number directly. i.e. 1234 ENTER. If the keyed in ID number does not exist, displays "NOT HERE". Press STEP to acknowledge and continue STEPing from last real ID. When desired ID is displayed press ENTER. Use the STEP key to move across the fields of the record (like a window moving across a tickertape).

Press CLEAR to step down the ID' 5 . Press ENTER to return to the more detailed level. At the outer level you see the IDs only, at the inner level the entire record.

\section{PRINT}

Dumps record log to external device via the serial connector. For "PRINT CD" Press PRINT, ENTER. For "PRINT LP" press PRINT, STEP, ENTER.

PRINT CD

Output formatted to upload record log to a computer directly or via modem. Ineludes a line count and with each data Line a checksum. Uses ACK, NACK software protocol to control transmission. 
PRINT LP

Output formatted for readability. Contains same information as Print CD dump without the line count and the checksums and does not wait for a response.

MENU

Step down the menu choices and press ENTER to select a choice.

SELFTEST

Runs a comprehensive hardware check on the gage consisting.6f RAM test, ROM test, Counter test, Timer test, Display test and Keyboard test. The RAM test is destructive. Do MOT run it if the LOG data is important. Selftest takes about 2 minutes. You must watch the display test to see that all segments are the same. The keyboard test echos the key pad you touch. To complete the keyboard test press CLEAR twice. A successful test will display "TST OK". If an error occurs, an error message will be displayed at the end of that test. To acknowlwedge it and continue the remaining tests press ENTER. To abort any test press CLEAR during the test.

BAUDRATE

Allows setting the baud rate for transmission on the serial connector. When first selected, displays the baud rate currently selected. Use the STEP key to step to a new rate and press ENTER.

\section{ATRIB}

Allows setting attributes into the PRINT LP output for specific external equipment. Has provisions for 3 each characters at the beginning of transmission (prefix) and three each at the end (suffix). A typical prefix would be to transmit an ASCII decimal 15 to an EPSON printer to place it in the compressed mode. A typical suffix would be an ASCII decimal 26, which is reconized by a CP/M system as the end of transmission of a file of data.

A TOD of Form character (TOF) is transmitted for each 60 lines of data.

$\begin{array}{lll}\text { "PX1 000" } & \text { 9st prefix character } \\ \text { "PX2 000" } & \text { 2nd prefix character } \\ \text { "PX3 000" } & \text { 3rd prefix character } \\ \text { "SX1 000" } & \text { 1st sufix character } \\ \text { "SX2 000" } & \text { 2nd sufix character } \\ \text { "SX3 026" } & \text { 3rd sufix character } \\ \text { "TOF 012" } & \text { TOP of Form character }\end{array}$

As shipped from the factory the prefix and two of the sufix characters are nulls $(0)$. One of the sufix characters is = control $Z$ (ASCII 26 for end of file in CP/M programs) and the TOF is a control $L$ (ASCII 12). The ASCII characters are entered as their decimal values $(0 . .127)$ by keying them in and pressing ENTER> 


\section{SERNO}

Displays the last four numbers of the probe serial number. Press CLEAR to return to the READY display. The serial number may be change by keying in new number followed by ENTER. This is useful when moving a surface electronic assembly from one gage shield/probe to another.

VERSION

Displays the gage software version (useful for service purposes).

Displays standard count information and/or take a new std count. Initially displays the current moisture standard count (MS). Step to display previous moisture standard count (MP). Step again to display the chi-square ratio of the current standard (MCHI).

(See section 2.6 for detailed procedures) To take a new standard count press STD followed by START. The DR will take 32 each 8 second counts, with a 2 second warmup delay between each count (4.27 minutes of counting). During each count period the previous count number and count are displayed e.9."24-1234". When the last count is finished a NEW standard count based on the average of the 32 counts is calculated and displayed (it is displayed as an equivalent 16 second count to be consistant with a normal count display, and so will be twice the previously displayed 8 second counts).

Use the STEP key to view the CURRENT standard count (identified as previous at this point) and NEW chi-sa ratio (continuing to press STEP will cycle the display around the three pieces of standard count information). If the difference between the new and current standard counts and the value of the new chi-sa ratio are acceptable press ENTER to update the standard count information. If they are not acceptable press CLEAR to abort and take another new standard count.

To abort a standard count in progress, press CLEAR several times until "READY" is displayed. The standard count information will rema in unchanged.

\begin{tabular}{ll} 
Display & \multicolumn{1}{c}{ Description } \\
"MS 1234" & $\begin{array}{l}\text { Current standard count } \\
\text { "MP } 1199\end{array}$ \\
"MCHI 1.16" & Chi-square ratio (std-dev)/(sqr-root of std)
\end{tabular}


Sets the record format, and clears the log space. On entry, displays the maximum number of possible records to be logged in the current format. e.9. "REC 279". Press ENTER. Key in the desired number of keydata entries ( 0 thru 15) and press ENTER. Do the same for depths ( 0 thru 15). When the gage displays "SET FMT?", be sure you want to do it and then press ENTER. This clear the log, sets the new record format, and start the storage at the top of the log area. Be sure you have dumped the previous information before clearing memory otherwise it will be erased. If you just wanted to view the current format but not clear it, press CLEAR to abort.

To display the maximum number of records under this new format, press FMT again. Press CLEAR to return the display to "READY" without changing or clearing the format.

\subsection{BATTERY}

The gage is powered by a replaceable NICAD battery pack $(500$ mAh capacity, 8 each cell(s). It is accessible by removing the four screws on the front panel. Also behind the front panel is a circuit breaker. The red indicator should be showing for the gage to operate.

-

The charger, which is supplied with the gage, charges at the rate of 50 $m A$. This $C / 10$ rate will charge the gage overnight ( 14 hours) yet will not damage the cells if left on indefinitely. The red lamp on the charger should be ON and the charger should be warm to the the touch.

The : 500 mah capacity of these cells will allow 3000 or more 16 second readings on a fuld charge.

The gage should be charged any time the decimal point in the lower left of the display blinks or turns $O N$, or before going into the field two or more weeks since the last charge (the cells have a self-discharge rate of $1 \%$ per day at room temperature). It should also be charged prior to making a computer dump. The nominal battery voltage is $10.0 \mathrm{~V}$. The gage turns the low battery indicator oN when the voltage drops to $9.60 \mathrm{~V}$. It will not turn back OFF until the voltage has increased to $10.60 \mathrm{~V}$ by recharging.

If the battery is allowed to discharge too low,. the gage will automatically go into a cutout condition to protect the battery cells. The display will show the lower vertical segement in the first character. The keyboard will be inoperative. The gage goes into futoff at $9.40 \mathrm{~V}$ (normally under load). The cutoff circuit is restored when the cells have been recharged to $10.40 \mathrm{~V}$. This takes about 20 minutes with a $6 / 10$ charger. If cutoff occurs, charge the gage overnight and press CLEAR.

Refer to the Service Section of this manual for more on battery cells. 


\subsection{OPERATING PROCEDURES}

\section{.5.1 TAKING A READING}

To take a reading lower the probe to the appropiate depth and press START. Before doing this you must select Units, Time and Calibration. If you select any units other than count, the gage must have a valid standard count.

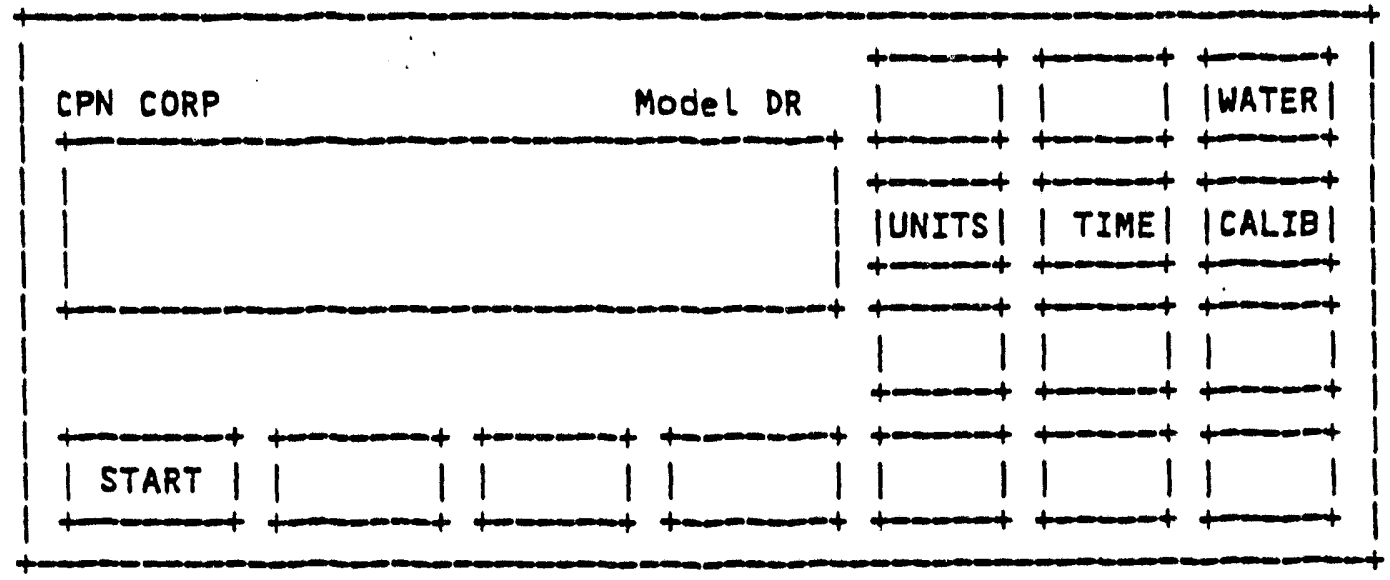

\section{UNITS}

The choice of display units will depend upon your use. Researchers will normally prefer grams per cubic centimeter or percent volume, while crop schedulers use inches per foot or centimeters per 30 centimeters. Counts are helpful for troubleshooting. It is the same data only differing by the conversion factor.

Once the units have been selected, then each time a count is taken the display will be in the units selected.

\section{TIME}

For a given counting rate the counting time interval determines the precision of the count. The longer the time, the more counts and thus the more precise the count. Correspondingly the longer the counting time the less measurement that can be made in a day. Thus the time interval is normally selected as the minimum time that will not sacrifice precision.

For scheduling type operation a count time of 16 seconds will provide sufficient precision to project the next irrigation date.

See the apendix section on Counting Statistics for a further discussion of precision. 


\section{CALIBRATION}

The calibration will have been determined previously and the slope (A) and intercept (B) coefficients stored in one of the eight calibrations. Select the one that is appropriate for the soil and type of access tube.

\section{TAKE A COUNT}

Lower the probe to the desired depth and press START. While taking a count the gage will display the time, calibration, and units selected.

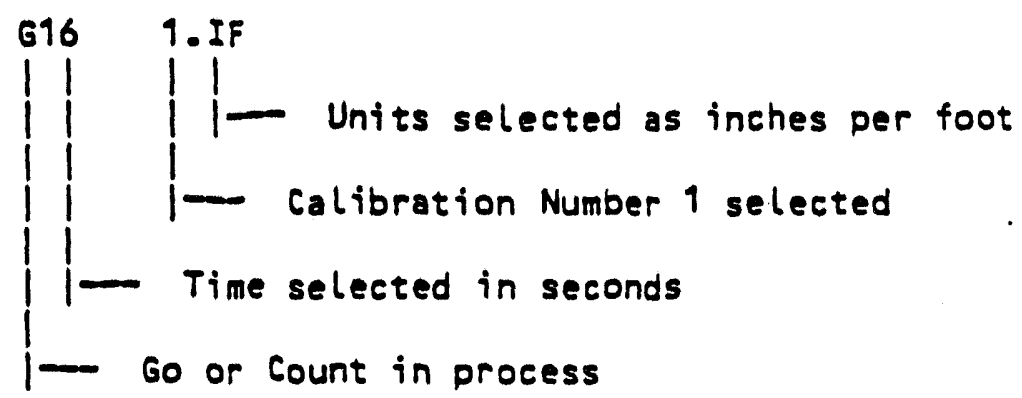

The gage will then display the measurement in the selected units. e.g. "3.88 IF".

The last reading may be displayed at any time, in the currently selected units and calibration, by pressing the WATER key.

\subsubsection{TO LOG READINGS}

Readings can be logged by the gage as they are taken in the field. Each tube site represents a record of information. Prior to storing any readings you must define the format of the tube site record. After readings have been logged, they can be recalled for display or dumped to an external device.

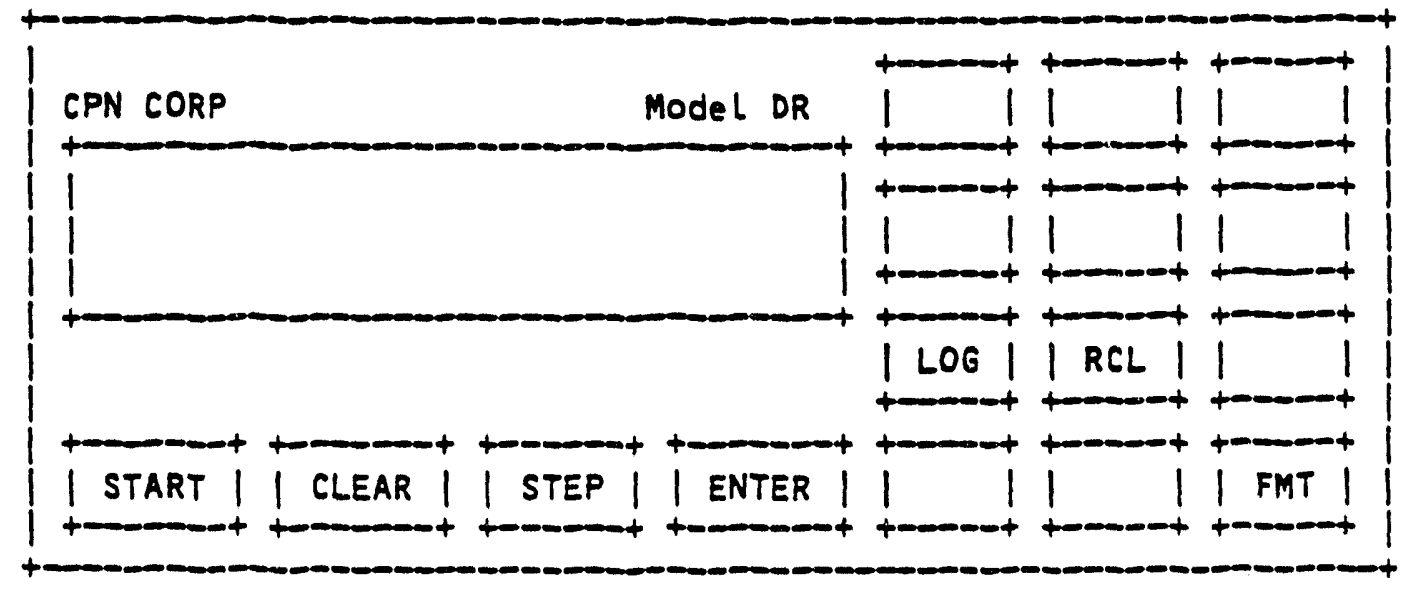

FORMAT

Use the FMT key to format the data storage area to agree with the 
tube conditions. For each access tube at which one record of data is stored, the format will allow 0 to 15 keydata entries and/or 0 to 15 tube depths (counts per tube). The gage always provides for an identifier number (ID) for each record and stores the selected calibration number $(9-8)$.

The total memory space available is 3072 bytes. The number of bytes required in a record for each tube site is as follows:

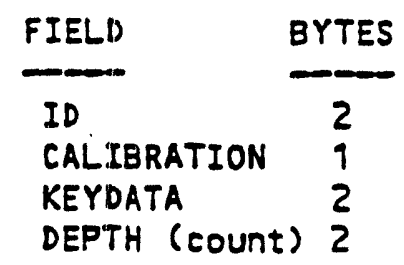

Thus a typical tube site record format of of one ID, one calibration number, one keydata, and three depths (counts), takes 19 bytes per record, and allows 279 records to be stored.

After the number of depths is entered the gage will display "SET FMT?". Press ENTER to set the new format or CLEAR to abort. Setting a new format clears all the data records. DO IT EACH TIME A NEW SET OF DATA IS TO BE STORED.

Press FMT, ENTER, ENTER, ENTER, CLEAR to view the number of records, keydatas and depths without clearing the data records.

\section{LOG/TAKE MEASUREMENTS}

Set units, time, calibration and format. Then to $\log$ a record of information, olace the gage on the access tube and press LOG. The gage will display the number of the current record into which data is to be logged. Since it down counts it is also an indication of how wany empty records remain.

You can use the gage generated number as the ID number to be stored by pressing ENTER. To enter your own ID number for this record (access tube) key in any number from 1 to 65,535 followed by ENTER. It may be meaningful to treat this number as more than one number. i.e. consider the first two digits as a farm number (allowing from 9 to 65 farms) and the last two digits as a farm field number (allowing from 9 to 99 fields on any farm). Enter the middle digit as 0 or use it to indicate an operator number from 1 to 9.

After entering the ID number the display will prompt for keydata entry e.g. "KI " (if selected by the FMT key previously). Keydata entries allow you to use the gage to key in auxiliary information sidin as temperature, rainfall etc. This feature helps eliminate errors in translating readings to note pads and then from the note pad. to the computer. (It is recommended that all field data be hand recorded to prevent loss due to subsequent equipment failure).

Enter the keydata as a number from 0 to 65,535 followed by ENTER. 
Again it may be treated as more than one piece of information. i.e. first two digits for temperature in degrees Celsius and the last two digits for rainfall in inches. A keydata field does not have provisions for decimal points. They must be implied, not entered directly.

Use any scheme whicin fits your field conditions. Just be consistant from record to record. The Irrigation Water Management Program available from CPN CorD. provides for entering the date for a series of readings by setting the ID to " $O$ " and the first keydata to the date. e.g. 412 for April the 12th. Press ENTER without taking counts to skip the depth readings for this special record. All records following until the next ID of "O" are then access tube readings. The IWM program expects that the farm number times 100 plus the field number will be stored in the ID and the site number in the first keydata. e.g. for farm "q", field " 2 " and site " 3 " the ID would be 102 and the first keydata would be 3 .

If you make an error in entering a number, press CLEAR and enter the correct number. If you press CLEAR more than once in succession it will cancel the record storage witliout saving any of the record and return to the "READY" display.

If the gage does not accept the number (e.g. you try and enter a

- decimal point) it will give a multiple beep and re-prompt. If it does accept the number it will prompt for the next keydata or if - all keydatas are entered it will prompt for a moisture reading by displaying "TAKE $M \# " ~(\#$ is the number of the depth position and will down count from the maximum number set via FORMAT to 1 ).

Lower the probe to the correct depth and press START. The gage will display during counting as follows:

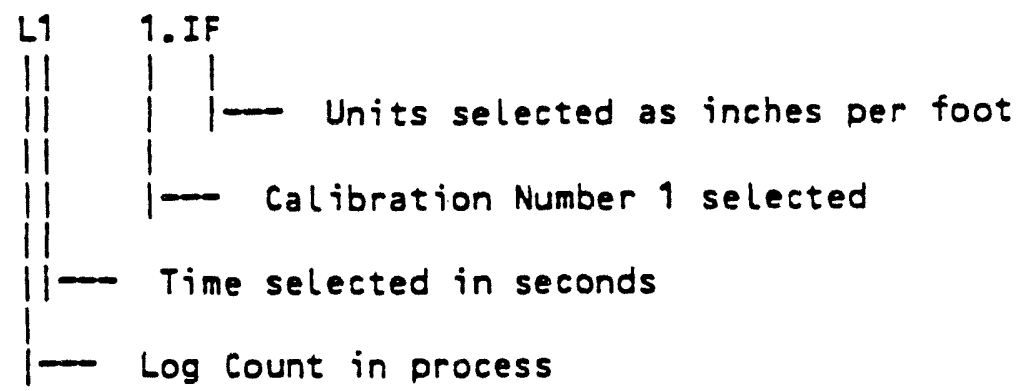

When the count is completed the gage will display "MH" and the value of the reading in the units selected e.g. "M3 2.6538 ". If the reading is acceptable press ENTER to store it. If not acceptable identify the reason and press START to take another. Thus the gage will only store a reading if you accept and enter it. The display will then prompt to take the next depth moisture reading. Move the probe and repeat the process by pressing START. Continue in this manner until all depths have been recorded.

If you want to skip a depth (e.g. the bottom depth is flooded) press STEP instead of START/ENTER. This is also useful if you have some tubes with five depths and some with only three. Format for five and skip two readings when on a tube with only three depths. 
If the units "IF" or "CC" have been selected then after the last depth has been entered or stepped over, the gage will totalize and display the TOTAL MOISTUPE in the root zone in inches or $\mathrm{cm}$ e.g. "TM 16.437".

Press STEP or ENTER and the gage will display "DATA OK?". Press ENTER to $\log$ all the data for this record. The display will return to "READY". If the data is not correct press STEP until the bad data is displayed (the display will start with the ID and skip across the record). Correct it by a keydata entry or taking and entering a new count. If you press CLEAR when "DATA OK?" is displayed then the logging of that record will be aborted and all data for that record eleared.

Use the ENTER or STEP key to skip to the keydata you want to change or take a new count. When you again reach the end of the record and "DATA OK?" is displayed press ENTER to log that record.

\section{RECALL A RECORD}

Normally the stored data will be dumped to a printer or computer. It may also be recalled to the display by the RCL key. When first entered it will point to the last ID stored. Either use the STEP key to step up the ID list (it steps back thru the list and circles around at the begining) or key in a specific ID and press ENTER. Use the step key to move across the record. If you continue pressing STEP the display will advance up to the next record and then across etc. 


\subsection{STANDARD COUNT}

The standard count is a measurement of the hydrogen in the wax in the shield. By taking it in exactly the same manner each time, it provides two means for checking the validity of the counting function.

1) By comparing it with the previous standard count to see that it has not changed more than an acceptable amount, it is an indication of acceptable drift of the electronics. Americium-241 has a one-half life of 458 years. It's decay rate is negligable.

2) By taking it as a series of short counts rather than one long count and verifying that its statistical distribution is normal, it is a means of checking that noise is not influencing the count.

\section{PREVIOUS STANDARD COUNT}

Whenever a new standard count is taken, the DR automatically holds the current standard count in the previous count memory. This allows for comparing the new count with the current count. The difference between the two counts should be within 0.707 of the square root of the average of the two counts $95 \%$ of the time.

Example: The current standard counts is 14400 while the previous standard count is 14450. The difference between the two numbers is 50. The square-root of the average of the current standard count is

120.1 (one standard deviation). Since 50 is less than $85,60.707$ times 120), the two counts are within acceptable limits and there is no reason to believe the gage is malfunctioning.

If it is more or if it is continuously changing in the same direction it indicates that service may be warranted.

TAKING A STANDARD COUNT

With the case on the ground, place the gage in the CPN nameplate depression on the top of the case. No other radioactive sources should be within 30 feet of the gage and no source of hydrogen should be within 10 feet of the gauge. The operator should stand back 10 feet after starting the reading.

To initate a new standard count press STD and then START.

The wax in the shield is not an infinite volume. Thus a standard count taken in this manner is subject to surrounding conditions. It is important that the standard count is taken in the same conditions as that used to estab-

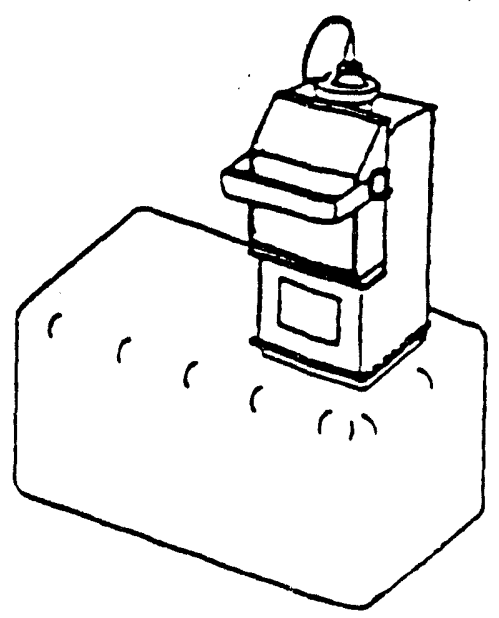

MaY 7, 1984 503DR MANUAL PAGE 18 
lish the calibration and that the conditions are the same each time.

more stable method to take a standard coumt in an access tube installed in a $30 \mathrm{gal}$ lon or larger water barrel. To use the factory calibration but change to a new method of taking a standard court, modify the " $A$ " calibration slope term by the ratio of the new standard count and the factory standard count. e.g. the original factory standard count was 11,000 with an "A" slope of 2.6, while the new water barrel standard is 33,000 . The new "A" coefficient should be:

\section{$2.6 *(33,000 / 91,000)=7.8$}

The verification reading of the wax in the shield as per section 1.3 .1 should be the same.

When a standard count is started, the gage will take 32 each 8.0 second counts, turning the HV power supply to the probe on with each count. With each count, the display will show the count number $(32,31,30, \ldots \ldots, 9)$ followed by the actual 8 second count e.g. "30 7200". When the 32nd count is completed the NEW standard count is displayed e.g. "MS 14400". It is hormalized to a 16 second count time, the same as a normal display count.

Press the STEP key to view the CURRENT standard count e.g. "PS 14500". Press the STEP key again to view the Chi-sa Ratio of the NEW count e.g. "MCHI 1.16". Continuing to press STEP will cycle the display thru the three pieces of standard count information. To update the standard count press ENTER while the display is in this LOOD. The eurrent standard count will be stored as the previous standard count and the new standard count will be stored as the current standard count.

(Note that the previous standard count viewed above is actually the current standard count until you press ENTER to accept the new standard count).

To exit STANDARD without updat ing the standard count press CLEAR. To abort a standard count in process press CLEAR several times until "READY" is displayed

If the gage is connected to a printer via the serial link (see section 2.9), individual counts and summary information will be printed

SN 4860

- STATCT-

32-4398

$3 \mid-446 !$

$30-1469$

$29-4267$

$28-1572$

$27-4349$

$26-1360$

$25-4467$

$24-4389$

$23-407$

$25-4498$

$21-4481$

20-4497

$19-1363$

$18-1575$

$17-4513$

$16-4471$

15-4407

$14-6390$

$13-4411$

$12-4157$

$11-463$

$16-477$

9- 414

$8-4508$

7- 452

$6-441$

5- 4489

4- 4410

3- 4454

2- 479

[- 423$]$

AVE $\$ 444$

CHIR 0.95

MSTD 8895 (AVE + 16/B)

FIG 2.6-1:<smiles>Cc1ccccc1C1CCCCC1</smiles> 
out as show in fig $2.6-1$

DISCUSSION

Taking such a series of 32 counts will result in a distribution of counts around a central value. The standard deviation is a measure of the spread of these counts about that central value. For a random device, such as the decay of a radioactive source, the ideal standard deviation should be equal to the square-root of the central value.

If the gage is working properly, then the measured standard deviation and the ideal standard deviation should be the same and their ratio should be 1.00. The Chi-squared test is used to determine how far the ratio can deviate from 1.00 and still be considered acceptable. This is similar to expecting heads and tails to come up equally when flipping an unbiased coin but but accepting other distributions when only fliping a small number of times.

For a sample of 32 counts, the ratio should be between 0.75 and 1.25 for 95 I of tha tests. Note that even a good gage will fail 5 out of every 100 tests. If the ratio falls too consistently outside, it may mean that the counting electronics is adversely affecting the counts. Generally the ratio will be high when the electronics is noisy. This might be due to breakdown in the high voltage circuits or a defective detector tube. The ratio will also be high if the detector tube counting efficiency or the = electronics is drifting over the measurement period. i.e. The average of the first five counts is significantly different than the average rof the last five counts.

It will be low when the electronics is picking up a periodic noise such as might occur due to failure of the high voltage supply filter. This should be accompanied by a significant increase in the standard count over its previous value. 


\section{.7 PRINT}

Outputs the contents of the record log to an external device (computer, CRT Terminal, modem, printer, ete) via the RS232C serial interface connector.

Twn forms are available:

PRINT CD - for dump to an active device such as a computer (or computer via a modem). Each line of data includes a check sum and requires a software response from the computer to insure proper transmission of data.

PRINT LP - for dump to a Dassive device such as a printer or CRT Terminal. Same as the Print CD except no checksum, and the next line of data is transmitted without waiting for a response from the receiving device. Also the data is formatted for ease of readability and header information is included.

PRINT CD

A simple software ACKNOWLEDGE/NEGATIVE-ACKNOWLWDGE handshaking scheme (ACK/control-F, NACK/control-U) allows the external device to control the dump: ACK echoed in response to a received line causes transmission of the next line, while NACK causes retransmission of the same line. NACK may be echoed as often as necessary to receive an error free line. Characters other than ACK, are by default NACK. If the DR does not receive a reply within 60 seconds after sending the carriage return and line feed (CRLF) at the end of each line, a default ACK is assumed and the next line is transmitted. The computer should not echo the DR transmission.

The following table shows how long in milliseconds it takes to transmit one character at each of the possible baud rates. The program in the computer must be fast enough to receive, process and be reaciy for the next character within this time to insure not missing any characters. (See also section 2.9 for hardware handshaking which allows control of the DR transmission on a charaeter by character basis).

\begin{tabular}{|c|c|c|}
\hline BAUD & BIT & CHARACTER \\
\hline RATE & ms & ms \\
\hline $\begin{array}{r}110 \\
300 \\
1200 \\
2400 \\
4800 \\
9600\end{array}$ & $\begin{array}{l}9.091 \\
3.333 \\
0.8333 \\
0.4167 \\
0.2083 \\
0.10417\end{array}$ & $\begin{array}{c}100.0 \\
36.67 \\
9.167 \\
4.583 \\
2.297 \\
1.1458\end{array}$ \\
\hline
\end{tabular}

It takes approximately $100 \mathrm{~ms}$ after an external device has transmitted an ACK or NACK, for the DR to respond and transmit another line of data.

May 7,1984 
Each dump line consist of a series of fields separated by commas, and terminated by CRLF. The fields are variable in number and width. The last field is a checksum determined by summing the ASCII decimal value of each of the characters in the line up to and including the last comma.

A received line whose computed checksum agrees with the transmitted checksum is good and should be echoed by ACK, else a loss of data is implied and NACK should be echoed.

As each line is being transmitted, (or retransmitted) its' line number is displayed on the $D R$. The line number down counts, giving an indication of the lines remaining, e.g. "LINE 123".

\section{PRINT CD Format}

The Print CD dump consists of 4 parts:
Line 1
Line count $(N+10)$, gage identification and record format.
Line $2-9$
8 calibrations.
Line $10-\mathrm{N}$
$N$ records

Line 1: Gage identification and format.

Lines, 503DR, Sn,Unit,Mstd,k,d,Chk

$=1$<smiles>II(I)I(I)I</smiles>

1 $111-$ Modulo 65536 checksum

1 - Depths per record $(0 . .15)$

1- Keydatas per record (0..15)

- Standard count (0..65535)

1 - Measurement unit (CT,RO,IF,CC,GC,ZV,PF)

$1-$ Probe serial number (0..9999)

- Gage model number

Total lines to be transmitted (10..1033)

Line 2 - 9: 8 Calibrations

$\mid \begin{gathered}\text { Cal,A,B,Chk } \\ \text { Calibration number (1..8) }\end{gathered}$ 
Line $10-N: N$ Records

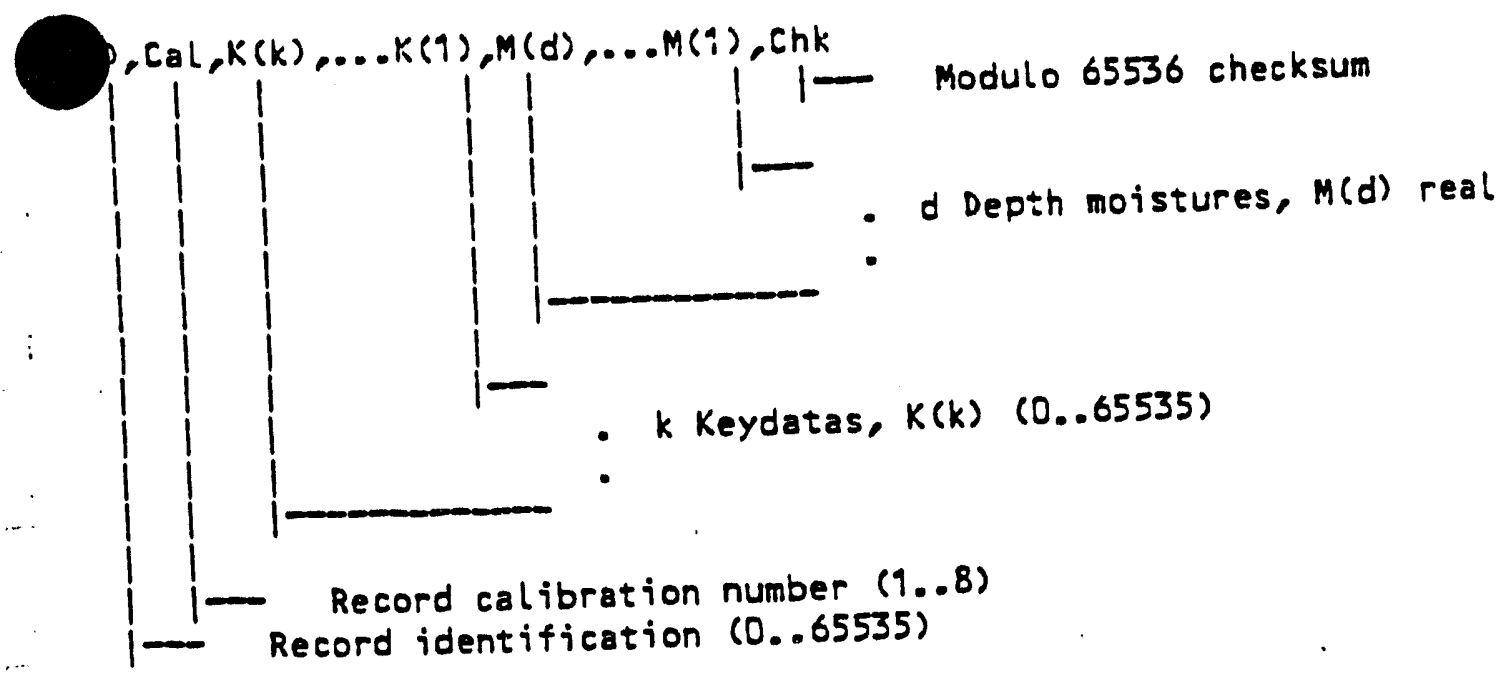

A file image of a PRINT CD dump is shown in Fig 2.7-1. Note that all data fields are separated by commas allowing easy use of input statements in BASIC or FORTRAN programs.

$16,5030 R, 9999,1 F, 10000,2,1,1427$

$1,3.0,-0.06,567$

$2,2.5,-0.055,624$

$3,3.0,-0.08,57 !$

$4,3.5,-0.06,575$

$5,2,8,0.0,457$

$6,0.0,0.0,470$

$7,0.0,0.0,471$

$8,0.0,0.0,472$

$5263,1,0,0,0.0,0.0,0.0,0.0,1273$

$11001,1,20,5,6.804,4.946,6.65 .3 .31,1716$

$11002,1,20,0,3.386,1.15,1.087,1.61,1699$

$11003, \downarrow, 20,1,2.275,1.822,1.826,1.702,1796$

$11004,1,21,5,2.887,1.855,1.966,2.191,1830$

$5273,1,2,0,0.0,0.0,0.0,0.0,1276$

$7003,1,22,15,3,11,4.442,4.135,4.082,1758$

FIG 2.7-1

The print program requires that the computer be up during the dump. This places a heavy drain on the battery. Charge the battery before dumping. If you believe the pack is up but not sure, connect the charger during the dump. If the cutout circuit turns on during the dump, the data is charge the gage for about an hour and then re-initiate the dump.

Some samples of computer programs as shown in Appendix $B$. The ones in BASIC will typically operate up to 300 baud. The assembly language call for the actual transfer of data that can be used to 9600 baud. 
Each computer system will require special considerations. Most operating systems and high level languages are not written for real time operation. For example one BASIC interperter does not require defining a string length but changes its length as data input requires it. For a business application this is convenient, but if it occurs while inputting a record of information from the DR, some characters will be lost. By including an assembly program control of the hardware ENABLE input to the OR this can taken care of, but such hardware control is not possible with a modem.

\section{PRINT LP}

A dump to a printer or a terminal contains the same information as the PRINT CD dump except that: no checksums, no line count and it is formatted for readability with a header and Top of Form command every 60 lines. Print LP also transmitts three control characters at the begining and three at the end of the transmission. These sign on and sign off characters may be used to set external devices such as a printer to a desired configuration. e.g. compressed print. These attributes along with the character reconized as tOD of form (TOF) are set via ATTRIB, a sub-menu of MENU.

Lines of data are transmitted one after another without waiting for an ACK/NACK response. The receiving device should NOT echo the transmission.

A typical PRINT LP printout is shown in Fig 2.7-2.

\begin{tabular}{|c|c|c|c|c|c|c|c|c|}
\hline $\begin{array}{l}\text { MODEL } \\
\text { SOJOR }\end{array}$ & $\begin{array}{l}5 / 1 \\
9999\end{array}$ & $\begin{array}{l}\text { WNIT } \\
\text { IF }\end{array}$ & $\begin{array}{l}\text { ISTD } \\
10000\end{array}$ & $\begin{array}{l}\text { XDATA } \\
2\end{array}$ & $\begin{array}{l}\text { DEPTH } \\
4\end{array}$ & & & \\
\hline CAL & A & $B$ & & & & & & \\
\hline 1 & 3.0 & -0.06 & & & & & & \\
\hline 2 & 2.5 & -0.055 & & & & & & \\
\hline 3 & 3.0 & -0.08 & & & & & & \\
\hline 1 & 3.5 & -0.06 & & & . & & & \\
\hline 5 & 8.8 & 0.0 & & & & & & \\
\hline 6 & 0.0 & 0.0 & & & & & & \\
\hline 7 & 0.0 & 0.0 & & & & & & \\
\hline 8 & 0.0 & 0.0 & & & & & & \\
\hline ID. & CAL & $K 2$ & $\mathrm{KI}$ & 14 & $n$ & 12 & $M$ & TOTAL \\
\hline 5263 & 1 & 0 & 0 & 0.0 & 0.0 & 0.0 & 0.0 & 0.0 \\
\hline 11001 & 1 & 20 & $5^{\circ}$ & 6.804 & 4.946 & 6.65 & 3.31 & 21.71 \\
\hline 11002 & 1 & 20 & 0 & 3.386 & 1.15 & 1.097 & 1.61 & 7.243 \\
\hline 11003 & 1 & 20 & 1 & 2.225 & 1.822 & 1.826 & 1.702 & 7.574 \\
\hline 11004 & 1 & 21 & 5 & 2.887 & 1.855 & 1.966 & 2.191 & 8.899 \\
\hline 5273 & 1 & 2 & 0 & 0.0 & 0.0 & 0.0 & 0.0 & 0.0 \\
\hline $700 j$ & 1 & 22 & 15 & 3.41 & 4.442 & 4.135 & 1.082 & 16.07 \\
\hline & & & FIG & $2.7-$ & & & & \\
\hline
\end{tabular}

While PRINT CD is prefered because of it's handshaking, PRINT LP can be used to make a passive data transfer to a computer. For a CP/M based 
- system with a reader port, set the baud rates to agree, set a Control $Z$ (ASCII decimal 12) attribute in one of the three suffix characters, connect the OR to the serial Dort defined as RDR: (see section 2.9), and ype in the computer while in CP/M:

>PIP DR503. DAT=RDR:

Then p!^ess PRINT, STEP and ENTER on the DR.

The data in the DR will transfer to the file named DR503.DAT. It will be a literal file. You will have to strip out header information etc. 


\subsection{REMOTE OPERATION}

The DR may be oderated remotely via the RS232C serial connector. Set the $D R$ and the external device to the same baud rate. If the $D R$ is in the command mode ("READY" is on the display) then any character on the serial input line will activate the DR without causing any action. The appropiate remote control character from the following table should then be sent to the DR. Because of the debounce software in the DR, the following character should be spaced at least 100 milliseconds but not more than 60 secondsfrom the activate character. The DR will perform the command and shut down.

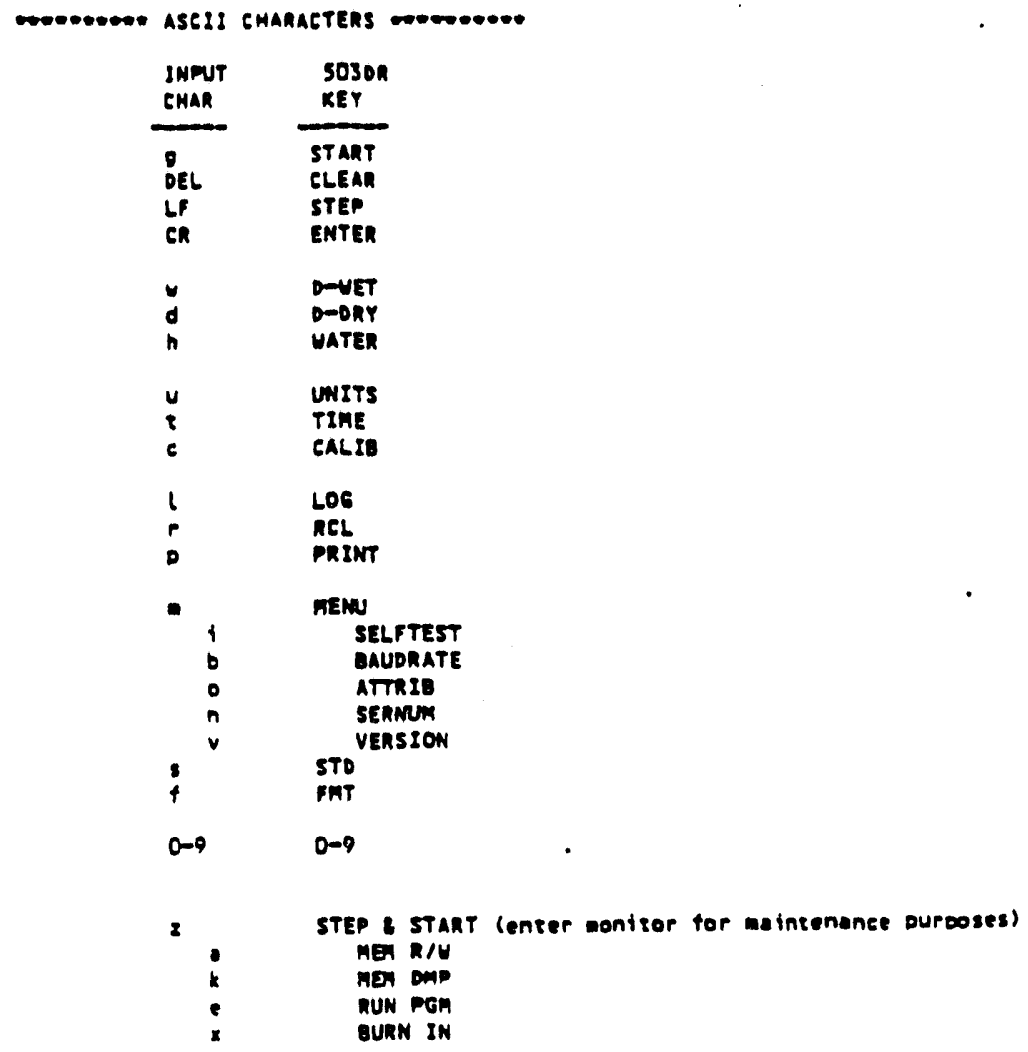

Control $R$ place the on in the terminal node loover wo allowing it to receive further Astl! comand characters. The Dh will return to the nombl bode 60 seconds ofter te receives any command unless. mow comeand is recetived within that rime. unile in the terminal mode all charoctery sent to sme disolay vill also be transateted on the seriel output (ime).

These commands can be used to control the DR from a CRT terminal or a special program can be written to control the DR from a computer.

Control-R is a special command that $p$ laces the $D R$ in the remote terminal mode. While in the remote terminal mode all information which appears on the DR display will also be sent on the serial output line where it can 
$\therefore$ be received by the control device. The DR will stay in this mode for 60 seconds after the last command. If a sequence of commands are separated aby more than 60 seconds it will be necessary to send another control-R. or CP/M based systems with a reader/modem serial port, the XCOM command can be used to control the DR from the computer keyboard.

Example: To cause the DR to start a count send:

$$
99 \text { (lower case "g", } 100 \text { millisecond delay then lower case " } g \text { ") }
$$

The first " $g$ " (or any character) activates the DR. The second " $g$ " is reconized as the equivalent of pressing the START key.

Example: To set the Time to 16 seconds:

$$
\begin{array}{cc}
\text { DR } & \text { SERIAL } \\
\text { DISPLAY } & \text { OUTPUT }
\end{array}
$$

$\begin{array}{lll}\text {-R } & \text { READY } \\ \text {-R } & \text { READY } & \\ t & \text { TIME } 1 & \text { TIME } 1 \\ \text { LF } & \text { TIME } 4 & -4 \\ \text { LF } & \text { TIME } 16 & -16 \\ \text { CR } & \text { READY } & \text { READY }\end{array}$

The first "aR" activates the DR. The second places the DR in the remote terminal mode. " $t$ " causes the current time to be displayed and sent to the external device. If the external device is a terminal then the user must view the display and key line-feeds (STEP commands) till the desired time is displayed then key a carrage return (ENTER command). If the external device is a computer, the program in the computer must do the same (the - shown above are actually backspace characters). The DR will return from the remote mode to the command mode 60 second after the $C R$ or last command is sent. 


\subsection{RSZZ2C SERIAL LINK (hardware description)}

The DR has the capability of two way communications over a serial link. This allows; 1 ) the data stored in the DR RAM memory to be converted to hard copy using a printer, 2) the data to be dumped to a computer for storage and further processing before printing a hard copy report, and 3) allows the DR to be controlled by the computer or a CRT terminal. The access to this serial link is through the ACCESSORY CONNECTOR located on the front of the surface electronic assembly.

\section{ACCESSORY CONNECTOR}

\begin{tabular}{|c|c|c|}
\hline PIN & FUNCTION & \\
\hline 1 & $+10 V$ & $\begin{array}{l}+10 \vee \text { from the DR for use by external } \\
\text { equipment (protected by the internal } \\
\text { circuit breaker) }\end{array}$ \\
\hline $\begin{array}{l}2 \\
3\end{array}$ & $\begin{array}{l}\text { GROUND } \\
\text { SERIAL DATA OUT }\end{array}$ & $\begin{array}{l}\text { Serial data from the } D R \text { to an external } \\
\text { device }\end{array}$ \\
\hline 4 & SERIAL DATA IN & $\begin{array}{l}\text { Serial data to the } D R \text { from an external } \\
\text { device }\end{array}$ \\
\hline 7 & ENABLE & $\begin{array}{l}\text { Control line from an external device to } \\
\text { the DR. When open or high the DR will } \\
\text { transmit the next character. When Low } \\
\text { the DR will hold transmission of the } \\
\text { next character until ENABLE goes high. }\end{array}$ \\
\hline
\end{tabular}

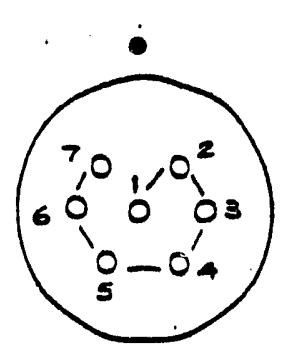

CABLE CONN. WIRING VIEW

The DR transmits -4.0 volts for a MARK and +10 volts (battery voltage actually ranges from 8 to 12 volts) for a SPACE.

\begin{tabular}{|c|c|c|c|}
\hline NAME & $\begin{array}{l}\text { VOLTS } \\
\star \star \star \star \star \star\end{array}$ & $\begin{array}{l}\text { LOGIC } \\
\star \star \star \star \star \star \star\end{array}$ & 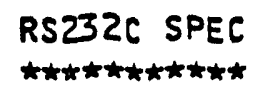 \\
\hline SPAC & $\begin{array}{l}10 V \\
-4 V\end{array}$ & $\begin{array}{l}\text { HIGH } \\
\text { LOW }\end{array}$ & $\begin{array}{r}+3 \text { To + } 25 V \\
-25 \text { to }-3 V\end{array}$ \\
\hline
\end{tabular}

The serial input to the DR accepts the standard RS232 voltage swing.

The ASCII code is 11 bits composed of: one start bit, 7 data bits, one parity bit which is always transmitted as a mark and ignored on receiving (some users refer to this as 8 data bits and no parity), and 2 stop bits (the DR sends two stop bits and will accept one or more stop 


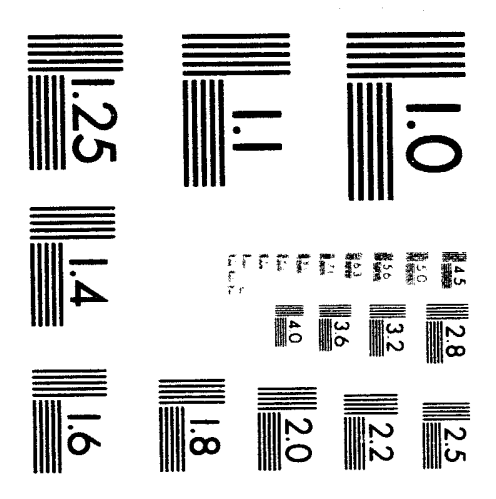



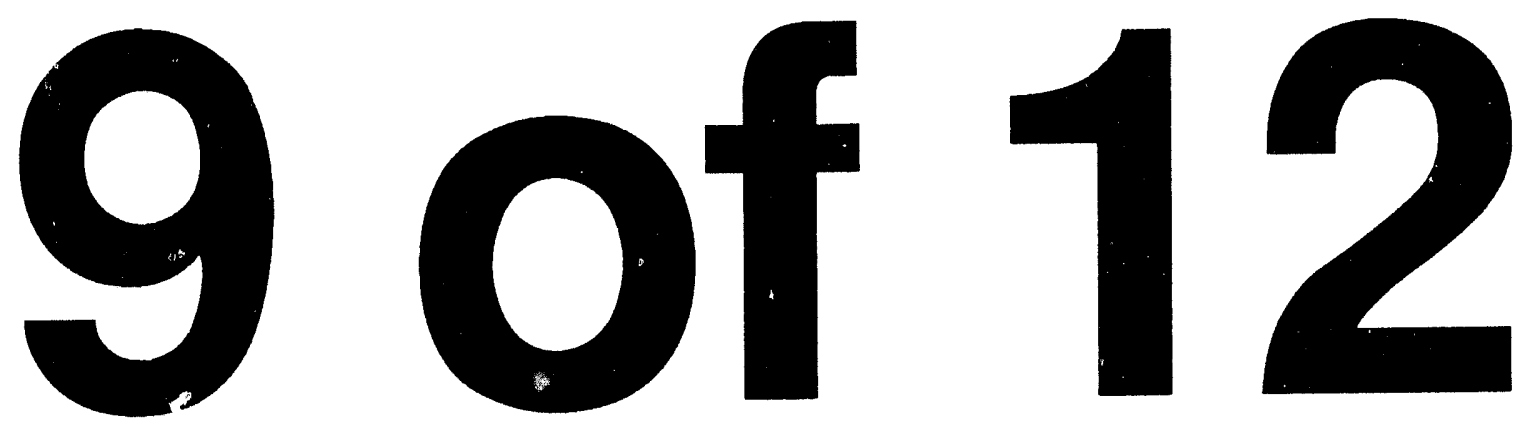
bits).

R5232C defines connector types, Din assignments, signal voltage levels o timing for communications beiween a piece of data communications quipment (DCE) and a piece of oata terminal equipment (DTE). The conneetor should be a DP25S (female) for the DCE and DP25P (male) for the DTE. The DTE transmits on pir. 2 and receives on pin 3 . The reverse is true for DCE equipment.

RS232C has worked well when a piece of terminal equipment was working with a modem. It has not been implemented consistantly when two pieces of terminal equipment are interconnected and the equipment designer must define one of them as communications equipment. e.g. when a computer drives a printer, which is the DCE and which is the DTE? ALL combinations of male/female connectors and transmission/receiving Dins are found.

The following $6 \mathrm{ft}$ long cables, including both a male and female version are available from CPN CorD. Also available are cable parts. Unless you have provisions for soldering the small terminals in the circular connector with 26 AWG wire, it is suggested that you order the unterminated cable for special external device connector applications.

\begin{tabular}{|c|c|c|}
\hline $\begin{array}{l}\text { PART NO } \\
\star \star \star \star \star \star \star \star\end{array}$ & 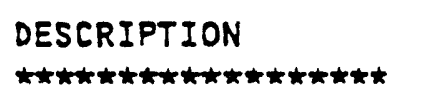 & 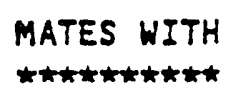 \\
\hline $\begin{array}{l}T 501544 \\
\text { C501516 } \\
T 501575 \\
\text { RGIB307 }\end{array}$ & $\begin{array}{l}\text { Cable, RS232C/DP25P } \\
\text { Cable, RS232C/DP25S } \\
\text { Cable, RS232C/unters } \\
\text { Connector, ch, 7pin }\end{array}$ & $\begin{array}{l}\text { DP25S } \\
\text { DP25P } \\
\text { ced }\end{array}$ \\
\hline
\end{tabular}

The wiring of these cables is shown in Appendix $C$, along with some specific hardware configurations. If your configuration does not. fit any of these cables available from CPN, order one with the proper connector type (male or female). Then rewire the pins as required.

The ENABLE LINE is available for hardware control of the transmission on a character basis. Most external equipment require the ENABLE line wired to pin 4 of the DPZ5 connector for an RTS protocol. On some it will be niecessary to wire it to pin 20 for a DTR protocol.

If hardware handshaking is not required, leave the ENABLE line open. The DR has an internal pull up to place it in the proper state. The circuit for the ENABLE input. (also the serial input) is shown in fig 2.9-9.

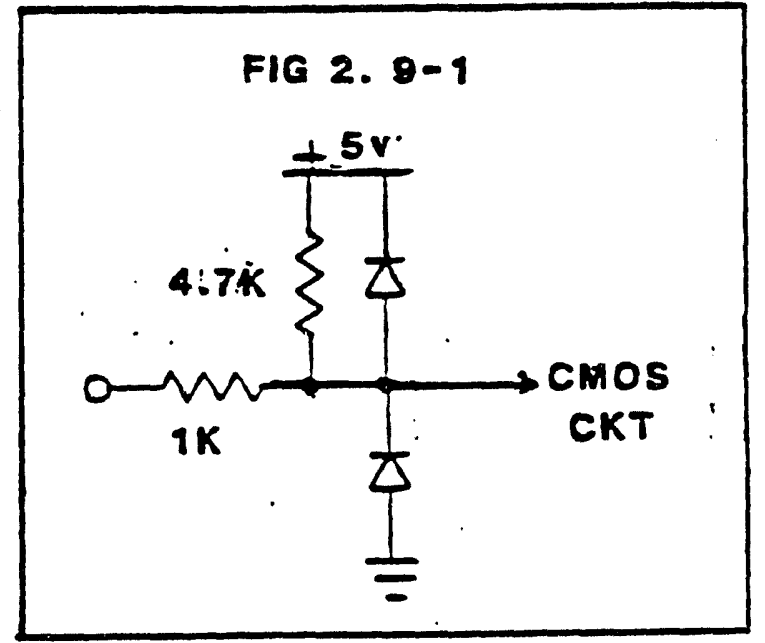

The input control pins to the external device should be wired to either the mark or space condition as appropiate to allow data to flow.. Typically this will involve wiring pin 5 (CTS) and pin 6 (DSR) to the space $(+10 \mathrm{~V})$ condition. 


\subsection{SPECIAL}

\subsection{CALIBRATION}

The neutron probe is a source of fast or high energy neutrons and a detector of slow or thermal neutrons. The fast neutrons are slowed down by collisions with the nucelus of matter in the soil and then absorbed by the soil matter. Since the mass of the nucleus of hydrogen is the same as that of a tree neutron, tile presence of hydrogen will result in a high field of thermal neutrons. Heaver elements will also slow down the neutrons but not nearly so effective. While it takes on the average only 18 collisions with hydrogen, it take 200 with the next element normally found in agro soil.

The thermal neutrons are continually being absorbed by the matter in the soil. Boron for example has a high affinity for thermal neutrons. The resulting thermal neutron flux will depend upon a number of factors both creating $\cdots$ and absorbing thermal neutrons, but most importantly will be how much hydrogen is present. The neutron probe may thus be used as a measuring device for moisture in the soil but it may require calibration for local soil conditions.

\section{FIELD CALIBRATION}

A field calibration requires the probe, a volume sampler, a scale and a drying oven. Install the access tube in a representative point in the soil. Take probe readings in the tube and volume samples in pairs around the tube. Take them at the same depth and within a foot or two of the tube.

Seal the volume samples in a sample can or plastic seal bag immediately after removing from the soil. Be carefull not to compact the surrounding soil when taking the samples. Ideally 20 such measurement pairs should be taken over a range of moisure conditions.

An alternate method is to use a sampler of smaller diameter than the tube and take volume samples at each depth while making the hole to install the access tube. Then take probe readings at the same depths. This has the advantage that the calibration is performed on the tube to be used for scheduling.

Another alternate, popular with irrigation schedulers is to only take two measurement pairs, cine pair at field capacity and a second at a soil moisture condition near $50 \%$ depletion. 


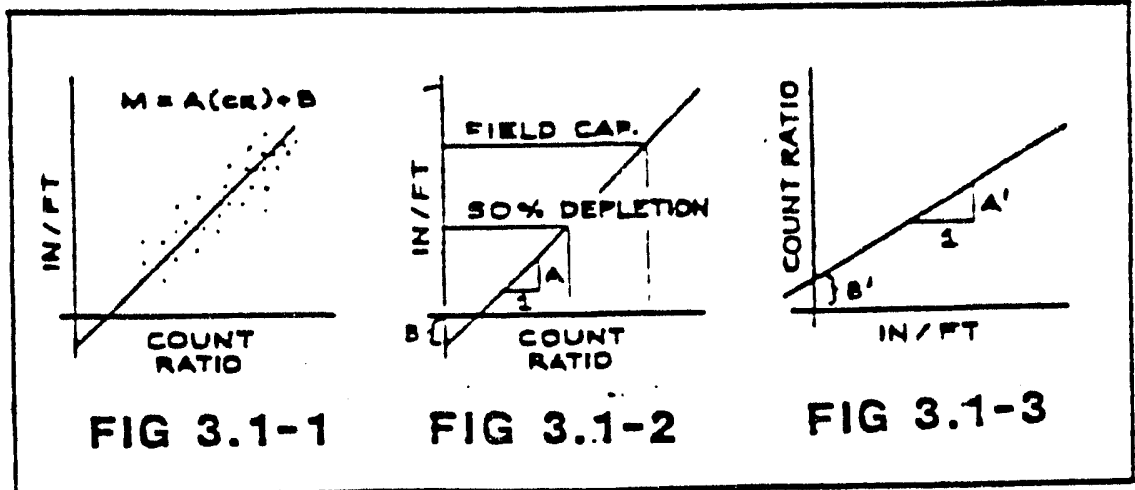

Weigh the soil samples wet and dry $(24 \mathrm{hrs}$ ot $105 \mathrm{deg} C$ in a vented oven). Calculate the moisture by weight and the dry soil density and then combine to determine the soil moisture content in inches per foot as follows:

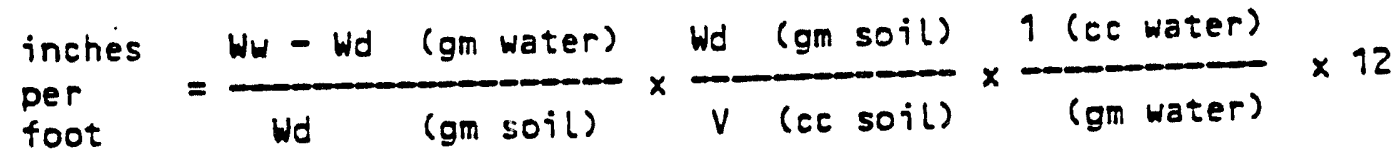

Using linear graph paper plot the probe readings in count ratio. versus the volume samples in inches per foot.

Fit the graph to a straight line. For a statter diagram of $10 \pm 020$ reading pairs do a linear regression on a hand calculator. For only two pairs use the following eqliations to determine the slope and intercept.

$$
\begin{aligned}
& \text { slope }=A=\frac{M H-M L}{R H-R L} \\
& \text { intercept }=B=M L-A \times R L \\
& \text { then: } m=(A \times r)+B
\end{aligned}
$$

where:

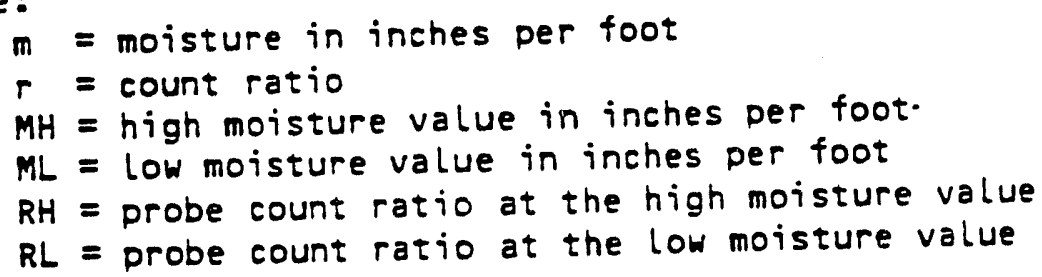


Example: A field capacity of $3.8 \mathrm{in} / \mathrm{tt}$ gives a ratio of 1.500 , while 50 percent depletion gives a ratio of 0.77 .

$$
\begin{aligned}
& A=\frac{3.8-1.90}{1.5-0.77}=2.603 \mathrm{in} / \mathrm{ft} / \text { countratio } \\
& B=1.9-2.603 \times 0.77=-0.1043 \\
& O R \\
& m=2.603 \times r-0.1043
\end{aligned}
$$

Perform the following keystrokes to enter this calibration into calibration number 2.

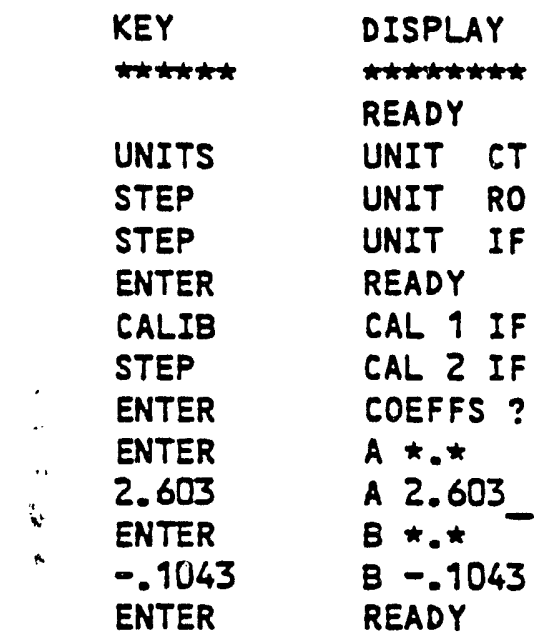

The DR defines the slope and intercept with water on the vertical axis and ratio on the horizontal axis as shown in Fig 3.1-2. If you data has been plotted with the axis reversed as shown in Fig 3.1-3, it will be necessary to transpose the slope and intercept terms before entering in the DR.

$$
\begin{aligned}
& A=1 / A^{\prime} \\
& B=B^{\prime} / A^{\prime}
\end{aligned}
$$


For a laboratory calibration two known calibration points are needed. A high calibration standard might be a barrel of sand saturated with water (typically $0.32 \mathrm{gm} / \mathrm{cc}$. i.e. 0.32 grams of water per cubic centimeter of soil or $32 \%$ water by volume or 3.84 inches of water per foot of soil). A Low standard of dry sand would be $0.0 \mathrm{gm} / \mathrm{cc}$.

Set the gage to the desired units and select a 256 sec count time. Use the SLFCAL feature of the probe. Place the probe in one of the two known moisture standards. The display will prompt for the known moisture density of the standard. Enter it in the units selected e.9. 3.88 ENTER. If count or ratio has been selected as the units, an error message will be displayed.

Pressing START will cause the gage to take and store a 256 second reading. When the count is completed move the probe to the second moisture standard. The display will prompt for the moisture density of that standard. Press START to take a 256 second reading in the second standard. When the count is completed, the gage will calculate and store the slope and intercept coefficients for the calibration in the selected units. Use "COEFF ?" ENTER to view them. Record them in your note book for future reference.

This is how the factory calibration was determined. It will be applicable for sandy soils with no significant minerals or organics. It can also be used for relative measurements. e.g. measuring a field before and after a known irrigation will allow determining how much was applied versus how much the probe measures.before and after, thus calibrating the probe to that field.

\section{RANGE}

The linear calibration supplied with the DR is useful over the most commonly used mositure range, $D$ to $33 \%$. For special operation in moisture percentages higher than this it is necessary to have a special calibration. Fig 3.9-4 shows a high range calibration. If field data is not available, a count in a water barrel can be used.

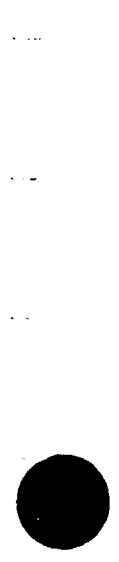

May 7,1984

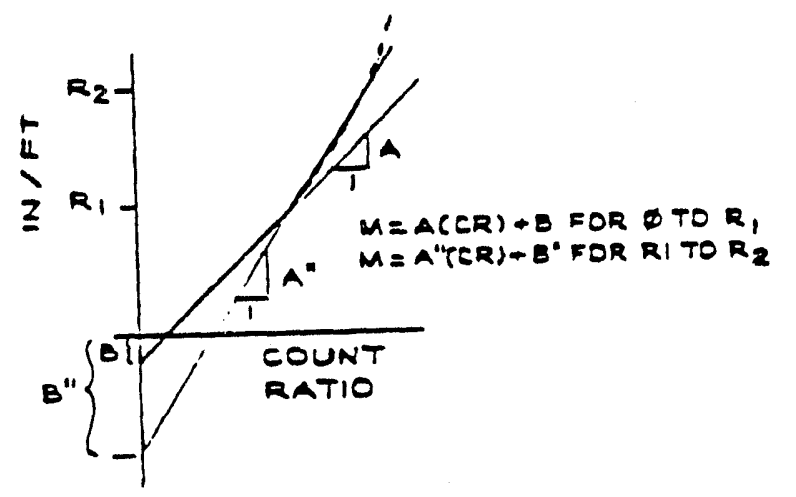

FIG $3.1-4$

5O3DR MANUAL 


\subsection{ERROR MESSAGES}

If an error occurs in the DR, then the function that was being performed is soorted, and an error deseription or number is displayed (the gage is actually in the READY mode). Errors that may occur in the normal operation of the gage, will display a descriptive message. You should take corrective action as appropiate.

Equipment errors that should seldom occur will display a descriptive message or "ERR" followed by a number of 100 or above. If such an error number occurs, contact the factory or local service center.

Operating errors:

NO STOCT

FULL

NOT HERE

EMPTY

NO DATA

SET UNIT
No moisture standard count. Take a new standard.

Record log full, PRINT-out record log, and clear viz FORMAT. ID entered into RECALL does not exist. Enter correct ID.

Record log empty whtn RECALL pressed. Load record(s) via LOG.

Record log empty when PRINT pressed.

Calibration coefficients underfined for CT or RO unit. Change UNIT.

Internad Check (hardware) errors:

$\begin{array}{ll}\text { RAM } & x \times X X \\ \text { ROM } & x \times X X \\ \text { EEP } & x \times X X \\ \text { CK } & X . X X X X \\ \text { MCTR } & \text { ERR }\end{array}$

Program traps:

I OIV 0

DIV BY 0

NEG ROOT

PBUS ERR

EEP $X X X X$

ERR 100

ERR 102

ERR 108

ERR 109

ERR 110

ERR 113

ERR 116

ERP 197

ERR 120

ERR 201

ERR 203

ERR 204

ERR 297
Non-destructive ram test failure at address $x \times x X$.

Program memory checksum error. $X X X X$ is the non-zero sum. EEPROM. read after write failure at address $x \times x X$.

Timer clock test period error. $|1-X . X X X X|>0.01$ seconds Moisture counter failure.

Integer divide by zero.

Real divide by zero.

Square root of negative number.

Pcode buss error.

EEPROM read after write failure at address $X X X X$.

Computer bus ersor, underfined restart.

Message number too big.

Real display roundup exponent underflow.

Onclear pstack pointer greater than current pstack pointer.

Onclear machine stack pointer greater than current machine stack pointe Undefined peode.

Spurious timeout.

Pcode pointer underflow (machine stack running peode).

Onclear stack underflow.

Depth index too big.

Recordindex too big.

Count too big to store in recoro.

Spurious initialization. 


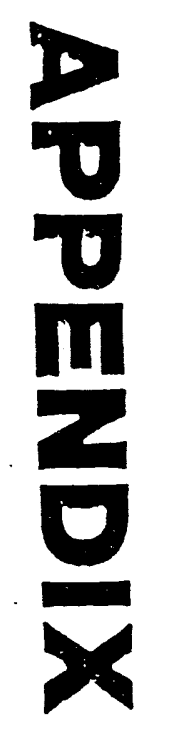




\subsection{General}

Radioactive decay is a chance process. For Cesium-137 which has a half-life of 30 years, it can be expected that in 30 years one-half of the material will have decayed, but in the next minute exactly which atoms will decay and exactly how many will decay is only by chance. Repeated measurements with the gage will thus most likely result in a different count for each measurement. A typical set of 32 such measurements is shown in Fig $A-1$.

Fig $A-2$, shows the distribution of these counts. The two characteristics of interest are: 1) The average value (also called measure of central tendency or mean) and 2) How wide the counts spread around this average.

Mathmatically the average value is defined as:

$$
\bar{x}=\frac{\sum_{x}}{n}
$$

SN 4660

The width of the spread is defined by a term called standard deviation.
$-5 T$ ATCT-
$32-4398$
$31-446 !$
$30-4169$
$29-1267$
$28-1572$
$27-4349$
$26-1360$
25-4467
$24-4389$
23-4407
22-4498
$21-418 i$
$20-4497$
19-4363
18-1575
$17-4513$
16-4471
$15-4407$
$1+-1390$
13-4411
$12-4457$
$11-4463$
$10-4471$
9- 4414
$8-4508$
7- 4452
$6-4411$
5- 4489
4- 4410
3- 444
2- 4479
1- 4123

$s=\sqrt{\frac{\sum(x-\bar{x})^{2}}{n-1}}$

Or an alternate form useful on calculators

$$
s=\sqrt{\frac{n\left(\sum x^{2}\right)-\left(\sum x\right)^{2}}{n(n-1)}}
$$

where:

$s=$ standard deviation of the sample

$x=$ count (value of each sample)

$\bar{x}=$ average of the sample

$n=$ number of measurements in the sample

The above describes the average value and the standard deviation of a sample from a population. They are an approximation to the true average value and true standard deviation of the population.

AVE 444

CHIR 0.95

$\$ 5 T 08895$ (AVS + 16/B)

FIG $A-1$ 
$\mu=$ average of the population

$\sigma=$ standard deviation of the population

The distribution from measurement samples of any process can be classified into expected shapes that have been previously observed. Three are applicable to radioactive decay: Binominal, Poisson and Normal (also called Gaussian).

The Binominal distribution applies when the measured event can take one of two states. Tossing a coin is an obvious case. It can also be applied to a given atom either decaying or not in a time period. It is difficult to deal with computationally.

Since the number of atoms is very large and the expected probability of a decay occuring is very low (source life in years and measurement time in minutes), we can use the poisson distribution which is a special case of the binominal distribution for these conditions. A special property of " the Poisson distribution is that the expected standard deviation is equal to the square-root of the average value.

$$
\sigma=\sqrt{\bar{x}}
$$

If the sample is large enough we can approximate for the standard deviation of the sample.

$$
\delta=\sqrt{\mu}
$$

This is an important relationship. It means that if repeated measurements are taken without moving the gage and the detector electronics are working properly, then the spread of the counts will only be dependent upon the average count rate. This is in contrast to most measurements where the spread will depend upon the process. Fig $\mathrm{A}-3$ shows the diameter of a part turned on a new lathe while Fig $A-4$ shows the same part turned on an old lathe. Both lathes produce. a part with the
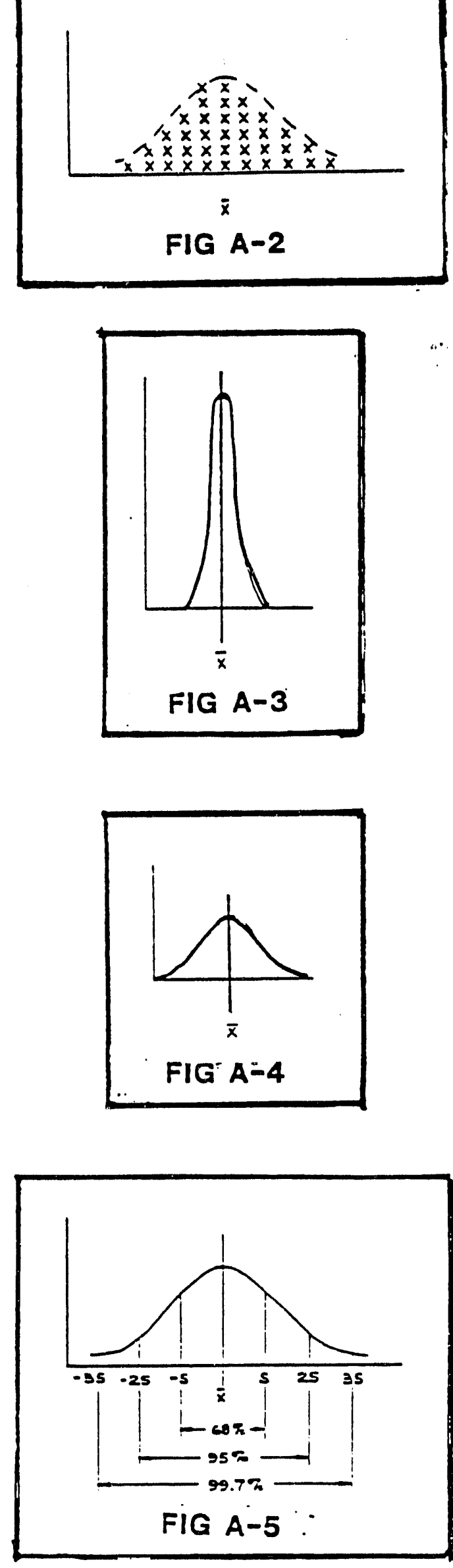

PAGE AZ 
same average diameter but a loose bearing caused the wider spread for parts manufactured on the older lathe.

The Poisson distribution applies to discrete measurements e.g. count or no count. Provided the average value is large enough ( 20 or greater), the Poisson distributions can be approximated by the Normal distribution.

Using the Normal distribution simplifies things even further. It is a continuous distribution. It is symmetrical about the average, and most important it can be completely described by its average and standard deviation.

As shown in Fig A-5, for a Normal distribution, $68.3 \%$ of all counts will be within one standard deviation, $95.5 \%$ of all counts will be within two standard deviations, and $99.7 \%$ of all counts will be within three standard deviations.

Thus these three distribution models become identical for the case with a small individual success probability but with a large number of trials so that the expected average number of successes is large. This allows the use of the best features of each distribution for three statistical situations concerning the gage:

1) Single measurement precision

2) Expected spread of measurements

3) Expected difference between two measurements

\subsection{Single Measurement Precision}

The expected variation for one standard deviation (68.3\%) of a single count can be expressed as a percent error as follows:

$$
\because E \text { ERROR }=100 \frac{\sqrt{x}}{x}=100 \frac{1}{\sqrt{x}}
$$

This expression reveals that the only way to improve the count precision (e.g. reduce the percent error) is to increase the size of $x$ (e.g. the gage manufacture selects components for a higher count rate while the gage user counts for a longer period of time).

The following table demonstrates that a minimum of 10,000 counts is required to achieve a count precision of 1.0 percent or better $68.3 \%$ of the time.

$\begin{array}{rccc}\text { COUNTS } & \text { SR RT } & \begin{array}{c}\text { COUNT } \\ \text { PRECISION } \\ (68.3 \%)\end{array} & \begin{array}{c}\text { COUNT } \\ \text { PRECISION } \\ (95.5 \%)\end{array} \\ 1 & 1 & 100.0 & \\ 10 & 3.16 & 39.6 & 63.2 \\ 100 & 10 & 10.0 & 20.0 \\ 1,000 & 31.62 & 3.16 & 6.32 \\ 10,000 & 100 & 1.00 & 2.0 \\ 100,000 & 316.22 & .32 & .63\end{array}$


The count precision improves with the square of the count. Thus taking four times the counts improves the count precision by a factor of two.

To provide a consistant frame of reference to the operator, the count displayed in the $D R$ is always an equivalent 16 second count regardless of the time base selected. It is necessary to correct a precision determination for other time base selections as follows.

$$
\text { 7.ERROR }=100 \frac{1}{\sqrt{x \cdot t / 16}}
$$

where $t$ is the selected time in seconds

Example: A 64 second direct count is taken and displays 3000 . The precision of the count is PCT $=100 /($ SQR $(3000 * 64 / 16)$ or $0.913 \%$.

The direct reading is $2.0 \mathrm{gm} / \mathrm{cm}^{3}$. To determine the end measurement precision it is necessary to multiply the count precision by the slope of the calibration curve. Assuming a slope of $0.0416 \mathrm{gm} / \mathrm{cm} 3$ per percent, the $2.0 \mathrm{gm} / \mathrm{cm} 3$ reading varies by $+1-0.038 \mathrm{gm} / \mathrm{cm} 3(68 \%$ of the time representing one standard deviation).

If you take repeat measurements but move the gage between reading then the standard deviation of that set of readings will include both the source random variation and the variation due to re-positioning the gage, and thus be larger.

\subsection{Expected spread of measurements}

An accepted quality control procedure for a random counting device is to record a series of 20 to 50 successive counts while keeping all conditions as constant as possible. By comparing the distribution of this sample of counts with the expected Normal distribution, abnormal amounts of fluction can be detected which could indicate malfunctioning of the gage.

The "Chi-squared test" is a quantitative means to make this comparsion. It can be used when a calculator is available to determine the standard deviation of the sample.

$$
x^{2}=\frac{(n-1) s 2}{82}
$$

where $x^{2}$ is from the Chi-squared tables.

By substituting that the expected standard deviation is equal to the square-root of the average count $(\sigma=\sqrt{\bar{x}})$, re-ir ranging terms and taking the square-root of both sides: 
Ideally the ratio on the right hand side of this expression should be 1.00. The degree to which this ratio departs from unity is an indicator of the extent to which the measured standard deviation differs from the expected standard deviation.

On the left hand side of the expression, the degree to which $x 2$ differs from $(n-1)$ is a corresponding allowance for the departure of the data from the predicted distribution (e.g. We flip a coin ten times and expect five heads and five tails but accept other distributions for a given sample). Chi-squared distribution tables are found in texts on statistics. The table values depend upon the degrees of freedom cone less than the number of counts) and the probability that a sample of counts would have a larger value of $x 2$ than in the table. The $x 2$ values for $2.5 \%$ and $97.5 \%$ (a $95 \%$ probability range) and 31 degrees of freedom are 17.54 and 48.23 substituting these value into the left hand side of the expression gives ratio limits between 0.75 and 1.25 for 32 samples and a $95 \%$ probability.

If the ratio on the right side is between these limits then there is no reason to suspect the gage is not performing properly. If the ratio is outside these limits then the gage is suspect and further tests are in order (even a properly working gage will fall outside the Chi-squared limits $5 \%$ of the time).

If a calculator is not available which can easly determine the standard deviation, a qualitative method to compare the observed standard deviation with the expected standard deviation is to take a series of 10 counts and determine their mean and the square-root of their mean (guess the square-root to 2 digits if not available on the simple calculator). If their distribution is normal then $68.3 \%$ of the readings will be within the mean $+1-$ the square-root of the mean (e.g. 7 out of 10 ). 


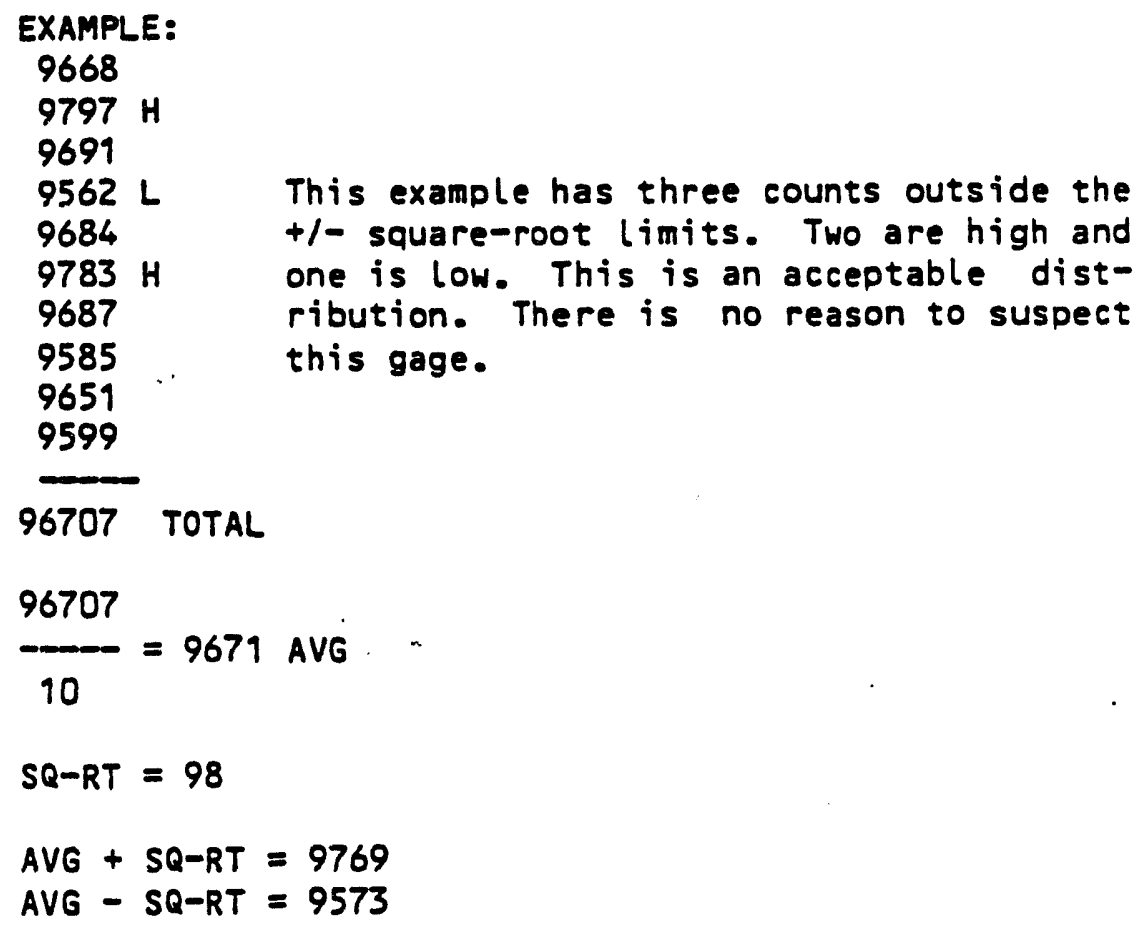

Since this test only involves a sample of ten counts it is not very predictable. Even a properly operating gage will only pass this test one out of four times. If the test fails repeat it to see if any adverse trends exist.

\subsection{Expected difference between two readings}

The standard count or some other reference count should be recorded on a regular basis to allow observing if it stays the same or if any adverse trends are present. If enough counts have been used to determined the average and thus also the standard deviation of the population, then the Normal distribution may te used.

$$
z=\frac{\bar{x}-\mu}{\sigma}
$$

Expressing the $\bar{x}$ value in terms of the $\mu$ value plus a factor of the deviation.

$$
\begin{aligned}
& \bar{x}=\mu+1-k \star \sigma \\
& z=+1-k \star \operatorname{sq}-r t(n)
\end{aligned}
$$

From the Normal tables, for $95 \%$ confidence, the $Z$ value is 1.96 .

$$
K=+1-\frac{z}{\sqrt{n}}=+1-\frac{1.96}{\sqrt{1}}=+1-1.96
$$


$\because \quad$ Thus the new reading should be equal to the average of the old reading plus/minus 1.96 times the square root of the old average.

This is true for the 16 second count which is direct. For another time base the $K$ term must be reduced by the square-root of the count pre-scaling. e.g. for a 64 second count which is 4 times as long as the direct 16 second count the new reading should be plus/minus 0.98 . A special case is the standard count which involves 32 each $(n=32) 8$ second counts. A new standard count should be equal to the old standard count plus/minus 0.49 times the square-root of the old standard count 95 percent of the time.

\section{EXAMPLE:}

The average of the daily standard count for the last month is 10,000. The square-root of this average is 100 . A new standard count ( 32 each at 8 seconds but displayed as 16 seconds) should be between 9,951 and 10,041 (95\% probability).

\subsection{TROUBLE SHOOTING}

\section{CONDITION}

Chi ratio too high, no change in the average count over previous.

Chi ratio too low with an increase in the average count over previous.

Chi ratio too low, no change in the average count.

Chi ratio OK but change in average count.

\section{POSSIBLE CAUSE}

Look for. a drift in the counts over the measurement time. e.g. the average of the first five counts is significantly different than the average of the last five counts.

Periodic noise occuring. Possibly an open filter capacitor in the HV power supply.

Procedure error. Possibly analyzing normalized counts. The standard deviation must be determined on direct counts.

Change in gage geometry. A change in counting efficiency will be normalized out by ratio technique. A change in gage geometry must either be corrected or the gage recalibrated. 


\section{INTERFACING THE 5O3DR TO MICROCOMPUTER DEVICES}

One of the features of the 5030R is to communicate with other computers as a remotely activated device or to download its logged data records to a disk or printer. There are two parts to this process; downloading software, and RS-232C serial communication hardware.

\section{DCWNLOADING RECORDED DATA}

Using the logging feature the gauge can record many records of site readings for recall later. It is extremely convenient if that data can be used in a program that can manipulate the data for the users needs. To get the data from the probe to the computer you may use the PRINT CD feature which stands for PRINT Computer Dump. Computer to computer communication requires a matched standard means of data communication implemented on both ends of the transfer. The gauge is capable of serial communication in an RS232 ASCII format which is standard for many computers and communication packages written to be used on them. One problem of serial communication is that it is not fool-proof and some form of error checking must be preformed on the data to insure that it is valid. The gauge uses a format where at the end of each line that is transmitted the computed checksum of the ASCII values of each character in the line is sent as the last data field. The receiving program must compare this value with the value it computes as the data is being received and send back an appropriate response. This format of communication is styled after ACK and NACK types of communication. The response is either ACK the line was received correctly and it is ACKnowledged or the response is NACK the line was not received correctly and it is Not ACKnowledged. The gauge receives the response and either transmits the line again or transmits the next line. The data records received are stored as a file on the current storage medium.

The program specifications that pertain to the gauge are:

RS232 type serial communication (TXD, RXD, GND).

1 start bit, 8 data bits, no parity, and 2 stop bits.

Baud rates; $110,300,1200,2400,4800,9600$ (programs in basic may not operate at speeds higher than 300 baud).

ACK character ASCII value 6 decimal.

NACK character ASCII value 21 decimal (any unrecognized character is treated as a NACK character).

Checksum computed by ASCII values up to and including the comma before the checksum field.

' $p$ ' is the ASCII character (value 112 decimal) that will remotely activate the PRINT feature of the gauge.

CR is the ASCII character (value 13 decimal) that will remotely acknowledge the PRINT CD prompt.

Included here are the descriptions and program listings for several types of computers that compose a fairly good cross-section of the microcomputers that are currently available. Even if the computer that you are currently using does not directly correspond to one of the ones listed, there is a good chance that with the information provided in the listings and a careful study of the programmers manual for your computer that you could write a downloading program of your own. The last listing DRINZDMP.BAS will convert the download file from the standard format 
to the format used by the IRRIGATION WATER MANAGEMENT system software from CPN CORP. It will operate as is on most of the microcomputers using variations of MIRCOSOFT BASIC for computers with disk drives.

LISTING \#1

;

This version of DRDUMP will operate on most IBM DC's and their close compatioles. It has been used on an IBM PC, HEATH/ZENITH 100 or 150, TELEVIDEO 1605, and with the replacement of line number 310 with the one listed below it will also operate on the APPLE MACINTOSH with MICROSOFT BASIC.

310 OPEN "COM1:300,N,8,2" AS \#1' Set port 300 baud, no parity, 8 bits, 2 stop bits

The requirement for this program to work is the inclusion of the OPEN "COM1:... statement in the BASIC language used. 


\section{LISTING \#2}

This version of DRDUMP was uritten for a CP/M based microcomputer that uses the port method of I/O addressing. It was specifically written to operated on a VECTOR GRAPHICS Model 4 as is or the following computers with their associated line modifications.

\section{VECTOR GRAPHICS 3}

No changes just use the printer port.

\section{VECTOR GRAPHICS VSX}

$1605 T \%=6$ ' Status port number

360 OUT ST\%,\&H18 ' Reset the port

362 OUT ST\%,\&H14 ' set port mode; (1 start), 8 data, no parity, 2 stop bits

364 OUT STK,\&H4C ' set por. for transmit and receive

366 OUT ST\%,\&H15

368 OUT ST\%,\&HEA

370 OUT ST\%,\&H11

372 OUT ST\%,\&HOO

374 OUT STK, \&H13

376 OUT STK,\&HC1

. 380 OUT ST\%,\&H1O

$\because 830$ OUT ST\%,\&H10: WHILE (INP(ST\%) AND 4) = IIP\%: WEND ' Wait for transmitter ready

$870 \mathrm{K \%}=1000:$ OUT ST\%,\&H10: WHILE ((INP(ST\%) AND ONE\%) =ZIP\%) AND (KZ > ZIP\%): K\% $=K \%$ - ONEZ: WEND

970 OUT ST\%,\&H10: WHILE (INP(ST\%) AND 1) = ZIP\%: WEND ' Wait for line feed character

$\therefore$ OSM ZEUS

$\because \quad 150 \mathrm{DPY}=2$ ' data port number

160 ST\% $=3$ ' status port number

220 PRINT CHRS(26) ' Clear screen

360 OUT. ST\%, \&H18 ' Reset the port

362 OUT ST\%,\&H14 ' set port mode; ( 1 start), 8 data, no parity, 2 stop bits

364 OUT STY, \&H4C' 'set port for transmit and receive

366 OUT ST\%, \&HO3

368 OUT ST\%, \&HE1

370 OUT ST\%,\&HO5

372 OUT ST\%,\&HEA

374 OUT STK, \&H11

376 OUT ST\%, \&HOO

378 OUT \&H61,\&HO5 ' Set to 300 baud

380 OUT \&H61, \&H34

655 IF PRNTR\% THEN LPRINT CHRS(26) ' Send to printer

830 OUT ST\%,\&H10: WHILE (INP(ST\%) AND 4) = ZIP\%: WEND ' Wait for transmitter ready

$870 \mathrm{KZ}=1000$ : OUT ST\%, \&H10: WHILE (CINP(ST\%) AND ONE\%) $=$ ZIP\%)

AND $(K \%>Z I P \%): K \%=K \%$ - ONE\%: WEND

970 OUT ST\%,8H10: WHILE (INP(ST\%) AND 1) = ZIP\%: WEND ' Wait for line feed character

1155 IF PRNTR\% THEN LPRINT CHRS(26) ' DUMP printer file 
LISTING $\# 3$

This is an assembly language upgrade to listing $\$ 2$ that will input data at speeds up to 9600 baud. The assembly language routine will have to be loaded at 40000 decimal before the basic program is run. The line changes required to implement it with listing $\# 2$ are:

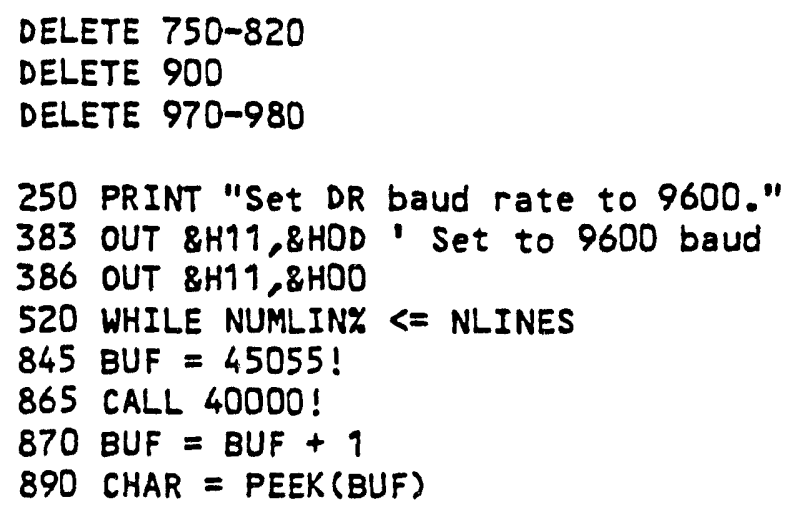

\section{LISTING \#4}

The special characteristics of the OSBORNE 1 and it's memory-mapped I/O that is page $f$ lipped with the program memory spawned this interesting use of the CP/M I/O byte at location 3 to swap the console with the serial port to input the data as though it was keyboard entry. It requires the OSBORNE'S SETUP program to set the configuration of the port to 300 baud before running the program.

LISTING \#5 and \#6

The APPLE II/II+/IIe/and IIc also have special considerations since in the beginning when the APPLE II was first designed there was no such thing as disk drives, serial communications, and printers available for microcomputers. The designers thought ahead enough to provide for 'plug-in' expansion that would include such things but INTEGER and APPLESOFT BASIC did not provide for direct interface with them. The result is a two part program in APPLESOFT BASIC and 6502 assembly Language that receives, the data through a SUPER SERIAL CARD (from APPLE) plugged in a card slot that is pointed to by the variable SLOT in the basic portion line 50 .

Type in the BASIC listing $\# 5$ and save it as DRDUMP. Type in the HEXDUMP for listing $\# 6$ by using the following steps:

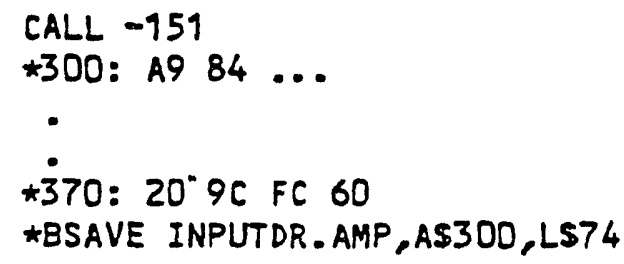




\section{LISTING $\#$}

This program is simular to the others but uses the ROM routines built into the TRS80 MODEL III \& 4 to input the data on the serial port. It will run on the MODEL III with no modifications and it will work on the MODEL 4 if it is brought up in MODEL III emulation mode (booted with a MODEL III disk).

\section{LISTING \#8}

This is the file converter that manipulates the data into the format expected by the IRRIGATION WATER MANAGEMENT program from CPN CORP. It will operate on most computers that use MICROSOFT BASIC for disk drives. 


\section{R8-232C SERIAL COMMUNICATION HARDWARE}

The SO3DR uses the RS-232C industry standard serial communication method. The 503DR cable connector is a special CPN CORP. configuration that can be linked to a 25 pin D-type connector on the opposite end of the cable. Most computers will have the DP25S (25 pin D-type sockets) connector built in although the IBM asynchronous communications card and a few others have a DP2.5P (25 pin D-type pins) connector built in.

Here are a collection of some of the more popular cable configurations: 


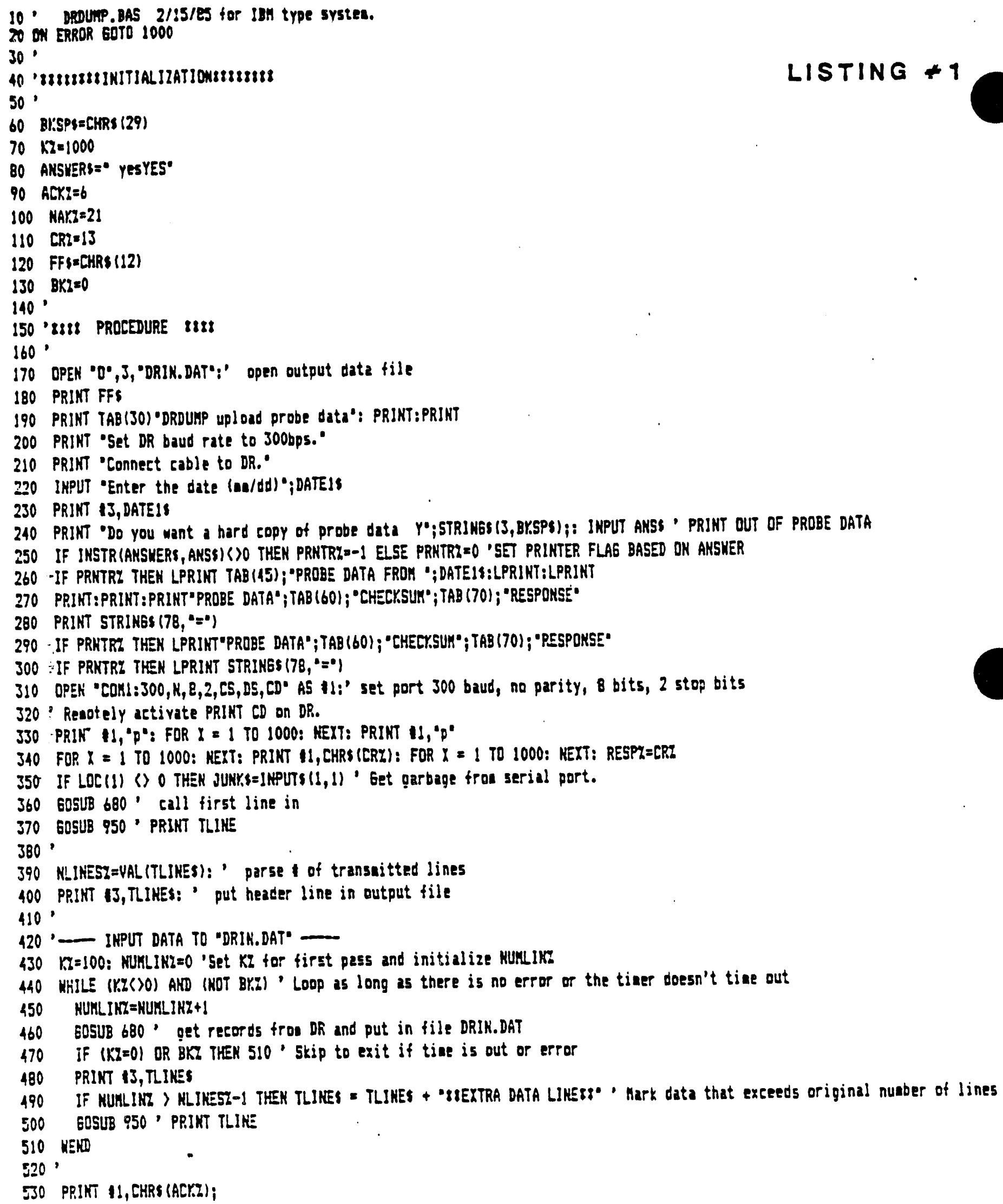


IF PRKTRZ THEN LPRINT FF' ' Output a forateed

CLOSE 11

CLOSE 13

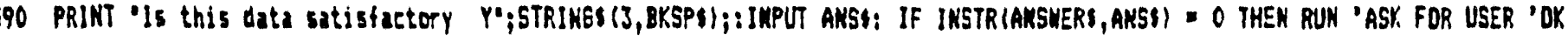

600 IF BK\% THEN ERD, Finish if erTor has Decured

610 CHAIK 'DRIN2DKP. BAS', ALL' CONYERT DATA STRIKE TO PARSED INFORAMTION.

620 END

$630^{\circ}$

$640^{\prime}$ 'ttttttsUBROUTIKEstttut!

$650^{\circ}$

$660^{\prime}$ 'SUB - INPUT LINE -

$670^{\circ}$

680 PRINT 11, CHRS (RESPZ);

690 TLINESE:-

700 CKSUKhz=0 ' reset cksun for nen record

710 KZ $=1000:$ WHILE (LOC(I) $=0)$ AND (KZ)0): $K Z=K Z$ Z -1 : NEND

720 IF R.I = O THEN RETURN

730 CHAR\&=IMPUTS $(1,11)$

740 IF ASC(CHARS) $=13$ THEN 6OTO $810^{\circ}$ is it the END DF RECORD?

750 IF INKEYS $(>$ " THEH ERROR

760 TLINES=TLINES+CHARS' if not, add it to the record string

770 NUKBY $=$ ASC (CHARS)

780 CXSUKI=CKSUmitAUKBBZ, and resord it in the eksun

- 790 60T0 $710^{\prime}$ get next character 800 .

$B 10^{\circ}$

END OF RECORD PrOCEsSING
IF LOC(1)=O THEN $820^{\circ}$ MAIT FOR A CHARACTER IN COH-BUFFER

CHARS $=$ INPUTS $(1,11) \cdot$ DISCARD LINE FEED

LASTCHARI ELEM(TLIMES) ' length of data string

LSTCOMAZZLASTCHARZ, injtialize CORE pOinter

COMCHARS=H!DS (TLINES, LSTCDHAZZ, I)

WHILE COMCHARS()",

CKSUAZ =CKSURI-ASC (COMCHARS)

LSTCOWMAL=LSTCOHKAI-1 ' renove checksun chars troe cksue and find

COMCHARS =KIDS (TLIKES, LSTCOMMAZ, 1)' the transuitted checksus fielo

MEND

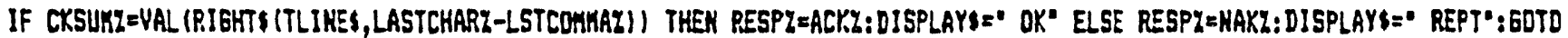

$680^{\prime}$ repeat line till right

930 RETURN

$940^{\circ}$

950, PRIKT TLIKE

$960^{\circ}$

970 PRINT TLINES;TAB(60); CYSUKZ;TAB (70); RESPI;DISPLAYS' DISPLAY DATA

980 IF PRNTRZ THEN LPRIKT TLIKES;TAB (60);CYSUMZ; TAB(70);RESPZ;DISPLAYS' PRIHT DATA

990 RETURM

1000 PRINT "COMHUNICATIOH INTERUPTER": BKZ=-1: RETURN' hANDLE ERROR IKCLUDINE KEYBOARD INTERUPTIONS 
10 - DRDUMP.BAS 2/15/85 FOR VECTOR 4 TYPE SYSTEMS.

20 ON ERROR GOTO 1170

3O' TRAMSFER FRON PROBE TO FILE DRIN.DAT

$40^{\circ}$

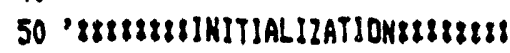

$60 \quad 12=1000$

70 AMSUERS : "YESYES"

80 BI:SPS = CHRS (23)

90 ACKZ $=6$

$100 \quad$ MAKZ $=21$

110 CR $=13$

120 ONEZ $=1$

130 TMOZ $=2$

$14021 \mathrm{PZ}=0^{\circ}$ nunerical constants

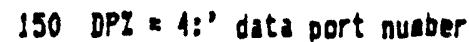

160 STZ $=5:^{\prime}$ status port nunber

170 BKZ $=0$

180,

190 ' 8418 PROCEDURE 8418

200 '

210 OPEN 'O",3, "DRIN.DAT":' open output data file

220 PRINT CHRS (4):' elear sereen

230 PRINT TAB(30) "DRDUAF upload probe data"

240 PRIKT: PRINT: PRINT

250 PRINT 'Set DR bayd rate to $3000^{\circ}$

260 "PRINT "Connest cable to DR."

270 INPUT 'Enter the date (m/dd) ; DATEls

2BO PRINT 13, DATEIS

290 PRINT DO you want a hard sopy of probe data Ye;STRINGS(3,BRSPS);: IKPUT ANSE 'PRIKT DUT OF PROBE DATA

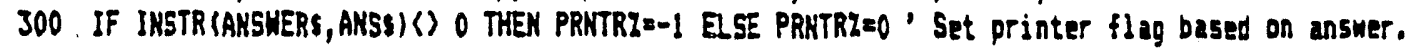

310 IF PRNTRZ THEN LPRINT TAB (45); "PROBE DATA FROH ";DATEIS: LPRINT:LPRINT

320. PRINT:PRINT:PRINT"PROBE DATA'; TAB (60); 'CHECYSUn'; TAB (70); 'RESPDKSE'

330 PRINT STRIHGS $\left(7 B_{1}=0\right.$ )

340 IF PRHTRZ THEY LPRINT'PRODE DATA';TAB(60); "CHECYSUR"; TAB(70); 'RESPOKSE"

350 IF PRHTRZ THEK LPRINT STRIHGS (7E, " $=$ ")

360 OUT ST\%, tH40: ' reset the port

370 OUT STZ, \&HCE: ' set port aode; (1 start), B data,no parity, 2 stop bits

380 DUT 5TZ, th25:' set port for transait and reseive

390 ' Resotely turnon the PRINT CD for data dura.

400 OUT DPY, ASC("P"):FOR $X=1$ TO 1000: NEXT: OUT DPY, ASC("p")

410 FOR $X=1$ TO 1000: NEXT: OUT DPF, CR:FOR $X=1$ TO 1000: NEXT: OUT DPY, CR

$420^{\circ}$

430' Input header line

410 60548 850 ' call first line in

450 ECSUB $1120^{\circ}$ PRINT TLINE

$460^{\circ}$

470 MLINES $=$ VAL (TLIKES) , parse 1 of transeitted lines

480 PRINT 13, TLIKES.' put header line in output file

$490^{\circ}$

500

Input data to "DRIN.DAT" 
KI = 100: KUKLIKZ = 0 ' Set $K 2$ for first pass and initialize NUnLIKZ

HHILE (KI (; O) AND (KOT BKZ) ' LOOP is long as there is ne erpor or the tiner boesn't tiae out

MUMLINZ = RURLIKR +1

605UB 830 ' get all the records froe the OR and put thes in the file DRIK.DAT

IF (Ki $=0$ ) OR BKI THEN 590 ' SKip to exit loop if tiae is out or error

PRINT 13, TLINES

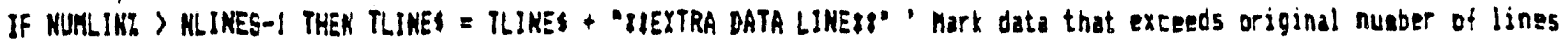

GOSUB 1120 ' PRIKT TLINE

590 HEND

$600^{\circ}$

610 OUT DPY, ACT.\%:' Do one nore ACK to sllor DR to finish

$620^{\circ}$

$630^{\circ}$

$640^{\circ}$ - QUIT -

650 IF PRNTRZ THEN LPRINT FFs' Output a forafeed

660 CLOSE 13

670 PRINT'Is this data satistactory Y";STRINGs (3, BKSPS); : INPUT AKSS: IF IKSTR (ANGMERS, AKSS) - O THEK 10: ' ASK USER FOR 'DY'

$6 B 0$ If BK2 THEN $700^{\circ}$ Skip converting data if error has occured

690 CHAIK "DRIN2DKP.BAS", ALL ' Convert dati string to parsed inforaation.

700 END

$710^{\circ}$

720 'tttttISUBROUTIHEStttt\&t

$730^{\circ}$

$740^{\circ}$ SUB - input first line -

$750^{\circ}$ LINES 860 THRU 920 HAVE TIHE CRITICAL CODE.

760' LINE 860 $\Rightarrow$ ) WAIT FOR CHARACTER TO BE RECEIYED (TIME MAITI

$770^{\circ}$ LINE 870 $\Rightarrow$ CHECK FOR OVER-RUN STATUS

780 ' LINE 880 $\Rightarrow$ GET CHARATER

$790^{\circ}$ LINE 890 $>>$ RETURK IF TIMER RAN OUT

$800^{\prime}$ LINE $900 \Rightarrow$ IS IT 'CR' THEK END OF RECORD

810' LINE 910 $\Rightarrow$ CHECK. FOR KEYBDARD INTERUPTIOK

820 ' LINE 920 $\Rightarrow$ MAKE TLINES, AND CHECKSUK. 60 EET MEXT CHARACTER

B3O WHILE (INP(STZ) AND II = ZIPY: NEND' Nait for transaitter ready

B40 OUT DPZ, RESPI', Send response to DQ

B5O TLIKES $="$ " Clear line record bufter

B60 CKSUKL = 23PY, Reset checksue for ner record

$870 \quad K Z=1000:$ WHILE (IINP (STZ) AND TNDZ) =2IPZ) AND (KZ > IIPZ): KZ = KZ - DNEZ: WEKD

B80 IF (IKP (STZ) AND I6) S) IIPZ THEN DUT STZ, \&H35: PRINT ' II O-R I8"

890 CHAR $=$ INP (DPZ)

900 IF KZ $=0$ THEK RETURH

910 IF CHAR = CR THEN 970

920 IF INKEYS $\langle>$ " THEN ERROR

930 TLINES = TLIKES + CHRS (CHAR): CXSUKZ = CYSUKZ + CHAR: $60 T 0870$

$940^{\circ}$

950 - END OF RECORD Processing

$960^{\circ}$

NHILE (INP (STZ) AND 2)=2IPZ: WEKD' mait for line teed char

CHAR = IMP(DPZ): ' get the line teed

LASTCHAR = LEN(TLIKES): ' get length of data string

1010 COMS $=$ MIDS (TLINES, LSTCOHMA, OKEZ) 


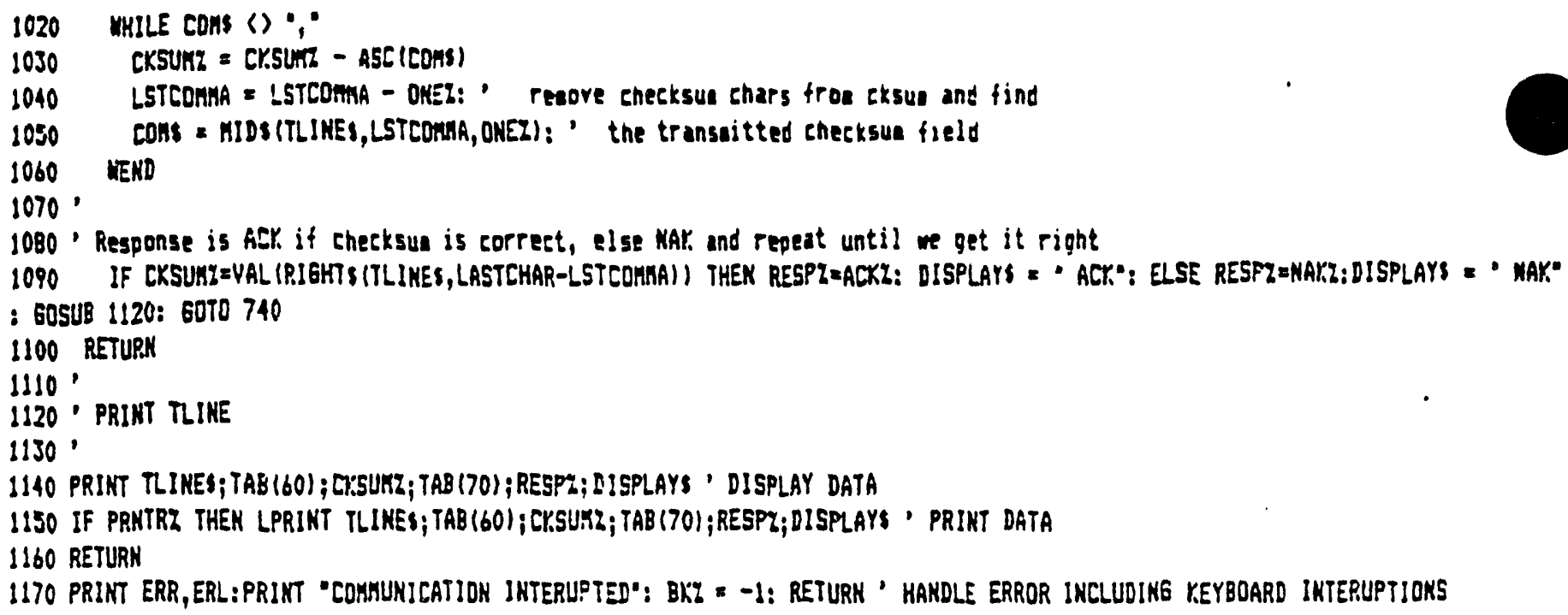

TYPE DRLIN.ASH

It888 P'BNi: ORLIN. ASY 288288188850304 28828288

;

;ASY SUBRDUTIKE FOR DATA INPUT FROK DR

¿PEN START AT 40000D, 9C4OH

;DATA STORAGE START AT 45056D, BOOOH

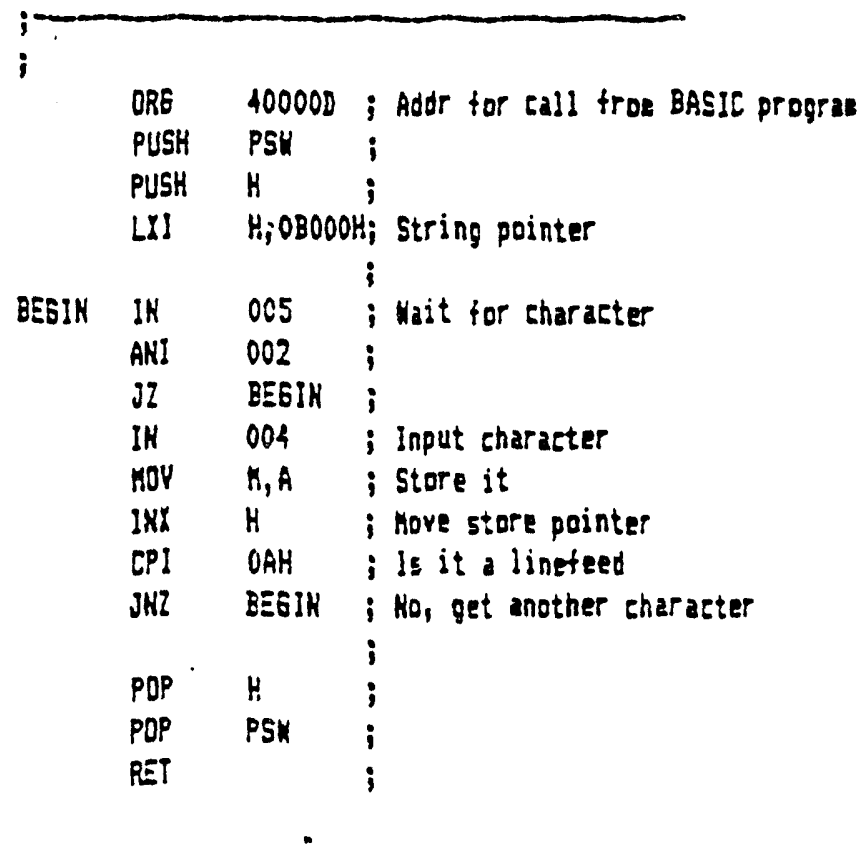


30 IOPORTZ=3

40 IONORKZ=PEEK(IOPORTZ)

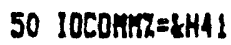

60 ACKS $=$ CHRS (6)

70 NACKs $=$ CHR $\$(21)$

80 CRS $=$ CHRS (13)

90 BKSPS=CHRS (B)

100 AHSWERS:" YESYES"

110 ZIPZ $=0$ : ONEI $=1:$ THOZ $=2$

120 BKI $=0:$ ERRFLAST $=0$

$130^{\circ}$

140 ' SETUP PORT AND PROMPT USER

$150^{\circ}$

160 OPEK ' $0 \circ, 3$, "DRIN.DAT' ' OPEN OUTPUT DATA FILS

170 PRINT CHRS (26) ' CLEAR THE SCREEK

180 PRINT TAB (15) "DRDUKP upload probe data":PRINT:PRINT

190 PRINT "Set DR beud rate to 300bps."

200 PRINT "Connect the cable to the DR."

210 IKPUT "Enter the date m/dd";DATEIS

220 PRINT 13, DATESS

230 PRINT:PRINT

240 PRIKT 'PROBE DATA2; TAB(45); 'RESPOKSE"

250 PRINT STRINGS $(52, "-1)$

$760^{\circ}$ Renotely activate PRINT CD on DR.

70 POKE JOPORTZ, 10COHKZ: PRINT 'P":FOR $X=1$ TO 1000: KEXT: PRINT ' $P$ '

280 FOR $X=1$ TO 1000: NEXT: PRINT CRS: FOR $X=1$ TO 1000: NEXT: RESPS = CRS

290 JUKKS=IMKEYs' Collect garbage off serial port.

$300^{\circ}$

$310^{\circ}$ - IMPUT DATA TO 'DRIK.DAT' -

$320^{\circ}$

330 WHILE BKZ $=0$. GET PECORDS TILL TIMEDUT

340 6OSUB 540 ' GET ALLL THE RECORDS FRDM THE DR AND PUT THEM IN THE FILE DRIN.DAT

350 IF BKZ $=1$ THEN 380 ' CHECK. FOR TIMEOUT

360 PRINT 13, TLLIKES

370 GOSUB 830 $:$ PRINT TLIKE

380 NEND

$390^{\circ}$

400 POKE IOPDRTZ, IOCOMKZ:PRINT ACKS:POYE IOPORTZ, IOKORKZ' DO OKE KORE ACK TO ALLOK DR TO FINISH

$410^{\circ}$

$420^{\circ}$

$430 \cdot$ - QUIT -

440 CLOSE 13

450 PRINT 'I5 this data satistastory $Y$ ';STRIKGS (3, BXSPS);: IMPUT AKSS:IF IKSTR (AKSUERS, AKSS) = O THEN RUN' ASK. FDR USER 'OK' 460 IF ERRFLAGZ THEK END

470 CHAIN 'DRIKODHP. BAS', ALL' CONYERT DATA STRIKE TO PARSED IKFDRKATIOX.

480 END

$490^{\circ}$

$500 \cdot$ - INPUT SUBROUTIKES —- 


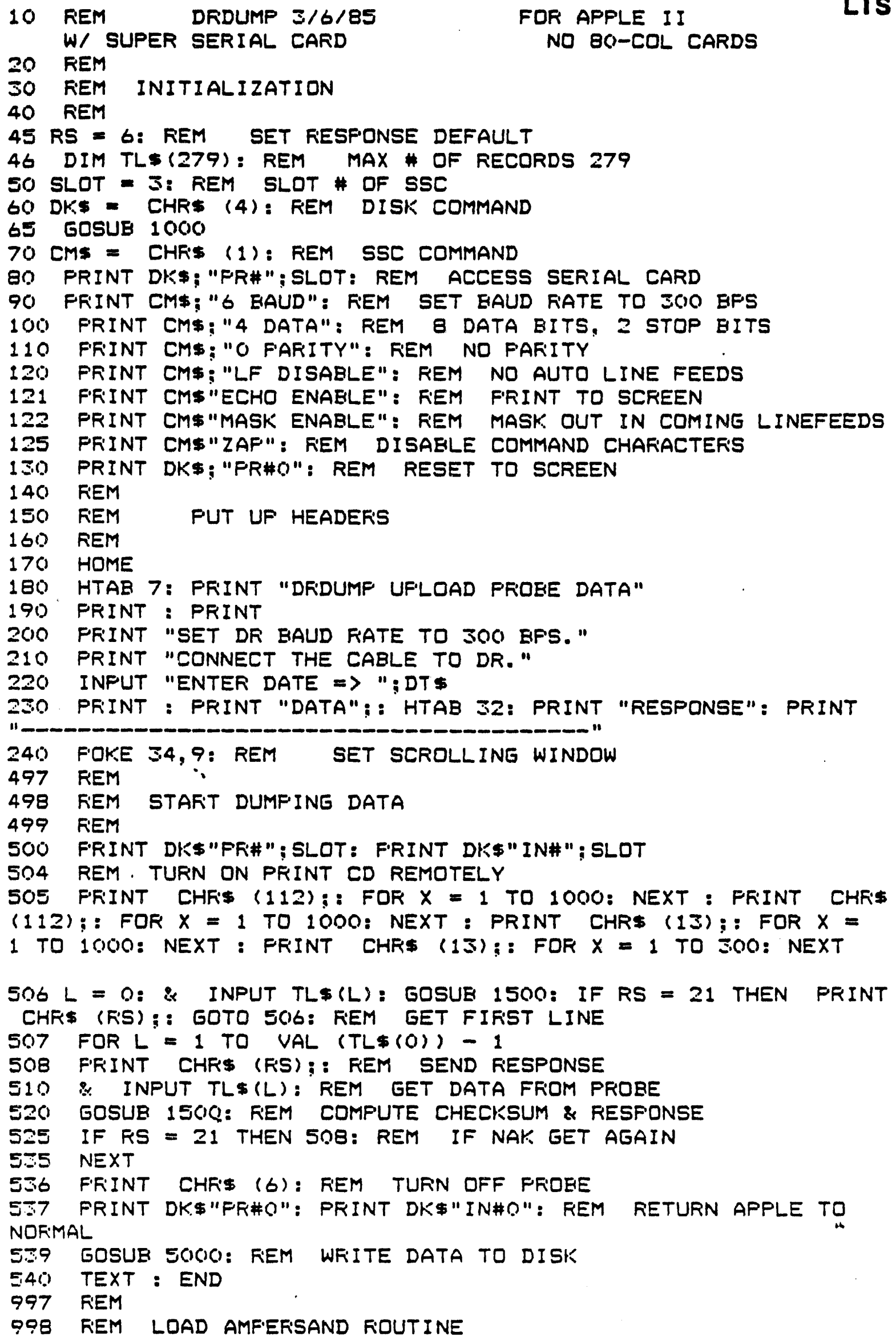


\$Q9 REM TO INFUT SEFIIAL DATA

1010 FIINT DKS"BLDAD INFUTDFI. AMF"

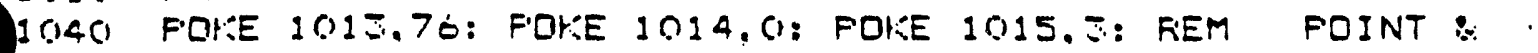

VECTOF: TO FAGE $\$ 3(1)(76 B$ DECIMAL)

1050 RETLINN

1457 FIEM

1498 FIEM COMFUTE CHECI:SUM \& DETEFMINE FIESFIONSE

1499 FIEM

$1500 \mathrm{CK}=0:$ FOF $X=1$ TO LEN (TLE(L)):CK: = CI: + ASC I MIDE.

(TL\$(L),X,1)): NEXT

150.19 FEMM LOOK FDK LAST CDMMA

1510 FOR LK $=$ LEN (TL\$(L)) TO O STEF - 1

1520 IF MID\$ (TL\$(L),LK, I) = ". "THEN CS = VAL (FIGHT\$ (TL\$(L),

LEN (TL\$(L)) - LY) I:LK = 0: EOTO 1550

$1530 \mathrm{CK}=\mathrm{CK}$ - $\operatorname{ASC}(\operatorname{MIDS}$ (TLS(L),LK:,1))

1550 NEXT

1559 FIEM IF CHECKSLIMS MATCH SEND AN ACK

1560 IF CK = CS THEN FIS = 6: GOSLIE 2010

1569 FEEM IF CHECI:SUMS DON'T MATCH SEND A NAK.

1570 IF CK < > CS THEN FIS = 21: GOSUR 2100

1580 FIETURN

1997 REM

1998 FEM SEND ACK: TO SCFEEN

1909 FEM

$2000 \mathrm{CH}=$ FEEK $(40)+$ FEEK $(41) * 256$

$2010 \mathrm{CH}=\mathrm{CH}+37$

2011 FOKE CH, ASE ("A") + 128

2012 FOKIE CH + 1, ASC ("C") + 128

2013 FPOKEE $\mathrm{CH}+2$, ASC ("K") + 12B

2020 CALL $-.920^{\circ}$

2030 FEETUFIN

2097 REM

ZOQB FEM SEND NAI: TO SCFEEN

2099 REM

$2100 \mathrm{CH}=$ PEEK $(40)+F E E K(41) * 256$

$2110 \mathrm{CH}=\mathrm{CH}+\$ 7$

2111 FOKEE CH, ASC ("N") + 128

2112 POKE $C H+1$. ASC ("A") + 128

2115 FOKE $\mathrm{CH}+2$, ASC ("K") + $12 B$

2120 CALL - 926

2130 REETURN

4997 REM

4998 FEM WFITE DATA TO DISK FILE DUMF.DATA

4999 REM

5000 FRIINT "WFITING DATA TD DISK..."

5005 FRINT DK\$ "OPEN DUMP. DATA"

5010 FFINT DKE"WRITE DUMF. DATA"

SO15 PRINT DT\$

5020 FOF $X=0$ TO VAL $(T L \$(0))$ - 1: FFIINT TLE(X): NEXT

5OEO F.RINT DKS"CLOSE"

50140 REETURNN 


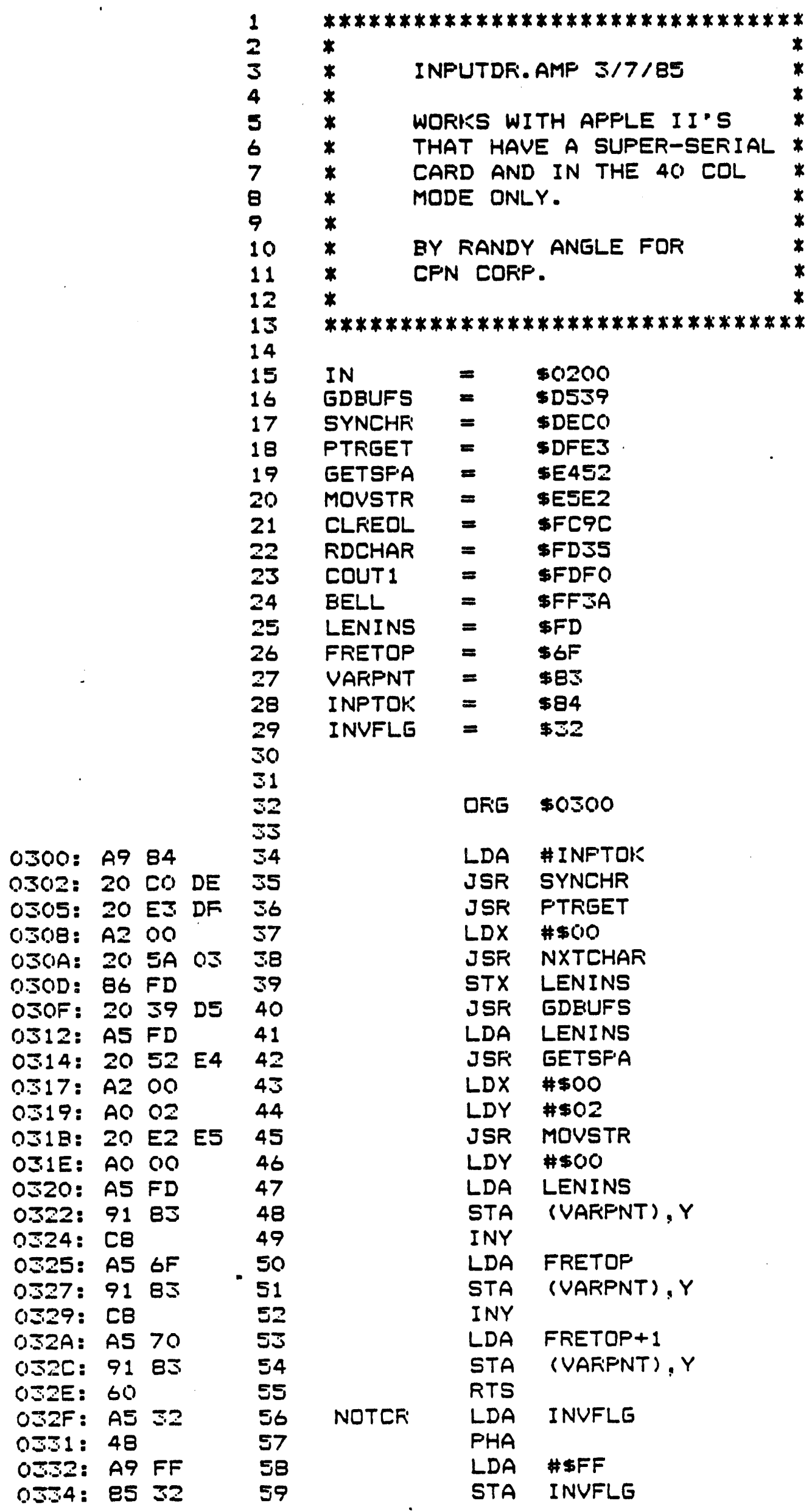




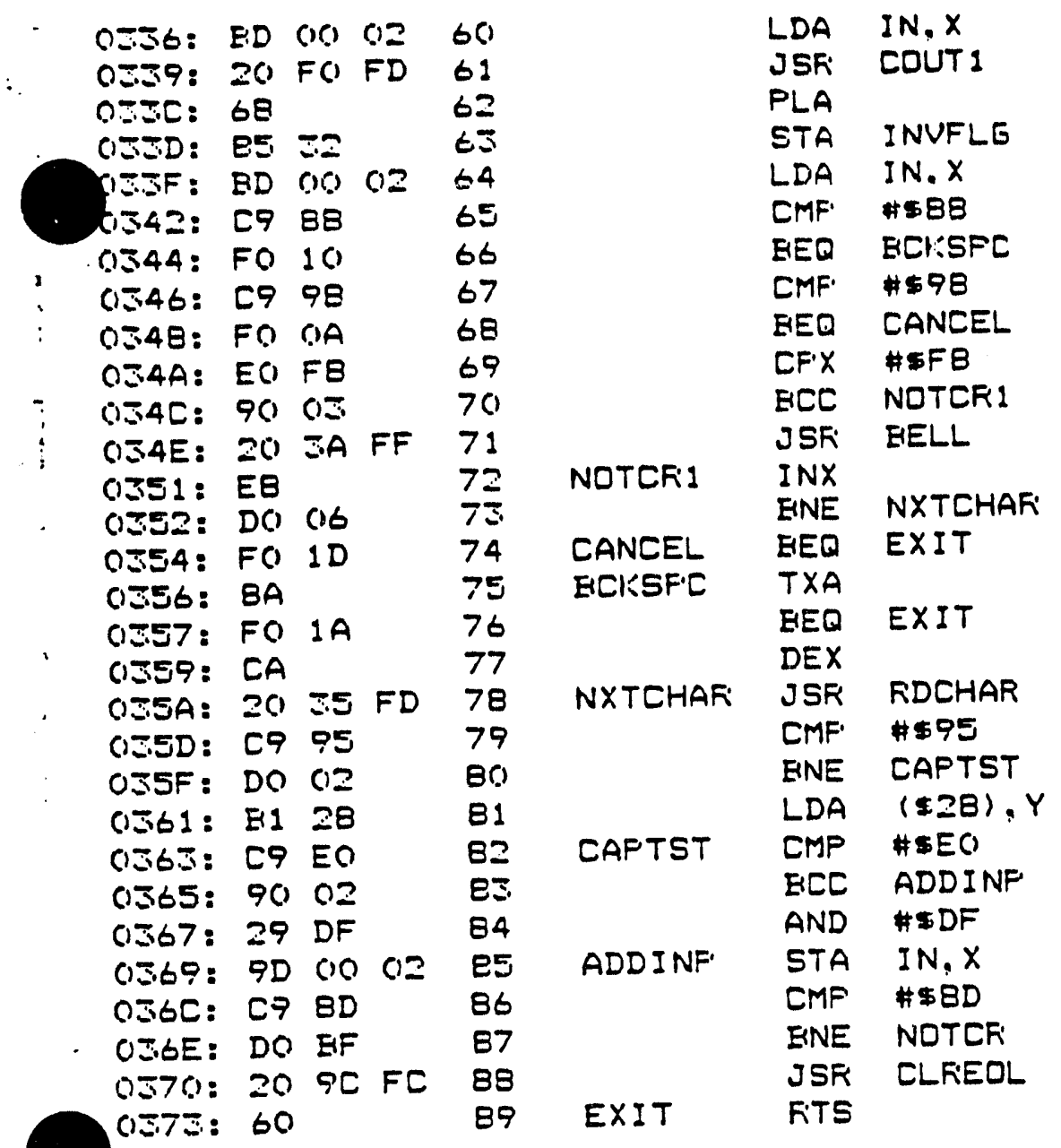

--End assembly--

116 bytes

\section{LISTING \#6 HEX DUMP}

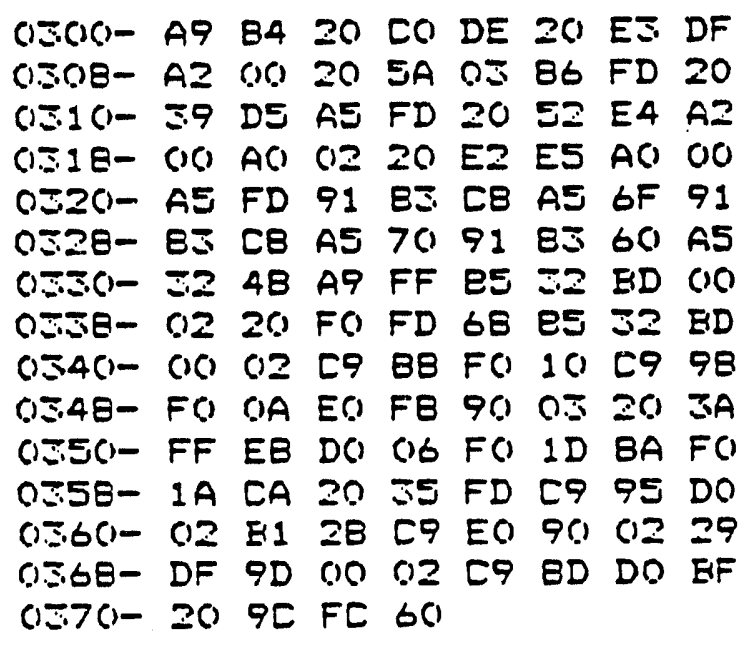


10' DRDUPP FOR TRS8O MODEL III/4 3/18/85

$20^{\circ}$

$30 \cdot$ IMITIALIZATION

$40^{\circ}$

50 DEFINT A-Z

60 CLEAR 10000

$70 \mathrm{TH}=500: \mathrm{WU}=0$

80 ACK $=6:$ MAK $=21: C R=13:$ NO $=0: M I=1: M Z=2$

$90 \mathrm{CI}=16872: \mathrm{CO}=16890$

$100^{\circ}$

$110^{\circ}$ SET UP SCREEM AND SET COMHUNICATION HODES

$120^{\circ}$

130 CLS

140 PRINT TAB(20), "DRDULP FROM HYDROPROBE": PRINT: PRINT

150 PRINT 'CONMECT CABLE TO DR"

160 PRINT 'SET DR TO 300 BAUD"

170 INPUT 'ERTER THE DATE"; DATEIS

180 INPUT 'DO YOU MANT A HARDCOPY'; ANSS

190 IF AKSS $=$ " $Y$ " OR AKSS $="{ }^{\circ}$ "THEN PRKT $=(1=1)$

200 PRINT: PRINT 'DATA';TAB (5B) 'RESPOKSE'

210 PRIKT STRINGS $(65, "-\circ)$

220 IF PRNT THEN LPRINT TAB (25) 'PROBE DATA FROM ";DATEIS

230 IF PRNT THEN LPRINT 'DATA'; TAB (5B) 'RESPOKSE'

240 IF PRNT THEN LPRINT STRINGS(65, ${ }^{\circ}$ ")

250 DPEK 'O', 13, 'DRIN.DAT'

260 PRINT 13, DATE1S

270 POKE 16889,119 ' B DATA 2 STOP MO PARITY

280 POKE $16888,(1625)+5$ - 300 BAUD

290 POKE 16890,0 ' DON'T WAIT

300 UEFUSRO $=$ LHSA: $X=$ USR $(0)$

310 DEFUSR1 $=$ \&H5O: DEFUSR2 $=8 H 55$

$320^{\circ}$

$330^{\circ}$ TURK OK DUAP REMOTELY

$340^{\circ}$

350 POKE CO,ASC ("p"): $X=$ USR2 (0):FOR $X=1$ TO 1000: NEXT:POKE CO,ASC("p"):FOR $\chi=1$ TO 1000: NEXT

360 POKE CO, CR: $X=U S R 2(0): F O R X=1$ TO 1000: NEXT:R $=C R$

$370^{\circ}$

380 ' EET THE FIRST LINE OF DATA

$390^{\circ}$

400 GDSUB 660

410 605UB 920

420 PRINT 13,TLS

$430 \mathrm{CT}=$ VAL (TLS)

$40^{\circ}$

450 ' EET THE REST OF TRE DATA

$460^{\circ}$

470 FOR CD $=C T-1$ TO 1 STEP -1

$480 \quad$ 6054B 660

490 GOSUB 920

500 PRINT 13, TLS 
510 KEXT

520 POKE CO, ACK

$530 X=U 5 R 2(0)$

$510^{\circ}$

$550^{\circ}$ CLDSE FILES AND DUIT

$560^{\circ}$

570 CLOSE 13

580 IF PRHT THEN LPRIKT CHRS (12)

590 PRINT 'IS THIS DATA SATISFACTORY';

600 ANSS = INKEEYI: IF AKSS = "T THEN 600 ELSE PRINT ANSS

610 IF ANSS \langle\rangle $"$ "Y" AND AKSS $\langle>$ " $Y$ " THEN 10

620 END

630 '.

640 DOHKLOAD DATA RECORD OKE LINE AT A TIME

$650^{\circ}$

66O POKE $C D, R$

$670 X=$ USR2101

680 TLS $=" 1$

$690 \mathrm{NU}=0$

$700 \mathrm{CK}=\mathrm{NO}$

$710 x=$ USRS (O) : CN $=$ PEEK(CI)

$720 \mathrm{IF}$ CN $=0$ THEN NU $=\mathrm{NU}+1$

730 IF NU > TK THEK NU $=0: R=$ HAK: 605UB 920: 6070660

740 IF $\mathrm{CN}=0$ 60T0 710

750 IF CN $=$ ER EOTO 820

760 TLS $=$ TLS + CHRS (CN)

$770 \mathrm{CK}=\mathrm{CK}+\mathrm{CN}$

7806070710

$790^{\circ}$

BOO ' COKPUTE CHECYSUM FIND RESPDMEE

$B 10^{\circ}$

B20 $X=$ USRI (O): CH = PEEY.(CI): IF CN = O GDTO E2O

830 LC $=$ LEN(TLS)

$840 \mathrm{CP}=\mathrm{LC}$

BSO CMSS $=$ KIDS ITLS, CP, KI)

860 If CAS \langle\rangle$\because$ THEN CK. CK. - ASCLCTS): $C P=C P$ - 1: $60 T 0850$

$B 70$ IF CK. $=$ VAL (RIEHTS (TLS, LC-CP)) THEN $R=$ ACK. ELSE $R=$ NAY: $60 T 0660$

8BO RETURH

$890^{\circ}$

9001 PRINT DATA RECDRD

$910^{\circ}$

920 PRINT TLS; TAE(63);:IF R = ACK. THEN PRINT -ACY. ELSE PRINT •KAY.

930 IF PRHT THEK LPRINT TLS;TAB(63);:IF $R=$ ACK THEN LPRIKT 'ACK' ELSE LPRINT 'KAK'

940 RETURH 
30 DIH K.DZ(1100),DP(100)

40 ' 8488 PROCEDURE 1848

5O ' CONYERT DRIK. DAT TO DRDUMP.DAT

$60^{\circ}$

70 OPEN ' $I$ ", 3, "DRIK.DAT" open probe info file

80 INPUT 13, DATEIS

90 IKPUT 13, NR, CDS, SK, UKS ,STC, KDATAZ, DEPTHS\%, CY.SUn\%' get header info

100 DASHY =INSTR (CDS, $-\cdots)$, FIND VERSIOH

110 IF DASHZ $=0$ THEN LASY $=(1=1)$ ELSE LHS\% $\approx(1=2)$ ' SET LINES NUMBERS FLAE FOR INPUT

120 IF LHST THEN NUMCALSZ $=9$ ELSE NUMCALSZ $\approx 17$ ' SET NUMBER OF CALIBRATIONS,

130 FOR $1 \%=2$ TO NUKCALS\%

140 IF LNSZ THEN JNPUT I3, CALZ,SL, YINT, CKSUM: ELSE INPUT 13,LNZ, CAL:, SL, YINT, CXSUH' 'get all the calibration into 150 NEXT

160 OPEN '0",2, 'DRDUMP.DAT" ' open the output file

170 PRINT 12, DATEIS

180 PRINT 12,DEPTHS\%", "KDATAZ-1' urite of data records, of depths per record

190 MHILE NOT EOF(3)' transter into

200 IF LHSZ THEN INPUT 13, ID\%, CALI ELSE IMPUT 13,LNZ, ID\%, CAL\%

210 FOR IZ=KDATAZ TO 1 STEP -1

220 INPUT 13,KDZ(IZ)' get all key fields

230 NEXT

240 FOR IZ=DEPTHSL TO \& STEP - 1

250 INPUT $13, D P(I Z)$ ' get the depth readings

260 HEXT

270 INPUT 13,CKSUK' ' get the checksu

280 FARM $=1 N T(10 \% / 100)$ ' parse the fare 1

290 FLDI=1DI-(100tFARMY)' parse the field

300 SITEZ=KDZ (K.DATAZ), get the site I

310 PRINT 12,FARKZ;",";FLD\%;"“;SITE\%;","; output tara,field, site into

320 FOR IZ=1 TO K.DATAZ- 1

330 PRINT 12,KDZ(II)",";

310 NEXT

350 FOR II $=1$ TO DEPTHSY

360 PRIKT 12,DP(IZ);"," ' output depth readings

370 KEXT

380 PAIHT 12,CK.SUA\%, output checksun (end of record)

390 HEND

400 CLDSE

410 RUM "DRMORKI" ' OR RUK MIATEEVER PROGRAK YOU HISH

420 EHD 


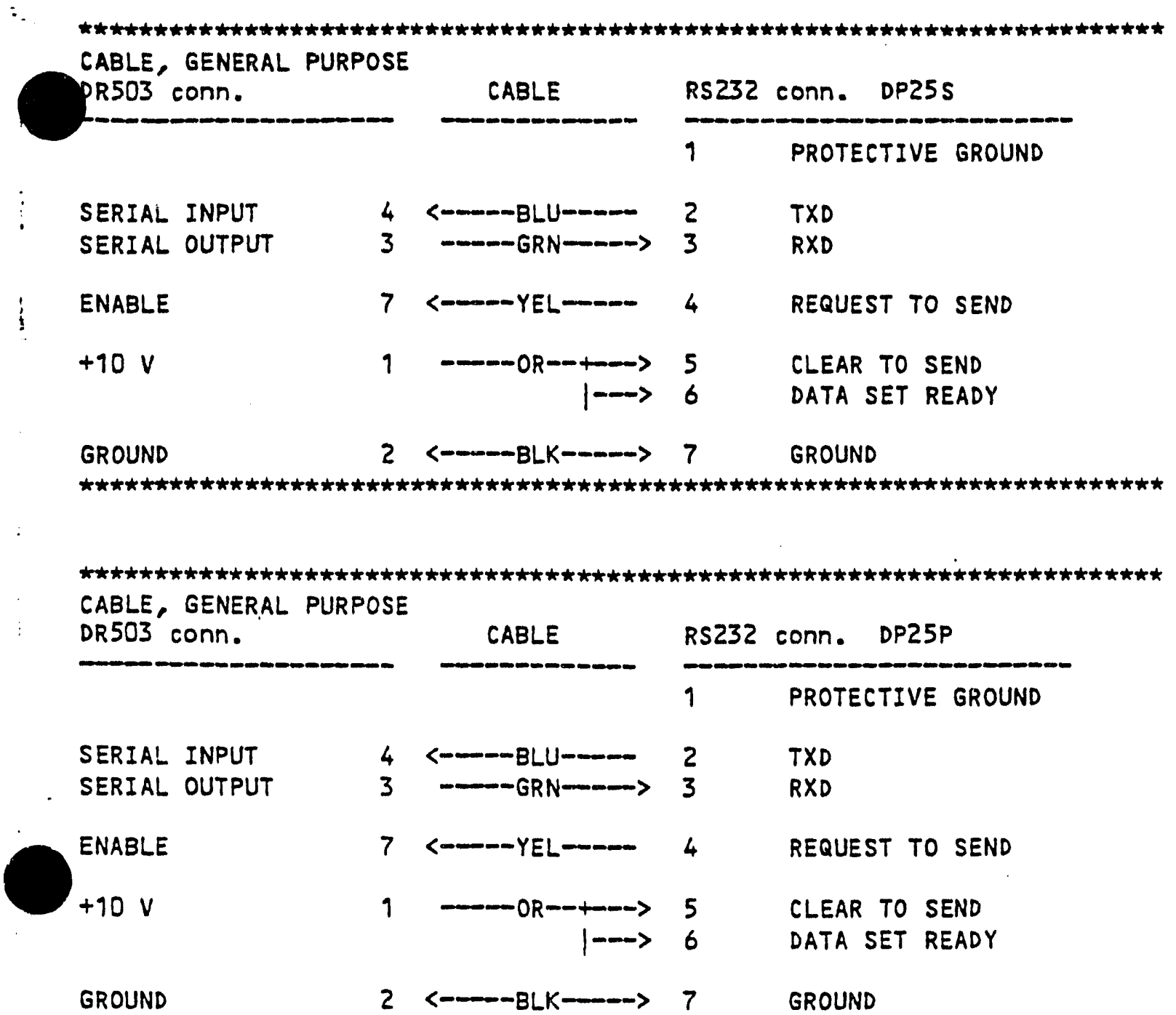

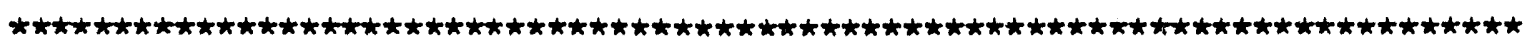

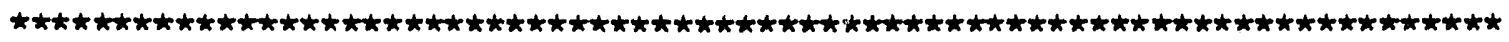
CABLE, VECTOR GRAPHICS 4, J1 MODEM CONNECTOR, 300 BAUD DR503 CONI. CABLE

$$
\text { RS232 conn. DP25P }
$$

\begin{tabular}{|c|c|c|c|c|}
\hline & & & 1 & PROTECTIVE GROUND \\
\hline SERIAL INPUT & 4 & $<-\infty$ & 2 & TXD \\
\hline \multirow[t]{2}{*}{ SERIAL OUTPUT } & 3 & -- GRN $\longrightarrow$ & 3 & $R \times D$ \\
\hline & & NC $\ll-$ & 4 & REQUEST TO SEND \\
\hline$+10 \mathrm{~V}$ & 1 & $-\infty \mathrm{OR}-$ & $\begin{array}{l}5 \\
6\end{array}$ & $\begin{array}{l}\text { CLEAR TO SEND } \\
\text { DATA SET READY }\end{array}$ \\
\hline \multirow[t]{2}{*}{ GROUND } & 2 & $\langle-B L K-\infty$ & 7 & GROUND \\
\hline & & $N C<-\infty$ & 20 & DATA TERMINAL READY \\
\hline ENABLE & 7 & $--Y E L \quad N C$ & & \\
\hline
\end{tabular}




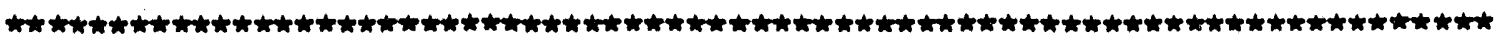
CABLE, VECTOR GRAPHICS 3, J2 PRINTER CONNECTOR, 300 BAUD

DR503 conn.

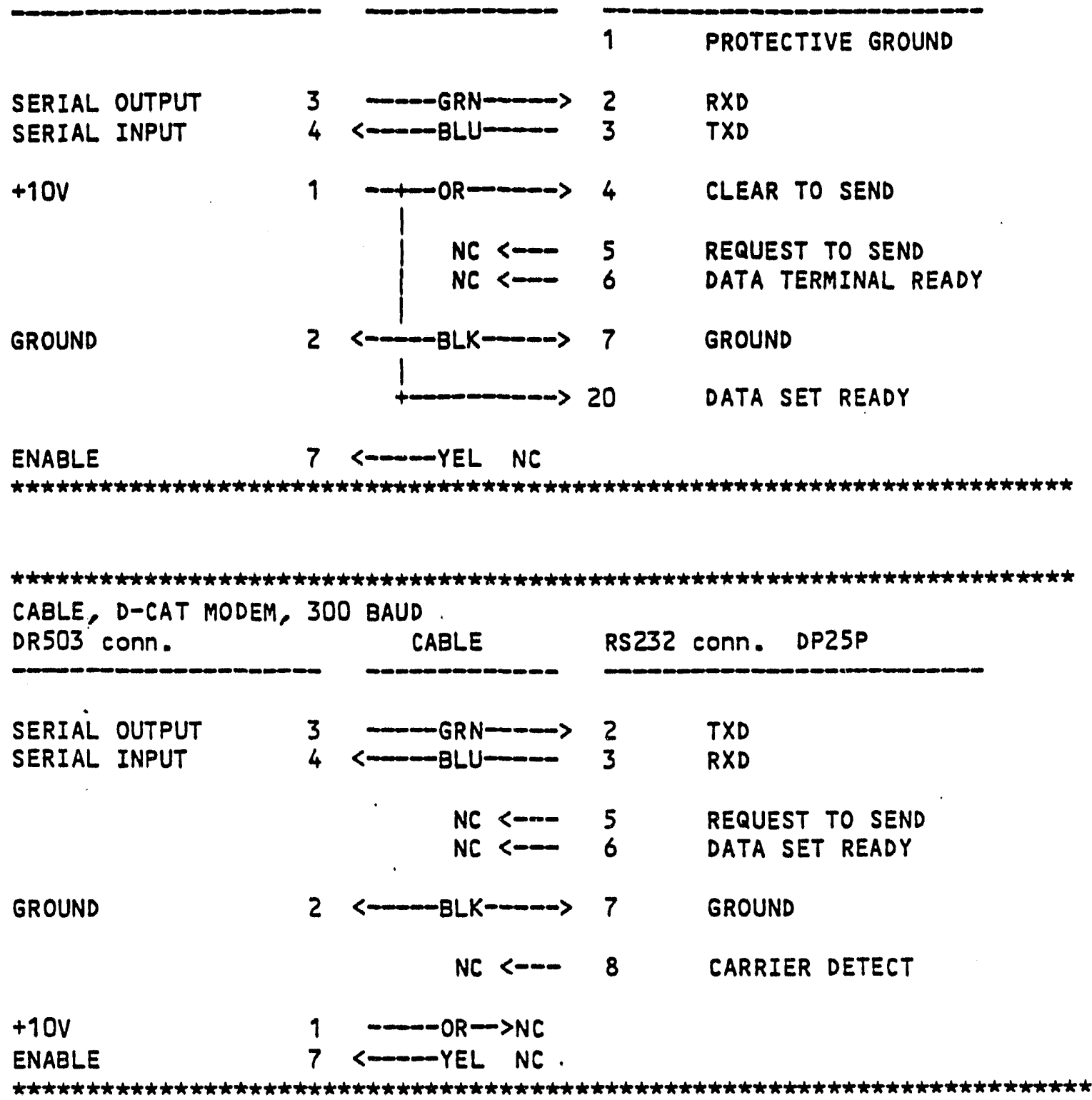

CABLE RS232 CONN. DP25P

RST2 CONח. DP25P 


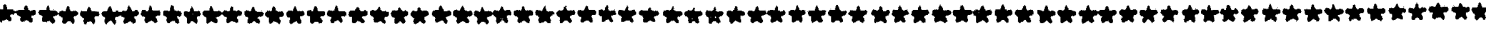

CABLE, SIGNALMAN MODEM, 300 BAUD

OR503 cONn.

CABLE

RS232 conn. DP25S

SERIAL OUTPUT

SERIAL INPUT

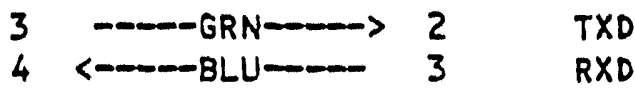

NC $<-\infty \quad 5 \quad$ REQUEST TO SEND

NC $--\infty \quad 6 \quad$ DATA SET READY

GROUND

$2 \longleftrightarrow-B L K-\longrightarrow 7$ GROUND

NC --8 CARRIER DETECT

$+10 \mathrm{~V}$

ENABLE

$O R \rightarrow N C$

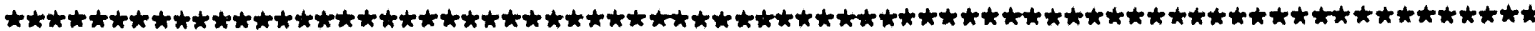

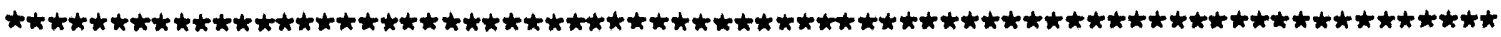

CABLE, IBM PC ASYNC COMM ADAPTER, 300 BAUD

DR503 conn.

CABLE

RS232 conn. DP25S

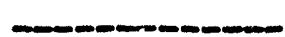

1 PROTECTIVE GROUND

SERIAL INPUT

SERIAL OUTPUT

$4<--B L U-\square-2$

$\longrightarrow 3$

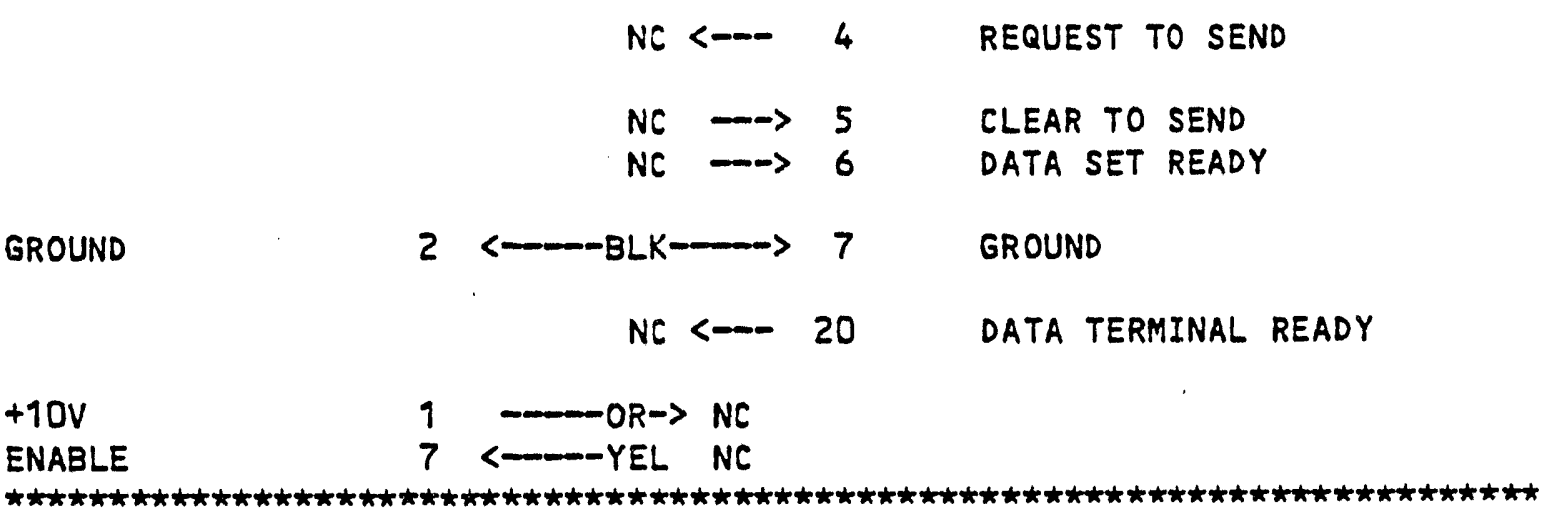


1.00 General: When used in occordance with Instructions, the CPI nuelear products represent no danger to the user or to the genersl public.

The general public is speciffeally restricted from access to the device by virtue of the operating procedures, locked storage and transportation ifmitations, and iegal restrictions imposed by 11 censing.

Operator protection is obtained through adequate training plus good gauge design for maximum bio-: shield useage.

Gann sources are relatively easy to shield. requiring only careful desion of heavy metal shielding (lead, spent uranium, tungsten, etc)."

Neurron sources are very diffleult to shield. Use of high hydrogen moderztors may provide shieiding but this is accomanted by defeat of the measurewent capacity of the gaupe. It is irpossible to moderate the neutrons with heavy plastic shielding and sttil expect the ground mo isture to then moderate more neutrons for measureaent.. Neutron shielding is further complicated in thet the thermal neutrons are captured by the moderating material with a resultant exisston of gama radiation of fairly high energy.

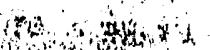

in tdeal neutron shield would be severil inches of plastie for fast neutron moderation. covered over with 17f" of cadirium sheet for thenul newtron capture. which in turn would be covered. Wth an inch of lead.to. stop the resultant gaumas. anch of Tead a Unfortunately, this combination rould be trapos-s. sible to lift and would no longer measure moisture.

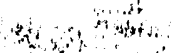

The best radiation protection progran of all is: a consentrated effort at maintaining the naxtmen distance from the source at all times combined wth expeditious use of the device. Operators should not stand unnecessarlly close to the untits during operation and should not carry them except by the eppropriate cartying handies.

and

CPN constantly reviews avaliable detectors and. source materials to permit reduction in source stze and reduction in external radiation lievels.

\subsection{Surface Gauoes: Nomal operation of the} PORTAPROBE requires the operator so ibe within 2' of the gauge for in perlod of approximately 10 seconds per test. There is ifttle reason to be closer than that distanes nor to work longer than this period to obtain :e tert. It may take longer than 10 seconds : to prepare the stte, however, the nuclear gauge should be remote from the site at this ttue...

A busy day can result in 30 tests being taken.

A busy work week would inciude five days of this extensive testing.
If we miliply this all together:

30 tests/day $\times 10$ seconds/test - 300 seconds or 5 minutes/dey of exposure within $2^{\prime}$

5 days $\times 5$ minutes. 25 minutes. This can be rounded off to $\$$ hour.

The average exposure level at $2^{\prime}$ from the gauge is 0.5 MREYHRR

2 hour $x y$ MREWHR -2 MREM accumulation in a busy work week witn the PORTAPROBE.

Operators are allowed weekly average accursyTation of 100 MPSY.

The dose to be expected from the PORTAPROBE using proper prosedures is only 1/400ih of the allowed dose. This is a large safery factor in the PORTAPROBE design limtetions and operating instruetions."."

1.02 Depth Probes: The radiation frow debeh probes can be higher be-

cause of the nork requirement of the depthprobe. Unilke the surface gauges, the depthprobes are carried around by the operator to a greater extent. The sources are. the same size, and the "shielding is equal, or even betcer, but the inmediate vieinity work requirement is higher.

CPN 500 Series depthprobes are designed to be carried with a sarap or handle. The source area is carried near the lower extrenities or ankles.

Density depthprobes are used primarily for research and the duty cycle is not high. The use of such gavge would be infrequent during 2 sotal year's tibe, and radiation accumblatien $r / 1)$ be low compared to other gauge uses.

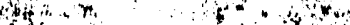

The major depthprobe consideration will be the Model 503 irOROPROBE for irrigation management. This unit will be used routinely, almost daily. throughout the growing season which may be afl year long in soeve iareas.

Garm output frow the HPDROPROBE is almost neglifible. The Americium 241/Be source has a 'iow energy gawn output which is not used for moisture measurement and wich is shieled out internally with a smaly lead sheath. Gauma. radiotion on the surface of the HYOROPROBE is approxtmate iy 1 MREV HR which reduces to less than 0.05 MREY/HR at 2 ' from the gauge.

Therual neutron ourput is approximately 0.2 MREVHR on the surface.

Fast neutron outpet is approximetely 4 MREW/HR on the surface of the gauge as measured with an Eberiline PNR-4 neutron counter.

Total garma and neutran radiation at mid-trunk 
of the human body, with the HYOROPROBE carried at the sioe by its handle, is approximetely $C .3$ MPEYH/HR.

The anticipated duty cycle in close proxinity to the gauge is approximately 2 hours/day during a full work day. The. operator will be driving par. of the time, performing some paderwork functicns part of the time, and trudging through the fields part of the time. We believe that the work cycle trudging through the fiteld carrying the: HYCROFROEE witl be approximaitely 2 hours out of $\mathrm{en} 8$. hour day.

Mulitpiying ous:

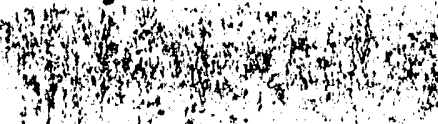

2. hours/day $x .5$ days $\times 0.3$ MREM/HR $=3.0$ MREM accumulationi in a week.

This is $1 / 3 c t h$ of the allowed weekly cose.

It is imporiant that the gauge be camied in iss approoriate cerrying location in the back of the venicle et meximum practicall distance from ine operacor, and that, allitise of the gauge be per

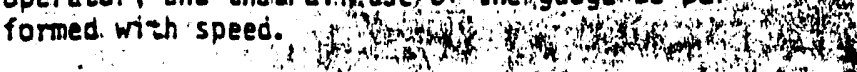

The gauge is at its safest when the probe is in the ground in the process of taking reading. No measureable riciation is present at the: gauge. electronics in this. operation.

$$
\text { - GOOD RADIATIOH PRACTICE }
$$

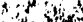

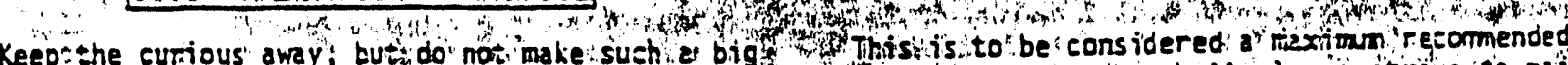

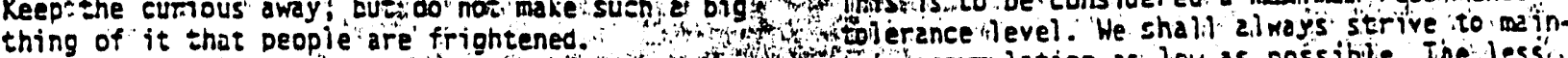

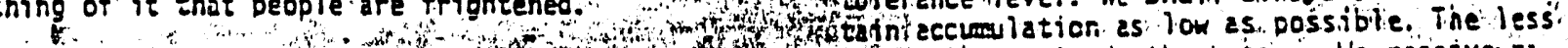

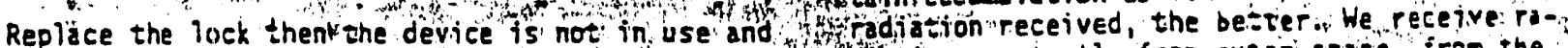
store uncer secure lock' and . Key. On'ly licensed operators should have the key to the gauge.

Place the handle of the surface gauge in SAFE po stition rmen not in actual use.

Petract the defthprobe into its stiveld when not in eztual use.

$$
\text { s.t. }
$$

Do not intentionelly expose any source in air.

Wear film badoes routinely. Do noti store then in heated envircriments, clove compertments, etc:

k'ork fist and keep distance between çauge and operacor. Distance and brevity are the two best operztor protective prograns:

han $y$ ?

and

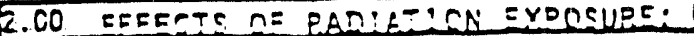

Radiation is net detectible by the bocy during 1 exposure. It cannot be seen, heare, smiellec, or felt.

Prolonged exposure wilt uoset: celli sitrueture. however, and the body will eventuelly react io. the insult as it mould. with ine cesack cf germ cellis er virus. Tre tody cefense mecherii sm wili carrect the insuliti or irjury: and will destroy any a anaged cellils. Whether from siveliness: or from

ifidiation exposiare.

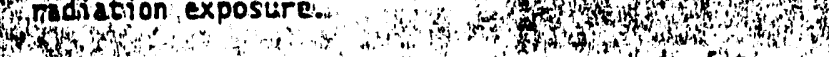
concentrated radiation in short deriod of time - fis more difficult for the tody sc handle than is: radiation spread over a longer pericd of time: siv rad

The Nuclear Regulacory Comission regulations allitow a maxinum accumulation of ratiation by workers in an occupationel wise of redioactive äterial's to be, 5:0 ReM per year:

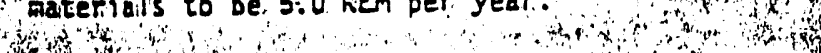
Whis reduces down to $14.25 \mathrm{k}=$ W Ot, which is a re- poiting, leveli. The Radiation Sar ety Officer ista reourred to report an exposure of thils revel to. Whis license jurisciction with a report on the manner in which it was received and reparding protective procedures to be talien so prevent. is

Acirom happening again.

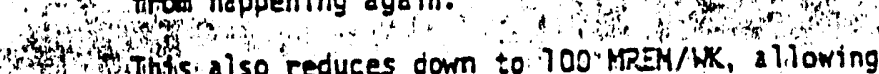
w y for twotweeks vacation for tres worker difation constantly from cuisr sizce, from the butiletings in which we live, irem inedical $x$-Rays, and from high energy radar and microwave enissions. Soil gauges are just cne more source of. eneroy added to the rest.. He shall diways strive to keep the total radiation at a minimus., however.
Exposure to radiation is relatively iomeasureto'a soil gauge under normal operating procedares.

Detection of rediation exposure is largety by. observation of reoorted sickness symoions, combined with cbserved cell count changes in a blcod samole, and also combined with derinite knowledge off the probabilitty of exposure.

$h$ change in cell count alone could occur from a cut finger as wuch es fran exposure. able in saal? coouncs accumul a ted from expestire

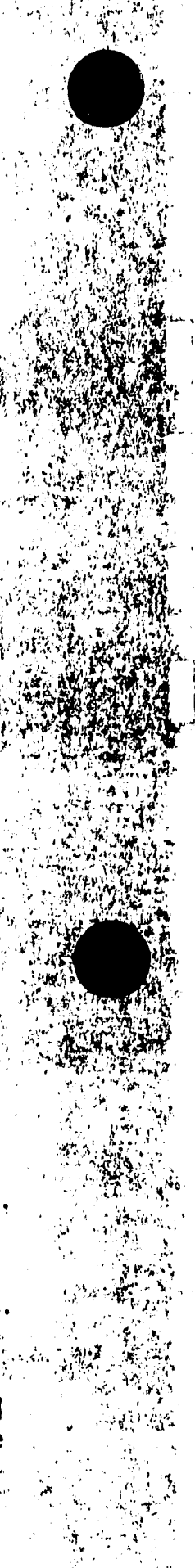

(n)

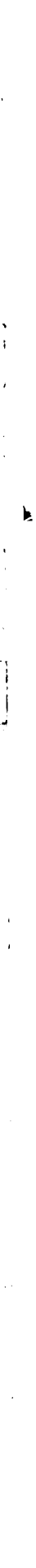




\begin{tabular}{|c|c|}
\hline $\begin{array}{lr}\text { Chest X-R.ay } & 100 \text { MREM } \\
\text { Tooth } \lambda \text {-Ray } & 10-90 \text { MREM } \\
\text { Comercial jet flight San Francisco }: 0 \\
\text { iver: Yorh } & 3 \text { MP.EM }\end{array}$ & 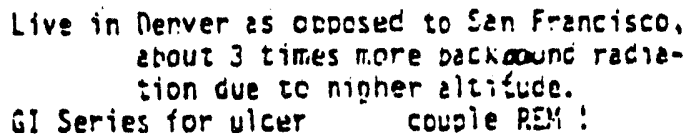 \\
\hline
\end{tabular}

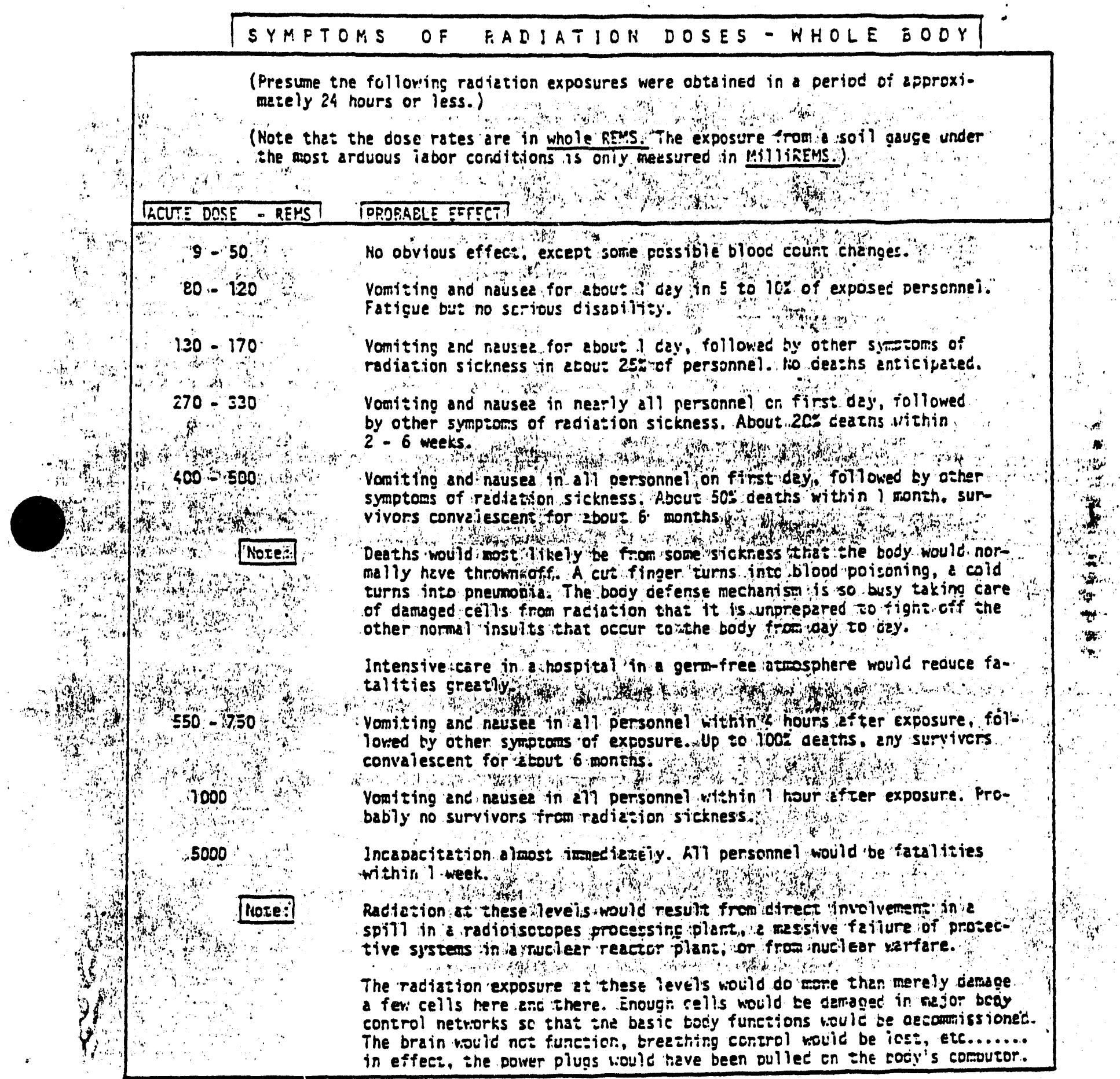


E.00 Generz: The ocerator must protec: numan life first. tnen proder:y from damage due to a radiazion incioens.

We must prevent the rati radjoactive materiai from escaping to the a conospnere or enviroment.

The source meterial is eneapsulated in two stainiess, vielded consainers, wniti is further: sesurely mounted ince. the gauçe encicsure. It is niçniy Hal ikely thet the moteriall could escape in the event of 2 severe accicent or fifre, however, aur protective proaram must thsure enat, we planitc-s this eveniuality.

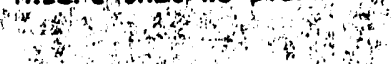

The first ecsion to be ik ken in the event of en

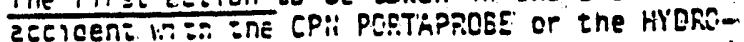
FROBE is io keep other people awey from the sicE.

Then exacreise ine foltowine oecison ocine:

- The gauge is superiticialiy oemaged dersed.

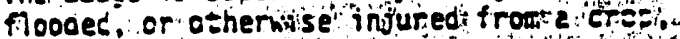

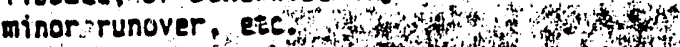

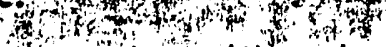

The enclosure is in one piese with a wiror threat or iwo in tre sheet metal or cassing and the source is obviously in piace, 2 : least the source loce'ign js not torn apert.

-7) Turn the gauge over to view the sounce area, if necessarys Do not walk tnrough the sice macerial where the gavge was

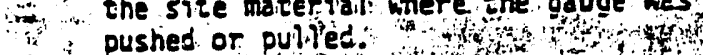
$\therefore$ inspect the souree area visua Tly so in Inspect the souree area visua Ty $t$ ?
sure no damage co stutiter or scures - nountins.

2) If source area is triet, pick uo cause, place in storave concainer and rezurn to permanent siorage arez.

3) Call the RSO, and CPN factory for assistance in stripping the oaude bezk to the factory for repair or disfosel.

DO NOT SHIP THE GOLGE WITHOUT FACTORT AFPROVAL OF MHOWLEDEE:

- The oauce is broken epars severely burnee. severely erusned with oarts strewn eroune, or the source arez: is visually damagec.

i) Freeze tha site. Rope off the cañoge site for $1 C^{\prime}$ around, Stoc the vericle and have the criver welk eway. DC ros: walk through the camage site. If recioactive meieria? is icose it cen de pickec up and-racke: eiserinere.

2) Cill the R.SO, arda/er :he nearesi oublic healih oedartment of ice for nelp. eall us. The ebjestive is to șe: an expert raoiacior. techriciar so ine site with as operating survey meter who can cetermine: if the rasioctive material is lost or is insoc:

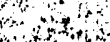

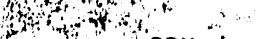

to. of does no: recomend ctiat cuscomers

$4 \mathrm{f}$ ourchase their orn survey meter for
of this purpose. There is liftelle.like-

of this purpose. There is liftelle. like-

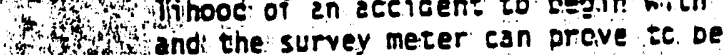

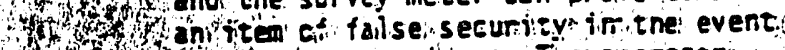

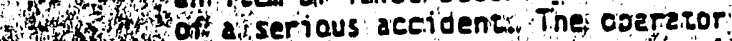

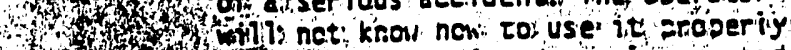

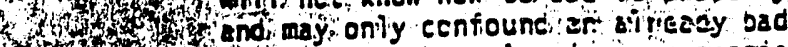

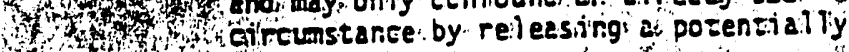

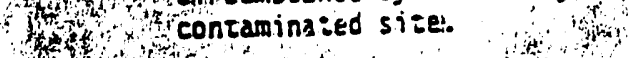

6. $3+5$

3i) The radicition expert wit dezer ine whether the sice is safe, viliil rmove

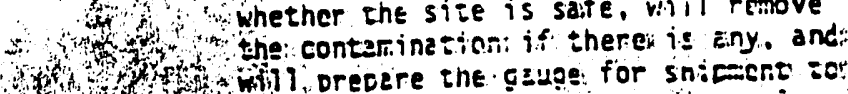

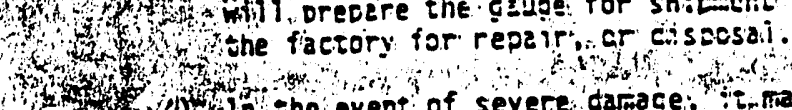

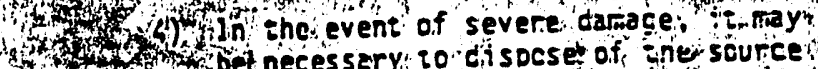
bet necessery 20 dispcse of ne scurce

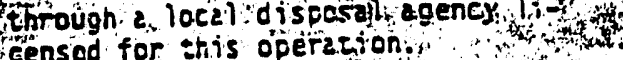

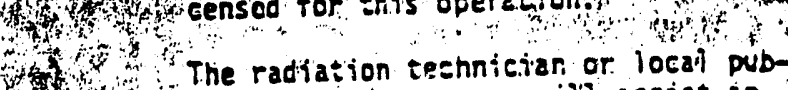
Why life health depowent will essist in

of this action.

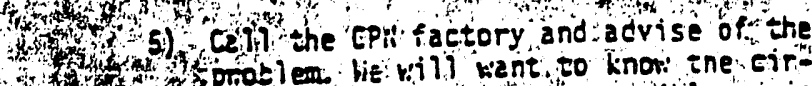

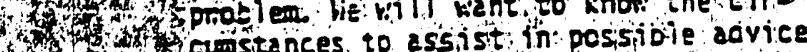

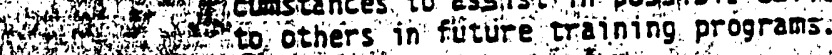
to ease the ands of oseretors in this regart. eppkitas never had $a$ canaged gauge requiring extreme security: frecalicion, a itrough he have had arneber of gaveses incroughiliy run ever in thet years: that: we riave manutiacureo many. units. atis

We know of no other canufacturer, either, who has experienced this degree of camage.

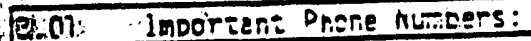

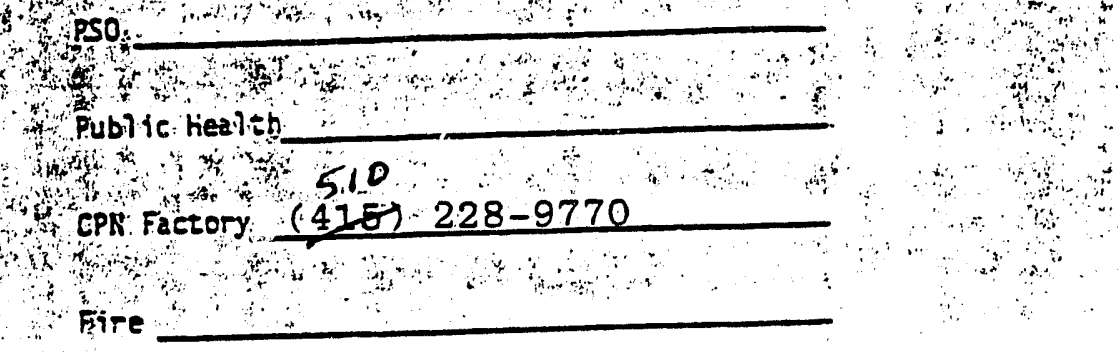

Police

Netify the oubitic neaith ofitice, colice ino our oifices innediateiy in ine case of a szoien cauce. 
4.00 LeEx Testinc: All radioactive sources nust be tested for cen-

zaminesion Deriodically. CPN sources are doutly encaosulater in stainiess steel and the likeihood of a leaking source is very remote, however tney still mast be leak tested in accordance with regulations, basically every six months.

CPN sources are approved for one year waiver uncer our CPM lícense, however, local juriscictions may still require the six month period.

It is important that your license be followed.

The user may test his own gauge following CPH instructions. Leak test kits are available from the factory and an initial kit was supplied with the gauge.

The leak test must be returned to our analysts lab. for processing. A certificate will be ire-: turned for the licensee's flle for inspeetion by licensing authorities at any tine.

6.01 Suriace Gavoes:

One test is reouired for the BRC MK II Series: two tests for the BRC MC Series.

EPC Mart I: Series:

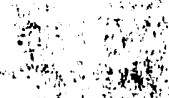

1) Stand gauge on end, leave shutzer closed.

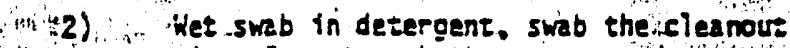

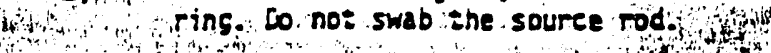

3) Return the swab to our analysis Tab in the envelope provided

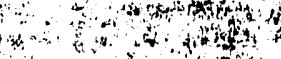

4) A certfficate will be returned for your? records.

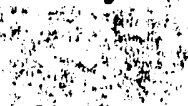

BRC MC Series:

3

The Ne Series uses two sebarate sourees to perwit the sirulizaneous counting at all times. Two sources mus: be leak tested therefore." However. only che swab need be used. $h=0$ sto

i) Stand cauge an end, leave shuter closed?

2) Het swab in detergent. swab the ciearourt ring. Do not swab the source rod.

3) Set gauge upricht, remove scriews and raise eleczronics to service poststion. Swab red spot at lower left of guidetube casting adjacent to the moisture detecsor and adjacent to the insernal

H. $\quad$ radiazion label.

i) (4) Return the swab to the enelyst's iab in the envelope provided.

5) A certifice te will be returned for file.

PadPrec: $7 / \pi$

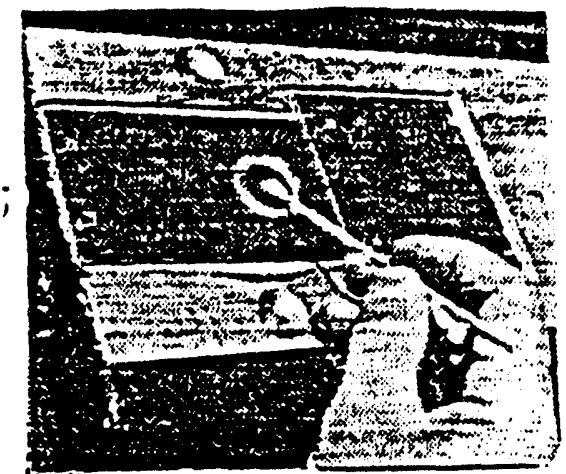

LEAK TESTING MODEL GRC MK II GAUEE AND CESIUM SOURCE OF A RNY MC SERIES GLUGE

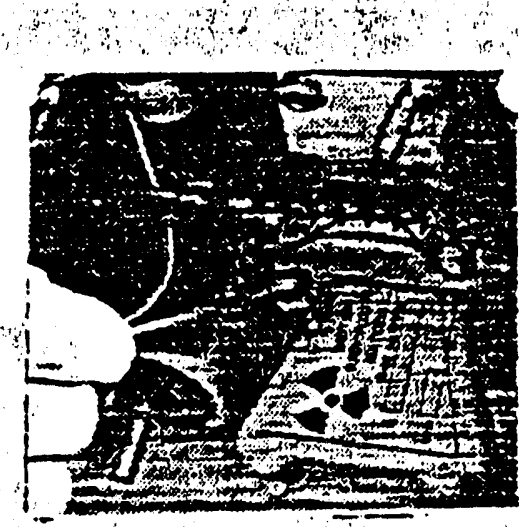

LEAK TESTIKG WOISTURE SOURCE OI MC GAUGE

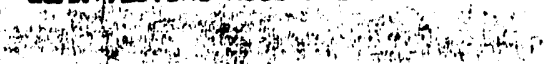

4.02 Depthorobes ifri of

1) Lay probe on itiss side. If source is leeking. concamination will be insige the shield tute.

2) Het swab and iswab instee of shield sube.

3) Return'swob to hab. certificate will be returned for your recores.

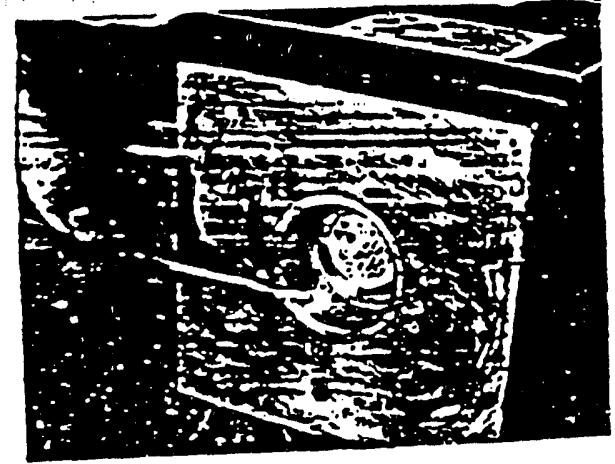

LEAK TESTING DEPTH PROBE IN SHIELD TUAE
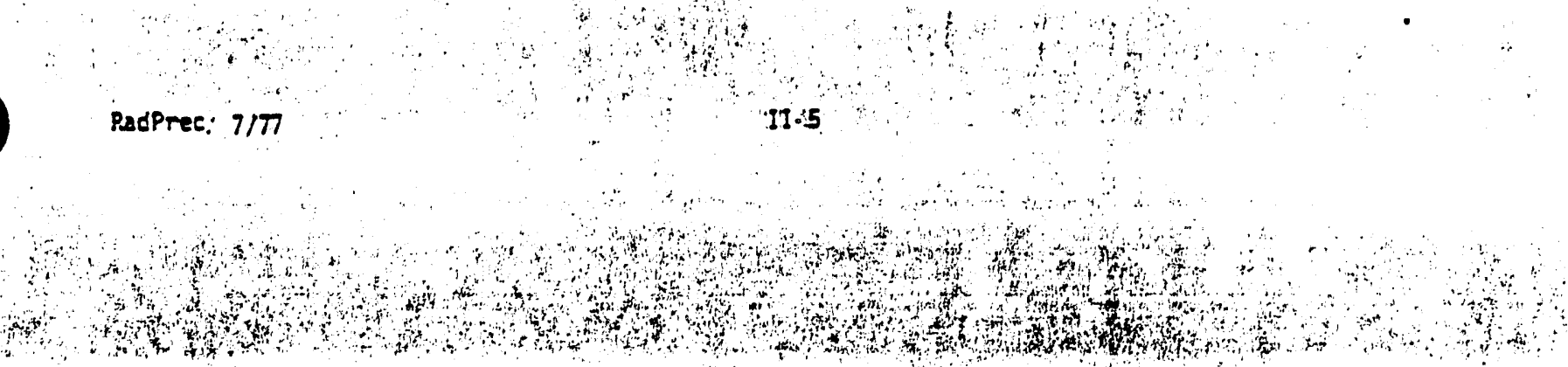
$\therefore \sin$

5.00 transportation of rean cave

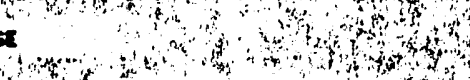

- Iransoortation vil comon carrier or th ortuste vericies

on bublte rnass at itiens conteining gooss. speoent amperous, suen as ine redioacsive cotertials. in your. ompe, is reoulated by Titile 69 code of inderal heoulat tons, parts 170-190:

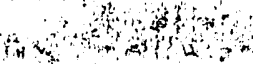

To tranadort namerous. acods rou nus aret: specitse

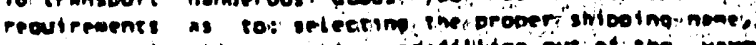

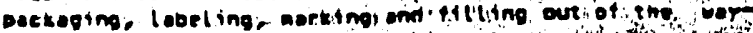

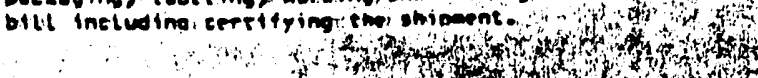
3.09 Proper shipPing wime

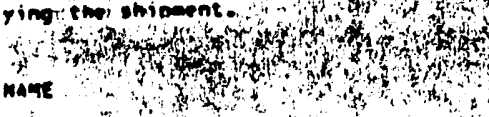

The ouvoe contoins 10 mest of Cesin-13T cowbly encessum lated in welded seninless sieel enclosures:. Sg" acti nt Anerictum-241/beryllitum is enelosed in: "secons melosure. Rodioecrive meterial mesosulated in ints

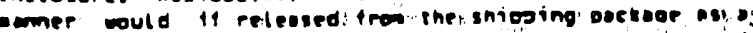

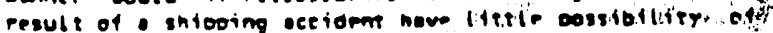
peleagtno ony rmoveslec conteatanstion and is classititied

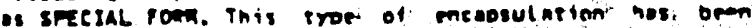

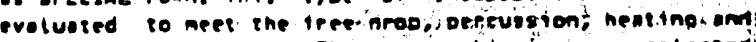

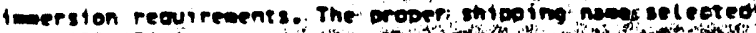
trow 172.101 ts:

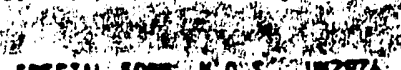

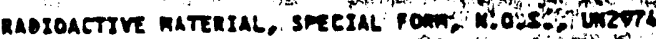

M.O.s. "stends tor not othervise soectitied.

$$
\text { x." }
$$

3.02 PReKAGine

$$
\text { a } 40+4
$$

S:OL MARTINS

intoietwop mast be marred with:

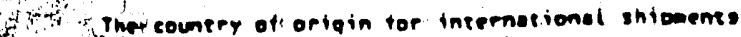
Theishiooing deeknar ipoe in 1/2" letrors The nroder stribotne name

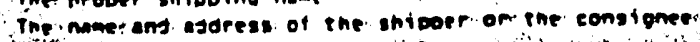
ing

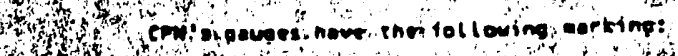

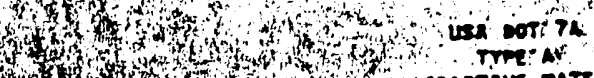

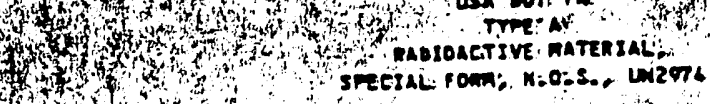
tistion

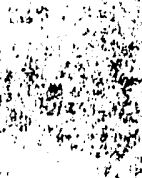

Wy intooing label orowides both ine shiooer

$\lim _{0}$

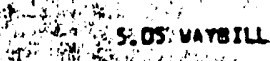

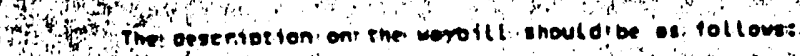

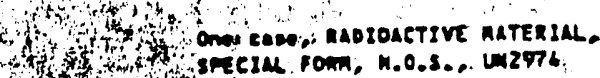

$\mathrm{g} y \mathrm{f}$ if

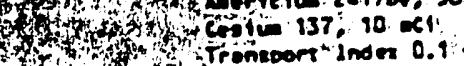

\section{Mabronetive TELLOW II Libel}

USA bot 7A, TTAE A PACEade

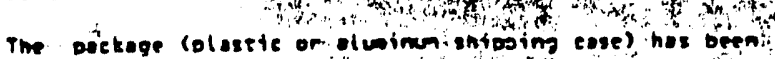

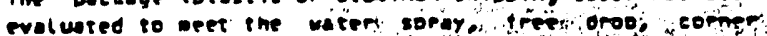

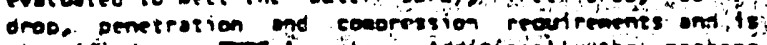

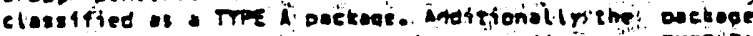

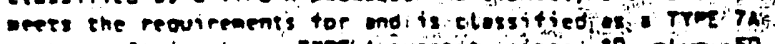

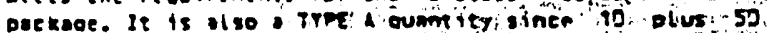

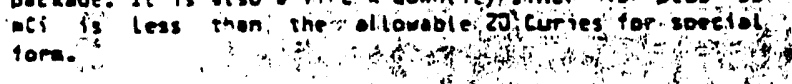

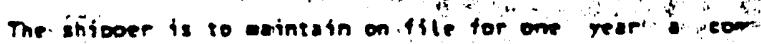
olete cerrfticaition ins suoporting sotety anslysis cover ing the daeksoing and soecial ford reouirements. Smot as the orfothal snioper sloolied suen - certtticaston with the gauge in a docket adgressed to the hadiation setety ofticer.$$
\text { thing }
$$

\section{S. IT LABELINE}

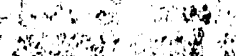

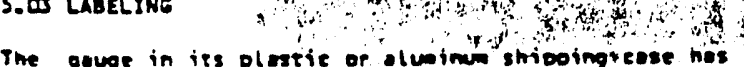

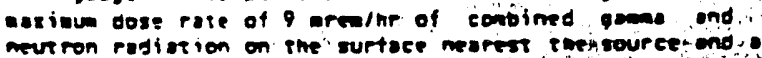
cose rate of .C25 mrowe as' 3 teet. It theretor shios.

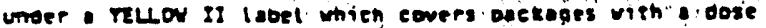
rete of nore than 2.3. and less than So ermathe on the

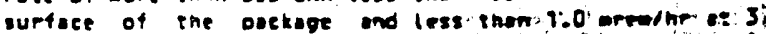

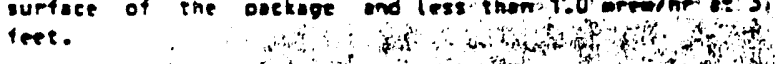

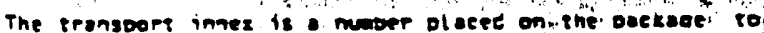
indiente to ine copripr the- oegree of control to bi esercised ouring sransoortation. It inticates the merisus pedistion sost pais at 3 tept from ine oucksoe surfose. for simolicity it is rounged wa to ine neoress tenth. The Dose pare at 3 toes tor the CPN gavoe is . 225 mrem/nr.

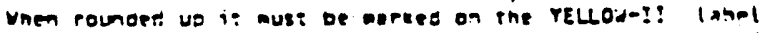
as a TRAMSTORT IMOEX of C.S.

Rodionesive rELLOU-II labels shoula be aftires to at leass two opnos:i. sians of the naeknoe. Entpret on ine lesels snoule to es-137 10met. An-261/e- $30 \mathrm{msi}$ ane : $=.9$ Transoors imaei. A: shudoen trom ine castory. Con's gavaes nave rellow-ll ladels on inree visible surthess.

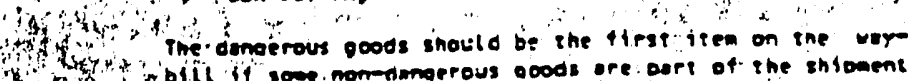

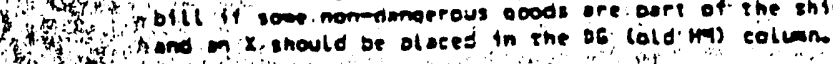

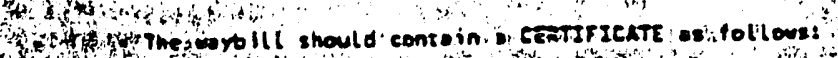

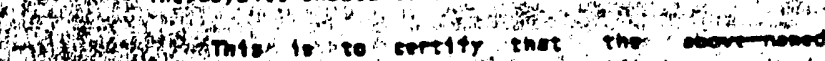

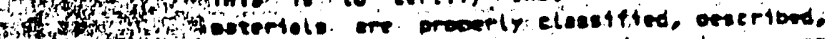

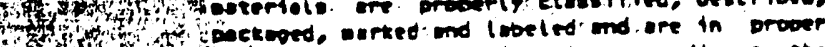

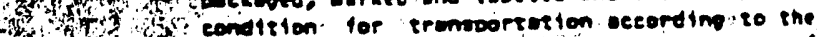

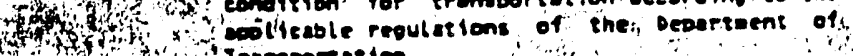
Y Trumportation.

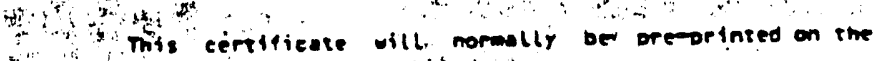

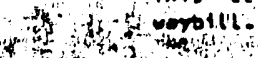

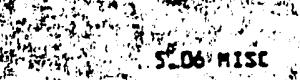

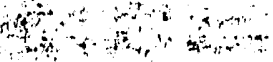

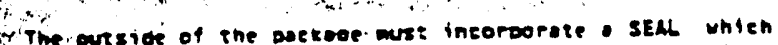

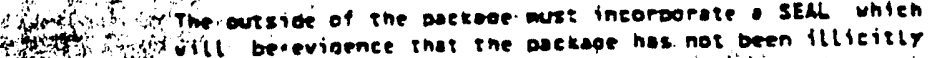

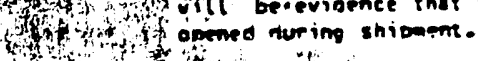

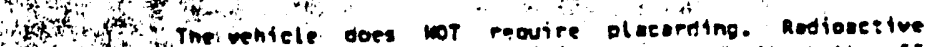

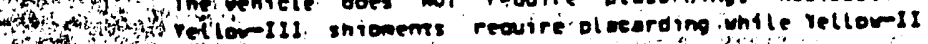

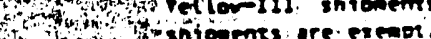

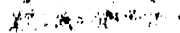

snigh

ine snlooing oopers must be reodily oesesstble for in joection or in the event of mo eccident. They wust be vithin the reach of the oriver and visible to person entering the driver's compertaent. Uhen the driver is not at the vehtele's controls the shiosing paoers must br on
ime drivers seat or in. noldet on the orivers door.

unem ermsoortiny the pawa by private ventele on publte

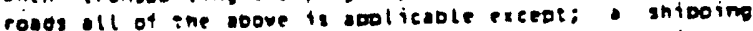
lasel virh ine snioper's or consiomee's nowe is not poouires. and cerpitication on ine stivesima onder is not requitec.

Yo eepe the requirement for aceessible shidoing onders. the nriver shoulo nave on the seat or on the neivers o0or

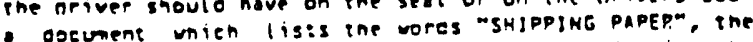
name ans sooress of the compony or oroenizotion he represents. and ine descriotion listed noove for truek shionents.
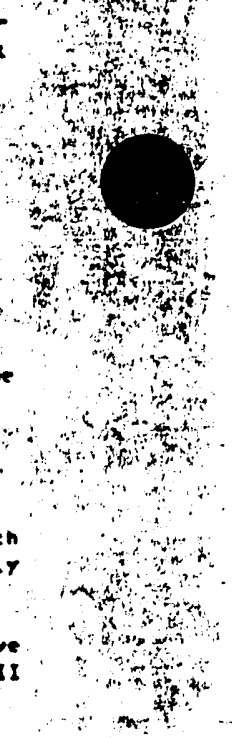


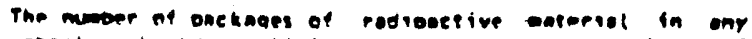
ventcle shoult be linted to a toral transoort inopr of Sa. A Detaoe with a iransonet inoes of 0.1 should be as least on toot teom ine neerest berzon. Also it should be at leost 3 tept from any unnevelooed fill for a tronste pioe wo to sours. The gavoe case should be locked. It the driver lonves the vehtele then ine thers should be removet trom the ionition ond the whicte lockeft. It the phe qoupe is stored in on ooen ares such os the bect of nictuo then the case should be chain locked or otherutae securat to the vehtele.

If the oaver is outside tis shioging case shen the gaupe liselt is consinered the hibotno petsege its gose pates ore hipher but still aness the Tellowli peculrements. It would not homper meet the recutrements for labeline ind noettina.

\section{(a)}

S.OT ATR SHTMENTs

aip shtomms mes conolly iutth ritle 49 ind the Intemational Aip Tramenort ascoetation's unnoerous coods Meoulations.

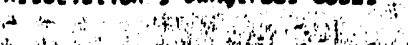

For vellowll lobel, on ofr enteoent aust hove erome sore imose nt 1.0 or less. catton of the tronsteres that he is autnorized oy

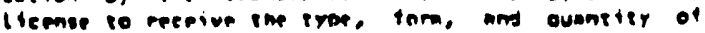

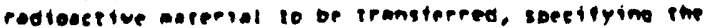
license numbet, issuing somcy om proipation outp.

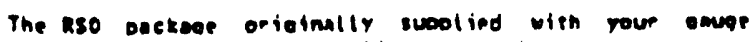
includes a cooy of CPW', lleense to allow you to returen your eowe to CPW for repaif or other reasons. Artatitional cooles ore ovailable woon reonest.

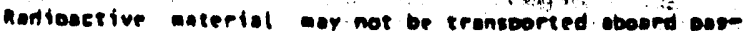
semaer corrying oircropt unless that eeteriol is intended. for use in, or incioent to, researeh, or eedteal diugnosis or treatome.

$$
\text { and } 12
$$

Under cost círeunstances rour gave ulll be liated te

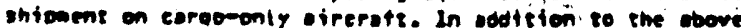
reovirements for transooptation by truet the following reatirements eust be wet to ship by enroomenty.etrerste

3

canco-areenaft-our

Tellowerl labels

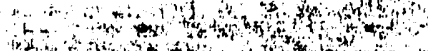

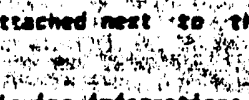

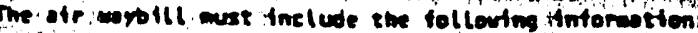
Damotnous cooos is is on

ATTACHED SHIPPER's DECLARATron.

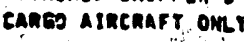

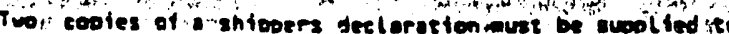
The carpier coetuslly it is best to nooly an aditionol? sooy tor eoch corrier unteh will be imol weds. Anendemes or eltermtions are not allowed enless they. ore siened toy twe the sane sianature as used to sim the coesnem. ...Th

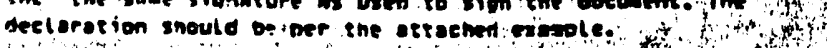

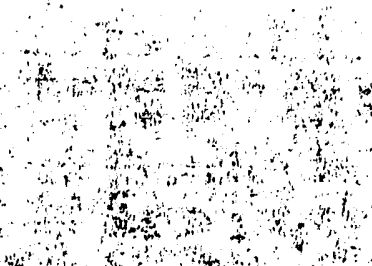

$t$

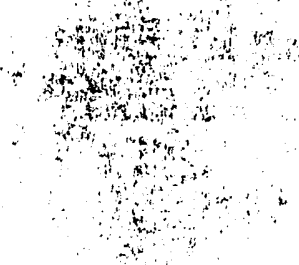

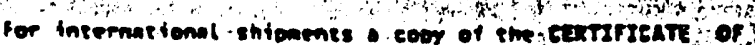

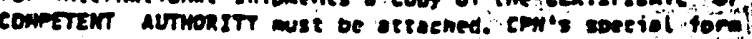

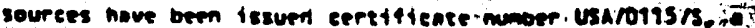
coor of wien is orinted on the back of the shiowern dreloration. in

\section{notsture}

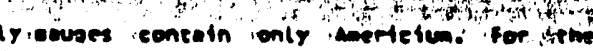
of these oncues the intormation is the "cons exeept delete all reterences ro Cr-137, 10 acl. The twois-

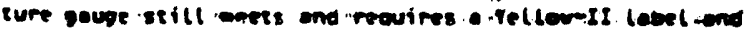
nos Tial. o4.0.1. . .

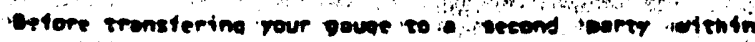

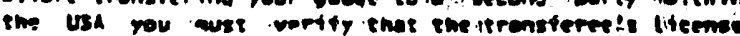

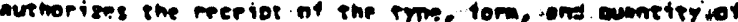

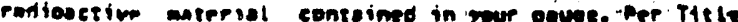
10. Chaoppr 1. CFR Port 30.61, there are two aceeotable artwots for vertificetion.

(1) You mey have in wour cossersien. and hove read current ceor of the tronsteree's license.

(2) Tou nop hove in rour possessien iupitren cerptit-

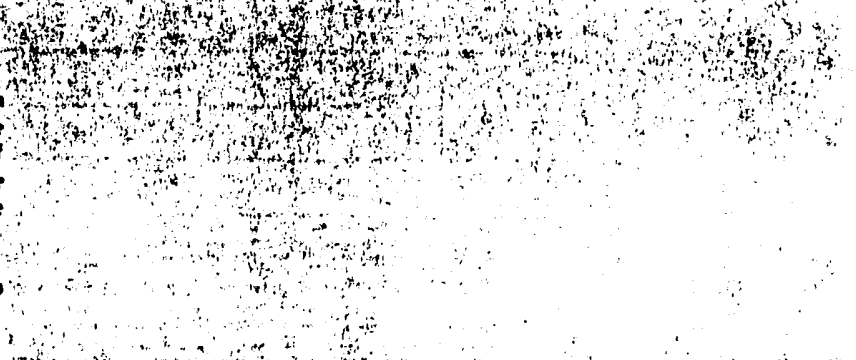

$$
\text { a }
$$
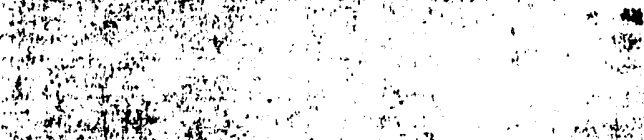


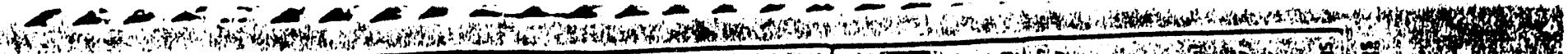

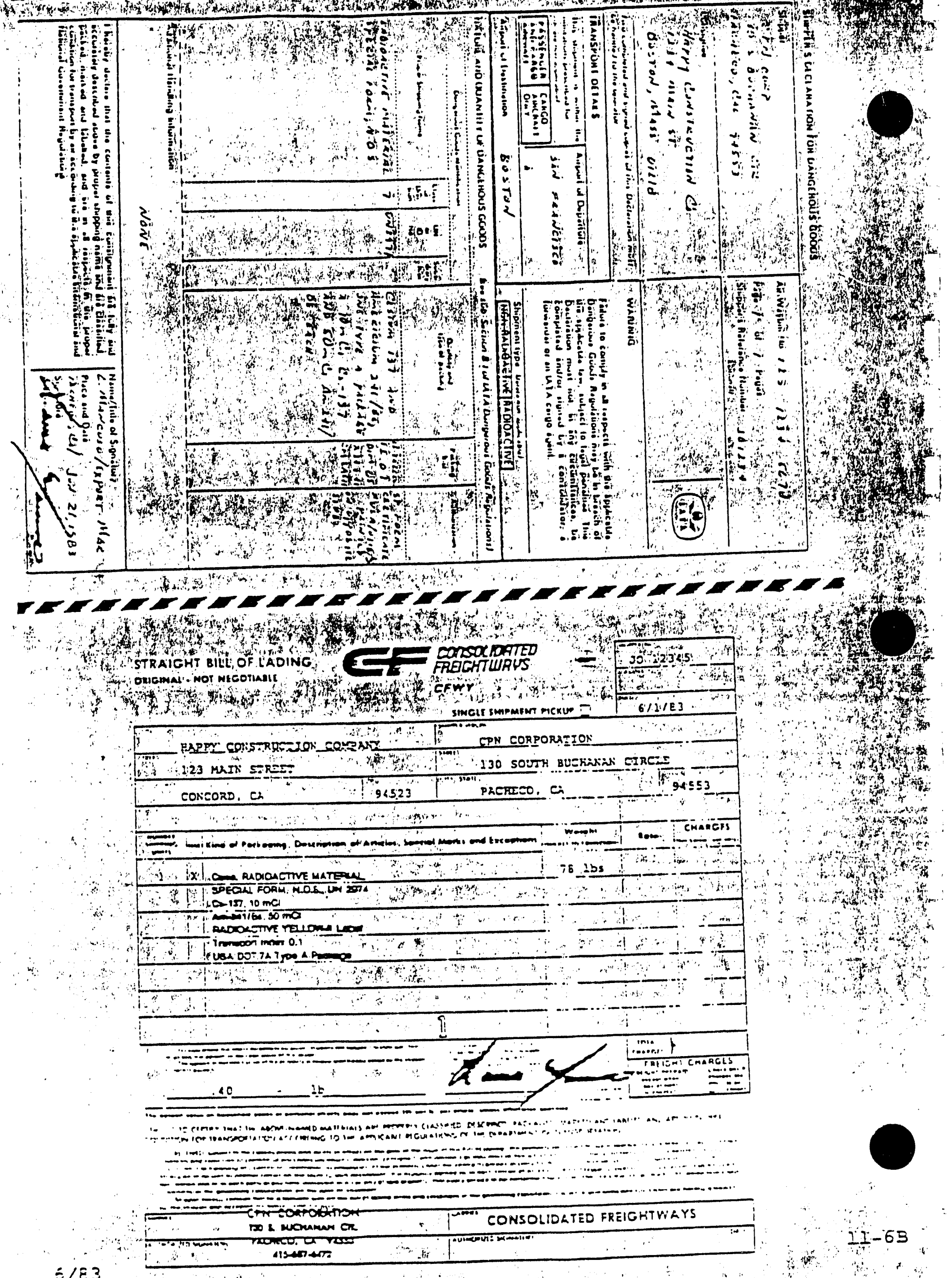




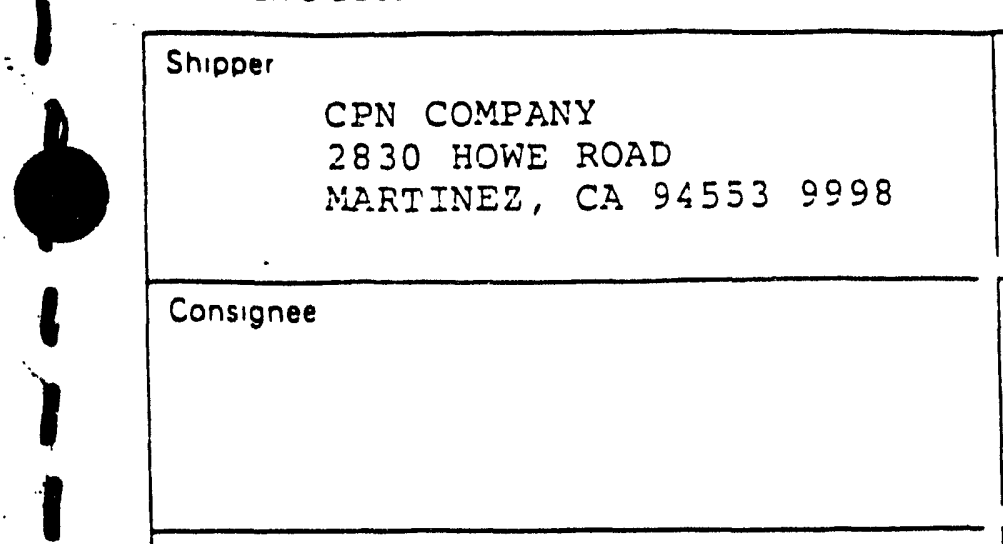

Two completed and signed copies of inis Declaration musi be handed 10 the operator

TRANSPORT DETAILS

This shipment is within the Airport of Departure limisations prescribed for:

loelere non-opolicablel

PASSENGER CARGO

AND CARGO AIRCRAFT

AIRCAAFT

Airport of Desination:

NATURE AND QUANTITY OF DANGEROUS GOODS

Proper Shipping Name. Class. UN Number or Identification Number, number of packages. packing instructions and all other required information as detailed in sub.Section 8.1 of IATA Dangerous Goods Regulations
Air Waybill No

Page of Pages

Shipper's Reterence Number loolionall

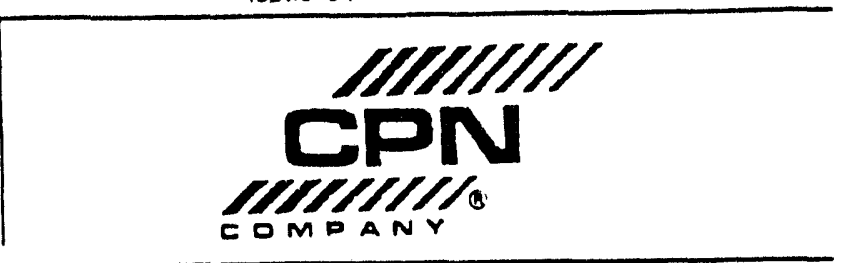

WARNING

Failure to comply in all respects with the applicable Dangerous Goods Regulations may be in breach of the applicable law, subject to legal penalties. This Declaration must not, in any circumstances, be completed and/or signed by a consolidator, forwarder or an IATA cargo agent.

Shipment type loelere non-soplicsole)

NON-RADIOACTIVE RADIOACTIVE

Additional Handling Information

I hereby declare that the contents of this consignment are fully and accurately described above by proper shipping name and are classified. packed, marked and labollad, and are in all respects in the propar condition for transport by air according to the applicable International and Netional Government Regulations.
Name/Tille of Signaiory

Place and Date

Signature

(see worning above) 
S. Depariment

400 Sevenin Sireel. $5 \mathrm{~W}$ or Tronsportarion

Research and Special Programs Administration

IAEA CERTIEICATE OF COMPETEKT AUTHORITY

SPECIAL PORM RADIOACTIVE MATERIALS ENCAPSULATION

CERTIFICAIE NUMBER USA/O115/S, REVISION 5

This certifies that the encapsulated source, as described, when loaded with the authorized radioactive contents, has been demonstrated to meet the regulatory requirements for special form radioactive material as prescribed in IAEA Regulations and USA regulations for the transport of radioactive materials.

1. Source Description - The source described by this certificate is idencified as Gulf Nuclear, Inc., Model VL-i which is a doubly encepsulated $17-4$ or 304 stainless steel welded capsule measuring $3.2 \mathrm{~mm}\left(0.125^{n}\right)$ to $12.7 \mathrm{~m}\left(0.5^{n}\right)$ diameter and $6.35 \mathrm{~mm}\left(0.25^{n}\right)$ to $19.05 \mathrm{~mm}\left(0.75^{n}\right)$ in length.

2. Radioactive Contents - The authorized radioactive contents of this source consist of not more than a total of $98.5 \mathrm{GBq}(500$ $m C i)$ of Americium-24i oxide in Beryllium metal powder and/or Cesium-137 in the form of Cesium chloride.

3. ExDiration Date - This certiricate expires October 10, 1992.

This certiricate is 1ssued in accordance with paragraph 803 of the IAEA Regulations and Section 173.476 of Iitle 49 of the Code of Federal Regulations, and in response to the September 29,1988 petition by CPN Corporation, Martinez, $C A$, and in consideration of the associated information therein.

Certiried by:
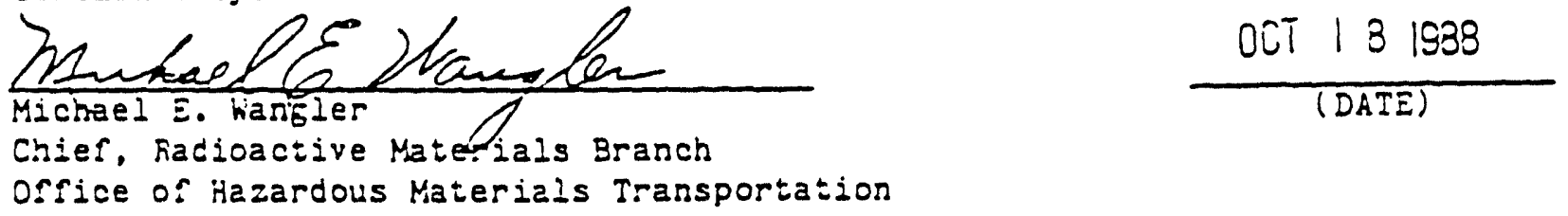

Revision 5 - issued to extend expiration date and to change capsuie dimensions and radioactuve concent.

1 "Safety Series No. 6, Regulations for the Safe Transport of Radioactive Materials, 1973 Revised Ed1tion" published by the International hioric Energy Agency $(I A E h)$, Vienna, Austria.

2 I:tle 49, Code of Federal Regulations, Parts 100 - 199, USA. 
U.S. Department

Research and Special Programs Adminisiration

IAEA CERTIEICATE OF COMPETENT AUTHORITY

SPECIAL FORM RADIOACTITE MATERIALS ENCAPSULATION

CERTIFICATE KUMBER USA/0400/S, REVISION $O$

This certifies that the encapsulated source, as described, when loaded with the authorized radloactive contents, has been demonstrated to meet the regulatory requirements for special form radioactive material as prescribed in IAEA Regulations and USA regulations for the transport of radioactive waterials.

1. Source Description - The source described by this certificate is identified as CPN Corporation Model No. T200156 neutron Eamma sourse. The doubly encapsulated type $316 \mathrm{~L}$ stainless steel welded capsule measures $9.0 \mathrm{~m}\left(.356^{n}\right)$ in diameter by $14.5 \mathrm{~mm}\left(.572^{n}\right)$ in length.

2. Radioactive Contents - The authorized radioactive contents of this source consist of not more than $0.37 \mathrm{GBq}(10 \mathrm{mC1})$ of Cesiuw-137 in the form of a ceramic and 1.85 GBq (50 mCi) of Americium oxide in a Beryllium metal powder pressed to form a pellet.

3. Expiration Date - Inis certificate expires April 30, 1990.

This certificate is 1ssued in accordance with paragraph 803 of the IAEA Fegulations and Section 173.476 of Ittle 49 of the Code of Federal Regulations, and in response to the August 31,1988 petition by CPN Corporation, Martinez, $C A$, and in consideration of the associated information therein.
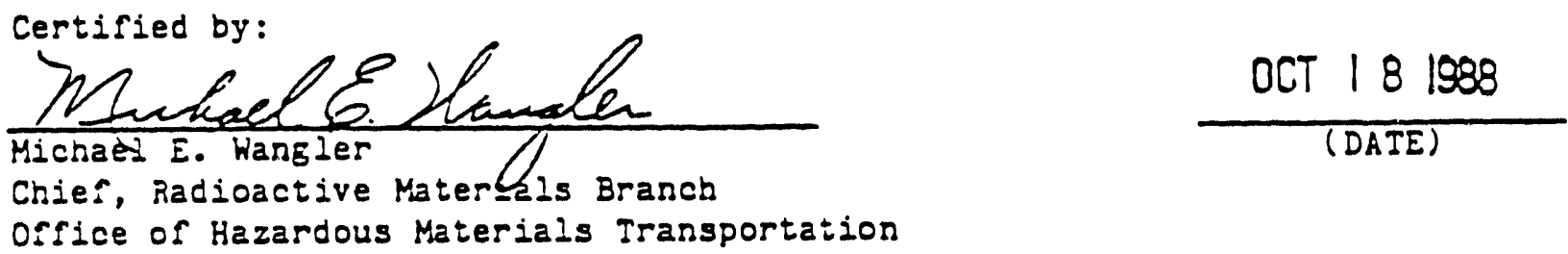

office of Hazardous Materials Transportation

1 "Safety Series No. 6, Regulations for the Safe Iransport of Radioactive Materials, 1973 Revised Ecition" published by the International Atomic Energy hgency (IAEA), Vienna, Austria.

2 Iitle 49, Code of Federal Regulations, Parts 100 - 199, USA. 
U.S. Deponment

100 Sevenin Sireet. $5 \mathrm{w}$ of Transponotion

Research and

SPECial Programs IAEA CERTIFICATE OF COMPEIENI AUTHORITY

Administration SPECIAL FORM RADIOACTIVE MATERIALS ENCAPSULATION

CERTIFICATE NUMBER USA/0399/S, REVISION $O$

This certifies that the encapsulated source, as described, when ioaded with the authorized radioactive contents, has been demonstrated to meet the regulatory requirements for special form radioactive material as prescribed in IAEA Regulations and USA regulations for the transport of radioactive materials.

1. Source Description - The source described by this certificate is identified as CPN Corporation Model No. T100405 neutron source. The doubly encapsulated type 3162 stainless steel welded capsule measures $9.0 \mathrm{~mm}\left(.356^{\mathrm{n}}\right)$ in diameter by $12.7 \mathrm{~mm}\left(.500^{\mathrm{n}}\right)$ in length.

2. Radioactive Contents - The authorized radioactive contents of this source consist of not more than 3.7 GBq (100 mCi) of Americium-241 oxide in a Beryllium metal pressed to form a pellet.

3. Expiration Date - This certificate expires May 31, 1990.

This certificate is 1ssued in accordance with paragraph 803 of the IAEA Regulations and Section 173.476 of Title 49 of the Code of Federal Regulations, and in response to the August 31,1988 petition by CPN Corporation, Martinez, $\mathrm{Ch}$, and in consideration of the associated information therein.
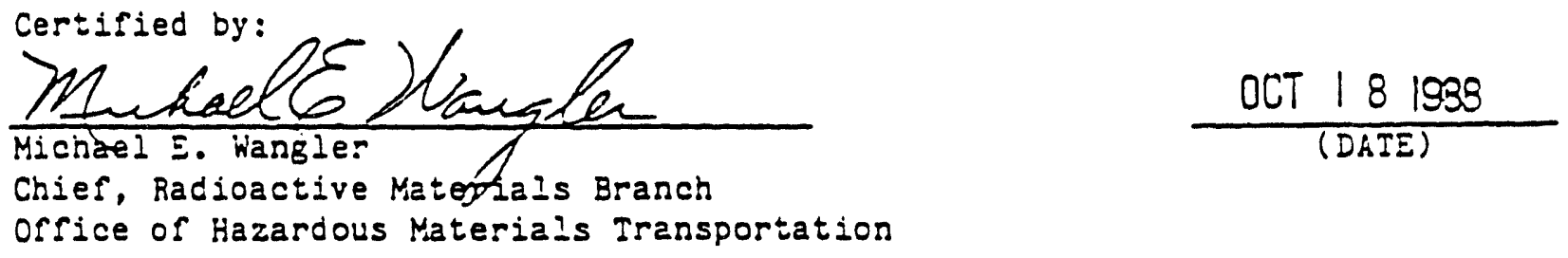

1 "Safety Series No: 6, Regulations for the Safe Transport of Radioactive Materiass, 1973 Revised Editionn published by the International htomic Energy Asency (IAEA), Vienna, Austriz.

2 :itle 49, Code of Federal Regulations, Parts 100 - 199, USA. 
US DeDorimen:

oi ironsporioikon

Researeh and

Special Programs

Administration

IARA CERTIPICATE OP COMPEFET AOTEORITY

SPECIAI PORA RADIOACNIVE MATERIALS

CRRTIFICATE UOMBER USA/0398/S, REVISION 1

This certifies that the source described hes been demonstiated to meet the reguiatory zequirements for special sorm radioactive material as prescribed in IAEA Regularions and USA reguiations for the transporr of radioactive materials.

1. Source Description - The source described by this certificate is identified as CPN Cozporation Model No. 131, Part No. :400521. The doubly encapsulated Type 3162 stainless steel welded capsule measures $9.0 \mathrm{~mm}\left(.356^{\circ}\right)$ in diameter by $12.7 \mathrm{~mm}\left(.500^{\circ}\right)$ in length.

2. Radioactive Contents - This souzce consists of not moze than 0.00185 TBq (50 mCi) of Americium-241 oxide in a befyllium metal powcer pressed to form a pellet.

3. Expiration Date - This certificate expires July 31, 1992.

This certificate is issued in accordance with paragraph 803 ot the Irst. pegulations and section 273.476 of $: i=1$ e 49 ct the code of Iedezal Regulations, and in =esponse to the kugust 3,1989 petition by CPN Corpcration, astinez, $C h$, and in consicezetion of ihe associated information thereis, and othez inforwation siled with this office.

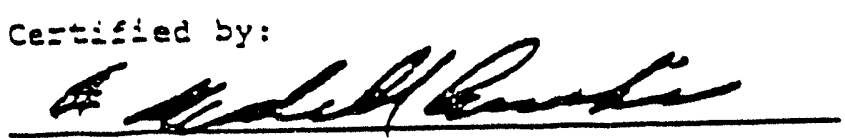

Michael E. Wangler

Chies, Radionctive Materials Braxch

office of Bazardons Materials rzansporteticn

Revision 1 - Issued to clarify the source nodel identification and to extend the expiration date.

1 - Safety Series No. 6, Regulations for the safe Transport of Racioactive raterials, 2073 Revised Edition" published by the Interausional hiomic Energy Agency (IfER), Vienna, Austria.

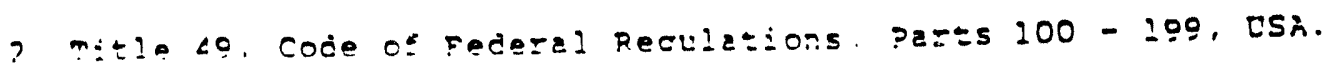


This certifles that the encapsulated source, as described, when loaded with the authorized radioactive contents, has been demonstrated to meet the regulatory requirements for special form rafloactive material as prescribed in IAEA Regulations and USA regulations for the transport of radioactive waterials.

1. Source Description - The source described by this certificate is ideneified as CPN Corporation Model No. T400378 gama source. The doubly encapsulated type $316 \mathrm{~L}$ stainless steel welded capsule measures $7.1 \mathrm{~mm}\left(.281^{\prime \prime}\right)$ in diameter by $13.3 \mathrm{~mm}\left(.525^{\mathrm{n}}\right)$ in lensth.

2. Radioactive Contents - The authorized radioactive contents of this source consist of not more than $0.37^{\circ} \mathrm{GBq}(10 \mathrm{mCi})$ Cesium-i37 in the form of a ceramic.

3. Expiration Date - This certificate expires october 10, 1992.

This certificate is issued in accordance with paragraph 803 of the IAEA Regulations and Section 173.476 of Title 49 of the Code of Federal Regulations, and in response to the August 31,1988 petition by CPN Corporation, Martinez, $C A$, and in consideration of the associated information therein.

Certified by:

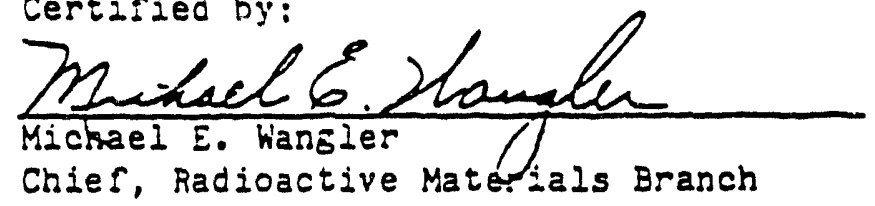

Chief, Radioactive Materials Branch

office of Hazardous Materials Transportation

1 "Safety Series No. E, Regulations for the Safe Iransport of Radioactive Materials, 1973 fevised Edition" published by the International Atomic Energy Agency (IAEA), Vienna, Austria.

2 Iitle 49, Code of Federal Regulations, Parts 100 - 199, USA. 
STATE OF CALIFOANIA

DEHARTMENT OF MEALTH

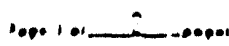

\section{RADIOACTIVE MATERIAL LUEENSE}

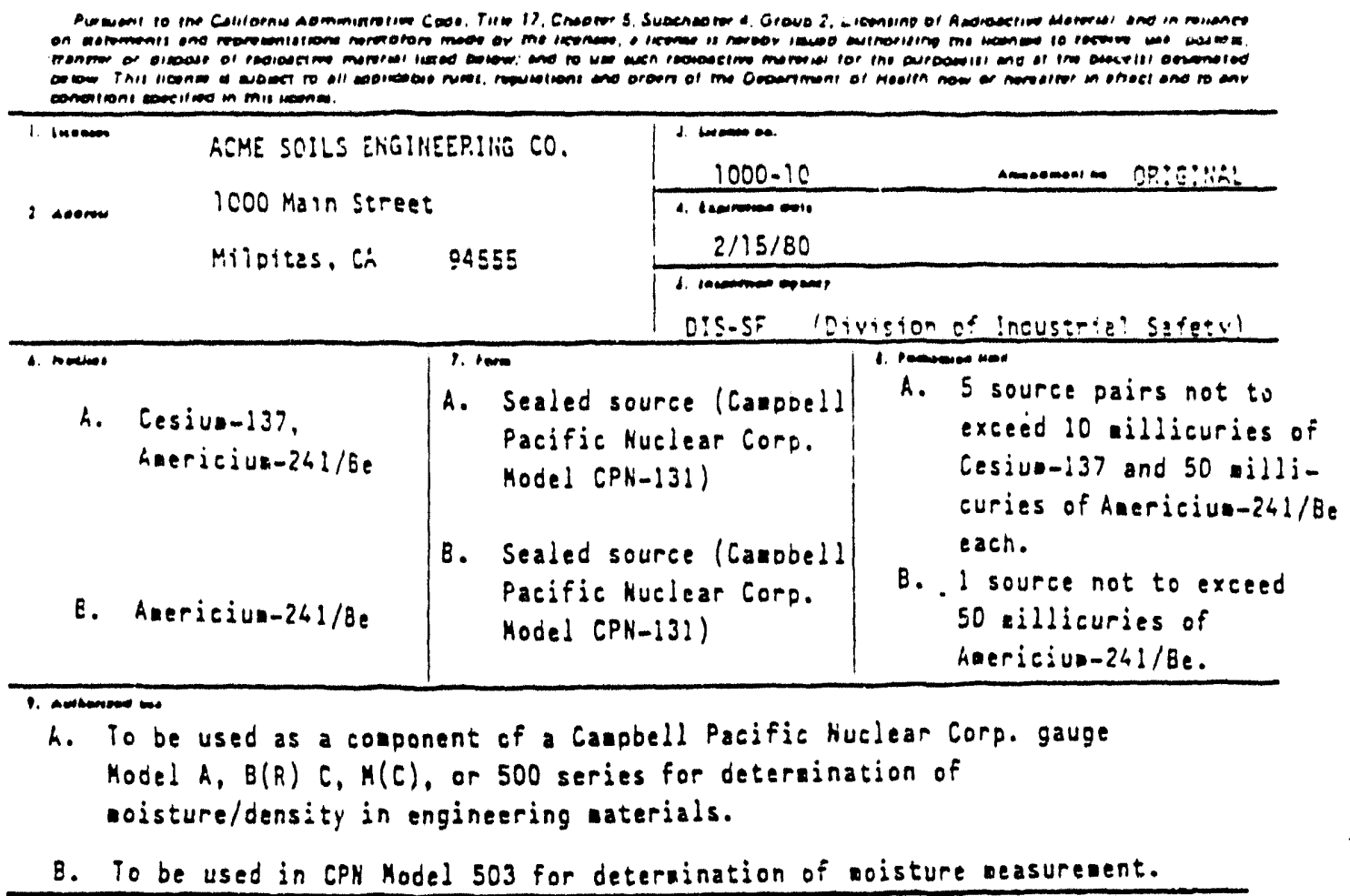

10. Fiadioactive materal may be used at temporary job sites of the licensee in areas not unde: exetustve fedeal jurisdicrion chroughout the State of Caliomia. Radioacsive materia may be permanenuy stored ony ai $\quad 1000$ Main Street, Milpitas. (h, B, \& C.)

500 Suburban avenue. Placervilie, Calif. (C.)

11. Racioacine material may be used only by, or unoer the supevision and in the phvsiaz presence of.

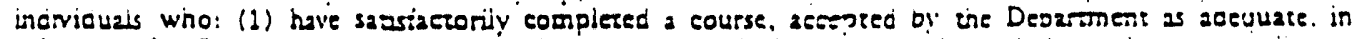
safe use of sol gages convaining racioarsive materal; and aiso (2) have been oesignates by tae raciauon

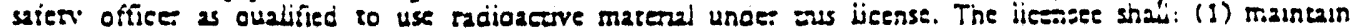

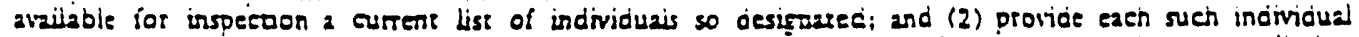
unth a starement, ausnorizing use of radioargive ma:erial unde inis license, signed by the radizeion sajery orisee. (Informasion on which courses have been aesered by the Deparment as zoeouzte iop ine purpose of inis condicion may be obeaned from the Depazenent.)

22. The raoiacion safery ofines in vis program shall be

hiexanoer Benowi=

For the Sute Debarmment of Healon

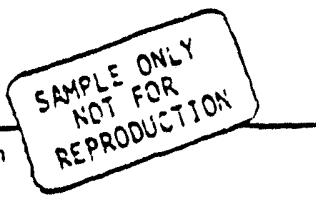

Dorn

by

on $2110.86112 \cdot 931$

Sumon Kinsmen. Pn. D.. Chiet

Fudiodoule Mealin Section

7ed P Sunet. Socraments. Call!. 85814

RAD PREC $6 / 83$ 
RADIOACTIVE MATERIAL LICENSE

Cunsınued

Thus lieense us subeer to sll numbered condisions below:

Condisions to which ths lieeme is not subject are marked N/A.

$y$ This lisense is sulivect 10 an annual tee vi THREE HUNDRE: oullars oue and pavabie on the annwersan. ul the oate of ussuc of tha iterne.

In aceordance with Section olus of the Califurnu Gerwernment Cove. inis license is not subjest in payment ui an annual license tee.

$y$ Sealed soures concained in suil pates snall be tested for leakape andlor sontamination al intenials not tu exceed one rear.

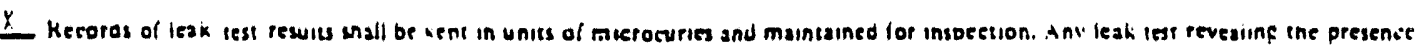

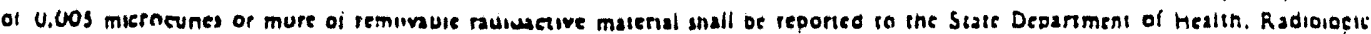

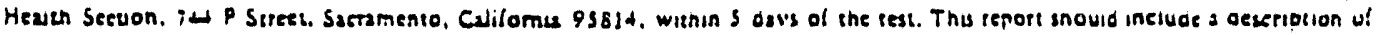

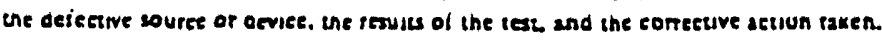

Terss for leskefe andlor contaminatiun of sealed souress shall be periormed onty hy persons soeejfiedly suthorized to pertorm those services.

Y. The followng indmousis are suenonzed to colleet wipe test a mpies of sealed source possezseo uncep enis license. usinf leak test kis secepuble to ine Staie Deparmeni of Heath:

Aiexander Benowizz

Y. Maintenance ano repar of coil guge shall be periormed only by persons specifielly anthopized to perform those services.

$x$

At any ume the licensee is enpged in making mesourements by authority of this lieense, at either a permanent of a temporary !nb

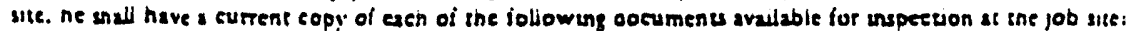

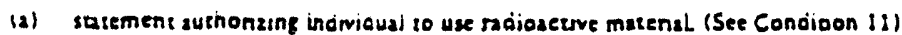

(b) this lueense

(c) Atanufacruret's instruction menual with esproptate emergeney procesures

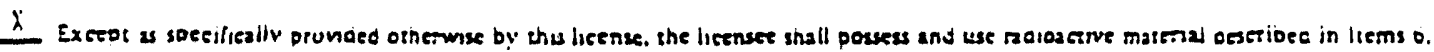

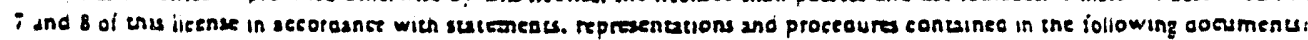

A.oplicazion and iester dazed $1 / 13 / 77$ and signed by Aiexander Eenoriz=.

RM 3551.50 16/751


Fadiation is similar to Light.

It INCREASES in strength by a factor of FOUR each time the distance from the source is cut in HALF.

It DECREASES in strength by a factor of ONE/FOURTH each time the distance from the source DOUBLES.

Millicurie:

Small QUANTIT of radioactive material

Milliroentgen: Small EXPOSURE of gamma radiation

Millirad:

Small DOSE of ABSORBED radiation

Millirem:

Small DOSE of ABSORBED radiation CORRECTED for its EFFECT on MAN

Millirem/hour:

The DOSE RATE of a radiation field

****

100 mrem: Weekly allowed dose for an industrial worker

( $5 \mathrm{rem}$ per year is maximum work dose)

$1 / 2$ mrem: Average heavy work week dose for a POFTAPROBE user ( $0.5 \%$ of allowed weekly dose)

3 mrem:

Average heavy work week dose for a HMDPOPROBE user

( $3 \%$ of allowed weekly dose) This is more than for a PORTAPROBE

due to longer close contact during field carrying of the HYDROPROBE

$1 / 4$ mrem:

Average heavy work week dose for a HYDPOTECTOR user

(0.25\% of allowed weekly dose)

$$
* * *
$$

$5 \mathrm{mrem} / \mathrm{hr}:$

Average dose rate at surface where hands must touch

$1 / 2 \mathrm{mrem} / \mathrm{hr}:$

Average GAMMA dose rate at 2 feet from PORTAPROBE

$1 / 3 \mathrm{mrem} / \mathrm{hr}$ :

Average NEUTRON dose rate at 3 feet from PORTAPROBE or

HYDROPROBE (source to midtrunk distance when carrying gauge)

EMERGENCY

Minor (Source intact)

1) Return the gauge to the storage container

2) Call the RSO and CPN factory

Major (Source damaged or Lost)

1) Stop the vehicle, if involved, get the driver out of the way

2) Rope off area

3) Call RSO and CPN factory

4) Call Public Health. They have the training and equipment required

5) Do not be unduly bold and brave.

RESPONSIBILTY: You are responsible for the safe and legal use, transportation and storage of the gauge.

You are responsible for the protection of the unwary general public from unnecessary exposure. 


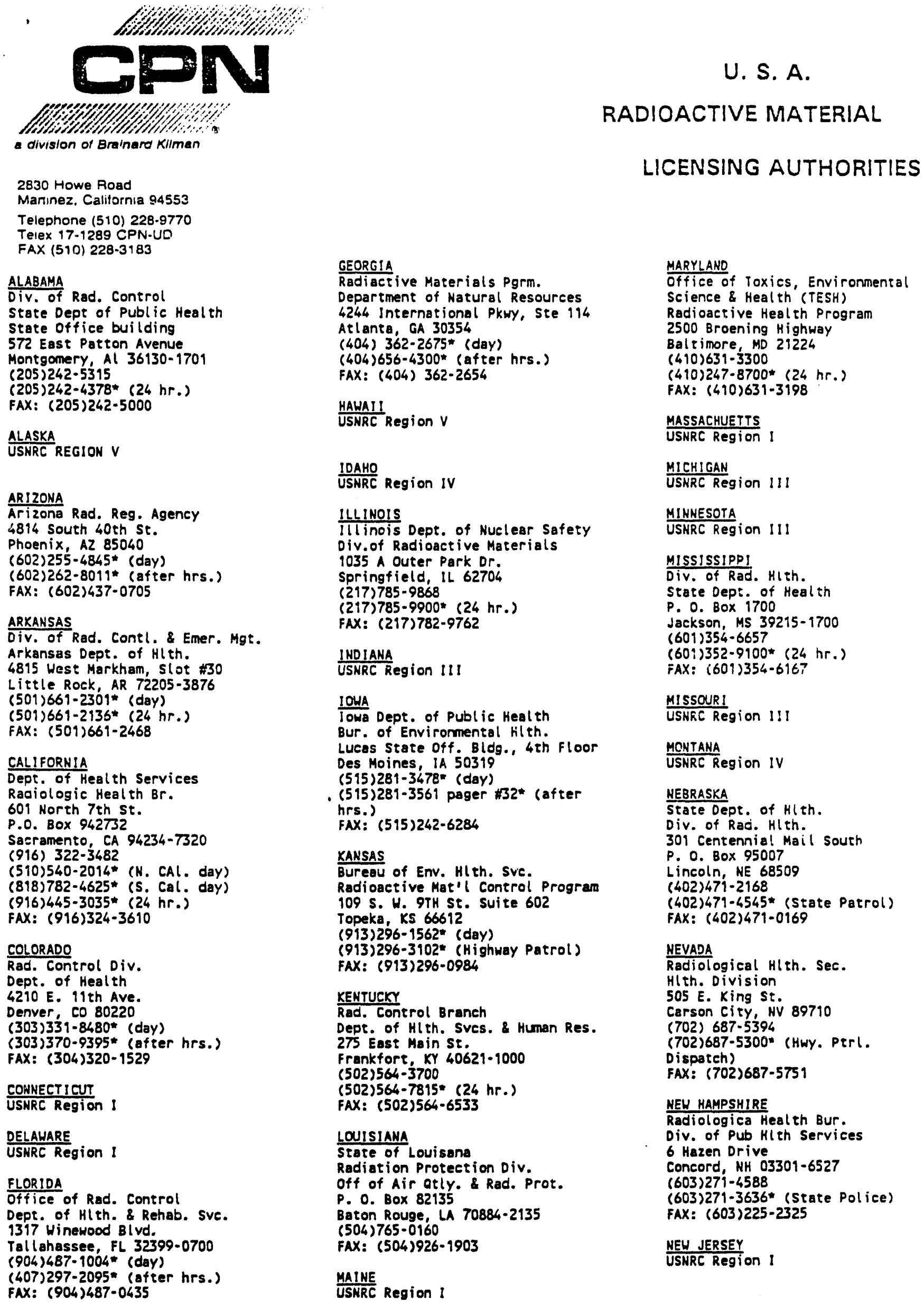

ot Comeany Committed to Quality and Relialitity. 


\section{NEU MEXICO}

Bur. Hazardous \& Rad. Mat'Is

Water \& Waste Management Div.

P.O. Box 26190

Santa Fe, NM 87502

(505) $827-4300$

(505)827-9329* (after hrs.)

FAX: (505)827-2736

\section{NEU YORK}

H. Y. State Dept. of Labor

Div. of Safety \& Hlth.

Radiological HIth. Unit

1 Main street, Room 813

Brooklym, NY 11201

(718) 797-7649

(518) $457-2200^{*}(24 \mathrm{hr}$.

(212) $340-4495 *(24 \mathrm{hr}$.

FAX: $(718) 797-7016$

NORTH CAROLINA

Dept, Env., HIth., \& Natrl. Res.

Div. of Rad. Protection

P. 0. Box 27687

Raleigh, NC 27611-7687

(919)571-4149

(800)662-7956*(24 hr.)

(919)733-3861* (out of state)

FAX: $(919) 733.0312$

NORTH DAKOTA

Dept. Of Heal th

Div. of Enviro. Engineering

1200 Missouri Ave. Rm 304

P. O. Box 5520

8 ismarck, ND $58502-5520$

(701) $221-25188$

(800)472-2121* (after hrs.)

FAX: (701)258-0052

OHIO

USARC Region III

OKLAHOMA

USNRC Region IV

OREGON

State Health Division

Radiation Control Section

800 NE Oregon $\# 21$, ste 705

Portland, OR 97232

(503)731-4279" (after hrs)

(503)731-4014

FAX: (503)731-4081

PENHSYLVANIA

USNRC Region !

PUERTO RICO

USNRC Region II

RHODE ISLAND

Div. of OCE. Hlth. \& Rad. Hlth. Dept. of Health

206 Cannon Bldg.

3 Capitol Hill

Providence, Rl 02908-5097

(401) 277-2438

(401)647-3311* (state Police)

FAX: (401)277-6953
SOUTH CAROLINA

Burem of Rad. Hlth.

Dept. of HIth \& Env. Cntl.

2600 Bull street

Columia, SC 29201

(803) $734-4700$

(803) 253-6497\% (24 hr.)

FAX: $(803) 799.6726$

$(803-734-4588$

SOUTH DAKOTA

USHRC Region IV

TENMESSEE

Div. Rad. Hlth.

Dept. of Hlth. and Env.

TERRA Building, sixth Floor

150 - 9th Avenue, North

Nashville, TN 37219-5404

(615) $749-7812$

(800)262-3300* (24 hr.)

FAX: $(615) 749-4608$

TEXAS

Bureau of Rad. Cntl.

Texas Dept. of Heal th

Exchange Building

8407 Hall Street

Austin, TX 78756-3189

(512)834-6688

(512)458-7460" (after hrs.)

FAX: (512)835-8109

UTAH

Div. of Radiation Cntl.

Dept. of Env. Heal th

288 North 1460 West

Salt Lake City, UT $84194-4850$

(801) $538-6734$

(801)538-6333*(24 hr.)

FAX: $(801) 531-8128$

VERMONT

USNRE Region I

VIRGINIA

USNRC Region 11

WASHINGTON

Dept of Heal th LE-13

Div. of Rad. Protection

Airdustrial Center Bldg. \#5

P.0. Box 47827

Olympis, WA 98504-7827

(206) 586-8949

(206) 686-0463* (after hrs.)

FAX: $(206) 753-1496$

HEST VIRGINIA

USHRC Region II

HISCONSIN

USNRC Region III

WYOMING

USNRC Region IV
U.S NUCLEAR REGULATORY COMMISSION

EMERGENCY (all regions)

(301) $951-0550$ (24 hr.)

USNRC, Region 1

Nuclear Material Section

475 Allendale Rd.

King of Prussia, PA 19406-1415

Jerry Johansen

(215) $337-5000$

FAX: (215)337.5324

USNRC, Region II

Material Rad. Prot. Sec.

101 Marietra st., NW, Suite 2900

Atlanta, GA 30323

Earl Wright

$(404) 331-4503$

FAX: $(404) 331-4449$

USHRC, Region III

Material License Section

799 Roosevelt Road

Glen Ellyn, IL 60137

Mike McCann

(708) $790-5500$

FAX: $(708) 790-5693$

(708) $790-5665$

USHRC, Region IV

Material Rad. Prot. Sec.

611 Ryan Plaza Drive, ste 400

Arlington, Tx 76011-8064

Jack Whiteen

(817) $860-8100$

FAX: $(817) 860 \cdot 8210$

( 817$) 860-8211$

USNRC Region $V$

Material Rad. Prot. Sec.

1450 Maria Lane ste 210

Walnut Creek, CA 94596-5368

Beth Prange

(510)975-0200

FAX: (510)975.0350 (510)975-0351

Canadian Atomic Eneray Commission $(613) 995-0479 *(24 \mathrm{hr}$.

* Emergency Asst. Phone Number

FOR ASSISTANCE:

CPN Company

2830 Howe Roed

Maninez. CA 94553

(510) 228.9770

Fax: 1510$) 228.3183$ 
OPEPATOR MAINTEATCE

HOOE 503 HMROPPROE

\section{SBETT}

General

Introduction

Deseription

Basic Maintenanee

Exchange Policy

Sazres Price List \& Exploded Viey

Troubleshooting Guide

Detailed Maintenance

$$
\begin{aligned}
& \text { Cable } \\
& \text { Surface Electronic Assenbly } \\
& \text { Description } \\
& \text { Renoval } \\
& \text { Battery Access } \\
& \text { Battery Cells } \\
& \text { Description } \\
& \text { Cell Testing } \\
& \text { Cell Recharsing } \\
& \text { Cell Overcharging } \\
& \text { Cell Replaceaent } \\
& \text { - Storage }
\end{aligned}
$$

Probe Assenbly

Description

Resoval

Probe Electronic Asseably

Probe Re-installation papactapai mo.

1

1.1

1.2

1.3

1.3

1.5

2

3

3.1

3.2

3.2.1

3.2 .2

3.2.3

3.2 .4

3.2 .4 .1

3.2.4.2

3.2 .4 .3

3.2.4.4

3.2 .4 .5

3.3 .4 .6

3.3

3.3.1

3.3 .2

3.3.3

3.3.4 


\section{EaP}

\section{1.) INTroouction}

Section III of this 503 Manual supplies basic information to jilow performing aintenance on a field level basis. The only required tools are the screwdriver and the spanner wrench which are supplied with the sauge. A voltater capable of readins to 15 vdc is recomended.

\subsection{DESCRIPTION}

The nodel 503 consists of four ajor asseablies:
1) Surface Mechanical Asseably
2) Surface Electronic Asseably
3) Cable
4) Probe Asseably

\subsection{BASIC MAINTEAHCE}

Using the troubleshooting suide in section 2 , isolate the problea to one of these maior assenblies. If a second gauge is avajlable the parts can be interchanged to easily isolate the defective asseably.

The Surface Mechanical Asseably is a pure nechanical asseably. Other than the latch wechanise which can be repaired br replacenent parts, no service other, than occasional cleaning is required.
If the cable is defective it should be replaced. It is recomended that a spart cable be kept on hand to andaize down time.

If the Surface Electronic Assenbly or the Frobe Asseably are found to be detective for reasons other than battery cells, then they require test equipment including an oscilloscope, signal generator and a digital voltaeter. As such ther should be returned to the factory for repair. As described ill section 3.3 the Probe Electronic Assenbly can be easily separated from the Source Tube Asseably aking it easy to ship the Probe Eiectronic Asseably by UPS or other convenient neans.

\subsection{Exahure PQICY}

Alternately the factory keeps on hand an inventory of exchange parts. Where ainioun dom tiae is iaportant CPN will ship an exchange asseably upon rour request. The time and terial cost to repair is oenerally less than the exchange cost, but with the exchange unit the repair tine and the one way shipping time are eliainated.

\subsection{SPAPES PRICE LIST \& EXPLOED VIEU}

see figures $1.5-1$ and $1.5-2$

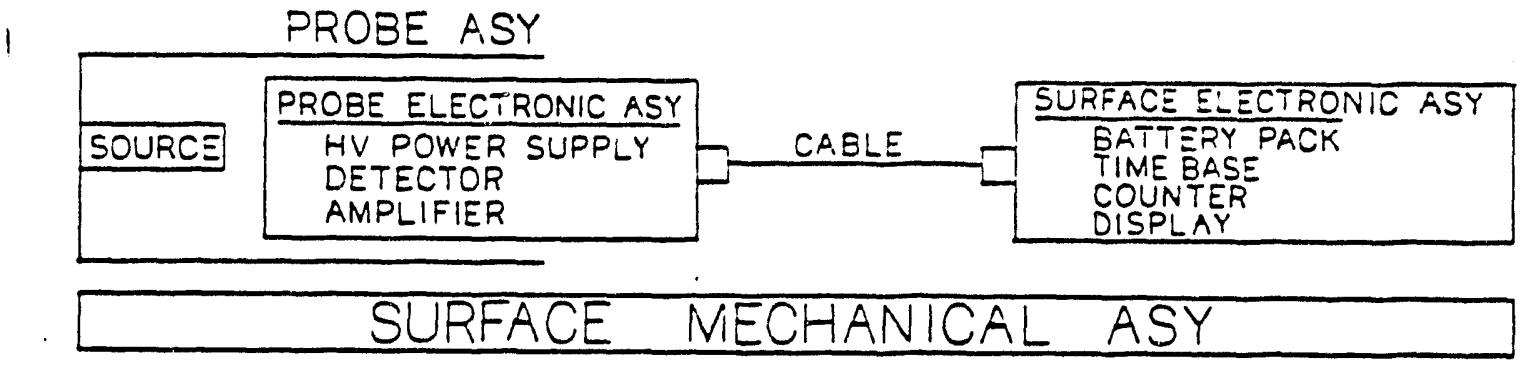




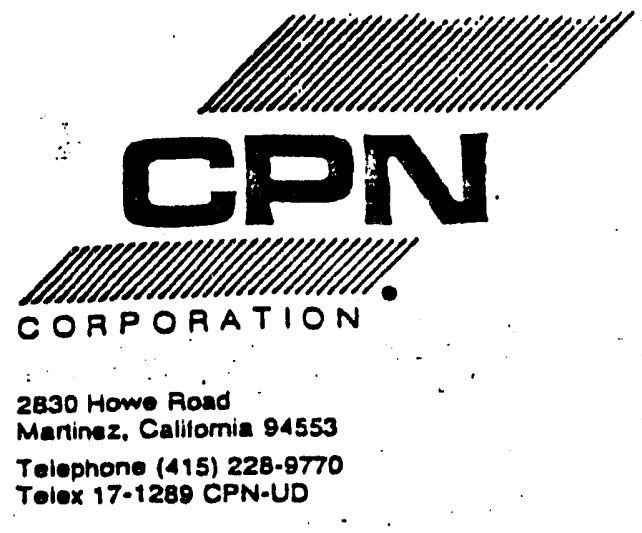

KOOEL SOJ MUOR SUP-ASSEMBLIES PARTS LIST

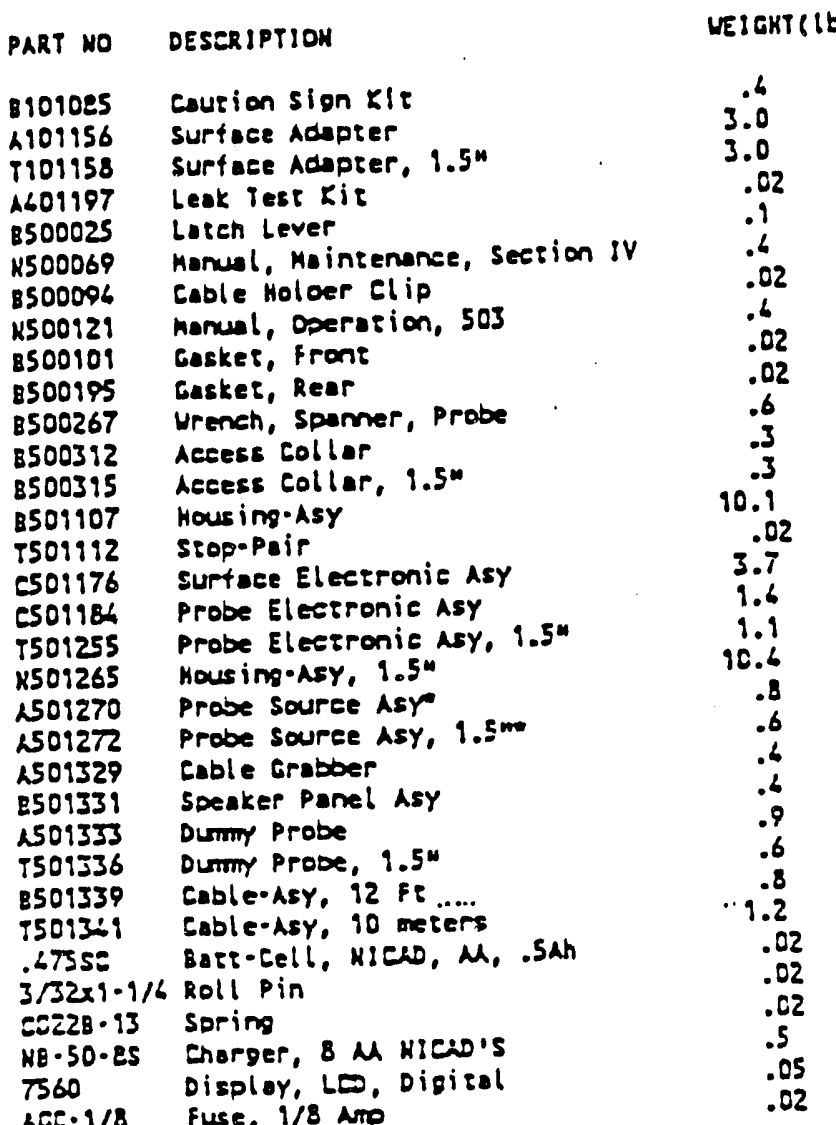

- These parts consain a rodionctive sourse. They are replacesble at the factory only.

EXEKAKEE PROSRAK W.T.

PRRT HO EXEKAHEE ITEM

309176 Surface Elestronic Asy

Es0i986 Prooe Eleseronic AsY

T50135s Prode Elestronic Asy, 1.5"

isoliss prosicional cos: if replacement reasired.

ine above parss are ovailable from CPH Jorp on an exenange price basis. in it she returned item is not in 9000 soncision or is nas returned so the iascory wishin 30 eeys, she eustomer will de billed ine full price.

m::25:23 


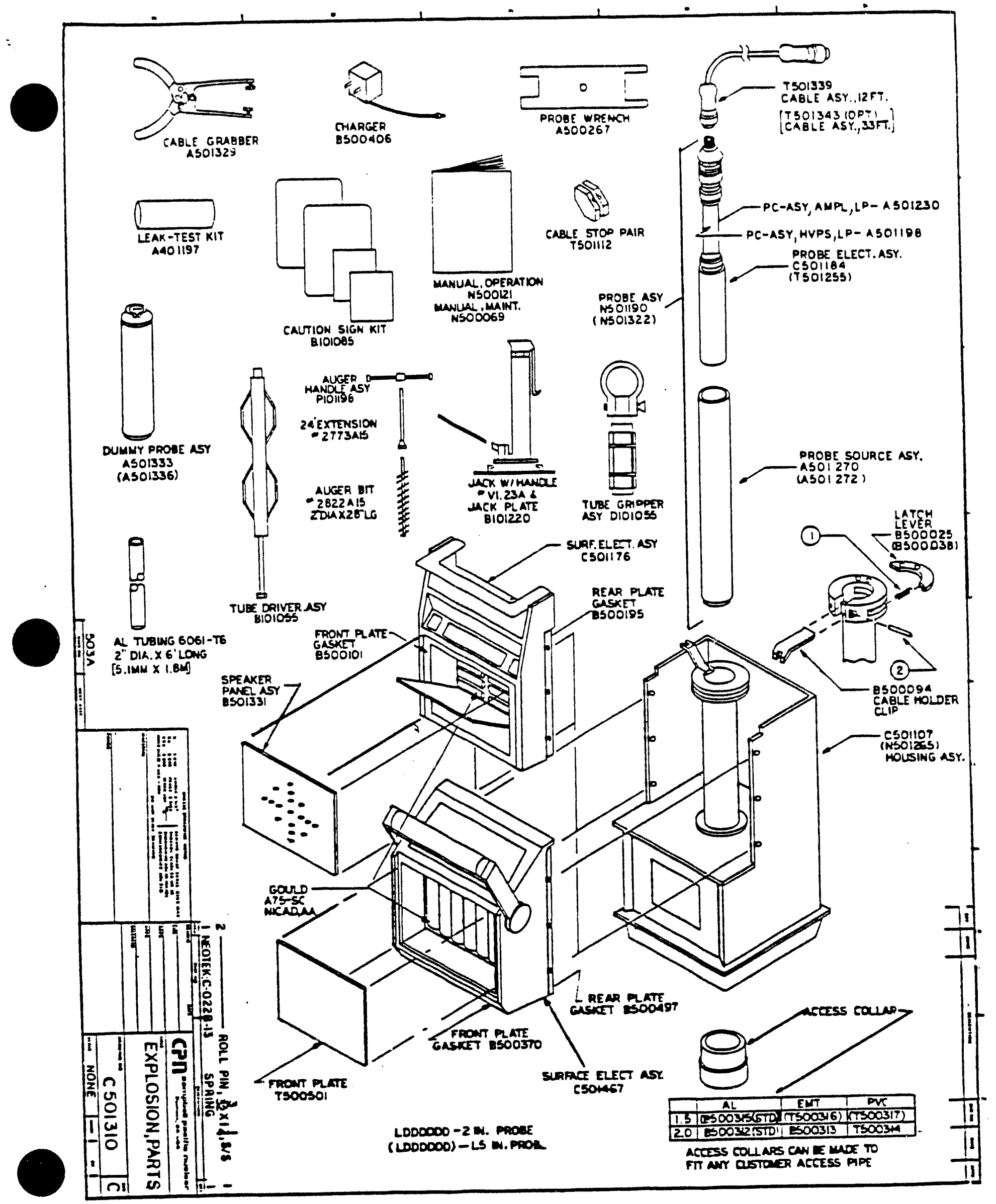




\subsection{Thateshotiks GuInE}

PROREA

CALSE

4** POHFR CIRCUIT H\#+

No display and no audjable hus at top of probe

No display and a $6 \mathrm{kHz}$ hus can be heard at top of the probe.

Gauge blows fuses

Cauge does not run for lons after a full charge

Blown fuse

QPen or dirty battery cell contact

Defective battery cell

Display defectiye

Stack defective (shorted caracitor, I.C. etc) Shorted cable

Open or dirty cell contact

Battery cell defective

Charger defective

Excessive load in surface or probe electronics

HH COWTING H*\$:

Displar reads 0 and the decial point flashes at a one second rate during the count and a $6 \mathrm{kHz}$ hus can be heard

Sane except no $6 \mathrm{kHz}$ hum

Display reads 0 and the decinal point to left of display flashes once each time the start switch is pressed

Iisplay reads 0 and no flashing decial point

Background segenents in display

Statistics test results in high ratio due to one or more vild counts

Statistics test resuits in high ratio due to shift of and during the test period

Statistics test results in low ratio with an increase in the standard count

\section{HHH PERTOMHANCE HHH}

Moisture reads high conpared with other nethods (2nd sauge, oven dry etc) wile statistics test standard count and all other functions are O.K.

Sane except noisture reads low

Sane axcept acconpanied by a shift in standard count

Probe defective

Cabie defective

Cable defective

Probe defective

Tiae base suitch not set or open

Oscillator circuit defective

LCD defective

HW supply noisy

HW supply drifting

Detector drifting

Perjodic noise bejng counted, nost likely due to open bypass capacitor in HW supply

Gave is reading both free water and bound water of hrdration. Apply correct bias Calitiration not applicable to the soil type or to the access tube type

Calibration not applicable to the soil type or to the access tube trpe

Probe geonetery changed

Defective detector 


\section{DETAILED MINTEAKCE}

\subsection{CABE}

The cable is constructed using a central stainiess steel vessinger surrounded by four insulated stranded copper wires. The nessinger is firmly clanped to the connector shell at each end to support the wight of the probe. The four copper conductors are wired point to point as shown in fis $3.1-1$.

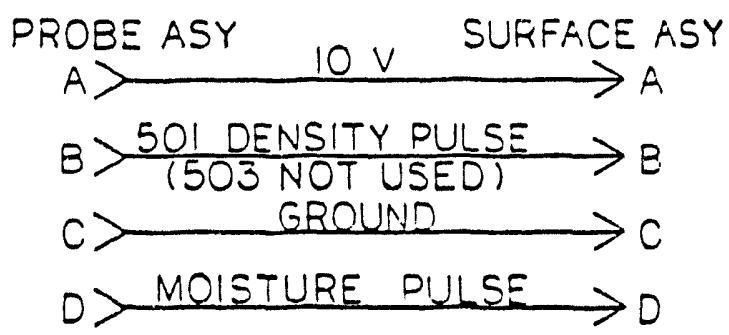

FIO 3.1-1

Undue stress or extended usage ay cause separation of the copper conductors. If the break is internitten it ay be located by operating the sauge and flex the cable while observing the display. Most often the break vili occur at the end near the probe. The cable is ade as a separate assembir so that it can be easir replaced. Breaks near the ends can be repaired by shortening the cable and resoldering the copper conductors. The inner chanber of the connector should be filled with RTV or an equivalent insulating conpound to prevent aisture intrusion. The essinger should be pre-stressed to take the pull off the copper conductors.

\subsection{STPFACE EETTROIC ASTRYY}

\subsubsection{DESCRIPTION}

The Surface Electronic Asseably consists of:

1) Surface PC-Asseubly

2) Battery Cells (8)

3) Display PC-Asseabir

4) Fuse Holder and Fuse

5) Charger Connector

6) Cable Connector
Field asintenaice of this unit vill normally be lanted to replacins the battery cells or the fust.

\section{2 .2 and}

The Surface Electronic Assembly can easly be renoved fron the Surface hechanical Assentily for convenience of return to the factory for repair of exchange by reanving the screus on each side of the assenbly.

\subsubsection{BATTER ACOESS}

To sain access to the printed circuit assembly and the battery cells, first remove the Speaker Panel Asseably yia the four corner screws. The speaker is connected to the PC-Assembly via a two pin connector. Polarity of this connector is not inportant when replacing the Speaker Panel Assembly. With the speaker panel renoved the Surface PC-Assenbly uill be exposed. The two botton standoffs are hinged. Br using a saall tool such 25 a flat bladed screudriver as shoun in figure $3.2-1$, the plastic catch at the top center of the PC-Assenbly ay be compressed allowing the PC-Assedbly to hinge down. This provides liaited access to the battery cells. If pore access is desired it way be more convenient to first remove the Surface Electronic Asseably from the Surface Mechanical Asseubly. Then the back panel can be reaoved fron the Surface Electronic Assenbly.

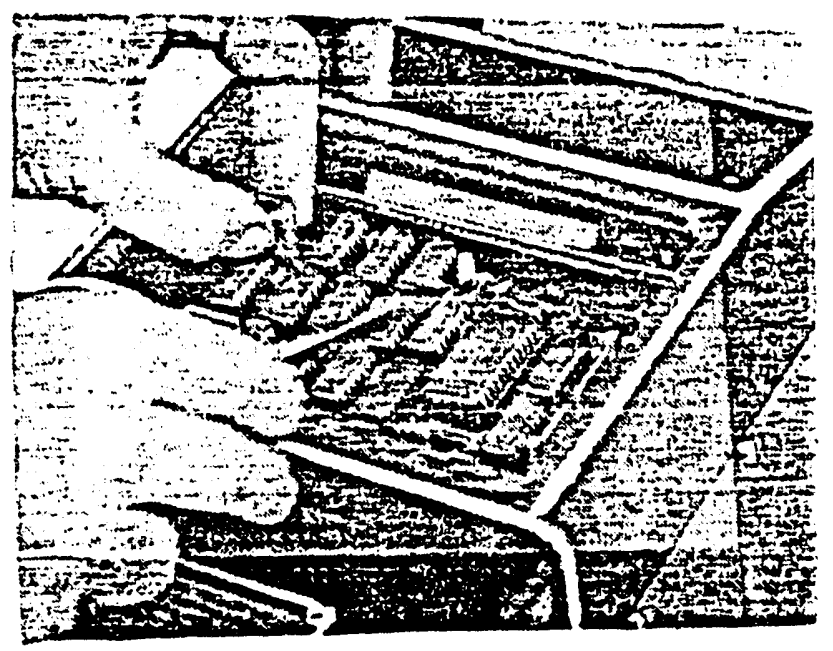

FIG 3.2-1

Then hinging the Surface PC-Assenbly back to its locked position, it way first be necessary to use your finger to for the locking plastic standoff dorm 50 that it will align vith the hole. 


\subsection{BTTEN CQUS}

\subsubsection{DEscription}

The Hodel-503: is supplied with 500 aillianre-hour nickel-cadaiu (NICAD) AA size rechargeable battery cells. The save draus approxinately 5 aillanpres then on. Assuaing 502 derating this means a fully charsed sause can operate 50 hours, or ake 3000 tests (one/half ninute count with one/half ainute display) before requiring charging.

\subsubsection{2 व्या TESTIN}

To test the cells remove the smaker panel and drop the PC-Asseably to the service position as described in section 3.2.3.

NICADS should nornally be tested under lagd. The best time to test the voltage of the cells is after the sauge has been operating for a long time and the $L$ is flashing (the $L$ starts flashing wen the battery voltage drops to 9.0 vde. The $L$ soes on pereanently and the sauge goes into cutout when the voltage drops to $8.4 \mathrm{rdcl}$. At this tian cells wich still have capacity vill atsure 1.2 vod thile ale cells will shor voltages 1.1 vde or le55. As shown in figure $3.2-2$ the NICAD cell has a flat voltage curve during its use and then drops off fast. In contrast to this an Alkaline cell starts higher and keers dropring during its use.

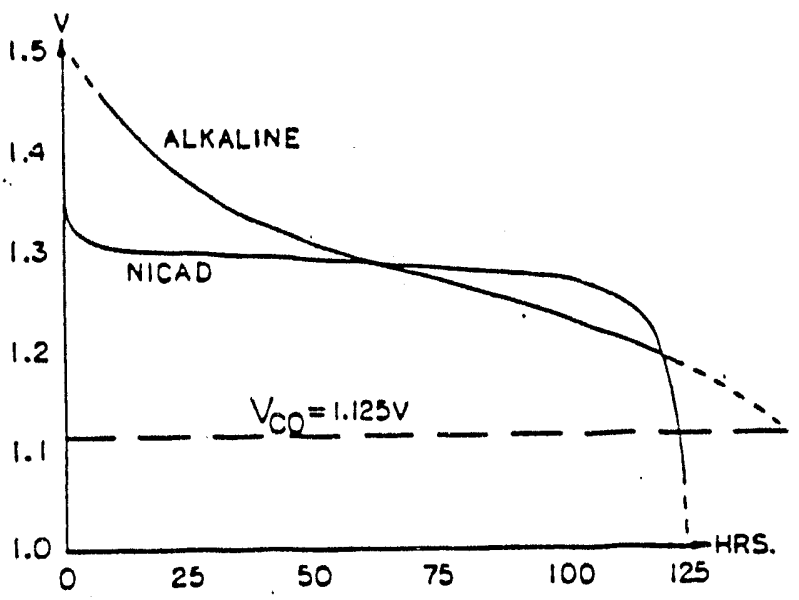

FIG 3.2-2

MICAD's then not used for long perjods of tice or are subject to only shallow discharges ar develop a evory hich only allows storing a partial anount of energy even though they are charged for 16 hours or ore. To return the cells to full energy storace caracity they should be rut thru a couple of deep charge-discharge creles. This cun be accomplished or learing the gaves on until the $L$ ammars, charaing it overnight and repeating until the save vill operate for 50 hours or more.

\section{3 call ReOApoing}

The cells have a rated energr storage caAacity of 500 aillianprethours. The charger supplied with the sauge charses at a pate of 50 A. Mominally this $\mathrm{C} / 10$ charge rate should take 10 hours to recharge the battery. However if the individual cells were then exenined some vould be fully charged while sone vould be undercharged. The undercharged cells would linit the batterr's capabilities. $A$ technique to balance the cells state of charge is to deliberately overcharae thea 140 to $160 \%$. To fully recharge the cells leave it on the charger 14 to 16 hours or overnight.

After charging the cells should feel ware to the touch indicating that they have gone onto the overcharged state (see sect. 3.2.3.4)

thile the gaves is charging and its OH-OFF switch is ar a bar vill appear in the upper left-hand portion of the display.

\subsubsection{Call OUERapoino}

Once a NICAD is fully charged any additional energr supplied uill be dissianted as hat. At the $\mathrm{C} / 10$ rate the cells can be overcharged for several reeks without it being a major cause of end of life. There is however a tendencr to dry out the electrolyte 50 it should not be done on a routine basis.

\section{cation}

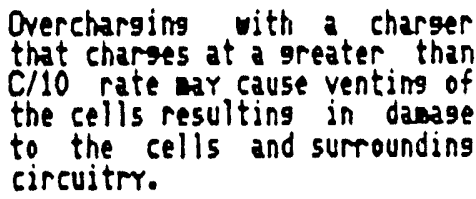

\subsubsection{COL RELACOETT}

A NICAD is rated for approxisately 1000 deep charge-discharge creles. Using your sauge during the aek and then charging it overnight every oek or two should result in approxinately 5 years of cell service.

What is suspected 25 a bad cell is sometimes really a contact problea. Before replacing a cell try ciezning its terainals. often just rotating the cell in its holder is sufficient.

In the event of the failure of one cell in a relatively ney set of cells, the single cell should be replaced and the cells balanced by a 1602 overcharge. The older the cell set the less justification for replacing individual 
cells since the resulting battery is no better than the worst rewaining cell.

The equge is equjerd initially with industrial graoe, 500 ah, rechargeabile NiCAD, A size cells. Replacenent cells of this type are available from con, radio-electronic surply stores and any photosraphy suppiy stores. Alternately in an energency one or more cells wa be replaced by fiashlight type $A$ cells available in drug stores and supermarkets. Norally these vill be of the carbon-zine or alkaline types. These are throw awey cell and should be discarded after discharged.

\section{cavtion}

Do not charge carbon-zine or alkaline cells.

The relative capacities of the three cell types are 25 follous:

$\begin{array}{lcc}\text { MPE } & \text { WOLTAOE } & \text { CAPACITY } \\ \text { in Ah } \\ \text { NICAD } & 1.2 & 500 \\ \text { Carbon-Zine } & 1.5 & 1000 \\ \text { Alkaline } & 1.5 & 1800\end{array}$

Thus a set of fresh alkalines should allow 360 hours of operation or 10,800 counts, enough for a full season.

Individual cells ay be replaced thru the front of the sauge by renoving the speaker Panel and hinging the PC-Assenbly to its ser-
vice position as described in section 3.2.3. The cell holders are very firs. It vill normally be necessary to use a fusepuller or short screwdriver to Pry the cells out of their holders.

When installine the new cells observe the polarity indication. The cells should be mounted with their + terminal to the operators left as he views the front of the sauge.

\subsection{4 .6 STLPACE}

Fully charged NICAD's standing idle at 20 degrees Celsius lose about 12 of their charge per day because of cheaical self-discharge. Thus if a gauge is not used for a month or core it uill have lost $30 \%$ or nore of its capacitr and should be recharged before using the sauge. The self-discharge rate increases vith ambient teaperature.

\subsection{Prose aseray}

\subsubsection{DESCRIPTION}

The Probe Assembly consists of:

1) Source Tube Asseably

2) Probe Electronic Assenbir

\subsection{2 ranal}

The Probe Electronic Asseably is easily renoved from the Source Tube Asseabiy. As shom in fisure 3.3-1, grasp the top of the Source Tube Asseably in the left hand and using the spanner rench in the right hand rotate the Probe Electranic Asseably counter-clockuise. After the threads are disengaged pull the Probe Electronic Assembly out of the Source Tube Asseably.

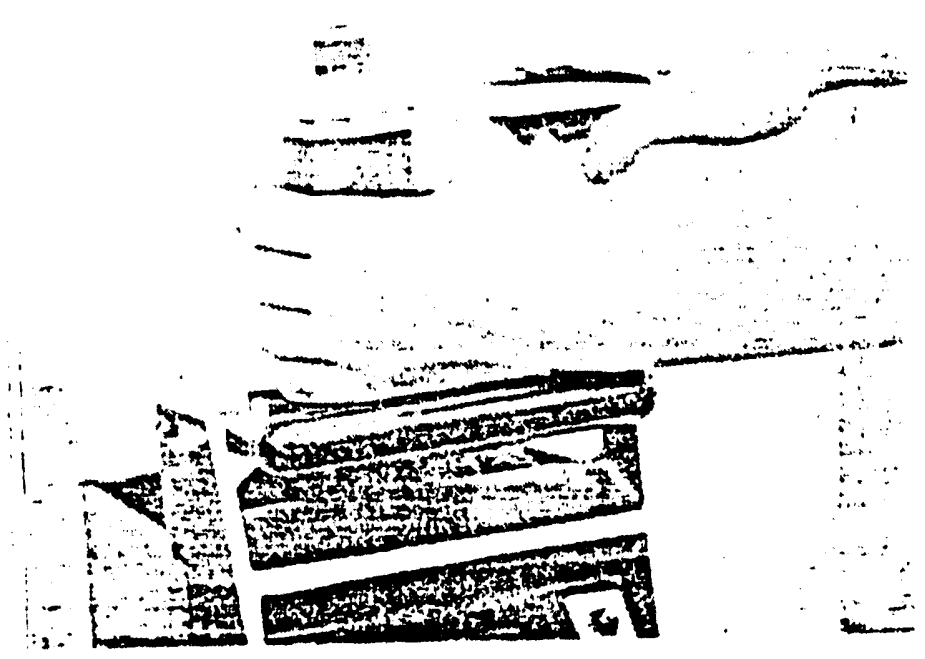

FIO $3.3-1$

\section{Hinums}

The radiactive source is Dounted in the base of the Source Tube Assenbir. Do not grasps the base with your hand. The Source Tube Asseably should be placed back in the Surface Mechanical Asseably during re pair of the Probe Electronic Asseably.

\subsubsection{PROE EECTROUSC ASSEXYY}

The Probe Electronic Asseably consists of the connector, brass plug housing a ferrite transformer, an aplifier PC-assenbly, an HPS PC-2sseable both ounted on a tray and the detector tube itself. These iteas are show in figure $3.3-2$. 


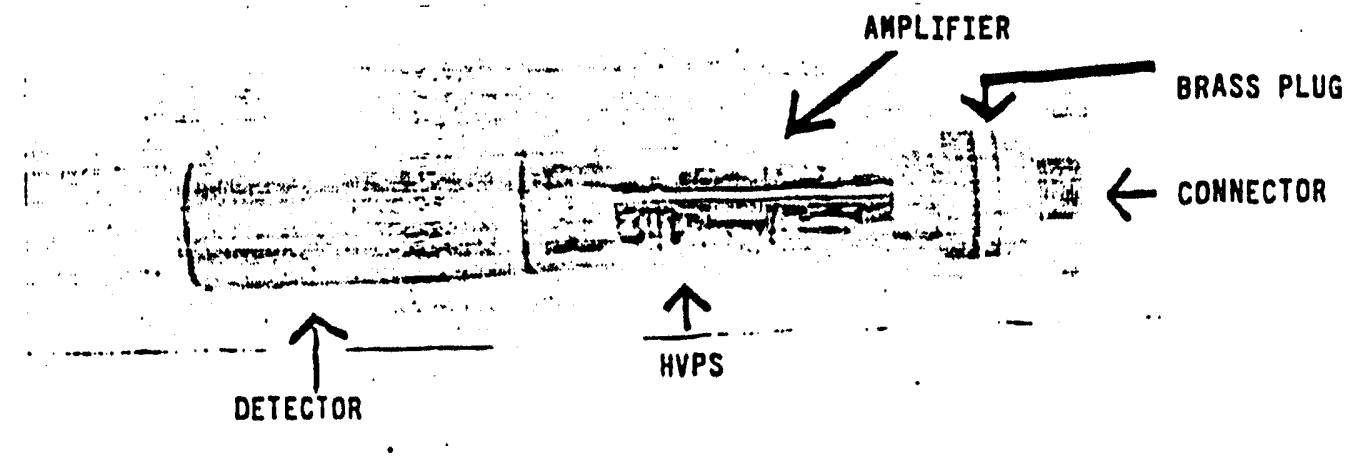

3

FIG 3.3-2

Field repair of the probe electronic asseably vili generally be ijited to a phrsical exarination for loose iteas. The connector pins at each end of the PC-2ssemblies should be engiaged and the brass rings on each end of the trar should be tight.

If noisture is observed inside the probe and no meranent dacige has occured it can be dryed by placing in a household oven for one hour on wark $600-70$ deg $\mathrm{C})$.

\subsubsection{PROE RE-DISTALATION}

When re-installing the repaired or exchanged Probe Electronic Asseably in the Source Asseably insure that the threads are properly engaged. If the probe has been roughly handed in shipping it way be mecessamy to bend the tray slightir to insure a correct fit. Thread together the asseablies al oost all the Dy br hand and then apply a thin costing of silicon grezse to the Oring. Use the spanner rench to coapress the o-ing to insure a wisture seal. 


\section{RADIATION SIMPLIFIED}

by

SIMON KINSMAN

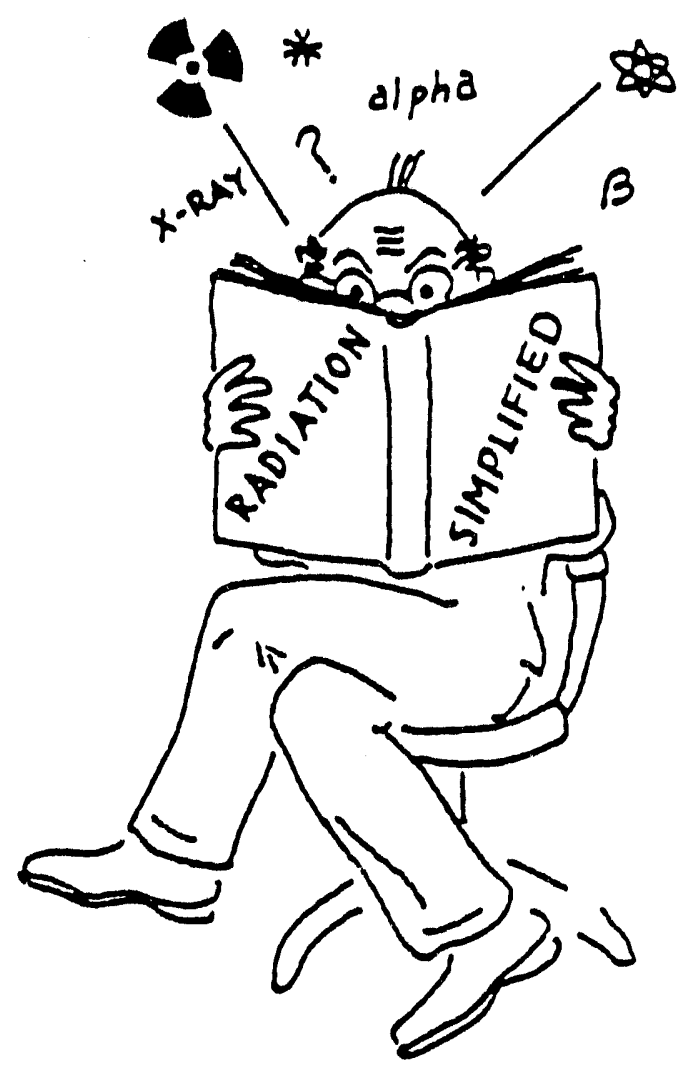

California State Denartment of Public Health

Campbell Pacific Nuclear Corporation would like to express our thanks to

Dr. Simon Kinsman. Ph.D.

Chief, Bureau of Radiological health, Caiifornia Stace

Department of Public Health, Berkeley, California for permission to reprint. 
Radiation in a variety of forms is familiar to all of us. Light is radiation we can see. lleat is radiation we can feel. Ultraviolet and $X$-Ray we neither see nor feel. None of the four can ve heard or smelled. All are like light because they do not continue after the source (bulb) is curned off or removed.

Radiation is as old as the universe. Stars are intenscly radioactive; our earth now only slishtly so. Ever since tis first appearance, man has been exposed to both visible and invisible radiation from the sun. Like sunshine and rain, thunder and lightning, radioactive substances until very recently occurred only in nature.

Radiation from radioactive material is a stream of fast flying particles or waves which come from tiny units of matter called atoms. Atoms of a single element often consist of different kinds which behave al ike chemically, yet have slichtly different weights. These varieties are called "isotopes". The atoms of stable isotopes are not radiosctive, but those of unstable or radioactive substancas give off portions of themselves, and change into other isotopes in the prosess.

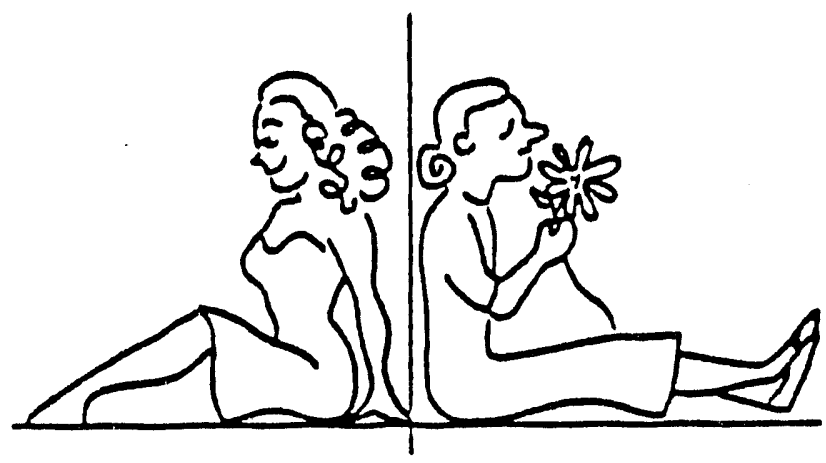

UNSTABLE

STABLE

Within the post sixty-five years we have learned much about radiation. Man-made radiation in the form of $y$-rays was discovered in Germany in 1895. In France, a year later, natural radioactivij' was first identified with uranium. H'ithin two years, one of its main sources was isolated--the naturally radioactive element, radium. Invisible rays from this element were soun found to be of three kinds: (1) heavy particles which travel but an inch or so in air, (2) 1ishier particles which travel a feir feet, and, (3) vaves similar to those of light, but too short to be seen by the human eye, which penescte to corisioerabile distances, even througn several inches of lead. These three forms of invisible raciation are called Alpha, Beta, and Garmia Rays.

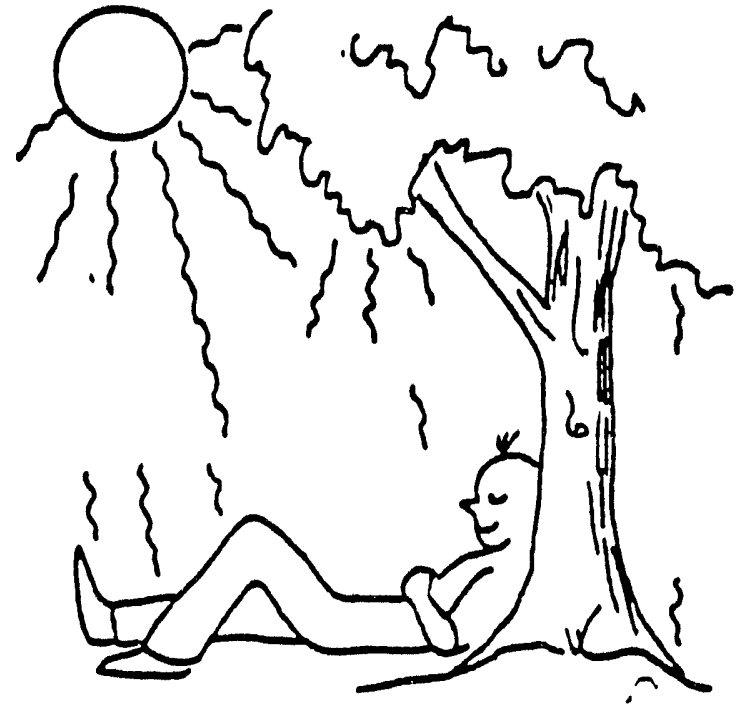

SUNLIGHT IS A FORM OF RADIATION

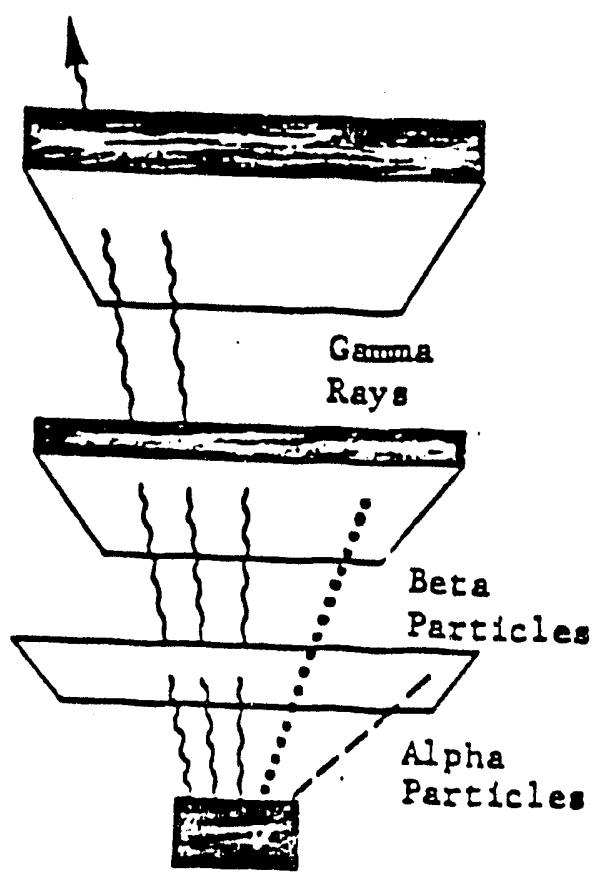


Shortly after the discovery of radium, man learned that for millions of years ancther form of invisitie radiction had been coming at him from yts ide the earth's atrosphere. The scurce of chese particles, calied "cosmic ray", is still unknosin. lie do know, however, that they are stopped to some extent by the earth's atmos- phere, and that if we were to itve in Deryer. Colorado, or elsekinere at nigh clevation, cosmic radiation would be tho to four times as incense as it is at sea levei. This is why scien:ists investigatino cosmic rays employ calloons and aireraft to collect information at high altitudes.

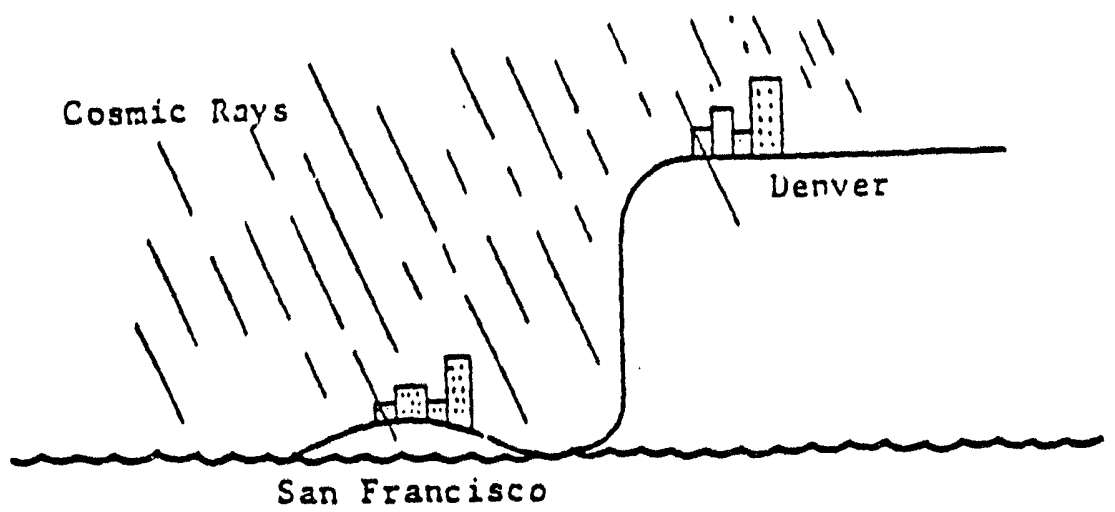

Natural radioactive substances are widely distributed. They exist in minute quantities in our bodies, in the water re drink, the air we breathe, the soll we cultivate, even in the materials we use for building. Along with the cosmic radiation from outer space, these tiny sources have been sending out invisible radioactive sionals for millions of jears. It is against this backround of natural radiation that man has lived in the past and lives today. This so-called "backround radiation" varies slightly from one locality to another, and also with rain or snowfalls.

Within ten years of their discovery, practical uses werc found for both $x$-rays and radium. $x$-rays proved valuable in locating bone fractures, in identifying diseases, arid as a supplenent to radium in the treatment of cancer. You probabiy had a chest $X=$ ray recently or a picture of your teeth. If so, you have been exposed to a relatively harm-

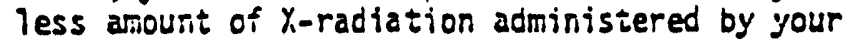
own doctor or dentist.

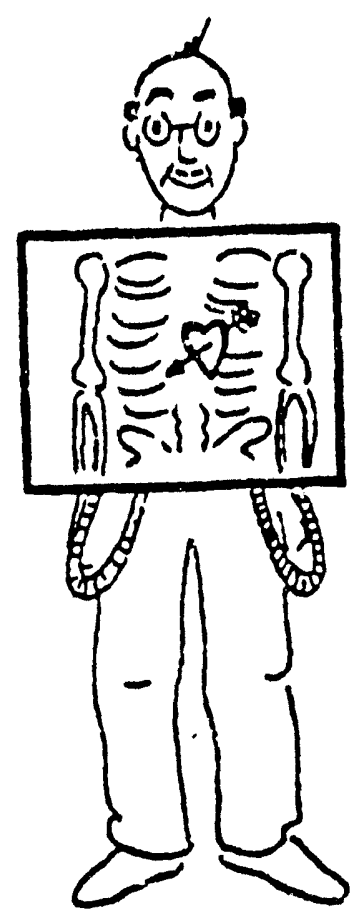


Within the past fifteen vears, man has learned to take naturally stablc atoms and make then ar tificially radioactive. It is these ladioactive varieties, or "radioisotopes", which are proving to be anong the most useful tools in the entire history of science. Because they are radioactive. their radiation tells where thes are, even if the amount is extrencly sma!l. Their location or movement $k^{\prime} i t h i n$ the plant or animal tissues. and in indutrial and chemical processes, can, therefore, be traced by sensitive recording instrumants. Used in this manner, radioisotopes are spoken of as "tracers".

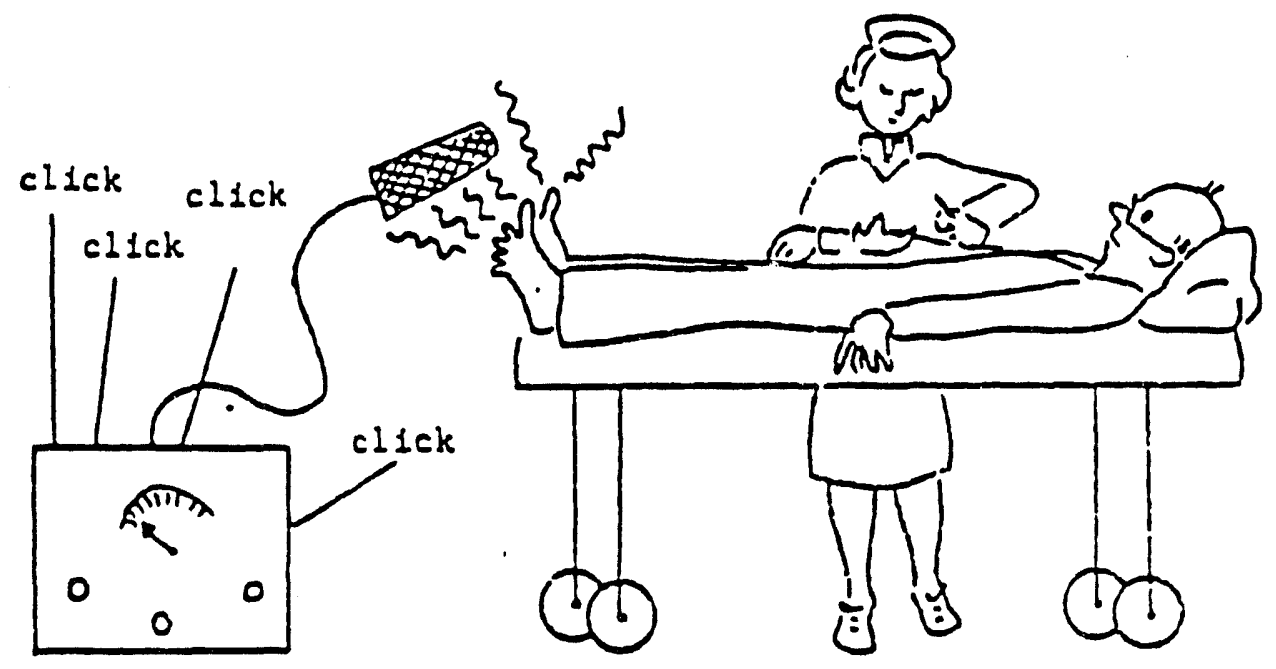

\section{IS RADIATION DANGEROUS TO YOU?}

It can be: it may be; but it need not be.

Danger from radiation depends upon the degree of exposure. Hor! dangercus is fire, or exposure to the sun? How dangerous is electricicy? It depends upon your exposure.
We all use clectricity, but we do not take chances. We have learned to live with these agents, and we call learn to live with radiation, too.

The chances of receiving an overexposure are slight. This depends upon how much you kriok' about

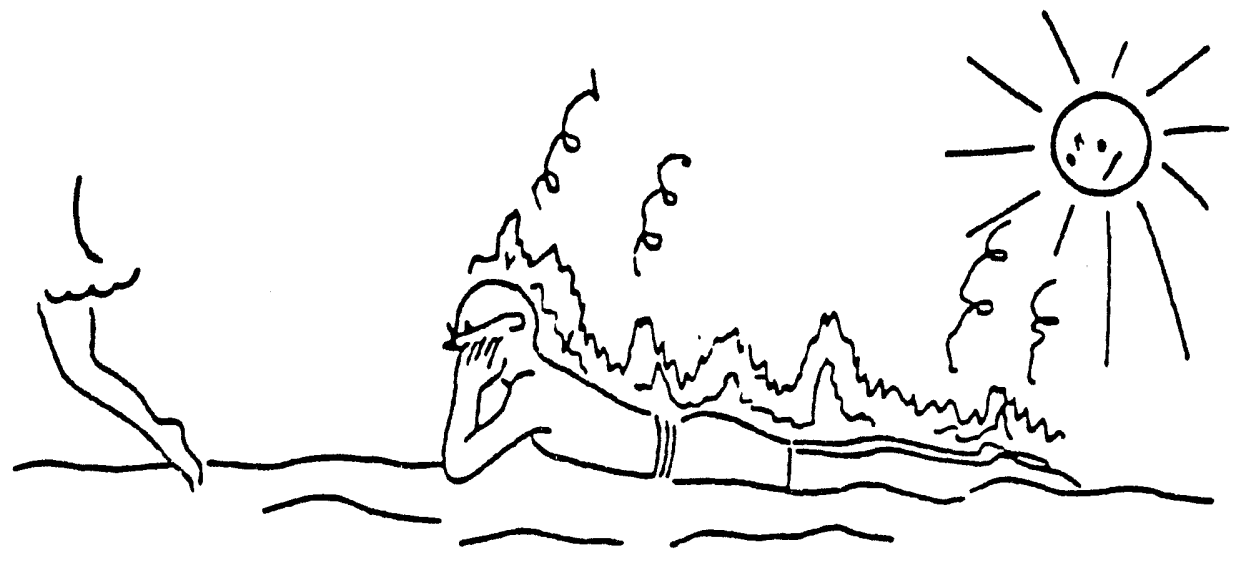


radiation and the common methods for protecting yourself icainst it, and upon whether you are reasonabie derson. You can set quite a burn rom a ratch, but you ridst be close te it. So witi radiation. There is little canger if you know: viliat you are doing, and if you follow the safety ruies.

Radiation effects on people were noticed shortIy after the discovery of $x$-rays. These effects resulted from extreme exposures due to ignorance. lie now! have special instrunents to detect and measure all tyoes of radiation. The "Roeritgen" has been adopted as the basic unit for radiation measurement. It is simply a label for a certain amount of radiation, just as the hord, "inch", is the label or word used to describe a certain cistance. Roentgens are commonly referred to as "r's", milliroentgens, or thousandths of a roentgen, as "mr's".

How much radiation can you stand? The important thing is that you do not take too much at one time. Small exposures with intervals in between can ado up to a fairly inigh amount without harmful effects because cclls either recover by tinenseives or can be replaced by other cells. Furthermore, you may safely expose a portion of your body to a mech higher amount than is permissible far the entire body.

Rapidly growinc cells are somewhat more sensitive to radiation than normal cells. Upon this principie is based the trcatment by radiation of certain types of cancer.

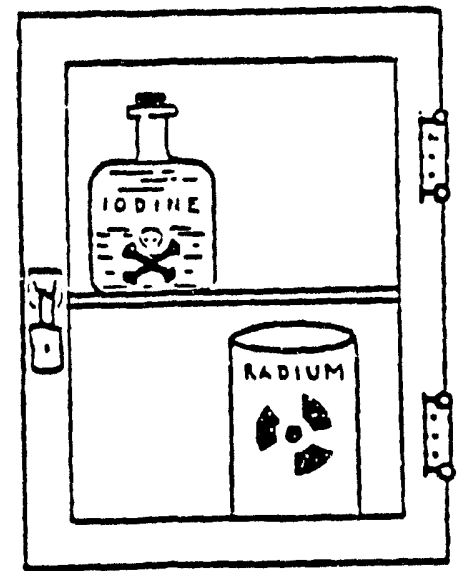

hith this unit of measurament, we are able to compare radiation exposure with its effests on livirg tissue. Years of experiments witn $x-l$ ays and redium and thousanos of exserimeris witr animia is have made it possible to jujoe how muen raciation we can stand or tolerate hiticut harm. This tolerance level is considerably highier than the amount of exposure which the fiomic Energy Comission Employees are nermitied to receive. (liow the liuclear Resulatory Comissicn, Ed.) The etandard operating limit is a continuing exposure ct no more than $100 \mathrm{mr}$ per week, not to be received in amiourits greater than $1 / 20 \mathrm{r}$ ( $50 \mathrm{mr}$ ) per day, except for unusual cases. Extensive experience indicates that an exposure of this amsunt can be given every vieei of your life without producing any detectable change whatsoever. This daily average is l/30 the wartime operating $i$ init established for the atomic bomb project.

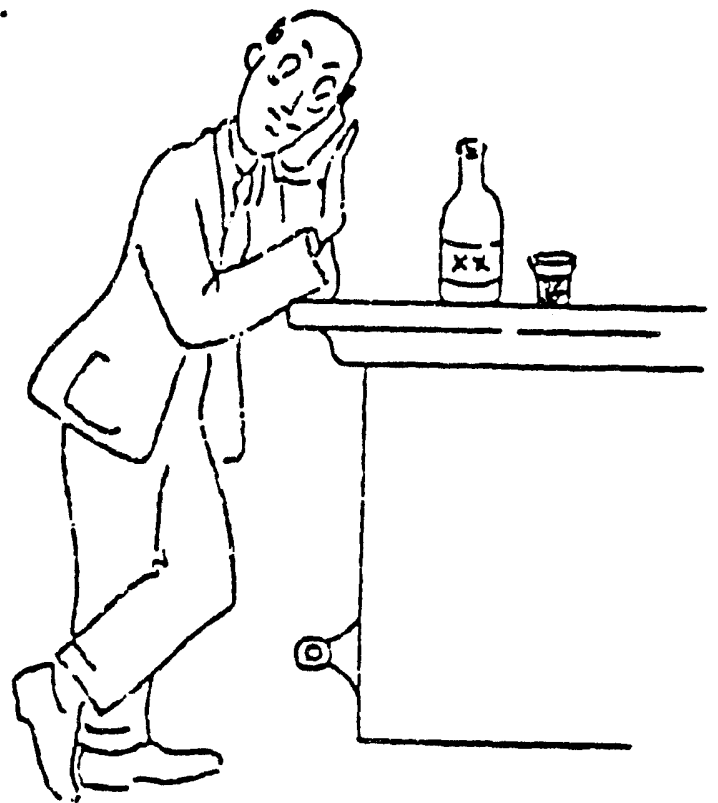

Radioactive materials can be harmful if within or on the body. You should, therefore, avoid inhaling radioactive substances or getting theili into your foct or drinii, just as you avoid taking in arseric, lead, or other poisonous substances.

The amount might be harmiess, but there is no need to take chances. 
Radioactive materials differ widely in the rate at which they lose their radiuactivity. The length of time they are kept in the booy also varies. Radium and plutcniun remain ective for thousanis of years and may be retained for long periods in the body, which such elements as radiosodium will be ouickiy eliminated and decay in a few days. Hatural$1 \%$, you must be careful tc avoid taking even small amounts of the more poisonous materials into your mouth or lungs. This is why eating or snicking is forbidden in some radiation areas.

When you have a chest $x-r a y$ taken with the curmon iupe of automatic equipment, you receive approximatcly $0.3 \mathrm{r}$. As far as the medical profession knows, this is harmless. Curing an examination of the stomach or intestines, patients frequently receive a series of expusures over a period of a few hours which may total 15 or $20 \mathrm{r}$. To render a person sterile, the sex organs alone would have to receive a single dose of $400-800 \mathrm{r}$, and even more if the total amount were not given at one time.

Setween 300 and $500 r$ of $X$ or gamma radiation given to the whole body at once would probably

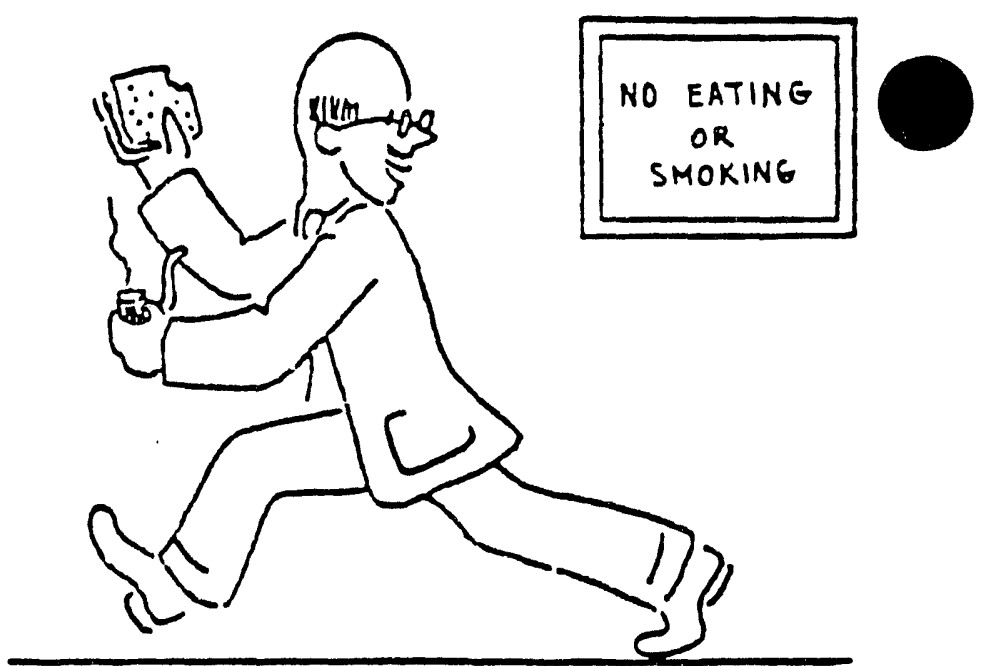

prove fatal. This, hovever, is a terrific anount, a fely thousand times the maximum fermissible deily occupational exposure ar tolerance.

As one radiation expert put it, "Tolerance is a poor word. Oneratinn limit is better. He can tolerate a lot more radiation that ine amount wich k'e have set as our operating limit".

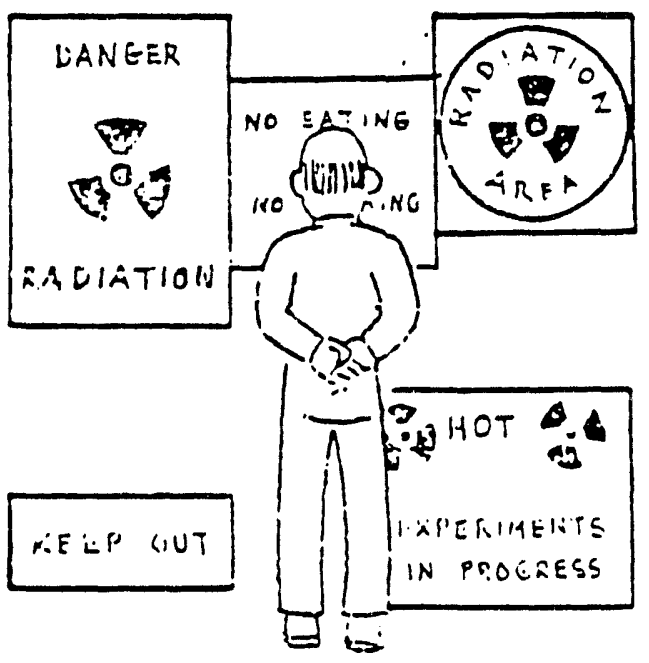

As a general precaution, sources of radiation. are confined to special "radiation areas" in which they are either roped off or are clearly indicated. All radiation arczs are marked with signs tearing the purpie radiation symbol on a white backround. liear the source itself villi be a warning sign with the purple symbel on a yeilow backround and a card stiting the tupe of radiation, its strength, and the precautions to be taken. 


\section{HOW TO LIMIT EXPOCURE TO PADILTICH}

xposure to radiation can be limited in thres ways:
(1) in time,
(2) by distance,
and (3)
by shieiding.

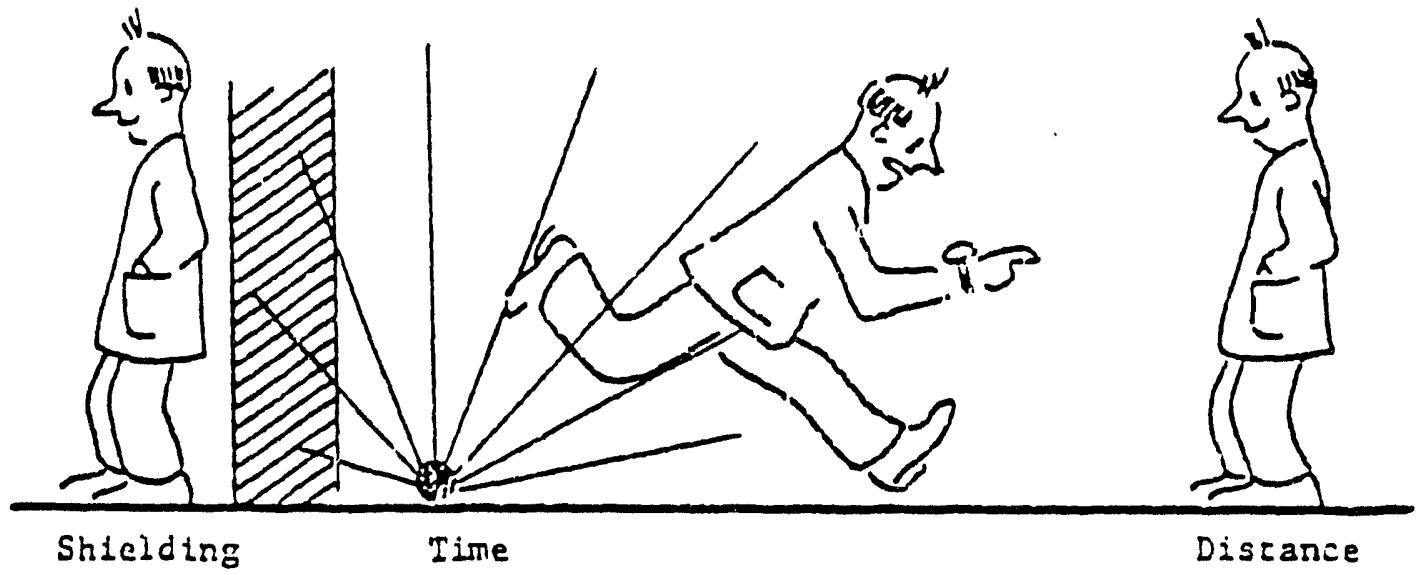

If you must work near radiation, the simplest way to limit your exposure is to stay in the vicirity as stort i time as possible. If there is a time lirrit on your job, observe it.

A second method is to maintain a safe distance hetiveen you and the source of radiation. If in doubt as to what oistance is saie, consult your supervisor or the Radiological Health unit. In gelleral, the eifect of radiation falls of sharp-: iy as you increase your cistance from its source. Double the distance and your exposure is cut to one-quarter.
Shielding varies in nature and thickness, depending upori the enrgy ind type of particles or waves. Alpha particles are stopned by a sheet of paper or the surface layer of skin on cur bodies; beta particles by a quarter of an inch of wood or an eighth of an inch of metal; gamma rays and neutrons by substintiai amounts of iead or concrete.

Special shielcing materials such as paraffin and cadmium can aiso be used effectively to slow down and stop neutron radiation.

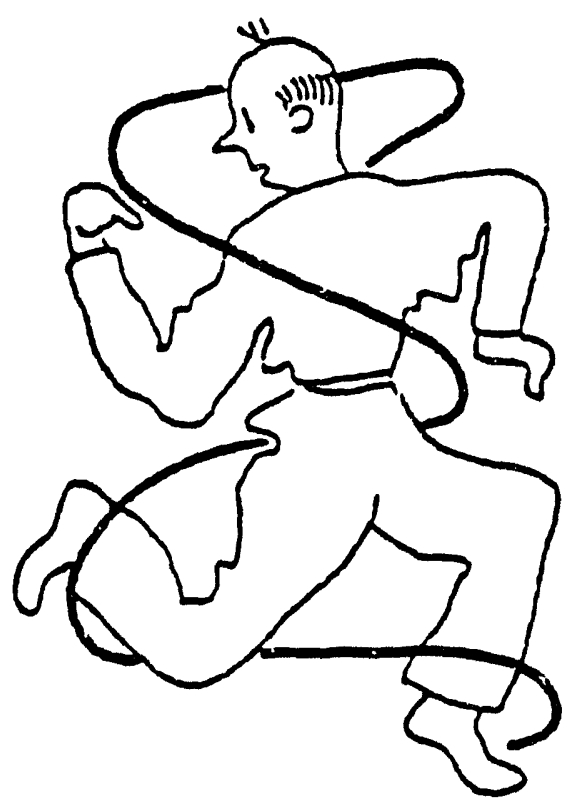

You can take it with you

\section{CONTAMIMATION}

Contamination is a more serious problem than exposure because it involves actual contact between you and a radioactive substance. An external source of radiation can be removed or stielcied, but you cannot run away from something inside of you or on you. If by accident you get a small amount of radioactive material on your hands, feet, or street clothes, it might possibly be deposited in your body from the end of a cigarette, through a cut, or in your food.

The longer lived materials, if not easily eliminated, might then cause trouble.

Fortunately: however, some isotopes with. shori halflives, or olles which are easily el iminaied, are valuable in the treatment of disease. Radioiocine, for example, is ta!:en by mouth for treatmeri of certain. thyroid conditions; rasioscoium is used ir measuring the circulation of the blooc; and rzoiophosphorous in treating some types of leukemia. 
To guard against contamination, special protective clothing is available in radiation areas. It use protects the wearer and helps to confine radioactive contamination within these areas. Laboratory coats or coveralls are widely used; in some locations caps, shoe covers, canvas or rubber gloves, masks, or respirators are also used. Piotective clothing worn where radioactive niaterials are present is specially marked and washed.

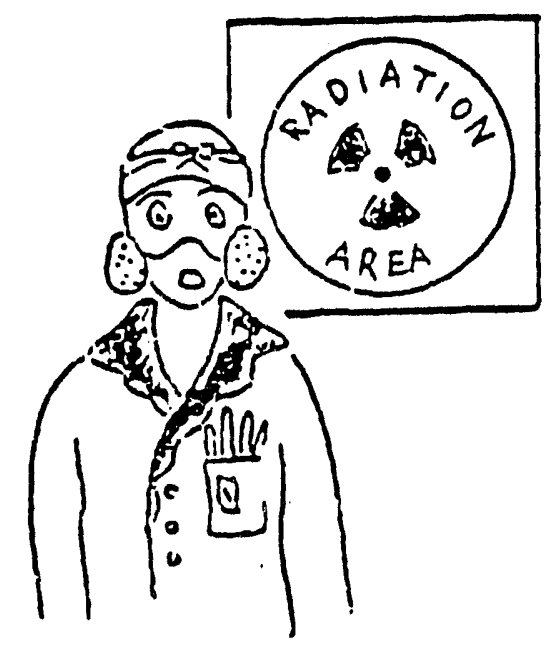

Alpha, beta, and garma radiation is not "catching" like a cold. Unless your hands, feet, or clothing are actually contaminated with materials which give off radiation, you

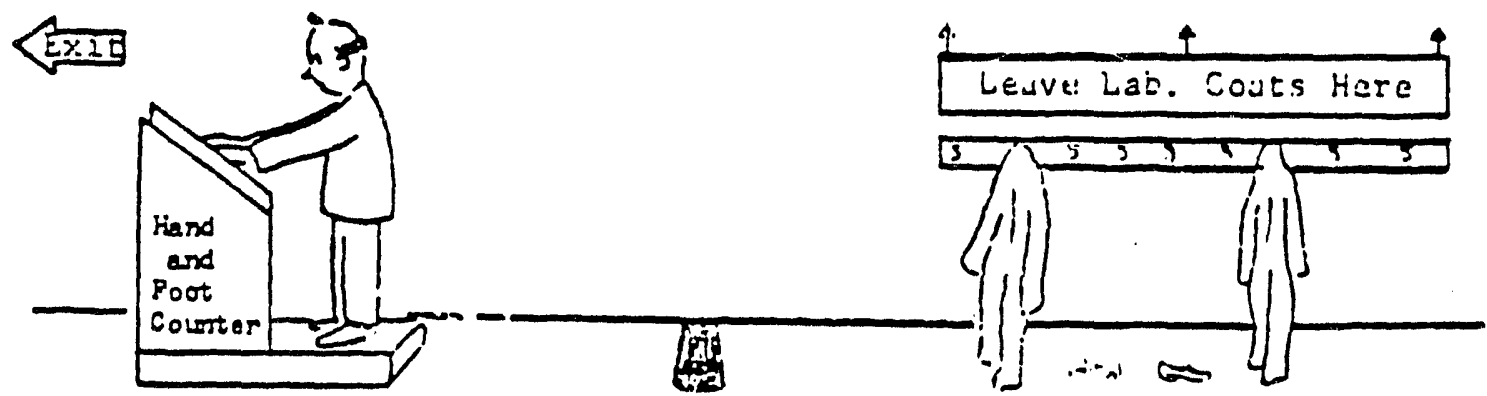

are quite safe. However, in working with radioactive materials wear protective clothing and upon removal of this clothing, wash with soap, check your hands, feet, etc., with instruments. 


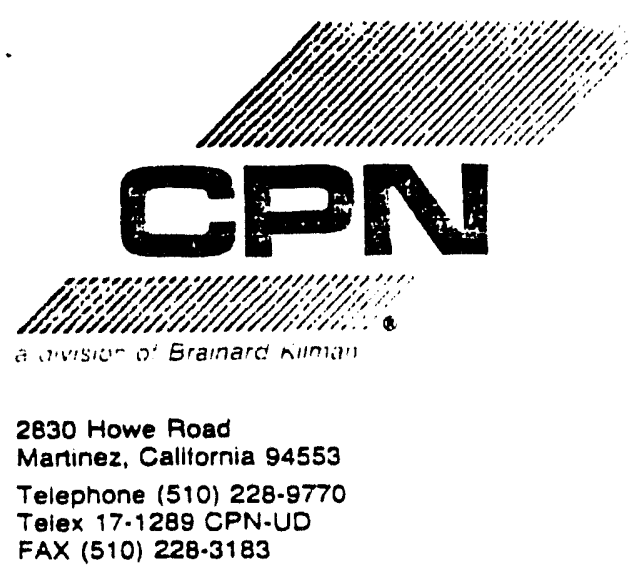

TECH MANUAL TM-1

Radiation Safety, Operating Procedures

and Technician Maintenance

Applicable to

All forms of Geophysical Testing

Equipment Utilizing Radioactive Sources

CAMPBELL PACIFIC NUCLEAR CORPORATION

$N \cup C L E A R$

OPERATOR TRAINING

COURSE

This course is a simple one day course designed to permit a soil gauge operator to use his gauge safely, in accordance with applicable legal restrictions, and accurately to abtain the desired field results. Completion of the course qualifies the operator to met NRC or agreement State licensing requirements. Operators are encouraged to attend other available radiation courses at local universities, highway departments or other agencies.

\section{AGENDA}

Morning: $\quad$ * Principles of Nuclear Physics of Soil Measurement

* Health Safety Regulations and Emergency Measures

* Application of Radioisotopes of Soil Gauge Design

Afternoon: * Field Use of Nuclear Gauges

* Calibration and Statistical Evaluation of Results

* Quiz

* Demonstration and Work Sheet Preparation 


PRINCIPLES OF NUCLEAR. PHYSICS RELATIVE TO SOIL MEASUREMENT

\subsection{General \\ Varlou: elements, both natur- aldy occuring (Radium) and re- actor produced (Cesium and Americium) are unsta- ble and are lowly decayine to a more stable st- ate. The act of decay produces emissions of en- ergy upon disintegration of the acoms. These em- lasions are either "zays" of electromagnetic ra- diation (Gamma Rays) or are actual particles of material (neutrons, for example). Other emis- szons are produced from varlous radioaceive ma- terials, however, we are concerned with only the gamma and neutron radiation for purposes of nu- clear soll gauging.}

Gama radiation occurs spontaneously from the Cesium or Radium source material.

Neutron emission occurs when an alpha particle enttter (Americium. Plutonium, or Radium) is mixed with Beryllium powder in a tightly compressed pellet. The aloha particles strike the Beryllium atoms to produce fast neutrons of an average energy of 5 million electron volts (MEV). The suffix "Be" is attached to the alpha source name to denote its use as a neutron source when it is mixed vrith Beryllium (RaBe, AmBe, PuBe).

These emissiors are detacted by appropriate detectors (Geiger Mueller tubes) for gamma and (Boron Tri-fiuoride-8F3 or Helium- 3 hi3 tubes) neltron measurements. The resultant signais are displayed electronically as an index of soil density and moisture.

Radioactivity, both gamma and neutron, may be thought of as being similar to light from an incandescent bulb. The light rays diminish rapidly as we move away from the bult (by the inverse square of the distance from the lamp). and they have the ability to penetrete various materials to some degree, ranging from nearly complete penetration (glass) to nearly complete blockage (metal shield).

Radlation obeye the ame roles, although 1 ts penetration capabilities are generally much greater than light. The farther we are from the source, the cafer we are, and the more absorbing material (shielding) we place between ourseives and the source, the safer we are. It is theoretically impossible to shield any radioactive source completely, however, careful gauge design and appropriate choice of shielding materials can reduce the radiation to an acceptable level with negligible absorption by the user under proper operating procedures. 0.01 Gamma Radiation: Gamma radiation is elec-
tromagnetic "photon" energy capable of penetration of several inches of most materials. It is essentially high energy "light ray" energy. It is useful for the total mass measurement of heavy materials and is used to determine total density of soil.
Gamsia radiation is emitted in several energy levels by a sealed Radium source anc in a single energy isvel by a Cesium source. The Cesium level is $0.6 e$ NiEl anc recuires less shielding than the multi-level output of the Radium source. The fixed spectrum emission is al so superior for soil denstity aetermination purposes. Cesium, a reacior produced isotope requires a licerise for lise anywhere in the U.S. and in foreign countries.

\subsection{Neutron Radieticn: Neutron radiatior con- sists of smail, nen-} charged particles enitted from the source at an average energy level of approximately 5.0 MEV. This is know'n as "fast" neutron emission. Neutron detectors "see" only slow, or "thermal" neutrons, therefore, the fast neutrons must slow down or they will be ignored by the detectors. Neutrons slow down by colliding with other objects much like a rifle bullet richocheting from rock to rock.

Collision of the fast neutrons with the nuclei of large atoms results in rebounding of the neutrons with little loss of energy. Collision with the orbiting electrons (approximately l/1840th the weight of a nestron produces little loss of energy. However, collision with an objec: of the same mass will produce a major loss of energy or slowing down.

The only atom which can markedly slow down a fast neutron, and winich we would likely see in soil. is hydrogen. The hydrogen nucieous is the same mess as the neutron and slows down the neutron immensely compared to collisjons with otner nuclei. The greatest loss of energy in any collision is wher two similar mass objects collide.

A simple anclogy is thet of a golf tall colliding with a bowling ball. The golf bell would. rebound with little loss of energy. The golf ball colliding with BB's (electrons floating around a nucleous) would push them aside. However, iwo colf balls colliding would produce a strong loss of energy in each of them, or a transfer of energy from one to the other.

This is what happens when a fast neutron hits a hydrogen atom. The neutron is markedly slowed down. A few collisions with hydrogen atoms reduces a fast neutron to the slow or "thermal" energy at which the moisture detectors in the soif gauge can "see" the slow neutron.

Thus, the moisture channel is in reality a "Hydrogen Analyser" and is responsive to any form of hydrogen present whether it be in the form of water, or of some organic matter. It is possible to measure water on a construction site because the only form of hydrogen we normally see on a soil site is free water, the very feature we are trying to measure. However, bound wazer within the mineral matrix, orcanic matter, roots, or 
asphait in an asphalt pavement would also provide hydrogen moderation and the neutron cauge would "read" it accordingly. If we know the quantity of extraneous hydrogen, we can account for 1t in calibration and the gauge can still be used for mo isture determination.

Neutron radiation is enitted by any alpha producing source when mixed with Beryllium. CPN uses Americium/Beryllium (AmBe) source in its soil gauges.

\subsection{Source Nomenclature: Certain terms are used to des-} cribe radiation factors important to us as users and we will explain them herein.

CURIE is a term used to describe the size of a racioactive source. It tells us that we have a quantity of meterial disintegrating at the rate of $3.7 \times 10^{10}$ disintegrations per second, or the same rate as one gram of Radium. This is not an index of how dangerous the source might be, oniy an index of quantity of the material in question.

We deal in small quantities of material and use only millicuries of radioactive material.

The potential danger of a source is not only a factor of the Curie size, but also of the type of material and the type of emissions it is produeing.

ROENTGEN is a term deseriting the amount of radiation accumulazed, or dose, or exposure. A roentgen of radiation could be accumulated by standing near a large radioactive, unshielded source for a short time or near a small, unshfelded source for a long time.

PEM is $\dot{a}$ superior term for human exposure accumulation than koentgen because it has been corrected to provide a common base for effeets on mankind. Some radiation is highly penetrating and would be more potentially cangercus than other forms. The description becomes eoual when we coreect them all to the common FIy bese.

He deal in small amounts of radiztion and shall work with millirems.

MILLIREN/HOUR (MREM/HR) is a term used to describe the "origniness" of a radioactive gama source. It is the strength of the radiation fieid at the poiri: of neasurement. This term is similar to foot candles of light when discussing light.

The brightness of a radiation field will be dictated by the type of radioactive material involved, the size of the source, the amourit of shielding preserit, and the distarice we are from the source. The total amourt of radiation we would accumulate would then become a factor also of how long we remained in thict fieid.
Because we deal with small sources, and because CPN PORTAPROBES and HYDROPROBES use small, well shielded sources, we will be involved with oniy Millirems of radiation and with levels which are only in the Millirem/Hour range.

FLUX is a term properly describing the strength of a neutron field. It properly describes the number of neutrons per square centimeter per second falling on a surface. CPN survey forms include the neutron readings in the form of MREM readings for ease of comparison and for determination of potential radiation levels or accumulation.

A conventional survey meter will read only the gamma or beta output of a device. Only special neutron meters will read neutron output. The CPM profiles in the manuals include the neutron levels converted to MREM readings.

\subsection{Dose Calculations: Radiation dose cal- culations are easily} done if the operator know's the radiation level in which he is working and the time or duration of exposure. Merely multiply the MREM/HR value times the duration of exposure. The result is MREM dose accumulated.

An accepted level for maximum occupational. accumulation, by edict of the Niuclear Regulatory Comission, is 5.0 REM/Year. This is approximately $100 \mathrm{kiREM} /$ Heek allowing for vacations.

We are always interested in minimizing radiation of any sort, however, the above level is a level established by statute as a legal limit.

\subsection{POPTAPROBE Calculations: The average ra- diation level at 21 from the PORTAPROBE is less than $0.5 \mathrm{MREM} / \mathrm{h}$.}

The average level on the surfaces is 5 MREM/HR.

He are primarily interested in whole body dose not just the fincers and feet. (Extremities can absorb 15 times the whole body allowances.) Accordingly', the $2^{\prime}$ distance represents the probable position of the body from the gauge when we are using the device.

Normal operating procedures require the user to hancle the oauge for approximately 10 seconds per test and a busy day would nomally result in approximately 30 tests per day being taken. Some days will be heavier in work load and others will be lighter. An operator can probably averaoe the 30 test figure. If he works at the testing for five days a week, then how much radiation nould he absorb?

10 seconds $\times 30$ tests $=300$ seconds $/$ day $=5 \mathrm{Min}$.

5 Minutes/day of close gauge contart $x 5$ days

$=25$ minutes/weet...... . round it off to $x$ hour. 
t hour $x 0.5$ MREN/TR $=0.25$ MREM accumulation.

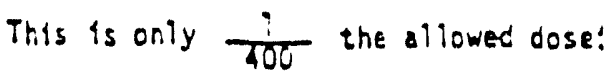

It is apperent that the operator, following the prescribec operacins procedures, would no: accumulate any excessive exposure. The gauge is sefe for the intended purpose.

The operetor ans: not sit the gauge in his lap, nor attemipt to repair the racioactive source in any fashion.

KEVER EXPOSE THE SOURCE UIMICCESSARILY:

DO NOT TOUCH THE EXPOSED SOURCE:

\subsubsection{Soll Gauge Sources: The most camon soll} gauge sources are:

Cesium 137 for garma enission

Americium 241/Be for neutron eamiston

Radium 226/8E for combined garma and neution emission.

Ail sources are supplied in a sealed stainless steel capsule, doubiy enczpsulated and further welded into a stainiess steel source rod or 10cated pemenently in the gauge housing.

Sources are manufactured for CPN by a number of manufacturers to CPH specifications. They are all toentical. They have been approved for manufacture by CPH's licensing jurisdiction, the State of California Public hea ith Dedartment. as well as for specific use in CPH products.

Soltce should never be removed from their mountings and no attempt should ever be made to repatr them. Advise the factory immediately in the event of camage to a source. See the section regarding Emergency Procedures in the Radiation Precaution Section (Red Section) of your gauge manual.

E.00 Licensine: The primary licensing agency in the U.S. is the Nuclear Regulatory Comission which has jurisdiction over reactor produced isotopes, but not Radium.

As of this writing, 25 states have undertaken their own licensing and are known as Agreenent States. "They have "agreed" to license in accordance with the NRE, can be somentiat more restrictive, but not less restrictive. In all cases they license Radium as well es other materiais. They elso include $X$-Ray machines as well as any other ionizing oevice or material.

Therefore, it is possibie to use Radium sources in sone statcs where only the Federal Law prevalls, but this is only due to a quirk of law and does not connote that Radium is safer than cther materials.
The Federel License applies for the reactor produced esterleis (cestian end Anertciur.) in all states not doing ineir own ifcensing and on ali Federal property regardiess of losation.

Accordingly, a Radium Source gauge coult be used on Federal proper:y ir. an Agreemen: Stete, although it: could not be taken off the federal property and used and, in this instance, no license would be required in that state.

The license spells out the condtions under which the material can be used and strietly limits che user.

IT IS ESSENTIAL THAT THE OPERATOR. READ AKD UNDERSTAND THE LICENSE UNDER WHICH HE PROPOSES TO USE RADIOACTIVE MATERIALS.

The license dictates the use of the materlais and this must not vary. Your 11 cense will stipuiate that the meterial is to be used for the measurement of toisture and density of construction materials". It cannot be used for other purposes. An attempt to $x-R a y$ an objest with the Wth the gauge might be moderately successful. but it is also petentiy 11 legal uniess your $11-$ cense specifically allows suen actions.

The license wlll require that one person be designeted as Radiation Safety officer (RSO). He is responsibie for the oeneral adherence to the iicense, the ma intenance of the required record flies, end is the person who should be consacted imediately in the even: of an erergency.

\subsection{Aoolication for License:}

Forms are avallable from CPN for tiRC or Agreenent states with the required nomenciature preprinted.

Additfora? information is fllled in for the spEcific applicant and this is subnjtted to the appropriate agency for the geographic location.

Upon return, the license must be followed to the ietter.

Contaet CPN for assistance in obteining an application or ilcense.

5.02 Transfer of Radiocetive Micterial to Others

Radioective material can only be transferred to others if the other party is appropriately liicensed to receive and possess or use the mater1al. The only sure proof is a copy of the other - party's iicense. That is why CPN requests a copy of our customer's licenses before we ship out 2 product to theri.

5.03 Pectorocity: Licensees can generally use their device in another license jurisdicion for a period not to exceed 180 days provided the other jurisdiction is notified of tive intruston and is notified of the nature of material, the device in which it is to 
be used, the duration of the use, and the location. Notify the other jurisdiction at least 5 days prior to your intenoed use. CPN Technical Data Sheet 5 lists the agreement states.

Use for a period of longer than 180 days will require the obtaining of a license in the other jurisdiction.

The new jurisdiction may require submission of - local reciprocity form with proof of your present license. It is wise to contact the other jurisdictional office well in advance of your planned use.

\subsection{Transportation: U.S. Government D.O.T. Regulations control} the transportation of radioactive materials on public accessways. All CPN products are furnished in 0.0.T. Yellow Label II, Type 7A shipping and storage containers. Yeilow II labeling means that the outside of the container has less than 10 MREMV/HR on any surface and less than 0.5 MREN/HR at $3^{\prime}$ from any surface. Under these conditions, no placarding of the vehicle is required. The devices themselves will generally not meet the Yellow II requirements when not in the shipping cases and, if transported on public roaoways, would require a placard stating, "RADIOACTIVE", in 4" high letters, front, back, and sides of the vehicle.

The case should be locked during transportation to prevent unauthorized entry to the device.

Anyone can transport the gauge, however, only properly treined and licensed operators can use the gauge.

When transported on commercial carriers. a "SHIPPER'S CERTIFICATION FOR RADIOACTIVE PHATERIALS" form should be affixed to the outside of the case. A copy of a suitable certification is included in the Radiation Precautions Section of this manual and in all gauge manuals.

Many airlines will not carry radioactive materials on passenger aircraft. Check before you ship or attempt to take a unit with you.

Contact the factory for assistance.

The reference for DOT Regulations is Title 49, Parts 17 through 173.350 .

Your vehicle should always be locked when the gauge is stored therein, and the gauge should be securely fastened down to the bed in an open pickup trucl:.

The CPN gauges are designed for easy field service and do not require shipment of the radioactive pertions to the factory. All CPN electronics may te removed for service withou: interfering $k^{\prime} i$ th the radioactive sources.
7.00 Storage: The CPN Gauges should be stored in their shipping cases

in a locked area with key access only by the licensed operators. CPN recomends that permanent storage be 10 feet from the nearest point of full time work requirements.

Post a permanent CAUTION-RADIOACTIVE MATERIAL sign on the storage area door. These are available from CPN.

CPN recommends that a sketch of the proposed storage area be furnished the licensing agency upon submission of the initial application. The agency may be able to comment on the proposal to insure compliance with local requirements. An tppropriate sketch form is included with all CPN iicense applications.

CPN recomends that the local fire department be called in for a review of the storage location and of the nature of the device stored therein. This may preclude frantic phone calls should a fire occur and the fire department not know the nature of the radioactive material.

\subsection{Health Safetv Considerations:}

Two major considerations are:

$$
\begin{aligned}
& \text { 1. Protection of the operator } \\
& \text { 2. Protection of the General Public }
\end{aligned}
$$

Protection of the operator is achieved through adherence to the manufacturer's instructions, successful completion of the Operator's Training Course, and good gauge design by the CPN factory.

Protection of the General Public is achieved through the factors in iten 1. combined with restriction of access to the device by untrained and uninformed individuals. The responsibility for this lies primarily with the licensed operators who keep the gauge safely away from unauthorized people.

\subsection{Film Badoes: Most licenses will require} the operators to wear film badges. These are similar to dental badges, carried in a small plastic holder, and processed monthly. They record the gamma absorption of the user with excellent accuracy. The neutron record is not so accurate and faoes with time. The record is a lifetime accumulation record and a copy of the accumulation to date should be obtained by each operator upon leaving his employment. He should present this record to the next employer, if nuclear devices are to be used, and the record can be continued in unbroken form.

A typical film badge service is:

$$
\begin{aligned}
& \text { R. S. Lanciauer Jr., \& Co. } \\
& \text { Qوo N. Sepulveda blva. } \\
& \text { El sequndo, li } 90245 \\
& \text { 213-640-1015 }
\end{aligned}
$$




\section{Q.O2 Piele sacty Frecosures:}

The ozuge is to be unoer coritrol of a ilcer.sec operator at all times. Altrouch interesied par. ties way wish to view the gauge in operation. they may not use it themseives uniess properiy irainec to do so.

A licensed operator may not train another person is. the use of the device unless his license specifically permits triis training.

There are no general regula:ions reoardino erection of barriers or sions while the gauge is in use. Local requirements of the em,oioyer ara differ. The gavoe has a secure radiation labei and the presence of the ilcensed operator would nomally be deemed adequate protection of the device from outsiders. CPli ooes not recormend the adoption of extraordinary precautions. It is the intention of the safety regulations to protect all parties, not frighten them with unnecessary restrictions or rituels.

See Section 11.00 on Emergencies.

\subsection{Leak Testinc: License regulations re- quire ind dil sealed} sources be "leak tested" cocasionally to insure that the radioactive materiel is all secure in the source and none has leaked ous. This is accomplished by swab test for CPli products and is generally performed a nnually by the PSO or any other authorized indivicual. Your license may require a six months inierval. READ YOUR LICENSE.

Leak test kits are ovailatle from the CPN factory at a nominal cost.

Your gauge instruction manual whll contain detalled instruetions regardir. leak testinc of your device. Follow them closely. It is MCT necessary to renove the source or open the shutter on CPN gauges to effect leak testing.

Up to date leak cest certificates must be maintained in the Radiation License flle for inspection at all times. This must certify that less than 0.005 microcuries of removatle contamination was removed from the gauge at its last test.

10.00 Records: The Radiation Safety Officer is responsible for maintenance of all records pertaining to the radioactive materials. CPN would recomend that a three ring notebook be mainteined with tabbed sections for:

a) License and supporting documentation.

b) Personnel records, training, etc.

c) Film Badge Pecorts.

d) Leak Test Records.

This is to be avallable to license inspection personnel at all times.

\section{i1.03 rugegriags}

The operator should a:iemp: to prevent exposure to himseit onc to ctners ane mus: insure tha: the raciodetive materials do no: escape the capsuie and coriarifinate the area.

Que to the methods of ensapsilaticri, 1t would require a very severe accider: ic damage the cafsule ard result in loss of raterials.

In the event of orvoe damage:

1. Protec: people....keep them away, keep them out of the immediate area until you ere certa in everything is alrighi.

2. Protect the gauce from further damage. Get it beck to its storage aree when you are certa in that it is moveable. Do no: de uncuiv dolu or brave.

3. Protest the surrounding area from contamination. Freeze the site in the event of a severe acefoent involving major gauge destruction.

4. Czll for help from your RSO who should have on imediateis avellable 11s: of 10cal emergency service offlces, the nearest Putlic llealth office, ine nearest hosfital or university where radiation specialists may be located, and the CPli factory pnone.

\section{A DECIS:Oli POIiT mav be exercised:}

A. The gavge is damaged, but is intact, and the source is otviously in place ane no: danaged. (Dropped, minor runover, floot.)

B. The gauge is damaged, is torn open or is not in a condtion to de:emine source integrity. (Fire, major runover, buried.)

In the event of $A$ : The operator should elose the snutzer if possicte, place the cauge in its shipping case, place the case in the storage location and call the factory for assisunce.

In the event of $B$ : The operator should freeze InE site, SICP inc vehicle if involved, get the driver off the vehicle, rope off the area around the site, keep people from waiking through the site, and should call for help from a traince nuclear investigator who KH:Ch'S HOH! TO USE A SURIEY HETEP. Contrary to loca requirements, CPN coes not recommend that operators have their onn survey meters for accident precautions. It is better to get an expert on the site rather than an amiateur if the source should realls be damaged.

Just keep people out of the damace area until it is cleared by the nuclear expcr:.

Play it safe if there is any doubt!

5. 
11.01 Theft: Call the police and the factory. Somebody usually calls the factory sooner or iater trying to escablish a price for this sort of commodity.

11.02 Fire: Call the fire department and advise them of the nature of

the radioactive materiais involved. Inform them that it is a sealed source. Your problems will be much simpler if you nave already had them in to view the device BEFORE you had a fire. Call the public health department for assistance in preparing the recovered source for shioment to the factory or to another authorized disposal service. DO NOT BURY IT OR OTHERHISE ATTEMPT TO DISPOSE OF THE SOURCE.

\subsection{Service and Maintenance:}

Detalled service sections are in the individual gauge manuais. For purposes of this course:

1. The CPN products are all field serviceable without removing the source.

2. The entire electronics and battery assemblies can be romoved with simple screwdrivers. These can be returned to the factory for service without shipping the source.

3. CPN does not recomend performing mechanical service other than the authorized cleaning of the shutter.

In the event of a severely damaged or jarmed shutter, contac: the factory.

13.00 Additionel Training Materials:

Radiation Dosimetry, (Hine and Brownell) Academic Press, Inc., 125 E. 23rd St., IIY NY

National Bureau of Standards Handbooks available from the Supt of cocuments, hiasnington, 25, DC:

\section{\& 92 SAFE HARDLING OF RADIOACTIVE ISOTOPES \\ * 54 PROTECTION AGAIIIST RHDIÁTIOH FROM RA- DIUH, COBALT 60, AND CESIUM 137. \\ t 59 PERM!SSIQLE COSE FPOM EXTERUIAL SOURCE OF IOKIZING RADIATIOK.}

Many other excellent references will be found in your public library or technical bookstores. Most public health departments have omphlets available on the subject of radiation. An excellent reprint of such a handbook is in the back of all CPN Instruction Manuals. and this training manual.

Hiany' colleṣes have short courses on nuclear matters, sciety, etc.

CPli recommenos an occasional refresher Operator iraining course for all operztors.
14.00 APPLICATION OF RADIOISOTOPES TO SOIL GAUGES.

14.01 Genera 1: If a gamma source is placed next to a geiger tube, the tube will produce a series of pulses which may be counted electronically and displayed numerically.

If an absorbing material is placed between the source and the detector, the count rate will be reduced. If the absorber is thick and very heavy, the count rate will be reduced to near zero.

If the source, intervening shield (lead) and the detector are placed on a surface, then some radiation will be reflected or "scattered back" to the detector from the source and the system will again produce counts. These counts will be proportional to the density of the reflecting material and we would have a "Backscatter Gauge".

If the source were dropped into a hole in the soil and the detectors left on top slightly avay from the hole, then we would have a transmission gauge with the radiation being "transmitted" through the soll. The signal is again proportional to density.

If a neutron source and a slow neutron detector are placed on a soll surface, then the count rate will be proportional to the amount of hydrogen present which should normally be proportional to moisture.

It makes little difference if the surface for either form of measurement is flat or is round as a result of being a larger hole drilled into the ground as in the case of "Ueptnprobes".

14.02 Surface Gavoes: Model A, Model BR, Model $M C$, etC.

Backscatter is the technique of placing source and oetector on the surface of a flat material. This technique measures only the top 2" of soil and is very sensitive to surface roughness or quality of site preparation.

Transmission is the technique of pre-drilling a a small hole in the ground and then inserting the radioactive source in the ground via the moveable source rod. This technique permits measurement to specific depths to $12^{\prime \prime}$ and is insensitive to surface roughness. It is very accurate anc is the preferred method of measurement for soils.

DENSSTY is measured by either of the two above tecnniques.

MOISTURE is measured by bacliscatter only. It is not a iransmission phenomenon. The tiodel M.C Series gavges use a separate source in the bottom casting to insure constant tacliscatter operation and permit simultaneous counting of both density and moisture. 
Moisture ettains adoroximately $g x^{*}$ cf its returned inemial neutrons from tria closest $E$ " of soil.

14.0? Oepth Gavoes Model 500 Series, HYDROPROEE, EES.

ine information under surfoce gauges has general application to desth vauces as well. Deoth gauges require a predrilied hole asproximately $2 "$ in ciamezer, cased with thir wall aluminum or steel, into which the probes are lowerec.

All depth gauge measurements are backscatter of nature and are subject to the surface rouchness effect of the walls of the access tube. This will have littie effect on moisture, but is influential in the density mode. Deoradation of the hole wall during drilling and casing will influence the accuracy of the density channel.

\subsection{Chemical Composition Cescorse}

All density gavges are influenced to some exien: by the varying outer electron ortit arrangement of the different soil elements. The major elements in soil are aluminum, silicon, calcium, oxvoen, and some hycrogen where moisture is present. Agricultural scils kill also have carbon and :race elements of other materiais in excess of those found in constr-ction soils.

Eama absorption is primarily effected by the mass of the atoms, thus the oensity channel is 2 mass channel. Hoviever, low energy gamma raj's below $200 \mathrm{KEV}$ are also influenced strongly by the atamic number of the atam as opfosed to its weight. Thus, the different atoms rocierate the high energy' gamas approximateiy in proportion to the keight of the atoms (scmeiring we are trying to measure). However, the low energy, gamas are influenced by the ortiting elestron arrangements proportional to atomic number. This is 2 iso a factor so the 5 in power of the atomic number, something we could well do without!

To avoid this, CPN uses special platinum cathode geiger tubes, internal filtering, and special geametrical design of the shutter arrangenient to cancel this potentioi error. It is no longer a serious factor in density cauges manufactured by CPN, a: least where the soils are the usual mix of silicon and caicium.

\subsection{Density effect on Moisture:}

Thermal neutrons are eventually captured by one atom or another and they disappear. This is accompanied, usually, by emission of a strong gamme ray which can then be detected by the density channel. This error is very small, if noticeable at all, and is not a soil measurement problem.

It has no bearing on Depth Probes aue to the geometry of these devices as compared to the surface devices.

\subsection{Chemicai Estes: or Moisture:}

This is nat comror, but mey be exizerictised ir scils naving a nign capiure cross section. for themal neutrons. Sush soils would have a lot of Bcror. in them or Cacmium. Tnese soils would absoro the thenici neutrons before they car ge: to the detectors and a low reating moisture error would resil:. These are uncomon anc are found in salt fiats or specific areas.

They are not a problem in agricultural soils. 14.07 Bound hieter Effect on Moisture:

This is very common..... it creates a major error in all moisture gauges. Whether surface or depth.

l'arious mineral combinations, laroely silicates. have a water of hyoration characteristic wherein they combine with water molecules in other than free water form. The hyorogen analyser neutron gauge correctly rieasures this bound water al. tnough this may not have any value whatsoever for either construction compaction or agriculture growth.

Correction for this error is made by measuring the desired water iraction by converitional means and then correcting the factory curve accoraingly.

In ag̣icultura! measuremerts, additiorial sourses of hydrogen from hydrcsarton compouncs in the humus and root structures may also incordorate errors. These are aiso easily corrected oy orawing a correction to the factory curve based on soil meesurements, or tensiometer readings.

Moisture gauges all tend to read higher then they should due to this misture error.

\subsection{Calibration Variations:}

The user should viev nis nuclear gauge as a tool and should not de reluctant to change its calibration to suit his needs if required in the fiele.

CPN feels that its calibration stamdards are accurately prepared and calibrated, however, we recognize thet our standards to not exactly agree with those of other manufacturers or of major users. We also recognize that other manufacturers do not agree with major users, eitner. In short. there is no universal standard at this time and any calibration is subject to change by the ultimate gauge buyer.

Our MC-2 series gavge even has a Bias provision to permit adjustment of the curve where this is felt necessary.

CPN would counsel the user, however, to be very sure in changing a calibration. The gauge is usually right. 


\subsection{Calibration Field Check using} Combaction Box:

Sometimes a surface unit will provide results in disagreement with conventional methods of testing (sandcones or baloon tests) and a dispute arises regarding accuracy of the nuclear test.

While complicated calibration programs have been developed for nuclear gauges, the only final answer must be based on actual field testing on real soil materials.

CPN products are chemically corrected for the usual soil elements of silicon, calcium, aluminum, and oxygen. However, the presence of a new element of substantial quantity could introduce an error of significant (measureable) amount.

(Fortunately, this new element, if in sufficient quantity to produce an error, would then increase the vaiue of the soll to the point where we would be mining the material for its intrinsic value and would not be building a freeway or a housing development on it.)

Where a dispute arises, however, there is a method of proofing of the calibration of the nuclear gauge, or of the conventional device, to produce an accurate sample ci the soil suitable for testing.

This technique revolves around the use of a comDaction box.

The user should build a sturdy, wooden or metal box measuring $18.6 \times 18.6 \times 11 "$ inside dimension. Allowing the last inch as "ireeooard" to retain loose material during preparation, the finished box will be $10^{\prime \prime}$ ceep and the volume is 2.00 Cubic Feet.

1) When a dispute arises, we will compact a boxful of material at a very specific densit:y...... de density appropriate for the material maximum weight. Choose a oensity of approximately ESE of Proctor for the typical sample. Any density can be compacted in the box, however, from pour point to 100\% compaction.

2) Presume we select a density of 130 PCF for the samole. The box holds two cubic feet, therefore, the firia $i$ box weight will be $2 \times 130$ PCF or 260 pounds total weignt.

We will compact this into a ten inch thickness, therefore, each inch will contain 25 pounds of $50 i l$.

3. Heigh out exactly 25 pounds of the materia), spread it around uriformly, and compast it bith a fiat ramer havinc a square bottom of approximetely $4 \times 4$ or $^{2} \times \Xi^{\prime \prime}$.

Measure down from the top at all points to insure even compaction to a depth of $10^{\prime \prime}$ froro the top of the box.

4)

Repeat this laver compaction until 10" of compacted soil is obtained.

Note:

Each layer NUST be exactly $1^{\prime \prime}$ thick. If you over compact by $1 / l E^{\prime \prime}$, this represents a $E$ : error in THAT lift, or an error of 8 PCF out of 130 PCF! That is unacceptable and the box should be dumped out and started over.

5) Take nuclear gauge readings on the box.

The gauge should read the box weight correctly and will do so $95 \%$ of the time!

The other 5: of disagreement will generally be due to miscalibration of the device and not due to some peculiarity of the soil.

6) Take conventional tests on the tox, including taking of sanocones in the box.

The usual result will be a disagreement between the sandcone and the box weight.

7) Since the box is a simple device from an engineering standooint, there can be little doub: as to its correzeness if the operator used care in preparation of the sample. Any disagreement must be in the devices.

For further information, write or call for CPN Technical Data Sheet 4, ACCURÁCY VERIF!CÁTION USING COHPACTION BOX ANO HAMMER.

\subsection{Deothorobe Calibratior:}

Density depthprobes tend to read low in the field due to abrasion of the access tube wall during drilling and casing. Untortunately, the depthprobe measurement is a backscatter measurement with the same errors as a surface unit.

Relative readings from one ievel to another, however, will terid to be accurate.

Moisture deptliprobes are more accurate than the density depthprobes due to the depoer reading of moisture as opoosed to density. Adjustments of the moisture calioration :o acsomocate bound water conditions, roots, or other organic hydrogen inclusions may be necessary.

For irrigation manaoment, a tensiometer reading is generally used for this starting point determination. 


\section{Field Sampling Method \\ for \\ Well Deviation Logging}

Well deviation logging at the INEL will be performed by a subcontractor. Their standard practices and procedures will be implemented. Subcontractor personnel will follow the health and safety practices being implemented for the RI (see the Addendum to the Health and Safety Plan in this Work Plan). Copies of the well deviation logs will be made part of the End-of-Well reports and will be incorporated into the $\mathrm{RI}$ report as an appendix. 


\title{
Standard Test Niethods for Density of Soil and Soil-Aggregate in Place by Nuclear Methods (Shallow Depth) ${ }^{1}$
}

\begin{abstract}
This standard is issued under the fixed des:znation D 2923: the number immediately following the desienation indicates the year of onginal adoption or, in the case of revision. : is lear of lasi revision. A number in parentheses indicates the year of last reappro:al. A superscript epsilon (t) indicates an editonai siange since the last revision or reapproval.

These methods have heen approved inr use' an atencles of the Deparment of Detere and for lisunc in the DoD Index of Speciticallons and Sianaiaris.
\end{abstract}

\section{INTRODLCTION}

These methods describe determination of the density of soil and soil-aggregate in place using nuclear equipment. In general. the total or wet density of the material under test is determined by placing a gamma source and a gamma detector either on. into. or adjacent to the material under test. These variations in test geometry are pre ented as the backscatter. direct transmission. or optional air gap approaches. The intensity of radiation detected is dependent in part upon the density of the material under test. The radiation intensity reading is converted to measured wet density by a suitable calibration surve. Principles of the nuclear test are discussed in the Appendix. as are some of its advantages and disadvantages. It should be noted that the density determined by these methods is not necessarily the average density within the volume involved in the measurement and that the equipment utilizes radioactive materials which may be hazardous to the health of users unless proper precautions are taken.

\section{Scope}

1.1 These methods cover the determination of the total or wet density of soil and soil-aggregate in place by the attenuation of gamma rays where the gamma source or gamma detector. or both. remain at or near the surface. The methods described are normally suitable to a test depth of approximately 2 to 12 in. $(50$ to $300 \mathrm{~mm})$. depending on the test geometry used.

1.2 Three methods are described as follow's:

$\begin{array}{lc} & \text { Section } \\ \text { Method A-Backscalter } & 0 \\ \text { Method B-Direct Transmission } & 10 \\ \text { Method C-Air Gap (Optional) } & 11\end{array}$

1.3 The values stated in incil-pound units are to be regarded as the standard. The metric equivalents of inch-pound units may be approximate.

\section{Referenced Documents}

2.1. AST:I Slandards

D 1556 Test Method for Density of Soil in Place by the Sand-Cone Method"

D216- Test Method for Density and Unit Weight of Soil in Place by the Rubber-Balloon. Method:

D 2216 Method for Laboratory Determination of Water (Moisture) Content of Soil. Rock. and Soil-Aggregate Mixtures:

D 301- Test Method for Moisture Content of Soil and SoilAggregate in Place by Nuclear Methods (Shallow Depth)"

'These methous are under the jurisdiction of $45 T$ T Comminee D- 18 on Soil and Rois.

Curtent acithon approved Ma: 29 . 1981. Publisned Sepienoer !981. Onginaily miblished as $0: 9: 2-9$ - Last previous edicion D $29: 2-78$

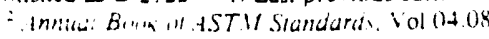

\section{Significance and Lise}

3.1 The methods described are useful as rapid. nondestructive techniques for the in-place determination of wet density of soil and soil-aggregate. The fundamental assumptions inherent in the methods are that Compton scattering is the dominant interaction and that the material within the zone of influence for each test is homogeneous.

3.2 Test results may be affected by chemical composition. sample heterogeneity. and the surface texture of the material being tested. The techniques also exhibit spatial bias in that the apparatus is more sensitive to certain regions of the material under test.

\section{Calibration}

4.1 Laboratory calibration of the gage is established by determining the nuclear count rate of each of several materials at different and known densities and establishing a relationship berween count rate and density. Sufficient data should be taken at each density to ensure a precision of at least twice the normal precision obtained in field use. Calibration can be accomplished by either laboratory or field methods. Laioraton methods are recommended due to the higher inherent accuracy of laboratory standards and instruments as opposed to field methods which require the volume measurement of an excasated hole.

VOTE :-Different chemical compositions of the blocks or other materials asn affect the count rate. Calibration curves may not be applicade to matenals not represented in establishing the calibration curve.

4.1.1 Laboratory calibration should involve the use of a minimum of five homogeneous blocks sufficiently large to represen: an infinite volume to the nuclear instrument. The densit: $O$ ithese standards shall be determined to an accurac: 
of $\pm 0.2 \%\left( \pm 0.3 \mathrm{lb} / \mathrm{ft}^{3}\right.$ at $160 \mathrm{lb} / \mathrm{ft}^{3}$ or $\pm 5 \mathrm{~kg} / \mathrm{m}^{3}$ at $2565 \mathrm{ig} /$ $\left.\mathrm{m}^{3}\right)$. Three of the standards shall be constructed of materais having gamma mass attenuation coefficients within $=1.01 \%$ of each other over the range of gamma energy utilized by the gage for density measurement. These standards uill be used to establish the gage response to density variations in the range from 100 to $170 \mathrm{lb} / \mathrm{ft}^{3}$ (1600 to $\left.2725 \mathrm{~kg} / \mathrm{m}^{3}\right)$. The last iwo standards shall be materials that equally bracket ine gamma mass attentuation coefficient of soils. Suggested materials are limestone and granite. The gage response will inen be rotated about the zero density point on a plot or graphical representation such that it falls halfway between the limestone and granite data. This final response will be used in the field for measurement.

4.1.2 Field calibration may be used where laboratory calibration facilities are not available or where it becomes necessary to calibrate the instrument for materials that chemically are different than soils. A minimum of ten field tests should be used for establishing calibration by the field metinod so that a range of densities and materials will be represented. The sand-cone method in accordance with Test Method D 1556 or the rubber-balloon method in accordance with Test Method D 2167 may be used to determine the wet range of density of carefully selected field sites on which density data have been previously determined. As an altername. nuclear data can be obtained on prepared containers of soil and soil-aggregate compacted to known densities. The containers must be sufficiently large to represent an ininite volume to the nuclear instrument. Data from either of inese methods may be used to establish a new gage response by isually fitting a line to a plot of gage response to density over the range of densities found in the samples. It is more cesirable to use the latter data. to rotate the laboratory calibration in 4.1.1. or a constant offset from this laboratory calibra:ion. to fit the field data. Extreme care must be exercised ir. the determination of wet density in either the field sites or prepared containers. Because of the variability and scatter irnerent in field tests and container preparation and compacion. inis method is considered less accurate than techniques using alibranon biocks.

VOT: =-Lise of blocks is advantageous hecause the are durabis and z:ovice stable densit! rejerences. Biocks and prepared contaners must $\because$ large enough not to change the onserved crunt rate lor count ra:101 if made large in an dimensions. Dimensions of approximatel! 12 is: $\$ 00$

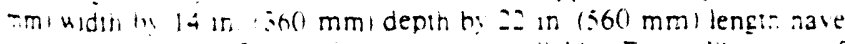
sover satusiactor io: equipment nou available. For calibraucr of winsaaner onl!. a derth of not less than 9 in. $(230 \mathrm{~mm}$ is adequate.

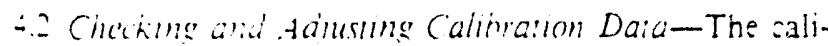
"ation data for newly acquired instruments, instruments for

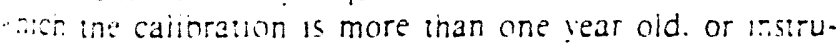
- ants that have ceen damaged and repaired mas be chesied using the meinod of 4.1 .2 . A minimum of ten fiele tests as: be used in compare whth the exsung calioration jata order to justify cranging the calibration.

$\therefore$. If each 0 ine field lests varies hy not more than :"

$\therefore$ ine nuclear densil and some of the field densitiss are

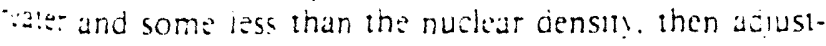

an of the calir:avon as noi necessar.

$\therefore$.: If all of ine field lests are more or all less thar :he

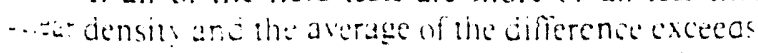

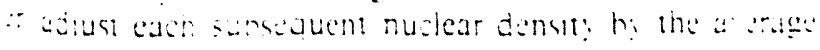

difference.

4.2.3 The average difference found in 4.2.2 may be used to determine a new calibration response. Either rotation about a zero point or constant offset from the original calibration may be used.

NOTE 3-Adjusting calibration curves is a complex task and it should be attempted only by those knowledgeable in this field. Most manufacturers etther provide this service or will offer assistance to the user.

\section{System Specifications}

5.1 Any equipment that is used under the requirements of this method shall satisfy the following specifications:

5.1.1 Precision-Precision of the system is determined from the slope of the calibration response and the statistical deviation of the count for the recommended period of measurement, which shall be at least $1 \mathrm{~min}$ :

$$
P=\sigma / S
$$

where:

$P=$ precision,

$\sigma=$ standard deviation. counts per measurement period. and

$S=$ slope. $\Delta$ counts per measurement period $/\left(\mathrm{lb} / \mathrm{ft}^{3}\right)$ or $(\mathrm{kg} /$ $\left.\mathrm{m}^{3}\right)$.

5.1.1.1 Determine the slope of the counts per measurement period at a unit weight of $125 \mathrm{lb} / \mathrm{ft}^{3}\left(2000 \mathrm{~kg} / \mathrm{m}^{3}\right)$. This can usually be determined from the calibration response and must be the true counts from the detector system before the display. Determine the counts per measurement period at the same density by the same method. The precision can be calculated by:

$$
P=\sqrt{C / P C} / S
$$

where:

$P=$ precision.

$C=$ counts per measurement period.

$S=$ slope. $\lrcorner$ counts per measurement period $/\left(\mathrm{lb} / \mathrm{ft}^{3}\right)$ or $(\mathrm{kg} /$ $\left.\mathrm{m}^{3}\right)$. and

$P C=$ amount of prescale $($ Vote 4$)$ applied to the detector counts prior to display. The manufacturer will suppiy. this value. If no prescale is built into the equipment. the value is 1 .

Note :-The value of pressale is the number hy which the total count rate of the deteciortsi is civided helore displas on the readout to elimi ate the need for disniaing non-significant digits. The value of $P$

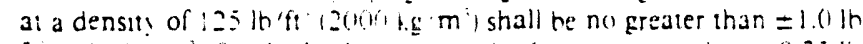
$\mathrm{ft}\left(=10 \mathrm{~kg} / \mathrm{m}^{3}\right)$ for the backsaziler methods nor greater than $\pm 0.35 \mathrm{lo}$ ft $( \pm 6 \mathrm{~kg} / \mathrm{m})$ for direct transmission method.

5.1 .1 .2 If 5.1 .1 cannot he performed as ahove. the precision can be computed hy determining the standard deviation of at least 20 repetitive measurements (gage not moved after the first measurement of material having density of $1250=50$ $\mathrm{Ih} / \mathrm{ft}(2)(1)()=80 \mathrm{~kg} / \mathrm{m}$ ) In order 10 periorm this procedure. the resolution of the count display. Calinration response. or other method of displayng densit! must he equal fo or hetter than $=0.1 \mathrm{hb} / \mathrm{fl}^{\circ}:=1.6 \mathrm{~h} \leqq \mathrm{~m}$ )

5.1.2 Cincmical Emw-The error due to changes in $6 \mathrm{sm}$ ical composition of materais haung gamma mass attenualion coefficients less irai ihose of grante or greater than those of limestone shali he not greater than $\pm 2 . . " \%$ of bact.seaner methods and $=2.2$, for direci transmusson with in:

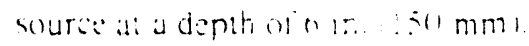


5.1.2.1 If the instrument was criginall: caliorated by the method described in 4.1.1. then the chemical error can be easily determined by using the standard block data which was taken to determine the gage response. Using the gage count rate on the limestone standard and the calibrated gage response. determine the gage density of the limestone standard. This will normally be higher than the true density. Compute the percent error. Repeat the same procedure for the granite standard. This will normally be lower than the true density. The difference between the two percent errors laking into account the sign of the error) divided by two will equal the chemical error.

5.1.2.2 If the gage was calibrated by some means other than 4.1 .1 or if the original calibration data are not available. then other means must be used to determine the chemical error. Using the procedure of 5.1.2.1. the relative densities of the limestone and granite standard blocks were not important. but using this procedure the standards should preferably be within $5 \mathrm{lb} / \mathrm{ft}^{3}\left(80 \mathrm{~kg} / \mathrm{m}^{3}\right)$ of each other in order to eliminate the possibility of an improper calibration which could cause good accuracy and small chemical error at the limestone and granite density but with large errors at all other densities. A high-density limestone or marble standard is suggested. First measure the density and compute the percent error on each standard. The chemical error is then the difference between the two errors divided by two. The sum of the two errors represents the calibration accuracy.

5.1.3 Surface Roughness Error-The error caused by a $0.050 \mathrm{in} .(1.3-\mathrm{mm})$ air gap introduced between the base of the gage and the surface of the material being measured should cause an error of no mure than $4 \%$ in the backscatter method nor more than $1 \%$ in the direct-transmission method with the source placed at a 6 -in. $(150-\mathrm{mm})$ depth. Older models of instruments currently in use may not meet this requirement. The users can minimize the effects of surface roughness by careful site preparation.

5.1.3.1 The effect of surface roughness can be measured by placing the instrument on a smooth flat surface after cleaning both the surface and the gage base and measuring the density. Next. elevate the gage by placing 0.050-in. (1.3$\mathrm{mm}$ ) spacers between the gage base and the material surface in such a way as to not interfere with the gage measurement zone. Remeasure the apparent density; the difference represents the gage error.

\section{Safety Precaution}

6.1 This equipment utilizes radioactive materials which may be hazardous to the health of the users. unless proper precautions are taken. Users of this equipment must become completely familiar with possible safery hazards and with all applicable government regulations. Effective operator instruction together with routine safety procedures such as source-leak tests, recording and elevation of film badge data. use of survey meters. etc.. are an essential part of the operation of equipment of this type.

\section{Apparatus}

7.1 The apparatus may consist of items to perform Method A-Backscatter. Method B-Direct Transmission. or a combination of both. Items listed are common to both apparatus except where noted as Method A or Method B only. Appa-

\section{2922}

ratus for Method C - Air Gap (optional) is detailed in Sectic. 11.

7.2 Gamma Source-An encapsulated and sealed radin ! active source meeting the specific form requirements of Tit:49 of the Code of Federal Regulations.

7.3 Gamma Detector-Any suitable type.

7.t Readou Device-A suitable scaler with a resolutioand range to display counts over the range of density fo; which the apparatus will be used. Usually the scaler will als: contain other electronic devices and the necessary electrica power for operation.

- 5 Housing-The source. detector. readout device. etc shall be in housings of rugged construction that are moisture. and dustproof. For Method B use. the housing shall contain a means of locating either the source of detector at a distance of 2 to $12 \mathrm{in}$. (50 to $300 \mathrm{~mm}$ ) into a preformed hole in the material to be tested. The probe containing the source or detector shall be sufficiently rigid so as to maintain a constant distance along the measuring path length and also contain markings to indicate the depth to which the probe has been placed.

VOTE 5-The gamma source. detector. readout device. and power supply may be housed separately or combined and integrated with a nuclear moisture-measuring system.

7.6 Reference Standard-A device that isolates the instrument and provides a means of allowing the instrument to make a measurement that is constant within the reproducibility of the system. All calibrations will be made as a ratio to the reference standard count and all field measurements will be taken as a ratio to the reference standard count.

-.7 Site-Preparation Devices:

7.7.1 Method A equipment shall include a flat plate straightedge. or other suitable tool to be used to level the test site to the required smoothness.

7.7.2 Method B equipment shall, in addition to the above. inciude a hole-forming device such as an auger or pin having a nominal diameter equal to or up to $1 / 8(3 \mathrm{~mm}$ ) larger than the probe and also a guide to ensure that the hole is perpendicular to the test site surface.

-8 Transport Case-Each system shall include a shipping and transport case to house the equipment and shall meet the U.S. Department of Transportation requirements in Title 49 of the Code of Federal Regulations. The exterior of the case shall contain all labels required by the regulations and radiation levels shall meet the "Yellow II" standards.

\section{Standardization}

3.1 All nuclear density instruments are subject to longterm aging of the radioactive source. detectors. and electronic systems. which may change the relationship between count rate and density. To offset this aging, all instruments are calibrated as a ratio of the measurement count rate to a count rate made on a reference standard. The reference count rate should be in the same order of magnitude or higher than the range of measurement count rates over the useful density range of the equipment.

3.2 Standardization of equipment on the reference standard is required at the stan of each day's use and a permanent record of these data should be retained.

s.j Turn on the equipment and allow for stabilization of 
the equipment in accordance with the manufacturer's recmmendations.

s. 4 Take at least four repetitive readings at the normal measurement period and obtain the mean. If available on the mstrument. one measurement at a period of four times the nomal period is acceptable. This constitutes one standard. intion check.

8.5 If the value obtained above is within the limits set by En 3. the equipment is considered to be in satisfactory contrion and may be used. If the value obtained is outside the tmits set by Eq 3. another standardization check should be made. If the second standardization check is within the limits. the equipment may be used: but if it also fails the test. the equipment should be checked as recommended by the manwacturer and the calibration checked (4.2) or recalibrated. or both.

$$
\therefore \quad \Lambda_{j}=\Lambda_{n} \pm 2.0 \sqrt{\Lambda_{o}} / P C
$$

where:

$A_{1}=$ value of current standardization check (8.4) on the reference standard (7.6).

$A_{r}=$ average of the past four values of $N_{3}$ taken for prior useage, and

$f C=$ amount of prescale applied to the detector counts prior to display. The manufacturer will supply this value. If no prescale is built into the equipment. the value is 1 .

8.6 The value of $\Lambda_{s}(8.4)$ will be used to determine the count ratios for the current day's use of the equipment. If. in any reason. measured densities become suspect during the day's use. another standardization should be performed.

TOTE 6-If the instrument is to be used either continuously or intermittently during the day. it is generally best to leave it in the "power 5: " or "standb!" condition durng the da! to prevent having to repeai tre standardization. This will provide more stable. consistent results.

SOTE 7-Standardization shall be performed in accordance uith the cunufacturer's recommendations and away from other radioactive xurces. large masses of metal or vertical objects. free water. or other coms that can affect the gage readings.

\section{S. Procedure. Method A-Backscatter}

9.1 Standardize the instrument (Section 8).

4.2 Select a test location where the gage in test position n:!l be at least 9 in. $(230 \mathrm{~mm})$ away from any verical Foluection.

4.3 Prepare the test site in the following manner:

4.3 .1 Remove all loose and disturbed material and addiunal material as necessary to expose the top of the material L c: tested.

Uite 8 - The snatial bias snouid he conscieres in deteminang the $=-2$ a which the gage is 10 be sialed.

?. Prepare a horizonia! area sufilelen: :n size lo accom

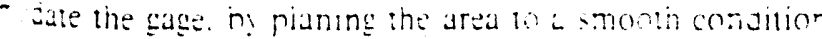
; as to obatin maximum contact nerwasl the sage and zilarial being lested

$\therefore \therefore$ The maximum void haneath the gage shall not cicecd

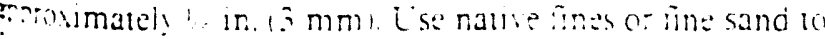

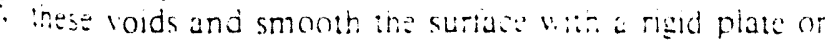
$\because \because$ suilatil: $100 !$

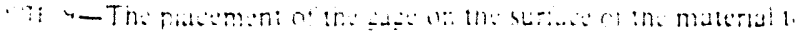

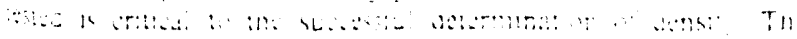

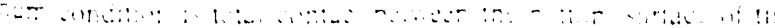

gage and the surface of the material being tested. This is not possible in all cases. To correct surface irregularities. use of native fines or sand as a filler is necessan. The depth of the filler should not exceed approximately is in. $(3 \mathrm{~mm})$ and the total area filled should not exceed $10 \%$ of the bottom area of the gage. Several trial seatings may be required to achieve these conditions.

9.4 Proceed with the test in the following manner:

9.4.1 Seat the gage firmly.

9.4.2 Keep all other radioactive sources away from the gage to avoid affecting the measurement

9.4.3 Secure and record one or more readings for the normal measurement period.

9.4.4 Determine the ratio of the reading to the standard count (9.1). From this ratio and the calibration. determine the in-place wet density.

NOTE 10-Some instruments have built-in provisions to compute the ratio. compute the in-place wet density. and display it to the operator automatically.

\section{Procedure, Method B - Direct Transmission}

10.1 Standardize the instrurnent (Section 8).

10.2 Select a test location where the gage in test position will be at least 9 in. $(230 \mathrm{~mm})$ away from any vertical projection.

10.3 Prepare the test site in the following manner:

10.3.1 Remove all loose and disturbed material. and remove additional material as necessary to expose the top of the material to be tested.

10.3.2 Prepare a horizontal area. sufficient in size to accommodate the gage. by planing the area of a smooth condition so as to obtain maximum contact between the gage and material being tested.

10.3.3 The maximum void beneath the gage shall not exceed approximately $1 / 8$ in. $(3 \mathrm{~mm})$. Lise native fines or fine sand to fill these voids and smooth the surface with a rigid plate or other suitable tool. The depth of the filler should not exceed approximately $1 / 8$ in. $(3 \mathrm{~mm})$.

10.3.4 Make a hole perpendicular to the prepared surface using the guide and the hole-forming device (7.6.2). The hole snall be of such depth and alignment that inserion of the probe will not cause the gage to tilt from the plane of the prepared area. The depth of the hole must $t$ at least 2 in. $150 \mathrm{~mm}$ ) deeper than the depth 10 which the probe will be placed. The guide shall be the same size as the base of the gage. with the hole in the same location on the guide as the probe on the gage. The corners of the guide are marked ti: scoring the surface of the soil.

10.4 Proceed with testing in the following manne:

10.4 .1 Set the gage on the soil suriace. carefull: aligning it with the marks so that tha prote will be directl over the prerormed hole.

$161 .+2$ lnsen the prona in the hoie.

10.4 .3 Seat the gage firmls hy rocating 11 about the probe with a hach and forth motion.

10.4 Pull sently on the suge in the direction thist "il! resne the side of the prothe agumst the sije of the hole clocest

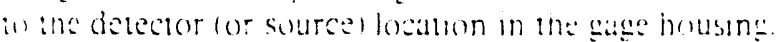

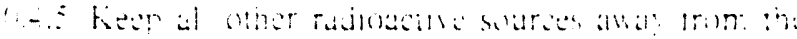

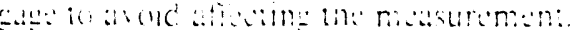

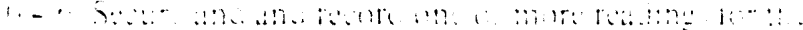
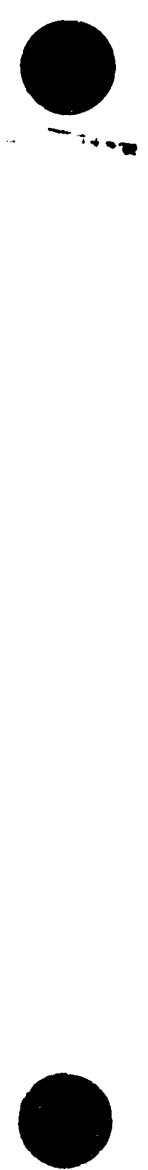
normal medsurement period

10.4.- Determine the ratio of the reading io the standard count $(10.1)$. From the ratio and the calibration. determine the in-place wet density.

\section{Procedure, Method C-Air Gap (Optional)}

11.1 If the nuclear gage errors due to calibration using materials with different chemical composition are in excess of maximum errors listed in 5.2. the air-gap method should be employed. It should be noted that the reciuired use of two different site measurements may decrease the precision due to the statistics of the air gap measurement.

11.2 .tpparatus:

11.2.1 All apparatus described in Section -

11.2.2 Cradle or Spacers, to suppor the gage at the optimum air gap above the material being tested. The cradles or spacers shall be so designed as to support the gage at optimum height without shielding the base of the gage. Figure ! shows a typical air-gap cradle that demonstrates the principle. The cradle shown in Fig. 1 is not the only satistactory method. Other methods which support the gage at the optimum air gap without shielding the base of the gage are satisfactory.

Nore 11-Air-gap calibration curves and optimum air gap may be furmished for each gage by the manufacturer and can re readily checked by the user.

11.3 Determination of Optimum Air Gap-To determine the optimum air gap for use in the air-gap method. proceed as follows:

11.3.1 Use three or more different areas on which to make detaminations. These areas may be either blocks (4.1.1) or fieid sites of compacted soil or soil-aggregate on which density data have been previously determined (4.1.2) or alternative prepared containers of soil or soil-aggregate compacted to known densities (4.1.2). The density of materials at the selected areas should vary through a range including the densities of the materials which will be tested.

11.3.2 Place the density gage over the test area. Support the gage by blocks placed at the extreme edges of the gage so as not to obstruct the space between the bottom of the gage and the surface of the test area.

11.3.3 Take and record readings for two normal measurement periods in counts per minute and determine the average of the readings.

11.3.+ By adding additional blocks or spacers. increase the air gap by $1 / 4$ in. $(6.3 \mathrm{~mm})$. Take and record. and average readings for two additional normal measurement periods.

11.3 .5 Continue increasing the air gap by increments of $1 / \%$ in.. securing average readings for each air gap (11.3.j) until there is a decrease in the counts per minute readings with an increase in air gap.

11.3.6 On an arithmetic scale. plot counts per minute as

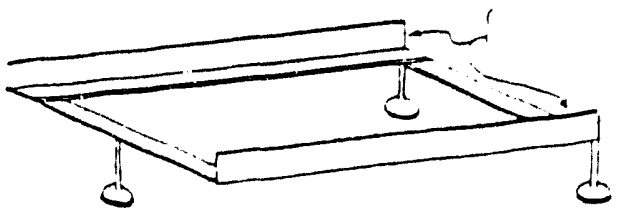

Weiced mital approx 1 by 1 by $1 / 8$ in. (25.4 by 254 jy $3.2 \mathrm{~mm}$ ) angle.

FIG. 1 Typical Air-Gap Cradle the ordinate iersus each air gap (in inches or millimetres) an draw a smooth curve through the resulting points. Recorc the peak air gap determined at the peak of the curve.

11.3.7 Repedt procedures 11.3.2 through 11.3 .6 over tws or more additional areas of materials of different density. anc record the peak air gap for each area.

11.3.8 Determine the average of the peak air gaps deter. mined on all areas. This is the optimum air gap. Use th: optimum air gap for establishing the calibration curce for the air-gap method. and for all determinations of density by th. air-gap method.

11.4 Procedure:

11.4.1 Standardize the instrument.

11.4.2 Select a test location where the gage in test position will be at least 12 in. $(300 \mathrm{~mm})$ away from any vertica projection. Plan sufficient area to accommodate the gage anc cradle.

11.4.3 Prepare the test site in the following manner:

11.4.3.1 Remove all loose and disturbed material. anc additional material as necessary to expose the top of the material to be tested. (See Note 8.)

11.4.3.2 Prepare a horizontal area. sufficient in size to accommodate the gage and cradle. by planing the area to 2 smooth condition so as to obtain maximum contact betweer. the gage and material being tested.

11.4.3.3 The maximum void beneath the gage shall no! exceed approximately $1 / 8$ in. $(3 \mathrm{~mm})$. Use native fines or fine sand to fill these voids and smooth the surface with a rigic plate or other suitable tool.

NOTE 12-The air-gap method requires taking one or more readirg in both the backscatter position and the air-gap position. The piacemen: of the gage on the surface of the material to be tested is critical to the successful determination of density. The optimum condition is tota contact between the bottom surface of the gage and the surtace of the material being tested. This is not possible in all cases and to correc. suriace irregularities use of sand or similar material as a filler is necessar: The depth of the filler should not exceed approximately $1 / 8 \mathrm{in} .(3 \mathrm{~mm}$ ! and the total area filled should not exceed $10 \%$ of the bottom area of the gage. Several trial seatings may be required to achieve these condi. tions.

11.4.4 Proceed with the test in the following manner:

11.4.4.1 Seat the gage firmly.

11.4.4.2 Keep all other radioactive sources away from the gage to avoid affec:ing the measurement so as not to affect the readings.

11.4.4.3 Secure and record one or more readings for the normal measurement period in the backscatter position.

11.4.4.4 Plice the cradle, set at optimum air gap. on the test site, and place the gage on the cradle so that the gage $1:$ directly over the same site used for backscatter reading. Wher. a direct-transmission type gage is used. set the probe in the retracted or backscatter position for this reading.

11.4.4.5 Take the same number of readings for the norma measurement period in the air-gap position as in the bach. scatter position (11.4.4.3).

11.4.4.6 Determine the air-gap ratio by dividing coun's per minute obtained in the airgap position (11.4.4.5) b! counts per minute obtained in backscatter position (11.4. +.3 )

11.4 .4 .7 Determine the in-place wet density by use of thit applicable calibration curve previously established.

Note 13-The arr.gap ratio may be determined by dividing count 
per minute obtained in the backscatter position by counts per mint: prtained in the air-gap position or vice versa. Whichever ratio is use - a calibration curve using the same ratio must also be used.

\section{Determination of Dry Density}

12.1 To obtain dry density, use one of the following altz:native methods:

12.1.1 If the water content is determined by nuclear me:ods. Test Method D 3017, subtract the pounds per cubic fos: $\left(\mathrm{kg} / \mathrm{m}^{3}\right)$ of moisture from the pounds per cubic foot $(\mathrm{kg} / \mathrm{m}:$ ) of wet density, and obtain dry density in pounds per cuais fool $\left(\mathrm{kg} / \mathrm{m}^{3}\right)$.

12.1.2 If the water content is determined by other methods. such as oven drying. Method D 2216. carbide method, ei:... and is in the form of percent, proceed as follows:

$$
\rho_{c}=\left[\rho_{m} /\left(w^{\prime}+100\right)\right] \times 100
$$

where:

$\rho_{\mathrm{d}}=\mathrm{dry}$ density, $\mathrm{lb} / \mathrm{ft}^{3}\left(\mathrm{~kg} / \mathrm{m}^{3}\right)$.

$\rho_{\mathrm{m}}=$ wet density, $\mathrm{lb} / \mathrm{ft}^{3}\left(\mathrm{~kg} / \mathrm{m}^{3}\right)$. and

${ }^{\prime \prime}=$ percent moisture in the specimen.

\section{Report}

13.1 The report shall include the following:

13.1.1 Location.

13.1.2 Elevation of surface.
13.1.3 Visual description of material.

13.1.4 Identification of test equipment (make. model. and serial number),

13.1.5 $N_{o}$, average of the past four values of $N_{s}$ taken for prior usage.

13.1.6 $\Lambda_{\mathrm{s}}$, value of the current standardization check (8.3) on the reference standard (7.5). and the method and date of standardization,

13.1.7 Count rate for each reading,

13.1.8 Count ratio,

13.1.9 Wet density.

NotE 14-The count rate for each reading (13.1.7) and the count ratio (13.1.8) may be omitted from the report for instruments that have built-in provisions to take the ratio. compute the in-place wet density, and display it to the operator automatically.

\section{Precision and Accuracy}

14.1 The precision and accuracy of this standard has not been determined. No methods are available that provide absolute values of the density of soil or soil-aggregate mixtures in place against which these methods can be compared. The variability of soil does not permit duplication of test results for obtaining a meaningful statistical evaluation. Accuracy is a function of the care exercised in performing the calibrations and steps of the test and of the variability of the material being tested.

\section{APPENDIX}

\section{X1. NOTES ON THE NUCLEAR TEST}

$X 1.1$ The equipment used in this method is of the surfa:e Iype as opposed to that designed for use in deep borings. In general. and neglecting the associated electronics. this equipment consists of three principal elements: ( $I$ ) a nuclear source emitting gamma rays. (2) a detector sensitive to these rays as they are modified by passing through the material beins lested. and (3) a counter or scaler with provisions for auts. matic and precise timing. for determining the rate at which ine modified gamma ray's arrive at the detector. While rate meters are suitable. in principle. scalers are commonly used. in general. any source of gamma rays that are sufficier:is numerous and properly energetic can be used in measurng ine density of soil and soil-aggregate. Source stability witin :tme. in terms of half-life. is an important design considerason and the sources most commonly used are cesium-i:and radium-226. The two detectors most commonly used 2:-2 "-3s-filled tubes of the Geiger-Muller type and scintillawn $\therefore$ stais. usually of sodium-iodide. Detectors of the latter t: pe Ser the potential of electronicall: varing the range $a$ targies of the gamma rays that are counted. With detectars the Geiger-Muller type this range is fined in the design. For most available equipment. the source-detector geome:? fixed for baciscatter gages and is adjustahle to various $\because$ relested depths of direct transmissiun guges.

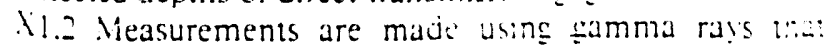
tyej: reflect at reduced energy h: sacisering in or hi dirs:

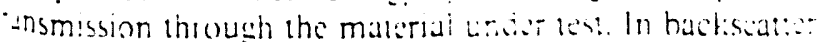

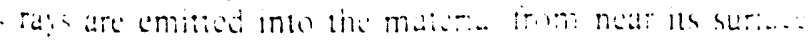

and some are deflected at reduced energy back to the detector, largely by Compton scattering. In direct transmission the source or detector is inserted in the test materials and. in contrast to the backscatter method. some of the emitted and unshielded ravs can presumably follow' a straight-line path to the detector. In either source-detector arrangement. the number of rays reaching the detector is. over-all. a nonlinear function of the density of the material being tested. For the usual range of soil and soil-aggregate densities the relationship is such that the higher the density of a given material. the low'er the count rate.

$\lambda 1.3$ The determination of density by the nuclear means of this method is indirect. To date no theoretical approach has been developed that predicts the count rate for given equipment. geometr. material. and density: As a result. the relationsin between material density and nuclear-count rate is determined bi correlation tests of materials at known average densities. Individual equipment manufacturers supply a calingation curve with each set of their equipment. It has been round that inese curves do not necessarily hold for all soils and soil-aggregates hecuuse of differences in chemical composition. Apparent variations in calibration may also be induced h! difierences in seatung. in hachground count. and niner lest variations. Because of these considerations. provisions are included in this methad for checking ior variations or changes. Different approdihe mas he used in cheching

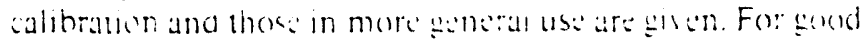

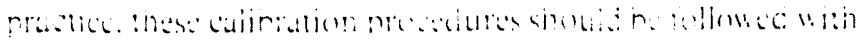




\title{
Standard Test Method for Capillary-Moisture Relationships for Coarse- and Medium- Textured Soils by Porous-Plate Apparatus ${ }^{1}$
}

\author{
This standard is issued under the fixed designation D 2325: the number immediately following the designatunn indicates the vear of \\ onginal adoption or. in the case of revision. the year of last revision. A number in parentheses indicates the year of last reapproval. A
superscript epsilon (e) indicates an editorial change since the last revision or reapproval. \\ Notr-Section 2 was added editorially and editorial changes were made throughout in Octoher 1984
}

\section{Scope}

1.1 This test method covers the determination of capillary. moisture relationships for coarse- and medium-textured soils as indicated by the soil-moisture tension relations for tensions between 10 and $101 \mathrm{kPa}(0.1$ and $1 \mathrm{~atm})$. Under equilibrium conditions. moisture tension is defined as the equivalent negative gage pressure. or suction, corresponding to a soil moisture content. This test method determines the equilibrium moisture content retained in a soil subjected to a given soil-water tension. This test method is not suitable for very fine-textured soils.

NOTE 1-For determination of capillary-moisture relationships for fine-textured soils, refer to Test Method D 3152.

\section{Referenced Documents}

2.1 . ASTM Standards:

D 421 Practice for Dry Preparation of Soil Samples for Particle-Size Analysis and Determination of Soil Constants ${ }^{2}$

D 698 Test Methods for Moisture-Density Relations of Soils and Soil-Aggregate Mixtures Using 5.5-lb (2.49-kg) Rammer and $12-\mathrm{in}$. (305-mm) Drop ${ }^{2}$

D 3152 Test Method for Capillary Moisture Relationships for Fine-Textured Soils by Pressure-Membrane Apparatus $^{2}$

\section{Summary of Method}

3.1 Saturated soil samples are placed in contact with a saturated porous plate installed within a pressure chamber. The bottom of each plate is covered by a rubber membrane. or otherwise sealed to be airtight. The bottom of each plate is maintained at atmospheric pressure by means of a small drain tube or opening through the side of the pressure chamber. A desired air pressure admitted to the pressure chamber. and consequently to the top of the porous plate, creates a pressure drop across the porous plate. The saturated soil samples on the plates establish equilibrium with the water in the plate. The water. held at a tension less than the pressure drop acioss the porous plate, will then move out of the soil, through the plate, and out through the drain tube. When water has ceased to flow from the sample and porous plate

\footnotetext{
'This test method is under the jurisdiction of ASTM Committee D-18 on Soil and Rock and is the direct responsibility of Subcommittee D18.04 on Hydrologic
Properies of Soils and Rocks.

Current edition approved Sept. 13, 1968. Originally issued as D 2325-64 T. Last previous edition D $2325-64 \mathrm{~T}$.

: tinnual Book of tSTM Slandards, Vol 04.08.
}

(indicating equilibrium for that particular tension), the mois ture content of each sample is determined. A series of thes. tests at various tensions is required to prepare a complet: curve of the capillary-moisture relationship for any particula:
soil.

\section{Apparatus}

4.1 An assembly of the apparatus is shown in Fig. 1.

4.1.1 Porous Plate Apparatus, consisting of the following:

4.1.1.1 Pressure Container. (such as a pressure cooker), ot approximately 15-L (16-qt) capacity.

4.1.1.2 Porous Ceramic Plates, 1 to 4 (see Fig. 2), approx. imately $280 \mathrm{~mm}$ (111/4 in.) in diameter and $6 \mathrm{~mm}(1 / 4 \mathrm{in}$.) in thickness. with an air entry value of $203 \mathrm{kPa}(2 \mathrm{~atm})$.

4.1.1.3 Brass Spout-The brass spout (one per porous plate) shall consist of a brass tube and associated washers. gaskets, and brass nuts. It shall provide an airtight joint when inserted through the porous plate $38 \mathrm{~mm}$ (1.5 in.) from the edge of the plate. The length of the unthreaded portion of the brass tube shall be $9.5 \mathrm{~mm} \mathrm{(3/8} \mathrm{in.);} \mathrm{the} \mathrm{length} \mathrm{of} \mathrm{the} \mathrm{threaded}$ portion shall be $15.8 \mathrm{~mm}(\% / \mathrm{s}$ in.); the inside diameter of the tube shail be $1.7 \mathrm{~mm}(1 / 16 \mathrm{in}$.); the outside diameter of the upper unthreaded portion shall be $4 \mathrm{~mm}(5 / 32 \mathrm{in}$.); the outside diameter of the lower threaded portion shall be $4.8 \mathrm{~mm}(3 / 16$ in.). The tap size for the hole through the porous plate shall be $5.5 \mathrm{~mm}(7 / 32 \mathrm{in}$.).

4.1.1.4 Disks of 10-mesh Brass Screen, from 1 to 4, of slightly smaller diameter than bottom of porous ceramic plates.

4.1.1.5 Rubber Membrane-The membrane shall consist of sheet neoprene, $0.79 \mathrm{~mm}(1 / 32 \mathrm{in}$.) in thickness, with a hardness of 35 by the Shore Durometer. Place a disk of brass screen over the bottom of each porous ceramic plate to provide space for the flow of water between the membrane and the ceramic plate (see Fig. 2). Then place the rubber membrane snugly over the brass screen. glue it securely to the outer edge of the ceramic plate, and wrap the edge tightly
with wire (see Fig. 2).

4.1.1.6 Tubing-A flexible tubing tube, $3 \mathrm{~mm}(1 / 8 \mathrm{in}$.) in diameter, to carry the outflow water from the brass spout on each porous piate to a short length of rigid tubing passing through a rubber stopper installed in the wall of the pressure container.

4.1.1.7 Assembly-Support and separate plates by means of plastic plugs approximately $15 \mathrm{~mm}(0.6 \mathrm{in}$.) in diameter by $25 \mathrm{~mm}$ ( 1 in.) in length.

4.1 .2 Sample Retainer Rings-Rigid plastic rings, $10 \mathrm{~mm}$ 

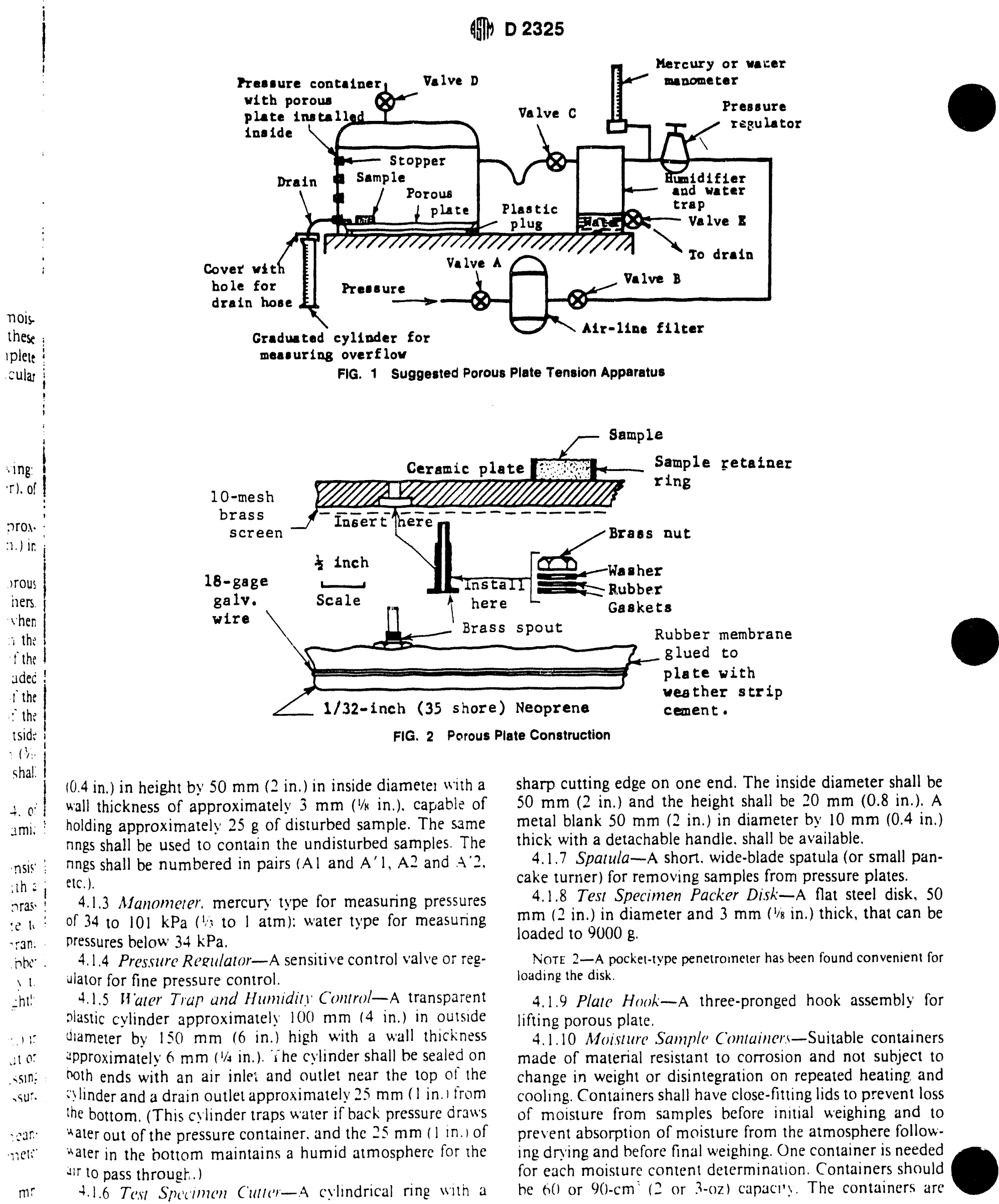

FIG. 2 Porous Plate Construction

$0.4 \mathrm{in.}$ ) in height by $50 \mathrm{~mm}(2 \mathrm{in}$.$) in inside diametes with a$ wall thickness of approximately $3 \mathrm{~mm} \mathrm{(1/8} \mathrm{in.).} \mathrm{capable} \mathrm{of}$ holding approximately $25 \mathrm{~g}$ of disturbed sample. The same nngs shall be used to contain the undisturbed samples. The nngs shall be numbered in pairs ( $A$ ) and $A^{\prime} 1, A 2$ and $A^{\prime} 2$,

4.1.3 Manometer, mercury type for measuring pressures easuring pessures below $34 \mathrm{kPa}$.

lator for fine pressure control.

4.1.5 Water Trap and Humidity Control-A transparent plastic cylinder approximately $100 \mathrm{~mm}(4 \mathrm{in}$.) in outside diameter by $150 \mathrm{~mm}$ (6 in.) high with a wall thickness pproximately $6 \mathrm{~mm}(1 / 4 \mathrm{in}$.). The cylinder shall be sealed on oth ends with an air inlei and outlet near the top of the alinder and a drain outlet approximately $25 \mathrm{~mm}(1)$ in. 1 from the bottom. (This cylinder traps water if back pressure draws ater out of the pressure container. and the $25 \mathrm{~mm} / 1$ in.) of 4.1.6 Test Spcimen Cutler-A cylindrical ring with a sharp cutting edge on one end. The inside diameter shall be $50 \mathrm{~mm}(2 \mathrm{in}$.) and the height shall be $20 \mathrm{~mm}(0.8 \mathrm{in}$.). A metal blank $50 \mathrm{~mm}(2 \mathrm{in.})$ in diameter by $10 \mathrm{~mm}(0.4 \mathrm{in}$. thick with a detachable handle. shall be available.

4.1.7 Spatula-A short. wide-blade spatula (or small pancake turner) for removing samples from pressure plates.

4.1.8 Test Specimen Packer Disk-A flat steel disk, 50 $\mathrm{mm}(2 \mathrm{in}$.) in diameter and $3 \mathrm{~mm}(1 / 8 \mathrm{in}$.) thick, that can be loaded to $9000 \mathrm{~g}$.

NOTE 2-A pocket-type penetrometer has been found convenient for loading the disk

4.1.9 Plate Hook-A three-pronged hook assembly for lifting porous plate.

4.1.10 Moisture Sample Container:-Suitable containers made of material resistant to corrosion and not subject to change in weight or disintegration on repeated heating and cooling. Containers shall have close-fitting lids to prevent loss of moisture from samples before initial weighing and to prevent absorption of moisture from the atmosphere following dring and before final weighing. One container is needed for each moisture content determination. Containers should he 6() or 9()$-\mathrm{cm}^{*}(2$ or $3-\mathrm{oz})$ capacily. The containers are 
CAPILLARY.MOISTLRE RELATIONS FOR SOILS

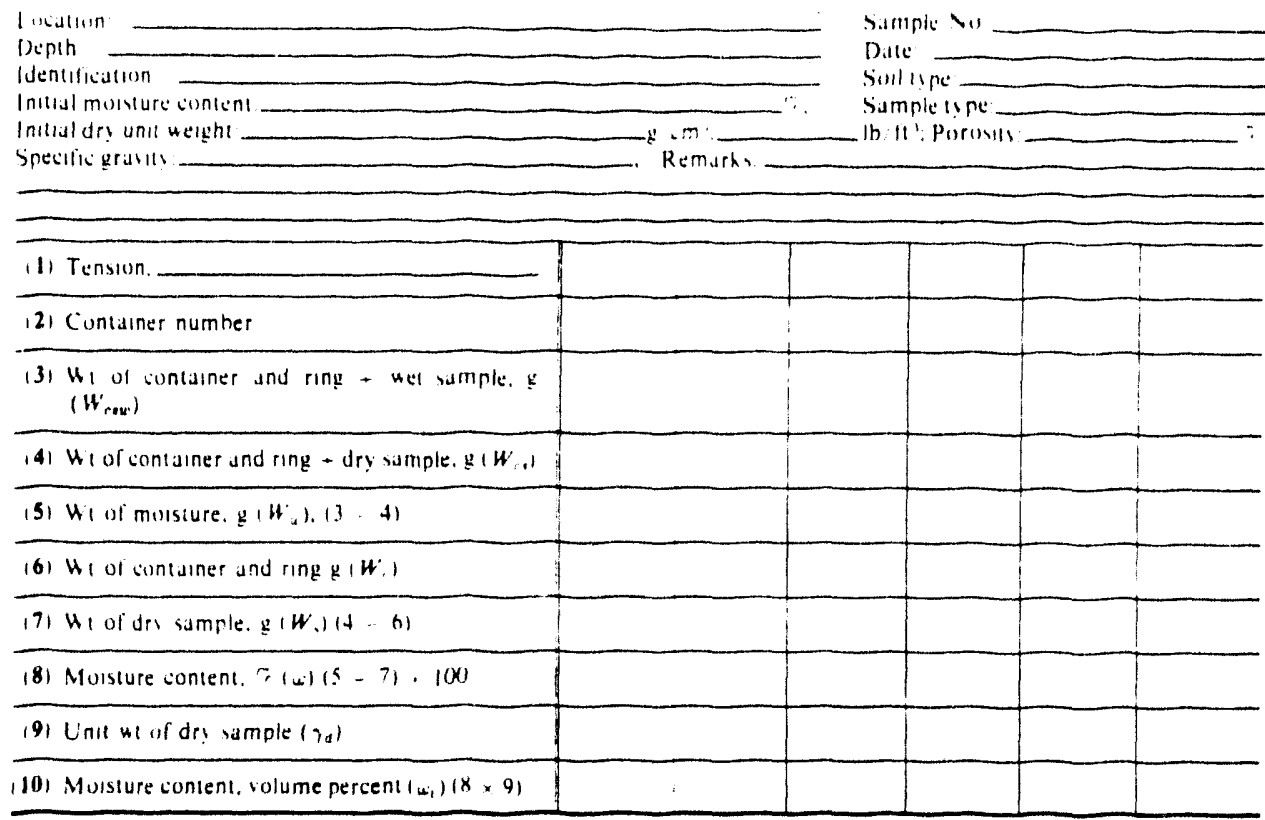

FIG. 3 Laboratory Form for Capillary Moisture Relations for Soils

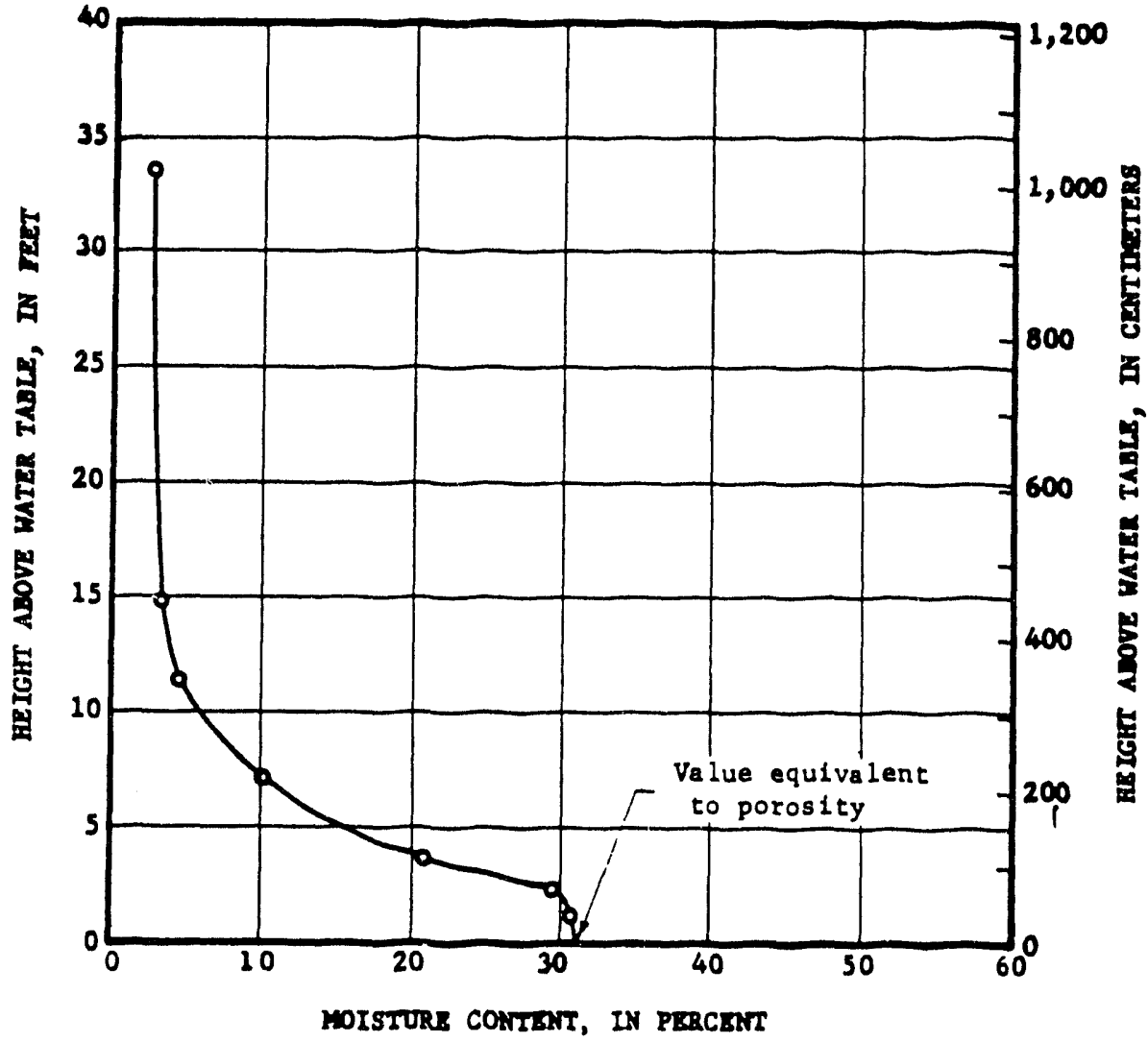

FIG. 4 Example of Data on Capillary-Moisture Relations of Soils 
numbered in pairs to coincide with the retainer rings.

4.1.11 Saturation Tray-A waterproof tray about $30 \mathrm{~mm}$ (1.2 in.) in depth, large enough to hold at least 4 porous plates while samples are being saturated thereon.

4.1.12 Balance-A balance with a capacity of at least 200 $\mathrm{g}$ and sensitive to $0.01 \mathrm{~g}$.

4.1.13 Desiccator-A desiccator of suitable size to hold samples for cooling after removal from the oven.

4.1.14 Oven-A thermostatically controlled drying oven capable of maintaining temperatures at $110 \pm 5^{\circ} \mathrm{C}(230 \pm$ $\left.9^{\circ} \mathrm{F}\right)$.

4.1.15 Trimmers-Wire saw, bevelled straightedge, spatula, and other small tools for trimming the test specimen.

\section{Samples}

5.1 Make tests in duplicate on specimens cut from undisturbed or remolded samples or on specimens packed from loose disturbed samples. Place duplicates in paired retainer rings diametrically opposite each other on the pressure plate.

\section{Preparation of Test Specimens from Disturbed Samples}

6.1 Take a sample weighing approximately $25 \mathrm{~g}$ from the thoroughly mixed portion of the air-dried soil passing the $2.00-\mathrm{mm}$ (No. 10) sieve, which has been obtained in accordance with Practice D 421.

6.2 Pour each sample into a retainer ring, pack, and level to fill the ring by pressing the top surface with the test specimen packer disk, using an applied force of $9000 \mathrm{~g}$. Record on the report form the sample type. disturbed, and the numbers of the paired sample retainer rings containing the duplicate samples.

\section{Preparation of Test Specimens from Undisturbed Samples}

7.1 Cut a block of the material, from which the test specimen is to be prepared, with two plane faces. Determine and record the natural moisture content and dry unit weight of the sample block. Also record the direction (perpendicular or transverse) of the sampling in relation to the structural or depositional lavers.

7.2 Place the test specimen cutter, with the cutting edge downward. on top of one of the plane faces and force the cutter down lightly and gradually as excess material is trimmed from the outside, using the minimum pressure required on the cutter. The trimming motions shall be from the cutter outward and downward, leaving a column of soil slightly larger than the outside diameter of the cutting edge. When the cutter is more than half full of soil. remove the excess at the bottom with the wire saw, invert the cutter and use a straightedge to make the soil flush. Invert the cutter again, place it on the smooth face of the metal blank. and carefully force it downward until the blank is flush. Remove the excess soil at the top with the wire saw. true the end with the straightedge, and remove the blank by means of the detachable handle.

7.3 Place the specimen cutter with the specimen downward. over a retainer ring, and use the metal blank to gently insert the test specimen into the ring. Record on the report the sample type, undisturbed. and the numbers of the paired sample retainer rings containing the duplicate samples.

7.4 Maintain the samples in closed containers until time for testing.

\section{Preparation of Test Specimens from Compacted Samples}

8.1 Compact the sample to a density and moisture content desired for anticipated service conditions in accordance with Test Methods D 698.

8.2 After the compacted sample has been ejected from the compaction mold, cut the test specimen by the process used for undisturbed samples as described in 7.1 through 7.4. Record on the report form, the sample type, compacted, and the numbers of the paired sample retainer rings containing the duplicate samples.

\section{Saturating and Testing of Porous Plates}

9.1 Install, by stacking with plastic plugs for spacers, as many porous plates in the pressure container as are to be used in the test. Fill the container with water, place the lid on the container and lock it in the closed position.

9.2 Close valves $C$ and $E$, open valves $A$ and $B$, and set the pressure at $101 \mathrm{kPa}$ or $776 \mathrm{mmHg}$ (1 atm or $15 \mathrm{psi}$ ) by adjusting the control valve on the pressure regulator and noting the pressure on the mercury manometer. Open valve $C$ on the water trap cylinder. The measured rate of water outflow from the porous plates should be at least $10 \mathrm{~mL} / \mathrm{min}$ for satisfactory operation of the plates.

9.3 Check the plates for air-entry value, as follows: release the air pressure by closing value $C$, and by opening valve $D$ on the lid, and empty the excess water from the pressure container and plates. Close and lock the lid of the container and apply the desired pressure. After approximately $10 \mathrm{~min}$ at this pressure, the outhlow of water from the plate outlets should cease and there should be no bubbling of air from these outlets. This will indicate that the entry values for the plates are above the value of the applied pressure.

9.4 If trouble is encountered in air-pressure control, submerge the pressure container in water, with the pressure still on, to check for leaks in the lid gasket or container connections.

9.5 Exercise care that the pressure is released by means of valve $D$ before the lid is opened or injury may occur to the operator or damage to the container.

\section{Procedure}

10.1 Place the required number of saturated porous plates in the saturation tray, one porous plate for approximately 12 sample retainer rings. Place the retainer rings containing duplicate samples prepared as described under Sections 6.7 and 8 , on a porous plate. locating duplicate samples diametrically opposite each other.

10.2 Place a control sample retainer ring in the center of the porous plate. In this retainer ring insert a disk of porous stone with standard sample dimensions, or pour and pack into this retainer ring a control sample consisting of a medium-textured soil with approximately equal parts of sand-, silt-, and clay-size particles. Record on the report form the number of the sample retainer ring containing the control sampit.

Ir 4 Thoroughly saturate the samples by pouring $3 \mathrm{~mm}$ $(\%, n$.) of water on each plate and gradually increasing the $\therefore$ pth of water over a minimum period of several hours until the water is at the top edge of the sampie. Hold the water at this depth for at least $24 \mathrm{~h}$. Place surcharge weights equivalent
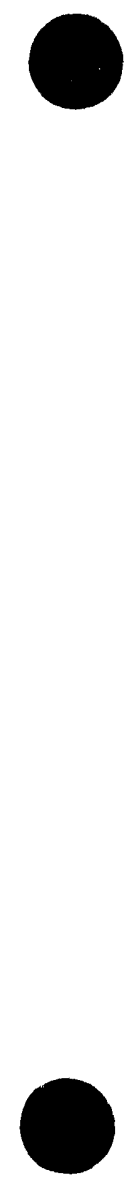
D 2325

TABLE 1 Pressure Conversion Factors

\begin{tabular}{|c|c|c|c|c|c|c|}
\hline \multirow[b]{2}{*}{ Tension (atmospherer } & \multicolumn{4}{|c|}{ Equivalent Pressure } & \multicolumn{2}{|c|}{ Capillary Head } \\
\hline & $\begin{array}{l}\text { Pounds per square } \\
\text { inch (psi) }\end{array}$ & $\begin{array}{c}\text { Millimetres of } \\
\text { mercury }\end{array}$ & Millimetres of water & Kilopascais & Feet of water & $\begin{array}{c}\text { Centimetres of } \\
\text { water }\end{array}$ \\
\hline $\begin{array}{l}0.1 \\
0.2 \\
0.3 \\
0.33 \\
0.4 \\
0.5 \\
0.6 \\
0.7 \\
0.8 \\
0.9 \\
1.0\end{array}$ & $\begin{array}{r}1.5 \\
2.9 \\
4.4 \\
5.3 \\
5.9 \\
7.4 \\
8.8 \\
10.3 \\
11.8 \\
13.2 \\
14.7\end{array}$ & $\begin{array}{r}76 \\
152 \\
228 \\
253 \\
304 \\
380 \\
456 \\
532 \\
608 \\
684 \\
760\end{array}$ & $\begin{array}{cc}1 & 033 \\
2 & 066 \\
3 & 099 \\
3 & 440 \\
4 & 132 \\
5 & 165 \\
6 & 198 \\
7 & 231 \\
8 & 264 \\
9 & 297 \\
10 & 330\end{array}$ & $\begin{array}{r}10 \\
20 \\
30 \\
33 \\
40 \\
51 \\
61 \\
71 \\
81 \\
91 \\
101\end{array}$ & $\begin{array}{c}3.4 \\
6.8 \\
10.2 \\
11.3 \\
13.6 \\
16.9 \\
20.3 \\
23.7 \\
27.1 \\
30.5 \\
33.9\end{array}$ & $\begin{array}{l}103.3 \\
206.6 \\
309.9 \\
340.9 \\
413.2 \\
516.5 \\
619.8 \\
723.1 \\
826.4 \\
929.7 \\
1033.0\end{array}$ \\
\hline
\end{tabular}

to field overburden weight on top of the samples during the soaking period.

10.4 Remove the excess water from the plates with a suction hose or syringe. Place the plates in the pressure container with each plate supported by the $25-\mathrm{mm}$ (1-in.) high plastic blocks. Insert the outflow tubes in perforated rubber stoppers in outlet holes where the plates are used; insert solid rubber stoppers in the holes where plates are not used. Place the container lid in position and lock it in the closed position.

10.5 Close valve $D$ on the container lid and valve $E$ on the water-trap outlet. Open air-control valves $A$ and $B$, and adjust the pressure regulator until the desired pressure (Table 1 ) is observed on the mercury or water manometer.

10.6 Open valve $C$ on the water trap outlet and admit the pressure to the pressure container. Allow the water from the outflow tubes to flow into $10-\mathrm{mL}$ graduates so it can be noted when moisture equilibrium is obtained, at which time the test is discontinued. It may take 18 to $48 \mathrm{~h}$ for some soils to reach this equilibrium. Consider equilibrium to be reached when no water flows out of the outlet tubes during $a, 1 / 2-h$ period. As each plate of samples reaches equilibrium, place a pinch clamp on each outflow tube to prevent backflow of water to the samples when the pressure is released.

10.7 Close valve $C$ and release the air pressure by opening valve $D$ on the lid of the pressure chamber and remove the lid.

10.8 Lift each plate out by means of the plate hook. By means of the wide-blade spatula, quickly transfer the samples to the sample containers, and immediately weigh them on a balance. Record the weight of moist sample plus container $\left(W_{c s w}\right)$ on the report form.
10.9 Dry the samples to constant weight in an oven at $1 / \mathrm{C}$ $\pm 5^{\circ} \mathrm{C}\left(230 \pm 9^{\circ} \mathrm{F}\right)$. Weigh the samples and record weights of oven-dry samples plus containers $\left(W_{c s}\right)$ on the report form.

10.10 Follow the above procedure until moisture contents have been obtained for at least 5 different tensions between 10 and $101 \mathrm{kPa}(0.1$ and $1 \mathrm{~atm})$.

\section{Calculations}

11.1 Calculate the moisture content of the soil, $w$, in percent as follows:

$$
W^{\prime}=\left[\left(W_{c s w}-W_{c s}\right) /\left(W_{c s}-W_{c}^{\prime}\right)\right] \times 100=\left(W_{w} / W_{s}\right) \times 100
$$

where:

$W_{c s w}=$ weight of container, ring and wet sample, $\mathrm{g}$,

$W_{c s}=$ weight of container, ring and dry sample, $\mathrm{g}$,

$W_{c}$ = weight of container and ring, $\mathrm{g}$,

$W_{w}=$ weight of water, $g$, and

$W_{s}=$ weight of dry soil, $\mathrm{g}$.

11.2 Obtain the moisture content in volume percent by multiplying the moisture content by the dry unit weight (g) $\left.\mathrm{cm}^{3}\right)$.

\section{Report}

12.1 Report the moisture content, tension data, and calculations on the form "Capillary-Moisture Relations for Soils" (Fig. 3).

12.2 Plot the moisture content and tension data on a graph similar to that shown in Fig. 4. Extrapolate the curve to the total porosity (converted to percent of dry weight) on the zero tension line. If desired, the moisture data can also be converted to moisture content in volume percent or to degree of saturation, but this should be clearly identified on the graph (Fig. 4). if not revised, either reapproved or withdrawn. Your comments are imvited either for revision of this standard or for additional standards and should be addressed to ASTM Headquarters. Your comments will recerve careful consideration at a meeting of the responsible technical committee, which you may attend. If you feel that your cemments heve not received a fair hearing you should make your views known to the ASTM Committee on Standards, 1916 Race St., Philadelphia, PA 19103. 


\title{
Standard Test Method for Permeability of Granular Soils (Constant Head) ${ }^{1}$
}

\author{
This standard is issued under the fixed designation D 2434: the number immediately following the designation indicates the year of \\ original adoption or, in the case of revision. the year of last revision. A number in parentheses indicates the year of last reapproval. A \\ superscript epsilon ( $($ ) indicates an editorial change since the last revision or reapproval. \\ "NotE-Section 2 was added editorially and subsequen: sections renumbered in July 1984.
}

\section{Scope}

1.1 This test method covers the determination of the coefficient of permeability by a constant-head method for the laminar flow of water through granular soils. The procedure is to establish representative values of the coefficient of permeability of granular soils that may occur in natural deposits as placed in embankments, or when used as base courses under pavements. In order to limit consolidation influences during testing, this procedure is limited to disturbed granular soils containing not more than $10 \%$ soil passing the $75-\mu \mathrm{m}$ (No. 200) sieve.

\section{Referenced Documents}

\subsection{ASTM Standards:}

D 42? Method for Particle-Size Analysis of Soils ${ }^{2}$

D 2049 Test Method for Relative Density of Cohesionless Soils ${ }^{3}$

\section{Fundamental Test Conditions}

3.1 The following ideal test conditions are prerequisites for e laminar flow of water through granular soils under contant-head conditions:

3.1.1 Continuity of flow with no soil volume change during a test,

3.1.2 Flow with the soil voids saturated with water and no air bubbles in the soil voids,

3.1.3 Flow in the steady stute with no changes in hydraulic gradient. and

3.1.4 Direct proportionality of velocity of flow with hydraulic gradients below certain values. at which turbulent now' starts.

3.2. All other types of flow involving partial saturation of soil voids. turbulent flow, and unsteady state of now are iransient in character and yield variable and time-dependent coefficients of permeability: therefore, they require special test conditions and procedures.

\section{Apparatus}

4.1 Permeamelers, as shown in Fig. 1, shall have specimen $\therefore$ linders with minimum diameters approximately 8 or 12 :mes the maximum particle size in accordance with Table 1. The permeameter should be fitted with: (I) a porous dist or

This test method is under the jurisdicuon of ASTM1 Commitlee D. 18 on Soll $\because$ Kook and is the direct responsibilily of Subcommultee DIX.14 on Hydrolingli "unies of Soil and Rocls

Curtent edition approved Sepl. 13, 1968. Originalls issued 1465. Reptaces $\therefore i-65 T$.

imnal Borsh of AST.M Slandards Vol (14.118.

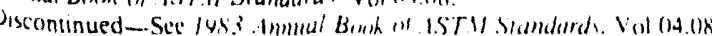

suitable reinforced screen at the bottom with a permeability greater than that of the soil specimen, but with openings sufficiently small (not larger than $10 \%$ finer size) to prevent movement of particles; ( 2 ) manometer outlets for measuring the loss of head, $h$, over a length, $l$, equivalent to at least the diameter of the cylinder; (3) a porous disk or suitable reinforced screen with a spring attached to the top, or any other device, for applying a light spring pressure of 22 to $45-\mathrm{N}$ (5 to $10-1 \mathrm{~b}$ ) total load, when the top plate is attached in place. This will hold the placement density and volume of soil without significant change during the saturation of the specimen and the permeability testing to satisfy the requirement prescribed in 3.1.1.

4.2 Constant-Head Filter Tank, as shown in Fig. 1, to supply water and to remove most of the air from tap water. fitted with suitable control valves to maintain conditions described in 3.1.2.

Note 1-De-aired water may be used if preferred.

4.3 Large Funnels, fitted with special cylindrical spouts 25 $\mathrm{mm}$ ( 1 in.) in diameter for $9.5-\mathrm{mm}(3 / 8$-in.) maximum size particles and $13 \mathrm{~mm}(1 / 2 \mathrm{in}$.) in diameter for $2.00-\mathrm{mm}$ (No. 10) maximum size particles. The length of the spout should be greater than the full length of the permeability chamberat least $150 \mathrm{~mm}$ (6 in.).

4.4 Specimen Compaction Equipment-Compaction equipment as deemed desirable may be used. The following are suggested: a vibrating tamper fitted with a tamping foot $51 \mathrm{~mm}$ ( 2 in.) in diameter; a sliding tamper with a tamping foot $51 \mathrm{~mm}(2 \mathrm{in}$.) in diameter, and a rod for sliding weights of $100 \mathrm{~g}(0.25 \mathrm{lb})$ (for sands) to $1 \mathrm{~kg}(2.25 \mathrm{lb})$ (for soils with a large gravel content). having an adjustable height of drop to $102 \mathrm{~mm}(4 \mathrm{in}$.) for sands and $203 \mathrm{~mm}$ ( $8 \mathrm{in}$.) for soils with large gravel contents.

4.5 l'acuum Pump or Water-Faucet Aspirator, for evacuating and for saturating soil specimens under full vacuum (see Fig. 2).

4.6 Manometer Tubes, with metric scales for measuring head of water.

4.7 Balance, of 2-kg (4.4-lb) capacity, sensitive to $1 \mathrm{~g}(0.002$ Ib).

$4.8 S c o(p)$, with a capacity of about $100 \mathrm{~g}(0.25 \mathrm{lb})$ of soil.

4.9 Miscellaneons dpparatus-Thermometers, clock with sweep second hand. $250 \mathrm{~mL}$ graduate. quart jar, mixing pan. etc.

\section{Sample}

5.1 A representative sample of air-dried granular soil, containing less than $10 \%$ of the material passing the $7.5-\mu \mathrm{m}$ (No. 
AfIN D 2434

\begin{tabular}{|c|c|c|c|c|}
\hline \multirow{3}{*}{$\begin{array}{c}\text { Maximum } \\
\text { Particle Size } \\
\text { Lies Between } \\
\text { Sieve Openings }\end{array}$} & \multicolumn{4}{|c|}{ Min!mum Cylinder Diameter } \\
\hline & \multicolumn{2}{|c|}{$\begin{array}{l}\text { Less than } 35 \% \text { of Total Soil } \\
\text { Retained on Sieve Opening }\end{array}$} & \multicolumn{2}{|c|}{$\begin{array}{l}\text { More then } 35:=0 \text { ! Tota: } S: \\
\text { Retained on Sieve Oderm }\end{array}$} \\
\hline & $200 \cdot \mathrm{mm}$ (No. 10) & $9.5-\mathrm{mm}(3 / \mathrm{s}-\mathrm{in})$. & $2.00-\mathrm{mm}$ (No. 10$)$ & $g=-m l_{1} a_{0}$. \\
\hline $\begin{array}{l}2.00-\mathrm{mm}(\text { No. } 10) \text { and } 9.5 \cdot \mathrm{mm}(3 / \mathrm{g}- \\
\text { in.) }\end{array}$ & $76 \mathrm{~mm}(3 \mathrm{in})$. & $\ldots$ & $114 \mathrm{~mm}(4.5 \mathrm{in})$. & \\
\hline $\begin{array}{l}9.5-\mathrm{mm}(3 / 0-\mathrm{in}) \text { and } 19.0-\mathrm{mm}(3 / 4 . \\
\text { in.) }\end{array}$ & & $152 \mathrm{~mm}$ ( $6 \mathrm{in})$. & $\therefore$ & $22=\mathrm{mm} 19-$ \\
\hline
\end{tabular}

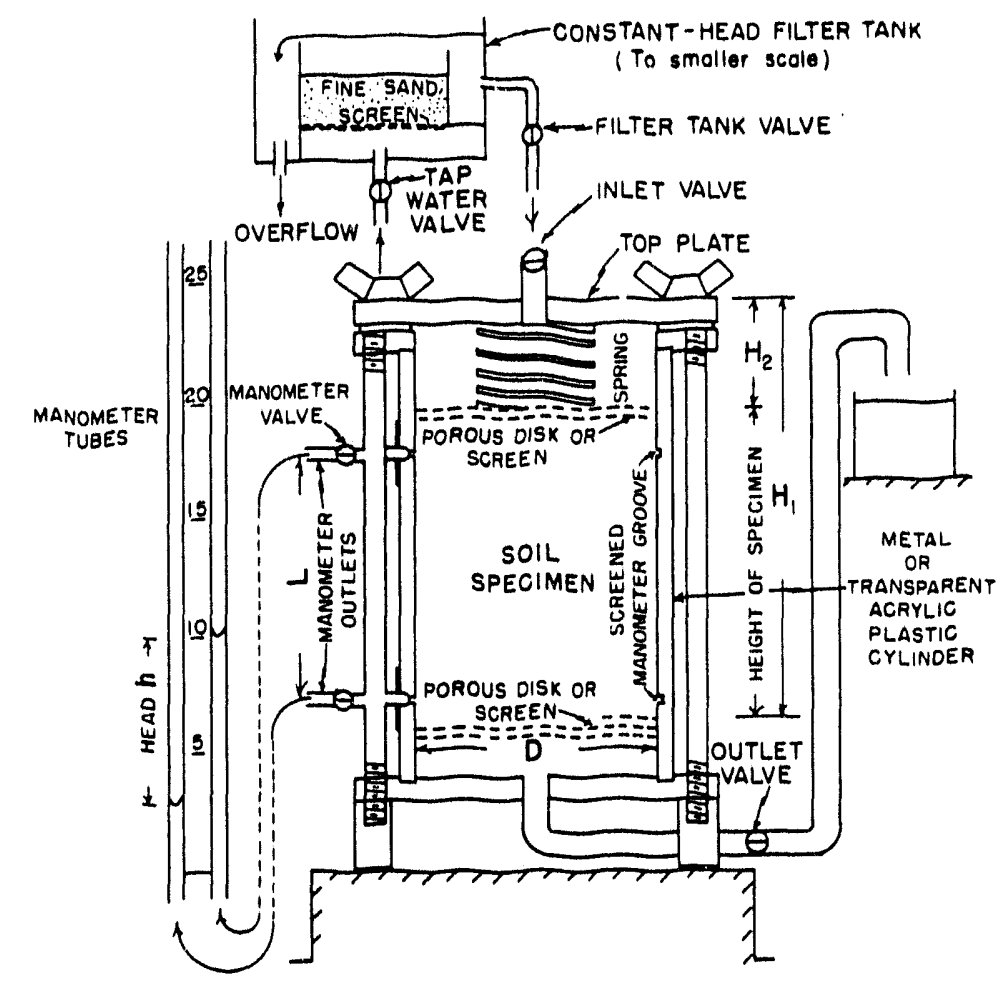

FIG. 1 Constant-Head Permeameter

200) sieve and equal to an amount sufficient to satisfy the requirements prescribed in 5.2 and 5.3, shall be selected by the method of quartering.

5.2 A sieve analysis (see Muthod D 422) shall be made on a representative sample of the complete soil prior to the permeability test. Any particles larger than $19 \mathrm{~mm}(3 / 4 \mathrm{in}$.) shall be separated out by sieving (Method D 422). This oversize material shall not be used for the permeability test, but the percentage of the oversize material shall be recorded.

Note 2-In order to establish representative values of coefficients of permeabilities for the range that may exist in the situation being investigated, samples of the finer. average. and coarser soils should be obtained for testing.

5.3 From the material from which the oversize has been removed (see 5.2), select by the method of quartering, a sample for testing equal to an amount approximately twice that required for filling the permeameter chamber.

\section{Preparation of Specimens}

6.1 The size of permeameter to be used shall be as prescribed in Table 1.
6.2 Make the following initial measurements in centimetres or square centimetres and record on the data sheet (Fig. 3); the inside diameter, $D$, of the permeameter; the length, $L$, between manometer outlets; the depth, $H_{1}$, measured at four symmetrically spaced points from the upper surface of the top plate of the permeability cylinder to the top of the upper porous stone or screen temporarily placed on the lower porous plate or screen. This automatically deducts the thickness of the upper porous plate or screen from the height measurements used to determine the volume of soil placed in the permeability cylinder. Use a duplicate top plate containing four large symmetrically spaced openings through which the necessary measurements can be made to determine the average value for $H_{1}$. Calculate the crosssectional area, $A$, of the specimen.

6.3 Take a small portion of the sample selected as scribed in $\mathbf{5 . 3}$ for water content determinations. Recor weight of the remaining air-dried sample (see 5.3), $W_{1}$ unit weight determinations.

6.4 Place the prepared soil by one of the following proce- 


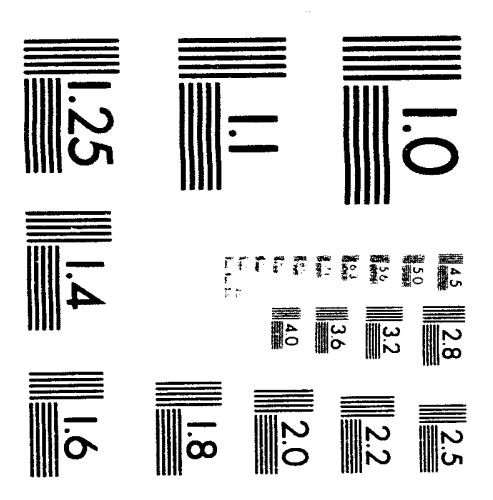



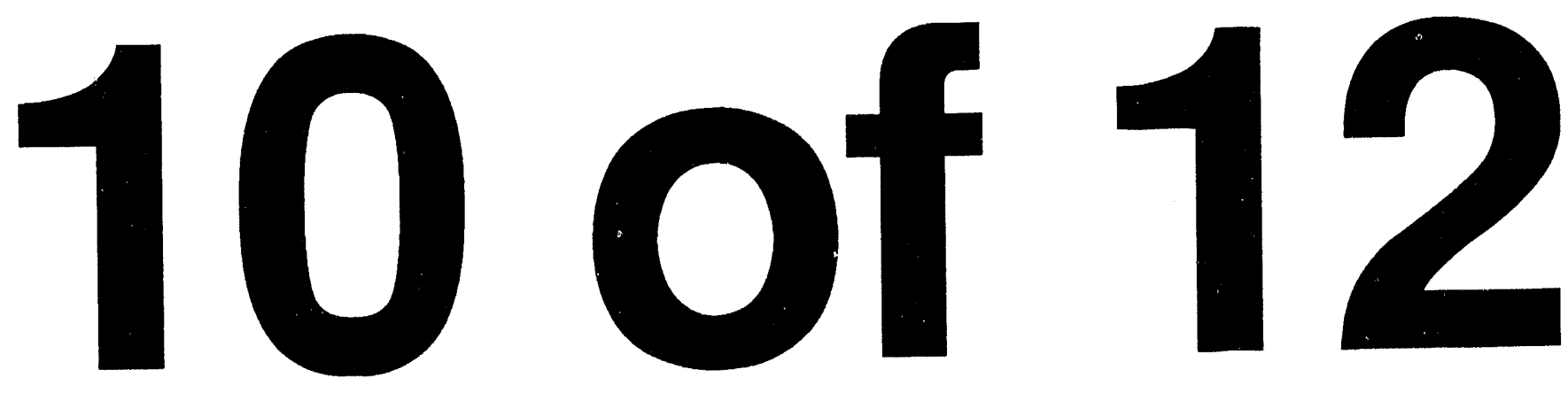


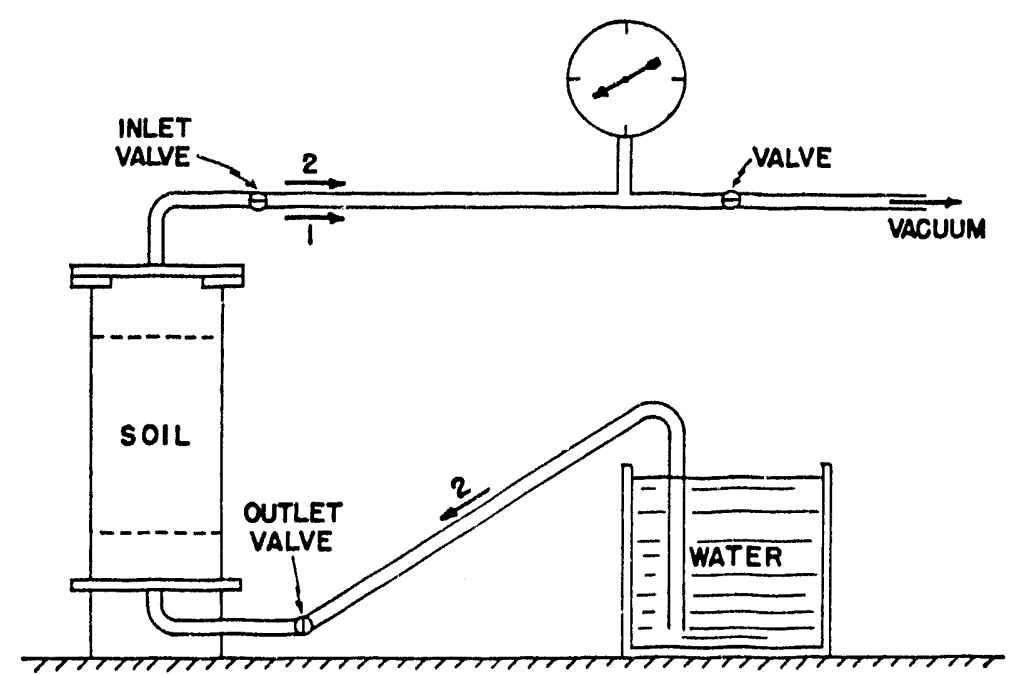

FIG. 2 Device for Evacuating and Saturating Specimen

dures in uniform thin layers approximately equal in thickness after compaction to the maximum size of particle. but not less than approximately $15 \mathrm{~mm}$ (0.60 in.).

6.4.1 For soils having a maximum size of $9.5 \mathrm{~mm} \mathrm{(3/8} \mathrm{in.)}$ : or less, place the appropriate size of funnel, as prescribed in 4.3 , in the permeability device with the spout in contact with the lower porous plate or screen, or previously formed layer. and fill the funnel with sufficient soil to form a layer. taking soil from different areas of the sample in the pan. Lift the funnel by $15 \mathrm{~mm}$ (0.60 in.), or approximately the unconsolidated layer thickness to be formed, and spread the soil a slow spiral motion, working from the perimeter of the ce toward the center, so that a uniform layer is formed. Remix the soil in the pan for each successive layer to reduce segregation caused by taking soil from the pan.

6.4.2 For soils with a maximum size greater than $9.5 \mathrm{~mm}$ ( $3 / 8$ in.), spread the soil from a scoop. Uniform spreading can be obtained by sliding a scoopful of soil in a nearly horizontal position down along the inside surface of the device to the hottom or to the formed layer. then tilting the scoop and drawing it toward the center with a single slow motion: this allows the soil to run smoothly from the scoop in a windrow uithout segregation. Turn the permeability cylinder sufficiently for the next scoopful, thus progressing around the inside perimeter to form a uniform compacted layer of a thickness equai to the maximum particle size.

6.5 Compact successive layers of soil to the desired relative density by appropriate procedures, as follows, to a height of about $2 \mathrm{~cm}$ ( $0.8 \mathrm{in}$.) above the upper manometer outlet.

6.5.1 Minimum Density (0\% Relative Density)-Conunue placing layers of soil in succession by one of the procedures described in 6.4.1 or 6.4.2 until the device is filled to the proper level.

6.5.2 Maximum Density (100\% Relarive Densiuy).

6.5.2.1 Compation hy. Vibrating Tamper-Compact each iver of soil thoroughly with the vibrating tamper, distributing th: light tamping action uniformly over the surface of the biter in a regular pattern. The pressure of contact and the fank of time of the vibrating action at each spot should not soil to escape from beneath the edges of the tamping foot, thus tending to loosen the layer. Make a sufficient number of coverages to produce maximum density, as evidenced by practically no visible motion of surface particles adjacent to the edges of the tamping foot.

6.5.2.2 Compaction by Sliding Weight Tamper-Compact each layer of soil thoroughly by tamping blows uniformily distributed over the surface of the layer. Adjust the height of drop and give sufficient coverages to produce maximum density, depending on the coarseness and gravel content of the soil.

6.5.2.3 Compaction by Other Methods-Compaction may be accomplished by other approved methods, such as by vibratory packer equipment, where care is taken to obtain a uniform specimen without segregation of particle sizes (see Test Method D 2049).

6.5.3 Relative Density Intermediate Between 0 and $100 \%$ - By trial in a separate container of the same diameter as the permeability cylinder, adjust the compaction to obtain reproducible values of relative density. Compact the soil in the permeability cylinder by these procedures in thin layers to a height about $2.0 \mathrm{~cm}(0.80 \mathrm{in}$.) above the upser manometer outlet.

NOTF 3-In order to bracket, systematically and representatively, the relative density conditions that may govern in natural deposits or in compacted embankments. a series of permeability tests should be made to bracket the range of field relative densities.

\subsection{Preparation of Specimen for Permeability Test:}

6.6.1 Level the upper surface of the soil by placing the upper porous plate or screen in position and by rotating it gently back and forth.

6.6.2 Measure and record: the final height of specimen. $H_{1}$ - $H_{2}$, by measuring the depth. $H_{2}$. from the upper surface of the perforated top plate employed to measure $H_{1}$ to the top of the upper porous plate or screen at four symmetrically spaced points after compressing the spring lightly to seat the porous plate or screen during the measurements: the final weight of air-dried soil used in the test $\left(H_{1}-W_{2}\right)$ by weighing the remainder of soil. $\boldsymbol{H}_{2}$. left in the pan. Compute and record the unit weights. void ratio. and relative density of the test specimen. 


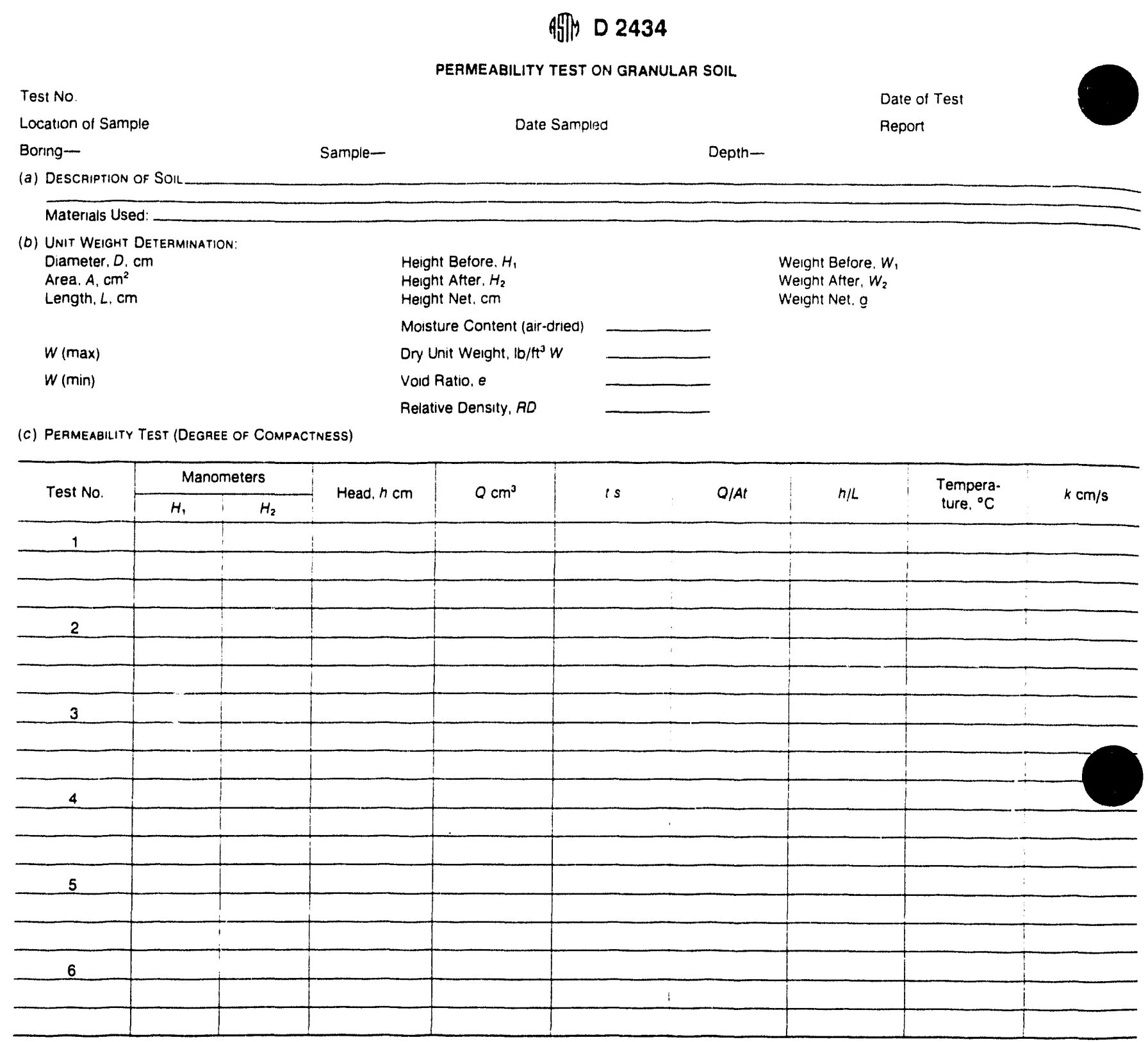

FIG. 3 Permeability Test Data Sheet

6.6.3 With its gasket in place, press down the top plate against the spring and attach it securely to the top of the permeameter cylinder, making an air-tight seal. This satisfies the condition described in 3.1 .1 of holding the initial density without significant volume change during the test.

6.6.4 Using a vacuum pump or suitable aspirator, evacuate the specimen under $50 \mathrm{~cm}$ (20 in.) $\mathrm{Hg}$ minimum for $15 \mathrm{~min}$ to remove air adhering to soil particles and from the voids. Follow the evacuation by a slow saturation of the specimen from the bottom upward (Fig. 2) under full vacuum in order to free any remaining air in the specimen. Continued saturation of the specimen can be maintained more adequately by the use of ( $l$ ) de-aired water, or (2) water maintained in an in-flow temperature sufficiently high to cause a decreasing temperature gradient in the specimen during the test. Native water or water of low mineral content (Note 4) should be used for the test, but in any case the fluid should be described on the report form (Fig. 3). This satisfies the condition described in 3.1.2 for saturation of soil voids.

Nore 4 Native water is the water occurring in the rock or soil in sim. It should be used if possible. but it (as well as de-aired water) may be a refinement not ordinarily feasible for large-scale production testing.

6.6.5 After the specimen has been saturated and the permeameter is full of water, close the bottom valve on the outlet tube (Fig. 2) and disconnect the vacuum. Care should be takien to ensure that the permeability flow system and the manometer system are free of air and are working satisfactorily. Fill the inlet tube with water from the constant-head tank by slightly opening the filter tank valve. Then connect the inlet tube to the top of the permeameter, open the valve slightly and open the manometer outlet cocks sl. to allow water to flow, thus freeing them of air. Connect the water manometer tubes to the manometer outlets and fill 
with water to remove the air. Close the inlet valve and open the outlet valve to allow the water in the manometer tubes to reach theii stable water level under zero head.

\section{Procedure}

7.1 Open the inlet valve from the filter tank slightly for the first run to conditions described in 3.1.3, delay measurements of quantity of flow and heat until a stable head condition without appreciable drift in water manometer levels is attained. Measure and record the time, $t$, head, $h$ (the difference in level in the manometers), quantity of flow. $Q$, and water temperature, $T$.

7.2 Repeat test runs at heads increasing by $0.5 \mathrm{~cm}$ in order to establish accurately the region of laminar flow with velocity, ' (where $v=Q / A t$ ), directly proportional to hydraulic gradient, $i$ (where $i=h / L$ ). When departures from the linear relation become apparent. indicating the initiation of turbulent flow conditions, $1-\mathrm{cm}$ intervals of head may be used to carry the test run sufficiently along in the region of turbulent flow to define this region if it is significant for field conditions.

Note $5-$ Much lower values of hydraulic gradient, $h / L$. are required than generally recognized, in order to ensure laminar fow conditions. The following values are suggested: loose compactness ratings, $h / L$ from 0.2 to 0.3 , and dense compactness ratings, $h / L$ from 0.3 to 0.5 , the lover values of $h / L$ applying to coarser soils and the higher values to finer soils.

7.3 At the completion of the permeability test. drain the specimen and inspect it to establish whether it was essentially homogeneous and isotropic in character. Any light and dark alternating horizontal streaks or layers are evidence of segregation of fines.

\section{Calculations}

8.1 Calculate the coefficient of permeability, $k$, as follows:

$$
k=Q L / A t h
$$

where:

$k=$ coefficient of permeability,

$Q=$ quantity of water discharged

$L=$ distance between manometers.

$A=$ cross-sectional area of specimen,

$l=$ total time of discharge,

$h=$ difference in head on manometers.

8.2 Correct the permeability to that for $20^{\circ} \mathrm{C}\left(68^{\circ} \mathrm{F}\right)$ by multiplying $k$ (see 8.1 ) by the ratio of the viscosity of water at test temperatui: to the viscosity of water at $20^{\circ} \mathrm{C}\left(68^{\circ} \mathrm{F}\right)$.

\section{Report}

9.1 The report of permeability test shall include the following information:

9.1.1 Project, dates, sample number, location, depth, and any other pertinent information,

9.1.2 Grain size analysis, classification. maximum particle size, and percentage of any oversize material not used,

9.1.3 Dry unit weight. void ratio, relative density as placed, and maximum and minimum densities,

9.1.4 A statement of any departures frorn these test conditions, so the results can be evaluated and used,

9.1.5 Complete test data, as indicated in the laboratory form for test data (see Fig. 3), and

9.1.6 Test curves plotting velocity, $Q / A t$, versus hydraulic gradient, $h / L$, covering the ranges of soil identifications and of relative densities.

\footnotetext{
The American Sociefy for Testing and Materials lakes no position respecting the validity of any patent rights asserted in connection with any item mentioned in this standard. Users of this stanoard are expressly advised that determination of the validity of any such patent rights, and the risk of intringement of such rights. are entirely their own responsibility.
}

This standard is subject to revision at any time oy the responsible technical committee and must be reviewed every five years and if not revised, either reapproved or withdrawn. Your comments are invited either for revision of this standard or for additional standards and should be addressed to ASTM Headquarters. Your comments will receive careful consideration at a meetıng of the responsible technical committee. which you may attend. It you teel that your comments have not recelved a fair hearing you should make your views known to the ASTM Committee on Standards, 1916 Race St., Philadelphia, PA 19103. 


\title{
Standard Method for Laboratory Determination of Water (Moisture) Content of Soil, Rock, and Soil-Aggregate Mixtures ${ }^{1}$
}

\begin{abstract}
This standard is issued under the fixed designation D 2216; the numcer immediately following the designation indicates the year of onginal adoption or, in the case of revision. the year of last revision 4 number in parentheses indicates the year of last reapproval. A superscript epsilon $(t)$ indicates an editorial change since the last revision or reapproval.
\end{abstract}

\section{Scope}

1.1 This method covers the laboratory determination of the water (moisture) content of soil. rock. and soil-aggregate mixtures by weight. For simplicity, the word "material" hereinafter refers to either soil, rock, or soil-aggregate mixtures, whichever is mosi applicable.

1.2 The water content of a material is defined as the ratio, expressed as a percentage, of the mass of "pore" or "free" water in a given mass of material to the mass of the solid material particles.

1.3 This method does not give true representative results for: materials containing significant amounts of halloysite, montmorillonite, or gypsum minerals; highly organic soils; or, materials in which the pore water contains dissolved solids (such as salt in the case of marine deposits). For a material of the previously mentioned types, a modified method of testing or data calculation may be established to give results consistent with the purpose of the test.

\section{Summary of Method}

2.1 The practical application in determining the water content of a material is to determine the mass of water removed by drying the moist material (test specimen) to a constant mass in a drying oven controlled at $110 \pm 5^{\circ} \mathrm{C}$ and to use this value as the mass of water in the test specimen. The mass of material remaining after oven-drying is used as the mass of the solid particles.

\section{Significance and Use}

3.1 For many soil types, the water content is one of the most significant index properties used in establishing a correlation between soil behavior and an index property.

3.2 The water content of a soil is used in almost every equation expressing the phase relationships of air, water, and solids in a given volume of material.

3.3 In fine-grained (cohesive) soils, the consistency of a given soil type depends on its water content. The water content of a soil, along with its liquid and plastic limit, is used to express its relative consistency or liquidity index.

\footnotetext{
'This method is under the jurisdiction of ASTM Committee D-18 on Soil and Rock and is the direct responsibility of Subcommittee D18.03 on Texture, Plasticity and Density Characteristics of Soils.

Current edition approved May 30, 1980. Published July 1980. Originally published as D 2216-63 T. Last previous edition D 2216-71.
}

3.4 The term "water" as used in geotechnical engineerine is typically assumed to be "pore" or "free" water and not that which is hydrated to the mineral surfaces. Therefore, the water content of materials containing significant amounts of hydrated water at in-situ temperatures or less than $110^{\circ} \mathrm{C}$ car. be misleading.

3.5 The term "solid particles" as used in geotechnical engineering, is typically assumed to mean naturally occurring mineral particles that are not readily soluble in water. Therefore. the water content of materials containing extraneous matter (such as cement, etc), water-soluble matter (such as salt) and highly organic matter typically require special treatment or a qualified definition of water content.

\section{Apparatus}

4.1 Dring Oven, thermostatically-controlled, preferably of the forced-draft type, and maintaining a uniform temperature of $110 \pm 5^{\circ} \mathrm{C}$ throughout the drying chamber.

4.2 Balances, having a precision (repeatability) of $\pm 0.01 \mathrm{~g}$ for specimens having a mass of $200 \mathrm{~g}$ or less. $\pm 0.1 \mathrm{~g}$ for specimens having a mass of between 200 and $1000 \mathrm{~g}$, or \pm 1 $\mathrm{g}$ for specimens having a mass greater than $1000 \mathrm{~g}$.

t. 3 Specimen Containers-Suitable containers made of material resistant to corrosion and a change in mass upon repeated heating, cooling, and cleaning. Containers with close-fitting lids shall be used for testing specimens having a mass of less than about $2 \mathrm{J0} \mathrm{g}$; while for specimens having a mass greater than about $200 \mathrm{~g}$, containers without lids may be used (Note 1). One container is needed for each water content determination.

VOTE 1-The purpose of close-fitting lids is to prevent loss of moisture from specimens before initial weighing and to prevent absorption of moisture from the atmosphere following drying and before final weighing.

t.4 Desiccator-A desiccator of suitable size (a convenient size is 200 to $250-\mathrm{mm}$ diameter) containing a hydrous silica gel. This equipment is only recommended for use when containers having close-fitting lids are not used. See 7.4.1.

\section{Samples}

5.1 Keep the samples that are stored prior to testing in noncorrodible airtight containers at a temperature between approximately 3 and $30^{\circ} \mathrm{C}$ and in an area that prevents direct contact with sunlight.

5.2 The water content determination should be done as 
soon as practicable after sampling, especially if potentially corrodible containers (such as steel thin-walled tubes. paint ans, etc.) or sample bags are used.

\section{Test Specimen}

6.1 For water contents being determined in conjunction aith another ASTM method, the method of specimen selecvon specified in that method controls.

6.2 The manner in which the test specimen is selected and its required mass is basically dependent on the purpose (application) of the test, type of material being tested, and the npe of sample (specimen from another test, bag, tube. splitbartel. etc.). In all cases, however, a representative portion of eering : the total sample shall be selected. If a layered soil or more nd no: than one soil type is encountered, select an average portion re, the or individual portions or both, and note which portion(s) was unts of tested in the report of the results.

$1^{\circ} \mathrm{C}$ car 6.2.1 For bulk samples, select the test specimen from the

ihnica:

curring

There.

uneous

uch as

treai. material after it has been thoroughly mixed. The mass of moist material selected shall be in accordance with the following table:

$\begin{array}{cc}\text { Seve Retaining More Than About } 10 \% \text { of } \\ \text { Sample } & \begin{array}{c}\text { Recommended Minimum Mass of } \\ \text { Moist Specimen, } B\end{array} \\ 2.0 \mathrm{~mm}(\text { No. 10) sieve } & 10010200 \\ 4.75 \mathrm{~mm} \text { (No. } 4 \text { ) sieve } & 30010500 \\ 19 \mathrm{~mm} & 500101000 \\ 38 \mathrm{~mm} & 1500103000 \\ 76 \mathrm{~mm} & 50001010000\end{array}$

6.2.2 For small (jar) samples, select a representative por-

ablyo:

ratur: tion in accordance with the following procedure:

6.2.2.1 For cohesionless soils, thoroughly mix the material. then select a test specimen having a mass of moist material 1.01 i in accordance with the table in 6.2.1. See Note 2.

g fo: 6.2.2.2 For cohesive soils, remove about $3 \mathrm{~mm}$ of material i \pm from the exposed periphery of the sample and slice it in half 110 check if the material is layered) prior to selecting the test de 0 specimen. If the soil is layered see 6.2. The mass of moist upo: material selected should not be less than $25 \mathrm{~g}$ or should be in w7: accordance with the table in 6.2 .1 if coarse-grained particles ing: are noted. (Note 2).

ang. 6.3 Using a test specimen smaller than the minimum mass me indicated previously requires discretion, though it may be

wate i dequate for the purpose of the test. A specimen having a mass less than the previously indicated value shall be noted in the repor of the results.

VOTE 2-In many cases, when working with a small sample containing a relatively large coarse-grained particle. it is appropriate not to include this particle in the test specimen. If this occurs. it should be soled in the report of the results.

nle: :

sili.: ! Procedure

7 Select representative test specimens in accordance with raction 6

.2 Place the moist specimen in a clean. dry container of

nown mass (Note 3), set the lid securely in position. and

$n::^{-}:$lermine the mass of the container and moist material using

"W: : appropriate balance (4.2). Record these values.

Jir. $\quad 3$ Remove the lid and place the container with moist

saterial in a drying oven maintained at $110 \pm 5^{\circ} \mathrm{C}$ and $\mathrm{dr}$.

$\therefore-:$ a constant mass (Notes 4. 5, and 6).
Note 3-To assist in the oven-drying of large test specimens, they should be placed in containers having a large surface area (such as pans) and the material broken up into smaller aggregations.

Note 4-The time required to obtain constant mass will vary depend. ing on the type of material, size of specimen, oven type and capacity, and other factors. The influence of these factors generally can be established by good judgment, and experience with the materials being tested and the apparatus being used. In most cases, drying a test specimen over night (about $16 \mathrm{~h}$ ) is sufficient. In cases where there is doubt concerning the adequacy of drying. drying should be continued until the mass after two successive periods (greater than $1 / 2 \mathrm{~h}$ ) of drying indicate an insignificant change (less than about $0.1 \%$ ). Specimens of sand may often be dried to constant mass in a period of about $4 \mathrm{~h}$. when a forced-draft oven is used.

Note 5-Oven-drying at $110 \pm 5^{\circ} \mathrm{C}$ does not always result in water content values related to the intended use or the basic definition especially for materials containing gypsum or other minerals having significant amounts of hydrated water or for soil containing a significant amount of organic material. In many cases, and depending on the intended use for these types of materials, it might be more applicable to maintain the drying oven at $60 \pm 5^{\circ} \mathrm{C}$ or use a vacuum desiccator at a vacuum of approximately $133 \mathrm{~Pa}(10 \mathrm{~mm} \mathrm{Hg})$ and at a temperature ranging between 23 and $60^{\circ} \mathrm{C}$ for drying. If either of these drying methods are used, it should be noted in the report of the results.

Note 6-Since some dry materials may absorb moisture from moist specimens, dried specimens should be removed before placing moist specimens in the oven. However, this requirement is not applicable if the previously dried specimens will remain in the drying oven for an additional time period of about $16 \mathrm{~h}$

7.4 After the material has dried to constant mass remove the container from the oven and replace the lid. Allow the material and container to cool to room temperature or until the container can be handled comfortably with bare hands and the operation of the balance will not be affected by convection currents. Determine the mass of the container and oven-dried material using the same balance as used in 7.2. Record this value.

7.4.1 If the container does not have a lid, weigh the container and material right after their temperatures are such that the operation of the balance will not be affected by convection currents or after cooling in a desiccator.

Note 7 -Cooling in a desiccator is recommended since it prevents absorption of moisture from the atmosphere during cooling.

\section{Calculation}

8.1 Calculate the water content of the material as follows:

$$
w=\left[\left(W_{1}-W_{2}\right) /\left(W_{2}-W_{1}^{\prime}\right)\right] \times 100=\frac{W_{u}}{W_{s}} \times 100
$$

where:

$w^{\prime}=$ water content. $\%$,

$H_{1}=$ mass of container and moist specimen, $\mathrm{g}$.

$W_{2}=$ mass of container and oven-dried specimen, $\mathrm{g}$.

$H_{c}=$ mass of container, $\mathrm{g}$.

$W_{n}^{\prime}=$ mass of water. $g$. and

$W_{s}=$ mass of solid particles, $\mathrm{g}$.

\section{Report}

9.1 The report (data sheet) shall include the following:

9.1.1 Identification of the sample (material) being tested, by boring number. sample number, test number. etc.

9.1.2 Water content of the specimen to the nearest $0.1 \%$ or $1 \%$ depending on the purpose of the test. 
9.1.3 Indication of test specimen having a mass less than the minimum indicated in Section 6.

9.1.4 Indication of test specimen containing more than one soil type (layered, etc).

9.1.5 Indication of the method of drying if different from oven-drying at $110 \pm 5^{\circ} \mathrm{C}$.

\section{2216}

9.1.6 Indication of any material (size and amount) cluded from the test specimen.

\section{Precision and Accuracy}

10.1 Requirements for the precision and accuracy of thi test method have not yet been developed.

The American Society for Testing and Materials takes no position respecting the validity of any patent rights asserted in connection with any item mentioned in this standard. Users of this standard are expressly advised that determination of the validity of any such patent rights, and the risk of intringement of such rights, are entirely their own responsibility.

This stanoard is subject to revision at any time by the responsible technical committee and must be reviewed every five years and it not revised, either reapproved or withdrawn. Your comments are invited either for revision of this standard or for additional standards and should be addressed to ASTM Headquarters. Your comments will receive careful consideration at a meeting of the responsible technical committee, which you may attend. If you teel that your comments have not received a fair hearing you should make your views known to the ASTM Committee on Standards, 1916 Race St., Philadelphia, PA 19103. 


\title{
Standard Method for Particle-Size Analysis of Soils ${ }^{1}$
}

\begin{abstract}
This standard is issued under the fixed designation D 422: the number immediately following the designatuon indicates the year of original adoption or, in the case of revision, the year of last revision. A number in parentheses indicates the year of last reapproval. A superscript epsilon ( $\epsilon$ ) indicates an editorial change since the last revision or reapproval.
\end{abstract}

"Nore-Section 2 was added editorially and subsequent sections renumbered in July 1984.

\section{Scope}

1.1 This method covers the quantitative determination of the distribution of particle sizes in soils. The distribution of particle sizes larger than $75 \mu \mathrm{m}$ (retained on the No. 200 sieve) is determined by sieving, while the distribution of particle sizes smaller than $75 \mu \mathrm{m}$ is determined by a sedimentation process, using a hydrometer to secure the necessary data (Notes 1 and 2).

Note 1-Separation may be made on the No. $4(4.75-\mathrm{mm})$, No. 40 $(425-\mu \mathrm{m})$, or No. $200(75-\mu \mathrm{m})$ sieve instead of the No. 10. For whatever sieve used, the size shall be indicated in the report.

NoTE 2-Two types of dispersion devices are provided: (I) a high-speed mechanical stirrer, and (2) air dispersion. Extensive investigations indicate that air-dispersion devices produce a more positive dispersion of plastic soils below the $20-\mu \mathrm{m}$ size and appreciably less degradation on all sizes when used with sandy soils. Because of the definite advantages favoring air dispersion, its use is recommended. The results from the two types of devices differ in magnitude, depending pon soil type, leading to marked differences in particle size distribu$\mathrm{n}$, especially for sizes finer than $20 \mu \mathrm{m}$.

\section{Referenced Documents}

2.1 ASTM Standards:

D 421 Practice for Dry Preparation of Soil Samples for Particle-Size Analysis and Determination of Soil Constants ${ }^{2}$

E 11 Specification for Wire-Cloth Sieves for Testing Purposes ${ }^{3}$

E 100 Specification for ASTM Hydrometers ${ }^{4}$

\section{Apparatus}

3.1 Balances-A balance sensitive to $0.01 \mathrm{~g}$ for weighing the material passing a No. $10(2.00-\mathrm{mm})$ sieve, and a balance sensitive to $0.1 \%$ of the mass of the sample to be weighed for weighing the material retained on a No. 10 sieve.

3.2 Stirring Apparatus - Either apparatus A or B may be used.

3.2.1 Apparatus A shall consist of a mechanically operated stirring device in which a suitably mounted electric motor turns a vertical shaft at a speed of not less than 10000 $\mathrm{pm}$ without load. The shaft shall be equipped with a

1 This method is under the jurisdiction of ASTM Committee D-18 on Soil and Rock and is the direct responsibility of Subcommittee D18.03 on Texture, Plasticity, and Density Characteristics of Soils.

Gurrent edition approved Nov. 21, 1963. Originally published 1935. Replaces

- 62 .

Annual Book of ASTM Standards, Vol 14.02.

4 Annual Book of ASTM Standards, Vol 14.01. replaceable stirring paddle made of metal, plastic, or hard rubber, as shown in Fig. 1. The shaft shall be of such length that the stirring paddle will operate not less than $3 / 4$ in. $(19.0$ $\mathrm{mm}$ ) por more than $1 \frac{1}{2}$ in. $(38.1 \mathrm{~mm}$ ) above the bottom of the dispersion cup. A special dispersion cup conforming to either of the designs shown in Fig. 2 shall be provided to hold the sample while it is being dispersed.

3.2.2 Apparatus B shall consist of an air-jet dispersion cup $^{5}$ (Note 3 ) conforming to the general details shown in Fig. 3 (Notes 4 and 5).

Note 3-The amount of air required by an air-jet dispersion cup is of the order of $2 \mathrm{ft}^{3} / \mathrm{min}$; some small air compressors are not capable of supplying sufficient air to operate a cup.

NoTE 4-Another air-type dispersion device, known as a dispersion tube, developed by Chu and Davidson at lowa State College, has been shown to give results equivalent to those secured by the air-jet dispersion cups. When it is used, soakjng of the sample can be done in the sedimentation cylinder, thus eliminating the need for transferring the slurry. When the air-dispersion tube is used, it shall be so indicated in the report.

Note 5-Water may condense in air lines when not in use. This water must be removed, either by using a water trap on the air line, or by blowing the water out of the line before using any of the air for dispersion purposes.

3.3 Hydrometer-An ASTM hydrometer, graduated to read in either specific gravity of the suspension or grams per litre of suspension, and conforming to the requirements for hydrometers $151 \mathrm{H}$ or $152 \mathrm{H}$ in Specifications E 100. Dimensions of both hydrometers are the same, the scale being the only item of difference.

3.4 Sedimentction Cylinder-A glass cylinder essentially $18 \mathrm{in} .(457 \mathrm{~mm})$ in height and $21 / 2 \mathrm{in.}(63.5 \mathrm{~mm})$ in diameter, and marked for a volume of $1000 \mathrm{~mL}$. The inside diameter shall be such that the $1000-\mathrm{mL}$ mark is $36 \pm 2 \mathrm{~cm}$ from the bottom on the inside.

3.5 Thermometer - A thermometer accurate to $1^{\circ} \mathrm{F}$ $\left(0.5^{\circ} \mathrm{C}\right)$.

3.6 Sieves -A series of sieves, of square-mesh woven-wire cloth, conforming to the requirements of Specification E 11. A full set of sieves includes the following (Note 6):

\footnotetext{
${ }^{5}$ Detailed working drawings for this cup are available at a nominal cost from the American Society for Testing and Materials, 1916 Race St., Philadelphia, PA 19103. Order Adjunct No. 12-404220-00.
} 

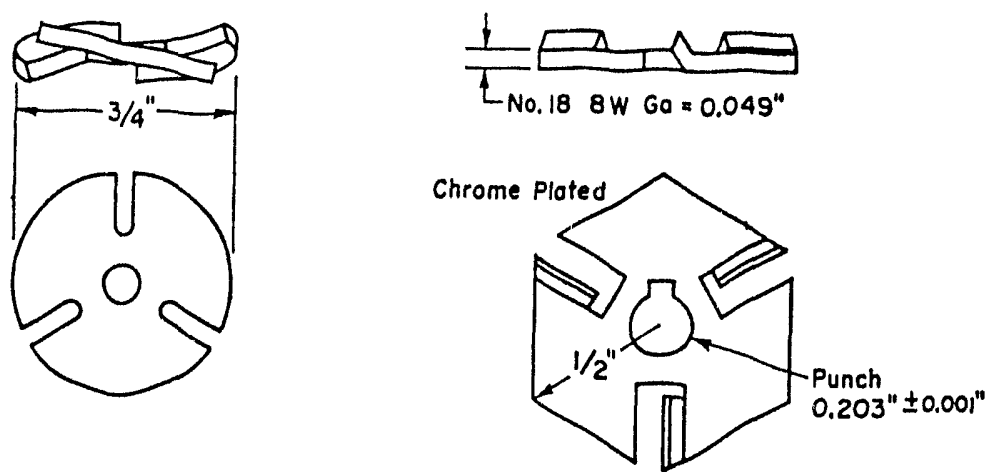

(a)

(b)

\begin{tabular}{llllll}
\multicolumn{5}{c}{ Metric Equivalents } \\
\hline in. & 0.001 & 0.049 & 0.203 & $1 / 2$ & $3 / 4$ \\
$\mathrm{~mm}$ & 0.03 & 1.24 & 5.16 & 12.7 & 19.0 \\
\hline
\end{tabular}

FIG. 1 Detail of Stirring Paddles

$\begin{array}{ll}3-\text { in. }(75-\mathrm{mm}) & \text { No. } 10(2.00-\mathrm{mm}) \\ 2-\text { in. }(50-\mathrm{mm}) & \text { No. } 20(850-\mu \mathrm{m}) \\ 1 / 2-\text { in. }(37.5-\mathrm{mm}) & \text { No. } 40(425-\mu \mathrm{m}) \\ 1-\text { in. }(25.0-\mathrm{mm}) & \text { No. } 60(250-\mu \mathrm{m}) \\ 3 / 4-\text { in. }(19.0-\mathrm{mm}) & \text { No. } 140(106-\mu \mathrm{m}) \\ 1 / 3-\text { in. }(9.5-\mathrm{mm}) & \text { No. } 200(75-\mu \mathrm{m}) \\ \text { No. } 4(4.75-\mathrm{mm}) & \end{array}$

Nore 6-A set of sieves giving uniform spacing of points for the graph, as required in Section 17, may be used if desired. This set consists of the following sieves:

$$
\begin{aligned}
& \text { 3-in. }(75-\mathrm{mm}) \\
& 1 / 2-\text { in. }(37.5-\mathrm{mm}) \\
& 1 / 4-\text { in. }(19.0-\mathrm{mm}) \\
& 1 / 8-\text { in. }(9.5-\mathrm{mm}) \\
& \text { No. } 4(4.75-\mathrm{mm}) \\
& \text { No. } 8(2.36-\mathrm{mm})
\end{aligned}
$$

$$
\begin{aligned}
& \text { No. } 16(1.18-\mathrm{mm}) \\
& \text { No. } 30(600-\mu \mathrm{m}) \\
& \text { No. } 50(300-\mu \mathrm{m}) \\
& \text { No. } 100(150-\mu \mathrm{m}) \\
& \text { No. } 200(75-\mu \mathrm{m})
\end{aligned}
$$

3.7 Water Bath or Constant-Temperature Room-A water bath or constant-temperature room for maintaining the soil suspension at a constant temperature during the hydrometer analysis. A satisfactory water tank is an insulated tank that maintains the temperature of the suspension at a convenient constant temperature at or near $68^{\circ} \mathrm{F}\left(20^{\circ} \mathrm{C}\right)$. Such a device is illustrated in Fig. 4. In cases where the work is performed in a room at an automatically controlled constant temperature, the water bath is not necessary.

3.8 Beaker - A beaker of $250-\mathrm{mL}$ capacity.

3.9 Timing Device-A watch or clock with a second hand.

\section{Dispersing Agent}

4.1 A solution of sodium hexametaphosphate (sometimes called sodium metaphosphate) shall be used in distilled or demineralized water, at the rate of $40 \mathrm{~g}$ of sodium hexametaphosphate/litre of solution (Note 7).

NOTE 7-Solutions of this salt, if acidic, slowly revert or hydrolyze back to the orhophosphate form with a resultant decrease in dispersive action. Solutions should be prepared frequently (at least once a month) or adjusted to $\mathrm{pH}$ of 8 or 9 by means of sodium carbonate. Bottles containing solutions should have the date of preparation marked on them.

4.2 All water used shall be either distilied or demineralized water. The water for a hydrometer test shall

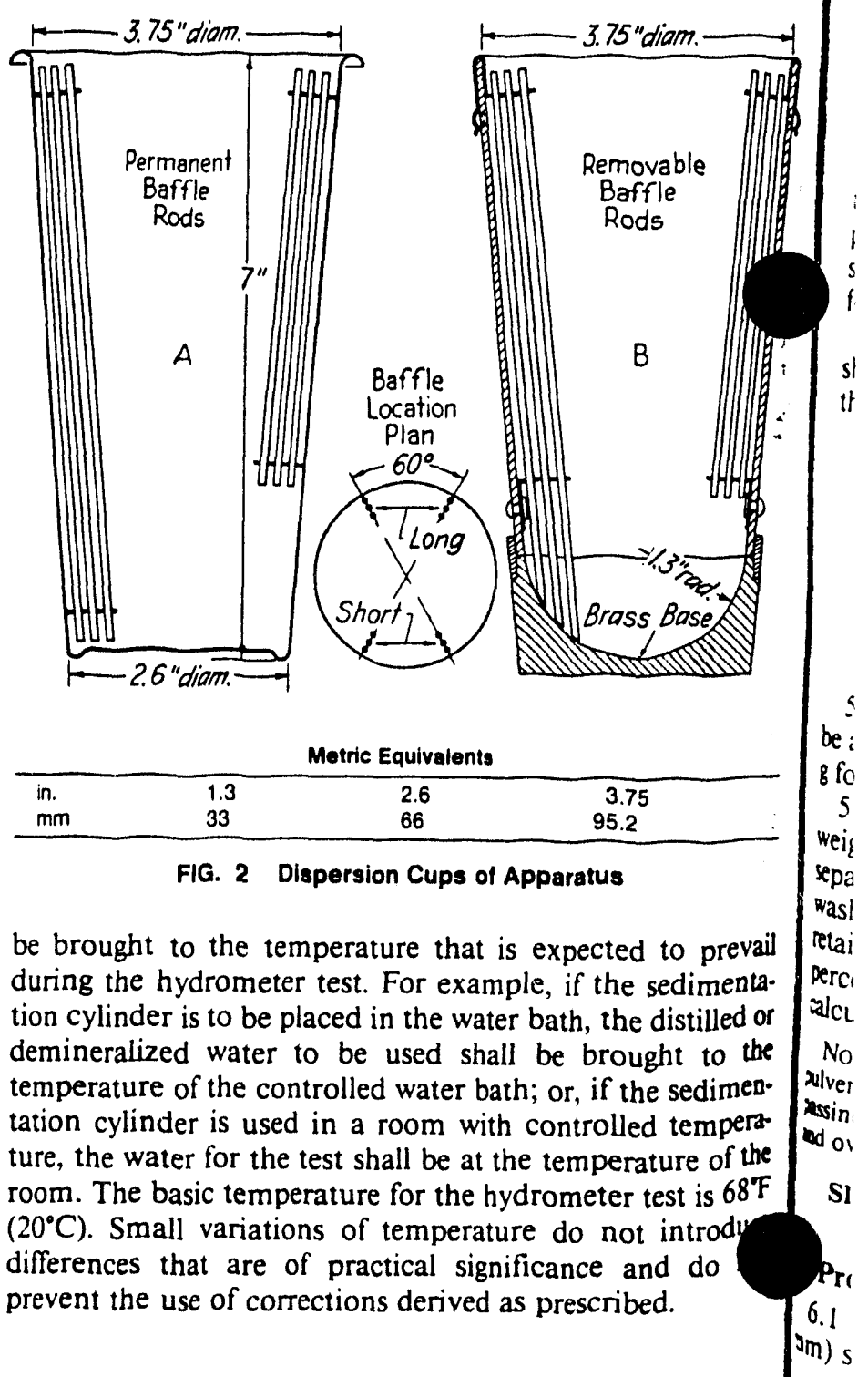



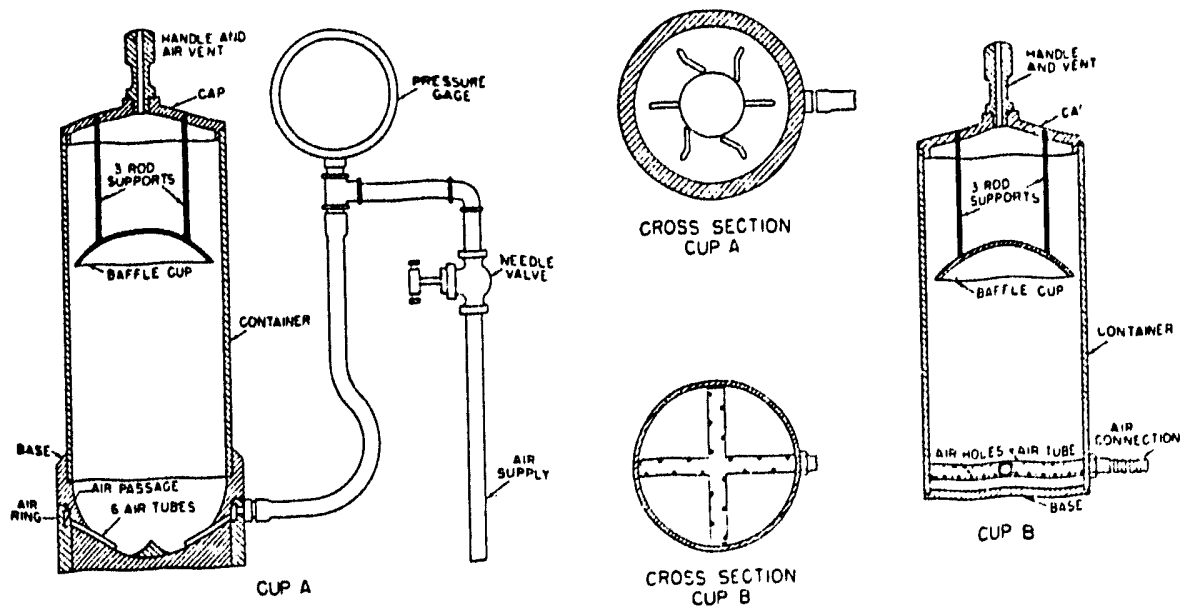

FIG. 3 Air-Jet Dispersion Cups of Apparatus B

\section{Test Sample}

5.1 Prepare the test sample for mechanical analysis as outlined in Practice D 421. During the preparation procedure the sample is divided into two portions. One portion contains only particles retained on the No. $10(2.00-\mathrm{mm})$ sieve while the other portion contains only particles passing the No. 10 sieve. The mass of air-dried soil selected for purpose of tests, as prescribed in Practice D 421, shall be sufficient to yield quantities for mechanical analysis as follows:

5.1.1 The size of the portion retained on the No. 10 sieve hall depend on the maximum size of particle, according to the following schedule:

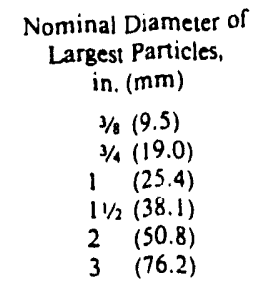

\section{Approximate Minimum} Mass of Portion, $B$

500
1000
2000
3000
4000
5000

5.1.2 The size of the portion passing the No. 10 sieve shall be approximately $115 \mathrm{~g}$ for sandy soils and approximately 65 $\mathrm{g}$ for silt and clay soils.

5.2 Provision is made in Section 5 of Practice D 421 for weighing of the air-dry soil selected for purpose of tests, the separation of the soil on the No. 10 sieve by dry-sieving and washing, and the weighing of the washed and dried fraction retained on the No. 10 sieve. From these two masses the percentages retained and passing the No. 10 sieve can be calculated in accordance with 12.1 .

Note 8-A check on the mass values and the thoroughness of pulverization of the clods may be secured by weighing the portion passing the No. 10 sieve and adding this value to the mass of the washed and oven-dried portion retained on the No. 10 sieve.

\section{SIEVE ANALYSIS OF PORTION RETAINED ON NO. 10 $(2.00-\mathrm{mm})$ SIEVE}

ice

$10 t$ 6. Procedure

6.1 Separate the portion retained on the No. $10(2.00$ $\mathrm{mm}$ ) sieve into a series of fractions using the $3-\mathrm{in} .(75-\mathrm{mm})$,

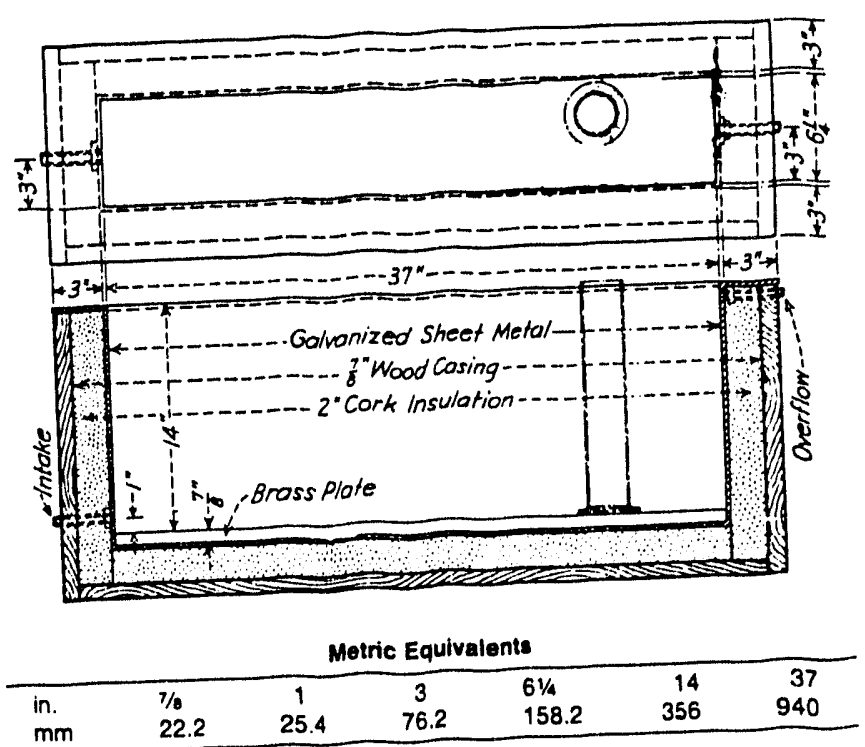

FIG. 4 Insulated Water Bath

2-in. (50-mm), 11/2-in. (37.5-mm), 1-in. (25.0-mm), 3/4-in. $(19.0-\mathrm{mm}), 3 / 8-$ in. $(9.5-\mathrm{mm})$, No. $4(4.75-\mathrm{mm})$, and No. 10 sieves, or as many as may be needed depending on the sample, or upon the specifications for the material under test.

6.2 Conduct the sieving operation by means of a lateral and vertical motion of the sieve, accompanied by a jarring action in order to keep the sample moving continuously over the surface of the sieve. In no case turn or manipulate fragments in the sample through the sieve by hand. Continue sieving until not more than 1 mass \% of the residue on a sieve passes that sieve during $1 \mathrm{~min}$ of sieving. When mechanical sieving is used, test the thoroughness of sieving by using the hand method of sieving as described above.

6.3 Determine the mass of each fraction on a balance conforming to the requirements of 3.1. At the end of weighing, the sum of the masses retained on all the sieves used should equal closely the original mass of the quantity sieved. 
HYDROMETER AND SIEVE ANALYSIS OF PORTION PASSING THE NO. $10(2.00-\mathrm{mm})$ SIEVE

\section{Determination of Composite Correction for Hydrometer Reading}

7.1 Equations for percentages of soil remaining in suspension, as given in 14.3, are based on the use of distilled or demineralized water. A dispersing agent is used in the water, however, and the specific gravity of the resulting liquid is appreciably greater than that of distilled or demineralized water.

7.1.1 Both soil hydrometers are calibrated at $68^{\circ} \mathrm{F}\left(20^{\circ} \mathrm{C}\right)$, and variations in temperature from this standard temperature produce inaccuracies in the actual hydrometer readings. The amount of the inaccuracy increases as the variation from the standard temperature increases.

7.1.2 Hydrometers are graduated by the manufacturer to be read at the bottom of the meniscus formed by the liquid on the stem. Since it is not possible to secure readings of soil suspensions at the bottom of the meniscus, readings must be taken at the top and a correction applied.

7.1.3 The net amount of the corrections for the three items enumerated is designated as the composite correction, and may be determined experimentally.

7.2 For convenience, a graph or table of composite corrections for a series of $1^{\circ}$ temperature differences for the range of expected test temperatures may be prepared and used as needed. Measurement of the composite corrections may be made at two temperatures spanning the range of expected test temperatures, and corrections for the intermediate temperatures calculated assuming a straight-line relationship between the two observed values.

7.3 Prepare $1000 \mathrm{~mL}$ of liquid composed of distilled or demineralized water and dispersing agent in the same proportion as will prevail in the sedimentation (hydrometer) test. Place the liquid in a sedimentation cyclinder and the cylinder in the constant-temperature water bath, set for one of the two temperatures to be used. When the temperature of the liquid becomes constant, insert the hydrometer, and, after a short interval to permit the hydrometer to come to the temperature of the liquid, read the hydrometer at the top of the meniscus formed on the stem. For hydrometer $151 \mathrm{H}$ the composite correction is the difference between this reading and one; for hydrometer $152 \mathrm{H}$ it is the difference between the reading and zero. Bring the liquid and the hydrometer to the other temperature to be used, and secure the composite correction as before.

\section{Hygroscopic Moisture}

8.1 When the sample is weighed for the hydrometer test, weigh out an auxiliary portion of from 10 to $15 \mathrm{~g}$ in a small metal or glass container, dry the sample to a constant mass in an oven at $230 \pm 9^{\circ} \mathrm{F}\left(110 \pm 5^{\circ} \mathrm{C}\right)$, and weigh again. Record the masses.

\section{Dispersion of Soil Sample}

9.1 When the soil is mostly of the clay and silt sizes, weigh out a sample of air-dry soil of approximately $50 \mathrm{~g}$. When the soil is mostly sand the sample should be approximately 100 g.
9.2 Place the sample in the $250-\mathrm{mL}$ beaker and cover with $125 \mathrm{~mL}$ of sodium hexametaphosphate solution $(40 \mathrm{~g} / \mathrm{L})$. Stir until the soil is thoroughly wetted. Allow to soak for at least $16 \mathrm{~h}$.

9.3 At the end of the soaking period, disperse the sample further, using either stirring apparatus A or B. If stirring apparatus $A$ is used, transfer the soil - water slurry from the beaker into the special dispersion cup shown in Fig. 2, washing any residue from the beaker into the cup with distilled or demineralized water (Note 9). Add distilled or demineralized water, if necessary, so that the cup is more than half full. Stir for a period of $1 \mathrm{~min}$.

NOTE 9-A large size syringe is a convenient device for handling the water in the washing operation. Other devices include the wash-water bottle and a hose with nozzle connected to a pressurized distilled water tank.

9.4 If stirring apparatus B (Fig. 3) is used, remove the cover cap and connect the cup to a compressed air supply by means of a rubber hose. A air gage must be on the line between the cup and the control value. Open the control valve so that the gage indicates $1 \mathrm{psi}(7 \mathrm{kPa})$ pressure (Note 10). Transfer the soil - water slurry from the beaker to the air-jet dispersion cup by washing with distilled or demineralized water. Add distilled or demineralized water, if necessary, so that the total volume in the cup is $250 \mathrm{~mL}$, but no more.

NoTE 10 -The initial air pressure of 1 psi is required to prevent the soil - water mixture from entering the airujet chamber when the mixture is transferted to the dispersion cup.

9.5 Place the cover cap on the cup and open the air control valve until the gage pressure is 20 psi $(140 \mathrm{kPa})$. Disperse the soil according to the following schedule:

Plasticity Index
Under 5
6 to 20
Over 20

Dispersion Period,
min
5
10
15

Soils containing large percentages of mica need be dispersed for only $1 \mathrm{~min}$. After the dispersion period, reduce the gage pressure to 1 psi preparatory to transfer of soil - water slurry to the sedimentation cylinder.

\section{Hydrometer Test}

10.1 Immediately after dispersion, transfer the soil - water slurry to the glass sedimentation cylinder, and add distilled or demineralized water until the total volume is $1000 \mathrm{~mL}$.

10.2 Using the palm of the hand over the open end of the cylinder (or a rubber stopper in the open end), turn the cylinder upside down and back for a period of $1 \mathrm{~min}$ to complete the agitation of the slurry (Note 11). At the end of 1 min set the cylinder in a convenient location and take hydrometer readings at the following intervals of time (measured from the beginning of sedimentation), or as many as may be needed, depending on the sample or the specification for the material under test: $2,5,15,30,60,250$, and $1440 \mathrm{~min}$. If the controlled water bath is used, the sedimentation cylinder should be placed in the bath between the 2 and 5 -min readings.

Nore 11 -The number of turns during this minute should be approximately 60 . counting the turn upside down and back as two turns. 
ith

L).

- at

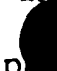

ing

ing

the

2 ,

ith

or

Jre

the

tter

iter :

he

by

ne

rol

te

he

or

if

sut

the

sie

tir

a).

12. Sieve Analysis Values for the Portion Coarser than the No. $10(2.00-\mathrm{mm})$ Sieve

12.1 Calculate the percentage passing the No. 10 sieve by dividing the mass passing the No. 10 sieve by the mass of soil originally split on the No. 10 sieve, and multiplying the result by 100 . To obtain the mass passing the No. 10 sieve, subtract the mass retained on the No. 10 sieve from the original mass.

12.2 To secure the total mass of soil passing the No. 4

\section{Percentages of Soil in Suspension}

14.1 Calculate the oven-dry mass of soil used in the ometer analysis by multiplying the air-dry mass by the oscopic moisture correction factor.
TABLE 1 Values of Correction Factor, $\alpha$, for Different Specific Gravities of Soll Particles^

\begin{tabular}{cc}
\hline Specific Gravity & Correction Factor ${ }^{A}$ \\
\hline 2.95 & 0.94 \\
2.90 & 0.95 \\
2.85 & 0.96 \\
2.80 & 0.97 \\
2.75 & 0.98 \\
2.70 & 0.99 \\
2.65 & 1.00 \\
2.60 & 1.01 \\
2.55 & 1.02 \\
2.50 & 1.03 \\
2.45 & 1.05 \\
\hline
\end{tabular}

${ }^{\wedge}$ For use in equation for percentage of soil remaining in suspension when using Hydrometer $152 \mathrm{H}$.

14.2 Calculate the mass of a total sample represented by the mass of soil used in the hydrometer test, by dividing the oven-dry mass used by the percentage passing the No. 10 $(2.00-\mathrm{mm})$ sieve, and multiplying the result by 100 . This value is the weight $W$ in the equation for percentage remaining in suspension.

14.3 The percentage of soil remaining in suspension at the level at which the hydrometer is measuring the density of the suspension may be calculated as follows (Note 13): For hydrometer $151 \mathrm{H}$ :

$$
P=\left[\left(100000 / W^{\prime}\right) \times G /\left(G-G_{1}\right)\right]\left(R-G_{1}\right)
$$

NoTE 13 -The bracketer portion of the equation for hydrometer $151 \mathrm{H}$ is constant for a series of readings and may be calculated first and then multiplied by the portion in the parentheses.

For hydrometer $152 \mathrm{H}$ :

$$
P=(R a / W) \times 100
$$

where:

$a=$ correction faction to be applied to the reading of hydrometer $152 \mathrm{H}$. (Values shown on the scale are computed using a specific gravity of 2.65 . Correction factors are given in Table 1),

$P=$ percentage of soil remaining in suspension at the level at which the hydrometer measures the density of the suspension,

$R$ = hydrometer reading with composite correction applied (Section 7),

$W=$ oven-dry mass of soil in a total test sample represented by mass of soil dispersed (see 14.2), g,

$G=$ specific gravity of the soil particles, and

$G_{1}=$ specific gravity of the liquid in which soil particles are suspended. Use numerical value of one in both instances in the equation. In the first instance any possible variation produces no significant effect, and in the second instance, the composite correction for $R$ is based on a value of one for $G_{1}$.

\section{Diameter of Soil Particles}

15.1 The diameter of a particle corresponding to the percentage indicated by a given hydrometer reading shall be calculated according to Stokes' law (Note 14), on the basis that a particle of this diameter was at the surface of the suspensior ${ }_{i}$ at the beginning of sedimentation and had settled to the level at which the hydrometer is measuring the density of the suspension. According to Stokes' law:

$$
D=\sqrt{\left[30 n / 980\left(G-G_{1}\right)\right] \times L / T}
$$


where:

$D=$ diameter of particle, $\mathrm{mm}$,

$n=$ coefficient of viscosity of the suspending medium (in this case water) in poises (varies with changes in temperature of the suspending medium),

$L=$ distance from the surface of the suspension to the level at which the density of the suspension is being measured, $\mathrm{cm}$. (For a given hydrometer and sedimentation cylinder, values vary according to the hydrometer readings. This distance is known as effective depth (Table 2)),

$T=$ interval of time from beginning of sedimentation to the taking of the reading, min,

$G=$ specific gravity of soil particles, and

$G_{1}=$ specitic gravity (relative density) of suspending medium (value may be used as 1.000 for all practical purposes).

NOTE 14-Since Stokes' law considers the terminal velocity of a single sphere falling in an infinity of liquid, the sizes calculated represent the diameter of spheres that would fall at the same rate as the soil particles.

15.2 For convenience in calculations the above equation may be written as follows:

$$
D=K \sqrt{L / T}
$$

where:

$K=$ constant depending on the temperature of the suspension and the specific gravity of the soil particles. Values of $K$ for a range of temperatures and specific gravities are given in Table 3. The value of $K$ does not change for a series of readings constituting a test, while values of $L$ and $T$ do vary.

15.3 Values of $D$ may be computed with sufficient accuracy, using an ordinary 10-in. slide rule.

Note 15-The value of $L$ is divided by $T$ using the $A$ - and $B$-scales, the square root being indicated on the $D$-scale. Without ascertaining the value of the square root it may be multiplied by $K$, using either the $C$ - or Cl-scale.

\section{Sieve Analysis Values for Portion Finer than No. 10} (2.00-mm) Sieve

16.1 Calculation of percentages passing the various sieves used in sieving the portion of the sample from the hydrometer test involves several steps. The first step is to calculate the mass of the fraction that would have been retained on the No. 10 sieve had it not been removed. This mass is equal to the total percentage retained on the No. 10 sieve (100 minus total percentage passing) times the mass of the total sample represented by the mass of soil used (as calculated in 14.2), and the result divided by 100 .

16.2 Calculate next the total mass passing the No. 200 sieve. Add together the fractional masses retained on all the sieves, including the No. 10 sieve, and subtract this sum from the mass of the total sample (as calculated in 14.2).

16.3 Calculate next the total masses passing each of the other sieves, in a manner similar to that given in 12.2.

16.4 Calculate last the total percentages passing by dividing the total mass passing (as calculated in 16.3) by the total mass of sample (as calculated in 14.2), and multiply the result by 100 .
TABLE 2 Values of Effective Depth Based on Hydrometer Sedimentation Cylinder of Specified Sizes ${ }^{\wedge}$

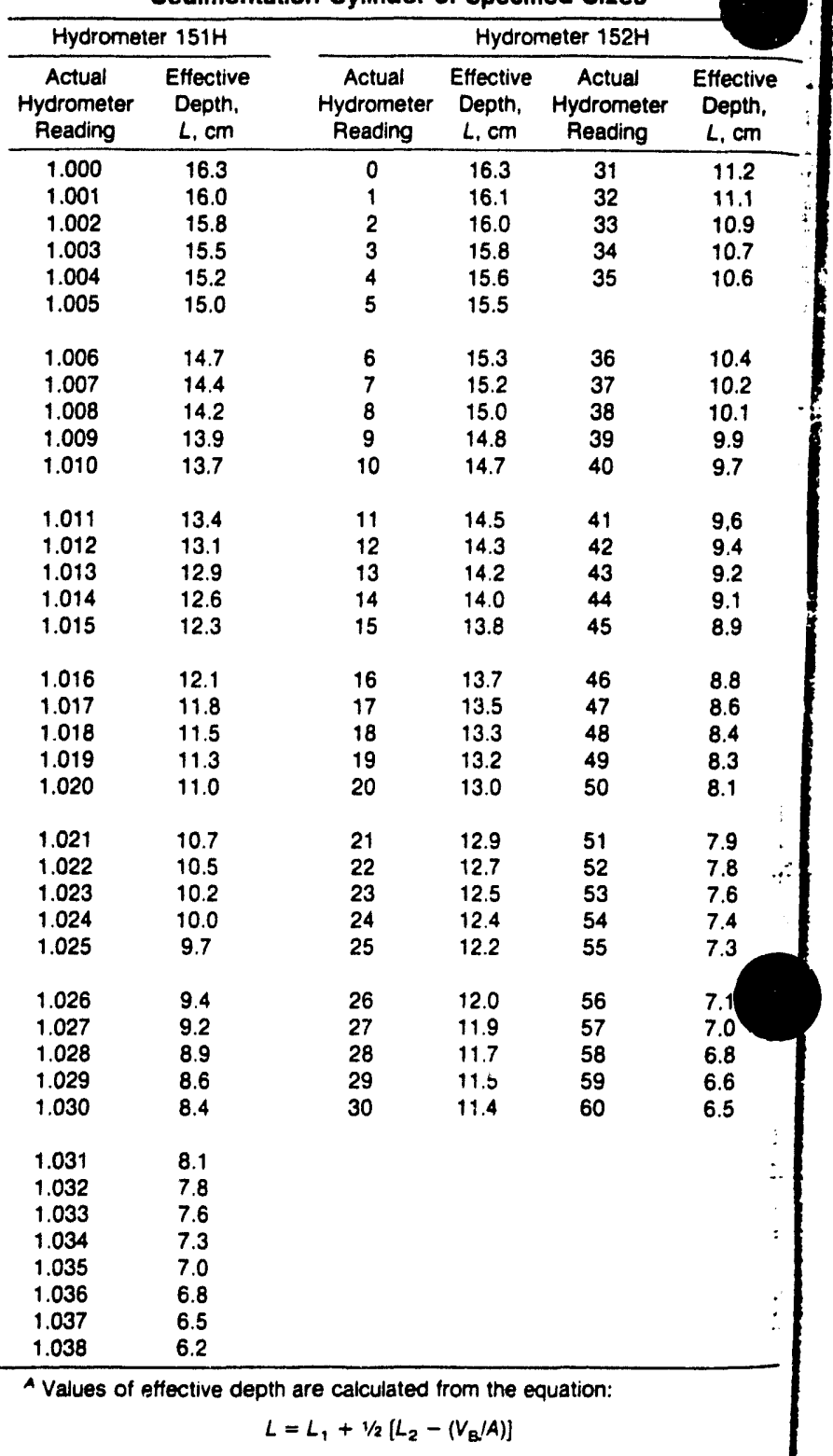

where:

$L$ = effective depth, $\mathrm{cm}$,

$L_{1}=$ distance along the stem of the hydrometer from the top of the bulb to the mark for a hydrometer reading, $\mathrm{cm}$.

$L_{2}=$ overall length of the hydrometer bulb, $\mathrm{cm}$,

$V_{B}=$ volume of hydrometer bulb, $\mathrm{cm}^{3}$, and

$A=$ cross-sectional area of sedimentation cylinder, $\mathrm{cm}^{2}$

Values used in calculating the values in Table 2 are as follows:

For both hydrometers, $151 \mathrm{H}$ and $152 \mathrm{H}$

$L_{2}=14.0 \mathrm{~cm}$

$V_{B}=67.0 \mathrm{~cm}^{3}$

$A=27.8 \mathrm{~cm}^{2}$

For hydrometer $151 \mathrm{H}$ :

$L_{1}=10.5 \mathrm{~cm}$ for a reading of 1.000

$=2.3 \mathrm{~cm}$ for a reading of 1.031

For hydrometer $152 \mathrm{H}$ :

$L_{1}=10.5 \mathrm{~cm}$ for a reading of $0 \mathrm{~g} /$ litre

$=2.3 \mathrm{~cm}$ for a reading of $50 \mathrm{~g} / \mathrm{litre}$

\section{Graph}

17.1 When the hydrometer analysis is performed, a graph 
TABLE 3 Values of $K$ for Use in Equation for Computing Diameter of Particle in Hydrometer Analysis

\begin{tabular}{|c|c|c|c|c|c|c|c|c|c|}
\hline \multirow{2}{*}{$\begin{array}{l}\text { Temperature, } \\
{ }^{\circ} \mathrm{C}\end{array}$} & \multicolumn{9}{|c|}{ Specific Gravity of Soll Particles } \\
\hline & 2.45 & 2.50 & 2.55 & 2.60 & 2.65 & 2.70 & 2.75 & 2.80 & 2.85 \\
\hline $\begin{array}{l}16 \\
17 \\
18 \\
19 \\
20\end{array}$ & $\begin{array}{l}0.01510 \\
0.01511 \\
0.01492 \\
0.01474 \\
0.01456\end{array}$ & $\begin{array}{l}0.01505 \\
0.01486 \\
0.01467 \\
0.01449 \\
0.01431\end{array}$ & $\begin{array}{l}0.01481 \\
0.01462 \\
0.01443 \\
0.01425 \\
0.01408\end{array}$ & $\begin{array}{l}0.01457 \\
0.01439 \\
0.01421 \\
0.01403 \\
0.01386\end{array}$ & $\begin{array}{l}0.01435 \\
0.01417 \\
0.01399 \\
0.01382 \\
0.01365\end{array}$ & $\begin{array}{l}0.01414 \\
0.01396 \\
0.01378 \\
0.01361 \\
0.01344\end{array}$ & $\begin{array}{l}0.01394 \\
0.01376 \\
0.01359 \\
0.01342 \\
0.01325\end{array}$ & $\begin{array}{c}0.01374 \\
0.01356 \\
0.01339 \\
0.1323 \\
0.01307\end{array}$ & $\begin{array}{l}0.01356 \\
0.01338 \\
0.01321 \\
0.01305 \\
0.01289\end{array}$ \\
\hline $\begin{array}{l}21 \\
22 \\
23 \\
24 \\
25\end{array}$ & $\begin{array}{l}0.01438 \\
0.01421 \\
0.01404 \\
0.01388 \\
0.01372\end{array}$ & $\begin{array}{l}0.01414 \\
0.01397 \\
0.01381 \\
0.01365 \\
0.01349\end{array}$ & $\begin{array}{l}0.01391 \\
0.01374 \\
0.01358 \\
0.01342 \\
0.01327\end{array}$ & $\begin{array}{l}0.01369 \\
0.01353 \\
0.01337 \\
0.01321 \\
0.01306\end{array}$ & $\begin{array}{l}0.01348 \\
0.01332 \\
0.01317 \\
0.01301 \\
0.01286\end{array}$ & $\begin{array}{l}0.01328 \\
0.01312 \\
0.01297 \\
0.01282 \\
0.01267\end{array}$ & $\begin{array}{l}0.01309 \\
0.01294 \\
0.01279 \\
0.01264 \\
0.01249\end{array}$ & $\begin{array}{l}0.01291 \\
0.01276 \\
0.01261 \\
0.01246 \\
0.01232\end{array}$ & $\begin{array}{l}0.01273 \\
0.01258 \\
0.01243 \\
0.01229 \\
0.01215\end{array}$ \\
\hline $\begin{array}{l}26 \\
27 \\
28 \\
29 \\
30\end{array}$ & $\begin{array}{l}0.01357 \\
0.01342 \\
0.01327 \\
0.01312 \\
0.01298\end{array}$ & $\begin{array}{l}0.01334 \\
0.01319 \\
0.01304 \\
0.01290 \\
0.01276\end{array}$ & $\begin{array}{l}0.01312 \\
0.01297 \\
0.01283 \\
0.01269 \\
0.01256\end{array}$ & $\begin{array}{l}0.01291 \\
0.01277 \\
0.01264 \\
0.01249 \\
0.01236\end{array}$ & $\begin{array}{l}0.01272 \\
0.01258 \\
0.01244 \\
0.01230 \\
0.01217\end{array}$ & $\begin{array}{l}0.01253 \\
0.01239 \\
0.01255 \\
0.01212 \\
0.01199\end{array}$ & $\begin{array}{l}0.01235 \\
0.01221 \\
0.01208 \\
0.01195 \\
0.01182\end{array}$ & $\begin{array}{l}0.01218 \\
0.01204 \\
0.01191 \\
0.01178 \\
0.01165\end{array}$ & $\begin{array}{l}0.01201 \\
0.01188 \\
0.01175 \\
0.01162 \\
0.01149\end{array}$ \\
\hline
\end{tabular}

of the test results shall be made, plotting the diameters of the particles on a logarithmic scale as the abscissa and the percentages smaller than the corresponding diameters to an arithmetic scale as the ordinate. When the hydrometer analysis is not made on a portion of the soil, the preparation of the graph is optional, since values may be secured directly from tabulated data.

\section{Report}

18.1 The report shall include the following:

18.1.1 Maximum size of particles,

18.1.2 Percentage passing (or retained on) each sieve, which may be tabulated or presented by plotting on a graph (Note 16),

18.1.3 Description of sand and gravel particles:

18.1.3.1 Shape-rounded or angular,

18.1.3.2 Hardness-hard and durable, soft, or weathered and friable,

18.1.4 Specific gravity, if unusually high or low,

18.1.5 Any difficulty in dispersing the fraction passing the No. $10(2.00-\mathrm{mm})$ sieve, indicating any change in type and amount of dispersing agent, and

18.1.6 The dispersion device used and the length of the dispersion period.

NoTE 16-This tabulation of graph represents the gradation of the sample tested. If particles larger than those contained in the sample were removed before testing, the report shall so state giving the amount and maximum size.

18.2 For materials tested for compliance with definite specifications, the fractions called for in such specifications shall be reported. The fractions smaller than the No. 10 sieve shall be read from the graph.

18.3 For materials for which compliance with definite specifications is not indicated and when the soil is composed almost entirely of particles passing the No. $4(4.75-\mathrm{mm})$ sieve, the results read from the graph may be reported as follows:

(l) Gravel, passing 3-in. and retained on No. 4 sieve

(2) Sand, passing No. 4 sieve and retained on No. 200 sieve
(a) Coarse sand, passing No. 4 sieve and retained on No. 10 sieve

(b) Medium sand, passing No. 10 sieve and retained on $\ldots \ldots \ldots \%$ No. 40 sjeve

(c) Fine sand, passing No. 40 sieve and retained on No. ........\% 200 sieve

(3) Silt size, 0.074 to $0.005 \mathrm{~mm}$

(4) Clay size, smaller than $0.005 \mathrm{~mm}$

Colloids, smaller than $0.001 \mathrm{~mm}$

18.4 For materials for which compliance with definite specifications is not indicated and when the soil contains material retained on the No. 4 sieve sufficient to require a sieve analysis on that portion, the results may be reported as follows (Note 17):

\section{SIEVE ANALYSIS}

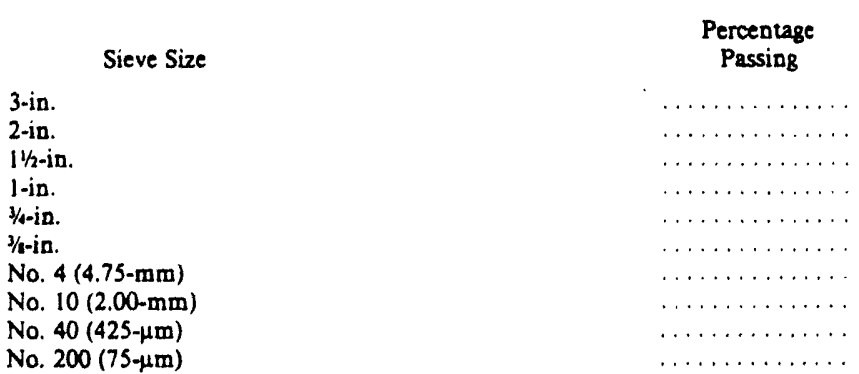

HYDROMETER ANALYSIS

$0.074 \mathrm{~mm}$

$0.005 \mathrm{~mm}$

$0.001 \mathrm{~mm}$

Note $17-$ No. $8(2.36-\mathrm{mm})$ and No. $50(300-\mu \mathrm{m})$ sieves may be substituted for No. 10 and No. 40 sieves.

The American Society for Testing and Materia/s takes no position respecting the validity of any patent rights asserted in connection with any hem mentioned in this standard. Users of this standard are expressly advised that determination of the validity of any such patent rights, and the risk of infringement of such rights, are entirely their own responsibility.

This stendard is subject to revision at any time by the responsibie technical commintee and must be reviewed every five years and If not revised, etther reapproved or withdrawn. Your comments are invited either for revision of this standard or for additional standards and should be addressed to ASTM Headquarters. Your comments will receive careful consideration at a meeting of the responsible technical committee, which you may attend. If you feel that your comments have not received a fair hoaring you should make your views known to the ASTM Committeo on Standards, 1916 Race St., Philadelphla, PA 19103. 


\title{
Standard Practice for Thin-Walled Tube Sampling of Soils ${ }^{1}$
}

\begin{abstract}
This standard is issued under the fixed designation D 1587: the number immediately following the designation indicates the year of original adoption or, in the case of revision, the year of last revision. A number in parentheses indicates the year of last reapproval. A superscript epsilon $(\epsilon)$ indicates an editorial change since the last revision or reapproval.
\end{abstract}

This practice has been approved for use by agencies of the Department of Defense and for listing in the DOD Index of Specifications and Slandards.

\section{Scope}

1.1 This practice covers a procedure for using a thin-walled metal tube to recover relatively undisturbed soil samples suitable for laboratory tests of structural properties. Thinwalled tubes used in piston, plug, or rotary-type samplers. such as the Denison or Pitcher, must comply with the portions of this practice which describe the thin-walled tubes (5.3).

NOTE 1-This practice does not apply to liners used within the above amplers.

\section{Referenced Documents}

\subsection{ASTM Standards:}

D 2488 Practice for Description and Identification of Soils (Visual-Manual Procedure) ${ }^{2}$

D 3550 Practice for Ring-Lined Barrel Sampling of Soils?

D4220 Practices for Preserving and Transporting Soil Samples ${ }^{2}$

\section{Summary of Practice}

I A relatively undisturbed sample is obtained by pressing 3 thin-walled metal tube into the in-situ soil, removing the wil-filled tube, and sealing the ends to prevent the soil from ring disturbed or losing moisture.

\section{Significance and Use}

\$.1 This practice, or Practice D 3550, is used when it is ascessary to obtain a relatively undisturbed specimen suitable $\therefore$ laboratory tests of structural properties or other tests that might be influenced by soil disturbance.

\section{: Ipparatus}

SI Drilling Equipment-Any drilling equipment may be sid that provides a reasonably clean hole: that does not -siurb the soil to be sampled: and that does not hinder the metration of the thin-walled sampler. Open borehole diimster and the inside diameter of driven casing or hollow $\because-$ auger shall not exceed 3.5 times the outside diameter of $\because$ inin-walled tube.

$:$ Sampler Insertion Equipmont, shall be adequate to - ide a relatively rapid continuous penetration force. For $\because$ formations it may be necessary, although not recomajed. to drive the thin-walled tube sampler.

- nractice is under the jurisdiction of ASTM Commituee D-18 on Soil and - 4.2 is the direct responsibility of Subcomminee D18.02 on Sampling and - $:$ ireld Testing for Soil Investigations.

$\because \because$ edinon approved Aug. 17. 1983. Published October 1483. Originalls $\therefore$ as D 1587-58T. Last previous editun D 1587-74.

-... Busk of ASTis Standards. Vol O4.018.
5.3 Thin-Walled Tubes, should be manufactured as shown in Fig. 1. They should have an outside diameter of 2 to 5 in. and be made of metal having adequate strength for use in the soil and formation intended. Tubes shall be clean and free of all surface irregularities including projecting weld seams.

5.3.1 Length of Tubes-See Table 1 and 6.4.

5.3.2 Tolerances, shall be within the limits shown in Table 2.

5.3.3 Inside Clearance Ratio, should be $1 \%$ or as specified by the engineer or geologist for the soil and formation to be sampled. Generally, the inside clearance ratio used should increase with the increase in plasticity of the soil being sampled. See Fig. 1 for definition of inside clearance ratio.

5.3.4 Corrosion Protection-Corrosion, whether from galvanic or chemical reaction. can damage or destroy both the thin-walled tube and the sample. Severity of damage is a function of time as well as interaction between the sample and the tube. Thin-walled tubes should have some form of protective coating. Tubes which will contain samples for more than $72 \mathrm{~h}$ shall be coated. The type of coating to be used may vary depending upon the material to be sampled. Coatings may include a light coat of lubricating oil. lacquer. epoxy, Teflon, and others. Type of coating must be specified by the engineer or geologist if storage will exceed $72 \mathrm{~h}$. Plating of the tubes or alternate base metals may be specified by the engineer or geologist.

5.4 Sampler Head, serves to couple the thin-walled tube to the insertion equipment and, together with the thin-walled tube, comprises the thin-walled tube sampler. The sampler head shall contain a suitable check valve and a venting area to the outside equal to or greater than the area through the check valve. Attachment of the head to the tube shall be concentric and coaxial to assure uniform application of force to the tube by the sampler insertion equipment.

\section{Procedure}

6.1 Clean out the borehole to sampling elevation using whatever method is preferred that will ensure the material to be sampled is not disturbed. If groundwater is encountered, maintain the liquid level in the borehole at or above ground water level during the sampling operation.

6.2 Bottom discharge bits are not permitted. Side discharge bits may be used, with caution. Jetting through an open-tube sampler to clean out the borehole to sampling elevation is not permitted. Remove loose material from the center of a casing or hollow stem auger as carefully as possible to avoid disturbance of the material to be sampled.

Not: 2-Roller bits are available in downward-jetting and diffused- 


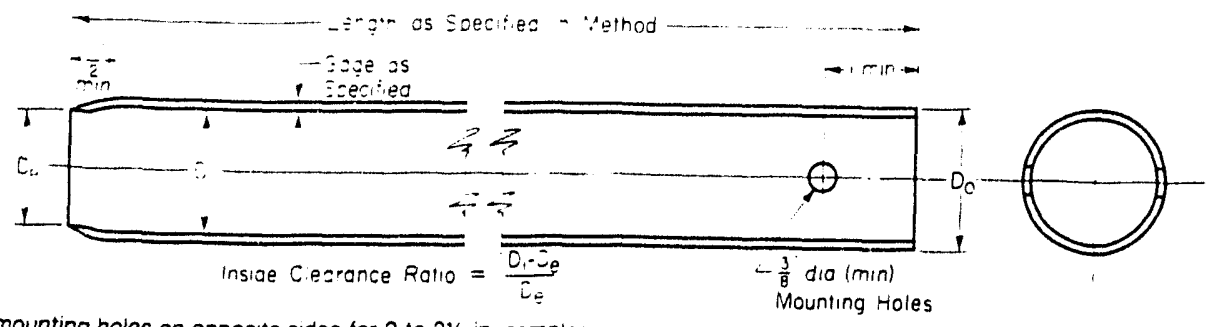

NOTE 1-Minimum of two mounting holes on opposite sides for 2 to $3 \frac{1}{2}$ in. sampler.

NoTE 2-Minimum of four mountıng holes spaced at $90^{\circ}$ for samplers 4 in. and larger

Note 3-Tube held with hardened screws.

NOTE 4-Two-Inch Outside-dia

advised that such tubing is difficult to locate and specified with an 18 -gage wall thickness to comply with area ratio criteria accepted for "undisturbed samples." Use is difficult to locate and can be extremely expensive in small quantities. Sixteen-gage tubes are generally readily available. Metric Equivalents

\begin{tabular}{cc}
\hline in. & $\mathrm{mm}$ \\
\hline $3 / 6$ & 6.77 \\
$1 / 2$ & 12.7 \\
1 & 25.4 \\
2 & 50.8 \\
$31 / 2$ & 88.9 \\
4 & 101.6 \\
\hline
\end{tabular}

FIG. 1 Thin-Walled Tube for Sampling

TABLE 1 Suitable Thin-Walled Steel Sample Tubes^

\begin{tabular}{llll}
\hline $\begin{array}{l}\text { Outside diameter: } \\
\text { in. }\end{array}$ & 2 & 3 & 5 \\
mm & 50.8 & 76.2 & 127 \\
Wall thickness: & 18 & 16 & 11 \\
Bwg & 0.049 & 0.065 & 0.120 \\
in. & 1.24 & 1.65 & 3.05 \\
mm & 36 & 36 & \\
Tube length: & 0.91 & 0.91 & 54 \\
in. & 1 & .1 & 1.45 \\
m & & 1 \\
Clearance ratio. \% & n The three diameters recommended in Table 1 are indicated tor purposes
\end{tabular}

The three diameters recommended in Table 1 are indicated for purposes of standardization, and are not intended to indicate that sampling tubes of intermediate or larger diameters are not acceptable. Lengths of tubes shown are illustrative. Proper lengths to be determined as suited to field conditions.

TABLE 2 Dimensional Tolerances for Thin-Walled Tubes

\begin{tabular}{lccc}
\hline \multicolumn{4}{c}{ Nominal Tube Diameters from Table t $^{\wedge}$ Tolerances, in. } \\
\hline $\begin{array}{c}\text { Size Outside } \\
\text { Diameter }\end{array}$ & 2 & 3 & 5 \\
\hline Outside diameter & +0.007 & +0.010 & +0.015 \\
& -0.000 & -0.000 & -0.000 \\
Inside diameter & +0.000 & +0.000 & +0.000 \\
& -0.007 & -0.010 & -0.015 \\
Wall thickness & \pm 0.007 & \pm 0.010 & \pm 0.015 \\
Ovality & 0.015 & 0.020 & 0.030 \\
Straightness & $0.030 / \mathrm{ft}$ & $0.030 / \mathrm{ft}$ & $0.030 / \mathrm{t}$ \\
\hline
\end{tabular}

A Intermediate or larger diameters should be proportional. Tolerances shown are essentially standard commercial manufacturing tolerances for seamless steel mechanical tubing. Specify only two of the first three tolerances; that is, O.D. and I.D.. or O.D. and Wall, or I.D. and Wall.

jet configurations. Downward-jetting configuration rock bits are not acceptable. Diffuse-jet configurations are generally acceptable.

6.3 Place the sample tube so that its bottom rests on the bottom of the hole. Advance the sampler without rotation by a continuous relatively rapid motion.

6.4 Determine the length of advance by the resistance and condition of the formation, but the length shall never exceed 5 to 10 diameters of the tube in sands and 10 to 15 diameters of the tube in clays.

Note 3-Weight of sample, laboratory handling capabilities, tr. portation problems. and commercial availability of tubes will gener limit maximum practical lengths to those shown in Table 1 .

6.5 When the formation is too hard for push-type in: tion, the tube may be driven or Practice D $3550 \mathrm{~m}$ us Other methods, as directed by the engineer or ged be used. If driving methods are used, the data regarding wei: and fall of the hammer and penetration achieved must shown in the report. Additionally, that tube must be prot nently labeled a "driven sample."

6.6 In no case shall a length of advance be greater than 1 sample-tube lengtl: minus an allowance for the sampler he and a minimum of 3 in. for sludge-end cuttings.

Nore 4-The tube may be rotated to shear bottom of the sam after pressing is complete.

6.7 Withdraw the sampler from the formation as carefu as possible in order to minimize disturbance of the sample

\section{Preparation for Shipment}

7.1 Upon removal of the tube, measure the length sample in the tube. Remove the disturbed material in $t$ upper end of the tube and measure the length again. Seal $t$. upper end of the tube. Remove at least 1 in. of material fro the lower end of the tube. Use this material for soil descriptic in accordance with Practice D 2488. Measure the over: sample length. Seal the lower end of the tube. Alternativel after measurement, the tube may be sealed without remov of soil from the ends of the tube if so directed by the engine or geologist.

Note 5-Field extrusion and packaging of extruded samples und the specific direction of a geotechnical engineer or geologist is permitte

Note 6-Tubes sealed over the ends as opposed to those expanding packers should contain end padding in end void. prevent drainage or movenient of the sample within the tube

7.2 Prepare and immediately affix labels or apply markin 


\section{明以 1587}

as necessary to identify the sample. Assure that the markings or labels are adequate to survive transportation and storage.

\section{Report}

8.1 The appropriate information is required as follows:

8.1.1 Name and location of the project,

8.1.2 Boring number and precise location on project,

8.1.3 Surface elevation or reference to a datum,

8.1.4 Date and time of boring - start and finish,

8.1.5 Depth to top of sample and number of sample,

8.1.6 Description of sampler: size, type of metal, type of coating,
8.1.7 Method of sampler insertion: push or drive,

8.1.8 Method of drilling, size of hole, casing, and drilling fluid used,

8.1.9 Depth to groundwater level: date and time measured,

8.1.10 Any possible current or tidal effect on water level,

8.1.11 Soil description in accordance with Practice

D 2488

8.1.12 Length of sampler advance, and

8.1.13 Recovery: length of sample obtained.

\section{Precision and Bias}

9.1 This practice does not produce numerical data; therefore, a precision and bias statement is not applicable.

The American Society for Testing and Materials takes no position respecting the validity of any patent rights asserted in connection with any item mentioned in this standard. Users of this standard are expressly advised that determination of the validity of any such patent rights, and the risk of infringernent of such rights, are entirely their own responsibility.

This standard is subject to revision at any time by the responsible technical committee and must be reviewed every five years and if not revised, either reapproved $c$ ' withdrawn. Your comments are invited either for revision of this standard or for additional standards and should be addressed to ASTWl Headquarters. Your comments will receive careful consideration at a meeting of the responsible technical committee, which you may attend. If you feel that your comments have not received a fair hearing you should make your views known to the ASTM Committee on Standards, 1916 Race St., Philadelphia, PA 19103. 


\title{
Standard Test Method for Moisture Content of Soil and Soil-Aggregate in Place by Nuclear Methods (Shallow Depth) ${ }^{1}$
}

\begin{abstract}
This standard is issued under the fixed desig:ation D 3017; the number immediately following the designation indicates the year of original adoption or, in the case of revision, the year of last revision. A number in parentheses indicates the year of last reapproval. A superscript epsilon (e) indicates an editorial change since the last revision or reapproval.

This method has been approved for use by agencies of the Department of Defense and for listing in the DoD Index of Specifications and Standards.
\end{abstract}

\section{INTRODUCTION}

This test method covers determination of the moisture content of soil and soil-aggregate in place through the use of nuclear equipment. The equipment is calibrated to determine moisture content, as weight of water per unit volume of material (pounds per cubic foot or kilograms per cubic metre). Moisture content as normally used is defined as the ratio, expressed as a percentage, of the weight of water in a given soil mass to the weight of solid particles. It is determined with this procedure by dividing the moisture content (pounds per cubic foot or kilograms per cubic metre) by the dry unit weight of the soil (pounds per cubic foot or kilograms per cubic metre). Therefore, computation of moisture content using the nuclear equipment also requires the determination of the dry unit weight of the material under test. Most available nuclear equipment has provision for measuring both moisture content (pounds per cubic foot or kilograms per cubic metre) and wet unit weight. The difference between these two measurements gives dry unit weight.

The moisture content determined by this test method is not necessarily the average moisture within the volume of sample involved in the measurement for reasons discussed in the Appendix. The principles of this test method as well as the advantages and limitations are also discussed in the Appendix.

The equipment utilizes radioactive materials which may be hazardous to the health of the users unless proper precautions are taken.

\section{Scope}

1.1 This test method covers determination of the moisture content of soil and soil-aggregate in place by moderation or slowing of fast neutrons where the neutron source and the thermal neutron detector both remain at the surface.

\section{Referenced Documents}

2.1 ASTM Standards:

D 1556 Test Method for Density of Soil in Place by the Sand-Cone Method ${ }^{2}$

D2167 Test Method for Density and Unit Weight of Soil in Place by the Rubber-Balloon Method ${ }^{2}$

D2216 Method for Laboratory Determination of Water (Moisture) Content of Soil, Rock, and Soil-Aggregate Mixtures $^{2}$

\section{Summary of Method}

3.1 The moisture content of the material under test is determined by placing a fast neutron source and a thermal neutron detector on or adjacent to the material under test. The intensity of slow or moderated neutrons detected is

\footnotetext{
' This test method is under the jurisdiction of ASTM Committee D-18 on Soil and Rock and is the direct responsibility of Subcommittee D18.08 on Special and Construction Control Tests.

Current edition approved Jan. 27, 1978. Published April 1978. Originally published as D 3017 - 72. Last previous edition D 3017 - 72 (1977).

innual Book of ASTM Standards, Vol 04.08 .
}

dependent upon the moisture content of the material under test. Moisture is determined by the relationship of nuclear count to weight of water per unit volume of soil.

\section{Significance and Use}

4.1 The test method described is useful as a rapid, nondes: tructive technique for the in-place determination of moisture content of soil and soil-aggregate. The fundamental assumptions inherent in the method are that the hydrogen present is in the form of water as defined by Method D 2216, and that the material under test is homogeneous.

4.2 The test method is suitable for control and acceptance testing of soils and soil-aggregate for construction, research. and development. Test results may be affected by chemical composition, sample heterogeneity, and, to a lesser degree. material density and the surface texture of the material being tested. The technique also exhibits spatial bias in that the apparatus is more sensitive to certain regions of the material under test.

\section{Safety Precaution}

5.1 This equipment utilizes radioactive materials which may be hazardous to the health of the users, unless proper precautions are taken. Users of this equipment must become completely familiar with possible safety hazards and with all applicable government regulations. Effective operator instruction together with routine safety procedures such as 


\section{3017}

source-leak tests, recording and evaluation of film badge data. use of survey meters, etc., are an essential part of the operation of equipment of this type.

\section{Apparatus}

6.1 Fast Neutron Source-A sealed isotope material such as americium-beryllium, radium-beryllium, or an electronic device such as a neutron generator.

6.2 Slow Neutron Detector-Any type of slow neutron detector such as boron trifluoride. a scintillator crystal, or a fission chamber.

6.3 Readout Device-A suitable scaler. Usually the readout device will contain the high-voltage supply necessary to operate the detector, and low-voltage power supply to operate the readout and accessory devices.

6.4 Housing-The source, detector, readout device, and power supply shall be in housings of rugged construction which shall be moistureproof and dustproof.

Note 1-The neutron source, detector, readout device. and power supply may be housed separately or may be combined and integrated with a nuclear density measuring system.

6.5 Reference Standard, for checking equipment operation and background count. and to establish conditions for a reproducible count rate.

6.6 Site Preparation Device-A steel plate, straightedge. or other suitable leveling tools may be used to plane the test site to the required smoothness.

\section{Calibration}

7.1 Calibration curves are established by determining by test the nuclear count rate of each of several samples of different known moisture content. plotting the count rate for count ratio) versus each known moisture content and placing a curve through the resulting points. The method and test procedure used in establishing the curve must be the same as used for determining moisture content of the material to be lested. The moisture content of materials used to establish the calibration curve must vary through a range to include :he moisture content of materials to be tested. The materials used for calibration must be of uniform density as well as uniform moisture content.

Vote 2-Due to the effect of chemical composition. some calibration - - res supplied with the apparatus may not be applicable to all materials -nder test. Therefore, calibration curves must he checked. and adjusted necessary, in accordance with 7.2 and 7.3

-1.1 Calibration curves can be established using the fol. .14ing methods:

-1.1.1 Prepared containers of compacted soil and soil- iregate of known moisture content. Prepared containers - ust be large enough to not change the observed count rate

$\because$ count ratio) if made larger in any dimension (Note 3 ). If

$\because$ nydrogen density of a material can be calculated from its suific gravity and chemical formula. provided these are - - urately known. a much more reliable calibration point $\therefore$ be obtained in comparison to oven drying methods.

-1.1.2 Volume determined by the sandcone (Test Method : $: 56$ ) or rubber balloon (Test Method D 2167) and weighi water by oven drying in conjunction with field compaction ects

.il 3-Dimensions of approximately 2t in. long hy is in. wide h:
18 in. deep (approximately 610 by 457 by $457 \mathrm{~mm}$ ) have proven satisfactory for equipment now available (1971)

7.2 Checking Calibration Curves-The calibration curves for newly acquired instruments and repaired instruments should be checked. Calibration curves should be checked prior to tests in materials that are distinctly different from material types previously used in obtaining the calibration curves. Calibration curves may be checked using the procedures given in 7.1.1.

7.3 Adjusting Calibration Curves-When prepared containers of material of known moisture are being used to check the calibration, plot the count rate (or count ratio) versus each known moisture content as described in 7.1. If the points do not fall in a random manner on each side of the previously established calibration curve, replace the original calibration curve with a curve through the plotted check points.

7.3.1 If the moisture content of each of the comparison tests determined by any of the above calibration methods is random about the previously established calibration curve and the average deviation does not exceed $\pm 0.5 \mathrm{lb} / \mathrm{ft}^{3}(8 \mathrm{~kg} /$ $\mathrm{m}^{3}$ ) of soil from the moisture content determined by the nuclear method, then adjustment of the calibration curve is not needed and should not be made.

7.3.2 If each of the moisture content determinations made by any of the above calibration methods is more than the corresponding moisture content by the nuclear method, adjust each subsequent nuclear determination by adding the average difference.

7.3.3 If each of the moisture content determinations made by any of the above calibration methods is less than the corresponding moisture content by the nuclear method, adjust each subsequent nuclear determination by subtracting the average difference.

7.3.4 The average difference obtained by 7.3 .2 or 7.3 .3 may be used to plot a corrected calibration curve which shall be parallel to the original calibration curve and offset by the amount and direction as indicated in 7.3.2 or 7.3.3.

\section{Procedure}

8.1 Standardization-Standardization of equipment on a reference standard is required at the start of each day's use as follows:

8.1.1 Warm up the equipment in accordance with the manufacturer's recommendations.

8.1.2 Take at least four repetitive readings of at least $1 \mathrm{~min}$ each with the gage on the reference standard. This constitutes one standardization check

8.1.3 If the mean of the four repetitive readings is outside the limits set by Eq 1 below, repeat the standardization check. If either the first or the second attempt satisfies Eq 1, the equipment is considered to be in satisfactory operation condition: continue with the procedure in 8.2 . The empirical relationship for the standardization check is as follows:

$$
\Lambda_{s}=\Lambda_{0} \pm 1.96 \sqrt{\Lambda_{0}}
$$

where:

$\Lambda_{s}^{\prime}=$ mean of repetitive readings (see 8.1 .2 ) and

$\Lambda_{0}=$ previously established count for the reference standard (mean of ten repetitive readings).

8.1.4 If the second attempt in 8.1.3 does rot satisfy Eq I. chect the system and repair the instrument if necessary. It is
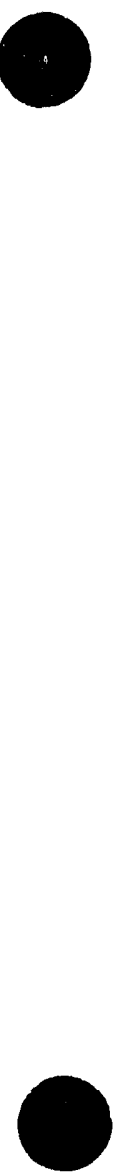
possible to use the instrument in this condition if a satistactory calibration relationship can bi: established.

8.1.5 Establish a new $N_{0}$ by computing the mean of ten repetitive readings on the reference standard.

8.1.6 Check the calibration curve in accordance with 7.2 and, if necessary, adjust the calibration curve in accordance with 7.3.

8.2 Test Site Preparation-Select a location for test where the gage in test position will be at least 6 in. (approximately $152 \mathrm{~mm}$ ) aw ay from any vertical projection.

8.2.1 Prepare the test site in the following manner:

8.2.1.1 Remove all loose and disturbed material. and remove additional material as necessary to reach the top of the vertical inten al to be tested (Note 4).

8.2.1.2 Prepare a horizontal area. sufficient in size to accommodate the gage, by planing to a smooth condition so as to obtain maximum contact between the gage and material being tested.

8.2.1.3 The maximum depressions beneath the gage shall not exceed approximately $1 / 8 \mathrm{in} .(3.2 \mathrm{~mm})$. Use native fines or fine sand to fill voids and level the excess with a rigid plate or other suitable tool.

NOTE 4-The spatial bias should be considered in determining the depth at which the gage is to be seated.

Note 5-The placement of the gage on the surface of the material to be tested is critical to the successful determination of moisture. The optimum condition is total contact between the bottom surface of the gage and the surface of the material being tested. This is not practically possible in all cases and therefore it becomes necessary to use a sand filler or similar material. The depth of sand fill should not exceed $1 / 8$ in. (approximately $3.2 \mathrm{~mm}$ ) anc the total area filled should not exceed $10 \%$ of the bottom area of the gage. Several crial seatings may be required to achieve these conditions.

8.3 Testing-Proceed with testing in the following manner:

8.3.1 Seat the gage firmly.

8.3.2 Keep all other radioactive sources away from the gage (as recommended by the manufacturer) so as not to affect the readings.

8.3.3 Use sufficient warm-up time as in standardization (8.1.1).
8.3.4 Place the source in the use position and take one... more I-min readings.

\section{Calculations}

9.1 Average the readings obtained in 8.3.4.

9.2 Determine the moisture content by use of an applicable calibration curve.

9.3 Calculate the moisture content. $w$. in percent of $d n$ weight of soil as follows:

$$
11=\left(W_{m} / H_{d}\right) \times 100
$$

where:

$\mathrm{II}_{\mathrm{m}}=$ moisture content of soil, $\mathrm{lb} / \mathrm{ft}^{3}\left(\mathrm{or} \mathrm{kg} / \mathrm{m}^{3}\right)$ and

$H_{\mathrm{d}}^{\mathrm{m}}=\mathrm{dry}$ weight of soil. $\mathrm{lb} / \mathrm{ft}^{3}\left(\mathrm{or} \mathrm{kg} / \mathrm{m}^{3}\right)$.

\section{Report}

10.1 The report shall include the following:

10.1.1 Location.

10.1.2 Elevation of surface.

10.1.3 Visual description of material.

10.1.4 Identification of test equipment (make. model. and serial number),

10.1.5 Count rate for each reading,

10.1.6 Moisture content in pounds per cubic foot (kilo. grams per cubic metre).

10.1.7 Wet unit weight,

10.1.8 Dry unit weight. and

10.1.9 Moisture content in percent of dry weight.

\section{Precision}

11.1 Determine the precision of the system. $P$, from the slope of the calibration curve, $S$, and the standard deviation. $\sigma$. of the signals (detected neutrons) in counts per minute, as follows:

$$
P=\sigma / S
$$

11.2 Where the slope of the calibration curve is determined at the $10-\mathrm{lb} / \mathrm{ft}^{3}\left(160-\mathrm{kg} / \mathrm{m}^{3}\right)$ point and the standard deviation is determined from ten repetitive readings of $1 \mathrm{~min}$ each (the gage is not moved after the first seating) taken on material having a moisture content of $10.0 \pm 0.6 \mathrm{lb} / \mathrm{ft}^{3}(160 \pm 10 \mathrm{~kg} /$ $\left.\mathrm{m}^{3}\right)$ the value of $P$ shall be less than $0.30 \mathrm{lb} / \mathrm{ft}^{3}\left(4.8 \mathrm{~kg} / \mathrm{m}^{3}\right)$.

\section{APPENDIX}

\section{(Nonmandatory Information)}

\section{X1. NOTES ON THE NLCLEAR TEST}

$\mathrm{X} 1.1$ The equipment used in this method is of the surface type as opposed to that designed for use in deep borings. In general, and neglecting the associated electronics, this equipment consists of three principal elements: (1) a suitable nuclear source emitting fast neutrons: (2) a detector sensitive to these neutrons after they are modified by passing through the material being tested; and (3) a counter, with provisions for automatic and precise timing, for determining the rate at which the modified neutrons arrive at the detector. While rate meters are suitable. in principle. counters are commonly used. In general, any source of sufficiently numerous and properly energetic neutrons can be used in measuring the moisture content of soil and soil-aggregate. The sources most commonly used, however, are americium-berylium and radium-beryllium with the latter normally being used in dualpurpose instruments that are designed to also determine wet or total density of soil and soil-aggregates. Detectors used are gas filled tubes of boron trifluoride and scintillation crystals or fission chambers. Detectors of the latter type offer the potential of varying the range of energies of the neutrons that are counted.

X1.2 Measurements are made using fast neutrons that 
reflect modification by backscattering through the material under test. When high-energy neutrons are scattered into the soil a loss in velocity of each neutron occurs as it collides with the nuclei of the atoms of the soil. The rate at which this slowing down process occurs depends upon: (1) the mass of the nucleus in collision with the neutron. and (2) the probability that the two will collide.

X1.2.1 The mass of the hydrogen nucleus is nearly equal to the neutron mass. Collision with hydrogen atoms therefore. reduces the velocity of neutrons more quickly than collision with heavier nuclei. The large difference between the masses of hydrogen atoms and those normally encountered in soils means that the relative effectiveness of hydrogen atoms in slowing down neutrons is very pronounced.

X1.2.2 The probability that a neutron will collide with the nucleus of an atom is dependent on the atom's scattering cross section. For most elements, this value is low. usually. increasing with decrease in neutron energy. The scattering cross section of the hydrogen atom for high energy' neutrons. however, is larger than for most of the other atoms present in soils.

X 1.2.3 These two features in combination make hydrogen the most effective medium for reducing the velocity of fast neutrons. If a detector of thermal (slow) neutrons is placed near a neutron source in a soil containing hydrogen. the activity registered is due almost entirely to neutrons that have been slowed down by hydrogen atoms. Other atoms present in the soil play a negligible part in this process. In natural soils hydrogen may be present in several forms but. with some exceptions. it occurs principally in the water held by the soil particles. Therefore, the "slow' neutron" activity registered hy. a suitable detector can be related to the concentration of water in a soil by calibration.

X1.3 The determination of moisture content by the nuclear means of this method is indirect. To date no theoretical approach has been developed that predicts the count rate for given equipment. geometry. material. density. and moisture content. As a result the relation between soil moisture and nuclear count rate is determined by correlation tests of ma. terials at known moisture content. Individual equipment manufacturers supply a calibration curve with each set of their equipment. It has been found that these curves do not necessarily hold for all soils and soil-aggregates because of differences in chemical composition. Apparent variations in alibration curves may also be induced by differences in seating. in background count. and other test variations. Beaduse of these considerations. provisions are included in this method for checking for variations or changes. Different spproaches may be used in checking calibration curves and hose in more general use are given. For good practice these should be followed with newly purchased equipment and "th major component replacement of in-service equipment.

X1.4 The moisture content determined by this method is ina amount of moisture that is contained in a given volume soil. It should be noted that the volume of soil and soil*gregate represented in the measurement is indeterminate $\therefore$ will vary with the source-detector geometry of the equip- ment used and with the characteristics of the material tested. In general, and with all other conditions constant, the greater the moisture content of the material, the smaller the volume involved in the measurement. Unlike oven drying tests, the moisture content so determined is not necessarily the average moisture within the volume involved in the measurement. For the usual surface test equipment and materials, for example, about $50 \%$ of the measured count rate is determined by the upper 3 to 4 in. (76 to $101 \mathrm{~mm}$ ) of soils and soilaggregate.

X1.5 The number of fast neutrons emitted from a given source over a given time period are statistically random and follow a Gaussian distribution. Hecause of this, the actual number of modified neutrons that are detected and counted in the moisture measuring process should be sufficiently large to minimize the probability that the observed count reflects unacceptable variations. This is reflected in the standard deviation which is the square root of the total count. The over-all system accuracy in determining moisture is also statistical in nature and appears to vary with equipment used. test conditions of laboratory versus field. materials, and operators. Because of these variables it is not possible to give precise numbers for system accuracy and precision for these test methods. It is believed. how'ever. that if the procedures herein are carefully followed. the standard deviation of the nuclear measured values. in terms of accuracy. will not be greater than on the order of some 0.5 to $1.0 \mathrm{lb}$ of water per cubic foot $\left(8\right.$ to $\left.16 \mathrm{~kg} / \mathrm{m}^{3}\right)$ of soil. Precision, determined without moving the test equipment. should be better than 0.3 lb of water per cubic fool $\left(5 \mathrm{~kg} / \mathrm{m}^{3}\right)$ of soil.

$X 1.6$ One of the fast neutron sources used. americium. is manmade and as such its use is regulated by the Federal government through the Atomic Energ! Commission as well as by some State and local governments. Because radium and beryllium are naturally occurring materials. their use is not now regulated by the Federal government but is regulated by some State and local governments. Among others. the objectives of these regulations are the use of radioactive materials in a manner safe to the operator and all others.

$X 1.7$ The in-place nuclear moisture tests of this method offer several advantages over conventional methods, such as oven drying of samples. particularly in tests performed for the continuing control of ccnstruction. Its greatest advantage is perhaps the shor time required to obtain a moisture content. An answer is available on the spot in a matter of minutes after completing the test. When conducting both moisture and density tests many more tests per das can be conducted than by older methods in current use. In addition. apparently erratic measurements can be immediately detected and checked since the nuclear tests are more nearly nondestructive. These advantages accrue to organizations that are engaged in moisture measurements on a more or less continuous hasis. Organizations that make infrequent or occasional moisture determinations may find that the advantages of the nuclear methods can be offiset hy maintenance and start up considerations such as periodically charging batteries, maintaining radiation exposure records. etc. 
The American Society for Testing and Materials takes no position respecting the validity of any patent rights asserted in connection with any item mentioned in this standard. Users of this standard are expressly advised that determination of the validity of any such patent rights, and the risk of infringement of such rights, are entirely their own responsibility.

This standard is subject to revision at any time by the responsible technical committee and must be reviewed every five years and if not revised, either reapproved or withdrawn. Your comments are invited either for revision of this standard or for additional standards and should be addressed to ASTM Headquarters. Your comments will receive careful consideration at a meeting of the responsible technical committee, which you may attend. If you teel that your comments have not received a fair hearing you should make your views known to the ASTM Committee on Standards, 1916 Race St., Philadelphia, PA 19103. 


\section{Appendix C}

\section{Field Logbooks}



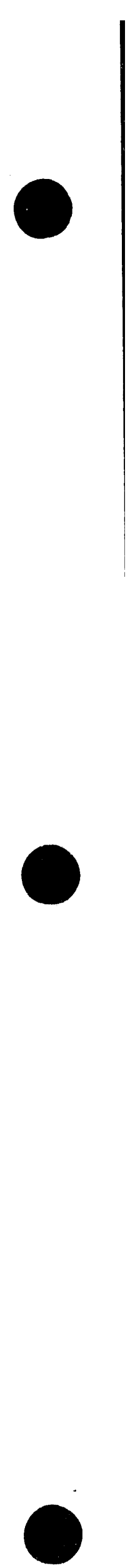

C-2 


\section{Field Logbooks}

1. Fisld Team Leader's Daily Logbook

2. Field Instrument Calibration/Standardization Logbook

3. Sample Logbook

4. Sample Shipping Logbook 
INEL RCRA/CERCLA INVESTIGATIONS

FIELD TEAM LEADER'S DAILY LOGBOOK

DATE START

19

DATE END

19

LOGBOOK NO:

LOGBOOK ASSIGNED TO:

SITE:

WHEN COMPLETED RETURN TO: D. KIRCHNER

MS: 3900 
INSTRUCTIONS FOR COMPLETING

FIELD TEAM LEADERS DAILY LOGBOOK

In some very large sampling activities e.g. Where there is a drilling crew, a sample collection crew, a shipping crew etc. and a field team leader that oversees all of the activities it may be necessary to record information on the daily activities of each of these crews. The size of the site and the preference of the field team leader will dictate whether or not this logbook is needed. This logbook should not be confused with the Sample Logbook. The purpose of the Sample Logbook is to record activities and conditions surrounding collection of a physical sample. This logbook is restricted to activities of a "sampling crew". The purpose of the Field Team Leader's Daily Logbook is to record information on field activities when several different crews are involved.

On the first page of the logbook record the name and signature of all field team members. You may wish to restrict this list to field workers whose name may appear on any field documentation e.g. field tags, labels, etc. The purpose is to be able to compare the handwriting on a field document to the signature in this logbook in the event a question should arise on the authenticity or reliability of an entry.

Should any visitors e.g. representatives from the State of Idaho or EPA or any auditors visit a site during field activities their name and purpose of visit should be recorded. 


\section{SAMPLING TEAM}

NAME (Print)

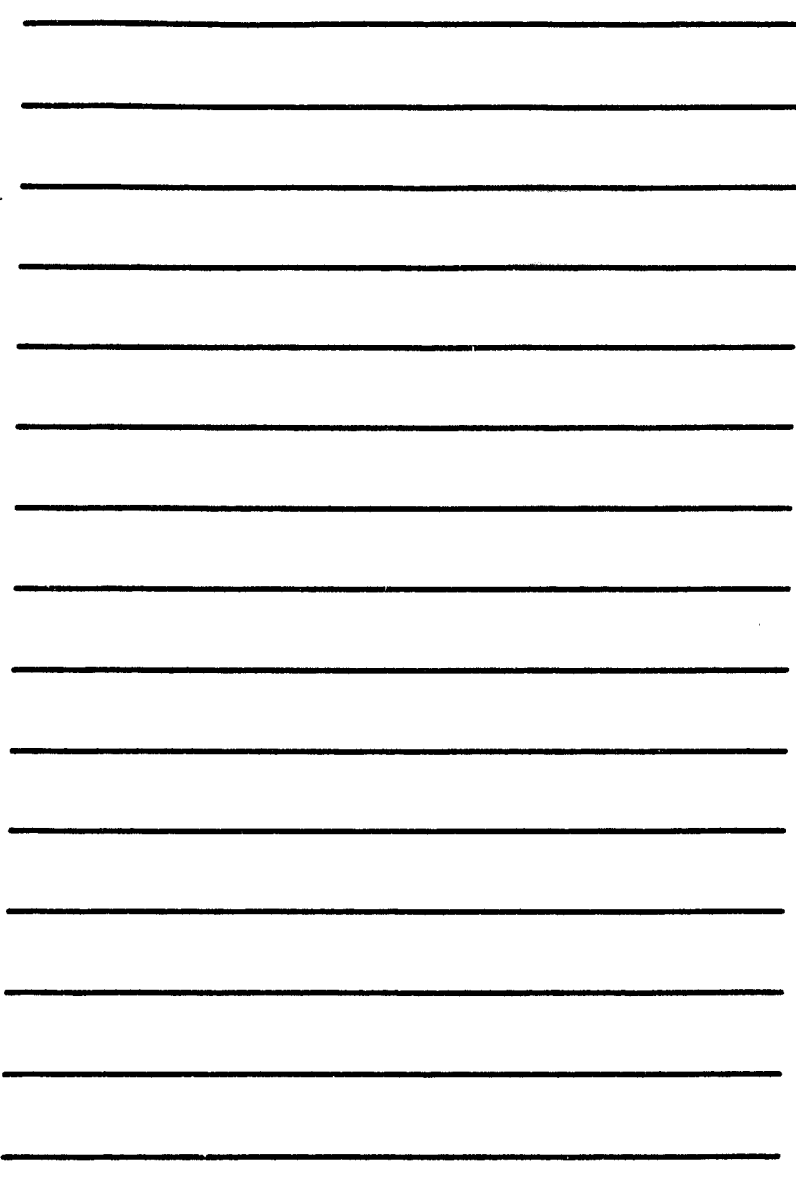

Visitors Name

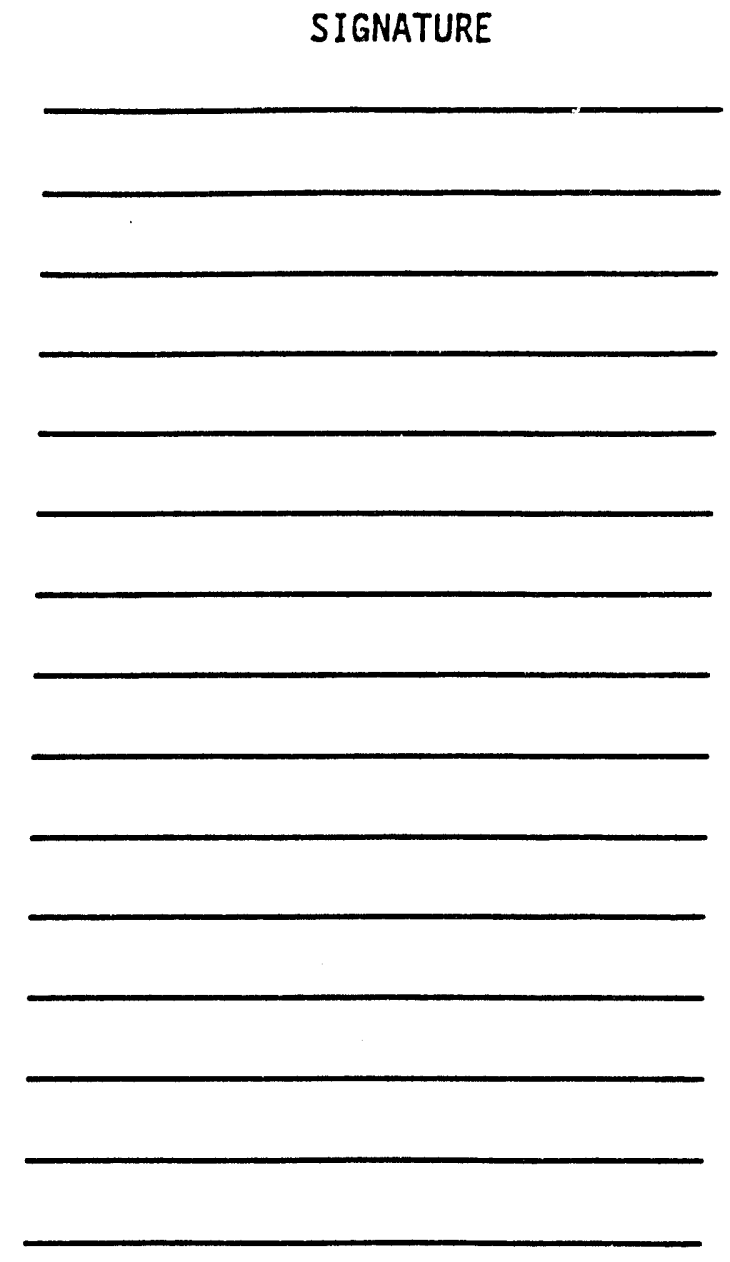

Reason for Visit 


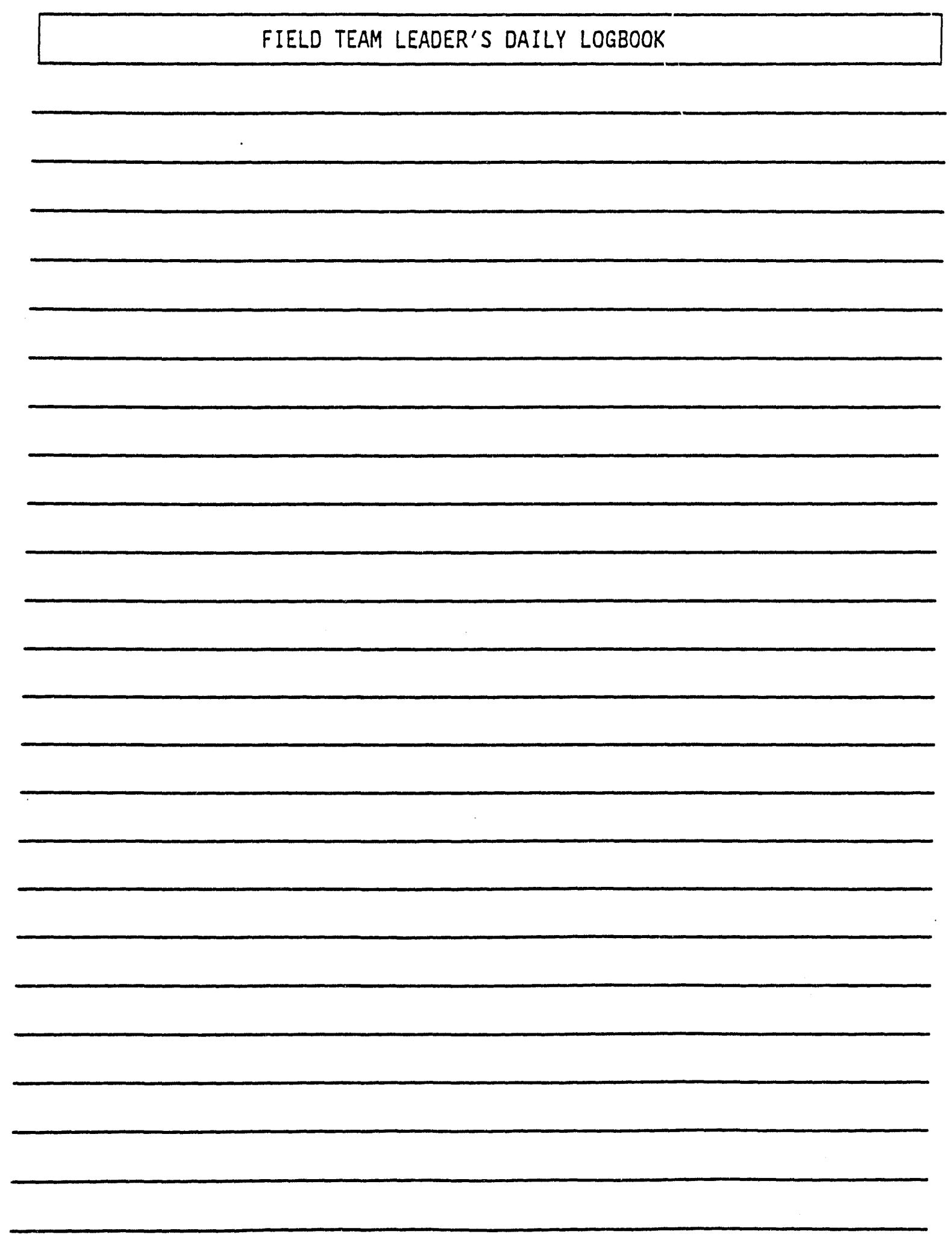




\section{INEL RCRA/CERCLA INVESTIGATIONS FIEEDATASTERUMENT FALIBRATION/}

DATE START

19

DATE END

19

LOGBOOK NO:

LOGBOOK ASSIGNED TO:

WHEN COMPLETED RETURN TO: D. KIRCHNER

MS: 3900 


\section{Instructions for Completing Field Instrument Calibration/ Standardization Logbook}

One logbook should be maintained for each piece of equipment. The logiook should be kept with the equipment at all times and periodically checked by equipment operators and field team leaders.

The logbook should contain a continuous log of the users of the instrument and any calibrations that were performed. List the date and time of calibration as well as the use of any calibration standards. 


\section{FIELD INSTRUMENT CALIBRATION/STANDARDIZATION LOG}

Instrument Property or Serial Number:

EG\&G Calibration Class: _ EG\&G Calib. Date: ____ Expires: _ _ _ _ _

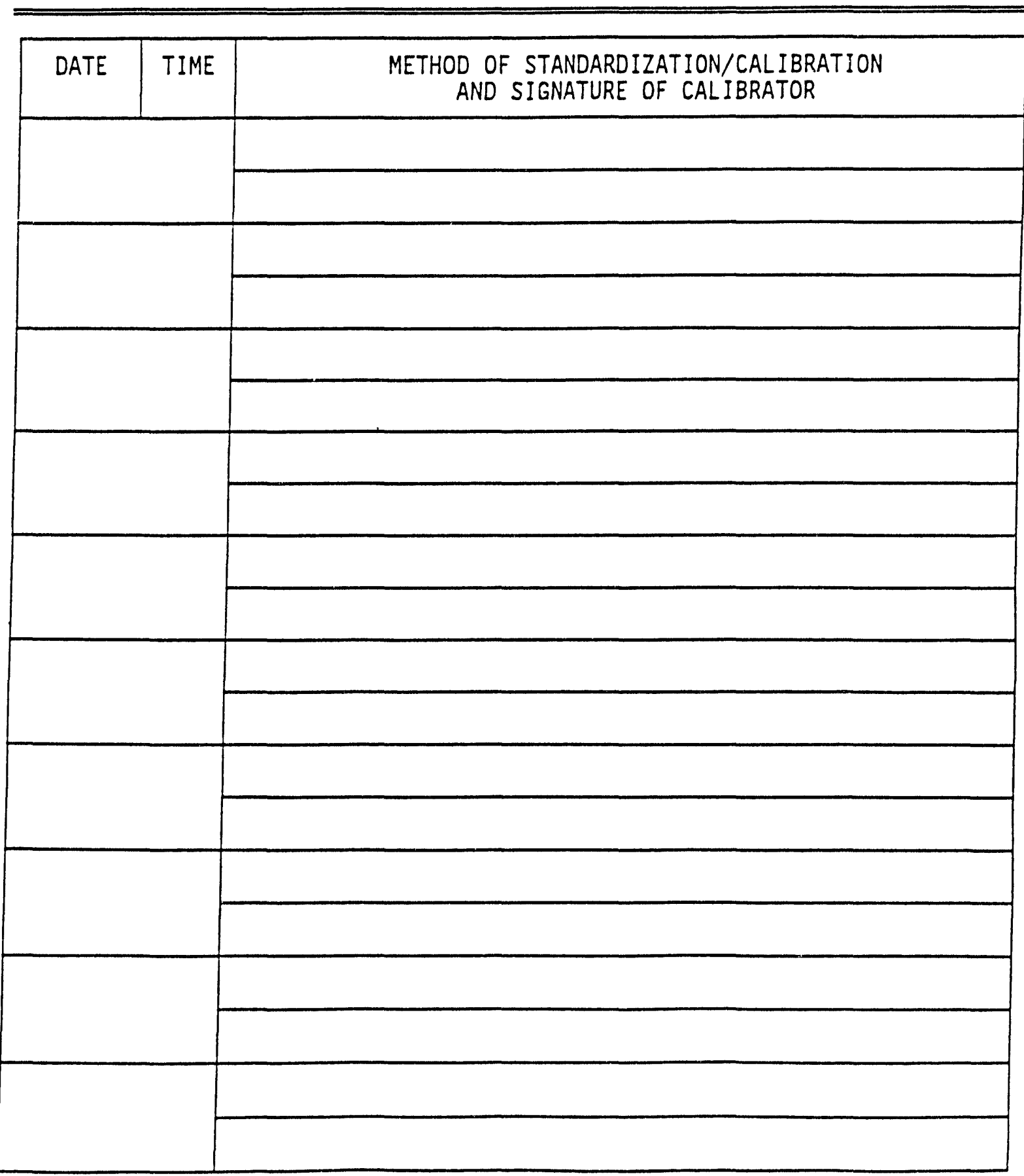

C-10 


\section{INEL RCRA/CERCLA INVESTIGATIONS \\ SAMPLE LOGBOOK}

Onte START

19

DATE END

19

LOGBOOK NO:

LOGBOOK ASSIGNED TO:

SITE:

WHEN COMPLETED RETURN TO: D. KIRCHNER

MS: 3900 


\section{DIRECTIONS FOR COMPLETING SAMPLE LOGBOOK}

Table of Contents: The TOC will be entered into a computer database to quickly retrieve and locate information from all sample logbooks. Therefore, the TOC must be complete.

Sampling Date: Enter the date the samples were collected.

Location: Enter the location (and when appropriate the ID number) of sample collection.

Description of Sample: Enter the media of the sample e.g. groundwater, soil

Page Number of Sample Logsheet: Enter the first page number of the Sample Logsheet

Narrative Sheet: The narrative section contains a description of the days activities of the sampling crew.

Team Members: Print the names of all sampling crew members.

Weather: Enter.a description of weather conditions at the start of sampling. If conditions change before sampling is complete then record the change under Narrative.

Narrative: Enter times and locations of the sampling crew. Enter a description of the sampling points identified on the map page of the logbook.

Map of Sampling Locations: Complete the orientation arrow on the top of the page. Draw a picture of sample collection points. Include the location of reference points.

Sample Logsheet: This logsheet was prepared for a sampling activity that collects sampies from one media e.g. pond or soil at several locations using one sampling method. The two pages of the sample logsheet will be entered into a computer database to perform a completeness check on the number and kinds of samples collected compared to the number and kinds stated in a sampling and analysis plan. It is important that these logsheets be completed fully. If a section does not apply to a particular activity then draw a single line and write NA through the section. 
Date $(\mathrm{mm} / \mathrm{dd} / \mathrm{yy}):$

Field Team Members:

Weather:

Narrative (description of field sampling activities with time and location, description of sampling point, and samples collected.): 


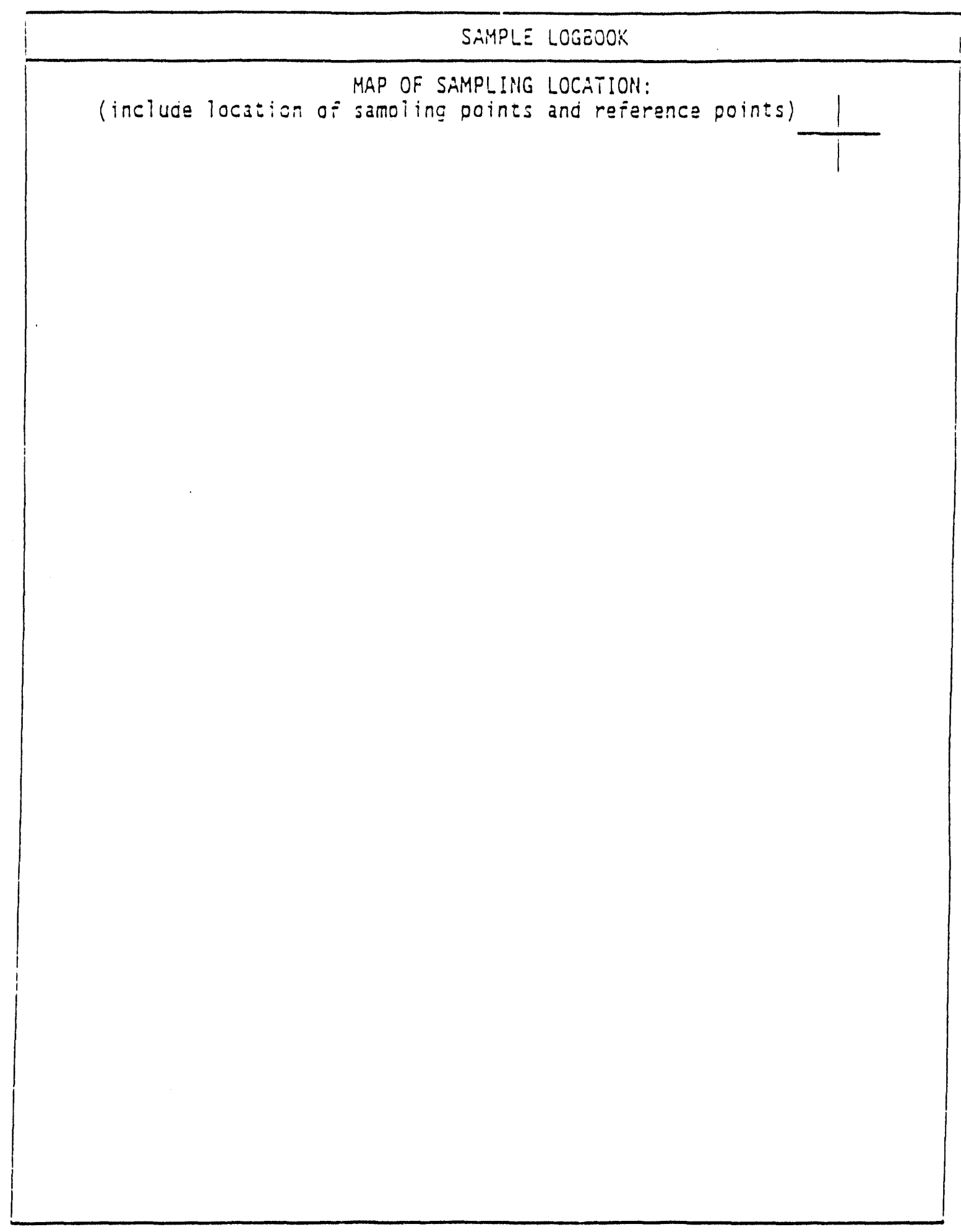

RE:OY:DEอ EY: QA CHECK BY: 
DATE (MM/DD/YY):

SAMPLE TYPE: (0) Normal (1) Equip. Blank (PRIOR) (2) Trip Blank (3) Replicate (4) Split (5) Equip. Blank (POST) (6) Spike (7) Other

ID NO.
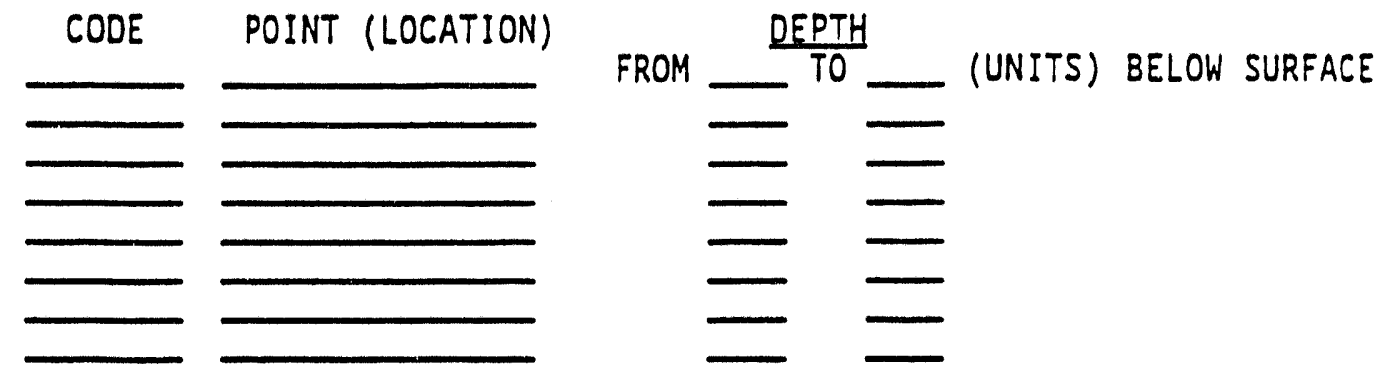

SAMPLE METHOD: CODE:

(0) Grab (1) Spatial Comp. (2) Time Comp. (3) Other

SAMPLE DESCRIPTION: CODE SOIL/ROCK

(00) Surf. Soil

(01) Sub. Surf. Soil

(02) Basalt

(03) Sediment interbed

(04) Other

Other:

SEDIMENT/SLUDGE

(05) Pono/Impoundment

(06) Drum/Tank

(07) Other AIR/GAS

(15) Soil Gas

(16) Other
LIOUIDS

(OB) Pond/Impoundment

(09) Drum/Tank

(10) Plant Discharge

(11) Spring/Seep

(12) Perched Aquifer

(13) Regional Aquifer

(14) Otner

EIELD MEASUREMENTS: (list field measurements of the samples) ID No. Measurement Units Instr. Make/Mode Instr. No.
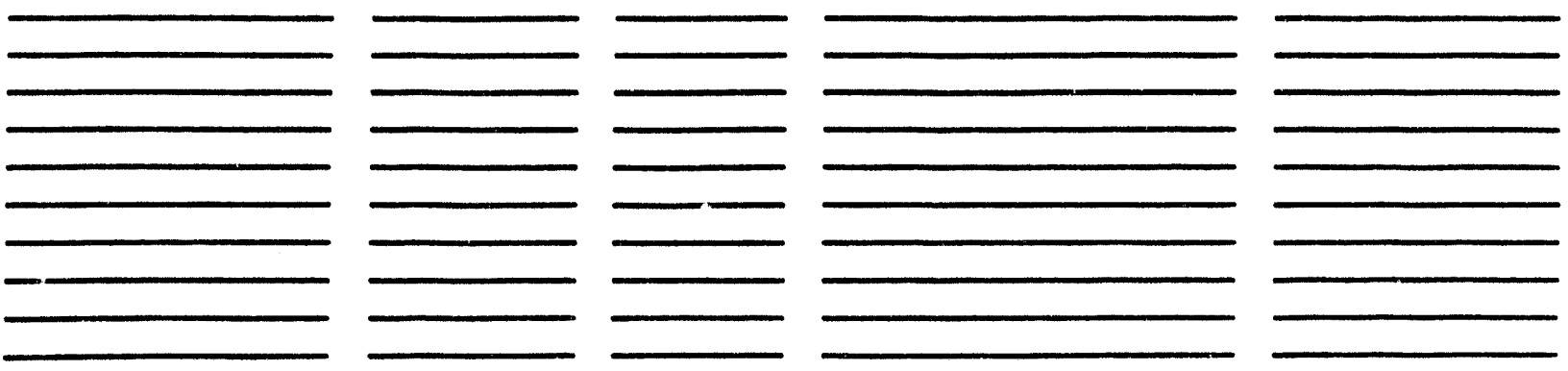

SAMPLINE AND ANALYSIS PLAN FOLLOWED: NO

YES IF HO EXPLAIN DEVIATIOHS:

RECORDED BY: QR CHECK EY: 
SAMPLE LOUDOOK

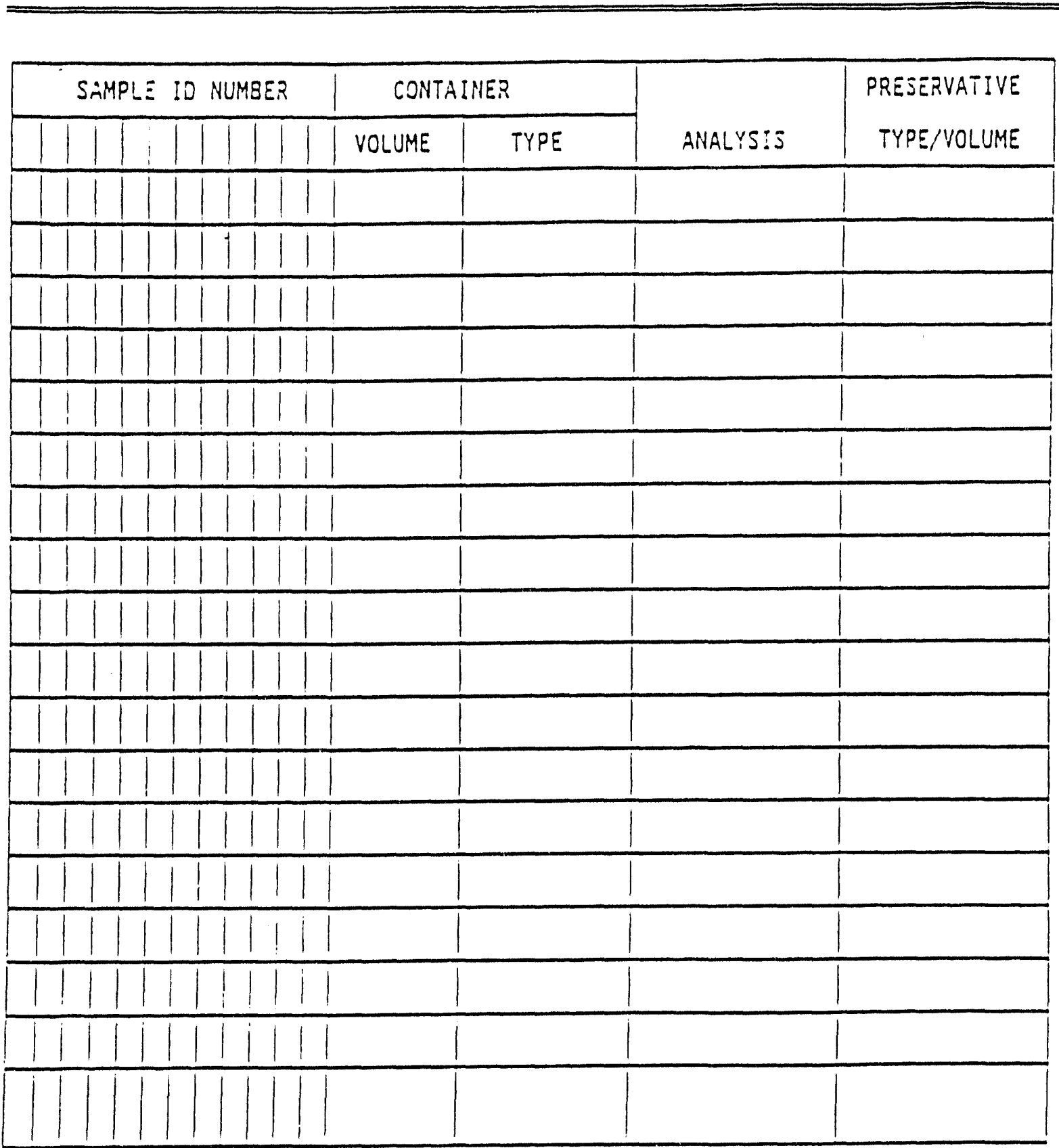

RESORDED BY: QA CHECK BY: 


\section{INEL RCRA/CERCLA INVESTIGATIONS SAMPLE SHIPPING LOGBOOK}

$\begin{array}{ll}\text { DATE START } & 19 \\ \text { DATE END } & 19\end{array}$

LOGBOOK NO:

LOGBOOK ASSIGNED TO:

WHEN COMPLETED RETURN TO: D. KIRCHNER

MS: 3900 


\section{DIRECTIONS TOR COHFLETING SAMPLE SIIIPPING LOGBOOK}

One line must be completed for each sample. Note that drilling cores are considered samples.

SAMPLE IDENTIFICAIION MUMBER: Enter the sample Identification number.

DAIE COLLECTED: Enter the date the sample was collected. This should correspond with the date entered on the sample label, tag, and in the Sample Logbook.

LAB OR STORAGE AREA: Enter the name of the analytical laboratory or core storage area that the sample was shipped to.

DATA SIIIPPED: Enter the date the sample was shipped to the analytical laboratory. Note: This includes both on-site (within INEL) and off-site laboratories. $\stackrel{D}{\infty}$ after the date shipped.

CHAIIN-OF-CUSTODY-HUMBER: Chain-of-custody form(s) must accompany each sample. Every coC form must have a serialized number. Enter the unique serialized number of the coc form.

SAHPLE SIIIPPING CLASSIFICATION: Enter the DOT or EPA classification of the sample.

SHIPPED BY: The qualified shipper responsible for the classification, packaging, and shipping of the sample should sign the sheet.

QA CIIECK BY: A second person who can attest to tlie correctness of the information should sign the sheet. 


\begin{tabular}{|c|c|c|c|c|c|}
\hline 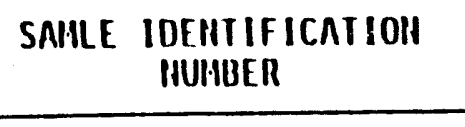 & $\begin{array}{l}\text { DATE } \\
\text { COLLECTED } \\
\end{array}$ & $\begin{array}{l}\text { LAB OR STORAGE } \\
\text { AREA SIIIPPED Tọ } \\
\end{array}$ & $\begin{array}{l}\text { DATE SIIIPPED } \\
\text { (COOLER No.) }\end{array}$ & $\begin{array}{c}\text { CINIH-OF-CUSTODY } \\
\text { MUNBER } \\
\end{array}$ & $\begin{array}{l}\text { COHMENTS ANO SAHPLE } \\
\text { SIIIPPING CLASSIFICAIIOH }\end{array}$ \\
\hline & & & & & \\
\hline & & & & & \\
\hline & & & & & \\
\hline & & & & & \\
\hline & & & & & \\
\hline & & & & & \\
\hline & & & & & \\
\hline & & & & & \\
\hline$-\dot{b}$ & & & & & \\
\hline & & & & & \\
\hline & & & & & \\
\hline 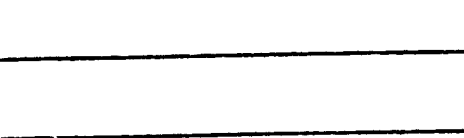 & & & & & \\
\hline & & & & & \\
\hline & & & & & \\
\hline & & & & & \\
\hline & & & & & \\
\hline
\end{tabular}

SIIIPPED BY:

(SIgnature) Q̣n Clleck $B Y:$ 
Remedial Investigation Sampling and Analysis Plan

Part II-Quality Assurance Project Plan for Operable Unit 4-12:

Central Facilities Area Landfills II and III at the Idaho National Engineering Laboratory

July 1993

Idaho National Engineering Laboratory

EG\&G Idaho, Inc.

Idaho Falls, Idaho 83415

Prepared for the

U.S. Department of Energy

Assistant Secretary for Environmental Restoration and Waste Management

Under DOE Idaho Operations Office

Contract DE-AC07-76ID01570 


\section{CONTENTS}

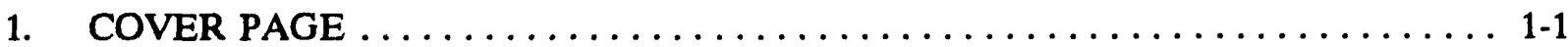

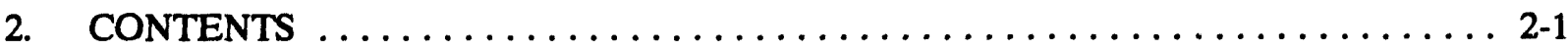

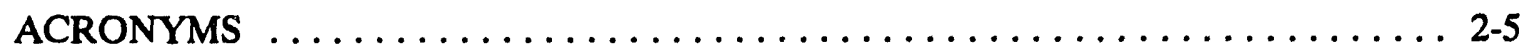

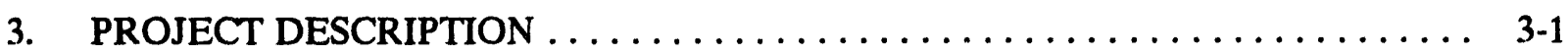

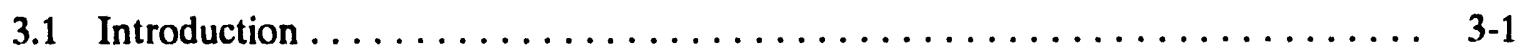

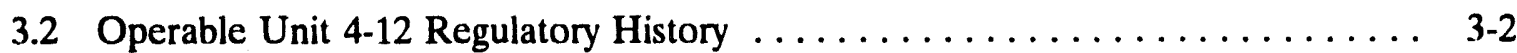

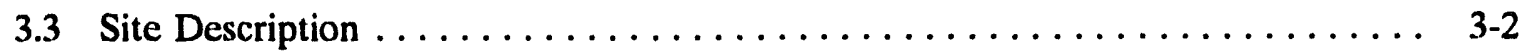

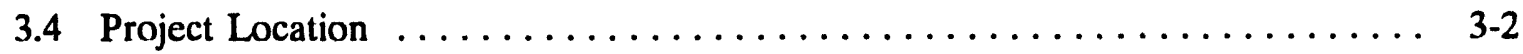

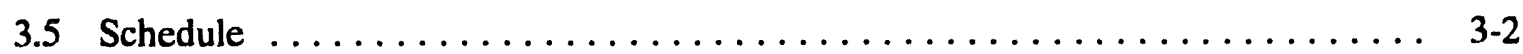

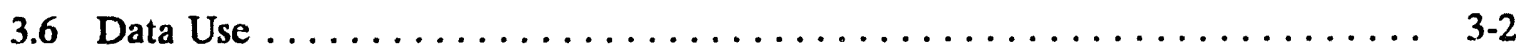

4. PROJECT ORGANIZATION AND RESPONSIBILITY $\ldots \ldots \ldots \ldots \ldots \ldots \ldots \ldots$

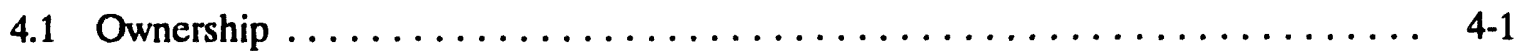

4.2 Organization of Project Personnel $\ldots \ldots \ldots \ldots \ldots \ldots \ldots \ldots \ldots \ldots \ldots \ldots \ldots \ldots$

Environmental Restoration and Waste Management Department Manager ... . 4-1

Site Remediation Group Manager . . . . . . . . . . . $\ldots \ldots \ldots \ldots \ldots$ 4-1

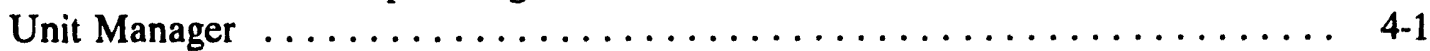

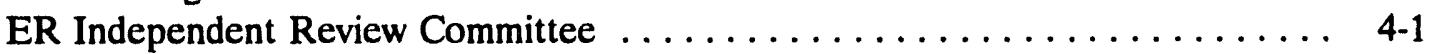

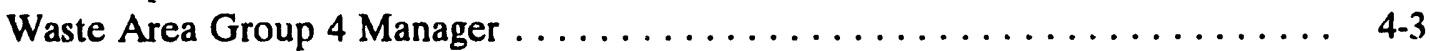

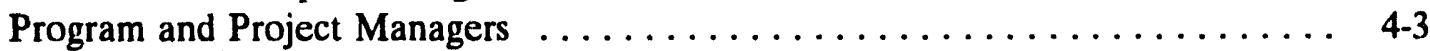

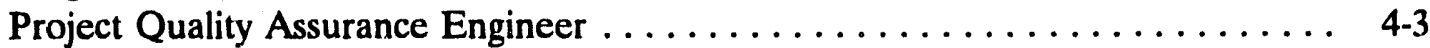

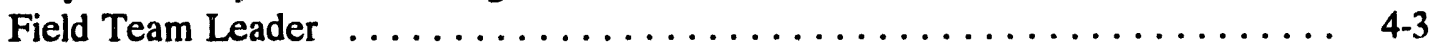

Field Team Members . . . . . . . . . . . . . . . . . . . . . . . . $4-4$

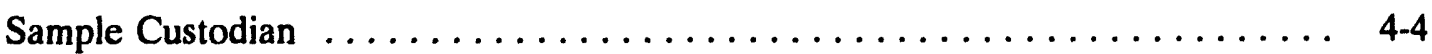

Health Physics Technician ....................... 4-4

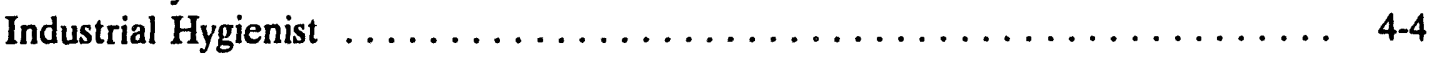

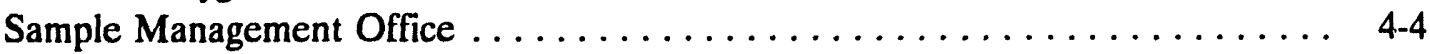

Administrative Record and Document Control Coordinator ........... 4-5

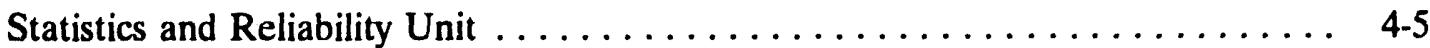


5. QA OBJECTIVES FOR MEASUREMENT DATA $\ldots \ldots \ldots \ldots \ldots \ldots \ldots \ldots \ldots$ 5-1

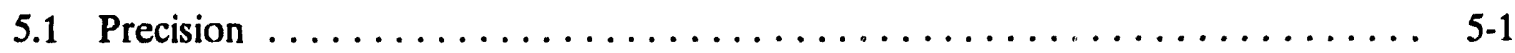

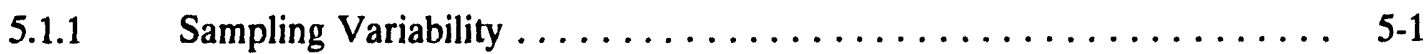

5.1.2 Measurement Reproducibility $\ldots \ldots \ldots \ldots \ldots \ldots \ldots \ldots \ldots . \ldots \ldots$

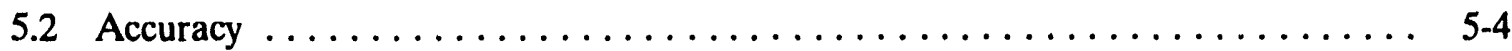

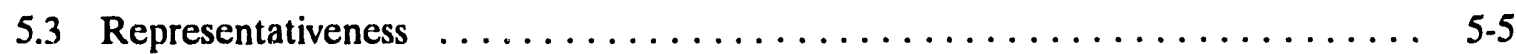

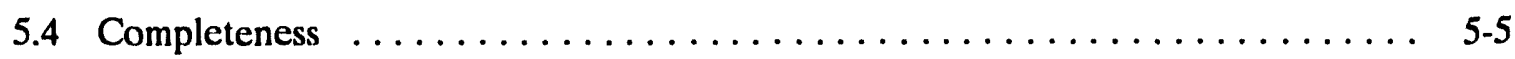

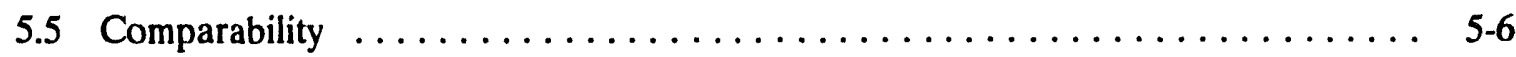

6. SAMPLING PROCEDURES $\ldots \ldots \ldots \ldots \ldots \ldots \ldots \ldots \ldots \ldots \ldots \ldots \ldots \ldots \ldots \ldots \ldots$

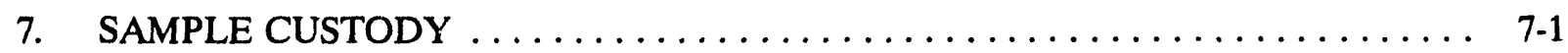

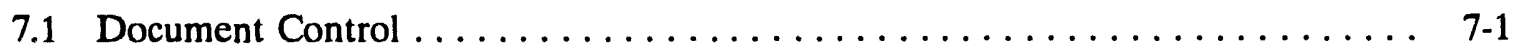

7.2 Chain of Custody Documentation $\ldots \ldots \ldots \ldots \ldots \ldots \ldots \ldots \ldots \ldots \ldots \ldots \ldots \ldots \ldots$

7.3 Chain of Custody Record $\ldots \ldots \ldots \ldots \ldots \ldots \ldots \ldots \ldots \ldots \ldots \ldots \ldots \ldots \ldots \ldots$

7.4 Field Logbook $\ldots \ldots \ldots \ldots \ldots \ldots \ldots \ldots \ldots \ldots \ldots \ldots \ldots \ldots \ldots \ldots \ldots \ldots \ldots \ldots$

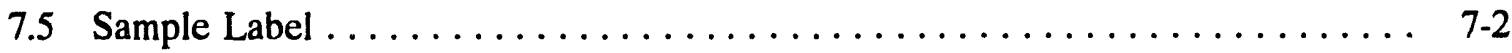

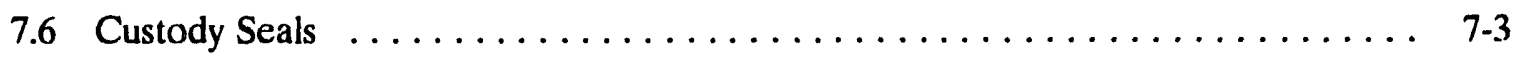

7.7 Document Corrections $\ldots \ldots \ldots \ldots \ldots \ldots \ldots \ldots \ldots \ldots \ldots \ldots \ldots \ldots \ldots \ldots \ldots$

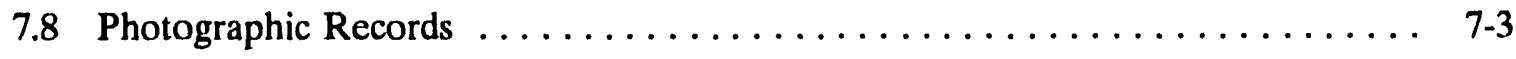

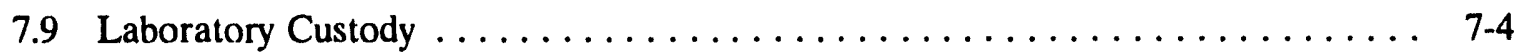

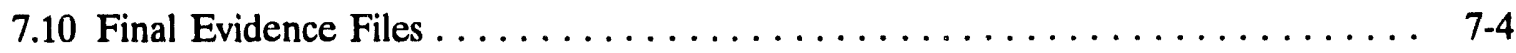

8. CALIBRATION PROCEDURES AND FREQUENCY $\ldots \ldots \ldots \ldots \ldots \ldots \ldots \ldots$ 8-1

9. ANALYTICAL PROCEDURES $\ldots \ldots \ldots \ldots \ldots \ldots \ldots \ldots \ldots \ldots \ldots \ldots \ldots \ldots$ 
10. DATA REDUCTION, VALIDATION, AND REPORTING $\ldots \ldots \ldots \ldots \ldots \ldots \ldots$ 10-1

11. INTERNAL QUALITY CONTROL CHECKS $\ldots \ldots \ldots \ldots \ldots \ldots \ldots \ldots \ldots \ldots$

11.1 Offsite Laboratory QA/QC $\ldots \ldots \ldots \ldots \ldots \ldots \ldots \ldots \ldots \ldots \ldots \ldots \ldots \ldots \ldots \ldots \ldots \ldots$

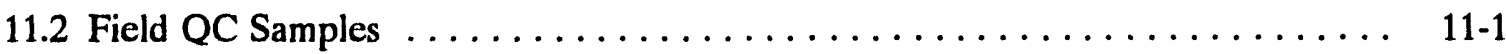

12. SYSTEM AND PERFORMANCE AUDITS $\ldots \ldots \ldots \ldots \ldots \ldots \ldots \ldots \ldots \ldots \ldots \ldots \ldots \ldots$

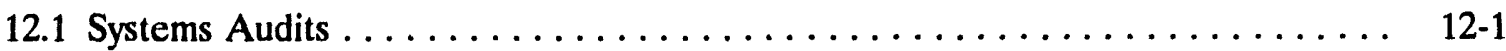

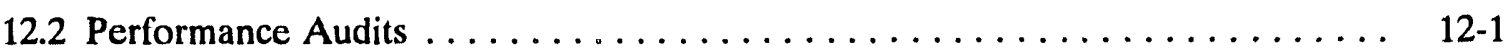

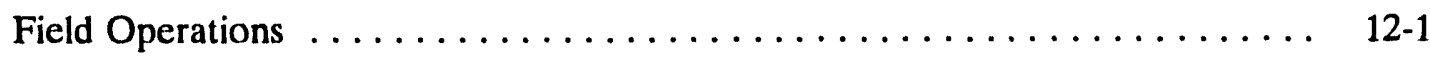

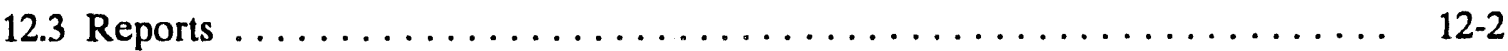

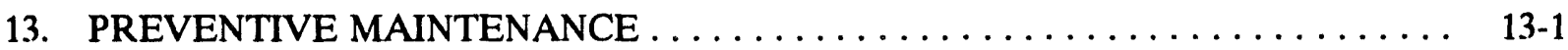

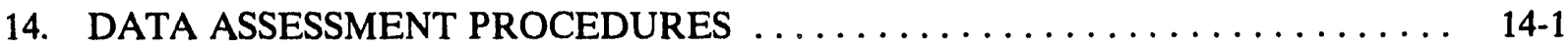

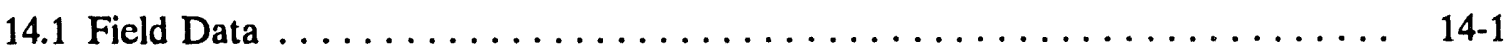

Procedures to Assess Field Data Precision . . . . . . . . . . . . . . . 14-1

Procedures to Assess Field Data Accuracy . . . . . . . . . . . . . . 14-1

Procedures to Assess Field Data Completeness .................. 14-1

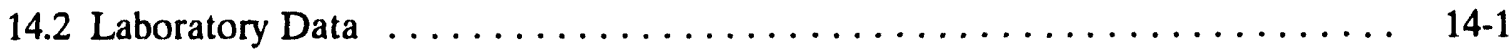

Procedures to Assess Laboratory Precision . . . . . . . . . . . . . . 14-2

Procedures to Assess Laboratory Accuracy . . . . . . . . . . . . . . . . . . . . 14- 14

Procedures to Assess Laboratory Completeness ................ 14-2

15. CORRECTIVE ACTION PROCEDURES $\ldots \ldots \ldots \ldots \ldots \ldots \ldots \ldots \ldots \ldots \ldots \ldots \ldots \ldots$

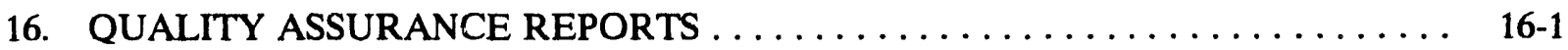

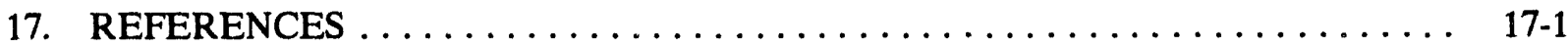

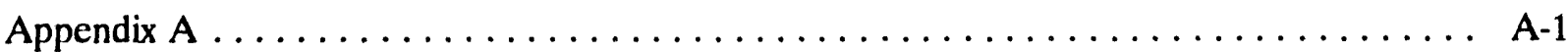




Section No.: $\frac{2}{0}$
Revision No.: $\frac{0}{\text { July } 1993}$
Date: $\frac{2-4}{\text { Page: }}$

\section{FIGURES}

4-1. Operable Unit 4-12 remedial investigation project organization $\ldots \ldots \ldots \ldots \ldots \ldots$ 4-2

\section{TABLES}

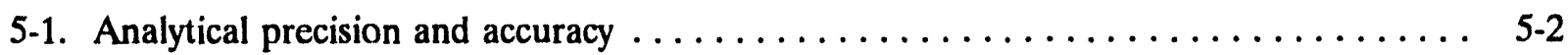

5-2. Relationships between statistical performance indicators and number of samples

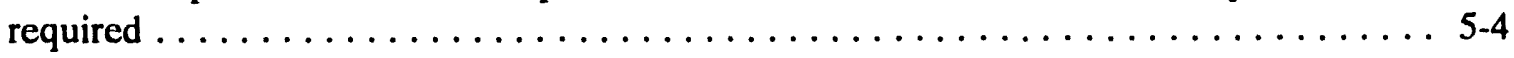

5-3. MDRD performance of analytical parameters $\ldots \ldots \ldots \ldots \ldots \ldots \ldots \ldots \ldots \ldots$ 


\section{ACRONYMS}

ARDC Administrative Records and Document Control

ASTM American Society for Testing and Materials

CERCLA Comprehensive Environmental Response, Compensation, and Liability Act

CFA Central Facilities Area

CFR Code of Federal Regulations

CL confidence level

CLP Contract Laboratory Program (EPA's)

COC chain of custody

DMP Data Management Plan

DOE U.S. Department of Energy

DOE-ID Department of Energy, Idaho Field Office

DOT Department of Transportation

DQO data quality objective

EPA U.S. Environmental Protection Agency

ER Environmental Restoration

ER\&WM Environmental Restoration and Waste Management

FFA/CO Federal Facility Agreement/Consent Order

FSP Field Sampling Plan

FTL Field Team Leader

HSP Health and Safety Plan

IH Industrial Hygienist

INEL Idaho National Engineering Laboratory

IRC Independent Review Committee

MDRD minimum detectable relative difference

MS matrix spike

MSD matrix spike duplicate

NEIC National Enforcement Investigation Center 


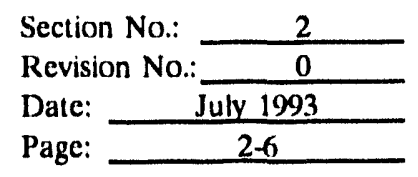

OU operable unit

PARCC precision, accuracy, representativeness, completeness, and comparability

PD program directive

QA quality assurance

QA/QC quality assurance/quality control

QAPjP Quality Assurance Project Plan

QC quality control

RCRA Resource Conservation and Recovery Act

RI/FS remedial investigation/feasibility study

RI remedial investigation

RPD relative percent difference

RSD relative standard deviation

SAP sampling and analysis plan

SD standard deviation

SMO Sample Management Office

SOP standard operating procedure

SOW Statement of Work

VOA volatile organic analysis

WAG waste area group 


Section No.: $\frac{3}{0}$
Revision No.: $\frac{0}{\text { July } 1993}$
Date: $\frac{3-1}{\text { Page: }}$

\section{PROJECT DESCRIPTION}

\subsection{Introduction}

This document presents the Quality Assurance Project Plan (QAPjP) for the remedial investigation of Operable Unit (OU) 4-12: Central Facilities Area (CFA) Landfills II and III at the Idaho National Engineering Laboratory (INEL) near Idaho Falls, Idaho. The QAPjP is written documentation of procedures that ensure precision, accuracy, representativeness, completeness, and comparability (PARCC) of data generated during an investigation. The QAPjP was prepared using the Environmental Protection Agency's (EPA's) guidelines, including Interim Guidelines and Specifications for Preparing Quality Assurance Project Plans (EPA 1983a) and the Quality Program Plan for the Environmental Restoration Program (ERP) (EG\&G Idaho 1991a). The ERP Quality Program Plan is utilized for all Environmental Restoration Program work. This QAPjP is developed specifically for OU 4-12 RI/FS activities. The QAPjP also addresses requirements set forth in 40 CFR 30, including procedures to ensure the PARCC of groundwater and field chemistry data collected during field investigations. The QAPjP is used by field, laboratory, and management personnel in all aspects of data collection, management, and control while onsite or offsite.

This QAPjP accompanies a set of other documents that constitutes background and guidance for performing the investigation. Specific issues that control data quality are found in a number of documents. Data quality objectives, sample location and frequency, and numbers of samples are described in the Field Sampling Plan (FSP). Quality control (QC) samples generation, chain of custody (COC), preservation and shipping, instrument calibration, quality assurance (QA) objectives, internal QC checks, audits, preventive maintenance, measurement of PARCC, corrective actions, and QA reporting are presented in this QAPjP.

Analytical procedures are presented in the FSP. Data reduction and reporting are described in the Data Management Plan (DMP) (EG\&G Idaho 1992). Data validation is discussed in Section 10 of this QAPjP.

The purpose of the Sampling and Analysis Plan is to guide the collection and analysis of samples for a remedial investigation (RI) of OU 4-12. This route of investigation has been chosen to facilitate data development in this remedial investigation/feasibility study (RI/FS) as a result of a Federal Facility Agreement/ Consent Order (FFA/CO) between the Department of Energy (DOE), the EPA, and the Idaho Department of Health and Welfare (IDHW). This FFA/CO guides the overall Comprehensive Environmental Response, Compensation, and Liability Act (CERCLA) response at the INEL.

The Sampling and Analysis Plan consists of three parts: the FSP, the QAPjP, and the DMP. These plans have been prepared pursuant to the National Contingency Plan (EPA 1990a) and Guidance for Conducting Remedial Investigations and Feasibility Studies Under CERCLA (EPA 1988a). 


Section No.: $\frac{3}{\text { Revision No.: } \frac{0}{\text { July } 1993}}$
Date: $\frac{3-2}{\text { Page: }}$

The FSP describes the field activities that will occur as part of the RI and the QAPjP describes the processes and programs that will be used to ensure the data generated will be suitable for its intended use. The guiding DMP for the OU 4-12 RI/FS will be the Data Management Plan for the EG\&G Idaho Environmental Restoration Department (EG\&G Idaho 1992). The DMP describes the flow of data from generation to use. Background information on CFA and the OU 4-12 RI/FS is found in Section 2 of the Work Plan.

\subsection{Operable Unit 4-12 Regulatory History}

Information on the regulatory history for OU 4-12 at CFA is contained in Section 1 of the Work Plan.

\subsection{Site Description}

The history of CFA operations that have an impact on OU 4-12 are discussed in detail in Section 2.2 of the RI/FS Work Plan. The reader is referred to this section for information on waste generation, processes, and disposal.

\subsection{Project Location}

OU 4-12 at the INEL is located approximately $42 \mathrm{mi}(68 \mathrm{~km})$ west of Idaho Falls, Idaho. Additional information on CFA facilities is given in Section 2 of the RI/FS Work Plan.

\subsection{Schedule}

A detailed schedule of RI/FS activities for OU 4-12 as proposed in the RI/FS Work Plan can be found in Section 6 of the Work Plan. RI/FS activities essentially started in August 1992 with development of the Scope of Work for OU 4-12, and will continue until approval of the Record of Decision, proposed for June 1995.

\subsection{Data Use}

The intended end uses of data gathered as part of the RI/FS are to determine the overall nature and extent of contamination, aid in the understanding of the hydrogeologic system at CFA, and ultimately to aid in the selection of an appropriate remedial alternative for the site.

Information needed to fill data gaps for the RI/FS has been identified in Section 4 of the Work Plan. Tasks planned to provide necessary data have been generally discussed in Section 5 of the Work Plan and are detailed in the FSP. 


\section{PROJECT ORGANIZATION AND RESPONSIBILITY}

\subsection{Ownership}

The INEL is managed by the U.S. Department of Energy, Idaho Field Office (DOE-ID). EG\&G Idaho, Inc., is the site contractor responsible for operations where the sampling unit is located. DOE-ID has primary responsibility and authority for Resource Conservation and Recovery Act (RCRA)/CERCLA EPA Regulatory Compliance activities at CFA.

\subsection{Organization of Project Personnel}

Figure 4-1 shows the project organizational structure and key personnel for the OU 4-12 Project.

\section{Environmental Restoration and Waste Management Department Manager}

The Environmental Restoration and Waste Management (ER\&WM) Department Manager is responsible for incorporating and implementing the Environmental Restoration Quality Program with this QAPjP and QPP-149. The ER\&WM Department Manager provides technical coordination and interface with the DOE-ID Technical Program Manager. The ER\&WM Department Manager ensures that all activities are conducted in accordance with program requirements; monitors the project budget and schedule; and ensures the availability of necessary personnel, equipment, subcontractors, and services.

\section{Site Remediation Group Manager}

The Site Remediation Group Manager is responsible for Waste Area Groups (WAGs) 1, 2, $4,5,6,10$, and the Decontamination and Decommissioning/Underground Storage Unit. Work includes management and coordination of both FFA/CO and non-FFA/CO projects.

\section{Unit Manager}

The Waste Area Groups 1, 4, and 5 Unit Manager is responsible for WAGs \#1, 4 and 5, and for management and coordination of the operable units associated with these WAGs under the FFA/CO.

\section{ER Independent Review Committee}

The Environmental Restoration (ER) Independent Review Committee (IRC) will review initial planning documents, such as sampling and analysis plans (SAPs) and standard operating procedures (SOPs), to ensure that the plans will produce data of the required level. 
Section No.:

Revision No.

Date:

Page: July 1993 4-2

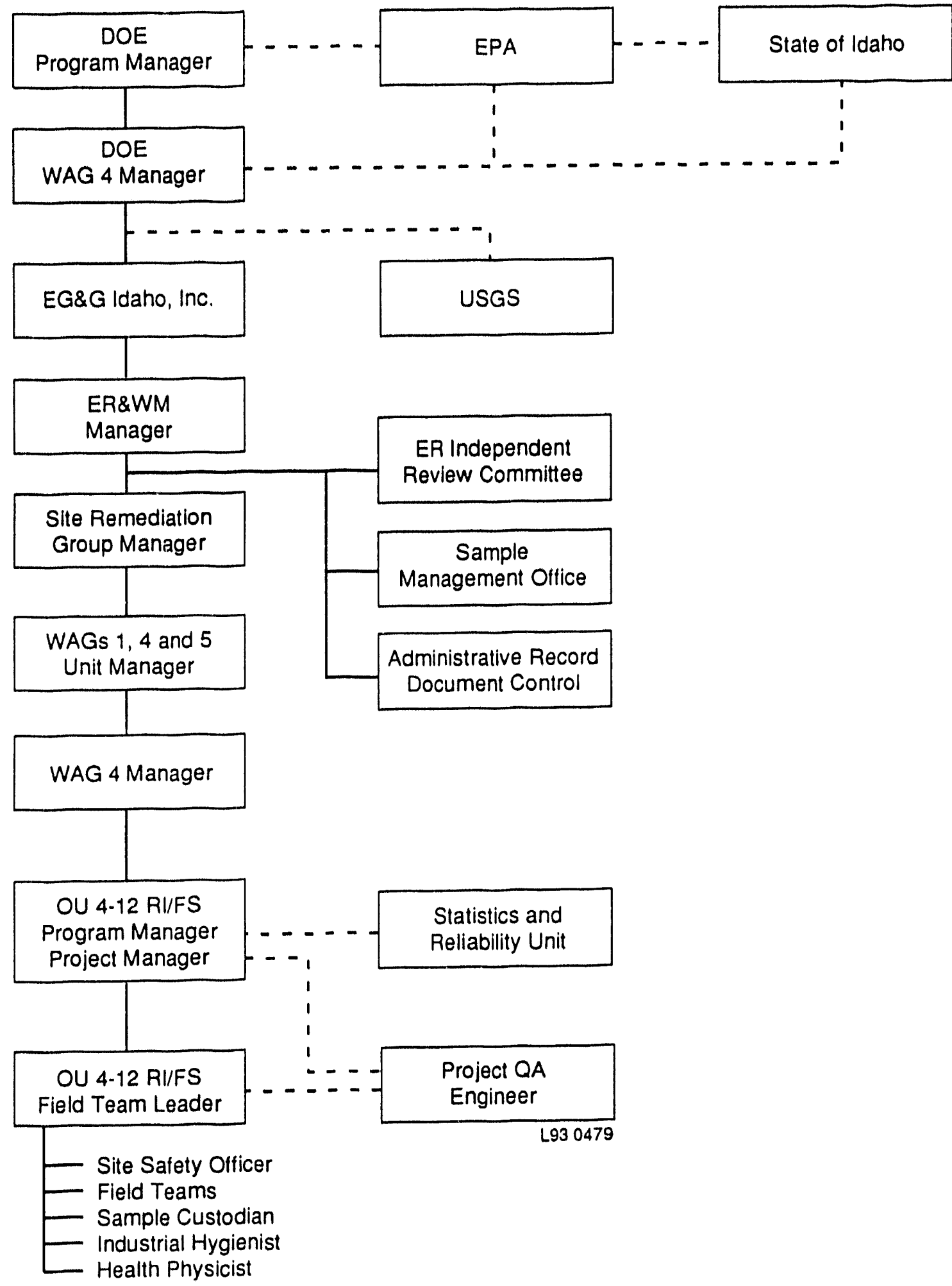

Figure 4-1. Operable Unit 4-12 remedial investigation project organization. 


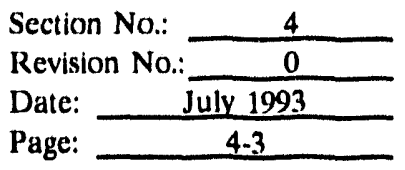

Waste Area Group 4 Manager

The WAG 4 Manager oversees WAG 4 projects and coordinates progress within the jurisdiction in which those projects are being investigated, including the OU 4-12 RI/FS.

\section{Program and Project Managers}

The Program Manager is responsible for the senior technical review of all project plans and deliverables. The Project Manager is responsible for ensuring that investigation activities are completed in accordance with the QAPjP, the QPP-149 document, the Health and Safety Plan (HSP), and the FSP. In addition, the individual will keep the WAG Manager informed of project status and any technical, administrative, contractual, and financial issues with the proposed resolutions.

\section{Project Quality Assurance Engineer}

The Project QA Engineer reports to the Project Manager and is responsible to see that project quality assurance requirements are met and that the QAPjP is implemented. The QA Engineer's responsibilities include, but are not limited to, surveillances of field activities and audits of laboratory activities.

\section{Field Team Leader}

The Field Team Leader (FTL) will oversee field operations and will report to the Project Manager. The FTL is responsible for implementing the FSP, the QAPjP, and the DMP for field activities (such as sampling).

The FTL has the primary responsibility for ensuring the fulfillment of technical and operational requirements of the sampling plan and for coordinating field team members during RI tasks. The FTL is also responsible to do the following:

- Locate support facilities outside of the areas where potentially contaminated samples are to be collected

- Integrate contact with facility safety and operations personnel and ensure that field team personnel are familiar with the location of the facility medical dispensary

- Instruct team personnel at a pretask briefing on technical, operational, quality, and safety requirements of the task

- Observe all site activities and ensure that the team meets all safety, quality, and operational requirements outlined in this document, the sampling plan, and the HSP 


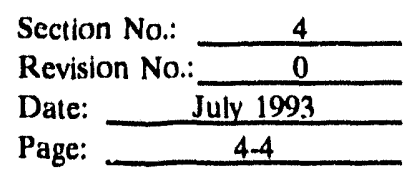

- Ensure that all safety equipment is available and in good working order before any potentially hazardous operation is initiated

- Control access to and departure from the working site

- Ensure compliance with field documentation, sampling methods, and COC requirements

- Determine, in conjunction with the Site Safety Officer, the level of personal protection necessary for the task being performed.

\section{Field Team Members}

Field Team Members perform the actual field data gathering in accordance with approved plans and procedures under the direction of the FTL.

\section{Samplo Custodian}

The Sample Custodian is responsible for documenting, handling, packaging, preserving, and shipping samples. This individual will document all sample descriptions and activity in a field logbook and fulfill COC procedures as described in the QAPjP.

\section{Health Physics Technician}

The Health Physics Technician will be the primary source of information and guidance for monitoring radiological hazards and will be on call and accessible by radio during sampling activities.

\section{Industrial Hygienist}

The Industrial Hygienist (IH) will be responsible for the adherence to all site safety requirements by the team members. The $\mathrm{IH}$ will assist in conducting briefings and in performing the final safety check of the area prior to each sampling event. The IH will also be responsible for ensuring that adequate health and safety monitoring is in place while field activities are being performed.

\section{Sample Management Office}

The Sample Management Office (SMO) conducts data validation. Its responsibilities are described in the Data Management Plan and in QPP-149. Data entry and manipulation are performed by the Statistics and Reliability Unit. Its responsibilities are also in the Data Management Plan and in QPP-149. 


Section No.: $\frac{4}{\text { Revision No.: } \frac{0}{\sqrt{\text { July } 1993}}}$
Date: $\frac{4-5}{\text { Page: }}$

\section{Administrative Record and Document Control Coordinator}

The Administrative Record and Document Control (ARDC) Coordinator for ER will maintain a supply of all controlled documents and have a documented filing system for storing all documents (e.g., reports, correspondence); all field laboratory data (e.g., field notebooks and raw data), including laboratory Contract Laboratory Program (CLP) data packages; and all references and final reports from the OU 4-12 RI/FS.

\section{Statistics and Reliability Unit}

The Statistics and Reliability Unit assists the Project Manager and the Sample Management Office in analyzing field data for statistical values (means, standard deviations) and in analyzing trends in the data. 


\section{QA OBJECTIVES FOR MEASUREMENT DATA}

The objective of this QAPjP is to ensure that the information collected for decision making during the OU 4-12 RI/FS is of known and adequate quality, and is technically sound, statistically accurate, and properly documented. By meeting this objective, these data will have the necessary quality for use in making the best possible decisions for the RI/FS. These data quality elements are essential for enforcement proceedings that may arise from RI/FS activities. QA is a management system for ensuring that all information, data, and decisions are technically sound and properly documented. QC is the mechanism by which the QA system is ensured. This usually consists of tests performed on the system for which quality is being ensured, using standards or known quantities. Specific QC procedures related to activities such as sampling, analysis, and engineering calculations will ensure that data are appropriate for risk assessment and enforcement use.

Data quality objectives (DQOs) are described in Section 4 of the Work Plan. Resulting QA objectives for analytical data (PARCC parameters) are defined in Table 5-1 and Sections 5.1 through 5.5 of this QAPjP.

\subsection{Precision}

Precision measures the reproducibility of measurements under a given set of conditions. Specifically, precision provides a quantitative measure of the variability of a set of measurements compared to the average value of the set. Two basic activities are performed in the assessment of precision: estimating sampling variability from the observed spatial variation of the sample population and estimating the measurement reproducibility attributable to the data collection process.

\subsubsection{Sampling Variability}

Three critical statistical performance measures are evaluated in the examination of sampling variability: confidence level, power, and minimum detectable relative difference. The minimum requirements for confidence level, power, and minimum detectable relative difference provided in Section 5.6 of Guidance for Data Useability in Risk Assessment (EPA 1990) are used in this investigation.

Confidence level (CL) will be $80 \%$ [i.e., the probability of a Type I error (taking action when no action is required) is $1-\mathrm{CL}$ or $100 \%-80 \%=20 \%]$.

The minimum value for power (P) will be $90 \%$ [i.e., the probability of a Type II error (taking no action when action is required) is $1-\mathrm{P}$ or $100 \%-90 \%=10 \%]$. 
Table 5-1. Analytical precision and accuracy.

\begin{tabular}{|c|c|c|c|c|c|}
\hline Parameter & Matrix & Method & $\begin{array}{l}\text { Analytical } \\
\text { Support } \\
\text { Level }\end{array}$ & Precision & Accuracy \\
\hline Volatiles & Groundwater & EPA 524.2 & IV & $<20 \%$ RSD & $\begin{array}{l}80-120 \% \text { spike } \\
\text { recovery }\end{array}$ \\
\hline Volatiles & Soil gas & EPA 601 & III & $-^{a}$ & $-{ }^{a}$ \\
\hline Volatiles & Surface soil & CLP SOW & IV & $\begin{array}{l}\text { Analyte } \\
\text { specific }\end{array}$ & $\begin{array}{l}\text { Analyte } \\
\text { specific }\end{array}$ \\
\hline Volatiles & $\begin{array}{l}\text { Subsurface } \\
\text { soil }\end{array}$ & CLP SOW & IV & $\begin{array}{l}\text { Analyte } \\
\text { specific }\end{array}$ & $\begin{array}{l}\text { Analyte } \\
\text { specific }\end{array}$ \\
\hline Semivolatile Organics & Surface soil & CLP SOW & IV & $\begin{array}{l}\text { Analyte } \\
\text { specific }\end{array}$ & $\begin{array}{l}\text { Analyte } \\
\text { specific }\end{array}$ \\
\hline Semivolatile Organics & $\begin{array}{l}\text { Subsurface } \\
\text { soil }\end{array}$ & CLP SOW & IV & $\begin{array}{l}\text { Analyte } \\
\text { specific }\end{array}$ & $\begin{array}{l}\text { Analyte } \\
\text { specific }\end{array}$ \\
\hline Metals & Groundwater & CLP SOW & IV & $\pm 20 \% \mathrm{RPD}$ & $\begin{array}{l}75-125 \% \text { spike } \\
\text { recovery }\end{array}$ \\
\hline Metals & Surface soil & CLP SOW & IV & $\pm 35 \%$ RPD & $\begin{array}{l}75-125 \% \text { spike } \\
\text { recovery }\end{array}$ \\
\hline Metals & $\begin{array}{l}\text { Subsurface } \\
\text { soil }\end{array}$ & CLP SOW & IV & $\pm 35 \%$ RPD & $\begin{array}{l}75-125 \% \text { spike } \\
\text { recovery }\end{array}$ \\
\hline $\begin{array}{l}\text { Hexavalent } \\
\text { Chromium }\end{array}$ & Groundwater & SW846-7195 & IV & - & - \\
\hline $\mathrm{Fl}^{-}$ & Groundwater & EPA 340.2 & IV & $0.03 \mathrm{mg} / \mathrm{L} \mathrm{SD}$ & $\begin{array}{l}99 \% \text { mean } \\
\text { recovery }\end{array}$ \\
\hline Alkalinity & Groundwater & EPA 310.1 & IV & $\begin{array}{l}1.27-5.36 \mathrm{mg} / \mathrm{L} \\
\mathrm{SD}\end{array}$ & $\begin{array}{l}+22.29 \text { to } \\
-8.19 \% \text { bias }\end{array}$ \\
\hline Anions & Groundwater & EPA 300.0 & IV & $--^{a}$ & $-{ }^{a}$ \\
\hline $\begin{array}{l}\text { Gravimetric Water } \\
\text { Content }\end{array}$ & Soil/rock & ASTM D-2216 & III & NA & NA \\
\hline Particle Size & Soil/rock & ASTM D-421 & III & NA & NA \\
\hline $\begin{array}{l}\text { Hydraulic } \\
\text { Conductivity }\end{array}$ & Soil/rock & ASTM D-2434 & III & NA & NA \\
\hline $\begin{array}{l}\text { Capillary Pressure- } \\
\text { Moisture Content }\end{array}$ & Soil/rock & ASTM D-2325 & III & NA & NA \\
\hline Density (in situ) & Soil/rock & ASTM D-2922 & II & NA & NA \\
\hline
\end{tabular}




$\frac{5}{\frac{0}{\text { July } 1993}}$

Table 5-1. (continued).

\begin{tabular}{|c|c|c|c|c|c|c|}
\hline \multicolumn{2}{|c|}{ Parameter } & Matrix & Method & $\begin{array}{l}\text { Analytical } \\
\text { support } \\
\text { level }\end{array}$ & Precision & Accuracy \\
\hline \multicolumn{2}{|c|}{$\begin{array}{l}\text { Moisture Content } \\
\text { (in situ) }\end{array}$} & Soil/rock & ASTM D-3017 & II & NA & NA \\
\hline \multicolumn{2}{|c|}{$\begin{array}{l}\text { Magnetic Field } \\
\text { (vertical gradient) }\end{array}$} & Soil/rock & $-b$ & II & \pm 10 gamma/m & $\begin{array}{l}\text { (Spatial) one } \\
\text { half the station } \\
\text { spacing }\end{array}$ \\
\hline \multicolumn{2}{|c|}{$\begin{array}{l}\text { Induced Electro- } \\
\text { magnetic Field } \\
\text { (terrain conductivity) }\end{array}$} & Soil/rock & $-b^{b}$ & II & $\pm 1 \mathrm{mmho} / \mathrm{m}$ & $\begin{array}{l}\text { (Spatial) one } \\
\text { half the station } \\
\text { spacing }\end{array}$ \\
\hline \multicolumn{2}{|c|}{ Seismic refraction } & Soil/rock & $-b$ & II & $\begin{array}{l}0.01 \% \\
\text { (time units) }\end{array}$ & $\begin{array}{l}\text { (Spatial) one } \\
\text { half the station } \\
\text { spacing }\end{array}$ \\
\hline \multicolumn{7}{|c|}{$\begin{array}{l}\text { a. Precision and accuracy values for this method are analyte-specific and are provided in the } \\
\text { Appendix. } \\
\text { b. Geophysical methods are performed in accordance with owner's manuals and manufacturers } \\
\text { specifications. }\end{array}$} \\
\hline ASTM & A & \multicolumn{5}{|c|}{ American Society for Testing and Materials } \\
\hline CLP SOW & - & \multicolumn{5}{|c|}{ Contract Lab Program Statement of Work for Inorganics (EPA 1988c and 1990b) } \\
\hline CLP SOW & - & \multicolumn{5}{|c|}{ Contract Lab Program Statement of Work for Organics (EPA 1990c) } \\
\hline EPA 601 & - & \multicolumn{5}{|c|}{40 CFR 136 Appendix A Method 601 Purgeable Halocarbons } \\
\hline EPA 524.2 & $-\quad \mathbf{N}$ & \multicolumn{5}{|c|}{$\begin{array}{l}\text { Methods for the Determination of Organic Compounds in Drinking Water (EPA } \\
\text { 1988b) }\end{array}$} \\
\hline EPA 300.0 & - & \multicolumn{5}{|c|}{$\begin{array}{l}\text { Test Method: The determination of Inorganic Anions in Water by Ion } \\
\text { Chromatography (EPA 1989) }\end{array}$} \\
\hline EPA 310.1 & - & \multicolumn{4}{|c|}{$\begin{array}{l}\text { Chromatography (EPA 1989) } \\
\text { Methods for Chemical Analysis of Water and Wastes (EPA 1983b) }\end{array}$} & Methods for Chemical Analysis of Water and Wastes (EPA 1983b) \\
\hline EPA 340.2 & - & \multicolumn{5}{|c|}{ Methods for Chemical Analysis of Water and Wastes (EPA 1983b) } \\
\hline
\end{tabular}

The minimum detectable relative difference (MDRD) is the percent difference required between observed site concentrations and action levels before the difference can be statistically detected. For this investigation, action levels are background concentration levels. The minimum detectable relative difference will be $20 \%$. Table 5-2 shows the number of samples necessary to achieve MDRD values ranging from 5 to 20\%. Table 5-3 gives the MDRD performance of the chemical analyses to be performed in this investigation. At moderately increased variability in the sample population (i.e., where the coefficient of variation is $35 \%$ ), the number of samples for each parameter remains sufficient to exceed the minimum requirement of a $20 \%$ MDRD

(Table 5-3). 


Section No:: $\frac{5}{\text { Revision No.: } \frac{0}{\text { July } 1993}}$
Date: $\frac{5-4}{\text { Page: }}$

Table 5-2. Relationships between statistical performance indicators and number of samples required (EPA 1989).

\begin{tabular}{cccccc}
\hline & \multicolumn{5}{c}{ Number of samples required to meet MDRD } \\
\hline $\begin{array}{c}\text { Coefficient of variation } \\
(\%)\end{array}$ & $\begin{array}{c}\text { Power } \\
(\%)\end{array}$ & $\begin{array}{c}\text { Confidence } \\
(\%)\end{array}$ & $5 \%$ & $10 \%$ & $20 \%$ \\
\hline 10 & 95 & 90 & 36 & 10 & 3 \\
15 & 95 & 90 & 78 & 21 & 6 \\
20 & 95 & 90 & 138 & 36 & 10 \\
25 & 95 & 90 & 216 & 55 & 15 \\
30 & 95 & 90 & 310 & 78 & 21 \\
35 & 95 & 90 & 421 & 106 & 28 \\
10 & 90 & 80 & 19 & 5 & 2 \\
15 & 90 & 80 & 41 & 11 & 3 \\
20 & 90 & 80 & 73 & 19 & 5 \\
25 & 90 & 80 & 114 & 29 & 8 \\
30 & 90 & 80 & 163 & 41 & 11 \\
35 & 90 & 80 & 222 & 56 & 15 \\
\hline
\end{tabular}

a. The number of samples is that required in a one-sided sample t-test to achieve a MDRD at the confidence level

Table 5-3. MDRD performance of analytical parameters.

\begin{tabular}{llcc}
\hline \multicolumn{1}{c}{ Parameter } & \multicolumn{1}{c}{ Matrix } & Samples & \multicolumn{1}{c}{$\begin{array}{c}\text { MDRD } \\
(\%)\end{array}$} \\
\hline Volatiles & Groundwater & 36 & $10<$ MDRD $<20$ \\
Volatiles & Soil gas & 214 & $5<$ MDRD $<10$ \\
Volatiles & Surface soil & 27 & $10<$ MDRD $<20$ \\
Volatiles & Subsurface soil & 16 & $10<$ MDRD $<20$ \\
Semivolatile organics & Subsurface soil & 16 & $10<$ MDRD $<20$ \\
Semivolatile organics & Surface soil & 16 & $10<$ MDRD $<20$ \\
Metals & Groundwater & 36 & $10<$ MDRD $<20$ \\
Metals & Surface soil & 27 & $10<$ MDRD $<20$ \\
Metals & Subsurface soil & 16 & $10<$ MDRD $<20$ \\
Cr ${ }^{6+}$ & Groundwater & 36 & $10<$ MDRD $<20$ \\
$\mathrm{Fl}^{-}$ & Groundwater & 36 & $10<$ MDRD $<20$ \\
Anions & Groundwater & 36 & $10<$ MDRD $<20$ \\
\hline
\end{tabular}

a. MDRD is at a $35 \%$ coefficient of variation, which corresponds to a power of $90 \%$ and a confidence of $80 \%$. 


\begin{tabular}{|c|c|}
\hline Section No.: & 5 \\
\hline Revision No.: & 0 \\
\hline Datc: & July 1993 \\
\hline Page: & 5.5 \\
\hline
\end{tabular}

\subsubsection{Measurement Reproducibility}

Variation attributed to the data collection process (i.e., measurement system) has four basic sources: sample collection procedures, sample handling and storage procedures, analytical procedures, and data processing procedures. Duplicate samples (a pair of collocated samples) allow estimation of measurement variability and will be collected during sample collection. Reproducibility of measurement will be expressed as relative percent difference (RPD) between duplicates or relative standard deviation (RSD).

Control limits for measurement variability at the OU 4-12 investigation sites are generated from Methods for the Chemical Analysis of Water and Wastes (EPA 1979), Test Methods for Evaluating Solid Waste Physical/Chemical Methods (EPA 1986a), Methods for the Determination of Organic Compounds in Drinking Water (EPA 1988b), Contract Laboratory Program Statement of Work for Organic Analysis (EPA 1990c), and the Contract Laboratory Program Statement of Work for Inorganic Analysis (EPA 1988c and 1990b).

Measurement variability will also be evaluated using site comparison samples. These samples consist of soil media that were spiked with semivolatile organic and metal analytes by the EPA in 1992.

A laboratory precision statement will be developed from laboratory duplicate analysis (split samples, split extractants/digestants, duplicate analyses, etc.). Precision goals for each method are listed in Table 5-1. The overall precision of the sampling and analysis program may be lower due to sampling error and matrix interference.

\subsection{Accuracy}

Accuracy measures the bias in a measurement system. Typically, it is difficult to completely measure accuracy for the entire data collection process. Errors of measurement are introduced through sampling methods, field contamination, methods of sample preservation and handling, sample matrix, and analytical procedures. Control limits for accuracy can be generated from Methods for Chemical Analysis of Water and Wastes (EPA 1979), Test Methods for Evaluating Solid Waste Physical/Chemical Methods (EPA 1986a), Methods for the Determination of Organic Compounds in Drinking Water (EPA 1988b), CLP SOW for Organics (EPA 1990c), and the CLP SOW for Inorganics (EPA 1988c and 1990b). Accuracy will be monitored with the use of surrogate recoveries, internal standard recoveries, and blank spike recoveries. Bias (i.e., accuracy) will be estimated for the measurement process by computing percent recovery of compounds spiked into the samples at known concentrations.

Blanks will be utilized to determine the introduction of artifact contamination into the sampling and analysis process. Field blanks will be prepared at a frequency of 1 per 20 samples collected. Equipment blanks will also be prepared at a frequency of 1 per 20 samples collected. Analysis of blanks will allow estimation of bias introduced from artifact contamination. This is not 


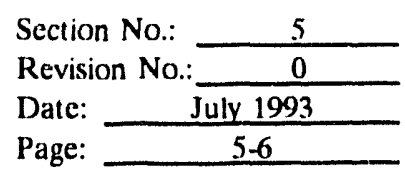

a true estimate of accuracy but will indicate whether artifact contamination resulted in bias. This parameter will be expressed as percent bias. Spikes will be utilized to determine the accuracy of positive results and no-detects in the analytical process.

QC samples will be used to assess laboratory accuracy. Laboratory matrix spikes are spikes prepared in the laboratory by splitting a sample and spiking one portion with a known amount of the analyte(s) of interest. The spiked sample result and sample result are compared, and the amount of spike recovered is calculated. The spike recovery is the measure of accuracy. Accuracy goals are presented in Table 5-1. Site comparison samples will also be used to assess laboratory accuracy. These samples were spiked with known concentrations of semivolatile and metal analytes by the EPA in April 1992. They will be submitted to the analytical laboratory along with the subsurface soil samples from Landfill II. The analytical results for these samples will be compared to the spike concentrations. The spike recovery is a measure of accuracy. The overall accuracy for the project may be less due to contributions of error.

\subsection{Representativeness}

Representativeness expresses the degree to which sample data accurately and precisely represent a population characteristic, parameter variation at a sampling point, or an environmental condition. Representativeness is a qualitative attribute and is a function of the proper design of the sampling strategy. The use of standard procedures for the collection and analysis of samples will help ensure representativeness.

The minimum requirements for representativeness are that the data are representative of the exposure area and that sample preparation procedures do not affect representativeness. This requires that sampling locations are truly representative of the site, and sample collection and preparation procedures do not affect representativeness by introducing bias. Representativeness during planning stages of this investigation was addressed in the DQO process described in Section 2 of the FSP and Section 4 of the Work Plan.

\subsection{Completeness}

Completeness is the amount of valid data obtained compared to the amount that was expected under normal conditions. Completeness can be expressed as the percentage of valid measurements. To achieve adequate completeness, a sufficient amount of valid data must be generated.

Completeness for this project will be assessed by comparing the number of planned sample analyses to the number of samples collected, analyzed, and validated. The completeness goals for various sampling activities are listed below: 


\section{Completeness Goal}

Data Collection Activity

Sampling and analysis of

wells

Shallow soil gas survey

Borehole soil gas survey

Surface and subsurface soils

Geophysical surveys

Cover material
(\%)

100

90

90

90

80

80

\subsection{Comparability}

Comparability is the confidence with which one data set can be compared with another. Comparability is a qualitative parameter and is attained by using standard methods for collecting and analyzing data as well as using standard units for reporting analytical results.

To assist in comparing data, all analyses will be accomplished utilizing an EPA-accepted method. These methods include the EPA CLP SOW for Organics (EPA 1990), EPA CLP SOW for Inorganics (EPA 1988c and 1990b), methods for the Determination of Organic Compounds in Drinking Water (EPA 1988b), Test Methods for Evaluating Solid Waste Physical/Chemical Methods (EPA 1986a), Index to EPA Test Methods (EPA 1988c), Methods for Chemical Analysis of Water and Wastes (EPA 1983b), or those listed in 40 CFR 136 (1984), or approved as an alternative test procedure in accordance with 40 CFR 136 . All analytical results will be reported in the concentration values and units required for entry into site-specific data bases and those values and units needed for use in models. In addition, so that data from subsequent sampling at the same site or facility can be compared, the specific sampling points will be established and documented.

Comparability will be assessed by comparing the following information on each data set:

- $\quad$ Field collection methods

- Field and laboratory QA/QC procedures (in accordance with previously established protocols)

- Laboratory detection limits

- Matrix. 
Section No.:

Revision No.:

Date:

Page: 58
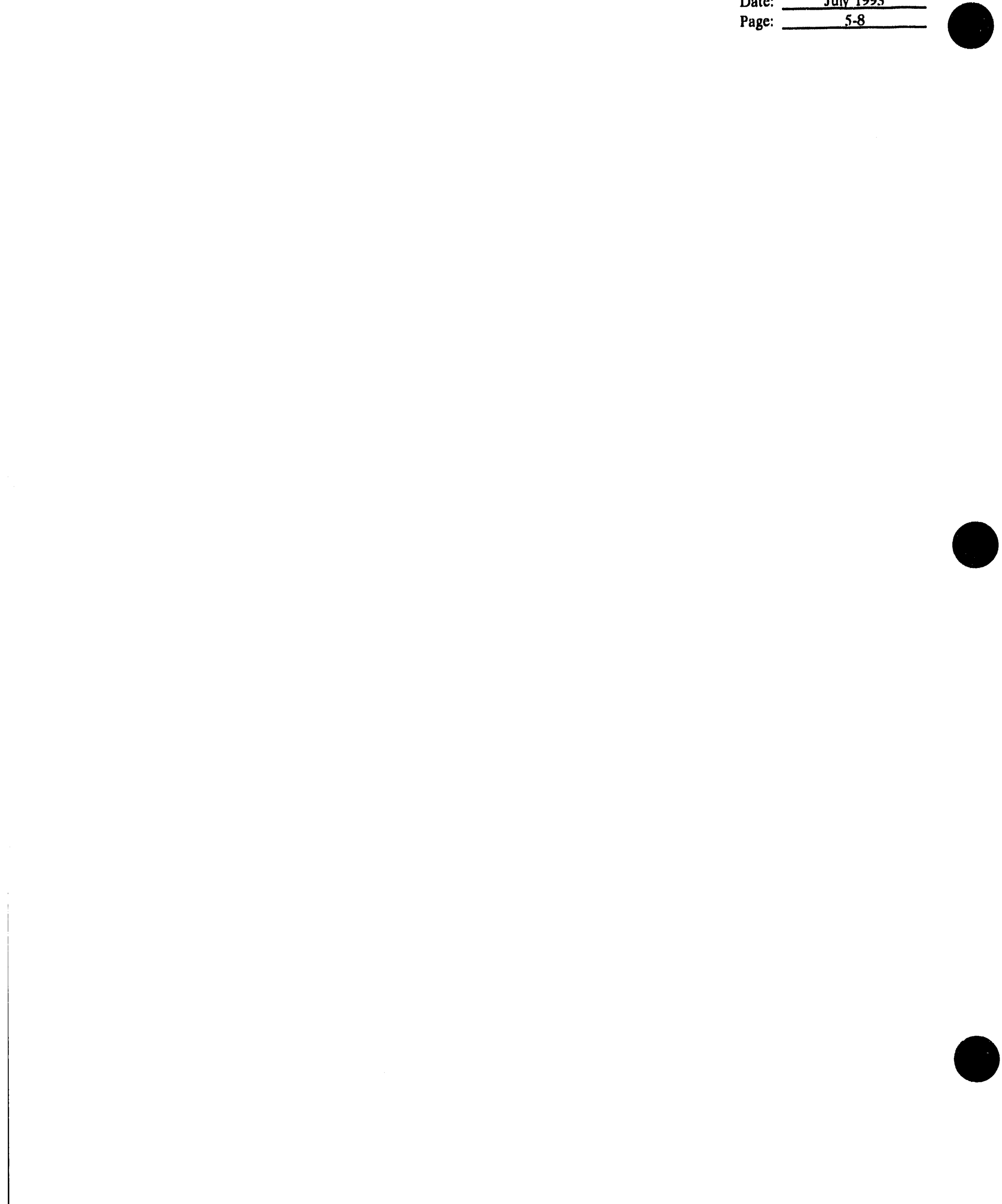


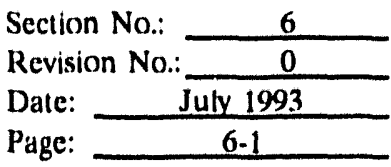

\section{SAMPLING PROCEDURES}

The objective of sampling procedures is to obtain samples that represent the environment being investigated. Trace levels of contaminants from external sources must be eliminated through the use of experienced field personnel, good sampling techniques, proper sampling equipment, and adequate decontamination.

Field measurements shall be performed in accordance with EPA-accepted procedures and the procedures listed and described in Appendix B of the FSP.

Details of sampling procedures are described in the FSP, Part I of the SAP. The standard operating procedures have been developed, and they are referenced and appended to the FSP (Appendix B).

All samples will be transported to the analytical laboratories in accordance with Department of Transportation (DOT) regulations (49 CFR) governing the shipment of hazardous materials and substances and EPA regulations governing the shipment of samples from hazardous waste site investigations. Originators of hazardous or radioactive shipments must be qualified and certified as Hazardous Material Shippers. Source documents for sample handling may include the User's Guide to the Contract Laboratory Program (EPA 1986b). Chain of custody and shipping requirements are detailed in ER Program Directive 5.7, "Chain of Custody Record," and in Section 6.2 of the FSP.

Project Managers or their delegated substitutes (such as field team leaders) are responsible for determining, to the best of their knowledge, whether samples planned for collection are environmental (contain hazardous or dangerous substances) or hazardous (per RCRA subtitle C) in nature. After collection (and prior to packaging and shipping), each sample will undergo an identification and classification process. A review of the Field Sampling Logbook, in which field measurements were recorded (radioactivity, $\mathrm{pH}$, organic vapors, explosivity, etc.) and other relevant information concerning the material within the sample container, will be conducted by the shipper and the Project Manager (or delegated substitute).

Most radioactive samples will meet the definition of limited-quantity radioactive material and are, therefore, exempt from the more stringent DOT requirements for greater activities of radioactive material. All samples will be screened for radioactivity before they are shipped to an analytical laboratory. The screening procedure is described in Section 6.2.3.1 of the FSP and will ensure that the samples are classified correctly and within DOT packaging and shipping requirements. Results from the screening will then be used to classify, package, and ship the samples to the appropriate analytical laboratory. Requirements for containers, procedures, and preservatives used for sample collection are detailed in Sections 5.3.3 and 6.2 of the FSP. Requirements for field records/documentation are detailed in Section 6.1 of the FSP and in ER PD 4.2, "Logbooks." 
Section No.:

Revision No.:

Date:

Page:

July 1993

6.2

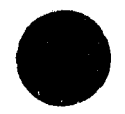




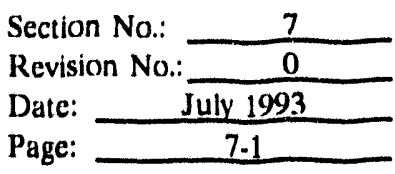

\section{SAMPLE CUSTODY}

\subsection{Document Control}

The procedures that govern document control for this project are ER Program Directives (PDs) 4.1, "Document Control," 1.8, "Administrative Record," 1.9, "Records Management, and 4.2, "Logbooks." Controlled documents will receive review and concurrence as directed by ER PD 4.8, "Internal and Independent Review of Documents."

\subsection{Chain of Custody Documentation}

A required part of any sampling and analytical program is the integrity of the sample from collection to data reporting. This includes the ability to trace possession and the handling of samples from the time of collection, through analysis and final disposition. This documentation of the sample's history is referred to as chain of custody (COC). Components of the sampling/field COC, which include a field logbook, sample labels, and custody seals, and of components the laboratory COC, which include a COC record, a laboratory sample log-in/log-out logbook, laboratory sample storage records, and laboratory sample disposal records are discussed in the following sections. Sample custody procedures will follow EPA CLP SOW for Organics (EPA 1990c), EPA CLP SOW for Inorganics (EPA 1988c and 1990b), National Enforcement Investigations Center (NEIC) procedures (NEIC 1980), and ER PD 5.7, "Chain of Custody Record."

A sample is considered to be under a person's custody if it is in that person's physical possession, in view of the person after he has taken possession, or secured by that person so that no one can tamper with it. A person who has samples under custody must comply with the procedures described in the following sections.

\subsection{Chain of Custody Record}

To establish documentation necessary to trace sample possession from the time of collection, a $\mathrm{COC}$ record will be completed and accompany every sample. The $\mathrm{COC}$ record to be used is exhibited in the FSP (Figure 6-4) and is distributed by ARDC. The record will be completed in ink by the sample custodian and shall contain the following minimum information:

- Sample number (traceable to a sampling location)

- Signature of collector

- Date and time of collection

- Signatures of people involved in the chain of possession

- Inclusive dates and times of possession

- Analyses requested. 


Section No.: $\frac{7}{0}$
Revision No.: $\frac{0}{\text { July } 1993}$
Date:
Page:

To maintain the COC, each person in custody of the sample will sign the form. Samples will not be left unattended unless placed in a secured and sealed container (custody seals) with the COC record inside the container.

\subsection{Field Logbook}

The field logbook is the written record of all field data, observations, field equipment calibrations, samples, and the COC, and is considered to be a legal document. The logbook will be site-specific and bound. The logbook sheets to be used are exhibited in Appendix $C$ of the FSP, and the logbooks will be distributed by ARDC. Pages will be sequentially numbered and firmly attached to the book. All entries will be made in ink. Any mistakes will be lined out with a single line and initialed and dated by the person making the correction. At a minimum, entries in the logbook include the following:

- $\quad$ Reference to the SOP used

- Purpose of sampling

- Location and description of sampling point

- Identification of sampling crew

- Type, number, preservative, and volume of sample

- Date and time of sampling

- Date and time of shipping

- Weather

- Ficld measurements

- Deviations from SOPs

- $\quad$ COC numbers.

\subsection{Sample Label}

All samples are identified by a sample label. The sample label to be used is exhibited in Figure 6-1 in the FSP. All sample labels shall be filled out using ink. Each sample shall be designated by a unique alphanumeric code that identifies the sample. When samples are transported from the sample location to the contract laboratory by common carrier, they shall be packaged and labeled according to procedures specified by the DOT (49 CFR) and as described in Section 6.2 of the FSP.

As appropriate, information recorded on the sample label shall include the following:

- Unit facility

- Location

- Sample type

- Sample date/time

- Sample number

- $\quad$ Sampling person 
- Radiation level (if applicable)

- Analysis requested.

\subsection{Custody Seals}

Custody seals are used to detect unauthorized tampering with samples prior to analysis. Gummed paper seals will be used for this purpose. The seal will be dated and signed, which makes it unique, and attached so that it must be broken to open the sample container/cooler. If samples are not contained in a shipping container with a custody seal, seals will be affixed to containers before the samples leave the custody of sampling personnel. Shipping containers will also contain seals to detect tampering.

\subsection{Document Corrections}

Documentation in logbooks, custody seals, and other accountable serialized documents will be completed with permanent ink. None of these documents will be destroyed or thrown away, even if they are illegible or if they contain inaccuracies that require they be replaced. They will be marked VOID and maintained in a file. A record of all voided documents will be maintained by ARDC.

If an error is made on an entry into an accountable document, the individual in error will draw a single line through the error, enter the correct information, and initial and date the change. This procedure also applies to words or figures inserted or added to a previously recorded entry.

If a COC record is lost in shipment or was never prepared for a sample, or if a properly labeled sample was not transferred with a formal COC record, a written statement will be prepared by the Field Team Leader detailing how the sample was collected. A copy of the statement will be sent to the project files.

\subsection{Photographic Records}

A photographic record will be made during field projects. When photographs are taken, the name of the photographer, date, time, sampling site or laboratory location, description of site or activity being photographed, and weather conditions (if appropriate) will be entered in the Field Team Leader's logbook. Special lens, film, filter, or other image-enhancement techniques will be noted in the photographer's logbook; however, the use of such techniques should be avoided because they can affect the admissibility of the photographs as evidence. Once developed, slides or photographic prints will be serially numbered (corresponding to logbook descriptions) and labeled. The ARDC Officer will maintain a supply of photograph logbooks and a file of all photographs taken. All photograph logbooks, slides, and prints will be controlled documents. 


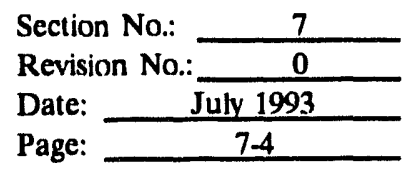

\subsection{Laboratory Custody}

Laboratory custody will conform to procedures established in ER PD 5.7, "Chain of Custody Record," and ER PD 5.5, "Obtaining Laboratory Services." These procedures include:

- Designation of a sample custodian

- Correct completion by the custodian of the COC record and laboratory request sheet, including documentation of sample condition upon receipt

- Laboratory sample tracking and documentation procedures

- $\quad$ Secure sample storage.

The sample will be delivered to the laboratory so requested analyses can be performed within the specified allowable holding time. The sample will be accompanied by the COC with an appropriate sample analysis request. The sample will be delivered to the person in the laboratory who is authorized to receive samples (Laboratory Sample Custodian). Samples will be packaged and shipped according to DOT and EPA regulations.

\subsection{Final Evidence Files}

The WAG Manager or Project Manager is responsible for active project files. At an appropriate time, the WAG or Project Manager will transfer files to ARDC. Final evidence files include all information and documentation developed in the field and laboratory.

Original final reports and analytical data will be maintained by ARDC and dispositioned in accordance with DOE Order 1324.2A, "Records Disposition." Copies of all analytical data and final reports will be retained in the laboratory files and stored on computer disk for a minimum of one year. Additional guidance on establishing administrative record data and data control is contained in the DMP. 


\section{CALIBRATION PROCEDURES AND FREQUENCY}

Field sampling procedures outline calibration procedures and the calibration frequency for field instruments. Guidance of the EPA CLP SOW for Organics (EPA 1990c), EPA CLP SOW for Inorganics (EPA 1988c and 1990b), Test Methods for Evaluating Solid Waste (EPA 1986a), Methods for the Determination of Organic Compounds in Drinking Water (EPA 1988b), and 40 CFR 136 Appendix A, Method 601 shall be followed in determining laboratory instrument calibration procedures and frequency for chemica! analysis. Measuring and testing equipment calibration may be performed internally using standards traceable to the National Institute of Standards and Technology, where applicable, or externally by the equipment manufacturer or approved calibration facility. If no nationally recognized standard exists for the equipment to be calibrated, the basis for calibration shall be documented.

Responsibility for calibrating laboratory equipment rests with the Laboratory Manager for onsite and offsite laboratories. The Field Team Leader is responsible for ensuring that equipment used by the sampling crew in the field is calibrated. Field calibration records will be collected by the Field Team Leader for the final evidence files where lab calibration records will also be maintained. The radiological and industrial hygiene equipment calibration responsibility rests with health physics and industrial hygiene personnel, respectively.

It is the responsibility of personnel using the equipment to check the calibration status prior to using it and to ensure that the equipment is operational prior to taking it to the sampling locations.

Documented and approved procedures shall be used to calibrate all measuring and testing equipment. Whenever possible, widely accepted procedures such as those published by EPA (1986a), or procedures provided by the equipment manufacturer shall be used.

Each piece of equipment shall be identified so that the pertinent calibration information can be retrieved. The equipment shall have an individual calibration log and be calibrated/standardized prior to use or as part of the operational use following the manufacturer's recommended calibration/standardization procedure(s). The frequency of calibration shall also be based on the requirements of the analytical method.

Measuring and testing equipment shall be calibrated at prescribed intervals or prior to use. Frequency shall be based on the type of equipment, inherent stability, manufacturer's recommendations, intended use, and experience.

Records shall be prepared and maintained for each piece of calibrated equipment to indicate that established calibration procedures have been followed. Calibration records for the equipment controlled by the various laboratories, offices, and groups shall be maintained by the respective organization. A copy of the instrument logbook shall be provided for the period the instrument was used for OU 4-12 samples. 


Section No.: $\frac{8}{0}$
Revision No.: $\frac{0}{\text { July } 1993}$
Date:
Page:

Equipment that fails calibration or becomes inoperable during use shall be removed from service and segregated to prevent inadvertent use or shall be tagged to indicate it is out of calibration. Such equipment shall be repaired or recalibrated prior to further use.

Data generated during downtime, failure of the instrument, or an instrument that requires adjustment during recalibration shall be evaluated by the Laboratory QA Officer for acceptability. Results of the evaluation shall be documented and retained in the project files. 


\section{ANALYTICAL PROCEDURES}

One or more analytical laboratories may be used. The choice of laboratory depends upon the DQOs for the task, the ability of the laboratory to perform a method, the acceptance criteria of a sample's radionuclide content, and the ability of the laboratory's QA/QC program to meet the RI/FS objectives. The analytical laboratory shall be approved prior to bid award based on audit results by ER prior to use. Audit procedures are described in Section 12 of this QAPjP. Analytical methods and respective analytical support levels are listed in Table 5-1. 
Section No.:

Revision No.:

Date:

Page: 


\section{DATA REDUCTION, VALIDATION, AND REPORTING}

Data reduction and reporting procedures are described in detail in the Data Management Plan, which describes management of field- and laboratory-generated data from point of generation to point of use (EG\&G Idaho 1992). The DMP is consistent with EPA Guidance or requirements per specific method and the EG\&G Idaho Sample Management Office SOPs for validating data (EG\&G Idaho 1991b). EG\&G Idaho ER SMO SOPs for the validation of inorganic and organic data are consistent with the requirements of the EPA functional guidelines for data validation.

Data from the chemical analysis of groundwater, surface soil, soil gas, and subsurface soil samples collected from the remedial investigation wells will be validated at Level $A$ as defined in SMO-SOP-12.1.1. The data from the analysis of geotechnical samples will all be validated at Level $B$ as defined in SMO-SOP-12.1.1.

Under level A vaiidation, analytical data are reviewed for the following:

- Data package completeness

- Requested versus reported analyses

- Analytical holding times

- Method blank criteria

- Matrix spike/matrix spike duplicate recoveries/precision

- Duplicate sample precision

- Surrogate spike recoveries

- Laboratory control sample recoveries (radiological methods)

- Any other method-specific quality control criteria. 
Section No.:

10

Revision No.:

Date:

Page: $10-2$ 


\section{INTERNAL QUALITY CONTROL CHECKS}

Several types of internal QC checks will be utilized during sampling. These include, but are not limited to the following:

- Decontamination (equipment rinsate) blanks

- Volatile organic analysis (VOA) trip blank-one per cooler shipped for groundwater samples

- Field and laboratory duplicates or replicates.

Amounts of each of the control samples listed above are specified in the FSP and EPA protocols. Internal QC check samples will be analyzed along with site samples.

\subsection{Offsite Laboratory QA/QC}

The laboratory QA/QC procedures used will be those submitted by the laboratory in its written QA plan. The submitted plan should provide for use of standards, laboratory blanks, duplicates, and spiked samples for calibration and the identification of potential matrix interferences. Laboratory results of matrix spike (MS) and matrix spike duplicate (MSD) sample analyses shall be provided in a manner that will allow assessment of accuracy and precision. Adequate statistical procedures will be used to monitor and document performance and to implement an effective program to resolve testing problems (e.g., instrument maintenance and operator training).

For soil gas samples carrier gas blanks will be analyzed prior to sample batch analysis and after every tenth sample analysis. Standards will be analyzed prior to sample batch analysis and at regular intervals during analysis. Duplicate samples will be run every tenth sample.

Specific routine procedures used to assess data precision, accuracy, and completeness are described in Section 14 of this QAPjP.

\subsection{Field QC Samples}

Blind QC samples listed below will be either collected in the field or generated in the lab and sent to the field, then shipped to the laboratories with other samples.

For groundwater sampling, QC samples will include:

- Trip blank-Trip blanks provide a measure of potential sample contamination from the presence of contaminants in the reagent water source, from preservative contamination 


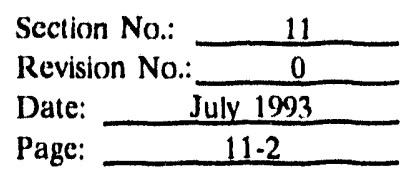

of the blank itself during blank preparation, from the shipment of the prepared blank to the field, and from the shipment from the ficld to the laboratory. The trip blank will be prepared using the lab's reagent water, with the addition of all appropriate preservative chemicals. Trip blanks accompany the sample shipping container and will remain unopened until after the laboratory receives them for analysis.

Trip blanks will be collected at a minimum frequency of one per shipping container for VOA and will be shipped blind to the laboratory(ies) with other samples and analyzed for volatile organics only.

- Decontamination (equipment) blank-A decontamination blank will be prepared and submitted for analysis at a minimum frequency of 1 per every 20 samples. This blank will consist of deionized rinse water collected after the water has rinsed decontaminated equipment and will be analyzed for the same parameters as the sample group it checks.

- Field blank-Field blanks provide a measure of potential errors that can be introduced from sources other than the sample. A field blank will also measure input from contaminated dust or air into the sample. A field blank is prepared in the field by pouring deionized or reagent-grade (analyte-free) water into the appropriate sample containers and includes all appropriate preservative chemicals. Field blanks are prepared at a minimum frequency of 1 per 20 .

- Field replicates-Field replicates are collocated samples collected identically and consecutively over a minimum period. Field replicates provide a measure of the total analytical bias (field and laboratory variance), including bias resulting from the heterogeneity of the replicate sample set itself. Field replicates will be collected at a minimum frequency of 1 per 20 samples or 1 per sampling round. Samples collected for VOA will not be homogenized because this increases volatilization.

For soil gas samples, ambient air filtered with an organic cartridge filter will be drawn through the sampling apparatus with the sampling probe attached at the following times:

- Prior to each day's sampling activities

- After every twentieth sample

- At the conclusion of each sampling day.

For surface soil samples, one field replicate and one decontamination (equipment) blank will be collected from each landfill and submitted for analysis. For subsurface soil samples, one field replicate and one decontamination blank will be collected and submitted for analysis. 


\section{SYSTEM AND PERFORMANCE AUDITS}

Audits and surveillances are systematic checks to determine whether or not personnel on a project are adhering to the requirements outlined and referenced in the QAPjP and the SAP. Audits of laboratory activities and surveillance of field activities are the responsibility of the ER Quality Engineer.

The Quality Engineer, in conjunction with the ER Project Manager, will determine the frequency of quality monitoring for this project. Checklists will be developed to accomplish the review of necessary items and to document results of the audit/surveillance.

Two types of audits will be performed: systems audits and performance audits. Details of these are contained in the following subsections.

\subsection{Systems Audits}

Systems audits consist of evaluating all components of the applicable measurement systems to determine their proper selection and use. At least one systems audit will be performed before or shortly after systems are operational to do the following:

- Verify that the QA organization is operational

- Verify that correct sampling methodologies have been chosen and that written procedures for sampling are available and being followed.

Systems audits of laboratories are qualitative audits of the measurement systems, ensuring they are properly maintained and used. These audits are performed before approval of a contract. A laboratory will be audited, and its QA plan will be reviewed and accepted by the ER Compliance Assurance Unit prior to being awarded a contract. The laboratory approval process is detailed in ER Program Directive 5.6, "Conducting Audits of Laboratories."

Technical experts will be assigned to the audit teams for laboratory audits. Audits of laboratories will be announced and planned. All audits will be conducted in accordance with approved project directives and an enhanced checklist modified from the checklist developed by EPA's CLP.

\subsection{Performance Audits}

Laboratory performance audits and field surveillance normally will be conducted after data production systems are operational and generating data. 


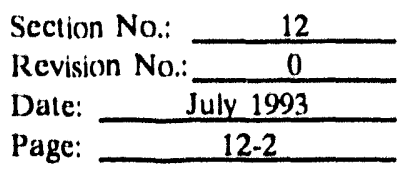

\section{Field Operations}

Surveillances will be conducted on field activities as ficld data are gencrated, reduced, and analyzed. The procedure for conducting field survcillance is detailed in ER PD 5.14, "Quality Monitoring and Surveillance." Items examined will include, but are not limited to, calibration records of field equipment, daily entries in logbooks, decontamination procedures, photographs, data logs, and sampling. At a minimum, one field surveillance will be performed every month while field activities are in progress.

After completion of the surveillance, any deficiencies will be discussed with the field staff, and corrections will be identified. If any of these deficiencies could affect the integrity of the samples being collected, the Program Manager will inform the field staff immediately so that corrective action can be implemented immediately. The Program Manager will submit a surveillance report to the Project Manager of the task and to the organization or subcontractor that was surveyed.

The Field Team Leader or the Project Manager will respond to findings listed in the surveillance report, in writing, to the ER WAG 4 Manager. The response will clearly state the corrective action taken or planned. If corrective actions have not been completed prior to issuance of the audit response, a scheduled date for completion will be provided. Requests for corrective action must be addressed to the satisfaction of the ER WAG 4 Manager.

Completion of the corrective action will be verified by the Program Manager through written communication, follow-up surveillance, or other appropriate means. After acceptance and verification of the corrective action, a surveillance closure will be issued to the same individuals receiving the surveillance report.

\subsection{Reports}

Following completion of an audit or surveillance, the Quality Engineer or Lead Auditor will prepare and submit a post-audit/surveillance report in accordance with ER PD 5.14, "Quality Monitoring and Surveillance."

The report will include the following information, when appropriate:

- Date(s) of the audit/surveillance

- Identification of audit/surveillance participants

- Identification of activities audited/surveyed

- Audit/surveillance results

- Description of items requiring corrective action

- Due date for completion of corrective actions and/or audit/surveillance response

- Means for audit/surveillance response (in writing). 
Section No.:

Revision No.

Date:

July 1993

Page:

$12-3$

A corrective action plan will then be prepared by the program being audited/surveyed. This will include a list of solutions or corrective actions that were taken to resolve problems identified by the auditors/quality engineers. When appropriate, a schedule for implementing corrective actions will be included in the corrective action report. Copies of the audit/surveillance report and corrective action responses will be sent to the Project Manager and Program Manager by the Quality Engineer. 
Section No.:

12

Revision No.

Date:

Page:

July

uly 1993 


\section{PREVEnTIVE MAINTENANCE}

Measuring and test equipment used in the ficld and laboratory will be controlled by a calibration program in compliance with "Control of Measuring and Test Equipment" (QP-12) of EG\&G Idaho (1990). Equipment of the proper type, range, accuracy, and precision will provide data compatible with project requirements and desired results. Calibration of measuring and test equipment may be performed internally using reference standards, or externally by agencies or manufacturers (see Section 8).

Equipment that fails calibration or becomes inoperable during use will be removed from service and segregated to prevent inadvertent use or will be tagged to indicate that it is out of calibration. Such equipment will be repaired and calibrated to the satisfaction of the manager of the laboratory or manager of the task, as appropriate, before further use. Equipment that cannot be repaired will be replaced.

Data generated from equipment that has failed calibration shall be evaluated and qualified for use on the project. The evaluation/qualification process is the responsibility of the cognizant manager and the Quality Engineer. The method of qualification and the results of the data evaluation will be documented.

Documented and approved laboratory procedures will be used to calibrate analytical instruments. These procedures will include, as a minimum:

- Type of equipment to be calibrated

- Calibration method and sequential actions

- Calibration data recording form/format

- A list of critical or replacement parts.

The information above will, in general, conform to the manufacturer's recommended procedures or explain the deviation from these procedures.

Laboratory equipment requiring routine maintenance will have an individual instrument file indicating the frequency of required maintenance history, spare parts maintained by the laboratory, directions for maintenance, and any external service contracts.

Analytical laboratory preventive maintenance will be the responsibility of the laboratory. However, at a minimum, the laboratory will be required to have the following:

- Service contracts on major instruments, when necessary

- Spare parts, as recommended by the instrument manufacturer

- Delineation of the above items in the laboratories' written QA/QC plan. 
Section No.:

Revision No.

13

Page:

13-2 
Section No.:

Revision No:

$\frac{14}{\frac{0}{\text { uly } 1993}}$

\section{DATA ASSESSMENT PROCEDURES}

Procedures to assess precision, accuracy, and completeness of data collected from sampling and analysis are different for field and laboratory data. Field and laboratory procedures are described in this section.

\subsection{Field Data}

Field data include all data recorded in field logbooks during field sampling activities. Field precision and accuracy will be assessed by conducting field audits to ensure use of uniform sample collecting, handling, and shipping procedures and by evaluation of field blanks.

\section{Procedures to Assess Field Data Precision}

Field precision will be assessed by field audits and checklists performed on a routine basis. These audits will document use (or nonuse) of uniform sampling methods and handling and shipping procedures. Field sampling precision will be assessed by analytical results of collocated (duplicate), split, or field blank samples, and through use of equipment blanks. These blanks will identify compounds inadvertently introduced onto the samples from contaminated sampling equipment.

\section{Procedures to Assess Field Data Accuracy}

Accurate sample collection will be evaluated from the results of field systems audits that include onsite evaluations of sample collection procedures, instrument performance, and calibration procedures. Field sampling accuracy cannot be assessed quantitatively because the "true" value is not available.

\section{Procedures to Assess Field Data Completeness}

Completeness of field data will be assessed by calculating the ratio of samples analyzed to the total number of samples planned, stated as a percentage.

\subsection{Laboratory Data}

The QAPjP and analytical laboratory methods describe precision, accuracy, and completeness. Accuracy and precision of data will be assessed for each sample lot using percent recovery and relative percent difference of MS/MSD pair analysis. 


\section{Procedures to Assess Laboratory Precision}

Precision of laboratory data will be measured by analysis of duplicates. Laboratory reagent blanks will be analyzed to monitor introduction of artifacts into the process. If data obtained are not within the control limits specified in EPA CLP SOW for Inorganics (EPA 1988c and 1990b), in EPA CLP SOW for Organics (EPA 1990c), in EPA (1988a), and in EPA (1986a), corrective action will be taken.

\section{Procedures to Assess Laboratory Accuracy}

Accuracy of chemical laboratory data will be assessed by examining the percent recovery of the MS/MSD and analytical spikes for organics, and surrogates and internal standards for inorganics. Accuracy of radiological laboratory data will be assessed by measuring the activity of known QC check samples and by demonstrating reasonable agreements (e.g., plus or minus three standard deviations) and demonstrating instances in which the known is traceable to a reputable standard (e.g., National Institute of Standards and Technology).

\section{Procedures to Assess Laboratory Completeness}

Completeness of laboratory data will be measured by the ratio of samples received at the laboratory to the total number of samples analyzed, stated as a percentage. 


\section{CORRECTIVE ACTION PROCEDURES}

Corrective action procedures are implemented when samples do not meet QA specifications. In all cases, corrective action procedures will be approved by the Project QA Engineer.

Limits and corrective actions for all analyses will be as specified in the laboratory analytical Statement of Work, as well as in Methods for the Determination of Organic Compounds in Drinking Water (EPA 1988b) and in the Test Methods for Evaluating Solid Waste (EPA 1986a). Limits and corrective action for organic and inorganic analyses will be as specified in the EPA CLP SOW (EPA 1988c and 1990b, EPA 1990c). Other corrective actions may have to be implemented based on performance, system, or evidence audits performed. These will be developed on a case-by-case basis.

Corrective action procedures that might be implemented from audits or detection of unacceptable data are developed on a case-by-case basis. Such actions may include altering procedures in the field, resampling or retesting, using a different batch of containers, or recommending an audit of laboratory procedures. The requirements identified in ER PD 5.13, "Corrective Action" will be followed. Corrective actions to any major nonconformances associated with field activities will be incorporated in addenda to field sampling plans.

Data will be validated as discussed in the DMP, which follows EPA procedures. Data that cannot be validated using procedures outlined in the DMP will be reviewed in detail in an attempt to evaluate each measurement.

Contract-required detection limits for parameters analyzed with the CLP SOW for Inorganics (EPA 1988c and 1990b), contract-required quantitation limits for parameters analyzed with the CLP SOW for Organics (EPA 1990c) and method detection limits for organics (524.2 and 601) (EPA 1988b, 1986a) are presented in Appendix A. 
Section No.:

15

Revision No:

Date:

Page: July 1993 15-2 


$\frac{16}{\frac{0}{\text { July } 1993}}$

\section{QUALITY ASSURANCE REPORTS}

A periodic performance report of the QA Program will be prepared by the Project QA Officer and presented to the Project Manager. When appropriate, analytical laboratory QA/QC reports will be included. At task completion and after data verification and validation, all $\mathrm{QC}$ data will be sent to ARDC to become part of the program files.

QA reports will include:

- Results of any systems and performance audits conducted during the period

- Assessment of precision, accuracy, representativeness, completeness, and comparability of Jata collected during the period

- Nonconformance reports issued during the period, related corrective actions undertaken, and an assessment of action results

- Significant QA problems and recommended solutions

- $\quad$ Summary of personnel training and QA objectives met.

The final report will have a section that summarizes the periodic reports. 
Section No.:

16

Revision No.:

Date:

Page: July 1993 16-2

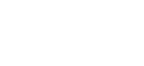




\section{REFERENCES}

Code of Federal Regulations, 40 CFR 136, 1984.

EG\&G Idaho (1990), Quality Manual.

EG\&G Idaho, 1991a, Quality Program Plan for the Environmental Restoration Program, Rev. 3, QPP-149, November.

EG\&G Idaho, 1991b, Sample Management Office Standard Operating Procedures for the Environmental Restoration Program, SOPs 1.12.1 through 1.12.5:

12.1.1 - SMO SOP Level of method validation, July 12, 1991

12.1.2 - SMO SOP Radiological data validation, August 1, 1991

12.1.3 - Validation of volatile and semi volatile organic gas chromatography/mass spectrophotometric data, August 9, 1991

12.1.4 - Validation of gas chromatographic data, August 9, 1991

12.1.5 - Validation of inorganic data validation, to be issued

EG\&G Idaho, 1992, EGG-WM-8676, Revision 1, September 1991, "Implementing Program Management Plan for the EG\&G Idaho Environmental Restoration Program," (no author is listed).

EG\&G Idaho, 1992, EGG-ERD-10227, Revision 0, June 1992, "Data Management Plan for the EG\&G Idaho Environmental Restoration Department," O. V. Hester, C. S. Watkins, D. J. Yurman.

EPA (Environmental Protection Agency), 1979, "Methods for the Chemical Analysis of Water and Wastes," EPA/600/4-79/002.

EPA, 1983a, "Interim Guidance and Specifications for Preparing Quality Assurance Project Plans," EPA/600/4-83/004, February.

EPA, 1983b, "Methods for Chemical Analysis of Water and Wastes," EPA-600/4-79-020, March.

EPA, 1986a, "Test Methods for Evaluating Solid Waste Physical/Chemical Methods," 3rứ edition, SW-846, November.

EP'A, 1986b, "User's Guide to the Contract Laboratory Program," Office of Emergency and Remedial Response, December.

EPA, 1988a, Guidance for Conducting Remedial Investigations and Feasibility Studies Under CERCLA, EPA/540/G-89/004, October. 


Section No:: $\frac{17}{0}$
Revision No.: $\frac{0}{\text { July } 1993}$
Date: $\frac{17.2}{\text { Page: }}$

EPA, 1988b, Methods for the Determination of Organic Compounds in Drinking Water (EPA 500 Methods), EPA/600/4-88/039, Environmental Monitoring Services Laboratory, Las Vegas, NV.

EPA, 1988c and 1990b, "Contract Laboratory Program Statement of Work for Inorganics Analysis Multi-media, Multi-concentration," SOW-788, Office of Emergency and Remedial Response, July 1988 and 1990.

EPA, 1989, Soil Sampling Quality Assurance User's Guide, EPA/600/8-89/046, U.S. Environmental Protection Agency, March.

EPA, 1990a, "National Oil and Hazardous Substances Pollution Contingency Plan," Federal Register, Volume 55, No. 46, pp. 8813-8865.

EPA, 1990c, "Contract Laboratory Program Statement of Work for Organics Analysis MultiMedia, Multi-concentration," SOW-390, Office of Emergency and Remedial Response, March.

NEIC (National Environment Investigations Center), 1980, "Enforcement Considerations for Evaluation of Uncontrolled Hazardous Waste Disposal Sites by Contractors," April. 


\section{Appendix A}

1. Contract required detection limits for metals.

2. Method detection limits for volatile organics (EPA Method 524.2).

3. Parameters and method detection limits - EPA Method 601.

4. Practical quantitation limits.

5. Method accuracy and precision as functions of concentration - EPA Method 601.

6. Method detection limits for standard anions - EPA Method 300.0.

7. Single-operator accuracy and precision of standard anions - EPA Method 300.0.

8. Single-operator accuracy and precision of by-products - EPA Method 300.0.

9. Contract required quantitation limits for volatile organics.

10. Contract required quantitation limits for semivolatile organics. 
Section No.: Appendix A

Revision No.

Date:

Page: uly 1993 
Section No.:

Revision No.:

Date:

Page:

$\frac{\frac{0}{\text { July } 1993}}{A-3}$

Table A-1. Contract required detection limits for metals.

\begin{tabular}{crc}
\hline Metal & $\begin{array}{r}\text { Water } \\
(\mu \mathrm{g} / \mathrm{L})\end{array}$ & $\begin{array}{c}\text { Soils } \mathrm{s}^{\mathrm{a}} \\
\text { approximate }) \\
(\mu / \mathrm{Kg})\end{array}$ \\
\cline { 2 - 3 } $\mathrm{Al}$ & 200 & 40 \\
$\mathrm{Sb}$ & 60 & 12 \\
$\mathrm{As}$ & 10 & 2 \\
$\mathrm{Be}$ & 200 & 40 \\
$\mathrm{Cd}$ & 5 & 1 \\
$\mathrm{Ca}$ & 5 & 1 \\
$\mathrm{Cr}$ & 5000 & 1000 \\
$\mathrm{Cr}$ & 10 & 2 \\
$\mathrm{Co}$ & 2 & - \\
$\mathrm{Cu}$ & 50 & 10 \\
$\mathrm{Fe}$ & 25 & 5 \\
$\mathrm{~Pb}$ & 100 & 20 \\
$\mathrm{Mg}$ & 3 & 1 \\
$\mathrm{Mn}$ & 5000 & 1000 \\
$\mathrm{Hg}$ & 15 & 3 \\
$\mathrm{Ni}$ & 0.2 & 0.04 \\
$\mathrm{~K}$ & 2 & 8 \\
$\mathrm{Se}$ & 5000 & 1000 \\
$\mathrm{Ag}$ & 5 & 1 \\
$\mathrm{Na}$ & 10 & 2 \\
$\mathrm{Ti}$ & 5000 & 1000 \\
$\mathrm{Vn}$ & 10 & 2 \\
$\mathrm{Cn}$ & 50 & 10 \\
& 20 & 4 \\
\hline
\end{tabular}

a. Based on one gram of soil digested to $200 \mathrm{ml}$ of digestant. Actual detection limits for soils will vary with quantity of soil digested (1 to $1.5 \mathrm{~g}$ ) and soil moisture content. Based on CLP SOW (EPA, 1988 and 1990). 
Section No.:

Appendix A

Revision No.

Date: July 1993

Page: A.4

Table A-2. Method detection limits for volatile organics (EPA Method 524.2).

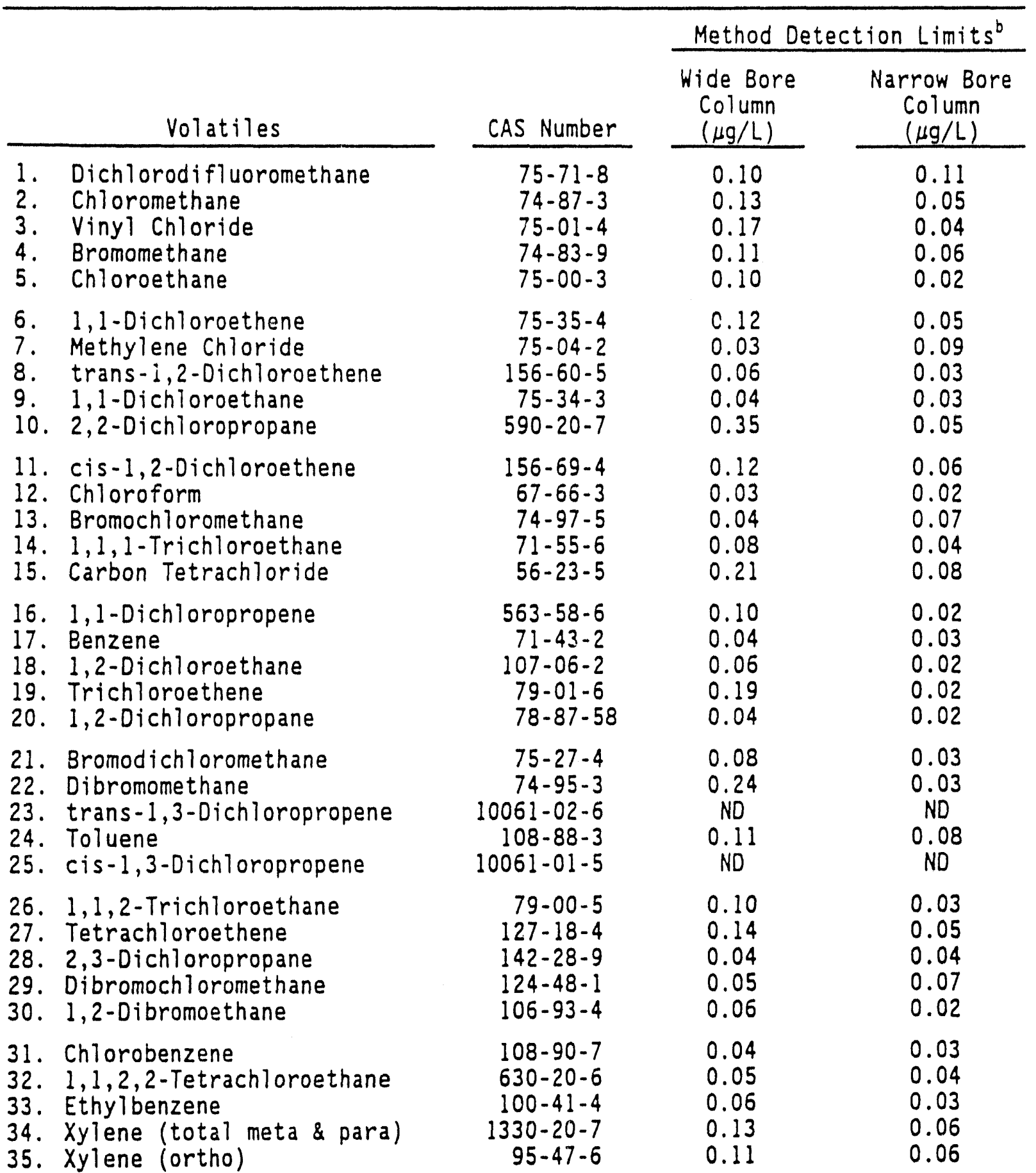




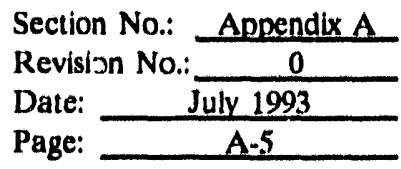

Table A-2. (continued).

\begin{tabular}{|c|c|c|c|}
\hline \multirow[b]{2}{*}{ Volatiles } & \multirow[b]{2}{*}{ CAS Numiver } & \multicolumn{2}{|c|}{ Method Detection Limits } \\
\hline & & $\begin{array}{l}\text { Wide Bore } \\
\text { Column } \\
(\mu \mathrm{g} / \mathrm{L}) \\
\end{array}$ & $\begin{array}{c}\text { Narrow Bore } \\
\text { Column } \\
(\mu \mathrm{g} / \mathrm{L})\end{array}$ \\
\hline $\begin{array}{l}\text { 36. Styrene } \\
\text { 37. Bromoform } \\
\text { 38. I sopropylbenzene } \\
\text { 39. 1,1,2,2-Tetrachloroethane } \\
\text { 40. Bromobenzene }\end{array}$ & $\begin{array}{r}100-42-5 \\
75-25-2 \\
98-82-8 \\
79-34-5 \\
108-86-1\end{array}$ & $\begin{array}{l}0.04 \\
0.12 \\
0.15 \\
0.04 \\
0.03\end{array}$ & $\begin{array}{l}0.06 \\
0.20 \\
0.10 \\
0.20 \\
0.11\end{array}$ \\
\hline $\begin{array}{l}\text { 41. 1,2,3-Trichloropropane } \\
\text { 42. n-Propylbenzene } \\
\text { 43. 2-Chlorotoluene } \\
\text { 44. 1, 3,5-Trimethylbenzene } \\
\text { 45. 4-Chlorotoluene }\end{array}$ & $\begin{array}{r}96-18-4 \\
103-65-1 \\
95-49-8 \\
108-67-8 \\
106-43-4\end{array}$ & $\begin{array}{l}0.32 \\
0.04 \\
0.04 \\
0.05 \\
0.06\end{array}$ & $\begin{array}{l}0.03 \\
0.06 \\
0.05 \\
0.02 \\
0.05\end{array}$ \\
\hline $\begin{array}{l}\text { 46. tert-Butylbenzene } \\
\text { 47. 1,2,4-Trimethylbenzene } \\
\text { 48. sec-Butylbenzene } \\
\text { 49. 1,3-Dichlorobenzene } \\
\text { 50. 1,4-Dichlorobenzene }\end{array}$ & $\begin{array}{r}98-06-6 \\
95-63-6 \\
135-98-8 \\
543-73-1 \\
106-46-7\end{array}$ & $\begin{array}{l}0.14 \\
0.13 \\
0.13 \\
0.12 \\
0.03\end{array}$ & $\begin{array}{l}0.33 \\
0.04 \\
0.12 \\
0.05 \\
0.04\end{array}$ \\
\hline $\begin{array}{l}\text { 51. n-Butylbenzene } \\
\text { 52. 1,2-Dichlorobenzene } \\
\text { 53. 1,2-Dibromo-3-chloropropane } \\
\text { 54. 1,2,4-Trichlorobenzene } \\
\text { 55. Hexachlorobutadiene }\end{array}$ & $\begin{array}{r}104-51-8 \\
95-50-1 \\
96-12-8 \\
120-82-1 \\
87-68-3\end{array}$ & $\begin{array}{l}0.11 \\
0.03 \\
0.26 \\
0.04 \\
0.11\end{array}$ & $\begin{array}{l}0.03 \\
0.05 \\
0.05 \\
0.20 \\
0.04\end{array}$ \\
\hline $\begin{array}{l}\text { 56. Naphthalene } \\
\text { 57. 1,2,3-Trichlorobenzene }\end{array}$ & $\begin{array}{l}91-20-3 \\
87-61-6\end{array}$ & $\begin{array}{l}0.04 \\
0.03\end{array}$ & $\begin{array}{l}0.04 \\
0.04\end{array}$ \\
\hline
\end{tabular}

a. Method detection limits are those published in the method and may not be achievable in all laboratories (see the Introduction to this section).

b. Method 524.2 s applicable to water samples only. The Method Detection Limits are listes for wide bore and narrow bore capillary columns. A wide bore capillary cilumn is defined as having an internal diameter of greater than $0.32 \mathrm{~mm}$. The data for the narrow bore column was obtained using the cryogenic trapping option in the method.

ND $=$ Not Determined for this compound. Use the laboratory determined MDL for reporting. 


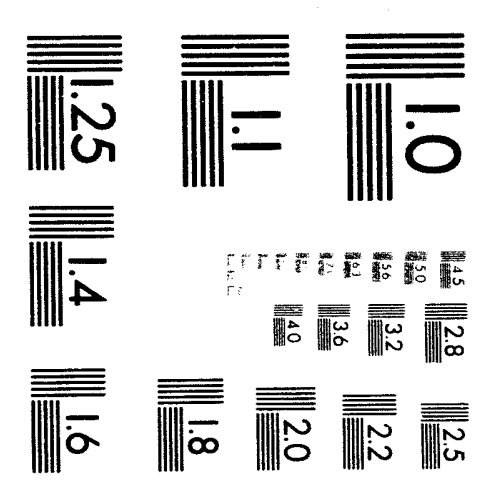



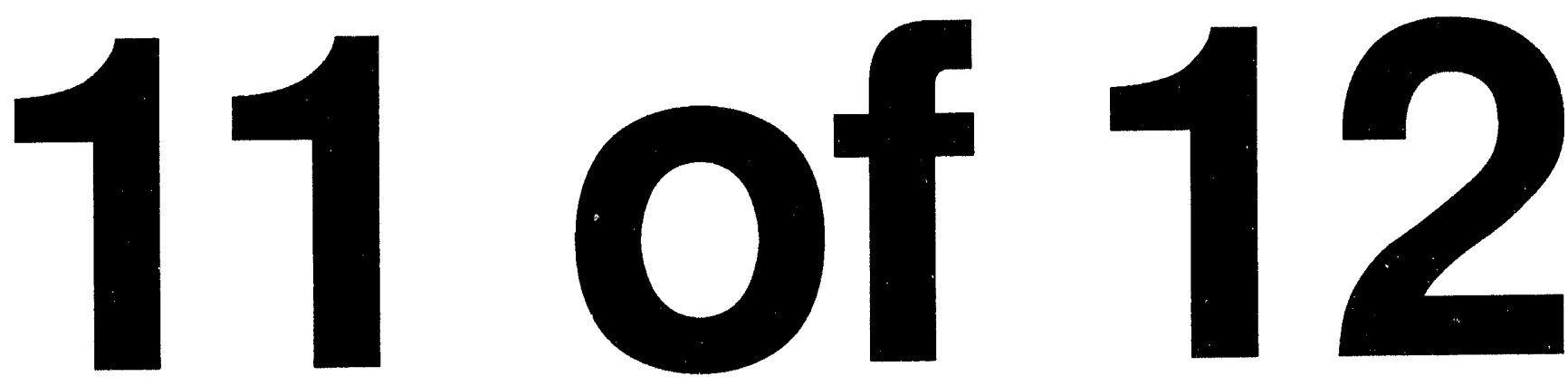
Section No.: Appendix A

Revision No.:

Date:

Page:

July 1993 A-6

Table A-3. Parameters and method detection limits - EPA Method 601.

\begin{tabular}{|c|c|c|c|}
\hline \multirow{2}{*}{ Parameter } & \multicolumn{2}{|c|}{ Retenuon ume (min) } & \multirow{2}{*}{$\begin{array}{l}\text { Method detecion } \\
\operatorname{limil}(\mu g / L)\end{array}$} \\
\hline & Column 1 & Column 2 & \\
\hline 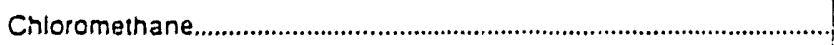 & 1.50 & 5.28 & 0.08 \\
\hline 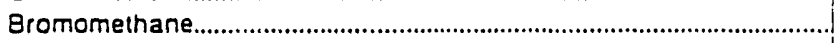 & 2.17 & 7.05 & 1.18 \\
\hline Dichlorodifluoromethane & 2.62 & nd & 1.81 \\
\hline Vinyl chloride & 2.67 & 5.28 & 0.18 \\
\hline 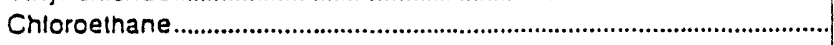 & 3.33 & 8.68 & 0.52 \\
\hline 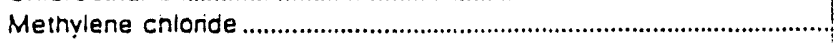 & 5.25 & 10.1 & 0.25 \\
\hline 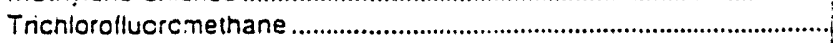 & 7.18 & nd & nd \\
\hline 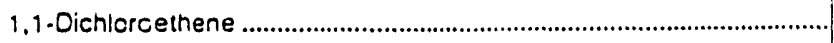 & 7.93 & 7.72 & 0.13 \\
\hline 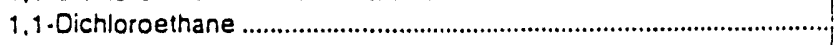 & 9.30 & 12.5 & 0.07 \\
\hline trans-1,2-Dichloroethene & 10.1 & 9.38 & 0.10 \\
\hline 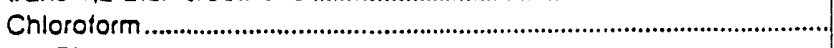 & 10.7 & 12.1 & 0.05 \\
\hline 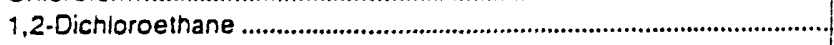 & 11.4 & 15.4 & 0.03 \\
\hline 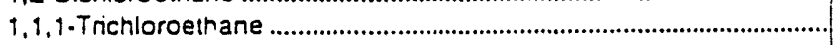 & 12.6 & 13.1 & 0.03 \\
\hline 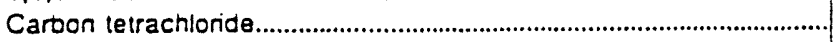 & 13.0 & 14.4 & 0.12 \\
\hline 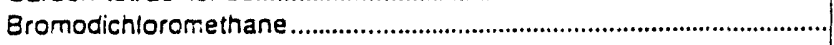 & 13.7 & 14.6 & 0.10 \\
\hline 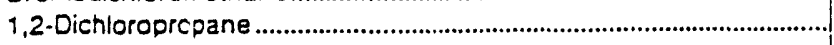 & 14.9 & 16.6 & 0.04 \\
\hline 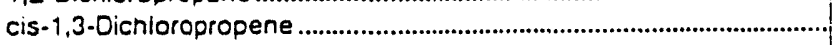 & 15.2 & 16.6 & 0.34 \\
\hline Trichloroethene & 15.8 & 13.1 & 0.12 \\
\hline 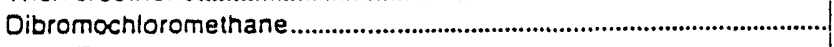 & 16.5 & 16.6 & 0.09 \\
\hline 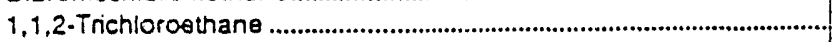 & 16.5 & 18.1 & 0.02 \\
\hline 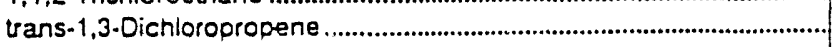 & 16.5 & 18.0 & 0.20 \\
\hline 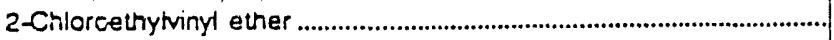 & 18.0 & nd & 0.13 \\
\hline Eromotor 1 . & 19.2 & 19.2 & 0.20 \\
\hline 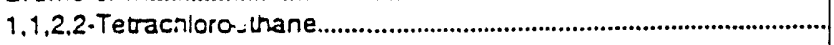 & 21.6 & nd & 0.03 \\
\hline 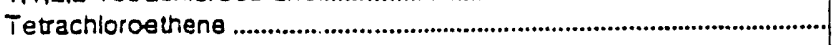 & 21.7 & 15.0 & 0.03 \\
\hline 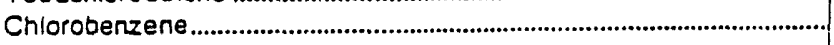 & 24.2 & 18.8 & 0.25 \\
\hline 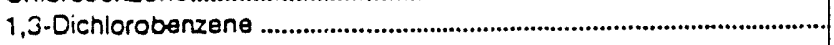 & 34.0 & 224 & 0.32 \\
\hline 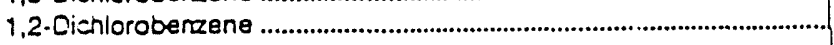 & 34.9 & 23.5 & 0.15 \\
\hline 1,4-Dichlorobenzene & 35.4 & 22.3 & 0.24 \\
\hline
\end{tabular}

Column I conditions: Carbopack 8 (60/80 mesh) coated with $1 \%$ SP-1C00 packed in an 8 H $\times 0.1$ in. ID stainless steel or glass column with helium camer gas at $40 \mathrm{~mL} / \mathrm{min}$ flow rate. Column temperature held at $45{ }^{\circ} \mathrm{C}$ lor $3 \mathrm{~min}$ then frogrammed at $8^{\circ} \mathrm{C} / \mathrm{min}$ to $220^{\circ} \mathrm{C}$ and held for $15 \mathrm{~min}$.

Column 2 conditions: Porisil-C (100/120 mesh) coated with n-octane packed in a $6 \mathrm{H} \times 0.1$ in. 10 stainless steel or glass column with helium camier gas at $40 \mathrm{~mL} / \mathrm{min}$ flow rate. Column temperature held at $50^{\circ} \mathrm{C}$ lor $3 \mathrm{~min}$ then programmed at $6^{\circ} \mathrm{C} /$ min $10170^{\circ} \mathrm{C}$ and held for $4 \mathrm{~min}$.

nd = not detemined.

Note: Acetone, benzene, and methane will be allalyzed according to EPA Method 601 using a flame ionization detector. The method detection limit for these three constiluents is $1.0 \mu \mathrm{g} / \mathrm{L}$. 
Section No::

Revision No:

Date:

Page: July 1993

Table A-4. Practical quantitation limits.

\begin{tabular}{|c|c|}
\hline Maci-ix & Factor ${ }^{b}$ \\
\hline $\begin{array}{l}\text { Groundwater } \\
\text { Low-level soil } \\
\text { Water miscible liquid waste } \\
\text { High-level soil and sludge } \\
\text { Non-water miscible waste }\end{array}$ & $\begin{array}{r}10 \\
10 \\
500 \\
1250 \\
1250\end{array}$ \\
\hline \multicolumn{2}{|c|}{$\begin{array}{l}\text { a. Sample PQLs are highly matrix-dependent. The PQLS listed herein are } \\
\text { provided for guidance and may not always be achievable. } \\
\text { b. PQL }=\text { Method detection iimit (Table A-1) X Factor (Table A-?). For } \\
\text { nonaqueous samples, the factor is on a wet-weight basis. }\end{array}$} \\
\hline
\end{tabular}


Section No.: Appendix A

Revision No:

Date: July 1993

Page: A-8

Table A-5. Method accuracy and precision as functions of concentration - EPA Method 601.

\begin{tabular}{|c|c|c|c|}
\hline Parameter & $\begin{array}{l}\text { Accuracy, as } \\
\text { recovery, } x^{\prime}(\mu g)\end{array}$ & $\begin{array}{c}\text { Single analyst } \\
\text { precision. si } \\
(\mu g / L)\end{array}$ & $\begin{array}{c}\text { Overall precision, } \\
S^{\prime}(\mu g / L)\end{array}$ \\
\hline 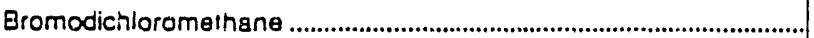 & $1.12 \mathrm{C}-1.02$ & $0.11 \bar{x}+0.04$ & $0.20 \bar{x}+1.00$ \\
\hline Bromolorm & $0.56 C-2.05$ & $0.12 \bar{x}+0.58$ & $0.21 \bar{x}+2.41$ \\
\hline Bromomathane & $0.76 C-1.27$ & $0.28 \bar{x}+0.27$ & $0.36 \bar{X}+0.94$ \\
\hline 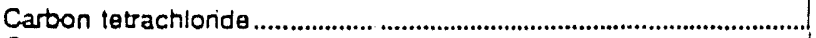 & $0.98 C-1.04$ & $0.15 \ddot{x} \div 0.38$ & $0.20 \bar{x}+0.39$ \\
\hline 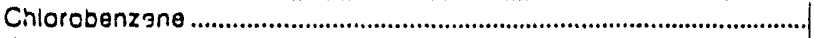 & $1.000-1.23$ & $0.15 \bar{x}-0.02$ & $0.18 \bar{x} \div 1.21$ \\
\hline Choroethane & $0.99 C-1.53$ & $0.14 \bar{x}-0.13$ & $0.17 \bar{x}+0.63$ \\
\hline 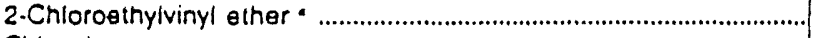 & 1.000 & $0.20 \bar{x}$ & $0.35 \bar{x}$ \\
\hline Chlorotom & $0.93 c-0.39$ & $0.13 \bar{x} \div 0.15$ & $0.19 \bar{x}-0.02$ \\
\hline 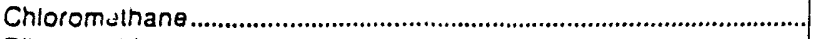 & $0.77 C \div 0.18$ & $0.28 \bar{x}-0.31$ & $0.52 \bar{x}+1.31$ \\
\hline Dibromochloromethane & $0.94 \mathrm{C}+2.72$ & $0.11 \bar{x}+1.10$ & $0.24 \bar{X} \div 1.68$ \\
\hline 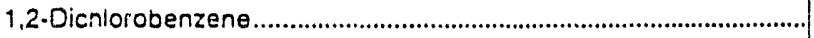 & $0.93 C \div 1.70$ & $0.20 \bar{x}+0.97$ & $0.13 \bar{x} \div 6.13$ \\
\hline 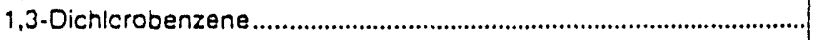 & $0.95 \mathrm{C} \div 0.43$ & $0.14 \bar{x}+2.33$ & $0.2 \epsilon \bar{X}+2.34$ \\
\hline 1.4-Dichlorobenzene & $0.93 C-0.09$ & $0.15 \bar{x}-0.29$ & $0.20 \bar{x}+0.41$ \\
\hline 1,1-Dichloroethane & $0.95 C-1.08$ & $0.09 \bar{x}+0.17$ & $0.14 \bar{X}+0.94$ \\
\hline 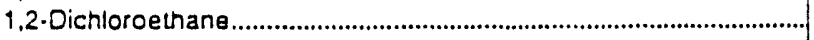 & $1.04 C-1.06$ & $0.11 \bar{x}+0.70$ & $0.15 \bar{x}+0.94$ \\
\hline 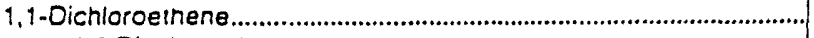 & $0.98 C-0.87$ & $0.21 \bar{x}-0.23$ & $0.29 \bar{x}-0.40$ \\
\hline trans-1,2-Dichloroethene & $0.97 C-0.16$ & $0.11 \bar{x} \div 1.46$ & $0.17 \bar{x}+1.46$ \\
\hline 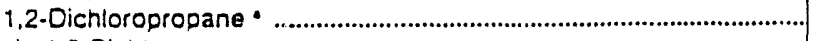 & $1.00 \mathrm{C}$ & $0.13 \bar{x}$ & $0.23 \bar{x}$ \\
\hline 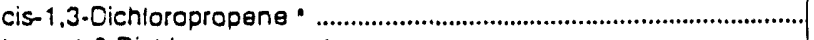 & $1.00 \mathrm{C}$ & $0.18 \bar{x}$ & $0.32 \bar{x}$ \\
\hline trans $-1,3$-Dichloropropene ${ }^{\circ}$.................. & $1.00 \mathrm{C}$ & $0.18 \bar{x}$ & $0.32 \bar{x}$ \\
\hline Methylene chionde................... & $0.91 C-0.93$ & $0.11 \bar{x} \div 0.33$ & $0.21 \bar{x}+1.43$ \\
\hline 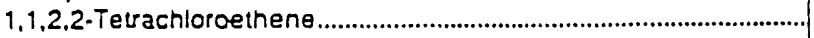 & $0.950 \div 0.19$ & $0.14 \bar{X}+2.41$ & $0.23 \bar{x}+2.73$ \\
\hline 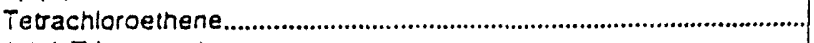 & $0.94 C \div 0.06$ & $0.14 \bar{x}+0.38$ & $0.18 \bar{x}+2.21$ \\
\hline 1,1,1-Trichloroethane............... & $0.90 \mathrm{C}-0.16$ & $0.15 \bar{x} \div 0.04$ & $0.20 \bar{x}+0.37$ \\
\hline 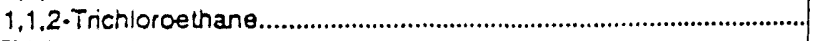 & $0.86 C \div 0.30$ & $0.13 \bar{x}-0.14$ & $0.19 \bar{x}+0.67$ \\
\hline 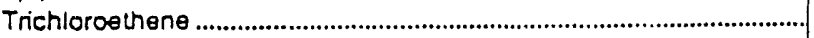 & $0.87 C \div 0.48$ & $0.13 \bar{x}-0.03$ & $0.23 \bar{x}+0.30$ \\
\hline 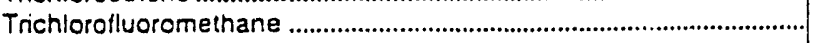 & $0.89 \mathrm{C}-0.07$ & $0.15 \bar{x}+0.67$ & $0.26 \bar{x} \div 0.91$ \\
\hline 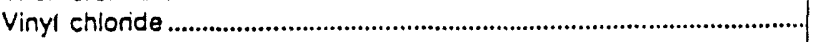 & $0.97 C-0.36$ & $0.13 \bar{x}+0.65$ & $0.27 \bar{X} \div 0.40$ \\
\hline
\end{tabular}

$\bar{X}^{\prime}=$ Expecied recovery for one or mort measurements of a sample containing a concentration of $\mathrm{C}$, in $\mu \mathrm{g} / \mathrm{L}$

$\mathrm{s}^{\prime}=$ =xpected single analyst standara deviation of measurements at an average concentration lound of $\bar{x}$, in $\mu \mathrm{g} / \mathrm{L}$ $S_{S}:=$ Expected intertaboratory stancard devation of measurements at an average concentracon lound of $\bar{X}$, in $\mu g / L$ $C=$ True value for the concentration, in $\mu \mathrm{g} / \mathrm{L}$

$\bar{x}=$ Average recovery found for measurements of samples containing a concentration of $\mathrm{C}$, in $\mu \mathrm{g} / \mathrm{L}$

- Esomales based upon the pertormance in a single laboralory. ${ }^{10}$ 
Table A-6. Method detection limits for standard anions - EPA Method 300.0.

\begin{tabular}{lccc}
\multicolumn{1}{c}{ Analyte } & Peak \# & $\begin{array}{c}\text { Retention time } \\
\text { (minutes) }\end{array}$ & $\begin{array}{c}\mathrm{MDL} \\
(\mathrm{mg} / \mathrm{L})\end{array}$ \\
\hline & & & \\
Chloride & 2 & 1.7 & 0.02 \\
Nitrate-N & 3 & 2.0 & 0.004 \\
Bromide & 4 & 2.9 & 0.01 \\
Nitrate-N & 5 & 3.1 & 0.002 \\
o-Phosphate-P & 6 & 5.3 & 0.003 \\
Sulfate & 7 & 6.8 & 0.02
\end{tabular}

Standard conditions:

Columns: as specified in 6.2.2.1

Detector: as specified in 6.2.4

Eluent: as specified in 7.3.1

Pump rate: $2.0 \mathrm{~mL} / \mathrm{min}$.

Sample loop: $50 \mu \mathrm{L}$

\begin{tabular}{lccc}
\multicolumn{1}{c}{ Analyte } & Peak \# & $\begin{array}{c}\text { Retention time } \\
\text { (minutes) }\end{array}$ & $\begin{array}{r}\text { MDL } \\
(\mathrm{mg} / \mathrm{L})\end{array}$ \\
\hline & & & \\
Chloride & 1 & 2.8 & 0.01 \\
Bromate & 2 & 3.2 & 0.02 \\
Chlorate & 3 & 7.1 & 0.003
\end{tabular}

Standard conditions:

Columns: as specified in 6.2.2.2

Detector: as specified in 6.2.4

Eluent: as specified in 7.3.2

Pump rate: $1.0 \mathrm{~mL} / \mathrm{min}$.

Sample loop: $50 \mu \mathrm{L}$

Attenuation: 1

Y-axis: $\mathbf{5 0 0 ~ n s}$

a. The MDL is calculated from data system using a y-axis selection of $1000 \mathrm{~ns}$ and with a stripchart recorder with an attenuator setting of 1 . 
Section No.: Appendix A

Revision No:

Date:

Page: July 1993 A-10

Table A-7. Single-operator accuracy and precision of standard anions - EPA Method 300.0.

\begin{tabular}{|c|c|c|c|c|c|}
\hline Analyte & $\begin{array}{c}\text { Sample } \\
\text { Type }\end{array}$ & $\begin{array}{l}\text { Known } \\
\text { Conc. } \\
\text { (ma/L) }\end{array}$ & $\begin{array}{l}\text { Number } \\
\text { of } \\
\text { Replicates } \\
\end{array}$ & $\begin{array}{c}\text { Mean } \\
\text { Recovery } \\
\%\end{array}$ & $\begin{array}{l}\text { Standard } \\
\text { Deviation } \\
\text { (ma/l) }\end{array}$ \\
\hline Bromide & $\begin{array}{l}\text { RW } \\
\text { DH! } \\
\text { SW } \\
W W \\
\text { GW } \\
\text { SD }\end{array}$ & $\begin{array}{l}5.0 \\
5.0 \\
5.0 \\
5.0 \\
5.0 \\
2.0\end{array}$ & $\begin{array}{l}7 \\
7 \\
7 \\
7 \\
7 \\
7\end{array}$ & $\begin{array}{r}99 \\
105 \\
95 \\
105 \\
92 \\
82\end{array}$ & $\begin{array}{l}0.08 \\
0.10 \\
0.13 \\
0.34 \\
0.34 \\
0.06\end{array}$ \\
\hline Chloride & $\begin{array}{l}R W \\
\text { OW } \\
\text { SW } \\
W W \\
\text { GW } \\
\text { SD }\end{array}$ & $\begin{array}{l}20.0 \\
20.0 \\
10.0 \\
20.0 \\
20.0 \\
20.0\end{array}$ & $\begin{array}{l}7 \\
7 \\
7 \\
7 \\
7 \\
7\end{array}$ & $\begin{array}{r}96 \\
108 \\
86 \\
101 \\
114 \\
90\end{array}$ & $\begin{array}{l}0.35 \\
1.19 \\
0.33 \\
5.2 \\
1.3 \\
0.32\end{array}$ \\
\hline Nitrate- N & $\begin{array}{l}R W \\
\text { DW } \\
\text { SW } \\
W W \\
\text { GW } \\
\text { SO }\end{array}$ & $\begin{array}{l}10.0 \\
10.0 \\
10.0 \\
10.0 \\
10.0 \\
10.0\end{array}$ & $\begin{array}{l}7 \\
7 \\
7 \\
7 \\
7 \\
7\end{array}$ & $\begin{array}{r}103 \\
104 \\
93 \\
101 \\
97 \\
82\end{array}$ & $\begin{array}{l}0.21 \\
0.27 \\
0.17 \\
0.82 \\
0.47 \\
0.28\end{array}$ \\
\hline Nitrite- N & $\begin{array}{l}R W \\
\text { DW } \\
\text { SW } \\
W W \\
\text { GW } \\
\text { SD }\end{array}$ & $\begin{array}{r}10.0 \\
10.0 \\
5.0 \\
5.0 \\
10.0 \\
2.0\end{array}$ & $\begin{array}{l}7 \\
7 \\
7 \\
7 \\
7 \\
7\end{array}$ & $\begin{array}{r}97 \\
121 \\
92 \\
91 \\
96 \\
98\end{array}$ & $\begin{array}{l}0.14 \\
0.25 \\
0.14 \\
0.50 \\
0.35 \\
0.08\end{array}$ \\
\hline o-Phosphate- $P$ & $\begin{array}{l}R W \\
\text { DW } \\
\text { SW } \\
W W \\
\text { GW }\end{array}$ & $\begin{array}{l}10.0 \\
10.0 \\
10.0 \\
10.0 \\
10.0\end{array}$ & $\begin{array}{l}7 \\
7 \\
7 \\
7 \\
7\end{array}$ & $\begin{array}{r}99 \\
99 \\
98 \\
106 \\
95\end{array}$ & $\begin{array}{l}0.17 \\
0.26 \\
0.22 \\
0.85 \\
0.33\end{array}$ \\
\hline Sulfate & $\begin{array}{l}R W \\
\text { DW } \\
\text { SW } \\
W W \\
\text { GW }\end{array}$ & $\begin{array}{l}20.0 \\
50.0 \\
40.0 \\
40.0 \\
40.0\end{array}$ & $\begin{array}{l}7 \\
7 \\
7 \\
7 \\
7\end{array}$ & $\begin{array}{r}99 \\
105 \\
95 \\
102 \\
112\end{array}$ & $\begin{array}{l}0.40 \\
3.35 \\
1.7 \\
6.4 \\
3.2\end{array}$ \\
\hline \multicolumn{2}{|c|}{$\begin{array}{l}R W=\text { Reagent Water } \\
D W=\text { Orinking Water } \\
\text { SW }=\text { Surface Water }\end{array}$} & \multicolumn{4}{|c|}{$\begin{array}{l}W W=\text { Mixed Domestic and Inoustrial Wastewater } \\
G W=\text { Groundwater } \\
\text { SD }=\text { USEPA QC Solid (shale) }\end{array}$} \\
\hline
\end{tabular}


Section No.: Appendix A

Revision No.:

Date:

Page: A-11

Table A-8. Single-operator accuracy and precision of by-products - EPA Method 300.0.

\begin{tabular}{|c|c|c|c|c|c|}
\hline Analyte & $\begin{array}{c}\text { Sample } \\
\text { Type }\end{array}$ & $\begin{array}{r}\text { Spike } \\
(\mathrm{mg} / \mathrm{L})\end{array}$ & $\begin{array}{c}\text { Number } \\
\text { of } \\
\text { Replicates }\end{array}$ & $\begin{array}{c}\text { Mean } \\
\text { Recovery } \\
\%\end{array}$ & $\begin{array}{l}\text { Standard } \\
\text { Deviation } \\
(\mathrm{mg} / \mathrm{L})\end{array}$ \\
\hline Bromate & $\mathrm{RW}$ & $\begin{array}{l}5.0 \\
1.0 \\
0.1 \\
0.05\end{array}$ & $\begin{array}{l}7 \\
7 \\
7 \\
7\end{array}$ & $\begin{array}{r}103 \\
98 \\
155 \\
122\end{array}$ & $\begin{array}{l}0.07 \\
0.04 \\
0.005 \\
0.01\end{array}$ \\
\hline : & DW & $\begin{array}{l}5.0 \\
1.0 \\
0.1 \\
0.05\end{array}$ & $\begin{array}{l}7 \\
7 \\
7 \\
7\end{array}$ & $\begin{array}{l}95 \\
85 \\
98 \\
98\end{array}$ & $\begin{array}{l}0.04 \\
C .02 \\
0.005 \\
0.005\end{array}$ \\
\hline \multirow[t]{2}{*}{ Chlorate } & $\mathrm{RW}$ & $\begin{array}{l}5.0 \\
1.0 \\
0.1 \\
0.05\end{array}$ & $\begin{array}{l}7 \\
7 \\
7 \\
7\end{array}$ & $\begin{array}{r}101 \\
97 \\
100 \\
119\end{array}$ & $\begin{array}{l}0.06 \\
0.01 \\
0.01 \\
0.05\end{array}$ \\
\hline & DW & $\begin{array}{l}5.0 \\
1.0 \\
0.1 \\
0.05\end{array}$ & $\begin{array}{l}7 \\
7 \\
7 \\
7\end{array}$ & $\begin{array}{l}101 \\
115 \\
121 \\
110\end{array}$ & $\begin{array}{l}0.04 \\
0.01 \\
0.005 \\
0.01\end{array}$ \\
\hline \multirow[t]{2}{*}{ Chiorite } & $\mathrm{RW}$ & $\begin{array}{l}5.0 \\
1.0 \\
0.1 \\
0.05\end{array}$ & $\begin{array}{l}7 \\
7 \\
7 \\
7\end{array}$ & $\begin{array}{r}100 \\
98 \\
86 \\
94\end{array}$ & $\begin{array}{l}0.04 \\
0.01 \\
0.01 \\
0.01\end{array}$ \\
\hline & DW & $\begin{array}{l}5.0 \\
1.0 \\
0.1 \\
0.05\end{array}$ & $\begin{array}{l}7 \\
7 \\
7 \\
7\end{array}$ & $\begin{array}{r}96 \\
100 \\
76 \\
96\end{array}$ & $\begin{array}{l}0.03 \\
0.02 \\
0.00 \\
0.01\end{array}$ \\
\hline
\end{tabular}




Section No:: $\frac{\text { Appendix A }}{0}$
Revision No.: $\frac{0}{\text { July } 1993}$
Date:
Page:

Table A-9. Contract required quantitation limits for volatile organics ${ }^{\mathrm{a}}$.

\begin{tabular}{|c|c|c|c|}
\hline \multirow[b]{2}{*}{ Volatiles } & \multirow[b]{2}{*}{ CAS number } & \multicolumn{2}{|c|}{ Quantitation Limits ${ }^{b}$} \\
\hline & & $\begin{array}{l}\text { Water } \\
\text { (ug/L) }\end{array}$ & $\begin{array}{l}\text { Low soil/sediment } \\
\qquad(\mathrm{ug} / \mathrm{Kg})\end{array}$ \\
\hline 1. Chloromethane & $74-87-3$ & 10 & 10 \\
\hline 2. Bromomethane & $74-83-9$ & 10 & 10 \\
\hline 3. Vinyl Chloride & $75-01-4$ & 10 & 10 \\
\hline 4. Chloroethane & $75-00-3$ & 10 & 10 \\
\hline 5. Methylene Chloride & $75-09-2$ & 5 & 5 \\
\hline 6. Acetone & $67-64-1$ & 10 & 10 \\
\hline 7. Carbon Disulfide & $75-15-0$ & 5 & 5 \\
\hline 8. 1,1-Dichloroethene & $75-35-4$ & 5 & 5 \\
\hline 9. 1,1-Dichloroethane & $75-34-3$ & 5 & 5 \\
\hline 10. 1, 2-Dichloroethene (total) & $540-39-0$ & 5 & 5 \\
\hline 11. Chloroform & $67-66-3$ & 5 & 5 \\
\hline 12. 1, 2-Dichloroethane & $107-06-2$ & 5 & 5 \\
\hline 13. 2-Butanone & $78-93-3$ & 10 & 10 \\
\hline 14. 1,1,1-Trichloroethane & $71-55-6$ & 5 & 5 \\
\hline 15. Carbon Tetrachloride & $56-23-5$ & 5 & 5 \\
\hline 16. Vinyl Acetate & $108-05-4$ & 10 & 10 \\
\hline 17. Bromodichloromethane & $75-27-4$ & 5 & 5 \\
\hline 18. 1, 2-Dichloropropane & $78-87-5$ & 5 & 5 \\
\hline 19. cis-1, 3-Dichloropropene & $10061-01-5$ & 5 & 5 \\
\hline 20. Trichloroethene & $79-01-6$ & 5 & 5 \\
\hline 21. Dibromochloromethane & $124-48-1$ & 5 & 5 \\
\hline 22. 1, 1, 2-Trichloroethane & $79-00-5$ & 5 & 5 \\
\hline 23. Benzene & $71-4.3-2$ & 5 & 5 \\
\hline 24. trans-1, 3-Dichloropropene & $10061-02-6$ & 5 & 5 \\
\hline 25. Bromoform & $75-25-2$ & 5 & 5 \\
\hline
\end{tabular}




Section No.: $\frac{\text { Appendix A }}{0}$
Revision No.: $\frac{0}{\text { July } 1993}$
Date: $\frac{A-13}{\text { Page: }}$

Table A-9. (continued).

\begin{tabular}{|c|c|c|c|}
\hline \multirow[b]{2}{*}{ Volatiles } & \multirow[b]{2}{*}{ CAS number } & \multicolumn{2}{|c|}{ Quantitation Limits ${ }^{b}$} \\
\hline & & $\begin{array}{l}\text { Water } \\
\text { (ug/L) }\end{array}$ & $\begin{array}{l}\text { Low soil/sediment }{ }^{\mathrm{c}} \\
\quad(\mathrm{ug} / \mathrm{Kg})\end{array}$ \\
\hline 26. 4-Methyl-2-pentanone & $108-10-1$ & 10 & 10 \\
\hline 27. 2-Hexanone & $591-78-6$ & 10 & 10 \\
\hline 28. Tetrachloroethene & $127-18-4$ & 5 & 5 \\
\hline 29. Toluene & $108-88-3$ & 5 & 5 \\
\hline 30. 1, 1, 2, 2-Tetrachloroethene & $79-34-5$ & 5 & 5 \\
\hline 31. Chlorobenzene & $108-90-7$ & 5 & 5 \\
\hline 32. Ethyl Benzene & $100-41-4$ & 5 & 5 \\
\hline 33. Styrene & $100-42-5$ & 5 & 5 \\
\hline 34. Xylenes (Total) & $1330-20-7$ & 5 & 5 \\
\hline \multicolumn{4}{|c|}{$\begin{array}{l}\text { a. Specific quantitation limits are highly matrix dependent. The quantitation limits listed } \\
\text { herein are provided for guidance and may not always be achievable. }\end{array}$} \\
\hline \multicolumn{4}{|c|}{$\begin{array}{l}\text { b. Quantitation limits listed for soil/sediment are based on net weight. The quantitation limits } \\
\text { calculated by the laboratory for soil/sediment, calculated on dry weight basis as required by the } \\
\text { contract, will be higher. }\end{array}$} \\
\hline $\begin{array}{l}\text { c. Medium Soil/Sediment Con } \\
\text { Compounds are } 125 \text { times the in }\end{array}$ & $\begin{array}{l}\text { Required Quar } \\
\text { al Low Soil/Se }\end{array}$ & $\begin{array}{l}\text { Limits } \\
\text { CRQL. }\end{array}$ & for Volatile TCL \\
\hline
\end{tabular}




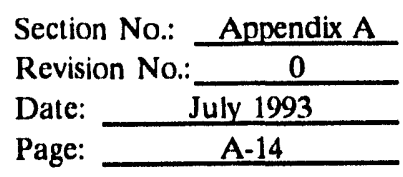

Table A-10. Contract required quantitation limits for semivolatile organics.

\begin{tabular}{|c|c|c|c|c|}
\hline & & & Qua & n Limits ${ }^{a}$ \\
\hline & & & Water & $\begin{array}{c}\text { Low } \\
\text { Soil/Sediment }^{b}\end{array}$ \\
\hline & Semivolatiles & CAS Number & $\mathrm{ug} / \mathrm{L}$ & $\mathrm{ug} / \mathrm{Kg}$ \\
\hline 1. & Phenol & $108-95-2$ & 10 & 330 \\
\hline 2. & bis(2-Chloroethyl) ether & $111-44-4$ & 10 & 330 \\
\hline 3. & 2-Chlorophenol & $95-57-8$ & 10 & 330 \\
\hline 4. & 1,3-Dichlorobenzene & $541-73-1$ & 10 & 330 \\
\hline 5. & 1,4-Dichlorobenzene & $106-46-7$ & 10 & 330 \\
\hline 6. & Benzyl alcohol & $100-51-6$ & 10 & 330 \\
\hline 7. & 1,2-Dichlorobenzene & $95-50-1$ & 10 & 330 \\
\hline 8. & 2-Methylphenol & $95-48-7$ & 10 & 330 \\
\hline 9. & bis(2-Chloroisopropyl) ether & $108-60-1$ & 10 & 330 \\
\hline 10. & 4-Methylphenol & $106-44-5$ & 10 & 330 \\
\hline 11. & N-Nitroso-di-n-dipropylamine & $621-64-7$ & 10 & 330 \\
\hline 12. & Hexachloroethane & $67-72-1$ & 10 & 330 \\
\hline 13. & Nitrobenzene & $98-95-3$ & 10 & 330 \\
\hline 14. & Isophorone & $78-59-1$ & 10 & 330 \\
\hline 15. & 2-Nitrophenol & $88-75-5$ & 10 & 330 \\
\hline 16. & 2,4-Dimethylphenol & $105-67-9$ & 10 & 330 \\
\hline 17. & Benzoic acid & $65-85-0$ & 50 & 1600 \\
\hline 18. & bis(2-Chloroethoxy) methane & $111-91-1$ & 10 & 330 \\
\hline 19. & 2,4-Dichlorophenol & $120-83-2$ & 10 & 330 \\
\hline 20. & 1,2,4-Trichlorobenzene & $120-82-1$ & 10 & 330 \\
\hline 21. & Naphthalene & $91-20-3$ & 10 & 330 \\
\hline 22. & 4-Chloroaniline & $106-47-8$ & 10 & 330 \\
\hline 23. & Hexachlorobutadiene & $87-68-3$ & 10 & 330 \\
\hline 24. & $\begin{array}{l}\text { 4-Chloro-3-methylphenol } \\
\text { (para-chloro-meta-cresol) }\end{array}$ & $59-50-7$ & 10 & 330 \\
\hline
\end{tabular}


Table A-10. (continued).

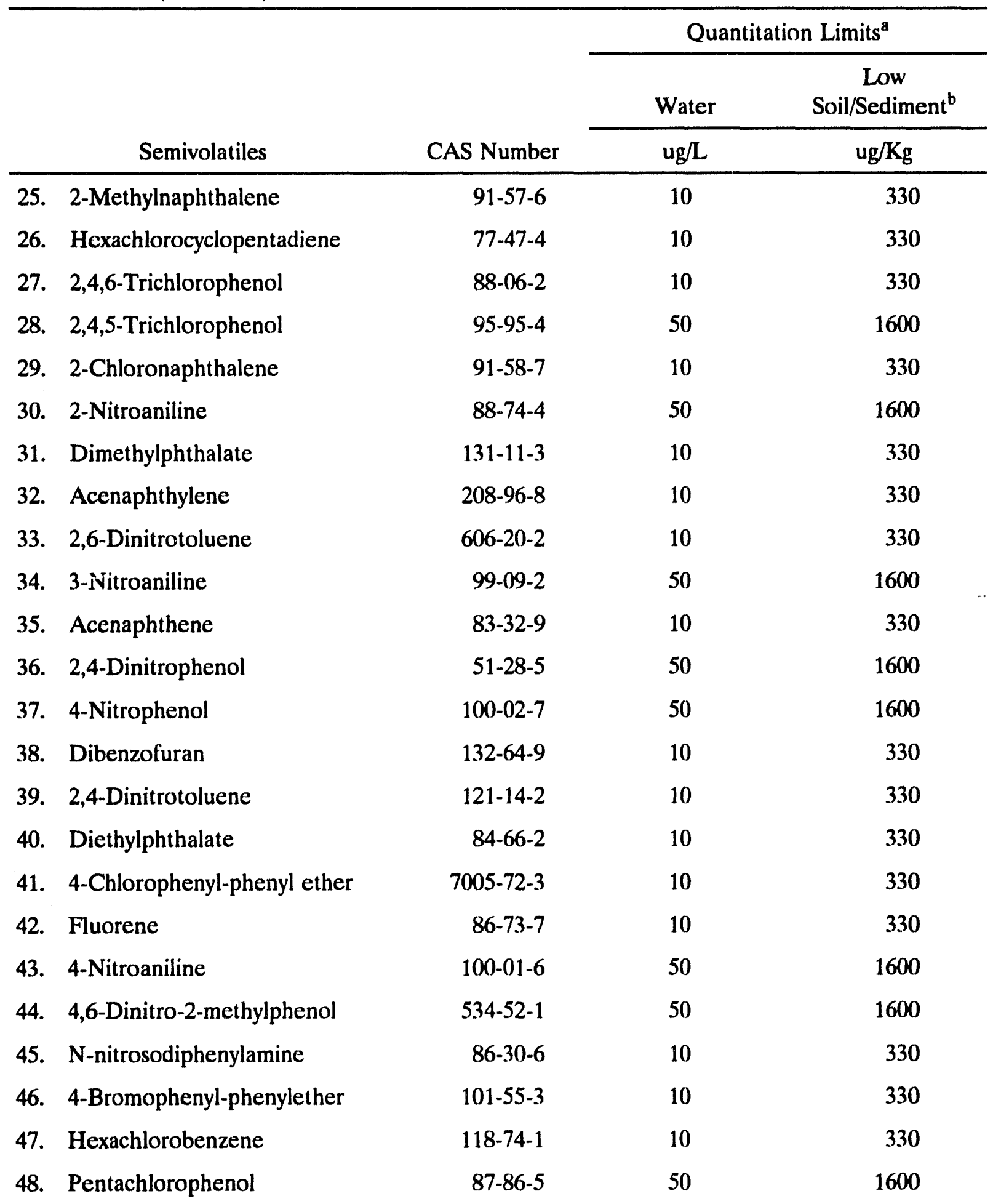




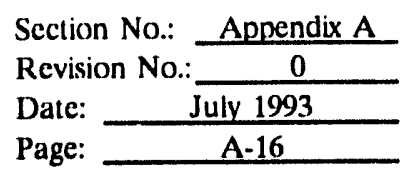

Table A-10. (continued).

\begin{tabular}{|c|c|c|c|c|}
\hline & & & Qua & Limits ${ }^{a}$ \\
\hline & & & Water & $\begin{array}{c}\text { Low } \\
\text { Soil/Sediment }^{b}\end{array}$ \\
\hline & Semivolatiles & CAS Number & $\mathrm{ug} / \mathrm{L}$ & $\mathrm{ug} / \mathrm{Kg}$ \\
\hline 49. & Phenanthrene & $85-01-8$ & 10 & 330 \\
\hline 50. & Anthracene & $120-12-7$ & 10 & 330 \\
\hline 51. & Di-n-butylphthalate & $84-74-2$ & 10 & 330 \\
\hline 52. & Fluoranthene & $206-44-0$ & 10 & 330 \\
\hline 53. & Pyrene & $129-00-0$ & 10 & 330 \\
\hline 54. & Butylbenzylphthalate & $85-68-7$ & 10 & 330 \\
\hline 55. & 3,3'-Dichlorobenzidine & $91-94-1$ & 20 & 660 \\
\hline 56. & Benzo(a) anthracene & $56-55-3$ & 10 & 330 \\
\hline 57. & Chrysene & $218-01-9$ & 10 & 330 \\
\hline 58. & bis(2-Ethylhexyl)phthalate & $117-81-7$ & 10 & 330 \\
\hline 59. & Di-n-octylphthalate & $117-84-0$ & 10 & 330 \\
\hline 60. & Benzo(b)fluoranthene & $205-99-2$ & 10 & 330 \\
\hline 61. & Benzo(k)fluoranthene & $207-08-9$ & 10 & 330 \\
\hline 62. & Benzo(a)pyrene & $50-32-8$ & 10 & 330 \\
\hline 63. & Indeno(1,2,3-cd)pyrene & $193-39-5$ & 10 & 330 \\
\hline 64. & Dibenz(a,h)anthracene & $53-70-3$ & 10 & 330 \\
\hline 65. & Benzo(g,h,i)perylene & $191-24-2$ & 10 & 330 \\
\hline
\end{tabular}

a. Quantitation limits listed for soil/sediment are based on wet weight. The quantitation limits calculated by the laboratory for soil/sediment, calculated on dry weight basis as required by the contract, will be higher.

b. Medium Soil/Sediment Contract Required Quantitation Limits (CRQL) for SemiVolatile TCL Compounds are 60 times the individual Low Soil/Sediment CRQL. 


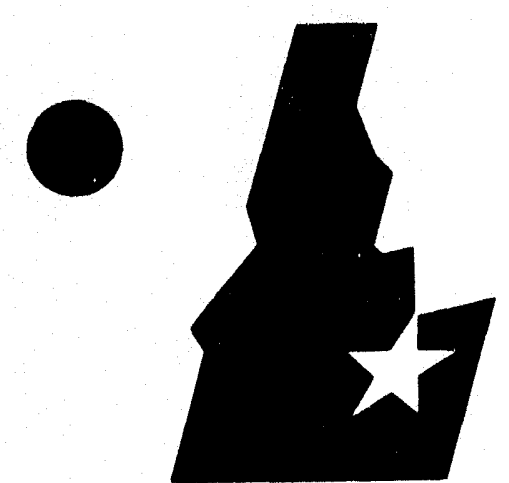

Idaho

National

Engineering Laboratory

Managed by the U.S.

Department of Energy
EGG-ERD-10452

July 1993

Health and Safety Plan for Operations Performed for the Environmental Restoration Department

Task: CFA Landfills II and III Remedial Investigation/ Feasibility Study

$\stackrel{n}{n} E E_{2} G_{\text {arno }}$

Work performed under

DOE Contract

No. DE-AC07-76/D01570 
Note: This Health and Safety Plan incorporates the Health and Safety Plan for Operations Performed for the Environmental Restoration Department, (EGG-WM-8771, Revision 2), with the Addendum completed for the CFA Landfill II and III Remedial Investigation/ Feasibility Study. 
Doc. No.: EGG-ERD-10452

Section No. H\&S Plan

Revision No.

Date $\frac{\text { July } 1993}{\text { Page No. i }}$

\section{Health and Safety Plan \\ for Operations Performed for the Environmental Restoration Program}

S. L. Morton

Published November 1991

Idaho National Engineering Laboratory

EG\&G Idaho, Inc.

Idaho Falls, Idaho 83415

Prepared for the

U.S. Department of Energy

Office of Environmental Restoration and Waste Management

Under DOE Idaho Fleld Office

Contract DE-AC07-76ID01570 
Doc. No.: EGG-ERD-10452

Section No. H\&S Plan

Revision No.

Date July 1093

Page No. ii

\section{Health and Safety Plan \\ For Operations Performed for the Environmental Restoration Program}

Approved by:

S. G. Stiger, Manager

Date

Environmental Restoration Program

Reviewed by:

J. P. Shea, Chairman

Date

ERP Independent Review Committee

D. E. Minner, Occupational Medical Program

Date

DOE-ID approval letter for this Health and Safety Plan is attached to DRR number ERP-340, dated 6/21/91. 
Doc. No.: EGG-ERD-10452

Section No. H\&S Plan

Revision No. $\quad 0$

Date July 1993

Page No. iii

\begin{abstract}
This document constitutes the generic health and safety plan for the Environmental Restoration Program (ERP). It addresses the health and safety requirements of the Comprehensive Environmental Response, Compensation, and Liability Act (CERCLA); Occupational Safety and Health Administration (OSHA) 29 CFR 1910.120 standard; and EG\&G Idaho, Inc. This plan is a guide to individuals who must complete a health and safety plan for a task performed for the ERP. It contains a task specific addendum that, when completed, specifically addresses task specific health and safety issues. This health and safety plan reduces the time it takes to write a task specific health and safety plan by providing discussions of requirements, guidance on where specific information is located, and specific topics in the Addendum that must be discussed at a task level. This format encourages a complete task specific health and safety plan and a standard for all health and safety plans written for ERP.
\end{abstract}


Doc. No.: EGG-ERD-10452

Section No. H\&S Plan

Revision No. 0

Date July 1993

Page No. iv 
Doc. No.: EGG-ERD-10452

Section No. H\&S Plan

Revision No. 0

Date July 1993

Page No. $v$

\section{CONTENTS}

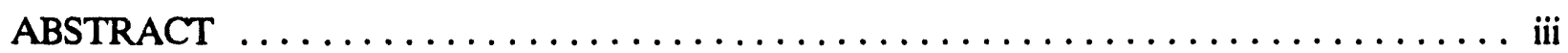

CONTENTS $\ldots \ldots \ldots \ldots \ldots \ldots \ldots \ldots \ldots \ldots \ldots \ldots \ldots \ldots \ldots \ldots \ldots \ldots, v$

ACRONYMS, ABBREVIATIONS, and DEFINITIONS $\ldots \ldots \ldots \ldots \ldots \ldots \ldots \ldots$ xi

1. INTRODUCTION $\ldots \ldots \ldots \ldots \ldots \ldots \ldots \ldots \ldots \ldots \ldots \ldots \ldots \ldots \ldots \ldots \ldots \ldots \ldots \ldots, 1-1$

1.1 H\&S Plan Task Specific Addendum $\ldots \ldots \ldots \ldots \ldots \ldots \ldots \ldots \ldots \ldots \ldots \ldots \ldots$

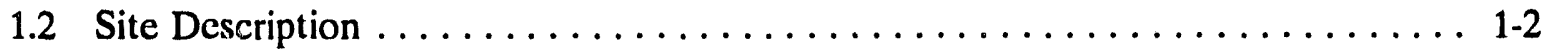

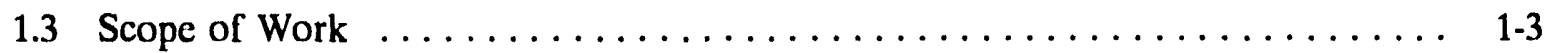

2. HEALTH AND SAFETY RESPONSIBILITIES $\ldots \ldots \ldots \ldots \ldots \ldots \ldots \ldots \ldots \ldots$

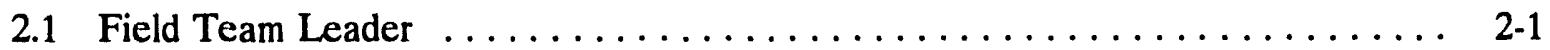

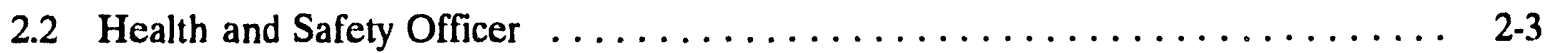

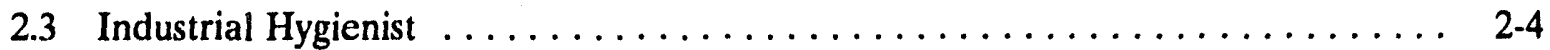

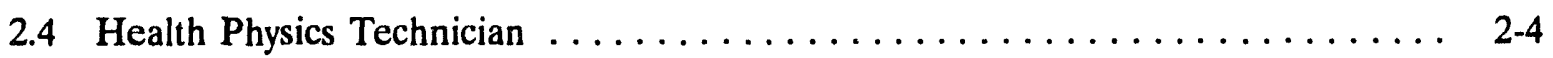

2.5 Radiological Engineer $\ldots \ldots \ldots \ldots \ldots \ldots \ldots \ldots \ldots \ldots \ldots \ldots \ldots \ldots \ldots \ldots$

2.6 Administrative Record and Document Control Office $\ldots \ldots \ldots \ldots \ldots \ldots$

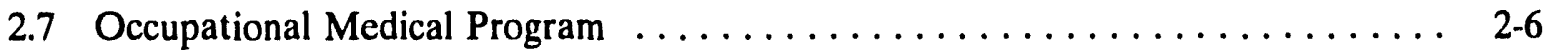

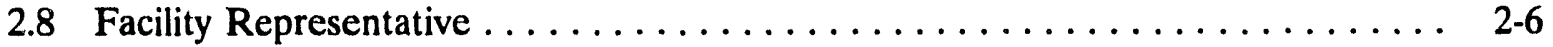

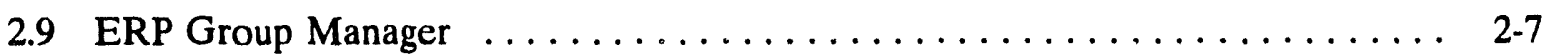

2.10 Project Manager $\ldots \ldots \ldots \ldots \ldots \ldots \ldots \ldots \ldots \ldots \ldots \ldots \ldots \ldots \ldots \ldots \ldots$

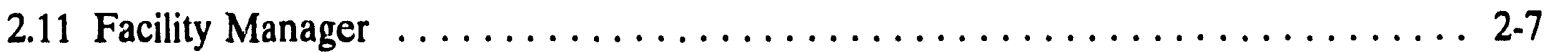

2.12 Environmental Hazardous Waste Engineer $\ldots \ldots \ldots \ldots \ldots \ldots \ldots \ldots \ldots$ 
Doc. No.: EGG-ERD-10452

Section No. H\&S Plan

Revision No. $\frac{0}{1093}$

Date July 1993

Page No. vi

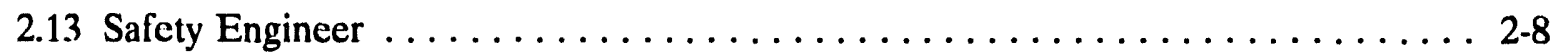

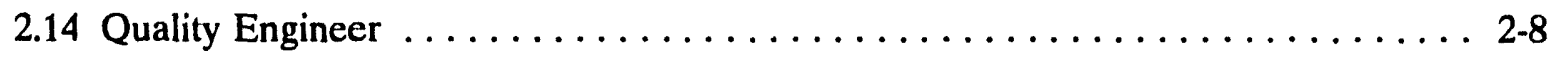

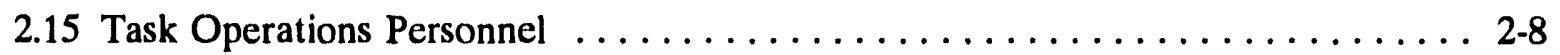

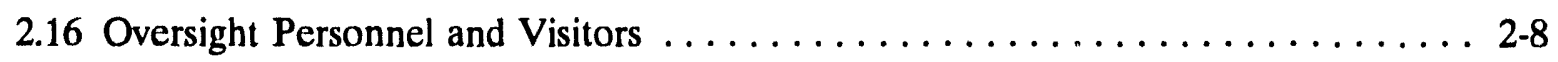

3. PERSONNEL TRAINING $\ldots \ldots \ldots \ldots \ldots \ldots \ldots \ldots \ldots \ldots \ldots \ldots \ldots \ldots \ldots \ldots$

4. MEDICAL SURVEILLANCE PROGRAM $\ldots \ldots \ldots \ldots \ldots \ldots \ldots \ldots \ldots \ldots$

5. HAZARD EVALUATION $\ldots \ldots \ldots \ldots \ldots \ldots \ldots \ldots \ldots \ldots \ldots \ldots \ldots \ldots$

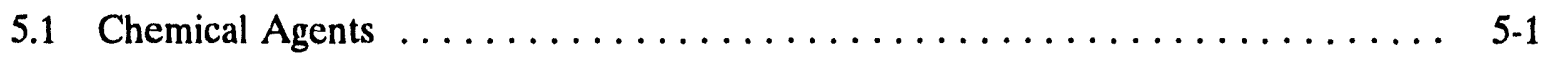

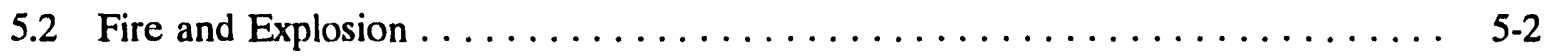

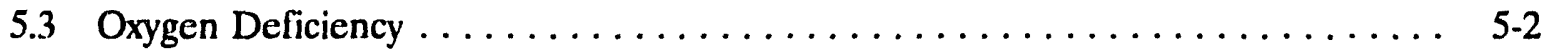

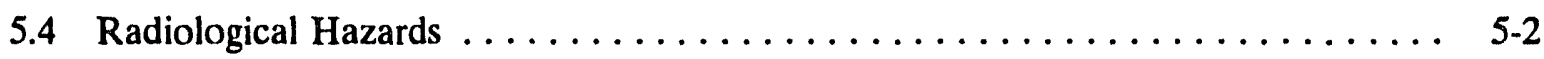

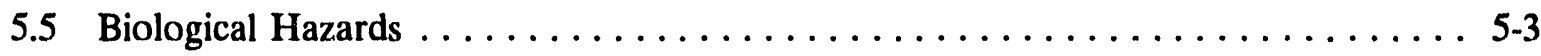

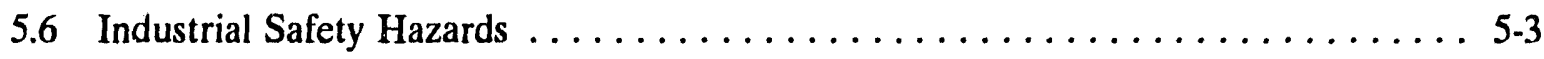

5.6.1 Existing Objects or Terrain $\ldots \ldots \ldots \ldots \ldots \ldots \ldots \ldots \ldots \ldots \ldots \ldots \ldots$

5.6 .2 Elevated Work Areas ....................... 5.4

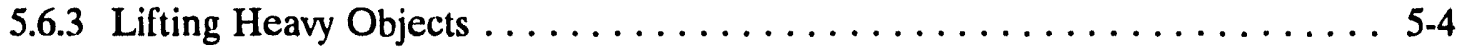

5.6 .4 Moving Machinery and Falling Objects $\ldots \ldots \ldots \ldots \ldots \ldots \ldots \ldots . \ldots \ldots$

5.6 .5 Personal Protective Equipment .................. 5.4

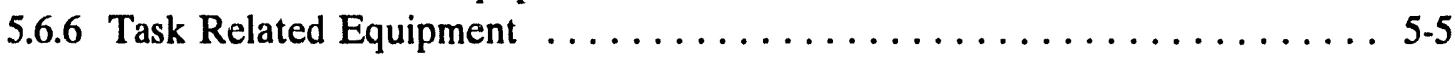

5.6.7 Excavation, Trenching, and Shoring $\ldots \ldots \ldots \ldots \ldots \ldots \ldots \ldots \ldots \ldots$

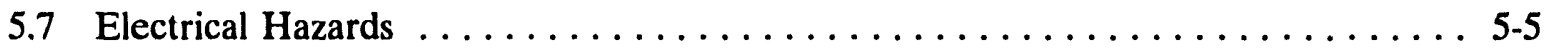

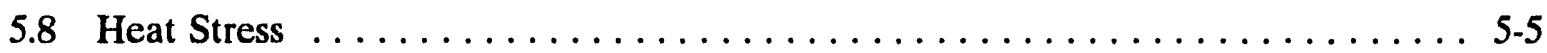

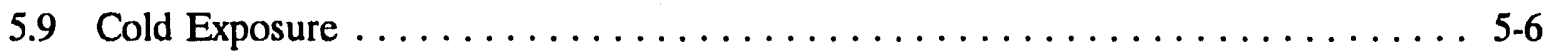

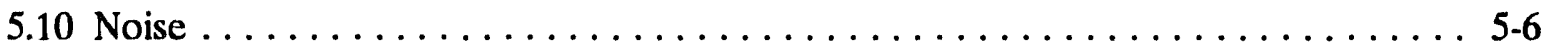


Doc. No.: EGG-ERD-10452

Section No. H\&S Plan

Revision No. 0

Date July 1993

Page No. vii

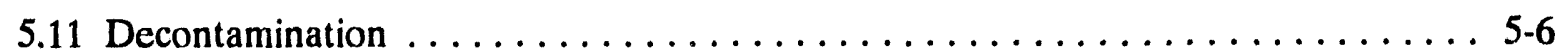

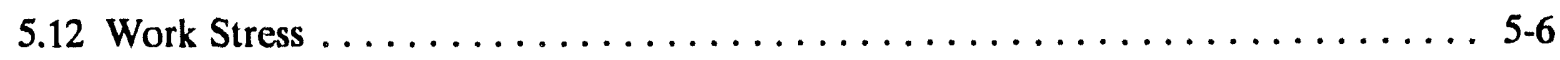

6. LEVELS OF PROTECTION AND PERSONAL PROTECTIVE EQUIPMENT . . . . 6-1

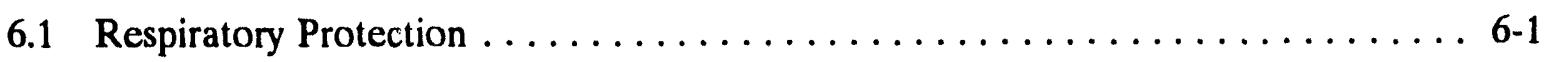

6.2 Level D Personal Protective Equipment .................. 6 -2

6.3 Level C Personal Protective Equipment . . . . . . . . . . . . $\ldots \ldots \ldots \ldots$

6.4 Level B Personal Protective Equipment $\ldots \ldots \ldots \ldots \ldots \ldots \ldots \ldots \ldots \ldots$

6.5 Level A Personal Protective Equipment . . . . . . . . . . . . . . 6-4

6.6 Personnel Protection in Radioactively Contaminated Areas ............ 6-5

6.6.1 Zone I - Low Level Contamination $\ldots \ldots \ldots \ldots \ldots \ldots \ldots \ldots \ldots$ 6-5

6.6.2 Zone II - Moderate Level Contamination ................. 6-5

6.6.3 Zone III - High Level Contamination $\ldots \ldots \ldots \ldots \ldots \ldots \ldots \ldots \ldots$

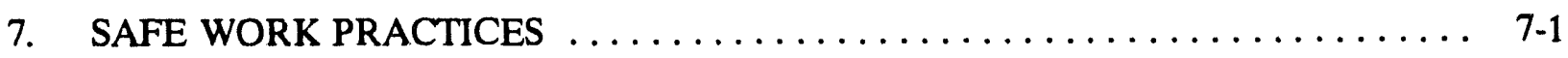

7.1 Working in Confined Spaces $\ldots \ldots \ldots \ldots \ldots \ldots \ldots \ldots \ldots \ldots \ldots \ldots \ldots \ldots \ldots \ldots \ldots$

7.2 Extended Working Schedules $\ldots \ldots \ldots \ldots \ldots \ldots \ldots \ldots \ldots \ldots \ldots \ldots \ldots \ldots \ldots$

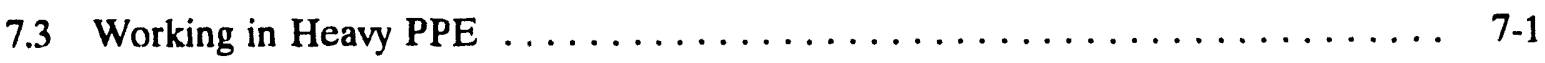

7.4 Working with Artificial Illumination $\ldots \ldots \ldots \ldots \ldots \ldots \ldots \ldots \ldots \ldots \ldots \ldots \ldots$

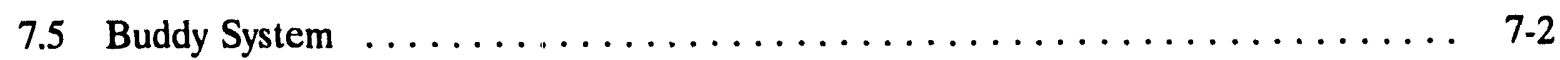

7.6 Handling Drums and Containers $\ldots \ldots \ldots \ldots \ldots \ldots \ldots \ldots \ldots \ldots \ldots \ldots \ldots \ldots \ldots \ldots .2$

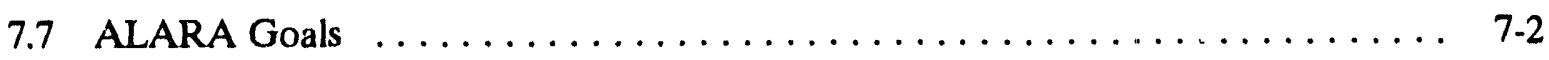

7.8 Radioactive Spill Control $\ldots \ldots \ldots \ldots \ldots \ldots \ldots \ldots \ldots \ldots \ldots \ldots \ldots \ldots \ldots \ldots$

7.9 General Safe Work Practices $\ldots \ldots \ldots \ldots \ldots \ldots \ldots \ldots \ldots \ldots \ldots \ldots \ldots \ldots \ldots \ldots$ 
Doc. No.: EGG-ERD-10452

Section No. H\&S Plan

Revision No. $\frac{H}{0}$

Date July 1993

Page No. viii

8. WORK/RADIATION ZONES, SITE ENTRY, AND SECURITY $\ldots \ldots \ldots \ldots$. $\ldots$-1

8.1 Work Zones $\ldots \ldots \ldots \ldots \ldots \ldots \ldots \ldots \ldots \ldots \ldots \ldots \ldots \ldots \ldots \ldots$

8.1.1 Exclusion Zone $\ldots \ldots \ldots \ldots \ldots \ldots \ldots \ldots \ldots \ldots \ldots \ldots \ldots$ 8-1

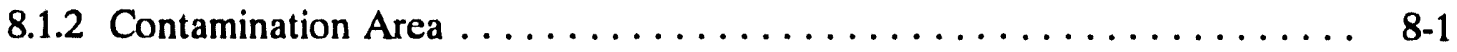

8.1.3 Contamination Reduction Corridor .................. 8-2

8.1.4 Contamination Reduction Zone $2 \ldots \ldots \ldots \ldots \ldots \ldots \ldots \ldots \ldots \ldots$ 8.2

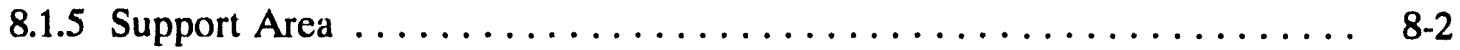

8.2 Radiological Control Zones $\ldots \ldots \ldots \ldots \ldots \ldots \ldots \ldots \ldots \ldots \ldots \ldots$ 8.2

8.2.1 External Radiation Exposure Control . . . . . . . . . . . . . 8-2

8.2.2 Radioactive Contamination Control $\ldots \ldots \ldots \ldots \ldots \ldots \ldots \ldots \ldots . . .6$ 8-3

9. ENVIRONMENTAL AND PERSONNEL MONITORING $\ldots \ldots \ldots \ldots \ldots \ldots$

9.1 Chemical Exposure Monitoring $\ldots \ldots \ldots \ldots \ldots \ldots \ldots \ldots \ldots \ldots \ldots \ldots$

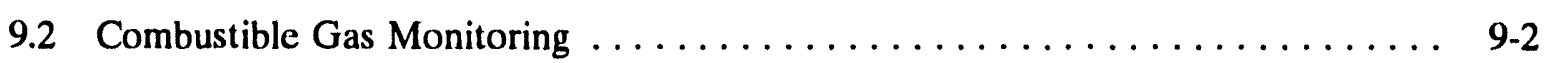

9.3 Radiological Monitoring $\ldots \ldots \ldots \ldots \ldots \ldots \ldots \ldots \ldots \ldots \ldots \ldots \ldots \ldots$

9.3.1 External Radiation Exposure Control . . . . . . . . . . . . . 9-2

9.3.2 Radioactive Contamination Control . . . . . . . . . . . . .

9.4 Heat and Cold Stress Control and Monitoring $\ldots \ldots \ldots \ldots \ldots \ldots \ldots$

9.5 Noise-Level Monitoring $\ldots \ldots \ldots \ldots \ldots \ldots \ldots \ldots \ldots \ldots \ldots \ldots \ldots$

9.6 Physical Hazard Control and Monitoring $\ldots \ldots \ldots \ldots \ldots \ldots \ldots \ldots . \ldots \ldots$

9.7 Record Keeping Requirements $\ldots \ldots \ldots \ldots \ldots \ldots \ldots \ldots \ldots \ldots \ldots \ldots$

10. DECONTAMINATION PROCEDURES $\ldots \ldots \ldots \ldots \ldots \ldots \ldots \ldots \ldots \ldots \ldots \ldots$ 10 1

10.1 Modified Level A and B Decontamination Procedures $\ldots \ldots \ldots \ldots \ldots \ldots \ldots$

10.2 Modified Level C Decontamination Procedures $\ldots \ldots \ldots \ldots \ldots \ldots \ldots \ldots$ 10 $\ldots \ldots$

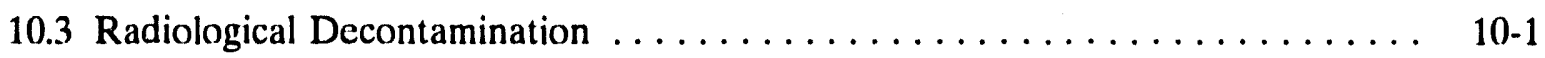


Doc. No.: EGG-ERD-10452

Section No. H\&S Plan

Revision No. $\quad 0$

Date July 1993

Page No. ix

10.4 Equipment Decontamination and Disposal of Contaminated Materials . . . . . 10-7

10.5 Decontamination During Medical Emergencies $\ldots \ldots \ldots \ldots \ldots \ldots \ldots \ldots$ 10-7

11. EMERGENCY PROCEDURES, EQUIPMENT, AND INFORMATION $\ldots \ldots \ldots$ 11-1

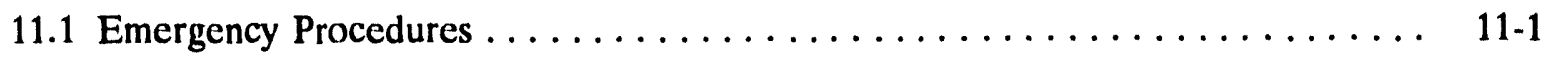

11.1.1 Personnel Occupational Injury or Illness in the Exclusion Zone . . . . 11-1

11.1.2 Personnel Occupational Injury or Illness in the Support Zone . . . . 11-2

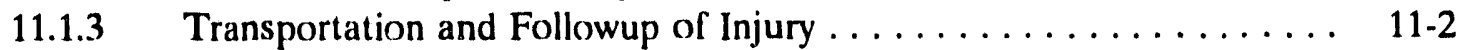

11.1.4 Fire/Explosion ......................... 11-2

11.1.5 Personal Protective Equipment Failure . . . . . . . . . . . $11-2$

11.1.6 Other Equipment Failure or Hazardous Material Spill . . . . . . . 11-2

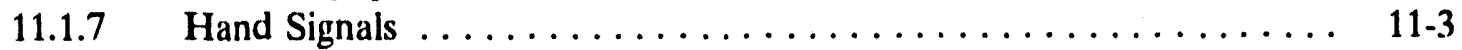

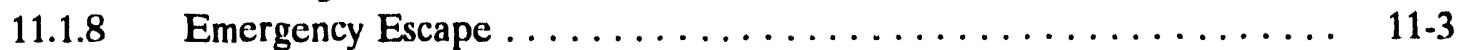

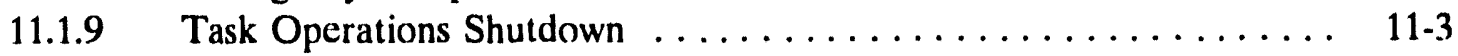

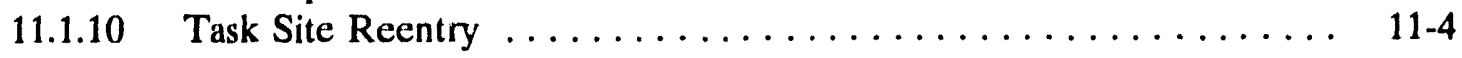

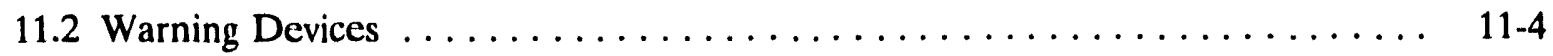

11.3 Emergency Equipment $\ldots \ldots \ldots \ldots \ldots \ldots \ldots \ldots \ldots \ldots \ldots \ldots \ldots \ldots \ldots$

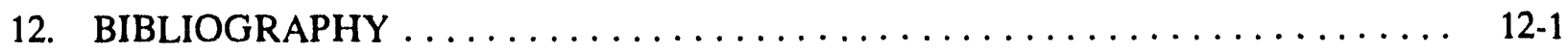

\section{FIGURES}

1-1. Map of INEL showing location of the major facilities. $\ldots \ldots \ldots \ldots \ldots \ldots$

2-1. Field organizational chart $\ldots \ldots \ldots \ldots \ldots \ldots \ldots \ldots \ldots \ldots \ldots \ldots \ldots \ldots \ldots \ldots$

10-1. Recommended modified Level A and B PPE hazardous chemical

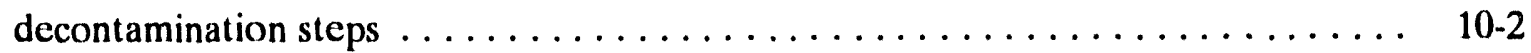

10-2. Recommended modified Level C PPE hazardous chemical decontamination steps .. 10-3

10-3. Anti-c removal steps for radiological control Zone I $\ldots \ldots \ldots \ldots \ldots \ldots \ldots \ldots$

10-4. Anti-c removal steps for radiological control Zone II $\ldots \ldots \ldots \ldots \ldots \ldots \ldots$

10-5. Anti-c removal steps for radiological control Zone III $\ldots \ldots \ldots \ldots \ldots \ldots \ldots$ 
Doc. No.: EGG-ERD-10452

Section No. H\&S Plan

Revision No.

Date July 1993

Page No. $x$

\section{TABLE}

3-1. Training topics for ERP hazardous material workers $\ldots \ldots \ldots \ldots \ldots \ldots \ldots \ldots$ 


\section{ACRONYMS, ABBREVIATIONS, and DEFINITIONS}

\begin{tabular}{|c|c|}
\hline ALARA & As Low As Reasonably Achievable \\
\hline ANSI & American National Standards Institute \\
\hline ARDC & Administrative Record and Document Control \\
\hline antic & Anti-contamination \\
\hline $\mathrm{CA}$ & Compliance Assurance \\
\hline CERCLA & Comprehensive Environmental Response Compensation, and Liability Act \\
\hline CFA & Central Facilities Area \\
\hline CFR & Code of Federal Regulations \\
\hline COCA & Consent Order and Compliance Agreement \\
\hline CPR & Cardiopulmonary Resuscitation \\
\hline DOE & Department of Energy \\
\hline DOE-ID & Department of Energy - Idaho Operations Office of DOE \\
\hline DOP & Detailed Operating Procedure \\
\hline DOT & Department of Transportation \\
\hline DRD & Direct Reading Dosimeter \\
\hline DRR & Document Revision Request \\
\hline EPA & Environmental Protection Agency \\
\hline ERP & Environmental Restoration Program \\
\hline FTL & Ficld Team Leader \\
\hline $\mathrm{H} \& \mathrm{~S}$ & Health and Safety \\
\hline HAZMAT & Hazardous Materials Response \\
\hline HPT & Hcalth Physics Technician \\
\hline HSO & Health and Safety Officer \\
\hline HW & Hazardous Waste \\
\hline IAG & Interagency Agreement \\
\hline IH & Industrial Hygienist \\
\hline INEL & Idaho National Engineering Laboratory \\
\hline LEL & Lower Explosive Limit \\
\hline MSDS & Material Safety Data Sheets \\
\hline NEPA & National Environmental Policy Act \\
\hline NIOSH & National Institute for Occupational Safety and Health \\
\hline NRTS & National Reactor Testing Station \\
\hline OMP & Occupational Medical Program \\
\hline OSHA & Occupational Safety and Health Administration \\
\hline $\mathrm{PD}$ & Program Directive \\
\hline PM & Project Manager \\
\hline PPE & Personal Protective Equipment \\
\hline $\mathrm{QE}$ & Quality Engineer \\
\hline QPP & Quality Program Plan \\
\hline
\end{tabular}


Doc. No.: EGG-ERD-10452

Section No. H\&S Plan

Revision No. 0

Date July 1993

Page No. xii

QAPjP Quality Assurance Project Plan

RCRA Resource Conservation and Recovery Act

RE Radiological Engineer

SAP Sampling and Analysis Plan

CBA Self-contained Breathing Apparatus

SE Safety Engineer

SOP Standard Operating Procedure

SSWP Special Safe Work Permit

SWIMS Solid Waste Information Management System

SWP Safe Work Permit

TLD Thermoluminescent Dosimeter

TRU Transuranic

USCG United States Coast Guard

Abbreviations:

NIOSH, 10/85-NIOSH/OSHA/USCG/EPA Occupational Safety and Health Guidance Manual for Hazardous Waste Site Activities, October 1985.

Definitions:

Action Limit-Any physical, chemical, or radiological limit set by a regulatory agency, EG\&G Idaho, Inc., or safety individual at the task site.

Area-A geographic subdivision of the INEL or a location outside the INEL dependent on the INEL for logistical or administrative support (e.g., TAN, TRA, CFA, IF).

Facility-The minimum complete and usable unit of Real Property designed to contain an organizational unit or operational function (e.g., building, central steam station).

Hazardous Material Response (HAZMAT) employee-Member of a group of employees, designated by management, who is expected to perform work to handle and control actual or potential leaks or spills of hazardous substances requiring possible close approach to the substance. The HAZMAT Team performs responses to releases or potential releases of hazardous substances for the purpose of control or stabilization of an incident. A HAZMAT Team is not a fire brigade nor is a typical fire brigade a HAZMAT Team. A HAZMAT Team, however, may be a separate component of a fire brigade or fire department.

Task Site-Immediate working area where ERP task operations are being performed. 
Doc. No.: EGG-ERD-10452

Section No. H\&S Plan

Revision No. 0

Date July 1993

Page No. 1-1

\section{Health and Safety Plan for Operations Performed for the Environmental Restoration Program}

\section{INTRODUCTION}

This Health and Safety (H\&S) Plan for operations performed for the EG\&G Idaho, Inc. Environmental Restoration Program (ERP) establishes the procedures and provides general guidelines to minimize health and safety risks to the worker and public. This plan, in conjunction with associated task specific information required by this plan, shall be used during selected activities aimed at assessing and remediating past hazardous waste and/or hazardous substance disposal at the Idaho National Engineering Laboratory (INEL).

This H\&S Plan and the associated task specific addendum required by this plan shall be in accordance with the Occupational Safety and Health Administration (OSHA), 29 CFR 1910.120 standard governing hazardous waste operations. It has been prepared in recognition of and is consistent with the NIOSH/OSHA/USCG/EPA Occupational Safety and Health Guidance Manual for Hazardous Waste Site Activities, October 1985 (hereafter referenced as NIOSH, 10/85); the EG\&G Idaho Company Procedures Manual; the EG\&G Idaho Safety Manual; and the EG\&G Idaho Radiological Controls Manual.

This H\&S Plan shall be used when work is performed at ERP task investigation sites by employees of EG\&G Idaho, subcontractors to EG\&G Idaho and employees of other firms, and Department of Energy (DOE) Laboratories. Occasional visitors and oversight personnel [DOE, State of Idaho, and Environmental Protection Agency (EPA) representatives] are subject to the requirements of Section 2.16 of this plan.

\subsection{H\&S Plan Task Specific Addendum}

This H\&S Plan must address the many diverse conditions encountered for each task included in the ERP investigations. Therefore, an addendum shall be written for each task that requires an H\&S Plan. The task addendum shall include any additions, omissions, or modifications to the main body of this H\&S Plan that can individualize this plan into a task specific plan. The task specific plan need not repeat EG\&G Idaho or ERP procedures for safety and health. However, these procedures shall be referenced in the Addendum.

NOTE: If an existing document meets the intent of the task specific plan [e.g., Detailed Operating Procedure (DOP)], it may be attached as the Addendum to this H\&S Plan. The following statement must then be included at the beginning of the task specific addendum: "The information contained in this document contains all the elements 
Doc. No.: EGG-ERD-10452

Section No. H\&S Plan

Revision No. 0

Date July 1993

Page No. 1-2

required by the task specific addendum and therefore replaces the stated addendum." If an existing document is used for the addendum, it does not have to be in the specified format of the addendum. The task specific H\&S Plan will be considered complete when the H\&S Plan task addendum is reviewed and approved per ERP Program Directive (PD) 2.2.

Upon request, a copy of this generic H\&S Plan and an electronic copy of the generic H\&S Plan task specific addendum may be obtained from the ERP Administrative Record and Document Control Office (ARDC, 526-2650). At a minimum, the generic H\&S Plan shall be reviewed annually and revised as required; therefore the requester shall verify the revision number of the generic H\&S Plan with ARDC. The electronic copy of the task specific addendum is provided as a guide in producing a task specific H\&S Plan. Pertinent topics referencing the main body of this H\&S Plan are provided in the electronic copy of the blank H\&S Plan task specific addendum to aid the author in writing a complete task specific H\&S Plan.

When the electronic guide is used to produce a task specific addendum, each topic must be evaluated to determine how it applies to the specific task requiring the addendum. If the topic does not apply to the subject task, "N/A" shall be written in that portion of the task specific addendum. If additional information is required to make a complete task specific H\&S Plan, additional blank pages may be added at the end of the task specific addendum. All technical information requested in the addendum must be obtained from knowledgeable individuals associated with the specific task [e.g., monitoring equipment information should be obtained from the task radiological engineer (RE) and/or industrial hygienist (IH)]. Once a task specific addendum is completed in accordance with ERP PD 4.4 and reviewed and approved in accordance with ERP PD 2.2, it shall be sent to ARDC, appended onto the generic H\&S Plan and processed. ARDC is responsible for maintaining the electronic copy and originals of the task specific H\&S Plans.

Any modifications to an approved task specific plan shall be implemented through a Document Revision Request (DRR), as described in ERP PD 4.1. If the change is made in an existing document used as a task specific plan but the document is not an ERP document, the changes will be made in accordance with the directives of the program/facility responsible for the document. Documentation of any changes made to documents external to ERP must be provided to ARDC.

\subsection{Site Description}

INEL is a multipurpose laboratory originally established in 1949 by the U.S. Government, under the direction of the Idaho Operations Office of the Department of Energy (DOE-ID). The primary mission of INEL is to support the engineering and operations efforts of DOE and other federal agencies in areas of nuclear safety research, reactor development, reactor operations 
Doc. No.: EGG-ERD-10452

Section No. H\&S Plan

Revision No. 0

Date July 1993

Page No. 1-3

and training, nuclear defense materials production, waste management and technology development, and energy technology/conservation programs.

INEL, formerly the National Reactor Testing Station (NRTS), encompasses 890 square miles and is located approximately 20 miles west of Idaho Falls, Idaho (Figure 1-1). The United States Atomic Energy Commission, now DOE, established the NRTS in 1949 as a site for building and testing a variety of nuclear facilities. INEL has also been the storage facility of transuranic (TRU) radionuclides and low-level radioactive wastes since 1952. DOE-ID has responsibility for the INEL and designates authority to operate the INEL to government contractors. The primary contractor for DOE-ID at INEL is EG\&G Idaho, Inc. which provides managing and operating services to the majority of INEL facilities. Other contractors who operate facilities at the INEL but are not covered by this H\&S Plan include Westinghouse Idaho Nuclear Company, Argonne National Engineering Laboratory, Westinghouse Electric Corporation, and Rockwell Corporation.

Tasks being performed for the ERP are scattered throughout INEL, and detailed facility or task site descriptions are too numerous to include in this generic portion of the H\&S Plan. Therefore, specific facility and/or task site descriptions shall be provided in the task specific addendum.

\subsection{Scope of Work}

ERP supports the following objectives identified in Chapter 2 of the Management Plan for the EG\&G Idaho Environmental Restoration Program:

- Identify and remediate all past waste units presenting a potential threat to human health or the environment.

- Comply with the Consent Order and Compliance Agreement (COCA), which will be the integration document for INEL cleanup activities, by implementing the COCA Action Plan. Comply with the Interagency Agreement (IAG) when approved by DOE, Environmental Protection Agency (EPA) Region 10, and the State of Idaho.

- Comply with the Comprehensive Environmental Response Compensation, and Liability Act (CERCLA) and the Resource Conservation and Recovery Act (RCRA) as established in an IAG among DOE-ID, EPA, and the State of Idaho.

- Support the DOE Environmental Restoration Program, as directed by DOE Headquarters in Washington, D.C.

A detailed work scope shall be included in the task-specific addendum. 
Doc. No.: EGG-ERD-10452

Section No. H\&S Plan

Revision No.

Date July 1993

Page No. 1 -4
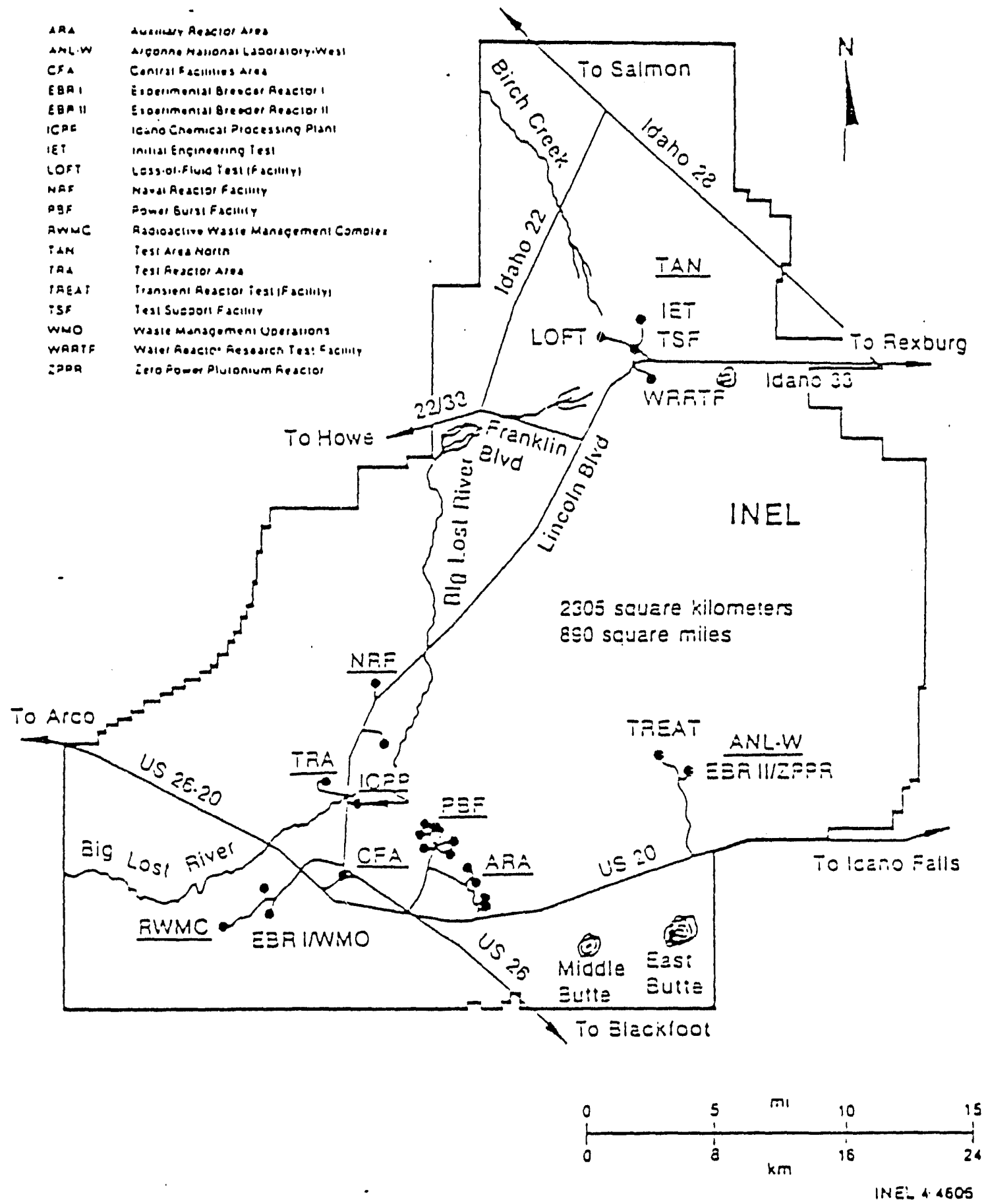

Figure 1-1. Map of INEL showing location of the major facilities. 
Field activities conducted during investigations may result in an exposure to hazardous and/or radioactive materials or wastes resulting from direct contact with contaminated soil, rock, groundwater, airborne particulates, and vapors. Protecting task site personnel from occupational health and safety hazards will be of major concern during the field activities. To this end, the ERP has identified a number of subjects that will provide protection to personnel and the environment. The following major subjects are addressed:

- Health and safety responsibilities

- Personnel training

- Medical surveillance program

- Hazard evaluation

- Levels of protection and use of personal protective equipment (PPE)

- Safe work practices

- Establishment of work zones, site entry, and security procedures

- Personnel and environmental monitoring and record keeping requirements

- Decontamination procedures

- Emergency procedures, equipment, and information.

Each subject is detailed in the following sections. 
Doc. No.: EGG-ERD-10452

Section No. H\&S Plan

Revision No. 0

Date July 1993

Page No. 1-6 
Doc. No.: EGG-ERD-10452

Section No. H\&S Plan

Revision No. $\frac{0}{1}$

Date July 1993

Page No. 2-1

\section{HEALTH AND SAFETY RESPONSIBILITIES}

Direct implementation of the H\&S Plan is the responsibility of the Field Team Leader (FTL). The FTL and/or Project Manager (PM) shall determine the task organizational structure and expertise required to perform the task while minimizing any risks to personnel health and safety. Expertise that may be required for the task includes but is not limited to industrial hygiene, health physics, industrial safety, and essential technical skills. The FTL shall develop a task organizational chart that identifies all key personnel. Both lines of command and lines of communication shall be identified by the task organizational chart (see Figure 2-1). The task organizational chart shall be located in the addendum. A qualified person may act in dual positions for a task [e.g., IH and health and safety officer (HSO)]. An HSO shall always be identified for a task per the requirements of OSHA 1910.120. The following subsections outline the responsibilities of the most common key personnel. If warranted by the requirements of the task, additional specialized positions may be necessary.

\subsection{Field Team Leader}

The FTL, the individual overseeing task activities, has ultimate responsibility for the safe and successful completion of task activities and for all phases of safety at the task site. If operations have been halted due to a potentially hazardous health and safety issue, the FTL will confer with the IH, HSO, RE, PM, health physics technician (HPT), safety engineer (SE), and facility representative, as required by the situation, to provide a safe solution to the problem. In addition, the FTL must remain responsive to health and safety issues raised by task operations personnel.

The FTL will ensure an orientation meeting is conducted before the start of a task to review and discuss operating procedures and the Task Specific H\&S Plan (including any attachments) with task operations personnel. If new team members arrive at the task site after initiation of the task, the FTL shall ensure this orientation is presented to them.

At the beginning of each work day, the FTL (or alternate) will meet with task operations personnel to discuss the day's activities and address any health and safety issues that may have arisen or potentially could arise that day.

The FTL will ensure that all task operations personnel have received the appropriate training as required by Section 3 of this H\&S Plan and that records of training for ERP personncl (including a copy of the signed Health and Safety Certification form as shown in the addendum) are submitted to the Training and Emergency Action Unit of the Waste Management Operations Support Group. 
Doc. No.: EGG-ERD-10452

Section No. H\&S Plan

Revision No. 0

Date July 1993

Page No. 2-2

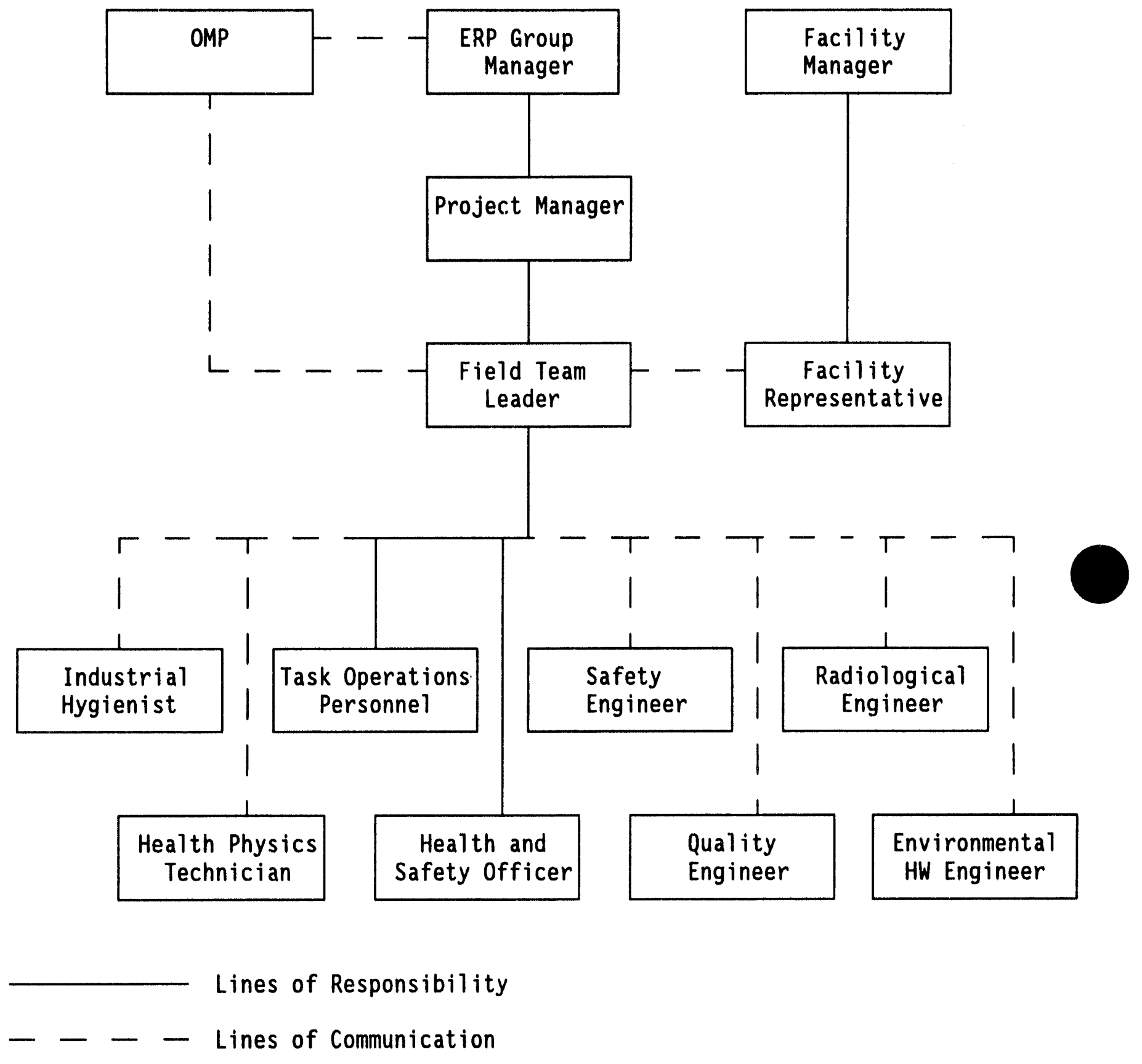

Figure 2-1. Field organizational chart. 
Additional responsibilities of the FTL include:

- Halting or modifying any task and/or evacuating the task site if work conditions are considered unsafe. This decision will be made after consulting with the HSO, IH, SE, and/or RE, as appropriate

- Reporting any accident, illness, or safety-related occurrence in accordance with Section 3 of the EG\&G Idaho Safety Manual.

- Notifying the facility representative (if applicable) of any modifications or suspension of the task

- Ensuring that an interface exists with the analytical laboratories regarding any analyses of personnel monitoring and/or ambient air samples and provisions are made with the laboratory for a 24 to 48-hour turnaround for analysis in the event of an exposure suspected of being above an action level.

- Ensuring that all task site personnel understand and comply with all safety requirements

- Initiating corrective action for observed safety violations

- Ensuring that safety training is implemented as described in this plan (Section 3).

An FTL not at the task site must appoint an appropriate alternate to act as FTL. This change must be communicated to the facility representative, when necessary, and recorded in the FTL logbook. Appropriate alternates shall be listed in the task specific addendum.

\subsection{Health and Safety Officer}

The HSO is responsible for ensuring compliance with and the execution of the health and safety procedures described in this plan and the associated task specific addendum. The HSO will be supported by those personnel necessary to effectively implement the task specific H\&S Plan and verify compliance (e.g., SE, IH, HPT, RE, and facility representative).

Responsibilities of the HSO include:

- Ensuring that all necessary safety equipment is located on or near the task site and properly maintained and calibrated by the appropriate personnel.

- Observing task site activities and reporting any deviations from the H\&S Plan to the FTL 
Doc. No.: EGG-ERD-10452

Section No. H\&S Plan

Revision No. 0

Date July 1993

Page No. $2-4$

- Initiating contact with the INEL emergency response agencies (security, fire, medical) at the beginning of the task, ensuring personnel and environmental monitoring requirements are established by the IH and RE (Section 9), and testing the emergency phone numbers to ensure accuracy.

\subsection{Industrial Hygienist}

The IH is the primary source of information regarding health issues at the task site. The IH is responsible for operations and maintenance of all monitoring equipment with the exception of radiological equipment and will maintain a daily logbook of monitoring activities. The IH will conduct task site health hazard assessments and advise the FTL on adequate health protection for task operations personnel. The IH will advise the FTL on changes to monitoring or PPE requirements throughout task activities and on any conditions necessitating task site evacuation and permitting personnel reentry to the task site.

The IH is responsible for designing a practical monitoring program to determine worker exposures to hazardous substances. The IH will also log results from field samples and observations.

NOTE: Much uncertainty is involved as to the chemical hazards that may be encountered. Not everything can be monitored, and professional judgment must be exercised at all times.

The IH shall aid the FTL in identifying employees experiencing adverse health effects that may have resulted from exposure to hazardous substances and environments and identifying such workers to the Occupational Medical Program (OMP).

\subsection{Health Physics Technician}

The HPT is the primary source of information and guidance with regards to radiological hazards. The HPT will be present at the task site before operations begin and at any point during task operations when a radiological hazard to operations personnel may exist or is anticipated.

Responsibilities of the HPT include:

- Ensuring radiological equipment is calibrated and functioning properly

- Radiological surveying of the task site, equipment (before and after decontamination), and samples

- Collecting and analyzing smears 
Doc. No.: EGG-ERD-10452

Section No. H\&S Plan

Revision No. 0

Date July 1993

Page No. 2-5

- Providing guidance and monitoring decontamination of equipment (radiological contaminants)

- Providing the FTL, OMP, and RE with radiological monitoring information as requested

- Immediately notifying the FTL of any radiological occurrence that must be reported as directed by the EG\&G Idaho Safety Manual, Section 3, Appendix II.

- Accompanying victim to the nearest INEL Medical Facility for evaluation if significant radiological contamination occurs.

\subsection{Radiological Engineer}

The RE is the primary source of information and guidance for radiological controls imposed on a task. The RE will make recommendations to minimize health and safety risks of task operations personnel if a radiological hazard exists or occurs at a task site.

The responsibilities of the RE include:

- Performing radiation exposure estimates using information provided by cognizant engineers, area HPTs, history of past work evaluations, bioassays, FTLs, etc.

- Identifying the type(s) of radiological monitoring equipment necessary to maintain safe working conditions for task operations personnel

- $\quad$ Attending pre-job briefings if required by the FTL

- Advising FTL and HPT of changes in monitoring or PPE and task site evacuation and reentry.

\subsection{Administrative Record and Document Control Office}

The ARDC is responsible for organizing and maintaining data and reports (safety, sampling, and operations) generated by ERP investigations. ARDC maintains a supply of all controlled documents and provides a documented checkout system for the control and relcase of controlled documents, reports, and records. A copy of the H\&S Plan and the associated electronic copy of the task specific addendum are available upon request by calling 526-2650. 
Doc. No.: EGG-ERD-10452

Section No. H\&S Plan

Revision No. 0

Date July 1993

Page No. 2-6

\subsection{Occupational Medlcal Program}

The OMP is mandated by DOE 5480.8 and uses the sciences related to preventive medicine and environmental health to determine the effects of environmental stress on human health or disease.

The OMP has responsibilities in the following areas:

- Review and comment on INEL emergency plans and operations

- Provide diagnosis, medical opinion, and treatment for INEL employees with occupational or nonoccupational illness or injuries

- Assist in the documentation and investigation of work-related illnesses or injuries

- Provide medical opinion whenever there is doubt by the FTL, advisors, or employee of the ability of the employee to perform assigned work or work being considered for assignment

- Plan and provide emergency medical care in support of individuals and Area emergency actions

- Maintain and operate a radiation and chemical decontamination facility at Central Facilities Area (CFA)

- Provide medical surveillance of workers who are identified by an IH as having been or are likely to be exposed over action levels to specific hazardous environments or substances.

\subsection{Facility Representative}

The facility representative serves as the Area Landlord representative and is responsible for the safety of personnel and safe completion of all project activities conducted within his/her Area. Therefore, the facility representative will be kept informed of all activities performed in the Area. Where applicable, the facility representative and FTL shall agree upon a schedule for reporting task progress and plans for work. The facility representative will serve as advisor to task operations personnel with regard to the Area operations when the task is performed in his/her Area. 
Doc. No.: EGG-ERD-10452

Section No. H\&S Plan.

Revision No. $\quad 0$

Date July 1993

Page No. 2-7

\subsection{ERP Group Manager}

The ERP Group Manager is responsible for investigation and remediation activities performed by ERP. This manager provides technical coordination and interfaces with the DOE-ID Environmental Support Office. The ERP Group Manager ensures that all activities are conducted in accordance with DOE, EPA, and State of Idaho requirements and agreements; monitors and approves program budgets and schedules; ensures the availability of necessary personnel, equipment, subcontractors, and services; and provides direction for the development of tasks, evaluation of findings, development of conclusions and recommendations, and production of reports. The ERP Group Manager has primary responsibility for the technical quality of all projects and safety of personnel.

\subsection{Project Manager}

The PM has the responsibility for ensuring that all tasks conducted during the project are in compliance with the Management Plan for the EG\&G Idaho Environmental Restoration Program and all applicable OSHA, EPA, DOE, Department of Transportation (DOT), and State of Idaho requirements. The $P M$ is responsible for ensuring tasks comply with the ERP Quality Program Plan (QPP) (QPP-149), Quality Assurance Project Plan (QAPjP), H\&S Plan, PDs, and Sampling and Analysis Plans (SAPs) of ERP. The PM coordinates all field, laboratory, and modeling activities.

\subsection{Facility Manager}

The Facility Manager is responsible for managing all aspects of the Area in his charge. The Facility Manager must be cognizant of work being conducted in the Area.

\subsection{Environmental Hazardous Waste Engineer}

The Environmental Hazardous Waste (HW) Engineer oversees, monitors, and advises EG\&G Idaho organizations performing field activities at the INEL. Responsibilities include ensuring compliance with DOE Orders, EPA regulations, and other regulations concerning effects of activities on the environment. Additional responsibilities of the HW Enginecr include:

- Acting as advisor for environmental concerns associated with ERP task activities

- Maintaining a library of applicable environmental information

- Disseminating applicable environmental information where/when needed. 
Doc. No.: EGG-ERD-10452

Section No. H\&S Plan

Revision No. 0

Date July 1993

Page No. 2.8

\subsection{Safety Engineer}

The SE offers guidance on all safety issues arising at the task site, observes tasks and advises the FTL on required safety equipment necessary to promote a safe work environment, advises FTL and HSO about safety concerns arising during task operations, and recommends solutions to any concerns.

\subsection{Quality Engineer}

The Quality Engineer (QE) provides guidance on task site quality issues when requested. The QE observes task site activities and verifies that task operations comply with quality requirements pertaining to these activities. The QE identifies activities that do not or have the potential for not complying with quality requirements and suggests corrective actions for such activities.

\subsection{Task Operations Personnel}

All task operations personnel, including EG\&G and subcontractor personncl, are responsible for understanding and complying with requirements of the task specific H\&S Plan. Task operations personnel will be briefed by the FTL before starting each day's activities. They should identify and discuss potentially unsafe task site activities or conditions with the FTL for corrective action. If unsafe conditions develop, task operations personnel are authorized to halt work and notify the FTL of the unsafe condition.

\subsection{Oversight Personnel and Visitors}

Oversight personnel (i.e., DOE-ID, EPA, and State of Idaho representative) and visitors shall be considered "workers on site only occasionally." To minimize risks that may result from task site activities, "workers on site only occasionally" must have official business and notify the FTL before entering the task site. All "workers on site only occasionally" shall follow the requirements of OSHA $1910.120(\mathrm{~d})(3)(\mathrm{ii})$ which states:

Workers on site only occasionally for a specific limited task (such as, but not limited to, groundwater monitoring, land surveying, or geo-physical surveying) and who are unlikely to be exposed over permissible exposure limits and published exposure limits shall receive a minimum of 24 hours of instruction off the site, and the minimum of one day actual field experience under the direct supervision of a trained, experienced supervisor.

If these individuals meet the requirements stated above, they may not proceed beyond the support zone without receiving a safety briefing and wearing the appropriate protective equipment. 
Doc. No.: EGG-ERD-10452

Section No. H\&S Plan

Revision No. 0

Date July 1993

Page No. 3-1

\section{PERSONNEL TRAINING}

Task operations personnel classified by the PM and/or FTL as hazardous material workers shall receive hazardous material worker training as specified by OSHA 29 CFR 1910.120 and the EG\&G Idaho Safety Manual, Section 8. Specific training requirements for each hazardous material worker may vary depending on the hazards associated with the job assignment (e.g., noise, radiation). All hazardous material workers must obtain OSHA Hazardous Waste Operator training. Additional training to be considered for hazardous material workers includes but is not limited to:

- Respirator Fit Test Qualification

- Radiation Worker

- Hearing Conservation.

In addition to the above mentioned training, at least one worker with Medic 1st [Cardiopulmonary Resuscitation (CPR) and First Aid] training shall be present at the task site when task operations personnel are present. Managers of hazardous material workers (e.g., PM, FTL) shall obtain Hazardous Waste Worker Supervisor Training. Additional safety training courses may be required as dictated by the job assignment. Section 8 of the EG\&G Idaho Safety Manual contains course numbers and descriptions for all EG\&G safety training courses.

Employees who attend training classes requiring an annual refresher course must attend the annual courses for as long as they remain active hazardous material workers. Proof of completion of all required training courses by employees and visitors must be provided to the Training and Emergency Action Unit of the Waste Management Operations Support Group. ERP personnel can also obtain information regarding ERP personnel training records (e.g., due dates of refresher courses) from the Training and Emergency Action Unit of the Waste Management Operations Support Group.

The FTL will ensure that all task operations personnel understand the specific site hazards associated with each task at the daily briefings. Each FTL will also design and ensure implementation of a task specific training orientation to inform task operations personnel about the unique hazards or procedures, task specific H\&S Plan, DOPs, etc. associated with the task at hand. Table 3-1 summarizes the above mentioned training requirements.

The following outline shall be used as a guideline for training and orientation before the start of a task. Personnel working at the task site shall be informed of the information listed in this outline, as applicable to the specific task. 
Doc. No.: EGG-ERD-10452

Section No. H\&S Plan

Revision No. 0

Date July 1993

Page No. 3-2

Table 3-1. Training topics for ERP hazardous material workers. ${ }^{a}$

\begin{tabular}{|c|c|c|c|}
\hline Training topic ${ }^{b}$ & $\begin{array}{l}\text { Personnel job } \\
\text { description }\end{array}$ & $\begin{array}{c}\text { Task } \\
\text { operations } \\
\text { personnel }\end{array}$ & $\begin{array}{c}\text { Field } \\
\text { managers }\end{array}$ \\
\hline $\begin{array}{l}\text { OSHA hazardous waste } \\
\text { operator }\end{array}$ & $\begin{array}{l}\text { Cleanup or operations of } \\
\text { hazardous waste sites }\end{array}$ & $\mathbf{R}$ & $\mathbf{R}$ \\
\hline $\begin{array}{l}\text { Respirator Fit Test } \\
\text { Qualification }\end{array}$ & $\begin{array}{l}\text { Work area requires use of } \\
\text { respirator }\end{array}$ & $\mathbf{R}$ & $\mathbf{R}$ \\
\hline Radiation Worker & $\begin{array}{l}\text { Level of radiation exposure } \\
\text { determines training category }\end{array}$ & $\mathbf{R}$ & $\mathbf{R}$ \\
\hline Medic 1st & First Aid, CPR & $\mathrm{R}^{*}$ & 0 \\
\hline $\begin{array}{l}\text { Personal Protective } \\
\text { Clothing and Equipment }\end{array}$ & $\begin{array}{l}\text { Required to wear Chemical } \\
\text { and/or Radiological }\end{array}$ & $\mathbf{R}$ & $\mathbf{R}$ \\
\hline $\begin{array}{l}\text { Site Specific Hazards } \\
\text { (FTL develops this } \\
\text { training) }\end{array}$ & $\begin{array}{l}\text { Encounters task specific } \\
\text { potential hazards }\end{array}$ & $\mathbf{R}$ & Develop \\
\hline Decontamination & $\begin{array}{l}\text { Chemical and/or } \\
\text { Radiological Procedures }\end{array}$ & $\mathbf{R}$ & $\mathbf{R}$ \\
\hline Hearing Conservation & $\begin{array}{l}\text { IH determines exposure to } \\
\text { noise above 8-hour } \\
\text { time-weighted average of } \\
85 \text { decibels }\end{array}$ & $\mathbf{R}$ & $\mathbf{F}$ \\
\hline Emergency Training & $\begin{array}{l}\text { Knowledge of Area drills, } \\
\text { rescue, response, } \\
\text { information }\end{array}$ & $\mathbf{R}$ & $\mathbf{R}$ \\
\hline \multicolumn{4}{|c|}{$\begin{array}{l}\text { R: Required } \\
\text { O: Optional } \\
\text { *: At least one worker with Medic 1st Training shall be at task site when task } \\
\text { operations personnel are present. }\end{array}$} \\
\hline \multicolumn{4}{|c|}{$\begin{array}{l}\text { a. Additional training may be required for each task or individual and should } \\
\text { be listed in the addendum. } \\
\text { b. These training topics include both the initial and refresher training } \\
\text { (See EG\&G Idaho Safety Manual, Section } 8 \text { for specific safety training course } \\
\text { descriptions and numbers). }\end{array}$} \\
\hline
\end{tabular}


Doc. No.: EGG-ERD-10452

Section No. H\&S Plan

Revision No. 0

Date July 1993

Page No. 3-3

A. WORK PLAN (SAP, Test Plan, etc.)

B. HEALTH AND SAFETY ITEMS

1. Personnel responsibilities

2. Medical program

3. Task site work zones

4. Vehicle operation and parking

5. Task site air and radiological monitoring

a. Monitoring equipment (task site and personal)

b. Calibration

c. Maintenance and decontamination procedures

6. Potential hazardous contaminants and chemical hazards (toxicity and symptoms) present at the task site

7. Potential radiological contaminants

8. Task Specific Hazard Communication (in addition to the General Hazard Communication) training
a. Inventory of hazardous agents
b. Material Safety Data Sheets (MSDSs)
c. Container labeling
d. Informing visitors
e. Contractor inventory and MSDSs

9. Contingency plans and responses
a. Spill control
b. Work stoppage

10. Use of field equipment and supplies
a. Drilling equipment
b. Work tools
c. Sampling equipment
d. Decontamination of equipment and supplies 
Doc. No.: EGG-ERD-10452

Section No. H\&S Plan

Revision No.

Date July 1993

Page No. $3-4$

B. HEALTH AND SAFETY ITEMS (continued)

11. Task site control and security

12. Buddy system and hand signals

13. Work limitations
a. Weather
b. Fatigue
c. Heat stress
d. Cold stress
e. Hours of work
f. Illumination
g. Lightning

C. PERSONAL PROTECTIVE EQUIPMENT AND CLOTHING

1. General

2. Availability

3. Level D PPE and clothing, as defined by OSHA, including limitations of protection
a. Work clothing
b. Eye protection
c. Foot protection
d. Head protection
e. Hearing protection

4. Level C PPE and clothing, as defined by OSHA, including limitations of protection
a. Respiratory protection
b. Work clothing
c. Eye protection
d. Foot protection
e. Head protection
f. Hearing protection
g. Skin/hand protection 


\section{PERSONAL PROTECTIVE EQUIPMENT AND CLOTHING (continued)}

5. Level B PPE and clothing, as defined by OSHA, including limitations of protection
a. Air supplied hood or self-contained breathing apparatus (SCBA)
b. Disposable, chemically resistant coveralls
c. Anti-contamination (anti-c) clothing as recommended by the RE
d. Chemically resistant safety shoes with steel toe
e. Chemically resistant shoe covers
f. Hard hat
g. Inner and outer chemically resistant gloves
h. Hearing protection, as required by $\mathrm{IH}$

6. Level A PPE and clothing, as defined by OSHA, including limitations of protection
a. SCBA
b. Fully encapsulating, chemically resistant suit
c. Additional anti-c clothing, as recommended by the RE
d. Chemically resistant safety shoes with steel toe
e. Chemically resistant shoe covers
f. Hard hat
g. Inner chemically resistant gloves
h. Hearing protection, as required by IH

7. Zone I anti-c clothing minimum requirements
a. One pair cloth anti-c coveralls (or disposable) (as required by HPT)
b. One yellow cloth hood (or disposable)
c. Two pair shoe covers
d. One pair latex gloves and cloth glove liners

8. Zone II anti-c clothing minimum requirements
a. One pair yellow cloth anti-c coveralls (or disposable)
b. One yellow cloth hood (or disposable)
c. Three pairs shoe covers (two pairs must be vinyl)
d. One pair latex gloves and cloth glove liners 
Doc. No.: EGG-ERD-10452

Section No. H\&S Plan

Revision No. 0

Date July 1993

Page No. 3-6

C. PERSONAL PROTECTIVE EQUIPMENT AND CLOTHING (continued)

9. Zone III anti-c clothing minimum requirements

a. One pair yellow cloth anti-c coveralls and head cover (hood)

b. One pair disposable anti-c coveralls (or plastic anti-c suit) with disposable hood

c. Three pairs shoe covers (two pairs must be vinyl)

d. Two pairs gloves and cloth glove liners

e. Respiratory protection commensurate with the contamination levels

10. Decontamination procedures
a. Chemical contaminants
b. Radiological contaminants
c. Mixed contaminants

D. EMERGENCY ASSISTANCE

1. Availability of emergency services and location of telephone and telephone numbers, MSDSs, and other emergency information

2. Transportation of emergency cases and accompanying medical monitoring procedures

3. Emergency assistance and review of hand and audible signals

\section{E. SPECIAL PRECAUTIONS DURING TASK SPECIFIC OPERATIONS}

1. Most dangerous times

2. Most dangerous conditions

3. Specific task checklist.

In addition, the FTL will conduct safety briefings (a) at the beginning of each shift, (b) whenever new personnel arrive at the task site, and (c) as significant changes to task site or work conditions occur. 
Doc. No.: EGG-ERD-10452

Section No. H\&S Plan

Revision No. 0

Date July 1993

Page No. 4-1

\section{MEDICAL SURVEILLANCE PROGRAM}

Employees identified as hazardous waste workers as defined by OSHA (29 CFR 1910.120) require medical surveillance examinations prior to beginning duties, annually, and at the termination of hazardous waste duties (if they have not had such an examination within a year). This includes (a) employees who are or who may be exposed to hazardous substances at or above established permissible exposure limits, without regard to respirator use, for $\mathbf{3 0}$ or more days per year; (b) those who wear a respirator for 30 or more days per year; and (c) all HAZMAT employees. In addition, employees who must use a respirator in their job or are required to take training to use a respirator to perform their duties under this plan must be medically evaluated for respirator use at lcast annually.

The OMP is responsible for evaluating the physical ability of a worker to perform the task assigned. The OMP provides medical clearance to the worker for the work to be performed. The OMP may impose restrictions on the employee by limiting the amount or type of work performed. The PM (or the IH and/or HP with the approval of PM) must provide the job related background information listed below to the OMP for each hazardous material worker. This information must be submitted to the OMP before work begins and annually, one month before birth date of the employee to maintain hazardous waste/hazardous material worker medical clearance. It may be submitted on EG\&G Form 3044, "Hazardous Material Worker Job Related Background Information;" EG\&G Form 735, "Industrial Hygiene Identification Of An Employec For A Medical Surveillance Program To OMP;" or by other means acceptable to the OMP.

- Medical history and physical examination

- Precmployment medical examination, for full-time employees

- Current comprehensive medical examinations, for full-time employees, in an INEL medical facility

- Records and reports from employecs' private physicians, as required by the Site Occupational Medical Director

- Medical evaluation by OMP on return to work following an absence in excess of one work weck ( 40 consecutive work hours) resulting from illness or injury

- Medical evaluation in the eve, a supervisor questions the physical condition of an employec

- Medical evaluation in the event the employee questions his/her physical condition

- Job related background information (Form EG\&G-3044) 
Doc. No.: EGG-ERD-10452

Section No. H\&S Plan

Revision No. 0

Date July 1993

Page No. 4-2

- What type of job does the individual perform?

- When was the individual first exposed to hazardous substances or working in an environment with potential hazardous exposure at the INEL?

- Relevant environmental monitoring (IH and HPT) data including sample dates and places (if the employee has been exposed to substances or physical agents above an action level)

- How and when was/will the employee (bc) trained in PPE including respirators?

- What type of respiratory protective device is to be used?

- How many days per month is respiratory protection to be used?

- How long is this work to continue?

The above information and examinations are used to determine the following for each employee:

- Ability to perform routine occupational tasks

- Work in protective equipment and/or heat stress environments

- Use of respiratory protection

- Need to be entered into additional specific medical surveillance examination programs.

Employees are cleared as hazardous material workers with or without specific restrictions relating to heat stress, certain job tasks, and/or use of respirators. If the OMP does not have sufficient information at the time of request for clearance for respirator training, the supervisor is notified and clearance is withheld until the needed information is provided and any necessary additional examination or testing is completed.

Results of the following tests shall be made available to the OMP when any abnormal exposure is noted or a radiological contamination incident occurs:

- Whole body count (baseline, annual, and on actual or suspected radiological contamination incident)

- Bioassay (baseline, as required to assess internal radiation dose, and on actual or suspected radiological contamination incident).

Subcontractors are responsible for being in compliance with health and safety requirements as stated in 29 CFR 1910.120. All medical data collected pursuant to hazardous material worker 
qualification of a subcontractor worker shall be made available to the OMP. Background information about the subcontractor worker will assist the OMP in assessing the medical ability of the subcontractor worker to work should doubt arise during task operations. This information is also required from the subcontractor in order for the OMP to clear the subcontractor worker as a hazardous material worker. Subcontractor past radiation exposure history shall be submitted to the Operational Dosimetry Unit of EG\&G Idaho (Section 3.5 of Chapter 2 in the Radiological Controls Manual).

It is the policy of the OMP to examine all workers, including subcontractors, when they are injured on the job or there is reason to believe that they have been exposed, over an action level, to toxic substances or physical agents.

Before initiation of any task where a chemical/radiological hazard exists, the appropriate medical facility will be notified of the start of the task, anticipated schedules, and task site locations by the HSO. In addition, the OMP shall be supplied with an inventory of the known hazardous constituents located at the task sites.

In the event of an IH and/or RE documented exposure to a hazardous substance or physical agent over an action level, the worker(s) shall be transported to the nearest medical facility for evaluation. Further medical evaluation will be in accordance with the symptoms, specific hazard involved, exposure level, medical surveillance requirements, current health and safety directives, and sound medical practices.

The following information shall be provided to the OMP:

- Name, job title, work location, supervisor's name, and supervisor's phone number

- $\quad$ Substances/physical agents (e.g., noise) involved

- Date the employec was first exposed to the substance/physical agent on this task

- Monitoring data including locations of samples and dates samples were taken, if exposed over action level

- $\quad$ PPE in use during this task

- Number of days per month PPE has been in use

- How long this employee will be exposed to the substance or physical agent

- Training the employee has received in the use of PPE

- Type of respirator, if any, being used. 
Doc. No.: EGG-ERD-10452

Section No. H\&S Plan

Revision No. 0

Date July 1993

Page No. 4.4 
Doc. No.: EGG-ERD-10452

Section No. H\&S Plan

Revision No. $\frac{0}{10}$

Date July 1993

Page No. 5-1

\section{HAZARD EVALUATION}

Personnel may be exposed to a variety of substances and physical agents while working at the task site. Exposures may be a result of contacting materials stored, handled, or disposed; equipment being used; weather conditions or time of day; environmental surroundings; and/or task specific working conditions. The FTL should asterisk all suspected hazardous materials pertinent to the task on the list provided in the task specific addendum. Any additional stress agents should also be listed in the task specific addendum. A job hazard analysis using EG\&G Idaho Company Procedure, Number 11.9 shall be used as appropriate to perform portions of the task site hazard evaluation. All personnel working at a task site should be aware of existing hazards.

The following sections provide general information on the types of potential exposures that may be encountered while working at task sites.

\subsection{Chemical Agents}

Exposure to chemical agents may result when personnel come in contact with gaseous, liquid, or solid materials encountered at the investigation sites. Personnel shall make every effort to avoid direct contact with disposed or hazardous materials. Task operations personnel may be exposed to contamination through inhalation, ingestion, absorption (skin/eye), and injection (puncture wound).

- Inhalation of hazardous materials can occur due to lack or improper use of respiratory equipment, malfunctioning monitoring equipment, presence of undetected chemicals, or chemicals in quantities greater than the respiratory equipment protection limits.

- Digestive system may be affected by hazardous substances when workers do not practice good personal hygiene habits (e.g., washing hands thoroughly after completion of work and before smoking, eating, drinking, and chewing gum or tobacco). Inhaling or swallowing airborne hazardous substances may also produce adverse effects to the digestive system.

- Skin absorption of solid, liquid, or gaseous hazardous substances can occur through cuts or abrasions. Skin absorption can occur when a worker does not wear proper protective clothing or when a breach of protective clothing has occurred.

- Eye irritation may develop from solid, liquid, gaseous contaminants. This irritation may occur when a worker does not wear proper eye protection or when unwashed hands come in contact with the eyes.

- Hazardous substances may be injected into the body through puncture wounds occurring from contaminated equipment with sharp edges or protrusions. 
Doc. No.: EGG-ERD-10452

Section No. H\&S Plan

Revision No. 0

Date July 1993

Page No. 5-2

\subsection{Fire and Explosion}

Explosions and fires may occur as a result of activities such as moving drums, accidentally mixing incompatible chemicals, introducing an ignition source into an explosive or flammable environment, or refueling equipment. Intense heat, open flame, smoke inhalation, flying objects, and the release of toxic chemicals into the environment can result.

\subsection{Oxygen Deficiency}

Oxygen deficiency can result from the displacement of oxygen by another gas or the consumption of oxygen by a chemical reaction. Confined spaces or low-lying areas such as pits or trenches are particularly susceptible to oxygen deficiency. The EG\&G Idaho Safety Manual, Section 20 Appendix $A$ and the EG\&G Idaho Company Procedures Manual, Number 11.3, should be reviewed by those working in a confined space.

\subsection{Radiological Hazards}

The potential exists for radiation exposure and radiological contamination to task operations personnel. Contamination is the presence of uncontained radioactive material on any object or surface or in the atmosphere, especially where the presence of radioactive material may be harmful or could be spread if disturbed by an outside agent.

Types of contamination are discussed below.

- Loose contamination is easily spread to adjacent areas and can be ingested or inhaled.

- Fixed contamination is the presence of uncontained radioactive material on surfaces which cannot be easily removed by normal decontamination techniques.

- Airborne contamination is normally in particulate form and is of concern because it can be ingested or inhaled. When inhaled, airborne particulate can deposit in the lungs and diffuse to other parts of the anatomy causing an internal exposure hazard (respiratory protection must be worn when entering an airborne contamination area.)

Contamination may enter the body through

- Absorption

- Injection

- Ingestion

- Inhalation. 
Doc. No.: EGG-ERD-10452

Section No. H\&S Plan

Revision No. 0

Date July 199.3

Page No. 5-3

Radiation is energy emitted from a source that travels in electromagnetic waves or very small particles at various speeds or encrgies. Ionizing radiation is energy emitted from an unstable atom in the form of particles (alpha, beta, neutron) and/or electromagnetic wave or photons (gamma and $x$-ray) which has enough encrgy to interact with ot ter atoms and change their charge. Personnel may be irradiated without contamination but cannot be contaminated without being irradiated.

\subsection{Biological Hazards}

Waste from rescarch facilitics, garbage, and animal feces may contain discase-causing organisms. If these agents are present, they could infect task operations personnel and be dispersed in the environment by water and wind. It is recommended (not required) that operations personnel be immunized against tetanus bacteria, which live in the soil, to minimize the effects of possible exposure.

Encounters with wildlife may be possible at the task site. Snakes, insects, and other animals can and will bite if disturbed and avoidance is the best solution. Prompt first aid should be performed if this type of injury occurs.

\subsection{Industrial Safety Hazards}

Numerous unsafe conditions or actions may be encountered. These may include:

- Existing objects and terrain

- Elevated work arcas

- Lifting heavy objects

- Moving machinery and falling objects

- Personal protective equipment

- Task related equipment

- Excavation, trenching, and shoring.

Task operations personnel should look for potential hazards and immediately inform the FTL of those hazards so that action can be taken to minimize injury due to an unsafe condition or action.

\subsubsection{Existing Objects or Terrain}

Existing objects and terrain can present safety hazards such as:

- Holes and ditches

- Precariously positioned objects (c.g., drums or boards that may fall)

- Sharp objects (e.g., nails, metal shards, and broken glass) 
Doc. No.: EGG-ERD-10452

Section No. H\&S Plan

Revision No. 0

Date July 1993

Page No. 5-4

- $\quad$ Slippery surfaces

- Overhead power lines

- Steep grades

- Uneven terrain

- Unstable surfaces (e.g., walls that may collapse or flooring that may give way)

- Ladders/stairs.

Additional safety hazards introduced by the task should be listed in the task specific addendum.

\subsubsection{Elevated Work Areas}

During the course of task activities, personnel may be required to work on elevated equipment. When such work must be performed, the provisions stated in Section 16 of the EG\&G Idaho Safety Manual shall be followed. In addition, personnel required to work under these conditions shall be trained on the use of elevated equipment.

\subsubsection{Lifting Heavy Objects}

Operations personnel may risk injury by lifting heavy objects. All operations personnel should be trained in the proper method of lifting heavy equipment and cautioned against lifting objects that are too heavy. Mechanical and hydraulic assists will be used whenever possible to minimize lifting dangers.

\subsubsection{Moving Machinery and Falling Objects}

Task operations personnel may be subject to lacerations and contusions (cuts and bruises) when activity involves contact with moving machinery and falling objects. Injury can be minimized by wearing protective clothing, hard hats, steel-toed boots, and using mechanical assists whenever possible. Loose clothing or neck chains for security badges should not be worn and hair should be secured when personnel work around equipment with moving parts or any other potentially hazardous piece of equipment. All moving and rotating machinery must be properly guarded and guarding must remain in place.

\subsubsection{Personal Protective Equipment}

Wearing PPE may reduce a worker's ability to move freely, see clearly, and hear directions and noise that might indicate a hazard. Also, PPE can increase the risk of heat stress. Personnel must adjust their work activities to accommodate limitations. 
Doc. No.: EGG-ERD-10452

Section No. H\&S Plan

Revision No. $\frac{0}{\text { Not }}$

Date July 1993

Page No. 5-5

\subsubsection{Task Related Equipment}

Hazardous equipment and/or situations not mentioned above shall be listed in the task specific addendum. The FTL shall make all personnel aware of possible dangers associated with use of hazardous equipment and/or situations.

\subsubsection{Excavation, Trenching, and Shoring}

Work at hazardous waste sites may involve excavations for purposes of positioning equipment, removal of contaminated soils, removal of underground tanks, or retrieval of containers such as drums, piping systems, or other buried materials. Tasks which involve work in any excavation present serious potential hazards to personnel; personnel protective systems, barricades, signs, and daily inspections are some of the safeguards required for excavation work. For more detail, see the OSHA requirements outlined in 29 CFR 1926, Subpart P - Excavations. In addition, EG\&G Idaho Safety Manual Section 20.0, Appendix B, contains additional requirements which may be more restrictive than those of the OSHA standard.

\subsection{Electrical Hazards}

Overhead power lines, downed electrical wires, and buried cables all pose the danger of shock or electrocution of workers. Electrical equipment may also pose a hazard to workers. Careful observation for overhead electrical hazards will be performed by operating personnel before raising masts on drill rigs or using cranes. Underground utility clearances must be obtained before drilling or excavating operations by contacting Telecommunications (526-1591/526-2512). The EG\&G Idaho Safety Manual, Supplement 2.2 "Safe Work Permits (SWPs)/Special Safe Work Permits (SSWPs)," and Section 10, "Electrical Safety," shall be followed for all work performed near overhead electric lines and electrical work.

\subsection{Heat Stress}

Workers may be required to wear protective clothing that could prevent the body from cooling naturally, thus causing a rise in body temperature. High body temperatures can result in heat fatigue, physical discomfort, and death. The IH must inform the FTL of signs and symptoms of heat stress to preserve safe work conditions at the task site. Work scheduled for summer months is subject to higher ambient temperatures than in winter. Radiant heat can create a hazard in the summer. EG\&G Idaho Company Procedures Manual, Number 11.10 discusses the hazards of heat stress. 
Doc. No.: EGG-ERD-10452

Section No. H\&S Plan

Revision No. 0

Date July 1993

Page No. 5-6

\subsection{Cold Exposure}

Exposure to low temperatures may be a factor if work is done in the evening hours, if winds are high, if unpredictable weather moves in, and in the winter months (e.g., at $50^{\circ} \mathrm{F}$, with a 25 $\mathrm{mph}$ wind, the equivalent chill temperature is $32^{\circ} \mathrm{F}$ ). EG\&G Idaho Company Procedures Manual, Number 11.10 discusses the hazards of cold stress.

\subsection{Noise}

Task operations personnel may be exposed to high levels of noise generated by heavy equipment and other sources.

\subsection{Decontamination}

The chemical and radiological decontamination processes for tools, equipment, clothing, and personnel to remove contaminant generated by the task site activities have the potential for spreading contamination and increasing the exposure to personnel if care is not exercised when decontamination activities are taking place. High pressure hot water and steam used in the process can present a hazard if blasts of either agent rebound into the face or onto the body. In addition, airborne contaminants may result from this process. Decontamination procedures shall be followed and appropriate personal protection shall be used during decontamination activities. Good housekeeping measures will be followed, so that decontamination liquids do not present a hazard.

\subsection{Work Stress}

Hazardous activities that rely on a high degree of personal alertness shall be performed under controlled conditions of job performance as outlined in Section 20 of the EG\&G Idaho Safety Manual. The FTL assumes responsibility of good judgment in the assignment of personnel fatigued by excessive hours of work in psychologically and possibly physiologically stressful environments. 
Doc. No.: EGG-ERD-10452

Section No. H\&S Plan

Revision No. 0

Date July 1993

Page No. 6-1

\section{LEVELS OF PROTECTION AND PERSONAL PROTECTIVE EQUIPMENT}

Selection of PPE is based on the recommendations contained in NIOSH, 10/85. Each work location will be evaluated for potentially hazardous contaminants by the SE, IH, and HPT before entry. Due to the types of known contaminants and the likelihood of unknown contaminants being present, several recommended levels of PPE are described in this section. The levels are Level A, Level B, Level C, and Level D. Radiological control levels include Zone I, Zone II, and Zone III. The required level for PPE will depend on the IH and RE task site hazard assessment, physical conditions, and monitoring data. The level of PPE used at the task site shall be specified by the IH and RE. Changes in PPE level shall be documented in the FTL logbook.

Without compromising the protection from chemical and radiological exposure, and considering the comfort of the workers, Level B and Level C clothing may be modified as stated in Chapter 8 of the referenced NIOSH, 10/85. That decision will be made by the IH and HPT and documented by the FTL in the task specific addendum.

\subsection{Respiratory Protection}

All personnel shall wear only those respirators for which they have been trained and acceptably fit-tested. Respirators shall be used under the recommendation of the IH and HPT. Also, guidelines for respirator use, emergency use, storage, cleaning, and maintenance, as stated in EG\&G Idaho Company Procedures Manual, Number 11.1, shall be followed.

Inspection procedure performed before respirators are used:

- $\quad$ Check to ensure that bag containing respirator is intact and that expiration date stamped on bag has not expired.

- Check to make sure respirator is clean.

- Look for breaks or tears in the headband material. Stretch the bands to ensure sufficient elasticity.

- Ensure that all headbands, fasteners, and adjusters are in place and not bent.

- Check the facepiece for dirt, cracks, tears, or holes. Ensure that the rubber is flexible, not stiff.

- Check the shape of the facepiece for possible distortion that may occur if the respirator is not properly stored. 
Doc. No.: EGG-ERD-10452

Section No. H\&S Plan

Revision No. 0

Date July 1993

Page No. 6-2

- Check the exhalation valve located near the chin between the cartridge holders by:

- Unsnapping the cover,

- Lifting the flexible rubber valve and the valve seat to check for cracks, tears, dirt, and distortion, and,

- After replacing the cover, ensuring that it spins freely.

- Check both inhalation valves located under the respirator cartridges for the same items listed above.

- Check the cartridge holders to ensure that they are clean, necessary gaskets are in place, threads are not worn, and there are no cracks or other visible signs of damage and ensure that they are the correct type of cartridge required for the job.

- Check cartridges (especially the threaded portions) for dents or other damage.

\subsection{Level D Personal Protective Equipment}

Personnel working inside the task site and wearing Level D PPE shall wear, as appropriate:

- Safety glasses (see Section 16 of EG\&G Idaho Safety Manual)

- Safety shoes as described in Supplement 16.4 of the EG\&G Idaho Safety Manual

- Hard hat (see Section 16 of the EG\&G Idaho Safety Manual)

- Hearing protection as required in the EG\&G Idaho Company Procedures Manual, Number 11.7.

\subsection{Level C Personal Protective Equipment}

Personnel working inside the task site and wearing Level C PPE shall wear, as appropriate:

- $\quad$ Full-face or half-face air-purifying respirator (with appropriate filters and eye protection) as required by INEL Health Physics and the IH.

NOTE: The use of half-face respirators is not permitted in a radiological environment without authorization of the program/facility manager in consultation with a radiological engineer per EG\&G Idaho Company Procedures Manual, Number 11.1. 
Doc. No.: EGG-ERD-10452

Section No. H\&S Plan

Revision No. $\quad 0$

Date July 1993

Page No. 6-3

- Disposable chemical-resistant coveralls

- Anti-c clothing as recommended by RE if radiological hazards exist (see Section 6.6 below)

- Safety shoes as described in Supplement 16.4 of the EG\&G Idaho Safety Manual

- Chemically resistant shoe covers

- Hard hat (see Section 16 of the EG\&G Idaho Safety Manual)

- Inner chemically resistant gloves

- Outer chemically resistant gloves

- Hearing protection as required in the EG\&G Idaho Company Procedures Manual, Number 11.7

- Eye protection as required by SE (see Section 16 of the EG\&G Idaho Safety Manual)

- Emergency egress respirator.

\subsection{Level B Personal Protective Equipment}

Level $B$ is the same as Level $C$ except the respiratory protection is upgraded to air supplied hood or SCBA. Personnel working inside the task site with designated Level B PPEs shall wear, as appropriate:

- $\quad$ Air supplied hood or SCBA

- Emergency egress respirator

- Disposable chemically resistant coveralls

- Anti-c clothing as recommended by the RE if radiological hazards exist (see Section 6.6 below)

- Safety shoes as described in Supplement 16.4 of the EG\&G Idaho Safety Manual

- Chemically resistant shoe covers

- Hard hat (see Section 16 of the EG\&G Idaho Safety Manual) 
Doc. No.: EGG-ERD-10452

Section No. H\&S Plan

Revision No. 0

Date July 1993

Page No. 6-4

- Inner chemically resistant gloves

- Outer chemically resistant gloves

- Hearing protection as required in the EG\&G Idaho Company Procedures Manual, Number 11.7.

\subsection{Level A Personal Protective Equipment}

In rare circumstances, it may be necessary for operating personnel to wear Level A PPE. Level $\mathrm{A}$ has the same maximum respiratory protection as Level $\mathrm{B}$; however, the highest available skin and eye protection are required for Level A. All personnel required to wear Level A PPE should include, as appropriate:

- $\quad$ SCBA

- $\quad$ Escape SCBA

- Fully encapsulating, chemically resistant suit

- Additional anti-c clothing as recommended by the RE if radiological hazards exist (see Section 6.6 below)

- Safety shoes as described by Supplement 16.4 of the EG\&G Idaho Safety Manual

- Chemically resistant shoe covers (if applicable)

- Hard hat (if applicable)

- Inner chemically resistant gloves

- Hearing protection as required in the EG\&G Idaho Company Procedures Manual, Number 11.7.

\subsection{Personnel Protection in Radioactively Contaminated Areas}

Anti-c clothing shall be worn in contamination control zones. Personal clothing other than underwear and shoes shall not be worn in Zones II and III. Health Physics personnel (HPT and RE) shall define the anti-c requirements for working in areas on the basis of contamination levels determined by surveys and the guidelines below. For entry into Zones II and III, all openings between the coveralls and shoe covers, gloves, and hood shall be taped. Anti-c clothing shall be donned only at or near the contamination control point of the area to be entered. Guidelines for 
Doc. No.: EGG-ERD-10452

Section No. H\&S Plan

Revision No. 0

Date July 1993

Page No. 6-5

personnel protection in radioactively contaminated areas are contained in the EG\&G Idaho Radiological Controls Manual, Chapter 4, Section 3.5.1. The minimum anti-c personal protection for each contamination zone is presented below.

\subsubsection{Zone I - Low Level Contamination}

The minimum requirements for Zone I anti-c personal protection include:

- One pair of cloth anti-c coveralls (or disposable)

Note: This requirement may be deleted by the HPT for walk-through entries or health physics surveys.

- One pair of shoe covers

- One pair of latex gloves.

\subsubsection{Zone II - Moderate Level Contamination}

The minimum requirements for Zone II anti-c personal protection include:

- One pair yellow cloth anti-c coveralls (or disposable)

- One yellow cloth hood (or disposable)

- Three pairs of shoe covers (two pairs must be vinyl)

- One pair latex gloves.

\subsubsection{Zone III - High Level Contamination}

The minimum requirements for Zone III anti-c personal protection include:

- One pair yellow cloth anti-c coveralls and hood

- One pair disposable anti-c coveralls (or plastic anti-c suit) with disposable hood

- Three pairs of shoe covers (two pairs must be vinyl)

- Two pairs of latex gloves

- Respiratory protection commensurate with contamination levels. 
Doc. No.: EGG-ERD-10452

Section No. H\&S Plan

Revision No.

Date July 1993

Page No. 6-6 


\section{SAFE WORK PRACTICES}

An SWP or SSWP may be required for a task as described in Section 2.4 of the EG\&G Idaho Safety Manual. That section along with Supplement 2.2 describe the types of work that require an SWP or SSWP.

Several factors may affect the safe working environment in the ficld (c.g., inclement weather, confined work space, extended working schedules, work in heavy PPE, temperature, and work done under artificial illumination). These factors can compromise the work performance of task operations personnel. The FTL is responsible for communicating with task operations personnel to ensure safe and efficient work conditions.

\subsection{Working in Confined Spaces}

If work is to be performed in a confined space, the FTL will ensure the area is safe for entry, work, and egress in accordance with EG\&G Idaho Company Procedures Manual, Number 11.3. If appropriate, specific task site instructions for working in confined spaces shall be presented in the task specific addendum. Task operations personnel shall not enter the confined space until safety personnel and the FTL can ensure it to be safe and the SWP is approved.

\subsection{Extended Working Schedules}

If work schedules must be extended, Section 20 in the EG\&G Idaho Safety Manual offers the guidelines and managerial approval needed for personnel working more than a 48-hour week. The FTL is responsible for the safety of task operations personnel; however, when work weeks are in excess of 48 hours, the FTL must realize that physiological and psychological stresses reduce the safety and efficiency of the field operations. Ultimate responsibility for safety of operations belongs to the FTL.

\subsection{Working in Heavy PPE}

Work performed in heavy PPE creates additional stresses which severely limit the ability of operations personnel to work long shifts. The FTL should be aware of such limitations and adjust schedules accordingly. The IH and HPT will advise the FTL on this issue.

\subsection{Working with Artificial Illumination}

If hot and/or windy conditions exist during the regular work shift, schedules may be changed to perform operations at night. Artificial illumination, although a necessity, can create an environment of reduced visibility for the workers. Task operations personnel must be alert and cautious as they maneuver around work areas. 
Doc. No.: EGG-ERD-10452

Section No. H\&S Plan

Revision No.

Date July 1993

Page No. $7-2$

\subsection{Buddy System}

The buddy system is an effective way to ensure each worker is monitored as to his mental and physical well being during the course of a work day. By using the buddy system, task operations personnel can reduce the chance of being ill or injured and not be noticed. This is particularly crucial for workers in the exclusion zone (Section 8.1 of this H\&S Plan). The FTL will pair workers to regularly check on one another during the day's activities. Each member of the pair will observe the other for alertness, motor functions, and coherence.

\subsection{Handling Drums and Containers}

Drums and containers handled during the task shall be addressed in the task specific addendum. Each drum or container shall meet the appropriate DOT, OSHA, and/or EPA regulations for the wastes they contain. The addendum shall address inspection, labeling, handling operations, waste characterization, spill containment, and transportation. EG\&G Idaho Company Procedures Manual, Numbers 8.1, 8.2, and 8.3 address many of the above items. In addition, if the work plan associated with the task addresses the handling and disposing of waste, the work plan shall be referenced in the addendum.

\subsection{ALARA Goals}

The as low as reasonable achievable (ALARA) policy objective is to reduce personnel and environmental radiation exposures and doses to the lowest levels in keeping with good operating practices. The ALARA program establishes annual radiation dosage goals and management commitments to assist in meeting these goals.

Personnel working at the task site must strive to keep his or her radiation exposure ALARA through the following practices:

- Adhere to all written radiological requirements and verbal guidance

- Be aware of personal radiation exposure history

- Work within ALARA guidelines and make suggestions as needed

- Minimize the production of all radiological waste

- Minimize personal radiation exposure by these basic protection techniques:

- Time - exposure is minimized as time is minimized 
Doc. No.: EGG-ERD-10452

Section No. H\&S Plan

Revision No. 0

Date July 1993

Page No. 7-3

- Distance - maintain a maximum distance from radiation source

- Shielding - use any solid material (e.g., lead, steel, concrete) as a shield (Exposure amounts will vary depending on thickness and type of material.)

- Limits - radiation exposure limits are contained in the EG\&G Idaho Radiological Controls Manual, Chapter 2, Section 3.2

- Adhere to general safe work practices discussed in Section 7.9 of this plan.

\subsection{Radioactive Spill Control}

Contamination in uncontrolled areas is designated as a "spill"; if a spill is noticed, task operations personnel shall initiate the SWIMS approach:

- Stop the spill

- Warn area personnel and notify Health Physics

- Isolate the area

- Minimize exposure to the spill

- Secure any ventilation paths and Health Physics surveys the extent of the spill.

Radioactive spill response is discussed in greater detail in the EG\&G Idaho Radiological Controls Manual, Chapter 4, Section 3.8.2.

\subsection{General Safe Work Practices}

The following are general safe work practices to be followed on each task (if work practices vary from those described below, the FTL must record changes in the task specific addendum):

- Contact lenses shall not be worn in company designated eye-hazard areas unless they are essential to correct a vision defect not correctable by prescription safety glasses. Additional restrictions apply as per the EG\&G Idaho Safety Manual, Section 16, paragraph 3.7.

- Eating, drinking, chewing gum or tobacco, smoking, and any other practice that increases the probability of hand-to-mouth transfer and ingestion of material are prohibited within the work/radiation zones. Approved eating areas shall be established or are designated at each Area facility. 
Doc. No.: EGG-ERD-10452

Section No. H\&S Plan

Revision No.

Date July 1993

Page No. $7-4$

- Do not perform work where contaminated substances may be present with an open wound. If a wound is received, report to the HPT and/or IH for further direction.

- Avoid contact with potentially contaminated substances. Do not walk through puddles, pools, mud, etc. Avoid kneeling, leaning, or sitting on equipment or the ground.

- Task operations personnel should watch for dangerous situations (the presence of strong, irritating, and/or nauseating odors, high airborne concentrations of dust, breached drums, etc.). Personnel should report all potentially dangerous situations to the FTL.

- Prevent releases of oil or hazardous materials used in task operations to the extent possible. If spillage occurs, contain it; report it to the facility representative, where applicable; and immediately clean it up in accordance with the Emergency Preparedness Procedures for the Area. Guidelines in Appendix III of the EG\&G Idaho Company Procedures Manual, Number 11.6 for spill cleanup may be useful.

- Prevent splashing of contaminated materials during decontamination.

- Keep all potential ignition sources at least $50 \mathrm{ft}$ from an explosive or flammable environment and use non-sparking, explosion-proof equipment.

- Task operations personnel will familiarize themselves with the physical characteristics of the task site including but not limited to:

- Wind direction

- Accessibility to fellow workers, equipment, and vehicles

- Communications at and near the task site

- Exclusion zones (areas of known or suspected contamination)

- Site access (both Area and Task)

- Nearest water sources

- Warning devices

- Nearest emergency assistance. 
Doc. No.: EGG-ERD-10452

Section No. H\&S Plan

Revision No. 0

Date July 1993

Page No. 7-5

- At all times, a worker in the exclusion zone shall be in line-of-sight contact with his partner.

- Observe your coworker. Look for signs of exhaustion, heat or cold stress, or exposure to harmful vapors. Ask regularly if he/she is okay. Talk to your partner.

- All wastes generated during the task site investigation shall be managed in accordance with the EG\&G Idaho Safety Manual, Section 15.

- Adhere to strict personal hygiene practices such as washing face, neck, and hands before eating, drinking, smoking, or using the restroom. Keep hands away from mouth and eyes when working in an exclusion zone or after handling samples or sample containers. A complete shower may be required at the end of a work shift (IH or HPT discretion).

- Proceed directly to a survey station upon leaving a radiological contamination zone. Care should be taken not to touch the face, mouth, and eyes before a survey has been performed. 
Doc. No.: EGG-ERD-10452

Section No. H\&S Plan

Revision No.

Date July 1993

Page No. 7.6 
Doc. No.: EGG-ERD-10452

Section No. H\&S Plan

Revision No.

Date July 1993

Page No. 8-1

\section{WORK/RADIATION ZONES, SITE ENTRY, AND SECURITY}

Based on the expected levels of contamination and work activity anticipated by each task, several work/radiation zones may be established for the task site. If it is determined that specific zones must be established for a particular task, then entry shall be controlled. Unnecessary personnel shall be excluded. Visitors must (a) notify the FTL in advance of the visit, (b) obtain the required training as specified in Section 3 of this H\&S Plan, and (c) have business at the task site to obtain access.

Figure 8-1 provides an example of an approved work site and its established work zones as recommended by NIOSH, 10/85. If work zones are deemed necessary by the FTL upon the advice of the HPT, SE, and/or IH, each project's established work zones should be documented in the task specific addendum. Several work zones required for Levels A, B, C, and D work activities are:

- Exclusion zone

- Contamination area

- Contamination reduction corridor

- Contamination reduction zone

- Support area.

Radiological control zones will be established or incorporated into the work zones as required by the RE. Task site areas with radiological contamination in excess of the limits established in Chapter 4 of the EG\&G Idaho Radiological Controls Manual shall be posted or labeled as specified in that chapter of the manual.

\subsection{Work Zones}

\subsubsection{Exclusion Zone}

The exclusion zone includes the immediate work area around the contamination area. The minimum number of personnel required to safely perform the required operations will be allowed into the exclusion zone. The cordon around the exclusion zone is called the "Hot Line."

\subsubsection{Contamination Area}

The contamination area is the immediate area inside the exclusion zone where investigation activities are taking place. In this area, operations personnel may be subject to the hazards listed in Section 5 of this H\&S Plan. 
Doc. No.: EGG-ERD-10452

Section No. H\&S Plan

Revision No. 0

Date July 1993

Page No. 8-2

\subsubsection{Contamination Reduction Corridor}

The contamination reduction corridor is a transition area between the exclusion zone and the support zone. This area will serve as a decontamination area for equipment and a PPE removal area for task operations personnel. In addition, this area may contain emergency response equipment, equipment resupply, and a worker temporary rest area. Due to potential contamination, sample packaging and preparation equipment should not be stored here, but rat? $x$, in a contamination free area.

\subsubsection{Contamination Reduction Zone}

The contamination reduction zone is an area that surrounds the exclusion zone and contamination reduction corridor. This area may consist of several work stations (i.e., sampling, handling, and record keeping) as well as staging areas for equipment. The cordon around the contamination reduction zone is called the "contamination control line."

\subsubsection{Support Area}

The support area is the area outside the contamination reduction zone. It may contain the equipment trailer, command post, vehicle parking, equipment staging, or any support activity related to the task at hand. All personnel not trained in hazardous material work and visitors are restricted to this area.

\subsection{Radiological Control Zones}

External radiation control areas and radioactive contamination zones are identified and posted as radiological hazards through the use of barriers and postings. Barriers are used to help confine radiological hazards to a specific area. Yellow and magenta ribbons, ropes, tags, and signs are used to keep unauthorized personnel out of the area. External radiation control areas and radioactive contamination zones shall be posted in accordance with the EG\&G Idaho Company Procedures Manual, Number 10.10 and the EG\&G Radiological Controls Manual, Chapters 2 and 4. Task specific radiation control areas and contamination zones shall be determined by the RE and HPT and documented in the addendum.

\subsubsection{External Radiation Exposure Control}

External exposure control is accomplished by identifying areas containing sources of radiation and controlling personnel access into these areas. Section 2 of the EG\&G Idaho Radiological Controls Manual discusses external radiation exposure control requirements. These areas shall be posted in accordance with the provisions stated above. External exposure control is achieved through the following: 
Doc. No.: EGG-ERD-10452

Section No. H\&S Plan

Revision No.

Date July 1993

Page No. 8-3

a. Controlled Area - Any area where radioactive materials or elevated radiation fields may be present shall be clearly and conspicuously posted as a controlled area.

b. Radiation Area - Any area within a controlled area where an individual can receive a dose equivalent greater than $5 \mathrm{mrem}$ but less that $100 \mathrm{mrem}$ in $1 \mathrm{hr}$ at $30 \mathrm{~cm}$ from the radiation source.

c. High Radiation Area - Any area within a controlled area where an individual can receive a dose equivalent of 100 mrem or greater but less than $5 \mathrm{rem}$ in $1 \mathrm{hr}$ at $30 \mathrm{~cm}$ from the radiation source.

d. Very High Radiation Area - Any area within a controlled area where an individual can receive a dose of $5 \mathrm{rem}$ or greater in $1 \mathrm{hr}$ at $30 \mathrm{~cm}$ from the radiation source. Access to these areas shall be maintained, locked, or physically guarded.

\subsubsection{Radioactive Contamination Control}

Radioactive contamination controls limit the amount of radioactive surface contamination which individuals are exposed to minimize possible inhalation, ingestion, or absorption of radioactive material; to minimize the potential for release of radioactivity to the environment; and to prevent external contamination of personnel. Contamination limits are set primarily to define "detectability" or the lower limit of detection under ideal conditions. The fundamental philosophy is that no "detectable" contamination will be released to uncontrolled areas. The limits are not based on hazards to personnel but to maintain a high degree of control, restricting radioactive contaminants by engineered barriers. Chapter 4 of the EG\&G Idaho Radiological Controls Manual discusses radioactive contamination control in more detail.

Contamination is classified as Zone I, II, or III based on contamination levels as follows:

- Zone I Limits of Contamination

- $\quad 200$ to $5,000 \mathrm{dpm} / 100 \mathrm{~cm}^{2}$ beta-gamma

20 to $50 \mathrm{dpm} / 100 \mathrm{~cm}^{2}$ alpha

- Zone II Limits of Contamination

- $\quad>5,000$ to $20,000 \mathrm{dpm} / 100 \mathrm{~cm}^{2}$ beta-gamma

$>50$ to $250 \mathrm{dpm} / 100 \mathrm{~cm}^{2}$ alpha

- Zone III Limits of Contamination

- $\quad>20,000 \mathrm{dpm} / 100 \mathrm{~cm}^{2}$ beta-gamma

$>250 \mathrm{dpm} / 100 \mathrm{~cm}^{2}$ alpha. 
Doc. No.: EGG-ERD-10452

Section No. H\&S Plan

Revision No. 0

Date July 1993

Page No. 8-4 
Doc. No.: EGG-ERD-10452

Section No. H\&S Plan

Revision No. $\frac{0}{1}$

Date July 1993

Page No. 9-1

\section{ENVIRONMENTAL AND PERSONNEL MONITORING}

Employee exposure to contaminants and physical hazards will be monitored during all task site activities using an appropriate combination of techniques. The FTL in conjunction with the appropriate personnel (e.g., IH, HSO, RE, SE, and HPT) for each task shall list any monitoring equipment requirements for specific potential hazards in the task specific addendum. An example of items that may be monitored is:

1. Organic vapor using an organic vapor monitor

2. Combustible gas using a combustible gas indicator

3. Heat or cold stress using field measurements and observations and, if necessary, body temperature measurements

4. Radiation and contamination surveys using radiological monitoring equipment

5. Personal exposure to organic vapors, particulate contamination (heavy metals) using personal monitoring pumps and appropriate filter collection media (active sampling)

6. Personal exposure to radiation using thermoluminesent dosimeters (TLDs) and direct reading dosimeters (DRDs)

7. Mercury vapors using a mercury vapor detector

8. Noise levels using a sound level meter and/or noise dosimeter

9. Loose radiological contamination using smears or large area wipes.

\subsection{Chemical Exposure Monitoring}

Selective monitoring of high-risk task operations personnel at the chest or face level for organic vapors may be recommended by the IH. The monitoring devices used, frequency of monitoring, designated high-risk jobs to be monitored, and action levels for hazardous contaminants shall be discussed in the task specific addendum.

Equipment for monitoring organic vapors at the task site shall be identified by the IH. The equipment, monitoring schedule, and calibration methods shall be discussed in the task specific addendum. The monitoring activities shall be initially based on the job hazard analysis results. 
Doc. No.: EGG-ERD-10452

Section No. H\&S Plan

Revision No. 0

Date July 1993

Page No. 9-2

\subsection{Combustible Gas Monitoring}

If deemed necessary by the SE, the task site will be monitored for combustible gases at time intervals recommended by the SE. Elevated readings from the organic vapor detector might indicate the presence of combustible gases. The SE and IH will advise the FTL on circumstances when work at the task site will be suspended and the course of corrective action, and ensure the task site is safe before work continues. Action levels for combustible gases shall be documented in the task specific addendum.

\subsection{Radiological Monitoring}

The RE and HPT will be responsible for radiological monitoring in accordance with the EG\&G Idaho Radiological Controls Manual, Chapters 2 and 4; and Section 10 of the EG\&G Idaho Company Procedures Manual.

\subsubsection{External Radiation Exposure Control}

Personnel exposures are monitored by TLDs and DRDs. Personnel are responsible for properly wearing the specified dosimetry while in radiologically controlled areas. If the TLD (or other dosimetry) is lost, task operations personnel shall immediately notify the FTL and HPT. TLDs are supplied and processed by the Operational Dosimetry Unit.

Radiation surveys shall be performed by the HPT to determine the extent and magnitude of radiation levels and to enable posting of radiation areas. Surveys shall be performed in accordance with the EG\&G Idaho Radiological Controls Manual, Chapter 2, Section 3.8.

\subsubsection{Radioactive Contamination Control}

All surfaces or areas with contamination levels in excess of those levels stated in Section 8.2 of this plan shall be monitored and controlled to prevent the spread of contamination. Contamination surveys shall be performed by the HPT in accordance with the EG\&G Idaho Radiological Controls Manual, Chapter 4, Section 3.3.

All personnel shall obtain a whole body survey after exiting a contamination zone; the whole body survey must be done for two to three minutes. The following portable instruments are most commonly used to detect personnel contamination: (a) Ludlum 2a, (b) Eberline RM-14, and (c) Ludlum 177 with pancake probe (frisker), for beta-gamma contamination; and (d) Ludlum Model 61 and (e) Eberline Pac-4s, for alpha contamination. In addition to portable field instruments, the following personnel contamination monitors may be used: (a) large area detectors, (b) portal monitors, (c) personnel contamination monitors, and (d) hand and foot monitors. 
Doc. No.: EGG-ERD-10452

Section No. H\&S Plan

Revision No. $\quad 0$

Date July 1993

Page No. 9-3

\subsection{Heat and Cold Stress Control and Monitoring}

The FTL will set work/rest schedules as recommended by the IH. Depending upon the ambient weather conditions or work conditions and physical response of task operations personnel, the IH will suggest adjustments of the work/rest cycle to the FTL. The FTL, HSO, and/or IH will ensure that operations personnel follow established work and break schedules, adequately replace body fluids, and keep body temperatures in a normal range in accordance with the EG\&G Idaho Company Procedures Manual, Number 11.10 .

Workers will be interviewed by the IH and/or HSO periodically to ensure that the controls are effective and excessive heat exposure is not occurring. Workers will be encouraged to monitor their body signs and take a break if symptoms of heat stress occur.

Task operations personnel shall be aware of the following signs and symptoms of heat stress:

$\begin{array}{ll}\text { - } & \text { Confusion } \\ \text { - } & \text { Fainting } \\ \text { - } & \text { Clurred speech } \\ \text { - } & \text { Dizziness } \\ \text { - } & \text { Fatigue } \\ \text { - } & \text { Nausea } \\ \text { - } & \text { Profuse sweating } \\ \text { - } & \text { Skin color change } \\ \text { - } & \text { Vision problems. }\end{array}$

Task operations personnel who exhibit any of these symptoms will be immediately removed from the task site. An individual who shows any of the symptoms that are capitalized and underlined, or any other evidence of change in level of consciousness, will be transported to an OMP facility for medical evaluation. Mental confusion and decreased level of consciousness must always be considered an emergency requiring medical evaluation and treatment. Transportation to a medical facility or use of an ambulance should be considered normal procedure in this situation. Individuals showing any of the remaining symptoms listed will be provided cool water and allowed to rest. On any occasion when the FTL, worker experiencing the heat stress symptoms, or $\mathrm{IH}$ believes the heat stress is severe or desires medical evaluation, the employee may be brought to an OMP medical facility.

Rest breaks shall include the following preventive measures:

- Drink adequate liquids

- Rest in a cool, shaded area

- Remove protective clothing to allow evaporative cooling 
Doc. No.: EGG-ERD-10452

Section No. H\&S Plan

Revision No. $\frac{0}{0}$

Date July 1993

Page No. 9.4

- Do not perform other work during the break.

If personnel are wearing semipermeable or impermeable PPE, the work/rest schedule may be adjusted and monitoring of individual personnel temperatures may be required by the $\mathrm{IH}$. If ambient temperatures are considered excessive by the $\mathrm{IH}$ and/or symptoms outlined above exhibited, workers must be monitored for heat stress and recovery. This includes measuring heart rates and temperatures. Temperatures can be obtained using disposable thermometers. The HSO will ensure that sufficient liquids (electrolyte replacement fluids such as Gatorade) are provided and that they are consumed only in the designated and approved eating/drinking area.

Adequate protective clothing as required by IH should be worn to protect against the cold. Extra care must be exercised while working in this environment. Workers should observe each others facial extremities (ears and nose) for signs of frostbite (whitening of the skin surface). Decreased mental coherence and body movements are signs of hypothermia. Individuals with suspected hypothermia or other significant cold injury (e.g., frostbite) will be taken to an OMP medical facility.

Finally, the FTL or IH will refer a worker to the OMP for medical evaluation whenever there is doubt concerning the medical ability of an employee to continue in the assigned task.

\subsection{Noise-Level Monitoring}

If high noise levels are encountered by operations personnel at the task site, worker exposure will be assessed by the IH. A hearing conservation program must be developed by the IH when the sound levels exceed an 8-hour time weighted average of $85 \mathrm{dBA}$. Noise level monitoring, PPE requirements, and audiometric tests shall be outlined in the hearing conservation program for the task or employee. Requirements shall be imposed by the PM based on the advice of the IH and the requirements stated in the EG\&G Company Procedures Manual, Number 11.7 and the EG\&G Industrial Hygiene Manual, Section 26.

\subsection{Physical Hazard Control and Monitoring}

The FTL will have the primary responsibility for ensuring the task site is maintained in a safe condition by requiring maintenance of barriers and signs, correction of unsafe conditions, and cleaning of debris and trash. The appropriate personnel (e.g., IH, SE, and HPT) will inspect and recommend changes in work habits to the FTL.

Individuals working on a task have a specific responsibility to use safe work techniques, report unsafe working conditions, and exercise good personal hygiene and housekeeping habits throughout the course of their job. 
Doc. No.: EGG-ERD-10452

Section No. H\&S Plan

Revision No. $\frac{0}{\text { Not }}$

Date July 1993

Page No. 9-5

\subsection{Record Keeping Requirements}

ERP is required to maintain the following information in the ARDC program file in accordance with 29 CFR 1910.120:

- Copies of the Management Plan for the Environmental Restoration Program, Task Specific H\&S Plan, QPP, QAPjP, and work plan.

In addition, ERP shall track the following information for each ERP hazardous material worker through the Training and Emergency Action Unit of the Waste Management Operations Support Group:

- Proof of training in health and safety hazard recognition, radiation worker training, respirator training, and any other training specific to the employee

- $\quad$ Required training and updates

- Copy of the signed Health and Safety Certification Form.

The $\mathrm{IH}$ is required to maintain a logbook of air monitoring data, personal sampling data, times of sampling intervals, calibration of instruments, and identity of personnel wearing the monitoring equipment. Instrumentation detection ranges and uncertaintics should also be recorded in the IH logbook. The HPT is required to keep a logbook of all radiological monitoring, daily operational activities, and instrument calibrations. All project records and logbooks, except HPT logbooks, shall be forwarded to ARDC within 30 days after completion of the task. 
Doc. No.: EGG-ERD-10452

Section No. H\&S Plan

Revision No.

Date July 1993

Page No. 9.6 
Doc. No.: EGG-ERD-10452

Section No. H\&S Plan

Revision No. 0

Date July 1993

Page No. 10-1

\section{DECONTAMINATION PROCEDURES}

Decontamination procedures for personnel and equipment are necessary to control contamination and to protect operations personnel. Both chemical and radiological decontamination are discussed in this section. However, combined chemical and radiation decontamination procedures are not discussed here and must be developed by the IH and RE if required for a specific task. Decontamination procedures shall be presented in the task specific addendum. These procedures can be amended upon recommendations by the IH, RE and/or HPT. When chemically hazardous material decontamination or radiological decontamination is required, the following procedures are suggested.

\subsection{Modified Level A and B Decontamination Procedures}

If Level A or B PPE is required, then two decontamination stations will be used at the task site--one at the hotline between the exclusion zone and the contamination reduction corridor and one at the contamination control line, which is the personnel access point to the support zone from the contamination reduction corridor. Decontamination Station A supports personnel and equipment exiting the exclusion zone. Figure 10-1 lists the recommended decontamination procedures. Steps 1 through 8 shall be completed at Station A. Coveralls shall be removed at Station B.

\subsection{Modifled Level C Decontamination Procedures}

Decontamination Station B should be located at the personnel access to the contamination reduction corridor. It is to be used by personnel working in the contamination reduction corridor. Figure 10-2 lists the modified Level $C$ decontamination procedures. If Tyveks are worn (if recommended by the $\mathrm{IH}$ ), they are decontaminated and removed at Station $\mathrm{A}$.

\subsection{Radiological Decontamination}

Radiological decontamination shall be done under the direct supervision of Health Physics (RE and/or HPT) and in accordance with the EG\&G Idaho Company Procedures Manual, Number 10.4. Figures 10-3, 10-4, and 10-5 provide the anti-c removal steps for the three contamination control zone designations. Any personnel and personal property contamination may be removed with tape, vacuuming (vacuum must be equipped with a high efficiency particulate air filter), washing with soap and water, or by mechanical means (grinding, etc.). 
Doc. No.: EGG-ERD-10452

Section No. H\&S Plan

Revision No.

Date July 1993

Page No. 10-2

1. Remove equipment

2. Wash and rinse boot covers and gloves

3. Remove tape

4. Remove boot covers, outer gloves, and hood

5. Disconnect air hose and tape end

6. Wash and rinse suit and boots

7. Remove suit

8. Wash, rinse, and remove inner gloves

9. Remove coveralls

10. Field wash/shower

11. Put on personal clothing
EXCLUSION

ZONE

HOT LINE

(STATION A)

CONTAMINATION

REDUCTION

CORRIDOR

CONTAMINATION CONTROL LINE (STATION B)

SUPPORT ZONE

Figure 10-1. Recommended modified Level A and B PPE hazardous chemical decontamination steps. 
Doc. No.: EGG-ERD-10452 Section No. H\&S Plan Revision No. 0 Date July 1993 Page No. 10-3

1. Remove equipment

CONTAMINATION

2. Wash and rinse boot covers and gloves (if worn)

3. Remove tape

4. Remove boot covers and outer gloves

5. Wash, rinse, and remove boots and suit (if worn)

6. Remove and drop respirator

7. Wash, rinse, and remove inner gloves

8. Remove coveralls CORRIDOR

9. Field wash/shower CONTROL LINE (STATION B)

10. Put on personal clothing

Figure 10-2. Recommended modified Level C PPE hazardous chemical decontamination steps. 
Doc. No.: EGG-ERD-10452

Section No. H\&S Plan

Revision No. 0

Date July 1993

Page No. $10-4$

1. Remove outer shoe covers

2. Remove gloves

3. Remove coveralls

4. Remove shoe covers (during the process of stepping through barrier)

5. Remove cloth glove liners

Figure 10-3. Anti-c removal steps for radiological control Zone I. 
Doc. No.: EGG-ERD-10452 Section No. H\&S Plan

Revision No. 0

Date July 1993

Page No. 10-5

\section{Zone II Removal Steps}

1. Remove outer shoe covers

2. Remove latex gloves

3. Remove hood, coveralls, and shoe covers (remove shoe covers during process of stepping through barrier)

4. Remove cloth glove liners

Figure 10-4. Anti-c removal steps for radiological control Zone II. 
Doc. No.: EGG-ERD-10452

Section No. H\&S Plan

Revision No.

Date July 1993

Page No. 10-6

\section{Zone III Kumoval Barrier}

1. Remove outer shoe covers

2. Remove latex gloves

3. Remove disposable hood, coveralls, and shoe covers (remove shoe covers during process of stepping through barrier)

4. Pull inner hood back and remove respirator

EGRESS POINT A

5. Remove inner latex gloves

6. Remove cloth hood, coveralls, and shoe covers (remove shoe covers during process of stepping through barrier)

7. Remove cloth glove liners

EGRESS POINT B

Figure 10-5. Anti-c removal steps for radiological control Zone III. 
Doc. No.: EGG-ERD-10452

Section No. H\&S Plan

Revision No. 0

Date July 1993

Page No. 10-7

\subsection{Equipment Decontamination and Disposal of Contaminated Materials}

Decontamination procedures for equipment shall be recorded or referenced in the task specific addendum. All waste generated by performing decontamination must be disposed in accordance with Section 15 of the EG\&G Safety Manual for hazardous nonradioactive waste and radioactive mixed waste. Radioactive waste shall be handled in accordance with the EG\&G Idaho Radiological Controls Manual, Chapter 6. Disposable clothing, tools, buckets, brushes, and other contaminated equipment shall be secured and disposed as stated in the task specific addendum. Unused contaminated equipment that can be used at a later time shall be placed in plastic bags and stored at the task site. Decontamination of monitoring equipment should also be addressed.

Radioactive waste shall be handled in accordance with the EG\&G Idaho Radiological Controls Manual, Chapter 6. Decontamination operations for equipment and areas shall be performed in accordance with approved procedures.

\subsection{Decontamination During Medical Emergencies}

If a person is injured or becomes ill and lifesaving care is required, the situation will be evaluated by the appropriate personnel (e.g., first aid personnel) on a case-by-case basis. Emergency care will be initiated in accordance with the emergency preparedness procedure for the facility at which the task is being performed. Medical care necessary to save life or limb is not delayed for decontamination. In such cases, decontamination may be performed at the medical facility. The IH and/or HPT will accompany the employee to the medical facility and relay information requested by medical personnel. 
Doc. No.: EGG-ERD-10452

Section No. H\&S Plan

Revision No. 0

Date July 1993

Page No. 10-8 
Doc. No.: EGG-ERD-10452

Section No. H\&S Plan

Revision No. 0

Date suly 1993

Page No. 11-1

\section{EMERGENCY PROCEDURES, EQUIPMENT, AND INFORMATION}

Work at hazardous waste sites makes emergencies a continuous possibility, no matter how infrequently emergencies may occur. Emergencies happen quickly, unexpectedly, and require immediate response. The reporting requirements of Section 3 of the EG\&G Idaho Safety Manual shall be followed by personnel at the task site. Locations and telephone numbers of emergency personnel and facilities will be posted at places specified in the task specific addendum. The appropriate emergency facilities will be notified by telephone at the beginning of the task to inform personnel at the facilities that work has begun at the task site. The following sections describe the procedures used during emergency situations; equipment that will be available for emergency situations; and agencies, facilities, and personnel who must be notified in case of emergency.

\subsection{Emergency Procedures}

The following procedures will be used if an emergency arises:

- $\quad$ FTL will be notified of accidents or conditions that have the potential for adversely affecting or threatening personnel safety, property, or environment. The FTL is responsible for ensuring that the EG\&G Idaho Safety Manual and the emergency action procedure for the facility are followed in the event of an accident or unusual condition.

- All safety related occurrences will be recorded in a field logbook and reported as indicated in Section 3 of the EG\&G Idaho Safety Manual.

\subsubsection{Personnel Occupational Injury or IIIness in the Exclusion Zone}

In the event of an occupational injury or illness in the exclusion zone, an assessment of the situation shall be made by the FTL using the advice of appropriate personnel (e.g., IH, SE, personnel trained in first aid). If the situation is deemed reportable as described in Section 3 of the EG\&G Idaho Safety Manual, the FTL is responsible for initiating reporting procedures. In addition, task personnel shall act in accordance with the emergency preparedness procedures for the facility at which the task is being performed. In the event that the task site is shut down due to an injury, task operations perronnel shall not reenter the exclusion zone until the cause of the injury or illness is identified and corrective action implemented. Decontamination shall be performed in accordance with the above mentioned emergency procedures and with recommendations made by the IH, HPT, and/or first aid personnel. 
Doc. No.: EGG-ERD-10452

Section No. H\&S Plan

Revision No. 0

Date July 1993

Page No. 11-2

\subsubsection{Personnel Occupational Injury or Illness in the Support Zone}

If an occupational injury or illness occurs in the support zone, the same procedures as described in Section 11.1.1 shall be followed. If the FTL determines the cause of the occupational injury or illness and the absence of the injured or ill party does not affect the performance of other personnel, task operations will continue.

\subsubsection{Transportation and Followup of Injury}

An injured worker transported to a medical facility will be accompanied by at least one worker (preferably the IH and/or HPT) to inform medical personnel of the level of decontamination performed before leaving the task site and provide specific details about the illness or injury.

\subsubsection{Fire/Explosion}

Before initiating task activities, brush and grass will be cleared from the task site to eliminate the risk of fire. The EG\&G Idaho Safety Manual, Section 11 and any applicable facility emergency preparedness procedures shall be reviewed.

In the event of a fire or explosion, all personnel not essential to controlling the situation will be evacuated from the task site, and fire and/or explosive experts will be notificd. In addition, Section 11 of the EG\&G Idaho Safety Manual and applicable emergency action procedures for the facility at which the task is being performed shall be followed.

\subsubsection{Personal Protective Equipment Failure}

If any task site worker experiences a failure or alteration of PPE, that person and his workmate shall immediately leave the exclusion zone. The HPT and IH will assess the situation and determine if exposure to hazardous substance or radiological uptake has occurred. Reentry will not be permitted until the equipment has been repaired or replaced.

\subsubsection{Other Equipment Failure or Hazardous Material Spill}

If task site equipment fails to operate properly, the FTL will be notified and will determine the effect of the failure on continuing operations. If the failure affects the safety of personnel or prevents completion of the tasks described in the SAP or other work plan, operations personnel shall leave the task site until the situation is evaluated and appropriate actions are taken.

If hazardous or potentially hazardous material is spilled, refer to the emergency preparedness procedure for the Area in which the task is being performed and report the spill to Area 
personnel as directed. Spillage of petroleum products, decontamination solutions, calibration material, equipment fuels, and other liquids containing hazardous materials must be assessed.

\subsubsection{Hand Signals}

Hand signals shall be used if an emergency situation arises and communication becomes impossible or unsafe. The following hand signals will be used in an emergency:

- Hand gripping throat - signals that the person is out of air or cannot breathe

- Grip partner's wrist or both hands around waist - means leave area immediately

- Hands on top of head - signals that assistance is needed

- Thumbs up - okay, I am all right, I understand

- Thumbs down - no, I am not all right, I do not understand.

\subsubsection{Emergency Escape}

In cases of life-threatening emergencies such as fire or explosion, personnel should leave the vicinity using the shortest possible route without regard for decontamination at that time and move upwind of the affected area. When the situation has stabilized, personnel will take necessary steps to decontaminate themselves, equipment, and other affected areas.

\subsubsection{Task Operations Shutdown}

Task operations may be suspended for several reasons as indicated below. However, the reasons for operations shutdown are directly related to the degree of hazard each task possesses. Specific reasons for suspending task operations should be listed in the task specific addendum. Examples include excessive vapor/gas concentrations, radiological hazards, uncovering waste, inclement weather, etc.

- If a combustible gas indication $>10 \%$ of the lower explosive limit (LEL) occurs indicating a buildup of explosive vapors, work shall stop. Evaluation of the situation will be made and a course of action determined by the FTL in conjunction with the IH and/or SE.

- When significant radiological hazards are identified by an HPT at the sampling site.

- When unexpected hazardous material is uncovered or found in soil samples, even when the appearance of such material may not be associated with a rise in detected contamination levels. 
Doc. No.: EGG-ERD-10452

Section No. H\&S Plan

Revision No.

Date July 1993

Page No. $11-4$

- In addition, drilling, sampling, instrumentation, and other weather sensitive activities will stop during consistent high winds (i.e., $>25 \mathrm{mph}$ ), electrical storms, or other inclement weather that may affect the work.

\subsubsection{Task Site Reentry}

In all situations, when a task site emergency results in evacuation of the task site, personnel shall not reenter until authorized to do so by the FTL. The FTL will ensure that:

1. The hazards have been reassessed by the HSO, IH, SE, and/or the RE.

2. The conditions resulting in the emergency have been corrected.

3. The task specific H\&S Plan, SAP, Operational Safety Requirements/Safety Assessments, Standard Operating Procedures (SOPs), DOPs, and the Facility Emergency Action Plan have been reviewed as appropriate.

4. Site personnel have been briefed on any changes in the ERP task specific H\&S Plan.

Reentry into an evacuated zone to monitor or collect air samples requires the more restrictive of Level C PPE or the level used by those individuals who evacuated the task site. The IH may upgrade to Level B or A if deemed necessary.

\subsection{Warning Devices}

Warning lights and/or audible alarms shall be installed in areas where needed to warn personnel against remaining in or entering a hazardous area. An explanatory sign or tag shall be posted immediately adjacent to a warning device to describe the hazardous condition and indicate the action to be taken. Table 12.1 in Section 12 of the EG\&G Idaho Safety Manual lists various audible warning devices, their meanings, and the required personnel action. Specific warning devices for the task shall be listed in the task specific addendum. Warning devices for radiological hazards (e.g., remote air monitors) shall also be listed.

\subsection{Emergency Equipment}

The following emergency equipment shall be available at the task site during field operations as appropriate. (A complete emergency equipment list shall be provided in the task specific addendum.)

Fire Extinguishers: Because of the potential threat of fire at hazardous waste sites, at least one 20-lb (minimum) $\mathrm{ABC}$ fire extinguisher will be readily available and at hand throughout the task 
activities. Additional fire extinguishers may be necessary. This should be indicated in the task specific addendum.

SCBA: Two SCBAs will be available for emergen 'es such as reentering a contaminated zone to retrieve injured personnel.

First Aid Kits: An industrial first aid kit with sufficient supplies for five people shall be kept in the support zone. The OMP will advise on the selection of first aid supplies to be included at each task site. The HSO will be responsible for maintaining the proper level of first aid supplies in the task site first aid kit.

Eye Wash: Portable eyewash fountains with sufficient potable water for flushing will be readily available for the duration of the task. The location of the eyewash will be determined by the IH.

Communications: Emergency telephone numbers shall be included in the task specific addendum and posted for all operations personnel. Emergency communication shall be discussed in the safety training prior to initiation of site investigation activities. A two-way radio or telephone with capability to contact emergency personnel shall be located on cach task site.

Personal Hygiene: A sufficient supply of clean wster, hand soap, and towels will be provided at the task site.

Radiological Contamination Spill Kit: Depending on the location of the task and recommendation from $\mathrm{RE}$, a spill kit shall be prepared in advance and located in appropriate work areas. These kits shall contain, at a minimum, the following radiological control equipment:

- $\quad$ Plastic Bags

- $\quad$ Absorbent materials (e.g., paper or rags)

- Latex gloves and glove liners

- Plastic shoe covers and/or rubber overshoes

- Smear paper and holders

- Pencils, grease pencils, and paper

- Radiological tags and signs and radiation rope or ribbon

- Yellow plastic sheeting and duct tape. 
Doc. No.: EGG-ERD-10452

Section No. H\&S Plan

Revision No. 0

Date July 1993

Page No. 11-6 
Doc. No.: EGG-ERD-10452

Section No. H\&S Plan

Revision No. 0

Date July 1993

Page No. 12-1

\section{BIBLIOGRAPHY}

American Conference of Governmental Industrial Hygienists, Threshold Limit Values and Biological Exposure Indices for 1989-1990, Second Printing.

EG\&G Idaho, EG\&G Idaho Company Procedures Manual.

EG\&G Idaho, EG\&G Emergency Action Manual.

EG\&G Idaho, Industrial Hygiene Manual.

EG\&G Idaho, Radiological Controls Manual.

EG\&G Idaho, Safety Manual.

EG\&G Idaho Environmental Restoration Program, Health and Safety Plan for Operations Performed for the Buried Waste Program, Environmental Restoration Program, EGG-WM-8504, May 1989.

EG\&G Idaho Environmental Restoration Program, Management Plan for the EG\&G

Environmental Restoration Program, EGG-WM-8676.

EG\&G Idaho Environmental Restoration Program, Program Directives.

National Archives and Records Administration, Code of Federal Regulations, 29 CFR 1910.120, "Hazardous Waste Operations and Emergency Response."

National Archives and Records Administration, Code of Federal Regulations, 29 CFR 1926, Subpart P, "Excavations."

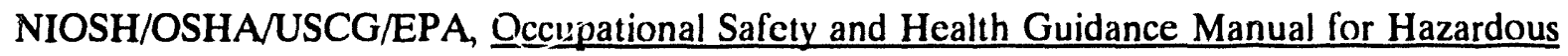
Waste Site Activities, October 1985.

Hazardous Material References:

Buried Sludge Waste Characterization, TLC-29-88, T. L. Clements, Jr. Itr to C. J. Bonzon, May 2, 1988.

Engineering Design File BWP-ISV-004 Jetailed Estimate of Radioactive Contents for Pit 9, E. C. Garcia and J. L. Knight. 
Doc. No.: EGG-ERD-10452

Section No. H\&S Plan

Revision No.

Date July 1993

Page No. 12-2

Estimate of Rocky Flats Plant Organic Wastes Shipped to the RWMC, D. E. Kudera, July 24, 1987.

Radioactive Waste Management Information System content code material listing, 1954 to 1970. 
Doc. No.: EGG-ERD-10452

Section No. H\&S Plan Revision No.

Date Julv 1993

Page No. A-1

Addendum

Health and Safety Plan

for the CFA Landfills II and III

Remedial Investigation/Feasibility Study 
Doc. No.: EGG-ERD-10452

Section No. H\&S Plan

Revision No.

Date July $\overline{993}$

Page No. A-2 


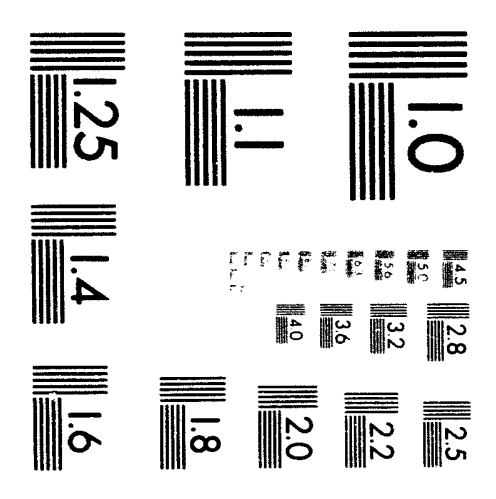



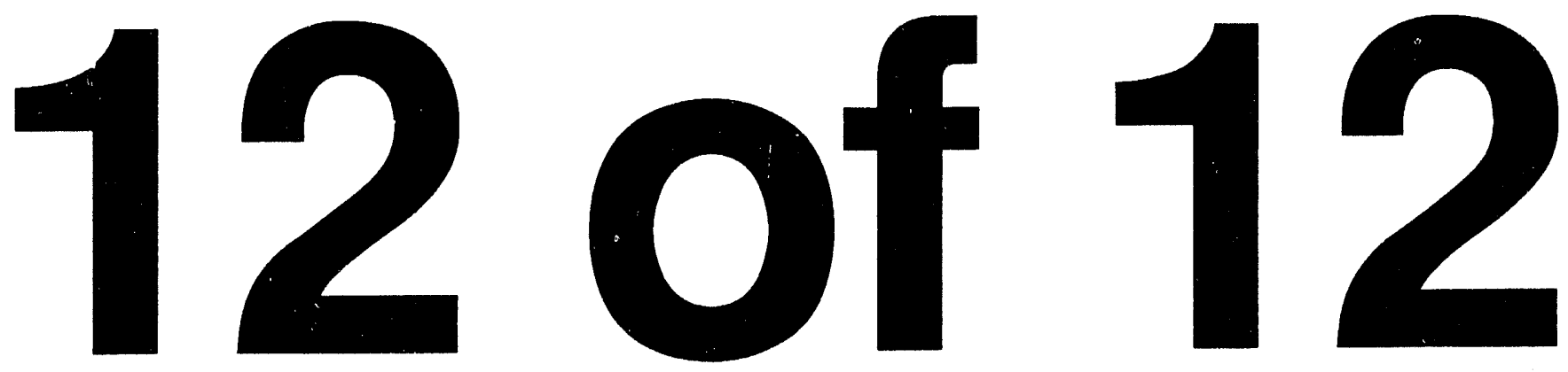
Doc. No.: EGG-ERD-10452

Section No. H\&S Plan Revision No. 0 Date July 1993 Page No. $A-3$

\section{Addendum}

\section{Health and Safety Plan for the CFA Landfills II and III Remedial Investigation/Feasibility Study}

Reviewed by:

J. P. Shea, Chairman, ER Independent Review Committee Date

W. R. Pigott, Manager, WAG 4

Date

W. N. Wyland, Landlord, Central Facilities Area

Date

P. N. Creighton, Occupational Medical Program

Date

Approved by:

R. P. Wadkins, Unit Manager, WAGs 1,4 , and 5

Date 
Doc. No.: EGG-ERD-10452

Section No. H\&S Plan

Revision No.

Date July 1993

Page No. A-4 
Doc. No.: EGG-ERD-10452

Section No. H\&S Plan

Revision No. 0

Date July 1993

Page No. $A-5$

\section{CONTENTS}

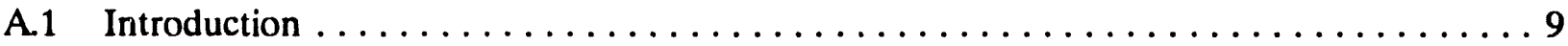

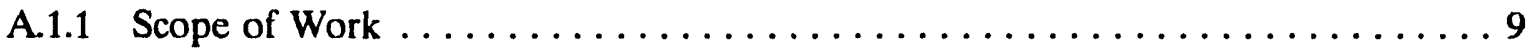

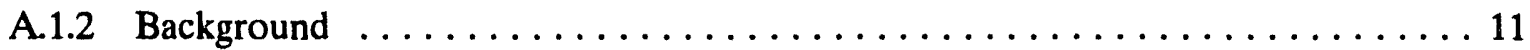

A.2 Responsibilities $\ldots \ldots \ldots \ldots \ldots \ldots \ldots \ldots \ldots \ldots \ldots \ldots \ldots \ldots \ldots \ldots \ldots$

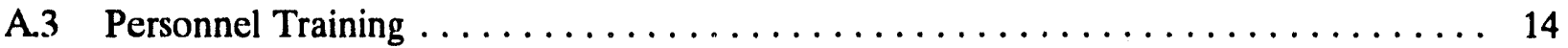

A.4 Medical Surveillance Program $\ldots \ldots \ldots \ldots \ldots \ldots \ldots \ldots \ldots \ldots \ldots \ldots \ldots$

A.5 Hazard Evaluation $\ldots \ldots \ldots \ldots \ldots \ldots \ldots \ldots \ldots \ldots \ldots \ldots \ldots \ldots \ldots$

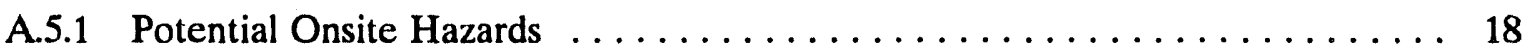

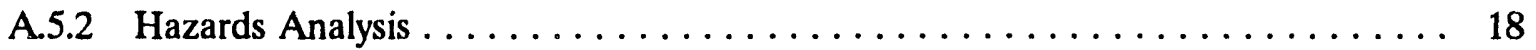

A.6 Levels of Protection and Personal Protective Equipment $\ldots \ldots \ldots \ldots \ldots \ldots \ldots$

A.6.1 Personal Protection Used on Previous Site Visits $\ldots \ldots \ldots \ldots \ldots \ldots \ldots \ldots$

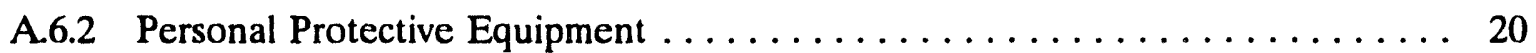

A.6.3 Action Levels Regarding Limitations in Tasks Assigned, PPE Requirements, and Withdrawal from Site $\ldots \ldots \ldots \ldots \ldots \ldots \ldots \ldots \ldots \ldots \ldots \ldots \ldots$

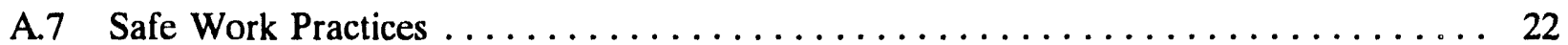

A.7.1 Variations to Safe Work Practices Listed in Section 7 of the Main Body of

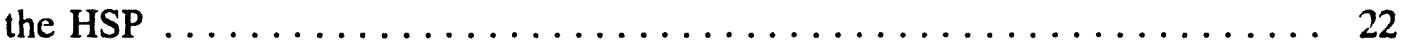

A.7.2 Additional Safe Work Practices for the Task Site $\ldots \ldots \ldots \ldots \ldots \ldots \ldots 22$

A.8 Work/Radiation Zones, Site Entry, and Security $\ldots \ldots \ldots \ldots \ldots \ldots$

A.8.1 Perimeter Establishment $\ldots \ldots \ldots \ldots \ldots \ldots \ldots \ldots \ldots \ldots \ldots \ldots \ldots \ldots$

A.8.2 Description of Work/Radiation Zones Including Site Entry and Security . . . . 23

A.9 Environmental and Personnel Monitoring $\ldots \ldots \ldots \ldots \ldots \ldots \ldots \ldots \ldots \ldots \ldots$

A.9.1 Operations and Monitoring Equipment Checklist $\ldots \ldots \ldots \ldots \ldots \ldots \ldots$

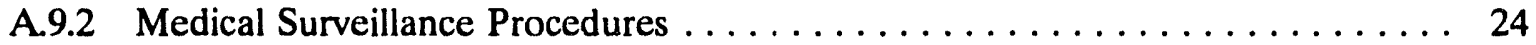

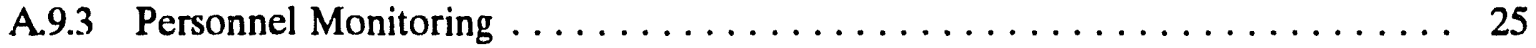

A.9.4 Operating Procedures and Methods for Surveillance $\ldots \ldots \ldots \ldots \ldots \ldots \ldots$ 
Doc. No.: EGG-ERD-10452

Section No. H\&S Plan

Revision No. 0

Date July 1993

Page No. $A-6$

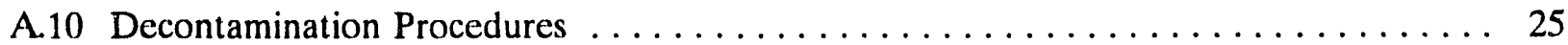

A.10.1 Personnel Decontamination Procedures $\ldots \ldots \ldots \ldots \ldots \ldots \ldots \ldots \ldots \ldots$

A.10.2 Decontamination of Sampling and Monitoring Equipment $\ldots \ldots \ldots \ldots \ldots$

A.10.3 Decontamination Modification (e.g., personnel, surfaces, materials,

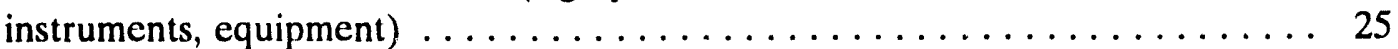

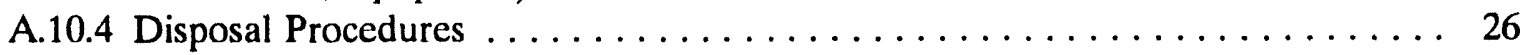

A.11 Emergency Procedures, Equipment, and Information $\ldots \ldots \ldots \ldots \ldots \ldots \ldots$

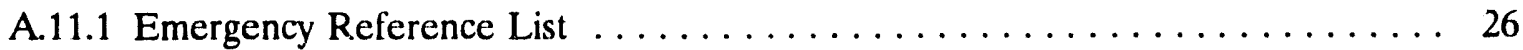

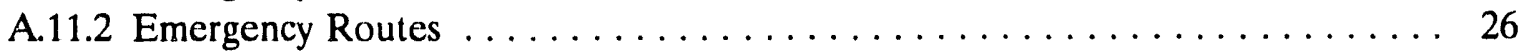

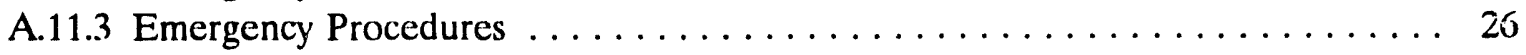

A.11.4 Emergency Equipment $\ldots \ldots \ldots \ldots \ldots \ldots \ldots \ldots \ldots \ldots \ldots \ldots \ldots \ldots \ldots$

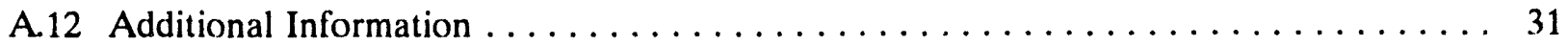

A.13 Health and Safety Certification Form $\ldots \ldots \ldots \ldots \ldots \ldots \ldots \ldots \ldots \ldots \ldots \ldots$

FIGURES

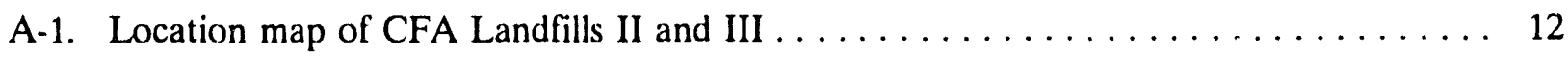

A-2. Field organization chart $\ldots \ldots \ldots \ldots \ldots \ldots \ldots \ldots \ldots \ldots \ldots \ldots \ldots \ldots \ldots$

A-3. Emergency route to the medical facility at CFA $\ldots \ldots \ldots \ldots \ldots \ldots$

\section{TABLES}

A-1. Known onsite contaminants and their concentrations at CFA Landfills II and III $\ldots \ldots 13$

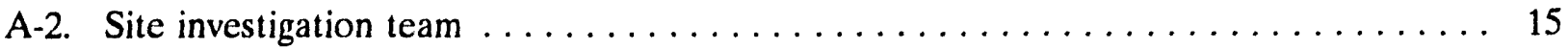

A-3. Training required for task site personnel $\ldots \ldots \ldots \ldots \ldots \ldots \ldots \ldots \ldots$

A-4. Values using a photoionization detector $\ldots \ldots \ldots \ldots \ldots \ldots \ldots \ldots \ldots \ldots \ldots$

A-5. Operations and monitoring equipment checklist $\ldots \ldots \ldots \ldots \ldots \ldots \ldots \ldots$

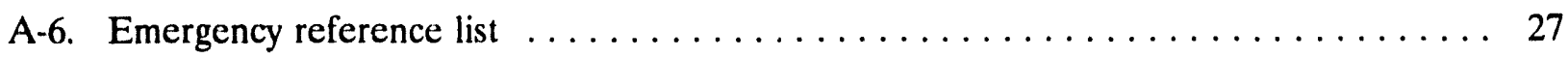

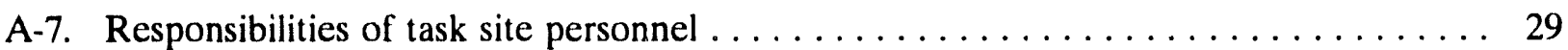


Doc. No.: EGG-ERD-10452

Section No. H\&S Plan

Revision No. 0

Date July 1993

Page No. $A-7$

A-8. Emerger.y equipment $\ldots \ldots \ldots \ldots \ldots \ldots \ldots \ldots \ldots \ldots \ldots \ldots \ldots \ldots \ldots \ldots$

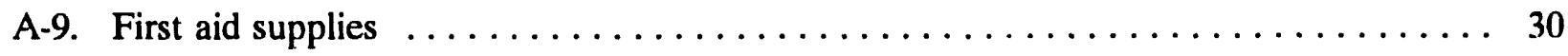


Doc. No.: EGG-ERD-10452

Section No. H\&S Plan

Revision No. 0

Date July 1993

Page No. A-8 
Doc. No.: EGG-ERD-10452

Section No. H\&S Plan

Revision No. 0

Date July 1993

Page No. $A-9$

\section{Addendum}

\section{Health and Safety Plan \\ for the CFA Landfills II and III \\ Remedial Investigation/Feasibility Study}

\section{A.1 Introduction}

Task: CFA Landfill II and III RI/FS

Project No. EGG-ERD-10453

DOE Operations Office: DOE-ID

Project Manager: S. H. McCormick

Phone No.: 5-3941

Other Contact: G. J. Stormberg

Phone No.: 6-1241

Date Plan Requested: $11 / 1 / 92$

Purpose of Task: The objective of this task is to adequately characterize OU 4-12 under the Comprehensive Environmental Response, Compensation, and Liability Act (CERCLA) process.

Proposed Dates of Work: January 1, 1993-December 8,1995

\section{A.1.1 Scope of Work}

The scope of this project is to characterize CFA Landfills II and III and associated media at the Idaho National Engineering Laboratory (INEL). The project will support the remedial investigation/feasibility study (RI/FS) of CFA Landfills II and III, as required by the Federal Facility Agreement/Consent Order (FFA/CO). Primary activities will include the collection of soil gas samples, groundwater samples from the landfill monitoring wells, landfill cover material for chemical, physical, and hydraulic properties, and subsurface soil (and leachate) soil samples. A brief description of the tasks can be found as follows.

Task 1: $\quad$ Soil Cover Investigation. This task involves (a) collecting samples for chemical analysis at Landfills II and III, (b) collecting samples for hydraulic and physical property analysis at Landfill III, and (c) determining the thickness of the soil cover at Landfill III. Up to 12 holes will be augered (using a gasoline-powered or hand auger) through the cover material to determine cover thickness across the landfill if the planned geophysical survey cannot determine this parameter. From these holes, field measurements of density and moisture content will be taken, and samples will be collected for physical and hydraulic property analyses. Samples for chemical analysis for volatile organics, semivolatile organics and metais will also be collected from both landfills (minimum of 
Doc. No.: EGG-ERD-10452

Section No. H\&S Plan

Revision No. 0

Date July 1993

Page No. A-10

1 per acre) using a shelby tube or similar device. During sample collection activities, boreholes will be surveyed periodically with field instruments (e.g., $\mathrm{HNu}$, Ludlum $2 \mathrm{~A}$, and combustible gas meter). Cover material mixed with bentonite will be used to backfill the boreholes. Landfill waste will not be removed from the boreholes.

1 ask 2: Geophysical Surveys. This task involves performing seismic refraction, induced electromagnetic, and magnetic field surveys across the surface of Landfill III to (a) define the boundaries of the landfill and the topography of the basalt beneath the landfill, (b) delineate concentrations of buried metal material, and (c) determine cover thickness.

Task 3: Soil Gas Surveys. This task will involve collecting shallow soil gas samples $(<5 \mathrm{ft})$ and collecting soil gas samples from existing, instrumented boreholes at both Landfills II and III. Surface flux chambers will also be used to estimate soil gas flux across the surface of Landfills II and III. Samples will be collected and shipped to an analytical laboratory for volatile organic and methane analysis. During sample collection activities, field instruments will be used to monitor site conditions (e.g., $\mathrm{HNu}$, Ludlum 2A, combustible gas meter).

Task 4: Monthly Water Level Measurements and Well Deviation Surveys. This task involves removing groundwater sampling pumps from wells in the vicinity of the landfills, performing well deviation surveys, and collecting water levels on a monthly basis as defined in the FSP. Water level measurements will be taken with an electronic or steel tape.

Task 5: Topographic Survey of Landfill III. A survey will be performed to define surface topography at Landfill III. The topographic survey will not disturb landfill waste.

Task 6: Groundwater Sampling. This task will involve sampling existing wells after the dedicated pumps have been removed to characterize the groundwater quality below CFA Landfills II and III, as described in the FSP. Groundwater from a network of approximately 10-15 wells will be sampled. Analyses may include volatile organics, contract laboratory program metals, and inorganics. Well purge water will be managed as described in the FSP. A portable groundwater sampling pump will be used to purge the wells and collect samples.

Task 7: Moisture Monitoring. This task involves taking measurements for moisture using a neutron probe via existing access tubes at Landfills II and III. Measurements will be taken every 6 in. to the total depth of the access tube on a monthly basis. Personnel performing these measurements will be radiation workers and will have been trained to use the neutron probe instrument. 
Doc. No.: EGG-ERD-10452

Section No. H\&S Plan

Revision No. 0

Date July 1993

Page No. $A-11$

Task 8: Subsurface Soil Sampling. Seven auger holes will be drilled and continuously splitspooned in Landfill II. A minimum of two samples will be submitted for VOC, SVOC, and metal analysis: one from within the waste unit and one beneath the waste. During augering and sampling activities, field instruments (e.g., $\mathrm{HNu}$, Ludlum $2 \mathrm{~A}$, combustible gas meter) will be used to monitor site conditions.

\section{A.1.2 Background}

A.1.2.1 Task Site Description. CFA Landfills II and III are located north of the Central Facilities Area. See Figure A-1 for additional information.

A.1.2.2 Waste Description (type and location). Both landfills received primarily cafeteria, office, and construction waste during their period of operation. Actual landfill waste will be disturbed only during the Landfill II augering and subsurface sampling. Potential contaminants encountered during this project will be in the form of soil, soil gas, and contaminated groundwater. Primary contaminants expected to be encountered in the groundwater may include metals (chromium) and tritium (documented in the groundwater and sourced from ICPP). Contaminants in the soil gas may include chlorinated volatile organics and methane. Contaminants in the soil may include metals, semivolatile organics, and volatile organics. A list of contaminants, their concentrations, and applicable environmental parameters is provided in Table A-1.

A.1.2.3 Unusual Features (e.g., containers, buildings, dikes, power lines, terrain). Power lines are oriented east-west along the southern border of each landfill. An underground fiber-optic cable runs parallel to the western edge of Landfill II. Although planned work will be away from buildings, power lines, etc., clearance approvals will be obtained prior to the start of field activities.

A.1.2.4 Status of Task Site (active, inactive, unknown). Landfill II is inactive. The eastern portion of Landfill III is inactive and is the area to be investigated under this HSP. The western portion of Landfill III is still active, and no work is planned in this area.

\section{A.1.2.5 History (worker or nonworker injury, complaints from public, previous} agency action). None.

A.1.2.6 Previous Onsite Monitoring; Previous Sampling Data. Previous groundwater, soil, and soil gas sampling data can be found in the Appendices to the RI/FS Work Plan. Previous industrial hygiene and health physics measurements using field instruments have found no detectable levels of radionuclides or organics in worker breathing zones during previous characterization tasks. Level D protection has always been appropriate. 


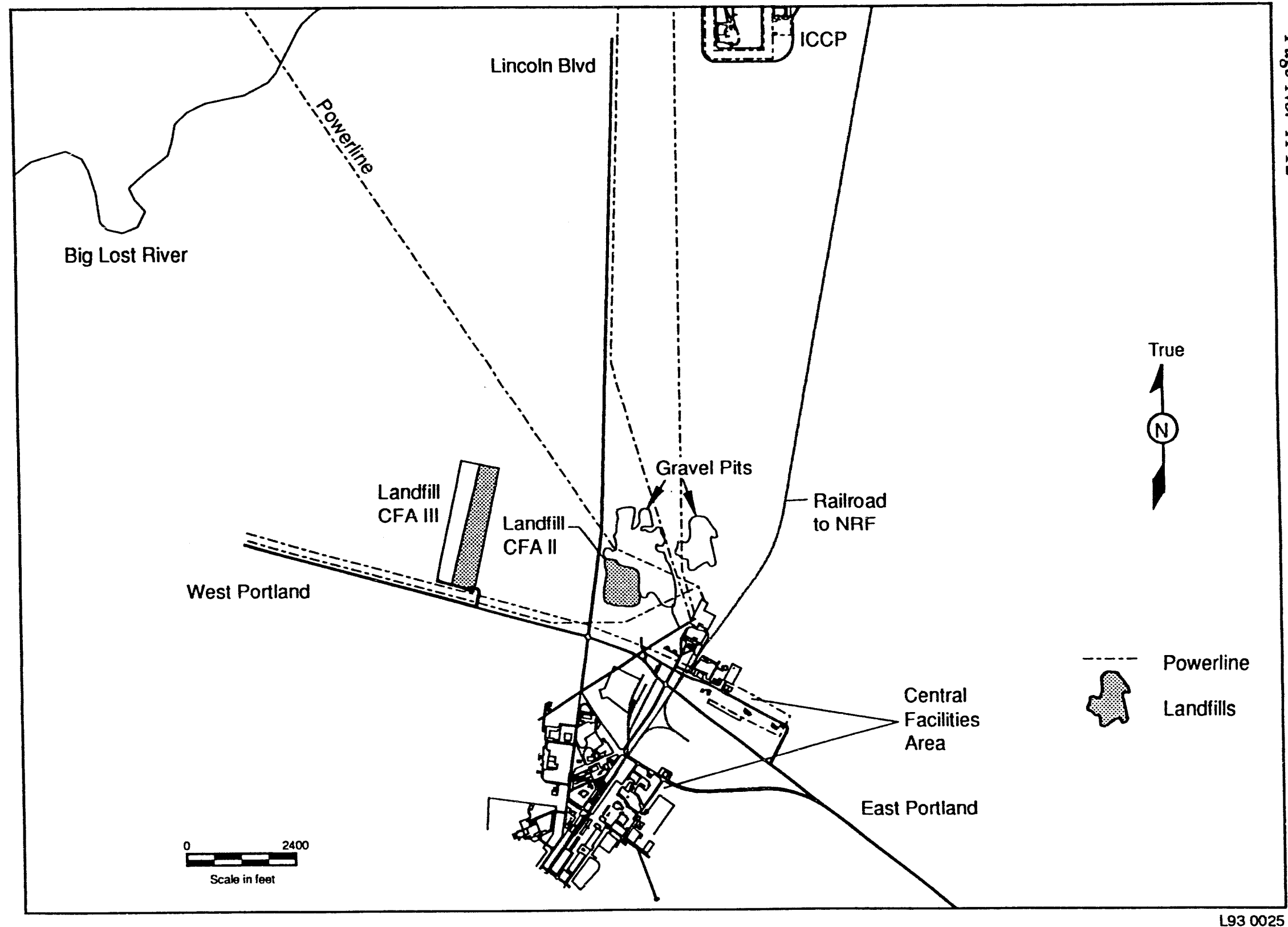

Figure A-1. Location map of CFA Landfills II and III. 


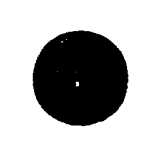

Table A-3. Training required for task site personnel.

\begin{tabular}{|c|c|c|c|c|c|}
\hline & $\begin{array}{l}\text { Operations } \\
\text { Personnel }\end{array}$ & $\begin{array}{c}\text { Task/Project } \\
\text { Manager }\end{array}$ & $\begin{array}{l}\text { Industrial } \\
\text { Hygienist }\end{array}$ & $\begin{array}{l}\text { Health } \\
\text { Physicist }\end{array}$ & $\begin{array}{l}\text { Field Team Leader/ } \\
\text { Job Site Supervisor }\end{array}$ \\
\hline Training ${ }^{\mathrm{a}}$ & required & required & Required & required & required \\
\hline Task site orientation & $\mathbf{X}$ & $\mathbf{X}$ & $\mathbf{X}$ & $\mathbf{X}$ & $\mathbf{X}$ \\
\hline Decontamination $^{\mathbf{b}}$ & $\mathbf{X}$ & - & - & - & $\mathbf{X}$ \\
\hline Hazard communication $^{b}$ & $\mathbf{X}$ & - & $\mathrm{X}$ & $\mathrm{X}$ & $\mathbf{X}$ \\
\hline Signs, tags, warning devices ${ }^{b}$ & $\mathbf{X}$ & - & $\mathrm{X}$ & $\mathrm{X}$ & $\mathbf{X}$ \\
\hline Hazardous waste operator & $\mathbf{X}$ & - & $\mathrm{X}$ & $\mathbf{X}$ & $\mathbf{X}$ \\
\hline Hazardous waste supervisor & - & - & - & - & $\mathbf{X}$ \\
\hline Medic $1 s t^{c, d}$ & $\mathbf{x}$ & - & $\mathrm{X}$ & - & $\mathbf{X}$ \\
\hline Respirator fit test qualification & $\mathrm{X}^{\mathrm{e}}$ & - & $\mathbf{X}$ & $\mathbf{X}$ & $\mathbf{X}$ \\
\hline Radiation worker & $X^{f}$ & - & - & $\mathrm{X}$ & $\mathrm{X}$ \\
\hline
\end{tabular}

a. Training should be current through original or refresher certification and requalification. The field team leader will verify current training prior to the start of each task.

b. May be part of task site orientation.

c. At least 2 people with medic/first aid training will be onsite during investigation activities.

d. Medic 1st training includes blood born pathogen training.

e. Respirator fit test certification is required only for personnel involved with the soil gas survey and the subsurface sampling.

f. Radiation worker training is only required for operations personnel performing moisture rnonitoring with a neutron probe.

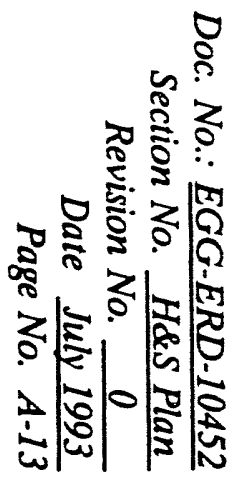


Doc. No.: EGG-ERD-10452

Section No. H\&S Plan

Revision No. 0

Date July 1993

Page No. A-14

\section{A.2 Responsibilities}

Table A-2 shows the proposed site investigation team (see Figure A-2). It is the responsibility of the Health and Safety Officer, the Job Site Supervisor, and the Field Team Leader (FTL) to ensure that all requirements stated in the base Health and Safety Plan (HSP) and this addendum are complied with and that the effectiveness of this HSP is evaluated. This project will comply with all applicable Occupational Safety and Health Administration (OSHA) regulations, American National Standards Institute standards, and the American Conference of Governmenial Irdustrial Hygienists threshold limit values for exposures to chemical and physical agents as declared in Department of Energy (DOE) 5480.10, "Contractor Industrial Hygiene Program," DOE/ID 5483.1A, "Occupational Safety and Health Standards," and DOE/ID 5480.4 "Environmental Protection, Safety, and Health Protection Standards (Industrial Hygiene Manual, Section 3).

\section{A.3 Personnel Training}

The following is an outline of site orientation tasks based on the outline presented in Section 3 of this Health and Safety Plan.

All field personnel will be trained per 29 CFR 1910.120 and the EG\&G Company Procedures Manual, Section 1.11, "Safety Certifications, Training, and Meetings." Additional required training may include, but is not limited to, respirator fit test qualification and radiation worker training.

In addition to the training mentioned above, at least two workers with Medic First (CPR and first aid) training shall be present at the task site when task site work is being performed. The Field Team Leader/Job Site Supervisor shall have hazardous waste worker supervisor training. Table A-3 shows the training required for task site personnel.

The Field Team Leader/Job Site Supervisor will ensure that all task site personnel understand site-specific hazards through daily briefings. The Field Team Leader will also ensure that task site personnel are trained about any unique hazards or procedures and this HSP.

\section{A.4 Medical Surveillance Program}

The requirements for medical surveillance are adequately discussed in Section 4 of the main body of this HSP and in Section A.9.2. 
Doc. No.: EGG-ERD-10452

Section No. H\&S Plan

Revision No.

Date July 1993

Page No. A-15

Table A-2. Site investigation team.

Personnel

S. H. McCormick

C. F. Hersley

To be determined (TBD) ${ }^{\mathrm{a}}$

$\mathrm{TB}^{\mathrm{a}}$

J. R. Lord

D. Haney

On-site IH/C. F. Hersley

C. F. Hersley

J. F. Kaminsky/A. H. Wylie

TBD
Discipline/Tasks Assigned

\section{Project Manager}

Field Team Leader/Job Site Supervisor

Industrial Hygienist

Health Physicist

Technician

Technician

Health and Safety Officer

Geologist

Hydrogeologist

Soil Gas subcontractor

a. EG\&G Idaho Industrial Hygienists and Health Physicists (CFA Facilities and Maintenance Support) will be used as available. Names of specific personnel will be entered into the field logbooks and safe work permit at the start of each task. 
Doc. No.: EGG-ERD-10452

Section No. H\&S Plan

Revision No.

Date July 1993

Page No. A-16

\section{FIELD ORGANIZATIONAL CHART}

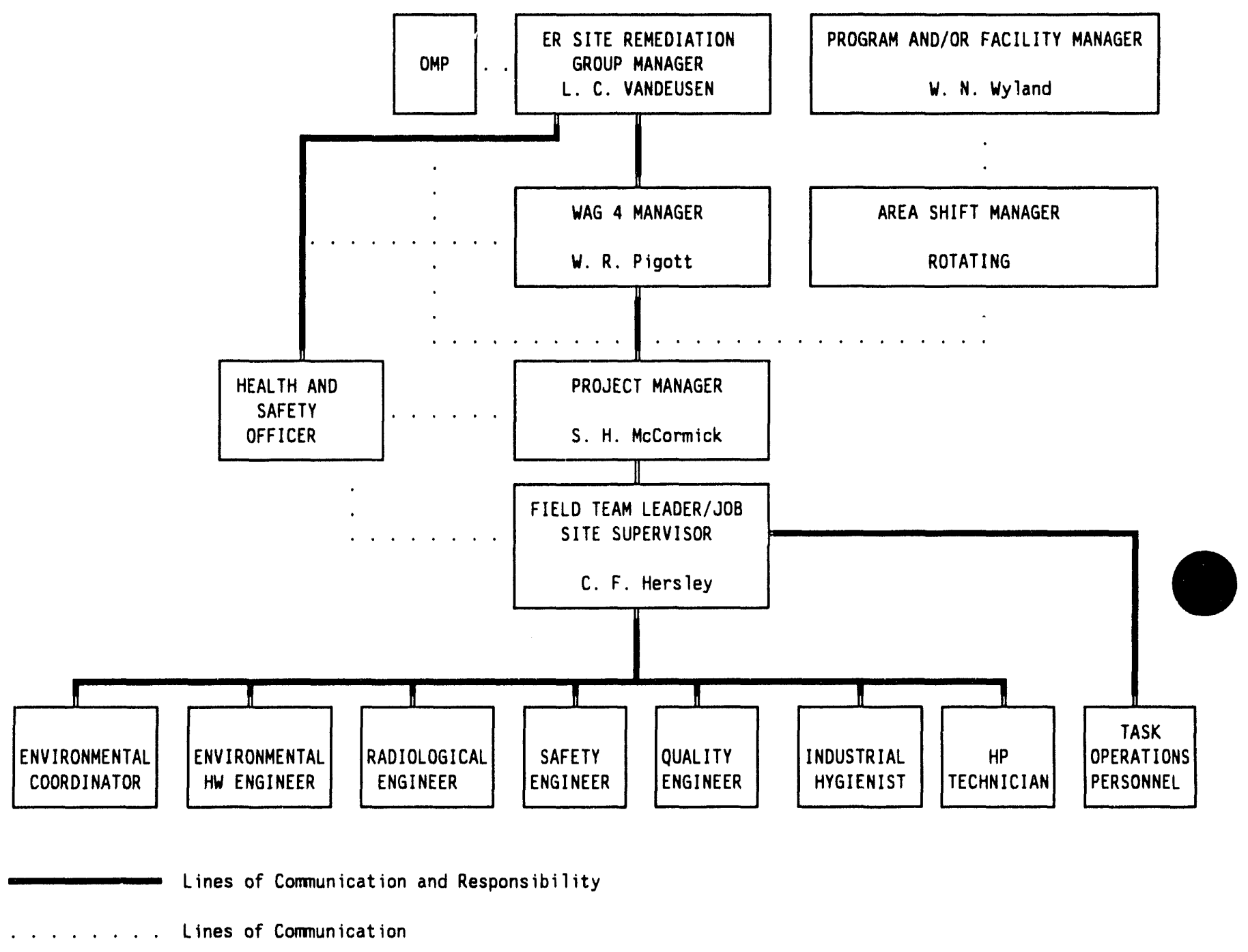

Figure A-2. Field organization chart. 


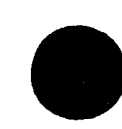

Table A-1. Known onsite contaminants and their concentrations at CFA Landfills II and III. ${ }^{a}$

\begin{tabular}{|c|c|c|c|c|c|c|c|}
\hline Substance & CAS No. & $\begin{array}{l}\text { Environmental } \\
\text { Concentration } \\
\text { (w/units) }\end{array}$ & Media & $\begin{array}{c}\mathrm{TLV} \\
\left(\mathrm{mg} / \mathrm{m}^{3}\right)\end{array}$ & Route of exposure & Symptoms & $\begin{array}{l}\text { EPA weight of evidence } \\
\text { classification for } \\
\text { carcinogenicity }\end{array}$ \\
\hline Trichloroethylene & $79-01-6$ & $12 \mathrm{mg} / \mathrm{m}^{3}$ & Soil gas & 269 & Inhalation/skin & c & $\mathbf{B 2}$ \\
\hline Tetrachloroethylene & $127-18-4$ & $111 \mathrm{mg} / \mathrm{m}^{3}$ & Soil gas & 339 & Intralation/skin & c & B2 \\
\hline 1,2-dichloroethylene & $540-59-0$ & $1 \mathrm{mg} / \mathrm{m}^{3}$ & Soil gas & 200 & Inhalation/skin & c & $\mathbf{D}$ \\
\hline Chloroform & $67-66-3$ & $357 \mathrm{mg} / \mathrm{m}^{3}$ & Soil gas & 49 & Inhalation/skin & c & B2 \\
\hline 1,1,1-trichloroethane & $71-55-6$ & $60 \mathrm{mg} / \mathrm{m}^{3}$ & Soil gas & 1,910 & Inhalation/skin & c & $\mathrm{D}$ \\
\hline Methylene chloride & $75-09-2$ & $300 \mathrm{mg} / \mathrm{m}^{3}$ & Soil gas & 174 & Inhalation/skin & c & B2 \\
\hline Benzene & $71-43-2$ & $130 \mathrm{mg} / \mathrm{m}^{3}$ & Soil gas & 32 & Inhalation/skin & c & $\mathbf{A}$ \\
\hline \multirow[t]{2}{*}{ 1,1-dichloroethane } & $75-34-3$ & $82 \mathrm{mg} / \mathrm{m}^{3}$ & Soil gas & 810 & Inhalation/skin & c & C \\
\hline & & & & $\begin{array}{c}\text { Safe risk-based } \\
\text { concentration } \\
(\mathrm{mg} / \mathrm{kg})\end{array}$ & & & \\
\hline Acetone & $67-64-1$ & $<800 \mathrm{ppb}$ & Soil & - & Ingestion & $\begin{array}{l}\text { Skin and } \\
\text { eye irritant }\end{array}$ & $\mathbf{D}$ \\
\hline Xylene & - & $4 \mathrm{ppb}$ & Soil & 50,000 & Ingestion & - & D \\
\hline 2-butanone & $78-93-3$ & $73 \mathrm{ppb}$ & Soil & 1,000 & Ingestion & - & D \\
\hline Benzoic acid & - & $90 \mathrm{ppb}$ & Soil & 100,000 & Ingestion & - & $\mathbf{D}$ \\
\hline 4-methylphenol & $1319-77-3$ & $110 \mathrm{ppb}$ & Soil & - & Ingestion & - & - \\
\hline \multirow[t]{2}{*}{ Methylene chloride } & $67-56-1$ & 73 ppb & Soil & - & Ingestion & $\begin{array}{l}\text { Skin and } \\
\text { eye irritant }\end{array}$ & B2 \\
\hline & & & & $\mathrm{MCL}$ & & & \\
\hline Tritium & - & $42,000-48,000 \mathrm{pCi} / \mathrm{L}$ & Groundwater & $20,000 \mathrm{pCi} / \mathrm{L}$ & Ingestion/inhalatiot & - & $\mathbf{A}$ \\
\hline Chromium & - & ND $-902 \mu \mathrm{g} / \mathrm{L}$ & Groundwater & $50 \mu \mathrm{g} / \mathrm{L}$ & Ingestion/skin & - & $\mathrm{A}(\mathrm{CrVI})$ \\
\hline
\end{tabular}

a. Low-levels of 1,2-dichloroethane, carbon tetrachloride, 1,1-dichloroethylene, 1,1-dichloroethane, toluene, and chloroform were detected in some soil gas samples. As a precaution, provisions have been made for onsite monitoring for organics by the onsite IH. Data were obtained from 1989-1991 sampling (see Appendices A-C of the RI/FS Work Plan).

b. A - Human carcinogen

B2 - Probable human carcinogen; sufficient evidence in animals, but inadequate or no evidence in humans

C - Possible human carcinogen

D - Not classified as a human carcinogen

c. Irritation of eyes or mucous membranes, dizziness, nausea, lack of coordination, elevated feelings. 
Doc. No.: EGG-ERD-10452

Section No. H\&S Plan

Revision No. 0

Date July 1993

Page No. $A-18$

\section{A.5 Hazard Evaluation}

\section{A.5.1 Potential Onsite Hazards}

Potential onsite hazards include those listed in Table A-1.

\section{A.5.2 Hazards Analysis}

Substances listed in Table A-1 have the hazard potentials listed below.

A.5.2.1 Chemical Agents. Hazards which may be encountered during the flux chamber tests and soil gas survey consist of the potential inhalation of organic vapors from volatile organics [such as trichloroethylene (TCE), benzene, and methylene chloride]. The hazard associated with groundwater sampling is possible skin contact with various contaminants (e.g., chromium and tritium). Hazards which may be encountered during surficial and subsurface soil sampling may include metals and volatile organics.

A.5.2.2 Fire and Explosion Hazards. There is a possibility for the presence of combustible gases in the landfills. The onsite industrial hygienist or designee will monitor the atmosphere during the soil cover, subsurface augering and sampling, and soil gas sampling tasks. The industrial hygienist will stop operations if combustible gases exceed $10 \%$ of the lower explosive limit. Since these operations will be outdoors, natural ventilation will be used to reduce the potential hazard.

A.5.2.3 Oxygen Deficiency/Confined Space. Based on available information, oxygen deficiency and work in a confined space environment are not anticipated during this project.

A.5.2.4 Radiological Hazards. Based on previous well drilling and testing, sampling, and logging activities at CFA, there are no radiation concerns at either landfill or any of the existing groundwater wells.

The groundwater contamination includes tritium. The concentration of tritium is approximately 42 to $48 \mathrm{pCi} / \mathrm{ml}$. Because there is not a "real-time" method for monitoring tritium, site personnel will avoid skin contact with the contaminated groundwater during the groundwater sampling task. RI/FS activities will also be surveyed for gamma-emitting radionuclides, and if radiation levels exceed $100 \mathrm{cpm}$ above background, operations will stop until the Health Physicist (HP) can evaluate the situation and recommend appropriate personal protective equipment.

Moisture monitoring (via existing access tubes) using a neutron probe will be conducted monthly from June 1993 through December 1993. Personnel performing the measurements will have radiation worker and neutron probe training 
Doc. No.: EGG-ERD-10452

Section No. H\&S Plan

Revision No. 0

Date July 1993

Page No. A-19

A.5.2.5 Biological Hazards. Biological hazards have not been a problem in the past and based on the tasks described, should not be encountered during the performance of the field activities.

A.5.2.6 Industrial Safety Hazards. Industrial hazards associated with auguring are anticipated. Safety measures in accordance with Section 5.6 of the main body of this HSP will be taken. Because the augering and subsurface sampling task at Landfill II will involve drilling through the waste unit, extra care will be taken due to the potential for drilling into unknown materials (concrete, steel, lumber, drums, etc.).

A.5.2.7 Electrical Hazards. The only electrical hazard anticipated is associated with the use of portable generators to power field equipment. Ground-fault interrupter circuits will be used on all outdoor connections. Care will be taken to keep the generator and associated equipment out of and away from any water (if present at the work site). Although no work is planned in the vicinity of power lines, clearance approvals will be obtained prior to starting field activities.

A.5.2.8 Heat/Cold Stress. During extreme weather conditions, proper exposure monitoring, clothing, fluid intake, and/or work/rest regiments will be implemented by the onsite IH or the Field Team Leader (FTL) as directed by the IH per the Industrial Hygiene Manual, Section 20. Some of the tasks in this project will probably be completed when there is a potential for heat stress resulting from the ambient air temperature. The IH or designee will monitor the temperature and adjust work/rest cycles according to the response of sampling personnel. Any team member who exhibits heat stress symptoms such as dizziness, profuse sweating, skin color change, vision problems, or confusion will be removed immediately from the work area and allowed to rest. If symptoms persist the team member will be taken to the nearest medical facility. The work schedule can be altered to take advantage of cooler ambient temperatures that occur in the late night or early morning periods of the day.

Exposure to low temperatures may also be a factor if work is done in the evening hours, if winds are high (e.g., at $50^{\circ} \mathrm{F}$, with a $25 \mathrm{mph}$ wind, the equivalent chill temperature is $32^{\circ} \mathrm{F}$ ), if unpredictable weather moves in, or in the winter months. EG\&G Idaho Company Procedures Manual, Number 11.10, discusses the hazards of cold stress. The FTL and the onsite IH will monitor workers for symptoms of cold stress such as whitening of the skin, especially at the extremities (nose, ears, fingers, etc.).

A.5.2.9 Noise Hazards. High noise levels are not anticipated during this project; however, safety measures in accordance with Section 5.10 of the main body of the HSP will be taken. The only activity with any hazard is during auguring activities. The IH will monitor this activity and determine the appropriate level of hearing protection (if necessary).

A.5.2.10 Other Hazards. Based on available information, other hazards include snakes, ticks, and spiders near the wells. Steam will be used to decontaminate sampling equipment. Standard industrial procedures (e.g., work/exclusion zones) will be used to protect workers. 
Doc. No.: EGG-ERD-10452

Section No. H\&S Plan

Revision No. 0

Date July 1993

Page No. A-20

\section{A.6 Levels of Protection and Personal Protective Equipment}

\section{A.6.1 Personal Protection Used on Previous Site Visits}

Level D personal protective equipment (PPE) was worn during previous field activities at CFA that are similar to the proposed activities. Previous drilling, soil gas surveys, soil cover investigations, subsurface sampling through the landfill, and groundwater sampling occurred in FY-88, FY-89, FY-90, and FY-91. Level C PPE was not required based on field measurements by the onsite IH and HP, and available lab data.

\section{A.6.2 Personal Protective Equipment}

Tasks $1,2,3,4,5,6,7$, and 8 : The level of protection for tasks 1 through 8 is Level $D$.

Sampling personnel must wear the following Level D PPE unless the project HP or IH instructs otherwise:

- Safety glasses or goggles

- Latex gloves (optional inner cloth gloves)

- $\quad$ Steel-toed, leather boots or shoes (except for geophysical survey personnel)

- Suitable work clothing (coveralls or Tyvek if recommended)

- Thermo-luminescent detectors (TLD) badges (if specified by the HP).

A.6.2.1 Respiratory and Dermal Requirements-PPE. Personnel must wear Level D PPE. Any upgrades or downgrades of PPE for work activities will be based on measurements taken by the onsite HP and IH.

A.6.2.2 Selection Criteria. PPE selection is based on recommendations contained in the Occupational Safety and Health Guidance Manual for Hazardous Waste Site Activities (NIOSH, 1985) and 29 CFR 1910.120. PPE selection is based primarily on hazard assessment data and work task requirements.

Based on INEL history, on types and quantities of known contaminants, and on the low probability of encountering significant unknown contaminants at CFA Landfills II and III, the recommended level of PPE is Level D.

A.6.2.3 Modification for Personal Protection Requirements. If photoionization readings exceeding the levels listed in Section A.6.3 are encountered, work will stop, and detector (Draeger) tubes for potential contaminants (TCE, benzene, methylene chloride) will be used to 
verify the presence or absence of those contaminants. The IH may recommend altered work practices or PPE based on that information.

Similarly, the onsite HP will check radiation levels using the field instruments listed in Section A.9.1. If readings exceed those listed in Section A.6.3, work will stop, and the HP may recommend altered work practices or PPE. Dermal contact with tritium contaminated groundwater will be prevented through the use of PPE.

Samples collected during field tasks, as well as the immediate work zone, will be monitored using instruments such as photoionization detectors, radiation survey meters, and combustible gas indicators in order to verify adequacy of personal protective equipment. Action limits requiring PPE upgrade are given for sustained organic vapor readings in the parts per million (ppm) range as listed in Section A.6.3. Sustained measurements in the breathing zone near or exceeding the levels listed in Section A.6.3 will require continued monitoring with both the $\mathrm{HNu}$ and combustible gas detector. If elevated ievels do not dissipate, work will be stopped until the Industrial Hygienist, the task area Health and Safety Officer, and the Field Team Leader decide on a course of action that will allow safe operations.

In addition, all samples collected will be checked by an HP. Any samples exceeding the levels listed in Section A.6.3 will be double bagged and handled as radioactively contaminated samples.

Periodic sampling for organic vapors using sampling methods approved by NIOSH will be conducted for the soil gas, groundwater, and soil sampling tasks in order to verify direct instrument readings and to provide more accurate personnel exposure data. In addition, lapel air samplers that indicate the presence of organic vapor may be used in the work zone breathing space. If initial analytical results are negative, the continued use of lapel samplers will be determined by the IH.

A.6.2.4 Levels of Protection. Engineering controls such as exclusion zone design and/or administrative controls will be used whenever feasible to minimize use of personal protective equipment. Each work location may be monitored for hazardous contaminants using appropriate instruments such as an $\mathrm{HNu}$ photoionization detector, a combustible gas indicator, and radiation survey meters.

\section{A.6.3 Action Levels Regarding Limitations in Tasks Assigned, PPE Requirements, and Withdrawal from Site}

If $\mathrm{HNu}$ readings exceed $5 \mathrm{ppm}$ sustained for 5 minutes or $50 \mathrm{ppm}$ for 2 minutes in the worker's breathing zone, all work in the exclusion area will be stopped, and the situation will be assessed by the IH, and the FTL. The values shown below in Table A-4 are for sustained measurements using an $11.7 \mathrm{eV}$ photoionization detector. 
Doc. No.: EGG-ERD-10452

Section No. H\&S Plan

Revision No. $\frac{0}{0}$

Date July 1993

Page No. A-22

Table A-4. Values using a photoionization detector. ${ }^{\mathrm{a}}$

\begin{tabular}{ll} 
Reading of & Requires \\
\hline $0-5 \mathrm{ppm}$ & \\
$5-10 \mathrm{ppm}$ & Level D PPE \\
$10-500 \mathrm{ppm}$ & Level C PPE \\
$500 \mathrm{ppm}+$ & Level B PPE \\
& Level A PPE
\end{tabular}

a. Some constituents (i.e., benzene, methylene chloride) should be monitored for using contaminant specific instruments (i.e., draeger tubes) as the above action level of $5 \mathrm{ppm}$ may not be protective of worker health for contaminants such as benzene (PEL $=1 \mathrm{ppm})$.

If field radiation instruments exceed $100 \mathrm{cpm}$ (beta/gamma) above background, all work will be stopped, and the situation will be assessed by the IH, the HP, and the FTL. Dermal contact with tritium contaminated groundwater will be prevented by the use of PPE.

If the combustible gas indicator exceeds $10 \%$ of the lower explosive limit, the onsite IH will stop operations until the level drops.

If any worker faints, becomes dizzy or sick, or generally incoherent, all work in the exclusion zone will be stopped. All workers will leave the exclusion zone, and the situation will be assessed by the IH, and the FTL.

\section{A.7 Safe Work Practices}

\section{A.7.1 Variations to Safe Work Practices Listed in Section 7 of the Main Body of the HSP}

The safe work practices listed in Section 7 of the main body of the HSP are excellent general practices. These safe work practices will be adhered to for all tasks.

\section{A.7.2 Additional Safe Work Practices for the Task Site}

A safe work permit will be obtained for all tasks. Drums and containers will be handled in accordance with company procedures. Drums containing waste solids or any waste liquid will be handled with care to avoid a release to the environment. The FTL will remind operating personnel about the hazards associated with auguring. 


\section{A.8 Work/Radiation Zones, Site Entry, and Security}

\section{A.8.1 Perimeter Establishment}

Site secured:

Perimeter identified: $\overline{\mathrm{X}}$
Containment zones mapped:

Containment zones identified:

Requirements for work zones for field activities will be based on FTL, IH, and HP recommendations for each task. Tasks 2,4 , and 5 will not use work or exclusion zones because these tasks require frequent/continuous movement and no hazards have been encountered for similar tasks at the CFA landfills in the past. Tasks $1,3,6,7$, and 8 will establish working zones using cones or roped areas established a minimum of $15 \mathrm{ft}$ from the sample/investigation site.

\section{A.8.2 Description of Work/Radiation Zones Including Site Entry and Security}

Chapter 8 of this HSP will be followed. EG\&G Idaho personnel will use site control zones as established by the IH and the HP when doing all tasks.

\section{A.9 Environmental and Personnel Monitoring}

\section{A.9.1 Operations and Monitoring Equipment Checklist}

Table A-5 shows the checklist used for operations and monitoring equipment.

Task 1: Periodic monitoring by the IH and HP or designees (FTL or jobsite supervisor) will be performed during the soil cover investigation. If readings are above the levels discussed in Section A.6.3, or if contaminant specific monitoring detects a contaminant above health-based values, work will be stopped until the readings return to acceptable levels or appropriate modifications to work practices are implemented.

Tasks 2, 4, and 5: The geophysical surveys and the topographic survey will not disturb the ground surface and thus Health and Safety monitoring will not be required. Similarly, water level measurements will not result in exposure to any known hazards and does not require Health and Safety monitoring. Contact with the groundwater will be avoided through the use of PPE.

Tasks 3,6, and 7: All monitoring during groundwater sampling, soil gas sampling and flux chamber tests, and neutron probe measurements will be intermittent (approximately 2 hours/day) unless levels indicate otherwise. The IH and HP will open the field site daily, check the calibration of instruments, perform periodic monitoring, and update the safe work permit and/or the job hazard assessment as necessary. The intermittent monitoring at CFA will be done by field personnel trained by the IH and HP. If readings are above the levels listed in Section A.6.3, or if contaminant specific monitoring detects contaminants above health-based values, all work will be shut down until readings return to acceptable levels. If high readings persist, work will not begin 
Doc. No.: EGG-ERD-10452

Section No. H\&S Plan

Revision No. 0

Date July 1993

Page No. A-24

Table A-5. Operations and monitoring equipment checklist.

Type of Equipment

$\mathrm{HNu}$ or equivalent-with

an $11.7 \mathrm{eV}$ probe or lamp

Ludlum-2A or equivalent

$\mathrm{LEL} / \mathrm{O}_{2}$ meter

Pump/draeger tube
Number Needed

1

Daily

1

1

1
Calibrated $^{\mathrm{a}}$

Field-Ready

Yes

Biannually

Yes

Weekly

Yes

Annually

Yes

a. Calibration methods are contained in the company procedures or are available from the IH or HP personnel.

until IH and/or HP support is available. Dermal contact with tritium contaminated groundwater will be prevented through the use of PPE. Training of the field personnel will be documented in the project file.

Task 8: The IH and HP will continuously monitor augering and subsurface sampling at Landfill II. Although similar activities performed in 1988 did not encounter any notable hazards, the potential exists for encountering physical and chemical hazards and combustible gas. Therefore, full-time monitoring is warranted as a best management practice.

\section{A.9.2 Medical Surveillance Procedures}

All workers at the site with the potential for ongoing exposures to hazardous substances or some other hazard that requires medical surveillance will be entered by their managers into the Occupational Medical Program (OMP) in accordance with company procedures. The files of workers already in the OMP will be checked to see if the appropriate form (EGG-735) is available. OMP will be contacted to see if any updates to the form are required.

When notified of the need for a hazardous waste worker or other specific medical surveillance examination program, the OMP will cnter the affected employee into the appropriate surveillance program. The elements of these examinations can be tailored to the specific agent involved, but all include at least annual occupational, medical, and exposure history; a complete physical examination; and appropriate clinical laboratory studies. 


\section{A.9.3 Personnel Monitoring}

The following personal monitoring device(s) shall be used:

- Breathing zone samples will be taken using the $\mathrm{HNu}$. Sampling pumps, draeger tubes, or organic vapor badges may also be used if the IH deems additional monitoring to be necessary. The IH will perform any additional monitoring.

- Combustible gas indicators, while not personal monitoring devices, will be used for situations in which combustible gases may be built up. These situations may be encountered during sampling/auguring during the soil eover investigation, the subsurface sampling task, and during soil gas sampling.

\section{A.9.4 Operating Procedures and Methods for Surveillance}

The Field Team Leader will be responsible for following the guidelines set forth in the main body of this HSP for (a) heat and cold stress (Section 9.4), (b) work stress (Section 9.4), (c) barriers, signs, and tags (Section 9.6), and (d) physical hazard control and monitoring (Section 9.6).

\section{A.10 Decontamination Procedures}

\section{A.10.1 Personnel Decontamination Procedures}

Decontamination for chemical and radiological contaminants will be handled in accordance with Section 10 of the main body of the HSP. However, similar past activities have not required personnel decontamination due to the low hazard level. All personnel will wash with soap and water upon leaving the work zone.

\section{A.10.2 Decontamination of Sampling and Monitoring Equipment}

Sampling equipment will be decontaminated as described in Appendix B of the FSP. Decon water will be contained in car boys or polyethylene tanks depending on the volume of water generated.

\section{A.10.3 Decontamination Modification (e.g., personnel, surfaces, materials, instruments, equipment)}

If necessary, personal protective equipment will be cleaned of soil or decontaminated with tap water before it is removed. The cleaned PPE will be surveyed for organic and radiological contamination with field instruments and disposed of as cold waste if no contamination is detected. Respirators will be surveyed with field instruments, bagged, and sent for cleaning. It should be noted that contaminated PPE has not been generated during past landfill investigations. 
Doc. No.: EGG-ERD-10452

Section No. H\&S Plan

Revision No. 0

Date July 1993

Page No. A-26

\section{A.10.4 Disposal Procedures}

Onsite:

Materials and equipment for which decontamination is not feasible shall be surveyed for radiological contamination by the Project HP, and for organic contamination by the Project IH. Hazardous nonradioactive contaminated materials, radioactive mixed contaminated materials, and noncontaminated materials will be bagged separately, labeled, and stored in the corresponding drums at the site to await final data results for disposal. All wastewater will be contained pending analysis and then disposed depending on the analytical results according to the Investigation Derived Waste Plan (see the FSP). Soil and landfill material will be returned to the boreholes after sampling is completed.

Offsite:

There will be no offsite disposal.

\section{A.11 Emergency Procedures, Equipment, and Information}

\section{A.11.1 Emergency Reference List}

Table A-6 shows the emergency numbers currently in use.

\section{A.11.2 Emergency Routes}

During dayshift, Monday through Thursday, the CFA medical facility located at CF-603 (phone 526-2356) is staffed by a full-time nurse and physician. In addition, DOE-ID firemen at CF-666 (phone 526-2212) are qualified to render first-aid assistance.

A requirement of this HSP is that workers not already familiar with the location of the facilities mentioned above must tour the facilities before doing any work described in this plan. The field team leader will conduct any necessary tours.

Figure A-3 shows the CFA Landfill areas and should be referenced for the location of CF-603 (the CFA medical facility). This information will be posted at each site.

\section{A.11.3 Emergency Procedures}

In general, emergency procedures given in Section 11 of the main body of this HSP will be followed. In case of a large-scale emergency or major personnel injury, the CFA Area Emergency Plan will be implemented by the Facility Manager. 
Doc. No.: EGG-ERD-10452

Section No. H\&S Plan Revision No. 0

Date July 1993

Page No. $A-27$

Table A-6. Emergency reference list.

Warning Communications Center (WCC)

777

Area Emergency Action Director

6-6206

First Aid

Occupational Medical Program

Ambulance

Fire

Security

Safety Engineer Support

CFA Industrial Hygiene

Explosives expert (Richard Green)

Health Physics

Area Safety (Jim Southwick)

CFA Landlord

Field Team Leader (C. F. Hersley)

Project Manager (S. H. McCormick)
6-2356

6-2356

777

777

777

6-6004

6-4369

6-2702

6-2284

6-6347

6-2150

6-1917

5-3941

a. This emergency reference list will be posted at each site.

A.11.3.1 Additional or Modified Emergency Procedures. The F-net radio communication system will be available at the work site at all times, and will be provided by the Field Team Leader. Additionally, a portable telephone will be available at the task site for emergency notifications. A portable eye-wash station, a first aid kit, and a 20-lb ABC fire extinguisher will be available at the work site at all times. A vehicle will also be in close proximity and accessible during the entire project. Hand signals and the buddy system per Section 11.1.7 in the main body of the HSP will be reviewed as part of the task site orientation and will be followed in an emergency.

A.11.3.2 Requirements for Task Site Evacuation. The FTL will evaluate and establish evacuation routes prior to the start of each task or sampling activity. The evacuation routes will follow the shortest distance over accessible roads to the gathering point for an evacuation. Notification will be performed by the FTL by notifying the medical dispensary directly, by calling the warning communication center (WCC), or by contacting the shift manager. This information will be posted in the field logbook, which will be at the task site during all investigation activities.

Prior to starting work, the FTL will brief site personnel nn the location of the emergency equipment and the evacuation procedures and route. Evacuation routes and gathering points may 
Doc. No.: EGG-ERD-10452

Section No. H\&S Plan

Revision No.

Date July 1993

Page No. $A-28$

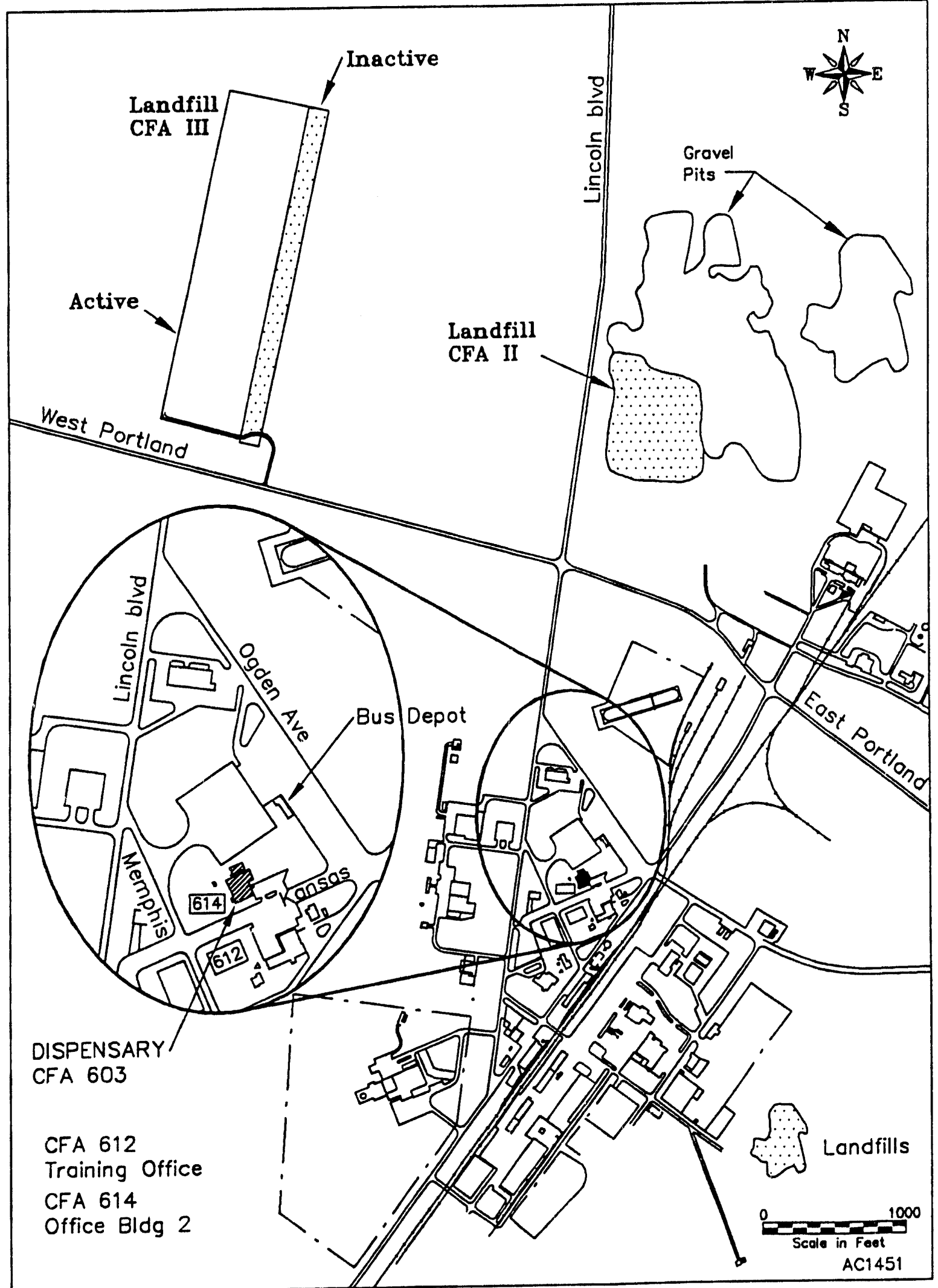

Figure A-3. Emergency route to the medical facility at CFA. 
vary depending on where activities are taking place on any given day. In the event of an evacuation, WCC will be contacted to determine the evacuation route and/or gathering point. The FTL will put a copy of emergency phone numbers and Figure A-3 in the field logbook.

\section{A.11.3.3 Task Site Warning Devices.}

- $\quad$ Portable two-way F-net radio (will be available at all times at each site)

- Facility sirens for general area emergencies

- Hand and voice signals for task specific emergencies.

A.11.3.4 Task Site Emergency Responsibilities. Table A-7 shows the responsibilities of task site personnel.

A.11.3.5 Procedures for Inclement Weather. Operations will cease in the event of winds exceeding $25 \mathrm{mph}$ on a sustained basis, electrical storms, heavy rains, or heavy snow if the activities are weather sensitive as determined by the Field Team Leader and onsite IH or HP.

A.11.3.6 Reentry Procedures. If a task site emergency results in the evacuation of the task site, personnel shall reenter the task site only as directed by the FTL as specified in Section 11.1.10 of the main body of the HSP.

\section{A.11.4 Emergency Equipment}

Equipment will be inspected and maintained by the technicians. The FTL will check all emergency equipment prior to starting operations (Tables A-8 and A-9).

Table A-7. Responsibilities of task site personnel.

\section{Name}

C. F. Hersley

C. F. Hersley

EG\&G Idaho personnel

J. R. Lord

W. N. Wyland
Responsibility

Field Team Leader

Field Team Leader

$\mathrm{HP} / \mathrm{IH}$

Technician

Facility Manager
Action

Direct Task Emergency Site

Direct evacuations

Recommend protective measures

Call for help as directed

Initiate large scale emergency action as needed 
Doc. No.: EGG-ERD-10452

Section No. H\&S Plan

Revision No.

Date July 1993

Page No. A-30

Table A-8. Emergency equipment.

Fire extinguishers -

Number Available:

Location:

Maintenance schedule:
1

On the transportation vehicle/at the task site Monthly inspections

First Aid Kits (see Table A-9) -

Number Available:

1

Location:

On the transportation vehicle/at the task site

Maintenance schedule:

Inventoried prior to sampling and monthly thereafter

Portable eyewashes -

Number Available:

1

Location:

On the transportation vehicle/at the task site

Maintenance schedule:

Shall be stored empty. Prior to sampling activities, it shall be filled with clean water.

Table A-9. First aid supplies.

Task

Date

Location

Kit No.

All

January 1993

Transport vehicle/at task site 1

Items:

2 boxes of triangular bandages

1 box of gauze pads

3 boxes of 1 -in. adhesive bandages

1 box of iodine wipes

1 box of scissors and forceps

1 box of eye pads

1 microshield
2 boxes of 4 -in. bandage compress

1 box of knuckle bandages

1 box of wound wipes

1 box of burn compound

1 box of adhesive tape

1 box of ammonia inhalers

2 pair of latex gloves 
Doc. No.: EGG-ERD-10452

Section No. H\&S Plan

Revision No. $\frac{0}{1}$

Date July 1993

Page No. A-31

\section{A.12 Additional Information}

Offsite personnel requiring entrance to the work zone for a limited time, but not performing work that involves the potential disturbance of or contact with contaminated materials, will be allowed access if escorted by the Site Health and Safety Officer or the FTL, but only during periods when no hazardous substances can be encountered, no hazardous operations are occurring, and hazardous operations have ceased.

Personnel with only incidental contact with the task will not be required to read this HSP but will be briefed on site hazards and emergency procedures. The decision concerning incidental classification will be made by the Field Team Leader in consultation with the Health and Safety Officer. If the requirement of reading this HSP is waived, the worker will be escorted to the task site while his job is being performed.

The Health and Safety Officer will not be required to be onsite whenever operations are performed, except during the subsurface augering and sampling task. However, this individual or designee will be available by at least radio or telephone for consultation concerning health and safety matters whenever operations are ongoing. When the Health and Safety Officer is not present, the FTL will be responsible for site safety. 
Doc. No.: EGG-ERD-10452

Section No. H\&S Plan

Revision No. 0

Date July 1993

Page No. $A-32$

\section{A.13 Health and Safety Certification Form}

Task Title:

Project Manager:

Field Team Leader:

I certify that I have been given a copy of the task-specific ERP Health and Safety Plan for the CFA Landfill II and III RI/FS Task and agree to comply with the procedures described therein. I further certify that I understand the potential health and safety hazards of the program (as outlined in this Health and Safety Plan) and have been trained in the use of personal protective equipment selected for this task.

Employee:

(Print)

(Signature)

(Date)

Company of Employment:

Field Team Leader:

(Print)

(Signature)

(Date)

Health and Safety Officer:

(Print)

(Signature)

(Date) 


\section{Remedial Investigation/Feasibility Study Community Relations Plan Supplement for Operable Unit 4-12: \\ Central Facilities Area Landfills II and III at the Idaho National Engineering Laboratory}

July 1993

Idaho National Engineering Laboratory

EG\&G Idaho, Inc.

Idaho Falls, Idaho 83415

Prepared for the

U.S. Department of Energy

Assistant Secretary for Environmental Restoration and Waste Management Under DOE Idaho Operations Office

Contract DE-AC07-76ID01570 


\section{CONTENTS}

ACRONYMS $\ldots \ldots \ldots \ldots \ldots \ldots \ldots \ldots \ldots \ldots \ldots \ldots \ldots \ldots \ldots \ldots$

1. OVERVIEW OF COMMUNITY RELATIONS PLAN SUPPLEMENT $\ldots \ldots \ldots \ldots$ 1-1

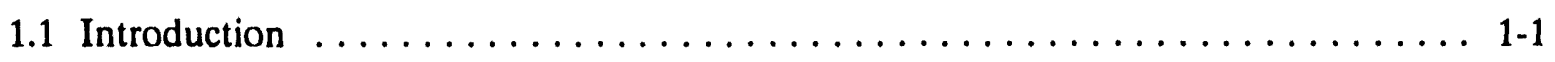

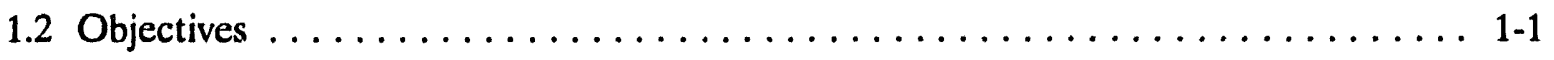

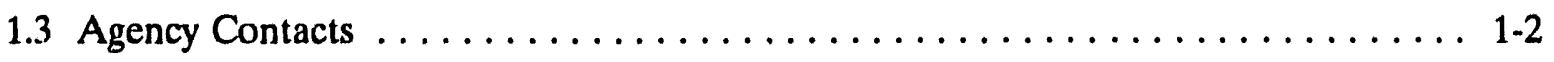

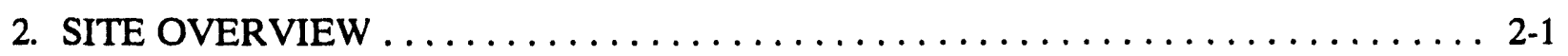

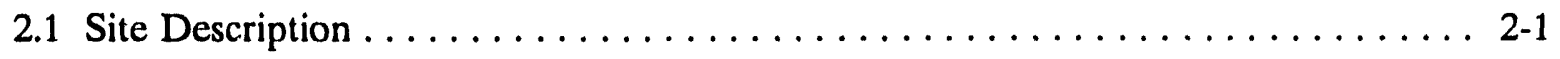

2.2 Environmental Investigations $\ldots \ldots \ldots \ldots \ldots \ldots \ldots \ldots \ldots \ldots \ldots \ldots \ldots \ldots \ldots \ldots$

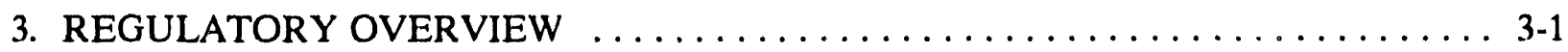

3.1 Resource Conservation and Recovery Act, and Consent Order and Compliance Agreement ............................ $3-1$

3.2 National Priorities Listing and Federal Facility Agreement . . . . . . . . . 3-1

3.2.1 Response Actions . . . . . . . . . . . . . . .

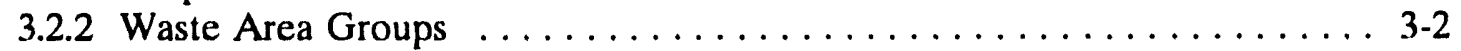

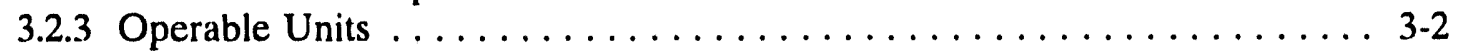

3.3 National Environmental Policy Act (NEPA) $\ldots \ldots \ldots \ldots \ldots \ldots \ldots \ldots \ldots \ldots \ldots$

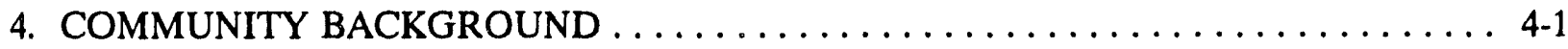

5. COMMUNITY RELATIONS ACTIVITIES $\ldots \ldots \ldots \ldots \ldots \ldots \ldots \ldots \ldots \ldots$

5.1 CERCLA Requirements For Community Relations $\ldots \ldots \ldots \ldots \ldots \ldots \ldots \ldots$.

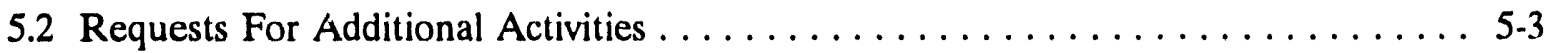

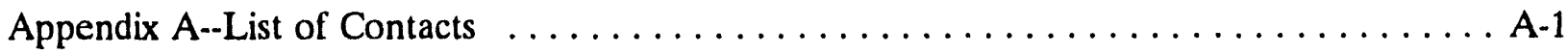

Appendix B--INEL Information Repositories $\ldots \ldots \ldots \ldots \ldots \ldots \ldots \ldots \ldots \ldots \ldots$ B-1

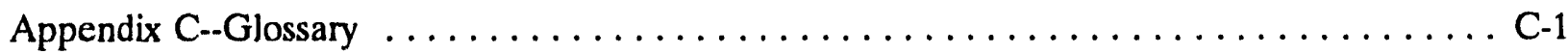




\section{FIGURES}

2-1. Map of Central Facilities Area Landfills II and III $\ldots \ldots \ldots \ldots \ldots \ldots \ldots \ldots$

3-1. Location of CFA within INEL boundaries $\ldots \ldots \ldots \ldots \ldots \ldots \ldots \ldots$

\section{TABLES}

5-1. Community relations activities for the CFA Landfills II and III RI/FS $\ldots \ldots \ldots$. 


\section{ACRONYMS}

CERCLA Comprehensive Environmental Response, Compensation, and Liability Act

CFA Central Facilities Area

COCA Consent Order and Compliance Agreement

CRP Community Relations Plan

DOE Department of Energy

DOE-ID Department of Energy Idaho Field Office

EPA Environmental Protection Agency

FFA/CO Federal Facility Agreement/Consent Order

IDHW Idaho Department of Health and Welfare

INEL Idaho National Engineering Laboratory

NCP National Contingency Plan

NEPA National Environmental Policy Act

NPL National Priorities List

RCRA Resource Conservation and Recovery Act

RI/FS remedial investigation/feasibility study

ROD Record of Decision

WAG waste area group 


\section{Remedial Investigation/Feasibility Study Community Relations Plan Supplement for Operable Unit 4-12: \\ Central Facilities Area Landfills II and III at the Idaho National Engineering Laboratory}

\section{OVERVIEW OF COMMUNITY RELATIONS PLAN SUPPLEMENT}

\subsection{Introduction}

This Community Relations Plan Supplement has been taken from the interim plan developed by the Department of Energy Idaho Field Office (DOE-ID) in March 1991. This supplement has been written specifically for the Central Facilities Area (CFA) Landfills II and III remedial investigation/feasibility study (RI/FS) Work Plan. Activities conducted under this plan will be integrated with activities being done under the installation-wide Community Relations Plan where feasible. Both plans will be used to establish a process to help DOE-ID, the Environmental Protection Agency (EPA), and the State of Idaho Department of Health and Welfare (IDHW) communicate information to the public and to help the public communicate concerns back to DOE-ID, EPA, and IDHW. These communications are intended to inform and involve interested citizens, public officials, agencies, groups, and organizations in the State of Idaho on the CFA Landfills II and III RI/FS.

\subsection{Objectives}

The RI/FS-Specific Community Relations Plan includes the following specific objectives for accomplishing remedial investigations at CFA:

- Address concerns expressed by the community during interviews and public meetings

- Comply with legal requirements of Comprehensive Environmental Response, Compensation, and Liability Act (CERCLA) and the National Environmental Policy Act (NEPA) as required by DOE-ID policy

- Provide the public with accurate and understandable information on the RI/FS and related remediation work at CFA

- Establish two-way communication with the public to achieve community involvement

- Provide an opportunity for the public to become involved in key decisions regarding the RI/FS and related remediation work.

Since the Community Relations Plan is intended to be a "working" document, it can be amended to provide additional community relations activities as needed. 


\subsection{Agency Contacts}

A current list of officials from the DOE-ID Environmental Restoration Division, the INEL Community Relations Plan Office, the INEL Public Affairs Office, EPA Region 10, and the Idaho State INEL Oversight Program is shown in Appendix A. Representing various offices and agencies, these individuals have a common interest in the activities described in this Plan, which is to ensure public involvement in remedial activities at CFA.

Inquiries or comments concerning any aspect of these INEL environmental investigations, including monitoring and remedial activities, or the content of this supplement, can be directed to the Federal and state regulatory agency contacts listed in Appendix A or to the following address:

Environmental Restoration Division

DOE Idaho Field Office

P.O. Box 2047

Idaho Falls, ID 83403-2047 


\section{SITE OVERVIEW}

\subsection{Site Description}

The INEL is located in southeastern Idaho near the center of the eastern portion of the Snake River Plain. The INEL encompasses $890 \mathrm{mi}^{2}$ of semi-arid land near the Lemhi and Lost River mountain ranges. The nearest city with a large population center is Idaho Falls, located about $45 \mathrm{mi}$ to the east. INEL employment at the present time is about 11,000 persons. Most employees live in Idaho Falls. Others live in the surrounding areas of Pocatello, Blackfoot, Rexburg, Arco, and other nearby towns. CFA Landfills II and III are located in the south central section of the INEL. Disposal in Landfill II began in 1970 and continued until September 1982. Disposal in Landfill III began in October 1982 and continued until December 1986, when part of the landfill was closed. A more detailed description of the CFA area is found in Section 2 of the RI/FS Work Plan.

The Eastern Snake River Plain is an area of complex geologic structure and history. Studies show basaltic lava flows to a depth of at least $2,440 \mathrm{ft}$. The flows are interbedded with cinders, silt, sand, and clay. The basalt has high permeability resulting from fractures and joints. Age-dating techniques estimate the youngest basalt at INEL to be between 45,000 and 145,000 years old.

The principal groundwater feature at INEL is the Snake River Plain Aquifer, a continuous body of groundwater underlying most of the Eastern Snake River Plain. The aquifer consists of basalt flows with sedimentary interbeds. The depth from the surface of the ground to the top of the aquifer at CFA is about $480 \mathrm{ft}$. Most of the water pumped from the aquifer in the CFA area is used for domestic and industrial purposes.

\subsection{Environmental Investigations}

Several types of hazardous substances have been produced, stored, and disposed of at the INEL during four decades of operation. Most waste treatment and disposal practices of the past have proven to be adequate. However, some practices that were approved at the time have released hazardous substances to the environment. At CFA Landfills II and III, sanitary and construction waste as well as some hazardous wastes were buried in both landfills during their operable periods. Consequently, one of the main objectives of the RI/FS is to determine if the hazardous contaminants have been released from the landfills and, if so, does this release pose a significant threat to human health and the environment.

Figure 2-1 is a map showing CFA Landfills II and III. More details on contaminant types and other site-specific information can be found in Section 2 of the RI/FS Work Plan. 


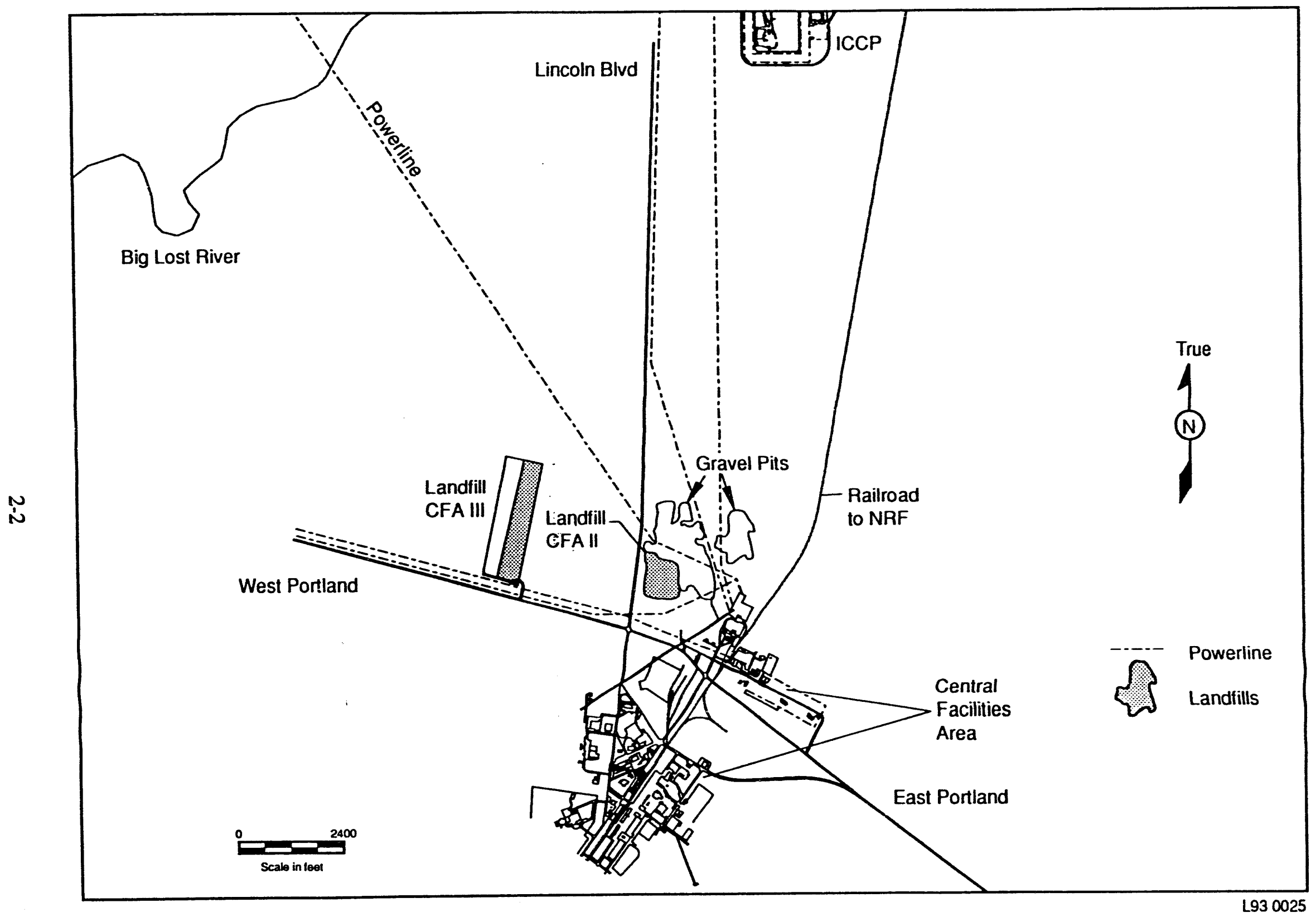

Figure 2-1. Map of Central Facilities Area Landfills II and III. 


\section{REGULATORY OVERVIEW}

\subsection{Resource Conservation and Recovery Act, and Consent Order and Compliance Agreement}

Originally, the environmental investigation at CFA was conducted under the authority of the Resource Conservation and Recovery Act (RCRA), 42 USC 6901 et. seg. RCRA is a law that regulates the generation, transportation, treatment, storage, and disposal of hazardous wastes. Monitoring well systems were installed around each landfill, and closure plans and cap designs were prepared in order to close and monitor the landfills. These actions were implemented as outlined in a Consent Order and Compliance Agreement (COCA) between DOE-ID, EPA Region 10, and the United States Geological Survey.

\subsection{National Priorities Listing and Federal Facility Agreement}

On November 15, 1989, the INEL was added to the EPA's National Priorities List (NPL) under CERCLA, 42 USC 9601 et. seg., also known as "Superfund," based on the detection of contaminants in the environment at INEL sites. The NPL identifies sites of high priority for investigation and remediation of hazardous materials.

Since INEL is a federal facility, CERCLA requires DOE, as the managing agency, to enter into an agreement with EPA to coordinate the remedial effort. In order to avoid potential conflict between CERCLA and RCRA and overlapping jurisdiction by different agencies, a Federal Facility Agreement/Consent Order (FFA/CO) between DOE-ID, EPA Region 10, and the State of Idaho was negotiated and became effective December 9, 1991.

This FFA/CO superseded COCA and outlined the remedial action process that encompasses all investigations of hazardous substances and remedial activities at INEL sites. The FFA/CO will integrate CERCLA response obligations with RCRA and State of Idaho Hazardous Waste Management Act corrective action obligations. All investigations and remedial activities being conducted under the FFA/CO will be conducted in accordance with the revised National Contingency Plan (NCP), which implements CERCLA, as amended by the Superfund Amendments and Reauthorization Act (SARA).

\subsubsection{Response Actions}

CERCLA Section 104 provides broad authority for a federal program to respond to releases of hazardous substances, pollutants, or contaminants. There are two major types of response actions: removal action and remedial action. A removal action is an immediate action that is taken to protect public heaith and welfare and the environment from a release or threatened release of a hazardous substance. A remedial action is a more permanent remedy taken to prevent or minimize the release or threat of release of hazardous substances that present current or future danger to public health or welfare, or to the environment. The objective of the CFA RI/FS is to (a) evaluate the potential risk to human health and to the environment from the CFA landfills, and (b) evaluate remedial action alternatives, if unacceptable risks are identified. 


\subsubsection{Waste Area Groups}

INEL is a large installation with a number of operating facilities, each containing a number of potentially hazardous locations. For management purposes, INEL has been divided into ten smaller parts called "waste area groups" (WAGs). WAGs 1 through 9 correspond to operating facilities at INEL, while WAG 10 corresponds to site-wide concerns, including the Snake River Plain Aquifer. WAG 10 also add resses miscellaneous surface and subsurface areas not included in the other nine WAGs. WAG 4 includes all the facilities at CFA. The map shown in Figure 3-1 illustrates where CFA is located within the boundaries of INEL.

\subsubsection{Operable Units}

WAG 4 has been broken down into 13 working "operable units" (of which Operable Unit 4-12 is CFA Landfills II and III) to focus investigation and remedial efforts. All potentially hazardous areas identified under COCA were assigned to an operable unit. Each operable unit may contain one or more potentially hazardous areas. The operable units under WAG 4 are described in the FFA/CO.

\subsection{National Environmental Policy Act (NEPA)}

As stated previously, all CFA investigations and remedial activities will be conducted in accordance with the DOE-ID policy of integrating NEPA requirements with CERCLA regulations. Such a practice is permitted and advocated by 40 CFR 1506.4, which states, "Any environmental document in compliance with NEPA may be combined with any other agency document to reduce documentation and paperwork."

Under NEPA, a more formal approach is required to inform the public earlier in the process. Where appropriate, DOE-ID will coordinate public involvement activities prescribed by NEPA, with the public participation requirements of CERCLA. In order to inform the public on how compliance with NEPA and CERCLA requirements will be achieved, this Community Relations Plan Supplement was prepared. 


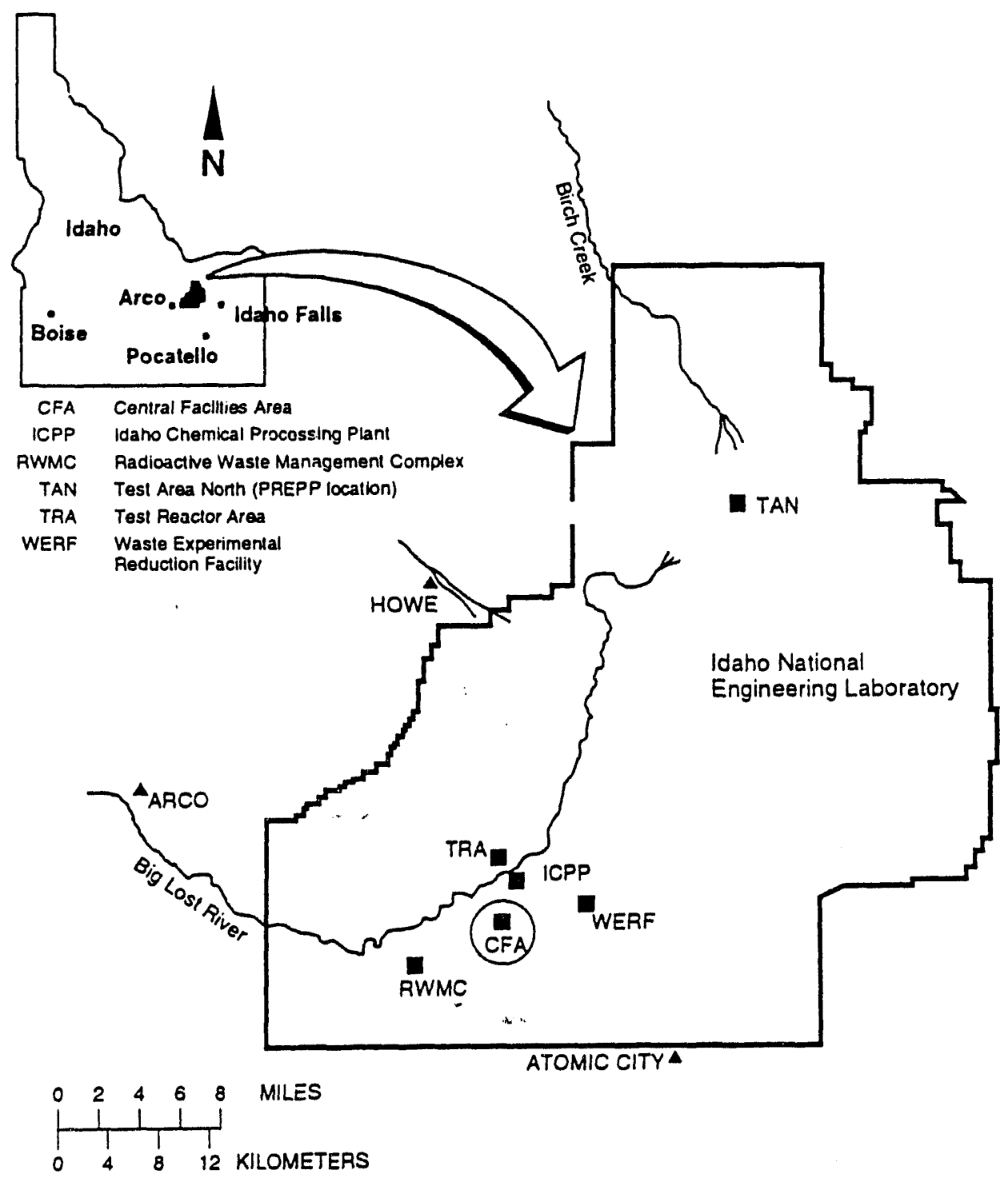

R92 1390

Figure 3-1. Location of CFA within INEL boundaries. 


\section{COMMUNITY BACKGROUND}

Detailed information on community background can be found in the September 1991 Community Relations Plan for Remedial Investigations at the INEL, Chapter 4, page 21. 


\section{COMMUNITY RELATIONS ACTIVITIES}

\subsection{CERCLA Requirements For Community Relations}

This Community Relations Plan Supplement, required under CERCLA, was developed pursuant to the INEL-wide CRP prepared by DOE-ID. This Plan describes public involvement requirements and public participation activities that will occur during the implementation of the CFA Landfills II and III RI/FS. Table 5-1 provides a list of the key community relations milestones. needs:

Community relations activities outlined in this plan are intended to meet the following

- Understanding the complexity of environmental restoration issues

- Identifying problems and issues that should be addressed

- Identifying alternative solutions to those problems and issues

- Addressing conflicts and misconceptions

- Pursuing actions and decisions in the best overall interest of the public and environment.

Interested citizens will be encouraged to look over Table 5-1 to find opportunities for involvement such as reading reports, brochures, and fact sheets; attending an informal briefing; visiting the nearest Information Repository; or sending in written comments or questions.

The following paragraphs add detail to some of the public involvement activities highlighted in Table 5-1.

1. Information repositories. Information repositories have been established in the public libraries of each of the following Idaho cities: Idaho Falls, Pocatello, Twin Falls, Boise, and Moscow. Appendix B lists the address of each repository and the days and hours they are open. Additional information repositories may be established if sufficient community interest is expressed.

The following types of documents for the CFA Landfills II and III RI/FS (and other INEL activities) will be put into the information repositories as the documents are completed:

- Index to the Administrative Record

- CFA Landfills II and III RI/FS Work Plan

- Remedial Investigation Report with Baseline Risk Assessment

- RI/FS Report

- Record of Decision

- Proposed Plan 
Table 5-1. Community relations activities for the CFA Landfills II and III RI/FS.

Activity

Schedule

Place Scope of Work in Administrative Record

January 1992

Publish and mail fact sheet on RI/FS Work Plan

June-July 1993

Public briefing on RI/FS Work Plan

May 1993

Prepare press release on RI/FS Report and publish Notice

January 1995

of Availability on the RI/FS report and the Proposed Plan

Public comment period on RI/FS and Proposed Plan

April-May 1995

Publish Notice of Availability on Record of Decision

November 1995

- Brochures

- Press releases

- Fact sheets

- Newsletters.

The following types of documents have been completed for the CFA Landfills II and III RI/FS (and other INEL activities) and are contained in the information repositories:

- $\quad$ Consent Order and Compliance Agreement

- Federal Facility Agreement/Consent Order and Action Plan

- DOE-ID Community Relations Plans

- DOE Environmental Restoration and Waste Management Five-Year Plan

- INEL Environmental Restoration and Waste Management Site-Specific Plan

- CFA Landfills II and III RI/FS Scope of Work.

2. Administrative Record. An Administrative Record containing all information used in the decision-making process for remedial activities will be available for review and copying at the Woodruff Avenue Complex, 200 South Woodruff Avenue, Idaho Falls. For convenient public access in the future, a branch of the Administrative Record will be established at the INEL Technical Library at 1776 Science Center Drive in Idaho Falls. Written and oral comments received during public comment periods will become a part of the Administrative Record. An index to the Administrative Record will be placed in information repositories and updated regularly.

3. Public Comment Periods. A Public comment period will be held for the Proposed Plan prior to the Record of Decision (See Table 5-1). The Plan will describe remedial alternatives and the preferred alternative for the CFA project. When the Plan has been completed, a public notice will be issued two weeks prior to the beginning of the comment period. At a minimum, this notice will consist of display advertisements in local newspapers describing procedures for submitting comments. Following that, a 30-day comment period will be provided. Comments can be provided in writing or given verbally. The comment 
period may be extended an additional 30 days if a request is made in writing 10 days prior to the close of the comment period.

Public meetings will be held during the comment period regarding final selection of a remedial alternative. The number and location of these meetings for comments will be determined before the scheduled start date of the public comment period. Public notification will be provided through news releases and direct mailing of the meeting schedule. Verbal comments received during those meetings and written comments received during the comment period will be given equal consideration by DOE-ID, EPA, and IDHW in selecting a remedial alternative. Transcripts will be prepared from the meetings and made a part of the Administrative Record and information repositories.

4. Responsiveness Summary. Following a public comment period, comments will be compiled, and a response to each comment will be documented in a Responsiveness Summary, which will be part of the ROD. Comments received during the public comment period will be considered in the remedial action decision for the CFA Landfills II and III RI/FS.

5. Record of Decision. Following the Responsiveness Summary and explanation of significant changes to the plan (if any), a ROD specifying the selected remedial alternative will be prepared by DOE. The ROD, which includes the responsiveness summary, is issued following EPA approval and concurrence by IDHW. If the selected remedy is different from alternatives listed in the RI/FS report and the Proposed Plan, the differences will be explained in the ROD.

An additional public comment period will be provided if the selected remedial alternative is significantly different from alternatives in the RI/FS Report and Proposed Plan.

\subsection{Requests For Additional Activities}

This part of the supplemental Community Relations Plan describes the extra public involvement activities in Table 5-1 in greater detail.

1. Briefings, Presentations, or Discussions. Semiannual briefings, presentations, or discussions will be conducted with interested individuals, groups, organizations, and agencies. More frequent briefings or presentations will be provided as remedial efforts at CFA intensify. The DOE-ID Environmental Restoration Division and INEL Public Affairs offices will invite and seek group discussions, briefings, meetings, and presentations regarding remediation issues.

2. Tours. Interested individuals and groups will be provided tours of facilities at CFA. For example, some tours may be conducted to visit areas associated with environmental restoration; waste technology demonstration; and waste treatment, storage, and disposal.

3. Public Involvement Meetings. Semiannually, or as requested, public involvement meetings will be held in interested communities. Where possible, meetings will be held to inform the public about the RI/FS process or to give an update on the status of the RI/FS. 
4. Newsletter. The INEL Environmental Restoration Program will publish the INEL Reporter on a quarterly basis. Its purpose is to inform the public about environmental investigation and remedial activities. The newsletter will be distributed via the mailing list described below.

5. Brochures. Nontechnical brochures will be prepared and distributed via the mailing list and through other means to give the public a better understanding of the Snake River Plain Aquifer, facts about radiation, waste management, environmental monitoring, waste units being remediated, and technology used to complete the work.

6. Fact Sheets. Fact sheets will be prepared and distributed via the mailing list to provide summaries of environmental investigations and related technical reports or background information helpful in understanding technical documents.

7. Press Releases. Press releases will be prepared and distributed to the news media announcing public meetings and public comment periods, and to publicize the latest developments of the RI/FS.

8. Mailing List. A mailing list will be developed to distribute information to the public and the news media. That list will be developed by adding the names of those interviewed, others expressing an interest in INEL, public officials, and names gathered during the development of other Community Relations Plan activities. Persons interested in being added to the mailing list can contact DOE-ID/INEL personnel listed in Appendix A.

9. News Media. DOE-ID will keep the news media informed of remedial activities by providing brochures, press releases, newsletters, fact sheets, and final reports. In addition, officials will meet with news media representatives to provide them with accurate information. Reporters will be invited and encouraged to visit CFA.

10. Displays and Exhibits. The INEL Environmental Restoration Program will utilize public gatherings, meetings, open houses, and other opportunities to set up displays and exhibits covering topics requested by members of the general public. Officials from INEL will be on hand to answer questions and listen to public concerns.

11. Employee Communications. The community relations activities described in this plan are available to employees at INEL as well as the general public. In addition, employees will be kept informed via the INEL Reporter, various company newsletters, computer mail systems, and presentations by management.

These activities will be conducted routinely throughout the course of CFA Landfills II and III RI/FS activities. Additional activities may be added to the list if requested by the community. All comments received from the public concerning ways to improve communication and public participation will be considered when updating this plan. 


\section{Appendix A}

\section{List of Contacts}



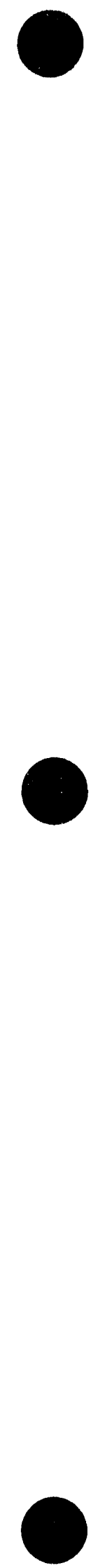

A-2 


\section{Appendix A}

\section{List of Contacts}

DOE-ID Environmental Restoration Program

Jerry L. Lyle

(208) $526-1148$

Acting Deputy Assistant Manager

Environmental Restoration and Waste Management

DOE Idaho Field Office

P.O. Box 2047

Idaho Falls, ID 83403-2047

Alice C. Williams

(208) 526-0972

Director

Environmental Restoration Division

DOE Idaho Field Office

P.O. Box 2047

Idaho Falls, ID 83403-2047

INEL Community Relations Plan

Reuel Smith

(208) 526-6864

CRP Coordinator

INEL Environmental Restoration Program

P.O. Box 2047

Idaho Falls, ID 83403-2047

\section{INEL Public Affairs}

Kathy Whittaker

(208) $526-9586$

Chief of Public Affairs and Tribal Liaison

INEL Public Affairs

P.O. Box 2047

Idaho Falls, ID 83403-2047

Nick Nichols

(208) $526-1693$

Media Contact

INEL Public Affairs

P.O. Box 2407

Idaho Falls, ID 83403-2047 
U.S. Environmental Protection Agency

Wayne Pierre

(206) 553-7261

Federal Facilities Superfund Branch

EPA Region 10, HW-124

1200 Sixth Avenue

Seattle, WA 98101

Bub Loiselle

(206) $553-1283$

Community Relations Coordinator

EPA Region 10, HW-117

Park Place Building

1200 Sixth Avenue

Seattle, WA 98101

State of Idaho Division of Environmental Quality

Terry Smith

(208) 334-5761

Public Information Officer

INEL Oversight Program

Idaho Department of Health and Welfare

1410 N. Hilton

Boise, ID 83706

Shawn Rosenberger, Remediation Technical Supervisor

(208) $525-7300$

Idaho Department of Health and Welfare

Division of Environmental Quality

900 North Skyline

Idaho Falls, Idaho 83402

a. Toll-free calls can be made by calling 1-800-232-INEL. 


\section{Appendix B}

INEL Information Repositories

B-1 

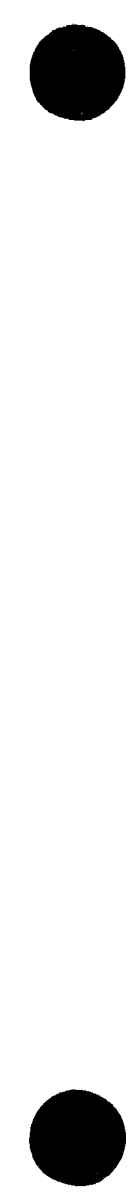

B-2 


\section{Appendix B}

\section{INEL Information Repositories}

Location, Days, and Hours Open:

INEL Technical Library, 1776 Science Center Drive, Idaho Falls, ID 83415; (208) 526-1185

Hours:8:00 a.m.-7:00 p.m. Monday through Thursday 8:00 a.m.-5:00 p.m. Friday

9:00 a.m.-1:00 p.m. Saturday

Idaho Falls Public Library, 457 Broadway,

Idaho Falls, ID 83402; (208) 529-1450

Hours: $\quad$ 9:00 a.m. - 9:00 p.m. Monday through Thursday

9:00 a.m. - 5:30 p.m. Friday, Saturday

Twin Falls Public Library, 434 2nd Street East,

Twin Falls, ID 83301; (208) 733-2964

Hours: $\quad$ 10:00 a.m. - 6:00 p.m. Monday, Friday

10:00 a.m. - 9:00 p.m. Tuesday, Wednesday, Thursday

12:00 p.m. - 5:00 p.m. Saturday

Pocatello Public Library, 812 East Clark,

Pocatello, ID 83201; (208) 232-1263

Hours: $\quad$ 10:00 a.m. - 9:00 p.m. Monday through Thursday 10:00 a.m. - 6:00 p.m. Friday, Saturday

Boise Public Library, 715 South Capitol Blvd.,

Boise, ID 83706; (208) 384-4076

Hours: $\quad$ 10:00 a.m. - 6:00 p.m. Monday, Friday 10:00 a.m. - 9:00 p.m. Tuesday, Wednesday, Thursday 1:00 p.m. - 5:00 p.m. Saturday, Sunday 
University of Idaho Library, Government Documents

University of Idaho Campus

Rayburn Street

Moscow, ID 83843; (208) 885-6344

Hours: $\quad$ 8:00 a.m. - 5:00 p.m. Monday through Friday 


\section{Appendix C}

\section{Glossary}

C-1 


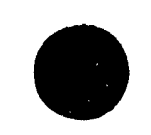

-

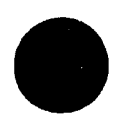

C-2 


\section{Appendix C}

\section{Glossary}

Argonne National Laboratory-West (ANL-W) - INEL facility for testing breeder reactor technology. ANL-W houses Experimental Breeder Reactor II, the first pool-type liquid-metal reactor. The facility has four other reactors and two fuel examination facilities.

Central Facilities Area (CFA) - INEL facility serving as headquarters for environmental laboratories, security, fire protection, medical, communications systems, warehouses, cafeteria, vehicle and equipment pools, bus system, and laundry.

Comprehensive Environmental Response, Compensation, and Liability Act (CERCLA) - Also known as Superfund, the Federal statute enacted in 1980 and reauthorized in 1986 that provides the statutory authority for cleanup of hazardous substances that could endanger public health or welfare or the environment.

Community Relations Plan (CRP) - A report that assesses and defines a community's informational needs concerning potential hazards posed by conditions at hazardous waste sites. The CRP also encourages and ensures two-way communications between an affected community and the public agency overseeing cleanup.

Feasibility Study (FS) - The step in the CERCLA process in which alternatives for a remedial action system are investigated and screened.

National Priorities List (NPL) - The EPA's list of the top priority hazardous waste sites eligible for investigation and cleanup under the federal Superfund program.

Proposed Plan - A summary of the Agency's preferred cleanup strategy, the rationale for the preference, a review of the alternatives presented in the detailed analysis of the RI/FS, and a presentation of any waivers to cleanup standards, if any are proposed.

Radioactive Waste Management Complex (RWMC) - INEL facility established in 1952 as a controlled area for disposal of solid radioactive wastes generated in INEL operations. Since 1954, the facility has received defense waste for storage.

Record of Decision (ROD) - The CERCLA report documenting the selection of remedial action to be implemented at a site after the RI/FS and Proposed Plan have been completed. The ROD is placed in the Administrative Record for the lead agency. The lead agency must 1. publish a notice of the availability of the ROD in a major local newspaper of general circulation; and 2. make the ROD available for public inspection and copying at or near the facility at issue prior to the commencement of any remedial action. 
Remedial Investigation (RI) - The CERCLA process that determines the extent of hazardous substance contamination and includes, as appropriate, treatability investigations. The RI is done in conjunction with the feasibility study.

Removal Action - An immediate action taken over the short-term to address a release or threatened release of hazardous substances.

Resources Conservation and Recovery Act (RCRA) - A Federal law enacted in 1976 (and amended in 1980 and 1984) that regulates the generation, transportation, treatment, storage, and disposal of hazardous wastes. In this Federal Facilities Agreement, RCRA is defined as being "functionally equivalent" to CERCLA.

Responsiveness Summary - A summary of oral and written public comments received during a comment period on key documents and DOE-ID responses to those comments. The Responsiveness Summary is especially valuable during the decision process at a site because it highlights community concerns about the proposed decisions.

Superfund - The common name used for the Comprehensive Environmental Response, Compensation, and Liability Act (CERCLA), also referred to as the Trust Fund. 

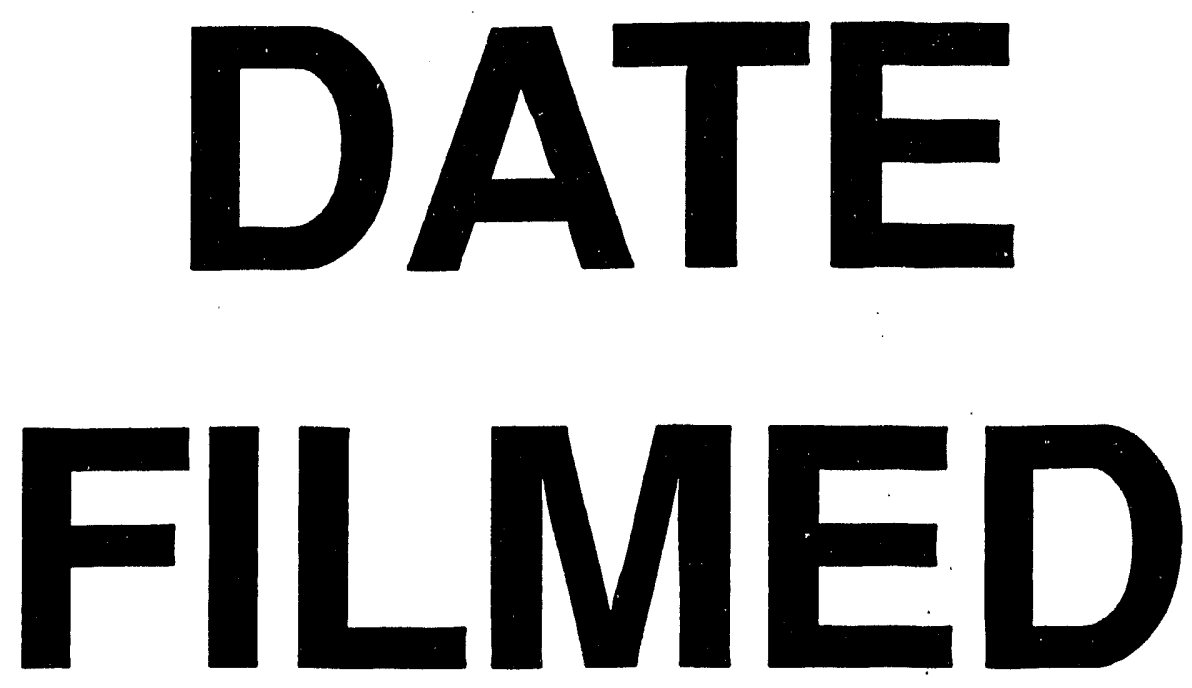

$12 / 28 / 93$
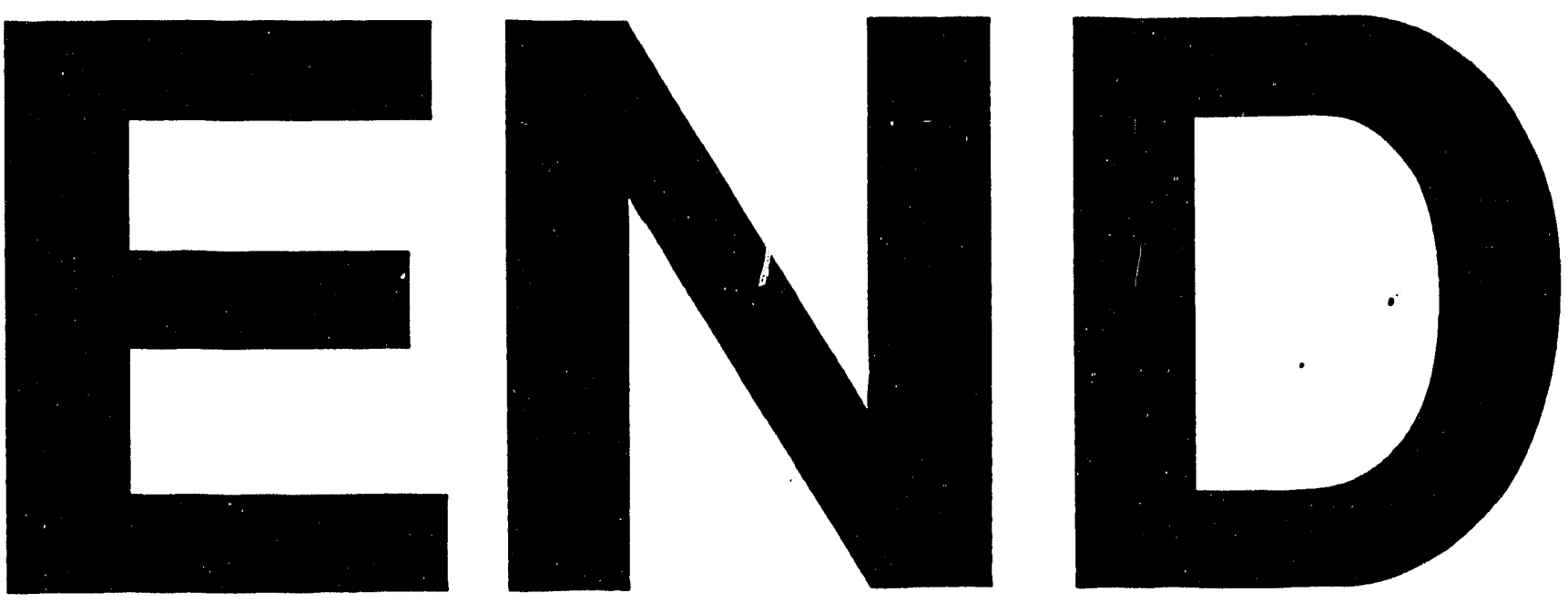

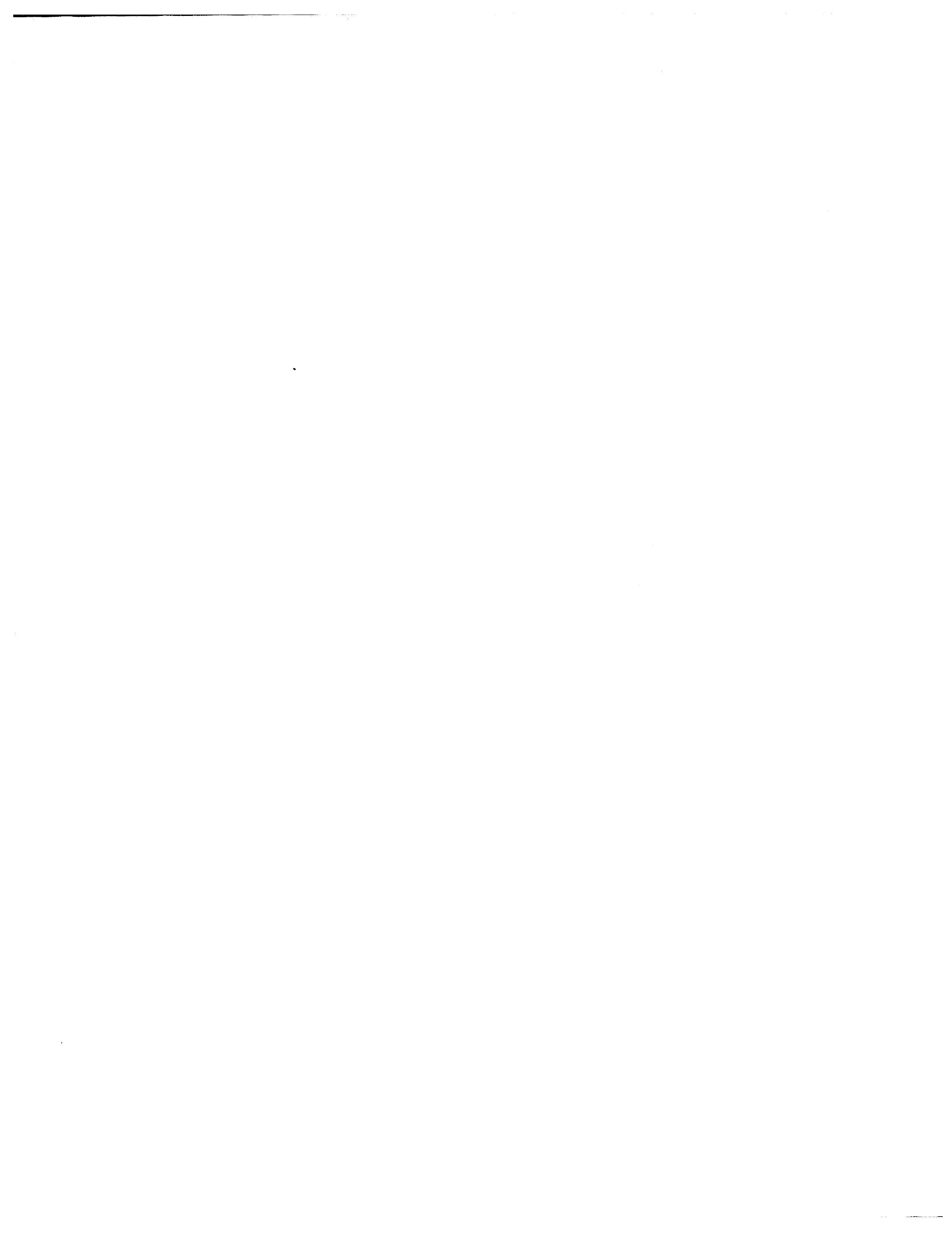